\title{
Abstracts of the 14th International Congress of the European Geriatric Medicine Society
}

\author{
10-12 October 2018, Berlin, Germany
}

\section{Contents}

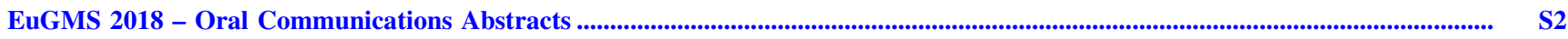

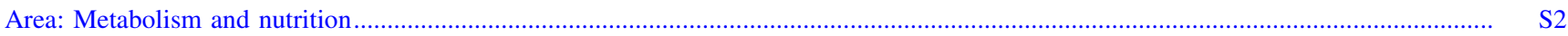

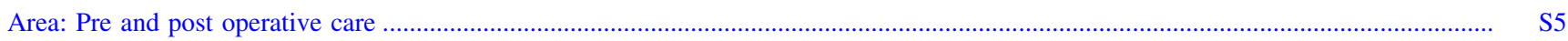

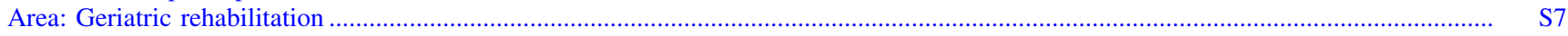

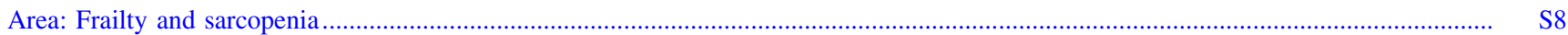

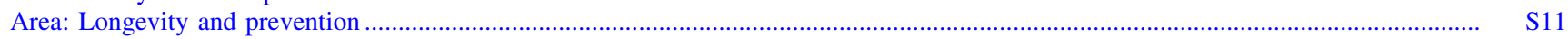

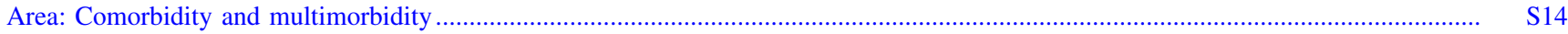

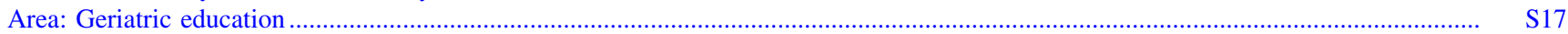

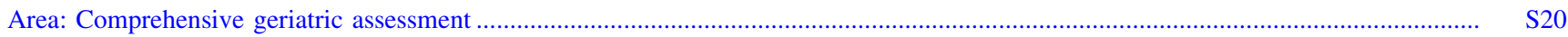

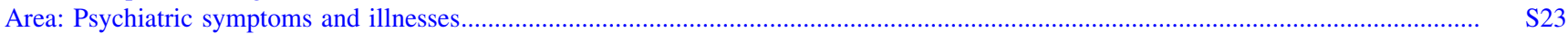

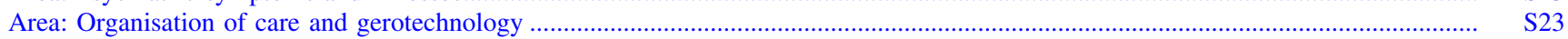

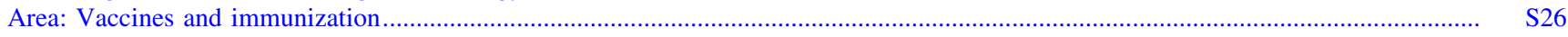

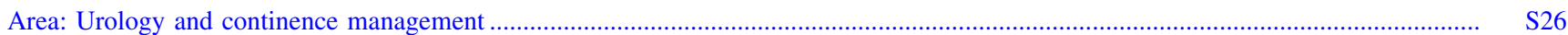

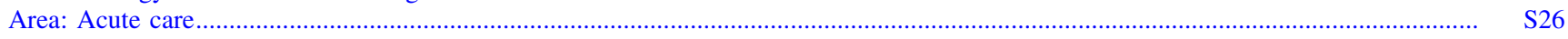

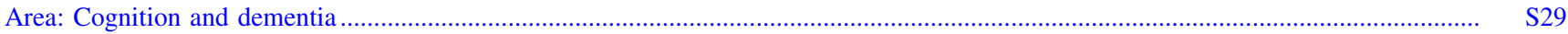

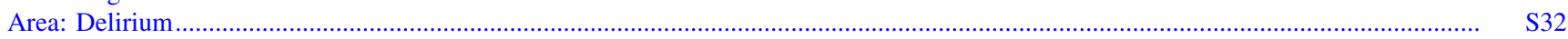

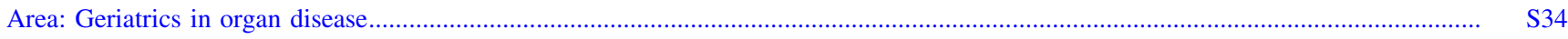

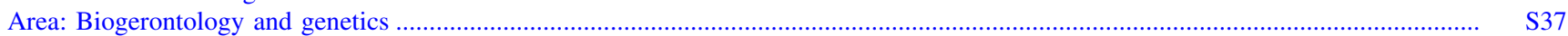

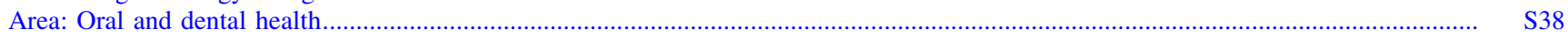

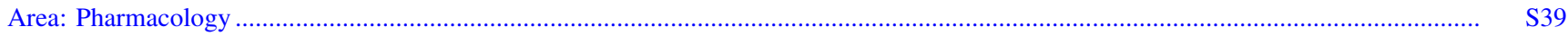

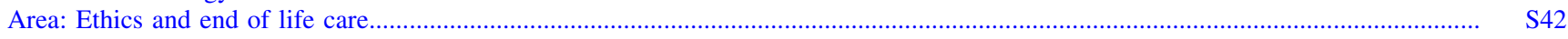

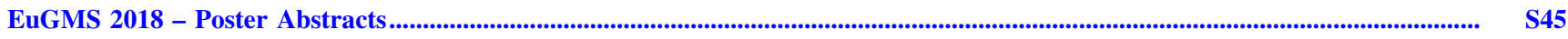

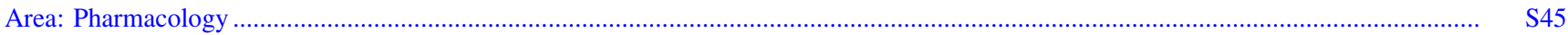

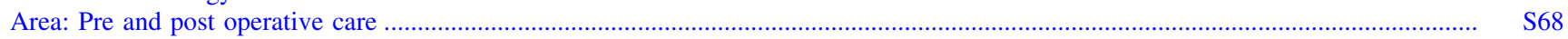

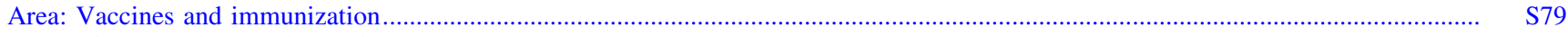

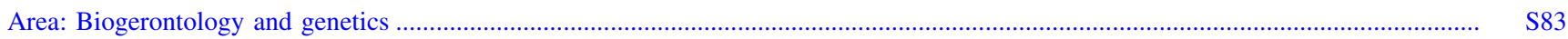

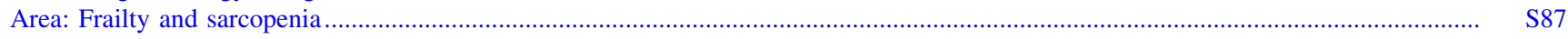

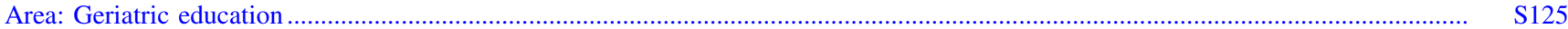

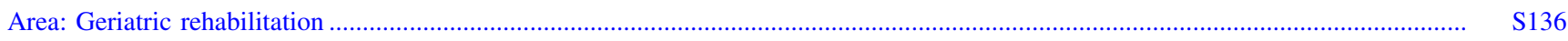

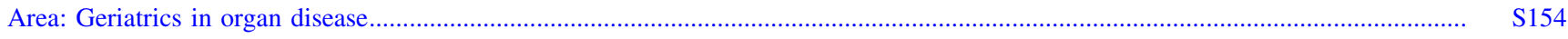

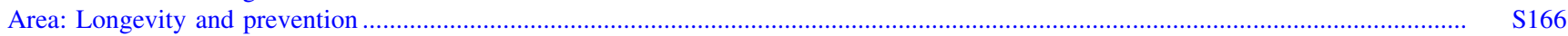

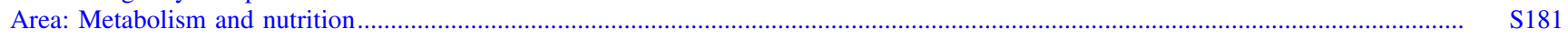

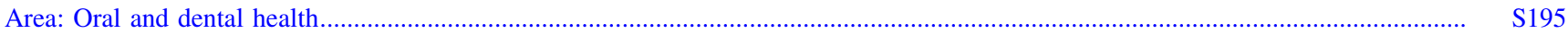

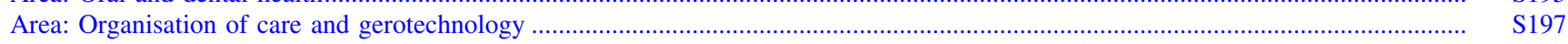

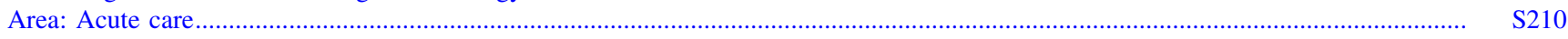

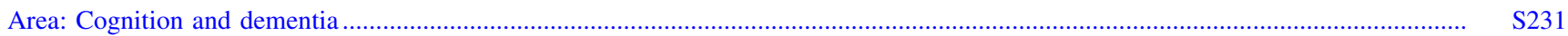

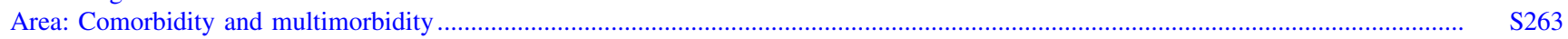

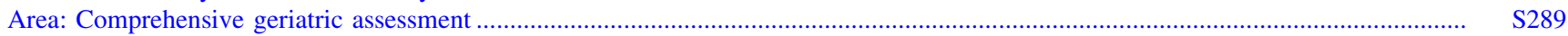

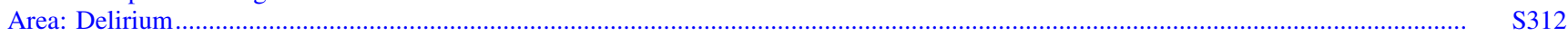

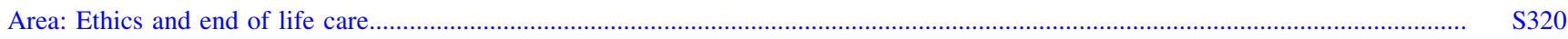

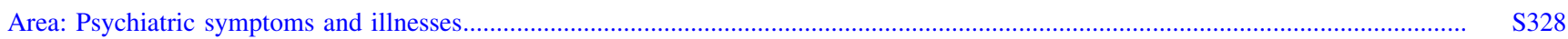

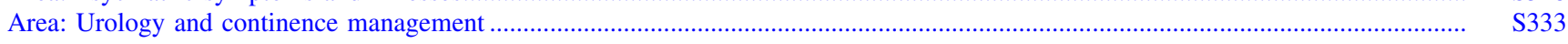

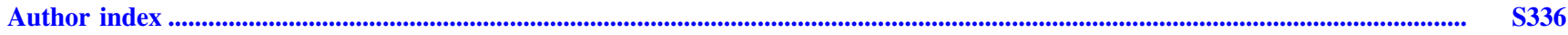




\section{EuGMS 2018 - Oral Communications Abstracts}

\section{Area: Metabolism and nutrition}

\section{0-1}

Prevalence of malnutrition using harmonized definitions in older adults from different settings in Europe and New Zealand: a MaNuEL study

Dorothee Volkert ${ }^{1}$, Melanie Streicher ${ }^{1}$, Eva Kiesswetter ${ }^{1}$, Gabriel Torbahn $^{1}$, Eibhlis M. O'Connor ${ }^{2}$, Mary O'Keeffe ${ }^{2}$, Mary Kelly ${ }^{2}$, Eileen O'Herlihy ${ }^{3}$, Paul W. O'Toole ${ }^{3}$, Suzanne Timmons ${ }^{4}$, Emma $\mathrm{O}^{\prime} \mathrm{Shea}^{4}$, Patricia Kearney ${ }^{5}$, Judith van Zwienen-Pot ${ }^{6}$, Marjolein Visser $^{6}$, Isabelle Maître ${ }^{7}$, Virginie Van Wymelbeke ${ }^{8}$, Claire SulmontRossé $^{9}$, Gabriele Nagel ${ }^{10}$, Marion Flechtner-Mors ${ }^{11}$, Sabine Goisser ${ }^{1}$, Ruth Teh $^{12}$, Antje Hebestreit ${ }^{13}$

${ }^{1}$ Institute for Biomedicine of Aging, Friedrich-Alexander-Universität Erlangen-Nürnberg, Nuremberg, Germany, ${ }^{2}$ Dept Biological Sciences, and Health Research Institute, University of Limerick, Limerick, Ireland, ${ }^{3}$ School of Microbiology and Alimentary Pharmabiotic Centre, University College Cork, Ireland, ${ }^{4}$ Centre for Gerontology and Rehabilitation, School of Medicine, University College Cork, Cork, Ireland, ${ }^{5}$ Dept Epidemiology and Public Health, University College Cork, Cork, Ireland, ${ }^{6}$ Dept of Health Sciences, Faculty Sciences, Vrije Universiteit Amsterdam, Amsterdam Public Health research institute, The Netherlands, ${ }^{7}$ USC 1422 GRAPPE, Ecole Supérieure d'Agricultures (ESA), SFR 4207 QUASAV, INRA, Angers, France, ${ }^{8}$ Centre Hospitalier Universitaire Dijon Bourgogne, Unité de Recherche Pôle Personnes Âgées, Dijon, France, ${ }^{9}$ Centre des Sciences du Goût et de l'Alimentation, AgroSup Dijon, CNRS, INRA, Université Bourgogne Franche-Comté, Dijon, France,

${ }^{10}$ Institute of Epidemiology and Medical Biometry, Ulm University, Ulm, Germany, ${ }^{11}$ Medical Center, Division of Sports and Rehabilitation Medicine, University of Ulm, Ulm, Germany, ${ }^{12}$ University of Auckland, School of Population Health, General Practice and Primary Health Care, Auckland, New Zealand, ${ }^{13}$ Leibniz Institute for Prevention Research and Epidemiology_BIPS, Bremen, Germany

Introduction: Prevalences of malnutrition in older people vary widely because of different diagnostic criteria. This study compares prevalences using harmonized definitions.

Methods: Prevalences of low BMI $\left(<20 \mathrm{~kg} / \mathrm{m}^{2}\right.$; age-specific $<20$ if $<70$ years and $<22$ if $\geq 70$ years), weight loss (WL), severe decrease in food intake (SDFI) and of combined BMI $<20$ and/or WL in participants aged $\geq 65$ years were compared.

Results: Fifteen samples with 5956 participants were included: 7 consisting of community-dwelling persons, 2 in geriatric day hospitals, 3 in hospitalized patients and 3 in nursing homes. Mean age of participants was between 67 and 86 years. Prevalences increase from communitydwellers to nursing homes residents and varied between and within the settings. Compared to BMI $<20 \mathrm{~kg} / \mathrm{m}^{2}$, using age-specific cut-offs doubled the prevalence. The criteria age-specific BMI and WL showed opposing prevalences. WL and SDFI appear to be important criteria to detect malnutrition in men but they seem to be no sensitive markers in higher age groups. Prevalence of BMI $<20 \mathrm{~kg} / \mathrm{m}^{2}$ and WL did not exceed $2.6 \%$. The highest prevalences were observed based on combined definitions when only one of the criteria had to be present.

Conclusion: Prevalences for different criteria vary which may be explained by functional status. Prevalences double when using an age-specific BMI versus a BMI $<20 \mathrm{~kg} / \mathrm{m}^{2}$. The criteria age-specific BMI and WL showed opposing prevalences. WL and SDFI appear to be important criteria to detect malnutrition in men but do not seem to be sensitive in higher age groups.

\section{0-2}

MNA $^{\circledR}$ Nutritional screening identifies COPD patients with higher rate of complications

Kala Kaspar ${ }^{1}$, Behnaz Shakersain ${ }^{1}$

${ }^{1}$ Nestlé Health Science, Lausanne, Switzerland

Introduction: This review aimed to describe the evidence on clinical screening and prognostic relevance of nutritional status evaluated by Mini Nutritional Assessment $\left(\mathrm{MNA}^{\circledR}\right)$ in chronic obstructive pulmonary disease (COPD) patients.

Methods: A comprehensive literature search in major electronic databases, including MEDLINE, EMBASE, Web of Science, and Cochrane Database of Systematic Reviews, irrespective of date and language was performed by two independent reviewers. Published prospective and cross-sectional studies of patients diagnosed according to the Global Initiative for Chronic Obstructive Lung Disease (GOLD) criteria, that evaluated nutritional status by the full or short MNA ${ }^{\circledR}$ tool were included.

Results: All of the 27 relevant search hits were included ( 22 focused on COPD patients, 1 on pulmonology ward patients, 1 on assisted living facility residents, and 3 on community dwelling older adults). A study of COPD clinic patients (mean age 71 years, $75 \%$ men) found $69 \%$ were malnourished or at-risk, and higher rates were observed in more severe COPD stages. Poor MNA ${ }^{\circledR}$ score could identify COPD patients with: (1) more severe COPD symptoms (9/9 studies), (2) compromised body composition (15/17 studies), (3) poor physical performance (10/11 studies), and (4) higher rate of complications (5/5 studies). Those with poor MNA ${ }^{\circledR}$ score had $31 \%$ higher risk of COPD exacerbations, 3-fold higher risk of hospital readmissions in 6 months [HR: 2.9]; and 3-times higher risk of mortality within 3 years [OR: 3.33 ].

Conclusions: Timely nutritional screening and intervention is recommended for optimal COPD management. $\mathrm{MNA}^{\circledR}$ is a useful screening tool with prognostic value in COPD patient care.

\section{0-3}

The role of health considerations in food choice of communitydwelling older adults with functional limitations

Hanna Maria Rempe ${ }^{1}$, Eva Kiesswetter ${ }^{1}$, Dorothee Volkert ${ }^{1}$, Gudrun Sproesser $^{2}$, Cornel Christian Sieber ${ }^{1}$, Ellen Freiberger ${ }^{1}$

${ }^{1}$ Institute for Biomedicine of Aging, Friedrich-Alexander-University of Erlangen-Nürnberg, Germany, ${ }^{2}$ Department of Psychology, University of Konstanz, Germany

Background: Interventions focusing on an adequate nutrition usually imply a health-oriented eating behavior. However, little is known about the role of health considerations in food choice of functionally impaired older persons and furthermore about other motives underlying their eating behavior. Hence, aims of this study were a) to identify the main motives influencing food choice and b) to investigate factors related to a weak health-oriented eating motivation (WHEM).

Methods: This sub-study of the European trial "SPRINT-T" included 166 independently-living adults aged $\geq 70$ years with functional limitations ("Short Physical Performance Battery" 3-9) in the area of Nuremberg (Germany). Using "The Eating Motivation Survey" (TEMS), 15 basic motives were recorded on a 7-point Likert scale ranging from 1 "never" to 7 "always applicable to eating behavior". In addition, gender ( $70.5 \%$ female), age $(80.4 \pm 5.2$ years), body 
mass index (29.5 \pm 5.5$)$, and frailty-status (Fried-phenotype) were assessed.

Results: "Appetite" $(5.4 \pm 1.4)$ was the most important eating motive, followed by "health" $(4.7 \pm 1.6)$ and "natural concerns" $(4.6 \pm 1.8)$. The probability (OR) of WHEM was 4.63 times higher for men than for women (95\% CI 2.15-9.97). Adjusted for gender, frail participants $(n=50)$ compared to pre-frail participants $(n=99)$ were less likely to have WHEM $(\mathrm{OR}=0.20 ; 95 \%$ CI $0.10-0.43)$. No further factors were associated with WHEM.

Conclusions: The importance of health as motive for food choice indicates that older people with functional limitations appear to be generally susceptible to health-related nutritional interventions. Particularly in male and frail older adults personalized strategies and barriers towards a healthy diet should be considered.

\section{$0-4$}

Development and validation of a short food questionnaire to screen for low protein intake in community-dwelling older adults: the Protein Screener 55+ (Pro55+)

Hanneke A.H. Wijnhoven ${ }^{1}$, Liset E. Elstgeest ${ }^{1}$, Henrica C.W. de Vet $^{2}$, Mary Nicolaou ${ }^{3}$, Marieke B. Snijder ${ }^{3}$, Marjolein Visser ${ }^{1}$

${ }^{1}$ Department of Health Sciences, Faculty of Science, Vrije Universiteit Amsterdam, Amsterdam Public Health research institute, Amsterdam, The Netherlands, ${ }^{2}$ Department of Epidemiology and Biostatistics, VU University Medical Center, Amsterdam Public Health research institute, Amsterdam, The Netherlands, ${ }^{3}$ Department of Public Health, Academic Medical Center, University of Amsterdam, Amsterdam Public Health research institute, Amsterdam, The Netherlands

This study is conducted within PROMISS. In old age, sufficient protein intake is important to preserve muscle mass and function. Around $50 \%$ of older adults $(65+$ years) consumes $\leq 1.0 \mathrm{~g} / \mathrm{kg}$ adjusted body weight $(\mathrm{BW}) /$ day (d). There is no rapid method available to screen for low protein intake in old age. Therefore, we aimed to develop and validate a short food questionnaire to screen for low protein intake in community-dwelling older adults. We used data of 1348 older men and women (56-101 years) of the LASA study (The Netherlands) to develop the questionnaire and data of 563 older men and women (55-71 years) of the HELIUS study (The Netherlands) for external validation. In both samples, protein intake was measured by the 238-item semi-quantitative HELIUS food frequency questionnaire (FFQ). Multivariable logistic regression analysis was used to predict protein intake $\leq 1.0 \mathrm{~g} / \mathrm{kg}$ adjusted $\mathrm{BW} / \mathrm{d}$ (based on the HELIUS FFQ). Candidate predictor variables were FFQ questions on frequency and amount of intake of specific foods. In both samples, $30 \%$ had a protein intake $\leq 1.0 \mathrm{~g} / \mathrm{kg}$ adjusted BW/days. Our final model included adjusted body weight and 10 questions on the consumption (amount on average day or frequency in 4 weeks) of: slices of bread (number); glasses of milk (number); meat with warm meal (portion size); cheese (amount and frequency); dairy products (like yoghurt) (frequency); $\operatorname{egg}(\mathrm{s})$ (frequency); pasta/noodles (frequency); fish (frequency); and nuts/peanuts (frequency). The area under the receiver operating characteristic curve (AUC) was 0.889 (95\% CI 0.870-0.907). The calibration slope was 1.03 (optimal slope 1.00). At a cut-off of $\leq 0.8 \mathrm{~g} / \mathrm{kg}$ adjusted $\mathrm{BW} / \mathrm{d}$, the AUC was $0.916 \quad(96 \% \mathrm{CI}$ 0.897-0.936). Applying the regression equation to the HELIUS sample, the AUC was 0.856 (95\% CI $0.824-0.888$ ) and the calibration slope 0.92 . Regression coefficients were therefore subsequently shrunken by a linear factor 0.92 . To conclude, the short food questionnaire (Pro55+) can be used to validly screen for protein intake $\leq 1.0 \mathrm{~g} / \mathrm{kg}$ adjusted BW/d in community-dwelling older adults.
An online version can be found at http://www.proteinscreener.nl. External validation in other countries is recommended.

\section{$0-5$}

\section{Physical function after resistance training among old adults with metabolic syndrome}

Olof Gudny Geirsdottir ${ }^{1}$, Alfons Ramel ${ }^{2}$, Milan Chang ${ }^{3}$, Palmi V Jonsson $^{4}$, Inga Thorsdottir ${ }^{5}$

${ }^{1}$ The Icelandic Gerontological Research Center, Reykjavik, Iceland and Faculty of Food Science and Nutrition, University of Iceland, Reykjavik, Iceland, ${ }^{2}$ Faculty of Food Science and Nutrition, University of Iceland, Reykjavik, Iceland and The Icelandic Gerontological Research Center, Reykjavik, Iceland, ${ }^{3}$ School of Education, University of Iceland, Reykjavik, Iceland and The Icelandic Gerontological Research Center, Reykjavik, Iceland, ${ }^{4}$ Department of Geriatrics, National University Hospital of Iceland, Reykjavik, Iceland and The Icelandic Gerontological Research Center, Reykjavik, Iceland, ${ }^{5}$ Faculty of Food Science and Nutrition, University of Iceland, Reykjavik, Iceland

After attending this session, participants will know that older adults with metabolic syndrome improve significantly less in mobility function compared with those without after a resistance exercise programme. However, this obeseved difference is not explained by differences in physical activity and body mass index. Metabolic dysregulation affects physical performance among older adults. Metabolic syndrome (MetS) is an index of collected risk factors for cardiovascular disease/diabetes and the prevalence of MetS increases with aging. However, there is little information for the longitudinal association between MetS and physical function. We investigated the effects of a 12-week resistance exercise programme on physical function among community dwelling old adults with and without MetS. Total 236 subjects $(73.7 \pm 5.7$ years, $58.2 \%$ female) participated in the exercise intervention and 210 people completed the program. MetS was defined according to the NIH criteria (having $\geq$ three risk factors, i.e., increased waist circumference, triglycerides, HDL cholesterol, blood pressure, fasting glucose). Objectively measured physical function included 6-min-walk-fordistance (6MWD), grip strength, timed-up-and-og (TUG) and knee extension strength. The prevalence of MetS was 33\% $(\mathrm{n}=78)$. The improvement of 6MWD from baseline to endpoint was $39.0 \pm 33.2 \mathrm{~m}$ for people without MetS, and $22.1 \pm 35.5 \mathrm{~m}$ for people with MetS. The linear regression model was performed to examine the association between baseline MetS and improvement of each physical performance after the intervention. Compared with those without MetS, those with MetS improved significantly less in 6MWD ( $\beta=-14.6$, 95\% Confidence interval; -26.5 to -2.7 , $\mathrm{P}<0.017$ ) after adjusting for age, gender, physical activity and body mass index, and cognitive function, but no significant difference was found in other physical function. In conclusion, older adults with MetS improved significantly less in mobility function compared with those without MetS after the resistance exercise.

\section{0-6}

Happiness of the oldest old men is associated with fruit and vegetable intakes

Annele Urtamo ${ }^{1}$, Kaisu Pitkälä ${ }^{1}$, Timo Strandberg ${ }^{1}$

${ }^{1}$ University of Helsinki, Helsinki, Finland 
Background: Positive emotions and happiness may improve health and prolong life. Diet quality, Mediterranean dietary pattern, fruit and vegetable, chocolate as well as fish consumption have been linked to positive affect, improved mood and reduced risk of depression in various studies. We examined how diet quality, food intakes, and nutrition related issues are associated with perceived happiness in the oldest old men.

Methods: The participants of this cross-sectional analysis of a longitudinal study were the oldest old home-dwelling men $(n=338$, mean age 88 years, range $82-97$ years) from the Helsinki Businessmen Study cohort. A postal health and nutrition survey was sent to them in 2016. Happiness was evaluated using the Visual Analog Scale of Happiness $(0-100 \mathrm{~mm})$. The nutrition survey included a 3-day food diary, Mediterranean Diet Adherence score and Diet Quality Index which is designed to measure adherence to Finnish nutrition recommendations. The participants were divided into quartiles corresponding to their respective happiness scores. Diet quality scores, food intakes and other nutrition related indicators were classified into happiness quartiles.

Results: Perceived happiness was linearly associated with total fruit and vegetable intakes $(\mathrm{p}=0.002)$ and inversely associated with age $(\mathrm{p}=0.016)$, blood glucose levels $(\mathrm{p}=0.049)$, skipping lunch $(p=0.023)$, reduced food intake $(p=0.002)$ and weight loss $(\mathrm{p}=0.016)$.

Conclusions: Fruit and vegetable intakes indicated perceived happiness in the oldest old men while variables of poor appetite and ill health were inversely associated with happiness. Maintaining good nutrition and increasing fruit and vegetable consumption may be important for psychological health of older people.

\section{0-7}

Body-shape and falls among community-dwelling older adults in the Malaysian elders longitudinal research study

\section{S.H. Kioh ${ }^{1}$, Sumaiyah $\mathrm{M}^{2}$, G.J. $\operatorname{Tan}^{3}$, S.B. Kamaruzzaman ${ }^{3}$, M.P. $\operatorname{Tan}^{3}$}

${ }^{1}$ Ageing and Age-Associated Disorders Research Group, University of Malaya, Kuala Lumpur, Malaysia, ${ }^{2}$ Center for Innovation in Medical Engineering (CIME), Faculty of Engineering, University of Malaya, Kuala Lumpur, Malaysia, ${ }^{3}$ Department of Medicine, Faculty of Medicine, University of Malaya, Kuala Lumpur, Malaysia

Background: Results of existing studies on body mass index (BMI) and falls has been conflicting, which may be due to the value of BMI measurements that may be limited in this age group. The aim of this study was, therefore, to the relationship between waist: hip (WHR) and fall among community-dwelling older adults.

Methods: This was a cross-sectional study using the first-wave data from the Malaysian Elders Longitudinal Study (MELoR) among older adults aged 55 years and above. Basic demographic characteristics, medical history, anthropometric parameters and 12-month falls history were recorded. The overall population was categorized into three WHR categories: pear-shaped (WHR $<0.8$ (women)/WHR $<0.95$ (men), avocado-shaped $(0.81<$ WHR $<0.85$ (women) $/ 0.96<$ WHR $<1.0$ (men) and apple-shaped (WHR $>0.85$ (women)/WHR $>1.0$ (men).

Results: Of the 1335 participants with mean age $68.38 \pm 7.15$ years, $559(41.9 \%)$ were considered apple-shaped, 430 (32.2\%) pear-shape and $346(25.9 \%)$ with avocado-shaped. Logistic regression revealed significant increase in risk of fall in the apple-shaped $(\mathrm{OR}=2.026$, 95\% CI 1.478-2.778) compared to the pear-shaped group. After adjustment for potential confounders, being apple-shaped $(\mathrm{aOR}=$
1.646, 95\% CI 1.086-2.495) remained independently associated with falls.

Conclusions: Significant relationship was observed between body shape and falls, with those within the apple-shaped category more likely to falls. Using the WHR may be a more useful measure to determine the relationship between excess body fat and falls. Future studies should seek to determine the underlying mechanisms and to identify interventions which alter body shape as a potentially modifiable risk factor for falls in older adults.

\section{0-8}

From mitochondria to healthy aging: the role of branched-chain amino acids treatment: MATeR a randomized study

Fausto Giordano Pili ${ }^{1}$, Francesca Dutto ${ }^{1}$, Paola Porrino ${ }^{1}$, Patrizia D'Amelio $^{1}$, Giulia Carignano ${ }^{1}$, Cinzia Ferreri ${ }^{1}$, Massimiliano Massaia $^{1}$, Giovanni C. Isaia ${ }^{1}$, Francesca Sassi ${ }^{2}$, Ilaria Buondonno ${ }^{2}$, Enzo Nisoli ${ }^{3}$, Claudia Ravetta ${ }^{3}$, Chiara Riganti ${ }^{4}$

${ }^{1}$ Department of Medical Science, Gerontology and Bone Metabolic Diseases, University of Torino, Turin, Italy, ${ }^{2}$ Department of Medical Science, Gerontology and Bone Metabolic Diseases, University of Torino, Turin, Italy, ${ }^{3}$ Department of Medical Biotechnology and Translational Medicine, Centre for Study and Research on Obesity, University of Milano, Milan, Italy, ${ }^{4}$ Department of Oncology, University of Torino, Turin, Italy

Introduction: Protein energy malnutrition often affects old patients and contributes to determine frailty and sarcopenia, which increase disability, morbidity and mortality. An increased intake of branched chain amino acids (BCAAs) enhances muscle protein anabolism and physical performance. The aim of this study is to assess if BCAAs supplements improve physical performance and mitochondrial function and biogenesis in old malnourished patients.

Methods: 155 old malnourished outpatients were randomized to receive dietary counselling or BCAAs supplements. At baseline and after 1 and 2 months patients were evaluated for cognitive performance, perceived health status, nutritional status, muscle performance, appendicular muscle and fatty mass. Concurrently, we evaluated mitochondrial activity, biogenesis and fusion and oxidative stress. The effect of treatment has been evaluated by a two-way ANOVA for repeated measures, and the correlation between metabolic parameters and clinical features by a Pearson's coefficient correlation. A $p$ value $<0.05$ was considered statistically significant. Results: In both groups treatment has shown to improve nutritional status, cognitive performance and resistance to muscular fatigue, perceived health status and muscle performance including mobility and balance (with consequent decrease in risk of falls), BCAA treated patients improved more significantly. BCAAs increase mitochondrial activity and energy production, which appears to be related to improvement in cognitive and muscular performances. The increased expression of specific genes shows that BCAAs enhance mitochondrial biogenesis and fusion.

Conclusions: Administration of BCAAs is effective in increasing physical and cognitive performance in old malnourished patients: we suggest that this effect is due to the increase in mitochondrial biogenesis and performance. 


\section{0-9}

Determinants of malnutrition in older Persons: results from the European MaNuEL knowledge hub

Melanie Streicher ${ }^{1}$, Gabriel Torbahn ${ }^{1}$, Eva Kiesswetter ${ }^{1}$, Mary $\mathrm{O}^{\prime} \mathrm{Keeffe}^{2}$, Mary Kelly ${ }^{2}$, Marjolein Visser ${ }^{3}$, Eibhlís O'Connor ${ }^{2}$

${ }^{1}$ Institute for Biomedicine of Aging, Friedrich-Alexander-Universität Erlangen-Nürnberg, Germany, ${ }^{2}$ Department of Biological Sciences, and Health Research Institute, University of Limerick, Ireland, ${ }^{3}$ Department of Health Sciences, Faculty of Science, Amsterdam Public Health research institute, Vrije Universiteit Amsterdam, The Netherlands

Introduction: Malnutrition in older persons is multifactorial in origin with a multitude of factors from all areas of life involved. Present knowledge about determinants of malnutrition is still fragmentary and mainly based on cross-sectional studies using different definitions and assessment tools for both determinants as well as malnutrition.

Methods: Within the European Knowledge Hub "Malnutrition in older persons (MaNuEL)", a systematic review (SR) focusing on longitudinal studies relating potential determinants to incident malnutrition in older participants from all health-care settings was performed. Furthermore, secondary data analyses and meta-analyses of 6 community-based, longitudinal datasets from Germany, Ireland, The Netherlands and New Zealand (n between 209 and 1841, total $\mathrm{n}=4844$ ) were conducted using a harmonized set of 23 potential determinants and a uniform definition of malnutrition.

Results: The SR identified 23 articles revealing consistent evidence of low to moderate quality that poor appetite, difficulties feeding oneself, poor self-reported health, and hospitalization are determinants of incident malnutrition. Meta-analyses of the 6 datasets (malnutrition incidence after 1-3 years between 5.1 and $17.2 \%$ ) found higher age, marital status, walking limitations, limitations climbing stairs, and previous hospitalization as determinants of incident malnutrition.

Key conclusion: Regarding preventive approaches, poor appetite, functional limitations, poor self-reported health and previous hospitalization need specific attention. Further initiatives of data pooling and efforts to standardize the assessment of malnutrition and its determinants are warranted to further understand the etiology of malnutrition.

\section{Area: Pre and post operative care}

\section{0-10}

Variability in the detection of delirium among older patients with hip fracture: results from the Gruppo Italiano di Ortogeriatria (GIOG) National Registry

Elena Tassitro ${ }^{1}$, Piero Rapazzini ${ }^{2}$, Amedeo Zurlo ${ }^{3}$, Chiara Mussi ${ }^{4}$, Maurizio Corsi ${ }^{5}$, Maria Lia Lunardelli ${ }^{6}$, Anita Andreano ${ }^{1}$, Giuseppe Bellelli ${ }^{7}$, Patrizia Floris ${ }^{8}$, Monica Pizzonia ${ }^{9}$, Antonella Barone ${ }^{10}$, Albert March ${ }^{11}$, Andrea Ungar ${ }^{12}$, Valter Galmarini ${ }^{13}$, Piera Ranieri ${ }^{14}$, Giuseppe Bellelli ${ }^{1}$

${ }^{1}$ University of Milano-Bicocca, Milan, Italy, ${ }^{2}$ Orthogeriatric Unit, Circolo Hospital, Varese, Italy, ${ }^{3}$ Orthogeriatric Unit, Arcispedale S Anna, Ferrara University, Ferrara, Italy, ${ }^{4}$ Orthogeriatric Unit, University of Modena and Reggio Emilia, Modena, Italy,

${ }^{5}$ Orthogeriatric Unit, S Gerardo hospital, Monza, Italy, ${ }^{6}$ Orthogeriatric Unit, S.Orsola Malpighi, Bologna, Italy, ${ }^{7}$ Orthopedic Unit, ASST Vimercate, Carate Brianza hospital, Vimercate (MB), Italy, ${ }^{8}$ Orthogeriatric Unit, Hospital of Sondrio-ASST VAL, Sondrio, Italy, ${ }^{9}$ Orthogeriatric Unit, San Martino hospital, Genova, Italy,
${ }^{10}$ Orthogeriatric Unit, Galliera hospital, Genova, Italy,

${ }^{11}$ Orthogeriatric Unit, Bolzano hospital, Bolzano, Italy, ${ }^{12}$ Geriatrics and Intensive Care Unit, Florence, Italy, ${ }^{13}$ Orthopedic Unit, ASST Fatebenefratelli Sacco, Milano, Italy, ${ }^{14}$ Orthopedic Unit, Poliambulanza hospital, Brescia, Italy, Department of Medicine and Rehabilitation, S. Anna hospital, Brescia, Italy

Introduction: Recently, a network of Orthogeriatric and Orthopedic Units (Gruppo Italiano di OrtoGeriatria, GIOG) was created in Italy to collect data of older patients admitted to hospital wards after hip fracture $(\mathrm{HF})$. We report some data regarding the detection of postoperative delirium (POD).

Methods: 2570 patients $(76.3 \%$ females $)$ aged $>65$ years were recruited in 14 hospitals after HF from 2016 to March 2018. Information about residence before fracture, pre-operative cognitive impairment (POCI), type of anaesthesia and involvement of a geriatrician as consultant were collected. POD development was assessed on the 1st day after surgery with routinely employed instruments. Multivariate logistic p-values are reported.

Results: POD occurred in 636 patients (24.7\%), with huge heterogeneity among centers $(11.7-38.8 \%, \mathrm{p}<0.001)$. Delirium was significantly associated with gender (30.6\% female vs. $22.9 \%$ male, $\mathrm{OR}=1.6, \quad 95 \% \quad$ CI $1.2-2.1), \quad$ age $\quad(30.0 \% \geq 85$ years vs. $18.2 \%<85$ years, $\mathrm{OR}=1.7,95 \%$ CI $1.3-2.1)$; POCI (40\% severe POCI vs. no-POCI, $\mathrm{OR}=5.6,95 \% \mathrm{CI} 4.0-7.9$ ); geriatric involvement ( $25.1 \%$ yes vs. $19.2 \%$ no, $\mathrm{OR}=2.8,95 \%$ CI $1.3-6.4)$. No association was found between POD and type of anesthesia (24.8\% general vs $24.7 \%$ other, $\mathrm{p}=0.38)$ and living at home before fracture $(23.9 \%$ yes vs $33.5 \%$ no, $p=0.86$ ). No significant difference in median time from hospitalization to surgery was found between patients with POD (42.0 h; I-III quartiles 24.0-63.3) and those without POD (40.0 h, I-III quartiles 23.0-58.0; $\mathrm{p}=0.59$ ).

Conclusions: We found a huge heterogeneity in delirium detection among centres, at least partially related to patient and organizational characteristics. Diffusion of tools to systematically detect delirium is urgently required.

\section{0-11}

Factors associated with institutionalization after 1 year of hip fracture

Esther Lueje Alonso ${ }^{1}$, Lourdes Del Rosario Evangelista Cabrera ${ }^{2}$, Karina Liz Quiñones Huayna ${ }^{1}$, Lucía Fernández Arana ${ }^{1}$, Victoria Garay Airaghi ${ }^{1}$, Jesús Mora Fernández ${ }^{1}$

${ }^{1}$ Department of Geriatrics, Hospital Clínico San Carlos, Madrid, Spain, ${ }^{2}$ Department of Geriatrics, Hospital Universitario Severo Ochoa, Madrid, Spain

Introduction: Between fifteen and thirty percent of subjects living at home at the time of the fracture require institutionalization. The aim of this study is to determine the factors that influence institutionalization in elderly one-year after suffering hip fracture.

Methods: Prospective 1 year follow up study (September 2015March 2018). Patients aged $>65$ years admitted to the Orthogeriatric Unit with fragility hip fracture were included. Variables: baseline characteristics, comorbidity (CIRS-G, Charlson-Index), functional status (Barthel index-BI-, Functional ambulation classification-FAC-, Lawton index-LI-), haemoglobin upon admission (HbAd) and before discharge ( $\mathrm{HbDi})$, inhospital complications and destination at discharge. Multivariate logistic regression analysis was performed. SPSS version 25.0.

Results: 237 patients were included; age $85.46 \pm 6.88$, women $75.9 \%$. Number of drugs $6(\mathrm{IQR}=4-9)$, moderate-severe cognitive 
impairment $17.3 \%$, Charslon 5.9 (IQR $=4.90-7.25)$, CIRS-G 12 $(\mathrm{IQR}=9-16) ; \mathrm{BI} 90$ (IQR = 75-95), FAC 5 (IQR = 4-5), 56.5\% used walking aids, LI 4 (IQR = 1-7); HbAd $12.67 \pm 1.66$, HbDi $10.44 \pm 1.00$. Intracapsular fracture $50.2 \%$, time until surgery $3.97 \pm 2.22$ days, heart failure (HF) $14 \%$, delirium $22 \%$, length of stay $13.44 \pm 9.78$ days; $3.8 \%$ were institutionalized before the fracture and $20.9 \%$ after 12 months. Factors associated with institutionalization after 1 year: age $\geq 85(p=0.010)$, moderatesevere cognitive impairment $(\mathrm{p}<0.0005)$, FAC $<4$ ( $\mathrm{p}=0.001)$; HF $(\mathrm{p}=0.05)$, BI at admission $(\mathrm{p}=0.002)$ and at discharge $(p<0.0005)$, length of stay $(p=0.002)$. Multivariate analysis: IB at discharge (OR: $0.9 ; 95 \% \mathrm{CI} ; 0.94-0.99 ; \mathrm{p}=0.026$ ), length of stay (OR:1.1; 95\% CI; 1.1-1.2; $\mathrm{p}=0.002)$ and HF (OR:9.0; 95\% CI; $1.1-80.5 ; \mathrm{p}=0.049)$.

Conclusions: The main risk factors for institutionalization following a hip fracture are IB at discharge, length of stay and to develop HF during the hospitalization. As a consequence, age, comorbidity and complications should not be considered isolated variables in the prediction of institutionalization.

\section{0-12}

Predicting 12-month mortality in emergency surgery patients assessed by an elderly care liaison service: Salford POP-GS

Nicholas Springall ${ }^{1}$, Jenny Fox ${ }^{1}$, Areej Paracha ${ }^{1}$, Mollie Rowley ${ }^{1}$, Haroon Khan ${ }^{1}$, Luciana Miguel-Alhambra ${ }^{1}$, Alex Gomez-

Quintanilla $^{1}$, Genna Logue ${ }^{1}$, Amanda Pedersen ${ }^{1}$, Angeline Price $^{1}$, Mohammad Moatari ${ }^{1}$, Arturo Vilches-Moraga ${ }^{1}$

${ }^{1}$ Department of Ageing and Complex Medicine, Salford Royal Hospital, UK

Objectives: Almost half of patients admitted non-electively to general surgery are over 70-years of age. This study describes the demographic of older emergency general surgery (EGS) patients and factors that influence 12-month mortality in this population.

Methods: Prospective study of consecutive patients aged 75-years or older admitted non-electively under general surgery between 8th September 2014 and 30th March 2017 and reviewed by our elderly care liaison team.

Results: 598 patients were included, with a mean age of $82.8 \pm 5.6$ years, and a female predominance $(56.4 \%)$. At presentation, $145(24.5 \%)$ and $234(39.1 \%)$ were dependent for basic and instrumental activities of daily living (ADL) respectively. 43 (7.2\%) were residents in a care home and $288(49.6 \%)$ were frail (Clinical Frailty Scale score of $>4$ ) Biliary conditions were the most common diagnoses $(170,28.4 \%)$. Complications affected the majority (466, $77.4 \%)$, with delirium affecting $136(22.8 \%)$. Most (353, 59\%) were managed medically, with $103(17.2 \%)$ undergoing non-surgical procedures and only $142(23.7 \%)$ requiring surgery. Median length of stay was 8 -days with a 30 -day readmission rate of $9.2 \%$ (51). Inhospital mortality was $7 \%$ (42), rising to $29.2 \%$ (175) at 12 -months. Strong predictors of mortality were ASA score III-IV (HR 2.62 IC 1.73-3.97), dependency for basic ADLs (HR 2.47 IC 1.56-3.89) and frailty (HR 1.94 IC 1.32-2.86)

Conclusions: Although the majority of older EGS patients survive an index hospital admission, a third are dead within a year. ASA score, presence of functional impairment, and frailty (measured using the Clinical Frailty Scale) are strong predictors of 12-month mortality.
0-13

Association between anticholinergic load and urinary retention after hip fracture surgery in patients over 75 years

Rebecca Haddad $^{1}$, Charlotte Tomeo ${ }^{1}$, Judith Cohen Bittan ${ }^{1}$, Alice Gioanni $^{2}$, Cedric Villain ${ }^{2}$, Marc Verny ${ }^{2}$, Jacques Boddaert ${ }^{3}$

${ }^{1}$ Unit of Peri-Operative Geriatric care, geriatric department, Hôpitaux universitaires Pitié-Salpêtrière-Charles Foix. DHU FAST, Assistance Publique Hôpitaux de Paris (APHP), Paris, France, ${ }^{2}$ Sorbonne University, University Pierre and Marie Curie (UPMC) Paris 06, University Hospital, Paris, France, ${ }^{3}$ Sorbonne University, University Pierre and Marie Curie (UPMC) Paris 06, University Hospital, Paris, France; Unit of Peri-Operative Geriatric care, geriatric department, Hôpitaux universitaires Pitié-Salpêtrière-Charles Foix. DHU FAST, Assistance Publique Hôpitaux de Paris (APHP), Paris, France

Introduction: Urinary retention (UR) is common after hip fracture surgery (HFS), which could be explained by several factors, including drug utilization with anticholinergic effects. Only two studies with conflicting results evaluate the association of anticholinergic load (AL) with UR in older patients after HFS. The objective of this study was to evaluate the association of AL measured by all existing scales with UR in this population.

Methods: All patients admitted after HFS in the Geriatric Perioperative Unit (UPOG) were included. UR was defined as the failure to urinate after indwelling catheter removal or the necessity of an indwelling catheter at any moment. AL was measured based on patients' computerized prescriptions and considered all treatments received during hospitalization. AL was evaluated by several scales: Drug Burden Index (DBI-Ach), Anticholinergic Drug Scale (ADS), Anticholinergic Risk Scale (ARS), Anticholinergic Cognitive Burden Scale (ACB), Chew's scale (Chew), Anticholinergic Activity Scale (AAS), Anticholinergic Load Scale (ALS), Clinician-Rated Anticholinergic Scale (CrAS), Duran's scale (Duran) and Anticholinergic Burden Classification (ABC).

Results: On the 53 patients included (mean age $86.9 \pm 5.4$ years; $81.1 \%$ female), there was $18.9 \%$ of UR. The mean \pm SD AL (scale) was: $0.72 \pm 0.40$ (DBI-Ach), $1.39 \pm 0.95$ (ACB), $0.28 \pm 1.26$ (ARS), $\quad 0.55 \pm 0.89$ (ADS), $\quad 0.73 \pm 1.30$ (ABC), $0.79 \pm 1.12$ (Chew), $1.39 \pm 0.74$ (Duran), $0.38 \pm 0.86$ (AAS), $0.72 \pm 0.95$ (ALS) and $1.45 \pm 1.08$ (CrAS). There was a significant association between $\mathrm{AL}$ and $\mathrm{RU}$ measured by $\mathrm{ABC}(\mathrm{p}<0.05)$, and a trend of significance when measured by ACB $(p=0.13)$, ADS, Chew $(\mathrm{p}=0.08)$ and ALS $(\mathrm{p}=0.16)$.

Key conclusions: In patients after HFS managed in UPOG, there was an association between UR and AL measured by only one of the 10 existing scales. These preliminary results will be verified in a cohort of 764 patients.

\section{0-14}

Documentation of capacity and consent to surgery in a trauma unit

\section{EC Pulford ${ }^{1}$, Ralhan $\mathrm{S}^{1}$, K Shah ${ }^{1}$, B Yiu ${ }^{1}$, V Borkar ${ }^{1}$}

${ }^{1}$ Oxford University Hospitals NHS Foundation Trust, Oxford, UK

Introduction: Previous audits have confirmed high prevalence of cognitive impairment, dementia and delirium in trauma inpatients aged $>=60$ years. Mental capacity for decision-making cannot be automatically assumed in this frail group. Our local standard is to undertake and document formal capacity assessment with respect to 
consent for surgery if patient meets one or more of: known to have dementia, currently delirious, AMT $=<8$.

Methods: A follow up 'snapshot' audit was undertaken of inpatients aged $>60$ years on Trauma Unit, recording: pre operative Abbreviated Mental Test (AMT) scores, diagnosis of dementia, cognitive impairment, delirium, any recorded assessment of mental capacity with respect to planned operation. Consent Forms used-Form 1 (capacity assumed, no space to document process of capacity assessment); form 4 (patient lacks capacity, form has specific section to document process of capacity assessment).

Results: A third of admissions met our local criteria, of these $54 \%$ had a documented assessment of mental capacity. Most were prompted by and recorded on a Consent Form 4. The remaining $46 \%$ of patients had no documentation of the capacity assessment process in the notes although it is assumed/implicit in the completion of Consent Form 1.

Conclusions: There are a significant number of patients meeting our local criteria for capacity assessment who consent to surgery using a Form 1 and do not have the process documented except 'by assumption'. A revision of Form 1 and a re-audit is planned. This work received ethical approval from the Trust Audit Committee.

\section{Area: Geriatric rehabilitation}

\section{O-15}

Pre-discharge rehabilitation after hip surgery reduces 30-day readmissions in older adults: National Health Insurance ServiceSenior Cohort (2007-2012)

Nari $\mathrm{Bu}^{1}$

${ }^{1}$ Dept of Family Medicine, Kyung Hee Medical Center, Seoul, South Korea

Background: Rehabilitation programs before and after hip surgery can shorten the length of hospital stay, reduce the incidence of complications, and reduce the readmission rate after surgery in older adults aged 65 years or over. The present study aimed to investigate the status of readmissions within 30 days after discharge and related factors in elderly people through the big data analysis using the sample data from the National Health Insurance Service-Senior Cohort (NHIS-SC).

Methods: The subjects of the present study were patients aged 65 years or older who underwent hip surgery between 2007 and 2012 . The subjects undergoing hip surgery included those who underwent at least one surgery of the following during the period: replacement arthroplasty, revision arthroplasty, arthrodesis, internal fixation, reduction of fractured extremity and internal fixation. Each variable was selected for sociodemographic characteristics and clinical features of the subjects, and the characteristics of medical institutions according to 30-day readmission.

Results: Among 9008 study participants, 1628 (18.1\%) were readmitted within 30 days. Lower readmission rate was associated with longer hospital stay, greater number of hospital beds, and rehabilitation before discharge; whereas a higher rate was associated with provincially located hospitals, getting of medical aid, accompanying fractures, ICU admission, and comorbidities.

Conclusions: Pre-discharge rehabilitation in patients aged $\geq 65$ years who underwent hip surgery can reduce readmissions, and efforts to increase daily living functions such as muscle strength and walking ability are very important.

\section{0-16}

Increasing life-space mobility in multimorbid older persons with motor and cognitive impairment

Phoebe Ullrich $^{1}$, Christian Werner ${ }^{1}$, Tobias Eckert ${ }^{1}$, Martin Bongartz $^{1}$, Rainer Kiss ${ }^{2}$, Jürgen M. Bauer ${ }^{1}$, Klaus Hauer ${ }^{1}$

${ }^{1}$ Department of Geriatric Research, AGAPLESION Bethanien Hospital Heidelberg, Geriatric Center at the Heidelberg University, Heidelberg, Germany, ${ }^{2}$ Department of Health and Social Affairs, FHM Bielefeld, University of Applied Science, Bielefeld, Germany

Introduction: The study objective was to determine the effectiveness of a specific, standardized home-based training to improve life-space mobility (LSM) in community-dwelling, multimorbid, older persons with cognitive impairment (CI) after discharge from geriatric rehabilitation, a high risk group for LSM restrictions and institutionalization.

Methods: A double-blinded, randomized, controlled trial including older persons with mild to moderate cognitive impairment (CI) was conducted by a 12-weeks home-based training intervention and 12-weeks follow-up period. The intervention group (IG) received supervised recommendations for a home-based balance-, strengthand walking- training including a tailored motivational strategy. The control group (CG) received supervised recommendations for lowintensity exercise while seated. LSM was evaluated by the validated Life-Space Assessment in Persons with Cognitive Impairment (LSACI).

Results: 118 older persons (age: $82.3 \pm 6.0$ years) with mild to moderate CI (Mini-Mental State Examination: $23.3 \pm 2.4$ ) were included. Analyses of covariance (ANCOVAs), with baseline LSM as a covariate, revealed a training-related significant increase in LSM as documented by the LSA-CI composite score and 3 sub-scores (change from baseline: $\mathrm{p} \leq 0.001-0.023$, partial eta ${ }^{2}: 0.06-0.20$ ), with female gender, low baseline LSM and increased physical activity during the intervention as significant predictors for increase in LSM. Training gains were sustained during follow-up for the composite score and one of three sub-scores ( $\mathrm{p} \leq 0.023-0.026$; eta ${ }^{2}$ : je 0.06 ).

Key conclusions: The training was feasible and sustainably improved LSM in multimorbid, older people with CI, thus representing a successful program for post ward rehabilitation to promote LSM in a high risk group for activity restriction and institutionalization.

\section{0-17}

Fear of falling in geriatric patients recovering from hip fracture: a matter of motor performance or emotion

Tobias Eckert ${ }^{1}$, Karin Kampe ${ }^{2}$, Michaela Kohler ${ }^{2}$, Diana Albrecht ${ }^{2}$, Klaus Hauer ${ }^{1}$, Clemens Becker ${ }^{2}$, Klaus Pfeiffer ${ }^{2}$

${ }^{1}$ Department of Geriatric Research, AGAPLESION Bethanien Hospital Heidelberg, Geriatric Center at the Heidelberg University, Heidelberg, Germany, ${ }^{2}$ Robert-Bosch-Hospital, Department of Clinical Gerontology and Geriatric Rehabilitation, Stuttgart, Germany

Objective: Aim of this study is to examine the relations between multidimensional FoF, psychological function and motor performance in geriatric patients with hip fracture after admission to inpatient rehabilitation.

Methods: Baseline data of a total of 115 cognitively intact (6-ItemCognitive-Impairment Test $\leq 10)$ geriatric patients with hip fracture (mean age: $82.5 \pm 6.8$ years) and present FoF confirmed to participate in a randomized controlled clinical trial. During first week after admission following measurements were conducted: 1-item FoF, fallrelated concerns (Short Falls Efficacy Scale-International, Short FES- 
I), perceived ability to manage falls (PAMF), fall-related posttraumatic stress (6 questions based on DSM-IV criteria) psychological inflexibility (Acceptance and Action Questionnaire, AAQ-2), history of falls and motor performance (Short Physical Performance Battery, SPPB). Cross-sectional data were analyzed using path analysis.

Results: Low motor performance had significant direct effects on FES-I ( $\mathrm{p} \leq 0.01$ ), whereas 1-item FoF was significantly and directly determined by fall-related posttraumatic stress $(p \leq 0.05)$. Posttraumatic stress significantly mediated the effects of high psychological inflexibility on 1 -item FoF (indirect effect, $\mathrm{p} \leq 0.05$ ). Being female $(p \leq 0.01)$ and history of falls $(p \leq 0.01)$ were significantly and directly associated with low perceived ability to manage falls.

Discussion: Results indicate that the assessment of fall-related concerns by FES-I during an early stage of stationary rehabilitation maybe is insufficient to capture FoF in its whole nature. FES-I targets fall-related concerns related to activities of daily living which cannot be performed by hip fracture during the early stage of recovery. In this context the relevance of psychological inflexibility and posttraumatic stress symptoms was emphasized.

\section{0-18}

Nutrition and functional outcomes in older adults admitted to rehabilitation units: a multi-centre cohort study

Diana Lelli ${ }^{1}$, Alicia Calle ${ }^{2}$, Laura Mónica Perez ${ }^{3}$, Graziano Onder ${ }^{4}$, Alessandro Morandi ${ }^{5}$, Elena Ortolani ${ }^{4}$, Miriam Colominas ${ }^{3}$, Claudio Pedone $^{1}$, Marco Inzitari ${ }^{3}$

${ }^{1}$ Campus Bio-Medico University of Rome, Rome, Italy, ${ }^{2}$ Vall d'Hebron University, Barcelona, Spain, ${ }^{3}$ Parc Sanitari Pere Virgili, Barcelona, Spain, ${ }^{4}$ Centro Medicina dell'Invecchiamento, Università Cattolica del Sacro Cuore, Rome, Italy, ${ }^{5}$ Department of Rehabilitation and Aged Care of the Fondazione Camplani, Ancelle Hospital, Cremona, Italy

Introduction: Evidence about the role of nutritional status (NS) on functional outcomes (FO) after rehabilitation in older adults is scarce. Our aim was to analyse the association between NS and FO in older adults admitted to geriatric rehabilitation units.

Methods: The Sarcopenia And Function in Aging Rehabilitation (SAFARI) multi-centre study enrolled patients aged $\geq 65$ years admitted to geriatric rehabilitation units in Italy and Spain. FO were absolute and relative functional gain (AFG-RFG) in Barthel Index (BI) at 1 and 3 months after admission. The association between NS (Mini Nutritional Assessment-Short Form) and FO was explored using linear regression and mixed models, adjusted for potential confounders. Analyses were then stratified for diagnosis at admission. Results: We included 415 patients [mean age 81.4 years (SD:7.7); $67 \%$ female; $9.4 \%$ malnourished (MN), $42.7 \%$ at risk of malnutrition (RM), and $48 \%$ well nourished (WN)]. Admission diagnoses were hip fracture $(39.5 \%)$, elective orthopaedic surgery (EOS) $(29.5 \%)$, and stroke $(31 \%)$. In adjusted linear mixed model, MN and RM participants had lower BI compared to $\mathrm{WN}(\mathrm{MN}: \beta$ : $-8.47, \mathrm{p}=0.023$; $\mathrm{RM}: \beta$ : $-5.22, \mathrm{p}=0.031)$, differences between groups remained stable over time. After stratification for admission diagnosis, only MN patients admitted after EOS had worse FO, both at 30 days (AFG: $\beta$ adjusted: $\quad-13.54, \quad \mathrm{p}<0.001$; RFG: $\beta: \quad-32, \quad \mathrm{p}<0.001)$ and 3 months (AFG: $\beta$ adjusted: $-17.79, \mathrm{p}<0.001$; RFG: $\beta$ : -26.77 , $\mathrm{p}=0.002)$.

Conclusions: In our sample, poor NS is associated with worse BI in older adults admitted to geriatric rehabilitation units; in patients undergoing EOS, MN is associated with worse FO. Our results documented for the first time the importance to assess nutritional status before EOS.
Area: Frailty and sarcopenia

\section{0-19}

Systematic review on pharmacotherapy for hypertension in functionally impaired elderly: sub-project of the Medication and Quality of Life in frail older persons (MedQoL) Research Group

Denkinger Michael ${ }^{1}$, Mühlbauer Viktoria ${ }^{1}$, Brefka Simone ${ }^{1}$, Bollig Claudia $^{2}$, Torbahn Gabriel ${ }^{3}$, Voigt-Radloff Sebastian ${ }^{2}$, Bauer Jürgen ${ }^{4}$, Haefeli Walter E. ${ }^{5}$, Dallmeier Dhayana ${ }^{1}$

${ }^{1}$ AGAPLESION Bethesda Clinic Ulm, Geriatric Research Unit, Ulm University and Geriatric Center Ulm/Alb-Donau, Germany, ${ }^{2}$ Institute for Evidence in Medicine (for Cochrane Germany Foundation), Medical Center-University of Freiburg, Faculty of Medicine, University of Freiburg, Germany, ${ }^{3}$ Institute for Evidence in Medicine (for Cochrane Germany Foundation), Medical Center-University of Freiburg, Faculty of Medicine, University of Freiburg and Institute for Biomedicine of Aging, FAU Erlangen-Nürnberg, Germany, ${ }^{4}$ Center for Geriatric Medicine, University of Heidelberg and AGAPLESION Bethanien Hospital, Heidelberg, Germany, ${ }^{5}$ Department of Clinical Pharmacology and Pharmacoepidemiology, University of Heidelberg, Heidelberg, Germany

Background: The 2013 guideline of the European Society of Cardiology on the management of hypertension leaves decisions on antihypertensive therapy in frail older patients to the treating physician, based on monitoring of the clinical effects of treatment (level of evidence: C). Due to the weak evidence, pharmacotherapy in this population is uncertain. As part of the "Medication and Quality of Life in frail older persons" study group we aim to review the evidence on pharmacotherapy for hypertension in functionally impaired older adults. Methods: We performed systematic literature searches for randomized-controlled trials (RCTs) and prospective cohort studies (PCS) in Medline, Embase and Central. Trial were included if functionality was assessed, and participants were characterized as at least moderately functionally impaired. Inclusion of trials, data extraction and assessment of risk of bias was carried out by two reviewers independently. Conflicts were resolved by discussion with a third person. Results: From 15389 citations for RCTs and 4570 citations for PCS, 35 and 36 full texts, respectively, were identified as potentially relevant. Only 2 RCT and 4 PCS included functionally impaired older adults. Due to high heterogeneity, meta-analyses were not performed. Some trials show methodological issues, which will be presented at the conference.

Conclusions: The current literature does not allow reliable recommendations on the pharmacotherapy of hypertension in functionally impaired older patients. The assessment of functionality as well as the inclusion of frail older people in RCTs is urgently needed to shift pharmacotherapy of vulnerable patients from intuition to compelling evidence.

\section{O-20}

The impact of intramuscular adipose tissue on the 4-years mortality risk of hospitalized geriatric patients

Eugénie Van Mieghem ${ }^{1}$, Scott Lamers ${ }^{1}$, Robin Degerickx ${ }^{1}$, Maurits Vandewoude $^{2}$, Stany Perkisas ${ }^{2}$

${ }^{1}$ School of Medicine, University of Antwerp, Antwerp 2610, Belgium, ${ }^{2}$ University of Antwerp, Belgium; University Centre for Geriatrics, ZNA (Ziekenhuis Netwerk Antwerp), Belgium 
Introduction: Intramuscular adipose tissue (IMAT) is an important factor in the decline of muscle strength and function, giving way to the process of sarcopenia. This study sought to determine the influence of IMAT on the four-year mortality risk and it's correlation with nutritional status and muscle mass, strength and physical performance.

Methods: All patients hospitalized at the geriatric department of the Saint-Elisabeth hospital in Antwerp (Belgium) from 01/08/2012-31/ 01/2013 were included. The IMAT and muscle mass were measured by a computed tomography (CT) scan of both upper legs. Subjects were divided into 4 groups according to percentage of IMAT in relation to the entire muscle bulk measured $(0-20 \%, 20-40 \%$, 40-60\%, 60-80\%). Muscle strength was measured by handgrip strength. Physical performance was measured by the Short Physical Performance Battery (SPPB). The nutritional risk status was assessed by the Mini-Nutritional Assessment-Short Form (MNA-SF).

Results: A total of 302 subjects were obtained. In male subjects $(\mathrm{n}=91)$, the IMAT has shown to be a significant factor $(\mathrm{HR}=1.037$; 95\% CI 1.013-1.063) in determining the 4 years mortality risk. When comparing the 4 different male IMAT groups a significant difference in survival was noted $(\mathrm{p}=0.047)$. In all subjects, a significant correlation was found between IMAT and muscle mass ( $P C=-0.606 ; p=0.00)$, muscle strength $(\mathrm{PC}=-0.305 ; \mathrm{p}=0.00)$ and physical performance $(\mathrm{PC}=-0.455 ; \mathrm{p}=0.00)$. No significant correlation was found between IMAT and nutritional status ( $\mathrm{PC}=-0.091 ; \mathrm{p}=0.203$ ).

Conclusion: The IMAT is a significant prognostic factor in determining the 4 years mortality risk in male hospitalized geriatric patients.

\section{$0-21$}

A cluster-randomized clinical trial of a daily physical activity combined with nutritional supplement in nursing home residents: the open study

H Grönstedt ${ }^{1}, \mathrm{~S}$ Vikström ${ }^{2}$, T Cederholm ${ }^{3}$, E Franzén $^{4}$, А̊ Seiger ${ }^{5}$, A Wimo ${ }^{6}$, G Faxén-Irving ${ }^{7}$, AM Boström ${ }^{8}$

${ }^{1}$ Stockholms Sjukhem R\&D unit, Stockholm; Allied Health Professionals, Function Area Occupational Therapy \& Physiotherapy, Karolinska University Hospital, Stockholm, Sweden, ${ }^{2}$ Department of Neurobiology, Care Science and Society, Division of Occupational Therapy, Karolinska Institutet, Stockholm, Sweden, ${ }^{3}$ Department of Public Health and Caring Sciences, Clinical Nutrition and Metabolism, Uppsala University \& Department of Geriatric Medicine, Uppsala University Hospital, Uppsala \& Karolinska University Hospital, Theme Aging, Stockholm, Sweden, ${ }^{4}$ Stockholms Sjukhem R\&D unit, Stockholm; Department of Neurobiology, Care Science and Society, Division of physiotherapy, Karolinska Institutet, Stockholm \& Allied Health Professionals, Function Area Occupational Therapy \& Physiotherapy, Karolinska University Hospital, Stockholm, Sweden, ${ }^{5}$ Stockholms Sjukhem R\&D unit, Stockholm \& Department of Neurobiology, Care Science and Society, Division of Clinical geriatrics, Karolinska Institutet, Stockholm, Sweden, ${ }^{6}$ Department of Neurobiology, Care Science and Society, Division of neurogeriatrics, Karolinska Institutet, Stockholm, Sweden, ${ }^{7}$ Stockholms Sjukhem R\&D unit, Stockholm, Department of Neurobiology, Care science and Society, Division of clinical geriatrics, Karolinska Institutet Stockholm \& Allied Health Professionals, Function Area Clinical Nutrition, Karolinska University Hospital, Stockholm, Sweden, ${ }^{8}$ Stockholms Sjukhem R\&D unit, Stockholm; Department of Neurobiology, Care science and Society, Division of nursing, Karolinska Institutet and Karolinska University Hospital, Theme Aging, Stockholm, \& Western Norway University of Applied Sciences, Haugesund, Norway
Introduction: Chair rise are strongly associated with the ability to perform Activities of Daily Living. Protein deficiency seems to play an important role in the development of sarcopenia in older persons. Our aim was to investigate the effects of a 12-week combined daily intervention consisting of a sit-to-stand exercise (STS) and an oral protein-rich nutritional supplement (ONS) on physical function in nursing home residents.

Methods: A two-arm cluster-randomized controlled trial was performed in eight Swedish nursing homes. The 30-s Chair Stand Test was used as primary outcome. Adherence to the combined intervention within the intervention group (IG; $n=60$ ) was divided into "high dose" ("HD") ( $>120$ occasions of STS and $>60$ bottles of ONS for 12 weeks) and "low dose" ("LD"). The control group (CG; $n=60$ ) received standard care. Data was analyzed using Student's t test, $\mathrm{p}<0.05$.

Results: 120 residents, (mean (SD) $86 \pm 5.4$ years), were recruited at baseline, and 102 were available for follow up. There were no statistical differences within or between the IG and CG regarding means of chair rises at baseline or follow-up. The mean number of chair rises increased significantly within the "HD-group" vs. the "LD-group". At baseline the mean numbers of chair rises were 6.8 for the "HDgroup" $(\mathrm{n}=21)$ and 5.7 for the "LD-group" $(\mathrm{n}=29)(\mathrm{p}=0.232)$. At follow-up the mean numbers of chair rise were 7.6 for the "HDgroup" and 5.4 for the "LD-group" $(\mathrm{p}=0.02)$.

Key conclusions: Residents who adhered to the intervention improved their capability to perform chair rise. Trial Registration: ClinicalTrials.gov Identifier: NCT02702037.

\section{O-22}

The functional continuum in relation to survival in older adults: the FRADEA study

Emiel Hoogendijk ${ }^{1}$, Luis Romero ${ }^{2}$, Pedro Sánchez-Jurado ${ }^{2}$, Teresa Flores Ruano $^{2}$, Silvia Lozoya Moreno ${ }^{2}$, Laura Plaza Carmona ${ }^{2}$, Borja Gil García ${ }^{2}$, Ana Pérez Fernández-Rius ${ }^{2}$, Melisa López Utiel ${ }^{2}$, Isabel Huedo Rodenas ${ }^{2}$, Leocadio Rodríguez-Mañas ${ }^{3}$, Pedro Abizanda ${ }^{2}$

${ }^{1}$ VU University Medical Center, Amsterdam, The Netherlands, ${ }^{2}$ Albacete University Hospital, Albacete, Spain, ${ }^{3}$ University Hospital of Getafe, Spain

Introduction: The aim of the current study was to investigate whether the functional continuum, based on a refined measure combining basic (BADL) and instrumental (IADL) activities of daily living and frailty, is associated with mortality in older adults during 10 years of follow-up.

Methods: Data were used from the Frailty and Dependence in Albacete (FRADEA) study, a population based sample of Spanish older adults. The current study included data of 924 participants over age 70 from the first measurement wave (2007-2009). At baseline, a functional continuum measure of 8 categories was constructed based on limitations in BADL using the Barthel Index, limitations in IADL using the Lawton IADL Index, and the criteria of the frailty phenotype. Associations with 10-year mortality were assessed using Kaplan-Meier curves and Cox proportional hazards models.

Results: The risk of mortality gradually increased towards the less functionally independent end of the continuum. The presence of mild, moderate or severe BADL impairment was associated with mortality, in models adjusted for age, sex, comorbidity and institutionalization. The analyses also revealed that those who were BADL independent, IADL dependent and pre-frail ( $\mathrm{HR}=2.27,95 \% \mathrm{CI}=1.22-4.20)$, and those who were BADL independent and frail $(\mathrm{HR}=3.74,95 \%$ $\mathrm{CI}=1.88-7.42)$ had an increased risk of mortality. 
Key conclusions: This study showed that a functional continuum measure based on a combination of BADL, IADL and frailty predicted survival in older adults. Interventions may be targeted at those who are becoming frail and are still functionally independent, as this group already showed an elevated risk of mortality.

\section{0-23}

\section{Muscle fiber atrophy in neurogenic sarcopenia}

Fabiana Tanganelli ${ }^{1}$, Stefanie Jarmusch ${ }^{1}$, Peter Meinke ${ }^{2}$, Uta Ferrari ${ }^{1}$, Fabian Hofmeister ${ }^{1}$, Stefan Hintze ${ }^{2}$, Benedikt Schoser ${ }^{2}$, Neuerburg $\mathrm{Carl}^{3}$, Mehaffey Stefan ${ }^{3}$, Drey Michael ${ }^{1}$

${ }^{1}$ Department of Medicine IV, geriatrics, University hospital, LMU Munich, Germany, ${ }^{2}$ Friedrich-Baur-Institute, Department of Neurology, University hospital, LMU Munich, Germany,

${ }^{3}$ Department of General-, Trauma- and Reconstructive Surgery, Munich University Hospital, Nußbaumstr. 20, 80336, Munich, Germany

Background: Neurogenic sarcopenia based on loss of motor neurons and degeneration of the neuromuscular junction seems to be a subgroup of sarcopenia. Nothing is known about histological changes in neurogenic sarcopenia. Therefore muscle fiber diameter and variability coefficient of type 1 and type 2 muscle fibers were measured in hip fracture patients.

Methods: Fiber diameters were measured from biopsies of the vastus lateralis muscle of patients with hip fracture $(n=19,8$ men and 11 women; $80.6 \pm 76$ years). To define neurogenic sarcopenia, Motor Unit Number Index (MUNIX) was measured. To define a metric measure for sarcopenia, a z-transformation from skeletal muscle index (SMI), derived from BIA and handgrip strength was calculated. All metric variables are presented as mean \pm standard deviation. Pearson correlation coefficient and software SPSS statistics version 25 was used for statistical analysis.

Results: Fiber diameters of type 1 were $60.7 \pm 9.5 \mu \mathrm{m}$ and of type 2: $46.3 \pm 10.2 \mu \mathrm{m}$. MUNIX mean $95 \pm 36$ was measured. BIA (mean SMI: $\quad 8.93 \pm 1.93 \mathrm{~kg} / \mathrm{m}^{2}$ ) and handgrip strength (mean: $23.4 \pm 9.4 \mathrm{~kg}$ ) was used to calculate $\mathrm{z}$-score. A correlation between $\mathrm{z}$-score of sarcopenia and MUNIX ( $\mathrm{r}=-0.510, \mathrm{p}=0.030)$, MUNIX and Variability Coefficient (VC) of fiber type 1 diameter $(r=0.538$, $\mathrm{p}=0.021)$ and $\mathrm{VC}$ of fiber type 1 diameter and $\mathrm{z}$-score of sarcopenia $(\mathrm{r}=-0.640, \mathrm{p}=0.030)$ could be revealed.

Conclusion: Neurogenic sarcopenia plays a role in the development of muscle loss in hip fractured patients. Predominantly atrophy of type 1 muscle fibers seems to be a histological marker for neurogenic sarcopenia. Further investigations of muscle histology in that cohort should give a deeper insight in the genesis of this subtype of sarcopenia.

\section{0-24}

Standard error of measurement and smallest detectable change of the SarQoL ${ }^{\circledR}$ questionnaire: an analysis of subjects from 8 validation studies

Anton Geerinck ${ }^{1}$, Charlotte Beaudart ${ }^{1}$, Ivan Bautmans ${ }^{2}$, Cyrus Cooper ${ }^{3}$, Fabiana De Souza Orlandi ${ }^{4}$, Jerzy Konstantynowicz ${ }^{5}$, Beatriz Montero Errasquín ${ }^{6}$, Eva Topinková ${ }^{7}$, Maria Tsekoura ${ }^{8}$, Jean-Yves Reginster ${ }^{1}$, Olivier Bruyère ${ }^{1}$

${ }^{1}$ Department of Public Health, Epidemiology and Health Economics, University of Liège, Liège, Belgium, ${ }^{2}$ Frailty in Aging Research Group, Vrije Universiteit Brussel, Brussels, Belgium, ${ }^{3} \mathrm{MRC}$ Environmental Epidemiology Unit, Southampton General Hospital,
Southampton, United Kingdom, ${ }^{4}$ Department of Gerontology, Federal University of São Carlos, São Carlos SP, Brazil, ${ }^{5}$ Department of Paediatric Rheumatology, Immunology, and Metabolic Bone Diseases, Medical University of Bialystok, Bialystok, Poland, ${ }^{6}$ Department of Geriatrics, University Hospital Ramón y Cajal, Madrid, Spain, ${ }^{7}$ Department of Geriatrics and Gerontology, 1st Faculty of Medicine, Charles University, Prague, Czech Republic, ${ }^{8}$ Department of Physical Therapy, Technological Institute of Western Greece, Aigio, Greece

Introduction: The Sarcopenia Quality of Life (SarQoL $\left.{ }^{\circledR}\right)$ questionnaire, a sarcopenia-specific patient-reported outcome measure, contains 55 items and produces 7 domain scores as well as 1 overall quality of life score (all between 0 and 100). This study aims to facilitate the interpretation of the $\mathrm{SarQoL}^{\circledR}$ scores by calculating its standard error of measurement (SEM) and smallest detectable change (SDC).

Methods: Subjects were included from 8 international studies that examined the test-retest reliability of the SarQoL ${ }^{\circledR}$. The SEM, a measure of the error in the scores that is not due to true changes, was calculated by dividing the standard deviation of the difference between test and retest scores (SDdiff) by $\sqrt{ } 2$. The SDC, defined as change beyond measurement error, was calculated by multiplying SDdiff by 1.96 .

Results: A total of 220 sarcopenic subjects, aged $77.0 \pm 7.8$ years and $65.0 \%$ female, were included. The SEM for the overall SarQoL ${ }^{\circledR}$ score ranged from 1.07 to 4.20 for the individual studies, and was 2.98 in the pooled analysis. The SDC for the overall score ranged from 2.96 to 11.65 for the individual studies, and was 8.26 for all subjects.

Conclusions: This study shows that, for individual subjects, a change in overall quality of life of at least 8.26 (on a scale from 0 to 100) would have to be observed to confirm that a true change has occurred. It also demonstrates that the $\mathrm{SarQoL}^{\circledR}$ is a precise instrument, with the observed scores within less than 3 points of the theoretical "true score".

\section{O-25}

Association between frailty and life-course obesity in communitydwelling elders: the GAZEL cohort

Landré Benjamin ${ }^{1}$, Czernichow Sébastien ${ }^{2}$, Zins Marie ${ }^{1}$, Goldberg Marcel $^{3}$, Ankri Joël ${ }^{1}$, Herr Marie ${ }^{1}$

${ }^{1}$ INSERM, U1168, ${ }^{2}$ Georges Pompidou European Hospital, ${ }^{3}$ INSERM, UMS011

Frailty, an indicator of decreased physiologic resources, has been positively associated with obesity in the elderly. However, few studies had tried to capture the association between life-course exposure to obesity and frailty. This study aimed to assess the association between obesity duration in adulthood and the risk of frailty in life over 60 years old. This longitudinal study included 13205 adults from the GAZEL cohort (mean age 70 years). Adapted Fried's frailty criteria were assessed by questionnaire. BMI categories were defined using self-reported height and annual self-reported weight from the 26 years follow-up and according to the World Health Organization cut-offs. Cross-sectional association between obesity and frailty was evaluated; among obese individuals, effect of obesity duration was estimated. We used multinomial regression adjusted for socio-demographic and medical confounders and odds-ratios (ORa) with $95 \% \mathrm{CI}$; results are presented separately for men and women. In 2015, $396(4.4 \%)$ men and $310(9.6 \%)$ women were considered as frail; $1351(15 \%)$ men and $457(14 \%)$ women were obese. A positive association was found between frailty and obesity status: $\mathrm{ORa}=4.4(3.2-6.2)$ in men and 8.1 (5.3-12) in women. Among the 1808 obese individuals, mean obesity duration was 16 years and each year spent in obese state increased the 
risk of being frail in old age for men $(\mathrm{ORa}=1.04 ; 1.01-1.1)$ and women $(\mathrm{ORa}=1.07 ; 1.02-1.13)$. In conclusion, our data shows that obesity duration increased the risk of being frail in life over sixty years old. Early weight control may participate to healthy aging.

\section{0-26}

Muscle function, muscle mass and sarcopenia and its relation to independent ageing. A report from the Uppsala Longitudinal Study of Adult Men (ULSAM)

\author{
Kristin Franzon ${ }^{1}$, Björn Zethelius ${ }^{1}$, Tommy Cederholm ${ }^{2}$, \\ Lena Kilander ${ }^{1}$ \\ ${ }^{1}$ Department of Public Health and Caring Sciences/Geriatrics, \\ Uppsala University, Sweden, ${ }^{2}$ Department of Public Health and \\ Caring Sciences/Clinical Nutrition and Metabolism, Uppsala \\ University, Sweden
}

Introduction: Preserved independence is crucial to maintain high quality of life. We investigated the longitudinal relationship between sarcopenia, its components and independent ageing in very old Swedish men.

Methods: Participants of The Uppsala Longitudinal Study of Adult Men (mean age of 87 years) were investigated. Baseline sarcopenia was defined according to criteria suggested by the European Working Group on Sarcopenia in Older People, i.e. a Skeletal Muscle Index $<7.26 \mathrm{~kg} / \mathrm{m}^{2}$ measured by dual energy X-ray absorptiometry and either gait speed $<0.8 \mathrm{~m} / \mathrm{s}$ or hand-grip strength $<30 \mathrm{~kg}$. At follow-up 5 years later, 129 of the participants were reinvestigated. The outcome, i.e. prevalent independent ageing at follow-up was defined as being community-dwelling, without a diagnosis of dementia, Mini Mental State Examination $\geq 25$ out of 30 possible points, independency in personal care and being able to outdoorwalking without assistance. Analyses were adjusted for age at baseline, smoking status, Charlson comorbidity index and total fat mass. Results: Baseline prevalence of sarcopenia was $19 \%(25 / 129)$. At follow-up, prevalence of independent ageing was 67\% (87/129). There was no association between sarcopenia, skeletal muscle mass and independent ageing. Higher gait speed (odds ratio (OR) per one standard deviation increase $1.86,95 \%$ confidence interval (CI) $1.21-2.86)$ and hand grip strength (OR $1.53,95 \%$ CI 1.02-2.30) were associated with independent ageing.

Conclusions: Muscle function, measured as gait speed and hand grip strength, were associated with independent ageing, whereas there was no association between muscle mass alone or sarcopenia and independent ageing in very old men.

\section{O-27}

\section{Telomere length and frailty: The Helsinki Birth Cohort Study}

Markus J Haapanen ${ }^{1}$, Mia-Maria Perälä ${ }^{2}$, Minna K Salonen ${ }^{2}$, Maria A Guzzardi $^{3}$, Patricia Iozzo ${ }^{3}$, Eero Kajantie ${ }^{2}$, Taina Rantanen ${ }^{4}$, Mika Simonen $^{1}$, Pertti Pohjolainen ${ }^{5}$, Johan G Eriksson ${ }^{1}$, Mikaela B von Bonsdorff

${ }^{1}$ University of Helsinki, Helsinki, Finland, ${ }^{2}$ National Institute for Health and Welfare, Helsinki, Finland, ${ }^{3}$ National Research Council, Pisa, Italy, ${ }^{4}$ University of Jyväskylä, Jyväskylä, Finland, ${ }^{5}$ Age Institute, Helsinki, Finland

Background: Telomere length is associated with aging-related pathologies. While the association between telomere length and frailty has been studied previously, only few studies assessing longitudinal changes in telomere length and frailty exist.

Methods: 1078 individuals born in Helsinki between 1934 and 1944 were measured twice for leukocyte telomere length (LTL) using quantitative real-time PCR at the average ages of 61 and 71 years, and at the latter they were assessed for frailty according to Fried criteria.

Results: The mean \pm SD relative LTLs were $1.40 \pm 0.29$ (average age 61 years) and $0.86 \pm 0.30$ (average age 71 years) for the cohort. A trend of shorter mean relative LTL across frailty groups was observed at 61 years $(p=0.016)$ and at 71 years $(p=0.057)$. Relative LTL at age 61 years was significantly associated with frailty: per 1-unit increase in relative LTL the corresponding relative risk (RR) of frailty was 0.28 (95\% CI $0.08,0.97)$, adjusting for several confounders. Also, LTL at age 71 years was associated with frailty (RR $0.18,95 \%$ CI $0.04,0.81$ ) after adjustment for sex, age and adult socioeconomic status, but further adjustment attenuated the association. No associations between telomere shortening and frailty were observed during the 10-year follow-up.

Key conclusions: Shorter relative LTL was associated with frailty in cross-sectional and longitudinal analyses but telomere shortening was not, suggesting that short LTL may be a biomarker of frailty.

\section{Area: Longevity and prevention}

\section{O-28}

Predicting the onset of functional decline in people aged 65-75 years old: pooled analysis of four European cohort studies

Nini H Jonkman ${ }^{1}$, Marco Colpo ${ }^{2}$, Jochen Klenk ${ }^{3}$, Chris Todd ${ }^{4}$, Trynke Hoekstra ${ }^{5}$, Vieri Del Panta ${ }^{2}$, Kilian Rapp ${ }^{3}$, Natasja M van Schoor ${ }^{6}$, Stefania Bandinelli ${ }^{2}$, Martijn W Heymans ${ }^{6}$, Dominique Mauger $^{4}$, Luca Cattelani ${ }^{7}$, Michael D Denkinger ${ }^{8}$, Dietrich Rothenbacher ${ }^{9}$, Jorunn L Helbostad ${ }^{10}$, Beatrix Vereijken ${ }^{10}$, Andrea B Maier $^{11}$, Mirjam Pijnappels ${ }^{1}$

${ }^{1}$ Department of Human Movement Sciences, Faculty of Behavioural and Movement Sciences, Vrije Universiteit Amsterdam, Amsterdam Movement Sciences, Amsterdam, The Netherlands, ${ }^{2}$ Laboratory of Clinical Epidemiology, InCHIANTI Study Group, LHTC Local Health Tuscany Center, Firenze, Italy, ${ }^{3} 1$ : Department of Clinical Gerontology, Robert Bosch Hospital, Stuttgart, Germany. 2: Institute of Epidemiology and Medical Biometry, Ulm University, Ulm, Germany, ${ }^{4}$ University of Manchester, Manchester, United Kingdom, ${ }^{5}$ 1: Department of Health Sciences, Vrije Universiteit Amsterdam, Amsterdam, The Netherlands. 2: Amsterdam Public Health Research Institute, Department of Epidemiology and Biostatistics, VU University Medical Center, Amsterdam, The Netherlands,

${ }^{6}$ Amsterdam Public Health Research Institute, Department of Epidemiology and Biostatistics, VU University Medical Center, Amsterdam, The Netherlands, ${ }^{7}$ Department of Computer Science and Engineering, University of Bologna, Bologna, Italy, ${ }^{8} 1$ : Institute of Epidemiology and Medical Biometry, Ulm University, Ulm, Germany. 2: Geriatric Research Unit Ulm University and Geriatric Center, Agaplesion Bethesda Hospital Ulm, Ulm, Germany, ${ }^{9}$ Institute of Epidemiology and Medical Biometry, Ulm University, Ulm, Germany, ${ }^{10}$ Department of Neuromedicine and Movement Science, Norwegian University of Science and Technology, Trondheim, Norway, ${ }^{11} 1$ Department of Human Movement Sciences, Faculty of Behavioural and Movement Sciences, Vrije Universiteit Amsterdam, Amsterdam Movement Sciences, Amsterdam, The Netherlands. 2: Faculty of Medicine Dentistry and Health Sciences, Medicine and Aged Care, University of Melbourne, Royal Melbourne Hospital, Melbourne, Australia. 
Introduction: Identifying people at risk of early functional decline in activities of daily living (ADL) is essential for initiating targeted preventive interventions. The aim of this study is to develop and validate a prediction model for the onset of functional decline in 3 -year follow-up in young older adults aged 65-75 years.

Methods: We conducted a pooled analysis of four population-based cohorts, including participants of 65-75 years old who reported no functional limitations at baseline. Functional decline was assessed with two items on basic ADL and three items on instrumental ADL. Multiple logistic regression analysis was used to develop a prediction model, with subsequent bootstrapping for optimism-correction. We applied internal-external cross-validation to assess model discrimination and calibration across the cohorts

Results: 2560 people were included (mean age $69.7 \pm 3.0$ years, $47.4 \%$ female) of whom $572(22.3 \%)$ reported functional decline at three-year follow-up. The final prediction model contained 10 out of 22 predictors: age, handgrip strength, gait speed, five-repeated chair stands time (non-linear association), body mass index, cardiovascular disease, diabetes, chronic obstructive pulmonary disease, arthritis, and depressive symptoms. The optimism-corrected model showed good discrimination with a $\mathrm{C}$ statistic of 0.72 . Calibration intercept was 0.06 and calibration slope was 1.05 . Internal-external cross-validation showed consistent performance of the model across the four cohorts. Conclusions: Based on pooled cohort data analyses we showed that the onset of functional decline in ADL in three years in people aged 65-75 years can be predicted by specific physical performance measures, age, body mass index, depressive symptoms, and chronic conditions.

\section{O-29}

"Active health promotion in old age": who benefited from participation? Compression of morbidity assessed in the Longitudinal Urban Cohort Ageing Study (LUCAS) over 13.8 years

\section{Ulrike Dapp ${ }^{1}$, Lilli Neumann ${ }^{1}$, Wolfgang von Renteln-Kruse ${ }^{1}$,} Christoph E. Minder ${ }^{2}$

${ }^{1}$ Albertinen-Haus, Centre of Geriatrics and Gerontology, Scientific Department at the University of Hamburg, Hamburg, Germany, ${ }^{2}$ Horten-Zentrum, University of Zürich, Zürich, Germany

Introduction: The WHO defined healthy ageing as development and maintenance of functional competence. Unfavourable ageing-described by the term frailty-is characterised by decline in functional reserves. The frailty process can be influenced positively. Previous interventions concerned mostly hospital patients and nursing home residents. Here, we examined the maintenance of functional competence in a non-institutional urban community setting.

Methods: In 2001, embedded in the Longitudinal Urban Cohort Ageing Study (LUCAS), the intervention ,Active health promotion in old age" was offered by a team of experts in ageing covering geriatrics, physiotherapy, nutritional science and social work to small groups of independent people 60 years and older without need of nursing care. Its effectiveness was evaluated in LUCAS over 13.8 years. Survival and disability free survival were calculated separately in persons with good functional competence (many functional reserves) and persons with few reserves, using Kaplan-Meier curves. Cox regression was used for adjustments (age, gender, educational level, chronic diseases and functional status using). This methodology facilitates the study of interrelationships between mortality and morbidity (compression of morbidity) including an impact from lifestyle interventions.
Results: The group of participants with good functional competence had longer disability-free lifes $(\mathrm{p}<0.001)$, and their average fraction of life-time with disability was shorter than either for non-participants, or those with low functional competence.

Key conclusions: Evidence from these analyses on compression of morbidity show strongest effects in the group of persons with high functional competence, i.e., exactly the target group of the health promotion programme.

\section{O-30}

Is healthy overweight in midlife also associated with successful aging? The Helsinki Businessmen Study

Timo Strandberg ${ }^{1}$, Satu Jyväkorpi ${ }^{2}$, Annele Urtamo ${ }^{2}$, Mikaela von Bonsdorff $^{3}$

${ }^{1}$ Universities of Helsinki and Oulu, Helsinki University Hospital, Helsinki, Finland, ${ }^{2}$ University of Helsinki, Helsinki, Finland,

${ }^{3}$ University of Jyväskylä, Jyväskylä, Finland

Introduction: The clinical significance of metabolically healthy overweight and obesity (MHO) is controversial. We studied this in a homogenous male cohort and related MHO, metabolically healthy normal weight (MHN), and metabolically unhealthy overweight (MUO) to health-related quality of life (HRQoL) and successful aging during a 32-year follow-up.

Methods: The Helsinki Businessmen Study (HBS, men born 1919-1934) cohort has been followed-up since the 1960-70s. In 1986 , overweight $\left(\mathrm{BMI}>25 \mathrm{~kg} / \mathrm{m}^{2}\right.$ ) and metabolic health (IDF definition) were determined in 1309 men with median age of 60 years. HRQoL was assessed in the years 2000 and 2007 using RAND-36/SF-36 (8 subscales), and total mortality up to 2018 whereupon the proportion of men reaching 90 years was calculated. Results: In 1986, 469, 538, and 276 men had MHN, MHO, and MUO, respectively; only 26 men had metabolically unhealthy normal weight and were excluded from main analyses. During the 32-year follow-up, $947(72.3 \%)$ men had died. MUO had highest mortality $(79.7 \%)$, no difference between MHN (69.2\%) and MHO (70.6\%). As compared to MUO ( $n=43,15.6 \%)$, odds of reaching 90 years was significantly increased for MHN ( $\mathrm{n}=120,25.6 \%$; OR $1.71,95 \%$ CI $1.14-2.55)$, but not for MHO ( $\mathrm{n}=103,19.1 \%$; OR $1.33,95 \%$ CI $0.89-1.99)$. Clinically meaningful impairment of several RAND-36 subscales was observed in MHO as compared to MHN, especially in 2000.

Key conclusions: Compared to normal weight, metabolically healthy overweight in midlife does not increase long-term mortality, but is associated with impaired quality of life in old age.

\section{0-31}

A new virtual ward: assessing its impact on elderly patients in the Poole North locality in Dorset, UK

Charles Jefferson-Loveday ${ }^{1}$

${ }^{1}$ Poole General Hospital, Poole, UK

Introduction: Dorset has an expanding elderly population above the national average. [1] The Poole North Locality Virtual Ward (VW) started on the 20th September 2017 replacing a standard model. Virtual wards allow community based multi-disciplinary team (MDT) discussion of vulnerable elderly patients and help early domiciliary intervention.

Methods: Unplanned admissions (UA), length of stay (LOS), total hospital bed days (THBD) and mortality (M) were compared in VW 
and standard MDT groups ( $\mathrm{n}=155$ per group) between May 2017 and January 2018 using computer records. Some patients had been discussed on both formats (crossed over). Baseline characteristics of VW and standard MDT patients were examined (90 from each group). Results: Baseline characteristics between the two groups were generally similar. Overall the VW patients showed a reduction in THBD, average LOS and total UA. However, mortality marginally increased. Analysis of the early stages of the VW showed an increase in THBD, average LOS and total UA. Sub-analysis of patients that did not cross over the two groups showed similar trends.

Key conclusions: The data suggests that our VW has potentially facilitated in the prevention of UA and helped reduce how long elderly patients are in hospital. However, an increase in outcomes was observed at the start of the VW and an overall marginal increase in mortality. Reasons could be seasonal changes (summer into autumn) or the VW selecting out more vulnerable patients at a time when it had not fully developed its service. [1] Dorset STP; http://www. dorsethealthcare.nhs.uk.

\section{0-32}

\section{Midlife predictors of reaching 90 years of age in the Helsinki} Businessmen Study

Annele Urtamo ${ }^{1}$, Satu Jyväkorpi ${ }^{1}$, Kaisu Pitkälä ${ }^{1}$, Timo Strandberg ${ }^{2}$

${ }^{1}$ University of Helsinki, Henlsinki, Finland, ${ }^{2}$ Universities of Helsinki and Oulu, Helsinki, Finland

Introduction: Previous studies of longevity have indicated the association of lifestyle and biological factors in midlife with survival until old age. However, less is known about various midlife factors associated with reaching oldest ages, 90 years or more. We studied midlife predictors of this extreme longevity among oldest-old businessmen, among whom socioeconomic effects on longevity are inherently adjusted for.

Methods: The Helsinki Businessmen Study cohort (born in 1919-1934) consists of men from the highest socio-economic class. Midlife factors were assessed in $1974(\mathrm{n}=2600)$, at an average age of 48 years. Cumulative total mortality was retrieved in March 2018 from the Finnish Population Information System, whereupon 409 men of the cohort had reached 90 years or more. Midlife factors between men reaching and not-reaching 90 years were compared, and analysed with logistic models (odds ratios [OR] with 95\% confidence intervals $[\mathrm{CI}])$.

Results: The following midlife factors were associated with reaching 90 years of age $(\mathrm{p}<0.001)$ : lower body mass index $(\mathrm{BMI})$, systolic and diastolic blood pressure, total cholesterol level, triglycerides, and one-hour post-load glucose. It was also associated with non-smoking, and lower consumption of alcohol and coffee in midlife. In adjusted analyses, the strongest midlife predictors of preventing extreme longevity were smoking (OR: 0.45 CI $0.34-0.61$ ) and higher BMI (OR per 1unit increase: 0.90 CI $0.85-0.94$ ).

Conclusions: Our study revealed that modifiable midlife factors, especially non-smoking and lower BMI were associated with reaching 90 years of age. The results show the importance of considering longterm predictors of longevity.
O-33

Prevention for senior citizens requiring care- Lübeck Model Worlds of Movement (Lübecker Modell Bewegungswelten)

S Krupp ${ }^{1}$, J Kasper ${ }^{1}$, F Balck ${ }^{2}$, A Hermes ${ }^{3}$, T Schmidt ${ }^{3}$, M Willkomm ${ }^{1}$, C Ralf ${ }^{1}$

${ }^{1}$ Research Group Geriatrics Lübeck, Hospital Red Cross Lübeck Geriatric Centre, Germany, ${ }^{2}$ Research Group Geriatrics Lübeck, Hospital Red Cross Lübeck Geriatric Centre, Germany, University Hospital Dresden, Technical University Dresden, Germany, ${ }^{3}$ Cancer Center North, University Hospital Schleswig-Holstein, Campus Kiel, Germany

Introduction: In 2015 the Research Group Geriatrics Lübeck developed a preventive program for seniors requiring care. Each of the training sessions held in nursing homes is a 1-h imaginary trip into a "world of movement" like "On the beach", "Shopping tour" or "House construction", offering exercises that are derived from movements well known to the participants. The effects have been evaluated.

Methods: The intervention group (IG) consisted of 171 participants in Lübeck $(81.9 \pm 9.2$ years, $21.6 \%$ male, Barthel Index $(\mathrm{BI})$ $76.3 \pm 15.8$ ). They got the training described above twice a week in ten groups. 84 citizens in nursing homes in Kiel served as control group (CG) (84.4 \pm 7.6 years, $23.8 \%$ male, BI $73.6 \pm 16.4)$. Participation in other activities was not limited. Assessment was performed at baseline and after 3, 6, 9 and 12 months.

Results: After 12 months the BI had fallen by $7.3 \pm 14.5$ points in the CG $(n=55)$, just $7.3 \%$ of the participants improved their results. In the IG $(\mathrm{n}=107)$ the $\mathrm{BI}$ had risen by $1.1 \pm 10.1$ points, $40.2 \%$ were better off than a year ago ( $\mathrm{p}=0.000$, intention-to-treat analysis). Persons in the IG had a better outcome than those in the CG concerning endurance, balance, mobility under dual task conditions and other parameters.

Key conclusions: Elderly that already need care have a good chance to prevent further deterioration of function and thereby stabilize performance in basic activities of daily living when they engage in standardized multimodal training like „Lübeck Model Worlds of Movement“.

\section{0-34}

The burden and mental health among singleton caregiver for multiple family members: a nationwide cross-sectional study

Miku Izutsu ${ }^{1}, \mathrm{Su} \mathrm{Ya}^{2}$, Fuhka Ito ${ }^{3}$, Yuhka Takahashi ${ }^{4}$, Kengo Hirayama ${ }^{5}$, Michiko Yuki $^{5}$

${ }^{1}$ Graduate School of Health Sciences, Hokkaido University, Hokkaido, Japan, ${ }^{2} \mathrm{Su}$ Ya, ${ }^{3}$ Tokyo Women's Medical University Hospital, Tokyo, Japan, ${ }^{4}$ Teine Keijinkai Hospital, Hokkaido, Japan, ${ }^{5}$ Faculty of Health Sciences, Hokkaido University, Hokkaido, Japan

Introduction: Because of the increasing older population in Japan, the cases of a single caregiver taking care of two or more family members have been increasing. The health of family caregiver has major influence on providing care for care recipient. This study aimed to clarify the degree of care burden and mental health of primary caregiver who take care multiple family members.

Methods: We conduct a survey of $20 \%$ of 5251 visiting nursing stations, 53 of which agreed to participate in the study. Station managers were requested to select caregivers and distribute anonymous self-administered structured questionnaire survey. The questionnaire included measures for caregivers' burden (Japanese version of Zarit Caregiver Burden Interview: J-ZBI) and mental 
health (Geriatric Depression Scale-Revised 15: GDS-15). Analysis compares the subjects by gender.

Results: Ninety-one singleton caregiver (17 men, 74 women; mean age $58.4 \pm 8.4$ years) gave reliable responses in this study. Singleton caregiver with 50-59 years old (42.9\%) were most frequently. There were no significant difference between genders in J-ZBI score $(\mathrm{t}=1.242, \mathrm{p}=0.05)$ and GDS-15 scores $(\mathrm{t}=0.422, \mathrm{p}=0.05) .82 .3 \%$ of men and $80.0 \%$ of women were suspected as depression, scoring 5 or higher on the GDS-15.

Conclusions: The majority of single caregivers taking care of multiple family members were women. However, there was no major difference of mental status between the genders. Moreover, there was no significant difference of depression, but more than $80 \%$ of caregivers in both genders showed a depressive tendency. Therefore it is important to develop support systems for singleton caregivers.

\section{0-35}

The pharmacist in the acute geriatric inpatient treatment teamimpact on hospital readmissions and trans-sectoral communication (AGITATE)

Mathias Freitag ${ }^{1}$, Olaf Krause ${ }^{2}$, Thea Laurentius ${ }^{1}$, Cornelius Bollheimer $^{1}$, Albrecht Eisert ${ }^{3}$

${ }^{1}$ Department of Geriatric Medicine, RWTH Aachen University Hospital, Germany, ${ }^{2}$ Centre for Medicine of the Elderly, DIAKOVERE Henriettenstift, Hannover, Germany; Institute for General Practice, Hannover Medical School, Hannover, Germany, ${ }^{3}$ Hospital Pharmacy, RWTH Aachen University Hospital, Germany

Introduction: This project aims to measure the effect of a clinical pharmacist on trans-sectoral adherence in terms of drug therapy safety and hospital readmissions.

Methods: The study is conducted as a before/after comparison and will end in 12/2019 (clinicaltrials NCT03412903). In the ongoing control phase, the pharmacist only surveys the patient's medication history. In the following second phase, he will additionally ascertain the patient's adherence and carry out a thorough medication review. Finally, he will prepare a comprehensive paragraph in the discharge report discussing medication changes and persisting drug interactions. This shall provide the general practitioner (GP) with all necessary information aiming a broader acceptance of discharge medication. Apart from the main outcome-i.e. number of hospital readmissions and days spent at home within 6 months after discharge - the time to readmission, differences between pharmaceutical/medical medication history ascertainment and the acceptance of the supposed medication regime by the GP will be analysed.

Preliminary results: 28 patients have been meanwhile included since 02/2018. In 10 cases the medication histories showed clinical relevant mistakes; three cases were inconclusive. The mean time spent at home was 16.8 days in the first month $(\mathrm{n}=15, \mathrm{SD}=12.32)$ with four reported hospital readmissions. In 6 of 17 cases, drug therapy recommendations were not accepted by the GP.

Clinical Relevance: First results of AGITATE show a relevant information loss regarding drug therapy at the interface of out- and inpatient care, which could potentially harm the patient. The first phase will serve as a comparison for the second phase, in which a clinical pharmacist provides counselling for both patients and medical professionals. The effect of this service on all outcomes will be evaluated.

\section{O-36}

Fifty percent reduction of falls and fractures among elderly in nursing homes

Christian Molnár ${ }^{1}$

${ }^{1}$ Familjeläkarna SÄBO

Fall-related injuries is one of the most common causes of death and reduced life quality among elderly in Sweden. With the goal to halve the incidence of falls and fractures we developed a new way of organising the multiprofessional team around patients living in nursing-homes, together with the establishment of a small central organisation collecting data and presenting regular feed-back to the team. Two year follow-up data shows a stable decline of the number of falls and fractures by more than $50 \%$ in the units working according to the new method as opposed to units not yet having implemented it. These results implicate a persistent and somewhat surprisingly great effect of the importance of how we organise the multiprofessional team around the patient in preventing falls and fallrelated injuries.

Area: Comorbidity and multimorbidity

O-37

Influence of cognitive impairment on cardiac mortality after percutaneous coronary intervention in very elderly patients

Tomoko Tomioka ${ }^{1}$, Kento Fukui ${ }^{1}$, Yoshitaka Ito $^{1}$, Hiroki Shioiri ${ }^{1}$, Jiro Koyama $^{1}$, Kanichi Inoue ${ }^{1}$

${ }^{1}$ Southmiyagi Medical Center, Miyagi, Japan

Introduction: Percutaneous coronary intervention (PCI) for patients with ischemic heart disease is a favorable strategy to obtain risk reduction of cardiac mortality, even for very elderly patients. However, influence of cognitive impairment (CI) on the mortality after PCI hasn't been estimated, so that we estimated it herein targeting very elderly patients.

Methods: We retrospectively examined 102 patients above 80 years old who were performed PCI from 2012 to 2014 at our facility. We categorized them into the CI group (less than 20 points by MiniMental State Examination and/or already diagnosed as CI by neurologists) and the non-CI group. We then evaluated the cardiac mortality during 1 year after PCI in both groups, and estimated the involvement of $\mathrm{CI}$ on the cardiac mortality by multivariate analysis adjusted for classical risk factors and CI. Furthermore, in the CI group, we estimated the prognostic factors including family structure on the cardiac mortality.

Results: Among 102 patients, 42 were in the CI group. The cardiac mortality was 68 and $24 \%$ in the CI and the non-CI groups, respectively (OR 4.5, 95\% CI 1.12-17.99, P < 0.05). In all subjects, CI was the independent predictor of the cardiac mortality with 12.9 of OR (95\% CI 1.5-211.5, P < 0.05). Additionally, in the CI group, patients, living only with a partner who was also suffering from $\mathrm{CI}$, were independently predisposed to higher risk on cardiac death $(\mathrm{P}<0.05)$. Conclusions: These results suggest the CI significantly influence the cardiac mortality after PCI, and we should consider the patient's living surroundings including family structure. 


\section{0-38}

Repeated cerebrospinal fluid removal procedure in older patients with Idiopathic Normotensive Hydrocephalus ineligible for surgical treatment

Paolo Dionigi Rossi ${ }^{1}$, Carolina Nani ${ }^{2}$, Mauro Pluderi ${ }^{3}$, Dario Consonni ${ }^{4}$, Matteo Cesari ${ }^{5}$, Sarah Damanti ${ }^{1}$

${ }^{1}$ Geriatric Unit, IRCCS Ca' Granda Ospedale Maggiore Policlinico, Milan, Italy, ${ }^{2}$ Elderly Care and General Medicine, King's College Hospital, London, UK, ${ }^{3}$ Neurosurgery Unit, IRCCS Ca' Granda Ospedale Maggiore Policlinico, Milan, Italy, ${ }^{4}$ Unit of Epidemiology, IRCCS Ca' Granda Maggiore Policlinico Hospital Foundation, Milan, Italy, ${ }^{5}$ University of Milan, Milan, Italy

Introduction: Shunt interventions represent the key treatment of Idiopathic Normotensive Hydrocephalus (iNPH) [1], but are complicated by high costs, risk of complications [2, 3], and temporary benefits $[4,5]$, especially in frail older people. Consecutive cerebral spinal fluid removal procedures (CSF TAP test) have been used to relieve patients' symptoms and predict surgery responsiveness. We evaluated the effect of repeated CSF TAP tests in older iNPH patients ineligible for surgery.

Methods: Prospective, monocentric, pilot study performed in a tertiary hospital of Milan, between 2011 and 2016. Inclusion criteria: age $\geq 75$ years; positive criteria for probable iNPH [6]; ineligibility for surgery. Exclusion criteria: secondary hydrocephalus, Mini Mental State Examination (MMSE) $<20$, gait disorders secondary to other causes. Pre-CSF TAP test evaluation included: Barthel index, continence, Performance-Oriented Mobility Assessment (POMA), Timed Up and Go (TUG) test, and MMSE. All the tests were periodically repeated during the follow-up. Every peri-procedural adverse event was recorded.

Results: Twenty-eight of the 39 enrolled patients (mean age $82.5 \pm 4.7$ years; women $43.5 \%$ ) showed an improvement in Barthel, TUG, POMA and MMSE scores after the drainage procedures, whereas continence was poorly influenced over the follow-up (median 31 months). No major adverse event was recorded from the total 83 procedures. The mean period of functional benefit between procedures was 7 months. Patients who chose to interrupt the study for subjective reasons showed a more rapid worsening of the health status over time.

Key conclusions: Periodic CSF TAP tests are safe, allow to control iNPH symptoms and improve functional status in geriatric patients. References:

(1) Rubén Martín-Láez et al. Incidence of Idiopathic Normal-Pressure Hydrocephalus in Northern Spain; Rube'n Martı'n-La'ez et al. Epidemiology of Idiopathic Normal Pressure Hydrocephalus: A Systematic Review of the Literature. World Neurosurg. (2015) 84, 6:2002-2009.

(2) M. Bergsneider et al. Surgical Management of Idiopathic Normal Pressure Hydrocephalus Neurosurgery. 2005.

(3) Halperin et al. Practice guidelines: idiopathic normal pressure hydrocephalus: response to shunting and predictors of response Neurology 2015; 85:2063-71.

(4) Malm J, Kristensen B, Stegmayr B, Fagerlund M, Koskinen LO. Three-year survival and functional outcome of patients with idiopathic adult hydrocephalus syndrome. Neurology. 2000; 55:576-8.

(5) Raftopoulos C, Massager N, Baleriaux D, Deleval J, Clarysse S, Brotchi J. Prospective analysis by computed tomography and longterm outcome of 23 adult patients with chronic idiopathic hydrocephalus. Neurosurgery. 1996; 38:51-9.

(6) Marmarou A, Black P, Bergsneider M, Klinge P, Relkin N; International NPH Consultant Group. Guidelines for management of idiopathic normal pressure hydrocephalus: progress to date. Acta Neurochir Suppl. 2005;95:237-40.

\section{0-39}

\section{Hypothyroidism and incidence of geriatric syndromes}

Mario Ulises Perez-Zepeda ${ }^{1}$, Matteo Cesari ${ }^{2}$, Ronald Camilo GomezArteaga $^{3}$, Miguel Borda ${ }^{3}$, Carlos Cano-Gutierrez ${ }^{3}$

${ }^{1}$ Instituto Nacional de Geriatria, Ciudad de México, Mexico,

${ }^{2}$ Geriatric Unit, Fondazione IRCCS Ca' Granda-Ospedale Maggiore Policlinico, Milan, Italy, ${ }^{3}$ Unidad de Geriatría, Departamento de Medicina Interna, Hospital Universitario San Ignacio, Bogotá, Colombia

Objective: To determine the incidence of geriatric syndromes in community dwelling older adults with subclinical hypothyroidism.

Methods: MHAS is a prospective study conducted in Mexico, consisting of four waves, (2001, 2003, 2012 and 2015), that sequentially included and described a large and representative sample of Mexican adults 50 years and older. We considered the surveys conducted in 2012 and 2015, from which a subsample of 2089 subjects with TSH test results derives. Form this last subsample, we included 1628 individuals, whose TSH leves were in the subclinical range $(4.5-10 \mathrm{ng} / \mathrm{ml})$.

Results: In the model adjusted by age, sex, educational level, marital status, years of education, and smoking, the multivariate analysis showed that when comparing data obtained from 2012 wave, with 2015 wave results, there was a significant greater incidence of some GS such as falls (OR 1.79 IC 1.16-2.77 P $=0.0116)$, fatigue (OR 2.17 IC $1.40-3.38, \mathrm{P}=0.0348$ ) and depression (OR $1.70 \mathrm{CI} 1.06-2.71$, $\mathrm{P}=0.0246$ among the subclinical hypothyroidism group.

Conclusions: This study showed a greater incidence of GS in subjects 50 years and older with sub-clinical hypothyroidism, when compared to those with normal thyroid function. These findings lead to generate proposals for the formulation of new studies.

\section{O-40}

Predictors of orthostatic hypotension in hypertensive patients: the role of hypotensive drugs

Giulia Rivasi ${ }^{1}$, Letizia Martella ${ }^{1}$, Giada Turrin ${ }^{1}$, Antonella Giordano ${ }^{1}$, Chiara Lorenzi ${ }^{1}$, Virginia Tortù ${ }^{1}$, Maria Flora D'Andria ${ }^{1}$, Giulia Casini ${ }^{1}$, Martina Rafanelli ${ }^{1}$, Andrea Ungar ${ }^{1}$

${ }^{1}$ Referral Centre for Hypertension in the Elderly, Department of Geriatrics and Geriatric Intensive Care Unit, Careggi Hospital and University of Florence, Florence, Italy

Introduction: orthostatic hypotension $(\mathrm{OH})$ is a common finding in hypertensive patients. Different predictors of $\mathrm{OH}$ are reported in Literature, but the role of anti-hypertensive drugs is unclear; moreover, several drugs with a different indication have hypotensive effects and may favor $\mathrm{OH}$. We evaluated prevalence and predictors of $\mathrm{OH}$ in hypertension, with a particular focus on anti-hypertensive and hypotensive drugs.

Methods: 386 consecutive patients referred to our Centre were evaluated for OH (January 2013-March 2017); a comparison between patients with and without $\mathrm{OH}$ was performed, analyzing clinical and pharmacological features.

Results: Our population had a mean age of 62 years (range 33-92; $51 \%$ male). Patients were taking $2.1 \pm 1.3$ anti-hypertensive drugs, mainly calcium-antagonists $(42.2 \%)$, angiotensin-receptor blockers (ARB, 37.8\%) and angiotensin-converting enzyme inhibitors (ACEi, $36.5 \%$ ). $\mathrm{OH}$ was diagnosed in the $32.6 \%$ of patients; those with $\mathrm{OH}$ were older $(\mathrm{p}<0.001)$ and more frequently received ARB $(p=0.008)$, nitrates $(p=0.008)$, alpha-blockers $(p=0.05)$, benzodiazepine $(\mathrm{p}=0001)$ and Levodopa $(\mathrm{p}=0.01)$; ACEi were more 
common in patients without $\mathrm{OH}(\mathrm{p}=0.02)$. At multivariate analysis, age (OR 1.03; $\mathrm{p}=0.02$ ), nitrates (OR 9.95; $\mathrm{p}=0.04$ ), alpha-blockers (OR 2.58; $\mathrm{p}=0.05)$ and benzodiazepine (OR 2.41; $\mathrm{p}=0.01$ ) were predictors of $\mathrm{OH}$; $\mathrm{ACEi}(\mathrm{OR} 0.53 ; \mathrm{p}=0.01)$ showed a protective role. If analysis was stratified by age, ARB were predictive of $\mathrm{OH}$ in patients aged 80 or older (OR 13.72; $\mathrm{p}=0.007$ ).

Conclusions: $\mathrm{OH}$ is frequent in hypertensive patients. Age, nitrates, alpha-blockers and benzodiazepine are predictive of $\mathrm{OH}$, whereas ACEi have a protective role. ARB are associated with a higher risk of $\mathrm{OH}$ in patients aged 80 or older.

\section{0-41}

QT Dispersion in masked hypertention: an arrhythmogenic risk factor (G-MASH-QT)

Mert Esme ${ }^{1}$, Cafer Balci ${ }^{1}$, Serkan Asil ${ }^{2}$, Bunyamin Yavuz ${ }^{3}$, Rana Tuna Dogrul ${ }^{1}$, Mustafa Kemal Kılıç ${ }^{4}$, Muhammed Cemal Kizılarslanoğlu ${ }^{5}$, Hacer Doğan Varan $^{6}$, Meltem Halil ${ }^{1}$, Mustafa Cankurtaran $^{1}$, Burcu Balam Yavuz ${ }^{1}$

${ }^{1}$ Hacettepe University Faculty of Medicine, Department of Internal Medicine Division of Geriatrics, Ankara, Turkey, ${ }^{2}$ Gulhane Education and Research Hospital, Department of Cardiology, Ankara, Turkey, ${ }^{3}$ Medical Park Hospital, Department of Cardiology, ${ }^{4}$ Ankara Education and Research Hospital, Ankara, Turkey, ${ }^{5}$ Gazi University Faculty of Medicine, Department of Internal Medicine Division of Geriatrics, Ankara, Turkey, ${ }^{6}$ Ankara Numune Education and Research Hospital, Ankara, Turkey

Introduction: Detection of blood pressure above normal limits with ambulatory blood pressure measurement (ABPM) when office measurements are normal is defined as masked hypertension $(\mathrm{MH})^{1}$. QT dispersion (QTd) is defined as the difference between the longest QT interval and the shortest QT interval in 12-channel electrocardiography (ECG), and it increases the risk of arrhythmia ${ }^{3}$. Hypertension is known to cause QTd, but the relationship between MH and QTd was not previously examined. Our aim was to examine the relationship between $\mathrm{MH}$ and QTd.

Methods: 74 normotensive patients were enrolled. $\mathrm{MH}$ was diagnosed by ABPM according to ESC13 guidelines. Patients with atrial fibrillation, branch block, ST segment changes, those using drugs that can affect QT, and patients with coronary artery disease were excluded. In the V1-V6 leads, the QT intervals were measured, the minimum-maximum values were determined and their difference was recorded in milliseconds. QTd was compared in patients with and without MH.

Results: $\mathrm{MH}$ was detected in $30(40 \%)$ patients with a mean age of $71.0 \pm 5.9$. QTd was significantly higher in the MH group than the normal group [52 (0-100) vs. $40(0-80)$, respectively; p: 0.025]. Logistic regression analysis showed that MH was associated with QTd and increased the risk 3.4-fold (p: 0.017; OR: 3.4; \%95 CI 1.2-9.2).

Conclusions: In this study, an arrhythmogenic indicator QTd was found to be significantly higher in masked hypertension. QTd is related to life span, cardiac mortality, and cerebrovascular events. Therefore, the relationship between QTd and MH is very striking. ECG of all MH patients should be examined and it should be known that they are at risk for arrhythmia.

References:

1. Mallion J-M, et al. Predictive factors for masked hypertension within a population of controlled hypertensives. Journal of hypertension. 2006;24: 2365-2370.2. Lepeshkin E. et al. Themeasurement of the QTinterval of the electrocardiogram Circulation 1952: 26:378-883. Day CP et al. QT dispersion:an indication of arrhytmia in patients with long QTintervals. Br Heart J 1990; 63: 342-44.
Yavuz B, et al. QT Dispersion Increases with Aging 2006; https://doi.org/10.1111/j.1542-474x.2006.00093.x

\section{$0-42$}

Profiling the hospital-dependent oldest-old patient: a case-series study of multiple readmissions at Parma University-Hospital, Italy

Andrea Ticinesi ${ }^{1}$, Fulvio Lauretani ${ }^{1}$, Beatrice Prati ${ }^{1}$, Antonio Nouvenne $^{1}$, Ilaria Morelli ${ }^{2}$, Claudio Tana ${ }^{2}$, Tiziana Meschi ${ }^{1}$

${ }^{1}$ Department of Medicine and Surgery, University of ParmaGeriatric Rehabilitation Department, Parma University-Hospital, Parma, Italy, ${ }^{2}$ Geriatric Rehabilitation Department, Parma UniversityHospital, Parma, Italy

Background: Hospital readmission risk is high in multimorbid patients aged $\geq 75$ years old. A condition of "hospital-dependence" may arise: patients get adapted to the hospital care and, once discharged, may experience quick health status decline, requiring readmission despite appropriate treatments. The clinical characteristics of this patient-category are still poorly investigated.

Aim: To retrospectively describe the characteristics of 118 patients aged $\geq 75$ who were urgently admitted to Parma University-Hospital, the only hospital facility of the district, at least 4 times in 2015 .

Methods: For each patient and admission, data on multimorbidity (Cumulative Illness Rating Scale Comorbidity Score, CIRS-CS, and Severity Index, CIRS-SI), frailty (Rockwood Clinical Frailty Scale, RCFS), functional dependence, mobility, polypharmacy, caregiver, length of stay (LOS) and interval between consecutive admissions were extrapolated from clinical records. Mortality during the years 2015 and 2016 was assessed on the institutional database.

Results: At the first admission, patients $(72 \mathrm{~F}$, age $83.7 \pm 4.9$ ) had a high burden of polypharmacy (median number of drugs 8.5 , IQR 6-11) and multimorbidity (CIRS-CS $15.8 \pm 4.1$, CIRS-SI $2.9 \pm 1.1$ ). However, most $(55.5 \%)$ were fit or pre-frail according to RCFS (score 1-4). At multivariate models, CIRS-SI was significantly correlated with $\operatorname{LOS}(\beta \pm$ SE $2.32 \pm 0.92, \mathrm{p}=0.01)$ and readmission interval $(\beta \pm \mathrm{SE}-22.92 \pm 9.84, \mathrm{p}=0.03)$. By the end of $2016,66 \%$ of patients had died. Baseline frailty (RCFS $>4$ ) was the only significant predictor of mortality (RR 1.62, 95\% CI 1.14-2.30, $\mathrm{p}=0.007$ ). Conclusions: At baseline, most patients with multiple readmissions had severe multimorbidity and polypharmacy, but exhibited an unexpectedly low prevalence of frailty/disability. When present, frailty represented the main risk factor for mortality.

\section{O-43}

Longitudinal trajectories of multimorbidity in old age: The role of childhood circumstances, education, life-long work stress, and late-life social network

Serhiy Dekhtyar ${ }^{1}$, Davide Vetrano ${ }^{1}$, Alessandra Marengoni ${ }^{2}$, Hui Xin Wang ${ }^{3}$, Kuan-Yu Pang ${ }^{1}$, Laura Fratiglioni ${ }^{1}$, Amaia CalderónLarrañaga $^{1}$

${ }^{1}$ Aging Research Center. Karolinska Institutet, Solna, Sweden, ${ }^{2}$ University of Brescia, Brescia, Italy, ${ }^{3}$ Stress Research Institute, Stockholm University, Stockholm, Sweden

Introduction: Life experiences, such as childhood socioeconomic circumstances, education, work-related stress, and characteristics of the social network are implicated in old age-health and well-being. In 
this study, we examine their association with the speed of multimorbidity development during aging.

Methods: The data are from the community-based longitudinal study of aging - the Swedish National Study on Aging and Care in Kungsholmen. Information on parental SES, highest-attained education, job strain in the longest-held occupation, and the size of the late-life social network were collected during comprehensive nurse interviews at baseline. Number of chronic conditions was assessed using physician examination and outpatient and inpatient medical records. Linear mixed models adjusted for health behaviors, the burden of medications, and the competing risk of death were used to assess the association between life experiences and the change in the number of chronic conditions over 9 years of follow-up.

Results: Secondary and university education was associated with a decelerated speed of multimorbidity development over time ( $\beta=-0.07$ and $\beta=-0.12$, respectively; both $p<0.05$; reference: elementary education). Multimorbidity development was also reduced in those with active life-long occupations $(\beta=-0.08, \mathrm{p}<0.05$, reference: high-strain jobs), as well as in those with both medium and large social networks in old age $(\beta=-0.06$ and $\beta=-0.11$, respectively; both $\mathrm{p}<0.01$; reference: small network size). Nonmanual parental occupation was protective in minimally-adjusted models, although its effect was fully attenuated by education.

Key conclusions: Longitudinal trajectories of multimorbidity in old age can be in part attributed to inter-individual differences in life experiences over the life course.

\section{0-44}

A James Lind alliance priority setting partnership for research about multiple conditions in later life

S G Parker ${ }^{1}$, L Corner ${ }^{1}$, K Laing ${ }^{1}$, G Nestor ${ }^{1}$, A Sayer ${ }^{1}$, J Frith ${ }^{1}$, $\mathrm{J}_{\text {Collerton }}^{2}$, D Craig ${ }^{1}, \mathrm{~K}$ Cowan ${ }^{3}$

${ }^{1}$ Newcastle University, UK, ${ }^{2}$ Academic Health Science Network for the North East and North Cumbria, UK, ${ }^{3}$ James Lind Alliance, UK

Introduction: In the UK by 2039 , over $8 \%$ of the population is projected to be aged 80 or older. Multiple conditions are a fact of life for this rapidly growing age group. Multiple conditions in later life has not (until recently) been prioritised as a topic for research. We have adapted the rigorous and inclusive methodology of a James Lind Alliance Priority Setting Partnership to identify the top 10 research topics in this field.

Methods: A multi-stakeholder steering group $(\mathrm{n}=24)$ oversaw a questionnaire survey and interviews with older people, their carers and health care professionals $(n=359)$ which identified 236 questions. These questions were collated, checked for relevance and reviewed against existing research evidence. The resultant 97 "unanswered questions" went to interim priority setting by survey and interviews. Finally a mix of people aged $80+$ living with 3 or more conditions, carers and health and social care providers considered a 21 questions short list to finalise and prioritise the top 10 .

Results: The organisation and delivery of health and social care and the reduction of social isolation were the top priorities for research. Prevention, assessment and treatment of multiple conditions and frailty were ranked alongside the maintenance of physical and psychological wellbeing for patients and carers

Conclusions: In an inclusive and methodologically rigorous priority setting process, older people, their carers and health care professionals identified issues from organisational, biomedical and psychosocial domains among the highest priority research topics, demonstrating the importance of multi-disciplinarity in research in this field.

\section{0-45}

Association between a higher drug burden index and the level of functional autonomy in seniors, 6 months after an emergency consultation for minor trauma in the ceti cohort

Kröger Edeltraut ${ }^{1}$, Simard Marilyn ${ }^{1}$, Sirois Marie-Josée ${ }^{1}$, Giroux Marianne ${ }^{2}$, Sirois Caroline ${ }^{3}$, Carmichael PierreHugues ${ }^{4}$, Kouladjian O'Donnell Lisa ${ }^{5}$, Reeve Emily ${ }^{6}$, Hilmer Sarah ${ }^{6}$, Émond Marcel $^{7}$

${ }^{1}$ Université Laval, Centre d'excellence sur le vieillissement de Québec, CIUSSSCN, ${ }^{2}$ Université Laval, ${ }^{3}$ Université Laval, Centre d'excellence sur le vieillissement de Québec, ${ }^{4}$ Centre d'excellence sur le vieillissement de Québec, CIUSSSCN, ${ }^{5}$ NHMRC Cognitive Decline Partnership Centre, University of Sydney, Kolling Institute of Medical Research, University of Sydney and Royal North Shore Hospital, St Leonards, NSW Australia, ${ }^{6}$ NHMRC Cognitive Decline Partnership Centre, University of Sydney, Kolling Institute of Medical Research, University of Sydney and Royal North Shore Hospital, St Leonards, NSW Australia, ${ }^{7}$ Université Laval, Centre d'excellence sur le vieillissement de Québec, Québec, Canada

Background: The Canadian Emergency Team Initiative (CETI) cohort showed that minor injuries like sprained ankles or small fractures trigger a downward spiral of functional decline in $16 \%$ of independent seniors up to 6 months post-injury. Seniors frequently receive medications with sedative or anticholinergic properties, as summarized by the Drug Burden Index (DBI), which are associated with decreased physical and cognitive functioning. We aimed at assessing the DBI's contribution to functional decline in the CETI cohort whose participants were assessed physically and cognitively at baseline, including medication use, and 6 months later.

Methods: Functional autonomy was measured with the Older Americans Rehabilitation Score (OARS). Medication data allowed calculating baseline DBI. Linear regression models assessed the correlation between DBI and functional autonomy at 6 months, adjusting for age, sex, baseline OARS, frailty level, comorbidities and mild cognitive impairment.

Results/conclusions: 557 study participants from CETI sites with medication data at baseline and complete follow-up data at 6 months were included in this study. In adjusted analyses, a higher DBI at the time of injury contributed to a lower level of functional autonomy, measured by the OARS at 6 months post injury: each additional point in the DBI lead to a loss of 0.2 points in the OARS, at $\mathrm{p}<0.001$. These results support those from other cohorts studied elsewhere. Medication taken during follow-up may be included in further analyses. Emergency visits are considered missed opportunities for optimal care interventions in seniors: identifying their DBI may contribute to less functional decline after minor injury.

\section{Area: Geriatric education}

\section{O-46}

Reducing antibiotic overprescribing in nursing homes (NHs): role of education, leadership, and variation in practice patterns

Philip Sloane $^{1}$, Sheryl Zimmerman ${ }^{1}$, Kimberly Ward ${ }^{1}$, Christopher Wretman ${ }^{1}$

${ }^{1}$ University of North Carolina at Chapel Hill, Chapel Hill, USA

Introduction: High antibiotic prescribing rates and growing numbers of multi-drug resistant bacteria in NHs have led to efforts worldwide to minimize antibiotic overuse. 
Methods: Results from two separate antibiotic stewardship interventions in the United States will be presented. One involved $27 \mathrm{NHs}$ in the state of North Carolina; the other involved $28 \mathrm{NHs}$ in Minnesota. Infection control nurses in each $\mathrm{NH}$ submitted antibiotic prescribing data monthly for a year, during which a standardized, multi-component antibiotic stewardship intervention was conducted. Results: Baseline antibiotic prescribing rates in participating North Carolina and Minnesota NHs respectively were 4.6 and 2.2 new prescriptions per resident per year. Baseline variation in prescribing between NHs was large: in North Carolina it ranged from 2.4 to 9.6 prescriptions per resident per year; in Minnesota it ranged from 0.7 to 4.9 prescriptions per resident year; and little of the variation was explained by differences in resident characteristics. Implementation of the intervention reduced prescribing across all North Carolina nursing homes by $20 \%$ and reduced intra-facility variation; in Minnesota, where baselines were much lower, preliminary data show reductions in the 3-5\% range. Marked prescribing fluctuations persisted, however, both between and within $\mathrm{NHs,} \mathrm{with} \mathrm{poor} \mathrm{response} \mathrm{to}$ the intervention associated with $\mathrm{NH}$ staff turnover and medical provider characteristics.

Key conclusions: Practice variation between NHs and regions is immense and indicates potential for significant reduction in highprescribing locations. Staff turnover is a major barrier to consistent application of antibiotic stewardship, but in spite of these barriers overall reductions can be achieved.

\section{$0-47$}

Why did they choose Geriatrics? A national survey among French medical students

Joaquim Prud'homm ${ }^{1}$, Aline Corvol ${ }^{1}$, Dominique Somme ${ }^{1}$

${ }^{1}$ Service de Gériatrie, Centre Hospitalier et Universitaire de Rennes, Rennes, Rennes, France

Introduction: Since 2017, French Medical students can choose Geriatrics as their future specialty from the 7 th year of study. We studied the motivation of the 171 students who have chosen Geriatric in 2017 after the end of the 6th years national exam.

Methods: We conducted a national survey in France among the French Geriatric Medicine residents, from 2017, December to 2018, April. We created a questionnary on the internet website Surveymonkey.fr and sent it by e-mail to the 171 students. The questionnary was composed of 53 quantitative questions-whom 35 questions with a 5-points Likert scale-and 7 qualitative questions.

Results: We received $139(81.2 \%)$ responses. The most important results are: most of the respondants $(82.6 \%)$ had part of their training in a Geriatric departement. $90.2 \%$ of the students claim that training in a Geriatric department influenced their choice. Geriatric medicine was the first discipline asked for $59.9 \%$ of them. If Geriatric medicine was a 2 nd choice, oncology was the most asked discipline $(18.3 \%)$. The proposals that had a strong or very strong influence were working with old people $(88.8 \%)$, former positive experiences with old people (70.4\%), interprofessional work $(83.2 \%)$, working on complex diagnosis and therapeutic cases $(85.6 \%)$, the wide range of work (acute geriatric unit, rehabilitation departement, long term chronic care facilities, ...) (77.6\%), extra-professional charms of the chosen regions $(62.4 \%)$. The status of Geriatrian had a low or very low influence for $65.5 \%$ of the students.

Key conclusions: Our survey is the first about the motivation of medical residents in Geriatrics in France. It highlights the importance of an internship in a Geriatric ward during the first years of study to reinforce students interest for Geriatrics. A 2016 United Kingdom survey reported similar results [1].

\section{Reference:}

[1] Fisher, James Michael, Mark J. Garside, Peter Brock, Vicky Gibson, Kelly Hunt, Sally Briggs, and Adam Lee Gordon. "Why Geriatric Medicine? A Survey of UK Specialist Trainees in Geriatric Medicine." Age and Ageing 46, no. 4 (July 1, 2017): 672-77. https://doi.org/10.1093/ageing/afx009.

\section{O-48}

Innovations in geriatric medicine: Lessons from the first panspeciality trainee-led national audit

Lauren McCluskey ${ }^{1}$

${ }^{1}$ Queen Elizabeth Hospital, Birmingham, UK

Introduction: We set up the first UK trainee-led Geriatric Medicine Research Collaborative (GeMRC) and conducted a pan-specialty national audit of delirium in March 2018. Primary aim. 1. To determine the feasibility of using GeMRC to design, promote and conduct a UK wide national audit. Secondary aims1. To determine variation in adherence to national guidelines for delirium screening in hospitalised older adults. 2. To pilot a national assessment of delirium. 2. To enable geriatric trainees to develop audit skills. 3. To provide guidelines for future collaboratives on conducting a national audit. Methods: For national "Delirium Audit Day" we used the GeMRC network to establish an audit committee comprised of experienced clinicians from broad spectrum of specialities. We created national and regional websites and utilised a combination of e-mail communication, social networking, word-of-mouth to disseminate project information. We provided a webinar, a phone line for support on the day and a user-friendly data collection form.

Results: We were successful in disseminating the information about the audit to a variety of specialities. Results were received from over 35 sites across the UK, including 1000 individual patients.

Conclusions: GeMRC offers an innovative approach to conducting research and audit, it facilitates the opportunity for trainees with limited experience to participate in research with trainee support and early impact upon patient care. The large social media network allows collection of much larger datasets and enhances the scientific validity of project outcomes. In the future collaboration with European trainees could increase the capacity of this collaborative.

\section{0-49}

\section{Geriatric medicine simulation for medical students}

Dr Nicola Linscott ${ }^{1}$, Dr Katherine White ${ }^{1}$, Dr David Ahearn ${ }^{1}$, Michael Smith ${ }^{1}$, AnnMarie Feeney ${ }^{1}$

${ }^{1}$ Manchester University Hospital Foundation Trust, Manchester, UK

Introduction: It is important that medical students are experienced in the management of frail and older patients. Simulation allows practice of the necessary clinical skills in a safe and controlled environment. Within medical education it is a proven valuable tool, however specifically within Geriatric medicine the literature is scarce. We were interested in the utility of simulation, to enable students to develop their abilities to manage older complex patients.

Methods: During 2017-2018, fourth year medical students on their Ageing and Complex Health rotation at Wythenshawe Hospital, Manchester, undertook a 180-min simulation session facilitated by a doctor and two simulation staff. In pairs, each student actively participated in one scenario and observed three. A high-fidelity, lifesized manikin was utilised in four sequential scenarios, following the 
journey of a patient with advanced Parkinson's disease. Themes included delirium, sepsis, reduced consciousness and ultimately the recognition and management of a dying patient. Ethical and communication themes were included. Following the session, students ranked learning outcomes on a Likert-type scale and provided qualitative feedback.

Results: Data was collected from seventy-three students from nine sessions $(100 \%$ return rate). $85 \%(n=62)$ "Strongly Agreed" and $15 \%(\mathrm{n}=11)$ "Agreed" that they gained significant knowledge. Additionally, 79\% $(\mathrm{n}=58)$ "Strongly Agreed" and 21\% $(\mathrm{n}=15)$ "Agreed" that they had gained confidence in management of similar situations. All (100\%) students found the environment conducive to learning.

Conclusions: Simulation is valuable tool for increasing knowledge and confidence of medical students in the management of complex older patients.

\section{$\mathbf{0 - 5 0}$}

Dementia communication skills course: improving medical students' confidence and patient-centred communication skills in dementia care

Jasmine Gan $^{1}$, Sanja Thompson ${ }^{1}$, Masanori Watanabe ${ }^{1}$, Pamina Mitter ${ }^{2}$, Helen Salisbury ${ }^{3}$, Lola Martos ${ }^{2}$, Zeinab Hussein ${ }^{1}$, Nicola Lovett ${ }^{1}$, Jennifer Challberg ${ }^{1}$, Emma Hawk ${ }^{2}$

${ }^{1}$ Department of Clinical Geratology, John Radcliffe Hospital, Oxford University Hospitals, Oxford, UK, ${ }^{2}$ Oxford Health NHS Foundation Trust, Oxford, UK, ${ }^{3}$ Department of Primary Care Sciences, University of Oxford, Oxford, UK

Introduction: Dementia is a progressive condition that can lead to communication difficulties and behaviours that challenge. Good communication skills are imperative to engage with such patients, enabling the delivery of high-quality, person-centred care. The Oxford Medical School dementia communication skills course aims to empower students to communicate effectively with a person with dementia (PwD).

Methods: Fourth-year medical students participate in four role-play scenarios with trained actors, facilitated by healthcare professionals. The scenarios focus on the importance of nonverbal interaction; manging an interview with a PwD and a carer; communication with a confused wandering patient; and de-escalation of aggressive behaviour. Anonymous written feedback were obtained before and after the course. A four-point Likert scale from "low" to "very high" was used to evaluate the change in student's perceived confidence to deal with PwD. Students were also asked to identify one key learning point.

Results: 96 responses were collected. Before the course, majority of students reported low $(41.7 \%)$ or moderate $(51.0 \%)$ confidence. After the course, $84.4 \%$ had increased confidence. $38.5 \%$ reported moderate confidence and $46.9 \%$ reported high confidence. Increased confidence by $>2$ Likert points was observed in $14.6 \%$. $13.5 \%$ had unchanged confidence. $2.1 \%$ had reduced confidence. Students listed the following key learning points: learning how to engage in "real situations" with PwD to maintain respectful rapport and communication, and how to adopt non-verbal techniques e.g. reassuring touch to connect with patients.

Key conclusion: The dementia communication skills course is an effective method to enhance students' ability to work respectfully and effectively with PwD.

\section{0-51}

Insight in residents and consultants applied knowledge of pharmacotherapy and polypharmacy

M van Haastrecht ${ }^{1}, \mathrm{~F}$ van den Hanenberg ${ }^{1}$, E Ozturk $^{2}$, J Tichelaar ${ }^{3}$, $\mathrm{H}$ van Goor $^{2}$, CJPW Keijsers ${ }^{4}$, MA van Agtmael $^{3}$

${ }^{1}$ Department of Geriatric medicine, MC Slotervaart, Amsterdam, The Netherlands, ${ }^{2}$ Department of Surgery, Nijmegen University MC, Nijmegen, The Netherlands, ${ }^{3}$ Department of internal medicine, section pharmacotherapy, VU University MC, Amsterdam, The Netherlands, ${ }^{4}$ Department of Geriatric Medicine, Jeroen Bosch Hospital, 's Hertogenbosch, The Netherlands

Introduction: Knowledge of pharmacotherapy in older patients is essential for all doctors. It is described that this knowledge is often insufficient, but the level of this insufficiency is unclear.

Aim: Gain insight into the current level of applied knowledge of pharmacotherapy and polypharmacy of residents and consultants. Secondarily, the trainability on the knowledge by an online course was studied.

Methods: The applied knowledge of pharmacotherapy and polypharmacy of residents and consultants from different clinical specialties were assessed, using formative multiple choice assessments as part of an online course regarding frail older patients. Explanatory variables to predict the level of knowledge were studied. The effects of an online course were studied using repeated ANOVA. Results: 448 residents and 331 consultants were included in 2016-2017. They had a mean pre-test score in pharmacotherapy of $74 \%$ (SD 15.5) vs 72\% (SD 15.2) and polypharmacy pre-test score of $75 \%$ (SD 14.3) and 74\% (SD 16.5), respectively ( $p=0.01$ ). Surgical specialties had lower pre-test scores compared to those with a medical specialty $(\mathrm{p}<0.001)$. There were no predicting variables for higher scores. With the course as intervention, an increase in scores of both groups (within groups, $\mathrm{p}<0.001$ ) was obtained with a betweengroup difference between surgical and medical specialty $(\mathrm{p}=<0.001)$. Clinical specialty appeared to influence the increase of knowledge of pharmacotherapy $(\mathrm{p}=0.006)$.

Conclusions: Differences in basic applied knowledge of pharmacotherapy and polypharmacy were seen between clinical specialties, with surgical specialities having a slightly lower level. No differences were found between residents and consultants. An online course can increase the level of knowledge in both medical and surgical specialties.

\section{$0-52$}

Clinical uncertainty in geriatrics: how the enhance coping by residents

Marianne van Iersel ${ }^{1}$, Dieneke van Asselt ${ }^{1}$, Natasja Looman ${ }^{1}$, Ellen Bazelmans ${ }^{1}$, Marieke de Visser ${ }^{1}$, Marjolein van de Pol $^{1}$

${ }^{1}$ Radboudumc, Nijmegen, The Netherlands

Introduction: Clinical uncertainty is an undeniable and common part of practising medicine and even more in geriatrics. Multimorbidity and frailty, superspecialisation and technical possibilities make health care more and more complex. This causes even more uncertainty leading to distress and extensive testing. Surprisingly, residents do not learn how to cope with clinical uncertainty. Our goal was to develop interventions to enhance coping with uncertainty by residents.

Methods: We used validated scales and psychoeducation sessions to raise awareness of clinical uncertainty. These sessions resulted in tips for the residents how to deal with uncertainty given their personal traits and experiences. These interventions were extended with semi- 
structured interviews, small group educational sessions and transfer of knowledge for example by explicitly discussing clinical uncertainty in clinical case conferences.

Results: Recognition of clinical uncertainty is the first step: labelling the vague, unpleasant feeling as such. Some residents link that feeling to personal functioning, others to the complexity of the patient. Everyone recognises uncertainty as a source of distress. To enhance coping discussions with fellow residents, in broader discussion groups and one-on-one, are helpful. Explicitly labelling the aspect of clinical uncertainty in discussions with faculty provides space for reflection and diminishes a feeling of loneliness in complex situations.

Conclusions: With simple interventions we aim to improve coping of residents with clinical uncertainty to ultimately improve wellbeing of residents and quality of care for patients.

\section{0-53}

How to organize a geriatric curriculum during financial crisis? Geriatric training from scratch

\author{
G Soulis $^{1}$, M Kotsani $^{1}$, P.P Sfikakis ${ }^{2}$, Y Ellul $^{1}$ \\ ${ }^{1}$ Hellenic Consortium For Geriatric Medicine, ${ }^{2} 1$ st Dept of \\ Propedeutic Internal Medicine, National Kapodistrian University of \\ Athens Medical School, Athens, Greece
}

Introduction: Greece is one of the few European countries where Geriatrics is not a recognized medical specialty. This is burdensome, since on one hand medical doctors that have been trained in Geriatrics elsewhere cannot have official recognition of their capacities and older people are deprived of the best available care and medical services. Recently healthcare authorities invited experts in the field to provide a theoretical context that can support the necessity to develop Geriatrics in Greece, while they asked to recommend a feasible and solid curriculum that is going to be the basis to develop Geriatrics. Methods: We proposed to adopt a Geriatric curriculum corresponding to the training standards in the field, while using existing infrastructures since the development of new ones fitting to this aim is unattainable.

Results: We considered as adequate a 2 year post specialty training period, consisted of 4 core rotations namely geriatric emergencies, inpatient management and assessment, outpatient geriatric assessment and psychogeriatrics, with 2 optional rotations depending on the availability for instance geriatric cardiological consultation-heart failure, rehabilitation, end of life care, home care, osteoporosis and falls clinic. We suggested to empower clinical training with seminars and webinars twice per year, that will cover both basic and advanced topics in Geriatrics.

Conclusions: The aim will be to train a critical mass of medical doctors in geriatrics and at the same time promote geriatric approach within hospital setting as well as in community level where there is little geriatric culture in order to offer the most to older people.

\section{O-54}

\section{Gero-Parcours: a teaching unit in dental education}

\author{
Ina Nitschke ${ }^{1}$ \\ ${ }^{1}$ University Zurich, Zurich, Germany
}

Introduction: Undergraduate gerodontology teaching is important. This study describes the acceptance of the "Gero-Parcours" among dental students. The low-level half-day course is based on selfawareness of ageing through sensitivity training exercises.
Methods: Undergraduate dental students in the 8th semester $(n=144)$ were trained in groups of two in a station run (10-20 min per station) by 19 stations: mobility (age and stroke simulationsuit), patient transfer (with wheelchair, patient lift), sensory limitations (bodyperception, tactility, hearing, vision, taste), dealing (ethics, communication with caregivers, emotion recognition, foreign toothbrushing, nursing aids, virtual reality dementia simulation), care and therapy (medical history, drug interaction, oral care aids for seniores, clinical nutrition, mobile treatment equipment). Standardized written feedback of 4 courses was evaluated.

Results: The undergraduate dental students were astonished by the diversity of bodyselfawareness triggered by simulations. They reported feeling well prepared for initial contact with geriatric patients and judged "age simulation suit" and "limited view simulation" to be the most impressive and "patient transfer" and "age simulation suit" most useful. Only $3.5 \%$ of the students would not recommend the course.

Key conclusions: There is great acceptance of "Gero-Parcours" among dental students reporting having gained understanding, knowledge and techniques in dealing with seniors. The willingness of the course program directors to include the training in the curricula, a course guide handbook, acquisition of teaching materials (partly rentable) and course tutors who want to deal intensively with the topic are required to establish the sensitivity training course prior to the first contact with the geriatric patient.

\section{Area: Comprehensive geriatric assessment \\ 0-55}

Presence of a geriatrician on site in a nursing home: what does it change? A before/after study

Graf Christophe ${ }^{1}$, Zekry Dina ${ }^{1}$, Genton Laurence ${ }^{1}$, Herrmann Francois ${ }^{1}$

${ }^{1}$ Geneva University Hospitals, Geneva, Switzerland

Background: In Switzerland, general practitioners (GP) assume standard medical care in the nursing home $(\mathrm{NH})$. They are generally working at their own office and are not physically present in $\mathrm{NH}$. In case of emergency, $\mathrm{NH}$ residents are often unnecessary transferred to the emergencies as the GP is not available. This retrospective study examined the effect of the presence vs. absence of a geriatrician in $\mathrm{NH}$ on outcome.

Methods: This retrospective study included all patients living in a single NH of the Lausanne area between 2010 and 2014. We compared the rate of hospitalization, the rate of falls, and the quality and the number of prescribed drugs between the two-year period without geriatrician (2010-2011) and the three-year period with a geriatrician working in the NH (2012-2014). Poisson regressions were performed and results are reported as incidence rate (IR). The statistical unit is expressed as stay by year.

Results: Mean age was 85.8 years \pm 6.5 and $69 \%$ of female in $2010-2011$, and 86.0 years \pm 7.6 and $73 \%$ of female in $2012-2014$. IR of hospitalization was 16.8 per 100 stays-year for 2010-2011 vs. 8 per 100 stays-year for 2012-2014 (i.e. 52\% of reduction, 95\% CI $35.2-64.8, \mathrm{p}<0.001)$. IR of falls was not statistically different between the two periods (51.5 vs. 43.5 per 100'000 stays-year, $\mathrm{p}=0.178$ ). The number of drugs was the same without or with the geriatrician (5.00 vs. 5.00 medications, $\mathrm{p}=0.421)$, but IR of antipsychotics was reduced by $29 \%$ (95\% CI $0.17-0.39, \mathrm{p}<0.001)$, of anxiolytics by $42 \%(95 \%$ CI $0.29-0.53, \mathrm{p}<0.001)$ and of hypnotics by $54 \%$ ( $95 \%$ CI $0.45-0.61, \mathrm{p}<0.001$ ).

Conclusion: The presence of a geriatrician in $\mathrm{NH}$ decreased the rate of hospitalization and the prescription of antipsychotics, anxiolitics 
and hypnotics. This could potentially improve quality of care by keeping residents in their environment and their awareness.

\section{0-56}

Validity of sensor-based, habitual physical activity and gait analysis in older persons with cognitive impairment after discharge from geriatric rehabilitation

Martin Bongartz ${ }^{1}$, Rainer Kiss ${ }^{2}$, Andrè Lacroix ${ }^{1}$, Phoebe Ullrich ${ }^{1}$, Tobias Eckert ${ }^{1}$, Sabato Melone ${ }^{3}$, Jürgen M. Bauer ${ }^{1}$, Klaus Hauer ${ }^{1}$

${ }^{1}$ Department of Geriatric Research Agaplesion Bethanien-Hospital, Geriatric Centre at the University of Heidelberg, Germany,

${ }^{2}$ Department of Health and Social Affairs, FHM Bielefeld, University of Applied Sciences, Germany, ${ }^{3}$ Department of Electrical, Electronic, and Information Engineering, University of Bologna, Italy

Introduction: The aim of the study was to investigate the biometrical quality of a newly developed activity monitor (uSense) to document established physical activity (PA) parameters as well as innovative qualitative and quantitative gait characteristics for habitual activity behavior in multi-morbid, older adults.

Methods: Validity, test-retest reliability of established PA parameters as well as gait characteristics which have not comprehensively been documented before for habitual assessment and feasibility of measurement have been analyzed for multi-morbid, geriatric patients with cognitive impairment $(n=110)$ discharged from geriatric ward based rehabilitation.

Results: Spearman correlations (rho) with established and innovative uSense parameters indicating construct validity were, on average, moderate to good for clinically relevant motor performance parameters (range rho: 0.02-0.63) and for life space (range rho: 0.01-0.59), and low to moderate for cognitive status and for age (range rho: $0.01-0.30$ ). Concurrent validity was high as PA parameters measured by the uSense showed consistently high correlations with equivalent parameters measured by another well-established ambulatory motion sensor (PAMSYS) (range rho: 0.59-0.91). Moderate to excellent testretest reliability was shown for all uSense parameters (Intra Class Correlation: 0.68-0.97) and good feasibility could be shown, as $85.5 \%$ of all measurements were completed without failure.

Conclusion: The uSense monitor allows documentation of established and innovative qualitative and quantitative parameters for habitual PA and gait behavior which have so far only been assessed in laboratory settings in multi-morbid, cognitively impaired, older adults with moderate to good validity and high test-retest reliability.

\section{0-57}

Sex differences in physical functioning while aging in The Netherlands: influence of age, generation and education

Lena D. Sialino ${ }^{1}$, L.A. Schaap ${ }^{2}$, S.H. van Oostrom ${ }^{2}$, H.S.J. Picavet ${ }^{2}$, W.M.M. Verschuren ${ }^{3}$, M. Visser ${ }^{3}$, H.A.H. Wijnhoven ${ }^{2}$

${ }^{1} \mathrm{MSc},{ }^{2} \mathrm{Dr},{ }^{3} \operatorname{Prof}$

Across countries women have a higher life expectancy, but, paradoxically, experience more years with functional disabilities than men. Systematic (longitudinal) research regarding this "female disadvantage" is lacking. This prospective study investigates sex differences in physical functioning while aging in The Netherlands by generation and education level. Data of the Longitudinal Aging Study Amsterdam $(n=4109$, age 55-85 years) and cross-sectional data of the Doetinchem Cohort Study ( $n=4018$, age $40-81$ years) were used. In LASA, follow-up measurements were performed every 3 years for 25 years. Mixed model analysis showed that women aged $40+$ years had a lower gait speed $(-0.08 \mathrm{~m} / \mathrm{s}, \mathrm{p}<0.001)$, chair stand speed $(-0.03$ stand/s, $\mathrm{p}<0.001)$, handgrip strength $(-16.12 \mathrm{~kg}, \mathrm{p}<0.001)$ and ability to perform the tandem balance test $(\mathrm{OR}=0.52, \mathrm{p}<0.001)$ compared to men in both cohorts. The female disadvantage in chair stand speed increased (interaction sex*age*age, $p=0.024)$ during aging, decreased for handgrip strength (interaction sex*age, $p<0.001$ ) and remained stable for gait speed and balance. Generation only modified the female disadvantage in handgrip strength, were it decreased more rapidly in the younger generation (2002/2003 versus 1992/1993, interaction generation*sex*age, $p=0.030$ ). Education level only modified the female disadvantage in balance (interaction education*sex*age, $\mathrm{p}=0.011$ ). In low educated persons the female disadvantage in balance increased during aging, while it remained stable for middle and high educated persons. In conclusion, there is a female disadvantage in physical functioning during aging in The Netherlands in persons aged $40+$ years, which is conditionally influenced by age, generation and education level. Further research investigating underlying mechanisms is advised.

\section{0-58}

Predicting frailty and geriatric interventions in older patients with cancer: performance of two screening tools for six frailty definitions in the prospective ELCAPA cohort

Claudia Martinez-Tapia ${ }^{1}$, Marie Laurent ${ }^{1}$, Elena Paillaud ${ }^{2}$, Philippe Caillet $^{2}$, Emilie Ferrat ${ }^{1}$, Jean-Léon Lagrange ${ }^{3}$, Mylène Allain $^{4}$, Anne Chahwakilian ${ }^{5}$, Pascaline Boudou-Rouquette ${ }^{6}$, Sylvie Bastuji-Garin ${ }^{7}$, Etienne Audureau ${ }^{7}$

${ }^{1}$ UPEC EA 7376 — CEpiA (Clinical Epidemiology And Ageing Unit), F94010 Créteil, France, ${ }^{2}$ Henri-Mondor Hospital, Internal Medicine and Geriatric Department, F94010 Créteil, France, ${ }^{3}$ Henri-Mondor Hospital, Department of Medical Oncology, F94010, France, ${ }^{4}$ HenriMondor Hospital, Clinical Research Unit (URC Mondor), F94010 Créteil, France, ${ }^{5}$ Broca Hospital, Oncogeriatrics, Geriatric Department, F75013 Paris, France, ${ }^{6}$ Cochin Hospital, Department of Medical Oncology, F75014 Paris, France, ${ }^{7}$ Henri-Mondor Hospital, Public Health Department, F94010 Créteil, France

Introduction: Screening tools have been developed to identify patients warranting a complete geriatric assessment (GA). However, GA lacks standardization and doesn't capture important aspects of geriatric oncology practice. We measured and compared the diagnostic performance of the screening tools G8 and modified-G8 according to multiple clinically relevant reference standards.

Methods: We included 1136 cancer patients $\geq 70$ years old referred for GA (ELCAPA cohort). Area under the receiver operating characteristic curve (AUROC) estimates were compared between both tools against: (1) detection of $\geq 1$ or (2) $\geq 2$ GA impairments, (3) prescription of $\geq 1$ geriatric intervention and identification of an unfit profile according to (4) a latent class typology or expert-based classifications from (5) Balducci and (6) the International Society of Geriatric Oncology-task force (SIOG).

Results: AUROC values were $\geq 0.80$ for both tools under all definitions tested. They were significantly higher for the modified-G8 for five reference standards: $\geq 1$ GA impairment (0.93 vs. 0.90), $\geq 2$ GA impairments ( 0.90 vs. 0.87$), \geq 1$ geriatric intervention ( 0.85 vs. 0.81 ) and unfit according to Balducci (0.86 vs. 0.80$)$ and SIOG classifications $(0.88$ vs. 0.83$)$.

Conclusion: Our findings demonstrate the robustness of both screening tools against different reference standards, with evidence of 
better diagnostic performance of the modified-G8. Since the choice of the most appropriate definition to operationalize the concept of frailty remains difficult and without consensus, we hope our findings may offer a practical response for daily practice with an instrument able to detect any potential risk problem regardless of the definition.

Keywords: Geriatric assessment, Reference Standard, Surveys and Questionnaires, Frailty, Sensitivity and Specificity.

\section{0-59}

Predictors of deprescribing in nursing home residents on polypharmacy: results from a Multi-Centre European Study

Emanuele Rocco Villani ${ }^{1}$, Davide Liborio Vetrano ${ }^{2}$, Eva Topinkova ${ }^{3}$, Henriëtte van der Roest ${ }^{4}$, Harriet Finne-soveri ${ }^{5}$, Roberto Bernabei ${ }^{1}$, Graziano Onder ${ }^{1}$

${ }^{1}$ Department of Geriatrics, Catholic University of Rome, Rome, Italy, ${ }^{2}$ Aging Research Center, Department of Neurobiology, Care Sciences, and Society (NVS), Karolinska Institutet and Stockholm University, Stockholm, Sweden; Department of Geriatrics, Catholic University of Rome, Rome, Italy, ${ }^{3}$ Department of Geriatrics and Gerontology, 1st Faculty of Medicine, Charles University, Prague, Czech Republic; Faculty of Health and Social Sciences, University of South Bohemia, Ceske Budejovice, Czech Republic, ${ }^{4}$ Department of General Practice and Elderly Care Medicine, Amsterdam Public Health Institute, VU University Medical Center, Amsterdam, The Netherlands, ${ }^{5}$ National Institute for Health and Welfare, Helsinki, Finland

Introduction: Complex drug regimens and inappropriate prescriptions are frequent in nursing home $(\mathrm{NH})$ residents and are associated to negative health-related outcomes. Deprescribing may reduce polypharmacy, limiting the use of potentially inappropriate medications and buffering their negative consequences. The present study investigates the predictors of deprescribing among $\mathrm{NH}$ resident on polypharmacy $(5+$ drugs $)$.

Methods: Longitudinal multi-centre cohort study based on data from the Services and Health for Elderly in Long TERm care (SHELTER) study. Participants were assessed through the interRAI-LTCF tool. One-year trajectories of deprescribing where detected through linear mixed models and predictor of deprescribing via multivariate logistic regressions.

Results: Among 2095 participants on polypharmacy (age 84 years; $74 \%$ women), $1142(55 \%)$ presented a deprescribing pattern over 1 year. In fully adjusted analyses, deprescribing was positively associated with cognitive decline (severe vs. none-mild OR 1.75; $95 \%$ CI 1.29-2.38), and with the presence of a geriatrician in the facility (OR 1.31; 95\% CI 1.07-1.61). A negative association was detected with age, cancer, dyspnea and severe dependency ( $\mathrm{P}<0.05$ for all). An interaction between age and the presence of a geriatrician was detected.

Conclusions: Cognitive impairment and the availability of a geriatrician in the facility is associated with deprescribing in $\mathrm{NH}$ residents on polypharmacy. However, several clinical characteristics hinder this outcome. Further studies investigating predictors and outcomes of deprescribing in frail older adults are warranted.

\section{0-60}

Predictive ability of physical and cognitive deficits in the Kihon Checklist for incident dependency and mortality in Japanese community-dwellers

Shosuke Satake ${ }^{1}$, Hiroshi Shimokata ${ }^{2}$, Kazuyoshi Senda $^{1}$, Hidenori Arai ${ }^{1}$, Kenji Toba ${ }^{1}$

${ }^{1}$ National Center for Geriatrics and Gerontology, Obu, Japan,

${ }^{2}$ Institute of Health and Nutrition, Nagoya University of Arts and Science, Nisshin, Japan

Introduction: The Kihon Checklist (KCL) is a self-administered questionnaire for older adults to assess their multiple functions in their daily livings. In this study, we examined predictive abilities of deficits in physical and cognitive domains of the KCL for independency and survival during 3 years.

Method: Of all senior residents in the Higashiura town, the municipal government identified independent older residents who were uncertified by the long-term care insurance (LTCI). We sent the KCL to the residents and asked them to send it back to us after answering all questions. We finally selected older residents who filled in all questions of the KCL as the eligible subject. Baseline characteristics and the data on KCL of the subjects were registered. Information about an incident LTCI certification and death in 3 years was given by the municipal government. Results: We classified the subjects into 4 groups based on the deficits in physical and cognitive domains of the KCL (no deficit [ND]; $\mathrm{n}=3286$, only cognitive deficit $[C D] ; \mathrm{n}=1413$, only physical deficit $[\mathrm{PD}] ; \mathrm{n}=387$, and both deficits $[\mathrm{BD}] ; \mathrm{n}=456$ ). Compared with ND, the Cox proportional hazard model adjusted for age and sex indicated that $\mathrm{CD}, \mathrm{PD}$ and $\mathrm{BD}$ significantly predicted the risk of dependency, with the hazard ratios (HRs) of 1.633, 2.470, and 3.642, respectively. On the other hand, the mortality could be significantly predicted in the BD with a HR of 2.764 .

Conclusion: Both physical and cognitive deficits in the KCL showed a significant predictive ability of the incident dependency and mortality.

\section{0-61}

Effect of cumulative exposure to medications with anticholinergic and sedative properties on dynamic gait characteristic in geriatric patients

Claudine Lamoth ${ }^{1}$, Hans Wouters ${ }^{2}$, Marloes Kuitert ${ }^{1}$, Katja Taxis ${ }^{3}$, Tibor Hortobagyi ${ }^{1}$, Jos van Campen ${ }^{4}$

${ }^{1}$ University of Groningen, University Medical Center Groningen, Center of Human Movement Science, The Netherlands, ${ }^{2}$ University Medical Center Groningen, Dept. of General Practice and Elderly Care Medicine, The Netherlands, ${ }^{3}$ University of Groningen, Dept. Department of Pharmaco Therapy, Epidemiology \& Economics (PTEE), The Netherlands, ${ }^{4} \mathrm{MC}$ Slotervaart, Dept. of geriatric medicine, Amsterdam, The Netherlands

Introduction: Medications with anticholinergic and sedative properties act on brain areas that are involved in cognitive and physical function [1]. Because physical functions, including gait dynamics, are often impaired in geriatric patients [2], the objective of the present study was to examine whether anticholinergic and sedative medications have additive unfavorable effects on gait dynamics.

Methods: From 3-dimensional accelerometer data collected during 3 min walking, 23 dynamic gait variables were calculated for 184 participants (Mean age $79.8 \pm 5.8$ ). Functional components were extracted from these gait variables with Principal Component Analysis (PCA). Medications were coded using the ATC classification. Cumulative exposure to anticholinergic and sedative drugs was quantified 
with the Drug Burden Index (DBI)[3], (DBI $=0$; $0>$ DBI $<1$; DBI $\geq 1$ ). Multivariate analyses with functional components as the dependent variables and the DBI as the independent variable were conducted. Analyses were adjusted for comorbidities, cognitive status, Timed Up and Go, Geriatric Depression Scale and frailty as covariates.

Results: Five functional components were extracted representing, 'Regularity', 'Pace', 'Synchronization', 'Stability', and 'Variability'. The DBI was associated with 'Pace' $(\mathrm{F}(2,181)=4.07, \mathrm{p}=0.019)$. When controlling for covariates, the DBI effect $(\mathrm{F}(2,179)=3.9$, $\mathrm{p}=0.021)$ remained significant.

Conclusion: The use of anticholinergic and sedative drugs, in addition to co-morbidities and frailty, significantly affect gait dynamics (pace). A limitation of the DBI is that it does not take into account the effects of other drugs and their interactions. The DBI is a valuable tool for identifying and deprescribing potentially inappropriate medications and could be included in comprehensive geriatric assessment. References:

[1] Morris R, et al Neurosci Biobehav Rev 2016; 64: 326-345.

[2] Kikkert LHJ, et al., J Neuroeng Rehabil 2017; 14: 1-1.

[3] Hilmer SN et al., Am Geriatr Soc 2007; 167: 781-787.

\section{Area: Psychiatric symptoms and illnesses}

\section{O-62}

Symptomatic orthostatic hypotension predicts incident depression in a large cohort of community-dwellling older people. Data from TILDA

\section{Robert Briggs ${ }^{1}$, Daniel Carey ${ }^{1}$, Sean Kennelly ${ }^{1}$, Rose Anne Kenny ${ }^{1}$ \\ ${ }^{1}$ The Irish Longitudinal Study on Ageing, Trinity College Dublin, Dublin, Ireland}

Background: There is an established cross-sectional association between orthostatic hypotension $(\mathrm{OH})$ and late life depression. The aim of this observational longitudinal study was to clarify the longitudinal association between baseline symptomatic $\mathrm{OH}(\mathrm{sOH})$ and incident depression in a sample of over 3000 people aged $\geq 50$ years. Methods: This study was embedded within the Irish Longitudinal Study on Ageing, and data was analysed from Waves 1, 2 and 3. Participants with baseline depression at Wave 1 were excluded. At 2 and 4 year follow-up, a score $\geq 9$ on the 8 -item Centre for Epidemiological Studies Depression Scale was used to define incident depression. SOH was defined as a drop in systolic blood pressure (BP) by $\geq 20 \mathrm{mmHg}$ or diastolic BP by $\geq 10 \mathrm{mmHg}$ at 30 s post standing in conjunction with orthostatic symptoms such as dizziness, using beat-to-beat BP measurements.

Results: 7\% (226/3174) of the study sample had sOH-30. One tenth (300/3174) had incident depression by 4-year follow-up. Participants with incident depression were almost twice as likely to have $\mathrm{sOH}$ at baseline compared to those without incident depression (Linear regression: 0.13 (95\% CI: 0.08-0.19) vs. 0.07 (95\% CI: 0.06-0.08)). Weighted logistic regression models demonstrated that $\mathrm{sOH}$ predicted incident depression with an odds ratio of 1.94 (95\% CI: 1.18-3.19) after controlling for covariates including subthreshold depressive symptoms. Asymptomatic $\mathrm{OH}$ did not predict incident depression.

Conclusion: This study demonstrates that $\mathrm{sOH}$ predicts incident depression in a population-representative sample of older people and may therefore represent a potentially modifiable risk factor for late life depression.

\section{O-63}

\section{Deprescribing made easier}

Denis Curtin ${ }^{1}$, Tim Dukelow ${ }^{1}$, Kirstyn James ${ }^{1}$, Desmond O’Donnell ${ }^{1}$, Denis O'Mahony ${ }^{1}$, Paul Gallagher ${ }^{1}$

${ }^{1}$ Department of Medicine, University College Cork, Cork, Ireland and Department of Geriatric Medicine, Cork University Hospital, Cork, Ireland

Introduction: Deprescribing is the process of discontinuing longterm medications with the goal of managing polypharmacy and improving outcomes. This is particularly important for older patients with advanced frailty where quality of life may be the priority over stringent chronic disease management. However, many of these patients are cared for by physicians who do not have expertise in Geriatric Medicine or Clinical Pharmacology. In this context, the process of balancing risks and benefits of medications to reach a deprescribing decision is likely to be challenging. Valid and effective methods are needed to assist non-specialist physicians with deprescribing decisions.

Methods: STOPPFrail, a concise, easy-to-use, deprescribing tool based on explicit criteria, was compared with gold standard, geriatrician-led deprescribing using 100 standardized clinical cases involving 1024 medications.

Results: STOPPFrail demonstrated substantial agreement with gold standard deprescribing [cohen's kappa 0.60]. Overall, $70.2 \%$ of potentially inappropriate medications were correctly identified by STOPPFrail. The positive predictive value of STOPPFrail was $89.3 \%$ indicating that the vast majority of deprescribing decisions were safe and aligned with gold standard care.

Key conclusions: STOPPFrail removes an important barrier to deprescribing by explicitly highlighting circumstances where commonly used medications can be safely deprescribed in older people with advanced frailty. Our results indicate that the use of STOPPFrail is safe, effective and a reasonable alternative to expert geriatric consultation.

\section{Area: Organisation of care and gerotechnology}

\section{O-64}

Elderly patients' experience of participation in the hospital discharge: a qualitative metasummary

Ingvild Lilleheie $^{1}$, Jonas Debesay ${ }^{1}$, Asta Bye ${ }^{1}$, Astrid Bergland ${ }^{1}$

${ }^{1}$ Oslo Metropolitan University, Oslo, Norway

Background: Aging patients are discharged from the hospital "quicker and sicker" than before. Hospital discharge is a critical step in patient care. The involvement of patients in healthcare is influenced by government policy, and an important part of quality improvement of care. Knowledge about participation in hospital discharge planning for elderly patients is limited, thus we want to gain an overview of the research evidence regarding patients experiences of participation in the discharge process.

Objective: To integrate international findings of older patients' experience of participation in their discharge process from hospital, and to explore the patients' rights and opportunities to influence the decision-making process through dialogue attuned to their preferences and potential.

Methods: A systematic review and a meta-summary were conducted. Data from eighteen qualitative studies were included.

Results: Findings show that older patients experience little impact in the discharge process. Participation are not sufficiently embedded in 
the process. Analysis revealed six themes influencing the patient's experiences of participation: (1) The complexity of the elderly patient's state of health, (2) Management and hospital routines, (3) The norm and preferences for returning home, (4) The challenges of communication, (5) The asymmetric relationship between health personnel and the patient, (6) The significance of a care network.

Conclusions: The results demonstrate that there is a gap between published health policy regarding elderly patients' participation and the professionals' opportunities to fulfil this policy in clinical work. To promote elderly patients' experience of participation in discharge processes, effective approaches that facilitate participating are needed.

\section{O-65}

Is the term 'Care of the Elderly' (CoE) pejorative, outdated and needs to be replaced? A patient and staff survey in a district general hospital in United Kingdom on patient and public perception

Rashid Mahmood ${ }^{1}$, Agustin Aranda-Martinez ${ }^{1}$, Amir Manzoor ${ }^{1}$, Peter Enwere ${ }^{1}$, Kamal Soliman ${ }^{1}$, Keefai Yeong ${ }^{1}$, Radcliffe Lisk ${ }^{1}$, Clarence Chikusu $^{1}$, Emma Wilkinson ${ }^{1}$

${ }^{1}$ Ashford and St Peters NHS Foundation Trust, Chertsey, UK

Introduction: The term "Care of the Elderly" is synonymous with medicine for the older people in National Health Services. Older people are sometime perceived in a positive light but negative perceptions predominate. This can have a significant negative impact on the quality of care elderly patients receive. Continuous service review, need for change with innovative ideas is therefore essential to deliver highest standard of care to our older population.

Methods: Patient feedback suggested that term 'elderly' is derogatory. To obtain wider public and patient's understanding, a survey was conducted. Patient/staff were asked about their thoughts of term 'Care of the Elderly'. List of options to choose from initially was generated by multidisciplinary team. Department of Medicine for Older People- (DMOP)Department of Older Person Care(DOPC)Department of Care of Whole Person- (DCWP) Department of Ageing and Health- (DOAH) Senior Adult Medical Service(SAMS) Acute Medicine for Mature Adults-(AMMA)Geriatric Medicine-(GM)

Results: Total 183 respondents, $42 \%$ patients/relatives, $58 \%$ staff $72 \%$ respondents suggested change. $36 \%$ of change group were patient/ relatives. 93\% chose a name from available options. $7 \%$ suggested other names. $31 \%$ respondents in change group also said the term 'care of the elderly' was discriminatory, derogatory or outdated and it inferred frailty when many older people don't feel elderly. 59\% change group proposed Senior Adult Medical Service- SAMS.

Conclusion: Survey suggested strong reservations about the term 'Care of the Elderly' and therefore a need to change and rebrand our services to older population within National Health Services.

\section{$0-66$}

Empowered signposting using a Local Asset Mapping Project (LAMP): a new era of social prescribing?

Gerard Boyle ${ }^{1}$, Christopher Soraghan ${ }^{1}$, Jennifer Feighan ${ }^{1}$, Luis Dominguez-Villoria ${ }^{1}$, Darren Clarke ${ }^{1}$, David Robinson ${ }^{1}$

${ }^{1}$ St James's Hospital, Dublin, Ireland
Background: Intersectoral health tries to bridge the medical and nonmedical approaches to wellbeing. Social prescribing is a means for a health professional to refer to a link worker who can encourage or signpost a patient to sources of practical and emotional support. These supports are often community based, and provided by the voluntary sector. Examples could be dance classes, social outings, and debt or counselling services. An up-to-date, easily accessible database is vital for such signposting.

Methods: We engaged school students, volunteers and work experience programmes to conduct an on-street census of every business, service and amenity in our hospital catchment area. These data were organised and made available in an online web portal of services that can be used to generate a personalised social prescription.

Results: $700 \mathrm{kms}$ of streets were mapped (population 198,000). 6084 individual assets were recorded. Of these, 463 were health-related, 302 were sport and fitness related, and 323 were community supports. These assets were made available at an online portal to generate social prescriptions. The mapping exercise resulted in new links between the hospital and local schools, colleges, and community centres.

Conclusion: There is a wealth of assets in the community that can be ultilised to supplement the traditional medical approach to health with a social prescription that addresses the social, emotional, or practical needs of patients.

\section{0-67}

Measuring the impact of communicative robots on older people in care facilities: do age, gender and stage of dementia matter?

Kazuko Obayashi ${ }^{1}$, Naonori Kodate ${ }^{2}$, Shigeru Masuyama ${ }^{3}$

${ }^{1}$ Social Welfare Corporation Tokyo Seishin-kai, Tokyo, Japan,

${ }^{2}$ School of Social Policy, Social Work and Social Justice, University College Dublin, Dublin, Ireland, ${ }^{3}$ Tokyo Medical University, Tokyo, Japan

The effectiveness of using socially assistive technologies in care settings has not been sufficiently assessed, despite much discussion on the inevitability of their use. The purpose of this paper is to examine how age, gender and stage of dementia can affect the effectiveness of using communicative robots in nursing facilities. The participants were 51 older people cared for in three nursing homes (43 females and 8 males; aged $86.6 \pm 8.0$ years) in Tokyo. The control group consisted of 14 females (aged $86.5 \pm 6.3$ years). Three types of communicative robots were assigned to the participants for eight successive weeks. Five goals for nursing care were selected for each participant prior to the allocation of a robot, and the changes were evaluated every day. Overall, the positive effects on residents' quality of life were observed [1]. We examined the correlations between age, gender and stage of dementia, and the degree of improvements. Statistical data were analyzed using software package R. The intervention group showed a greater improvement in its assigned goals than the control group. There was no difference between the genders. Concerning the stage of dementia, greater improvements were found in participants with moderate dementia (Stage IIb or more) than those with mild dementia (Stage IIa or lower). Communicative care robots have great potential for enhancing older people's capabilities, even among those with middle stage dementia.

\section{Reference:}

[1] Obayashi K, Kodate N, Masuyama S. Socially assistive robots and their potential in enhancing older people's activity and social participation. JAMDA, May 2018;19 (5), 462-463. 


\section{0-68}

Concurrent validity of a mobile sensor-based gait analysis system on a smart walker in oder adults with gait impairments

\section{Werner ${ }^{1}$, G. Chalvatzaki ${ }^{2}$, C.S. Tzafestas ${ }^{2}$, J. M. Bauer ${ }^{1}$, K. Hauer ${ }^{1}$}

${ }^{1}$ Department of Geriatric Research, Agaplesion Bethanien Hospital Heidelberg, Geriatric Center at the University of Heidelberg, Heidelberg, Germany, ${ }^{2}$ School of Electrical and Computer Engineering, National Technical University of Athens, Athens, Greece

Introduction: Gait tracking is an emerging functionality of smart walkers (SW). Based on real-time recorded gait parameters, SW may provide assistive actions and behaviors (e.g. velocity adjustments) specifically tailored to the individual user. An objective, on-board measurement of gait parameters represents a prerequisite for such adaptive, context-aware SW assistance. The aim of this study was to evaluate the concurrent validity of a SW-integrated gait analysis system.

Methods: Twenty-seven older rollator users ( $\geq 65$ years) with no severe cognitive impairment (Mini-Mental State Examination $\geq 17$ ) were asked to walk on a $6 \mathrm{~m}$ walkway with assistance of the SW. Spatio-temporal gait parameters (step time and length, stance and swing time, gait speed) were simultaneously recorded with the SWintegrated, sensor-based gait analysis system and the GAITRite ${ }^{\circledR}$ system. Concurrent validity was assessed by calculating betweenmethod differences, percentage errors (PE), and interclass correlation coefficients for absolute agreement (ICC).

Results: Low between-method differences were found for step time $(<0.01 \mathrm{~s})$, swing time $(0.05 \mathrm{~s})$, and stance time $(0.09 \mathrm{~s})$. For step length $(0.16 \mathrm{~m})$ and gait speed $(0.13 \mathrm{~m} / \mathrm{s})$, these differences were slightly higher. PE were acceptable for step time, step length, and gait speed (12.2-20.1\%). Stance and swing time showed PE of 39.6 and $40.6 \%$, respectively. ICCs were good to excellent for step time and gait speed $(\mathrm{ICC}=0.79-0.95)$ and moderate for step length, stance time, and swing time (ICC $=0.58-0.61$ ).

Conclusions: The SW-integrated, sensor-based gait analysis system is a valid tool for measuring spatio-temporal gait characteristics of potential SW users.

\section{0-69}

Addressing frailty in smart cities through unobtrusive data collection, data analytics and empowerment technologies. Experimental results from the City4Age project

\section{Letizia Venturini ${ }^{1}$, Giovanni Ricevuti ${ }^{1}$, Sergio Copelli ${ }^{2}$, Franco Mercalli ${ }^{2}$}

${ }^{1}$ University of Pavia, Italy, Postgraduate Course in Geriatrics, Dpt. Of Internal Medicine, Horizon 2020 City4age Project, ${ }^{2}$ Multimed Engineers Srls, Strada Felice Cavallotti 11, 43121 Parma, (Italy), Horizon 2020 City4age Project

This abstract presents a step towards the development of a datacentric approach for addressing frailty in elderly populations living in smart-cities, as experimented within the City4Age Project [1] [2]. Datasets have been collected at six Pilot sites-in Athens, Birmingham, Lecce, Madrid, Montpellier and Singapore-through a mix of advanced unobtrusive technologies. An appropriate computational model [3] has been used for mapping such datasets to relevant frailtyrelated domains, used in geriatrics (such as in the Lawton IADL scale or Fried Frailty Index). Analysis of the experimental results allowed to derive preliminary insights into the effectiveness of technologybased data collection-e.g. in terms of relative accuracy, data interdependency, etc. This is important to support smart-cities' digital policies for the deployment of age friendly environments [4]. Moreover, ground truth data has been collected at the Athens and Birmingham Pilot sites, using the Functional Ability Index [5], to label datasets with a relevant frailty status. This allowed to investigate the application of supervised machine learning algorithms for the automatic detection of early frailty onset. A preliminary proof-ofconcept demonstrated the feasibility of this approach, to support costeffective screening of the elderly population. Finally, digital communication technologies (e.g. smartphone-based messaging apps, such as WhatsApp) have been tested at the Lecce Pilot, in order to "coach" elderly participants and nudge them towards healthier behaviour through an appropriate mix of messages, conveying educational material (e.g. on nutrition, physical activity, etc.), information about local socializing or cultural events, or data about own performance along various domains.

\section{References:}

[1] Paolini P et al., City4Age: Smart Cities for Health Prevention, IEEE 2nd International Smart Cities Conference, 2016.

[2] http://www.city4ageinsight.org/.

[3] Ricevuti G et al., Data driven MCI and frailty prevention: geriatric modelling in the City4Age project, IEEE 3rd International Forum on Research and Technologies for Society and Industry, 2017.

[4] https://ec.europa.eu/eip/ageing/actiongroup/index/d4_en.

[5] Dapp U et al., Long-term prediction of changes in health status, frailty, nursing care and mortality in community-dwelling senior citizens - results from the longitudinal urban cohort ageing study (LUCAS), BMC Geriatrics, 2014.

Acknowledgement: The City4Age project has received funding from the European Union's Horizon 2020 research and innovation programme under grant agreement No 689731.

\section{O-70}

Robotic balance evaluation with hunova in older people: correlation with Short Physical Performance Battery (SPPB)

Alberto Cella ${ }^{1}$, Valentina Squeri ${ }^{2}$, Alice De Luca ${ }^{2}$, Ekaterini Zigoura $^{1}$, Francesco Vallone ${ }^{1}$, Barbara Senesi ${ }^{1}$, Angela Giorgeschi ${ }^{1}$, Katerin Leslie Quispe Guerrero ${ }^{1}$, Valentina Garofalo ${ }^{1}$, Lorenzo De Michieli $^{3}$, Jody Saglia ${ }^{3}$, Carlo Sanfilippo ${ }^{3}$, Alberto Pilotto ${ }^{1}$

${ }^{1}$ Dept. Geriatric Care, Orthogeriatrics and Rehabilitation, E.O. Galliera Hospital, Genova, Italy, ${ }^{2}$ Movendo Technology, Genova, Italy, ${ }^{3}$ Italian Institute of Technology (IIT), Genova, Italy

Introduction: Introhunova is a new robotic device developed and validated to perform functional evaluation and sensorimotor rehabilitation of lower limbs and trunk in static and dynamic conditions. This study aims to evaluate correlation between robotic balance parameters in different condition and SPPB in older community-dwelling people. Methods: 100 elderly subjects (mean age $77.17 \pm 6.49$ years) were enrolled. According to the SPPB score, participants were classified as normal (30, SPPB between 10 and 12), with intermediate functional impairment (29, SPPB 8 or 9) or severe functional impairment (37, SPPB $<8$ ). Balance was evaluated by hunova in static, dynamic and perturbating conditions, in both standing/seated positions and with open $(\mathrm{OE}) /$ closed eyes (CE). Parameters were: sway area (SA), path length (PL), range of oscillations in anterior-posterior (OAP) and medio-lateral directions (OML) (platform and trunk oscillations). Statistical analysis: Kruskal-Wallis test and Spearman correlation test.

Results: The three different SPPB classes showed significant differences in postural control parameters in standing static (SA: $p=0.03$; OAP: $\mathrm{p}=0.04 ;$ OML: $\mathrm{p}=0.03$, PA: $\mathrm{p}=0.01, \mathrm{CE}$ condition) and 
standing dynamic conditions (SA: $\mathrm{p}=0.01$; OAP: $\mathrm{p}=0.04$; OML: $\mathrm{p}=0.006 ;$ PL: $\mathrm{p}=0.004$, OE condition). Trunk control was more impaired in subjects with severe functional impairment in standing dynamic condition-OE (OAP: $p<0.001$, OML: $p<0.001$ ), in standing perturbating condition-OE (OAP: $\mathrm{p}=0.01$; OML: $\mathrm{p}<0.001$ ), and in seated perturbating condition-CE (OAP: $\mathrm{p}=0.01$, OE; OAP: $\mathrm{p}<0.001$; OML: $\mathrm{p}=0.01$ ).

Key conclusions: Robotic balance parameters assessed in static, dynamic and perturbating conditions by means of hunova showed a significant strong correlation with SPPB score in older communitydwelling people.

\section{Area: Vaccines and Immunization}

\section{0-71}

Determinants of General Practitioners' Attitude Towards Zoster Vaccination Among People Aged 65 and Over in France

Juliette Gautier ${ }^{1}$, Annick Bosseray ${ }^{2}$, Olivier Epaulard ${ }^{3}$, Caroline Landelle ${ }^{4}$, Gaëtan Gavazzi ${ }^{5}$

${ }^{1} \mathrm{CHU}$ Grenoble Alpes, France, ${ }^{2}$ Internal Medicine, CHU Grenoble Alpes, France, ${ }^{3}$ Infectious Disease Unit, CHU Grenoble Alpes, France, ${ }^{4}$ Hospital Hygien Unit, CHU Grenoble Alpes, France, ${ }^{5}$ Geriatric Medicine, CHU Grenoble Alpes, France

Introduction: Herpes zoster is a common disease among older population, which can cause severe complications. Since 2016, it has been recommended to vaccinate against it for French people aged 65-74; nevertheless, vaccine coverage remains very low in France $(<5 \%)$. Health prevention is performed by primary care providers, which is why it was necessary to analyse general practitioners' reasons to vaccinate the senior population or not.

Methods: A survey about zoster vaccine determinants was sent to 940 French general practitioners. Univariate and multivariate analysis were performed, both aiming to compare these determinants between physicians who do or do not recommend the vaccine.

Results: $17.9 \%$ of general practitioners answer the survey. $24.8 \%$ of them recommend the vaccine. 50.9\% don't fully understand the disease's complications, but this is not a significant barrier to vaccination. The most important barrier to vaccination is the vaccine's perceived lack of efficiency at reducing the incidence of zoster $(p=0.028)$. The major argument in favour of vaccination is the perception that the quality of life of elderly people is severely reduced by zoster $(\mathrm{p}=0.017)$.

Conclusion: The lack of knowledge about zoster does not appear to be a barrier to vaccination, but the lack of perception of vaccine's efficiency is. On the other hand, the negative impact of zoster on one's quality of life is a motor for vaccination. In order to improve vaccine coverage, it seems general practitioners' training is highly necessary and should encompass a demonstration of zoster's complications and of the vaccine's efficiency.

\section{Area: Urology and continence management}

\section{O-72}

Thermo-expandable metallic urethral stent for managing elderly people with indwelling urinary catheter: a compelling option to avoid long-term catheterization

Rambaud Cyrielle ${ }^{1}$, Gonfrier Sebastien ${ }^{1}$, Livigni Deborah ${ }^{1}$, Fallot Jeremy ${ }^{2}$, Arlaud Cyprien ${ }^{3}$, Leborgne Fanny ${ }^{1}$, Durand Matthieu ${ }^{2}$, Guerin Olivier ${ }^{1}$
${ }^{1}$ Geriatrics, CHU Nice, Nice, France, ${ }^{2}$ Urology, CHU Nice, ${ }^{3} \mathrm{CHU}$ Nice, Nice, France

Introduction: Management of chronic urinary retention in elderly people is challenging and time consuming, leading to high risk to place indwelling urinary catheter (IUC). The use of thermo-expandable metallic urethral stent may be an option in selected patients to avoid long-term catheterization. We assess the efficacy and treatmentrelated complications.

Methods: All consecutive patient over 70 yo with failed removal of long-term urinary catheter were proposed to a multidisciplinary decision-making including urologists and geriatricians. Alternative treatment (AT) was offered after decision process. 045-TW Memokath stent was indicated for treatment of patients without other alternatives.

Results: 96 mens were enrolled with a mean age of 85.4 yo. AT were offered to $65.6 \%(\mathrm{n}=63)$, including 25 urethral stents, 9 catheter withdrawals, 25 photovaporizations of the prostate (PVP), 2 prostatic artery embolizations and 2 other surgeries. Overall, the success rate was $85.7 \%$ at 7 days and $88.9 \%$ at 1 month. Urethral stents were significantly more offered for men with neuroleptics (OR:5.4 [1.9-15.5], $\mathrm{p}=0.002)$, neurologic comorbidities (OR: 5.4 [1.5-19.6], $\mathrm{p}=0.005)$, loss of autonomy (ADL $<2$, OR: 6.1 [2.1-18.3], $\mathrm{p}<0.001$ ) and a risk to tear off the catheter (OR: 14.9 [4.8-46.4], $\mathrm{p}<0.001$ ). The success rate of urethral stent, at 7 days, was $92 \%$, with two stent removed for an acute urinary retention. In all, 11 stents were still functioning at the end of follow-up after a mean 291 days [82-663]. 10 patients had died with a functioning stent in situ after a mean of 52 days [12-554], only one died of urinary tract infection. One stent was removed after 192 days due to an infection, and one after 170 days to make a PVP. No other serious infections or stent migrations has been reported. Residual urine was $<200 \mathrm{ml}$ after placement of the stents.

Conclusion: The multidisciplinary analysis may offer a better chance to deal with IUC in elderly people. In our cohort, urethral stents allowed to $94.4 \%$ of frailty patients to avoid an indwelling catheter. Urethral stents are indicated for dependent patients with neurologic comorbidities and a risk to tear off their catheters.

\section{Area: Acute care}

\section{O-73}

Geriatric vulnerability in older emergency department patients according to electronic health records

L.C. Blomaard ${ }^{1}$, J.A. Lucke $^{2}$, J. de Gelder ${ }^{1}$, prof. J. Gussekloo ${ }^{1}$, dr. B. de Groot ${ }^{2}$, dr. S.P. Mooijaart ${ }^{1}$

${ }^{1}$ L.U.M.C. Department of Geriatrics, ${ }^{2}$ L.U.M.C. Emergency Department

Introduction: Older emergency department (ED) patients often have complex care needs and are at increased risk of adverse outcomes. Guidelines indicate that evaluation of geriatric vulnerability can lead to improved outcomes for older patients by optimizing care. The aim of this study was to assess the current registration of the risks in social, physical and cognitive domains as a proxy for clinician's awareness of geriatric vulnerability.

Methodology: A prospective observational cohort study was conducted in ED patients aged 70 years or older. Electronic health records were evaluated by 2 independent data abstractors who used pre-defined descriptions of geriatric vulnerability in the social, physical and cognitive domain. Records were classified in one of three categories: in the lowest category no descriptions were registered on geriatric vulnerability and in the highest category 
descriptions were registered and clearly taken into account in ED management.

Results: In 100 included older ED patients ( 135 health records) interrater agreement was good (Cohen's kappa of $\kappa=0.753$ ). In most records $(\mathrm{N}=72,53 \%)$ no descriptions were registered on geriatric vulnerability. In 50 records $(37 \%)$ at least one of three domains was described, but it was not clear if this contributed to the clinicians' policy. Only 13 records (10\%) were classified in the highest category. Conclusions: Despite guidelines' recommendations clinicians working with older patients in the ED rarely register signs of geriatric vulnerability. This lack of registration may reflect inappropriate clinician awareness and perhaps hampers adequate treatment of older ED patients. Future studies should investigate how to improve guideline adherence, clinician awareness and registration of geriatric vulnerability. Due to ongoing analyses, during the conference data will be presented on $n=998$ patients, including analyses of physician and patient sub-groups.

\section{0-74}

Usefulness of $\mathrm{C}$ reactive protein and procalcitonin to rule out bacteremia in older patients: retrospective study on 776 blood cultures

Gaetan Gavazzi Gavazzi ${ }^{1}$, Lucille Meyrignac ${ }^{2}$, Sabine Drevet $^{2}$, Nabil Zerhouni $^{2}$, Nathalie Mitha ${ }^{2}$, Clara LOpez ${ }^{2}$, Patrice François ${ }^{3}$

${ }^{1}$ University Clinic of Geriatric Medicine, ${ }^{2}$ University clinic of Geriatric Medicine, University of Grenoble-Alpes, Grenoble cedex, France, ${ }^{3}$ Quality of Care Unit, University of Grenoble-Alpes, Grenoble cedex, France

Introduction: When bacteremia is suspected, despite few clinical signs, but because of potential severity, antibiotic therapy is often begins; this may leads to antibiotic over-prescriptions as $8-20 \%$ of Blood cultures (BC) are positives. Biomarkers should help in these situations. We aim to study clinical performances of $\mathrm{C}$ reactiveProtein (CRP) and procalcitonin (PCT) to rule out bacteremia occurring in older patient $>75$ years.

Methods: It is a retrospective study carried out in 5 geriatric wards between 2012 and 2016. All patients with negative and positive BC, CRP and PCT dosages at the same time of BC have been included. We compared ROC curves and specifically negative predictive value (NPV) performance between CRP and PCT.

Results: We included 623 patients, mean 86.7 years of age, analysed $776 \mathrm{BC}$, out of them 118 were positives (15.2\%), 109 monomicrobial andt 9 polymicrobial (2 germes), with 127 different germs. $\mathrm{a}<0.3 \mathrm{ng} / \mathrm{ml}$ PCT threshold had significant better clinical performance than a $<100 \mathrm{mg} / \mathrm{l}$ CRP threshold ( $\mathrm{p}<0.0001$ ), 0.78 (IC 0.73 0.82 ) et de 0.62 (IC $0.57-0.68$ ). NPV were respectively at $95 \%$ and $91 \%$. With such thresholds antiobitic therapy should have been avoided for 353/776 BC (45.5\%) with PCT and for only 110/776 BC (14.2\%) for CRP.

Conclusions: In our aged population, a $<0.3 \mathrm{ng} / \mathrm{ml}$ PCT threshold could rule out bacteremia for $95 \%$. Better than CRP $(<100 \mathrm{mg} / \mathrm{l})$. We need futher demontrate in a prospective real life study if an algorithm with a such threshold may avoid antibiotic initiation in theses situations.
0-75

Assessment of anemia and functionality in a geriatric acute care unit

J Corrales Cardenal ${ }^{1}$, C Elías de Molíns Peña ${ }^{1}$, G Jimenez Clemente ${ }^{1}$, S Solsona Fernández ${ }^{1}$

${ }^{1}$ Servicio de Geriatría, Hospital Provincial Nuestra Señora de Gracia, Zaragoza, Spain

Introduction: Describe the patients profile with anemia that are admitted in a geriatric acute care unit (GACU) and assess the association between anemia and functional status.

Methods: Observational, descriptive, transversal study. Included all the patients admitted in the GACU who were diagnosed with anemia in the blood test at admission (according to WHO criteria) November-December 2017. Variables: association between anemia, nutritional status and functionality (Barthel index). Statistical analysis: SPSS.

Results: 163 patients were admitted in GACU, 73 patients $(48.5 \%)$ were diagnosed with anemia. Mean age was 87.6 years $( \pm 6.2)$. $63.3 \%$ were women. Mean previous Barthel index is 35, Pfeiffer 5 errors, MNA 8.2 and Charlson index $2.87 .48 \%$ had previous diagnosis of anemia, types of anemia: chronic process $31.2 \%$; ferropenic $24 \%$; mixed $23.4 \%$; folic acid deficiency $7.8 \%$; vitamin B12 deficiency $5.2 \%$; associated with renal pathology $5.2 \%$; others: $2.6 \% .78 \%$ had mild anemia, $14 \%$ moderate and $7.6 \%$ severe. $41 \%$ had total dependence previously and $12.8 \%$ was severe. $42.4 \%$ had malnutrition and $44.1 \%$ were in risk of malnutrition. In the bivariate study the severity of anemia was related with malnutrition $\mathrm{p}=0.037$, more dependence $\mathrm{p}=0.058$ and intrahospital mortality $\mathrm{p}=0.002$.

Conclusions: Anemia in elderly patients admitted in GACU is prevalent, its profile is a woman, severely dependent, cognitive impairment and malnutrition. Greater degree of anemia was associated with worst functional status, malnutrition and mortality. Half of the patients with anemia didn't have previous diagnosis, which motivates the necessity to do a better diagnose assessment.

\section{0-76}

The acute care of the elderly unit: providing rapid specialised care for frail older people

Towhid Imam ${ }^{1}$, Robert Goldsby-West ${ }^{1}$, Wallace $\operatorname{Tan}^{1}$, Joe Rehman ${ }^{1}$, Joanne Bramble $^{1}$, Alan Earnshaw ${ }^{1}$, Nima Hashemi ${ }^{1}$

${ }^{1}$ Croydon University Hospital, Thornton Heath, UK

Introduction: The aim of the Acute Care of the Elderly Unit was to deliver early comprehensive geriatric assessment providing robust discharge plans resulting in more efficient and safer care. It formed part of a whole system transformation in the management of the ageing population of Croydon to prevent unnecessary hospital admissions and enable specialist care in the right place at the right time for the older person in or approaching crisis.

Methods: A short-stay inpatient facility with daily consultant ward rounds of 14 beds seeing was created. A multidisciplinary approach was integral involving doctors, therapists, pharmacy and social worker support, old age psychiatry and the voluntary sector. A GP/ED hotline to a consultant geriatrician with rapid access outpatient clinic was available. Appropriate patients were chosen from the emergency and acute medical services to relieve pressure on the front door.

Results: Approximately 100 patients were seen per month over a oneyear period. The impact was a $10 \%$ increase in patients discharged within $24 \mathrm{~h}$ of admission. There a reduction in the average length of stay in the hospital of 5 days. A reduction in the re-admission 
numbers was observed. This enabled 80 inpatient beds to be closed over a three-year period.

Key conclusions: Discharges were more streamlined for several reasons. Social care provision was efficient as sections were not required. Pharmacists participated in ward rounds to ensure medications were promptly arranged. The unit streamlined referrals to community services improving the care of the discharged patient and preventing readmission.

\section{0-77}

\section{The Frailty Index in the emergency department}

\section{E. Patrizio ${ }^{1}$, L.C. Bergamaschini ${ }^{2}$, M. Cesari ${ }^{3}$}

${ }^{1}$ Università degli Studi di Milano, Milan, Italy, ${ }^{2}$ Università degli Studi di Milano; ASP IMMeS e Pio Albergo Trivulzio, Milan, Italy, ${ }^{3}$ Università degli Studi di Milano; Fondazione IRCCS Ca' Granda Ospedale Maggiore Policlinico, Milan, Italy

Introduction: The number of frail patients admitted to Emergency Departments (ED) is increasing. It is important to identify individuals in the need of adapted care since their first contact with the hospital system. The Frailty Index (FI), based on the age-related accumulation of deficits model might be computed from routinely collected data and support clinical decisions.

Methods: Data are from medical records of patients admitted to our ED in 3 random days of 2017. A 42-item FI was computed from vital signs, physical examination, anamnestic data, and blood tests. Length of hospital stay (from the day of admission to ED till the hospital discharge or death) and institutionalization of the patient at the discharge were the dependent variables of interest.

Results: A total of 40 patients (mean age $=65.1 \pm 10.6$ years) was considered. Mean lenght of stay was $13.0 \pm 10.6$ days. Institutionalisation rate at discharge was $19.4 \%$. The median FI was 0.23 $(\mathrm{IQR}=0.15-0.31)$. The FI was significantly correlated with the lenght of stay $(r=0.48, p=0.002)$. This finding was confirmed after adjustment for age and gender $(\mathrm{p}=0.001)$. The FI was associated with the risk of institutionalization at discharge $(\mathrm{OR}=1.11,95 \%$ $\mathrm{CI}=1.01-1.22, \mathrm{p}=0.028)$. No association of age with lenght of stay or institutionalization was reported.

Conclusions: The creation of a FI from data part of the routine ED visits is possible. As such, the tool can be computed without burdening the clinical practice. Its use may support clinical decisions since the first contact of the individual with the hospital system.

\section{O-78}

Acute heart failure: clinical characteristics and in-hospital outcomes in elderly patients. Realworld data from ATHENA registry

Andrea Herbst ${ }^{1}$, Francesco Orso ${ }^{1}$, Alessandra Pratesi ${ }^{1}$, Giulia Biagioni ${ }^{1}$, Filippo Fedeli ${ }^{1}$, Silvia Parlapiano ${ }^{1}$, Camilla Ghiara ${ }^{1}$, Aldo Lo Forte ${ }^{1}$, Camilla Tozzetti ${ }^{1}$, Gabriele Ciuti ${ }^{1}$, Francesca Bacci ${ }^{1}$, Mauro Di bari ${ }^{1}$, Niccolò Marchionni ${ }^{1}$, Samuele Baldasseroni ${ }^{1}$, Andrea Ungar ${ }^{1}$, Anna Chiara Baroncini ${ }^{1}$

\section{${ }^{1}$ Azienda Ospedaliera Universitaria Careggi}

Introduction: Acute heart failure (AHF) is a common disease in elderly. Our purpose was to compare clinical characteristics and inhospital prognosis of elderly patients hospitalised for AHF in cardiology, internal medicine and geriatrics.
Methods: data derived from ATHENA, a retrospective observational study, which included elderly patients ( $\geq 65$ years) admitted for AHF to the Emergency department (ED) of a tertiary University teachinghospital and transferred to this settings of care in the period 01.12.2014-01.12.2015.

Results: 401 patients were enlisted; $15 \%$ from cardiology, $15 \%$ from geriatrics and $70 \%$ from internal medicine. Mean age was 83.5 years, resulting higher in geriatrics (86.9 years) versus internal medicine (83.4 years) and cardiology (81.0 years), $\mathrm{P}=0.001$. Females were $52.6 \%$ without statistically significant differences between the three settings. Patients with HFpEF were $47.4 \%$ : $36.8 \%$ from cardiology, $55.1 \%$ from geriatrics and $44.8 \%$ from internal medicine, $\mathrm{P}=0.147$. In-hospital mortality was $8.9 \%$ and it was higher in geriatrics $(18.6 \%)$ compared to cardiology $(14.8 \%)$ and internal medicine $(5.7 \%)$, $(\mathrm{P}=0.002)$. Independent predictors of in-hospital mortality were related with Barthel index (OR 0.98, 95\% CI 0.97-1.00, $\mathrm{p}=0.028$ ), systolic blood pressure in ED (OR 0.96, CI $=0.93-0.99, \mathrm{p}=0.014)$, cognitive impairment (OR 5.33, CI $=1.16-24.40, \mathrm{p}=0.031)$ and the setting of care; it was significantly lower in patients hospitalized in cardiology (geriatrics vs cardiology OR 13.23, 95\% CI 1.80-97.07, $\mathrm{p}=0.011$; internal medicine vs cardiology OR $12.32 ; 95 \% \mathrm{CI}$ 2.25-67.54).

Key conclusions: elderly patients with AHF differ significantly in terms of clinical characteristics and in-hospital prognosis according to the different settings of care.

\section{0-79}

\section{Effect of systemic intensive care unit triage on long-term mortality among critically ill elderly patients in France: a randomized clinical trial}

Hélène Vallet ${ }^{1}$, Caroline Thomas ${ }^{2}$, Laura Moisi ${ }^{2}$, Jacques Boddaert ${ }^{1}$, Ariane Boumendil ${ }^{3}$, Bertrand Guidet ${ }^{3}$

${ }^{1}$ Service de Gériatrie aigüe, unité périoopératoire gériatrique, DHU FAST, Groupe Hospitalier Pitié Salpêtrière Charles Foix, Paris, France, ${ }^{2}$ Service de Gériatrie aigüe, hôpital saint Antoine, Paris, France, ${ }^{3}$ Service de réanimation médicale, hôpital saint Antoine, Paris, France

Introduction: The high mortality rate in critically ill patients has led to questioning of the beneficial effect of intensive care unit (ICU) admission. The objective of this study was to determine whether a recommendation for systemic ICU admission in critically ill elderly patients reduces 6-month mortality compared with usual practice. Material: Multicenter, cluster-randomized clinical trial of critically ill patients (age $\geq 75$ years), free of cancer, with preserved functional status and nutritional status, was performed. Centers were randomly assigned either to use a program to promote systematic ICU admission of patients ( $\mathrm{n}=1519$ participants) or to follow standard practice ( $\mathrm{n}=1518$ participants). The primary outcome was death at 6 months. Secondary outcomes included ICU admission rate, in-hospital death, functional status, and quality of life at 6 months.

Results: 3036 patients were included in the trial (median age, 85 years). Patients in the systematic strategy group had an increased risk of death at 6 months (45\%vs 39\%; relative risk [RR], 1.16; 95\% CI, 1.07-1.26) despite an increased ICU admission rate (61\% vs $34 \%$; RR, 1.80 ; 95\% CI, 1.66-1.95). After adjustments, patients in the systematic strategy group were more likely to be admitted to an ICU (RR, 1.68; 95\% CI, 1.54-1.82) and had a higher risk of in-hospital death (RR, 1.18; 95\% CI, 1.03-1.33) but had no significant increase in risk of death at 6 months (RR, 1.05; 95\% CI, 0.96-1.14). Functional status and physical quality of life at 6 months were not significantly different between groups. 
Conclusion: Among critically ill elderly patients in France, a program to promote systematic ICU admission increased ICU use but did not reduce 6-month mortality.

\section{O-80}

Is the emergency department fast-track system effective and safe for older patients? Beatrice Gasperini ${ }^{1}$, Andrea Fazi ${ }^{2}$, Gianfranco Maracchini ${ }^{2}$,
Antonio Cherubini

${ }^{1}$ Emergency Department Azienda Ospedaliero Universitaria Ospedali Riuniti, Ancona, Italy, ${ }^{2}$ Emergency Department Ospedale Principe di Piemonte, Senigallia, AN, Italy, ${ }^{3}$ Geriatrics and Geriatric Emergency Care, IRCCS-INRCA, Ancona, Italy

Introduction: Emergency department (ED) fast-track system is a pathway for patients admitted with non-urgent complaints to decrease ED length of stay (LOS). Although its efficacy is proven in the general patient population, its role in the management of older adults is still unclear. The primary outcome measure was ED LOS for fasttrack older patients. Secondary outcome was their rate of early unplanned readmissions.

Methods: A retrospective single-center cohort study. Patients aged at least 65 years treated by the ED fast-track (cases) between 1 January and 31 December 2012 were compared with patients treated by the usual ED processes (controls) from 1 January and 31 December 2010 ( $n=546$ matched pairs), when the fast-track system was not active. Propensity score method was used for matching by age, gender, priority level at admission and ICD9 diagnosis.

Results: Time between admission and discharge was $136+92 \mathrm{~min}$ in the fast-track group vs $304+506$ in the control group ( $<<0.001)$. The time between admission and clinical examination was $117+71 \mathrm{~min}$ in the fast-track group vs $104+91$ in the control group $(\mathrm{p}<0.001)$. Time between clinical examination and discharge was $19+60 \mathrm{~min}$ in the fast-track group versus $199+501$ in the control group $(\mathrm{p}<0.001)$. There were 69 unplanned readmissions within a week from discharge. Among these, 3.8\% occurred in the fast-track group $(n=21)$ compared to $8.8 \%$ in the control group $(n=48)$ $(\mathrm{p}<0.001)$

Key conclusions: The fast-track system reduces the time spent to the ED of older adults admitted for non-urgent complaints with a low rate of unplanned readmissions.

\section{0-81}

Rapid cognitive assessment with $4 \mathrm{AT}$ in acutely ill patients aged $\geq 65$ years with suspected infection

Marius Myrstad ${ }^{1}$, Srdan Alaburic $^{1}$, Guri Hagberg ${ }^{1}$, Bjørn Erik Neerland ${ }^{2}$, Leiv Otto Watne ${ }^{2}$

${ }^{1}$ Department of Medical Research, Bærum Hospital, Oslo, Norway, ${ }^{2}$ Oslo Delirium Research Group, Department of Geriatric Medicine, Oslo University Hospital, Oslo, Norway

Background: Quick Sequential Organ Failure Assessment (qSOFA) is recommended for acute sepsis management. Altered mental state, respiratory rate $\geq 22 / \mathrm{min}$, and systolic blood pressure $\leq 100 \mathrm{mmHg}$ give one point each, and qSOFA $\geq 2$ is associated with poor outcome. With the aim to improve cognitive assessment in the Emergency Room (ER), we used 4AT (rapid screening of alertness, cognition, attention and fluctuation of symptoms) in addition to
qSOFA. $4 \mathrm{AT} \geq 4$ indicates delirium or cognitive impairment, and a 4AT of 1-3 might indicate cognitive impairment.

Methods: From October 23rd 2017 to May 14th 2018, patients aged $\geq 65$ years with suspected infections admitted to a local Norwegian hospital were screened by ER nurses with qSOFA and 4AT. Time spent on 4AT was reported. Delirium diagnoses and in-hospital mortality were retrieved from patient records.

Results: 4AT- and qSOFA-scores were available for 111 patients (mean age 81, range 65-98). Median time spent on the assessment with 4AT was two Minutes (mean 2.6). Among 39 patients with a qSOFA-point given for altered mental state, 4AT revealed signs of cognitive impairment in $37(95 \%)$. 4AT revealed signs of cognitive impairment in 26 out of 72 patients $(36 \%)$ where qSOFA did not reveal altered mental state. In total, 29 patients $(26 \%)$ had delirium during the hospital stay. The overall in-hospital mortality was $4.5 \%$. Conclusions: 4AT is a rapid assessment of cognitive impairment feasible for use in the ER. 4AT improved the assessment of cognitive impairment in patients aged $\geq 65$ years with suspected infection. We found a high prevalence of delirium.

\section{Area: Cognition and dementia}

\section{O-82}

Association between blood pressure variability and findings on magnetic resonance imaging in memory clinic patients

LM van den $\operatorname{Ingh}^{1}$, RAA de Heus ${ }^{2}$, AL Meendering ${ }^{3}$, JAHR Claassen ${ }^{2}$

${ }^{1}$ Radboudumc, department of Geriatric Medicine, Nijmegen, The Netherlands, ${ }^{2}$ Radboudumc, department of Geriatric Medicine, Nijmegen, The Netherlands. Donders Institute for Brain, Cognition and Behaviour, Nijmegen, The Netherlands, ${ }^{3}$ Radboudumc, department of Geriatric Medicine, Nijmegen, The Netherlands

Introduction: Emerging evidence suggests a role for high blood pressure variability (BPV) in the association between blood pressure (BP) and cognition, but the pathophysiological mechanisms remain unclear. The aim of this study was to investigate the association of day-to-day BPV with white matter hyperintensities (WMH) and cerebral atrophy on magnetic resonance imaging (MRI).

Methods: In this cross-sectional study, data from 93 memory clinic patients were analyzed. BP was measured twice in the morning and evening for 1 week. BPV was expressed as coefficient of variation (COV). WMH were assessed with the Fazekas scale (0-3). Additionally, the global cortical atrophy (GCA) and medial temporal lobe atrophy (MTA) were scored. ANCOVA was conducted to investigate BPV in different groups of Fazekas, GCA or MTA, corrected for age and mean BP. As a second step, smoking status, diabetes, use of antihypertensives, medical history of hypertension and hypercholesterolemia were added in the analysis.

Results: Higher Fazekas score was significantly related to BPV (COV of systolic BP), controlling for age, $\mathrm{F}(3,77)=3.176, \mathrm{p}=0.029$. Controlling for mean systolic BP reduced the statistical significance of this association, $\mathrm{F}(3,76)=2.640, \mathrm{p}=0.056$. Planned contrasts showed higher COV of systolic BP for Fazekas 1, 2 or 3 compared to Fazekas 0 ( $\mathrm{p}=0.047,0.010$ and 0.019 , respectively). Secondary analysis showed that smoking status and hypercholesterolemia significantly influenced the correlation between COV and Fazekas ( $p=0.040$ and 0.036 , respectively). No significant associations were present for GCA and MTA with BPV.

Conclusion: These results suggest an association between BPV and WMH, but not with other markers of neurodegeneration. More research is recommended to further explore this association. 


\section{0-83}

Efficacy of hearing aids on the cognitive status of patients with Alzheimer's disease and hearing loss: a multicenter controlled randomized trial

Nguyen Marie-France ${ }^{1}$, Bonnefoy Marc ${ }^{2}$, Adrait Arnaud ${ }^{1}$, Gueugnon Marine ${ }^{3}$, Petitot Charles ${ }^{1}$, Collet Lionel ${ }^{4}$, Roux Adeline ${ }^{3}$, Perrot Xavier

${ }^{1}$ Geriatric Medicine unit, Lyon Sud Hospital, Hospices Civils de Lyon, Pierre Bénite, France, ${ }^{2}$ Geriatric Medicine unit, Lyon Sud Hospital, Hospices Civils de Lyon, Pierre Bénite, France, University Claude Bernard Lyon 1, Faculty of Medicine Lyon Sud, Pierre Bénite, France, ${ }^{3}$ Clinical Research unit, Pole IMER, Hospices Civils de Lyon, France, ${ }^{4}$ Department of Audiology, Edouard Herriot Hospital, Hospices Civils de Lyon, Lyon, France, ${ }^{5}$ University Claude Bernard Lyon 1, Faculty of Medicine Lyon Sud, Pierre Bénite, France; Department of Audiology, Edouard Herriot Hospital, Hospices Civils de Lyon, Lyon, France

Introduction: This study evaluated the cognitive benefit of hearing aids (HA) in older patients with Alzheimer'sdisease (AD) and hearing loss (HL) after a 6- and 12-month period of utilization.

Methods: A multicenter double-blind randomized placebo-controlled trial was conducted in patients aged more than 65 years. A group was equipped with active HA for 6 months (active group) and a second group had placebo HA for 6 months (placebo group) followed by a secondary activation phase for a further 6 months (semi crossover procedure). Both groups were retested after a 12-month period. The primary endpoint was the change from baseline of the Alzheimer's Disease Assessment Scale-Cognitive subscale (ADAS Cog) after a 6-month period in both groups and after 6 months of secondary HA activation in the placebo group. A smaller cognitive decline should be obtained with HA use; an increase in ADAS Cog score of less than 6 points was defined a success.

Results: Fifty-one patients aged 68 to 99 years were included; 38 attended the 6-month visit: 18 in the active group and 20 in the placebo group. At 6 months, $14(82.4 \%)$ successes were noticed in the active group, and $15(88.2 \%)$ in the placebo group $(\mathrm{p}=1.0)$; delta ADAS Cog in the active group was $1.8 \pm 5.3$ and $1.3 \pm 5.3$ in the placebo group $(\mathrm{p}=0.8)$. In the placebo group, after the secondary HA activation, no significant improvement was observed.

Conclusion: No significant effect of HA use was observed after 6 months of follow-up in patients with AD and HL.

\section{O-84}

Delirium and other complications during hospital stay related to femoral nerve block vs conventional pain management among patients with hip fracture: A randomised controlled trial

Anna Unneby ${ }^{1}$, Olle Svensson ${ }^{1}$, Britt-Marie Lindgren ${ }^{1}$, Ulrica Bergström ${ }^{1}$, Yngve Gustafson ${ }^{1}$

${ }^{1}$ Umeå University, Umeå, Sweden

Background: The aim of this study was to investigate whether Femoral Nerve Block (FNB) can reduce complications during hospital stay, with special focus on delirium, compared to conventional pain management with opioids among patients with hip fracture, including those with dementia.

Patients and methods: In a prospective randomized controlled trial, 236 consecutive patients with hip fracture $>70$ years were assigned to preoperatively either receive FNB and opioids if required (intervention group, $\mathrm{n}=116$ ) or conventional pain management using opioids if required (control group, $n=120$ ). The FNB was given as soon as possible after admission. Delirium was set according to DSMIV-TR criteria based on Nursing Delirium Screening Scale, Mini Mental State Examination, Organic Brain Syndrome Scale and documentations in the medical records.

Preliminary results: Most participants, 157 (66\%), were women, mean age was $84( \pm 6.7)$ years and $109(46 \%)$ participants had dementia disorders. Forty-four patients $(38.9 \%)$ developed delirium preoperatively in the intervention group compared with $59(49.2 \%)$ patients in the control group $(\mathrm{p}=0.116)$. Common postoperative complications were postoperative delirium, malnutrition, anaemia, constipation and urinary infection with no significant difference between the groups. A subgroup analysis among patients with dementia, intervention group had significantly less number of complications $(6.1 \pm 1.9$ vs $6.8 \pm 1.6, \quad \mathrm{p}=0.038)$ compared with controls.

Conclusion: Femoral nerve block is a safe and efficient pain management for patients with hip fracture but did not reduce the incidence of complications. However, patients with dementia that received FNB suffered less number of complications.

\section{O-85}

Symptom profiles in Alzheimer's disease patients with and without cerebrovascular disease

Rannveig Eldholm ${ }^{1}$, Maria Barca ${ }^{2}$, Karin Persson ${ }^{2}$, Anne-Brita Knapskog ${ }^{3}$, Geir Selbæk ${ }^{2}$, Knut Engedal ${ }^{2}$, Eva Skovlund ${ }^{4}$, Ingvild Saltvedt ${ }^{1}$

${ }^{1}$ Department of Neuromedicine and Movement Science, NTNU, Trondheim, Norway, ${ }^{2}$ Norwegian National Advisory Unit on Ageing and Health, Tønsberg, Norway, ${ }^{3}$ Department of Geriatric Medicine, Memory Clinic, Oslo University Hospital, Ullevaal, Oslo, Norway, ${ }^{4}$ Department of Public Health and Nursing, NTNU, Trondheim, Norway

Introduction: Alzheimer's disease (AD) patients often have concomitant cerebrovascular disease (CVD) and could therefore be expected to display symptoms of both conditions. We aimed to investigate whether symptom profile differs in $\mathrm{AD}$ patients with and without concomitant CVD. According to diagnostic criteria, medial temporal lobe atrophy (MTA) is a biomarker of AD. Our second aim was to study differences in MTA among AD patients with and without CVD.

Methods: AD patients $(n=192)$ with mild dementia or amnestic mild cognitive impairment underwent cognitive assessment and depression screening in memory clinics. Results of cognitive tests for memory, executive function, processing speed, and measures of depression were compared in patients with and without CVD. Cerebral MRIs were assessed for MTA, white matter hyperintensities (WMH) and infarcts. CVD was defined as WMH Fazekas scale $\geq 2$, lacunae or cortical infarcts. Multiple regression analyses were carried out with CVD, age and sex as independent variables, and cognitive test scores, depression scores and MTA as dependent variables, respectively.

Results: In unadjusted analyses, patients with CVD $(n=121)$ scored lower on processing speed, executive function and immediate recall, compared to patients without CVD $(n=71)$. In regression analyses adjusted for age and sex, there was no association between concomitant CVD and scores on cognitive tests or depression rating scales. A statistically significant association between CVD and more pronounced MTA was identified.

Key conclusions: Contrary to what we expected, cognitive profile did not differ between AD patients with and without CVD, and MTA was more pronounced among $\mathrm{AD}$ patients with $\mathrm{CVD}$. 


\section{0-86}

The relationship between peripheral inflammation and progression of Alzheimer's Disease

Josje Hazen $^{1}$, Maria Lage Barca ${ }^{2}$, Rannveig Eldholm ${ }^{3}$, Karin Persson ${ }^{4}$, Maria Vistnes ${ }^{5}$, Ingvild Saltvedt ${ }^{6}$, Geir Selbæk ${ }^{7}$, Knut Engedal $^{4}$, Anne-Brita Knapskog ${ }^{8}$

${ }^{1}$ The Faculty of Medicine, Utrecht University, Utrecht, The Netherlands, ${ }^{2}$ Norwegian National Advisory Unit on Ageing and Health, Tønsberg, Department of Geriatric medicine, Oslo University Hospital, Ullevål, Norway, ${ }^{3}$ Department of Neuromedicine and Movement Science, Norwegian University of Science and Technology (NTNU), Trondheim, Department of Geriatrics, St Olav Hospital, University Hospital of Trondheim, Trondheim, Norway, ${ }^{4}$ Norwegian National Advisory Unit on Ageing and Health, Tønsberg, Department of Geriatric medicine, Oslo University Hospital, Ullevål, Norway, ${ }^{5}$ Department of Internal Medicine, Diakonhjemmet Hospital, Institute for experimental medical research, Oslo University Hospital, University of Oslo, Oslo, Norway, ${ }^{6}$ Department of Neuromedicine and Movement Science, Norwegian University of Science and Technology (NTNU), Trondheim, Department of Geriatrics, St Olav Hospital, University Hospital of Trondheim, Trondheim, Norway, ${ }^{7}$ Centre for Old Age Psychiatric Research, Innlandet Hospital Trust, Ottestad, Institute of Health and Society, University of Oslo, Oslo, Norway, ${ }^{8}$ Department of Geriatric medicine, Oslo University Hospital, Ullevål, Norway

Introduction: Neuroinflammation may play an important role in the pathogenesis of Alzheimer's disease (AD). We wanted to examine if inflammatory activity at baseline could predict cognitive and functional decline among patients with amnestic mild cognitive impairment (MCI) and AD dementia after 2 years.

Methods: Samples of serum were collected from 245 patients with $\mathrm{aMCI}$ and $\mathrm{AD}$ dementia at baseline, and analyzed for 14 inflammatory markers (interleukin (IL)-1 $\beta,-1 \mathrm{ra},-6,-10,-12 \mathrm{p} 40,-17 \mathrm{a},-18,-22,-33$, tumor necrosis factor (TNF), CD40 ligand, interferon (IFN)- $\gamma$, CCL2 and -4 ) by bead-based multiplex immunoassay. All patients were followed up after approximately 2 years (18-36 months). Disease progression was measured by the annual increase in the Clinical Dementia Rating Scale Sum of Boxes (CDR-SB) and annual decrease in the Mini-Mental State Examination (MMSE) test.

Results: The CDR-SB score increased with a mean of 1.63 (1.83 SD), the MMSE sum score decreased with a mean of 1.67 (2.44 SD) per year. The markers IL-1 $\beta$, IL-10, IL-12p40, IL-17a, IL-22, IL-33 and IFN- $\gamma$ were below the lowest standard curve point in more than $50 \%$ of the patients and were excluded from further analysis. We did not find a significant association between increased levels of inflammatory markers and either CDR-SB or MMSE change, by using univariate and multiple regression analysis.

Key conclusions: Increased levels of IL-1ra, IL-6, IL-18, CD40L, CCL2, CCL4, TNF $\alpha$ and CRP at baseline were not significantly associated with a faster cognitive decline measured by annual change in CDR-SB- and MMSE after two years of follow -up.

\section{0-87}

\section{A multi-level, mixed methods evaluation of Irish national dementia strategy implementation plan}

Irene Hartigan ${ }^{1}$, Laura O' Philbin ${ }^{1}$, Tom Andrews ${ }^{1}$ Jonathan Drennan $^{1}$, Jenny van der Steen ${ }^{2}$, Kevin Brazil ${ }^{3}$, Niamh O' Regan ${ }^{4}$, Catherine Buckley ${ }^{5}$, Corina Naughton ${ }^{1}$, Alice Coffey ${ }^{6}$, Kieran O' Connor ${ }^{7}$, Rónán O'Caoimh ${ }^{8}$, Nicola Cornally ${ }^{1}$
${ }^{1} \mathrm{UCC},{ }^{2} \mathrm{LEI},{ }^{3} \mathrm{QUB},{ }^{4} \mathrm{UWO},{ }^{5} \mathrm{NHERC},{ }^{6} \mathrm{UL},{ }^{7} \mathrm{MUH},{ }^{8} \mathrm{UHG}$

Background: Ireland's first National Dementia Strategy (NDS) was launched in 2014, aiming to meet the needs of people living with dementia in Ireland, now and in the future. This was followed by the $€ 27.5 \mathrm{~m}$ National Dementia Strategy Implementation Plan (NDSIP), a joint initiative between the Irish Department of Health, Health Service Executive, and Atlantic Philanthropies to implement significant elements of the Irish NDS between 2014 and 2017. Aim: The aim of this research is to evaluate the multi-level impact and progress-to-date of the NDSIP.

Methods: A mixed-methods approach was used. Perspectives of stakeholders on executive, strategic, and operational levels were explored through semi-structured focus groups, interviews, and online surveys. Stakeholders included people living with dementia, caregivers of people living with dementia, health and social care providers, not-for-profit organisations, representatives of government departments, and other stakeholders who were involved in the development, monitoring and implementation of both the NDS and NDSIP.

Results/conclusions: Results are discussed in relation to the progress and multi-level impact of the NDSIP from the perspectives of all stakeholders. Comparisons are drawn between the experiences of people with dementia, caregivers, not-for-profit organisations, and health and social care service providers in 'resource intensive' and 'resource-limited' regions in Ireland.

\section{O-88}

Cognitive decline and survival in Parkinson's disease dementia and dementia with Lewy bodies: longitudinal data from the Swedish dementia registry

Maria Eriksdotter ${ }^{1}$, Johan Lökk ${ }^{1}$, Anders Wimo ${ }^{2}$, Mohammad Fereshtehnejad ${ }^{3}$

${ }^{1}$ Division of Clinical Geriatrics, Department of Neurobiology, Care Sciences and Society (NVS), Karolinska Institutet, Stockholm,

Sweden, ${ }^{2}$ Theme Aging, Karolinska University Hospital, Huddinge, Sweden, ${ }^{3}$ Division of Neurogeriatrics, Department of Neurobiology, Care Sciences and Society (NVS), Karolinska Institutet, Huddinge, Sweden, ${ }^{4}$ Department of Neurology and Neurosurgery, McGill University, Montreal, QC, Canada.

Background: It is not yet fully understood whether dementia with Lewy bodies (DLB) and Parkinson's disease dementia (PDD) are distinct disorders or two subtypes of the same entity. There is a gap of knowledge on differences in longitudinal clinical outcomes between DLB and PDD. Objective: In DLB and PDD patients compare the longitudinal trend of cognitive decline and mortality.

Methods: 1110 DLB and 764 PDD patients who were registered in the Swedish dementia registry (SveDem, http://www.svedem.se) at the time of diagnosis during 2007-2015 were included. Cognitive status using mini-mental state examination (MMSE) was assessed at baseline and each follow-up visit. The rate of cognitive decline and mortality risk were compared between the two groups.

Results: The rate of cognitive decline did not differ between DLB (1.1 MMSE units/year) and PDD (1.2 MMSE units/year) groups after 2-years follow-up. Antipsychotic drug use in DLB and higher comorbidity burden in PDD were the most important determinants of faster annual cognitive decline. There was no significant difference in the median survival time between DLB (4.0 years) and PDD (4.1 years) groups.

Conclusion: There were no differences between the rate of cognitive decline and mortality between DLB and PDD in clinical routine settings at least over 2 years suggesting that DLB and PDD may be 
similar disorders with different phenotypes. However, comorbidity burden and antipsychotic use influence the rate of cognitive decline.

\section{0-89}

Development of the highly reproducible standardized evaluation system for dementia care by thinking model of artificial intelligence

\section{Yuki Sasaki ${ }^{1}$, Shogo Ishikawa ${ }^{1}$, Shinya Kiriyama ${ }^{1}$, Yves Gineste $^{2}$, Miwako Honda ${ }^{3}$ \\ ${ }^{1}$ Shizuoka University, Shizuoka, Japan, ${ }^{2}$ Kyoto University, Kyoto, Japan, ${ }^{3}$ National Hospital Organization Tokyo Medical Center, \\ Tokyo, Japan}

Background: As population of dementia increase, need of educations for caregivers became crucial. A French multimodal care methodology, Humanitude, has shown improvement of Behavioral Psychological Symptoms of Dementia, reduction of incidence of delirium in elder patients, and reduction of the burden of caregivers. Since traditional on-site training has limitation to numbers of trainees, effective standardized mass training is needed to educate highly skilled healthcare professionals.

Methods: A multiple-thinking-model based on the concept of Marvin Minsky was developed for interaction between people with dementia and their caregivers. The video analysis system, consisted of hierarchical evaluations about 9 basic elements of Humanitude, was created with the thinking model of artificial intelligence. The videos which contain 5 min oral care by healthcare professionals were used for the evaluation. The certified trainers of Humanitude watched the videos and made hierarchical and narrative evaluation based on the multiplethinking-model. They independently watched the videos and gave 3 -grade evaluation of 9 elements of Humanitude and narrative 6 -grade evaluation of the multiple-thinking-model. There was no interaction among trainers during the evaluation.

Results: 6 certified trainers of Humanitude enrolled the study. In 3 -grade evaluation, the kappa statistic was 0.711 . The narrative evaluation with Minsky's 6-grade thinking model showed the significant correlation among the trainers.

Conclusion: Video analysis of care by artificial intelligence thinking model is highly reproducible and standardized for the trainers to teach. Since video based remote training can reduce time-cost and financial-cost of training, this evaluation system for dementia care contributes to mass training.

\section{0-90}

Sub-typing of dementia after TIA and stroke: comparison of clinical diagnostic criteria for vascular dementia in a longitudinal population-based study

\section{Aubretia McColl ${ }^{1}$, Linxin $\mathrm{Li}^{1}$, Peter M Rothwell ${ }^{1}$, Sarah T Pendlebury ${ }^{1}$ \\ ${ }^{1}$ Centre for Prevention of Stroke and Dementia, University of Oxford, UK}

Introduction: Sub-typing of post-TIA/stroke dementia may be aided by application of vascular dementia (VD) diagnostic criteria. We examined rates of VD obtained using the well-established NINDSAIREN criteria versus the recently developed VASCOG criteria in a population-based study of TIA/stroke.

Methods: In a longitudinal study of all TIA/stroke occurring within a defined population of 92,728 (Oxford Vascular Study/2002-2012) baseline clinical assessment and CT/MR brain imaging were performed with follow-up to 5 years. Multiple overlapping methods including face-to-face interview were used to ascertain dementia. VASCOG and NINDS-AIREN criteria were applied in patients with dementia and agreement assessed with kappa statistic.

Results: Among 2305 TIA/stroke patients (mean/SD age 73.7/13.0), $432(18.7 \%)$ had dementia during follow-up. Using VASCOG criteria, $194(44.9 \%)$ patients fulfilled criteria for probable VD versus 90 (20.8\%) using NINDS-AIREN criteria. Rates of possible VD varied even more widely at $21(4.9 \%)$ by VASCOG criteria vs $293(67.8 \%)$ by NINDS-AIREN. Many patients did not fulfil criteria because of failure to meet imaging requirements $(\mathrm{n}=123 / 51.6 \%$-VASCOG and $\mathrm{n}=175 / 54.3 \%$-NINDS-AIREN), followed by no history/signs of stroke $(\mathrm{n}=84 / 35.3 \%$-VASCOG) or lack of a temporal relationship between cerebrovascular disease and dementia $(n=274 / 85.1 \%$ NINDS-AIREN). Agreement between NINDS-AIREN and VASCOG for the diagnosis of probable/possible VD was low at $36.6 \%$ (kappa $0.22, \mathrm{p}<0.001$ ), but increased to $75.0 \%$ for the diagnosis of probable VD (kappa 0.47, p < 0.001).

Conclusion: The proportion of post-TIA/stroke dementia cases classified as VD differs depending on the applied diagnostic criteria. A history of TIA (rather than stroke) and strict imaging requirements are key reasons for failing to meet criteria for VD diagnosis.

\section{Area: Delirium}

O-91

Anticholinergic drug burden and delirium: a systematic review

Angelique Egberts ${ }^{1}$, Rafael Moreno-Gonzalez ${ }^{2}$, Gijsbertus Ziere ${ }^{3}$, Francesco Mattace-Raso ${ }^{3}$

${ }^{1}$ Dept. of Internal Medicine, section of Geriatric Medicine, Erasmus University Medical Center, Rotterdam, The Netherlands; ${ }^{2}$ Dept. of Hospital Pharmacy, Franciscus Gasthuis \& Vlietland, Rotterdam \& Schiedam, The Netherlands, ${ }^{3}$ Dept. of Internal Medicine, section of Geriatric Medicine, Bellvitge University Hospital, Barcelona, Spain, ${ }^{4}$ Dept. of Internal Medicine, section of Geriatric Medicine, Erasmus University Medical Center, Rotterdam, The Netherlands

Introduction: The aim of this review was to assess the association between anticholinergic drug burden, measured with anticholinergic drug scales, and delirium.

Methods: A systematic literature search was performed in Medline, Embase, PsycINFO, Web of Science, CINAHL, Cochrane library and Google Scholar. Studies evaluating the association between anticholinergic drug burden (measured as a total score), and delirium or delirium severity, published in English, were eligible for inclusion.

Results: Ten studies, including 21152 persons, were included. Nine studies investigated delirium (delirium on admission $n=3$, incident delirium $\mathrm{n}=6$, delirium during 1-year follow-up $\mathrm{n}=1$ ). Anticholinergic drug burden was measured with the Anticholinergic Risk Scale (ARS, $\mathrm{n}=5$ ), the Anticholinergic Cognitive Burden scale $(\mathrm{n}=3)$, the list of Chew $(\mathrm{n}=1)$, and the Anticholinergic Drug Scale (ADS, $n=3$ ). A high anticholinergic drug burden, measured with the ARS, was associated with delirium on admission (1/1), incident delirium (3/3) and delirium during follow-up (1/1). No associations were found using the other anticholinergic drug scales. Two studies investigated the possible association between anticholinergic drug burden (ADS $n=2$, Summers Drug Risk Number $\mathrm{n}=1$ ) and delirium severity. One study found an association between a high anticholinergic drug burden, measured with the ADS, and an increase in delirium severity.

Conclusions: There is limited evidence that a high anticholinergic drug burden is associated with delirium or delirium severity. 
However, in all studies using the ARS, an association was found between anticholinergic drug burden and delirium.

\section{0-92 \\ Is there a role for vitamin $\mathrm{D}$ in preventing delirium? Evidence from mendelian randomization}

\author{
Kirsty Bowman ${ }^{1}$, David Melzer ${ }^{2}$ \\ ${ }^{1}$ University of Exeter Medical School, ${ }^{2}$ University of Exeter Medical \\ School UK, University of Connecticut Health Center USA
}

Introduction: Delirium is associated with increased mortality risks, admittance to nursing homes and high health care costs. Many delirium episodes may be preventable. There has been increased interest in the role of vitamin D in cognitive impairment. A recent analysis showed that genetically determined higher levels of vitamin $\mathrm{D}$ were associated with reduced risks for Alzheimer's disease. Evidence is limited for the role of vitamin D with delirium using large community-based studies.

Methods: We used 313,121 European participants from the UK Biobank, a healthy volunteer cohort, who were aged 60 years by the end of the follow-up. We used a Mendelian randomization approach to minimize confounding and reverse causation. We tested the associations between individual vitamin $\mathrm{D}$ altering variants and a vitamin D genetic risk score with incident hospital delirium episodes during $\leq 9.9$ years of follow-up.

Results: The mean age of the participants was 61.7 years (s.d. 4.7 years), 544 subjects had a delirium episode. We found genetically determined higher levels of vitamin $\mathrm{D}$ were associated with a reduced risk of incident delirium (Hazard Ratio 0.74 [95\% CI 0.62, 0.87] per $10 \mathrm{nmol} / \mathrm{L}$ increase in genetically instrumented vitamin D), with a dose-response across the variants. Results were little altered after adjusting for time spent outdoors or a calcium genetic risk score.

Key conclusions: We found that those with vitamin D increasing genetic variants have a reduced risk of delirium, providing evidence of causality. Our results may pave the way for trials on increasing low vitamin D levels to potentially preventing delirium.

\section{O-93}

\section{Our evidence base for delirium: is there age discrimination?}

\author{
Desmond O’Neill ${ }^{1}$, Sean Kennelly², Emmet Jordan ${ }^{2}$ \\ ${ }^{1}$ Tallaght University Hospital, Dublin, Ireland, ${ }^{2}$ Tallaght University \\ Hospital, Dublin, Ireland
}

Introduction: Delirium among hospital inpatients is highly prevalent and is associated with a variety of adverse outcomes. One of the strongest risk factors for developing delirium is advanced age. Age bias has been identified in a number of studies of conditions related to geriatric medicine-whether it is present in trials examining delirium is currently unknown. This study aims to systematically review the literature and identify the extent to which ageism is present in delirium studies.

Methods: All randomised control trials (RCT) in systematic reviews with 'delirium' in their title on the Cochrane Database were assessed. Patient gender and exclusion criteria were also recorded. These were compared to prevalence studies of delirium and assessed for discrepancies.

Results: 45 RCTs were identified, 38 of which were eligible for inclusion, comprising 16,276 participants. The mean age of patients was 71.7 years, at least 6 years younger than the mean age of patients developing delirium in hospitals internationally. In addition to this, 16 trials $(42.1 \%)$ excluded patients based on pre-existing cognitive impairment and 2 trials excluded based on advanced age.

Key conclusions: This study identifies a clear difference between patients included in delirium studies and patients experiencing delirium in clinical practice. This is particularly troubling as the difference lies in patient age and cognitive impairment-two of the strongest predictors for developing hospital delirium. To ensure evidence based practice, future trials must include a larger sample of our older population, and include patients with cognitive impairment.

\section{0-94}

Delirium assessment in dementia patients: relationship with anticholinergic burden

Alba Malara ${ }^{1}$, GIuseppe Andrea De Biase ${ }^{1}$, Francesco Bettarini ${ }^{1}$, Francesco Ceravolo ${ }^{1}$, Serena Di Cello ${ }^{1}$, Michele Garo ${ }^{1}$, Francesco Praino ${ }^{1}$, Vincenzo Settembrini ${ }^{1}$, Fausto Spadea ${ }^{1}$, Vincenzo Rispoli ${ }^{1}$

${ }^{1}$ Scientific Committee of National Association of Third Age Residences (ANASTE) Calabria, Italy

Introduction: Delirium is a frequent clinical complication in elderly people. The objective of this study was to evaluate the prevalance of delirium and its relationship with anticholinergic burden in patients with dementia admitted in LTC facilities.

Methods: A prospective observational study was performed in seven ANASTE LTC facilities over a 2-month period. The screening for delirium was performed by the Confusion Assessment Method (CAM) and by the Delirium Rating scale (DRS). Diagnosis of delirium was performed according to DSMV criteria. The anticholinergic burden was evaluated according to score of the Anticholinergic Burden Scale (ACB). We used MMSE to evaluate the cognitive impairment and Neuropsichiatric Inventory to evaluate the behavioural problems. Hypokalemia $(\mathrm{K}<3.5 \mathrm{mEq} / \mathrm{l})$ and hypernatremia (NA $>145 \mathrm{mEq} / \mathrm{l}$ ) were also assessed. Statistical analysis was performed using Fisher Test and Odds Ratio (OR) with related confidence interval (C.I.) at $95 \%$ estimated for the primary endpoint.

Results: We have enrolled 268 patients $(61.1 \% \mathrm{~F}$, mean age $83.3 \pm 8.9 ; 31.3 \% \mathrm{M}$, mean age $81.3 \pm 8.8)$ of these $68 \%$ suffer from dementia. In dementia patients the prevalance of delirium was $43.7 \%$. We found a positive correlation between delirium and anticholinergic burden (OR: 2.566; C.I. 1.5, 4.3; $\mathrm{p}<0.005$ ), severe cognitive impairment (OR:2.75; C.I. 1.57, 4.81; $\mathrm{p}<0.005)$, NPI item for Delusions (OR: 5.42; C.I. 3.08, 9.5) and hypernatremia (OR:1.47; C.I. 0.84, 2.59). Among ACB1drugs, Furosemide, Alprazolam, Digital Trazodone, Risperidone were often prescribed. Among ACB 2-3 drugs, promazine quetiapine and olanzapine were the most prescribed. Finally, the CAM had a sensibility of $89 \%$ and specifity of $96 \%$ and DRS 56 and $82 \%$ respectively to detect delirium in dementia patients.

Conclusion: There is a close correlation between anticholinergic burden and delirium in people with dementia, recognize this causal link can improve the management of delirium. 


\section{$0-95$}

Delirium and dementia in elderly persons in the Emergency Department (ED): prevalence and long-term outcomes. Results from the "Anziani in DEA" (AIDEA) study

Francesco Tonarelli ${ }^{1}$, Daniela Balzi ${ }^{2}$, Ilaria Giannini ${ }^{1}$, Alessandra Pratesi $^{1}$, Enrico Mossello ${ }^{1}$, M. Chiara Cavallini ${ }^{1}$, Enrico Benvenuti ${ }^{3}$, Luciano Gabbani ${ }^{4}$, Stefano Grifoni ${ }^{5}$, Andrea Ungar ${ }^{1}$, Mauro Di Bari ${ }^{1}$

${ }^{1}$ Dept. of Experimental and Clinical Medicine, Research Unit of Medicine of Aging, Univ. of Florence and AOU Careggi, Florence, Italy, ${ }^{2}$ Epidemiology Unit, Central Tuscany Local Healthcare Unit, Florence, Italy, ${ }^{3}$ Unit of Geriatrics, SM Annunziata Hospital, Florence, Italy, ${ }^{4}$ Unit of Geriatrics, AOU Careggi, Florence, Italy, ${ }^{5}$ Emergency Department, AOU Careggi, Florence, Italy.

Introduction: Delirium is frequently observed in older patients presenting to the Emergency Department (ED), either as a de novo condition secondary to somatic diseases or superimposed to dementia. The aim of this study was to evaluate: 1) prevalence of delirium in older patients presenting to the ED, comparing subjects with and without pre-existing dementia; 2) long-term prognostic implications of delirium detected in the ED.

Methods: In AIDEA, 3722 participants aged 75 + years, accessing the ED of two hospitals in Florence, Italy were interviewed. History of dementia was collected from caregivers; delirium was identified from a 4AT score $>4$. Long-term mortality (median follow-up duration: 4 months) was compared between patients with probable delirium and those in whom delirium was absent or possible.

Results: Mental status prior to the ED visit was known in 2494/3722 participants. Delirium was observed in $13 \%$ of participants and was more frequent in the 329 participants with than in the 2165 without history of dementia ( 60 vs. $15 \%$; $p<0.001$ ). Long-term mortality was significantly greater in participants with probable delirium than in those without $(41 \%$ vs. $14 \%$; $\mathrm{p}<0.001)$. The HR $(95 \% \mathrm{CI})$ for death in participants with vs. without probable delirium was $2.2(1.8-2.7)$, adjusting for interview variables, including history of dementia.

Conclusions: In this sample of older patients accessing the ED, delirium was frequently associated with history of dementia and was a strong, independent risk factor for long-term mortality. Sponsored by the Italian Ministry of Health, RF-2010-2321801.

\section{O-96}

Prevalent versus incident delirium in acute medicine: observational prospective study

Ana Sofia Pessoa ${ }^{1}$, Filipa Queresma ${ }^{1}$, Catherine Ashton ${ }^{2}$, Nicola Lovett ${ }^{2}$, Sarah Smith ${ }^{2}$, Sarah T Pendlebury ${ }^{1}$

${ }^{1}$ Centre for Prevention of Stroke and Dementia, Nuffield Department of Clinical Neurosciences, University of Oxford and Departments of General (Internal) Medicine and Geratology, John Radcliffe Hospital, Oxford, UK, ${ }^{2}$ Departments of General (Internal) Medicine and Geratology, John Radcliffe Hospital, Oxford, UK

Introduction: Reliable epidemiologic studies in delirium are required to inform service development but the majority of existing data are from selective cohorts focussed on incident delirium. We therefore determined rates of prevalent versus incident delirium in all consecutive admissions to acute (internal) medicine.

Methods: Admissions to our acute medical team during 2 month blocks over five periods $(2010,2012,2015,2016,2017)$ were screened on admission with a short cognitive test and the confusion assessment method (CAM) and at least every $48 \mathrm{~h}$ thereafter. Delirium was diagnosed using DSM-IV criteria and defined as prevalent (present within $48 \mathrm{~h}$ of admission) or incident (occurring at least $48 \mathrm{~h}$ after admission).

Results: Among 1463 patients (mean age $=69.4 / 19.6$ years, 689 (47\%) male, $212(14.5 \%)$ with a known dementia diagnosis), delirium occurred in $350(23.9 \%)$ overall but was strongly related to age ranging from $32 / 484(6.6 \%)$ at $<65$ years, to $44 / 228(19 \%)$ at 65-74 years and $274 / 746(36.6 \%)$ at $\geq 75$ years, ptrend $<0.001$. Prevalent delirium occurred in 239/350 (68\%), incident delirium in 64 $(18 \%)$ and $47(13 \%)$ had both prevalent and incident delirium. Patients with delirium had more dementia (102/350 (29\%) vs 75/1113 $(6.7 \%), \mathrm{p}<0.0001)$ but were as likely to be living at home as nondelirious patients $(274 / 350(78 \%)$ vs $820 / 1113$ (74\%).

Conclusions: Most delirium occurs in non-institutionalised patients without a known dementia diagnosis and is much more likely to be present on admission than to occur during admission. Delirium screening should therefore be routine at first assessment of (older) patients presenting to acute medicine.

\section{Area: Geriatrics in organ disease}

\section{O-97}

Clinical examination of peripheral arterial disease and ankle: brachial index in a nation-wide cohort of older subjects. Practical implications

Karolina Piotrowicz ${ }^{1}$, Jarosław Królczyk ${ }^{1}$, Jerzy Chudek $^{2}$, Monika Puzianowska-Kuźnicka ${ }^{3}$, Małgorzata Mossakowska ${ }^{4}$, Aleksandra Szybalska $^{4}$, Tomasz Grodzicki ${ }^{1}$, Anna Skalska ${ }^{1}$, Jerzy Gąsowski ${ }^{1}$

${ }^{1}$ The Department of Internal Medicine and Gerontology, Faculty of Medicine, Jagiellonian University Medical College, Kraków,

${ }^{2}$ Department of Internal Medicine and Oncological Chemotherapy, School of Medicine in Katowice, Medical University of Silesia in Katowice, ${ }^{3}$ Department of Human Epigenetics, Mossakowski Medical Research Centre, PAS, Warsaw; Department of Geriatrics and Gerontology, Medical Centre of Postgradual Education, Warsaw, Poland, ${ }^{4}$ International Institute of Molecular and Cell Biology in Warsaw

Introduction: Prevalence of peripheral arterial disease increases with age and is related to increased morbidity and mortality, especially in older adults. The clinical diagnosis includes the measurement of ankle-brachial index (ABI). We aimed to check the prevalence of abnormal $\mathrm{ABI}$, and the value of physical examination of arterial system in detection of $\mathrm{ABI}<0.9$.

Methods: We performed subgroup analysis of patients included in the PolSenior survey in whom we measured ABI, performed detailed physical examination of arterial system, and assessed laboratory and questionnaire factors related to atherosclerosis. Patients were divided according to ABI strata of $<0.9,0.9-1.4$ and $>1.4$ and summary clinical score of abnormalities on physical examination was proposed. Using logistic regression we obtained areas under the curve (AUC). Results: The mean (SD) age of 860 participants (53.5\% men) was 74.7 (10.6) years. ABI $<0.9$ was found in $19.9 \%$ participants and it was linked to higher overall cardiovascular risk. In the $<0.9 \mathrm{ABI}$ group $62.7 \%$ group vs. $73.9 \%$ in normal $\mathrm{ABI}$ group, and $75.7 \%$ in ABI $>1.4(p=0.02)$ could walk more than 200 meters. Higher clinical score was associated with lower ABI. Full physical examination (AUC $=0.67$ ) followed by examination of lower extremities $(\mathrm{AUC}=0.65)$ showed strongest diagnostic value for presence of $\mathrm{ABI}$ based PAD (nie rozumiem!). Neither ABI nor clinical examination were good predictors of inability to walk over 200 meters. 
Key conclusions: Full clinical examination, although moderately, may add to detection of PAD. The ability to walk 200 meters is not a good measure of PAD in older subjects.

\section{0-98}

Pre-transplant polypharmacy: an alert associated with a higher risk of hospitalizations after kidney transplantation in older recipients

Barange Emmanuel ${ }^{1}$, Guerville Florent ${ }^{1}$, MERVILLE Pierre ${ }^{1}$, Couzi Lionel $^{1}$, MOREAU Karine ${ }^{1}$

${ }^{1} \mathrm{CHU}$ Bordeaux, Bordeaux, France

Background: Age is no longer a limit to kidney transplantation (KT) access for patients with end stage renal disease, but recipients older age and frailty are associated with poor outcome after KT, especially hospitalizations for complications. Pre-KT comorbidity, polypharmacy and sarcopenia could be contributors to frailty. Our objective was to identify factors associated with a higher number of days of hospitalizations during the first year post-KT.

Methods: Recipients older than 70 years who underwent KT between 2009 and 2016 in our center were included in this retrospective study. The burden of complications was assessed by the number of days of hospitalization during the first year post-KT. We tested pre-KT comorbidities, polypharmacy and sarcopenia, as well as KT characteristics, as potential factors associated with post-KT complications. Muscle mass was assessed by measuring the L4 psoas area on abdominal computed tomography.

Results: A hundred and thirteen patients were included. Median age was $74 \pm 3$ (70-85). Median number of days of hospitalization during the first year post-KT was $32 \pm 25$ (2-162). Pre-KT polypharmacy $>5$ and 10 drugs was the only factor associated with a number of days of hospitalization higher than the median $(\mathrm{OR}=3.7$, $95 \% \mathrm{CI}=1.2-14.3$ and $8.3,95 \%$ CI $2.3-36.7$, respectively), even after adjusting for age and Charlson comorbidity index in a multivariate analysis. Low muscle mass was not associated with an increased risk of hospitalization.

Conclusion: in older KT recipients, pre-transplant polypharmacy is associated with post-transplant complications and hospitalizations, independently of comorbidity. Pre-KT geriatric interventions should be implemented to reduce polypharmacy.

\section{0-99}

Age is an individual risk factor for not being referred to adjuvant chemotherapy in resected UICC III colorectal cancer patients: a nation wide cohort study

MF Marie Friis ${ }^{1}$, RDB Rasmus Dahlin Bojesen ${ }^{2}$, IG Ismail Gögenur ${ }^{3}$

${ }^{1}$ Department Of Geriatric Medicine, Bispebjerg University Hospital, Copenhagen; Center For Surgical Science (CSS), Department Of

Surgery, Zealand University Hospital, Denmark, ${ }^{2}$ Center For Surgical Science (CSS), Department Of Surgery, Zealand University Hospital; Department Of Surgery, Slagelse Hospital, Denmark, ${ }^{3}$ Center For Surgical Science (CSS), Department Of Surgery, Zealand University Hospital; University Of Copenhagen, Denmark

Introduction: International guidelines recommend that resected stage III colorectal cancer (CRC) patients should be treated with adjuvant chemotherapy without age restriction. The aim of this study was to assess the association between age and referral to an oncologist for postoperative adjuvant chemotherapy for patients with stage III CRC.
Methods: This was a nation wide cohort study with prospectively collected data. Study period was from 2014 to 2016. Data were provided by the Danish Colorectal Cancer Group Registry, which has a completeness of $99 \%$. Univariate analysis was performed and significant factors were used in a multivariate logistic regression (comorbidity, performance status (PS)).

Results: A total of 2791 patients with resected stage III CRC were included. In the fully adjusted model, patients $>80$ years of age had an increased risk (OR $(95 \% \mathrm{CI}))$ of not being referred to an oncologist (10.85 (7.70-15.28)) compared with patients $<75$ years of age. Other significant factors associated with no referral in the model included American Society of Anesthesiologists score (ASA), PS, postoperative complications, and geographic region.

Conclusion: Patients $>80$ years have an increased risk of not receiving the recommended postoperative adjuvant chemotherapy.

\section{0-100}

Comparison of tools for the identification of frail communitydwelling older people in primary care settings: frail, but according to which tool?

Itziar Vergara ${ }^{1}$, Maider Mateo-Abad ${ }^{2}$, Carmen Saucedo-Figueredo $^{3}$, Mónica Machón ${ }^{4}$, Alonso Montiel-Luque ${ }^{5}$, Kalliopi Vrotsou ${ }^{1}$, Antonia Nava-del Val ${ }^{6}$, Ana Díez-Ruiz ${ }^{7}$, Carolina Güell ${ }^{8}$, Francisco Rivas-Ruiz ${ }^{9}$

${ }^{1}$ Unidad de Investigación APOSIs Gipuzkoa, Osakidetza, DonostiaSan Sebastian, Spain. 2. Instituto Biodonostia, Donostia-San Sebastian, Spain, ${ }^{3}$ Red de Investigación en Servicios de Salud en Enfermedades Crónicas (REDISSEC), Spain, ${ }^{2} 1$. Unidad de Investigación APOSIs Gipuzkoa, Osakidetza, Donostia-San Sebastian, Spain. 2. Instituto Biodonostia, Donostia-San Sebastian, Spain 3. Kronikgune, Barakaldo, Spain, ${ }^{3}$ Unidad Gestión Clínica Los Boliches, Fuengirola. Distrito de Atención Primaria Costa del Sol, Málaga, Spain, ${ }^{4} 1$. Unidad de Investigación APOSIs Gipuzkoa, Osakidetza, Donostia-San Sebastian, Spain. 2. Instituto Biodonostia, Donostia-San Sebastian, Spain 3. Red de Investigación en Servicios de Salud en Enfermedades Crónicas (REDISSEC), Spain, ${ }^{5}$ Unidad Gestión Clínica San Miguel, Torremolinos. Distrito de Atención Primaria Costa del Sol, Málaga, Spain, ${ }^{6}$ Unidad Gestión Clínica Las Albarizas, Marbella. Distrito de Atención Primaria Costa del Sol, Málaga, Spain, ${ }^{7} 1$. Instituto Biodonostia, Donostia-San Sebastian, Spain 2. Centro de Salud Beraun, OSI Donostialdea, Osakidetza, Renteria, Spain, ${ }^{8} 1$. Unidad de Investigación de Atención PrimariaOSIs Gipuzkoa, Osakidetza, Spain; 2. Centro de Salud de Alza, Osakidetza, Spain, ${ }^{9} 7$. Unidad de Investigación. Agencia Sanitaria Costa del Sol, Marbella, Málaga, Spain Red de Investigación en Servicios de Salud en Enfermedades Crónicas, REDISSEC

Introduction: No consensus has been reached regarding which tools are the most adequate to identify frail patients in primary care (PC). We aimed to analyse the characteristics of community-dwelling frail individuals aged $\geq 75$ years as identified by four tools (Timed up and Go test -TUG, Short Physical Performance Battery -SPPB, Tilburg Frailty Indicator-TFI and Gérontopôle Frailty Screening Tool -GFST) in order to assess the performance of these tools when applied in PC. Methods: A prospective cohort study of 2 years of follow up was performed in two regions of Spain. The analysis reported here is based on baseline data, collected via face-to-face interviews. Multiple correspondence analysis (MCA) and cluster analysis were used to analyse groups of individuals. Cohen's Kappa was calculated to measure the concordance.

Results: 865 individuals were recruited, $53 \%$ women, with a mean age of 78 years. The tools yielded different prevalence rates of frailty: 
$38,55,29$ and $31 \%$ for the TUG, SPBB, TFI and GFST, respectively. TUG and SPPB showed a moderate concordance (Cohen's Kappa 0.49 (95\% CI $0.44,0.54)$ ). Four clusters of participants emerged. Cluster $1(\mathrm{~N}=263)$ contained patients categorized as robust by most of the tools studied, whereas clusters $2(\mathrm{~N}=199), 3(\mathrm{~N}=183)$ and 4 $(\mathrm{N}=220)$ grouped patients classified as frail by at least one of the tools. Significant differences were found between clusters.

Key conclusions: frailty is highly prevalent. The studied tools identify different profiles of frail patients. TUG and SPPB constitute a sound approach for the identification of frailty in PC.

\section{0-101}

Association between frailty tools and functional status in frailty and no frailty subjects (frailtools study): preliminary results

Jimmy Martin Gonzales Turin ${ }^{1}$, Tania Guevara Guevara ${ }^{2}$, Marta Checa Lopez ${ }^{1}$, Shirley Soledad Alamo Ascencio ${ }^{1}$, Jose Antonio Carnicero Carreño ${ }^{1}$, Myriam Del Rosario Oviedo Briones ${ }^{1}$, Armando Pardo Gomez ${ }^{1}$, Sara Artola Menendez ${ }^{3}$, Carmen Perez Calpena ${ }^{3}$, Angel Flores Auñon ${ }^{4}$,

Leocadio Rodriguez Mañas ${ }^{5}$

${ }^{1}$ Fundacion Para La Investigacion Biomedica Del Hospital Universitario De Getafe, ${ }^{2}$ Fundacion Par Ala Investigacion Biomedica Del Hospital Universitario De Getafe, ${ }^{3}$ Servicio Madrileño De Salud, ${ }^{4}$ Residencia Parque De Los Frailes, ${ }^{5}$ Servicio Madrileño De Salud, Fundacion Para La Investigación Biomedica Del Hospital Universitario De Getafe

Introduction: The Frailtools Project (FP) was designed to assess the usefulness of frailty tools for detecting frailty in clinical and social settings.

Methods: FP was developed in five countries (Spain, United Kingdom, Poland, Italy and France). The setting were: Outpatient and Geriatric ward, Nursing home and Primary care. Eligibility criteria: People $\geq 75$ years. Exclusion criteria: MMSE $<20$, terminal illness, Barthel Index $<40$ in nursing homes and $<90$ in all other settings. Our purpose is to assess the association between Frailty and functional status. Frailty was defined: Fried and FRAIL scale $(\geq 3)$, SHARE-FI (Female $<6$, male $<7$ ), Clinical Frailty Scale (CFS) $(>4)$, Rockwood-35 (>0.25), FTS 3 (>15), Gérontopole Frailty Screening Tool (GFST) (Yes). Functional status was evaluated by Short Physical Performance Battery score (SPPB).

Results: 654 subjects were included. Mean age was $82.4( \pm 5.3)$ years; $59.5 \%$ were women. The association between FRAILTY and functional status (SPPB) were: $(\mathrm{p}<0.05)$ [FTS 3 (SPPB: 1.38 $( \pm 2.08$ ); Fried (SPPB: 5.72 ( \pm 3.07); FRAIL (SPPB: 3.83 ( \pm 3.41 ); GFST (SPPB: $4.19( \pm 3.68)$; CFS (SPPB: $2.89( \pm 2.92)$; SHARE (SPPB: 3.59 ( \pm 3.74); Rockwood-35 (SPPB: 3.68 ( \pm 3.42$)$ ]. NO Frailty and SPPB: $(\mathrm{p}<0.05)$ [FTS 3 (SPPB: 6.97 ( \pm 3.05$)$; Fried (SPPB: 4.07 ( \pm 4.25); FRAIL (SPPB: $5.96( \pm 4.10)$; GFST (SPPB: 5.95 ( \pm 3.80$)$; $\mathrm{P}<0.05)$; CFS (SPPB: 6.63 ( \pm 3.69$)$; SHARE (SPPB: 6.27 ( \pm 3.85 ); Rockwood-35 (SPPB: 6.57 ( \pm 3.86 )]

Conclusion: According to the tool used, frailty subjects showed different SPPB scores, however in non-frailty subjects no many differences punctuation in SPPB were found.
0-102

Socio-demographic and health characteristics associated with transitions between frailty states over three years in the MAPT study

Herr Marie $^{1}$, Cesari Matteo ${ }^{2}$, Landré Benjamin ${ }^{3}$, Joel Ankri ${ }^{3}$, Sandrine Andrieu ${ }^{1}$

${ }^{1}$ LEASP, UMR 1027, INSERM, UPS, Université de Toulouse, France, ${ }^{2}$ Geriatric Unit, Fondazione IRCCS Ca' Granda-Ospedale Maggiore Policlinico, Università di Milano, Milan, Italy, ${ }^{3}$ INSERM, U1168, VIMA: Aging and chronic diseases. Epidemiological and public health approaches, Villejuif, F-94807, France; Univ Versailles St-Quentin-en-Yvelines, UMR-S 1168, F-78180, Montigny le Bretonneux, France

Introduction: The epidemiology of frailty has mostly been described in cross-sectional studies. Using repeated measures of frailty over 3 years, this work aimed to describe transitions between frailty states and associated factors.

Methods: This study used the data from the Multidomain Alzheimer Prevention Trial (MAPT) and included the 842 participants aged 70 and over who did not receive the multidomain intervention. Frailty was assessed using the phenotype model proposed by Fried et al. at baseline and at $6,12,24$, and 36 months. Factors influencing the transitions across frailty states were examined using multistate modelling.

Results: The study population included 548 women and 294 men, mean age $75.4 \pm 4.5$ years. At baseline, $430(53 \%)$ participants were robust, $349(43 \%)$ pre-frail, and $28(4 \%)$ frail. A total of 2271 transitions were observed during the 3 years of follow-up, without change in the frailty status for 1548 of them (68\%), a worsening of the frailty status for 426 of them (19\%), and an improvement of the frailty status for the remaining 297 (13\%). Polypharmacy (i.e. 6 drugs) and probable depression were associated with the occurrence of pre-frailty. Female gender was systematically associated with a lower probability of recovering from pre-frailty and frailty. Older age, polypharmacy, probable depression, overweight and obesity, comorbidity, and abnormal CRP reduced also the probability of recovering from frailty or pre-frailty.

Key conclusions: This study sheds light on populations and factors that should be given particular attention in the prevention and management of frailty.

\section{0-103}

Frailty predicts medication-related harm requiring healthcare: A UK multicentre prospective cohort study

Nikesh Parekh ${ }^{1}$, Khalid Ali ${ }^{1}$, Jennifer Stevenson ${ }^{2}$, Graham Davies ${ }^{2}$, Rebekah Schiff ${ }^{3}$, Jatinder Harchowal ${ }^{4}$, Tischa Van der Cammen ${ }^{5}$, Chakravarthi Rajkumar ${ }^{1}$

${ }^{1}$ Department Of Elderly Medicine, Brighton And Sussex Medical School, Brighton UK, ${ }^{2}$ Institute Of Pharmaceutical Science, King's College London, London UK, ${ }^{3}$ Department Of Ageing And Health, Guy's and St. Thomas' NHS Foundation Trust, London UK,

${ }^{4}$ Department Of Pharmacy, The Royal Marsden NHS Foundation Trust, London UK, ${ }^{5}$ Department of Industrial Design, Delft University of Technology, Delft, Netherlands

Introduction: Frailty has been under investigated as a risk factor for medication-related harm (MRH) in older adults [1]. We sought to determine whether frailty is independently associated with MRH in a large multicentre prospective cohort, the PRIME study. 
Methods: The PRIME study recruited 1280 older adults at hospital discharge from 5 hospitals in England between 2013 to 2015 [2]. MRH and associated healthcare use within 8-weeks post-discharge were identified by senior pharmacists using ${ }^{1}$ hospital readmission data, ${ }^{2}$ primary care records, ${ }^{3}$ patient telephone interviews. Based on the Rockwood approach [3], we developed a frailty index including 55 deficits from multiple domains (morbidity, cognition, mood, strength and mobility, nutrition, daily function). Frailty was defined using the established cut-off of $20 \%$ deficits [4], and internally validated using Kaplan-Meier plots comparing survival in frail and nonfrail patients. We then used logistic regression analysis to investigate the relationship between frailty and MRH requiring healthcare.

Results: 1116 patients completed follow-up (median age 81.9 years, range $65-103$ years, $58.4 \%$ female). 446 patients (40\%) were frail in our cohort. $36 \%$ of frail patients experienced MRH compared with $25 \%$ in non-frail patients. There was a strong relationship between frailty and MRH (OR 1.67, 95\% CI 1.29-2.17, p <0.001). A significant relationship between frailty and MRH remained on multivariable regression, adjusting for polypharmacy, age and gender (OR 1.37, 95\% CI 1.04-1.81, p = 0.027). Frail patients had significantly reduced 18-month survival (Log-Rank test $\mathrm{p}<0.001$ ).

Key conclusions: Frailty is a predictor of MRH requiring healthcare, independent of polypharmacy.

References:

[1] Parekh N, Ali K, Page A, Roper T, Rajkumar C. Incidence of medication-related harm in older adults following hospital discharge: a systematic review. J Am Geriatr Soc 2018;in press.

[2] Stevenson J, Parekh N, Ali K, Timeyin J, Bremner S, Van Der Cammen T, et al. Protocol for a Prospective (P) study to develop a model to stratify the risk (RI) of medication (M) related harm in hospitalized elderly (E) patients in the UK (The PRIME study). BMC Geriatr 2016;16:22. https://doi.org/10.1186/s12877-016-0191-8.

[3] Searle SD, Mitnitski A, Gahbauer EA, Gill TM, Rockwood K. A standard procedure for creating a frailty index. BMC Geriatr 2008;8:24. https://doi.org/10.1186/1471-2318-8-24.

[4] Mitnitski A, Song X, Skoog I, Broe G, Cox JL, Grunfeld E, et al. Relative Fitness and Frailty of Elderly Men and Women in Developed Countries and Their Relationship with Mortality. J Am Geriatr Soc 2005;53:2184-9.

\section{Area: Biogerontology and genetics}

\section{0-104}

\section{Acute cytokine production upon stimulation} with lipopolysaccharide associates with cardiovascular mortality risk independent of circulating markers of chronic inflammation

Karel Beenakker ${ }^{1}$, Sijia Chen ${ }^{1}$, Maarten Rozing ${ }^{2}$, Stella Trompet ${ }^{3}$, Wouter Jukema $^{3}$, Rudi Westendorp ${ }^{2}$

${ }^{1}$ Department of Gerontology and Geriatrics, Leiden University Medical Center, Leiden, The Netherlands, ${ }^{2}$ Department of Public Health and Center for Healthy Aging, University of Copenhagen, Copenhagen, Denmark, ${ }^{3}$ Department of Cardiology, Leiden University Medical Center, Leiden, The Netherlands

Background: The risk of a cardiovascular event is transiently increased after infection, which suggests a role for the immune response in its pathogenesis. The aim of this study is to investigate whether acute cytokine production upon stimulation with lipopolysaccharide associates with cardiovascular, non-cardiovascular and all-cause mortality and whether this association is dependent on chronic inflammation.

Methods: In 403 subjects from the PROspecitve Study of Pravastatin in the Elderly at Risk (PROSPER) trial, with a mean age of
75.1 years, we determined acute cytokine production at baseline by ex vivo stimulating whole-blood samples with lipopolysaccharide and measuring the production of interleukin (IL)-6, tumor necrosis factor (TNF)- $\alpha$, IL-12, IL-1 $\beta$, IL-10, and IL-1RA production within $24 \mathrm{~h}$. A composite acute cytokine production score was calculated by averaging the individual acute cytokines production values after sexspecific standardisation. Serum IL-6 and high-sensitivity C-reactive protein (hs-CRP) were measured as circulating markers of chronic inflammation. Subjects were followed for 12.8 years and cardiovascular, non-cardiovascular and all-cause mortality was recorded.

Results: A higher acute IL-6, TNF- $\alpha$, IL-1 $\beta$, and IL-1RA production was associated with a significantly higher cardiovascular and allcause mortality risk. The hazard ratio $(95 \%$ confidence interval; P-value) per standard deviation increment in the acute cytokine production composite score was $1.89(1.26-2.85 ; \mathrm{P}=0.002)$ for cardiovascular mortality risk and $1.39(1.12-1.74 ; \mathrm{P}=0.003)$ for allcause mortality risk. Adjusting these relations for circulating markers of chronic inflammation did not change the results.

Conclusion: A higher acute cytokine production associates positively with cardiovascular and all-cause mortality risk independent of chronic inflammation.

\section{0-105}

Association of a regulatory anti-oxidant and drug-metabolising gene with multi-morbidity and adverse drug reactions in older adults

Greg Scutt ${ }^{1}$, Andy Overall ${ }^{1}$, Prijay Bakrania ${ }^{1}$, Elisaveta Krasteva ${ }^{2}$, Nikesh Parekh ${ }^{3}$, Khlaid $\mathrm{Ali}^{3}$, Graham Davies ${ }^{4}$, Chakravarthi Rajkumar $^{3}$

${ }^{1}$ School of Pharmacy and Biomolecular Sciences, University of Brighton, UK, ${ }^{2}$ Dartford and Gravesham NHS Trust, UK, ${ }^{3}$ Brighton and Sussex Medical School, UK, ${ }^{4}$ Institute of Pharmaceutical Science, King's College London, UK

Introduction: Multimorbidity and adverse drug reactions (ADR) are problems associated with ageing populations. Exploring underlying genetic predispositions might help to risk-stratify patients for early intervention. The nuclear factor erythroid 2-like 2 (Nrf2) protein regulates antioxidant and de-toxifying effectors in the cell. Nrf2 expression declines with age, potentially increasing vulnerability to multimorbidity and ADR. We hypothesise that single nucleotide polymorphisms (SNPs) at 3 loci in the Nrf2 gene are associated with multimorbidity and ADR in older adults.

Methods: One-hundred and twenty-seven patients were recruited from a sub-population of the PRIME study (a multicentre prospective cohort study that followed older adults over 8-weeks post-discharge to determine ADR status). Donated genetic material was sequenced to determine genotype at 3 loci: rs6721961, rs35652124 and rs6706649 and then analysed for association with ADR (Naranjo Algorithm) and multimorbidity ( $>2$ conditions defined by the Charlson Index $(\mathrm{CI})$ ). Results: One-hundred and twelve patients (mean age $76.6 \pm 7.3$ years, $55.4 \%$ female) were successfully genotyped. In patients aged 65-79, those with the rs35652124 A allele showed increased odds of having $>2$ co-morbidities (OR 9.03 95\% CI $1.16-70.2, p=0.0127)$. Individuals with the CGG haplotype in this age-group showed reduced odds of multimorbidity (OR $0.11,95 \% \mathrm{CI}$ $0.01-0.86, p=0.001)$. No association between Nrf2 geno/haplotype and ADR was identified.

Conclusions: Polymorphisms in the Nrf2 gene are associated with multimorbidity, but not ADR, in older adults. 


\section{O-106}

\section{Telomere length according to age and sex in adults} and in children

Athanase Benetos ${ }^{1}$, Carlos Labat ${ }^{2}$, Simon Toupance ${ }^{3}$, Masayuki Kimura $^{4}$, Sylvie Gautier ${ }^{3}$, Patrick Rossignol ${ }^{3}$, Abraham Aviv ${ }^{4}$

${ }^{1}$ CHRU Nancy, Vandœuvre-lès-Nancy, France, ${ }^{2}$ INSERM U1116 Nancy, Vandœuvre-lès-Nancy, France, ${ }^{3} \mathrm{CHRU}$ de Nancy, Vandœuvre-lès-Nancy, France, ${ }^{4}$ Rutgers University NJ, USA

Background: People with shorter leucocyte telomere length (LTL) are at higher risk for mortality and age-related diseases. Since LTL is longer in women than in men and decreases with age, it is important to define its usual values according to age and sex. The aim of this study was to present the age/LTL relationship in adults men and women and in children.

Methods: We present data of 1421 adults (928 men and 493 women) aged $53 \pm 15$ years included by our research group in 3 different cohorts (ERA; $\mathrm{n}=427$, TELARTA; $\mathrm{n}=452$ and STANISLAS; $\mathrm{n}=542$ ) and having telomere measurements and a complete clinical evaluation including phenotypes of cardiovascular aging. We also studied the age/LTL relationship in children $<15$ years of the STANISLAS cohort $(n=110$ mean age $12 \pm 3$ years $)$. LTL was measured using TRF Southern blot analysis, which is recognized as the most reproducible method.

Results: In adults the linear relationship age/TL was LTL $(\mathrm{Kb})=$ $8.22-0.026 \times$ age in men and 8.55-0.028 $\times$ age in women. In both sexes this relationship was very significant $(\mathrm{p}<0.00001)$ and the slopes were similar. Mean LTL differed significantly between men $(6.74 \pm 0.74 \mathrm{~Kb})$ and women $(7.22 \pm 0.77 \mathrm{~Kb})(\mathrm{p}<0.0001)$. In children also the decrease in LTL with age was highly significant ( $\mathrm{p}<0.005$ ) but the slope was almost threefold steeper as compared to the adults: $\mathrm{LTL}(\mathrm{Kb})=9.10-0.073 \times$ age.

Conclusions: This study presents LTL reference values according to sex and age. Age-related decrease is similar in adult men and women ( 26 vs. 28 bases/year), but it is much stronger in children (73 bases/ year) pointing out the important role of early life in the determination of LTL later in life.

\section{O-107}

Pronostic value of routine biomarkers in older patients with cancer: pooled analysis of three prospective cohorts

Nadia Oubaya ${ }^{1}$, Pierre Soubeyran ${ }^{2}$, Nicoleta Reinald ${ }^{1}$, Marianne Fonck $^{2}$, Mylène Allain ${ }^{1}$, Sonia Zebachi ${ }^{3}$, Damien Heitz ${ }^{4}$, Marie Laurent ${ }^{5}$, Hubert Rousselot ${ }^{6}$, Philippe Caillet ${ }^{1}$, Jérôme Dauba ${ }^{7}$, Sylvie Bastuji-Garin ${ }^{1}$, Gilles Albrand ${ }^{8}$, Michael Bringuier ${ }^{9}$, Muriel Rainfray ${ }^{10}$, Etienne Brain ${ }^{9}$, Thomas Grellety ${ }^{2}$, Elena Paillaud ${ }^{11}$, Simone Mathoulin-Pélissier ${ }^{2}$, Carine Bellera (equally) ${ }^{2}$, Florence Canouï-Poitrine (equally) ${ }^{1}$

${ }^{1}$ AP-HP, Henri Mondor Hospital, Créteil, France, ${ }^{2}$ Institut Bergonié, Bordeaux, France, ${ }^{3}$ Université Paris-Est, UPEC, CEpiA (Clinical Epidemiology And Ageing), Créteil, France, ${ }^{4}$ Strasbourg University Hospital, Hautepierre Hospital, Strasbourg, France, ${ }^{5}$ APHP, Albert Chenevier Hospital, Créteil, France, ${ }^{6}$ Institut de Cancérologie de Lorraine Alexis Vautrin, Vandoeuvre les Nancy, France, ${ }^{7}$ Mont de Marsan Hospital, Mont de Marsan, France, ${ }^{8}$ Antoine Charial Hsopital, Francheville, Lyon, France, ${ }^{9}$ Institut Curie, Saint-Cloud, France,

${ }^{10}$ Bordeaux University Hospital, Bordeaux, France, ${ }^{11}$ APHP, Hôpital Européen Georges Pampidou, Paris, France

Introduction: To assess pronostic value of routine biomarkers in older patients with cancer.
Methods: A pooled analysis of three prospective multicentre cohorts, ELCAPA, PHRC Aquitaine and ONCODAGE was conducted. Patients aged 70 years or older, with cancer were included. Biomarkers collected were plasmatic C-reactive protein, albumin and a combined score: Glasgow Prognostic Score (GPS). The GPS comprised three categories $(0$ : $C R P \leq 10 \mathrm{mg} / \mathrm{L}$, albumin $\geq 35 \mathrm{~g} / \mathrm{L} ; 1$ : $\mathrm{CRP} \leq 10 \mathrm{mg} / \mathrm{L}$ and albumin $<35 \mathrm{~g} / \mathrm{L}$, or CRP $>10 \mathrm{mg} / \mathrm{L}$ and albumin $\geq 35 \mathrm{~g} / \mathrm{L} ; 2: \mathrm{CRP}>10 \mathrm{mg} / \mathrm{L}$ and albumin $<35 \mathrm{~g} / \mathrm{L}$ ). The primary endpoint was overall survival at 12 months. Multivariable Cox models were used, adjusting for age, sex, localisation, metastatic status, performance status, frailty screening index, the G8. Discriminative properties were assessed using Harrell C index and NRI (Net Reclassificatio Improvement).

Results: Overall 1800 patients were analysed (ELCAPA: N $=543$, PHRC Aquitaine: $\mathrm{N}=253$, ONCODAGE: $\mathrm{N}=1004$; mean age: $78.5 \pm 5.5$ years; $61.7 \%$ of men; $28.9 \%$ metastatic; most frequent localisations: breast (34.9\%) and colon-rectum (17.7\%); $70.7 \%$ of patients screened at risk of frailty with G8). Overall survival was $71.1 \%$. GPS was independently associated with death (among normal G8: GPS 1: Hazard Ratio $(\mathrm{HR})=4.48 ; 95 \%$ Confidence Interval $(95 \%$ CI $)=[2.03 ; 9.89]$, GPS 2: 11.64 [4.54; 29.81], among anormal G8: GPS 1: 2.45 [1.79; 3.34], GPS 2: 3.97 [2.93; 5.37]. The addition of GPS to the clinical model (Harell C: 0.82 [0.80; 0.83]) improved discrimination (Harell C: 0.84 [0.82; 0.85], NRI: 11\% [5; 19]).

Conclusion: GPS could be used in older patients with cancer to help decision-making and prognosis assesment.

\section{Area: Oral and dental health}

\section{O-108}

The association of oral health with body weight within 10 years in community-dwelling older adults

Eva Kiesswetter ${ }^{1}$, Bart Keijser ${ }^{2}$, Dorothee Volkert ${ }^{3}$, Marjolein Visser ${ }^{4}$

${ }^{1}$ Department of Health Sciences, Faculty of Science, Amsterdam Public Health research institute, Vrije Universiteit Amsterdam, The Netherlands and Institute for Biomedicine of Aging, FriedrichAlexander-Universität Erlangen-Nürnberg, Germany, ${ }^{2}$ Research Group Microbiology and Systems Biology, TNO, Utrechtseweg 48, 3704HE Zeist, The Netherlands and Department of Preventive Dentistry, Academic Center for Dentistry Amsterdam (ACTA), University of Amsterdam, Amsterdam, The Netherlands, ${ }^{3}$ Institute for Biomedicine of Aging, Friedrich-Alexander-Universität ErlangenNürnberg, Germany, ${ }^{4}$ Department of Health Sciences, Faculty of Science, Amsterdam Public Health research institute, Vrije Universiteit Amsterdam, The Netherlands

Introduction: To develop preventive strategies for malnutrition the knowledge about factors affecting body weight (BW) is essential. Therefore, we aimed to investigate the longitudinal associations of multiple oral health aspects with BW within 10 years in communitydwelling older adults.

Methods: This analysis is based on prospective data of 657 Dutch community-dwelling older adults (age $66.4 \pm 5.8$ years) from the Longitudinal Aging Study Amsterdam. Participants characteristics, BW and 12 oral health variables (edentulism, dentures, nine oral problems, self-rated oral health (1-very unhealthy to 5-very healthy)) were assessed in 2005/07 and 2015/16. The association between oral health and BW was analyzed by mixed models and adjusted for demographic, socio-economic, lifestyle, health, and functional aspects considering data of both time points.

Results: Mean BW was $79.1 \pm 13.3 \mathrm{~kg}$ at baseline (B) and $77.6 \pm 13.8 \mathrm{~kg}$ at follow-up (FW). Most participants rated their oral status as healthy (B:65.2\%, FW:66.9\%). At baseline $29.6 \%$ of the 
participants reported being edentulous (FW:34.4\%) and 55.8\% to wear dentures (FW:62.3\%). Toothache while chewing was the oral problem with the lowest (B:5.2\%, FW:6.6\%) and xerostomia with the highest prevalence at both time points (B:24.3\%, FW:30\%). In the long term better self-rated oral health was positively $(b=0.650$, $\mathrm{SE}=0.298, \mathrm{p}=0.029)$ and being edentulous $(\mathrm{b}=-1.739, \mathrm{SE}=$ $0.753, \mathrm{p}=0.021)$ as well as wearing complete dentures $(\mathrm{b}=-1.591$, $\mathrm{SE}=0.779 \mathrm{p}=0.041)$ were negatively associated with $\mathrm{BW}$. No further oral health variables showed an association with BW.

Key conclusions: In community-dwelling older adults poorer selfrated oral health, being edentulous and wearing complete dentures may contribute to weight loss in the long term.

\section{Area: Pharmacology}

\section{0-109}

Association of individual antidepressants and hip fracture in older adults: a cohort study with nested case-control analysis based on the German Pharmacoepidemiology Research Database

Federica Edith Pisa ${ }^{1}$, Jonas Reinold ${ }^{1}$, Bianca Kollhorst ${ }^{1}$, Ulrike $\operatorname{Haug}^{2}$, Tania Schink ${ }^{1}$

${ }^{1}$ Leibniz Institute for Prevention Research and Epidemiology-BIPS, Bremen, Germany, ${ }^{2} 1$ Leibniz Institute for Prevention Research and Epidemiology_BIPS, Bremen, Germany. 2. Faculty of Human and Health Sciences, University of Bremen, Bremen, Germany

Background: Evidence on the risk of hip fracture (HF) associated with individual antidepressants (ADs) is scarce, because most studies addressed only $\mathrm{AD}$ classes. However, risk profiles can vary widely between individual agents. We assessed the risk of HF associated with individual ADs in older adults.

Methods: We conducted a case-control study nested in a cohort of new users of ADs aged $\geq 65$ years, based on the German Pharmacoepidemiological Research Database (GePaRD) during 2005-2014. Incident cases of hospitalization for HF were identified and matched to up to 100 controls per case using incidence density sampling. Use of $\mathrm{AD}$ was ascertained at index date (ID; cases: date of HF; controls: matched date) based on the duration of the last dispensation. Current use was defined as use overlapping ID. We estimated adjusted odds ratios (aORs), with 95\% confidence interval (CI), using conditional logistic regression with remote users of any $\mathrm{AD}$ as reference category, adjusting for co-morbidities and co-medications.

Results: Among 706,561 cohort members, 39,853 cases were identified and matched to $3,979,510$ controls (in both groups: $79.8 \%$ women; median age 81 years). Current users had aOR $(95 \% \mathrm{CI})$ of $1.51(1.47-1.56)$ for citalopram, $1.51(1.38-1.65)$ for duloxetine, 1.48 (1.34-1.63) for escitalopram, $1.47(1.35-1.61)$ for venlafaxine, 1.46 (1.39-1.53) for amitriptyline, $1.45(1.35-1.55)$ for sertraline and 1.45 $(1.27-1.66)$ for fluoxetine. The aOR was lower for paroxetine $(1.36$; $1.20-1.55)$, trimipramine $(1.14 ; 1.04-1.26)$ and mirtazapine (1.13; $1.08-1.17)$.

Conclusions: In this large cohort, mirtazapine and trimipramine had a lower risk than most examined individual SSRI, SSNRI and amitriptyline.

\section{0-110}

Poly-de-prescribing to treat polypharmacy: efficacy and safety of the Garfinkel method

\section{Doron Garfinkel ${ }^{1}$}

${ }^{1}$ Wolfson Medical Center Holon and Homecare Hospice, Isarael Cancer Association

Background: This study evaluated efficacy and safety of the Garfinkel method of poly-de-prescribing (PDP) in older people with polypharmacy.

Methods: In a longitudinal, prospective study in Israel; PDP of $\geq 3$ prescription medications recommended to 177 people age $\geq 66$ with polypharmacy. $\geq 3$ years later, Likert scale questionnaires were used to evaluate clinical outcomes including changes in functional, mental and cognitive status, sleep quality, appetite, continence, major complication, the rate of hospitalizations, mortality, and family doctor's cooperation.

Results: PDP was achieved by 122 participants; $\leq 2$ drugs were discontinued by 55 'non-responders' (NR group). The average age was $83.4 \pm 5.3$ in PDP and $80.8 \pm 6.3$ in NR ( $p=0.0045)$. Follow up $\geq 3$ years in all. The prevalence of most diseases/symptoms was comparable. The main barrier to de-prescribing was the family doctor's unwillingness to adopt PDP recommendations ( $p<0.0001)$. The average number of medications at baseline in the NR and PDP, was 10.49 vs.10.20 (NS), and in the last follow-up 10.67 vs. 3.84, respectively $(\mathrm{p}=0.0001)$. PDPs showed significantly less deterioration in functional, mental and cognitive status, sleep quality, appetite, sphincter control, and less major complications ( $p<0.002$ in all). The rate of hospitalizations and mortality was comparable. Health improvement occurred within 3 months after PDP in $83 \%$ and persisted for $\geq 2$ years in $68 \%$.

Conclusion: This longitudinal research proves that in older people poly-de-prescribing is both safe and beneficial, being associated with significantly better quality of life, as compared to clinical outcomes of those who adhere to conventional recommendations and take all medications based on all specialists' clinical guidelines.

\section{0-111}

Simplification of medications prescribed to long term care residents (SIMPLER): a cluster randomised controlled trial to reduce unnecessary medication regimen complexity in residential aged care

Janet Sluggett ${ }^{1}$, Esa Chen ${ }^{1}$, Jenni Ilomäki ${ }^{2}$, Megan Corlis ${ }^{3}$, Sarah Hilmer ${ }^{4}$, Jan Van Emden ${ }^{3}$, Choon Ean $\mathrm{Ooi}^{5}$, Kim-Huong Nguyen ${ }^{6}$, Tracy Comans ${ }^{7}$, Michelle Hogan ${ }^{3}$, Tessa Caporale ${ }^{3}$, Susan Edwards ${ }^{8}$, Lyntara Quirke $^{9}$, Allan Patching ${ }^{10}$, J Simon Bell ${ }^{5}$

${ }^{1}$ Centre for Medicine Use and Safety, Faculty of Pharmacy and Pharmaceutical Sciences, Monash University, Parkville, VIC, Australia; and NHMRC Cognitive Decline Partnership Centre, Hornsby Ku-ring-gai Hospital, Hornsby, NSW, Australia, ${ }^{2}$ Centre for Medicine Use and Safety, Faculty of Pharmacy and Pharmaceutical Sciences, Monash University, Parkville, VIC, Australia, ${ }^{3}$ Helping Hand, North Adelaide, SA, Australia; and the NHMRC Cognitive Decline Partnership Centre, Hornsby Ku-ring-gai Hospital, Hornsby, NSW, Australia, ${ }^{4}$ Kolling Institute of Medical Research, Royal North Shore Hospital, Northern Clinical School, School of Medicine, University of Sydney, NSW, Australia; and the NHMRC Cognitive Decline Partnership Centre, Hornsby Ku-ring-gai Hospital, Hornsby, NSW, Australia, ${ }^{5}$ Centre for Medicine Use and Safety, Faculty of Pharmacy and Pharmaceutical Sciences, Monash University, 
Parkville, VIC, Australia; and the NHMRC Cognitive Decline Partnership Centre, Hornsby Ku-ring-gai Hospital, Hornsby, NSW, Australia, ${ }^{6}$ Menzies Health Institute Queensland, Griffith University, Meadowbrook, QLD, Australia; and the NHMRC Cognitive Decline Partnership Centre, Hornsby Ku-ring-gai Hospital, Hornsby, NSW, Australia, ${ }^{7}$ Centre for Health Services Research, The University of Queensland, Woolloogabba, QLD, Australia; Menzies Health Institute Queensland, Griffith University, Meadowbrook, QLD, Australia; and the NHMRC Cognitive Decline Partnership Centre, Hornsby Ku-ring-gai Hospital, Hornsby, NSW, Australia, ${ }^{8}$ Drug \& Therapeutics Information Service, GP Plus Marion, Oaklands Park, SA, Australia, ${ }^{9}$ Consumer representative, Alzheimer's Australia, Scullin, ACT, Australia, ${ }^{10}$ Helping Hand Consumer and Carer Reference Group, Helping Hand, North Adelaide, SA, Australia

Introduction: The high prevalence of polypharmacy, together with a multiplicity of medication administration times, formulations and special dosing instructions, means that complex medication regimens are common in residential aged care facilities (RACFs). Complex regimens can be burdensome for residents and nursing staff. The aim of SIMPLER study is to investigate the application of a structured process to simplify medication administration in RACFs.

Methods: SIMPLER is a non-blinded, matched-pair, 36-month cluster randomised controlled trial of a single multidisciplinary intervention delivered to South Australian RACFs. A validated, fiveitem tool was used to assess medications taken by residents in the intervention arm and identify opportunities to reduce medication complexity (e.g. by administering medications at the same time, or through use of longer-acting or combination formulations). The primary outcome is the total number of medication administration times per day at 4 months post-study entry. Secondary outcomes include time spent administering medications, incidents, resident satisfaction and quality of life, hospitalisations, falls and mortality.

Results: 242 permanent residents were recruited from eight RACFs between April and October 2017. The median age of participating residents was 87 years and $179(74 \%)$ were female. Of the 96 residents who received the intervention, opportunities for medication regimen simplification were identified for $62(65 \%)$ residents. Many recommendations were able to be actioned by registered nurses employed at the RACFs.

Key conclusions. Opportunities exist to reduce medication regimen complexity in RACFs. SIMPLER will quantify the impact of medication regimen simplification on outcomes that are important for residents and aged care providers.

\section{0-112}

\section{Antiepileptic drugs and accumulation of hospital days among persons with Alzheimer's disease}

Piia Lavikainen ${ }^{1}$, Heidi Taipale ${ }^{1}$, Antti Tanskanen ${ }^{2}$, Marjaana Koponen ${ }^{1}$, Jari Tiihonen ${ }^{2}$, Sirpa Hartikainen ${ }^{1}$, Anna-Maija Tolppanen ${ }^{1}$

${ }^{1}$ School of Pharmacy, University of Eastern Finland, Kuopio, Finland, ${ }^{2}$ Department of Clinical Neuroscience, Center for Psychiatry Research, Karolinska Institutet and Stockholm County Council, Stockholm, Sweden

Introduction: Antiepileptic drug (AED) use among older persons may lead to adverse drug events, and therefore to higher number of hospital days. We compared the accumulation of hospital days between AED initiators and non-initiators among persons with Alzheimer's disease (AD) as well as between individual AEDs.

Methods: We conducted an exposure-matched cohort study among persons diagnosed with AD in 2005-2011 $(n=70,718)$ as identified from Finnish health care registers. For each AED initiator, one noninitiator matched on age, sex and time since AD diagnosis was selected. Accumulation of hospital days was measured during a twoyear follow-up. Association between AED initiation or use of individual AEDs and accumulation of hospital days was assessed using negative binomial model.

Results: AED initiators $(n=4878)$ were hospitalized on average for 42.3 (SD: 87.9) days and matched non-initiators 28.0 (SD: 66.0) days during the two-year follow-up. Zero hospital days were observed for $31.4 \%$ of the AED initiators and for $41.4 \%$ of the non-initiators. Number of accumulated hospital days during the follow-up was $52 \%$ higher (adjusted incidence rate ratio, aIRR: 1.52, 95\%, CI: $1.38-1.67$ ) among AED initiators than the non-initiators. Among pregabalin (aIRR: 0.61, 95\% CI: 0.52-0.72), gabapentin (aIRR: $0.59,95 \% \mathrm{CI}$ : $0.44-0.80$ ) and clonazepam (aIRR: $0.72,95 \%$ CI: $0.54-0.96$ ) initiators the number of accumulated hospital days was $28-41 \%$ lower than among initiators of valproic acid.

Key conclusions: AED initiators are at increased risk of higher number of hospital days than non-initiators. Pregabalin and gabapentin were associated with lower number of hospital admissions than valproic acid.

\section{0-113}

Use of drugs among the older adult population: a pharmacoepidemiologic study

LD Christensen ${ }^{1}$, M Reilev ${ }^{2}$, HG Juul-Larsen ${ }^{1}$, LM Jørgensen ${ }^{1}$, $\mathrm{S} \mathrm{Kaae}^{3}, \mathrm{O}$ Andersen $^{1}$, A Pottegård ${ }^{4}$, J Petersen ${ }^{1}$

${ }^{1}$ Optimized Senior Patient Program (Optimed), Clinical Research Centre, Amager and Hvidovre Hospital, University of Copenhagen, Denmark, ${ }^{2}$ Clinical Pharmacology and Pharmacy, Department of Public Health, University of Southern Denmark, ${ }^{3}$ Section for Social and Clinical Pharmacy, Department of Pharmacy, University of Copenhagen, Denmark, ${ }^{4}$ Hospital Pharmacy, Odense University Hospital, Denmark

Introduction: Multi-morbidity and polypharmacy are common among older people. However, population-based utilization data in this age group is limited. Leveraging the Danish nationwide health registries, we aimed to characterize the drug use among Danish people $\geq 60$ years.

Methods: A descriptive study assessing the drug use in 2015 in the Danish population age $\geq 60$ years. Drug use at specific therapeutic subgroups and chemical subgroups and its dependence on age were described using descriptive statistics. The patterns of combination of drugs were analysed by using latent class analysis.

Results: We included $1,424,775$ persons (median age 70 years, interquartile range (IQR) $65-77 ; 53 \%$ women). The median number of drugs groups was five per person (IQR, 2-8). The most used single drug groups were paracetamol (5.9\%), statins $(5.7 \%)$, and platelet aggregation inhibitors $(4.1 \%)$. The most used drug groups were cardiovascular drugs (18\%), nervous system drugs (14\%) and alimentary tract and metabolism (11\%). The distribution of the therapeutic groups was in large consistent with age. Eighteen classes with different drug combination patterns were identified. One class without any drugs ( $21 \%$ of the population), and 11 classes with an expected number of drugs over five were identified.

Conclusions: The use of drugs is extensive both at the population level and increasing with age at an individual level. Cardiovascular, analgesics, and psychotropic drugs were the most prevalent drug classes. Eighteen different drug patterns were identified. The drug patterns have the potential to be used in further studies about risk prescriptions. 


\section{0-114}

Non-adherence to cardiovascular drugs in older patients with depression: a population-based cohort study

Hans Wouters ${ }^{1}$, Richard Oude Voshaar ${ }^{1}$, Karin Hek $^{2}$, Francois Schellevis ${ }^{2}$, Liset van Dijk ${ }^{2}$, Huibert Burger ${ }^{1}$, Peter Verhaak ${ }^{1}$, Floor Holvast ${ }^{1}$

${ }^{1}$ RUG, UMCG, Netherlands, ${ }^{2}$ NIVEL, Utrecht, Netherlands

Background: Depression is common among patients with cardiovascular disease and has been associated with both drug nonadherence and increased mortality. Non-adherence can occur because of non-initiation, suboptimal implementation, or non-persistence. We aimed to determine if depression increased the risk of any of these components of non-adherence among older patients prescribed cardiovascular drugs in primary care.

Methods: A longitudinal analysis of routine primary care data from the NIVEL Primary Care Database was performed using data for 2011-2013. Patients aged $\geq 60$ years diagnosed with depression in 2012 were compared with age- and sex-matched groups with either other psychological diagnoses or mentally healthy controls. Regression analyses were performed to determine the association between mental health status and non-initiation, suboptimal implementation, and non-persistence.

Results: Mixed-effects logistic regression analyses showed increased odds for suboptimal implementation of beta-blockers among depressed patients $(2.18 ; 95 \%$ CI 1.29-3.69). For non-persistence, a clustered Cox regression analysis demonstrated that, compared with controls, there was an increased hazard ratio for depressed patients to discontinue beta-blockers $(2.31 ; 95 \%$ CI $1.58-3.37)$ and calcium antagonists (1.74; 95\% CI 1.23-2.46).

Key conclusions: It is likely that older patients in primary care diagnosed with depression are at increased risk of non-persistence with cardiovascular drug therapy. Because non-adherence is associated with increased cardiovascular mortality, it is important that physicians ensure that older depressed patients persevere with therapy.

\section{O-115}

Polypharmacy in patients admitted to an Orthogeriatric Unit: a retrospective study

Cristina Carvalho Gouveia ${ }^{1}$, Sofia Duque ${ }^{1}$, Filipa Bianchi-DeAguiar $^{1}$, Inês Miguéis Ferreira ${ }^{2}$, Ana Lopes Santos ${ }^{1}$, Sara Trevas ${ }^{1}$, André Ferreira $^{1}$, Joana Lopes ${ }^{1}$, Mafalda Sequeira ${ }^{1}$, Ana Pedroso ${ }^{1}$, José Guimarães Consciência ${ }^{1}$, Luís Campos ${ }^{1}$

${ }^{1}$ São Francisco Xavier Hospital, Occidental Lisbon Hospital Centre, Portugal, ${ }^{2}$ Faculty of Medicine, University of Lisbon, Portugal

Introduction: Hip fracture (HP) is associated with considerable morbidity and mortality, especially in the elderly. Polypharmacy is considered an important risk factor for falling and has been demonstrated to predict outcome after HP. We aim to study polypharmacy in this population and its possible association with adverse outcomes during a hospital admission for HP.

Methods: Retrospective study of elderly admitted to an Orthogeriatric Unit for HF between June 2016 and August 2017. Patients were included in two groups accordingly to the number of drugs at admission: group $\mathrm{A}-$ no polypharmacy (less than 6 drugs) and $\mathrm{B}-$ polypharmacy (6 or more drugs).

Results: 257 patients were included. $58 \%$ of them met the criteria for polypharmacy. The most prescribed drugs in group B were: proton pump inhibitors (55\%), diuretics $(43 \%)$ and benzodiazepines $(42 \%)$.
Patients in group B had worse functional status before hospital admission, as demonstrated by a lower Barthel index (78.8 points vs A: 93.3), lower Holden classification (3.9 points vs A: 4.17) and higher Cumulative Illness Rating Scale-Geriatrics (10.5 points vs A: 6.5). Group B had also more risk factors for falling (mean 8.8 vs A: 5.9), longer preoperative time (4.9 days vs A: 3.5 ) and hospitalization time (11.9 days vs A: 7.8) and more complications during hospital admission (mean 2.8 vs A: 2.1 ).

Key conclusions: Polypharmacy is prevalent in this population and is associated with worse outcomes after hospital admission. It is essential to review the patients' drugs, in order to provide them a better quality of life.

\section{0-116}

Predictors of self-reported adherence to direct oral anticoagulation in a population of elderly men and women with non-valvular atrial fibrillation

Andrea P Rossi ${ }^{1}$, Roberto Facchinetti ${ }^{2}$, Sofia Rubele ${ }^{1}$, Nicole Nori ${ }^{1}$, Anna De Caro ${ }^{1}$, Chiara Guerra ${ }^{1}$, Francesco Fantin ${ }^{1}$, Elena Bortolazzi $^{1}$, Stefano Gattazzo ${ }^{1}$, Mauro Zamboni ${ }^{1}$

${ }^{1}$ University of Verona, Verona, Italy, ${ }^{2}$ AOUI di Verona, Verona, Italy

Introduction: There is a general lack of studies evaluating medication adherence with self-report scale in elderly patients in treatment direct oral anticoagulants (DOACs). The aim of the study was to assess the degree of adherence to DOAC therapy in a population of elderly outpatients 65 years of age or older affected by non-valvular atrial fibrillation (NVAF) using the 4-item Morisky Medication Adherence Scale, and to identify potential factors, including the geriatric multidimensional evaluation, which can affect adherence in the study population.

Methods: A total of 103 subjects, anticoagulated with DOACs for NVAF in primary or secondary prevention, were eligible; 76 showed adequate adhesion to anticoagulant therapy, while 27 showed inadequate adherence. Participants underwent biochemical assessment and Morisky scale, Instrumental Activities of Daily Living, CHA2DS2VASc, HAS-BLED, mental status and nutritional evaluation.

Results: $2 \%$ of subjects assumed Dabigatran at low-dose, while $7.8 \%$ at standard dose, $9.7 \%$ assumed low-dose of Rivaroxaban and $30.1 \%$ at standard dose, $6.8 \%$ assumed Apixaban at low-dose and $39.7 \%$ at standard dose, finally $1 \%$ assumed Edoxaban at low-dose and $2.9 \%$ at standard dose. Most subjects took the DOACs without help (80.6\%), while 16 subjects were helped by a family member $(15.5 \%)$ and 4 were assisted by a caregiver (3.9\%). Binary logistic regression considered inappropriate adherence as a dependent variable, while age, male sex, polypharmacotherapy, cognitive decay, caregiver help for therapy assumption, duration of DOAC therapy and double daily administration were considered as independent variables. The double daily administration was an independent factor, determining inappropriate adherence with an OR of 2.88 ( $\mathrm{p}=0.048$, CI 1.003-8.286). Key conclusions: Double daily drug administration seems to be the main factor for inappropriate adherence to anticoagulant therapy.

\section{0-117}

Stimulating innovation management of polypharmacy and adherence in the elderly across the EU

Alpana Mair ${ }^{1}$

${ }^{1}$ Head of Effective Prescribing and Therapeutics 
Introduction: Populations are aging, with people over the age of 50 living with multiple long-term conditions and taking multiple medications (polypharmacy). Medication is the single most common healthcare intervention, generating the third highest cost of health expenditure. Up to $11 \%$ of all unplanned hospital admissions are attributable to medicines related harm. The European Union has identified the reduction of avoidable harm in healthcare as a key priority. The SIMPATHY consortium explored how polypharmacy programmes can be implemented to improve medication safety and prevent patient harm by addressing the appropriate polypharmacy.

Methods: This was achieved by case studies of $9 \mathrm{EU}$ countries and how programmes were implemented, a literature review, benchmarking survey across the EU and also a modified Delphi to determine the consensus across the EU.

Results: Within the nine case studies, polypharmacy management was only addressed in 4 countries and only in one nationally. Over a 1000 participants completed the EU wide benchmarking survey including 100 patients. These findings were sometimes at odds with the case studies highlighting the lack of programmes to manage polypharmacy. From the Delphi, Consensus was obtained for statements relating to management, development and measurement but not the time taken to implement such a change.

Conclusions: Results highlight importance of change management and theory-based implementation strategies, and collaborative working of policy makers and clinicians to develop, implement or scaling up programs to address polypharmacy. Delphi indicated the vision statements were too ambitious and not achievable by the specified timeframe of 2025 .

\section{Area: Ethics and end of life care}

\section{0-118}

Educating nursing home staff in palliative care to improve end-oflife care and to reduce burdensome hospitalisations: baseline findings and feasibility of a randomised, controlled trial

Pauli Lamppu ${ }^{1}$, Harriet Finne-Soveri ${ }^{2}$, Marja-Liisa Laakkonen ${ }^{3}$, Jouko Laurila $^{1}$, Kaisu Pitkälä ${ }^{4}$

${ }^{1}$ Helsinki University, Department of General Practice and Primary Health Care, Helsinki, Finland, ${ }^{2}$ National Institute ofHealth and Welfare, Helsinki, Finland, ${ }^{3}$ Department of Social Services and Health Care, Laakso Hospital Geriatric Clinic, Helsinki, Finland, ${ }^{4}$ Helsinki University Hospital, Unit of Primary Health Care and Helsinki University, Department of General Practice and Primary Health Care, Helsinki, Finland

Introduction: Varying efforts have been made to support good quality end-of-life care in nursing homes. One prior educational trial has shown favorable outcomes in reducing hospitalizations among dying nursing home residents. However, these positive outcomes have not been replicated in larger scale trials. Our aim is to investigate in this cluster-randomized educational trial whether staff education in palliative care will 1) improve the residents' health-related quality of life (HRQOL), 2) reduce unnecessary hospitalizations during a two year follow-up compared to control group in usual care. Secondary endpoints include symptoms and proxies' satisfaction.

Methods: We recruited 340/625 residents in five nursing homes in Helsinki. At baseline, all participants were assessed for HRQOL (15D), symptoms (ESAS, PAINAD), hospital use, advance care plans and proxies' satisfaction of care. The staff in intervention wards was given four 4-hour education sessions discussing the principles of palliative care (introduction, advance care planning, adverse effects of hospitalizations, symptom management, communication, supporting proxies, challenging situations) using constructive learning methods with staffs' own patient cases.

Results: Mean age of participants was 84 years, $76 \%$ were females. The intervention and control groups did not differ in respect to demographics, terminal diseases, comorbidities, nutritional status, MMSE or functioning. Of about 180 staff members, 102 completed the educational intervention. The educational sessions had lively discussions and the participants gave an overall score of $4.6 / 5$ for the education.

Conclusions: We have successfully randomised nursing home wards in this palliative care education trial and completed staff education with very positive feedback.

\section{0-119}

The knowledge and attitudes of Flemish geriatric nurses regarding tiredness of life and euthanasia: a survey study

Nele Van Den Noortgate ${ }^{1}$, Liesbeth Van Humbeeck ${ }^{1}$, Let Dillen ${ }^{1}$, Ruth Piers ${ }^{1}$

${ }^{1}$ Ghent University Hospital, Ghent, Belgium

Introduction: The prevalence of thoughts of possible death to self, increases from $6.4 \%$ in 80 years to $13.4 \%$ in 90 years old. Flemish nurses are frequently confronted with euthanasia requests of older persons being tired of life, although euthanasia is not legalized for this indication in Belgian law. Therefore we wanted to explore the knowledge and attitudes of nurses regarding euthanasia and tiredness of life.

Methods: A survey, consisting of four case vignettes and five multiple choice questions, was send to nurses employed in acute (2 hospital wards) and chronic care setting (1 nursing home and 1 home care organisation).

Results: A sample of 151 nurses $(92.7 \%$ female, median age 42 years) was obtained. Nurses in chronic care were significantly more confronted with tiredness of life $(n=76 ; 32 \%$ at least once a week) than nurses in acute care $(\mathrm{n}=75 ; 20 \%)(\mathrm{p}=0.02)$. The mean knowledge score was $62.1 \% \pm 24.4$ in chronic care and $73.6 \% \pm 21.6$ in acute care $(\mathrm{p}=0.01)$. Although $79.5 \%$ of the nurses were aware of the fact that euthanasia was legally not an option, $43 \%$ would agree upon the act. No significant differences were noticed between settings and level of education.

Conclusion: The law on euthanasia is rather well known among Flemish nurses. A discrepancy is noticed between the knowledge of the illegality and the positive attitude to proceed to euthanasia in older people with tiredness of life. Physicians should be aware of this attitude of nurses and foresee ongoing discussions with nurses on this topic.

\section{0-120}

Impact of spousal bereavement on health and mortality among older adults: nationwide, matched cohort study

Lucas Morin ${ }^{1}$, Jonas Wastesson ${ }^{1}$, Neda Agahi ${ }^{1}$, Kristina Johnell ${ }^{1}$

${ }^{1}$ Karolinska Institutet, Solna, Sweden

Background: The present study aimed to investigate the effects of spousal loss on health outcomes and mortality among older adults. Methods: Nationwide, longitudinal matched cohort study. Data was extracted from national registers with full-population coverage in Sweden. All surviving spouses of older adults ( $\geq 65$ years) who died in Sweden in 2013-2014 were matched 1:1 (sex, age) with married 
older adults who did not experience spousal loss and followed-up for 1 year. Risk of adverse event was calculated with adjusted conditional fixed effect Poisson regression modelling.

Results: 42918 bereaved older adults were included and matched with an equal number of married controls. Mean age was 78.9 years $(\mathrm{SD}=7.2), 68.3 \%$ were women. All-cause mortality significantly higher among bereaved older adults (49.2 per 1000 person-years) than among matched controls (30.8 per 1000 person-years). Adjusted incidence risk ratio for 1-year mortality was 2.36 (95\% CI 2.17-2.58). Excess risk of mortality was higher among men (IRR $=2.62,95 \% \mathrm{CI}$ 2.32-2.97) than among women $(2.24,95 \%$ CI $1.97-2.54)$. Bereaved older adults were also at increased risk of acute cardiovascular event $(\mathrm{IRR}=1.72,95 \%$ CI $1.58-1.88)$, hip fracture $(1.88,95 \%$ CI $1.63-2.16$ ), pneumonia (IRR $=1.31,95 \%$ CI $1.18-1.46$ ), acute renal failure (IRR $=1.67,95 \%$ CI 1.26-2.22), and self-harm $(I R R=18.73$, 95\% CI 9.83-35.7). Risk of unplanned hospitalization during the first year after spousal loss was higher among bereaved older adults than among controls (IRR $=1.31,95 \%$ CI 1.28-1.35). During the first 90 -day period after the index date, we observed a $65 \%$ increase in the use of benzodiazepines and a $10 \%$ increase in the use of selective serotonin reuptake inhibitors among cases, while no variation was noted in the control group.

Conclusion: Bereavement has a significant and rapid impact on both fatal and non-fatal health outcomes among surviving spouses. This demonstrates the need for appropriate support of recently bereaved older adults.

\section{0-121}

Physical restraining is a common practice in end-of-life management of nursing home residents with dementia: a crosssectional study

Antoine Elyn ${ }^{1}$, Sandrine Sourdet ${ }^{2}$, Lucas Morin $^{3}$, Fati Nourhashemi ${ }^{2}$, Nicolas Saffon ${ }^{1}$, Philip DE Souto Barreto ${ }^{4}$, Yves Rolland ${ }^{2}$

${ }^{1}$ Palliative care, Geriatrics and Internal Medicine, Universitary Hospital of Toulouse, Toulouse, France, ${ }^{2}$ Geriatrics and Internal Medicine, Universitary Hospital of Toulouse, Toulouse, France, ${ }^{3}$ Aging Research Center, Karolinska Institutet, Stockholm, Sweden, ${ }^{4}$ INSERM UMR1027 "Aging and Alzheimer disease: from observation to intervention", University of Toulouse, Toulouse, France

Introduction: End-of-life care is a central issue in nursing homes and is frequently pointed at because of a lack of resources. Poor care outcomes have been often reported especially for residents with dementia. Our aims were twofold: first to assess whether the diagnosis of dementia was associated with specific patterns of end-of-life care during the last 6 months of life, and second to compare care pattern of residents with dementia according to living status at 6 month.

Methods: Secondary, cross-sectional analysis and prospective study IQUARE. 175 nursing homes in south-west France. Residents at baseline, stratified according vital status at 6-month follow-up and dementia diagnosis.

Results: Among the 6275 residents enrolled in IQUARE study, 494 (7.9\%) were dead at 6-months of follow-up; among them, 240 (48.6\%) had a diagnosis of dementia. Among all residents with dementia $(n=2688), 240(8.9 \%)$ were dead at 6-months of follow-up. Among residents who died during the first 6 months, compared to residents without dementia, residents with dementia were more disabled and malnourished. They are more likely to be exposed to physical restraints $(\mathrm{OR}=1.65,95 \%$ IC $=1.08-2.51)$, with a prevalence of $55.4 \%$; and less likely to have anxiolytic prescription $(\mathrm{OR}=0.58,95 \% \mathrm{IC}=0.38-0.88)$. Among residents with dementia, residents dying within a delay of 6 months are more likely to be identified with "end-of-life" status $(\mathrm{OR}=5.71,95 \% \quad \mathrm{IC}=$ $3.48-9.37)$, but with only $15 \%$ of identification. They are more likely to experience pain ( $\mathrm{OR}=1.43,95 \% \mathrm{IC}=1.04-1.97)$ and to be physically restrained $(\mathrm{OR}=1.46,95 \%$ IC $=1.08-1.98)$. However, pain and psychological distress management are not improved.

Key conclusions: Poor quality indicators such as physical restraints are associated with end of life care for residents with dementia.

\section{0-122}

Pain as a challenge in nursing home residents with cognitive impairment

Agnieszka Neumann-Podczaska ${ }^{1}$, Tomasz Nowak ${ }^{2}$, Ewa Deskur-

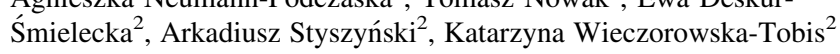

${ }^{1}$ Department of Geriatrics and Gerontology, Poznan University of Medical Sciences, Poland, ${ }^{2}$ Laboratory for Geriatric Medicine, Department of Palliative Medicine, Poznan University of Medical Sciences, Poznań, Poland

Introduction: In patients with dementia observational scales are recommended for use in the assessment of pain. Unfortunately, their application is rare-in consequence, pain is frequently underdiagnosed and undertreated in these subjects. The aim of the study was to assess analgesic treatment in nursing home residents (NHR) with cognitive impairment and to delineate the relationship between pain and behavioral disturbances.

Method: The research was conducted in two nursing homes in Wielkopolska, Poland. The analyzed group consisted of 96 residents (78 female) with moderate and severe cognitive impairment in whom pain was assessed with the Abbey Pain Scale (APS) and agitation with the Cohen-Mansfield Agitation Inventory (CMAI). Medical files related to drug prescriptions were analyzed.

Results: Analgesics were consumed by 33 individuals (34\%); 24 $(25 \%)$ received regular pain treatment, 7 -as needed only $(\mathrm{PRN})$ and $2(2 \%)$ - both regular and PRN. In regards to non-opioid analgesia 13 persons $(14 \%)$ received them regularly. No one received monotherapy with analgesics from the second step of the analgesic ladder, 2 (2\%)received a strong opioid Relationship was found between the APS and CMAI $(r=0.45, p<0.0001)$. Subjects with a higher CMAI received sedative drugs more frequently $(\mathrm{p}<0.001)$ and despite having a higher APS ( $p=0.001)$, this did not correlate with higher analgesia. Conclusion: Our study suggests that pain can be an important underlying cause of behavioral disturbances in older subjects with dementia. To reduce their frequency and to avoid excessive usage of sedatives, proper pain assessment and management is essential.

\section{0-123}

\section{Vascular risk factors and CSF findings in MCI}

Arne Nakling ${ }_{1}^{1}$, Dag Aarsland ${ }^{2}$, Tormod Fladby ${ }^{3}$, Hogne Sønnesyn ${ }^{4}$, Halvor Næss ${ }^{1}$

${ }^{1}$ University of Bergen, Bergen, Norway, ${ }^{2}$ King's College, London, UK, ${ }^{3}$ University of Oslo, Oslo, Norway, ${ }^{4}$ Stavanger University Hospital, Stavanger, Norway

Introduction: The dementia disease initiation study (DDI) is a mainly Norwegian, longitudinal cohort study established to explore the neuropsychological features and early biological pathways of Alzheimer's Disease (AD) and other neurodegenerative diseases leading to dementia. 
Methods: Individuals aged 40-80 years with subjective cognitive decline (SCD) were recruited via advertisements and news-bulletins, and through referral to memory clinics. Normal controls were recruited through spouses of participants, or volunteers from the community. Participants underwent physical examination, medical history, cognitive testing with a standardized battery, biobanking, genetics, spinal tap, MRI and FDG-PET. Participants were classified as either SCD or mild cognitive impairment (MCI) according to cognitive screening. MCI was determined if score was $\leq 1.5 \mathrm{SD}$ below the normative mean on either CERAD delayed recall, Trailmaking B, COWAT, VOSP silhouettes, or MMSE $\leq 27$.

Results: 721 participants were included. The MCI group was older (mean 65.1 vs mean $60.9 \mathrm{yr}$ ). The SCD group had lower Body Mass Index. In the MCI group, there were significantly elevated levels of $\mathrm{CRP}$ and pro-BNP, and also diabetes and coronary heart disease. There were more smokers in the MCI group. BMI of $\leq 25$ was associated with higher levels of CSF total/phospho-tau. Total cholesterol of 7.0 or more was associated with pathological levels of CSF Abeta42-amyloid, P- and T-tau.

Conclusion: The association between elevated levels of CRP and proBNP and a higher total cholesterol in the MCI group could be interpreted as presence of inflammatory response in these individuals who are at risk for developing $\mathrm{AD}$.

\section{0-124}

Socioeconomic risk factors for neurocognitive disorders in older people

G.I. Prada ${ }^{1}$, RM Nacu ${ }^{2}$, AG Prada ${ }^{2}$, AC Ilie ${ }^{3}$, ID Alexa ${ }^{3}$, AC Ilie $^{3}$, AI Pislaru ${ }^{3}$, R Stefaniu ${ }^{3}$, IA Sandu ${ }^{3}, \mathrm{C} \mathrm{Nuta}^{1}$, OL Bajenaru ${ }^{1}$, AM Herghelegiu ${ }^{2}$

1"Ana Aslan" National Institute of Gerontology and Geriatrics, Bucharest, Romania, "Carol Davila" University of Medicine and Pharmacy, Bucharest, Romania, ${ }^{3 " G r}$.T. Popa" University of Medicine and Pharmacy, Iasi, Romania

Introduction: Many factors influence cognitive function. We evaluated influence of some socioeconomic factors upon cognitive disorders in elderly.

Materials and methods: 975 consecutively admitted older patients were investigated. Gender distribution: $67 \%$ women, $33 \%$ men; age range $65-97$, mean 81 years. Sample was divided into three age groups: young-old (65-74 years), old-old (75-84 years), very-old (85-97 years). Parameters considered: gender, age, level of education, place of residence (urban/rural), income, marital status, comorbidity, cognitive function at admission. Tests used for screening cognitive disorders: Mini Mental Status Examination (MMSE), Clock Drawing Test and Five Words Test.

Results: Two neurocognitive disorders were evaluated: mild neurocognitive disorders (former mild cognitive impairment -MCI) and moderate to severe neurocognitive disorder (dementia). Higher risk of MCI in men than women: Odds Ratio (OR) 1.47. Adjusting for age and education OR was higher 1.67, adjusting for marital status OR was 1.71 in favor of men. Adjusting for disease burden we obtained 1.54 in favor of men. Higher risk of dementia for women, OR 1.61. Adjusting for age and education Odds Ratio increased to 2.2 in favor of women; adjusting for marital status value decreased to 1.3. Adjusting for comorbidity, risk of developing dementia in women as compared to men was 1.9. Odds of having dementia was higher in rural as compared to urban patients 1.3 .

Conclusions: Risk of MCI was higher in men, with low education and without spouse. Risk of dementia was higher in women, with lower education and higher comorbidity and from rural area.

\section{0-125}

Can minimum nurse-to-patient ratios reduce patient mortality in acute care hospitals: A cohort study

\section{Rochefort Christian ${ }^{1}$}

${ }^{1}$ University of Sherbrooke, Sherbrooke, Canada

Introduction: In 2004, California became the first state to implement mandatory minimum nurse-to-patient ratios in hospitals. Since then, several other jurisdictions worldwide have implemented or are considering implementing such ratios. We examined whether failure to meet minimum nurse-to-patient ratios as set in California is associated with an increased risk of death.

Methods: A dynamic cohort of adult medical, surgical, and intensive care unit (ICU) patients admitted between 2010 and 2017 to a large university health network in Quebec (Canada) was followed to examine the associations between patient cumulative exposure to work-shifts where nurse-to-patient ratios were below the minima set in California (i.e., 1:5 on medical/surgical wards, and 1:2 in the ICU) and the risk of death. The association between these ratios and the risk of death was assessed using a Cox regression model which adjusted for patient (e.g., age, sex, comorbidities), nursing unit (e.g., unit type) and other nurse staffing characteristics (e.g., nursing experience).

Results: A total of 124,832 patients were followed, of which 4975 died during their hospitalization. Patients who died where, on average, older, had more comorbidities, and a higher severity of illness on admission than those who survived. After adjusting for patient, nursing unit, and other nurse staffing characteristics, we found that each 3-additional work-shifts where the nurse-to-patient ratios on medical-surgical wards were less than 1:5 was associated with an increased risk of death of $1.5 \%$ (HR: 1.015, 95\% CI 1.008-1.022). We also noted that very few work-shifts in the ICU were below the minimum ratio set in California (i.e., 1:2), and found that the cumulative number of shifts where the ICU nurse-to-patient ratios were below this threshold was not significantly associated with the risk of death.

Discussion: Failure to meet the minimum nurse-to-patient ratio set in California for medical and surgical units increases the risk of death among patients admitted to these units. There is a pressing need for policies that will attract and retain greater number of nurses in hospitals to satisfy minimum staffing ratios.

\section{0-126}

\section{Is acute care ageist?}

Graham Ellis ${ }^{1}$, Phyo Kyaw Myint ${ }^{2}$

${ }^{1}$ Glasgow Caledonian University, Glasgow, UK, ${ }^{2}$ University of Aberdeen, Aberdeen, UK

Background: In the UK the 4-h standard in the emergency department (ED) is accepted as the acute standard of hospital care and breaches of the 4-h targets are associated with poorer clinical outcomes and higher mortality.

Methods: We looked ED attendance for Scotland by age bracket and four, eight and 12-h targets from 1st January 2017 to 31st January 2017.

Results: 1,500,800 people attended an ED in Scotland between 1/1/17 and $31 / 12 / 17$. $22 \%$ of these attenders were over the age of 65 . Analysis by five-year age bracket showed that the lowest breaches were in the 5-9-year age group $(1.4 \%)$ and the highest were in the over 85 age group $(16.5 \%)$. Looking at the 12 -hour target, $0.001 \%$ of 5-9-year olds breached compared to $0.1 \%$ of $20-24$-year olds and $0.5 \%$ of over 85 s. This means that $1 / 6$ over 85 -year olds in Scotland 
waited more than $4 \mathrm{~h}$ in the ED in 2017. These differences become more extreme at longer lengths of stay where $1 / 200$ adults 85 or older waited more than $12 \mathrm{~h}$ in the ED, compared to $1 / 1000$ 20-24-year olds and 1/100,000 5-9-year olds.

Conclusion: While multiple factors may explain reasons for a delay in the over $85 \mathrm{~s}$ group, disproportionate waits for vulnerable older adults raise difficult questions about the configuration of acute services. Scotland is launching the Scottish Care of Older People (SCoOP) benchmarking network to identify national variations in acute care for older people.

\section{EuGMS 2018 - Poster Abstracts}

\section{Area: Pharmacology}

P-1

Effects of artemisinin on pressure overload induced arrhythmias in rats comparison with verapamil

Xue $\mathrm{Xu}^{1}$, Yuansheng $\mathrm{Liu}^{2}$

${ }^{1}$ Department of Cardiology, Peking University People's Hospital, Beijing, China, ${ }^{2}$ Emergency Department, Peking University People's Hospital, No.11 Xizhimen South Street, Xicheng District, Beijing, 100044, China

Introduction: Arrhythmias induced by a change in ventricular wall stress might be important in clinical situations, for example, arterial blood pressure is more labile in hypertensive patients. Whether artemisinin (ART) can prevent ventricular tachyarrhythmias induced by pressure overload and induce atrioventricular block (AVB) compared with verapamil (VER) in SH rats was never studied.

Methods: Transverse aortic constriction (TAC) surgery increased the left ventricular pressure, left ventricular wall stress, and, eventually, caused ventricular tachyarrhythmias. The SH rats that underwent TAC surgery comprised the ST group. The ventricular arrhythmia scores (VASs) and the rates of the different types of AVB were determined in the absence and presence of ART and VER.

Results: PVC and VT were detected promptly and no VFs occurred after increases in wall stress in hypertrophic hearts. The SH rats that had undergone TAC had significantly reduced VASs when they were pretreated with $150 \mathrm{mg} / \mathrm{kg}$ ART (1.40 vs $4.30, \mathrm{P}<0.001), 300 \mathrm{mg} /$ $\mathrm{kg}$ ART (2.11 vs 4.30, P < 0.001), $0.5 \mathrm{mg} / \mathrm{kg}$ VER (2.75 vs 4.30, $\mathrm{P}<0.05)$, and $1.0 \mathrm{mg} / \mathrm{kg}$ VER $(0.29$ vs $4.30, \mathrm{P}<0.001)$ compared with the VAS in ST group (4.30). AVBs were only detected in the groups that had undergone TAC and had been pretreated with ART and VER.

Key conclusions: Based on these findings, ART may be a promising medication for ventricular arrhythmias prevention during acute pressure overloads in SH rats. Like VER, ART tended to be associated with the occurrence of AVBs.

\section{P-3}

The effect of hospitalization on potentially inappropriate medication use in older adults with chronic kidney disease

Wubshet Tesfaye ${ }^{1}$, Syed Tabish Zaidi ${ }^{1}$, Ronald Castelino ${ }^{2}$, Barbara Wimmer $^{1}$, Gregory Peterson ${ }^{1}$, Matthew Jose ${ }^{1}$, Charlotte McKercher ${ }^{1}$

${ }^{1}$ University of Tasmania, Hobart, Australia, ${ }^{2}$ The University of Sydney, Sydney, Australia
Background: Potentially inappropriate medications (PIMs) use has been associated with increased morbidity and mortality in patients with chronic kidney disease (CKD). However, little has been published about PIMs use in hospitalized older adults ( $\geq 65$ years) with CKD. Therefore, we aimed to measure the impact of hospitalization on PIMs use in older CKD patients.

Setting: Australian tertiary care hospital.

Participants: Older adults ( $\geq 65$ years) with CKD (estimated glomerular filtration rate [eGFR)] of $<60 \mathrm{~mL} / \mathrm{min} / 1.73 \mathrm{~m}^{2}$ ) admitted to hospital between January and June 2015 .

Measurements: Inappropriate medication use was measured using the medication appropriateness index (MAI) and Beers criteria for medications recommended to be avoided in older adults and under certain conditions.

Results: The median age of the 204 patients was 83 years (interquartile range (IQR): 76-87 years) and most were men (61\%). Overall, the level of PIMs use (MAI) decreased from admission to discharge [median (IQR): $6(3-12)$ to 5 (2-9)]. More than half of the participants $(55 \%)$ had at least one PIM per Beers criteria on admission, which reduced by discharge $(48 \%)$. People admitted with a higher number of medications ( $\beta 0.72,95 \%$ CI 0.56 to 0.88 ) and lower eGFR values $(\beta-0.11,95 \% \mathrm{CI}-0.18$ to -0.04$)$ had higher MAI scores after adjusting for age, sex and Charlson's comorbidity index (CCI).

Conclusion: PIMs were commonly used in older patients with CKD. Hospitalization appears to lead to a reduction in PIMs use, but there is still considerable scope for improvement in these susceptible individuals.

\section{P-4}

Potential inappropriate prescribing and the correlates among older persons in Nigeria and South Africa: a comparative study

Sule Ajibola Saka ${ }^{1}$, Frasia Oosthuizen ${ }^{1}$, Manimbulu Nlooto ${ }^{1}$

${ }^{1}$ University of Kwa-Zulu Natal, Durban, South Africa

Introduction: Potential inappropriate prescribing (PIP) impacts negatively on the quality of life among older persons worldwide. However, despite PIP being a global phenomenon, cross-country data that can influence interventions on a global or regional level are scarce. This study aimed to compare the prevalence of PIP among older persons in Nigeria and South Africa and to determine the risk factors for PIP in both countries.

Methods: A retrospective evaluation of medical chart of older persons in one teaching hospital each, in Nigeria and South Africa was carried out. Older persons aged $\geq 60$ years that attended the outpatients' clinics of the hospitals between January and December 2016 were included. Information including patients' socio-demographics, medical histories, past and current medications were extracted from the patients' medical records. The PIP was evaluated using the 2015 updated American Geriatric Society-Beers Criteria. The PIP in both countries was compared, and the risk factors for PIP were determined using a multivariate regression model.

Results: A total of 352 and 328 older persons, mean age $(69 \pm 7.35$ years vs $68.21 \pm 7.42$ years, $\mathrm{CI}-0.29$ to $1.93, \mathrm{p}=0.15)$ were evaluated in Nigeria and South Africa respectively. There was no significant difference in PIP among the study participants in Nigeria and South Africa [124/352 (35.2\%) vs 97/328, (29.6\%), 95\% $\mathrm{CI}-0.14$ to $0.13, \mathrm{p}=0.12]$ respectively. Female gender was significantly associated with PIP in South Africa [OR 1.69, CI 1.03-2.8, $\mathrm{p}=0.04]$. 
Conclusions: The PIP among Nigerian and South African older persons was comparable. Female gender was a risk factor for PIP among South African older persons. Keywords: Beers, prescribing, Nigeria, older persons, hospital.

\section{P-5}

Polypharmacy and its influence on 5-years mortality in older adults: results of the Moscow 85+ observational study

\author{
Yuliya Kotovskaya ${ }^{1}$, Olga Tkacheva ${ }^{1}$ \\ ${ }^{1}$ Pirogov Russian National Research Medical University, Russian \\ Clinical and Research Center of Gerontology, Moscow, Russia
}

Background: The prevalence of chronic diseases and polypharmacy increases with aging. However, influence of polypharmacy on mortality risk in older adults is still controversial.

Purpose: To evaluate influence of polypharmacy on 5-years mortality in very old patients in Moscow population.

Methods: Two hundred and thirty-seven patients (56 men) aged $85-98$ (mean $88.2 \pm 2.8$ ) years were included in the observational prospective study during their planned admission to the hospital from 2011 to 2013. All patients had stable condition without evidence of acute illness or decompensation of chronic diseases. We estimated the number of any medications during hospitalization. The follow-up period was 5 years. Endpoint was all-cause mortality.

Results: Median of follow-up was 3.67 years (min 2 weeks, max 6.03 years, IQR $2.72-4.54$ years). Ninety-two patients (40.2\%) died. During hospitalization, all patients received 4-21 (mean $10.8 \pm 3.2$ ) of any medications, including 1-11 (mean $5.1 \pm 2.1$ ) of injectable medications and $0-12$ (mean $5.6 \pm 2.2$ ) of oral medications. Kaplan-Meier analysis showed that use of $\geq 5$ injectable medications was associated with increase of 5-years mortality (Chi square $5.8 ; \mathrm{p}=0.016$ ). 5 -years mortality in patients who received $1-4$ injectable medications was $28.7 \%$, and in patients who received $\geq 5$ injectable medications was $46.3 \%(\mathrm{p}=0.011)$. Cox regression with age and sex adjustment showed that use of $\geq 5$ injectable medications during hospitalization is associated with a 1.8 fold increase of 5-years mortality risk (HR 1.78; 95\% CI 1.11-2.88; $\mathrm{p}=0.018$ ).

Conclusions: Thus, use of $\geq 5$ injectable medications during hospitalization may increase 5-years mortality risk in very old patients.

\section{P-6}

A case report of Memantine-induced bradycardia in an 83 year old male

\author{
Matthew Formosa ${ }^{1}$, Antoine Vella ${ }^{2}$ \\ ${ }^{1}$ Mater Dei Hospital (MDH), Msida, Malta, ${ }^{2}$ Rehabilitation Hospital \\ Karen Grech (RHKG), Pieta, Malta
}

Introduction: To highlight a rare case of bradycardia as an adverse effect of Memantine therapy.

Methods: Mr. JV, a 91 year old gentleman was seen by the Geriatrics Outreach team in a community residential home because of deterioration in his cognitive state as well as a worsening, recent onset hoarding syndrome. He had a MMSE score of 16/30 and met the criteria for the commencement of Memantine at $5 \mathrm{mg}$ per day with a plan to escalate the dose to $10 \mathrm{mg}$ if no untoward side effects were noted. It was felt prudent to check a resting ECG prior to commencing Memantine. The patient was seen 6 weeks later at a routine follow-up appointment where his cognitive state was noted to have improved slightly. An ECG was taken at this time and it was compared with his previous ECG, where an elongation of the patients PR interval as well as his QTc was noted.

Results: Memantine was stopped and behavioural interventions were tried instead of medication. The ECG was repeated and the PR and QTc intervals returned to normal once Memantine was stopped.

Conclusions: The authors recommend that the patients pulse rate be documented together with an ECG prior to starting Memantine. A repeat ECG and the patient's pulse rate should be checked 1 month after the commencement of Memantine or before if patient is noted to develop bradycardia.

\section{P-7}

The linked anticholinergic risk scale: building a clinically relevant comprehensive listing of drugs with anticholinergic properties

Lars Druiven ${ }^{1}$, Derek Dyks ${ }^{2}$, Allen Huang ${ }^{3}$

${ }^{1}$ LUMC, Leiden, The Netherlands, ${ }^{2}$ The Ottawa Hospital, Ottawa, Canada, ${ }^{3}$ The Ottawa Hospital, University of Ottawa, Ottawa, Canada

Introduction: The Anticholinergic Risk Scale [1], Beers [2] and START/STOPP criteria [3] and a systematic review [4] all highlighted potentially inappropriate drugs with anticholinergic properties. This study was done to link and validate the data and develop a working list of these drugs that can potentially be used for both clinical decision-making and research purposes.

Methods: A master list of drugs with anticholinergic properties was compiled including data from previous studies [1-4]. We used a modified Delphi technique and a web questionnaire to collect expert opinions from hospital pharmacists and geriatricians. Round one asked participants to rank 42 drugs on a categorical scale (high $=3$, moderate $=2$, low $=1$, don't know) that was based on their clinical experience. Round 2 focused on drugs with blank or "don't know" responses and a basic drug monograph was attached as reference. Rating categories were compiled and means and standard deviations (SD) calculated.

Results: The master list contains 92 drugs. 11/109 pharmacists and $5 / 12$ geriatricians responded. The mean number of responses for each drug was 8.0 in round 1 and 5.4 in round 2. There was strong agreement for some drugs such as protryptyline (all raters scored 3) and less for others such as tropicamide (mean 2.28, SD 0.89).

Key conclusions: We compiled a Linked Anticholinergic Risk Scale (LARS) of drugs with anticholinergic properties and a category of the potential severity of their clinical effects. The next step is a study using the LARS tool to explore the association of exposure to these drugs with patient outcomes.

\section{References:}

1. Rudolph JL (2008) The anticholinergic risk scale and anticholinergic adverse effects in older persons. Arch Intern Med 168(5):508.

2. By the American Geriatrics Society 2015 Beers Criteria Update Expert Panel (2015) American Geriatrics Society 2015 updated beers criteria for potentially inappropriate medication use in older adults. J Am Geriatr Soc 63(11):2227-46.

3. O'Mahony D, O'Sullivan D, Byrne S, O'Connor MN, Ryan C, Gallagher P (2015) STOPP/START criteria for potentially inappropriate prescribing in older people: version 2. Age Ageing 44(2):213-8.

4. Duran CE, Azermai M, Vander Stichele RH (2013) Systematic review of anticholinergic risk scales in older adults. Eur J Clin Pharmacol 69:1485-1496. 
P-8

Prevalence and incident prescribing of drugs with anticholinergic properties in older adults admitted to an academic hospital

Lars Druiven ${ }^{1}$, Derek Dyks ${ }^{2}$, Allen Huang ${ }^{3}$

${ }^{1}$ LUMC, Leiden, The Netherlands, ${ }^{2}$ The Ottawa Hospital, Ottawa, Canada, ${ }^{3}$ The Ottawa Hospital, University of Ottawa, Ottawa, Canada

Introduction: Medications with anticholinergic properties belong to potentially inappropriate medications (PIMs) as described in the Beers [1] and STOPP/START [2] criteria. The prevalent use and incident prescribing of these drugs was studied in older adults admitted to hospital.

Methods: A random sampling of 14,880 admissions of patients 65-years and older admitted during 2015 to a 1000-bed academic hospital in Canada was done. The cohort consisted of 100 patients admitted to the General Surgery (GS) and Internal Medicine (IM). The electronic records of their community meds and in-hospital prescribing were analysed. Index drugs were listed in the anticholinergic risk scale [3].

Results: Compared to patients admitted to IM, GS patients were younger (mean, 76 vs. 80 -years), had shorter lengths of stay ( 8 vs. 13-days), were less often admitted from the Emergency Department (71 vs. $98 \%$ ) and had lower documented dementia (5 vs $25 \%$ ). GS patients used less anticholinergic drugs in the community (mean number 0.56 vs. 0.87 ), were prescribed more in hospital (mean 2.14 vs. 1.87 ) and were administered similar amounts (mean 1.10 vs 1.45 ). Hospitalization resulted in an increase in the use of these drugs: average ratio of 1.60 for GS and 1.01 for IM.

Key conclusion: The use of drugs (prevalent and incident) with anticholinergic properties in an academic hospital is high. This situation potentially places older patients at higher risk for adverse events. Factors leading to these findings need to be studied and interventions designed to improve the safety and quality of care of hospitalized older patients.

References:

1. American Geriatrics Society 2015 Beers Criteria Update Expert Panel (2015) American Geriatrics Society 2015 updated beers criteria for potentially inappropriate medication use in older adults. J Am Geriatr Soc 63(11):2227-46.

2. O'Mahony D, O'Sullivan D, Byrne S, O'Connor MN, Ryan C, Gallagher P (2015) STOPP/START criteria for potentially inappropriate prescribing in older people: version 2. Age Ageing 44(2):213-8.

3. Rudolph JL (2008) The anticholinergic risk scale and anticholinergic adverse effects in older persons. Arch Intern Med 168(5):508.

\section{P.9}

Diazepam as an oral hypnotic increases nocturnal blood pressure in healthy elderly

R. Fogari ${ }^{1}$, A. Costa $^{1}$, A. D'Angelo ${ }^{2}$, M. CottaRamusino ${ }^{1}$, D. Bosone ${ }^{3}$

${ }^{1}$ Center of Neurological Medicine, IRCCS Mondino, Pavia, Italy, ${ }^{2}$ Department of Internal Medicine, University of Pavia, Pavia, Italy, ${ }^{3}$ Center of Neurological Medicine, Pavia, Italy

Introduction: No study has evaluated the cardiovascular effects of diazepam in elderly subjects. Aim of this study was to evaluate the effects of chronic oral administration of diazepam as hypnotic drug on blood pressure (BP) and heart rate (HR) in healthy elderly subjects. Methods: Twenty-five healthy elderly subjects, aged 65-74 years, were treated with diazepam $5 \mathrm{mg}$ or placebo both taken in the evening for 4 weeks according to a randomized, double-blind, cross-over design. At the end of each period 24-h ambulatory BP and HR were evaluated.

Results: At the end of 4-week diazepam treatment 24-h BP monitoring showed that during night-time systolic BP values under diazepam were $7.6 \%$ higher than under placebo, with a mean difference of $7.9 \mathrm{mmHg}(\mathrm{p}<0.01)$, diastolic $\mathrm{BP}$ values were $5.8 \%$ higher, with a mean difference of $3.7 \mathrm{mmHg}$ ( $\mathrm{p}<0.05$ vs placebo) and HR values were $6.6 \%$ higher with a mean difference of $4.2 \mathrm{~b} / \mathrm{min}$ $(\mathrm{p}<0.05)$. The HR increase observed with diazepam persisted also during the morning hours.

Key conclusions: In elderly subjects chronic assumption of diazepam as hypnotic agent produced an increase of BP during night-time and of HR during night-time and morning hours. These effects, which probably depend by a diazepam mediated increase in noradrenaline positive inotropic effect and decrease in vagal tone, might be of clinical relevance due to the role of increased BP and HR as independent cardiovascular risk factors.

\section{P-10}

FORTAMET study: the impact of FORTA on disease-related over- and under-treatment in older hospitalized patients

Martin Wehling ${ }^{1}$, Heinrich Burkhardt ${ }^{2}$, Helmut Frohnhofen ${ }^{3}$, Christel Weiss ${ }^{4}$, Christina Throm ${ }^{5}$, Alexandra Kuhn-Thiel ${ }^{6}$, Farhad Pazan ${ }^{1}$

${ }^{1}$ Institute for Clinical Pharmacology, Medical Faculty Mannheim, Heidelberg University, Heidelberg, Germany, ${ }^{2}$ IV. Medical Department, Geriatrics, University Hospital Mannheim, Heidelberg University, Heidelberg, Germany, ${ }^{3}$ Faculty of Health, Department of Medicine, University Witten-Herdecke, Witten, Germany

Clinical studies on the efficacy and safety of drugs often do not include older people. Hence, there is a lack of evidence as to the appropriateness of numerous drug treatments in the elderly. Undoubtedly, these circumstances often turn the drug therapy of older people into a risky experiment. To address this issue, the FORTA classification (A: Absolutely; B: Beneficial; C: Careful; D: Don't) was proposed as a clinical aid for physicians. The FORTA List was validated and expanded in a Delphi consensus procedure. To evaluate the effectiveness of FORTA we conducted a prospective randomized controlled trial in hospitalized elderly patients. Nearly half of our patients received standard care and the other half received standard care plus the FORTA method. In this study, we separately tested changes of drug prescriptions at the anatomical-therapeutic-chemical system (ATC) level for important diagnoses and compared over- and undertreatment rates between the groups. At the individual drug/drug group level, two items (e.g. loop diuretics) were significantly altered by FORTA when overall use changes between admission and discharge were compared between groups. In addition, FORTA also significantly improved undertreatment (e.g. beta receptor blocking agents or phenprocoumon to treat atrial fibrillation) or overtreatment (e.g. loop diuretics to treat arterial hypertension) for nineteen drugs/drug groups. Based on this study, FORTA is the first combined positive/negative labelling approach at the individual drug level which is effective in eliminating inappropriate prescribing and certainly ameliorates the drug treatment of older people.

\section{P-11}

Medication management of osteoporosis prevention in nursing home residents in Europa: the shelter study

Hein van Hout ${ }^{1}$, Graziano Onder ${ }^{2}$, Rob van Marum ${ }^{3}$ 
${ }^{1} \mathrm{PhD}$, Department of General Practice and Elderly Care Medicine and Amsterdam Public Health research institute, VU University Medical Center, Amsterdam, The Netherlands, ${ }^{2} \mathrm{MD}, \mathrm{PhD}$, Universita

Cattolica del Sacro Cuoro, Rome, Italy, ${ }^{3}$ Prof, MD, PhD, Department of General Practice and Elderly Care Medicine and Amsterdam Public Health research institute, VU University Medical Center, Amsterdam, The Netherlands; and Department of Geriatric Medicine, Jeroen Bosch Hospital, 's-Hertogenbosch, The Netherlands

Introduction: Nursing home residents are at increased risk of fractures due to high fall risk, multimorbidity and high prevalence of osteoporosis but often also have a limited life expectancy limiting appropriateness for preventive osteoporosis medication (OM).

Objective: To assess the prevalence of drug treatment for osteoporosis in European nursing homes.

Methods: We used data from the SHELTER study, conducted Czech Republic, England, Finland, France, Germany, Italy, The Netherlands and Israel. We assessed the overall prevalence of OM use (vitamin D, calcium and bisphosphonates) in residents with stratification for recent falls, recent fractures, health instability, cognitive impairment. Results: Of 3832 residents, vitamin D, calcium and bisphosphonates were used by $16.2,10.4$, and $4.5 \%$ respectively. All 3 classes of OM together were used by $1.5 \%$ of all residents. Of residents with a fracture $9.5 \%$ used a bisphosphonate (and $2.7 \%$ all 3 OMs). In patients with recent falls, $20.8 \%$ used vitamin D and $15.3 \% \mathrm{Ca}$. In residents with severe cognitive impairment, $15.5 \%$ used vitamin $\mathrm{D}$ and $9.3 \%$ used $\mathrm{Ca}$. Of bisphosphonate users $33.7 \%$ also used both vitamin $\mathrm{D}$ and calcium, $25.8 \%$ only used $\mathrm{Ca}$ and $17.4 \%$ only vitamin D. Any OM use varied largely across countries from 42.7 in Finland to $2.1 \%$ in Israel.

Conclusion: The prescription of OM varies strongly between countries. There seems to be underprescribing in high risk groups for fractures (recent fracture, recent falls), but also overprescribing in patients with severe cognitive impairment.

Keywords: Osteoporosis, medication, prevention, Nursing home resident, vulnerable population, Shelter study.

\section{P-12}

Multidisciplinary approach for medication review to older inpatients with polypharmacy

Fumihiro Mizokami ${ }^{1}$, Masato Kato ${ }^{1}$, Hidetoshi Endo ${ }^{2}$, Shosuke Satake $^{2}$, Atsuya Shimizu ${ }^{3}$, Akiko Yamamoto ${ }^{4}$, Yumiko Izuka ${ }^{5}$, Keiji Nomoto ${ }^{6}$, Tomoharu Kobayashi ${ }^{1}$, Hidenori Arai $^{2}$

${ }^{1}$ Department of Pharmacy, National Center for Geriatrics and Gerontology, Obu, Japan, ${ }^{2}$ Department of Comprehensive Geriatric Medicine, National Center for Geriatrics and Gerontology, Obu, Japan, ${ }^{3}$ Department of Cardiology, National Center for Geriatrics and Gerontology, Obu, Japan

Introduction: Polypharmacy is one of the most important issues in geriatric pharmacotherapy. To tackle with polypharmacy in an acute care hospital, we started a clinical medication review team to optimize the medications and investigated the effect of the intervention on older patients with polypharmacy.

Methods: We organized "Geriatric Pharmacotherapy Optimization Team" consisting of doctors, pharmacists, nurses, dieticians and rehabilitation therapists. Our team routinely reviewed the medication regimen and adherence based on comprehensive geriatric assessment (CGA) and medication review followed by a multidisciplinary team conference. From September 2016 to December 2017, we reviewed 58 cases, which were divided into two groups; three or more medication reduction group $(\geq 3 \mathrm{R})$ and less than three reduction group
$(<3 R)$. The incidence of adverse drug reactions (ADRs) within 60 days was analyzed using the Chi square test.

Results: In the $\geq 3 R$ group $(n=32)$, the average age was 79.6 (7.2(SD)) years old, and the average number of medications was 11.6 (3.4) at admission and 6.7 (3.5) at discharge. In the $<3 \mathrm{R}$ group $(n=26)$, the average age was 78.9 (6.4) years old, the average number of medications was 9.9 (1.9) at hospitalization and 9.5 (3.0) at discharge. The incident of ADRs was significantly different between $\geq 3 \mathrm{R}$ and $<3 \mathrm{R}$ groups; $53.1 \%$ vs. $34.6 \%$ at admission, and $6.3 \%$ vs. $26.9 \%(\mathrm{p}=0.031)$ after 60 days.

Conclusions: Multidisciplinary medication review and a team approach for in-patients with polypharmacy can reduce the number of medications and ADRs. Therefore, this approach could be recommended for older in-patients with polypharmacy.

\section{P-13}

Analysis of evolution of appropriate prescribing antidepressants and cholinesterase inhibitors in a psychogeriatric ward in a Belgian hospital in order to decrease the use of antipsychotics

L. Maufort ${ }^{1}$, J. Peeterbroeck ${ }^{1}$, C. Lelubre ${ }^{1}$, S. Higuet ${ }^{2}$

${ }^{1} \mathrm{CHU}$ Charleroi, Charleroi, Belgium, ${ }^{2} \mathrm{CHU}$ Charleroi, Charleroi, Belgium and GBHI (Ireland)

Introduction: The treatment of psycho-behavioral symptoms associated with dementia (BPSD) presents a major challenge in the management of patients with cognitive disorders. Neuroleptics are usually overused and are the cause of many side effects. Some molecules: acetylcholinesterase inhibitors (Cohen-Mansfield 2013), memantine, or some antidepressants (Gauthier 2008) have been shown to be effective for certain symptoms of BPSD. (Seitz 2011; Pollock 2007).

Methods: This is a monocentric retrospective observational study based on the systematic review of computerized hospitalization records of patients who stayed in the Psychogeriatric ward at André Vésale University Hospital (Belgium) during two periods. First from January 1st 2013 to January 1st 2014 and, second, from January 1st 2016 to January 1st 2017. We studied the prescription of antidepressants, memantine and acetylcholinesterase inhibitors, the type of molecule used, the daily dose prescribed and the daily dose defined "DDD".

Results: There is no statistically significant difference in the groups for reasons of admission or for the type of dementia. In 2013, at admission, $38 \%$ of patients were treated with antidepressants, this proportion increasing to $44.2 \%$ at discharge. There was also an increase in antidepressant coverage in 2016 from $37.2 \%$ to $57 \%$. Prescription of Cholinesterase Inhibitors or memantine decreased between admission and discharge (26.4\% vs. $17.1 \%)$ in 2013 . On the other hand, the opposite trend is observed in 2016 (4.7\% vs. $15.5 \%$ ). Conclusions: In this study, we observed an increase of the coverage and consumption of antidepressants. Additional analyses should investigate the possible link with the decrease in neuroleptic consumption. The prescribing of Cholinesterase Inhibitors to treat BPSD increased between admission and discharge in 2016. It should be noted that in general, patients are subsequently examined again at the memory clinic and that cholinesterase inhibitor treatment is rarely prescribed during acute hospitalization. 


\section{P-14}

Comparative safety of individual antidepressants regarding traumatic brain injury in older adults

Federica Edith Pisa ${ }^{1}$, Jonas Reinold ${ }^{1}$, Bianca Kollhorst ${ }^{2}$, Ulrike Haug $^{3}$, Tania Schink ${ }^{2}$

${ }^{1}$ Leibniz Institute for Prevention Research and Epidemiology-BIPS, Bremen, Germany, ${ }^{2}$ Leibniz Institute for Prevention Research and Epidemiology-BIPS, Bremen, Germany, ${ }^{3} 1$ Leibniz Institute for Prevention Research and Epidemiology-BIPS, Breme, Germany

Background: Antidepressants (ADs), a useful therapeutic tool in depression and anxiety disorders, can increase the risk of Traumatic Brain Injury (TBI) because of adverse effects such as sedation or dizziness. Evidence on the comparative safety of individual AD in this regard is scarce. We assessed the risk of TBI associated with ADs in older adults.

Methods: We conducted a case-control study nested in a cohort of new users of ADs aged $\geq 65$ years, based on the German Pharmacoepidemiological Research Database (GePaRD) during 2005-2014. Incident cases of hospitalization for TBI were identified and matched to up to 100 controls per case using incidence density sampling. Use of $\mathrm{AD}$ was ascertained at index date (ID; cases: date of TBI; controls: matched date) based on the duration of the last dispensation. Current use was defined as duration overlapping ID. We estimated adjusted odds ratios (aORs), with 95\% confidence interval (CI), using conditional logistic regression with current users of mirtazapine as reference category, adjusting for co-morbidities and co-medications. Results: Among 701,309 cohort members, 18,224 cases were identified and matched to $1,820,362$ controls (in both groups: $70.4 \%$ women; median age 80 years). Current users had aOR $(95 \% \mathrm{CI})$ of $1.64(1.54-1.75)$ for citalopram, $1.60(1.44-1.77)$ for sertraline, 1.60 (1.41-1.82) for duloxetine, 1.58 (1.37-1.81) for escitalopram, 1.45 (1.19-1.76) for fluoxetine, $1.41(1.18-1.69)$ for paroxetine, 1.19 $(1.09-1.30)$ for amitriptyline, $0.90(0.81-1.01)$ for opipramol, 0.73 (0.60-0.88) for doxepin.

Conclusions: In this large cohort, opipramol and doxepin had a lower risk than most examined individual SSRI, SSNRI and amitriptyline.

\section{P-15}

Sensitivity and specificity of STOPP/START criteria to detect drug-related problems in older adults

Marta Gutiérrez-Valencia ${ }^{1}$, María Teresa Acín-Gericó ${ }^{2}$, Beatriz Contreras-Escámez ${ }^{3}$, María Fernanda Ramón-Espinoza ${ }^{3}$, Itxaso Marín-Epelde $^{3}$, Álvaro Casas-Herrero ${ }^{3}$, Nicolás Martínez-Velilla ${ }^{3}$

${ }^{1}$ Department of Health Sciences, Public University of Navarra, Pamplona, Spain, ${ }^{2}$ Primary Care Pharmacy Unit, Navarra Health Service, Pamplona, Spain, ${ }^{3}$ Complejo Hospitalario de Navarra, Pamplona, Spain

Introduction: The STOPP/START criteria are one of the most used methods to detect potentially inappropriate medications (PIM) in older people. However, it has certain disadvantages, such as not taking into account the individual characteristics, and questionable validity. The aim was to asses the sensitivity and specificity of STOPP/START criteria to detect drug-related problems (DRPs) in older adults admitted to an acute geriatric unit (AGU).

Methods: A pharmacist-led medication review was performed to 250 patients admitted to an AGU to detect DRP in their chronic medication. A clinical interview by the pharmacist and a Comprehensive Geriatric Assessment by the geriatric team were made before the medication review. All chronic medications and the presence of
STOPP/START criteria (2nd version) were registered from clinical records. Patients' DRPs were registered as the reference method, considering their individual characteristics, as well as whether DRPs were associated with STOPP/START criteria. True positives (TP) were DRPs associated with STOPP/START and false positives (FP) were STOPP/START criteria that were not considered a DRP. False negatives (FN) were DRPs not associated with STOPP/START and true negatives (TN) were those drugs not associated with DRPs or STOPP/START criteria.

Results: The mean age was 87.6 years (SD 4.6) and $60.0 \%$ were female. The mean number of medications was 10.5 (SD 4.1). Sensitivity $=\mathrm{TP} /(\mathrm{TP}+\mathrm{FN})=259 /(259+760)=25.4 \% . \quad$ Specificity $=$ $\mathrm{TN} /(\mathrm{TN}+\mathrm{FP})=1114 /(1114+485)=69.7 \%$.

Key conclusions: STOPP/START criteria have low specificity and sensitivity. These findings suggest that application of STOPP/START criteria for medication review in older adults should preferably be combined with more comprehensive and individualized methods.

\section{P-16}

Polypharmacy among the elderly patients in nursing and supportive treatment hospital

Jurate Macijauskiene ${ }^{1}$, Agne Jakavonytè-Akstiniené ${ }^{2}$

${ }^{1}$ Medical Academy, Faculty of Nursing, Department of Geriatrics, Lithuanian University of Health Sciences, Kaunas, Lithuania, ${ }^{2}$ Faculty of Health Care, Department of Nursing, Vilniaus Kolegija/ University of Applied Sciences (VIKO), Kaunas, Lithuania

Introduction: With the aging of society, polypharmacy becomes extremely important in health care.

Methods: The study was conducted in 2015 from January to December in one of nursing and supportive treatment hospitals in Vilnius with the aim to analyze the relationship of cognitive and physical functions of elderly patients with their nursing needs. The data on prescribed drugs (prescription date and duration, name of medication, dosage, method of administration, frequency of use and side effects) were obtained from medical records. A permission from Vilnius Regional Biomedical Research Ethics Committee to conduct study was obtained.

Results: There were 177 research participants, out of which $40.1 \%$ $(\mathrm{N}=71)$ were males and $59.9 \%(\mathrm{~N}=106)$ females. The average of participants' age was $78.93 \pm 8.95$ years. During the entire treatment period each patient used for an average of $7.76 \pm 0.9$ medications. On average, the lowest number $(6.91 \pm 3.34)$ of medications were administered to the patients who were treated in the hospital less than 120 days and highest number of medications $(8.64 \pm 4.16)$-in patients who were treated at nursing and supportive treatment hospital for 120 calendar days $(\mathrm{F}=3.41, \mathrm{df}=2, \mathrm{p}=0.036)$.

Conclusions: Polypharmacy was common in nursing and supportive treatment hospital. The majority patients were discharged from the hospital with severe polypharmacy.

\section{P-17}

Possible benefit of contact between the discharging doctor and the patient's GP - a feasibility study

Jens-Ulrik Rosholm $^{1}$, A. Pottegård ${ }^{1}$, P. M. Christensen ${ }^{2}$, T. Graabæk ${ }^{3}$

${ }^{1}$ Odense University Hospital, Odense, Denmark, ${ }^{2}$ General Practice, Otterup, Denmark, ${ }^{3}$ Hospital Pharmacy of Funen, Denmark 
Background: Previous register studies have shown poor compliance after discharge with changes made to the patients' medicine during admission. Follow up interview with the GPs found a wish for contact between the hospital doctor and the GP for discussion of eventual changes. The aim of the study was to enlighten the feasibility of a study of discussion of the patient's medicine between the hospital doctor and the GP on discharge proceeded by a comprehensive clinical pharmacist-led medication review.

Method: Patients admitted to the Department of Geriatric Medicine, Odense University Hospital, Denmark during spring 2018 were screened by a clinical pharmacist. Main exclusion criteria were cognitive impairment or patients isolated due to infection or less than 5 drugs (inclusion criteria). Following the medication review suggestions for drug changes were given to the doctor. Within the first days after discharge the pharmacist contacted the GP for setting up a telephone-contact for discussion of the drug treatment.

Results: Of the invited 62 patients, 51 accepted to participate. The clinical pharmacist suggested medication changes for 16 of the 25 intervention patients. For the 11 patients eligible for telephone-contact with GP, only 9 contacts were offered mostly due to practical problems. For 7 of these patients the GP wanted a discussion with the hospital doctor, and for 6 patients a contact between the hospital doctor and the GP was established (Data collection still going on May 2018).

Discussion: Even though the GPs previously have expressed a wish for discussion of discharged patients' medicine with the hospital doctor it was very difficult to establish this contact due to practical problems. A large scale RCT seems hardly feasible.

\section{P-18}

Methods to assess patient preferences in geriatric pharmacotherapy-a systematic review

Annette Eidam ${ }^{1}$, Anja Roth ${ }^{1}$, André Lacroix ${ }^{1}$, Sabine Goisser ${ }^{2}$, Jürgen M. Bauer ${ }^{3}$

${ }^{1}$ AGAPLESION Bethanien Hospital Heidelberg, Heidelberg, Germany, ${ }^{2}$ Network of Aging Research (NAR), University of Heidelberg, Heidelberg, Germany, ${ }^{3}$ AGAPLESION Bethanien Hospital Heidelberg/Network of Aging Research (NAR), University of Heidelberg, Heidelberg, Germany/Center of Geriatric Medicine

Introduction: When trying to individualize geriatric pharmacotherapy the elucidation of the older patient's treatment and outcome preferences should be considered during the prescription process. A systematic review was conducted to identify methods used to explore patient preferences in old age pharmacotherapy and to evaluate their appropriateness in the context of multi-morbidity.

Methods: We searched three electronic databases (PubMed, Web of Science, PsycINFO) for studies reported in English or German. Studies were eligible for inclusion if they assessed individual treatment and outcome preferences in a population aged $\geq 65$ years or with a mean/median age of $\geq 75$ years. The current review focused on long-term pharmacological treatment options. We excluded qualitative studies as well as studies targeting preferences for lifesustaining treatments.

Results: A total of 60 articles met our eligibility criteria, reporting 55 different instruments to assess patient preferences. Discrete choice experiments, medication willingness, health outcome prioritization tools, ranking exercises and Likert scale based questionnaires were the most commonly used preference elicitation techniques. Single studies investigated the feasibility of the analytical hierarchy process, adaptive conjoint analysis and maximum difference scaling. The majority of the instruments were designed for disease-specific or context-specific settings, only three instruments (Health outcome prioritization tool, STEP assessment, MediMol questionnaire) targeted the issue of geriatric multi-morbidity.

Key conclusions: Up to now a broad range of elicitation methods have been applied to assess medication preferences in older patients. However, few instruments exist that aim at clarifying patient preferences in multi-morbidity-related polypharmacy, demonstrating a need for future tools tailored to this context.

\section{P-19}

Introduction of non-vitamin $K$ antagonist anticoagulants strongly increased the rate of anticoagulation in geriatric patients

Marija Djukic ${ }^{1}$, Larissa Maria Braun ${ }^{1}$, Claudius Jacobshagen ${ }^{2}$

${ }^{1}$ Geriatric Center, Protestant Hospital Göttingen-Weende, Göttingen, Germany, ${ }^{2}$ Department of Cardiology and Pneumology, University Medicine Göttingen, Göttingen, Germany

Introduction: The benefit of anticoagulative treatment to prevent thromboembolism has been established in patients with atrial fibrillation and flutter (AF) of all age groups. Traditionally, anticoagulation was underused in geriatric patients with AF.

Methods: Hospitalized geriatric patients treated in 2015 were retrospectively studied for the presence of $\mathrm{AF}$ and the use or non-use of anticoagulation. The risk of stroke and the indication for permanent anticoagulation were assessed using the CHA2DS2-VASc score.

Results: 512 of 1320 patients showed a clear indication for therapeutic anticoagulation $(38.8 \%)$. Of these, 431 patients $(84.2 \%)$ had long-standing persistent ( $>1$ year)/permanent $\mathrm{AF}$ or paroxysmal/ persistent ( $>7$ days) AF as well as a CHA2DS2-VASc score of $\geq 2$ in men and $\geq 3$ in women. In this group, 378 patients $(87.7 \%)$ received anticoagulation. Of all patients anticoagulated for AF, 221 received NOACs (58.5\%), 176 received apixaban (46.6\%), 32 rivaroxaban $(8.5 \%)$, and 13 dabigatran $(3.4 \%) .107$ patients received the vitamin $\mathrm{K}$ antagonist phenprocoumon $(28.3 \%)$ and 50 patients high-dose low-molecular weight heparins $(13.2 \%)$. In 26 patients $(5.7 \%$ of all anticoagulated patients, $\mathrm{n}=459)$ complications were documented. 13 complications $(50.0 \%$ of those treated) occurred during treatment with NOACs, $5(19.2 \%)$ during anticoagulation with phenprocoumon and $8(30.8 \%)$ during treatment with low molecular weight heparins. No intracranial hemorrhages occurred, and 9 patients required transfusion or surgery to stop bleeding.

Conclusion: The introduction of NOACs and an increased awareness of their benefits led to an increased use of anticoagulation from $52.8 \%$ (2011) to $87.7 \%$ (2015) in geriatric patients with AF at our institution.

\section{P-20}

Current status of potentially inappropriate medication and polypharmacy researches in Turkey

Fatma Özge Kayhan Koçak ${ }^{1}$, Seval Ay ${ }^{1}$, Yasemin Kılıç Öztürk ${ }^{2}$, Sevnaz Şahin ${ }^{1}$

${ }^{1}$ Department of Internal Medicine, Division of Geriatrics, Ege University Hospital, İzmir, Turkey, ${ }^{2}$ Medical Sciences University Tepecik Training and Research Hospital, Family Medicine Department, Izmir, Turkey

Introduction: Increasing medical treatment options leads to widespread drug use and potentially inappropriate medication (PIM) prescription. Due to the change in prescription trends; necessity of rational drug use 
researches, where PIM and polypharmacy assessed, increased. The aim of the study is to evaluate the number of PIM publications in Turkey. Methods: The number of published articles on Electronic PubMed records and Google Scholar engine about polypharmacy and PIM use from 2005 to 2018 in Turkey is evaluated.

Results: There were 3 in 306 publications found from Turkey by the search result for 'potentially inappropriate medication' ( 2 observational, 1 retrospective). The 5 in 5307 articles were from Turkey according to the search of term 'polypharmacy', and all were observational. Search result for 'potentially inappropriate medication' words at Google Scholar, showed 15900 articles, where 36 were from Turkey (20 observational, 5 retrospective, 11 review). The term polypharmacy was included in all inappropriate drug use publications so that not needed to be re-scanned. Only one-third of the studies were about PIM.

Key conclusions: Although observational studies are often easier and cheaper to conduct, polypharmacy and PIM publications are lacking. There are several reasons why not doing study on polypharmacy and use of inappropriate medications. Guideline for observational studies conducted on drugs in Turkey may be one of the most important reasons. The statutory and regulatory requirements are needed to be assessed in order to increase the number of observational drug studies.

\section{P-21}

\section{Optimizing pharmacotherapy on geriatric wards in Belgium-} stronger together?

Julie Hias ${ }^{1}$, Lorenz Van der Linden ${ }^{2}$, Karolien Walgraeve ${ }^{1}$, JeanClaude Lemper ${ }^{3}$, Isabel Spriet ${ }^{2}$, Jos Tournoy ${ }^{4}$

${ }^{1}$ Pharmacy Department, University Hospitals Leuven, Leuven, Belgium, ${ }^{2}$ Pharmacy Department, University Hospitals Leuven, Leuven, Belgium and Department of Pharmaceutical and Pharmacological Sciences, KU Leuven, Leuven, Belgium, ${ }^{3}$ President of the Belgian College for Geriatrics, Federal Public Service, Belgium, ${ }^{4}$ Department of Geriatric Medicine, University Hospitals Leuven, Leuven, Belgium and Department of Chronic Diseases, Metabolism and Ageing, KU Leuven, Leuven, Belgium

Introduction: Suboptimal prescribing remains highly prevalent in adults admitted to geriatric care wards. Several approaches have been developed that could be implemented to manage suboptimal or even inappropriate drug use in older adults. The aim of this investigation, initiated by the Belgian College for Geriatrics, was to assess the implementation of strategies to optimize pharmacotherapy on geriatric wards in Belgium.

Methods: A systematic literature search was performed to identify determinants for appropriate drug use in very old inpatients. These determinants were subsequently reviewed by an expert panel, using a Delphi consensus technique, in order to develop a national survey. The survey, counting 61 questions, was then distributed to the heads of the geriatric departments of all Belgian hospitals.

Results: The survey was sent to 100 hospitals. Within 3 months a response rate of $57 \%$ was achieved. On average, the number of admissions for the reference year (2016) was 1427 (IQR 944-2080). In the minority $(26 \%)$ of the hospitals, a clinical pharmacist was directly involved in the multidisciplinary geriatric team. Ward-based activities for clinical pharmacists were reported as participating in medication reconciliation on admission $(73 \%)$ and discharge $(67 \%)$, medication review (87\%) and patient education (80\%). The majority of the hospitals (86\%) used electronic prescriptions; $33 \%$ also implemented clinical decision support systems (CDSS).

Key conclusion: Uptake of ward-based clinical pharmacy services for older inpatients is still limited in Belgium. Given the current body of evidence, implementation of strategies such as clinical pharmacy services and CDSS should be increased.

\section{P-22}

Type and determinants of clinical pharmacy recommendations prior to discharge: a Leuven experience

Lorenz Van der Linden ${ }^{1}$, Julie Hias ${ }^{2}$, Karolien Walgraeve ${ }^{2}$, Silke Loyens $^{3}$, Johan Flamaing ${ }^{4}$, Isabel Spriet ${ }^{1}$, Jos Tournoy ${ }^{4}$

${ }^{1}$ Pharmacy Department, University Hospitals Leuven, Leuven, Belgium and Department of Pharmaceutical and Pharmacological Sciences, KU Leuven, Leuven, Belgium, ${ }^{2}$ Pharmacy Department, University Hospitals Leuven, Leuven, Belgium, ${ }^{3}$ Faculty Of Biomedical Sciences, KU Leuven, Leuven, Belgium, ${ }^{4}$ Department of Geriatric Medicine, University Hospitals Leuven, Leuven, Belgium and Department of Chronic Diseases, Metabolism and Ageing, KU Leuven, Leuven, Belgium

Introduction: A clinical pharmacy $(\mathrm{CP})$ program was installed at the acute geriatric wards of a teaching hospital. The study aim was to derive a model to identify patients more likely to receive CP recommendations at discharge.

Methods: Inpatients receiving a CP consultation at discharge were selected from April to December 2016. Information about medical conditions, patient demographics, and drug use was collected retrospectively. Number and type of the $\mathrm{CP}$ recommendations were determined. A multivariable Poisson regression analysis was performed to identify determinants for the number of $\mathrm{CP}$ recommendations. The predictive performance of the final model was evaluated, using several thresholds.

Results: 524 patients were included, aged 85 (IQR 82-89) years. On admission, 10.31 (SD 4.49) drugs were taken. During hospital stay, 3 (IQR 1-4) drugs were initiated. At discharge, 3 (IQR 2-4) CP recommendations were provided per patient, of which $70.2 \%$ targeted drug discontinuation. A model was derived, containing the following determinants: number of drugs on admission (IRR 1.063; 95\% CI 1.052-1.074), number of previous contacts with the geriatric department (IRR 0.869; 95\% CI 0.816-0.926), presence of left-ventricular dysfunction (IRR $1.179,95 \%$ CI $1.023-1.360$ ), the number of new drugs (IRR 1.046; 95\% CI 1.021-1.071) and the use of colecalciferol (IRR 1.22 ; 95\% CI 1.088-1.367). Using a threshold of at least five CP recommendations, a positive likelihood ratio of 9.32 was found $(95 \%$ CI 5.05-17.22), signifying good performance.

Key conclusion: A model, consisting of five determinants for the number of $\mathrm{CP}$ recommendations at discharge, was derived. It can readily be used to increase the efficiency of the medication review process at discharge.

Mechanism of subcutaneous insulin resistance by localized insulin amyloidosis

Yohei Misumi $^{1}$, Toshiya Nomura ${ }^{1}$, Yukio Ando ${ }^{1}$

${ }^{1}$ Department of Neurology, Graduate School of Medical Sciences, Kumamoto University, Kumamoto, Japan

Introduction: The world's aging is a significant driver of the number of diabetic patients, and the number of insulin users continues to increase. Localized insulin-derived amyloidosis, also known as insulin balls, appear occasionally at the site of repeated insulin injections in patients with insulin-dependent diabetes, and are 
reported to be associated with insulin resistance. In this study, we aimed to clarify the mechanism of insulin resistance at the site of amyloidoma using a mouse model.

Methods: We generated an insulin amyloidoma mouse model by repeated subcutaneous injection of insulin amyloid fibrils, and performed insulin tolerance test at the site of amyloidoma. The interaction between insulin amyloid and native insulin was analyzed in vitro.

Results: Subcutaneous insulin amyloidoma in mice revealed features similar to those of human insulin amyloidoma, and remained to be similar feature for a long time. Native insulin showed no significant hypoglycemic effect when administered into the site of amyloidoma. In vitro analysis showed that insulin amyloid fibrils strongly adsorbed native insulin.

Conclusions: The effect of preformed insulin amyloidoma on trapping native insulin may be a cause of insulin resistance and poor blood glucose control in clinical cases with insulin amyloidoma.

\section{P-24}

\section{Quality and determinants of anticoagulation therapy by AVK in elderly subjects}

Sanaa Asgassou ${ }^{1}$, Adrien Hardel ${ }^{1}$, Mélanie Dipanda ${ }^{1}$, Julien Gauthier $^{1}$, Sofia Da Silva ${ }^{1}$, Patrick Manckoundia ${ }^{1}$, Pierre Jouanny ${ }^{1}$

${ }^{1}$ University Hospital Geriatric Department Dijon-Champmaillot, Dijon, France

Objective: AVK are prescribed in elderly patients over 75 years for the prevention of thromboembolic complications of atrial fibrillation (AF). The objective of this study was to study the quality of the anticoagulation balance by AVK and its determinants.

Method: Inclusion of all patients $\geq 75$ years of age treated with AVK for an AF hospitalized in an acute geriatric ward. The balance of the AVK treatment was determined by the calculation of the TTR. The last four INRs prior to admission were retrospectively collected for its calculation, as well as the input INR. Each patient had a standardized geriatric evaluation (EGS). The variables collected were lifestyle, MMSE, nutritional status, polypathology, level of dependence and existence of bleeding complication.

Results: 155 patients aged over 75 years $(87 \pm 5.6$ years, 88 women and 67 men) were included The way TTR calculated was $55.4 \% \pm 31.2 \%$. Only $46 \%$ of patients had a satisfactory TTR $(\geq 75 \%)$. The balance was significantly worse in women than in men $(49.3 \pm 29.5$ vs $60.1 \pm 31.8 \%, p=0.0326)$, and in case of haematological pathology $(41.7 \pm 27.1$ vs $57.2 \pm 9.8, \mathrm{p}=0.047)$ but better with high BMI $(\mathrm{r}=0.416, \mathrm{p}=0.001)$. No EGS parameters were associated with the quality of anticoagulation.

Conclusion: The balance of AVK is insufficient in geriatric elderly subjects. No modifiable geriatric factor has been identified. If AVK remains the old therapeutic option, the TTR must be monitored. Direct oral anticoagulants should be considered as the best first choice

\section{P-25}

Genetic polymorphisms affecting diuretics efficacy and diureticrelated fall risk

Lotta Seppälä ${ }^{1}$, Stephan Luiting ${ }^{1}$, Katerina Trajanoska ${ }^{2}$, Sadaf Oliai Araghi $^{3}$, Natasja van Schoor ${ }^{4}$, Lisette de Groot ${ }^{5}$, Andre Uitterlinden ${ }^{6}$, Fernando Rivadeneira ${ }^{2}$, Bruno Stricker ${ }^{2}$, Nathalie van der Velde ${ }^{1}$

${ }^{1}$ Department of Internal Medicine, Section of Geriatric Medicine, Amsterdam UMC, University of Amsterdam, The Netherlands,
${ }^{2}$ Department of Epidemiology, Erasmus MC, University Medical Center, Rotterdam, The Netherlands, ${ }^{3}$ Section of Geriatrics, Department of Internal Medicine, Erasmus MC, University Medical Centre Rotterdam, The Netherlands, ${ }^{4}$ Department of Epidemiology and Biostatistics, Amsterdam UMC, VU University Medical Centre, Amsterdam, The Netherlands, ${ }^{5}$ Department of Human Nutrition, Wageningen University \& Research, Wageningen, The Netherlands, ${ }^{4}$ Department of Internal Medicine, Erasmus MC, University Medical Center, Rotterdam, The Netherlands

Introduction: Cardiovascular drugs like diuretics are risk factors for falling in older people. Our objective was to assess whether genetic polymorphisms affecting the diuretics efficacy modify the association between diuretics and falls.

Methods participants: $\geq 55$ years from B-PROOF (primary study) and the Rotterdam Study (validation study). Cox proportional hazards models with diuretic exposure as time-varying determinant were used to calculate fall hazard ratios (HRs). Interaction terms between diuretic use and single nucleotide polymorphisms (SNPs) were created to identify relevant SNPs. The participants were stratified into categories according to genotype and use/non-use of diuretics. Cox models were applied to determine fall risk of the categories.

Results: Loop diuretics were associated with increased fall risk in the meta-analysis (HR 1.20, 95\% confidence interval 1.04-1.39), whereas low-ceiling diuretics use was protective in the Rotterdam Study (HR 0.77 [0.65-0.92]), but not in B-PROOF. Three of the 32 assessed interaction terms were significant. In loop diuretic users, our results indicated a trend of higher fall risk (non-significant) in increased response-allele carriers of rs17268282. In B-PROOF, normal response-genotype carriers of rs1458038 using low-ceiling diuretics had increased risk (HR 1.60 [1.01-2.54]), and in the Rotterdam Study, decreased risk (HR 0.30 [0.12-0.73]), compared to nonusers. In lowceiling diuretic users, a lower fall risk was shown (HR 0.62 [0.42-0.91]) in increased response-allele carriers compared to normal response-allele carriers.

Conclusions: SNPs may have a role in diuretic-related fall risk. However, this is the first study to address this issue. Genetic risk scores or a genome-wide association study could be next steps in further research.

\section{P-26}

Falls, fractures and osteoporosis treatment among elderly outpatients in Moscow

Olga Tkacheva ${ }^{1}$, Nadezda Runikhina ${ }^{2}$, Valentina Ostapenko ${ }^{3}$, Natalia Sharashkina $^{4}$, Ekaterina Dudinskaya ${ }^{5}$, Yliya Onuchina ${ }^{6}$, Yliya Kotovskaya $^{2}$

${ }^{1}$ Head of Russian Clinical and Research Center of Gerontology, Moscow, Russia, ${ }^{2}$ Deputy Head of Russian Clinical and Research Center of Gerontology, Moscow, Russia, ${ }^{3}$ Aging Disease Department Assistant of Pirogov Russian National Research Medical University, Moscow, Russia, ${ }^{4} \mathrm{Head}$ of outp

Object: There is a need for studies on the prevalence of geriatric syndromes and age-associated diseases due to the rapid growth of life expectancy and active development of the geriatric service in the last few years in Russia.

Materials and methods: The study included 356 patients 65-93 years (average age was $74.9 \pm 6.1$ years, $80.4 \%$ were women), who were treated in four community clinics in Moscow. Information was collected when performing patients interviewing.

Results: The prevalence of falls during the last year was $35.4 \%$ $(\mathrm{n}=126)$. One third of them was repeated falls ( $\geq 2$ times) $-11.0 \%$ $(\mathrm{n}=39)$. The prevalence of low-traumatic fractures was $20.5 \%$ $(n=73)$. Radial bone fractures prevalence was $78 \%(n=57)$, 
humerus - $17.8 \%(\mathrm{n}=13)$, proximal femur-6.9\% $(\mathrm{n}=5)$-as well as compression fractures of the vertebrae. Among patients who underwent low-traumatic fractures only $8.2 \%$ received bisphosphonates, $5.5 \%$ received only vitamin D and $2.7 \%$ - calcium with vitamin D.

Conclusions: In our study, a high prevalence of repeated falls and osteoporotic fractures has been revealed. In spite of this, no more than $10 \%$ of patients with severe osteoporosis received anti-osteoporotic therapy. Further research is needed to determine whether this is due to the low detectability of osteoporosis and the prescription of the drugs or to the low adherence of patients to anti-osteoporotic therapy.

\section{P-27}

Vancomycin dosing optimisation-lessons learnt from a PK/PD study

Hana Suchankova ${ }^{1}$, Karolina Lecbychova ${ }^{2}$, Tomas Fürst ${ }^{2}$

${ }^{1}$ University Hospital Olomouc, Olomouc, Czech Republic, ${ }^{2}$ Palacky University, Olomouc, Czech Republic

Introduction: The process of aging is associated with altered pharmacokinetics (PK) and pharmacodynamics (PD) of many drugs. Dosing strategies derived from studies including younger and/or healthy individuals might not be appropriate. The aim of our study was to evaluate the frequency of optimal initial vancomycin dosing (with regard to the PK/PD parameters, i.e. the ratio of area under the concentration versus time curve to the minimum inhibitory concentration) in University Hospital Olomouc and to identify covariates enabling to predict optimal individual dosage regimen.

Patients and methods: A retrospective analysis of vancomycin plasma levels determined during a 5-year period in patients aged $\geq 65$ years treated with i.v. vancomycin was performed. Pharmacokinetic modelling using MWPharm++ software was performed to assess individual PK/PD indices.

Results: A total of 794 vancomycin concentrations obtained in 208 patients were included. Pharmacokinetic simulations showed suboptimal and supratherapeutic concentrations in $14 \%$ and $42 \%$ patients, respectively. Data were in clear contrast to those observed in individuals aged $<65$ yrs. During the same study period, 44 and $22 \%$ non-geriatric patients would be considered underdosed and overdosed, respectively. Creatinine clearance was the only covariate predicting over- and underdosing. Increased creatinine clearance by $1 \mathrm{ml} / \mathrm{s} / 1.73 \mathrm{~m}^{2}$ was associated with 8 times lower risk of overdosing. Conclusion: Optimal initial vancomycin dosing still remains challenging in clinical practice. Simple nomograms with creatinine clearance could improve vancomycin prescribing.

Disclosure of interest: None declared. Approved by institutional ethics committee, Nr. 183/14. Supported by MH CZ-DRO (FNOl, 00098892).

\section{P-28}

Anticholinergic burden before and after hospitalization in older adults with dementia: large increase due to antipsychotic medications

\section{Jonas Reinold ${ }^{1}$, Oliver Riedel ${ }^{1}$, Federica Edith Pisa ${ }^{1}$ \\ ${ }^{1}$ Leibniz Institute for Prevention Research and Epidemiology-BIPS, Bremen, Germany}

Background: Hospitalizations can lead to increased use of medications, particularly in older adults with dementia who frequently use multiple medications including some with anticholinergic activity (MAC), despite strongly discouraged.
Objectives: To evaluate changes in MAC use and anticholinergic burden during hospitalization in older adults with dementia and to evaluate factors associated with an increased burden.

Methods: Retrospective cohort, including all inpatients aged $\geq 65$ years with a discharge diagnosis of dementia hospitalized at the University Hospital of Udine, Italy, 2012-2014. Only first hospitalization was included. Medications were identified in communitypharmacy dispensations within 3 months before hospitalization while medications prescribed at discharge were collected from Hospital Electronic Medical Records (EMR). Anticholinergic burden was assessed using the Anticholinergic Cognitive Burden score (ACBscore).

Results: Among 1908 patients (64\% women, median age 86 years), $68.6 \%$ used $>1 \mathrm{MAC}$ at discharge, $49.1 \%$ before and $45.7 \%$ after hospitalization, and $38.4 \%$ had increased ACB-score at discharge. While cardiovascular medications (e.g. furosemide, digoxin and warfarin), were among the most commonly used MAC both before (29.2, 12.0 and 6.4\%) and at discharge $(29.7,10.5$ and 5.8\%), use of antipsychotics, especially quetiapine and promazine, strongly increased at discharge (33\%) compared to before hospitalization (12\%). Patients with psychiatric conditions (1.91; 1.52-2.39), discharged from surgical $(1.75 ; 1.09-2.80)$ or medical department $(1.50$; $1.04-2.17)$ and with cardiac insufficiency $(1.41 ; 1.00-1.99)$ had greater risk of increased $\mathrm{ACB}$ at discharge.

Conclusions: Use of MAC and ACB were considerably higher at discharge than before hospitalization and antipsychotics were the main drivers of this increase.

\section{P-29}

Research priorities for optimizing geriatric pharmacotherapy: an international consensus

Edwin $\operatorname{Tan}^{1}$, Janet Sluggett ${ }^{1}$, Kristina Johnell ${ }^{2}$, Graziano Onder ${ }^{3}$, Monique Elseviers ${ }^{4}$, Lucas Morin ${ }^{2}$, Davide Vertrano ${ }^{2}$, Jonas Wastesson $^{2}$, Johan Fastbom ${ }^{2}$, Heidi Taipale ${ }^{5}$, Antti Tanskanen ${ }^{5}$, Simon Bell ${ }^{1}$

${ }^{1}$ Monash University, Clayton, Australia, ${ }^{2}$ Karolinska Institutet, Solna, Sweden, ${ }^{3}$ Università Cattolica del Sacro Cuore, Milan, Italy, ${ }^{4}$ University of Antwerp, Antwerp, Belgium, ${ }^{5}$ University of Eastern Finland, Joensuu, Finland

Introduction: Medication management is becoming increasingly challenging for older people, and there is limited evidence to guide medication prescribing and administration for people with multimorbidity, frailty, cognitive and functional impairment, or at the end of life. The aim of this study was to investigate the priorities for conducting research in the field of geriatric pharmacotherapy.

Methods: Twelve international experts from five research groups in geriatric pharmacotherapy and pharmacoepidemiology research (representing Australia, Belgium, Finland, Italy, and Sweden) were invited to attend the inaugural Optimizing Geriatric Pharmacotherapy through Pharmacoepidemiology Network (OPPEN) workshop. A modified nominal group technique was used to explore and consolidate the priorities for conducting research in this field.

Results: Eight research priorities were elucidated: underuse, overuse and misuse of medications; medications in frail and vulnerable patient groups; understanding and informing prescribing in people with polypharmacy and multimorbidity; person-centered practice and research; deprescribing and regimen simplification; methodological development; understanding unexplained variability in prescribing and medication use; and national and international comparative research. Participants identified current research gaps and future actions for addressing these priorities. 
Key conclusions: Many key areas in geriatric pharmacotherapy are currently understudied. These priorities highlight areas for future research in this field to improve medication outcomes in older people.

\section{P-30}

Use of nonbenzodiazepine hypnotics is associated with falls in nursing home residents

\section{B. Westerlind ${ }^{1}$, C. J. Östgren ${ }^{2}$, P. Midlöv ${ }^{3}$, S. Hägg ${ }^{4}$}

${ }^{1}$ Department of Clinical and Experimental Medicine, Linköping University, Linköping, Sweden, ${ }^{2}$ Department of Medical and Health Sciences, Linköping University, Linköping, Sweden, ${ }^{3}$ Department of Clinical Sciences in Malmö, Center for Primary Health Care Research, Lund University, Malmö, Sweden, ${ }^{4}$ Department of Medical and Health Sciences, Linköping University, Linköping, Sweden

Introduction: Falls and fall-related injuries are common among older people. This study aimed to investigate associations between the use of certain drug classes (antipsychotics, anxiolytics, hypnotics, antidepressants, antihypertensives, and vasodilators) and falls in older nursing home residents in Sweden, and relate these to different age groups.

Methods: Information on regular drug use and occurred falls the last year were collected in 331 nursing home residents during 2008-2011. The following 6 months the occurrence of serious falls, requiring a physician visitor hospital care, was registered. Associations between serious falls and drug use were then compared between an older and a younger group.

Results: In $93 \%$ of the study subjects (aged 65-101 years, median age 85 years) an increased fall risk (Downton Fall Risk Index $\geq 3$ ) was found. Baseline data indicated an association between falls the previous year and regular use of non-benzodiazepine hypnotics ( $p=0.005$ ), but no association with the other studied drug classes was observed. During the following 6 months, an association between use of non-benzodiazepine hypnotics and serious falls in the older (above median age) group $(p=0.017$, Odds Ratio $=4.311)$ was found. No associations were found between the other studied drug classes and serious falls.

Conclusions: A great majority of nursing home residents had increased fall risk. Regular use of non-benzodiazepine hypnotics was associated with a history of falls and was also prospectively associated with serious falls in the older age group, indicating that caution is advocated when using these drugs regularly in older people living in nursing homes.

\section{P-31}

Fluctuation of the renal function after discharge from hospital and its effects on drug dosing in elderly patients

J. A. M. Altena ${ }^{1}$, H. J. Derijks ${ }^{2}$, R. M. J. Hoedemakers ${ }^{3}$, W. Wester ${ }^{1}$, W. L. Eppenga ${ }^{2}$, R. J. van Marum ${ }^{4}$

${ }^{1}$ Department of Geriatrics, Jeroen Bosch Ziekenhuis, Den Bosch, The Netherlands, ${ }^{2}$ Department of Clinical Pharmacy, Jeroen Bosch Ziekenhuis, Den Bosch, The Netherlands, ${ }^{3}$ Department of Clinical Chemistry and Haematology, Jeroen Bosch Ziekenhuis, Den Bosch, The Netherlands

Introduction: Impaired renal function is common in elderly patients, and their glomerular filtration rate (GFR) should be taken into account when prescribing renally excreted drugs. Normally, GFR at hospital discharge is used for drug dosing. However, GFR might change in the weeks following discharge.

Methods: We conducted a prospective observational study. Patients $\geq 70$ years with MDRD $<60 \mathrm{ml} / \mathrm{min} / 1.72 \mathrm{~m}^{2}$, admitted to the geriatric ward of a large teaching hospital and discharged to a primary care setting were eligible to participate. MDRD was measured at three time points: at discharge from the hospital, 14 days and 2 months after discharge. We used 3 renal function groups: MDRD 50-60, MDRD $30-49$ and $<30 \mathrm{ml} / \mathrm{min} / 1.72 \mathrm{~m}^{2}$. The primary outcome is the percentage of patients that change from renal function group after discharge. Secondly, we analysed risk factors for fluctuation of the renal function to identify this vulnerable group.

Results: In total 65 patients were included with an average age of 84 years. Forty patients $(62 \%)$ changed from renal function group at either the first or the second time point after discharge. Twenty-five patients $(38 \%)$ remained stable during the whole period. At the second time point renal function increased in 11 patients (19\%), decreased in 15 patients (26\%) and remained stable in 31 patients (54\%) compared with discharge. Risk factors were dehydration at the time of admission $(\mathrm{p}=0.012)$ and patients who are mentally competent $(\mathrm{p}=0.046)$.

Key conclusions: $62 \%$ of the patients older than 70 years with an MDRD $<60 \mathrm{ml} / \mathrm{min} / 1.72 \mathrm{~m}^{2}$ during admission fluctuate in renal function group after discharge potentially leading to ineffectiveness or toxicity of drugs

\section{$\mathbf{P}-32$}

Chronic noncommunicable diseases and drug use in primary care

Cris Renata Grou Volpe ${ }^{1}$, Diana Lucia Moura Pinho ${ }^{1}$, Anna Cecília Soares Santos ${ }^{1}$, Valdiane Dutra Oliveira ${ }^{1}$, Marina Morato Stival ${ }^{1}$, Tania Cristina S. B. Rehem ${ }^{1}$

${ }^{1}$ University of Brasilia, Brasilia, Brazil

Objective: To analyze electronic and manual prescriptions regarding the occurrence of polypharmacy and potential types of medication errors in the context of primary care. Method: a descriptive, transversal and retrospective study, based on the evaluation of prescriptions filed at the pharmacy of the Basic Health Unit of the Federal District. We analyzed in the study 1500 s-line prescriptions that had at least one drug prescribed for chronic non-communicable diseases. The collection occurred between July 18 and September 29, 2017.

Results: The total of the evaluation criteria proposed in this study were: illegibility $(98.2 \%)$, abbreviations $(97.7 \%)$, pharmaceutical form $(57.6 \%)$, concentration (32.4\%), polypharmacy was identified in $46 \%$ of users and this was directly related to the age of the user. The presence of all non-transmissible chronic diseases were positively and significantly associated with polypharmacy, among the ones that presented the greatest risk for the presence of polypharmacy were: arterial hypertension and dyslipidemia, increasing the risk of polycoding at 5.47 and 6.40 times respectively.

Conclusion: Measures that improve the prescriptions and that involve the professionals involved in the care to the patient with the safety in the prescriptions and other actors involved in this process on the prescription of medicines. Strategies aimed at this profile of patients that are increasingly present today are needed, public policies that insist on promoting health and quality of life of this population profile in primary care are essential. 
P-33

Proton pump inhibitor use in residential aged care services: does it pass the acid test?

Ivanka Hendrix ${ }^{1}$, Amy Page ${ }^{2}$, Maarit Korhonen ${ }^{3}, \mathrm{~J} \mathrm{Simon} \mathrm{Bell}^{4}$, Edwin Tan ${ }^{5}$, Renuka Visvanathan ${ }^{6}$, Tina Cooper ${ }^{7}$, Leonie Robson ${ }^{7}$, Janet Sluggett $^{8}$

${ }^{1}$ Department of Pharmacy, Southern Adelaide Local Health Network, Bedford Park, SA, Australia; and NHMRC Centre for Research Excellence in Frailty and Healthy Ageing, Adelaide, SA, Australia, ${ }^{2}$ Centre for Medicine Use and Safety, Faculty of Pharmacy and Pharmaceutical Sciences, Monash University, Melbourne, VIC, Australia; and Centre for Optimisation of Medicines, School of Allied Health, Crawley, WA, Australia, ${ }^{3}$ Centre for Medicine Use and Safety, Faculty of Pharmacy and Pharmaceutical Sciences, Monash University, Melbourne, VIC, Australia; and Institute of Biomedicine, University of Turku, Finland, ${ }^{4}$ Centre for Medicine Use and Safety, Faculty of Pharmacy and Pharmaceutical Sciences, Monash University, Melbourne, VIC, Australia; NHMRC Centre for Research Excellence in Frailty and Healthy Ageing, Adelaide, SA, Australia; and NHMRC Cognitive Decline Partnership Centre, Hornsby Kuring-gai Hospital, Hornsby, New South Wales, Australia, ${ }^{5}$ Centre for Medicine Use and Safety, Faculty of Pharmacy and Pharmaceutical Sciences, Monash University, Melbourne, VIC, Australia, ${ }^{6}$ NHMRC Centre for Research Excellence in Frailty and Healthy Ageing, Adelaide, SA, Australia; School of Medicine, Faculty of Health and Medical Sciences, University of Adelaide, SA, Australia; and The Queen Elizabeth Hospital, Central Adelaide Local Health Network, SA Health, Adelaide, SA, Australia, ${ }^{7}$ Resthaven Incorporated, Adelaide, SA, Australia, ${ }^{8}$ Centre for Medicine Use and Safety, Faculty of Pharmacy and Pharmaceutical Sciences, Monash University, Melbourne, VIC, Australia; and NHMRC Cognitive Decline Partnership Centre, Hornsby Ku-ring-gai Hospital, Hornsby, NSW, Australia

Introduction: While proton pump inhibitors (PPIs) are generally well tolerated, frail older people who take PPIs long-term may be susceptible to dose-dependent adverse events. The objective of this study was to determine factors associated with high-dose PPI use in residential aged care services (RACSs).

Methods: A cross sectional study of 383 residents (mean age 87 years) in six Australian RACSs was conducted. Clinical, diagnostic and medication data were collected by study nurses. The proportions of residents who took PPIs for $>8$ weeks and without documented indications were calculated. Age and sex-adjusted logistic regression models were used to identify factors associated with high-dose PPI use compared to standard/low doses.

Results: $196(51 \%)$ residents received a PPI, with 46 (23\%) prescribed a high dose. Overall, 173 (88\%) PPI users had documented clinical indications or received medications that can increase bleeding risk. Three quarters of PPI users with GORD or dyspepsia had received a PPI $>8$ weeks. High dose PPI use was associated with increasing medication regimen complexity (odds ratio (OR) 1.02, 95\% CI 1.01-1.04 per one point increase in Medication Regimen Complexity Index score) and a greater number of regular charted medications (OR 1.11, 95\% 1.01-1.21 per additional medication).

Key conclusions: Most PPI use was consistent with guidelines but confirmation of clinical indications for residents taking PPIs $>8$ weeks and 'step-down' approaches for high dose PPI users may reduce the likelihood of adverse events.

\section{P-34}

Patient involvement and inter-professional collaboration in medication reviews conducted in nursing homes

Hans Wouters ${ }^{1}$, Anne Ensink ${ }^{2}$, Lisa Kouladjian O’Donnell ${ }^{3}$, Sytse Zuidema ${ }^{1}$, Froukje Boersma ${ }^{1}$, Katja Taxis ${ }^{2}$

${ }^{1}$ Univ. of Groningen, UMCG, Dept. General Practice and Elderly Care Medicine, Groningen, The Netherlands, ${ }^{2}$ Univ. of Groningen, Fac. Science and Engineering, Dept. Pharmaco Therapy,

-Epidemiology \& -Economics (PTEE), Groningen, The Netherlands, ${ }^{3}$ Univ. of Sydney, Royal North Shore Hospital, Dept. Clinical Pharmacology and Aged Care, Sydney, NSW, Australia

Introduction: Inappropriate prescribing remains persistent in nursing home residents. Insight into barriers and facilitators of medication reviews aimed at improving prescribing is needed, because findings about their effectiveness were equivocal.

Methods: Embedded in a cluster RCT, showing medications reviews to be successful in discontinuing inappropriate medication, we conducted semi-structured interviews about purposively sampled medication reviews ( 8 with 5 pharmacists, 8 with elder care physicians, 10 with nursing staff, and 6 with residents/relatives). Transcribed interviews were analysed with the constant comparison method.

Results: The main theme on patient involvement was "Fidelity" (e.g. the patient perspective as a leverage whether residents perceived their medications as inappropriate vs. distortions by residents' bereavement, cognitive impairment, or submissive attitudes, requiring specific strategies e.g. consistent gauging). Themes on inter-professional collaboration were "Clinicians" Tendentiousness" (e.g. physicians valuing pharmacists making pharmacotherapeutic assessments independent from patient data and presuming pharmacists were able to infer indications from prescriptions vs. pharmacists valuing access to residents' medical charts; medication reviews being useful for reducing overprescribing possibly linked to physicians' treatment inclination), "Level of Comprehensiveness" (e.g. utility of inappropriate prescribing criteria vs. overload of impracticable alerts), and "Interprofessional alliances" (e.g. no consensus about involving nursing staff in medication review discussions vs. their pivotal role while executing changes; physicians' commitment to discontinue inappropriate medications being impeded by residents' dependence on medication in which case pharmacists could substantiate physicians' efforts).

Conclusions: These insights underline the need for meta-communication with residents/relatives about their involvement and among clinicians about inter-professional collaboration to implement medication reviews in practice.

\section{P-35}

Appropriateness of new oral anticoagulant dose adjustment in older adults

Tugba Erdogan ${ }^{1}$, Onur Erdogan ${ }^{2}$, Gülistan Bahat ${ }^{1}$, Mehmet Akif Karan $^{1}$

${ }^{1}$ Istanbul University Istanbul Medical School Department of Internal Medicine Division of Geriatrics Istanbul, Turkey, ${ }^{2}$ Istinye Public Hospital, Istanbul, Turkey

Aim: Atrial-fibrillation (AF) remains the major cause of stroke. Nonvitamin $\mathrm{K}$ antagonist oral anticoagulants (NOACs) usage for stroke prevention in non-valvular-AF, increasing rapidly. However current guidelines recommend dose-adjustment according to certain criteria for each NOAC, physician judgement may be a strong influence for reduced NOAC. Our aim is to determine if use of inappropriate 
NOAC prescribing is a significant problem and whether this is related to the existing diseases, demographic-data, CHADSVASc and HASBLED score, frailty and falls/fear-of-falls.

Method: Older adults between the ages of 60-99 who have AF and under the treatment of NOACs admitted to outpatient clinic were included. Demographic data, clinical data were obtained. Frailty was evaluated by FRAIL-scale. For each patient the doses of NOACs were evaluated according to the recommendations of the guideline and it was categorized as appropriate or inappropriate-dose.

Results: A total of 295 older-adults were included. The mean-age was $75.4 \pm 7.6$ years, and $64.4 \%$ of patients were female. Rivaroxaban was the most commonly used NOAC observed in 169 patients (57.3\%). A total of $104(35.6 \%)$ were prescribed a dose lower than recommended. Factors associated with inappropriate-low-dose use were weight, cerebrovascular disease and being older than 70 years of age. In regression analysis independent factor was age older than 70 years.

Conclusion: Our study suggests that dose reduction is performed only in consideration of the age factor, contrary to the guideline recommendations in clinical practice. We observed that the use of inadequate low-dose medication was common and this condition was not related to falling/falling-fear or frailty.

\section{P-36}

Antidepressants in French nursing homes for dependent elderly patients: the cross sectional associations of institutional factors with prescription conformance

\section{A. A. B. Abdeljalil Anne-Bahia ${ }^{1}$, Rolland Yves $^{1}$, Arbus Christophe ${ }^{1}$ \\ ${ }^{1}$ Gérontopôle Toulouse, Toulouse, France}

Introduction: Depression is diagnosed in approximately $30 \%$ of the nursing homes $(\mathrm{NH})$ residents but the pertinence of the high use of antidepressants is often criticized. Our aim was to determine the proportion of non-conforming antidepressant prescriptions among $\mathrm{NH}$ residents and the associated demographic and institutional factors.

Method: Descriptive cross-sectional study of 2651 residents in 175 French NH participating in the IQUARE study (Impact of a QUAlity process on the development of practices and the functional decline of EPHAD Residents) and treated with antidepressants. Diagnosis of depression was obtained from the medical records. Antidepressant treatments were identified on the prescriptions and their conformance was analyzed in light of the international recommendations.

Results: A total of 1017 residents (38.4\%) had a non-conforming prescription. The presence of a recommended list of medications for elderly subjects (OR 1.39, 95\% CI [1.15; 1.68], p < 0.001) and regular intervention by a psychiatrist or psychiatric nurse (OR 1.50, 95\% CI $[1.26 ; 1.77], \mathrm{p}<0.001)$ were associated with better conformance of antidepressant prescription. Women (OR 0.76, 95\% CI [0.61; 0.93 ], $\mathrm{p}<0.05$ ) and polymedicated residents (OR 0.64, 95\% CI $[0.50 ; 0.80], \mathrm{p}<0.001)$ were at higher risk for non-conforming antidepressant prescriptions. A history of dementia (OR $1.54,95 \%$ CI [1.29; 1.84], $\mathrm{p}<0.001$ ), co-prescription of neuroleptics (OR 4.23, 95\% CI [2.20; 8.12], $\mathrm{p}<0.001)$ and a known date of psychotropic initiation (OR 2.58, 95\% CI [2.10; 3.16], p < 0.001) were associated with better antidepressant prescription conformance.

Conclusion: Our results suggest that a "quality process" by the $\mathrm{NH}$ combining accessible psychiatric care in facilities, pharmacological monitoring and prescription improvement actions might optimize antidepressant treatment for residents.
P-37

Iatrogenic Cushing's syndrome following topical steroid therapy for psoriasis

Roberta Vella Azzopardi ${ }^{1}$, Elaine Vella Seychell ${ }^{1}$, Antoine Vella ${ }^{1}$

${ }^{1}$ Karin Grech Rehabilitation Hospital, Pieta, Malta

Introduction: Due to their anti-inflammatory properties, topical corticosteroids are widely used in the management of dermatological conditions including eczema, atopic dermatitis and psoriasis. Topical corticosteroid potency ranges from level 1 (ultra-high) to 7 (lowest). Potency should be chosen based on the type and anatomical distribution of the dermatological pathology.

Case report: A 78-year-old lady presented to the Geriatrics Day Hospital with difficulty rising from a chair, difficulty managing stairs, deterioration in mobility and recurrent falls. On physical examination she was noted to have a puffy hirsute face; a fat hump on the nape of her back; central obesity and bilateral proximal myopathy affecting lower limb girdle. She admitted to using a 15 g-tube of hydrocortisone butyrate $0.1 \%$ cream daily/alternate days particularly in the flexures due to uncontrolled psoriasis. Therefore the patient was diagnosed with iatrogenic Cushing's syndrome secondary to systemic absorption of a topically applied steroid.

Discussion: Hydrocortisone butyrate is a medium potency topical steroid. Only low potency topical corticosteroids should be used in intertriginous/flexural areas affected by psoriasis, as absorption of steroids at these sites is rapid thereby increasing likelihood of systemic adverse effects such as iatrogenic Cushing's syndrome due to suppression of the hypothalamic-pituitary axis. This can be avoided by applying the right amount of adequately potent topical steroid measured in fingertip units once/twice daily for a period not exceeding 3 months.

Conclusion: In adulthood, iatrogenic Cushing's syndrome should be considered when stronger than low potency topical steroids are applied sparingly on damaged skin especially in the flexures.

\section{P-38}

Does clinical pharmacy have an impact on the re-hospitalization rate in a geriatric emergency department (MUPA-unit)? Preliminary results of MUPAPHARM OBS study

\section{A. Clementz ${ }^{1}$, J. Jost ${ }^{1}$, A. Lacour ${ }^{2}$, V. Ratsimbazafy ${ }^{1}$, A. Tchalla ${ }^{2}$}

${ }^{1}$ Pharmacy Unit, Limoges Teaching Hospital, Limoges, France, ${ }^{2}$ Geriatric Medicine, Limoges Teaching Hospital, Limoges, France

Introduction: Iatrogenic issues are responsible for $20 \%$ of elderly frail hospitalizations [1], while $25 \%$ might be avoided [2]. Falls are one of major symptoms. Clinical pharmacy can reduce risk factors such as polypharmacy, medication complexity [3]. Objective: to evaluate the impact of pharmacist-led interventions at the MUPA-unit on re-hospitalization rate after discharge.

Methods: 12 weeks longitudinal study in a teaching hospital; primary endpoint were 72-h and 30-day re-admissions rate. We compared two groups: (1) group with patients followed by a pharmacist (FPH) and benefited from medication reconciliation, pharmaceutical analysis, town-hospital network and (2) group with patients without pharmacist follow-up (wFPH). Pharmaceutical interventions (PI) acceptation by geriatrician was also assessed. Inclusion criteria: 75 years and older, admitted for fall at the MUPA-unit, more than two diseases and medications.

Results: 142 patients (47 male, 75-108 years), 72 in FPH group and 70 in wFPH group. The two groups are comparable concerning age, sex, number of medications, number of drugs causing falls, patient's 
orientation, MUPA-unit length of stay. 91\% PI were accepted by geriatrician. Two (2.8\%) re-hospitalization after 72-h in FPH group compared to six $(8.6 \%)$ in wFPH group. Seven $(9.7 \%)$ re-hospitalizations after 30 days in FPH group compared to twelve $(17.1 \%)$ in wFPH group.

Key conclusion: Pharmaceutical interventions were well accepted by geriatrician. We found that $72 \mathrm{~h}$ and 30 days re-admissions rate were two time higher in the group without pharmacist follow-up. These results might be confirmed by larger further randomized controlled trial.

\section{References:}

1. Beijer HJ, de Blaey CJ (2002) Hospitalisations caused by adverse drug reactions (ADR): a meta-analysis of observational studies. Pharm World Sci 24(2):4654

2. Gurwitz JH, Field TS, Harrold LR, Rothschild J, Debellis K, Seger $\mathrm{AC}$ et al (2003) Incidence and preventability of adverse drug events among older persons in the ambulatory setting. JAMA 289(9):1107-1116

3. Cooper JA, Cadogan CA, Patterson SM et al (2015) Interventions to improve the appropriate use of polypharmacy in older people: a Cochrane systematic review. BMJ Open 5

\section{P-39}

Association between anticholinergic burden and nutritional status in older patients living in nursing homes: study of a subpopulation of the idem cohort

\section{Jullien Adeline ${ }^{1}$, Pagès Arnaud ${ }^{1}$, Cantet Cécile ${ }^{2}$, Rolland Yves ${ }^{3}$, McCambridge Cécile ${ }^{1}$ \\ ${ }^{1}$ Pharmacist, CHU Toulouse, Toulouse, France, ${ }^{2}$ Statistician, INSERM Toulouse, Toulouse, France, ${ }^{3}$ Geriatrician, CHU Toulouse, Toulouse, France}

Introduction: This cross-sectional study was conducted on baseline data from the IDEM cohort (Benefit of systematic tracking of dementia cases in nursing homes) subgroup $(\mathrm{N}=585)$. The aim of this study was to explore the association between anticholinergic burden and nutritional status among elderly nursing home residents. Methods: Malnutrition risk was determined using the Mini Nutritional Assessment (MNA) and high anticholinergic burden (level $\geq 3$ ) was defined using the Anticholinergic Drug Scale (ADS). Multivariate logistic regression was used to examine the contribution of high anticholinergic burden and associated potential confounding factors on the nutritional status of participants.

Results: The average anticholinergic burden was $1.39 \pm 1.46$. After adjusting for potential confounding factors, there was no significant association between high anticholinergic burden and risk of malnutrition $(\mathrm{OR}=1.03,95 \%$ CI $[0.52-2.03], \mathrm{p}=0.925)$. However, the risk of malnutrition was significantly higher for women, patients with a high Charlson Comorbidity Index score, history of fracture or falls, a low score on the MMSE (Mini Mental State Examination), AGGIR (Autonomie Gérontologique Groupe Iso-Ressources) and QOL-AD (Quality of Life in Alzheimer's Disease) assessments.

Conclusions: The use of existing scales in clinical practice remains a challenge because of the complexity of calculating atropine exposure, the updating of scales, and the differences observed between anticholinergic burden and clinically proven adverse events.

\section{$\mathbf{P}-40$}

Perceptions on the interest and feasibility: community pharmacists-led medication reviews in elderly in primary care

Qassemi Soraya ${ }^{1}$, Tesquet Nathalie ${ }^{1}$, Eyvrard Frédéric ${ }^{2}$, Pagès Arnaud $^{2}$, McCambridge Cécile $^{2}$, Cestac Philippe ${ }^{3}$

${ }^{1}$ Pharm D. Department of Pharmacy, Toulouse University Hospitals, Purpan Hospital, Toulouse, France, ${ }^{2}$ Pharm. D, Department of Pharmacy, Toulouse University Hospitals, Purpan Hospital, Toulouse, France, ${ }^{3}$ Pharm. D, Ph.D., Department of Pharmacy, Toulouse,

France

Introduction: To identify potentially inappropriate prescriptions and reduce iatrogenic events, different actions may be proposed. Among them, a structured medication review (MR) can be provided in France by community pharmacists for elderly patients in collaboration with general practitioners. MR is supported by a professional Network for the Community Pharmacy (REIPO) structured around the city of Toulouse (Occitanie area, France). The main objective of this study is to evaluate community pharmacists' perceptions on the interest and feasibility of pharmaceutical interviews targeting elderly patients with polymedication.

Methods: An enquiry was sent to 8939 pharmacies via the LGPI software of pharmagest in the whole country. The survey was conducted by REIPO, during a 2 week period from April 7-22, 2017. The main barrier identified was the fear of physicians' non-adherence by $69.7 \%$ of pharmacists interviewed $(\mathrm{n}=276 / 396)$. However, $71.5 \%$ $(n=283 / 396)$ of them think that they can identify physicians with whom they could collaborate. The second obstacle identified was the human resources lack by $52 \%$ of pharmacists $(n=205 / 396)$. Then there was the lack of financial resources $(n=163 / 396)$, the fear of poor patient adhesion $(n=153 / 396)$, and the lack of knowledge about therapeutics optimization in elderly patients $(n=108 / 396)$. From the perspective of the interviewed pharmacists, the main advantage is an improvement in the patient's medication management $(90.7 \%$, $\mathrm{n}=359 / 396)$.

Conclusion: This study allowed to discuss with community pharmacists their expectations and needs to achieve MR. These results will have to be taken into account by REIPO and sanitary authorities to include representative of general practitioners into brainstorming workshops with pharmacists to improve collaboration. REIPO offers a wide range of training for health professionals wishing to upgrade their skills in therapeutics optimization in elderly patients. REIPO's aim is to integrate MR in the daily pharmaceutical activity of the largest number of community pharmacists in collaboration with physicians.

\section{P-41}

Inhalation technique of dry powder inhalers in hospitalized geriatric patients: a cross-sectional study

Karine Vigestad Rauff ${ }^{1}$, Jesper Ryg ${ }^{2}$

${ }^{1}$ Hospital Lillebaelt, Kolding, Denmark, ${ }^{2}$ Department of Geriatric Medicine, OUH Odense University Hospital, Odense, Denmark

Introduction: To ensure optimal treatment benefit it is important that patients prescribed with dry powder inhalers (DPIs) uses these correctly. This study assessed the prevalence of correct inhalation technique (InTec) among hospitalized geriatric patients and investigated whether InTec was assessed by doctors.

Method: All patients using DPIs at admission when the author was on call were included. Tests of inspiratory capacity (IC) and InTec using own device were performed $\leq 48 \mathrm{~h}$ before expected discharge. 
Background variables included age, sex, cognition (Orientation-Memory-Concentration (OMC)-score), comorbidity (Charlsoncomorbidity-index (CCI)), and ADL (Barthel-index). The record was checked for information regarding doctors InTec assessment.

Results: A total of 39 patients (64\% women) were included. Patients were old (80.6 (5.5) years (mean(SD)), had moderately reduced ADL (Barthel-Index 59 [34-80] (median[IQR])) and cognitive function (OMC-score 17 [10-24]), and high comorbidity (CCI 3 [2-5]). Of these, $18(46 \%)$ had sufficient IC. In patients bringing own device $(n=29) 7(24 \%)$ used this correctly and $6(20 \%)$ had sufficient IC. Doctors had tested $3 / 33(9 \%)$. Patients with sufficient IC were younger (78.7 (4.8) vs $82.3(5.6)$ years, $\mathrm{p}=0.038)$ and more likely to be men $(71.4 \%$ vs $32.0 \%, p=0.018)$. No significant differences were found between patients when addressing InTec in terms of IC, Barthelindex, OMC-score, or CCI. Patients with correct InTec were significantly younger (76.7 (3.9) vs 82.6 (5.6) years, $p=0.017)$, which stayed borderline significant when adjusting for sex, Barthel-Index, OMC-score, and CCI ( $\mathrm{p}=0.086)$.

Conclusion: Only few patients had sufficient IC and used their DPIs correctly. This was not recorded by doctors. Focused attention is necessary to identify patients who might benefit from different treatment.

\section{P-42}

EURO-FORTA: consensus validation of the FORTA (Fit fOR The Aged) list in several European countries

\section{Farhad Pazan ${ }^{1}$, Christel Weiss ${ }^{2}$, Martin Wehling ${ }^{1}$ \\ ${ }^{1}$ Institute for Clinical Pharmacology, Medical Faculty Mannheim, Ruprecht-Karls-Heidelberg University, Mannheim, Germany, ${ }^{2}$ Department of Medical Statistics, Biomathematics and Information Processing, Medical Faculty Mannheim, Heidelberg University, Mannheim, Germany}

Numerous trials have shown that older people are often subjects to inappropriate and unsafe drug treatment. The main reason for this problem is that for most of the medications there is no evidence regarding efficacy and safety in older patients. This problem is worsened by the high prevalence of multimorbidity and therefore polymedication in this group. To raise the appropriateness of drug therapy in the aged, we have developed a clinical aid called the FORTA List. FORTA was originally invented by Wehling and validated and expanded by twenty experts in a Delphi consensus procedure in Germany/Austria. Subsequently, we evaluated FORTA's usefulness in a pilot clinical trial as well as in a controlled prospective trial. These trials revealed that FORTA significantly improves the quality of pharmacotherapy and reduces the frequency of adverse drug reactions. Based on these results, Delphi consensus validations of country/region-specific FORTA Lists were conducted in seven European countries/regions. 47 experts in total agreed to participate in our study. For each country/region, the overall mean consensus coefficient was higher than 0.9. FORTA Lists from six countries/ regions plus the German FORTA List were collated into the EUROFORTA List including 264 items in 26 main indication groups. For only $4.2 \%$ of the proposed items the consensus results were different from the original/proposed FORTA class. This study produced seven new country/region-specific FORTA Lists as well as the EUROFORTA List. The results of this project are going to increase the applicability of the FORTA List for international use.

\section{P-43}

Non-adherence to antidepressants among older patients with depression: a longitudinal cohort study in primary care

Richard Oude Voshaar ${ }^{1}$, Hans Wouters ${ }^{1}$, Floor Holvast ${ }^{1}$, Karin Hek ${ }^{2}$, Francois Schellevis ${ }^{2}$, Huibert Burger ${ }^{1}$, Peter Verhaak ${ }^{1}$

${ }^{1}$ RUG, UMCG, Utrecht, The Netherlands, ${ }^{2}$ NIVEL, Utrecht, The Netherlands

Background: Depression is common among older adults and is typically treated with antidepressants. Adherence is important for antidepressants to be effective. We aimed to determine the non-adherence rates to antidepressants among older adults in primary care, based on non-initiation, suboptimal implementation or nonpersistence.

Methods: We selected all patients aged $\geq 60$ years and diagnosed with depression in 2012, from the NIVEL Primary Care Database. Non-adherence was divided into three components: non-initiation was defined as no dispensing within 14 days of the first prescription; suboptimal implementation, as fewer than $80 \%$ of the days covered by dispensed dosages; and non-persistence, as discontinuation within 294 days after first dispense. First, we determined the antidepressant non-initiation, suboptimal implementation and non-persistence rates. Second, we examined whether comorbidity and chronic drug use were associated with non-adherence by mixed-effects logistic regression (non-initiation or suboptimal implementation as dependent variables) and a clustered Cox regression (time to non-persistence).

Results: Non-initiation, suboptimal implementation, and non-persistence rates were $13.5,15.2$ and $37.1 \%$, respectively. As the number of chronically used drugs increased, the odds of suboptimal implementation (odds ratio 0.89 ; 95\% CI $0.83-0.95$ ) and of non-persistence (hazard ratio $0.87 ; 95 \%$ CI $0.82-0.92$ ) reduced.

Key conclusions: Non-adherence to antidepressants was quite common. Adherence is better when patients are accustomed to taking larger numbers of drugs, but this only provides partial explanation of the variance. General practitioners should be aware of the high rates of nonadherence. Elaborating on the efficacy and optimal length of antidepressant therapy might be prudent first steps to improve adherence.

\section{P-44}

Rivaroxaban in real geriatric clinical practice: prescribing pattern and inappropriate dose prescription in patients with nonvalvular atrial fibrillation. Experience of a center

\section{Tall Fatoumata ${ }^{1}$, Pautas Eric ${ }^{2}$}

${ }^{1}$ Division of Geriatric Medicine, Dr Grancher, Rothschild HospitalAssistance Publique Hôpitaux de Paris, Paris, France, ${ }^{2}$ Division of Geriatric Medicine, Pitié-Salpêtrière-Charles Foix-DHU FASTAssistance Publique Hôpitaux de Paris, Ivry-sur-Seine, France

Rivaroxaban is gaining currency in clinical practice to reduce the risk of stroke in patients with non-valvular atrial fibrillation (NVAF). Elderly are under-represented in the trials that proved efficacy and safety of rivaroxaban, with $18 \%$ of patients over 80 -years-old. Our goal was to report use of rivaroxaban in real geriatric clinical practice describing the pattern of prescription in a teaching-hospital. From January 2017 to April 2018, 53 patients, with a median age of 85, treated with rivaroxaban for NVAF were included. Demographic, biologic, therapeutic data with doses, coprescription and comorbidities were collected from the hospital's electronic records. They were then compared to ROCKET patient's profile. Patients were at low embolic risk compared to ROCKET study patients (mean CHADS2 $2.72 \pm 1.18$ vs $3.5 \pm 0.9 ; \mathrm{p}<0.0001)$. They had lower rates of 
coexisting illnesses, regarding stroke histories (18.9\% vs $54.9 \%$, $\mathrm{p}<0.0001)$, diabetes $(22.6 \%$ vs $40 \%, \mathrm{p}=0.013)$ and heart failure $(37.7 \%$ vs $62.6 \%, \mathrm{p}=0.0003)$ anyway more ischemic cardiopathies $(30.2 \%$ vs $16.6 \%, p=0.014)$, and renal impairment (Cockcroft creatinine clearance 48 vs $67 \mathrm{~mL} / \mathrm{min}, \mathrm{p}<0.0001$ ) were reported. Fourteen patients $(26.4 \%)$ received an inappropriate dose despite guidelines that suggest adjusting prescription to renal function; 9 of them $(64 \%)$ received a reduced dose instead of a standard dose. No predictive factors of underdosing were underlined. Inappropriate dose prescription of rivaroxaban is frequent in elderly patients treated for NVAF. Direct oral anticoagulants need to be prescribed with doses tailored to renal function if we want to reach efficacy and safety previously described in large clinical trials and registries.

\section{$\mathbf{P}-45$}

A survey on polypharmacy of older population in Japan; an analysis of health care claims data from a local city

\section{Masahiro Akishita ${ }^{1}$ \\ ${ }^{1}$ The University of Tokyo, Tokyo, Japan}

Aim: To clarify the status of drug prescriptions of general population including older adults in super-aged society, this study investigated the status of drug prescriptions by using the claim data of older adults in a local city in Japan.

Methods: Drug prescriptions of older adults aged 65 years or older in a Japanese city (approximately 230,000 of population and 65 years or older was 34\%) were investigated using health care claims data. Age group, sex, number of medical facilities used, and 8 most frequent diseases were assessed and whether these factors were associated with polypharmacy ( $>5$ drugs) or not was analyzed using logistic regression analysis.

Results: Total of 50,983 older adults ( $\geq 65$ years) were analyzable and polypharmacy was seen in $35.3 \%$. Polypharmacy was most frequent in the age group of 85-89 years (51.6\%) and the number of medical facilities used was 1: $55.2 \%, 2: 30.7 \%, 3: 10.4 \%$, and 4 or more: $3.7 \%$. The prevalence of 8 major diseases was hypertension: 59.9\%, dyslipidemia: $43.0 \%$, peptic ulcer diseases: $41.5 \%$, diabetes: $29.3 \%$, neuralgia: $22.5 \%$, arthritis $21.0 \%$, osteoporosis: $18.9 \%$, and coronary disease: $18.4 \%$. In multiple regression analysis, older age, multiple use of medical facilities, and each disease were independently associated with polypharmacy.

Key conclusion: In a city of super-aged society, the prevalence of polypharmacy was high and increased with age, multiple use of medical facilities, and diseases. Surprisingly, polypharmacy was most seen in the age group of $85-89$, which is similar to the mean life expectancy of Japan.

\section{P-46}

Overdose in vitamin $\mathrm{K}$ antagonist's administration in the elderly: clinical aspects

Bani Wifek ${ }^{1}$, Zaghbi Nesrine ${ }^{1}$, Ben Selem Thouraya ${ }^{1}$, Said Fatma ${ }^{1}$, Khanfir Monia ${ }^{1}$, Ben Ghorbel Imed ${ }^{1}$, Lamloum Mounir ${ }^{1}$, Houman Mohamed Habib ${ }^{1}$

${ }^{1}$ The Department of Internal Medicine of the University Hospital of La Rabta, Tunis, Tunisia

Introduction: Vitamin $\mathrm{K}$ antagonists (VKA) are widely used for the curative treatment of thromboembolic events. The aim of this study is to describe the clinical aspects of overdose in VKA administration and determine its hemorrhagic factors in elderly.

Methods: A retrospective analysis was carried out with elderly patients treated with VKA. All patients with an INR greater than 4 were included. We studied patients gender and age, VKA use period, other associated drugs, indications, INR value, presence of hemorrhage and immediate management.

Results: There were 31 patients complicated with 35 events of VKA overdose. Acenocoumarol was the only prescribed VKA. Sex ratio favored women (0.82). The average age of patients was 73.7 years. Forty one percent were smokers $(n=13)$. Multi- morbidity was seen in $19.35 \%$ of patients $(n=6)$. Indications were deep vein thrombosis in $83.87 \%(\mathrm{n}=26)$, superficial thrombophlebitis in one patient, pulmonary embolism in $19.35 \%(n=6)$, portal vein thrombosis in on patient, inferior vena cava thrombosis in one patient. High level of homocysteine was found in $25.8 \%(\mathrm{n}=8)$, tumors in 2 patients, Behçet's disease in one patient, antiphospholipid syndrome in two patient, activated protein $\mathrm{C}$ resistance in one patient and indeterminate causes in $54.83 \%(\mathrm{n}=17)$. Most of events happened in the first 6 months of use: $40 \%(\mathrm{n}=14)$. Medium value of INR was 8.16 (4-17). Overdose was complicated with hemorrhagic signs in $48.57 \%$ of events $(n=17)$. Hemorrhagic symptoms were mainly represented by hematuria $(\mathrm{n}=8)$ and bruising $(\mathrm{n}=5)$. Major bleeding episodes were found in $25.71 \%$ of events $(n=9)$ and they were represented by melena in 4 events $(11.42 \%)$ and deep muscle hematoma in 5 events $(14.28 \%)$. Miss use was observed in $31.42 \%(\mathrm{n}=11)$, indeterminate causes in $40 \%(\mathrm{n}=14)$, Drug interaction in 4 events (antibiotics, fibrate, nonsteroidal anti-inflammatory drugs). Kidney failure was found in $19.35 \%$ of patients $(n=6)$. VKA assumption was suspended transiently in all patients and vitamin $\mathrm{K}$ was prescribed in $34.28 \%$ of events $(\mathrm{n}=12)$.

Conclusion: VKA can cause serious side effects. The reduction in VKA overdose requires caregivers to manage overdose factors and provide proper patient education.

\section{P-47}

A prospective three-year study of risk factors for repeated hospitalizations in patients of 65 years old and older

Olga Tkacheva ${ }^{1}$, Anton Pereverzev ${ }^{1}$, Nadezda Runikhina ${ }^{1}$, Yulia Kotovskaya ${ }^{1}$, Sergey Zyryanov ${ }^{2}$, Anastasia Pereverzeva ${ }^{1}$, Daniella Shevchenko ${ }^{2}$, Natalia Temnenko ${ }^{2}$, Elena Ushkalova ${ }^{2}$

${ }^{1}$ Pirogov Russian National Research Medical University of the Ministry of Health of the Russian Federation, Russian Clinical and Research Center of Gerontology, Moscow, Russia, ${ }^{2}$ RUDN University, Moscow, Russia

Introduction: The development of effective and safe schemes of pharmacotherapy in elderly patients is an important problem of healthcare.

Methods: A prospective three-year (2015-2017) observational study was conducted to analyze the impact of co-morbidities and different regimes of drug therapy in the prehospital stage for the frequency of repeated hospitalizations in patients 65 years old and older regardless of sex.

Results: One hundred and forty seven patients (36.7\%) from 401, included in the study had the repeated hospitalizations. The highest frequency (69 cases) of repeated hospitalizations was seen in participants of 75-84 years old. Anemia (OR 16.68, 95\% CI 2.089-133.195, $\mathrm{p}=0.00793)$, bronchial asthma (OR 7.55, 95\% CI 1.77-32.2, $\mathrm{p}=0,0063$ ), diabetes mellitus (OR 0.4, 95\% CI 0.24-0.69, $\mathrm{p}=0.00091$ ), arthropathy (arthritis, arthrosis) (OR 2.5, $95 \% \mathrm{CI} 1,37-4.55, \mathrm{p}=0.00294)$ and pain $(\mathrm{OR} 4.48,95 \% \mathrm{CI}$ 
$1.92-10.42, p=0.0005)$ were associated with a higher risk of repeated hospitalizations. Prehospital pharmacotherapy analysis revealed that the use of acetylsalicylic acid (OR 1.91, 95\% CI 1.29-2.84, $\mathrm{p}=0.00115)$; losartan (OR: 1.92, 95\% CI 1.17-3.17, $\mathrm{p}=0.00952)$; enalapril (OR 1.97, 95\% CI 1.16-3.35, $\mathrm{p}=0.01)$ were also associated with the increased risk of re-hospitalization. Polypharmacy (defined as simultaneous administration of 5 or more drugs) was observed in $86(58.5 \%)$ re-hospitalized patients and most often in persons 85 years old and older.

Key conclusions: Further studies are required to assess the effect of the combination of risk factors for repeated hospitalizations in these patients.

\section{P-48}

Association between socioeconomic status and medication-related harm in elderly patients: a multicentre prospective cohort study in England

Nikesh Parekh ${ }^{1}$, Talalah $\mathrm{Ali}^{2}$, Khalid $\mathrm{Ali}^{1}$, Jennifer Stevenson ${ }^{3}$, Graham Davies $^{3}$, Rebekah Schiff ${ }^{4}$, Anjum Memon ${ }^{5}$, Chakravarthi Rajkumar ${ }^{1}$

${ }^{1}$ Department Of Elderly Medicine, Brighton And Sussex Medical School, Brighton UK, ${ }^{2}$ Brighton And Sussex Medical School, Brighton UK, ${ }^{3}$ Institute Of Pharmaceutical Science, King's College London, London UK, ${ }^{4}$ Department Of Ageing And Health, Guy's and St. Thomas' NHS Foundation Trust, London UK, ${ }^{5}$ Department of Primary Care and Public Health, Brighton And Sussex Medical School, Brighton UK

Introduction: Older adults are at high risk of medication-related harm (MRH)[1]. Lower socioeconomic status (SES) is associated with polypharmacy and inappropriate medication use [2]. Our study investigated the relationship between SES and MRH in older adults. Methods: Methods for this study are published [3]. Patients aged $65+$ years were recruited at hospital discharge from 5 teaching hospitals in England between 2013 and 2015. Patients were followed up for 8-weeks by senior pharmacists to identify MRH using 3 data sources: hospital readmissions, GP records and patient interviews. Based on residential postcodes, Index of Multiple Deprivation quintiles [4] were used as a standard proxy measure for patient SES (1 least deprived, 5 most deprived). Logistic regression models, adjusting for confounders (age, gender, number of medicines, Charlson index, Barthel index), were used to examine the association between SES and MRH.

Results: 1116 patients (median age 82; 58\% female) were included. 413 patients experienced MRH, of which $301(73 \%)$ experienced MRH from adverse drug reactions, 45 (11\%) from non-adherence, and $67(16 \%)$ from combinations of these and medication errors. In the univariate analysis, lower SES was significantly associated with $\mathrm{MRH}$ due to non-adherence (OR 1.27, 95\% CI 1.08-1.49, $\mathrm{p}=0.004)$. Multivariable logistic regression, controlling for confounders, found this relationship to remain significant (OR 1.39, 95\% CI $1.07-1.82, \mathrm{p}=0.015)$. There was no association between lower SES and adverse drug reactions (OR 0.92 , 95\% CI $0.84-1.01$, $\mathrm{p}=0.092$ ).

Key conclusions: Lower SES is independently associated with MRH due to non-adherence, but not ADR. This association might be mediated by lower health literacy in patients with low SES.

References:

1. Parekh N, Ali K, Stevenson J, Davies JG, Schiff R, Cammen T Van Der et al (2018) Incidence and cost of medication harm in older adults following hospital discharge: a multicentre prospective study in the UK. Br J Clin Pharmacol (in press)
2. Guthrie B (2011) High risk prescribing in primary care patients particularly vulnerable to adverse drug events: cross sectional population database analysis in Scottish general practice. BMJ 342:d3514 3. Stevenson J, Parekh N, Ali K, Timeyin J, Bremner S, Van Der Cammen $T$ et al (2016) Protocol for a prospective (P) study to develop a model to stratify the risk (RI) of medication (M) related harm in hospitalized elderly (E) patients in the UK (The PRIME study). BMC Geriatr 16:22. https://doi.org/10.1186/s12877-016-0191-8

4. Department for Communities and Local Government (2015) The English Index of Multiple Deprivation (IMD) 2015-Guidance

\section{P-49}

Drug-associated falls in community-dwelling older adults and potential health impact of pharmacogenomics biomarkersresults from the ActiFE Ulm study

Katja S. Just ${ }^{1}$, Miriam Böhme ${ }^{1}$, Michael Steffens ${ }^{1}$, Dietrich Rothenbacher ${ }^{2}$, Michael D. Denkinger ${ }^{3}$, Jürgen Brockmöller ${ }^{4}$, Julia C. Stingl ${ }^{1}$

${ }^{1}$ Research Division, Federal Institute for Drugs and Medical Devices, Bonn, Germany, ${ }^{2}$ Institute of Epidemiology and Medical Biometry, Ulm University, Ulm, Germany, ${ }^{3}$ AGAPLESION Bethesda Clinic, Geriatric Research Ulm University, Geriatric Center Ulm/Alb, Ulm, Germany

Introduction: Falls are a major health problem in older adults and are often associated with prescription drug use [1]. In many fall-risk increasing drugs large differences in the required doses can be due to genetic polymorphisms in drug-metabolizing enzymes [2]. We analyzed the drug-related risk of falling in community-dwelling elderly and the potential pharmacogenetic impact.

Methods: Data of the ActiFE Ulm study were analyzed [3]. Participants were grouped as fallers and non-fallers according to a prospective recording over 1 year using fall calendars. Characteristics and use of drugs was compared using logistic regression analysis. Those drugs significantly associated with falls were classified according to their dependence on highly variable metabolism and transport based on information in drug labels and the pharmacogenomics databases.

Results: In total, 1506 community-dwelling people aged 65 and older were enrolled. Of this cohort, $458(32.3 \%)$ reported at least one fall with $162(11.4 \%)$ reporting multiple falls, whereas $958(67.7 \%)$ reported not being fallen. Polymorphic CYP enzymes were nominally more often involved in metabolism of substances taken by fallers, although not significant. Many drugs associated with falling were metabolized by phase II enzymes.

Key conclusions: The impact of pharmacogenomics variation in the older adult appears to be moderate only. But confirmatory studies concerning the influence of pharmacogenomics interacting with age, drug metabolism, multimorbidity, and polypharmacy on drug-associated falls in the older adult are urgently needed [4].

\section{References:}

1. (2012) Drugs Aging 29:359-376

2. (2005) Nat Rev Drug Discov 4:639-647

3. (2010) BMC Geriatr 10:50

4. (2017) Pharmacogenomics 18:1281-1297 


\section{P-51}

Potential use of polypill in cardiogeriatrics: effectiveness of an algorithm to reduce pill load

\section{Witold Jarzebowski ${ }^{1}$, Carmelo Lafuente ${ }^{1}$ \\ ${ }^{1} \mathrm{CHU}$ Charles Foix, Ivry-sur-Seine, France}

Background: Cardiovascular diseases are frequent in elderly populations. Patients often need to take several drugs.

Objectives: We aim to evaluate the frequency of cardiovascular drugs and assess a computer algorithm to reduce the number of drugs by substituting with polypill.

Methods: Extraction of drug orders from patients admitted in a cardiogeriatric ward during 1 year (2015). We listed all combinedfixed dose available on the market then using ATC code, spotted all cardiovascular drugs and combined every possible associations of two and three components; We created an algorithm proposing available combined-fixed dose and applied it into a random sample of 100 patients. The main criterion of judgment was the diminution of the number of drugs that would be obtained by substituting with combined fixed dose using the algorithm into the random sample (Student Test on matched data).

Results: 505 patients were included. They took an average of 2.2 cardiovascular drugs. The most prescribed drugs were $\beta$-blockers (48\%), statins (45\%), anti-platelet agents (42\%) and Angiotensin Converting Enzyme Inhibitor (42\%). In France there were 87 fixed dose combined drugs marketed. In the 100 random patients sample there were 162 combinations of three components and 252 of two components. The algorithm showed that an available association could be employed in $10 \%$ of patients, reducing the number of pills from 220 to $210(\mathrm{p}<0.01)$.

Conclusion: Systematic use of available fixed-dose drug combinations can reduce the number of pills taken by elderly patients with cardiovascular diseases. However, available commercial associations do not match well the most frequent combinations of drugs actually prescribed to these patients.

\section{P-52}

Main title: Treatment of sleeping problems in acutely hospitalized older patients. Subtitle: Results from a survey among Dutch geriatricians

Fiona Stenveld ${ }^{1}$, Nynke Smidt ${ }^{2}$, Sjanne Bosman ${ }^{1}$, Barbara C. van Munster ${ }^{3}$, Sophia E. de Rooij ${ }^{1}$

${ }^{1}$ Department of Geriatric Medicine, University of Groningen, University Medical Center Groningen, Groningen, The Netherlands, ${ }^{2}$ Department of Geriatric Medicine, University of Groningen, University Medical Center Groningen, Groningen, The Netherlands

Introduction: Sleeping problems among older patients are a hospital wide problem. There is insufficient evidence to support a specific treatment of sleeping problems in acutely hospitalized older patients. Based on the little evidence available, a national guideline was published about this topic in The Netherlands in 2015. We investigated how sleeping problems are treated in Dutch hospitals and whether medical doctors followed the recommendations in the guideline.

Methods: A survey was conducted among geriatricians in Dutch hospitals between February and April 2018. It consisted of questions regarding non-pharmacologically and pharmacologically treatment preferences, whether geriatricians were informed and had knowledge of the guideline and acted accordingly.
Results: In the survey $36 \%$ of the Dutch hospitals were represented. First choice of treatment was non-pharmacological treatment, such as treatment of symptoms associated with sleeping problems (e.g. pain, dyspnoea) and improvement of sleep environment. Pharmacological treatments were indicated as last option and prescribed sometimes. First choice of medication was benzodiazepines (47.5\%) and second choice melatonin $(18.3 \%)$. Only $32.8 \%$ of participating geriatricians had ever been informed about the guideline, the publication of the guideline has changed their treatment of older patients with sleeping problems. Treatment decisions were mostly based on advice of a more experienced colleague $(47.5 \%)$ or on the Dutch national pharmacological manual $(50.8 \%)$.

Key conclusions: Geriatricians were not well informed about the guideline and acted partially accordingly. Besides adherence to the guideline more evidence based treatment is needed, as most treatments occur now practice based with a rather large practice variation.

\section{P-53}

Reducing use of inappropriate medication by personalizing pharmaceutical care and optimal use of professional resources in nursing homes: PEP, a controlled demonstration study

Kröger Edeltraut ${ }^{1}$, Guénette Line ${ }^{2}$, Demers Marie-France ${ }^{2}$, Voyer Philippe $^{3}$, Guertin Jason ${ }^{2}$, Carmichael Pierre-Hugues, ${ }^{4}$, Rouleau Rachel ${ }^{5}$

${ }^{1}$ Université Laval, Centre d'excellence sur le vieillissement de Québec, Québec, Canada, ${ }^{2}$ Université Laval, Québec, Canada, ${ }^{3}$ Université Laval, Centre d'excellence sur le vieillissement de Québec, Québec, Canada, ${ }^{4}$ Centre d'excellence sur le vieillissement de Québec, Québec, Canada

Background: Increasingly, frail seniors in Canadian nursing homes $(\mathrm{NH})$ receive polypharmacy comprising inappropriate medications. PEP, initiated by the pharmacists of the greater Quebec City Health Board $(30 \mathrm{NH})$, is an evidence based intervention to optimize pharmaceutical and nursing care, facilitated by expanded competencies empowering pharmacists and nurses. The objective was to evaluate whether PEP is: (1) reducing polypharmacy/inappropriate medications; (2) beneficial regarding adverse outcomes (emergency visits, hospitalization, falls); (3) cost-effective.

Methods: PEP updates practices to recent legal changes using (1) tailored knowledge exchange sessions for pharmacists, nurses and physicians, (2) short information sessions for personal care workers and (3) leaflets for residents/their families. Pharmacists perform medication reviews in consultation with nurses, residents/families and physicians using published criteria aimed at deprescribing inappropriate medications. They use collective prescribing for a more timely treatment of minor illness such as constipation. The project was approved by the local ERB.

Results: The intervention concerned 601 residents in $3 \mathrm{NH}$, a pilot $(\mathrm{n}=96)$, an intervention $(\mathrm{n}=263)$ and a control $\mathrm{NH}(\mathrm{n}=242)$. 10 months data show that PEP is feasible and support objective 1: the mean number of regular medications per resident fell from 12.0 in May 2017 to 10.2 medications in March 2018 in the intervention NH and from 9.9 to 9.3 in the pilot $\mathrm{NH}$, as compared to 13.7 and 13.4 in the control $\mathrm{NH}$. Discussion/conclusion: Preliminary data are creating a momentum to unroll PEP in further NH in the province of Quebec/Canada. Results from PEP may help to decrease inappropriate medication use in $\mathrm{NH}$. 


\section{P-54}

Estimation of glomerular filtration rate in elderly hospitalised patients using cockcroft-gault and modification of diet in renal disease (MDRD) equations

\author{
K Nagaratnam ${ }^{1}$, J Akerman ${ }^{1}$ \\ ${ }^{1}$ Royal Berkshire Hospital, Reading, UK
}

Introduction: Dosing of several medications including antibiotics and anticoagulants is adjusted according to renal function. Glomerular filtration rate is the best index of renal function. Cockcroft-Gault (CG) equation is traditionally used to estimate Glomerular Filtration Rate (eGFR). Four variable Modification of Diet in Renal Disease (4vMDRD) equation has become available recently. Estimated GFR using 4vMDRD equation is readily available with routine lab results in our hospital. We aim to assess the impact of using CG and 4vMDRD equations to estimate GFR in elderly hospitalised patients. Methods: In this cross-sectional study we calculated the eGFR using CG equation (CG-eGFR) for inpatients in acute elderly care wards and compared it with eGFR reported with the lab results using 4vMDRD equation (MDRD-eGFR).

Results: Total of 102 patients were recruited with the median age of 85 -years, of which $60.7 \%$ (62/102) were females. Mean CG-eGFR vs MDRD-eGFR was $53 \mathrm{ml} / \mathrm{min} / 1.73 \mathrm{~m}^{2}$ vs $67 \mathrm{ml} / \mathrm{min} / 1.73 \mathrm{~m}^{2}$ $(\mathrm{p}<0.00)$. On average the difference between two estimates was $22 \mathrm{ml} / \mathrm{min} / 1.73 \mathrm{~m}^{2}$. Only $31 \%$ of the patients were categorised in the same stage of Chronic Kidney Disease (CKD) by both methods. MDRD-eGFR overestimated renal function in CKD stages 30-44, $45-59,60-89,>90 \mathrm{ml} / \mathrm{min} / 1.73 \mathrm{~m}^{2}$ compared to CG-eGFR; the discordance was $42,87,63$ and $79 \%$ respectively.

Conclusions: Our study demonstrated high discordance between the estimates of GFR using CG and 4vMDRD equations. Although it is readily available with the lab results judicious consideration is warranted when using MDRD-eGFR for drug dosing in elderly patients. Larger studies are needed to further evaluate this discrepancy in older patients.

\section{P-55}

Observational study of management of curative anticoagulant therapy with direct oral anticoagulant among a hospitalized geriatric population

S. Higuet ${ }^{1}$, J. Bourgeois ${ }^{2}$, J. Peeterbroeck ${ }^{2}$, C. Lelubre ${ }^{2}$, J. C. Wautrecht ${ }^{2}$

${ }^{1} \mathrm{CHU}$ Charleroi, Charleroi, Belgium and GBHI, Dublin, Ireland, ${ }^{2} \mathrm{CHU}$ Charleroi, Charleroi, Belgium

Introduction: With the use of new oral direct anticoagulants (NOACs) and their different guidelines it seemed interesting to analyze practices in real life in a geriatric hospitalized population.

Methods: This is a retrospective study about all patient over 75 years old who was hospitalized in Geriatric ward (CHU Charleroi) between 01/10/2015 and 31/08/2017 and who received at least one dose of low molecular weight heparin(LMWH) and one dose of NOAC. Demographical data, reasons of anticoagulation, reasons of bridging and bleeding complications were analysed.

Results: Patients were anticoagulated for the following reasons: atrial fibrillation $(83.75 \%)$, pulmonary embolism $(8.75 \%)$, atrial flutter $(3.75 \%)$, deep vein thrombosis $(3.75 \%)$. One quarter had a bridging, $31.25 \%$ received a new treatment and $43.75 \%$ had already a chronic treatment. Mean CHA2DS2-VASc was 5. The average length of stay (LOS) was 27.3 days. At the discharge, the majority (84\%) had a NOAC. The main reason for "bridging" were gastroscopy and colonoscopy (55\%) There were $8.75 \%$ bleeding complications. These bleeds were essentially digestive $(3.75 \%)$ or intracranial $(2.5 \%)$.

Conclusion: Our results show that the main reason for anticoagulation is atrial fibrillation and there is a low rate of bleeding complications in geriatrics but the LOS is elevated. This study confirms that NOACS, used in geriatrics, give few complication but in reason of the different guidelines for the different molecules, a dissemination of the recommendations (of each NOAC as well as the algorithm of bridging) within the units would improve our practices.

\section{P-56}

In-hospital adverse drug reactions in hospitalised older adults-a systematic review

Emma Jennings ${ }^{1}$, Kevin Murphy ${ }^{2}$, Paul Gallagher ${ }^{3}$, Denis O'Mahony ${ }^{3}$

${ }^{1}$ Department of Medicine, Cork University Hospital, Cork, Ireland, ${ }^{2}$ School of Pharmacy, University College Cork, Cork, Ireland, ${ }^{3}$ Geriatric Medicine, Cork University Hospital, Cork, Ireland

Introduction: Studies indicate 1 in 4 older people experience hospital-related adverse drug reactions [ADRs]. This systematic-review aims to evaluate in-hospital ADRs in hospitalised older-adults in terms of incidence, prevalence, most commonly involved drug classes, severity, and consequences.

Methods: Using PRISMA methodology [PROSPERO CRD42018079095], we searched PubMed, Embase, Ebsco-CINAHL, Cochrane Library, library hosted sources, Google ${ }^{\circledR}$ scholar, and 'grey' literature, using terms; aged, ADRs, hospitalized, multi-morbid, polypharmacy and hospital-acquired. References of editorials and systematic reviews were hand searched. Studies of all languages and dates until 15/01/2018 were included. All studies reporting ADRs outcomes, $\geq 65$ years, hospitalised at time of ADR occurrence were included. Two researchers screened all papers for inclusion, risk of bias and data extraction.

Results: Initial search yielded 1721 abstracts, 200 underwent full text screening. 60 were potentially suitable for inclusion; 48 papers reported combined ages, 12 papers reported directly on ADRs in our age cohort [2 papers reported the same data]. 11 studies [4424 patients] were analysed; 24\% [1064] experienced ADRs. 7 reported severity $(\mathrm{n}=707) ; 31 \%$ [220] being severe. 5 reported on post-ADR outcomes i.e. length of stay $[\mathrm{n}=3]$, death $[\mathrm{n}=1]$ and functional decline $[\mathrm{n}=1]$. Frequency of culprit drug-groups were described in 6 [672 ADRs]; 43\% [291] cardiovascular system, 17\% [114] central nervous system, 16\% [112] clotting pathways, 13\% [90] antimicrobials.

Conclusions: One in four over 65 years experience an ADR during hospitalisation, one third being severe, and almost half cardiovascular system drugs. Clinical outcomes associated with ADRs are generally poorly described in the literature.

\section{P-57}

Benefit of statins after a first myocardial infarction in the oldest old. A cohort study using general practitioner data from the CPRD

Geert Lefeber ${ }^{1}$, Huiberdina Koek ${ }^{1}$, Wilma $\mathrm{Knol}^{1}$, Marcel Bouvy ${ }^{2}$, Anthonius de Boer ${ }^{2}$

${ }^{1}$ Department of Geriatrics, UMC Utrecht, Utrecht, The Netherlands, ${ }^{2}$ UIPS, Department of Pharmacoepidemiology and Clinical 
Pharmacology, Faculty of Science, Utrecht University, Utrecht, The Netherlands

Introduction: Currently, in patients aged 80 and above, 50-80\% use statins post myocardial infarction (MI). However, evidence to support initiation and use of statins in this population lacks. Therefore our aim was to investigate effects of cumulative statin use on MI, stroke or cardiovascular mortality (primary outcome) and on overall mortality in patients 80 years and older after first MI.

Methods: A cohort study in the Clinical Practice Research Datalink was conducted between January first, 1999 and February 26, 2016. A total of 3900 patients aged 80 and above, hospitalised for first MI, surviving 30 days after discharge, without statin treatment 1 year before hospitalisation, were included. Time varying Cox regression was used to estimate HRs and $95 \%$ confidence intervals (CI) of statin treatment on the primary outcome and mortality, adjusted for confounders including frailty. HRs were converted into numbers needed to treat (NNT) and adjusted for 2 year mortality.

Results: Comparing over 2 years statin treatment to no/less than 2 years statin treatment on the primary outcome resulted in a HR of 0.81 (95\% CI $0.66-0.99)$ and a NNT of 59 over 3 years, increasing to 93 after adjusting for $36.2 \%$ mortality. Over 2 years statin treatment decreased mortality (HR 0.84; 95\% CI 0.73-0.97).

Conclusion: Protective effects of statins initiated after a first MI and continued for at least 2 years were found in patients aged 80 and older. When considering the mortality during and after hospitalisation and competing risks thereafter in old age, initiating statins may not benefit all.

\section{P-58}

Clinical impact of potentially inappropriate prescriptions identified using STOPP/START criteria version 2 in older patients according to discharge setting: an Italian multicenter prospective study

Enrico Brunetti ${ }^{1}$, Maria Luigia Aurucci ${ }^{1}$, Matteo Sappa ${ }^{1}$, Maddalena Gibello ${ }^{1}$, Edoardo Boietti ${ }^{1}$, Dario Bianca ${ }^{1}$, Gianfranco Fonte ${ }^{1}$, Yolanda Falcone ${ }^{1}$, Giorgetta Cappa ${ }^{2}$, Mario $\mathrm{Bo}^{1}$

${ }^{1}$ SCDU Geriatria e Malattie Metaboliche dell'Osso, AOU Città della Salute e della Scienza, and Dipartimento di Scienze Mediche, Università degli Studi di Torino, Turin, Italy, ${ }^{2} \mathrm{SC}$ Geriatria e Cure Intermedie, ASO Santa Croce e Carle, Cuneo, Italy

Introduction: Poly-pathology exposes older patients to polytherapy, potentially leading to adverse outcomes. The STOPP/START criteria are intended to optimize prescription in older patients [1]. Few studies have investigated potential inappropriate prescribing (PIP) using their updated version (STOPP/STARTV2), mainly retrospectively and in community-dwellers.

Methods: Multicenter prospective observational study on patients $\geq 65$ years discharged from geriatric and medicine wards between March-June 2017. STOPP/STARTv2 were applied at discharge to identify potentially inappropriate medications (PIMs) and potential prescribing omissions (PPOs). Death and readmissions at 6 months were investigated through telephone interviews. Variables associated with outcomes according to discharge setting (home vs medium/longterm care facility, MLTCF) were identified through multivariate logistic regression. Terminally-ill patients were excluded.

Results: On a sample of 611 patients (mean age 81.6 years, $48.4 \%$ females, $34.2 \%$ MLTCF-discharged, mean drugs at discharge $7.7 \pm 3.2$ ), we recorded a $71.7 \%$ PIP prevalence, with PIMs being more common than PPOs (54.9\% vs $47.3 \%$ ). Among other variables, a higher number of drugs at discharge was associated with both PIMs and PPOs, while geriatric discharge was protective for both. At follow-up we observed a $25.0 \%$ mortality and a $30.9 \%$ readmission rate. Neither PIMs nor PPOs were found to be associated with overall mortality both in home-discharged and in MLTCF-discharged patients. A higher number of PIMs was significantly associated with hospital readmission in home-discharged patients (OR 1.37, 95\% CI 1.13-1.68), along with cardiac (OR 1.68, 95\% CI 1.03-2.74) and renal (OR 2.38, 95\% CI 1.52-3.71) comorbidities, while the only variables associated with readmission in MLTCF-discharged patients were a higher drug burden (OR 1.27, 95\% CI 1.13-1.42) and the presence of neurological comorbidities (OR 2.37, 95\% CI 1.17-4.81). Key conclusions: In this study we observed a high prevalence of PIP, involving both PIMs and PPOs. On MLTCF-discharged patients, with worse health and functional statuses, PIP according to STOPP/ STARTv2 was neither associated with death nor with readmissions. Among home-discharged patients, a higher number of PIMs was associated with hospital readmission.

Reference:

1. O'Mahony D et al (2015) Age Ageing 44:213-218

\section{P-59}

Use of direct oral anticoagulants (DOACs) in the Norwegian population and focus on the very elderly

\section{L. Stokes ${ }^{1}$}

${ }^{1}$ Department of Medical Biochemistry and Pharmacology, Haukeland University Hospital, Bergen, Norway

Introduction: DOACs (dabigatran, rivaroxaban, apixaban and edoxaban) were introduced in Norway from 2012, and are replacing warfarin as main oral anticoagulant in Norway. Although DOACs are thought to have less drug interactions than warfarin, interactions do exist and they may have most impact on the oldest, most vulnerable patients, due to polypharmacy and multimorbidity [1]. Methods Data is collected from The Norwegian prescription registry which is nationwide and includes all pharmacy dispensings. A case from Norwegian Medicines Agency adverse drug reaction database is presented.

Results: Changes in the dispensing of oral anticoagulants from 2012 to 2017 (\%): warfarin 18.87-9.71, dabigatran $0.82-2.49$, rivaroxaban: $0.27-5.83$, apixaban: $0.07-10.23$ and edoxaban: $0.00-0.08$. Changes in the age group 85-90 years, warfarin: 160.64-95.87, all DOACs: 5.87-149.41. We received a report about an 87-year-old woman using dabigatran who 9 days after the start of Helicobacter Pylori eradication treatment with clarithromycin, amoxicillin and pantoprazole, was admitted to hospital. She had acute on chronic kidney failure and INR (international normalized ratio) was very high, a sign of dabigatran accumulation suspected caused by drug-drug (dabigatran-clarithromycin) and drug-disease (dabigatran-kidney failure) interactions. She was treated with dialysis and antidote idarucizumab.

Key conclusions: We urge medical practitioners to be aware of the risk of drug interactions in the increasing elderly population using a DOAC.

\section{Reference:}

1. Stöllberger C (2017) Drug interactions with new oral anticoagulants in elderly patients. Expert Rev Clin Pharmacol 10(11):1191-1202 


\section{P-60}

Analyse of reasons to keep potentially inappropriate medications after a multidisciplinary evaluation in an acute geriatric unit

Cindy Riffardy ${ }^{1}$, Armance Grevy ${ }^{1}$, Prudence Gibert ${ }^{1}$, Clara Lopez ${ }^{2}$, Nathalie Mitha ${ }^{2}$, Nabil Zerhouni ${ }^{2}$, Pierrick Bedouch ${ }^{1,3,4}$, Gaetan Gavazzi ${ }^{2,4}$

${ }^{1}$ Pharmacy Department, Grenoble Alpes University Hospital, F38000 , France, ${ }^{2}$ University clinic of geriatric medicine, Grenoble Alpes University Hospital, F-38000, France, ${ }^{3}$ ThEMAS, CNRS/UGA TIMC-IMAG UMR 5525, F-38000, France, ${ }^{4}$ University Grenoble Alpes, F-38000, France

Introduction: Potentially inappropriate medications (PIM) in elderly are an iatrogenic illness source. However, interventions to reduce PIM usually do not allow a complete stop of all PIM. This study's objective is to measure the rate of PIM, which are finally continued after multidisciplinary reevaluation and record the reasons why PIM still remain.

Method: Prospective study on prescriptions at admission (usual treatment) and discharge in patients over 75 years old hospitalized in acute geriatric unit from $01 / 25$ to $04 / 25 / 2018$. The aim of multidisciplinary intervention composed of a pharmacy students, pharmacy and medical residents and geriatricians, is to evaluate for each patient if PIM prescriptions according to v2 STOPP/START list are really inappropriate or not and to decide to stop or keep them.

Results: In the study, 101 patients were included and $70.3 \%(\mathrm{n}=71)$ had at least 1 PIM at home. In all, 147 PIM were found and $72.8 \%$ $(n=107)$ were discontinued after the multidisciplinary intervention. There are two main situations where PIM are continued: discontinuation proposed to the general practitioner at distance of hospitalization, and treatments with clinical situation that justifies their use. These cases concerns benzodiazepines $(40 \%, \mathrm{n}=16)$, neuroleptics $(20 \%$, $\mathrm{n}=8)$, and proton pump inhibitors (PPI) $(15 \%, \mathrm{n}=6)$.

Conclusion: This study shows a high prevalence of PIM in hospitalized geriatric population and a beneficial effect of multidisciplinary intervention for their reassessment. PIM maintained at discharge are complex treatments to stop in the context of hospitalisation. A medication review at distance should be proposed to general practitioner in the discharge letter.

\section{P-61}

Preventable iatrogenic acute renal failure leading to lactic acidosis: a still topical adverse effect!

Cindy Riffardy ${ }^{1}$, Armance Grevy ${ }^{1}$, Prudence Gibert ${ }^{1}$, Clara Lopez ${ }^{2}$, Nathalie Mitha ${ }^{2}$, Nabil Zerhouni ${ }^{2}$, Pierrick Bedouch ${ }^{1,3,4}$, Gaetan Gavazzi ${ }^{2,4}$

${ }^{1}$ Pharmacy Department, Grenoble Alpes University Hospital, F38000 , France, ${ }^{2}$ University clinic of geriatric medicine, Grenoble Alpes University Hospital, F-38000, France, ${ }^{3}$ ThEMAS, CNRS/UGA TIMC-IMAG UMR 5525, F-38000, France, ${ }^{4}$ University Grenoble Alpes, F-38000, France

Introduction: Although risk factors are well known, drug combinations leading to acute renal failure (ARF), which can cause metformin overdose, are still prescribed especially in geriatric population.

Methods: We report a typical avoidable iatrogenic lactic acidosis case managed in our acute geriatric unit.

Results: Mrs C, 89 years old, started suffering from lower back pain a month before hospitalization. Her usual treatment contains metformin for type 2 diabetes, valsartan/hydrochlorothiazide combination and rilmenidine for hypertension. A paracetamol/opium and non-steroidal anti-inflammatory (NSAIDs) drugs prescription (meloxicam and topical administration of diclofenac) is introduced. Due to an increase of pain 2 week later, a $30 \mathrm{mg}$ long-acting and $10 \mathrm{mg}$ immediate release morphine prescription is added. Three days later, Mrs $\mathrm{C}$ is admitted to the emergency department for delirium. Clinically, she presents a bradypnea $(7 / \mathrm{mn})$ and depressed conscious status. A lactic acidosis and a respiratory failure were diagnosed related to metformin overdose in an iatrogenic ARF context and a morphine overdose. She is then admitted to intensive care unit where she is dialyzed, an antagonization of morphine is done and her usual treatment is stopped. Three weeks later, we notice a partial recovery of the renal function $\left(\mathrm{CrCl}: 34 \mathrm{~mL} / \mathrm{min} / 1.73 \mathrm{~m}^{2}\right.$ (CKDEPI/3 months ago: $\mathrm{CrCl}$ : $\left.51 \mathrm{~mL} / \mathrm{min} / 1.73 \mathrm{~m}^{2}\right)$.

Conclusion: Although this adverse effect is well known, this case report reminds us the need for adapting metformin dosage with renal function, and to avoid combination of drugs at risk of ARF. Furthermore, NSAIDs drugs are potentially inappropriate medications in the elderly with a $\mathrm{CrCl}<50 \mathrm{~mL} / \mathrm{mn}$ according to the STOPP list.

\section{P-62}

Analyse of reasons to keep potentially inappropriate medications after a multidisciplinary evaluation in an acute geriatric unit

Riffard Cindy ${ }^{1}$, Grevy Armance ${ }^{1}$, Gibert Prudence ${ }^{1}$, Lopez Clara ${ }^{2}$, Mitha Nathalie $^{2}$, Zerhouni Nabil ${ }^{2}$, Bedouch Pierrick ${ }^{1,3,4}$, Gavazzi Gaetan ${ }^{2,4}$

${ }^{1}$ Pharmacy Department, Grenoble Alpes University Hospital, F-38000, France, ${ }^{2}$ University clinic of geriatric medicine, Grenoble Alpes University Hospital, F-38000, France, ${ }^{3}$ ThEMAS, CNRS/UGA TIMC-IMAG UMR 5525, F-38000, France, ${ }^{4}$ University Grenoble Alpes, F-38000, France

Introduction: Potentially inappropriate medications (PIM) in elderly are an iatrogenic illness source. However, interventions to reduce PIM usually do not allow a complete stop of all PIM. This study's objective is to measure the rate of PIM, which are finally continued after multidisciplinary reevaluation and record the reasons why PIM still remain.

Method: Prospective study on prescriptions at admission (usual treatment) and discharge in patients over 75 years old hospitalized in acute geriatric unit from $01 / 25$ to $04 / 25 / 2018$. The aim of multidisciplinary intervention composed of a pharmacy students, pharmacy and medical residents and geriatricians, is to evaluate for each patient if PIM prescriptions according to v2 STOPP/START list are really inappropriate or not and to decide to stop or keep them.

Results: In the study, 101 patients were included and $70.3 \%(\mathrm{n}=71)$ had at least 1 PIM at home. In all, 147 PIM were found and $72.8 \%$ $(n=107)$ were discontinued after the multidisciplinary intervention. There are two main situations where PIM are continued: discontinuation proposed to the general practitioner at distance of hospitalization, and treatments with clinical situation that justifies their use. These cases concerns benzodiazepines $(40 \%, \mathrm{n}=16)$, neuroleptics $(20 \%, \mathrm{n}=8)$, and proton pump inhibitors (PPI) $(15 \%$, $\mathrm{n}=6$ ).

Conclusion: This study shows a high prevalence of PIM in hospitalized geriatric population and a beneficial effect of multidisciplinary intervention for their reassessment. PIM maintained at discharge are complex treatments to stop in the context of hospitalisation. A medication review at distance should be proposed to general practitioner in the discharge letter. 


\section{P-63}

Preventable iatrogenic acute renal failure leading to lactic acidosis: a still topical adverse effect!

Riffard Cindy ${ }^{1}$, Grevy Armance ${ }^{1}$, Gibert Prudence ${ }^{1}$, Lopez Clara ${ }^{2}$, Mitha Nathalie ${ }^{2}$, Zerhouni Nabil ${ }^{2}$, Bedouch Pierrick ${ }^{1,3,4}$, Gavazzi Gaetan ${ }^{2,4}$

${ }^{1}$ Pharmacy Department, Grenoble Alpes University Hospital, F38000 , France, ${ }^{2}$ University clinic of geriatric medicine, Grenoble Alpes University Hospital, F-38000, France, ${ }^{3}$ ThEMAS, CNRS/UGA TIMC-IMAG UMR 5525, F-38000, France, ${ }^{4}$ University Grenoble Alpes, F-38000, France

Introduction: Although risk factors are well known, drug combinations leading to acute renal failure (ARF), which can cause metformin overdose, are still prescribed especially in geriatric population.

Methods: We report a typical avoidable iatrogenic lactic acidosis case managed in our acute geriatric unit.

Results: Mrs C, 89 years old, started suffering from lower back pain a month before hospitalization. Her usual treatment contains metformin for type 2 diabetes, valsartan/hydrochlorothiazide combination and rilmenidine for hypertension. A paracetamol/opium and non-steroidal anti-inflammatory (NSAIDs) drugs prescription (meloxicam and topical administration of diclofenac) is introduced. Due to an increase of pain 2 week later, a $30 \mathrm{mg}$ long-acting and $10 \mathrm{mg}$ immediate release morphine prescription is added. Three days later, Mrs $\mathrm{C}$ is admitted to the emergency department for delirium. Clinically, she presents a bradypnea (7/mn) and depressed conscious status. A lactic acidosis and a respiratory failure were diagnosed related to metformin overdose in an iatrogenic ARF context and a morphine overdose. She is then admitted to intensive care unit where she is dialyzed, an antagonization of morphine is done and her usual treatment is stopped. Three weeks later, we notice a partial recovery of the renal function $\left(\mathrm{CrCl}: 34 \mathrm{~mL} / \mathrm{min} / 1.73 \mathrm{~m}^{2}\right.$ (CKDEPI/3 months ago: $\mathrm{CrCl}$ : $51 \mathrm{~mL} / \mathrm{min} / 1.73 \mathrm{~m}^{2}$ ).

Conclusion: Although this adverse effect is well known, this case report reminds us the need for adapting metformin dosage with renal function, and to avoid combination of drugs at risk of ARF. Furthermore, NSAIDs drugs are potentially inappropriate medications in the elderly with a $\mathrm{CrCl}<50 \mathrm{~mL} / \mathrm{mn}$ according to the STOPP list.

\section{P-64}

\#PPWhy? - a quality improvement project to rationalise prescription of proton pump inhibitors

\author{
Matthew North ${ }^{1}$, Annie Pervaiz ${ }^{1}$, Clifford Lisk ${ }^{1}$ \\ ${ }^{1}$ Division of Medicine, Barnet Hospital, Royal Free London NHS \\ Foundation Trust, London, UK
}

Introduction: Polypharmacy is increasing in older adults due to multimorbidity. Deprescribing guidelines support healthcare providers and patients in reducing or stopping medications that may be harmful or no longer needed with the goal of reducing medication burden or harm while improving quality of life [1]. Proton pump inhibitors (PPI) are widely prescribed; they are associated with hyponatremia, hypomagnesemia, Clostridium difficile diarrhoea and pneumonia. This study aims to determine the extent of PPI prescribing in patients admitted to a UK District General hospital and determine the confidence of doctors in training within Medicine regarding deprescribing.

Methods: Demographic data, PPI prescribing and their indications were assessed for all new patients admitted to a Medical short stay unit. Doctors were surveyed with regards to their confidence in deprescribing.

Results: 56 patients were identified. The majority were male (35) with an age range of $27-93$ years. $31 / 56$ patients (55\%) were prescribed a PPI. Only 10 patients $(32 \%)$ had a documented indication in the clinical notes for a PPI. These included concomitant steroid use, hiatus hernia and peptic ulcer disease. Confidence of doctors in deprescribing was low.

Key conclusions: This study indicates that a third of patients on a PPI admitted acutely to hospital did not have a clear indication for ongoing prescription of a PPI and junior doctor confidence in deprescribing was low. Formal screening to identify patients for whom deprescribing PPI is appropriate together with education of doctors and pharmacists to address the knowledge gaps in deprescribing could lead to appropriate prescribing, reduction in patient harm and cost savings.

\section{P-65}

\section{Relevance of heparin anticoagulant activity monitoring}

Lind Sandra $^{1}$, Potier Arnaud ${ }^{1}$, Toussaint-Hacquard Marie ${ }^{2}$, Demoré Béatrice $^{1}$, Perret-Guillaume Christine ${ }^{3}$, Benetos Athanase ${ }^{3}$

${ }^{1}$ Pharmacy, University Hospital, Nancy, France, ${ }^{2}$ Hematological Laboratory, University Hospital, Nancy, France Geriatrics,

${ }^{3}$ Geriatrics and Internal Medicine Unit, University Hospital, Nancy, France

Introduction: Anticoagulant activity monitoring is expected with unfractionated heparin (UFH), yet low molecular weight heparin (LMWH) monitoring by antifactor Xa (AFXa) is only recommended on therapeutic regimen among patients with renal insufficiency, extreme body weight or hemorrhagic history. AFXa should not be used for dose adjustment but only for overdose detection. This study aims at assessing relevance of anticoagulant activity monitoring as regards indication, implementation and interpretation in a 60-bed acute geriatric unit.

Method: A prospective observational study was carried out from February to April 2018. Data were reported on every admitted patient being prescribed LMWHs or UFHs regarding clinico-biological condition, dosing, indication and anticoagulant activity monitoring. Relevance of dosage indication, its implementation and results interpretation were determined according to summaries of product characteristics and literature recommendations.

Results: 81 patients of the 285 admitted during the study had either LMWH or UFH prescription: $53(65 \%)$ at thrombo-prophylactic doses and $28(35 \%)$ at therapeutic doses. $97(76 \%)$ of 127 anticoagulant activity tests performed were relevant according to the indication. 49 (39\%) were non-compliant with implementation recommendations: 29 (59\%) resulted from biological sampling timing error, $5(10 \%)$ in performed tests selection and $15(39 \%)$ resulted from both. These non-compliant samplings couldn't be meaningfully interpreted.

Key conclusions: Overall, a number of AFXa monitoring were nonrelevant according to the indication, due to wrong test prescription or sampling timing. This underlines the risk of hemorrhagic or thromboembolic events if non-relevant monitoring is followed by inappropriate dosing adjustment caused by misinterpretation. 


\section{P-66}

Which are the best known drugs in elderly patients?

Elena Valle Calonge ${ }^{1}$, Pablo Enrique Solla Suárez ${ }^{1}$, Javier Montero Muñoz ${ }^{1}$, Eva María López Álvarez ${ }^{1}$, Iratxe Molinuevo $\mathrm{Alba}^{1}$, Juan José Solano Jaurrieta ${ }^{1}$

${ }^{1}$ Hospital Monte Naranco, Oviedo, Spain

Objectives: To study elderly's knowledge of different drug groups. Materials and methods: Descriptive cross-sectional study in April/ 2018. Included admitted patients from an Acute Geriatric Unit. Exclusion criteria was cognitive impairment or no collaboration.

Variables: Age, sex, Barthel Index, education level, drugs number, drug names, dose and indication. For analysis, Chi squared test was used.

Results: Drugs: PPIs (71.2\%), diuretics (66.7\%), ACEIs-ARBs $(45.5 \%)$, oral anticoagulants $(42.4 \%)$, benzodiazepines $(39.4 \%)$. Drug names knowledge: anticoagulants (82.1\%), antiepileptics $(75 \%)$, minor analgesics $(71.4 \%)$ and antiarrhythmics $(71.4 \%)$. Insulin was used in $9.1 \%$ and this was known in $66.7 \%$. Oral antidiabetics were used in 24.2 and $43.8 \%$ knew the drug name; among these, $71.4 \%$ $(\mathrm{p}=0.049)$ managed the dose well, and $100 \%$ knew the indication $(\mathrm{p}=0.002)$. Among those who consumed PPIs, $44.7 \%$ knew the drug, of which $90.5 \%$ knew the drug's function $(p=0.001)$. In the group that used diuretics, $63.6 \%$ knew the drug, from which $89.3 \%$ knew the indication $(\mathrm{p}<0.001)$. Among those taking ACEIs-ARBs, $45.5 \%$ knew the drug, without finding relationship to the knowledge of the indication. Among those using anticoagulants, $82.1 \%$ knew the drug, from which $91.3 \%$ knew what for $(\mathrm{p}<0.001)$. Among those who consumed benzodiazepines, 50\% knew the drug, from which $92.3 \%$ knew what it was for $(\mathrm{p}=0.027)$. Among those who used minor analgesics $(21.2 \%)$ and knew the name (71.4\%), 90\% knew what it was for $(\mathrm{p}=0.015)$.

Conclusion: In our study, anticoagulants were the drugs best known by the patients. PPIs were the most used, but less than half of the users knew what was for. Although less than $50 \%$ of oral antidiabetics users knew the drug, all of them knew the indication.

\section{P-67}

Quality of care for geriatric patients: can we improve discharge prescriptions?

Marion Collignon ${ }^{1}$, Nathalie Marie-Nelly ${ }^{2}$, Gita Motamed ${ }^{2}$, MarieChristine Sagnier ${ }^{1}$, Jean-Philippe David ${ }^{2}$, Vanida Brunie ${ }^{1}$

${ }^{1}$ Pharmacy, University Hospital Henri Mondor, Geriatric site Emile Roux, Limeil-Brévannes, France, ${ }^{2}$ Geriatric Unit, University Hospital Henri Mondor, Geriatric site Emile Roux, Limeil-Brévannes, France

Introduction: Elderly patients are at risk of drug-related iatrogenia and are often prescribed a large amount of medicines at hospital discharge. The aim of this study is to evaluate the admissibility and clarity of these prescriptions.

Methods: This non-interventional study has 1 inclusion criteria: every prescription of patients returning home after hospital discharge. The analysis focuses on 2 criteria and 11 indicators: 1/Admissibility according to legislation (patient's name, age and weight, physician's name and signature, date, prescription in international non-proprietary name), 2/Understandability (posology, route of administration, vocabulary, unit conversion).

Results: Prescriptions of 180 geriatric patients were analyzed. They contained in average $9.38 \pm 3.16$ drugs and most of them (78\%) had issues regarding either admissibility or understandability. Indeed, $43 \%$ were admissible regarding our 7 indicators. In $68 \%$ of the prescriptions, patients' weight was filled out whereas $61 \%$ of the prescriptions were completely written in international non-proprietary name. Concerning clarity, $42 \%$ were understandable. Posology was not clear for $37 \%$ of the prescriptions, $17 \%$ raised questions about route of administration, $26 \%$ contained unintelligible vocabulary and $0.47 \%$ did not explain unit conversion. Since $27 \%$ of our elderly patients return home without nurse assistance, issues about their drug intake and compliance need to be identified.

Conclusions: Only $22 \%$ of prescriptions met all the criteria. Health professionals (physician, nurse and pharmacist) have to ensure understandability of patients' treatments. The implementation of medication reconciliation and a pharmaceutical consultation at hospital discharge could improve prescription writing and drug intake security at home, especially for patients without assistance.

\section{P-68}

The proton pump inhibitors use on elderly-a faster way to death?

M. A. Fernandes ${ }^{1}$, M. T. Torres ${ }^{1}$, D. D. Borges ${ }^{1}$, R. V. Alves ${ }^{1}$, I. R. Figueiredo ${ }^{1}$, A. M. Antunes ${ }^{1}$, H. Gruner ${ }^{1}$, A. Panarra ${ }^{1}$

${ }^{1}$ Centro Hospitalar de Lisboa Central, EPE, Lisbon, Portugal

Introduction: The proton pump inhibitors (PPIs) are largely prescribed since the first one was released on $80 \mathrm{~s}$. According to several studies, pneumonia, vitamin B12 deficiency, dementia, $C$. difficile infection, kidney disease and osteoporosis-related fractures have been addressed as long term adverse effects of using PPIs. The elderly population accounts for approximately one half of patients who are currently taking this class of drugs and almost $40 \%$ have no indication for being on PPI therapy.

Methods: The population of this cross-sectional study included patients aged 65 years and older (mean age 81 years) admitted to an Internal Medicine Department between 1st January and 31st December of 2017. Data obtained included epidemiologic data, drug history with focus on acid suppression therapy, past medical history and comorbid conditions with determination of Charlson Comorbidity Index. Pneumonia as reason of admission, fracture, $C$. difficile infection, dementia, chronic renal disease and level of vitamin B12, as well as the length of stay and mortality constituted the outcomes.

Results: Of the 286 patients, 16 patients were excluded for not meeting the eligibility criteria. From 270 patients analysed, 147 patients were women $(54.4 \%)$ and 123 were men (45.6\%). One hundred and twenty three patients $(45.6 \%)$ have been on IBP therapy. About $55 \%$ of pneumonia cases on admission were in patients taking IBPs (vs $15.6 \%$ on patients not being on IBP; $p=0.137$ ). On the contrary, fractures were more common in patients not being at IBP therapy $(56.8 \%$ vs $43.2 \%$; $=0.730)$. Almost $59 \%$ of patients with dementia were under IBP $(\mathrm{p}=0.007)$. Only 66 patients studied have chronic kidney disease and more than half $(53 \%)$ have been on IBP therapy $(p=0.161)$. Mean level of vitamin B12 were higher on people on PPI (603.5 vs $567.1 \mathrm{pg} / \mathrm{mL})$. The only patient who developed $C$. difficile infection was not on PPI.

Key conclusions: Aging is intrinsically associated with modifications in the organ functions increasing the risk of adverse effects of drugs and mortality, as we show on our study. Therefore, the elderly people should be submitted to a periodic evaluation regarding the need for being on PPI therapy. 


\section{P-69}

What are my patients taking and what should $I$ be doing about it

\author{
Y. L. Yasmin Latief ${ }^{1}$, C. L. Clifford Lisk ${ }^{2}$ \\ ${ }^{1}$ PBH Potters Bar Hospital, Potters Bar, UK, ${ }^{2}$ BH Barnet Hospital, \\ Barnet, UK
}

Older adults are increasingly at risk because of multimorbidity. Polypharmacy and potentially inappropriate medications (PIMS) have been associated with many negative health outcomes; reduced quality of life, adverse drug reactions, falls, nonadherence, hospitalisations and mortality. Hospital physicians have an opportunity to prioritize essential medications and deprescribe PIMS. This study assessed the medication consumption and the prevalence of PIMS on all patients discharged from a UK rehabilitation hospital. A retrospective discharge summary review of all patients above 65 years old discharged from a community hospital in the first quarter of 2017 was undertaken. Medication consumption was determined by examining hospital discharge summaries and PIMs were identified using the European Union (7)-PIM (EU7-PIM) list. 66 patients were assessed with a preponderance of females (43). 21 patients were aged 91 and above. The total number of medicines being taken was 433 with analgesics (50), laxatives (42), Adcal D3 (39), Cardiac medications (38) and PPIs (30) the commonest prescribed medicines. The Commonest PIMs were PPIs and Ranitidine (36), Opiates (23) Senna (19) and anticholinergics (15). Antidepressants (7), Digoxin (7) benzodiazepines (5), antibiotic prophylaxis ${ }^{4}$, high dose iron supplements ${ }^{4}$ and verapamil (1) featured less prominently. Full implementation of the EU(7)-PIM list would have resulted in one-in-four medications being discontinued. High levels of medication consumption amongst older patients discharged from hospital reflects high symptom burden and continued prescribing of PIMs. Deprescribing tools focussing on common PIMs should be implemented widely as part of a comprehensive geriatric assessment to reduce patient harm from inappropriate prescribing.

\section{P-70}

The OptimaMed intervention study to reduce medications of questionable benefit among nursing home residents with advanced dementia: first results

Kröger Edeltraut ${ }^{1}$, Wilchesky Machelle ${ }^{2}$, Voyer Philippe ${ }^{1}$, Champoux Nathalie $^{3}$, Monette Johanne ${ }^{4}$, Giguère Anik ${ }^{1}$, Morin Michèle ${ }^{1}$, Carmichael Pierre-Hugues ${ }^{5}$, Aubin Michèle ${ }^{1}$, Arcand Marcel ${ }^{6}$

${ }^{1}$ Université Laval, Centre d'excellence sur le vieillissement de Québec, CIUSSSCN, Québec, Canada, ${ }^{2}$ McGill University, Donald Berman Maimonides Geriatric Research Centre, Montreal, Canada, ${ }^{3}$ Université de Montréal, Institut Universitaire de Gériatrie de Montréal, Montréal, Canada, ${ }^{4} \mathrm{McGill}$ University, Montréal, Canada, ${ }^{5}$ Centre d'excellence sur le vieillissement de Québec, CIUSSSCN, Québec, Canada, ${ }^{6}$ Université de Sherbrooke, Institut Universitaire de Gériatrie de Sherbrooke, Sherbrooke, Canada

Background: Nursing home (NH) residents with advanced dementia receive multiple medications. With disease progression care goals shift to palliative care and medications may need to be discontinued because of changes in their harm-benefit ratio. Building on a successful pilot study we performed this controlled demonstration study to evaluate the effects of an inter-professional knowledge exchange (KE) intervention with a focus on antipsychotics use to reduce medication of questionable benefit in these residents.

Methods: A 6-month intervention was implemented in $4 \mathrm{NH}$ in Quebec while $3 \mathrm{NH}$ served as controls. (1) Families of participating residents received an information leaflet on optimal medication use in advanced dementia; (2) the facilities' nurses, pharmacists and physicians participated in $2 \mathrm{KE}$ sessions. The pharmacists performed 1 medication review for each participant using lists of "mostly", "sometimes" or "exceptionally" appropriate medications on which a multidisciplinary Delphi panel had agreed in prior research. These lists were tailored for NHs. Recommendations from the review were discussed with nurses, physicians and families. Residents' agitation and pain levels were monitored using the CMAI and PACSLAC scales.

Results: 124 out of 298 eligible residents participated in follow-up. 28 health professionals participated in the 1 st KE session. The intervention was well received in all $\mathrm{NH}$. Numbers, types and appropriateness categories of medications used by the residents at baseline and follow-up will be presented, as well as levels of agitation and comfort during follow-up.

Conclusions: This interdisciplinary intervention for $\mathrm{NH}$ residents with advanced dementia was feasible and may have reduced inappropriate medication use.

\section{P-71}

Knowledge of older patients about pharmacological treatment at discharge

Neus Gual ${ }^{1}$, Gabriela Bernal ${ }^{2}$, Ana Maria de Andrés ${ }^{1}$, Laura Mónica Perez $^{1}$, Manuel Arévalo ${ }^{1}$, Miriam Corominas ${ }^{1}$, Mónica Mesas ${ }^{1}$, Marco Inzitari ${ }^{1}$

${ }^{1}$ Parc Sanitari Pere Virgili, Barcelona, Spain, ${ }^{2}$ Universitat Autònoma de Barcelona, Barcelona, Spain

Objective: Explore the knowledge of older patients about their pharmacological treatment at hospital discharge and detect where they do more mistakes.

Methods: We performed a short interview to older patients (or caregivers) being discharged home from an intermediate care hospital in Barcelona. We asked them to explain the pharmacological treatment they had been told to follow at discharge. We asked for the drugs name, dose, dosage, and associated risks.

Results: 44 patients (68.9\% female), mean age of $83+7$ years, had a mean number of prescribed drugs of $7.6+3.4$. The mean number of mistakes was $6.1+7.8$ at discharge, decreasing to $4.7+6.2$ after one week at home. $77.8 \%$ of the patients $(n=35)$ didn't have a correct knowledge about their treatment. When analyzing the different drugs, we observed significant differences in the number of mistakes $(67.9 \%$ of prescribed cardiovascular drugs had errors on the part of patients, compared to $13.4 \%$ of psychotropic drugs and $3.7 \%$ of painkillers) and in the type of mistakes (regarding doses knowledge, cardiovascular and psychotropic drugs had the highest error rate regarding: 22.7 and $16.7 \%$ respectively; regarding dosage, respiratory agents had the highest error rate $(26.3 \%)$ ). There is a high rate of ignorance regarding the risk of cardiovascular $(89.1 \%)$ and psychotropic drugs $(53.30 \%)$.

Conclusions: Almost $80 \%$ of older patients do not have an accurate knowledge of their pharmacological treatment at hospital discharge. Cardiovascular and psychotropic treatments present greater errors of understanding; and highlights a significant ignorance of the risk that these drugs present, something to be taken into account especially in this elderly and polymedicated population. 


\section{Area: Pre and post operative care}

P-72

Multidimensional Prognostic Index is associated with mortality in older patients undergoing transcatheter aortic valve implantation

J. A Goudzwaard ${ }^{1}$, S. Chotkan ${ }^{1}$, M. J. A. G de Ronde-Tillmans ${ }^{2}$, N. M. van Mieghem ${ }^{2}$, A. Pilotto ${ }^{3}$, N. El Faquir ${ }^{2}$, M. J. Lenzen ${ }^{2}$, P. P. T. de Jaegere ${ }^{2}$, F. U. S. Mattace-Raso ${ }^{1}$

${ }^{1}$ Section of Geriatrics, Department of Internal Medicine, Erasmus University Medical Center, Rotterdam, the Netherlands, ${ }^{2}$ Department of interventional cardiology, ThoraxCenter, Erasmus University Medical Center, Rotterdam, the Netherlands, ${ }^{3}$ Department of Geriatric Care, E.O. Galliera Hospital, Genoa, Italy

Background: Predicting outcome after Transcatheter Aortic Valve Implantation (TAVI) for older, mostly frail patients is still challenging since traditional cardiovascular risk scores are appear to be unreliable in this population. The multidimensional prognostic index (MPI) is a validated predictive tool for mortality based on information in a standardized Comprehensive Geriatric Assessment (CGA). The aim of this study was to evaluate whether the MPI was associated with mortality in older patients undergoing TAVI.

Methods: TAVI Care and cure is an observational ongoing study including consecutive patients undergoing TAVI at the Erasmus Medical Center. Prior to TAVI, a complete CGA was performed. Baseline demographic and clinical data were collected. The MPI score was calculated and divided into three groups, low-risk (MPI-1 value $\leq 0.33$ ), moderate-risk (MPI-2 value $0.34-0.66$ ), and severerisk (MPI-3 $\geq 0.67$ ), based on earlier established cut-offs. Mortality data were obtained from the Dutch Civil Registry and was assessed at one and 12 months.

Results: 295 patients were included. Mean age was $81.0 \pm 6.2$ years. 121 patients $(37.0 \%)$ belonged to the MPI-1 group, 184 patients $(56.3 \%)$ to the MPI-2 group, and 11 patients $(3.4 \%)$ to the MPI-3 group. Mean MPI score was $0.39( \pm 0.15)$. 1-year mortality rates were significantly different between the MPI groups $(p=0.03)$, 30 -day mortality rates were not different $(\mathrm{p}=0.14)$. Belonging to MPI-2 of MPI-3 group at baseline was associated with increased risk of 1-year mortality (OR 2.7 (95\% CI 1.17-6.4).

Discussion: In this study we found that in older patients undergoing TAVI the MPI score can be used to predict prognosis.

\section{P-73}

Consultants of the week model in orthogeriatric care $(\mathrm{CoW})-$ 1 year data

\section{Radcliffe Lisk ${ }^{1}$, Hazel Watters ${ }^{1}$, Keefai Yeong ${ }^{1}$ \\ ${ }^{1}$ Ashford and St. Peter's NHS Trust, Surrey, UK}

Objectives: There are various models of orthogeriatric care. This includes older models such as the traditional model of orthopaedic care and post-operative geriatric care. Newer models include orthopaedic patients having routine orthogeriatric review, admitted under geriatricians and shared care (patients are managed by a named orthopaedic surgeon and a named orthogeriatrician within a defined orthogeriatric team).

Methods: We use the shared care model but noted that the orthogeriatrician focused on the hip fracture patients $(\mathrm{NoFs})$ whereas the orthopaedic surgeon focused on the other trauma patients. The medical needs of the trauma patients were not addressed early and at times the orthopaedic needs of the hip fracture patients were delayed. In November 2016, we implemented the CoW, whereby the same orthopaedic surgeon and orthogeriatrician saw patients on the ward round.

Results: 1 year before the CoW model (Nov-Oct 2016), 1531 patients (including NOFs and other trauma) were admitted with 14,191 bed days and Length of Stay (LOS) - 9.27 days. 1 year after the CoW model (Nov-Oct 2017), 1553 patients with 12,969 bed days, LOS-8.35 days 1447 bed days saved; cost savings (£300/bed) is $£ 434,100$ Readmissions: $11.3 \%$ before CoW, $14.4 \%$ after CoW. (50 extra staying 8 days costing $£ 120,000$ ). Net saving $£ 314,100$. For NoFs, LOS remained stable -13.25 days (403 NoFs) and 1 year after the CoW model, LOS 13.35 days (381 NoFs).

Conclusion: This model of care addresses the needs of older population with frailty and complex co-morbidities whereby continuity and working together achieves cost savings.

\section{P-74}

Length of hospital stay after hip fracture associated with shortterm mortality after hospital discharge among persons with Alzheimer's disease

Piia Lavikainen ${ }^{1}$, Heidi Taipale ${ }^{1}$, Marjaana Koponen ${ }^{1}$, Antti Tanskanen ${ }^{2}$, Jari Tiihonen ${ }^{2}$

${ }^{1}$ University of Eastern Finland, Kuopio, Finland, School of Pharmacy, ${ }^{2}$ Department of Clinical Neuroscience, Center for Psychiatry Research, Karolinska Institutet and Stockholm County Council, Stockholm, Sweden and Department of Forensic Psychiatry, Niuvanniemi Hospital, Kuopio, Finland

Introduction: Persons with Alzheimer's disease (AD) are in higher risk of hip fractures than dementia-free persons and have worse prognosis after hip fracture. Hospital stays after hip fracture have shortened along time. We aimed to investigate the association between length of hospital stay after hip fracture and mortality after discharge among persons with AD.

Methods: We identified persons newly diagnosed with $\mathrm{AD}$ in 2005-2011 ( $\mathrm{n}=70,718)$ who experienced first hip fracture after AD diagnosis in 2005-2015 $(\mathrm{n}=5754)$ from Finnish health care registers. Length of hospital stay was defined as a sum of the consecutive days spent in hospital after hip fracture until discharge measured from the Finnish Care Register. Deaths within 30-days after discharge were identified from the Causes of Death Register. Association between deciles of length of hospital stay after hip fracture and short-term death was assessed using Cox's proportional hazards regression model.

Results: Mean length of hospital stay after a hip fracture decreased from 52.9 (SD 63.3) days in 2005 to 19.4 (SD 23.6) days in 2015. First (1-4 days; adjusted hazard ratio (HR) $1.93,95 \%$ CI $1.10-3.40$ ), second (5-6 days; adjusted HR 1.85, 95\% CI 1.05-3.27) and third (7-10 days; adjusted HR 1.86, 95\% CI 1.07-3.24) deciles of lengths of hospital stays were associated with an increased risk of mortality after discharge when compared to the sixth decile of length of stays (21-26 days).

Conclusions: $\mathrm{Sh}$

orter length of hospital stay after hip fracture is associated with an increased risk of death after hospital discharge among persons with AD. 


\section{P-75}

The introduction of the Cumulated Ambulatory Score (CAS) as an outcome measure in the acute rehabilitation of patients with hip fracture

\author{
Nathan Swingewood ${ }^{1}$, Atef Michael ${ }^{2}$ \\ ${ }^{1}$ Russells Hall Hospital, Dudley, UK, ${ }^{2}$ Russells Hall Hospital, \\ Dudley, UK
}

Introduction: The CAS is a validated functional outcome measure that allows an assessor to award an objective score based on a patients ability to complete three functional activities depending on the level of assistance they need to do so. The activities are: Get in and out of bed, Sit to stand to sit from chair and to walk. The score for each is 0 if not able to perform the task despite human assistance or cueing, 1 if able to with human assistance or cueing or 2 if able to with no human assistance or cueing. The aim of this study was to investigate how useful the CAS could be for measuring functional progress during rehabilitation of hip fracture patients.

Methods: Prospective study of consecutive hip fracture patients admitted in a 6 months period to a UK teaching hospital. The CAS prior to fracture, on the first postoperative day and on discharge were collected.

Results: 240 patients were admitted in the study period 18 patients were excluded; 4 who were treated conservatively and 14 who died. 222 were included; 163 females and 59 males with mean age of 83 and 79.6 years respectively. The average CAS before fracture was 5.6, on the first postoperative day was 2.2 and on discharge was 3.6. On discharge patients were generally able to do the activities with less assistance and mobilise further but the tool does not allow measurement of this level of improvement.

Conclusion: The utilisation of the CAS for hip fracture patients allowed to evaluate the pre-fracture activity status retrospectively and provided the therapists with an objective and easy tool to assess the functional status and monitor the postoperative progress during the acute rehabilitation phase. However the tool is not sensitive enough to monitor small improvement in function.

\section{P-76}

Experiences with community services in surgical and non-surgical hip fracture patients post-discharge in Singapore

\section{Y. L. Foong ${ }^{1}$, S. H. G. $\mathrm{Lau}^{2}$, J. M. Yi ${ }^{2}$, H. M. Ng${ }^{3}, \mathrm{~S} \mathrm{Su}^{1}$, Y. H. Ang ${ }^{1}$, J. U. Mallya ${ }^{1}$ \\ ${ }^{1}$ Khoo Teck Puat Hospital, Yishun, Singapore, ${ }^{2}$ Community Care Team, Yishun, Singapore, ${ }^{3}$ Hip Fracture Unit, Yishun, Singapore}

Introduction: The hip fracture unit in Khoo Teck Puat Hospital, Singapore, has been in operation since 2015. During this time, the Community Care Team (CCT) has provided step-down care in the home to both post-operative and non-surgical patients.

Methods: This retrospective analysis compares outcomes between surgical and non-surgical patients who are cared for at home, looking at readmission and mortality rates. It also describes patient demographics and the categorization of their medical complexity, to illustrate how their needs are determined and met.

Results: Of 73 patients seen by CCT, $68.5 \%$ were very elderly (81110 years old), with high Clinical Frailty Scale (CFS) scores (78.1\% CFS $>5$ ). 24 patients $(33 \%)$ had surgery. Patients were medically complex, with $71.2 \%$ having at least 4 comorbidities. $85 \%$ required a high level of care ( 2 or 3 ) and the services provided included medical stabilization, nursing care, rehabilitation and carer training. Nonsurgical patients had readmission rates of 0.33 at 1 month and 0.55 at
3 months post-discharge. In comparison, surgical patients had rates of 0.13 at 1 month post-discharge and 0.21 at 3 months. In non-surgical patients, mortality was $32.7 \%$ at 6 months post-discharge, compared to $4.7 \%$ in surgical patients.

Conclusion: CCT is useful for the management of frail, medically complex patients who sustained hip fractures. Even within this frail group, surgical intervention seems to give much better outcomes. This continues to strengthen the case for encouraging operative management as it can prevent negative outcomes in this vulnerable population.

\section{P-77}

Geriatrician case-note summarising improves income-dependent clinical coding in older patients undergoing vascular surgery

Emma Mitchell ${ }^{1}$, Nicola Beauvois ${ }^{2}$, James Rainbow ${ }^{2}$, Katie Rainey ${ }^{3}$, Rebecca Winterborn ${ }^{4}$, David Shipway ${ }^{5}$

${ }^{1}$ Geriatric Medicine Trainee, North Bristol NHS Trust, Bristol, UK, ${ }^{2}$ Department of Clinical Coding, North Bristol NHS Trust, Bristol, UK, ${ }^{3}$ Consultant Geriatrician, North Bristol NHS Trust, Bristol, UK, ${ }^{4}$ Consultant Vascular Surgeon, North Bristol NHS Trust, Bristol, UK, ${ }^{5}$ Consultant Geriatrician, North Bristol NHS Trust, Bristol, UK

Introduction: NHS hospitals are reimbursed for care provided based on clinical codes generated from patients' records. This is termed 'Payment by Results (PbR)' and is based on a national tariff system [1]. Whilst PbR was designed to reward efficiency and to focus care towards quality and innovation, there are inherent difficulties related to coding accuracy, which is largely dependent on clinical record quality [1-3]. Coding is performed by clinical coders, who are not permitted to interpret records. Failure to document secondary diagnoses and complications is a common source of under-coding and lost revenue [2, 4-5]. We hypothesised that physician case-note summarisation would improve documentation and accuracy of coding in older vascular patients.

Methods: Case-notes for 47 randomly selected patients aged $>65$ admitted to vascular surgery between January-March 2017 were summarised by 3 geriatricians. Repeated coding was then conducted and the revised coding outcome updated to determine effect on episode tariff.

Results: Using the revised coding tariffs, departmental income was uplifted by $£ 34,610$. This represents an average increase of $£ 736.38$ per case-note summary. Coding discrepancies occurred mostly due to inadequately documented comorbidities and laboratory results. In 2017-2018, 1207 patients aged $>65$ were admitted under vascular surgery. Extrapolating these data to this population indicates potential missed revenue exceeding $£ 800,000$.

Conclusions: Thorough case-note summarisation is central to Comprehensive Geriatric Assessment (CGA) and is part of routine geriatrician practice. These data indicate that note summarisation as part of CGA may generate income to justify financial investment in Geriatric liaison services for older surgical patients.

References:

1. Mahbubani K, Georgiades F, Lin Goh E et al (2018) Cliniciandirected improvement in the accuracy of hospital clinical coding. Future Healthc J 5(1):47-513. https://assets.publishing.service.gov.uk/ government/uploads/system/uploads/attachment_data/file/213150/PbRSimple-Guide-FINAL.pdf2

2. Martinou E, Shouls G, Betambeau N (2014) Improving the accuracy of operation coding in surgical discharge summaries. BMJ Qual Improv Rep u202053.w19904. Chks.co.uk. (2018). [online]. http://www.chks.co.uk/m/userfiles/The_quality_of_clinical_coding_ in_the_NHS.pdf5 
3. Nouraei S, Virk J, Hudovsky A, Wathen C, Darzi A, Parsons D (2015) Accuracy of clinician-clinical coder information handover following acute medical admissions: implication for using administrative datasets in clinical outcomes management. J Public Health 38(2):352-362

\section{P-78}

Fit for surgery? Developing a perioperative geriatric service for frail older people undergoing surgery

\section{Zeinab Majid ${ }^{1}$, Leo Feinberg ${ }^{1}$, Nigel Page ${ }^{1}$, Huma Naqvi ${ }^{1}$ \\ ${ }^{1}$ Sandwell and West Birmingham Hospitals NHS Trust, Birmingham, UK}

Introduction: There are increasing number of older patients undergoing surgery. Older patients are a known group with increased complications, length of stay, and mortality. Frailty is a marker of risk requiring recognition in ageing populations. We outline a review of a surgical liaison service at a Birmingham district general hospital established from 2015, designed to provide geriatric input for perioperative general surgical patients.

Methods: A spot audit of elective and emergency general surgical patients over 65 years was undertaken in 2015 , evaluating the quality of frailty screening and assessment; furthermore, identifying those requiring medical input. Subsequently, this led to development of the service, which was evaluated at 12 and 24 months, with an re-audit carried out in February 2018.

Results: We initially identified prevalence of frailty at $52 \%$. The surgical teams had poor recognition of frailty, with minimal medical input, despite identifying $79 \%$ of the cohort warranting a medical review. Up until December 2017, 230 referrals were made and 184 individuals seen. The majority of patients were more complex than initial referral reasons. $80 \%$ of patients were appropriately referred, with the majority discharged home. 6 month mortality was 33 and $7 \%$ at 12 and 24 months respectively. 14\% of patients transferred for ongoing geriatric care. The re-audit in 2018 identified similar rates of frailty prevalence, assessment and screening.

Conclusions: We have identified surgical frail patients are multimorbid with complex frailty-associated syndromes. A surgical liaison service has been invaluable in a semi-collaborative setting providing geriatric input, laying the foundations for joint team working in the future.

\section{P-79}

Implementation of coMplex PeRi-operatiVe intervEntion in olDer patients with cancer (IMPROVED program)

Frederique Peschaud ${ }^{1}$, Tristan Cudennec ${ }^{2}$, Philippe Caillet ${ }^{3}$, Mathilde Gisselbrecht ${ }^{4}$, Pauline Occeli ${ }^{5}$, Denis Angelique ${ }^{6}$, De Laure Decker ${ }^{7}$, Juliette Podevin ${ }^{8}$, Dominique Fletcher', Nicola De Angelis ${ }^{10}$, Pierre Cattan ${ }^{11}$, Jane Poincenot ${ }^{12}$, Benoit Plaud $^{13}$, Christophe Tournigand ${ }^{14}$, Thomas Aparicio ${ }^{15}$, Sandrine Touzet $^{16}$, David Moszkowicz ${ }^{1}$, Marie Laurent ${ }^{3}$, Florence CanouiPoitrine $^{17}$, Elena Paillaud ${ }^{4}$

${ }^{1}$ Hopital Amboise Paré, Service Chirurgie, Boulogne Billancourt, ${ }^{2}$ Hopital Amboise Paré, service de gériatrie, Boulogne-Billancourt, ${ }^{3} \mathrm{CHU}$ Henri Mondor, Service Geriatrie, Creteil, ${ }^{4}$ Hopital Europeen Georges Pompidou, service de gériatrie, Paris, ${ }^{5} \mathrm{CHU}$ Lyon, Service de santé publique, ${ }^{6} \mathrm{CHU}$ Lyon, Service santé publique, ${ }^{7} \mathrm{CHU}$ de Nantes, service de gériatrie, Nantes, ${ }^{8} \mathrm{CHU}$ de Nantes, service de chirurgie, Nantes, ${ }^{9}$ Hopital Amboise Paré, service d'anesthesie,
Boulogne-Billancourt, ${ }^{10} \mathrm{CHU}$ Henri Mondor, Service chirurgie, Creteil, ${ }^{11} \mathrm{CHU}$ Saint Louis, service chirurgie, Paris, ${ }^{12} \mathrm{Centre}$ Hospitalier Intercommunal de Creteil, service de chirurgie, ${ }^{13} \mathrm{CHU}$ de Saint Louis, service d'anesthesie, ${ }^{14} \mathrm{CHU}$ Henri Mondor, service d'oncologie, ${ }^{15} \mathrm{CHU}$ Saint Louis, Oncologie Digestive, Paris, ${ }^{16} \mathrm{CHU}$ Lyon, service de santé publique, ${ }^{17} \mathrm{CHU}$ Henri Mondor, Santé publique, Creteil

Introduction: Nearly $50 \%$ of patients are older than 70 years at diagnosis of digestive cancer. Surgical resection is the first line strategy of treatment. Despite improvement in surgical techniques and development of rehabilitation programs, the rate of postoperative complications remains high. Peri-operative involvement of geriatricians may improve care management older cancer patients.

Methods: During a 6 months run-up period (emerging project), we structured a multi-professional network (digestive surgeons, anesthetists, geriatricians, digestive oncologists, epidemiologists), we elaborated a innovative peri-operative geriatric intervention (Improved program) in digestive surgery setting based on evidence-based data. We build a dedicated evaluation plan by determinate the best design for assessing geriatric intervention in this complex context and choose the more appropriate endpoints.

Results: We will include 554 patients aged 75 or more with resectable digestive cancer in a randomized controlled trial. The intervention is based on (1) a preoperative geriatric assessment, focusing on frailty parameters and developing a coordinated program of tailored geriatric interventions (2) a postoperative shared care with an integrated care model where both surgeon and geriatrician share responsibility for the patient management in surgical ward. This geriatric postoperative management will be focus on prevention and correction of complications, early mobilization, optimal nutritional support. The main endpoint is Grade II or higher post-surgical complications rate according Clavien-Dindo classification within 30 days after the surgical procedure.

Conclusion: We expected to demonstrate a benefit of a peri-operative shared management model to decrease the risk of post-surgical complications in older patients with digestive cancer.

\section{P-80}

\section{Pelvic ring fractures in geriatric patients}

Ioana Dana Alexa ${ }^{1}$, Bogdan Velniceasa ${ }^{1}$, Anca Iuliana Pislaru ${ }^{1}$, Ramona Stefaniu ${ }^{1}$, Teodor Stefan Gheorghevici ${ }^{1}$

${ }^{1}$ University of Medicine and Pharmacy "Grigore T Popa" Iasi, Iasi, Romania

Introduction: Due to increasing life expectancy, more and more pelvic fractures occur in elderly patients. These fractures are a different entity-fragility fracture.

Materials and methods: In the period 2013-2016, 123 patients with fragility pelvic fractures were treated in our clinic. The mean age of patients was 86.5 years. 108 patients were women and 15 men. All patients received pelvic X-ray and CT scan. Fractures were categorized by Rommens [1] in 56 fractures type Ia, 38 fractures type Ib, 4 fractures type IIa and 25 fractures type IIc. As mechanism, lateral compression (LC) was encountered 6 times more frequently than antero-posterior compression (AP). The mortality rate at 30 days was $13 \%$.

Therapy and progression: For both types of fracture treatment was conservative. For type I fractures primary care was walking with weight bearing as tolerated. 30-days X-ray showed fracture consolidation in most cases, when full weight bearing was allowed. For type II fractures started with bed rest for 30 days followed by walking with weight bearing. For the other cases walking with weight bearing was 
allowed when radiological evidence of fracture consolidation. In 12 cases of type I fractures and 14 cases of type II fractures, a minor displacement of the fracture was observed at the 30 days X-ray control.

Conclusions: Fragility pelvic fractures are a relatively new entity. Type I and II fractures can be treated conservatively, but in type II, a minimally-invasive fixation would be desirable that would allow greater fracture stability and a faster mobilization of patients.

\section{P-81}

The effect of timing of surgery and comorbidities on in-hospital mortality of the geriatric hip fracture population

Ioana Dana Alexa ${ }^{1}$, Bogdan Veliceasa ${ }^{1}$, Bogdan Puha ${ }^{1}$, Ovidiu Alexa ${ }^{1}$, Ioana Alexandra Sandu ${ }^{1}$

${ }^{1}$ University of Medicine and Pharmacy "Grigore T Popa" Iasi, Iasi, Romania

Purpose: Our objective was to assess in-hospital mortality and factors that may influence it in geriatric patients with hip fracture.

Methods: We evaluate retrospectively 1660 patients hospitalised within three years in our Trauma Clinic with hip fractures and age above 65 years in terms of demographic characteristics, the preoperative interval, the in-hospital mortality and the ASA score.

Results: We found a slight predominance of intracapsular fractures. According to the AO classification of 2018, 51.2\% of patients had fractures $31 \mathrm{~B}(1-3), 38.45 \% 31 \mathrm{~A} 1,2.5 \% 31 \mathrm{~A} 2$ and $7.85 \% 31 \mathrm{~A} 3$. The sex ratio of $1.8 / 1$ and the mean age for male patients was $72.4 \pm 14.8$ years, and for females $79 \pm 9.7$ years. In-hospital overall mortality was $4.45 \%$. In the study group, $88.4 \%$ of patients were surgically treated and $11.6 \%$ benefited from functional therapy due to refusal of surgery or increased operator and anesthetic risks. The preoperative interval was significantly lower in the deceased patients ( 4 vs 5 days, $p=0.05$ ), along with the duration of the hospitalization ( 11 vs 12 days, $p=0.380)$. The main factors influencing mortality were fracture type $(\mathrm{p}=0.023)$, ASA score $(\mathrm{p}=0.005)$ and operator interval greater than 3 days $(\mathrm{p}=0.005)$.

Conclusion: Despite the large number of comorbidities, surgical delay is associated with increased mortality of geriatric patients with hip fractures.

\section{P-82}

Post-discharge complications in frail older patients after surgery for colorectal cancer

Nina Ommundsen ${ }^{1}$, Arild Nesbakken ${ }^{2}$, Torgeir Bruun Wyller ${ }^{1}$, Eva Skovlund $^{3}$, Arne Olav Bakka ${ }^{4}$, Marit Slaaen Jordh $\varnothing y^{5}$, Siri Rostoft ${ }^{1}$

${ }^{1}$ Department of Geriatric Medicine, Oslo University Hospital, Oslo, Norway, ${ }^{2}$ Department of Gastrointestinal Surgery, Oslo University Hospital, Oslo, Norway, ${ }^{3}$ Department of Public Health and Nursing, NTNU, Trondheim, Norway, ${ }^{4}$ Department of Digestive Surgery, Akershus University Hospital, Lørenskog, Norway, ${ }^{5}$ Institute of Clinical Medicine, Faculty of Medicine, University of Oslo, Nydalen, Oslo, Norway

Background: The incidence of postoperative complications after colorectal cancer surgery varies between publications, and complications occurring after discharge from hospital are often not reported. The aims of this study were to investigate the proportion of frail older colorectal cancer patients who developed complications after discharge from hospital, the severity of post-discharge complications, and the time point at which the most frequent complications occurred.
Methods: Patients were included if they were 65 years or older, screened positively for frailty and were scheduled for colorectal cancer surgery. Included patients were followed prospectively in hospital and after discharge for 30 days after surgery. Complications were graded according to the Clavien-Dindo classification.

Results: We included 114 patients. Median age was 79 years. Twenty-two patients (19\%) were discharged without complications, but developed complications after discharge. These patients had shorter length of stay (6.5 versus 10 days), were more often discharged to their own home without assistance, and had higher 5-year survival (76\% vs 54\%) than patients who developed complications inhospital. Post-discharge complications were most frequently grade II. The most common types of complications that were diagnosed late in the postoperative course were urinary tract infections and superficial surgical site infections.

Conclusions: Complications after colorectal cancer surgery in frail older patients frequently arise after discharge from hospital. Doctors should be aware of this and inform their patients. This is increasingly important as length of stay after surgery decreases. When complications are used as a quality measure, it should be clear whether only inhospital complications are registered.

\section{P-83}

Protein energy malnutrition as predictor of poor outcome after hip arthroplasty

Sandra Eminovic ${ }^{1}$, Doris Eglseer ${ }^{2}$, Regina Riedl ${ }^{3}$, Andreas Leithner ${ }^{1}$, Gerwin Bernhardt ${ }^{1}$

${ }^{1}$ Department of Orthopedics and Trauma, Medical University of Graz, Graz, Austria, ${ }^{2}$ Institute of Nursing Science, Medical University of Graz, Graz, Austria, ${ }^{3}$ Institute for Medical Informatics, Statistics and Documentation, Medical University of Graz, Graz, Austria

Introduction: Successful rehabilitation after total hip arthroplasty is determined by patient's preoperative health status. Additionally elderly patients show a higher risk for malnutrition. For those affected, this can lead to longer hospital stays with increased morbidity and higher mortality. A few studies indicated the nutritional status and postoperative outcome in patients undergoing elective arthroplasty. On the basis of studies demonstrating the relevance of preoperative nutritional status in surgical patients this study aims to assess the prevalence of Protein Energy Malnutrition (PEM) and to assess whether preoperative nutritional parameters are associated with clinical outcome parameters.

Methods: We retrospectively evaluated the nutritional status of 220 patients aged over 65 years undergoing hip arthroplasty during an 8 year study period. PEM was assessed using serum albumin and total lymphocyte count as predictors of clinical outcome. Studied outcome parameters were length of postoperative stay, 6-month postoperative re-admission due to complications, and 12-month postoperative mortality.

Results: Patients with PEM ( $\mathrm{n}=27,12.3 \%$ ) were significantly older (mean age $81.3 \pm 7.0, \mathrm{p}<0.001$ ), had a lower body mass index $\left(24.7 \pm 4.1 \mathrm{~kg} / \mathrm{m}^{2}, \mathrm{p}=0.022\right)$ and showed more comorbid conditions (mean Charlson Comorbidity Index (CCI) $2.8 \pm 2.0, p=0.002$ ) compared to well-nourished patients (age 75.6 \pm 6.2 , BMI $26.8 \pm 4.3 \mathrm{~kg} / \mathrm{m}^{2}$, CCI: $\left.1.7 \pm 1.7\right)$. Length of preoperative stay differed significantly $(\mathrm{p}<0.001)$ between PEM (median $=7$, range: 1-36 days) and non PEM (median: 1, range: 1-22 days). However, for the length of postoperative stay no differences were observed between the groups (PEM: median = 14, range: 4-36 days; non PEM: median $=14$, range: $1-50$ days). Within 6 months after OP, 12 (44.4\%) patients in the PEM group were readmitted and $15(7.8 \%)$ patients without PEM. Adjusted for preoperative characteristics, we 
observed a hazard ratio of 6.3 (95\% confidence interval: 1.7-23.1) for PEM. Only three patients with PEM died 12 months after OP.

Conclusions: We observed a higher 6-month re-admission rate for malnourished patients undergoing elective hip arthroplasty compared with well-nourished patients. We suggest that serum albumin and total lymphocyte count are valuable clinical markers evaluating the nutritional status and the clinical outcome. These results underline the importance of preoperative nutritional assessment of patients undergoing elective hip arthroplasty.

\section{P-84}

Prognostic significance of chronic kidney disease and acute kidney injury in elderly patients after hip fracture surgery

Cédric Villain ${ }^{1}$, Sara Thietart ${ }^{2}$, Judith Cohen-Bittan ${ }^{2}$, Mathieu Raux ${ }^{3}$, Bruno Riou ${ }^{4}$, Anthony Meziere ${ }^{5}$, Frederic Khiami ${ }^{6}$, Marc Verny ${ }^{7}$, Jacques Boddaert ${ }^{7}$

${ }^{1}$ Unit of Peri-Operative Geriatric Care, Hõpitaux Universitaires PitiéSalpõtriére-Charles Foix, DHU FAST, Assistance Publique Hõpitaux de Paris (APHP), INSERM U-1018, CESP Equipe 5, EpRec, UVSQ, Sorbonne Universités, UPMC Paris 6, Paris, France, ${ }^{2}$ Unit of PeriOperative Geriatric Care, Hõpitaux Universitaires Pitié-SalpétriéreCharles Foix, DHU FAST, Assistance Publique Hépitaux de Paris (APHP), Paris, France, ${ }^{3}$ Department of Anesthesiology and Critical Care, Groupe Hospitalier Pitié Salpétriére-Charles Foix, APHP, Sorbonne Universités, UPMC Paris 6, Paris, France, ${ }^{4}$ Department of Emergency Medicine and Surgery, Hõpitaux Universitaires Pitié Salpétriére-Charles Foix, APHP, UMR INSERM 1166, IHU ICAN, Sorbonne Universités, UPMC Paris 6, Paris, France, ${ }^{5}$ Department of Rehabilitation, Hõpitaux Universitaires Pitié Salpétriére-Charles Foix, APHP, Ivry-sur-Seine, France, ${ }^{6}$ Department of Orthopedic Surgery and Trauma, Hõpitaux Universitaires Pitié SalpétriéreCharles Foix, APHP, Sorbonne Universités, UPMC Paris 6, Paris, France, ${ }^{7}$ Unit of Peri-Operative Geriatric Care, Hõpitaux Universitaires Pitié-Salpétriére-Charles Foix, DHU FAST, Assistance Publique Hõpitaux de Paris (APHP), UMR CNRS 8256, Sorbonne Universités, UPMC Paris 6, Paris, France

Introduction: Chronic kidney disease (CKD) and acute kidney injury (AKI) have been found associated with poor prognosis after various types of surgery. However, the prognosis associated with these disorders has been seldom studied in elderly patients after hip-fracture surgery.

Methods: Patients admitted after hip-fracture surgery in a PostOperative Geriatrics Unit between 2009 and 2017 were included in the analysis. Having an estimated glomerular filtration rate $<60 \mathrm{ml} / \mathrm{min} / 1.73 \mathrm{~m}^{2}$ using MDRD equation defined CKD; AKI was diagnosed if the maximal post-operative serum creatinine was superior to $150 \%$ of the minimal creatinine, according to current guidelines. Statistical analysis used multivariate logistic and linear regression.

Results: A total of 744 patients were analyzed (median age 87.0 years), of whom 95 had CKD (12.8\%) and 234 experienced AKI $(31.5 \%)$. The occurrence of AKI during hospitalization was more frequent in the CKD group as compared to the non-CKD group $(65.3 \%$ vs $26.5 \%$, respectively, $\mathrm{p}<0.001)$. Having CKD was associated with higher AKI severity. After adjustment for various confounders, AKI but not CKD was significantly associated with higher occurrence of delirium (OR 1.46, 95\% CI [1.03-2.08]), blood transfusion (OR 2.56, 95\% CI [1.81-3.65]), acute heart failure (OR $5.09,95 \%$ CI [2.95-8.97]), infection (OR 2.25, 95\% CI [1.49-3.40]), and death (OR 6.31, 95\% CI [2.13-23.20]) during hospitalization.
Acute kidney injury was also associated with increased duration of hospitalization (+ 2.24 days, 95\% CI [1.24-3.23]).

Conclusion: In elderly patients after hip-fracture surgery, CKD was associated with the incidence of post-operative AKI. However, AKI but not CKD was associated with poor prognosis. Interventions to prevent post-operative AKI are needed in elderly patients.

\section{P-85}

Short-use post-operative administration of nonsteroidal antiinflammatory drugs in elderly patients with hip fracture

Sara Thietart ${ }^{1,2}$, Cédric Villain ${ }^{1,3}$, Judith Cohen-Bittan ${ }^{1,2}$, Mathieu Raux ${ }^{4}$, Bruno Riou ${ }^{5}$, Frederic Khiami ${ }^{6}$, Anthony Meziere ${ }^{7}$, Marc Verny ${ }^{1,2,8}$, Héléne Vallet ${ }^{1,2}$, Loréne Zerah ${ }^{1,2}$, Jacques Boddaert ${ }^{1,2}$

${ }^{1}$ Unit of Peri-Operative Geriatric care, Department of Geriatrics; Groupe Hospitalier Pitié-Salpétriére - Charles Foix, Assistance Publique - Hpitaux de Paris (APHP), Paris, France, ${ }^{2}$ Sorbonne Université, DHU FAST, UMRS INSERM 1166, IHU ICAN, UMRS INSERM 1158, CNRS UMR 8256, Paris, France, ${ }^{3}$ CESP, INSERM UMRS 1018, Univ. Paris-Sud, UVSQ, Univ. Paris-Saclay, F-94800, Villejuif, France, ${ }^{4}$ Department of Anesthesiology and Critical Care, Groupe Hospitalier Pitié-Salpétriére - Charles Foix, Assistance Publique-Hôpitaux de Paris (APHP), Paris, France, ${ }^{5}$ Department of Emergency Medicine and Surgery, Groupe Hospitalier PitiéSalpétriére - Charles Foix, Assistance Publique-Hôpitaux de Paris (APHP), Paris, France, ${ }^{6}$ Department of Orthopedic Surgery and Trauma, Groupe Hospitalier Pitié-Salpétriére - Charles Foix, Assistance Publique-Hôpitaux de Paris (APHP), Paris, France, ${ }^{7}$ Department of Rehabilitation, Groupe Hospitalier Pitié-Salpétriére Charles Foix, Assistance Publique-Hôpitaux de Paris (APHP), Ivry sur Seine, France, ${ }^{8}$ Sorbonne Universités, Université Pierre et Marie Curie (UPMC) et UMR8256 (CNRS), Team Neuronal Cell Biology \& Pathology, Paris, France

Introduction: Nonsteroidal anti-inflammatory drugs (NSAIDs) are an efficient post-operative analgesic. However, their use is associated with potentially life-threatening side-effects, especially in elderly patients. The objective of this study was to evaluate the safety of a single administration of NSAIDs in a geriatric population after a hipfracture surgery.

Methods: Patients admitted after hip-fracture surgery in a PostOperative Geriatrics Unit between 2009 and 2017 were included in the analysis. Peri-operative administration of NSAIDs was collected and patients were prospectively followed-up. The primary end-point was defined by the occurrence of acute kidney failure (AKI) or blood transfusion during hospitalization in our unit. Logistic regression was performed with adjustment on confounders.

Results: Seven hundred and forty-six patients were included (median age 87 years [82.0-91.0], $180(24.1 \%)$ males, CIRS2 score 9.5 [6.0-12.0], duration of hospitalization 9 days [7-12]). Fifty-four patients $(7 \%)$ received peri-operative NSAIDs. Characteristics of the NSAIDS group were similar to the non-NSAIDs group, except for ischemic cardiopathy ( $7.4 \%$ vs $19.4 \%$, respectively, $\mathrm{p}=0.03$ ). Primary end-point occurred in 22 patients $(40.7 \%)$ in the NSAIDs group and $359(52.1 \%)$ patients in the non-NSAIDS group $(\mathrm{p}=0.19$, after adjustment for confounders). No significant difference was found in the frequency of post-operative AKI, transfusion, heart failure, and infections. A trend towards faster recovery of walk was noted in the NSAIDs group as compared to the non-NSAIDs group (median 1 day [1.0-3.0] vs 2.0 days [1.0-3.0], $\mathrm{p}=0.06$ ). 
Conclusion: A single administration of NSAIDs was not associated with an increase in adverse-effects in elderly patients after hip-fracture surgery.

\section{P-86}

\section{Before-and-after hip fracture in tose who live alone}

Esther Álvarez- Gómez ${ }^{1}$, Daniel Schadegg-Peña ${ }^{1}$, María Pilar MesaLampré $^{1}$

${ }^{1}$ Geriatric Department, Hospital Real Nuestra Señora de Gracia, Spain

Introduction: The prognosis for a hip fracture can includes a great loss of functionality and high mortality. Patients who lived previously alone could have much more autonomy loss.

Methods: Observational and retrospective cohort study in patients $>75$ years old, hospitalized by hip fracture from February 2009 to February 2018. Comorbidity, functionality and medical, surgical and environmental complications are analyzed in patients who previously lived alone(I) and in those who lived with another person(II). Collected variables are: prior fracture, at hospital discharge, at 6 months and at 1 year.

Results: Of 1326, 184 lived previously alone. Lawton-Brody index $(\mathrm{p}<0.05)$ prior hospital admission/6 months/1 year (median): 6/3/ 5(I) and 1/0/0(II). Barthel index $(\mathrm{p}<0.05)$ prior/hospital discharge/ 6 months/1 year (median): 95/55/80/85(I) and 75/35/60/60(II). Pfeiffer test $(\mathrm{p}<0.05)$ prior/6 months/1 year (median):2/1/0.5(I) and 4/3/3(II). MiniMNA ( $<0.05)$ prior/6 months/1 year (median): 12/13/12(I) and 11/11/12(II). Charlson index (p < 0.05): 1.27(I) and 1.70(II). More local infection, delirium, fecal impact, heart failure and in-hospital death in II $(\mathrm{p}<0.05)$ and new institutionalization in I $(\mathrm{p}<0.05) .39 \%$ of group I returned home. Hospital stay in Orthogeriatric Service: 13 days(NS).

Conclusions: 1. A better prior situation was found in group I (comorbidity, cognition, mobility, functionality and nutrition). This difference was maintained throughout the first year except in regards to nutricional status, which was equal in both groups (possibly due to the implementation of more intensive programs in group II). 2. The new institutionalization (temporary in many cases) was higher in group I. The inpatient stay is similar in both cases.

\section{P-87}

Why are older people excluded from clinical studies? A real-life example

Marta Moreno Iturriaga ${ }^{1}$, Mónica Gœmez Valent ${ }^{2}$,

Sara Ortonobes Roig ${ }^{2}$, Bárbara González Ávila ${ }^{1}$, Esperança Antón Nieto ${ }^{1}$, Neus Muñoz Gost ${ }^{1}$, $\mathrm{M}^{\mathrm{a}}$ Teresa Vilarmau Dolcet ${ }^{1}$, Carme Nogueres Rimblas ${ }^{1}$, Elena Canta López ${ }^{1}$, Monica-Tudorita Bojor ${ }^{1}$

${ }^{1}$ Medium-Stay Unit. Corporació Sanitária Universitária Parc Taulí, Sabadell, Barcelona, Spain, ${ }^{2}$ Pharmacy Department, Corporació Sanitária Universitária Parc Taulí, Sabadell, Barcelona, Spain

Introduction: The upward trend in life expectancy places the ageing population under an increasing focus of clinical care. Nevertheless, older patients are under-represented in clinical research, as well as their pain management after surgery. Due to this, we aimed to perform a Randomized Control Trial (RCT) evaluating postoperative pain treatment in elderly people. Unfortunately, 18 months after, the amount of patients included is far below our expectations. Here we describe the reasons for excluding these patients.
Methods: We conducted a RCT to compare safety and effectiveness of metamizole, ibuprofen and tramadol added to paracetamol to management post-surgical pain after a fracture intervention in the proximal third of the femur in patients $>70$ years old admitted to a Medium-Stay Unit. EudraCT code: 2015-004482-88. The main exclusion criteria were: admission $>120 \mathrm{~h}$ after surgery, dementia, renal failure, haematological abnormalities or chronic analgesic treatment.

Results: Patients potentially included in RCT: 297. Age $86.5( \pm 6.3)$ years old. Female 212 (71.4\%). Patients excluded: 267 (89.9\%). Reasons for exclusion: dementia, $100(37.5 \%)$; renal failure, 62 $(23.2 \%)$; $>120 \mathrm{~h}$ after surgery, $52(19.5 \%)$; chronic analgesic treatment, 25 (9.4\%); haematological abnormalities, 10 (3.7\%); other 18 $(6.8 \%)$.

Conclusions: Our attempt to carry out this RCT has been unsuccessful mostly because nearly $90 \%$ of patients that could be included met at least one exclusion criteria. Our experience supports that older patient's inherent features often exclude them from participating in RCTs, and therefore remain under-represented in clinical research. As a result of this, we are considering to discontinue the study.

\section{P-88}

Emotional stress does not affect heart rate variability in older surgical inpatients

\section{E. P. Schuurmans ${ }^{1}$, M. G. M. Olde Rikkert ${ }^{1}$}

${ }^{1}$ Radboud University Medical Center, Nijmegen, The Netherlands,

Introduction: Elderly patients indicate that wellbeing is important to them, but they tend to be less focused on perceived stress. Heart Rate Variability (HRV) could be used to monitor stress levels during hospital stay. Increased stress has been correlated with sympathetic dominance, whereas positive mood is associated with a shift toward parasympathetic activity. Unobtrusive wearables allow continuous monitoring of HRV data, which can be analysed by calculating the relative contribution of the parasympathetic branch (high frequency or HF) to autonomic regulation, as well as the sympathovagal balance (low frequency/high frequency or LF/HF) [1].

Methods: In this pilot study 35 patients aged 65 and up, admitted for planned and unplanned surgery were included. Anxiety was measured 4 times a day using the trait part of the State-Trait Anxiety Inventory (STAI-T). Heart rate variability was measured using a sensor patch (Health Patch). Log transformed HF and LF/HF values in the hour before the highest STAI-T were compared to the values in the hour before the lowest STAI-T scores using paired sample $T$ Test.

Results: Complete data was available for 24 participants. In the hour before the highest STAI-T Mean HF was significantly lower in 5 subjects and mean LF/HF significantly higher in 4 subjects. In most subjects there was no significant difference in HRV measurements. Conclusions: Perceived stress was not reflected in $\mathrm{HF}$ and $\mathrm{LF} / \mathrm{HF}$ values.

Discussion: HRV is not a good measure for pre surgery stress in elderly; this could be due to loss of complexity in the heart rate signal with ageing [2].

\section{References:}

1. Kimhy D, Delespaul P, Ahn H, Cai S, Shikhman M, Lieberman JA, Malaspina D, Sloan RP (2009) Concurrent measurement of "realworld" stress and arousal in individuals with psychosis: assessing the feasibility and validity of a novel methodology. Schizophr Bull 36(6):1131-1139

2. Lipsitz LA (2002) Dynamics of stability: the physiologic basis of functional health and frailty. J Gerontol Ser A Biol Sci Med Sci 57(3):B115-B125. 


\section{P-89}

Elderly patients are remarkably resilient to anxiety: perioperative stress does not significantly impact wellbeing

\section{E. P. Schuurmans ${ }^{1}$, M. G. M. Olde Rikkert ${ }^{1}$ \\ ${ }^{1}$ Radboud University Medical Center, Nijmegen, The Netherlands}

Introduction: Older patients have stated that subjective well-being is one of their most important treatment outcomes [1,2]. We hypothesized that peri-operative anxiety could be an important factor in changes in well-being during chirurgical trajectories.

Methods: Thirty-five patients aged 65 and up, admitted for planned and unplanned surgery were included. Wellbeing was measured shortly after admission and one month after discharge using the Personal Wellbeing Index (PWI: 0-100 (= best)). Anxiety was measured 3 to 4 times a day during hospital stay using the trait part of the State-Trait Anxiety Inventory (STAI-T: 6-24 (= most anxiety)). Change in PWI and STAI-T were correlated by Pearson's R. Group analysis was performed by comparing STAI-T among participants with an increased PWI to participants with a decrease.

Results: Complete data was available for 29 participants. Average Pre-operative PWI was 75 (SD 7.58). Average STAI-T was 9.75 (SD 1.62). Mean change in pre-operative to 1 month post operative PWI was -1.19 (SD 7.84). There was no statistical relationship between change in PWI and average STAI-T $(\mathrm{R}=-.1, \mathrm{p}=.5)$ or maximum STAI-T $(\mathrm{R}=-.2, \mathrm{p}=.3)$. Participants with a decrease in PWI did not have statistically higher STAI-T than participants with an increase in PWI. Conclusions: Although elderly patients undergoing surgery perceive significant anxiety they seem to be resilient to this and it does not affect their overall wellbeing. Patient's resilience, their anxiety and wellbeing evaluation should be taken into account in shared decisions making about whether or not to undergo surgery.

References:

1. Robben SH, Perry M, Olde Rikkert MG, Heinen MM, Melis RJ (2011) Care-related goals of community-dwelling frail older adults. J Am Geriatr Soc 59(8):1552-1154

2. van Kempen JA, Robben SH, Zuidema SU, Rikkert MG, Melis RJ, Schers HJ (2012) Home visits for frail older people: a qualitative study on the needs and preferences of frail older people and their informal caregivers. Br J Gen Pract 62(601):e554-e560

\section{P-90}

Predicting 12-month mortality in older patients undergoing emergency laparotomy: a prospective study

Mollie Rowley ${ }^{1}$, Jenny Fox ${ }^{1}$, Areej Paracha ${ }^{1}$, Haroon Khan ${ }^{1}$, Nicholas Springall $^{1}$, Luciana Miguel-Alhambra ${ }^{1}$, Alex GomezQuintanilla $^{1}$, Genna Logue ${ }^{1}$, Amanda Pedersen ${ }^{1}$, Angeline Price ${ }^{1}$, Arturo Vilches-Moraga ${ }^{1}$

${ }^{1}$ Department of Ageing and Complex Medicine, Salford Royal NHS Foundation Trust, Salford, UK

Objectives: Older patients comprise half of those admitted for emergency general surgery, and have worse outcomes. Whilst high rates of in-hospital mortality have been described, less is known about longer term outcomes in this population. This study describes factors associated with 12-month mortality in older people who undergo emergency laparotomy.

Methods: All general surgical patients aged 75 years or older who underwent emergency laparotomy between 8th September 2014 and 30th March 2017 were included in this prospective non-randomised study. The primary outcome measure was 12 -month mortality.
Results: 113 patients were included, with a mean age of $81.9 \pm 4.65$ years, and a female predominance $(53.1 \%)$. At presentation, $91.2 \%$ were independent of basic activities of daily living, 92\% were continent and $2.7 \%$ were permanent residents in a care home. The most common indication for laparotomy (46\%) was bowel obstruction, as a consequence of cancer or adhesions. Following surgery, 95(84.1\%) were admitted to the high-dependency unit (HDU). 88(77.9\%) patients survived to discharge. In our centre, $81(89 \%)$ received comprehensive geriatric assessment (CGA) led by a geriatrician. $25(22.1 \%)$ patients died in hospital, the majority of these in HDU. The 12-month mortality rate was $38.9 \%$ (44/113). Strong predictors of mortality were ASA score III-IV ( $p<0.001)$, frailty ( $p$ 0.002 ) and dependency for instrumental activities of daily living $(\mathrm{p}=0.037)$.

Conclusions: One-year mortality is high in older people undergoing emergency laparotomy. ASA class, clinical frailty and functional status can be employed to predict those at highest risk

\section{P-91}

The impact of frailty on hip fracture management and mortality outcomes in nonagenarians and centenarians admitted to an orthogeriatric unit

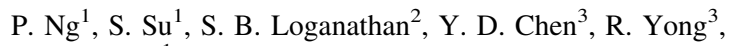
J. U. Mallya ${ }^{1}$

${ }^{1}$ Geriatric Medicine, KTPH, Singapore, ${ }^{2}$ Anaesthesia, KTPH, Singapore, ${ }^{3}$ Orthopaedic Surgery, KTPH, Singapore

Introduction: Increasing numbers of the oldest old present with hip fractures, driven by population ageing and high rates of injurious falls. Frailty and multimorbidity often lead to hesitation in offering surgery. Better data on outcomes can assist clinicians in evaluating the risks and benefits of operating on these vulnerable individuals.

Methods: We retrospectively reviewed 93 patients aged 90 and above admitted to our orthogeriatric unit with hip fractures between January 2015 and February 2017. Mortality at 30 days and 1 year was analysed in relation to demographics, comorbidities, Modified Barthel Index (MBI), Clinical Frailty Scale (CFS), and American Society of Anaesthesiologists (ASA) scores.

Results: Seventy-six females and seventeen males with a mean age of 93.3 (90-108) were studied. Fifty-four $(58.1 \%)$ underwent surgical fixation. Thirty-day mortality was $4.3 \%$, with three of four patients from the non-operative group. Overall 1-year mortality was $19.4 \%$, $33.3 \%$ in the non-operative group compared to $9.3 \%$ among surgical patients. Frailty $(\mathrm{p}=0.019)$, ASA $(\mathrm{p}=0.016)$, and comorbidities $(\mathrm{p}=0.035)$ were independently associated with mortality in univariate analysis. Sex $(p=0.358)$ and fracture type $(p=0.201)$ did not show a significant association. In multivariate logistic regression, non-operative management was a significant determinant of 1-year mortality with odds of 4.65 (95\% CI 1.09-19.7, $\mathrm{p}=0.034)$. Surgical patients were less frail (CFS 5.2 vs $6.3, p<0.001$ ) and higherfunctioning (MBI 88.6 vs 61.0, p < 0.001) than non-operative patients.

Key conclusions: Careful preoperative evaluation and counselling, with attention to frailty and function, can enable selected patients even among the oldest old to benefit from hip fracture surgery with up to $90 \%$ 1-year survival. 


\section{P-92}

Barriers of mechanical prophylaxis for deep vein thrombosis among elderly with hip fracture

\author{
Khalid Al-Mugheed ${ }^{1}$, Nurhan Bayraktar ${ }^{1}$ \\ ${ }^{1}$ Near East University Faculty of Nursing, Mersin, Turkey
}

Introduction: Deep vein thrombosis (DVT) is a common complication following lower extremity fracture surgery. Mechanical prophylaxis is effective way for prevent deep vein thrombosis, and commonly used to reduce venous congestion. There are several barriers that affect profilactic measures. This systematic review provides an overview to relevant literature on barriers of mechanical prophylaxis among elderly patients with hip fracture.

Methods: Databases searched included Science direct, Scopus, Pub Med, Web of Science and Near East University Grand Library. Databases were searched using the following term (elderly hip fracture, mechanical prophylaxis, DVT, barriers). We included all full papers with English Version. Also we excluded studies that were published only in abstract form.

Results: Recent studies show that disparate percentage of patient's non-compliance and adherent to mechanical prophylaxis. Too much pressure, pump activated too frequently, feet feel too hot and disturbed sleep patterns are barriers for mechanical prophylaxis. Skin breakdown and oedematous state is considered as a major problem in elderly patients. There are hospital factors, that include availability and timely replacement of mechanical prophylaxis. Also patient selfreported to assess response related satisfaction play an important role in non-compliance.

Key conclusions: Deep vein thrombosis among elderly patients with hip fracture is preventable. Health care providers can overcome of these barriers to prevent complications. In-service education for health care providers and patient education are vital to enhance knowledge.

\section{P-93}

Risk of bilateral femoral fractures in elderly patients with bisphosphonates therapy: a case report

Ioana Dana Alexa ${ }^{1}$, Ramona Stefaniu ${ }^{1}$, Ioana Alexandra Sandu ${ }^{1}$, Ovidiu Alexa $^{1}$

${ }^{1}$ University of Medicine and Pharmacy "GRIGORE T POPA" Iasi, Iasi, Romania

Introduction: The widespread use of bisphosphonate therapy as an affordable solution against osteoporosis has led to the occurrence of a complication of atypical fractures in the femur. The way these fractures appear and develop can include them in the category of stress fractures. The global population aging phenomenon will bring a new challenge for the medical system, namely establishing optimal diagnostic recommendations and treatment.

Methods: The case presented is of a 64-year-old female patient who was diagnosed with a left subtrochanteric fracture following a low energy trauma. Bisphosphonate treatment was initiated 6 years ago, at menopause, the patient receiving $70 \mathrm{mg}$ of alendronic acid weekly. After a period of 2 years, the patient developed a similar fracture at the right hip level.

Results: The initial fracture, the one at the level of the left hip, was operated and a Dynamic Condylar Screw (DCS) system was used for osteosynthesis. Consolidation evolved with difficulty and healing was achieved after 6 months. In case of fracture at the right hip we chose a centromedular implant represented by the Gamma nail, healing occurring much faster, after a 3-month interval.
Conclusions: Atypical fractures of the femur are a consequence of changes in micro and macro-architecture of bone induced by longterm treatment with bisphosphonates. These patients require rigorous follow-up because they often have similar lesions in the controlateral femur. Changes in bone metabolism cause the delay of the cure and a high rate of pseudarthrosis, the centromedular implant providing superior results to the DCS.

\section{P-94}

Delirium in patients with acute hip fracture admitted to a multidisciplinary orthogeriatrics unit: Incidence, characteristics and associated risk factors

Isabel Arnau-Barrés ${ }^{1}$, Óscar Miranda ${ }^{1}$, Lizzeth Canchucaja ${ }^{2}$, Olga Vázquez ${ }^{1}$, Juan Carlos Monllau ${ }^{3}$, Santos Martinez ${ }^{4}$, María José Robles ${ }^{1}$

${ }^{1}$ Geriatrics Department, Hospital del Mar, Barcelona, Spain, ${ }^{2}$ Geriatrics Department, Hospital del Mar, Bracelona, Spain, ${ }^{3}$ Department of Orthopaedic Surgery, Hospital del Mar, Barcelona, Spain, ${ }^{4}$ Department of Orthopaedic Surgery, Hospital del Mar, Barcelona, Spain

Introduction: To determine the incidence and associated factors for delirium in patients $\geq 65$ years old with hip fracture admitted to an orthogeriatrics unit of a university hospital.

Methods: Observational study of patients admitted to the unit between November 2017 and January 2018. We registered variables related to demographic, social, clinical characteristics, blood test results and perioperatory data. The primary outcome was to determine the incidence of delirium based on the Confusion Assessment Method (CAM) and DSM-V criteria. Multivariate logistic regression analysis was performed to analyse the variables. In-hospital mortality associated with the incidence of delirium was also evaluated.

Results: We included 111 patients. Seventy-one women (64\%), average age $85.5 \pm 7.76$. Procedence: Home 100 (90.1\%), nursing home $6(5.4 \%)$, others $5(4.5 \%)$. Dementia: 43 (38.7\%). Barthel $<60$ : $27(24.3 \%)$. Charlson $\geq 2$ : $75(67.6 \%)$. Sensory deficit: $52(46.8 \%)$. Polypharmacy/psychotropic use: $90(81.1 \%)$. Surgery in the first $48 \mathrm{~h}$ : $57(51.4 \%)$. Spinal anesthesia: $87(78.4 \%)$. Use of sedation: 65 (58.6\%). Postoperative complications: renal failure 55 (49.5\%), dehydration 45 (40.5\%), dyselectrolytemia 28 (25.2\%), anaemia 107 (96.4\%), malnutrition 92 (82.9\%), urinary retention/constipation/urinary catheter $94(84.7 \%)$. During hospital admission 4 (3.6\%) patients died. A total of 50 patients presented delirium, with an incidence of $45 \%$ (95\% CI 35.7-54.3). In the multivariate analysis the factors associated with the incidence of delirium were being female (OR 0.20 [95\% CI0.71-0.61]), dementia (OR 7.97 [95\% CI2.61-24.83]) and use of sedation (OR 3.89 [95\% CI1.19-12.69]). Patients with delirium had a risk of in-hospital mortality of 4.2 (95\% CI3.4-5.3).

Conclusions: Despite the comprehensive geriatric intervention, the incidence of delirium is very high in elderly patients admitted for hip fracture, especially among the male patients and those with dementia. Suffering delirium increases the risk of mortality. Therefore, next to comprehensive geriatric intervention, the implementation of additional preventive measures to decrease the incidence of delirium in the acute phase of hip fracture is required. 


\section{P-95}

Management of perioperative anticoagulation in patients with hip fracture and atrial fibrillation

C. M. Zárate-Sáez ${ }^{1}$, C. Sanchez-Castellano ${ }^{1}$, M. A. Parro-Martín ${ }^{1}$, E. Delgado-Silvela ${ }^{1}$, M. Muñoz-García ${ }^{1}$, L. A. Hernandez-Sanchez ${ }^{1}$, A. Merello-De Miguel $^{1}$, A. Cruz-Jentoft ${ }^{1}$

${ }^{1}$ Ramón y Cajal Hospital, Madrid, Spain

Aim: To investigate perioperative management of anticoagulation in old patients with hip fracture and atrial fibrillation (AF), and it's association with the prevalence of hemorrhagic and cardiovascular complications, transfusions and mortality.

Methods: 18-month observational retrospective study of hospitalized patients over 70 years old with hip fracture who were on anticoagulant therapy on admission and had no history of severe anemia in the previous 6 months.

Results: 79 patients, mean age $86.4 \pm 5.3$ years, were included. $82.1 \%$ received treatment with coumadin. All patients were switched to enoxaparin in the first $24 \mathrm{~h}$ after admission, $89.9 \%$ received a full therapeutic dose (as opposed to prophylactic dose) although many had an INR within normal range or overdose $(53.9 \%)$. $46.8 \%$ received vitamin $\mathrm{K}$ in the first $24 \mathrm{~h}$. Perioperative severe anemia happened in $20.3 \%$. Transfusion of 1t-4 RBC units was required before or during surgery in $38 \%$ and after surgery in $32.9 \%$. Other complications observed: perilesional hematoma (55.7\%), heart failure $(32.9 \%)$, uncontrolled AF (30.4\%), gastrointestinal bleeding (3.8\%), stroke $(1.3 \%)$, acute arterial ischemia (1.3\%), and early infection of the surgical wound $(1.3 \%)$; with an overall mortality of $8.9 \%$. There were no statistically significant differences in the development of complications related to the administration of either prophylactic or therapeutic doses of enoxaparin or Vitamin K.

Conclusions: One in five hospitalized patients for HF on anticoagulant treatment due to $\mathrm{AF}$ suffered from severe perioperative anemia and almost two thirds required RBC transfusion. Notably, full anticoagulation bridge therapy was used in most patients, when more than half presented with a high INR. Anticoagulation practices did not impact on complication rates.

\section{P-96}

Influence of frailty in older patients undergoing emergency laparotomy: a prospective study

Areej Paracha ${ }^{1}$, Mollie Rowley ${ }^{1}$, Jenny Fox ${ }^{1}$, Haroon Khan ${ }^{1}$, Nicholas Springall $^{1}$, Luciana Miguel-Alhambra ${ }^{1}$, Alex GomezQuintanilla $^{1}$, Genna Logue ${ }^{1}$, Amanda Pedersen ${ }^{1}$, Angeline Price ${ }^{1}$, Mohammad Moatari ${ }^{1}$, Arturo Vilches-Moraga ${ }^{1}$

${ }^{1}$ Department of Ageing and Complex Medicine, Salford Royal Hospital, Salford, UK

Introduction: Frailty is an established predictor of poor outcomes in older people undergoing emergency laparotomy. However, there is limited evidence supporting the use of frailty scores in predicting mortality. This study describes the influence of frailty, measured using the Clinical Frailty Scale (CFS), in older people undergoing emergency laparotomy.

Methods: All patients aged 75-years or older who underwent emergency laparotomy between 8th September 2014 to 30th March 2017 were included in this prospective non-randomised study. Frailty was defined as CFS score of 5 or more. The primary aim was to establish if there was a relationship between CFS score and mortality.

Results: 113 patients were included and $37(32.7 \%)$ had a CFS score of 5-9. Frailty became more prevalent with increasing age. There was no significant difference in length of stay between the cohorts but mean time to readmission was significantly longer in those with a CFS score of 1-4 compared to those with a CFS 5-9 (451 days vs 266 days, $\mathrm{p}=0.009$ ). $77.9 \%$ of all patients survived to discharge (82.9\% CFS $1-4$ vs $67.6 \%$ CFS 5-9 p $=0.065$ ) and 12 -month mortality rate was significantly higher in the frail population $(59.5 \%$ vs $28.9 \%, \mathrm{p}=0.002$ ). Multivariate analysis showed that death within 12-months was 7.068 times more likely in frail patients.

Conclusion: Frail patients undergoing emergency laparotomy have a shorter time to readmission and a higher 12-month mortality rate. We advocate the use of CFS in conjunction with other predictive factors in guiding decision making in older people requiring emergency laparotomy.

\section{P-97}

Baseline characteristics, clinical outcomes and long-term survival of older patients admitted non-electively to general surgery with liver and biliary conditions

Rachel Khaw ${ }^{1}$, Jenny Fox ${ }^{2}$, Haroon Khan ${ }^{2}$, Nicholas Springall ${ }^{2}$, Mollie Rowley ${ }^{2}$, Luciana Miguel-Alhambra ${ }^{2}$, Areej Paracha ${ }^{2}$, Genna Logue $^{2}$, Amanda Pedersen ${ }^{2}$, Alex Gomez-Quintanilla ${ }^{2}$, Arturo Vilches-Moraga ${ }^{2}$

${ }^{1}$ Medical Student, Salford Royal NHS Foundation Trust, Salford, UK, ${ }^{2}$ Salford Royal NHS Foundation Trust, Salford, UK

Introduction: Biliary tract diseases are common in older people. Little is known about the impact of functional status on long-term survival and readmission rates in patients aged 75 -years or older. This study describes baseline characteristics and clinical outcomes in older patients reviewed by our elderly care in-reach service.

Methods: Prospective cohort study of all patients aged over 75-years admitted non-electively under general surgery with a diagnosis of biliary or liver disease between 8th September 2014 and 31st March 2017.

Results: 171-patients were identified with a mean age of $83.1 \pm 6.14$-years and female predominance $(60.8 \%) .98 .2 \%$ had 2 or more comorbidities. $41.2 \%$ took 10 or more medications. $21 \%$ and $46.7 \%$ were dependent for basic and instrumental activities of daily living (ADLs) respectively. $29.2 \%$ and $15.2 \%$ had impaired mobility or cognition, and $47.6 \%$ were frail (Clinical Frailty Scale (CFS) score $5-9)$. The majority $(63.7 \%, \mathrm{n}=109)$ were managed medically/noninvasively. 50 underwent endoscopic retrograde cholangiopancreatography (ERCP), 12 had a cholecystostomy and 12 had an inpatient cholecystectomy. Median length of stay was 9-days, with a 12-month readmission rate of $57.3 \%$. Meantime from discharge to readmission was 232.7-days (3-1085, SD 256.3). 35-patients (20.5\%) were dead 12 -months after admission to hospital. Strong predictors of mortality at 12-months included CFS score of 5-9, polypharmacy, ASA (American Society of Anaesthesiologists) class III-V and dependency for instrumental ADLs $(\mathrm{p}<0.05)$.

Conclusions: The majority of older people admitted with biliary disorders are managed non-invasively. A fifth are dead at 1-year. Presence of frailty, dependency for ADLs, polypharmacy and ASA classification may be useful predictors of 12 -month mortality. 


\section{P-98}

Factors that promote motor recovery in patients with hip fracture: data from the Italian Orthogeriatric Group (GIOG) study

A. Ceccofiglio ${ }^{1}$, A. Ungar ${ }^{2}$, C. Rostagno ${ }^{1}$, B. Govoni ${ }^{3}$, G. Mantovani ${ }^{3}$, S. Volpato ${ }^{4}$, P. Rapazzini ${ }^{5}$, A. Zurlo $^{3}$, C. Mussi ${ }^{6}$, M. Corsi ${ }^{7}$, ML. Lunardelli ${ }^{8}$, A. Andreano ${ }^{9}$, G. Castoldi ${ }^{10}$, P. Floris ${ }^{11}$, M. Pizzonia ${ }^{12}$, A. Barone ${ }^{13}$, A. March ${ }^{14}$, V. Galmarini ${ }^{15}$, S. Franzoni ${ }^{16}$, P. Ranieri ${ }^{17}$, G. Bellelli ${ }^{7,9}$

${ }^{1}$ Internal Medicine and Post-surgery, Orthopedic Unit, Florence, Italy, ${ }^{2}$ Geriatrics and Intensive Care Unit, Florence, Italy, ${ }^{3}$ Orthogeriatric Unit, Arcispedale S Anna, Ferrara University, Ferrara, Italy, ${ }^{4}$ Department of Medical Sciences, Ferrara University, Ferrara, Italy, ${ }^{5}$ Orthogeriatric Unit, Circolo Hospital, Varese, Italy, ${ }^{6}$ Orthogeriatric Unit, University of Modena and Reggio Emilia, Modena, Italy, ${ }^{7}$ Orthogeriatric Unit, S Gerardo hospital, Monza, Italy,

${ }^{8}$ Orthogeriatric Unit, S.Orsola Malpighi, Bologna, Italy, ${ }^{9}$ School of Medicine and Surgery, Milano-Bicocca University, Milan, Italy,

${ }^{10}$ Orthopaedic Unit, ASST Vimercate, Carate Brianza hospital, Vimercate (MB), Italy, ${ }^{11}$ Orthogeriatric Unit, Hospital of SondrioASST VAL, Sondrio, Italy, ${ }^{12}$ Orthogeriatric Unit, San Martino hospital, Genova, Italy, ${ }^{13}$ Orthogeriatric Unit, Galliera hospital, Genova, Italy, ${ }^{14}$ Orthogeriatric Unit, Bolzano hospital, Bolzano, Italy, ${ }^{15}$ Orthopedic Unit, ASST Fatebenefratelli sacco, Milano, Italy,

${ }^{16}$ Orthopedic Unit, Poliambulanza hospital, Brescia, Italy,

${ }^{17}$ Department of Medical Sciences, Ferrara University, Ferrara, Italy

Introduction: The Italian Orthogeriatric Group (GIOG) started in 2016 a database on hip fracture in elderly. The present analysis aimed to identify the factors that promote a complete recovery of the prefracture motor function.

Methods: Of 2570 enrolled, we considered 587 patients (mean age $84 \pm 7$ years, 453 female) who were able to walk before the fracture and who had a follow-up. We compared data on pre-fracture motor function with that referring at 30 and 120 days of follow-up.

Results: At 30 days of follow-up, the $24 \%$ of patients (140/587) recovered a complete pre-fracture motor function, while the percentage grown up to $44 \%(181 / 391)$ at 120 days. We found that a short term complete recovery (30 days) was significantly associated with continuous geriatric management, compared to only pre-operative or post-operative involvement (29\% vs $19 \%, \mathrm{p}=0.07)$. Conversely, a long term complete recovery (120 days) occurred significantly in patients with fewer comorbidities (ASA score $<355 \%$ vs ASA score $\geq 342 \%, p=0.02$ ), and those in osteo-metabolic treatment $(51 \%$ vs $39 \%, \mathrm{p}=0.02)$. We also observed that patients who experienced delirium during hospitalization had a worse longterm motor recovery.

Key conclusion: The geriatric management could facilitate a complete short-term motor recovery when its occurred since hospitalization and continues throughout the length of hospital stay. Conversely, other factors could influence long-term motor recovery, such as comorbidity, osteo-metabolic therapy and the development of delirium, emphasizing a greater frailty of the elderly patient.

\section{P-99}

\section{Elderly hip fracture and cognitive impairment degrees}

Sonia Jiménez Mola ${ }^{1}$, María Pilar López Viñas ${ }^{2}$, Antonio Rodríguez Rosa $^{3}$, Javier Idoate Gil ${ }^{4}$, Jesús Seco Calvo ${ }^{5}$, Carmen Benítez González
${ }^{1}$ Complejo Asistencial Universitario de León, León, Spain,

${ }^{2}$ Residencia Mixta de Personas Mayores, León, Spain, ${ }^{3}$ Hospital San Juan de Dios, León, Spain, ${ }^{4}$ Complejo Asistencial Universitario de León, León, Spain, ${ }^{5}$ Universidad de León, León, Spain

Introduction: The aim of this study is to determine the influence of the cognitive impairment grades in the descriptive characteristics, complications and features at hospital discharge of older adults with hip fracture.

Methods: We enrolled 534 patients with hip fracture, aged 75 years or older in an Orthogeriatric Unit of the University Hospital from León (Spain), between December 2013 and November 2014. The cognitive impairment grades were divided into 3 groups (severe/moderate; mild; and no impairment). Descriptive characteristics, complications and ambulation capacity at discharge were collected.

Results: Among the 534 participants, 499 (93\%) underwent surgery. The mean age was $86.1 \pm 7.3$ years $(75-105$ years). Regarding the cognitive impairment grades, no impairment $(n=293 ; 77$ men and 216 women; 121 subcapital and 172 pertrochanteric fractures; $11.49 \pm 7.41$ days), mild impairment $(\mathrm{n}=109 ; 30$ men and 79 women; 57 subcapital and 52 pertrochanteric fractures; $11.90 \pm 5.28$ days) and moderate/severe impairment $(\mathrm{n}=132 ; 28$ men and 104 women; 62 subcapital and 69 pertrochanteric fractures; $10.37 \pm 5.81$ days) did not show any statistically significant differences for sex $(\mathrm{P}>.05)$, fracture type $(\mathrm{P}>.05)$ or total hospital staying days $(\mathrm{P}>.05)$. With respect to complications statistically significant differences were shown for delirium $(\mathrm{P}<.001)$. With regards to features at hospital discharge, statistically significant differences were shown for destination $(\mathrm{P}<.001)$, home move $(\mathrm{P}<.05)$ and ambulation capacity $(\mathrm{P}<.001)$.

Conclusions: The cognitive impairment grades may determine the features and complications of older adults who suffer hip fracture.

\section{P-100}

Assessing pre-operative frailty index as indicator of 90 daysmortality in a cohort of older vulnerable adults with proximal femur fracture

\section{Chiara Giannotti ${ }^{1}$, Fiammetta Monacelli ${ }^{1}$, Monica Pizzonia ${ }^{1}$}

${ }^{1}$ Hospital Policlinic San Martino, Section of Geriatric Medicine, Department of Internal Medicine and Medical Specialties (DIMI), University of Genoa, Genoa, Italy

Introduction: Hip fracture is often a fatal event in older people, associated with increased disability, morbidity and mortality, posing a significant public health concern. Predictors of adverse outcomes after hip fracture is essential to guide treatments, plan discharge and use of healthcare resources. Therefore, we sought to evaluate the ability of 25-items Frailty Index (FI) to assess the clinical variables associated with 90-days mortality in a group of elders undergoing hip fracture surgery.

Materials and methods: A consecutive series of 284 elders over 65 years with proximal femur fracture was recruited in the Orthopaedic and Trauma Unit, Department of Emergency, Hospital Policlinic San Martino, Genoa, Italy. All patients underwent com- 
prehensive geriatric assessment pre-operatively. We retrospectively stratified patient's vulnerability on the basis of Frailty Index (FI), calculated using 25 items of the 70 provided by the list of the Canadian Study of Health and Aging (CSHA).

Results: The mean age was 86.6 years \pm 5.9 (range $72-103$ years); $76 \%$ were females and $94 \%$ were community-dwelling. Elders presented a predominantly vulnerable phenotype (FI $0.445 \pm 0.227$ ) with fair functional status (Barthel Index 70.1 \pm 25.1 ; ADL $4.06 \pm 1.94$ ), a severe risk for malnutrition (MNA-SF $9.19 \pm 3.14$ ) and multimorbidity (CIRS comorbidity index $3.99 \pm 1.76$; severity index $1.91 \pm 0.33$ ). After surgery, $88 \%$ reported at least one postoperative complication, $30 \%$ at least three complications. The inhospital and 90-days mortality were $2.8 \%$ and $15.2 \%$, respectively. Surgical timing $(\mathrm{t}-4.03, \mathrm{p}<0.0001)$, functional (Barthel Index $\mathrm{t}$ 4.4, p < 0.0001; IADL t 3.35, p < 0.0009) and cognitive decline (SPMSQ $\mathrm{t}-4.2, \mathrm{p}<0.00001$ ), poor nutritional status (MNA-SF $\mathrm{t}$ 3.99, $\mathrm{p}<0.0001$ ), decreased grip strength (Hand Grip $\mathrm{t} 4.3$, $\mathrm{p}<0.0001$ ) and multimorbidity (CIRS comorbidity $\mathrm{t}-3.28$, $\mathrm{p}<0.001$; CIRS severity $\mathrm{t}-4.38, \mathrm{p}<0.0001)$ were mostly associated with increased 90 -days mortality. Patients who died presented a more significant impairment on Frailty Index (FI 0.141 vs 0.615 ; $\mathrm{t}$ $-5.5, \mathrm{p}<0.00001)$, resulting all extremely frail.

Conclusions: Because among vulnerable elders high mortality after hip fracture surgery goes beyond the peri-operative, the assessment and stratification of frailty according to FI might be an useful tool able to delineate a possible trajectory of disability and survival.

\section{P-101}

Assessing pre-operative frailty index as indicator of 90 daysmortality in a cohort of older vulnerable adults with proximal femur fracture

\section{Chiara Giannotti ${ }^{1}$, Fiammetta Monacelli ${ }^{1}$}

${ }^{1}$ Hospital Policlinic San Martino, Section of Geriatric Medicine, Department of Internal Medicine and Medical Specialties (DIMI), University of Genoa, Genoa, Italy

Introduction: Hip fracture is often a fatal event in older people, associated with increased disability, morbidity and mortality, posing a significant public health concern. Predictors of adverse outcomes after hip fracture is essential to guide treatments, plan discharge and use of healthcare resources. Therefore, we sought to evaluate the ability of 25-items Frailty Index (FI) to assess the clinical variables associated with 90-days mortality in a group of elders undergoing hip fracture surgery.

Materials and methods: A consecutive series of 284 elders over 65 years with proximal femur fracture was recruited in the Orthopaedic and Trauma Unit, Department of Emergency, Hospital Policlinic San Martino, Genoa, Italy. All patients underwent comprehensive geriatric assessment pre-operatively. We retrospectively stratified patient's vulnerability on the basis of Frailty Index (FI), calculated using 25 items of the 70 provided by the list of the Canadian Study of Health and Aging (CSHA).

Results: The mean age was 86.6 years \pm 5.9 (range 72-103 years); $76 \%$ were females and $94 \%$ were community-dwelling. Elders presented a predominantly vulnerable phenotype (FI $0.445 \pm 0.227$ ) with fair functional status (Barthel Index 70.1 \pm 25.1 ; ADL $4.06 \pm 1.94$ ), a severe risk for malnutrition (MNA-SF $9.19 \pm 3.14$ ) and multimorbidity (CIRS comorbidity index $3.99 \pm 1.76$; severity index $1.91 \pm 0.33$ ). After surgery, $88 \%$ reported at least one postoperative complication, $30 \%$ at least three complications. The inhospital and 90-days mortality were $2.8 \%$ and $15.2 \%$, respectively. Surgical timing $(\mathrm{t}-4.03, \mathrm{p}<0.0001)$, functional (Barthel Index $\mathrm{t}$
4.4, $\mathrm{p}<0.0001$; IADL $\mathrm{t} 3.35, \mathrm{p}<0.0009)$ and cognitive decline (SPMSQ $\mathrm{t}-4.2, \mathrm{p}<0.00001$ ), poor nutritional status (MNA-SF $\mathrm{t}$ 3.99, $\mathrm{p}<0.0001$ ), decreased grip strength (Hand Grip $\mathrm{t}$ 4.3, $\mathrm{p}<0.0001$ ) and multimorbidity (CIRS comorbidity $\mathrm{t}-3.28$, $\mathrm{p}<0.001$; CIRS severity $\mathrm{t}-4.38, \mathrm{p}<0.0001)$ were mostly associated with increased 90 -days mortality. Patients who died presented a more significant impairment on Frailty Index (FI 0.141 vs 0.615 ; t $-5.5, \mathrm{p}<0.00001)$, resulting all extremely frail.

Conclusions: Because among vulnerable elders high mortality after hip fracture surgery goes beyond the peri-operative, the assessment and stratification of frailty according to FI might be an useful tool able to delineate a possible trajectory of disability and survival.

\section{P-102}

Does previous functional status determine mortality after suffering hip fracture? Associated factors in 1 year followup

Victoria Garay Airaghi ${ }^{1}$, Esther Lueje Alonso ${ }^{2}$, Yolanda Parada de Freitas $^{2}$, Lucía Fernández Arana ${ }^{2}$, Jesús Mora Fernández ${ }^{2}$

${ }^{1}$ Geriatrics and Gerontology Service, Hospital Clinico San Carlos, Madrid, Spain, ${ }^{2}$ Geriatrics and Gerontology Service, Hospital Clínico San Carlos, Madrid, Spain

Introduction: The mortality rate after osteoporotic hip fracture can reach $10 \%$ during admission in hospital and 30\% after 12 months [1, 2]. Aim: to describe the factors that influence long-term mortality after a fragility hip fracture (FHF).

Methods: 3-years retrospective study (January 2015 and February 2017) 1-year follow-up. Patients aged $>65$ years admitted with surgical management of FHF were included. Baseline characteristics (Age, sex, comorbidity, previous radiotherapy (RT)), functional status (Barthel Index (BI), Lawton Index (LI), Functional Ambulation Classification (FAC)), cognitive impairment, and in-hospital complications. Multivariate logistic regression analysis and 3 logistic regression model (BI, LI, FAC) has been performed respectively. Statistical significance $\mathrm{p}<0.005$. SPSS 23.0.

Results: 680 patients were included; $\geq 90$ years $30.1 \%$, women 20.6\%. 53.5\% BI > 80. Severe cognitive impairment $6.3 \%$. $4.4 \%$ death before discharge. BI regression model: Sex $(\mathrm{p}<0.001)$, age $>90(\mathrm{p}=0.003)$, RT $(\mathrm{p}=0.029)$, BI $(\mathrm{p}=0.039)$, Respiratory infections $(p<0.001)$, delirium $(p<0.001)$, renal failure $(p=0.026)$, heart failure $(p=0.002)$. FAC regression model: Sex $(\mathrm{p}<0.001)$, age $>90(\mathrm{p}=0.005), \mathrm{RT}(\mathrm{p}=0.032)$, FAC $(\mathrm{p}=0.017)$, Respiratory infections $(\mathrm{p}<0.001)$, delirium $(\mathrm{p}<0.001)$, renal failure $(p=0.026)$, heart failure $(p=0.001)$. LI regression model: Sex $(\mathrm{p}<0.001)$, age $>90(\mathrm{p}=0.004)$, RT $(\mathrm{p}=0.032)$, LI $(\mathrm{p}=0.016)$, Respiratory infections $(\mathrm{p}<0.001)$, delirium $(\mathrm{p}<0.001)$, renal failure $(\mathrm{p}=0.025)$, heart failure $(\mathrm{p}=0.001)$.

Conclusions: Being a female, nonagenarian, having received previous RT, having developed in-hospital complications, and poor functional status (BI, FAC, LI) before the fracture determine the longterm mortality in our sample.

\section{References:}

1. Roche JJ, Wenn RT, Sahota O (2005) BMJ 331(7529):1374

2. Morris AH, Zuckerman JD (2002) J Bone Jt Surg Am 84A(4):670-674 


\section{P-103}

\section{Associated factors in 30-days mortality after fragility hip fracture}

Esther Lueje Alonso ${ }^{1}$, Yolanda Parada de Freitas ${ }^{1}$, Victoria Garay Airaghi $^{1}{ }^{1}$, Lucía Fernández Arana ${ }^{1}$, Jesús Mora Fernández ${ }^{1}$

${ }^{1}$ Geriatrics and Gerontology Service, Hospital Clínico San Carlos, Madrid, Spain

Introduction: The mortality rate after osteoporotic hip fracture can reach $10 \%$ during admission in hospital and $30 \%$ after 12 months [1, $2]$. The aim of the study is to describe the factors that influence short term mortality after a fragility hip fracture (FHF).

Methods: 3 years retrospective study (January 2015 and December 2017). Patients aged $>65$ years admitted to the Orthogeriatric Unit with surgical management of FHF were included. Variables: Baseline characteristics (Age, sex, comorbidity, previous radiotherapy (RT)), functional status (Barthel Index (BI), Lawton Index (LI), Functional Ambulation Classification (FAC), walking aids), cognitive impairment and social assessment. Surgery characteristics (ASA) and inhospital complications (IC) (Infections, organ failure, acute confusional syndrome). Multivariate logistic regression analysis and 3 logistic regression model (BI, LI, FAC) has been performed. Statistical significance $\mathrm{p}<0.005$. SPSS 23.0.

Results: 963 patients were included; $>90$ years $31.4 \%$, women $21.3 \%$. Severe cognitive impairment $6.4 \%$. $4.4 \%$ death before discharge. BI regression model: Sex $(p=0.035)$, age $>90(p=0.00)$, RT $(p=0.001)$, BI $(p=0.040)$, IC $(p=0.018)$. FAC regression model: Sex $(p=0.47)$, age $>90(p=0.00)$, RT $(p=0.002)$, FAC $(p=0.069)$, IC $(p=0.013)$. LI regression model: Sex $(p=0.064)$, age $>90(p=0.00)$, RT $(p=0.002)$, IC $(p=0.008)$.

Conclusions: Being a female, nonagenarian, having received previous RT, having developed in-hospital complications, and poor functional status (BI, FAC) before the fracture, determine the short term mortality. References:

1. Roche JJ, Wenn RT, Sahota O (2005) BMJ 331(7529):1374

2. Morris AH, Zuckerman JD (2002) J Bone Jt Surg Am 84A(4):670-674

\section{P-104}

\section{Impact of diabetes on functional outcome after hip fracture}

Lourdes Del Rosario Evangelista Cabrera ${ }^{1}$, Esther Lueje Alonso ${ }^{2}$, Victoria Garay Airaghi ${ }^{2}$, Lucía Fernández Arana ${ }^{2}$, Karina Liz Quiñones Huayna ${ }^{2}$, Jesús Mora Fernández ${ }^{2}$

${ }^{1}$ Department of Geriatrics, Hospital Universitario Severo Ochoa, Madrid, Spain, ${ }^{2}$ Department of Geriatrics, Hospital Clínico San Carlos, Madrid, Spain

Introduction: The objective of this study was to describe the influence of diabetes mellitus on functional outcome after hip fracture.

Methods: Prospective one-year follow-up study. Patients aged $>65$ admitted with fragility hip fracture(September 2015-March 2017). Variables: Functional(Barthel index-BI-, Functional ambulation classification-FAC-, Lawton index-LI-) at baseline(b), at discharge(d) and at 1-year follow-up(f), comorbidity (CIRS-G, Charlson-Index), biochemical parameters and complications. The 75th percentile value of the improvement of the BI(f), respect to the $\mathrm{BI}(\mathrm{d})$ was used as the cutoff point to define satisfactory functional outcome( $>38$ points). Statistical analysis: Logistic regression analysis. SPSS 25.0.

Results: 237 patients were included; age $85.46 \pm 6.88$, women $75.9 \%$, number of drugs 6.6 (IQR 4-9), cognitive impairment $36.7 \%$, Charlson 5.9 (IQR 4.90-7.25), CIRS-G 12 (IQR 9-16). Diabetes
$25.7 \%$. The $86.9 \%$ use antidiabetic drugs (insulin $23.0 \%$, oral antidiabetic $70.5 \%$ ). Glycated haemoglobin (HbA1c) $6.5 \pm 1.2$. The mean $\mathrm{BI}(\mathrm{b}), \mathrm{BI}(\mathrm{d})$ and $\mathrm{BI}(\mathrm{f})$ for diabetics was $82.49 \pm 15.2$, $44.85 \pm 18.2, \quad 64.69 \pm 29.4$ and $85.82 \pm 14.7, \quad 45.22 \pm 19.3$, $70.61 \pm 26.6$ for nondiabetic patients. Diabetic patients have higher comorbidity $(p<0.0005)$, lower levels of haemoglobin $(p=0.009)$ and Vitamin-D ( $\mathrm{p}=0.018)$, use more drugs $(\mathrm{p}<0.0005)$, and have a time to surgery $\geq 48 \mathrm{~h}(\mathrm{p}=0.031)$. Factors associated with satisfactory functional outcome after 1-year: normal cognitive $(\mathrm{p}=0.023), \mathrm{BI}(\mathrm{b})(\mathrm{p}=0.001)$, non-diabetes $(\mathrm{p}=0.018), \mathrm{LI}(\mathrm{b})(0=$ $0.004), F A C(d)(p=0.014)$ and non-Institutionalization $(p=0.023)$. In multivariate analysis the only independent predictors were BI(b) (OR 1.04; 95\% CI 1.01-1.07; p = 0.007) and non-diabetes(OR 2.7; 95\% CI 1.08-7.11; $\mathrm{p}=0.033$ ).

Conclusions: The correction of anemia and levels of vitamin-D should be optimized in diabetic patients with hip fracture. There is no difference in the functional outcome at discharge of diabetic and nondiabetic patients with hip fracture, however they have worst functional outcome during the follow-up.

\section{P-105}

\section{Psychotropic drug use among older hip fracture patients}

Sonia Jiménez Mola ${ }^{1}$, María Piar López Viñas ${ }^{2}$, Antonio Rodríguez Rosa $^{3}$, Javier Idoate Gil ${ }^{4}$, Encarna Martín Pérez ${ }^{5}$, Pilar Sáez López

${ }^{1}$ Complejo Asistencial Universitario de León, León, Spain,

${ }^{2}$ Residencia Mixta Personas Mayores, León, Spain, ${ }^{3}$ Hospital San Juan de Dios, León, Spain, ${ }^{4}$ Complejo asistencial Universitario de León, León, Spain, ${ }^{5}$ Hospital san Juan de Dios, León, Spain

Introduction: Drugs with effects on the central nervous system have been associated with an increased hip fracture risk through several mechanisms. Sedatives, anxiolytics and antidepressants may increase the risk of fall. Neuroleptics inhibit dopaminergic receptors and may therefore increase prolactin secretion and thus decrease bone mineral density (BMD). We aim to investigate the intake of these drugs in patients admitted for hip fracture in our Hospital and its relationship with evolution.

Method: Observational, longitudinal, prospective study of 534 patients, aged 75 years or older, admitted for hip fracture during 2014. Variables: age, sex, cognitive and functional baseline and discharge status and taking of psychotropic drugs. The possible relationship between these variables was analyzed. SPSS ${ }^{\circledR}$, v.22.0.

Results: Among the 534 participants, 499 (93\%) underwent surgery. $75.4 \%$ were women and the mean age $86.1 \pm 7.3$ years. Only 132 (25\%) had a previous diagnosis of dementia. 64\% walk independently. $26 \%$ live in nursing homes prior to fracture. $69 \%$ had Barthel $>60$. They take benzodiazepines $35 \%$, antidepressants $32 \%$ and neuroleptics $9.7 \%$. All were related $(\mathrm{p}<.001)$ with worse ambulation at admission and discharge $(\mathrm{p}<.01)$. Benzodiazepines were more frequent $(49 \%)$ in the nonagenarian group $(\mathrm{p}<.001)$ and antidepressants in women (35\% vs. $23 \%$ ). No relation was found $(p>.05)$ with in-hospital mortality or with the presentation of delirium.

Conclusions: Patients with psychotropic drugs at admission have worse cognitive and functional status and also worse ambulation. The intake of benzodiazepines is high in the group of nonagenarians. Geriatric assessment during hip fracture admission improves the prescription of these drugs.

\section{Area: Vaccines and immunization}




\section{P-106}

\section{Challenges in adult vaccination}

Eduardo de Gomensoro ${ }^{1}$, Giuseppe Del Giudice ${ }^{1}$, T. Mark Doherty ${ }^{1}$ ${ }^{1}$ GSK, Brentford, UK

In older adults infectious diseases remain a major cause of illness and death, and life-long primary preventive interventions, including healthy diet and exercise, are key to meeting the healthcare challenges that accompany demographic changes. Vaccination also has a role to play, but adult vaccine coverage is lower than target rates in most developed countries. In 2015 44.8, 24.6 and $23.1 \%$ of US adults were vaccinated against seasonal influenza, hepatitis B or tetanus-diphtheria-pertussis, respectively; $23 \%$ of adults at-risk of pneumococcal disease were covered; $30.6 \%$ of $\geq 60$-year-olds received herpes zoster immunization. Unawareness by the public and some healthcare providers (HCPs) of vaccination's benefits, inconsistent providers' recommendations, cost-benefit uncertainties and lower vaccine efficacy (VE) in older adults all play a part. Unadjuvanted influenza vaccines have lower VE in $\geq 65$-year-old subjects $(17-50 \%)$ compared with younger adults (70-90\%). Similarly, VE with liveattenuated herpes zoster vaccine falls as age increases: from $69.8 \%$ in $50-59$-year-olds to $18.3 \%$ in $\geq 80$-year-olds. Improvement of VE in older adults is required and could encourage HCPs to recommend vaccination. Adjuvantation of influenza vaccine was shown to improve immune responses in elderly, and an adjuvanted recombinant zoster vaccine showed high VE regardless of age: VE for adults aged $50-59,60-69,70-79$, or $\geq 80$ years was $96.6,97.4,91.3$ and $91.4 \%$, respectively. To achieve optimal vaccine uptake in adults, economic analyses demonstrating the overall value of vaccination, and acceptance of adult vaccination by the public and HCPs are needed to allow people to live healthier, longer lives.

\section{P-107}

Impact of frailty on the immunogenicity of the varicella-zoster vaccine in community-dwelling older adults: a prospective cohort study

Jung-Yeon Choi ${ }^{1}$, Kwang-il Kim ${ }^{1}$, Kyoung-Ho Song ${ }^{1}$, Min-gu Kang ${ }^{1}$, Cheol-Ho Kim ${ }^{1}$, Hong Bin Kim ${ }^{1}$, Kyoung Un Park ${ }^{1}$, Wan Beom Park $^{2}$

${ }^{1}$ Seoul National University Bundang Hospital, Seongnam, South Korea, ${ }^{2}$ Seoul National University Hospital, Seoul, South Korea

Objectives: We aimed to evaluate the immunogenicity of the herpes zoster live-attenuated vaccine (ZVL) in elderly and to determine whether it is influenced by frailty status and baseline cytokine levels. Methods: Community-dwelling elderly aged $\geq 65$ years were prospectively enrolled and administered ZVL after assessing their frailty status and cytokine levels. Humoral and cellular immunogenicity were assessed using enzyme-linked immunosorbent assays (ELISAs) for the varicella-zoster virus (VZV)-specific IgG antibody and VZV-specific enzyme-linked immunospot (ELISPOT) assays, respectively, before and 6 -week after vaccination.

Results: Sixty-nine participants (mean age, 72.3 years) were enrolled; 31 (44.9\%) were prefrail-to-frail (P-F). Thirty-seven participants $(53.6 \%)$ had a $>2$ geometric mean fold rise (GMFR) of IgG antibody 6 -week after vaccination, $31.9 \% \mathrm{p}$ more participants had $\geq 10$ spotforming cells (SFCs) and 42 subjects $(60.9 \%)$ experienced $>2$-fold increase of GMFR of ELISPOT assay. The GMFRs of the antibody titres were similar in the non-frail and P-F groups (2.71 vs. 3.55, respectively, $\mathrm{P}=0.193$ ); moreover, the baseline ELISPOT assay geometric mean values were not significantly different before $(29.7$ vs. 49.6, respectively, $\mathrm{P}=0.296)$ and 6 -week after vaccination $(21.4$ vs. 41.0 , respectively, $\mathrm{P}=0.460)$. We observed no significant correlations between baseline cytokine levels and immunogenicity as assessed by antibody GMFR and difference of ELISPOT levels before and 6-week after vaccination.

Conclusion: Approximately half of our community-dwelling elderly experienced a twofold rise in antibodies, and one-third more subjects showed $\geq 10$ SFCs ELISPOT response after ZVL administration. Our data suggest that the ZVL can boost humoral and cellular immunity to similar extents in P-F and non-frail elderly patients.

\section{P-108}

\section{Elderly living with HIV_portrait of a Portuguese population}

J. E. Mateus ${ }^{1}$, S. $\operatorname{Costa}^{1}$, R. Sá ${ }^{2}$ I. Ramos ${ }^{2}$, J. Oliveira ${ }^{2}$, J. Saraiva da $\mathrm{Cunha}^{2}$, M. T. Veríssimo ${ }^{1}$

${ }^{1}$ Internal Medicine Department, Centro Hospitalar e Universitário de Coimbra, Coimbra, Portugal, ${ }^{2}$ Infectious Disease Department, Centro Hospitalar e Universitário de Coimbra, Coimbra, Portugal

Introduction: The epidemiology of the HIV infection is changing, with significant increase in the number of elderly patients, due to prolonged survival following antiretroviral therapy (ART) but also to the rising of new diagnosis at an older age. Managing the HIV/AIDS elderly population presents numerous clinical and immunological challenges.

Methods: HIV infected patients aged 65 or more years currently followed by the Infectious Disease Department at a tertiary center in Portugal were included in the study. Hospital records were reviewed. Results: There are 303 HIV infected geriatric patients (representing $15 \%$ out of 2017$)$ at our center $(73 \%$ men $)-96 \%$ transmission by sexual contact. Viral co-infection has low expression (HBV 9\%; HCV $1 \%$ ). At diagnosis median viral load was $37,670 \mathrm{copies} / \mathrm{mL}$ and median CD4 cell count was 249 cells/mcL. Most patients (58\%) were diagnosed following symptomatic infection, $17 \%$ of these had an AIDS-defining condition. In this study, $22 \%$ had one or more comorbidities (dyslipidemia 66\%, diabetes 22\%, kidney disease $20 \%$, non-AIDS-defining malignancy $11 \%$ ), with an average of 2.0 comorbid conditions/person. Our population shows a high adherence to ART $(97 \%)$ and $88 \%$ have viral control.

Key conclusions: As the HIV population ages and the rate of newly detected infections in the elderly rise, clinicians should be aware of the increasing need to balance HIV care and the management of comorbid conditions commonly associated including the potential interactions between ART and drugs used to treat age-related pathologies. The impact of the HIV/AIDS elderly population on the health-care system needs greater recognition and study.

\section{P-109}

Immunization coverage in elderly: primary care physicians views and practices

Florio Dorothee ${ }^{1}$, Drevet Sabine ${ }^{2}$, Bioteau Catherine ${ }^{2}$, Gavazzi Gaetan $^{2}$

${ }^{1}$ Grenoble Prevention Center, Grenoble, France, ${ }^{2}$ Grenoble Alpes University Hospital, Geriatric Department, Grenoble, France

Introduction: Vaccination is an important part of prevention in elderly, especially to prevent some serious infection that could lead to death. In most cases primary care physicians are responsible for the management of community dwelling old patients. Despite availability 
of the vaccination schedule and digital tools, immunization practice remains inadequate. The main objective of the study was to assess general practitioners (GPs) opinions and practices regarding older populations.

Method: This study sought to identify the views of a sample of 250 GPs contacted through a local mailing list. A self-administered questionnaire was assessing socio-demographic characteristics of GPs, their views about vaccination, vaccine recommendations, barriers encountered, references they use routinely, their continuing education status.

Results: 180/250 (72\%) responded. GP's mean age was 42.5 years, $50 \%$ were urban GPs, $2 / 3$ worked in a group. Two-thirds used a vaccine record. $99.5 \%$ were in favor of vaccination, $98.2 \%$ recommended influenza vaccination, $95.5 \%$ for the DTP, but only $52.2 \%$ for pneumococcal, $50 \%$ for pertussis, $21.1 \%$ for herpes zoster, Twothirds considered the illness ranges in severity, but only $5 \%$ recognized a collective interest and recommended at same level pneumococcal and pertussis vaccination. $40 \%$ used a vaccination record, $25.5 \%$ was awarded of the electronic health card. $48.8 \%$ were trained in vaccinating, $25 \%$ have taken part in a vaccinal campaign. Conclusions: Most of GPs are in favour of vaccination for elderly but very few recommended all vaccine and numerous discrepancies appeared between intentions and behaviours. Both theoretical and practical education should be enhanced on all aspect of vaccination.

\section{P-110}

Impact of the adjuvanted recombinant zoster vaccine on pain and use of pain medication in adults aged $\geq 50$ years

Robert Johnson on behalf of the ZOE-50/70 Study Group ${ }^{1}$

${ }^{1}$ University of Bristol, Faculty of Health Sciences, Bristol, UK

Introduction: To determine the efficacy of a adjuvanted Recombinant Zoster Vaccine (RZV) in reducing the burden of illness (BOI, i.e. pain severity*duration*incidence) and use of pain-related medication in herpes zoster (HZ) episodes.

Methods: The assessments were integrated in two Phase III trials, ZOE-50 (subjects $\geq 50$ years of age [YOA], NCT01165177) and ZOE-70 (subjects $\geq 70$ YOA, NCT01165229). Pain was assessed by the Zoster Brief Pain Inventory (ZBPI) instrument. We report results of the ZOE-50 and a pooled analysis of subjects $\geq 70$ YOA from the two trials combined. In addition, use of HZ-related pain medication was recorded.

Results: The estimated vaccine efficacy (VE) in reducing the HZ BOI was $98.4 \%$ and $92.1 \%$ in the ZOE-50 study and the pooled analysis of subjects $\geq 70$ YOA, respectively. In confirmed cases, RZV significantly reduced the maximal ZBPI worst pain score in the pooled analysis $(p=0.032)$ and the maximal ZBPI average pain scores in both the ZOE-50 $(\mathrm{p}=0.049)$ and the pooled analysis $(\mathrm{p}=0.043)$. The use of medication for $\mathrm{HZ}$ pain was reported for six $(66.6 \%)$ and $190(74.8 \%)$ patients in the RZV and placebo groups, respectively for the ZOE-50 study ( $\mathrm{p}=0.697)$. Corresponding numbers in the pooled analysis were $11(44.0 \%)$ and $205(72.2 \%)$ patients, respectively $(\mathrm{p}=0.006)$.

Conclusions: RZV significantly reduced the HZ BOI, particularly by a very high $\mathrm{VE}$ in preventing $\mathrm{HZ}$. For $\mathrm{HZ}$ breakthrough cases, the results suggest that RZV mitigated the severity of HZ-related pain compared to placebo $\mathrm{HZ}$ cases. Additionally, use of pain medication was reduced significantly in adults $\geq 70$ YOA.
P-112

Real-world effectiveness of live zoster vaccine: a systematic review

Patricia Saddier ${ }^{1}$, Morgan A. Marks ${ }^{1}$, Shawna R. Calhoun ${ }^{1}$, Xiaoyan $\mathrm{Lu}^{1}$, Kelly D. Johnson ${ }^{1}$, Yola Moride ${ }^{2}$

${ }^{1}$ Merck Sharp and Dohme, Corp., Center of Observational and RealWorld Evidence (CORE), Kenilworth, NJ, USA, ${ }^{2}$ YolaRx

Consultants, Montreal, Canada

Background: Several studies of the real-world effectiveness of Zostavax $^{\mathrm{TM}}$, a live zoster vaccine (ZVL), have been published since its licensure in 2006. The objective of this review was to summarize available evidence on vaccine effectiveness (VE) of ZVL against herpes zoster (HZ) and post-herpetic neuralgia (PHN) in the general population.

Methods: An extensive literature search was performed in Embase and Medline for the period January 2007 to January 2018 to identify peer-reviewed, original, English study manuscripts reporting the results of observational studies of ZVL VE in the general population. Results: Seven original studies were identified ( 5 from the United States and 1 each from the United Kingdom and Canada) that assessed $\mathrm{HZ}$ effectiveness in the general population. Five of these studies also assessed PHN effectiveness. VE against $\mathrm{HZ}$ was similar across studies in the early years following vaccination, but tended to diverge in the later years (overall VE against $\mathrm{HZ}$ ranged from 33 to $62 \%$, clustering around $\sim 50 \%$ across studies providing this information). Overall VE against PHN ranged from 55 to $88 \%$, clustering around $\sim 65 \%$.

Conclusion: Real-world observational studies assessing the effectiveness of ZVL in preventing $\mathrm{HZ}$ and PHN in the general population reported generally similar results. Differences in VE estimates across studies were likely driven by differences in study design and methods, including sample size and age of study population, HZ and PHN definition, follow-up duration, and methods of adjustment. We are currently conducting a meta-analysis to assess potential heterogeneity across studies and calculate summary VE estimates.

\section{P-113}

Vaccination rates among patients 65 years and older who require interpreter services in Minnesota

Maria Mendoza-De la Garza ${ }^{1}$, Stephanie Quigg ${ }^{1}$, Ericka Tung ${ }^{1}$, Paul Takahashi ${ }^{1}$

${ }^{1}$ Division of Primary Care Internal Medicine, Mayo Clinic College of Medicine, Rochester, MN, USA

Background: Profound disparities are faced by the growing population of older adults with Limited English Proficiency (LEP) in the US. Previous research on vaccination of older adults has been based on self-report data, without clinical verification. We aim to compare vaccination rates of 13-valent pneumococcal conjugate vaccine (PCV13) and 23-valent pneumococcal polysaccharide vaccine (PPSV23) among LEP patients with English speakers and determine the predictors of vaccination completion.

Methods: This is a population-nested matched cohort from Minnesota, US of individuals 65 years and older. LEP patients were identified by an electronic alert within the electronic medical record, designed to determine the need for an interpreter. Cases were matched 1:1 on the basis of age, sex, and Charlson Comorbidity Index.

Results: 24052 patients were identified. Of those 617 (2.6\%) were individuals with LEP, $60 \%$ were females with a mean age of $74 \pm 7$ years. The most common primary languages were Somali (24\%), Vietnamese (15\%) and Spanish (13\%). Univariate analysis 
revealed higher rates of non-vaccination with PCV13 in the LEP group vs English speakers (62\% vs. 77\% OR 2.07 95\% CI 1.61, 2.66 p-value $<0.0001)$. Results were similar for PPSV23 (60\% vs. $75 \%$ OR $1.9795 \%$ CI 1.54, 2.51 p-value < 0.0001).

Discussion: The data suggest that older adults who required an interpreter during medical encounters were less likely to be vaccinated with PCV13 and PPSV 23 as compared to older adults who do not require an interpreter. There is a need for effectiveness studies to determine which interventions can help this vulnerable group.

\section{P-114}

\section{A 86-Year-old man with Varicella (chickenpox)}

Vanessa Álvarez Vidal, MD; Laila Dib, MD; Gianina Emily Sedano Gómez, MD and RocÚo Malfeito Jiménez, MD

Department of Geriatrics, University Hospital Lucus Augusti, Lugo, Spain

Introduction: Chickenpox is an unsual condition but life-threating disease in elderly individuals. Vaccination is recommended for adults, yet not many have had access to this treatment.

Clinical case: Man aged 86 appears with a 72 hour rash in face, limbs and torso (back and chest). He never suffered from chickenpox before, therefore he is not vaccined for so. Aciclovir was prescribed, and yet an acute respiratory failure, anuria and hypotension were later developed. After admitted to intensive care unit (ICU), intubation and vasoactive drugs were required. He deceased mainly due to hemorragic chickenpox associated with dissiminated intravascular coagulation, pneumonia, hepatitis and glomerulonephritis.

Methods and results: Laboratory dates of renal failure and stroke, as trombocytopenia, consumption of fibrinogen and complement with elevation of transaminases and lactate. Serum was positive for VZV (Varicella-Zoster Virus).

Conclusions: Weakened cell-mediated immunity expected in elderly individuals could lead to more severe diseases. Delayed or weakened virus specific T-cell recognition has been shown to correlate with a more serious outcome in chickenpox (pneumonia, ataxia, encephalitis, hepatitis, and hemorrhagic conditions) that is much more common in geriatric population. Since the clinical features of breakthrough varicella are often mild, an accurate diagnosis of the clinical presentation is a difficult task. Chickenpox rash must be treated with antivirals applied onset within 24 hours. Aciclovir prescription is recommended for inmunosupressed patients.

\section{P-115}

Vaccination for influenza and pneumococcus in an acute geriatric unit

Jaime Rodríguez Salazar ${ }^{1}$, Lourdes Del Rosario Evangelista Cabrera ${ }^{1}$, Juan García Martinez ${ }^{1}$, Soledad Dominguez Mendoza ${ }^{1}$, María Del Rosario García Martín ${ }^{1}$, Carmen Navarro Ceballos ${ }^{1}$, María Jesús Molina Hernández ${ }^{1}$

${ }^{1}$ Department of Geriatrics, Hospital Universitario Severo Ochoa, Madrid, Spain

Introduction: To determine the prevalence of vaccination for influenza and pneumococcus in an acute geriatric unit (AGU) in patients diagnosed with respiratory infection, influenza or pneumonia.

Methods: We select patients admitted to AGU in 2017 with diagnoses of respiratory infection (RI), pneumonia and confirmed influenza. The following data were obtained: age, sex, history of chronic obstructive pulmonary disease (COPD), history of diabetes mellitus (DM). Functionality (Barthel Index (BI), Functional Ambulation Categories (FAC)), mental red cross scale (CRM) baseline and at discharge (b- and d-), influenza and pneumococcal vaccination, and main diagnose: RI, pneumonia (P), invasive pneumococcal disease (IND), length of stay (LOS) and death during hospitalization. Statistical analysis: Chi square, Mann-Whitney U, T-Student, SPSS 25.0. Results: 213 patients were included (46.9\% men, 53.1\% women). COPD: $28.6 \%$. DM2: $29.6 \%$ b-BI: $48.95 \pm 35.95$, d-BI: $44.31 \pm 34.63$; b-FAC: $2.25 \pm 1.881$, d-FAC: $1.94 \pm 1,771$; b-CRM $1.52 \pm 1.46$, d-CRM $1.69 \pm 2.12$ ). Influenza vaccine (IV) 164 (77\%). 23 pneumococcal vaccine (P23 V): $170(79.8 \%)$. Second P23V: 10 (4.7\%). 13 pneumococcal vaccine (P13): 8 (3.8\%). Diagnoses: Flu: 4(1.9\%). RI: 129(60.6\%). P: 90(42.3\%). IND: 4(1.9\%). Death: 15 (7\%). LOS: $8.48 \pm 6.061$. We obtained statistical significance in: greater IV in men versus women $(p=0.009)$, greater IV in COPD patients $(\mathrm{p}=0.004), \mathrm{P} 23 \mathrm{~V}$ and IV $(\mathrm{p}<0.0005)$, death and IV $(\mathrm{p}=0.004)$.

Conclusions: In our AGU, the rates of influenza and pneumococcal vaccine are over the WHO recommendations. Men and COPD patients are more vaccinated for flu. P23V is related with more IV. IV is related with lower rates of mortality. Vaccination is important for maintaining health status in elderly people.

\section{P-116}

Cervical HPV infection in peri- and postmenopausal women at a reference center in Northeast Brazil

Leonardo Victor Galvão-Moreira ${ }^{1}$, Joyce Pinheiro Leal Costa ${ }^{1}$, Haissa Oliveira Brito ${ }^{1}$, Maria Bethânia da Costa Chein ${ }^{1}$, Flávia Castello Branco Vidal ${ }^{1}$, Maria do Desterro Soares Brandão Nascimento $^{1}$, Débora Sousa ${ }^{1}$, Ismael Dale Cotrim Guerreiro da Silva $^{2}$, Luciane Maria Oliveira Brito ${ }^{1}$

${ }^{1}$ Federal University of Maranhão, São Luís, Brazil, ${ }^{2}$ Federal University of São Paulo, São Paulo, Brazil

Introduction: Although the occurrence of cervical HPV infection in peri- and postmenopausal women remains widely controversial, this population has been targeted by screening programs for cervical cancer.

Objectives: To investigate the occurrence of cervical HPV infection among peri- and postmenopausal women.

Methods: A cross-sectional study was carried out and included a total of 70 women, aged 35-65 years (mean age $51.1 \pm 8.4$ ), who attended outpatient clinics of a public research hospital in São Luís, Brazil, in the period from 2015 to 2017. A questionnaire was applied to the participants regarding sociodemographic, behavioral and clinical variables. After completing the questionnaire, patients underwent the collection of endocervical material for identification and genotyping of HPV DNA using polymerase chain reaction (PCR) assay, followed by collection of material for oncotic cytology.

Results: The prevalence of positive HPV DNA was $40 \%$, being HPV 16 the most prevalent subtype (28.6\%) and women aged 51-65 the most affected age group. Nevertheless, there was no statistically significant association between age and HPV-DNA status $(p=0.10)$. Women either positive or negative for HPV DNA showed similar characteristics regarding number of partners, condom use, smoking status, and history of sexually transmitted infections. Most non-infected women $(59.5 \%)$ reported having a fixed partner, and this was shown to be a protective factor for HPV infection ( $\mathrm{p}=0.02$; prevalence ratio $=0.50$ ).

Conclusion: Even though a high prevalence of high risk HPV was found in postmenopausal women, age was not associated with the occurrence of cervical HPV infection. 


\section{P-117}

Update on the use of herpes zoster vaccination (HSV) among older persons from Qatar

Navas Nadukkandiyil ${ }^{1}$, Hanadi Al Hamad ${ }^{1}$, Mahmoud Al Refae ${ }^{1}$, Marwan Ramadan ${ }^{1}$, Mohammed Al Husami ${ }^{1}$

${ }^{1}$ Hamad Medical Corporation, Doha, Qatar

Introduction: The vaccine for shingles (Zostavax ${ }^{\circledR}$ ) is recommended for use in people 60 years old and older to prevent shingles. The shingles vaccine is specifically designed to protect people against shingles and will not protect people against other forms of herpes, such as genital herpes. AIM: To improve the vaccination rates and documentation of Herpes Zoster Vaccination (HSV) from 0 to $90 \%$ of geriatric in and outpatient by the end of December 2015 and $100 \%$ by end of June 2016 and to reassess the effectiveness of the program by December 2016.

Methods: Introduced HSV, in addition to existing adult vaccines like pneumococcal and influenza vaccines. Staff education by hand out and presentation about Zoster vaccinations indication, and implementation of adult vaccine card. Data extraction from patient files to determine baseline adult vaccination and documentation rates. New registry for Zoster vaccination in geriatric setting and prepare an addendum to the antimicrobial policy as vaccination program for preventable infectious disease.

Results: Vaccination program achieved highest rates Dec 2015 and it exceeded the goal of $90 \%$ for inpatients and outpatients.

Conclusions: Implementation to Long-term specialized care and Home care services elderly patients into this program. Meeting with the pharmacy for creating alertness for prescriptions to identify non vaccinated elderly patients.

\section{P-118}

Prevalence of influenza vaccine hesitancy in healthcare worker at hospital's elderly: a new "vaccinal packing"

Cyprien Arlaud ${ }^{1}$, Frederic Prate $^{1}$, Isabelle Bereder ${ }^{1}$, Veronique Mondain ${ }^{1}$, Olivier Guerin ${ }^{1}$

\section{${ }^{1}$ Medical Doctor}

Introduction: Infections from the Influenza virus represent a hazard vulnerable subjects aged $\geq 75$ years who can lead to high morbidity and mortality. Because they work proximity to these patients, healthcare workers are at risk of infecting elderly patients. We aimed to determine the prevalence of influenza vaccine hesitancy and motors of vaccine acceptance.

Material: The study was conducted during the 2017-2018 winter season. We determine rates to influenza infection confirmed in individuals aged 75 years or over and prevalence of influenza vaccine in health care worker. We propose them to completed a validated questionnaire on influenza vaccine to determine flu why vaccine uptake remains low among.

Results: We report a case series of 56 elderly patients with influenza virus infection (55\% were infected with influenza B virus) the leading cause of death in $15 \%$. Vaccination coverage among health professionals was $19.5 \%$ : physicians $(30 \%)$, nurses $(29 \%)$ and administrative staff $(25 \%)$. A validated questionnaire will be put in place on the web platform of the hospital center. It will be feasible in $120 \mathrm{~s}$, participants were asked whether they had been vaccinated or not against the flu in the 2017/2018 flu season, and will allow a sociological analysis and behavior to refusal of vaccination.

Conclusion: We expect to increase the vaccination coverage in health care worker putting in a "new vaccine packing" adapted to specific behavior by promoting citizen behavior $£$.

\section{P-119}

Octogenarian women with an extensive skin rash on the leg

Halina Kusz ${ }^{1}$, Ali Ahmad ${ }^{1}$, Shujing Lin ${ }^{1}$

${ }^{1}$ McLaren Flint/Michigan State University, East Lansing, USA

Introduction: Herpes zoster (HZ), or shingles, is a debilitating condition that predominantly affects older adults. It is caused by the reactivation of the varicella zoster virus (VZV) due to decline in cellmediated immunity over time. Older adults often will present with an atypical symptoms, leading to delayed or inaccurate diagnosis, and thereby, causing more complications.

Case presentation: An 87 year-old Caucasian female with a previous history of hypertension and chronic atrial fibrillation presented to the clinic with a skin rash on her left lower extremity which spontaneously appeared $48 \mathrm{~h}$ ago. She denied any preceding symptoms, such as itching, pain, fever or chills. She also denied similar episodes, dietary changes, or the introduction of new medications, detergents or skin products. Her immunization history revealed no prior vaccination for HZ. Physical examination demonstrated a frail older female in the wheelchair, not in acute distress. Vital signs were stable. On skin examination, there were clusters of vesicular and bullous eruptions with an underlying erythematous base in the L1-L2 dermatomal distributions. HZ was ascertained and antiviral therapy was initiated. The infection resolved in 2 weeks without complications.

Discussion: HZ often presents in individuals with underlying immunosuppression, however it may spontaneously occur. Atypical presentations are infrequent, but observed more commonly in older individuals as seen in our patient with multiple dermatomal involvement. Prevention with vaccination is imperative, but continues to be underutilized.

Conclusion: The use of appropriate vaccination to prevent shingles and annual skin examinations should be encouraged to all older adults.

Area: Biogerontology and genetics

\section{P-120}

\section{Gut microbiota and vascular aging biomarkers}

Daria Kashtanova ${ }^{1}$, Olga Tkacheva ${ }^{1}$, Yulia Kotovskaya ${ }^{1}$, Ekaterina Doudinskaya ${ }^{1}$

${ }^{1}$ Russian Clinical Research Center for Gerontology, Moscow, Russia

Aim: To study the association between the gut microbiota composition and arterial wall properties in patients without clinical manifestation of cardiovascular disease.

M\&M: The study included 92 Moscow residents, 66 women and 26 men aged 25 to $76 y / o(52 \pm 13 y / o)$ carefully selected through exclusion of CVD by means of clinical and laboratory evaluation, ECG, treadmill test, ECHOCG, carotid ultrasound examination with IMT measurement. PWV measurement was performed by using the SphygmoCor. Gut microbiota was studied by $16 \mathrm{~S}$ rRNA sequencing. Statistical analysis was performed using the R3.1.0., generalized linear models (FDR, age and sex adjusted) and clustering methods.

Results: About $50 \%$ of the total microbial abundance was represented by five genera: Blautia, Bacteroides, Prevotella, Faecalibacterium, and Clostridium. Blautia was the most dominant genus. The composition of the gut microbiota was quite rich, alpha diversity index was higher than the average in Western studies. The average value of IMT was $0.76 \pm 0.20 \mathrm{~mm}$. IMT $>0.9 \mathrm{~mm}$ was diagnosed in 20 patients. The average value of PWV was $10.9 \pm 2.6 \mathrm{~m} / \mathrm{s}$. PWV of more than $10 \mathrm{~m} / \mathrm{s}$ was observed in 55 participants and was elevated in 
patients with high Bacteroides abundance (FDR $<0.001)$. IMT was strongly associated with higher Blautia (FDR 0.004) and Serratia (FDR 0.009) representation. Carotid artery stenosis was directly associated with high abundance of Serratia (FDR 0.002). Moreover, systemic inflammation was more pronounced in patients with high Serratia abundance (FDR 0.0001).

Conclusion: Results show that there is relationship between early vascular wall changes and higher representation of opportunistic pathogens in the gut microbiome even in apparently healthy participants. We concede that gut microbiota composition shifts along with classical risk factors act together to influence vascular aging.

\section{P-121}

Immune capacity determines outcome following surgery or trauma: a systematic review and meta-analysis

\author{
Ruiyi Jia ${ }^{1}$, Camilla Tuttle ${ }^{1}$ \\ ${ }^{1} @$ AgeMelbourne, The University of Melbourne, \\ Melbourne, Australia
}

Background: Immunological functions are altered following physical injury due to surgery or trauma. The inflammatory response elicited after injury is dependent on the magnitude of the initial injury. However, variability exists in the immune response induced where potentially only some patients are at risk of developing complications such as systemic inflammatory response syndrome after injury. This systematic review and meta-analysis examines whether lipopolysaccharide (LPS) induced cytokine production capacity of leucocytes can be used as a functional test to predict risk of developing complications after injury.

Methods: Medline, Embase and Web of Science were systematically searched to identify original research articles that investigated the association between LPS induced cytokine production capacity in leucocytes and any clinical outcome or surrogate outcome measures after surgery or trauma. Meta-analysis was performed for clinical outcomes that have been investigated by two or more studies if sufficient information was supplied.

Results: A total of 25 articles out of 6765 abstracts identified through the literature search were included in this review. Majority of the literature investigated the association of cytokine production capacity with the development of inflammatory complications (15 out of 25 articles). Meta-analyses demonstrated a significant standardized difference in means of cytokine production capacity between patients who developed an infectious complication compared to patients who did not for tumour necrosis factor alpha (TNF $\alpha$ ), interleukin 6 (IL-6) and interleukin 8 (IL-8) on the first day of hospital admission for trauma patients or first post-operative day for surgical patients. No significant difference was observed for IL-1 $\beta$.

Conclusion: Based on the evidence currently available, it appears that at least for infectious complications, there is a potential role for cytokine production capacity as a prognostic marker. However, further research is required to establish a standardized LPS stimulation method and reporting system in order to determine a clinically meaningful stimulated cytokine production threshold which can be used in prognostic testing.

\section{P-122}

Are skin senescence and immunosenescence associated intraindividually?

Mariette Waaijer ${ }^{1}$, Rudi Westendorp ${ }^{2}$, Pawelec Pawelec ${ }^{3}$, Andrea Maier ${ }^{4}$

${ }^{1}$ Department of Gerontology and Geriatrics, Leiden University Medical Center, the Netherlands, ${ }^{2}$ Department of Public health and Center of Healthy Aging, University of Copenhagen, Copenhagen, Denmark, ${ }^{3}$ Department of Internal Medicine II, Centre for Medical Research, University of Tübingen, Germany, ${ }^{4}$ Department of Human Movement Sciences, @AgeAmsterdam, Vrije Universiteit; Department of Medicine and Aged Care, @ AgeMelbourne, Royal Melbourne Hospital, University of Melbourne, Melbourne, Australia

With advancing age organs display functional deterioration. One of the factors that might contribute to this deterioration is an accumulation of senescent cells, cells that lost their proliferative capacity, but which are metabolically active. Whilst cellular senescence has been described in several organ systems, such as the skin and the immune system, it is less understood whether the senescence load, the number of senescent cells, in different organs is linked within the individual. To approach this question, we correlated senescence in dermal fibroblasts and in T-cells in 80 middle-aged and older individuals. The number of epidermal and dermal p16INK4a-positive cells in skin biopsies and immunosenescent CD4+ and CD8+ blood T-cells (i.e. end stage differentiated/senescent T-cells, including CD45RA-CCR7CD28-CD27-,CD57+ and KLRG1+ T-cells) were compared using linear regression. Epidermal p16INK4a positivity was associated with neither CD4+ nor CD8+ immunosenescence composites. Dermal p16INK4a positivity was associated with the CD4+ immunosenescence composite. This association remained after adjusting for age, gender and cytomegalovirus serostatus. Dermal p16INK4a positivity was not associated with the CD8+ immunosenescence composite. Although we did find one significant association between skin senescence and immunosenescence, the results are not consistent over different skin layers and over T-cell subsets. We conclude that there is little evidence for a link between skin senescence and immunosenescence within individuals.

\section{P-123}

Cellular senescence and chronological age in various human tissues: a systematic review and meta-analysis

Mariette Waaijer ${ }^{1}$, Camilla Tuttle ${ }^{2}$, Rudi Westendorp ${ }^{3}$, Andrea Maier ${ }^{4}$, M.S. Slee-Valentijn ${ }^{5}$

${ }^{1}$ Department of Gerontology and Geriatrics, Leiden University Medical Center, the Netherlands, ${ }^{2}$ Department of Medicine and Aged Care, @ AgeMelbourne, Royal Melbourne Hospital, University of Melbourne, Melbourne, Australia, ${ }^{3}$ Department of Public health and Center of Healthy Aging, University of Copenhagen, Copenhagen, Denmark, ${ }^{4}$ Department of Human Movement Sciences, @ AgeAmsterdam, Vrije Universiteit Amsterdam; Department of Medicine and Aged Care, @AgeMelbourne, Royal Melbourne Hospital, University of Melbourne, ${ }^{5}$ Department of Medical Statistics and Bioinformatics, Leiden University Medical Center, Leiden, The Netherlands

Background: Cellular senescence, a stable growth arrest of cells, is increasingly recognized as a driver of the aging process and of agerelated diseases. Our aim was to systemically summarize the literature on the association between senescence and chronological age in human tissue. 
Methods: We searched Pubmed, Web of Science and Embase for articles that reported on senescence markers dependent on age in any human tissue. Out of 4838 unique articles, 51 articles were included in a systematic review, and 45 of these were suitable for meta-analysis. Data was extracted on the origin of tissue, senescence markers, age and gender distribution of donors and correlation coefficients. The Z-scores, transformed from the correlation coefficients through Fisher's method, were pooled under a random effects model using Comprehensive Meta-Analysis Software. Heterogeneity was reported with the I-squared test.

Results: Overall, the correlation coefficient between senescence and age was 0.276 (95\% CI 0.225-0.325), with differences between tissue types. Highest mean correlation coefficients was found for brain $(0.701)$, followed by lung (0.670). Mean correlation coefficient were lower for other tissues: artery (0.199), blood (0.290), eye $(0.100)$, kidney $(0.235)$, prostate $(0.263)$ and skin $(0.287)$. The I2 value was $67.7 \%$.

Conclusion: Senescence is significantly associated with chronological age in all human tissues. The effect size of association varies widely between tissues.

\section{P-125}

\section{Telomere dynamics relation with obesity}

Simon Toupance ${ }^{1}$, Mirna Chahine ${ }^{2}$, Irini Tzanetakou ${ }^{3}$, Carlos Labat ${ }^{4}$, Sylvie Gautier ${ }^{5}$, Cécilé Lakomy ${ }^{4}$, Pascal M. Rossi ${ }^{6}$, Toufic Moussallem ${ }^{7}$, Pierre Yared ${ }^{7}$, Didier Quilliot ${ }^{5}$, Evangelos Menenakos $^{8}$, Roland Asmar ${ }^{2}$, Athanase Be

${ }^{1}$ Inserm UMRS 1116 and CHRU Nancy, Université de Lorraine, Nancy, France, ${ }^{2}$ Foundation-Medical Research Institutes, Beirut, Lebanon, ${ }^{3}$ European University of Cyprus, School of Sciences, Engomi, Cyprus, ${ }^{4}$ Inserm UMRS 1116, Université de Lorraine, Nancy, France

Introduction: The relation between telomere dynamics and obesity remains unclear. Cross-sectional studies found associations between short leukocyte telomere length (LTL) and high body mass index (BMI) but longitudinal studies did not find any association between LTL attrition and BMI. In two parallel studies, we aimed to assess the relationship between obesity and telomere dynamics in different tissues.

Methods: Study 1: Measurements of LTL and TL in skeletal muscle (MTL) were performed in 53 subjects with severe obesity (BMI > $35)$ and in 53 age- and sex-matched without obesity $(21<$ BMI $<$ 30). MTL is a proxy of TL at birth and the LTL/MTL ratio represents life-long telomere attrition. Study 2: Measurements of TL in subcutaneous fat (SCF), a high proliferative adipose tissue, and visceral fat (VF), a low proliferative one in 50 severe obese bariatric patients. TL measurements were performed by Southern blot.

Results: Study 1: In younger ( $<55$ years), but not in older, LTL and LTL/MTL were shorter in obese patients vs controls $(7.16 \mathrm{~kb}$ vs $7.54 \mathrm{~kb}, \mathrm{p}<0.05$ for LTL and $81 \%$ vs $84 \%, \mathrm{p}<0.05$ for LTL/MTL). Study 2: Within obese bariatric patients, SCF/VF TL ratio was lower in those with early onset obesity ( $96 \%$ for obesity since childhood vs $97 \%$ since adolescence vs $100 \%$ for adult development of obesity; $\mathrm{p}<0.05)$.

Key conclusions: Early life obesity is associated with higher TL attrition leading to shorter TL in high proliferative tissues.

\section{P-126}

\section{The individual's signature of telomere length distribution}

Simon Toupance ${ }^{1}$, Denis Villemonais ${ }^{2}$, Daphné Germain ${ }^{3}$, Anne Gegout-Petit ${ }^{4}$, Eliane Albuisson ${ }^{5}$, Athanase Benetos ${ }^{1}$

${ }^{1}$ Inserm UMRS 1116 and CHRU Nancy, Université de Lorraine, Nancy, France, ${ }^{2}$ IECL and Ecole des Mines, Université de Lorraine, Nancy, France, ${ }^{3}$ Ecole des Mines, Université de Lorraine, Nancy, France, ${ }^{4}$ IECL, Université de Lorraine, Nancy, France

Introduction: Mean telomere length in DNA samples of human leukocytes reflects the different lengths of telomeres at the ends of the 23 chromosomes and an admixture of cells. However, only rudimentary information is available about the distribution of the lengths of telomeres in all chromosomes and the different cell types in leukocyte samples. Understanding the configuration of leukocyte telomere length distribution (LTLD) might be helpful in capturing intrinsic elements that are not provided by the mean leukocyte telomere length (mLTL).

Methods: LTLD and its temporal variation were analysed in leukocyte samples donated on two occasions ( 8 years apart) by 72 participants in the ADELAHYDE study. Telomere length was measured by Southern blot of the terminal restriction fragments. Differences between distributions were characterized by the Kolmogorov distance (KD).

Results: Individuals with comparable mLTLs displayed different configurations of LTLDs. Inter-individual variation in the LTLD configuration across individuals was much larger than intra-individual variation in LTLD configuration between baseline and follow-up leukocyte samples (inter $\mathrm{KD}=0.064$ vs intra $\mathrm{KD}=0.026$; $\mathrm{p}<0.0001)$.

Key conclusion: Inter-individual variation in the LTLD configuration are wide and change minimally during the course of 8 years. Thus, each individual might have a unique signature (configuration) of LTLD that is independent of mLTL.

\section{P-127}

Age-related changes to the expression of nuclear factor erythroid 2-like 2 (Nrf2), a regulatory antioxidant and xenobiotic defence gene

Greg Scutt ${ }^{1}$, Andy Overall ${ }^{1}$, Prijay Bakrania ${ }^{1}$, Eliseveta Krasteva ${ }^{2}$, Nikesh Parekh ${ }^{3}$, Khalid Ali ${ }^{3}$, Graham Davies ${ }^{4}$,

Chakravarthi Rajkumar ${ }^{3}$

${ }^{1}$ School of Pharmacy and Biomolecular Sciences, University of Brighton, UK, ${ }^{2}$ Dartford and Gravesham NHS Trust, UK,

${ }^{3}$ Brighton and Sussex Medical School, UK, ${ }^{4}$ Institute of Pharmaceutical Science, King's College London, UK

Introduction: Multimorbidity, polypharmacy and susceptibility to adverse drug reactions (ADR) are common problems in old age. Understanding the age-related biological changes that occur at a cellular level, may assist in identifying novel therapeutic targets. The nuclear factor erythroid 2-like 2 (Nrf2) transcription factor regulates antioxidant and drug metabolising pathways in the cell. Data from rodent models showed that Nrf2 protein expression declines with age. If similar findings are observed in humans, it may help to explain why older people are vulnerable to multimorbidity and ADRs. This study investigates whether Nrf2 expression (both mRNA and protein) decreases with increasing age in humans.

Methods: Fifty-five adults were recruited to our study (age range: 18-75 years). Participants donated $6 \mathrm{~mL}$ of venous blood, from which peripheral blood mononuclear cells (PBMCs) were separated 
and analysed for Nrf2 mRNA and protein expression (real time quantitative polymerase chain reaction and enzyme-linked immunosorbent assay respectively).

Results: Our data showed that Nrf2 protein expression was approximately $50 \%$ lower in individuals aged $>30$ years $(0.065 \pm 0.014$ $\mathrm{EU}<30$ years vs. $0.033 \pm 0.0060 \mathrm{EU}>30$ years, $\mathrm{p}<0.05)$. Similarly, mRNA expression declined with advancing age (log2-fold change compared to $18-29$ year-olds: $1.08 \pm 1.19,2.14 \pm 1.25$, $-0.69 \pm 1.15, \quad 0.45 \pm 2.03, \quad-4.76 \pm 2.06$, in $30-39, \quad 40-49$, $50-59,60-69,>70$ year age categories respectively; $\mathrm{p}<0.0001)$.

Conclusions: The expression of Nrf2 transcription factor is reduced in old age, potentially contributing to the increased risk of multimorbidity and adverse drug reactions.

\section{P-128}

Steroid metabolome and Alzheimer's disease: new biomarkers?

Hana Vankova ${ }^{1}$, Iva Holmerova ${ }^{2}$, Eva Jarolimova ${ }^{2}$, Marketa Vankova ${ }^{3}$

${ }^{1}$ The Third Faculty of Medicine, Charles University, Prague, Czech Republic, ${ }^{2}$ Charles University, Prague, Czech Republic, ${ }^{3}$ Institute of Endocrinology, Prague, Czech Republic

Objective: Pathogenetic relations common for both Alzheimer's disease and diabetes mellitus type 2 on the metabolic level [1] inspire our study searching for molecular and biochemical markers that enable early diagnosis of Alzheimer's Disease (AD). Previous studies indicate that serum concentrations of selected steroid hormones and amino-thiols could serve as effective biomarkers of $\mathrm{AD}[2,3]$.

Methods: The analysis of steroid hormones is managed mostly with the use of gas chromatography-mass spectrometry, GC-MS. Steroid metabolome in patients with Alzheimer's disease and in controls is being compared and its relationship to glucose metabolism is being assessed. The aim of our study is to build a predictive model based on steroid data.

Results: Some components of steroid metabolome differ between the group of Alzheimer's disease patients and healthy controls of the same age. Significant differences in levels of $\mathrm{C} 21$ and $5 \beta$-reduced metabolites of $\mathrm{C} 19$ steroids were found.

Conclusions: According to preliminary results, some components of steroid metabolome are very promising in prediction of Alzheimer's disease. Our new study aims to simplify the prediction model and make it applicable to clinical practice, thanks to detection of neuroactive biomarkers in peripheral blood. Supported by the grant NV18-01-00399 of the Ministry of Health of the Czech Republic.

\section{References:}

1. de la Monte SM (2012) Triangulated mal-signaling in Alzheimer's disease: roles of neurotoxic ceramides, ER stress, and insulin resistance reviewed. J Alzheimers Dis 30:S231-S249

2. Hampl R, Bičíková M (2010) Neuroimmunomodulatory steroids in Alzheimer dementia. J Steroid Biochem Mol Biol 119(3):97-104

3. Vaňková M et al (2016) Preliminary evidence of altered steroidogenesis in women with Alzheimer's disease: have the patients "older" adrenal zona reticularis? J Steroid Biochem Mol Biol 158:157-177

\section{P-129}

Monocytes-induced activation as a biomarker of pernicious clinical outcome after hip fracture in elderly

Hélène Vallet ${ }^{1}$, Martin Larsen ${ }^{2}$, Charles Bayard ${ }^{2}$, Judith CohenBittan $^{3}$, Victor Appay ${ }^{2}$, Jacques Boddaert ${ }^{3}$, Delphine Sauce ${ }^{2}$

${ }^{1}$ INSERM U1135, Cimi-Paris, Paris, France, Pitié Salpétrière Hospital, Geriatric Department, Paris, France, ${ }^{2}$ INSERM U1135, Cimi-Paris, Paris, France, ${ }^{3}$ Pitié Salpétrière Hospital, Geriatric Department, Paris, France

Hip fracture (HF) is a common and devastating injury as well as a major health issue in old age. HF has a one-year mortality $>30 \%$ in the elderly and is a frequent cause of institutionalization. The reasons for such poor outcomes in this trauma are multifactorial but we aim here at identifying immunological factors, which can influence and/or predict the outcome of hip trauma in elderly patients post-surgery. We analyze immunological parameters evocating of the Immune Risk Phenotype in sequential pre- and post-surgical blood samples collected from HF patients over 75 years of age. The study revealed that $\mathrm{HF}$ is associated with an immune scar depicting a transient T-cell leucopenia and an acute hyper-inflammation. Importantly, we show that blood level of a molecule released by activated monocytes (neopterin) is predictive of one-year mortality in these patients. Its plasmatic level correlated negatively with the time of survival after HF surgery. In conclusion, HF patients exhibit transient changes in innate and adaptive immunity. Meanwhile, profound acute inflammatory processes measurable pre-surgery occur, which are predictive of long-term survival after HF surgery. We propose to use the identified predictive biomarker to improve medical interventions and follow-up of patients at risk of death.

\section{P-130}

The Diet-microbiota-intestinal permeability axis in older subjects: rationale of the MaPLE project

A. Cherubini ${ }^{1}$, S. Guglielmetti ${ }^{2}$, P. Kroon ${ }^{3}$, C. Andres-Lacueva ${ }^{4}$, P. Riso ${ }^{2}$

${ }^{1}$ IRCCS-INRCA, Geriatria, Accettazione Geriatrica e Centro di ricerca per l'invecchiamento, Ancona, Italy, ${ }^{2}$ Università degli Studi di Milano, DeFENS, Milan, Italy, ${ }^{3}$ Quadram Institute Bioscience,

Norwich, UK, ${ }^{4}$ BIomarker and Metabolomic Lab

Introduction: Gut barrier function can be significantly compromised in older subjects. It is plausible that an increased intestinal permeability (IP) could induce inflammation through the translocation of pro-inflammatory bacterial factors into the blood stream. Promising candidate food bioactives could represent a potential strategy to modulate intestinal permeability both affecting microbial ecology and cell function.

Methods: The European MaPLE (Microbiome mAnipulation through Polyphenols for managing gut Leakiness in the Elderly) project aims to evaluate whether a polyphenol rich dietary pattern can modulate IP, promoting an overall protective metabolic phenotype in older subjects. A randomised, controlled, cross-over dietary intervention study (8-week polyphenol-rich diet versus 8-week control diet, separated by a wash-out period) has been carried out in subjects living in a wellcontrolled setting (i.e. nursing home).

Results: Zonulin, a protein involved in tight junction modulation, has been exploited as IP marker and revealed an increased permeability. The effect of the intervention has been evaluated on numerous markers related with IP and inflammation, including the gut microbial 
ecosystem (i.e. gut microbiota composition and related metabolites), blood bacterial loads and the serum/urine metabolomics.

Key conclusions: Standard methodologies for IP evaluation cannot be easily applied in the older population, thus zonulin and total bacterial load can be considered valuable candidate biomarkers. The MaPLE project will contribute to identify non-invasive strategies to improve IP and related disorders in the older population.

Funding: INTESTINAL-MICROBIOMICS JPI HDHL ( http://www.healthydietforhealthylife.eu/). Biotechnology and Biological Sciences Research Council (UK) Grant BB/R012512/1. MINECO (Spain), PCIN-2015-238. Mipaaf (Italy) D.M. 8245/7303/ 2016.

\section{Area: Frailty and sarcopenia}

\section{P-131}

The impact of simple frailty scale use on the discharge in older adults admitted to acute geriatric medicine ward

\author{
Hyun Suk Lee ${ }^{1}$, Aneeka Alexander ${ }^{1}$, Ngie Law ${ }^{1}$ \\ ${ }^{1}$ Fiona Stanley Hospital, Murdoch, Australia
}

Background: Two different simple frailty scales were used to identify the prevalence and severity of frailty in older adults with acute medical conditions and geriatric syndromes admitted to acute geriatrics ward to assess the effect of frailty on discharge planning without additional clinical resources.

Methods: Clinical frailty scales (CFS) and reported Edmonton frailty scales (REFS) were completed based on electronic medical records (EMR) and REFS questionnaire for 107 patients. Patients' demography, medical conditions and main outcomes (length of stay (LOS), 30-day readmission rates, discharge accommodations) were analysed divided into frail versus non-frail groups and according to frailty severities. Variables with $\mathrm{P}<0.2$ in univariate analysis were included for multi-regression analysis to assess the impact of frailty on outcome measures.

Results: Prevalence of frailty was 75 percent for both scales out of which approximately 50 percent have mild to moderate frailty. LOS was increased in the frail group and correlated with the severity of frailty. Demographic data and medical comorbidities were not statistically different. LOS, 30-day readmission rate and dementia were increased based on CFS severity. Patients transferred to rehabilitation units were not necessarily frail and same outcome as non-frail group. Frailty was the only significant variable for LOS in univariate analysis but not for 30-day readmission rates and discharge destination in univariate analysis.

Conclusion: Severity of frailty is correlated with increased LOS. CFS may be more useful to detect frailty.

\section{P-132}

\section{The impact of frailty on discharge in an acute geriatric ward}

\author{
Hyun Suk Lee ${ }^{1}$, NC Law ${ }^{1}$, Aneeka Alexander ${ }^{1}$ \\ ${ }^{1}$ Fiona Stanley Hospital, Murdoch, Australia
}

Background: To analyse prevalence and severity of frailty in patients admitted to an acute geriatric ward. To assess the effect of frailty and its severity on discharge.

Methods: Clinical frailty scale (CFS) and Reported Edmonton frailty scale (REFS) were determined based on electronic medical records (EMR) and REFS questionnaire for 107 patients admitted to an acute geriatric ward over a 2 months period. Patients' demography, medical conditions, geriatric syndromes and main outcomes (length of stay (LOS), 30-day readmission rates, discharge accommodations) were analysed, divided into frail and non-frail groups and according to severity of frailty using two different scales. Patients transferred to rehabilitation units were analysed in the same way as described.

Results: Prevalence of frailty was 75 percent from which moderately severe frailty $(\mathrm{CFS}=29$ percent, REFS 27.1 percent $)$ was most common in both scales. Main admission diagnoses were falls $(\mathrm{n}=35)$ and related fractures $(n=18)$, delirium $(n=14)$ and pneumonia $(n=14)$. Dementia $(P=0.01)$ and Parkinson's disease $(P=0.03)$ were more common in frail group with CFS but not with REFS. LOS increased in frail group (CFS P $=0.02$, REFS $P=0.02$ ) and correlated with severity in both scales (CFS not frail 7.9 days, mildmoderately 9.7 days, severe 11.5 days). Discharge destination (CFS $\mathrm{P}=0.14$, FEFS $\mathrm{P}=0.10$ ) and readmission rates $(\mathrm{CFS} \mathrm{P}=0.96$, REFS $P=0.37$ ) were not associated with frailty.

Conclusion: CFS and REFS are useful to detect frailty which predicts prolonged hospital stay. CFS was easier to complete and its outcome was similar to REFS. Frailty needs to be incorporated into discharge plan.

\section{P-133}

Vitamin B12 deficiency might be related to sarcopenia in older adults

Esra Ates Bulut ${ }^{1}$, Pinar Soysal ${ }^{2}$, Ali Ekrem Aydin ${ }^{1}$, Ozge Dokuzlar ${ }^{1}$, Suleyman Emre Kocyigit ${ }^{1}$, Ahmet Turan Isik ${ }^{1}$

${ }^{1}$ Department of Geriatric Medicine, Dokuz Eylul University, Faculty of Medicine, Izmir, Turkey, ${ }^{2}$ Geriatric Center, Kayseri Education and Research Hospital, Kayseri, Turkey

Introduction: Sarcopenia and dynapenia are related to repeated falls, depression, frailty, increased mortality and morbidity. Although malnutrition is the most blamed factor for sarcopenia, the role of micronutrients is unclear. The aim of this study is to evaluate relationship between vitamin B12 deficiency and sarcopenia in older adults.

Methods: 742 patients, who attended to geriatric clinics, included study. All cases' skeletal muscle mass, walking speed and hand grip strength were recorded by bioimpedance, $4 \mathrm{~m}$ walking test and hand dynamometer respectively. The diagnosis of sarcopenia was defined according to European Working Group on Sarcopenia in Older People criteria.

Results: The prevalence of sarcopenia and dynapenia was 28.7 and $32.5 \%$, respectively. In the patients with sarcopenia, mean age, number of used drugs osteoporosis, falls in the last year, congestive heart disease, dementia and frailty were higher, and Mini Mental State Examination(MMSE), instrumental activity of daily living scores were lower $(\mathrm{p}<0.05)$. The vitamin B12 levels in patients with sarcopenia were similar to the patients without sarcopenia $(\mathrm{p}>0.05)$. The frequency of sarcopenia and dynapenia was higher in the patients with vitamin B12 levels less than $400 \mathrm{pg} / \mathrm{mL}$, besides lean body mass, total skeletal mass and bone weight were lower $(\mathrm{p}<0.05)$.

Key conclusions: Sarcopenia, which results in lots of negative clinical outcomes in older adults, might be related to vitamin B12 deficiency. Therefore, these patients should be periodically examined for vitamin B12 deficiency due to the potential negative clinical outcomes such as sarcopenia in older adults. 


\section{P-134}

Disentangling the relationship between muscle mass and frailty status: Insights from the GERILABS study

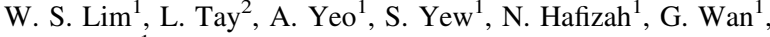 \\ Y. Y. Ding ${ }^{1}$ \\ ${ }^{1}$ Tan Tock Seng Hospital, Malacca, Malaysia, ${ }^{2}$ Sengkang Hospital, \\ Singapore
}

Introduction: Although low muscle mass has been linked to early manifestations in the natural history of frailty, contradictory findings exist about the impact of different definitions on frailty status. We aim to study the relationship between three widely used muscle mass definitions with frailty status in an Asian population.

Methods: We studied 200 community-dwelling older adults from the GERILABS cohort study. We compared three DXA-based definitions of muscle mass: appendicular lean mass (ALM) adjusted for height squared (ALM/Ht2) using Asian Working Group cutoffs; ALM adjusted for body mass index [ALM/BMI]; and residuals of linear regression on ALM adjusted for fat mass and height (ALM-res). Frailty phenotype (robust, pre-frail and frail) was defined according to modified Fried criteria. We examined the relationship between frailty status and muscle mass using scatter plot with Lowess smoothing and multinomial logistic regression adjusted for age and gender.

Results: Prevalence of frailty among those with low muscle mass was 8.8\% (ALM/Ht2), 5.5\% (ALM/BMI), and 4.4\% (ALM-res) respectively. Conversely, among frail participants, the prevalence of low muscle mass was $72.7,27.3$ and $18.2 \%$ respectively. A linear relationship with frailty was observed for ALM/Ht2, but not ALM/BMI or ALM-res. In multinomial logistic regression, each unit increase in $\mathrm{ALM} / \mathrm{Ht} 2$ was associated with $41 \%(95 \% \mathrm{CI} 12-61, \mathrm{p}=0.010)$ lower odds of being pre-frail, and $70 \%(95 \%$ CI 9-90, $\mathrm{p}=0.034)$ lower odds of being frail as opposed to robust. ALM/BMI and ALM-res were not associated with pre-frailty/frailty after adjustment.

Conclusion: Our results support sarcopenia and frailty as inter-related but distinct entities, with muscle mass-frailty association inferences altered substantially by the definition employed. Contrary to earlier non-Asian studies, ALM/Ht2 was strongly, linearly and inversely related to pre-frailty/frailty status in our Asian cohort.

\section{P-135}

Impact of sarcopenia on 1-year mortality among older hospitalized patients with impaired mobility

M. Pourhassan ${ }^{1}$, K. Norman ${ }^{2}$, M. J. Müller ${ }^{3}$, R. Dziewas ${ }^{4}$, R. Wirth ${ }^{1}$

${ }^{1}$ Department of Geriatric Medicine, Marien Hospital Herne, Ruhr University Bochum, Germany, ${ }^{2}$ Charité Research Group on Geriatrics, Charité - Universitätsmedizin Berlin, Germany, ${ }^{3}$ Institute of Human Nutrition and Food Science, Christian-AlbrechtsUniversity, Kiel, Germany, ${ }^{4}$ Department of Neurology, University Hospital Münster, Germany

Objectives: Aim of the present study was to investigate the associations between sarcopenia and 1-year mortality in a prospectively recruited sample of geriatric inpatients with different mobility and dependency status.

Design and setting: Sarcopenia was diagnosed using the criteria of the European Working Group on Sarcopenia in Older People (EWGSOP). Hand grip strength and skeletal muscle mass were measured using Jamar dynamometer and bioelectrical impedance analysis, respectively. Physical function was assessed with the Short Physical Performance Battery. Dependency status was defined by Barthel-Index (BI). Mobility limitation was defined according to walking ability as described in BI. The survival status was ascertained by telephone interview.

Results: The population comprised 198 patients from a geriatric acute ward with a mean age of $82.8 \pm 5.9(70.2 \%$ females). $50(25.3 \%)$ patients had sarcopenia, while $148(74.7 \%)$ had no sarcopenia. 14 (28\%) patients died among sarcopenic subjects compared with 28 (19\%) non-sarcopenic subjects $(\mathrm{P}=0.229)$. After adjustment for potential confounders, sarcopenia was associated with increased mortality among patients with limited mobility prior to admission $(\mathrm{n}=138$, hazard ratio, HR: $2.52,95 \%$ CI 1.17-5.44) and at time of discharge $(\mathrm{n}=162$, HR $1.93,95 \%$ CI $0.67-3.22)$. In a sub-group of patients with pre-admission $\mathrm{BI}<60(\mathrm{n}=45),<70(\mathrm{n}=73)$ and $<80(\mathrm{n}=108)$, the risk of death was $3.63,2.80$ and 2.55 times higher in sarcopenic patients, respectively.

Conclusion: Sarcopenia is significantly associated with higher risk of mortality among sub-groups of older patients with limited mobility and impaired functional status, independently of age and other clinical variables.

\section{P-136}

Relationship of muscle function to circulating myostatin, follistatin and GDF11 in older women and men

Elizaveta Fife ${ }^{1}$, Joanna Kostka ${ }^{1}$, Agnieszka Guligowska ${ }^{1}$, Małgorzata Pigłowska $^{1}$, Bartłomiej Sołtysik ${ }^{1}$, Agnieszka Kaufman-Szymczyk ${ }^{1}$, Krystyna Fabianowska-Majewska ${ }^{1}$, Tomasz Kostka ${ }^{1}$

${ }^{1}$ Medical University of Lodz, Lodz, Poland

Introduction: Myostatin, its inhibitor follistatin, and growth/differentiation factor 11 (GDF11) have been proposed as factors that could potentially modify biological aging. The study aimed to test whether there is a relationship between these plasma circulating proteins and muscle strength, power and optimal shortening velocity (vopt) of older adults.

Methods: The cross-sectional study included 56 women and 45 men aged 60 years and older. Every participant underwent examination which included anthropometric and bioimpedance analysis measurements, functional and cognitive performance tests, muscle strength of upper and lower extremities, muscle power testing with two different methods and blood analyses.

Results: Women had higher plasma levels of myostatin and GDF11 than men. Men had higher plasma level of follistatin than women. In women, plasma level of myostatin was negatively correlated with left handgrip strength and vopt. Follistatin was negatively correlated with maximum power (Pmax), Pmax $\mathrm{kg}^{-1}$ and $\mathrm{P} 70 \%$ leg press, and positively correlated with the Timed Up and Go (TUG) test. GDF11 was negatively correlated with body mass, body mass index, waist circumference, fat mass and the percentage of body fat. In men, there were no significant correlations observed between circulating plasma proteins and muscle function measures.

Key conclusions: The circulating plasma myostatin and follistatin are negatively associated with muscle function in older women and the relationship between these proteins is more visible for muscle power than muscle strength. GDF11 has a higher association with the body mass and composition than muscle function in older women. 


\section{P-137}

Trends in frailty and its association with mortality across a period of 21 years: results from the longitudinal aging study Amsterdam

Emiel O. Hoogendijk ${ }^{1}$, Hans W. Jeuring ${ }^{1}$, Martijn Huisman ${ }^{1}$, Dorly J. H. Deeg ${ }^{1}$

${ }^{1}$ VU University Medical Center, Amsterdam, The Netherlands

Introduction: Frailty is an important predictor of mortality in older adults, but not much is known about cohort and period changes in frailty and its association with mortality. The aim of the current study was to investigate trends in frailty and the relationship between frailty and mortality across a period of 21 years.

Methods: Data from 1995 to 2016 were used from the Longitudinal Aging Study Amsterdam. A total of 7742 observations of 2874 respondents aged 65-84 years across 6 measurement waves were included. Frailty was measured with a 32 -item frailty index (FI), with a cut-off of 0.25 to indicate frailty. The outcome measure was 4-year mortality. Trends in frailty and its association with 4-year mortality were assessed using Generalized Estimating Equation (GEE) analysis. Results: The GEE analyses showed that the 4-year mortality rate declined between 1995 and 2016 in both people with and without frailty. In the same observation period, levels of frailty slightly increased. Across all measurement waves, frailty was associated with 4-year mortality (OR 2.57, 95\% CI 2.03-3.25). The OR slightly increased in more recent measurement waves. However, there was no statistically significant interaction effect between frailty and time on 4-year mortality, indicating that the association between frailty and mortality remained the same during the period of observation.

Key conclusions: Between 1995 and 2016, the prevalence of frailty increased among older adults aged 65-84 years, but the lethality of frailty did not change, as there was a stable trend in the frailtymortality relationship.

\section{P-138}

Association of the SARC-F Questionnaire with muscle mass, strength, and power: data from the African American Health (AAH) study

Fougère Bertrand ${ }^{1,2}$, Malmstrom Ted ${ }^{3}$, Miller Douglas ${ }^{4}$, Morley John ${ }^{2}$

${ }^{1}$ Division of Geriatric Medicine, Tours University School of Medicine, Tours, France, ${ }^{2}$ Division of Geriatric Medicine, Saint Louis University School of Medicine, St. Louis, Missouri, USA,

${ }^{3}$ Department of Psychiatry and Behavioral Neuroscience. Saint Louis University School of Medicine, St. Louis, Missouri, USA,

${ }^{4}$ Center for Aging Research, Indiana University School of Medicine and Regenstrief Institute, Indianapolis, Indiana, US

Background: Screening for sarcopenia in daily practice can be challenging. The SARC-F questionnaire may be considered as a suitable tool for community screening for sarcopenia. Our objective was to explore whether the SARC-F is related to muscle mass (MM), strength (MS) and power (MP).

Methods: In the population-based African American Health (AAH) study, MM, MS and MP were evaluated in a clinical testing center in a subsample of $\mathrm{n}=190$ women. MM was measured using dual energy X-ray absorptiometry (DEXA). MS and MP were evaluated by knee extensor and flexor tests (using an isokinetic dynamometer; Biodex Medical Systems, Inc., Shirley, NY). The SARC-F self-report questionnaire items were coded using these 5 items: sluggishness, assistance in walking, rise from a chair, climb stairs, falls. The questionnaire score ranged from 0 to 10 : [using a previously determined cut point] a sarcopenia was defined as a score $\geq 4$.

Results: Participants with SARC-F $\geq 4$ had lower household income, worse self-rated health and poorer income than those with SARC-F $<4$. They also had a higher BMI than participants with SARC-F $<4$ $(\mathrm{p}<.001)$. AAH women participants with SARC-F scores $\geq 4$ had significantly higher MM (p .022), lower MS (p .001) and lower MP $(\mathrm{p}<.001)$ than participants with SARC-F scores $<4$.

Conclusion: Participants who screened positive using the SARC-F questionnaire showed lower muscle function, predisposing them to greater vulnerability in functional activities. This, combined with the simplicity of use for SARC-F, justifies its use as the initial step in identifying the presence of sarcopenia.

\section{P-139}

A European joint action towards frailty prevention and management

Maria Angeles Caballero-Mora ${ }^{1}$, Inés García-Sánchez ${ }^{1}$, Graziano Onder $^{2}$, Branko Gabrovec ${ }^{3}$, Regina Roller-Wirnsberger ${ }^{4}$, Roberto Bernabei $^{2}$, Leocadio Rodríguez-Mañas ${ }^{1}$

${ }^{1}$ Hospital Universitario de Getafe, Getafe, Spain, ${ }^{2}$ Università Cattolica del Sacro Cuore, Milan, Italy, ${ }^{3}$ Nacionalni Inštitut Za Javno Zdravje, Ljubljana, Slovenia, ${ }^{4}$ Medizinische Universität Graz, Graz, Austria

Addressing the increasing demands for social and health care from the burden of chronic diseases and disability is a central priority for the European Union (EU). Frailty is at the centre of these two challenges. ADVANTAGE is the Joint Action (JA) co-financed by the EU and 22 Member States (MS) to build a common understanding on health and social care policies to prevent and manage frailty. ADVANTAGE conducted a systematic search on evidence supporting frailty prevention and management activities which crystalize on a State of the Art Report. Main results support that frailty management should be directed towards comprehensive and holistic treatment in multiple and related fields. Prevention calls for a multifaceted approach addressing life course factors. ADVANTAGE recommends that all persons older than 70 years attending health care services should be screened for frailty. Geriatric Comprehensive Assessment, incorporated for assessment of frailty and planning a personalized multidomain treatment render better outcomes. Therefore, we propose a number of instruments for the screening phase and others for the diagnostic one. Physical exercise (multidomain and strength training) in combination with adequate nutrition have proved to be the best treatment of frailty. Other useful actions are reduction of polypharmacy, moderate weight loss in combination with appropriate physical activity, Vitamin D supplementation in some cases, and use of ICTs.

\section{P-140}

Sarcopenia and osteoporosis are interrelated in geriatric inpatients and associated with poor nutritional and functional state

Jens Reiss ${ }^{1}$, Bernhard Iglseder ${ }^{1}$, Rainhard Alzner ${ }^{2}$, Brigitte MayrPirker $^{1}$, Chrisitan Pirich ${ }^{3}$, Helmut Kässmann ${ }^{3}$, Martina Kreutzer ${ }^{1}$, Ingrid Pfaffinger ${ }^{1}$, Peter Dovjak ${ }^{4}$

${ }^{1}$ Christian Doppler Klinik Salzburg, Salzburg, Austria, ${ }^{2}$ ChristianDoppler-Klinik Salzburg, Salzburg, Austria, ${ }^{3}$ Landeskrankenhaus Salzburg, Salzburg, Austria, ${ }^{4}$ Salzkammergutklinik Gmunden, Gmunden, Austria 
Introduction: Sarcopenia and osteoporosis have common underlying pathology and reinforce each other in terms of adverse outcomes. We looked into the degree of overlap between sarcopenia and osteoporosis in geriatric inpatients from the Sarcopenia in Geriatric Elderly (SAGE) study.

Methods: All geriatric inpatients from SAGE whose dataset allowed for the diagnosis of sarcopenia (EWGSOP definition) and osteoporosis (WHO definition) were included. In addition to dual energy X-ray absorptiometry derived muscle mass and bone mineral density, functional (gait speed, hand grip, Barthel index) and nutritional characteristics (mini nutritional assessment short form (MNA-SF), body mass index (BMI)) were measured for comparison between sarcopenic, osteoporotic osteosarcopenic and normal (reference group).

Results: $141 / 148$ of SAGE participants (84 women, 57 men; mean age $80.6+5.5)$ met inclusion criteria. 22/141 (15.6\%) were only osteoporotic, 19/141 (13.5\%) were only sarcopenic and 20/141 (14.2\%) osteosarcopenic (both conditions present). Prevalence of osteoporosis was higher in sarcopenic than non-sarcopenic $(51.3 \%$ vs. $21.6 \%, \mathrm{p}<0.001)$. Sarcopenic, osteoporotic and osteosarcopenic subjects had lower BMI, MNA-SF, handgrip and gait speed (all $\mathrm{p}<0.05)$ than the reference group. Barthel index was lower for sarcopenic and osteosarcopenic $(p<0.05)$ but not for osteoporotic individuals $(\mathrm{p}=0.07)$. BMI and MNA-SF were lower in osteosarcopenia compared to sarcopenia or osteoporosis alone $(\mathrm{p}<0.05)$ while there was no difference in functional criteria.

Conclusion: Osteoporosis and sarcopenia go along with poor nutrition and reduced function in geriatric inpatients. Co-occurrence (osteosarcopenia) is common and associated with a higher degree of malnutrition than osteoporosis or sarcopenia alone.

\section{P-141}

Association between gait speed with mortality, cardiovascular disease and cancer: a systematic review and meta-analysis of prospective cohort studies

Brendon Stubbs ${ }^{1}$, Alberto Pilotto ${ }^{2}$, Emanuele Cereda ${ }^{3}$, Nicola Veronese $^{4}$

${ }^{1}$ King's College London, London, UK, ${ }^{2}$ EO Galliera Genova, Genoa, Italy, ${ }^{3}$ Fondazione IRCCS Policlinico San Matteo, Pavia, Italy, ${ }^{4}$ Consiglio Nazionale delle Ricerche, Padua, Italy

Introduction: Slow gait speed is associated with premature mortality, cardiovascular disease (CVD) and cancer, although a comprehensive meta-analysis is lacking. In this systematic review and meta-analysis, we explored potential associations between gait speed and mortality, CVD and cancer.

Methods: A systematic search in major databases was undertaken until March 15th 2018 for prospective cohort studies reporting data on gait speed and mortality, incident CVD and cancer. The adjusted hazard ratios (HRs) and 95\% confidence intervals (CIs), based on the model with the maximum number of covariates for each study between gait speed (categorized as decrease in $0.1 \mathrm{~m} / \mathrm{s}$ ) and mortality, incident CVD and cancer were meta-analysed with a random effects model.

Results: Among 7026 papers, 44 articles corresponding to 48 cohorts were eligible. The studies followed-up a total of 101,945 participants (mean age 72.2 years; $55 \%$ women) for a median of 5.4 years. After adjusting for a median of 9 potential confounders and the presence of publication bias, each reduction of $0.1 \mathrm{~m} / \mathrm{s}$ in gait speed was associated with a $14 \%$ increased risk of earlier mortality (45 studies; HR $1.12 ; 95 \%$ CI $1.09-1.14 ; \mathrm{I} 222=90 \%$ ) and $8 \%$ increased risk of CVD
(13 studies; HR 1.08; 95\% CI 1.03-1.13; I223 = 81\%), but no relationship with cancer was observed.

Conclusions: Slow gait speed may be a predictor of mortality and CVD in older adults. Since gait speed is a quick and inexpensive measure to obtain, our study suggests that it may help identify people at risk of premature mortality and CVD.

\section{P-142}

Application of ultrasound for muscle assessment in sarcopenia: towards standardized measurements

Stany Perkisas ${ }^{\mathrm{a}, \mathrm{m}}$, Stéphane Baudry ${ }^{\mathrm{b}, \mathrm{m}}$, Jürgen Bauer ${ }^{\mathrm{c}}$,

David Beckwée ${ }^{\mathrm{d}}$, Anne-Marie De Cock ${ }^{\mathrm{a}}$, Hans Hobbelen ${ }^{2, \mathrm{n}}$, Harriët Jager-Wittenaar ${ }^{\mathrm{e}, \mathrm{o}}$, Agnieszka Kasiukiewicz ${ }^{\mathrm{f}}$,

Francesco Landi $^{\mathrm{g}}$, Ester Marco ${ }^{\mathrm{h}}$, Ana Merello ${ }^{\mathrm{i}}$, Karolina Piotrowicz ${ }^{\mathrm{j}}$, Elisabet Sanchez ${ }^{\mathrm{i}}$, Dolores Sanchez-Rodriguez ${ }^{\mathrm{k}}$, Aldo Scafoglieri ${ }^{\mathrm{l}, \mathrm{m}}$, Alfonso Cruz-Jentoft ${ }^{\mathrm{i}}$ and Maurits Vandewoude ${ }^{\mathrm{a}, \mathrm{m}}$

${ }^{\mathrm{a}}$ University of Antwerp, Antwerp, Belgium, ${ }^{\mathrm{b}}$ Laboratory of applied biology and neurophysiologie, Université libre de Bruxelles, Brussels, Belgium. Belgian Ageing Muscle Society, ${ }^{\mathrm{c} D e p a r t m e n t}$ of Geriatric Medicine, Klinikum, Carl von Ossietzky University, Oldenburg, Germany, ${ }^{\mathrm{d}}$ Rehabilitation Sciences Research Department, Vrije Universiteit Brussel, Brussels, Belgium. Department of Rehabilitation Sciences and Physiotherapy, Faculty of Medicine and Health Sciences, University of Antwerp, Antwerp, Belgium, ${ }^{\mathrm{e}}$ Hanze University of Applied Sciences; Research Group Healthy Ageing, Allied Health Care and Nursing, University of Groningen, Groningen, the Netherlands, ${ }^{\mathrm{f}}$ Department of Geriatrics, Medical University in Bialystok, Poland, ${ }^{g}$ Department of Geriatrics, Neuroscience and Orthopedics, Teaching Hospital "Agostino Gemelli", Catholic University of the Sacred Heart School of Medicine, Rome, Italy, ${ }^{\text {h}}$ Physical \& Rehabilitation Medicine, Parc de Salut Mar;

Rehabilitation Research Group, Hospital del Mar Research Institute; Universitat Autónoma de Barcelona, Barcelona, Spain, ${ }^{\mathrm{i}}$ University Hospital Ramœn y Cajal, Madrid, Spain, ${ }^{\mathrm{j}}$ Department of Internal Medicine and Gerontology, Faculty of Medicine, Jagiellonian University Medical College, Kraków, Poland, ${ }^{k}$ Geriatrics Dpt. Parc Salut Mar. Institut Hospital Del Mar d Investigacions Mèdiques (IMIM). Universitat Autónoma de Barcelona. Universitat Pompeu Fabra. Barcelona, Spain, ${ }^{1}$ Faculty of Medicine and Pharmacy, Vrije Universiteit Brussel, Brussels, Belgium, ${ }^{\mathrm{m}}$ Belgian Ageing Muscle Society, Belgium, ${ }^{\mathrm{n}}$ Department of general practice and elderly care medicine, University Medical Centre, University of Groningen, ${ }^{\circ}$ Department of Maxillofacial Surgery, University Medical Center Groningen, Groningen, the Netherlands

Introduction: Measurement of muscle mass is paramount in the screening and diagnosis of sarcopenia. Besides muscle quantity, also quality assessment is important. Currently used tools are not always feasible in daily practice (CT/MRI), or do not provide sufficient information (BIA/DEXA). Ultrasonography (US) could represent a relevant tool to give both quantitative and qualitative information on muscle. However, before its use in clinical practice, several methodological aspects still need to be addressed. Both standardization in measurement techniques as the availability of reference values are currently lacking. This study aims to provide an evidence-based standardization of assessing muscle with the use of US.

Methods: A systematic review was performed for ultrasonography, older people and muscle assessment. Pubmed, SCOPUS and Web of Sciences were searched. All manuscripts regarding the use of US in assessing appendicular muscle were used. Description of US-specific parameters, localization of measurement and a quality assessment of the manuscript were noted. 
Results: In total, 358 of 14.830 abstracts were withheld. In total, 5 items of muscle assessment were found: thickness, cross-sectional area, echogenicity, fascicle length and pennation angle. In all, quality of description of US-specific parameters was poor, whereas description of localization was good.

Key conclusion: With this study, a standardized method of assessing muscle and its specific components through means of ultrasonography is presented. This shapes a standardized design to use US on a large scale as a routine assessment for muscle screening. Further studies need to assess the usability of these proposed measurements in routine practice.

\section{P-143}

Three-year adverse health consequences of sarcopenia in community-dwelling older adults according to five diagnosis definitions

Médéa Locquet ${ }^{1}$, Charlotte Beaudart ${ }^{1}$, Manon Hajaoui ${ }^{1}$, Jean Petermans $^{2}$, Jean-Yves Reginster ${ }^{1}$, Olivier Bruyère ${ }^{1}$

${ }^{1}$ University of Liège, Liège, Belgium, ${ }^{2} \mathrm{CHU}$ of Liège, Liège, Belgium

Introduction: Few studies having assessed this, our aim was to highlight the major outcomes of sarcopenia over a 3-year follow-up and to determine the power of 5 different definitions of sarcopenia to predict these outcomes.

Methods: The SarcoPhAge project includes 534 community-dwelling older adults. Sarcopenia was defined as low muscle mass plus low grip strength and decreased physical performance. Data on adverse outcomes were recorded during a clinic visit or with a phone call. The association between sarcopenia and the occurrence of undesirable outcomes was tested using the Cox proportional hazards model or a logistic regression model. A sensitivity analysis was performed to determine the power of 5 definitions to predict outcomes.

Results: 534 subjects were recruited $(73.5 \pm 6.2$ years, $60.5 \%$ female). After 3 years, 33 participants were lost to follow-up. No association between sarcopenia and falls, fractures, disabilities or institutionalizations was highlighted. A higher number of deaths occurred in individuals with sarcopenia than in those who were not with $(16.2 \%$ versus $4.6 \%$, p-value $<0.001)$. The probability of death within 3 years when presenting sarcopenia showed an approximately threefold increase (for 4 out 5 definitions). A longer hospital stay was observed in subjects with sarcopenia only when defined using the EWGSOP algorithm or the Asian Working Group criteria.

Key conclusions: Over a 3-year period, sarcopenia at baseline was associated with an increased risk of mortality and with longer hospital stays. There were significant variations in the ability of the different definitions of sarcopenia to predict these outcomes.

\section{P-144}

Relationship between within-visit blood pressure variability and skeletal muscle mass among Korean older adults

\section{Kwang-il Kim ${ }^{1}$, Jung-Yeon $\mathrm{Choi}^{1}$, Sun-wook Kim ${ }^{1}$, Cheol-Ho Kim ${ }^{1}$ \\ ${ }^{1}$ Seoul National University Bundang Hospital, Seongnam, South Korea}

Background: Sarcopenia is an important health issue in aging society. Although, hemodynamic factor is considered to be an important contributor in the development of sarcopenia, there were few studies regarding this topic. Thus, we tried to investigate the relationship between blood pressure variability and skeletal muscle mass in nation-wide large population cohort.

Methods: This cross-sectional study was based on data acquired in the Korea National Health and Nutrition Examination Survey (KNHANES), conducted from 2009 to 2011 by the Korean Centers for Disease Control \& Prevention. We included 3069 participants (age $\geq 65$ years, male 1342) for the analysis who had both blood pressure and whole-body dual energy X-ray absorptiometry (DXA) scan data. As an intra-individual within-visit blood pressure variability index, we calculated standard deviation (SD), coefficient of variation (CV), and maximum minus minimum BP difference (MMD) of systolic and diastolic blood pressure, which was measured 3 times. Appendicular skeletal muscle mass (ASM) was the sum of lean masses of both arms and legs. We adjusted ASM by body mass index.

Results: Significant inverse relationship was observed between blood pressure variability index (SD, CV, and MMD) and adjusted ASM. Blood pressure variability index were significantly higher in the lowest quartile group both in male and female participants $(\mathrm{p}<0.05)$. In multivariate analysis, blood pressure variability index were significantly associated with ASM, even after adjusting confounding factors.

Conclusions: Using the national representative database, we showed significant inverse relationship between within-visit blood pressure variability and skeletal muscle mass in Korean older adults. Considering the underlying mechanism of increased blood pressure variability, hemodynamic influence may play an important role in the development of sarcopenia.

\section{P-145}

\section{Anthropometric measurements and mortality in frail older adults}

Mario Ulises Perez-Zepeda ${ }^{1}$, Jonathan Easton ${ }^{2}$, Christopher Stephens ${ }^{2}$, Heriberto Roman-Sicilia ${ }^{2}$, Matteo Cesari ${ }^{3}$

${ }^{1}$ Instituto Nacional de Geriatria, Mexico, ${ }^{2}$ Centro de Ciencias de la Complejidad, Universidad Nacional Autónoma de México, Ciudad de México, México, ${ }^{3}$ Geriatric Unit, Fondazione IRCCS Ca' GrandaOspedale Maggiore Policlinico, Milan, Italy

Background: As the number of older adults increases, so does the number of frail older adults. Although anthropometry has been widely used as a way to stratify the overall mortality risk of a person, the significance of these measurements becomes blurred in the case of frail older adults who have changes in body composition. Therefore, the aim of this study is to determine the association of anthropometric measurements (body mass index, knee-adjusted height body mass index, waist-to-hip ratio and calf circumference) with mortality risk in a group of older Mexican adults.

Methods: This is a longitudinal analysis of the Mexican Health and Aging sub-sample (with biomarkers, $n=2573$ ) from the first wave in 2001, followed-up to the last available wave in 2015. Only frail 50 -year or older adults (Frailty Index with a cut-off value of 0.21 or higher, was used) were considered for this analysis $(n=1298)$. A survival analysis was performed with Kaplan-Meier curves and Cox regression models (unadjusted and adjusted for confounding). Sociodemographic, health risks, physical activity and comorbidities were variables used for adjusting the multivariate models.

Results: From the total sample of 1298 older adults, 32.5\% $(n=422)$ died during follow-up. The highest hazard ratio in the adjusted model was for calf circumference 1.31 (95\% confidence interval 1.02-1.69, $\mathrm{p}=0.034)$. Other measurements were not significant.

Conclusions: Anthropometric measurements have different significance in frail older adults, and these differences could have 
implications on adverse outcomes. Calf circumference has a potential value in predicting negative health outcomes or likewise.

\section{P-146}

Association between antioxidant and frailty in elderly Tunisian patient; a cross sectional study

Sonia Hammami ${ }^{1}$, Imen Gzael ${ }^{1}$, Amira Zarrouk ${ }^{1}$, Mohamed Hammami ${ }^{1}$

${ }^{1}$ Biochemistry Laboratory, LR12ES05 LR-NAFS 'NutritionFunctional Food and Vascular Health' Faculty of MedicineUniversity of Monastir, Monastir, Tunisia

Introduction: With the increase in population aging, a frailty syndrome is highlighted. We intended to understand the evolution of this syndrome by an antioxidant status estimation on frail patients aged 65 years old and up.

Patients and methods: Frail subjects $(\mathrm{n}=40)$ and non-frail subjects $(n=30)$ were concerned. The frailty status was made by means of SEGA m scale. An analysis of the biochemical parameters (glycemia, creatinine, urea, lymphocyte, CRP), oxidative stress parameters (Glutathion peroxydase (GPX), Superoxyde dismutase (SOD), Catalase, Diène conjugué $\mathrm{CD}$, Malondialdéhyde (MDA), carbonylated proteins).

Results: Our results showed high plasmatic levels of renal biomarkers (creatinine) and inflammation markers (lymphocyte and CRP) in frail patients with regard to non-frail. Besides, the frail elder had higher rates of lipids and proteins oxidation markers (DC : $0.17 \pm$ $0.06 \mathrm{nmol} / \mathrm{mg}$ d'Hb vs $0.14 \pm 0.06$ ), MDA: $9.07 .10-3 \pm 3.85 \times$ $10^{-3} \mathrm{nmol} / \mathrm{mg}$ de protéine vs $8.89 \times 10^{-3} \pm 2.26 \times 10^{-3}$, and PC: $3.5 \pm 0.69 \mathrm{nmol} / \mathrm{mg}$ de protéine vs $3.1 \pm 0.9)$, compared with the non-frail. Higher activity $(\mathrm{p}<0.05)$ of antioxidant enzymes: GPX (83. $6 \pm 27.1 \mathrm{U} / \mathrm{mg} \mathrm{Hb}$ vs $73.3 \pm 17.8)$, SOD $(3.36 \pm 0.93 \mathrm{U} / \mathrm{mg}$ $\mathrm{Hb}$ vs $27 . \pm 21$, and catalase $(0.15 \pm 0.04 \mathrm{U} / \mathrm{mg} \mathrm{Hb}$ vs $0.12 \pm 0.03)$ were observed in frail elderly. Furthermore, a significant positive correlation was revealed between frailty score and catalase activity $(\mathrm{r}=0.36 ; \mathrm{p}<0.002)$, DC $(\mathrm{r}=0.2, \mathrm{p}<0.02)$ and MDA $(\mathrm{r}=0.46$, $\mathrm{p}<0.04^{\circ}$. In conclusion, this study suggests an installation of an oxidative stress and an inflammatory reaction in frail patients. A possible relation between these two phenomena could be suggested.

\section{P-147}

Glutamine supplementation for urinary incontinence: a prospective, randomized, controlled double-blind study

Gulistan Bahat ${ }^{1}$, Birkan Ilhan ${ }^{6}$, Nalan Capan ${ }^{2}$, Cihan Kılıc ${ }^{1}$, Cenk Yaşa ${ }^{3}$, Funda Gungor ${ }^{3}$, Ozlem Persil Ozkan ${ }^{4}$, Mehmet Akif Karan ${ }^{5}$, Ayşe Karan ${ }^{2}$

${ }^{1}$ Istanbul University, Istanbul Medical School, Division of Geriatrics, Istanbul, ${ }^{2}$ Istanbul University, Istanbul Medical School, Department of physical medicine and rehabilitation, ${ }^{3}$ Istanbul University, Istanbul Medical School, Department of Urogynecology, ${ }^{4}$ Istanbul Arel University, Nutrition and Dietetics, Istanbul, ${ }^{5}$ Istanbul University, Istanbul Medical School, Division of Geriatrics, ${ }^{6}$ Dr. Ersin Arslan Research and Training Hospital, Gaziantep, Turkey

Aim: The objective of this study is to determine the effect of additional oral glutamine supplementation to Kegel exercise on pelvic floor strength and clinical parameters of urinary incontinence in females.
Methods: It is a randomized, double-blind study. Digital test and a vaginal manometer were used for measuring the strength of the pelvic floor muscles. $24 \mathrm{~h}$ pad weight test was examined. Participants were randomized into 2 groups as oral Glutamine $30 \mathrm{~g} /$ day and placebo. It was asked to use the supplementation and Kegel exercises to all participants for 3 months. Basic and 3th month measurements were compared by Paired sample T-test and Wilcoxon tests in each group. The progression between measurements at basic and 3th months was compared between the groups by using Mann-Whitney-U test.(Clinical Trials protokol ID: 2014/1203).

Results: There were 11 patients in the glutamine arm and 18 patients in the placebo arm. Mean age was $58.2 \pm 6.6$ years. Mean body mass-index was $32.9 \pm 4.8 \mathrm{~kg} / \mathrm{m}^{2}$. There was no age difference between the groups [glutamine $59 \pm 3.8$, placebo $57.8 \pm 7.9$ years, $\mathrm{p}>0.05]$. In glutamine arm, vaginal muscle strength assessed by digital test was higher at the end of 3 months $[2.9 \pm 0.7$ vs $4 \pm 0.9$; $0-3$ months respectively, $\mathrm{p}=0.014]$; perinemeter measurements were not statistically different $[27.4 \pm 8.3$ vs $31.2 \pm 8.9 ; 0-3$ months respectively, $\mathrm{p}>0.05$ ]; $24 \mathrm{~h}$ pad weight was not different [ $\mathrm{p}>0.05]$. In placebo arm, there was statistically significant progress in vaginal muscle strength assessed by both digital test and perinemeter, and $24 \mathrm{~h}$ pad weight(p values: $0.005,0.011,0.002$, respectively). When we compare the progression scores between the groups; there was no statistically significant difference [p $>0.05]$.

Conclusions: Our study suggests that glutamine supplementation does not provide additional benefit in the treatment of pelvic muscle sarcopenia in patients without protein-energy malnutrition.

\section{P-148}

Efficacy of a primary care geriatric intervention model in reversing frailty-protocol of a randomized clinical trial

Miguel Marques Ferreira ${ }^{1}$

${ }^{1}$ NMS-UNL, Lisbon, Portugal

Keywords: Frailty; Primary care.

Introduction: Longevity should go with quality of life-reversing the frailty syndrome (SF) and its impact are central to this goal. SF is a risk factor for adverse health events: mortality, disability, falls and hospitalization. Most of the interventions to revert SF can't be implemented in primary care (PC). There is no standard model for the follow-up frail elderly in PC.

Objective: Evaluate the efficacy of a PC geriatric intervention model in reversion SF.

Methods: Randomized, unicentric, clinical trial, comparing usual care and a geriatric intervention model. 240 frail individuals followed in primary health care and community residents. Inclusion criteria: age $>70$ years; FS present; community residents and no institutionalization plan. Exclusion criteria: cognitive impairment, institutionalization, hospitalization $<1$ month. "blinding": The family doctor will not access to evaluations results. The evaluations will be available to Intervention geriatric team (IGT). Evaluators: can't be members of IGT; randomly assigned to evaluations; not have access to the results of previous evaluations.

Intervention: IGT by 12 months. Individualized care, starts with a global assessment and a home evaluation. Nurse appointment every month. Medical appointment $2 / 2$ months or $3 / 3$ if frailty reverts. If nutrition risk - nutritionist appointment. Individualized care plan by IGT, after each evaluation. Evaluations: basal, 6 months and final.

Conclusion: A model of geriatric follow-up that proves to be more effective in the reversion of frailty will be an advantage in PCs. The use of PC resources results in the operationalization of the model and possible immediate clinical application. 


\section{P-149}

Frailty, polypharmacy and potentially inappropriate medications in older patients in hospital: a cross-sectional study

Kinda Ibrahim ${ }^{1,2}$, Fiona F A Howson ${ }^{1,3}$, Avan A Sayer ${ }^{4,5}$, Helen C Roberts ${ }^{1,2,3}$

${ }^{1}$ Academic Geriatric Medicine, University of Southampton, UK, ${ }^{2}$ NIHR CLAHRC: Wessex, University of Southampton, UK, ${ }^{3}$ University Hospital Southampton NHS Foundation Trust, UK, ${ }^{4}$ AGE Research Group, Institute of Neuroscience, Newcastle University, UK, ${ }^{5}$ NIHR Newcastle Biomedical Research Centre, Newcastle upon Tyne Hospitals NHS Foundation Trust and Faculty of Medical Sciences, Newcastle University, UK

Introduction: Many older patients experience polypharmacy and risk taking potentially inappropriate medications (PIMs) leading to adverse events. Recent studies have demonstrated the association between frailty and an increase in PIMs among community-dwelling older people and those with cancer. We evaluated whether frailty in hospitalised older patients is associated with polypharmacy and PIMs. Methods: A cross-sectional study of inpatients aged $\geq 70$ years admitted to one UK hospital. Frailty was assessed using the Fried Frailty Phenotype and FRAIL Scale. Polypharmacy and hyperpolypharmacy were defined as the concomitant use of five+ and 10+ medications, respectively. PIMs were identified using the Beers and STOPP criteria.

Results: 201 participants (median age 80.7 years; 120 (60\%) men) were recruited. 1738 medications were prescribed in this cohort, median 9 medications/patient. Frailty was identified in 56 and 36\% using the Fried Frailty Phenotype and FRAIL scale, respectively. Polypharmacy (46\%) and hyper-polypharmacy (41\%) were also common. Frailty using both scales was significantly associated with polypharmacy $(\mathrm{P}<0.001)$. The Beers' criteria identified 90 PIMs in $57(28 \%)$ patients. $33(58 \%)$ received one and $24(42 \%)$ received $2+$ PIMs. 108 PIMs in $76(38 \%)$ patients were identified using the STOPP criteria. 53 patients $(70 \%)$ received one and 23 (30\%) received $2+$ PIMs. Both frailty tools were significantly associated with PIMs using the STOPP criteria but not using the Beers' criteria. Conclusions: Frailty in older inpatients was significantly associated with polypharmacy and PIMs using the STOPP criteria. Structured medication review is essential for older people in hospital.

\section{P-150}

Association of independence in daily activities with muscle structure, strength and physical function in nursing home residents

\section{Julia Wearing ${ }^{1,2}$, Maria Stokes ${ }^{3}$, Eling D. de Bruin ${ }^{4,5}$ \\ ${ }^{1}$ Faculty of Health, Medicine and Sciences, University Maastricht, The Netherlands, ${ }^{2}$ Adullam Stiftung, Basel, Switzerland; Email: j.wearing@bluewin.ch, ${ }^{3}$ Faculty of Health Sciences, University of Southampton, United Kingdom, ${ }^{4}$ Institute of Human Movement Sciences and Sport (IBWS), ETH Zurich, Switzerland, ${ }^{5}$ NVS, Karolinska Institute, Stockholm, Sweden}

Introduction: Older people are often institutionalized in nursing homes as a consequence of functional decline accompanied by restrictions in independent living [1]. Physical capacity is an important determinant of functional limitations in older adults [2], however, whether independence in activities of daily living (ADL) of nursing home residents is directly associated with a specific muscle parameter has not yet been investigated. This study explored the relationship between the level of independence in ADL in older, nursing home residents and muscle structure, strength as well as physical function. Methods: Independence in performing ADL was assessed in 30 older, nursing home residents (age, $85.6 \pm 7.06$ years; weight, $65.4 \pm 15.41 \mathrm{~kg}$; height, $1.60 \pm .06 \mathrm{~m}$ ), with the Resident Assessment Instrument. Residents were categorized as either independent or dependent in basic ADL. Muscle thickness and echo intensity, isometric grip-, quadriceps- and elbow-flexor strength, gait speed, timed sit-to-stand task and physical activity were determined by validated assessments. Relationships among variables were investigated using Spearman's correlation coefficient and logistic regression analysis.

Results: The category of dependence in ADL correlated with handgrip strength $(=-.38, \mathrm{p}=.038)$, quadriceps strength $(=-.67, \mathrm{p}<.01)$, elbow-flexor strength $(=-.42, \mathrm{p}=.032)$ and physical activity $(=-.44$, $\mathrm{p}=.015)$. After regression analysis, only quadriceps strength remained a significant determinant of dependence category $(\mathrm{R} 2=.66$; Wald $(1)=5.97, \mathrm{p}=.015$ ). For every $0.1 \mathrm{~kg}$ decrease in quadriceps strength the risk of being dependent in basic ADL increased by $10 \%$. Conclusion: Quadriceps strength has a strong, independent relationship with ADL performance in nursing home residents. Improving quadriceps strength may help to avoid further decline in physical function.

References:

1. Dos Santos L, Cyrino ES, Antunes M, Santos DA, Sardinha LB (2017) Sarcopenia and physical independence in older adults: the independent and synergic role of muscle mass and muscle function. J Cachexia Sarcopenia Muscle 8(2):245-250. https://doi.org/10.1002/ jcsm. 12160

2. Laffon de Mazieres C, Morley JE, Levy C, Agenes F, Barbagallo M, Cesari M, Rolland Y (2017) Prevention of functional decline by reframing the role of nursing homes? J Am Med Dir Assoc 18(2):105-110. https://doi.org/10.1016/j.jamda.2016.11.019

\section{P-151}

Is osteoarthritis predicted frailty? Association between Fried frailty phenotype and osteoarthritis in Iranian elderly community dwellers-a cohort of Bushehr Elderly Health (BEH) program

Farshd Sharifi ${ }^{1}$, Mahtab Alizadeh-Khoei ${ }^{1}$, Hossein Fakhrzadeh ${ }^{1}$, Masoud Arzaghi ${ }^{1}$, Gita Shaffeiee ${ }^{2}$, Ramin Heshmat ${ }^{2}$, Iraj Nabipour ${ }^{3}$, Afshin Ostovar ${ }^{4}$, Bagher Larijani ${ }^{5}$, Reyhaneh Aminalroaya ${ }^{6}$, Zahra Vahabi $^{6}$, Alireza Namazi ${ }^{6}$, Abolfazl Zendedel ${ }^{2}$, Alireza Sepyani ${ }^{2}$

${ }^{1}$ Elderly Health Research Center, Endocrinology and Metabolism Population Sciences Institute, Tehran University of Medical Sciences, Tehran, Iran, ${ }^{2}$ Department of Geriatric Medicine, Medical School, Ziyaiyan Hospital, Tehran University of Medical Sciences, Tehran, Iran, ${ }^{3}$ Chronic Disease Research Center, Endocrinology and Metabolism Population Sciences Institute, Tehran University of Medical Sciences, Tehran, Iran, ${ }^{4}$ The Persian Gulf Biomedical Research Institute, Bushehr University of Medical Sciences, Bushehr, Iran, ${ }^{5}$ Osteoporosis Research Center, Endocrinology and Metabolism Clinical Sciences Institute, Tehran University of Medical Sciences, Tehran, Iran, ${ }^{6}$ Endocrinology and Metabolism Research Center, Endocrinology and Metabolism Clinical Sciences Institute, Tehran University of Medical Sciences, Tehran, Iran

Introduction: Frailty and osteoarthritis (OA) cause functional and movement limitation in elderly, which both have common pathophysiology and risk factors that have not been completely defined yet. The purpose is figure out the relationship between frailty and osteoarthritis for preventive and therapeutic approach in a community base cohort of elderly household dwellers. 
Methods: This is a cross-sectional analysis of cohort Bushehr Elderly Health (BEH) program. The sampling was multistage stratified-cluster method that representative of the general population, aged $\geq 60$ by interviewing in southern of Iran. Clinical OA defined with any symptoms in knee or other joints without history of Rheumatic disease or diagnosis of osteoarthritis by clinicians. Based on Fried Frailty phenotype criteria, individuals with three or more criteria considered as frail that examine as dependent variable in a univariate and several multivariable logistic regression models.

Results: From older participants $(\mathrm{N}=2315)$ female were $50.8 \%$. The mean age was $69.27(\mathrm{SD}=6.30)$ years old. The prevalence of frailty was $7.7 \%(\mathrm{~N}=174)$ and osteoarthritis was $12.4 \%(\mathrm{~N}=286)$. In Univariate model odds ratio (OR) of osteoarthritis for frailty phenotype was 1.99 (95\% CI 1.35-2.93). In final multivariable model after adjustment for age, gender, BMI, and smoking the OR of osteoarthritis for frailty phenotype was 2.44 (95\% CI 1.57-3.78).

Conclusion: Based on our results, osteoarthritis is a strong independent predictor of frailty phenotype in community elderly dwellers. It is needed further evaluation to understand the causal relationship through a longitudinal study.

Key words: Osteoarthritis, Fried frailty phenotype, Elderly, Community dwellers

\section{P-152}

\section{Nutritional composition with active ingredients to improve muscle atrophy}

E. A. Pestana ${ }^{1}$, P. Poydenot ${ }^{2}$, M. Raul ${ }^{2}$, M. K. Bothe ${ }^{1}$, M. Westphal ${ }^{1}$

${ }^{1}$ Fresenius Kabi Deutschland GmbH, Else-Kroener-Strasse 1, 61352 Bad Homburg, Germany, ${ }^{2}$ CYTOO, 7 parvis Louis Néel, 38040 Grenoble, France

Introduction: In older adults frailty is linked to increase muscle atrophy due to inflammation, decline in humoral, cell-mediated immunity and hormone levels, overexpression of cytokines and sarcopenia. Due to poor appetite, reduced mobility and isolation, frail older people do not meet their caloric, protein and micronutrient targets with food alone, and often need the use of oral nutritional supplements (ONS) providing sufficient amounts of calories, protein and micronutrients in a relatively low volume to improve compliance. Polyunsaturated Fatty Acids (PUFA), vitamin E, vitamin D along with the protein bound amino acids glycine, arginine and tryptophan are proposed as active novel ingredients in the therapy of patients with sarcopenia and/or frailty; due to their combined effect in muscle atrophy thereby improving muscle protein synthesis (MPS). The study objective is to show efficacy of PUFA, vitamin E, vitamin D and the protein bound amino acids glycine, arginine and tryptophan as active ingredients to increase MPS in human myoblasts.

Methods: The MyoScreenTM muscle wasting platform is based on the treatment of human primary myoblasts with TNF-alpha to induce muscle atrophy. We tested the ability of the active ingredients of Fresubin $3.2 \mathrm{kcal}$ to increase whole protein synthesis measured by the Click-iT $^{\circledR}$ HPG Alexa Fluor ${ }^{\circledR}$ Protein Synthesis Assay (ThermoFisher) on the MyoScreenTM platform.

Results: The active ingredients of Fresubin $3.2 \mathrm{kcal}$ increased protein synthesis in primary human myoblasts after 6 days of treatment.

Conclusion: The active ingredients of low volume Fresubin $3.2 \mathrm{kcal}$, increased protein synthesis in TNF-alpha incubated myoblasts and thus may reduce TNF-alpha related atrophy in muscles.

\section{P-153}

Stages of frailty syndrome and mortality in older men and women 80+

Ewa Zasadzka ${ }^{1}$, Magdalena Roszak ${ }^{1}$, Tomasz Trzmiel ${ }^{1}$, Mariola Pawlaczyk $^{1}$

${ }^{1}$ Poznan University of Medical Sciences, Poznań, Poland

Objective: The objective of the paper was to determine the dependence between the stage of the frailty syndrome (FS) in women and men and the risk of limitation of activities of daily living (ADL), hospitalisation and death in the $80+$ age group.

Methods: The study included 213 respondents (59 men and 154 women) aged 80 and over. Respondents were assessed using the Fried criteria: weight loss, weakness, low physical activity, slowness and exhaustion. All underwent assessment using Mini-Mental State Examination (MMSE), Geriatric Depression Scale (GDS) and activities of daily living (ADL).

Results: Frailty was found in 117 persons in the studied group. The analysis showed that the results in the frail group were much worse than the results in the pre-frail and non-frail groups in all the criteria looked at. After 12 months a significant deterioration of ADL, an increased risk of hospitalisation and death was observed in the frail group, with additional higher mortality among men in this group.

Conclusion: The study shows that significant risk of hospitalisation, ADL deterioration and death occurs more often in the group of persons aged 80 or over with diagnosed FS than in the pre-frail and nonfrail group. Men are more at risk of death than women, especially in the frail group. The occurrence of the FS plays an important role in the prognosis and assessment of the need for health care among the elderly.

\section{P-154}

Influence of type 2 diabetes and insulin therapy on muscles

Uta Ferrari ${ }^{1}$, Marietta Rottenkolber ${ }^{1}$, Canan Selte ${ }^{1}$, Cornelia Then ${ }^{1}$, Jochen Seißler ${ }^{1}$, Birgit Linkohr ${ }^{2}$, Annette Peters ${ }^{2}$, Michael Drey ${ }^{1}$, Barbara Thorand ${ }^{2}$

${ }^{1}$ Department of Medicine IV; University Hospital, LMU Munich, ${ }^{2}$ Institute of Epidemiology, Helmholtz Zentrum München-German Research Center for Environmental Health $(\mathrm{GmbH})$, Neuherberg, Germany

Introduction: Loss of muscle mass and function is the main cause of immobility in old age. Geriatric patients with type 2 diabetes (T2D) often suffer from mobility limitations. The aim of the current study is to investigate the association of $\mathrm{T} 2 \mathrm{D}$ and the change in muscle mass or function in old age and whether it is influenced by insulin treatment in diabetics in the population-based KORA AGE study.

Methods: In a population-based cohort study (KORA Age) the skeletal muscle index (SMI $\left[\mathrm{kg} / \mathrm{m}^{2}\right]$ ) and the hand grip strength (HS $[\mathrm{kg}]$ ) were measured at baseline (2009) and timed Up and Go (TUG [s]) test performed after a follow-up time of 3 years (2012). Multiple linear regression models were used to investigate the influence of T2D and insulin therapy on the change of muscle parameters (difference of baseline and follow-up values).

Results: Among the 731 subjects (360 women (49.3\%)) with a mean age of $74.6 \pm 6.2$ years $)$ there were $16.1 \%(\mathrm{n}=118)$ with T2D (HbA1c $6.4 \pm 0.7 \%$ ), who were treated with insulin in $16.9 \%$ $(\mathrm{n}=20)$. Patients with T2D showed a greater decrease in HK (women: $-1.2 \pm 4.3$, men: $-1.8 \pm 5.4$ ) and SMI (women: $-0.2 \pm 0.7$, men: $-0.2 \pm 0.7$ ) subjects without T2D (women: $-0.5 \pm 3.9$, men: $-0.4 \pm 5.5)$. After adjustment for gender, age, 
BMI, physical activity, smoking, and multimorbidity, T2D remains significant with the change in SMI $(\mathrm{p}=0.02)$, but not in HS $(\mathrm{p}=0.07)$ and TUG $(\mathrm{p}=0.76)$. Insulin therapy was significantly associated with the change in SMI $(\mathrm{p}<0.001)$, but not with HS $(\mathrm{p}=0.18)$ and TUG $(\mathrm{p}=0.09)$, compared to treatment with oral antidiabetic alone in subjects with treated T2D.

Key conclusions: Patients with T2D showed an accelerated decline in muscle mass in subject over 65 years of age compared to subjects without T2D. Therefore, routine clinical muscle assessment in this high-risk population might be useful. Insulin therapy could be of benefit to the maintenance of muscle mass for the elderly with T2D. However, further studies are needed to confirm this result.

\section{P-155}

Eccentric versus concentric training for increases in muscle mass and strength?

Flemming Dela ${ }^{1}$, Filippa Orlien Lindskov ${ }^{2}$, Anna Karnoe Knudsen ${ }^{2}$, Signe Regnersgaard ${ }^{2}$, Eckart Pressel ${ }^{2}$

${ }^{1}$ University of Copenhagen, Copenhagen, Denmark, ${ }^{2}$ Department of Geriatrics, Bispebjerg University Hospital, Copenhagen, Denmark

The most effective training modality to prevent and treat sarcopenia is unknown. Traditionally concentric training is employed, but this may not be as effective/feasible as eccentric training. Twenty-two healthy men and women $(70 \pm 1$ year) were randomized to stair-case training as concentric ([CON] walking up; elevator down), eccentric ([ECC] walking down, elevator up) or eccentric training $+([\mathrm{ECC}+]$ carrying weights equivalent to $15 \%$ of bodyweight) 3 times/wk for 3 -weeks (all subjects) or 6-weeks (ongoing; $\mathrm{n}=5,5$ and 3 in CON, ECC and $\mathrm{ECC}+$, respectively). Leg muscle-mass was estimated by thigh circumference (TC) and DXA-scanning, strength by 6 min walk test (6MWT), leg press test (LP) and 30-sec chair-stand test (30CST). Rate of perceived exertion (RPE) measured by Borg scale (6-20). At 3 -week the subjects had stair-walked $2.8 \pm 0.5$ [CON, upwards], $3.4 \pm 0.2$ [ECC, downwards] and $2.2 \pm 0.4$ [ECC + , downwards] $\mathrm{km}$, which by 6 -week was $4.6 \pm 1.0,7.2 \pm 0.6$ and $7.3 \pm 1.5 \mathrm{~km}$, respectively. TC didn't change in either group; DXA revealed significant $(\mathrm{P}<0.05) 3$-wk increases in muscle mass (by $1.7 \pm 0.8$, $2.8 \pm 0.4$ and $2.5 \pm 1.0 \%$ in $\mathrm{CON}, \mathrm{ECC}$ and $\mathrm{ECC}+$, respectively), with no further significant increase by 6 week. $6 \mathrm{MWT}$ and leg press at 3-wk increased $(\mathrm{P}<0.05)$ only in ECC + (from $562 \pm 26$ to $619 \pm 34 \mathrm{~m} ; 94 \pm 16$ to $100 \pm 15 \mathrm{~kg})$. 30CST increased in all groups by 3 -week (from $15 \pm 1$ to $18 \pm 1$ times). RPE was lower $(\mathrm{P}<0.05)$ in $\mathrm{ECC}+(11.5 \pm 0.3)$ vs. $\mathrm{CON}(12.6 \pm 0.4)$ at 3 -week. $\mathrm{ECC}+$ is superior to $\mathrm{CON}$ for improvement in strength/performance, in spite of lower physical demand and should be considered a new training modality for frail elderly.

\section{P-156}

In-hospital sarcopenia: sarcopenia 7+ EAMA study. From research into clinical practice

Dolores Sanchez-Rodriguez ${ }^{1}$, Florence Benoit ${ }^{2}$, Dhayana Dallmeier ${ }^{3}$, Suzy Hope ${ }^{4}$, Ester Marco ${ }^{5}$, Asta Mastaviciute ${ }^{6}$, Murielle Surquin ${ }^{2}$, Miguel Toscano-Rico ${ }^{7}$, Nele Van Den Noortgate ${ }^{8}$, Francesco Landi ${ }^{9}$

${ }^{1}$ Geriatrics Department. Centre Fòrum del Hospital del Mar. Parc de Salut Mar. Rehabilitation Research Group. Institut Hospital del Mar $\mathrm{d}^{\prime}$ Investigacions Mèdiques (IMIM). School of Medicine, Universitat Autónoma de Barcelona, School of Medicine, Universitat Pompeu Fabra, Barcelona, Spain, ${ }^{2}$ Geriatrics Department. CHU Brugmann,
Université Libre de Bruxelles, Brussels, Belgium, ${ }^{3}$ Geriatric Center Ulm/Alb-Donau, Ulm University. Agaplesion Bethesda Clinic Ulm, Germany, ${ }^{4}$ University of Exeter Medical School, Healthcare for Older People Department, Royal Devon \& Exeter NHS Foundation Trust, Exeter, UK, ${ }^{5}$ Physical Medicine and Rehabilitation Department. Parc de Salut,Rehabilitation Research Group. Institut Hospital del Mar d'Investigacions Mèdiques (IMIM), School of Medicine, Universitat Autònoma de Barcelona, Universitat Internacional de Catalunya. Barcelona, Spain, ${ }^{6}$ Vilnius University, Faculty of Medicine, Clinic of internal diseases, family medicine and oncology, Lithuania, ${ }^{7}$ Nova Medical School, Centro Hospitalar Lisboa Central, Hospital de Santa Marta. Lisbon, Portugal, ${ }^{8}$ Department of Geriatrics, Ghent University Hospital, Ghent, Belgium, ${ }^{9}$ Department of Geriatrics, Neurosciences and Orthopedics, Catholic University of the Sacred Heart, Rome, Italy

Background: Sarcopenia is a nutrition-related syndrome related to worse clinical outcomes, physical impairment, and mortality. Data regarding its prevalence in hospital settings is limited. We aim to assess the prevalence of sarcopenia in patients admitted to geriatric acute care units in 7 European countries, identifying risk factors associated with its presence or incidence, and evaluating its association with further negative outcomes during hospitalization and postdischarge.

Methods: Design: Longitudinal, prospective European multi-centre study (Belgium, Germany, Italy, Lithuania, Portugal, Spain, United Kingdom). Settings: Acute-care geriatric units. Participants: Patients aged $>70$ admitted to acute geriatric units. Exclusion criteria: disabling conditions that could directly affect muscle weakness, general and/or cognitive condition preventing administration of the tests. Main outcome measures: (1) Prevalence of sarcopenia at admission; (2) Risk factors associated with presence of sarcopenia; (3) Incidence of sarcopenia during hospital stay; (4) Negative outcomes during hospitalization (hospital-acquired infections, falls, length-of-stay, mortality) and post-discharge (institutionalization, readmission to hospital, mortality). Sarcopenia will be defined according to the European Working Group on Sarcopenia criteria: low muscle mass (mid-calf circumference $<31 \mathrm{~cm}$ ) plus either low handgrip strength (men $<30 \mathrm{~kg}$, women $<20 \mathrm{~kg}$ ) or walking speed $<0.8 \mathrm{~m} / \mathrm{s}$. Information on demographics, comorbidities, medication, cognitive function, depression/anxiety, nutrition, and SARC-F will be collected. National and international ethics guidelines will be followed.

Conclusion: Our study will provide data regarding prevalence and incidence of sarcopenia in hospitalized patients across Europe, allowing the identification of risk factors. Our results should help to develop preventive and treatment strategies for sarcopenia in this vulnerable population.

\section{P-157}

Comparing SARC-F with SARC-CalF to screen sarcopenia in community living older adults

Gulistan Bahat ${ }^{1}$, Meryem Merve Oren ${ }^{1}$, Ozlem Yilmaz ${ }^{1}$, Cihan Kilic $^{1}$, Kubra Aydin ${ }^{2}$, Mehmet Akif Karan ${ }^{1}$

${ }^{1}$ Istanbul University Istanbul Medical Faculty, Istanbul, Turkey,

${ }^{2}$ Ataturk University Istanbul Medical Faculty, Istanbul, Turkey

Objectives: To compare the diagnostic value of the SARC-F combined with calf circumference (SARC-CalF) with the standard SARC$F$ to screen sarcopenia in community-dwelling older adults.

Design: Cross-sectional, diagnostic accuracy study. Setting: Geriatric outpatient clinic. Participants: Older adults $\geq 65$ years.

Measurements: Muscle mass, hand grip strength, and usual gait speed. Currently used diagnostic criteria EWGSOP, FNIH, IWGS, and SCWD were applied. SARC-CalF was performed by using two 
different calf circumference (CC) threshold: standard cut-off $31 \mathrm{~cm}$ (SARC-CalF-31) and national cut-off $33 \mathrm{~cm}$ (SARC-CalF-33). The sensitivity/specificity analyses of the SARC-CalF and SARC-F tools were run.

Results: We included 207 subjects; 67 male and 140 female with a mean age of $74.6 \pm 6.7$ years. The prevalence of sarcopenia ranged from 1.9 to $9.2 \%$. The sensitivity of SARC-F was between $25 \%$ (EWGSOP) and 50\% (IWGS); specificity was about $82 \%$. For SARCCalF-31 and SARC-CalF-33 sensitivity was similar-between $25-50 \%$-which pointed out that SARC-CalF was not superior to SARC-F for senstivity in this sample. Corresponding specificities for SARC-CalF-31 and SARC-CalF-33 were higher than SARC-F and were between $90-98 \%$. The AUC values, which indicates the diagnostic accuracy of a screening test, were in general higher for SARCCalF-33 than the SARC-F and SARC-CalF-31.

Conclusions: We reported that addition of $\mathrm{CC}$ item to SARC-F improved the specificity and diagnostic accuracy of SARC-F but it didn't improve the sensitivity in a community-dwelling Turkish older adult population sample that had low prevalence of sarcopenia. The performance of SARC-CalF tool to screen sarcopenia is to be studied in different populations and living settings.

\section{P-158}

Performance of SARC-F in regard to sarcopenia definitions, muscle mass and functional measures

\section{Gulistan Bahat ${ }^{1}$, Ozlem Yilmaz ${ }^{1}$, Cihan Kilic ${ }^{1}$, Meryem Merve Oren $^{1}$, Mehmet Akif Karan ${ }^{1}$ \\ ${ }^{1}$ Istanbul University Istanbul Medical Faculty Internal Medicine Department Geriatrics Division, Istanbul, Turkey}

Objectives: To assess the reliability and validity of Turkish version of SARC-F in regard to current definitions of sarcopenia, muscle mass and functional measures.

Design: Cross-sectional. Setting: Geriatric outpatient clinic. Participants: Patients aged $\geq 65$ years Measurements: Muscle mass (MM), handgrip strength (HGS), usual gait speed (UGS), chair sit-to-stand test (CSS), functional reach test (FRT), short physical performance battery (SPPB), SARC-F questionnaire, FRAIL questionnaire. Sarcopenia was evaluated with different definitions EWGSOP, FNIH, IWGS SCWD.

Results: After cross-cultural adaptation, 207 subjects were analysed. Mean age was $74.6 \pm 6.7$ years, $67.6 \%$ were women. Against EWGSOP, FNIH, IWGS and SCWD definitions of sarcopenia, sensitivity of SARC-F were $\% 25,31.6,50$ and $40 \%$; specificity were $81.4,82.4,81.8$ and $81.7 \%$, respectively. Positive predictive values (PV) were between 5.1-15.4\% and negative PVs were 92.3-98.2\%. Low MM, sensitivity were about $20 \%$ and specificity were about $81 \%$. For low HGS, sensitivity of SARC-F were $33.7 \%$ (Turkish cutoff); $50 \%$ (FNIH cut-off); specificity were $93.7 \%$ (Turkish cut-off) and $85.8 \%$ (FNIH cut-off). Against low UGS, poor performance in CSS, FRT, SPPB and presence of positive frailty screening sensitivity were $58.3 \%, 39.2 \%, 59.1 \%, 55.2 \%, 52.1 \%$ while specificity were $97.3 \%, 97.8 \%, 88.1 \%, 99.3 \%, 91.2 \%$, respectively.

Conclusion: The psychometric performance of Turkish SARC-F was similar to the original SARC-F. It revealed low sensitivity but high specificity with all sarcopenia definitions. Sensitivity and specificity were higher for muscle function (MF) tests reflecting its inquiry and input on MF. Our findings suggest that SARC-F is an excellent test to exclude MF impairment and sarcopenia. SARC-F is relatively a good screening test for functional measures.

\section{P-159}

Social frailty as a predictor for physical frailty and cognitive decline in community-dwelling elderly adults: from the Kashiwa Study

Katsuya Iijima ${ }^{1}$, Tomoki Tanaka ${ }^{2}$, Kyo Takahashi $^{1}$, Kenji Toba ${ }^{3}$, Koichi Kozaki ${ }^{4}$, Masahiro Akishita ${ }^{2}$

${ }^{1}$ Institute of Gerontology, The University of Tokyo, Tokyo, Japan, ${ }^{2}$ Department of Geriatric Medicine, The University of Tokyo, Tokyo, Japan, ${ }^{3}$ National Center for Geriatrics and Gerontology, Obu, Japan, ${ }^{4}$ Department of Geriatric Medicine, Kyorin

Introduction: Although social factors may contribute adverse health outcome in elderly adults, a little study addressed the longitudinal impact on physical weaken and cognitive decline. We aimed to examine the association between social frailty and new-onset frailty and cognitive decline in community-dwelling elderly adults.

Methods: The longitudinal surveys with randomly selected community-dwelling older adults aged $\geq 65$ years were performed in annually from 2012 to 2014 . We exclude physically frail individuals at baseline. Physical frailty was defined by the Cardiovascular Health Study index. Cognitive decline was determined using the Mini-Mental State Examination (MMSE) with deceased score of greater than 1.0 point per 1 year. Social frailty was operationally defined using a deficit accumulation model referred to previous study in Japan.

Results: Of 1422 elderly who were included in this study. $9.7 \%$ of participants experienced developing frailty, and $4.7 \%$ experienced cognitive decline during the follow-up period. Baseline prevalence of social prefrailty ( $1 / 5$ score) was $30 \%$ and it of social frailty $(\geq 2 / 5)$ was $21 \%$. Adjusted by confounding factors (such as age, instrumental activity of daily living), those who with social frailty were found to be significantly at risk of new-onset frailty (the hazard ratio, $2.10 ; 95 \%$ confidence interval, 1.2-3.4), and cognitive decline (the hazard ratio, 1.76; $95 \%$ confidence interval, 1.0-3.1).

Key conclusions: Our research showed strong impact of social frailty on the risk of new onset of physical frailty and cognitive decline in community-dwelling elderly adults. To prolong healthy life expectancy, considering interventions for social frailty via multidimensional approaches may be indispensable.

\section{P-160}

Identifying important items of quality of life of frail older subjects: a qualitative study among patients and professionals

Fanny Buckinx ${ }^{1}$, Charlotte Beaudart ${ }^{1}$, Yves Rolland ${ }^{2}$, Jean-Yves Reginster ${ }^{1}$, Matteo Cesari ${ }^{3}$, René Rizzoli ${ }^{4}$, Jean Petermans ${ }^{5}$, Bertrand Fougère $^{6}$, Olivier Bruyère ${ }^{1}$

${ }^{1}$ Department of Public Health, Epidemiology and Health Economics, University of Liège, Belgium, WHO Collaborating Centre for Public Health Aspects of Musculoskeletal Health and Ageing, ${ }^{2}$ Gérontopôle, University Hospital of Toulouse III, Toulouse, France

Introduction: In recent years, there has been an increased focus on placing patients at the center of health care research. In this sense, the aim of this study was to identify important items of quality of life of frail older subjects.

Methods: A Delphi survey was conducted among professionals in the field of frailty (i.e. members of the working groups on frailty from the EUGMS or ESCEO). We also performed two focus groups, one in frail community-dwelling older subjects and one in frail nursing home residents. Frailty was assessed using different definitions.

Results: The important items to assess quality of life related to frailty, highlighted by frail patients themselves $(n=14)$ or by professionals 
$(n=35)$, have been indexed in 6 domains, based on the concept of intrinsic capacity proposed by the WHO: locomotion, sensory, vitality, psychosocial, cognition and others. Respectively 78 and 52 items have been highlighted by the Delphi Approach and the focus groups. Globally, professionals and older patients cited a majority of items from the domain of vitality (i.e. 14 items cited by both professionals and patients). Moreover, community-dwelling elderly cited many items in the psychosocial field (i.e. 8 items) while nursing home residents did not (i.e. 1 item). Professionals also place a lot of importance on locomotion (i.e. 13 items).

Conclusion: The items identified both by the Delphi approach and the focus groups could be interesting to develop a frailty specific PatientReported Outcomes (PRO).

\section{P-161}

Serum Klotho and mortality risk in nursing home residents: results from the SENIOR cohort

Fanny Buckinx ${ }^{1}$, Olivier Bruyère ${ }^{1}$, Jean-Yves Reginster ${ }^{2}$, Alexia Charles $^{1}$, Etienne Cavalier ${ }^{3}$

${ }^{1}$ Department of Public Health, Epidemiology and Health Economics, University of Liège, Belgium, WHO Collaborating Centre for Public Health Aspects of Musculoskeletal Health and Ageing, ${ }^{2}$ Department of Public Health, Epidemiology, Liège, Belgium

Introduction: Several studies suggest that circulating Klotho, a single-pass transmembrane protein, could be associated with longevity. This study aimed to test the relationship between the level of serum Klotho and mortality among a specific population of nursing home residents.

Methods: We followed subjects of the SENIOR study, a cohort of nursing home residents in Liège, Belgium, during 24 months, for the occurrence of death. In this population, $\alpha$ Klotho was measured at baseline to assess the relationship between the level of serum Klotho and survival. The IBL ELISA kit was used to measure soluble $\alpha$ Klotho protein in blood. The subjects were categorized in 3 groups according to the lower, middle and upper tertiles of serum Klotho.

Results: Among residents followed for 2 years, $\alpha$ klotho dosages was available for 415 subjects. The mean age of these subjects was $82.9 \pm 9.24$ years and $332(74.8 \%)$ of them were women. Deceased subjects had a baseline serum klotho level of $487.6(385.9-599.4) \mathrm{pg} /$ $\mathrm{mL}$ and still alive subjects had a level of 510.2 (394.7-622.7) $\mathrm{pg} / \mathrm{mL}$ $(p=0.32)$. The number of deaths observed during the follow-up wasn't significantly different according to the tertiles $(p=0.72)$. At last, after adjustment on age and sex, the survival time wasn't different according to the tertiles $(\mathrm{p}=0.38$ ).

Conclusions: An isolated measurement of serum Klotho, at baseline does not seem to be a predictor of mortality at 24 months.

\section{P-162}

Sarcopenia and its association with falls and fractures in older adults: a systematic review and meta-analysis

Suey Yeung ${ }^{1}$, Esmee Reijnierse ${ }^{2}$, Marijke Trappenburg ${ }^{3}$, Carel Meskers ${ }^{4}$, Andrea B Maier ${ }^{5}$

${ }^{1}$ Department of Human Movement Sciences, Faculty of Behavioural and Movement Sciences, Vrije Universiteit Amsterdam, Amsterdam Movement Sciences, Van der Boechorststraat 7, 1081 BT

Amsterdam, The Netherlands, ${ }^{2}$ Department of Medicine and Aged Care, @AgeMelbourne, The Royal Melbourne Hospital, The University of Melbourne, 300 Grattan Street, Parkville, Victoria 3050,
Australia, ${ }^{3}$ Department of Internal Medicine, Section of Gerontology and Geriatrics, VU University Medical Centre, De Boelelaan 1117, 1081 HV Amsterdam, The Netherlands, ${ }^{4}$ Department of Rehabilitation Medicine, VU University Medical Center, De Boelelaan 1117, 1081 HV Amsterdam, the Netherlands, ${ }^{5}$ Department of Medicine and Aged Care, @ AgeAmsterdam, The Royal Melbourne Hospital, The University of Melbourne; @ AgeAmsterdam, Vrije Universiteit Amsterdam

Background: Sarcopenia is a potentially modifiable risk factor for falls and fractures in older adults, but the strength of the association between sarcopenia, falls and fractures is unclear. Purpose: To assess the literature and perform a meta-analysis of the association between sarcopenia with falls and fractures among older adults.

Data sources: A literature search of MEDLINE, EMBASE, Cochrane and CINAHL from inception to May 2017. Study Selection: Published in English, mean/median age $\geq 65$ years, sarcopenia diagnosis, falls and/or fractures outcomes, any study population. Data Extraction: Pooled analysis was conducted of the association of sarcopenia, falls and fractures, expressed in odds ratios (OR) and 95\% confidence intervals (CI). Subgroup analyses were performed by study design, population, sex, sarcopenia definition, continent and study quality. Heterogeneity was assessed using the I 2 statistics. Data Synthesis: The search identified 2511 studies. Thirty-one studies (43962 individuals) were included in the systematic review. Three studies reported both outcomes falls and fractures. Twelve out of 19 studies reported a significantly higher risk of falls in sarcopenic compared to non-sarcopenic individuals; 12 out of 15 studies showed a significant association with fractures. Twenty-five studies (34117 individuals) were included in the meta-analysis. Sarcopenic individuals had a significant higher risk of falls (OR 1.82; 95\% CI 1.61-2.05) and fractures (OR 1.73; 95\% CI 1.47-2.03) compared to non-sarcopenic individuals. This was independent of study design, population, sex, sarcopenia definition, continent and study quality.

Limitations: Results were expressed as crude or adjusted ORs with varying adjustments.

Conclusions: A strong and consistent association between sarcopenia with falls and fractures in older adults strengthens the need to invest in sarcopenia prevention and interventions to evaluate its effect on falls and fractures.

\section{P-163}

The effect of statin therapy on frailty status in elderly patients with atherosclerotic cardiovascular disease

Qing Lu ${ }^{1}$, Xuefei Tao ${ }^{1}$, Biao Cheng ${ }^{1}$, Dan Wang ${ }^{2}$

${ }^{1}$ Department of Geriatric Cardiology, Sichuan Provincial People's Hospital affiliated University of Electronic Science and technology, ${ }^{2}$ Department of Geriatric Medicine, Sichuan Provincial People's Hospital affiliated University of Electronic Science

Objective: To determine the effect of statin usage on frailty status in elderly patients ( $\geq 65$ years old) with Atherosclerotic Cardiovascular Disease (ASCVD).

Methods: 628 patients (average age $74.08 \pm 6.43$ years, $48.73 \%$ is female) were involved in this study. The presence of frailty was verified by Fried's Frailty Criteria. All patients were prescribed moderate dose statins. Patients who met a Proportion of days covered (PDC) $\geq 50 \%$ were assigned to the treatment group, while others were in the control group. After one year follow-up, the levels of frailty degree, walking speed and grip strength before and after treatment were analyzed, the changes between the treatment group and the control group were compared. 
Results: At baseline, $12.90 \%$ patients were frail $(n=81), 59.08 \%$ were pre-frail $(n=371)$ and $28.03 \%$ were robust $(n=176)$. Compared to non-frail patents (who are robust and pre frail), frail ones participants were more likely to be older, female and lower PDC $\geq 50 \%$ ( $\mathrm{P}<0.05)$. For non-frail patents, there were no statistically significant differences found in age, gender, BMI, disease constitution, comorbid disease number, baseline walking speed and grip strength between the treatment and control group $(\mathrm{P}>0.05)$. The mean scores of frailty was significantly decreased and the level of frailty were significantly improved after 12 -month statin treatment $(\mathrm{P}=0.000)$. Patients could benefit in grip strength from 6-month statin use (compared to baseline, $\mathrm{P}=0.000$; compared to the control group, $\mathrm{P}=0.012$ ). However, No changes were found in walking speed before and after statin usage $(\mathrm{P}>0.05)$.

Conclusion: A moderate-intensity statin treatment in elderly non-frail ASCVD patients would not cause frailty deterioration, but improve frailty status as well as grip strength. Considering the evidences that statin usage could provide significant reductions in cardiovascular events and death, Using a moderate-intensity statin in elderly nonfrail ASCVD patients is generally safe and beneficial.

\section{P-164}

A higher protein intake at breakfast does not compromise total daily protein intake in older adults

Amely M. Verreijen ${ }^{1}$, Martinet T. Streppel ${ }^{1}$, Ilse Rotteveel ${ }^{1}$, Daniëlle Heman $^{1}$, Jantine van den Helder ${ }^{1}$, Carliene van Dronkelaar ${ }^{1}$, Robert G. Memelink ${ }^{1}$, Michael Tieland ${ }^{1}$, Marjolein Visser $^{2}$, Mariëlle F. Engberink $^{1}$, Peter J. M. Wei ${ }^{1,2}$

${ }^{1}$ Faculty of Sports and Nutrition, Amsterdam University of Applied Sciences, Amsterdam, The Netherlands, ${ }^{2}$ Department of Health Sciences, Faculty of Sciences, Vrije Universiteit Amsterdam, Amsterdam Public Health research institute, Amsterdam, The Netherlands

Introduction: A protein intake of 25-30 g per meal is suggested to maximally stimulate muscle protein synthesis in older adults in order to prevent sarcopenia. Protein intake at breakfast is often low and therefore breakfast offers the potential for protein suppletion. Since protein is known for its satiating effects, we explored the association between the amount of protein intake at breakfast and total daily protein intake in older adults.

Methods: Baseline protein intake was assessed by a 3-day dietary record in 507 community dwelling older adults of 55 years and older participating in lifestyle interventions at the Amsterdam Nutritional Assessment Center. Multiple linear regression analysis was used to examine the association between protein intake at breakfast (in g) and total daily protein intake (in $\mathrm{g}$, and $\mathrm{g} / \mathrm{kg}$ body weight), adjusted for energy intake $(\mathrm{kcal} / \mathrm{d})$, sex, age and BMI. Interactions were tested for sex, age and BMI but were not significant ( $\mathrm{p}>0.80)$.

Results: Mean age was $67.6 \pm$ (SD) 7.3 years, $42 \%$ was female, and mean BMI was $30.0 \pm 5.6 \mathrm{~kg} / \mathrm{m}^{2}$. Total daily protein intake was $81 \pm 24 \mathrm{~g}$ which equals $0.96 \pm 0.3 \mathrm{~g} / \mathrm{kg}$ and $17.6 \pm 3.7$ percent of total energy intake. Protein intake at breakfast was $14 \pm 7 \mathrm{~g}$. A $10 \mathrm{~g}$ higher protein intake at breakfast was associated with a $6.7 \mathrm{~g}(\mathrm{SE}=$ $1.0 ; \mathrm{P}<0.001)$ and a $0.06 \mathrm{~g} / \mathrm{kg}(\mathrm{SE}=0.01 ; \mathrm{P}<0.001)$ higher total daily protein intake after adjustment for confounders.

Key conclusions: A higher protein intake at breakfast does not compromise total daily protein intake in community dwelling older adults.

\section{P-165}

Prevalence of sarcopenia among healthy ambulatory subjects: the sarcopenia begins from 45 years

Voronska Elena ${ }^{1}$, Lamberti Carla ${ }^{1}$, Kruiskamp Saskia ${ }^{2}$

${ }^{1}$ Institut Medecine et Physiologie de la Longevite, ${ }^{2}$ Institut de Medecine et Physiologie de la Longevite

Background and aims: Sarcopenia has been indicated as a reliable marker of frailty and poor prognosis among the oldest individuals. There are only few data on sarcopenia in healthy general population. We evaluated the prevalence of sarcopenia and its association with functional and clinical status in a population of healthy ambulatory subjects over 45 years living at home, in Paris (France).

Methods: This study was conducted selecting all ambulatory participants $(n=1445)$ aged 45 years and older from October 2008 to September 2011, consulting in the Institute of Physiology (Institut de Jaeger) from Paris (France) for a functional and muscular evaluation, and did not have limitations to moderate physical exercise. All were healthy people. All subjects performed a medical examination, associated with evaluation of muscle mass (body composition assessment using dual-energy X-ray absorptiometry) and of muscle function (by hand grip strength). Diagnosis of sarcopenia required the documentation of low muscle mass with low muscle strength according to the current international consensus definition of sarcopenia.

Results: From 1421 participants (553 males and 868 females) definitively enrolled, 221 subjects (135 females and 86 males) $(15.5 \%)$ were identified as sarcopenic. Results from multivariate logistic regression models showed that sarcopenia was inversely associated with BMI with those participants with BMI higher than $22 \mathrm{~kg} / \mathrm{m}^{2}$ showing a lower risk of sarcopenia relative to those with BMI less than $21 \mathrm{~kg} / \mathrm{m}^{2}$ (OR 0.72 ; 95\% CI $0.60-0.91$ ). Similarly, probability of sarcopenia was lower among subjects involved in leisure physical activities for $3 \mathrm{~h}$ or more per week (OR $0.45 ; 95 \% \mathrm{CI}$ $0.24-0.93)$. According to the category of age $[45-54 ; 55-64 ; 65-74$; $75-84$ and 85 years or more], the prevalence of sarcopenia in women increase from $9.1 ; 12.7 ; 14.5 ; 19.4 ;$ to $33.3 \%$, respectively. For the men, the percentage of sarcopenia increase with aging from 8.6; 15.6; 13.6; 63.8 to $45.5 \%$, respectively.

Conclusions: The present study suggests that among healthy ambulatory subjects over 45 years living at home, sarcopenia is frequent, even to the youngest subjects of the studied population, taking place from $9 \%$ from 45 years, until $64.3 \%$ for the subjects over 85 years. Our findings support the hypothesis that muscle mass and function are associated with BMI and physical activity, whatever the age of the subject.

\section{P-166}

Depressive symptoms, handgrip strength, and weight status in US older adults

Lee Smith ${ }^{1}$, Brendon Stubbs ${ }^{2}$, Nicola Veronese ${ }^{3}$, Lin Yang ${ }^{4}$

${ }^{1}$ The Cambridge Centre for Sport and Exercise Sciences, Anglia Ruskin University, Cambridge, UK, ${ }^{2}$ Physiotherapy Department, South London and Maudsley NHS Foundation Trust, UK, ${ }^{3}$ National Research Council, Neuroscience Institute, Aging Branch, Padua, Italy

Introduction: Handgrip strength is a valid indicator of broader physical functioning. Handgrip strength and weight status have been independently associated with depressive symptoms in older adults, but no study has yet investigated the relationships between all three in older US adults. This study investigated the relationship between 
physical function and depressive symptoms by weight status in older US adults.

Methods: Cross-sectional data were analysed from the National Health and Nutrition Examination Survey waves 2011-2012 and 2013-2014. Physical function was assessed using a grip strength dynamometer. Depressive symptoms were assessed using the selfreported Patient Health Questionnaire-9. Weight status was assessed using Body Mass Index (BMI) and participants were categorised as normal weight/underweight $\left(<25 \mathrm{~kg} / \mathrm{m}^{2}\right)$, overweight (25 to $\left.<30 \mathrm{~kg} / \mathrm{m}^{2}\right)$, and obese $\left(\geq 30.0 \mathrm{~kg} / \mathrm{m}^{2}\right)$. Associations between depressive symptoms and hand grip strength were estimated by gender-specific multiple linear regressions and BMI stratified multivariable linear regression.

Results: A total of 2851 adults (51\% female, mean age 69.2 years, mean BMI $29.0 \mathrm{~kg} / \mathrm{m}^{2}$ ) were included. Women with moderate to severe depressive symptoms had $1.55 \mathrm{~kg}$ (95\% CI $0.85-2.25)$ lower hand grip strength compared to women with minimal or no depressive symptoms. No such association was observed in men. Among those with obesity, men $(-3.72 \mathrm{~kg}, 95 \% \mathrm{CI}-7.00$ to -0.43$)$ and women $(-1.83 \mathrm{~kg}, 95 \% \mathrm{CI}-2.87$ to -0.78$)$ with moderate to severe depressive symptoms both had lower handgrip strength.

Conclusion: Among older US adults, women and people who are obese and depressed are at the greatest risk of decline in physical function.

\section{P-167}

Effects of whole-body vibration exercise in combination with parathyroid hormone (1-34) on physical performance measures in osteoporotic women: a secondary analysis from a randomized controlled trial

Ditte (D. B.) Jepsen ${ }^{1,2}$, Tahir (T.) Masud ${ }^{1,2,3}$, Anders Holsgaard (A.H.) Larsen ${ }^{2,4}$, Stinus (S.) Hansen ${ }^{2,5}$, Niklas Rye (N. R.) Jørgensen ${ }^{6,7}$, Jesper (J.) Ryg ${ }^{1,2}$

${ }^{1}$ Department of Geriatric Medicine, Odense University Hospital, Denmark, ${ }^{2}$ Institute of Clinical Research, University of Southern Denmark, Denmark, ${ }^{3}$ Department of Geriatric Medicine, Nottingham University Hospitals Trust NHS, United Kingdom, ${ }^{4}$ Department of Orthopaedics and Traumatology, Odense University Hospital, Denmark, ${ }^{5}$ Department of Endocrinology, Odense University Hospital, Denmark, ${ }^{6}$ Department of Clinical Biochemistry, Rigshospitalet, Denmark, ${ }^{7}$ OPEN- Odense Patient data Explorative Network, Odense University Hospital/ University of Southern Denmark

Introduction: Osteoporosis and falls are the leading causes of fragility fractures. Teriparatide (parathyroid hormone, PTH) is a bone anabolic agent [1] and whole body vibration exercise (WBV) has been shown to decrease the rate of falls [2]. The purpose of this assessor-blinded randomized controlled trial (RCT) was to investigate the effects of $\mathrm{WBV}+\mathrm{PTH}$ vs PTH on physical function in osteoporotic women.

Methods: This is a secondary analysis from a RCT with change in bone mineral density from baseline to 12 months as primary outcome yet to be obtained. Outcomes were short physical performance battery (SPPB; walking-speed, tandem balance-, five-times-sit-to-stand test), timed-upand-go (TUG), leg extension power, and handgrip strength at baseline, $3-$, and 6 months of follow-up. Participants received teriparatide $20 \mu \mathrm{g} / \mathrm{day}$ and WBV were performed three times per week. The analyses were performed with intention-to-treat and mixed linear regression.

Results: A total of 35 postmenopausal women (mean age $69 \pm 7$ ) were randomized to $\mathrm{WBV}+\mathrm{PTH}(\mathrm{n}=17)$ or PTH $(\mathrm{n}=18)$. After 6 months, 91.4\% completed follow-up. SPPB improved from baseline to 3 months $(\mathrm{p}=0.039)$ in the WBV + PTH compared to the PTH with a non-significant change at 6 months (median [IQR] 9 [7.5-10.5], 11 [9-12], and 10 [8-12] (WBV + PTH) vs 10 [10-12], 10 [10-12], and 10 [9-12] (PTH)). No differences between groups were observed for leg extension power, TUG, or handgrip strength. Conclusion: Adding WBV to PTH yields a significant improvement in physical performance after the first 3 months. This suggests that employing WBV to those undergoing PTH-treatment could have an added benefit to its direct effects on bone by improving physical function.

\section{References:}

1. Neer RM, Arnaud CD, Zanchetta JR, Prince R, Gaich GA, Reginster J-Y et al (2001) Effect of parathyroid hormone (1-34) on fractures and bone mineral density in postmenopausal women with osteoporosis. New Engl J Med 344(19):1434-1441

2. Jepsen DB, Thomsen K, Hansen S, Jorgensen NR, Masud T, Ryg J (2017) Effect of whole-body vibration exercise in preventing falls and fractures: a systematic review and meta-analysis. BMJ Open 7(12):e018342

Keywords: Lifestyle intervention (diet, physical activity, etc.), Prevention in vulnerable population (oldest old, minorities, women, etc.).

\section{P-168}

\section{Hour ambulatory blood pressure changes in sarcopenia}

Hande Selvi Öztorun ${ }^{1}$, Remzi Bahşi ${ }^{1}$, Tuğba Turgut ${ }^{1}$, Deniz Mut Sürmeli $^{1}$, Volkan Atmiş ${ }^{2}$, Ahmet Yalçin ${ }^{3}$, Sevgi Aras ${ }^{1}$, Murat Varli ${ }^{1}$

${ }^{1}$ Department of Geriatric Medicine, İbni-i Sina Hospital, Ankara Unıversity School of Medicine, Ankara, Turkey, ${ }^{2}$ Şevket Yılmaz Training and Research Hospital, Bursa, Turkey, ${ }^{3}$ Ankara Atatürk Training and Research Hospital, Ankara, Turkey

Introduction: Sarcopenia is used in older people to describe reduction in skeletal muscle mass and muscle strength. Blood pressure (BP) is directly and causally related to body size in the general population. It remains uncertain whether muscle mass is an important factor in determining blood pressure. The purpose of our study; the 24-hour ambulatory blood pressure changes in sarkopenic aged patients are different from those in non-sarcopenic patients.

Method: Forty-two patients aged between 63 and 94 who applied to the geriatric clinic were included in the study. Demographic data and laboratory values of the patients were recorded. Blood pressure measurements were made with a 24-h ambulatory blood pressure meter. Muscle strength was assessed by handgrip test, muscle performance by $4-\mathrm{m}$ walking test. Skeletal muscle mass was assessed by bioelectrical impedance analysis (BIA).

Result: The patients were divided into two groups, 16 sarcopenic and 34 non-sarcopenic patients with a mean age of $78.22 \pm 6.80$. In sarcopenic group, daytime systolic blood pressures were found to be significantly lower than in non-sarcopenic group. Muscle mass (muscle mass assessed by BIA alone, without hand strength and walking speed) and ambulatory blood pressure data were assessed by Pearlson correlation analysis. There was statistically significant and negative correlation between muscle mass and blood pressure change. Key conclusion: When antihypertensive treatment is given in the patients with sarcopenia, it should be considered that there may be decrease in systolic blood pressure and the dose should be titrated by starting the treatment at low doses and monitoring the blood pressure closely. 


\section{P-169}

Short physical performance battery and obesity in Old Icelandic adults

\author{
Alfons Ramel ${ }^{1}$, Olof G Geirsdottir ${ }^{1}$, Milan Chang ${ }^{1}$, Palmi V. Jonsson ${ }^{1}$ \\ ${ }^{1}$ University of Iceland, Reykjavik, Iceland
}

Introduction: Associations between obesity, physical function and falls in old adults are not clear. Obesity is defined using body mass index (BMI), which does not discriminate between body fat and muscle mass. More sophisticated measurements of body composition might be better suited to explore associations between body composition and function. The aim was to investigate BMI and other anthropometric variables in relation to physical function and falls.

Methods: Community dwelling subjects from Reykjavik, Iceland $(\mathrm{N}=108,78.3 \pm 6.4$ years, $76 \%$ female) participated in this crosssectional study. Anthropometrics (bio-electrical-impedance), grip strength, Short-Physical-Performance-Battery (SPPB), gait speed $(400 \mathrm{~m})$ and number of falls during the last 12 months were assessed. Results: Neither overweight $(40.2 \%)$ nor obesity $(35.3 \%)$ were associated with SPPB, gait speed or falls but with higher grip strength $(5.2 \pm 2.2 \mathrm{~kg}, \mathrm{P}=0.021$ and $5.6 \pm 2.3 \mathrm{~kg}, \mathrm{P}=0.021$, respectively) when compared to normal weight $(24.3 \%)$. BMI correlated significantly with indices of muscle mass $(r=0.4-0.7)$ and fat mass $(r=0.5-0.9)$ in both sexes. Measurements of segmental muscle mass were associated with better SPPB, grip strength and gait speed, corresponding measurements of body fat were associated with poorer function. No anthropometric variable was independently associated with number of falls.

Key conclusions: Obesity defined by BMI is not related to strength but not to physical function in old adults. A possible explanation is that BMI is both related to fat and muscle mass. A segmental analysis of body composition shows that muscle and fat are related to physical function in the expected way. However, body composition is not related to falls in old Icelandic adults.

\section{P-170}

Perspectives from patients attending a falls clinic-a qualitative study

Ann Sophia (A. S.) Bertelsen ${ }^{1}$, Jesper (J.) Ryg ${ }^{1}$, Dorthe (D.) Nielsen ${ }^{2}$

${ }^{1}$ Department of Geriatric Medicine, Odense University Hospital (OUH), Denmark, ${ }^{2}$ Center of Global Health, University of Southern Denmark (SDU), Denmark

Introduction: Falls are associated with increased morbidity, loss of skills, and increased need for assistance. Furthermore, having fallen once increases the risk of falling again. This study explored the perspectives of older persons experiencing a recent fall and how the fall affected everyday life.

Methods: A phenomenological-hermeneutical approach was used together with a qualitative explorative design. Semi-structured interviews were conducted and analyzed by systematic text condensation. Data was managed in NVIVO.

Results: A total of nine patients (five women, four men) attending a Danish Falls Clinic were included. Median [IQR] age was 78 [76-84] years. The analysis provided four themes: "Importance of getting professional help", "Maintaining meaningfulness in everyday life", "Help supplies", and "The silent patient". The analysis emphasized that wishes and needs varied depending on what the older person found most meaningful in life. However, all patients expressed a wish not to fall again. Some patients expressed specific requests for help supplies, which tended to be more frequent among women.
Whereas, "silent patients" rejected or didn't express any specific needs or wishes related to their fall.

Key conclusions: This study provides knowledge about the differences in older person's wishes and needs after a fall, which highlights the importance of person-centered treatment of older fallers. The expression of no needs could represent a lack of knowledge or a social and cultural norm, where need for help is considered as a threat to the personal identity. This indicates that there might be reasons for providing better information in the society about fall prevention.

Keywords: Health promotion, Prevention in vulnerable population

\section{P-171}

Comprehensive geriatric assessment based frailty index (CGA-FI) as predictor of health outcomes in a geriatric acute care centre

Ruth Paz-Maya ${ }^{1}$, Dacil Cabezas-Jaen ${ }^{1}$, Concepcion PeinadoGallego $^{1}$, Nuria Cristina Herrera-Fernandez ${ }^{1}$, Francisco Javier Balea-Fernandez ${ }^{1}$

${ }^{1}$ Lanzarote Insular Hospital, Canary Islands, Spain

Background: Frailty is closely linked to health results. Frailty indexes (FI) and Comprehensive Geriatric Assessment (CGA) are multidimensional tools. FIs serve as quantitatively measure for frailty levels. They have excellent correlation with mortality, however aren't used in routine clinical practice.

Objectives: Analyze frailty degree in patients admitted at our Geriatric Unit and assess how it influences their functional situation and correlation with mortality at 6 months after discharge from hospital. Methods: Retrospective, cross-sectional and descriptive study of a patient cohort admitted at an Acute Geriatric Unit from April to September 2017. We carried out a 6 month follow up and analyzed visits to emergency room, new hospital admissions, functional status and mortality. Statistical analysis was carried out with IBM SPSS.

Results: We included 160 patients, with an average age of 84.6 years and $62 \%$ were female. Respiratory infections were the main reason for admission $(32.5 \%)$. About $70 \%$ of patients came from the emergency service, $69 \%$ of them with severe dependence and $28 \%$ died during hospital stay. The average Frailty-CGA Index was 0.45 , having $48 \%$ of patients a moderate frailty. After 6 months $64 \%$ of patients went at the emergency room at least once and $17.8 \%$ of them died, of which $47 \%$ had a moderate frailty and $43 \%$ an advanced frailty.

Conclusions: Frailty assessment offers professionals a simple analysis to determine patient's reserve, vulnerability and situational diagnosis; it's crucial to establish therapeutic intensity intervention and measure efficacy afterwards. Frailty quantification methodizing will provide a framework in the interdisciplinary geriatric care teams.

P-172

Validation of SARC-F for the screening of sarcopenia in elderly patients with dementia: a cross-sectional study

\section{Zaid $\operatorname{Kasim}^{1}$}

${ }^{1}$ Department of Geriatric Medicine, University of Antwerp, Antwerp, Belgium

Introduction: Screening for sarcopenia is essential in a geriatric population including patients with dementia. The SARC-F is a validated questionnaire for the screening of sarcopenia in elderly patients. However, using this questionnaire in patients with cognitive problems might be a problem. The objective of this study was to determine 
whether the SARC-F questionnaire is useful in screening for sarcopenia in patients with dementia.

Methods: This study included patients aged 60 years or older with various grades and types of dementia, who were admitted to the ZNA Joostens PsychoGeriatric Hospital. The SARC-F questionnaire was completed by the patients themselves. Sarcopenia was defined using the EWGSOP's diagnostic criteria. Calf circumference, hand grip strength and gait speed were measured and used as the variables for muscle mass, strength and function respectively.

Results: This study included 174 patients, 59.2\% female. Mean age of 83.3 year (SD 7.1). Sarcopenia was identified in 110 patients, while SARC-F identified only 38 patients as possibly sarcopenic. In this sample SARC-F had $58 \%$ sensitivity and $33 \%$ specificity with a positive predictive value of $66 \%$ and a negative predictive value of $27 \%$.

Conclusion: SARC-F could not be used as a satisfactory screening tool for sarcopenia among patients with dementia who score the questionnaire themselves.

\section{P-174}

Prevention of hospitalization-associated disability: a new paradigm of care plan focused on frailty

E. Martín de Francisco Murga ${ }^{1}$, M. Herrera Abian ${ }^{1}$,

C. Palicio Martínez ${ }^{1}$, L. Bárcena Goitiandia ${ }^{1}$, J. M. Ortega Morente $^{1}$, A. Jimenez Rodriguez ${ }^{2}$

${ }^{1}$ Geriatría-Hospital Infanta Elena, Valdemoro, Madrid, ${ }^{2}$ Medicina Interna-Hospital Infanta Elena, Valdemoro, Madrid

Introduction: Population is getting older, what brings us to adapt the classical hospitals care plan to a comorbid, polymedicated and social poor supported most prevalent patient. This frail phenotype tends to present functional decline (FD) during the hospitalization, with many negative consequences and costs related.

Methods: We developed a strategy to prevent FD in patients above 75 years old admitted to medical wards of our hospital along 4 months (September to December 2017), and examine the results in functional terms. Patients at high risk of developing FD (Barthel 30-70) were included. An individual intervention was performed by a trained nurse enhancing the maintenance of ADLs, early ambulation, staff education to diminish the use of diapers and mechanical restraints, and participation of caregivers. Results were compared to a sample of patients admitted during April (classic care plan).

Results: We included 134 patients, with a mean age of 84 years old, similar percentage of male and female. In April we detected same baseline and discharged Barthel score in only $25 \%$, while in September it was $40 \%$, increasing until December reaching $65 \%$. Diapers in continent patients were removed in $77 \%$ in September, decreasing to $28 \%$ in December (in April $91 \%$ weared diapers). Mechanical restraints were used in $40 \%$ in April, $24 \%$ in September and descended to $5 \%$ in December.

Key conclusions: It's a priority to reorganize the hospitals elderly care plan working on FD besides treating the acute illness in order to get better health, functionality, life quality and economic outcomes.
P-175

How do older people with mild frailty experience health promotion based on behaviour change theory? A qualitative study

Christina Avgerinou ${ }^{1}$, Benjamin Gardner $^{2}$, Kalpa Kharicha ${ }^{1}$, Rachael Frost ${ }^{1}$, Ann Liljas ${ }^{1}$, Rekha Elaswarapu ${ }^{3}$, Jill Manthorpe ${ }^{4}$, Vari Drennan ${ }^{5}$, Claire Goodman ${ }^{6}$, Steve Iliffe ${ }^{1}$, Kate Walters ${ }^{1}$

${ }^{1}$ Department of Primary Care and Population Health, University College London, London, UK, ${ }^{2}$ Department of Psychology, Kings

College London, London, UK, ${ }^{3}$ Trustee, Age UK Ealing, UK, ${ }^{4}$ Social Care Workforce Research Unit, King's College London, London, UK, ${ }^{5}$ Centre for Health and Social Care Research, Kingston University and St George's, University of London, London, UK, ${ }^{6}$ Centre for Research in Primary and Community Care, University of Hertfordshire, Hatfield, UK

Background: While mild frailty is common, it has the potential to be reversible with health promotion interventions. The COM-B model (Michie et al. 2011) takes into account the impact of Capability, Opportunity and Motivation on Behaviour and has been applied in health promotion. Aims: We aimed to explore the experiences of recipients and providers of a health promotion intervention for older people with mild frailty, underpinned by behaviour change theory and COM-B, with a view to understanding how it was perceived by participants.

Methods: This qualitative study was nested within a randomised controlled trial testing the feasibility of a home-based health promotion service for older people with mild frailty in two diverse areas of South England. Clients developed personalised goals around maintaining assets and devised strategies to achieve these in conjunction with project workers, whose role was to use COM-B to help identify barriers and facilitators to these and provide information, emotional/practical support, teach skills, as well as provide feedback. We conducted semi-structured interviews with 3 service providers and 16 clients and thematically analysed data.

Findings: Interview data suggest that COM-B can be used to support behaviour change in mild frailty. Clients were able to identify a range of assets to improve/maintain. Project workers successfully worked with older people to assess their capability, opportunity and motivation to meet outcome goals, helped them develop related behavioural goals and work towards these. Most participants responded positively to this approach. However, further consideration needs to be made for people developing cognitive impairment, and the best approaches to support behaviour change in this group.

\section{P-176}

The relationship between frailty and sleep quality in oldest old patients

Cagatay Cavusoglu ${ }^{1}$, Olgun Deniz ${ }^{1}$, Rana Tuna Dogrul ${ }^{1}$, Suheyla Cotelli ${ }^{1}$, Ali Oncul ${ }^{1}$, Muhammet Cemal Kizllarslanoglu ${ }^{1}$, Berna Goker ${ }^{1}$

${ }^{1}$ Gazi University School of Medicine, Department of Internal Medicine, Division of Geriatrics, Ankara, Turkey

Introduction: Frequently sleep disorders increase with aging. Rate of frailty also increases with age and its association not only physical but also cognitive, mood and social vulnerability are reported. We aim to study the relationship between frailty and sleep quality in the oldest old geriatric patients.

Methods: 50 patients age 80 years and over were included. All patients underwent comprehensive geriatric assessment (CGA) including evaluation of Basic Activities of Daily Living (ADL), 
Instrumental ADL, Yesavage Depression Scale (YDS), Mini-Mental State Examination, handgrip strength, Mini Nutritional AssessmentShort Form. Sleep quality of the patients was assessed by Pittsburg Sleep Quality Index (PSQI). Frailty was assessed by Fried frailty index.

Results: The median age was 84 years (min-max $80-91)$ and $50 \%$ was female. There were 15 frail $(30 \%)$ and 35 non-frail $(70 \%)$ patients. Age, co-morbidities such as hypertension, diabetes mellitus, coronary heart disease, depression and chronic obstructive pulmonary disease and CGA test parameters were similar between two groups $(p>0.05)$. Female rate was higher in frail group compared to nonfrail group ( $73 \%$ vs. $40 \%, \mathrm{p}=0.031$ ). The median score of Pittsburg sleep quality index was higher in the frail group [11 points (min-max $3-17)]$ than non-frail group [6 points (min-max $1-19)](p=0.018)$. In a multivariate analysis model, when adjusted for age and gender, Pittsburg sleep quality index score was found to be independently associated with frailty (OR 1.197; 95\% CI 1.019-1.405; $\mathrm{p}=0.028$ ). Conclusion: Our results suggest that sleep quality may be worse in the frail oldest old patients compared to non-frail ones. Evaluation of sleep during CGA and might help improve quality of life in frail patients in daily geriatric practice. Prospective studies are needed to its management clarify the possible causality between sleep quality and frailty.

\section{P-177}

Beyond the chronological age: comparing the prognostic value of different health indicators in older adults

\author{
A. Zucchelli ${ }^{1}$, D. L. Vetrano ${ }^{2}$, A. Marengoni ${ }^{1}$, G. Grande ${ }^{2}$, \\ A. Calderon-Larranaga ${ }^{2}$, L. Fratiglioni ${ }^{2}$, D. Rizzuto ${ }^{2}$ \\ ${ }^{1}$ University of Brescia, Brescia, Italy, ${ }^{2}$ Karolinska Institutet, \\ Stockholm, Sweden
}

Introduction: The search for reliable indicators of biological age across different health outcomes has been ongoing for over three decades, and until recently, largely without success. We aimed to compare the prognostic accuracy of five geriatric health indicators: frailty index (FI), frailty phenotype (FP), walking speed (WS), multimorbidity, and the health assessment tool (HAT).

Methods: Data from the Swedish National Study on Aging and Care in Kungsholmen, an ongoing population-based study including 3363 people $60+$, were used. The ability of the five geriatric indicators to predict mortality (3- and 5-year), unplanned hospitalizations (1- and 3 -year), and $2+$ health provider contacts ( 6 months prior and after assessment) was compared using the area under the ROC curves (AUROC) from unadjusted logistic regressions. Predictive ability was tested also in younger $(<75)$ and older participants.

Results: FI, WS and HAT had the highest predictive power for mortality (3-year AUROC: 0.84, 0.85, 0.86 respectively; $\mathrm{p}<0.001$ ). The predictive ability of all indicators diminished for unplanned hospitalizations (AUROC: from 0.73 for HAT to 0.66 for FP). Contacts with health providers were better predicted by multimorbidity (AUROC: $0.66 ; 95 \% ; \mathrm{p}<0.001$ ). Multimorbidity had higher AUROC among younger subjects (mean AUROC: 0.65 vs 0.62; $\mathrm{p}<0.001$ ), while other indicators performed better among older ones.

Conclusion: Simple geriatric indicators as WS and HAT are comparable to the complex FI in predicting mortality and unplanned hospitalization. Multimorbidity performs better in predicting provider contacts. Being aware of the specificity of different measures toward different outcomes may help to provide more effective care.

\section{P-178}

Is increased neutrophil lymphocyte ratio remarking the inflammation in sarcopenia?

Zeynel Abidin Öztürk ${ }^{1}$, Seval Kul ${ }^{1}$, İbrahim Halil Türkbeyler ${ }^{1}$, Zeynel Abidin Sayiner ${ }^{2}$, Azer Abiyev ${ }^{2}$

${ }^{1}$ Associate Professor, ${ }^{2}$ Doctor

Objectives: Increasing data suggests that chronic inflammation has an essential role on development of muscle dysfunction and progression of sarcopenia in aging population. The aim of the present study was to compare Neutrophil Lymphocyte Ratio (NLR) levels in sarcopenic and non-sarcopenic individuals and to present the correlation between NLR and other inflammatory markers.

Methods: A total of 105 subjects with sarcopenia (male/female: $54 / 51$, mean age $72.8+7.3$ ) and 314 subjects as non-sarcopenic (male/female: $125 / 189$, mean age $71.44+5.4$ ) were enrolled in this cross-sectional study. Sarcopenia was diagnosed according to The European Working Group on Sarcopenia in Older People criteria. Comprehensive geriatric assessment was performed to participants. Complete blood count, biomarkers of inflammation (C-reactive protein (CRP), erythrocyte sedimentation rate (ESR)) of all patients were measured.

Results: NLR levels were found to be higher in the sarcopenic group $(2.52 \pm 1.30$ vs $2.21 \pm 0.93, \mathrm{p}<0.013$, respectively). Compared to non-sarcopenic participants white blood cell (WBC), ESR and CRP levels were also higher in sarcopenic group. There was a positive correlation between CRP, WBC, total body fat ratio and NLR (r: $0.433, \mathrm{p}<0.001 ; \mathrm{r}: 0.237, \mathrm{p}: 0.022 ; \mathrm{r}: 0.339$, p $<0.001$, respectively). A strong negative correlation was identified between fat free mass and NLR levels in sarcopenic individuals ( $\mathrm{r}$ : -0.755 , $p<0.001)$. The result of the logistic regression analysis depicted that NLR is an independent predictor for sarcopenia (OR 1.31; $95 \% \mathrm{CI}$ 1.06-1.62, P: 0.013).

Conclusion: Increased NLR levels may indicate that inflammation may have a significant role in development of sarcopenia in the elderly population.

\section{P-179}

Frailty and long-term outcomes in two hospital-based cohorts: admission avoidance is not enough

E. Keeble ${ }^{1}$, S. P. Conroy ${ }^{2}$, H. C. Roberts ${ }^{3}$, S. G. Parker ${ }^{4}$, S. Arora ${ }^{1}$, J. Neuburger ${ }^{1}$

${ }^{1}$ The Nuffield Trust, London, UK, ${ }^{2}$ Leicester University, Leicester, UK, ${ }^{3}$ Southampton University, Southampton, UK, ${ }^{4}$ Newcastle University, Newcastle upon Tyne, UK

Introduction: Frailty provides a useful lens into stratifying risk, beyond age, comorbidities or the presenting acute condition. If frailty can inform decisions about care versus cure, then clinicians need better data on longer term outcomes using appropriate frailty tools in different contexts.

Methods: We compared two contrasting hospital based populations: patients aged $70+$ years (1) admitted to an ambulatory care setting and (2) admitted for acute inpatient care. Comparisons of 2-year mortality, hospital use and costs by frailty status were determined using both clinical and data derived frailty measures.

Results: Mortality after 2 years was increased for frail compared to non-frail individuals in both the ambulatory and admitted cohorts. Patients in the ambulatory care cohort classified as frail by any scale were over twice as likely to die over the 2 years. There was also increased hospital use and costs in the ambulatory cohort with around 
twice as many bed days and over $£ 1000$ more hospital costs for frail individuals. Shorter survival times meant there was less evidence of an association between hospital use and frailty in the admitted cohort. Conclusions: A better response to the needs of frail older people in hospital is required but especially for ambulatory patients at high risk of poor outcomes where admission avoidance alone is insufficient. Health and social care systems might wish to examine their current care provision for ambulatory frail older people with urgent care needs which have received less focus than admitted cohorts.

\section{P-180}

Clinical variations between sarcopenic obesity and sarcopenia in geriatric patients: nomogram approach

\author{
Zeynel Abidin Öztürk ${ }^{1}$, Seval Kul ${ }^{2}$ \\ ${ }^{1}$ Gaziantep University, Faculty of Medicine, Department of \\ Geriatrics, Gaziantep, Turkey, ${ }^{2}$ Gaziantep University, Faculty of \\ Medicine, Department of Biostatistics, Gaziantep, Turkey
}

Purpose: We aimed to identify clinical parameters effected from sarcopenic obesity compared to sarcopenic geriatric patients and construct nomogram.

Methods: 105 sarcopenic patients admitted to Gaziantep University Hospital Geriatrics clinic between January 2016 and 2017 were included in this cross-sectional study. A multivariate model was built for the most important factor to differentiate sarcopenia and sarcopenic obesity. Afterwards, a nomogram was drawn with the aid of statistically significant variables.

Results: Significantly higher waist circumference (OR $1.38 \% 95 \%$ CI 1.17-1.64), increase in calf circumference (OR 1.21, 95\% CI 1.02-1.43), having higher IADL score (OR 2.89, 95\% CI 1.36-6.13), having GDS score of 5 or higher (OR $11.0195 \%$ CI 1.18-86.25), having lower MMSE score of 24 (OR 29.45, 95\% CI 3.28-64.49), being smoker (OR 0.06, 95\% CI 0.04-0.83) and having CRP higher than 5 (OR $16.89,95 \%$ CI 2.65-27.86) were found to be variables showed changes for sarcopenic obesity with sensitivity and specificity of 95 and $93.3 \%$, respectively.

Conclusions: A large number of meaningful variables were put together and an effective demonstration of the model on the nomogram was made and an answer to a clinical question was found.

\section{P-181}

Frailty and mortality, hospital use and costs over 7 years in the Newcastle 85+ cohort

E. Keeble ${ }^{1}$, J. Neuburger ${ }^{1}$, S. Arora ${ }^{1}$, C. Jagger ${ }^{2}$, R. Duncan ${ }^{2}$, A. L. Robinson ${ }^{2}$, S. G. Parker ${ }^{2}$

${ }^{1}$ The Nuffield Trust, London, UK, ${ }^{2}$ Newcastle University, Newcastle upon Tyne, UK

Introduction: Frailty is a significant determinant of health care utilisation and costs, which also increase in proximity to end of life. The over $85 \mathrm{~s}$ are the fastest growing age group in many countries. We report relationships between frailty, proximity to death, hospital use and costs in the Newcastle 85+ cohort.

Methods: Data from the Newcastle $85+$ Study were linked with Hospital Episode Statistics and death registrations. Using both Fried (F) and Rockwood (R) definitions of frailty we analysed the relationship between frailty and mortality, hospital use and hospital costs over 7 years using descriptive statistics, Kaplan-Meier survival curves, Cox's proportional hazards and negative binomial regression models.

Results: Those who were frail at baseline had a 2.8 (F)-2.9 (R)-fold increased risk of mortality after two years $(2.3$ (F) to 2.7 (R) over 5 and 7 years) compared to people who were non-frail. They spent 2.2 (F) to $2.4(\mathrm{R})$ times more days in hospital over 2 years $(1.3(\mathrm{~F}, \mathrm{R})-1.6$ (R) after 5 and 7 years) with associated increase in costs (R). However, baseline frailty $(F, R)$ was not associated with Increased time spent in hospital during the last 90 days of life.

Conclusions: Members of this $85+$ cohort who were frail at baseline experienced higher mortality and hospital use over 2, 5 and 7 years, with some evidence of increased costs compared to those who were not frail at baseline. However, increased hospital use at end of life was more closely associated with proximity to death than with baseline frailty status.

\section{P-182}

Prevalence of osteo-sarcopenia and osteo-sarcopenia obesity in healthy ambulatory subjects older than 45 years in France

Lamberti Carla $^{1}$, Kruiskamp Saskia ${ }^{1}$, Cherin Patrick ${ }^{2}$, Voronska Elena ${ }^{3}$

${ }^{1}$ Institut de Medecine et Physiologie de la Longevite, Paris, France, ${ }^{2}$ Groupe Hospitalier Pitie Salpetriere, Paris, France, ${ }^{3}$ Institut de Medecine et Physiologie de la Longevite-IDJ, Paris, France

Introduction: This study aims to determine the prevalence of sarcopenia and osteopenia/osteoporosis among a population of 1508 healthy ambulatory subjects over 45 years living at home, in Paris (France). Methods This study was conducted selecting all ambulatory participants aged 45 years and older, consulting in the Institute of Physiology from Paris, for a functional, muscle and bone mineral density evaluation, and did not have limitations to moderate physical exercise. All were healthy people. Lombar, femoral bone mineral density and muscle body composition were measured with dual-energy X-ray absorptiometry. Skeletal muscle mass index and handgrip strength were used for sarcopenia diagnosis. Independent samples $t$ tests determined group differences in body composition and functional ability according to recommended diagnostic cut points.

Results: From 1409 participants definitively enrolled, prevalence of sarcopenia was $16.1 \%$ (135 females and 86 males) according to the EWGSOP definition. The prevalence of osteopenia in the cohort was $33.5 \%$ and osteoporosis was $17.9 \%$, according to the standard WHObased T-score criterion. From the age of 45 years, more than $10 \%$ of the sarcopenic population suffers from osteoporosis. Osteosarcopenia begins from 45 years and the risk increases with the advanced in age. Sarcopenia increased as BMD decreased as follows: normal BMD: $5.7 \%$, osteopenia $27.1 \%$, and osteoporosis $34.2 \%$ respectively. After adjustment for age and BMI, the adjusted odds ratio (OR) (95\% confidence interval) for sarcopenia was respectively 2.21 (1.36-4.28) for the osteopenic group, and $1.88(1.15-3.84)$ for the osteoporotic group ( $\mathrm{p}<0.05)$. Using the WHO definition, the percentage of sarcopenic obesity was $35.6 \%$ of our sarcopenic female population, and $33.7 \%$ of the sarcopenic males. In this sarcopenic obesity sub-population, $21.5 \%$ of women $(29 / 135)$ and $22.1 \%$ of men (19/86) were osteoporotic. Brought back in the whole studied population, sarcopenic obesity and osteosarcopenic obesity were observed in respectively 5.6 and $3.4 \%$ of women; and in 5.3 and $3.5 \%$ of men.

Conclusions: The present study suggests that among healthy ambulatory subjects living at home, osteosarcopenia is frequent and begin from 45 years. The prevalence of osteosarcopenic obesity is less frequent. Attention for sarcopenia are needed in subjects showing low BMD to prevent and manage poor quality of life and specific morbidity. 


\section{P-183}

Ultrasound and electromyographic assessment of the multifidus spinae muscle: reliability, function and relevance for sarcopenia

Marco Narici ${ }^{1}$, Tereza Jandova ${ }^{2}$, Elena Monti ${ }^{1}$, Michela Brogi ${ }^{3}$, Sara Tagliaferri ${ }^{4}$, Andrea Ticinesi ${ }^{5}$, Buttò Valeria ${ }^{4}$, Colizzi Elena ${ }^{5}$, Lauretani Fulvio $^{5}$, Maggio Marcello ${ }^{6}$

${ }^{1}$ Department of Biomedical Sciences, University of Padova, ${ }^{2}$ University `G. d'Annunzio of Chieti and Pescara, Department of Neuroscience \& Imaging, Chieti, Italy, ${ }^{3}$ School of Sports Medicine, Department of Medicine, University of Padova, Italy, ${ }^{4}$ Department of Medicine and Surgery, ${ }^{5}$ Department of Geriatric-Rehabilitation,

${ }^{6}$ Department of Clinical and Experimental Medicine, University Hospital, University of Parma, Parma, Italy

Introduction: The multifidus spinae (MF) is an essential muscle for preserving an erect posture and for rotating the spine. This muscle undergoes significant atrophy with prolonged bed rest [1] and ageing [2]. The aims of the present study were to, (1) quantify the activity of the MF muscle during basic activities of daily living (ADL), (2) evaluate the reliability of ultrasound measures of MF cross-sectional area (CSA) and, (3) establish the degree of MF sarcopenia in community-dwelling sarcopenic older individuals.

Methods: Ten young controls (YC) (5 male, 5 female, aged 20-35 years) and 10 older individuals (OI) (5 male, 5 female, aged $70 \geq$ years) sarcopenic according to the EWGSOP definition [3], were tested in this study. MF CSA was measured at L 5 by ultrasound using a 3-11 MHz linear probe. Surface electromyographic (EMG) activity of the MF was recorded in YC only during the 5-repetitions chair stand test (CST) and the $4 \mathrm{~m}$ gait speed test (GST).

Results: Activity of the MF, normalised to its maximum voluntary contraction EMG, was $45 \%$ during the CST and $21 \%$ during the GST. ICC of MF CSA measurements at L-5 was good to excellent (0.851-0.998). MF CSA of the older participants was found to be $33 \%$ smaller of the younger controls $(\mathrm{p}<0.01)$.

Key conclusions: Given the considerable activation of the MF during basic ADLs, sarcopenia of this muscle, particularly when exacerbated by disuse, may seriously impact on mobility and independence of older people. Sarcopenia of the MF muscle may be reliably assessed by ultrasound imaging.

\section{References:}

1. Belavý DL, Armbrecht G, Richardson CA, Felsenberg D, Hides JA (2011) Muscle atrophy and changes in spinal morphology: is the lumbar spine vulnerable after prolonged bed-rest? Spine (Phila Pa 1976) 36(2):137-145

2. Cuellar WA, Wilson A, Blizzard CL, Otahal P, Callisaya ML, Jones G, Hides JA, Winzenberg TM (2017) The assessment of abdominal and multifidus muscles and their role in physical function in older adults: a systematic review. Physiotherapy 103(1):21-39

3. Cruz-Jentoft A, Bayens JP, Bauer JM et al (2010) Sarcopenia: European consensus on definition and diagnosis. Age Ageing $39: 412-423$

\section{P-184}

How may B-mode sonography help to assess muscle aspects in an acute care setting

Heinrich Burkhardt ${ }^{1}$, Laura Parigger ${ }^{1}$

${ }^{1}$ Universitätsmedizin Mannheim-Geriatrisches Zentrum, Mannheim, Germany
Background: There is an increasing interest in the assessment of muscular aspects to detect and quantify both sarcopenia and consequences of deconditioning in acute care medicine. At present the possible role of B-mode sonography in this context is discussed but has still to be evaluated.

Methods: 84 inpatients (aged 65 and above) were included. They attended the geriatric ward due to a variety of clinical causes (e.g. pneumonia, fluid imbalance, heart failure). All received B-mode sonography measurements of the quadriceps femoris muscle and the gastrocnemius muscle. Measurement was standardized with regard to anatomical landmarks. Reliability oft the measurements was analyzed utilizing three repeated measurements (intra-observer). Also assessment of muscle strength of those muscles was included in the study protocol applying a hand-hold dynamometer and finally BIA was used to assess the presence of sarcopenia following current recommendations. Measurement of muscle strength and results of the BIA were subsequently utilized for validation analysis.

Results: 44 women and 40 men, age 65-96 years (78) were included. 34 underwent an early rehabilitation protocol due to significant deconditioning. Activities of daily living (Barthel) ranged 0-100 (85). Complete data could be retrieved from 80 patients (4 declined the ultrasound examination). Cronbach alpha for the congruence of the repeated measurements showed 0.99 for $\mathrm{m}$. quadriceps and 0.98 for m. gastrocnemius. Further positive correlations were found between muscle diameter, grip strength and skeletal muscle index derived from the BIA-data (0.29-0.49).

Discussion: B-mode sonography can be easily applied in an acute care setting and reveals sufficient reliability. Further the expected correlation to established markers of muscle performance could be demonstrated.

\section{P-185}

The orthogeriatric prevention service improves the adherence to treatments among functionally resilient older persons

Marika Ferracci ${ }^{1}$, Valentina Prenni ${ }^{1}$, Marta Baroni ${ }^{1}$, Luca Parretti ${ }^{1}$, Jacopo Penzo ${ }^{1}$, Valentina Bubba ${ }^{1}$, Clara Tinarelli ${ }^{1}$, Maurizio Massucci ${ }^{2}$, Auro Caraffa ${ }^{3}$, Patrizia Mecocci ${ }^{1}$, Giuseppe Rinonapoli ${ }^{3}$, Carmelinda Ruggiero ${ }^{1}$

${ }^{1}$ Geriatrics, S.M. della Misericordia Hospital, University of Perugia, Perugia, Italy, ${ }^{2}$ Orthopedic Rehabilitation, Media Valle del Tevere Hospital, USL 1 Umbria, Italy, ${ }^{3}$ Orthopaedics and Traumatology ward, S.M. della Misericordia Hospital, University of Perugia, Perugia, Italy

Introduction: Despite technological and organizational-procedural advances, femoral fractures cause high mortality, severe permanent disability, contributing to an increase in the consumption of social and health resources. The aim of this study is to evaluate the effectiveness of an orthogeriatric outpatient service in the prevention of refractures.

Materials and methods: Observational clinical study conducted on 762 over- $65 \mathrm{~s}$ with hip fracture outcomes discharged from the trauma and orthopedics department between February 2016 and February 2017. Of these 271 cases (patients received at the orthogeriatric surgery) and 283 are the controls (patients who they followed the usual route). Indicators of the outcome of the intervention are the percentage of patients who receive therapeutic indications aimed at preventing falls and re-fractures, those who adhere to these indications at follow-up and those who develop complications, including falls, re-fractures and use of social and health services.

Results: The cases are more adherent to the indications received in particular as regards calcium/vitamin D supplementation and antifracture therapy. Finally, in this context, the indication received in 
orthogeriatric surgery presents a better prescriptive appropriateness compared to controls.

Conclusions: The results of our study suggest that an orthogeriatric outpatient management is potentially able to respond to the multiple care needs of the elderly with a fracture of the femur by taking care of the person. It is necessary to validate this model of assistance and verify its effectiveness in antifriction terms in order to structure corporate assistance paths.

\section{P-186}

Impact of the rapid access clinic for the elderly in supporting older people with "frailty" in the community

Giles Rolph ${ }^{1}$

${ }^{1}$ Chelsea and Westminster NHS Foundation Trust, London, UK

Introduction: People aged $75+$ are at high risk of acute hospital admission becoming medicalised with worse clinical outcomes. To minimise these risks, our Acute Frail Elderly Team manages the Frailty syndromes delivering the CGA within the first $72 \mathrm{~h}$ of admission. Suitable patients are selected for supported early discharge and their clinical risk managed in the Rapid Access Clinic for the Elderly (RACE). RACE also receives referrals from other specialties and GPs. Pre-Frail and Frail patients are identified at RACE building Resilience.

Methods: Level of Frailty assessed with locally WMUH-Clinical Frailty Score (CFS) [1] and Rockwood-CFS [2]. Clinical outcomes measured: successful management of Frailty Syndromes; Polypharmacy review; Readmissions within 7 and 30 days compared to the Medical Ambulatory Emergency Clinic (AEC) used for younger patients, Referrals to other Services and Mortality.

Results and conclusions: - Majority of patients are $85+, 80 \%$ female.-WMUH-CFS identified $56 \%$ of the patients as having "Frailty".-Approximately 300 referrals for Diagnostics, Treatments and Resilience-building.-Medications changed in 49\% patients; GP to adjust drugs doses advised for $66 \%$ patients.-RACE readmissions rate of $6 \%$ equivalent to AEC.-Data analysis of RACE suggests it reduces mortality in patients attending the service.

References:

1. Martin-Marero et al (2017) In: 13th EUGMS international congress 20-22 September 2017, France.

2. Rockwood et al (2005) A global clinical measure of fitness and frailty in elderly people. CMAJ 173:489-495/Canadian Study on Health \& Aging. Reviewed 2008

\section{P-187}

Validation of SARC-F by proxy (SARC-F-Proxy)

for the screening of sarcopenia in elderly patients with dementia: a cross-sectional study

Zaid Kasim ${ }^{1}$, WD Rodríguez-García ${ }^{2}$, Stany Perkisas ${ }^{1}$, Maurits Vandewoude $^{1}$

${ }^{1}$ Department of Geriatric Medicine, University of Antwerp, Antwerp, Belgium, ${ }^{2}$ Regional Center for Higher Education, Autonomous University of Guerrero, Mexico

Introduction: The SARC-F is a validated questionnaire for screening for sarcopenia in older patients. However, it is challenging when using this questionnaire in patients with dementia. Medical caregivers or family can fill in (by proxy) the SARC-F questionnaire for these patients. The aim of this study was to validate the SARC-F-Proxy as a surrogate for the SARC-F in older patients with dementia.
Methods: This study included patients aged 60 years or older with various grades and types of dementia, who were admitted to the ZNA Joostens PsychoGeriatric Hospital. SARC-F-Proxy was completed by medical caregivers and family members. Sarcopenia was defined using the EWGSOP's diagnostic criteria. Calf circumference, hand grip strength and gait speed were measured and used as variables for muscle mass, strength and function respectively.

Results: This study included 174 patients, $59.2 \%$ female. Mean age of $83.3 \mathrm{yr}$ (SD 7.1). Sarcopenia was identified in 110 patients with muscle measurements while SARC-F-Proxy identified 77 patients as possibly sarcopenic. SARC-F-Proxy had $70 \%$ sensitivity and $15 \%$ specificity with a positive predictive value of $59 \%$ and negative predictive value of $23 \%$.

Conclusion: SARC-F-Proxy is better than SARC-F as and has a good enough sensitivity to be used as a screening tool for sarcopenia among patients with dementia.

\section{P-188}

Screening for frailty in the specialist oncology setting

$$
\text { C. Royles }{ }^{1} \text {, C. } \mathrm{Ng}^{1} \text {, G. Long }{ }^{1} \text {, L. Wentworth }{ }^{1}
$$

${ }^{1}$ Department of Geriatric Medicine, Manchester University NHS Foundation Trust, London, UK

Introduction: Half of cancer cases in the United Kingdom are diagnosed in the elderly; $12.6 \%$ of older people are living with cancer. This is predicted to rise to $23.7 \%$ by 2025 [1]. Advancement in cancer treatments means more older adults are undergoing therapy and this cohort is more likely to be frail. Frail patients are less likely to tolerate cancer treatments, but screening for frailty is not routinely performed in the oncology setting. Current practice is to evaluate patients for treatment based on the Eastern Cooperative Oncology Group (ECOG) Performance Status (PS) score; a tool not validated to assess for frailty. We looked at the correlation between PS and frailty screening tools.

Methods: We evaluated in-patients aged $>65$ years across two wards at a specialist oncology center. Data was collected from electronic records and by undertaking the G8 frailty tool questionnaire.

Results: 36 patients were included in the study (mean age 73 years). $83 \%$ of patients were frail. There was no significant correlation (Spearman's correlation $\mathrm{p}=0.177$ ) between age and G8 score. The area under the ROC curve of 0.39 comparing G8 with PS $<2$ (not frail) and PS $\geq 2$ (frail) suggests that PS does not reflect the burden of frailty detected by the G8.

Conclusion: Frailty is highly prevalent in older cancer patients. The ECOG PS score does not correlate to frailty. A more suitable frailty assessment tool should be used to identify those who are most at risk of treatment-related toxicity and cancer mortality.

\section{Reference:}

1. Kingston A, Robinson L, Booth H, Knapp M, Jagger C, MODEM Project (2018) Projections of multi-morbidity in the older population in England to 2035: estimates from the Population Ageing and Care Simulation (PACSim) model. Age Ageing 47(3):374-380

\section{P-189}

Determination of the relation between age-related hyperkyphosis and muscle mass and muscle function

Gülçimen Soylu ${ }^{1}$

${ }^{1}$ Gaziantep University, Faculty of Medicine, Department of Internal Medicine, Division of Geriatrics, Gaziantep, Turkey 
Aim: The prevalence of hyperkyphosis is rising among elderly individuals. In previous studies, it was associated with paraspinal muscle composition, spinal extensor muscle weakness, and impaired lower extremity physical function. The aim of this study is to determine the relationship between hyperkyphosis and structural and functional muscle deterioration due to aging.

Methods: The study included 142 participants who applied to our university's geriatric outstanding clinic from January 2017 to January 2018. The kyphosis grade was measured using the block method, where the participants were told to lie in a supine position on a radiology table. Blocks with thickness of $1.7 \mathrm{~cm}$ were placed under their heads to keep participants' heads in neutral position. The block of one and above was considered as hyperkyphosis.

Results: Seventy-one of our participants were hyperkyphotic while the other 71 were normal. The mean age of the patients was $72.1 \pm 6.90$. Thirty-six of the participants were male $(25.3 \%)$ and 106 were female (74.6\%). The sarcopenia parameters are given in Table 1 . The walking speeds of the patients were significantly lower in the hyperkyphotic group than normal group $(p<0.001)$. There was no significant difference between smooth muscle mass (SMM) and smooth muscle mass index (SMMI) in the two groups (p: 0.260 and p: 0.733). There was also no statistically significant difference in hand strength assessment (p: 0.157).

Conclusion: Exercise-based treatments are preliminary for hyperkyphosis; however, there is no standard treatment yet. In addition to muscle strengthening exercises, new treatment modalities are needed. These may be promising in posture-related progressive kyphosis by increasing muscle strength.

\section{P-190}

Mobility disorders and cognitive impairment predict disability and mortality in community-dwelling older adults

\section{Mika Okura ${ }^{1}$, Mihoko Ogita ${ }^{2}$, Hirenori Arai $^{3}$}

${ }^{1}$ Kyoto University Graduate School of Medicine, Kyoto, Japan,

${ }^{2}$ Shiga University of Medical Science, Shiga, Japan, ${ }^{3}$ National Center for Geriatrics and Gerontology, Obu, Japan

Aim: This study aimed to examine whether the combination of mobility disorders and cognitive impairment was associated with mortality and the new long-term care insurance (LTCI) service requirement need.

Methods: We analyzed cohort data for older adults in Kami town, Japan. The response rate to the self-reported questionnaire was $94.3 \%$ $(\mathrm{n}=5094)$. The outcomes were new certifications for the LTCI service requirement and mortality in 3 years. The mobility disorders and cognitive impairment were determined by the Kihon checklist.

Results: The prevalence of no-mobility disorders and no-cognitive impairment (GG), no-mobility disorders and cognitive impairment (GB), and mobility disorders no-cognitive impairments (BG), and cognitive impairments and mobility disorders (BB) were 46.8, 20.0, 18.3 and $14.9 \%$, respectively. During the 3 -year follow-up period, $5.2 \%(\mathrm{n}=262)$ died and $13.9 \%(\mathrm{n}=708)$ individuals were newly certified for the LTCI service requirement. As determined by multivariate analyses, GB (hazard ratio adjusted sex and age (HR): 2.1 [95\% CI 1.9-2.3]), BG (HR: 2.8 [2.6-3.1]) and BB (HR 4.3 [4.0-4.7]) had a significantly higher risk of needing LTCI services than GG. Similarly, GB (HR 1.2 [1.0-1.4]), BG (HR 1.9 [1.7-2.2]) and BB (HR 2.7 [2.4-3.0]) had a significantly higher risk of mortality than GG did. Conclusion: The presence of mobility disorders and cognitive impairment was related to mortality and new certifications for LTCI services, and an additive effect of these factors was found for both outcomes consistent with the notion of cognitive frailty.

\section{P-191}

Frailty: critical term in critical care

A Orozco ${ }^{1}, \mathrm{O}^{\text {Duems }}{ }^{1}$

${ }^{1}$ Hospital General de Granollers, Granollers, Spain

Introduction: Frailty scales applied to patient's $\geq 70$ years of age on admission in Critical Care Unit (CCU) can be helpful to evaluate clinical severity and its association with short and medium term mortality.

Methods: Retrospective study of patients with non-surgical pathologies, admitted to CCU, period January 2010-January 2017. Acute Physiology and Chronic Health Evaluation II (APACHE II) and Simplified Acute Physiology Score (SAPS II) were compared as well as frailty scales (Clinical Frailty Scale (CFS), Modified frailty Index (MFI) and Frail-VIG index ("VIG" = Spanish abbreviation for Comprehensive Geriatric Assessment) assessing its relationship with mortality (during admission, at 6 and 12 months).

Results: 478 patients (mean age $78.83 \pm 5.16,61 \%$ males), mostly from emergency department (48\%), mean severity at admission by APACHEII $18.19 \pm 8.05$ and by SAPSII $33.07 \pm 15.68$ respectively. $1 / 3$ of sample classified as fragile with variations (33.3\% MFI, $31.8 \%$ CFS and $37.2 \%$ Frail -VIG index). 200 patients died in study period (17.8\% in CCU, $11.1 \%$ during hospitalization, 7.1 and $5.9 \%$ at 6 and 12 months after hospital discharge). High scores of APACHEII, SAPII and CFS were correlated significantly with mortality in CCU [RR 2.55 (1.78-3.63), $3.03(1.81-5.06)$ and 1.35 (1.09-1.66) respectively], with mortality at 6 months in $\geq 80$ years high scores of CFS [RR 2.09 (1.09-4.00)] and with mortality per year only CFS [RR 2.47 (1.3-4.45)], without evidencing a relationship with any scale in hospital mortality.

Conclusions: Short-term associated mortality scores were (APACHE II, SAPSII) and only (CFS) was associated with long-term mortality.

\section{P-192}

Association between timed up and go test and future dependence onset

Ji Eun Lee ${ }^{1}$, Jong Lull Yoon ${ }^{2}$, In Soon Kwon ${ }^{3}$

${ }^{1}$ Department of Family Medicine, Seoul National University Hospital, Republic of Korea, ${ }^{2}$ Department of Family Medicine, Hallym

University Medical Center, Seoul, Republic of Korea, ${ }^{3}$ Department of Internal Medicine, Inje University Seoul Paik Hospital, Seoul, South Korea

Introduction: As society gets older, it is important to predict and prevent elderly dependence. This study examined the association between baseline timed up and go (TUG) test results and occurrence of future dependence.

Methods: Korean health insurance system provides long-term care insurance service to elderlies who show a certain level of dependence. The dependence level criteria includes activities of daily living, cognitive function, and functional limitation. The subjects of this study were those who applied for the long-term care insurance service for 2009-2013 period among those who performed TUG test in $2007-2008$ by national health examination for the 66 years old. This study analyzed the relationship between the baseline TUG test results $(\geq 15 \mathrm{~s})$ and whether or not to receive long-term care service, which means dependence occurrence. Cox proportional hazard model was used for the analysis.

Results: Total 1694 were included to the analysis. Among them, 335 showed functional limitation in baseline TUG test ( $\geq 15 \mathrm{~s})$. Limitation in baseline TUG test increased future dependence occurrence in 
multivariable model including sex, history of stroke, baseline cognitive function, depressive mood (adjusted Hazard Ratio 1.32, 95\% Confidence Interval 1.10-1.59).

Key conclusions: Functional limitation in TUG test increased risk of future dependence occurrence. Elderly people with limitations in the TUG test should be provided with more efforts to prevent future dependence.

\section{P-193}

Calf circumference as a simple screening marker for diagnosing sarcopenia in older Korean adults: the Korean frailty and aging cohort study (KFACS)

\section{Chang Won Won ${ }^{1}$}

${ }^{1}$ Kyung Hee University Medical Center, Seoul, South Korea

Background: The purpose of this study is to explore the optimal cutoff point of calf circumference (CC) as a simple proxy marker of appendicular skeletal muscle mass (ASM) and sarcopenia in the Korean elderly and to test the criterion-related validity of $\mathrm{CC}$ by analyzing its relationships with the physical function.

Methods: The participants were 657 adults aged $70-84$ years who had completed both dual energy X-ray absorptiometry (DXA) and physical function test in the first baseline year of the Korean Frailty and Aging Cohort Study (KFACS).

Results: ASM and SMI (skeletal muscle index) were correlated positively with CC (male, ASM, $r=0.55$ and SMI, $r=0.54$; female, ASM, $r=0.55$ and SMI, $r=0.42$; all $\mathrm{P}<0.001$ ). Testing the validity of $\mathrm{CC}$ as a proxy marker for low muscle mass, an area under the curve (AUC) of 0.81 for males and 0.72 for females were found and their optimal cut-off values of $\mathrm{CC}$ were $35 \mathrm{~cm}$ for males and $33 \mathrm{~cm}$ for females. In addition, CC-based low muscle groups were correlated with physical functions even after adjusting for age and BMI. Also, the cut-off value of CC for sarcopenia was $32 \mathrm{~cm}$. (AUC; male, 0.82 and female, 0.72)

Conclusion: The optimal cut-off values of CC for low MM were $35 \mathrm{~cm}$ for males, $33 \mathrm{~cm}$ for females and $32 \mathrm{~cm}$ for sarcopenia. Lower CC based on these cut-off values was related with poor physical function. $\mathrm{CC}$ may be also a good indicator of sarcopenia in Korean elderly.

\section{P-194}

Trajectory of frailty over 4 years in community-dwelling older Japanese adults: a prospective longitudinal study

\section{Mihoko Ogita ${ }^{1}$, Mika Okura $^{2}$, Hidenori Arai $^{3}$}

${ }^{1}$ Department of Clinical Nursing, Division of Geriatric Nursing, Shiga University of Medical Science, Otsu, Japan, ${ }^{2}$ Department of Human Health Sciences, Kyoto University Graduate School of Medicine, Kyoto, Japan, ${ }^{3}$ National Center for Geriatrics an

Introduction: The aim of this study was to examine the 4-year trajectory of frailty of older community-dwellers.

Methods: The data was collected by mail and field interview in 2013 and 2017. Questionnaires including the Kihon checklist (KCL) were distributed to 5401 older adults. The response rate was $94.3 \%$ (in 2013) and $81.5 \%$ (in 2017). At baseline, participants were divided into two groups according to the total scores of the Kihon checklist and the mobility disorder and cognitive impairment were defined based on mobility and cognitive domains of KCL. The participants who responded to the questionnaires at baseline and follow-up were classified into four groups according to the change of frailty status;
RR (robust to robust), RF (robust to frail), FR (frail to robust), FF (frail to frail), and the percentage was calculated in each age group. Results: The percentage of mobility disorder was $59.2 \%$ (RR), $13.5 \%$ (RF), $8.9 \%$ (FR), $18.4 \%$ (FF), whereas that of cognitive impairment was $52.4 \%, 17.3 \%, 13.1 \%, 17.2 \%$, respectively. Additionally, the percentage of deterioration in both motor and cognitive functions was higher in older groups. However, the percentage of improvement was not different among each age group.

Conclusions: Approximately 10-20\% of older people in the community were found to have mobility disorder or cognitive impairment. Considering the proportion of improvement in mobility and cognitive function, it is important to examine what factors are involved in the improvement.

\section{P-195}

Assessment of bone and muscle measurements by peripheral quantitative computed tomography in geriatric patients

Michael Drey ${ }^{1}$, Michaela Henkel ${ }^{1}$, Stefanie Wei ${ }^{1}$, Sophie Petermeise $^{1}$, Uta Ferarri ${ }^{1}$, Marietta Rottenkolber ${ }^{1}$, Ralf Schmidmaier ${ }^{1}$

${ }^{1}$ Medizinische Klinik und Poliklinik IV, Schwerpunkt Akutgeriatrie, Klinikum der Universität München (LMU), München, Germany

Introduction: The loss of bone and muscle mass increases the risk of osteoporotic fractures. Dual Energy X-ray Absorptiometry (DXA) loses sensitivity in older age. The purpose of this study was to evaluate bone and muscle measurements of peripheral quantitative computed tomography (pQCT) in a geriatric cohort with osteoporosis. Methods: Bone mineral density (BMD) and muscle area (MA) of 168 patients aged 65 years and older $(76.3 \pm 6.5)$ were measured with pQCT at distal forearm additionally to an osteoporosis basic assessment consisting of anamnesis, blood test and DXA of lumbar spine and hip. Prior fractures were categorized in major osteoporotic fractures. Logistic regression was used to show the association of BMD and MA with major fractures.

Results: $54.8 \%$ of the participants had at least one major fracture. Only BMD measured with pQCT and MA are significantly associated with these fractures (total and trabecular BMD OR 2.243 and 2.195, $\mathrm{p}<0.01$; MA OR 2.378, $\mathrm{p}<0.05$ ), whereas DXA BMD shows no significant association. These associations remain after adjustment for age, sex, BMI, physical activity and other factors. In all models for patients $>75$ years only MA was significantly associated (OR 5.354, $\mathrm{p}<0.05)$ with major fractures.

Conclusions: Measurement of BMD and MA with pQCT seems to have advantage over DXA in fracture association in geriatric patients. Measuring muscle area also adds useful information to estimate the presence of (osteo)sarcopenia.

\section{P-196}

Frailty evaluation with Kihon Checklist (KCL) for COPD patients at Frailty Prevention Clinic: a prospective case-control study over 6 years in National Center for Geriatrics and Gerontology, Japan

Kazuyoshi Senda ${ }^{1}$, Shosuke Satake ${ }^{1}$, Izumi Kondo ${ }^{1}$, Haruhiko Tokuda $^{1}$, Hidetoshi Endo ${ }^{1}$, Hisayuki Miura ${ }^{1}$, Yasumoto Matsui ${ }^{1}$

${ }^{1}$ National Center for Geriatrics and Gerontology, Obu, Japan

Background: COPD is a prevalent chronic systemic inflammatory disease with multi-comorbidities. The Kihon Checklist (KCL) is a self-administrated, comprehensive 25 items questionnaire for assessing frailty in Japan. 
Methods: Stable outpatients with COPD at Frailty Prevention Clinic (FPC), National Center for Geriatrics and Gerontology (NCGG), underwent a comprehensive geriatric assessment and followed from October 2010 to April 2018 by a registered nurse. The cohort consisted of 40 male and 3 female; age: $74.9 \pm 5.9(65-87)$ years, BMI: $21.7 \pm 3.2 \mathrm{~kg} / \mathrm{m}^{2}$, appendicular skeletal muscle index (ASMI): $6.61 \pm 0.64 \mathrm{~kg} / \mathrm{m}^{2}$, Charlson Comorbidity Index (CCI): $1.7 \pm 1.2$. Control group was age- and gender-matched outpatients with geriatric syndrome (including sleep apnea syndrome, DM, dementia, etc.) at NCGG, age: $75.1 \pm 5.8$ (66-87) years, BMI: $22.8 \pm 3.3 \mathrm{~kg} / \mathrm{m}^{2}$, ASMI: $6.54 \pm 2.00 \mathrm{~kg} / \mathrm{m}^{2}$, CCI: $1.7 \pm 1.4$.

Results: Initial KCL of COPD group was 5.2/25 \pm 4.4 (0-18). With frailty evaluation by KCL, 9 were classified as frail (F, KCL $\geq 8), 17$ as pre-frail $(7 \geq P \geq 4)$, and 17 as robust $(3 \geq R)$. As J-CHS criteria, $\mathrm{F} / \mathrm{P} / \mathrm{R}$ in COPD group were $5 / 27 / 11$. In control group, KCL was $6.1 \pm 5.4$ (5-18), and F/P/R were 16/10/17 with KCL, and 10/19/14 with J-CHS. Eight of fatal 13 COPD cases were P $(K C L \geq 4)$ and all 4 fatal cases in control were $\mathrm{KCL} \geq 9$.

Conclusion: COPD patients showed same level of frailty as geriatric syndrome outpatients at FPC, NCGG. Fatal case in this study showed higher KCL, which indicated possibility of KCL to evaluate not only physical function but prognosis for COPD and geriatric syndrome patients in integrated care settings by interdisciplinary team.

\section{P-197}

\section{Validation of the walkway "SPEED-AGE"}

Esther López-Giménez ${ }^{1}$, Mariano Esbrí-Víctor ${ }^{1}$, Luis-Carlos Venegas-Sanabria ${ }^{2}$, Günnar Andersen ${ }^{1}$, Héctor Caulin ${ }^{1}$, Silvia Lozoya-Moreno $^{1}$, Laura Plaza-Moreno ${ }^{1}$, Borja Gil-García ${ }^{1}$, Ana Pérez Fernández-Rius ${ }^{1}$, Melisa López-Utiel ${ }^{1}$, Pedro Abizanda ${ }^{1}$

${ }^{1}$ Geriatrics Department, Hospital Universitario Perpetuo Socorro, Albacete, Spain, ${ }^{2}$ Geriatrics Department, Hospital Universitario San Ignacio, Bogotá, Colombia

Objectives: Validate the "SPEED-AGE" walkway, a technological device whose objective is to measure the gait speed in the elderly in an automated way.

Methods: The "SPEED-AGE" walkway is composed of two proximity sensors (start and end) that record the gait speed data in the patient's medical history through a computer program. We analyze 90 patients from the Falls Clinic of the Geriatrics Service. Participants walked first in "SPEED-AGE" and later in GAITRite, and gait speed test of SPPB. The GAITRite protocol of the Falls Clinic includes two measurements at usual pace, one at speed fast, one slow and one with dual-task. For the validation we use the walk to usual pace and the average of these 5 measurements of GAITRite. The association between gait speed determinations was performed by correlation analysis of Pearson, the Bland-Altman method and finally t-test was used to paired samples to quantify the differences between methods. Results: 90 patients, mean age 78.8 years (SD:5.3), 67.8\% women. Average of falls in the last year: 1.9 (DE:2.2). The average gait speed in "SPEED-AGE":0.70 m/s (DE:0.22), in the SPPB:0.68 m/s (DE:0.23) and in GAITRite first: $0.77 \mathrm{~m} / \mathrm{s}$ (DE:0.23), second: $0.81 \mathrm{~m} / \mathrm{s}$ (DE:0.23) and average of 5 walks: $0.71 \mathrm{~m} / \mathrm{s}$ (DE:0.21). Pearson's correlation index between "SPEED-AGE" and SPPB, GAITRite second walks, was 0.822 (linear-r2: 0.7676, p < 0.001) and 0.824 (linear-r2: 0.688, $\mathrm{p}<0.001)$ respectively. Through Bland-Altman, only 3 measures were outside the range of 2DE when comparing "SPEED-AGE" with GAITRite and 4 with SPPB. The average difference between "SPEEDAGE" and SPPB was $0.014 \mathrm{~m} / \mathrm{s}(95 \%$ IC -0.013 to 0.043$)$ and with GAITRite -0.107 ( $95 \%$ CI -0.136 to 0.078 ).

Conclusion: The "SPEED-AGE" walkway is a method valid to measure gait speed in clinical setting.

\section{P-198}

\section{Benefits of a multicomponent training program in elderly} with falls

Mariano Esbrí-Víctor ${ }^{1}$, Esther López-Giménez ${ }^{1}$, Rafael GarcíaMolina $^{1}$, Marta Carolina Ruiz-Grao ${ }^{1}$, Alicia Noguerón-García ${ }^{1}$, Melisa López-Utiel ${ }^{1}$, Silvia Lozoya-Moreno ${ }^{1}$, Isabel HuedoRodenas ${ }^{1}$, Laura Plaza-Carmona ${ }^{1}$, Pedro Abizanda-Sol ${ }^{1}$

${ }^{1}$ Hospital Universitario Perpetuo Socorro, Albacete, Spain

Objectives: To analyze the effects of multicomponent training program in elderly patients with falls.

Methods: 50 patients older than 64 years were included from the Falls Unit of the Geriatrics Service. Participants presented at least one fall in the last year. The multicomponent training program was based on 4 months of intervention with 2 weekly sessions, including strength and muscle power exercises, stretching and balance. The SPPB, walking-speed, frailty, falls, FES-I, hand-grip, leg-strength, GDS-Yesavage and lean-mass by BIA were determined before and after the program. Six months after the training, participants were asked about the presence of falls. The effectiveness of the intervention was determined by comparing means for paired data and Chi squared when agreed.

Results: Mean age 77.2 (SD 5.8). 39 women. Participants reduced the number of frailty criteria from 2.1 to 1.3 (95\% CI $0.4-1.1)$, improved their SPPB score from 8.3 to 9.7 (95\% CI 0.8- 2.0), leg strength from $66 \mathrm{~kg}$ to $86 \mathrm{~kg}$ (95\% CI 14.9-25.6), power to $60 \%$ load from 84.7watts to 136.6 watts (95\% CI 39.2-64.6), the walking speed from $0.65 \mathrm{~m} / \mathrm{s}$ to $0.82 \mathrm{~m} / \mathrm{s}(95 \%$ CI $0.11-0.22)$ and the GDS score from 5.3 to 4.4 (95\% CI 0.1-1.7). The BMI did not change, while the fat-free mass increased from $43.7 \mathrm{~kg}$ to $44.2 \mathrm{~kg}(95 \% \mathrm{CI} 0.1-1.0)$, and the fatmass percentage decreased from $36.7 \%$ to $36.0 \%$ (95\% CI $0.1-1.4$ ). Seventeen patients had some fall in the next six months, with a decrease from 3.3/year to $0.6 / 6$ months (95\% CI 19-3.5). The adherence to exercise was $75 \%$.

Conclusions: A multicomponent training program in elderly people with falls, improves physical function, affectivity, body composition and reduces the number of falls.

\section{P-199}

Gait kinematic parameters analysis using an inertial sensor unit after 1 month of multicomponent exercise training (vivifrail) in frail/prefrail older adults with cognitive impairment: preliminary results of a randomized controlled trial

Mikel Lopez Sáez de Asteasu ${ }^{1,6}$, Ivan Anton Rodrigo ${ }^{2}$, Fabricio Zambom Ferraresi ${ }^{1,6}$, Jaione Elexpuru Estomba ${ }^{2}$, Itxaso Marin Epelde ${ }^{3}$, Maria Fernanda Ramon Espinoza ${ }^{3}$, Roberto Petidier Torregrosa ${ }^{4}$, Juan Luis Sanchéz Sanchéz ${ }^{4}$, Nicolas Martinez Velilla ${ }^{3,5,6}$, Andres Gamboa ${ }^{7}$, Mikel Izquierdo ${ }^{1,5,6}$, Alvaro Casas Herrero ${ }^{3,5,6}$

${ }^{1}$ Department of Health Sciences, Public University of Navarre, Pamplona, Spain, ${ }^{2}$ Matia Fundazioa. San Sebastían, Spain, ${ }^{3}$ Division of Geriatric Medicine, Complejo Hospitalario de Navarra, Pamplona, Spain, ${ }^{4}$ Division of Geriatric Medicine University Hospital of Getafe. Madrid, Spain, ${ }^{5}$ IdiSNa, Navarra Institute for Health Research Pamplona, Navarra, Spain, ${ }^{6}$ CIBER of Frailty and Healthy Aging, Madrid, Spain, ${ }^{7}$ Consorci Sanitari de l'Anoia. Hospital de Igualada, Cataluña, Spain

Introduction: Frail/prefrail older adults with cognitive impairment have reduced functional and physiological reserves, which increases the vulnerability of this population to clinically important outcomes 
including functional decline, falls, and institutionalization. Gait velocity has been reported as one of the strongest criteria to predict adverse outcomes and the most useful for the identification of physical frailty. Nevertheless, gait is a complex motor behavior with many measurable parameters besides velocity.

Methods: In a randomized controlled trial, 48 community-living older adults aged older than 75 years with mild cognitive impairment or mild dementia were randomized into an intervention group (IG, $\mathrm{n}=22)$ and a control group $(\mathrm{CG}, \mathrm{n}=26)$. The intervention consisted of a multicomponent exercise-training program based on the clinical physical exercise guide "Vivifrail". Evaluations of 5-m gait velocity test (GVT), verbal GVT and arithmetic GVT were performed at baseline and after 1 month of intervention in both groups. An inertial sensor unit (IU) was attached over lumbar spine (L3) to record the acceleration data.

Results: The IG showed enhancements in the normal GVT after the intervention period compared with the baseline value in the root mean square (RMS) (mean change of $1.5 ; 95 \%$ confidence interval (CI) $-0.1,0.2)$, whereas no change was found in CG $(-0.1 ;-0.3$, $-0.1)(\mathrm{p}<0.05)$. No significant differences were found between groups in other parameters.

Conclusions: A no supervised multicomponent exercise-training program improves some gait-related parameter, such as RMS, in frail/ prefrail community-dwelling older adults with mild cognitive impairment or mild dementia compared with the control group.

\section{P-200}

The cut-off points and prevalence of low hand grip strength for sarcopenia in korean elderly based on the 7th KNHANES

Kwang Min Kim ${ }^{1}$, Kyu Nam Kim ${ }^{1}$, Sae Mi Park ${ }^{1}$

${ }^{1}$ Ajou University School of Medicine, Suwon, Korea

Introduction: Hand Grip strength (HGS) is an important index of low muscle strength to diagnose sarcopenia. We aimed to assess age- and gender-specific values of the HGS based on the Korean National Health and Nutrition Examination Survey (KNHANES) 7th and identify cut-off points for low muscle strength of HGS of Korean elderly.

Methods: 5253 from the age of $20-80$ years were subjected to measurements of HGS, including 2489 men and 2764 women. HGS was evaluated in both the dominant and non-dominant arm using the Digital hand dynamometer. HGS measurements were repeated three times, respectively and the greatest value was recorded. The cut-off value for low HGS was defined as below 2 SD values of healthy young adults in this study population.

Results: The mean maximum HGS was increased from the age of 20-39 years. The peak in maximum HGS was in the age of 30-39 years range for both men and women. It was then decreased after 39 years. The cut-off values of low HGS for sarcopenia in male and female elderly populations were 29.6 and $16.5 \mathrm{~kg}$, respectively. The prevalence of low HGS for sarcopenia was $25.7 \%$ in elderly men and $17.3 \%$ in elderly women respectively, and significantly increased with aging.

Key conclusion: These data suggest useful reference values to assess HGS and may help in defining sarcopenia among the Korean population.

\section{P-201}

Sit-to-stand and protein supplementation in the OPEN Study-a surprisingly feasible and welcome health concept for nursing home residents

Sofia Vikström ${ }^{1}$, Helena Grönstedt ${ }^{2}$, Tommy Cederholm ${ }^{3}$, Erika Franzén ${ }^{4}$, Åke Seiger ${ }^{5}$, Anders Wimo ${ }^{6}$, Gerd Faxén ${ }^{7}$, Anne-Marie Boström $^{8}$

${ }^{1}$ Department of Neurobiology, Care Science and Society, Division of Occupational Therapy, Karolinska Institutet, ${ }^{2}$ Stockholms Sjukhem R\&D unit, Stockholm; Allied Health Professionals, Function Area Occupational Therapy \& Physiotherapy, Karolinska University Hospital, ${ }^{3}$ Department of Public Health and Caring Sciences, Clinical Nutrition and Metabolism, Uppsala University \& Department of Geriatric Medicine, Uppsala University Hospital, Uppsala \& Karolinska University Hospital, Theme Aging, Stockholm, ${ }^{4}$ Stockholms Sjukhem R\&D unit, Stockholm; Department of Neurobiology, Care Science and Society, Division of physiotherapy, Karolinska Institutet, Stockholm \& Allied Health Professionals, Function Area Occupational Therapy \& Physiotherapy, Karolinska University Hospital, Stockholm, ${ }^{5}$ Stockholms Sjukhem R\&D unit, Stockholm \& Department of Neurobiology, Care Science and Society, Division of Clinical geriatrics, Karolinska Institutet, Stockholm, ${ }^{6}$ Department of Neurobiology, Care Science and Society, Division of neurogeriatrics, Karolinska Institutet, ${ }^{7}$ Stockholms Sjukhem R\&D unit, Stockholm, Department of Neurobiology, Care science and Society, Division of clinical geriatrics, Karolinska Institutet, ${ }^{8}$ Stockholms Sjukhem R\&D unit, Stockholm; Department of Neurobiology, Care science and Society, Division of nursing, Karolinska Institutet and Karolinska University Hospital, Theme Aging, Stockholm, \& Western Norway University of Applied Sciences, Haugesund, Norway

Introduction: In line with person-centered care, active ageing should be recommended all older residents when possible, e.g. in nursing homes $(\mathrm{NH})$ meaningful activities such as physical activity should be offered regularly. In spite of the well-known health-related benefits of daily physical activity a majority of $\mathrm{NH}$ residents are inactive with limited social interaction. Following a cluster randomized health intervention trial (the Older People Exercise and Nutrition (OPEN) Study), where residents' were offered to conduct sessions of sit-tostand (STS) exercises together with oral nutritional supplement (ONS) individual interviews were conducted. The aim was to describe the residents' perceptions and experiences of the health intervention introduced.

Methods: In-depth interviews were done in eight $\mathrm{NH}$ facilities with 20 residents having performed the 12 week ONS/STS intervention. The transcribed interviews were analyzed inductively, following a constant comparative method described in Grounded theory-a reliable technique to form theory and hypothesis in un-exploited areas. Results: This exercise and nutritional intervention was described highly feasible by the NH residents. Furthermore, the analysis displayed experiences ranging from neutral to mainly positive; from existential feelings of hope and trust; to driving forces to fulfil the health concept. Also, the intervention trial was described as a welcome contribution to fill the day and were perceived easy to perform and integrate in daily activities.

Key conclusions: Health component interventions such as OPEN that bear hope of improvement, and contribute to a more meaningful day, are of interest to - and potentially beneficial for - $\mathrm{a}$ broader nursing home clientele. 


\section{P-202}

Diabetes and falls in the elderly

Francois Puisieux ${ }^{1}$

${ }^{1}$ CHRU Lille, Lille, France

Falling and diabetes are important public health issues associated with a risk of functional decline. Diabetic patients are known to have an increased risk of falling. Our study aimed to determine the prevalence of diabetes in the population of elderly patients seen at the multidisciplinary falling consultation at the University Hospital (CHRU) of Lille and to study the characteristics of falling diabetic patients. This is a monocentric retrospective study with prospective data collection. All patients seen between 1995 to 2011 were included. For data analysis for quantitative variables, we used the student test or the Pearson correlation test. For qualitative variables to analysis of variance according to a Fischer test. 1179 patients benefited from a multidisciplinary consultation of the fall between 1995 and 2011. Of the patients interviewed, 205 patients (17\%) were type 2 diabetics. Among the significant differences identified between groups: diabetic consultants were on average younger, had a lower level of education, a lower ADL, a higher body mass index. The fear of falling was more often expressed by diabetics than non-diabetics and was accompanied by a greater restriction of leaving their homes. Diabetic patients consumed significantly more drugs and the high polypharmacy was almost twice as common in diabetics as in non-diabetics. Our study suggests that diabetics experience falling earlier in life. Once identified the subject at high risk of falling, appropriate care must be offered to reduce the risk of falls. For this, we can, with benefit, appeal to the geriatrician, an expert in this area.

\section{P-203}

The multidimensional prognostic index predicts 90-day mortality in older medical patients admitted to an Emergency Department

\section{Merete Gregersen $^{1}$, Else Marie Damsgaard ${ }^{1}$ \\ ${ }^{1}$ Department of Geriatrics, Aarhus University Hospital, Aarhus, Denmark}

Background: Only a minor stress factor as well as an acute illness may result in increased vulnerability to sudden changes of health. A state of frailty may develop or deteriorate in hospitalized older person. This fragility may increase the risk of death. We wanted to examine whether the degree of frailty can predict 90-day mortality in older patients with medical diseases.

Methods: All patients aged 65 years or more, consecutively admitted to the emergency department (ED) due to acute illness or relapse of chronic disease, were included. Terminal patients were excluded. The 'Multidimensional Prognostic Index' (MPI) based on comprehensive geriatric assessment was used to identify the moderately and severely frail patients. The 90-day mortality of these was compared to patients who were assessed to be non-frail. A trained interdisciplinary geriatric team collected the data from face-to-face interviews, patient charts, admission reports from home care, and relatives.

Results: A total of 624 patients was included. Of these $23 \%$ were nonfrail, $33 \%$ moderately frail, $44 \%$ severely frail. More than half were women $(52 \%)$. Mean age was 84 years $( \pm 7.40)$. Both age and gender were associated with frailty. The risk of death increased proportionally with the degree of frailty. In the moderately frail, Hazard Ratio adjusted for age and gender, was $2.54(95 \%$ CI 1.04-6.23, $\mathrm{p}=0.04)$, in the severely frail 7.38 (95\% CI 3.20-17.0, p < 0.001).

Key conclusion: MPI can predict short-term mortality in older medical patients acutely admitted to an ED. The tool is useful to identify frail patients for targeting comprehensive geriatric interventions.

\section{P-204}

Prevalence of frailty in clinical and social settings. Frailtools study: preliminary results

Marta Checa ${ }^{1}$, Tania Guevara ${ }^{1}$, Jimmy Gonzales ${ }^{1}$, S.Soledad Álamo ${ }^{1}$, Myriam Oviedo $^{1}$, Armando Pardo ${ }^{1}$, J.A. Carnicero 1, Stefan Walter ${ }^{1}$, Saul Martin ${ }^{2}$, Agnieszka Parnicka ${ }^{3}$, Barbara Wizner ${ }^{3}$,

Barbara Gryglewska ${ }^{3}$, Tomasz Tomasz $\mathrm{Gr}^{3}$, L. Rodriguez-Mañas ${ }^{1}$, on behalf of the Frailtools Team

${ }^{1}$ FIB-Hospital Universitario de Getafe. Madrid, Spain, ${ }^{2}$ Cetro de Salud Luis Vives, Alcalá de Heneres, Spain, ${ }^{3}$ Jagiellonian University Medical College, Poland

Background: To evaluate the usefulness of frailty scales as frailty detection instruments in clinical and social settings is the main objective of the FRAILTOOLS project.

Method: Frailtools Project is a European study developed in five countries (Spain, United Kingdom, Poland, Italy and France) in different clinical and social settings: Outpatient and Geriatric ward, Nursing home and Primary care. Eligibility criteria: People $\geq 75$ years. Exclusion: MMSE $<20$, terminal illness, Barthel Index $<40$ in nursing homes and $<90$ in all other settings. Frailty was defined as $\geq 3$ in Fried, FRAIL scale and SHARE- FI. $\geq 4$ in the Clinical Frailty Scale (CFS), Rockwood-35 $>0.25 \%$, FTS $3>15$, Gérontopole Frailty Screening Tool (GFST): Yes.

Results: 654 subjects, $59.5 \%$ were female. Mean age was $82 \pm 5.3$ (sd). Barthel Index $93.7 \pm 12.2$, MMSE $26.3 \pm 3$, Charlson Index $5.6 \pm 2$. Prevalence of frailty by setting: Geriatric Ward: Fried: 45.1\%; Frail: 57.3\% ; CFS: 49.2\%, Rockwood -35: 64.1\%, SHAREFI 63.6\%; FTS-3: $41.3 \%$, GFST: $67.5 \%$. Total: $51.5 \%$. Nursing home: Fried: 53\%; Frail: 42.7\%; CFS: 69.6\%, Rockwood -35: 93.8\%, SHAREFI $45.4 \%$; FTS-3: 60.8\%, GFST: $74.5 \%$. Total: $60 \%$, Primary care: Fried: 19.9\%; Frail: 6\%; CFS: 15.3\%, Rockwood -35: 40.1\%, SHAREFI 11.2\%; FTS-3: 9\%, GFST: 30\%. Total: 17\% Outpatient: Fried: $30.6 \%$; Frail: 13\%; CFS: $23.7 \%$, Rockwood -35: $51.8 \%$, SHAREFI 16.1\%; FTS-3:19.6\%, GFST: $48.7 \%$. Total: $26.1 \%$.

Conclusion: The prevalence of frailty was different depending on the tool used. Frailty was very prevalent in the geriatric ward though they present a good functional and cognitive status.

\section{P-205}

The relationship between cognition and physical performance in non-demented older adults with sarcopenia

Anna Skalska ${ }^{1}$, Karolina Piotrowicz ${ }^{1}$, Tomasz Grodzicki ${ }^{1}$

${ }^{1}$ Department of Internal Medicine and Gerontology Jagiellonian University Medical College, Krakow, Poland

Background: There is growing body of evidence on relationship of cognitive and motor performance in older adults. The aim of this study was to assess the relationship of cognitive function and physical performance in older adults with sarcopenia.

Methods: In subjects $\geq 70$ years with sarcopenia identified by densitometry sociodemographic data and body mass index (BMI) were collected. Muscle mass was presented as appendicular muscle mass (aLM) and aLM/BMI. Cognition was assessed by Mini Mental State Examination (MMSE) and Trail Making Test A and B (TMTa/ TMTb), physical function by handgrip strength, daily and 7 days steps count, gait speed, $400 \mathrm{~m}$ walk test, short physical performance battery (SPPB). Functional status was assessed by Katz's Index (ADL) and Lawton's Instrumental Activities of Daily Living scale (IADL). SARC-F scale on screening sarcopenia and Charlson Index to assess multimorbidity were used. 
Results: Mean age of 68 subjects was $78 \pm 5.9$ years. Mean value of MMSE was $28.12 \pm 1.6$, ADL $5.82 \pm 0.46$, IADL $7.19 \pm 1.5$. MMSE correlated negatively with age $(p<0.0001)$, SARC-F $(\mathrm{p}=0.002)$, chair stand time $(\mathrm{p}=0.047), 400 \mathrm{~m}$ walk test $(\mathrm{p}<0.0001)$, Charlson index $(\mathrm{p}=0.005)$, and positively with handgrip strength $(p=0.01)$, gait speed $(p=0.002)$, SPPB $(p<0.0001)$, daily steps $(p=0.04)$ and 7 days step count $(p=0.02)$. In multiple linear regression analysis with MMSE as dependent variable adjusted for age, sex, handgrip strength, gait speed, IADL, daily steps, SARC$\mathrm{F}$, Charlson index significantly associated with cognition were sex $(p=0.009)$, gait speed $(p=0.046)$ and SARC-F $(p=0.0009)$.

Conclusions: The non-demented subjects with sarcopenia present a decline in cognitive function related to deterioration in physical performance.

\section{P-206}

Physical frailty and adverse drug events (ADEs) in the elderly inpatients

Akane Oyama ${ }^{1}$, Yasushi Takeya ${ }^{1}$, Tsuneo Nakajima ${ }^{1}$, Miyuki Takeya ${ }^{1}$, Shuko Takada ${ }^{2}$, Yoichi Takami ${ }^{1}$, Ken Sugimoto ${ }^{1}$, Koichi Yamamoto ${ }^{1}$, Hiromi Rakugi ${ }^{1}$

${ }^{1}$ Geriatric and General Medicine, Osaka University Graduate School of Medicine, Osaka, Japan, ${ }^{2}$ Department of Clinical Gene Therapy, Graduate School of Medicine, Osaka University, Osaka, Japan

Introduction: It is well known that the risk of adverse drug events (ADEs) are higher in the elderly and it was reported that ADEs occur 3 times higher in frail elderly people than non-frail older people. So that we need to take special care to prescribe medicines to the elderly patients. Our study investigated what kind of patient characteristics or what kind of prescription were related to the occurrence of ADE in elderly for reducing ADEs.

Methods and materials: A retrospective observational study was conducted between September 2014 to October 2016, included 260 consecutive elderly inpatients (mean age $77.3 \pm 6.6$, male $29.4 \%$ ) admitted to the department of Geriatric and Hypertension of Osaka University hospital. We examined frequency of ADE and the related factors with the occurrence of ADEs and relation between physical frailty and ADEs.

Results: 69 ADEs were identified. By multiplex logistic-regression analysis, emergency admission, the number of the drugs and the comorbidities increase, and the phenotype of frailty; weight loss $(>2 \mathrm{~kg})$ in the past 6 months were associated with the occurrence of ADEs.

Conclusion: Our results suggest the above three factors may be potential useful for identifying individuals at higher vulnerability to ADEs, and it might be better to distinguish those patients as "Pharmaco-frailty". Dose reduction and medication change may be considered based on organ impairment and not only patient's body weight but also body loss over those past few months, special management for the patients would be helpful for reducing ADEs in elderly patients.

\section{P-207}

Dietary patterns and their relationship with functional capacity

Mónica Machón 1,2,3,4, Maider Mateo-Abad ${ }^{1,3,4}$, Kalliopi Vrotsou ${ }^{5}$, Xabier Zupiria $^{1,6}$, Carolina Güell ${ }^{1}$, Leonor Rico ${ }^{5}$, Itziar Vergara ${ }^{1}$

${ }^{1}$ Unidad de Investigación de Atención Primaria-OSIs Gipuzkoa, Osakidetza, San Sebastián, Spain, ${ }^{2}$ Red de Investigación en Servicios de Salud en Enfermedades Crónicas (REDISSEC), Barakaldo, Spain, ${ }^{3}$ Instituto de Investigación Sanitaria Biodonostia, San Sebastián, Spain, ${ }^{4}$ Kronikgune-Centro de Investigación en Cronicidad, Barakaldo, Spain, ${ }^{5}$ Centro de Salud de Pasajes Antxo, Osakidetza, Pasajes, Spain, ${ }^{6}$ Centro de Salud de Alza, San Sebastián, Osakidetza, Spain

Introduction: The impact of dietary patterns rather than single foods or nutrients on health outcomes is increasingly recognized. The objective of this work was to describe the dietary patterns and their relationship with functional capacity in community-dwelling elder people.

Methods: This was a cross-sectional study of 527 non-institutionalized functionally independent older people aged $\geq 70$ years from Gipuzkoa (Spain). Functional capacity (by the Timed-Up and Go testTUGT) anthropometric measures, presence of malnutrition (by the Mini Nutritional Assessment) and dietary data (type of food, frequency and amount) were collected. First, a multiple correspondence analysis (MCA) was performed to identify dietary patterns, considering frequency of food intake and compliance with food recommendations. Second, a cluster analysis was applied, based on MCA results, to identify groups of similar individuals.

Results: The mean age was 76.2 (SD 5.2) years, 55\% were women, $48 \%$ obese and only $3.3 \%$ were at risk of malnutrition. A $24.6 \%$ had a limited functional capacity according to the TUGT. Three groups of individuals were identified in the cluster analysis: cluster $1(\mathrm{n}=285)$, cluster $2(n=194)$ and cluster $3(n=48)$. A gradient of decreasing functional capacity, poorer health status and worse dietary pattern (not complying with recommendations) was observed from cluster 1 to cluster 3 .

Key conclusions: An association between dietary patterns and functional capacity is observed. It is necessary to include nutritional screening and a subsequent dietary assessment in primary care to provide adequate nutritional care to elder population as an intervention to protect and improve their functional capacity.

\section{P-208}

Pulse pressure amplification as a new clinical and hemodynamic phenotype marker of vascular frailty: a new concept in the elderly

I. Bulubas ${ }^{1}$, L. Admant ${ }^{1}$, S. Gautier ${ }^{2}$, M. Kotsani ${ }^{2}$, MJ. Fourrière ${ }^{2}$, L. Mongars ${ }^{2}$, V. Bourdaire ${ }^{2}$, A. Bénétos ${ }^{1}$, L. Joly ${ }^{1}$

${ }^{1}$ Université de Lorraine, CHRU de Nancy, France, ${ }^{2} \mathrm{CHRU}$ de Nancy, France

Introduction: The aging process is known to be associated with an increase in arterial stiffness and frailty. Some studies have already shown that pulse pressure amplification (PPA) has an impact on cardiovascular mortality as criteria of arterial stiffness among older. The aim of the present analysis is to show that arterial stiffness might be an indicator of severe vascular frailty.

Methods: 56 patients were enrolled in a cross sectional study for a 1 year period in the day hospital of Nancy university hospital. Cardiovascular check up, pulse wave velocity (PWV) and PPA were assessed with an arterial tonometer. Data were collected about Fried frailty criteria from patient's medical history. Subjects were then later classified as not frail, prefrail (one or two criteria) and frail (more than 2 criteria).

Results: A total of 56 individuals older than 75 years (mean aged $83.02 \pm 4.85,48 \%$ of women) were analysed. Comparison according Spearman rank correlation with a risk of $5 \%$ showed a significant relationship between PPA and Fried frailty criteria $(\mathrm{P}<0.05$, $\mathrm{R}^{2}=0.42$ ). After adjustment for age, gender and mean arterial pressure this relationship persisted $\left(\mathrm{p}=0.05, \mathrm{R}^{2}=0.23\right)$. There was 
also a significant correlation between the frailty degree and Lawton instrumental activities of daily living scale $(\mathrm{P}=0.01)$.

Conclusion: There is a significant relationship between PPA and frailty degree. Pulse pressure amplification is a mechanical biomarker of "vascular frailty" which can be modified by the mean of pharmacological treatments. Nevertheless, a prospective multicentre study on a larger population is necessary.

\section{P-209}

\section{Determinants of physical function in community dwelling old adults}

Milan Chang ${ }^{1}$, Alfons Ramel ${ }^{2}$, Olof Gudny Geirsdottir ${ }^{2}$, Palmi V. Jonsson ${ }^{3}$, Inga Thorsdottir ${ }^{2}$

${ }^{1}$ Faculty of Health Promotion, Sport, and Leisure studies, University of Iceland, Reykjavik, Iceland, ${ }^{2}$ Faculty of Food Science and Nutrition, University of Iceland, Reykjavik, Iceland, ${ }^{3}$ Department of Geriatrics, National University Hospital of Ice, Reykjavik, Iceland

Poor physical function is associated with adverse health outcomes in older adults. Several variables, e.g., nutrition, physical activity (PA), body composition, hematological variables, medication and cognitive function have been associated with physical function among older adults. However, findings from studies have been inconsistent and it is still unclear which factors are independent predictors of physical function. We conducted a cross-sectional study including 236 community-dwelling old people (age range: 65-92 years, 58.2\% female) in Reykjavik, Iceland. Timed-up-and-go (TUG), 6-minutes-walk-for distance (6MWD), nutrition, PA, body composition, blood chemical variables, medication and cognitive function were assessed. We found bivariate correlations between physical function with body composition, strength, physical activity, number of medications, hematological variables, cognitive function, energy and protein intake. According to hierarchical linear models, body composition, strength, number of medications, PA and cognitive function were predictors of physical function but not hematological variables. Results were similar for both 6MWD and TUG and the strongest modifiable predictors in the final models were quadriceps strength/bodyweight $\quad(\mathrm{N} / \mathrm{kg}) \quad((+16.1 \mathrm{~m}, \quad \mathrm{P}=0.001 ; \quad-0.4 \mathrm{~s}$, $\mathrm{P}=0.001)$, physical activity (h/week) $(+2.2 \mathrm{~m}, \mathrm{P}=0.001 ;-0.04 \mathrm{~s}$, $\mathrm{P}=0.021)$ and number of medications $(-8.1 \mathrm{~m}, \mathrm{P}=0.003 ;+0.2 \mathrm{~s}$, $\mathrm{P}=0.021)$. Physical function decreases with age. However, there are modifiable determinants of physical function among communitydwelling older adults, in particular strength for a given body weight, PA and number of medications, which might give the possibility to maintain or improve physical function in this age group.

\section{P-210}

Different profiles of frailty among older and younger heart failure patients: preliminary data from the deus ex machina study

Rui Valdiviesso ${ }^{1}$, Emília Moreira ${ }^{1}$, Rosário Ataíde ${ }^{2}$, Sónia Martins ${ }^{3}$, Luís Filipe Azevedo ${ }^{4}$, Lia Fernandes ${ }^{5}$, José Silva Cardoso ${ }^{6}$, Nuno Borges ${ }^{7}$

${ }^{1}$ Centro de Investigação em Tecnologias e Serviços de Saúde, Faculdade de Medicina da Universidade do Porto, CINTESIS / FMUP, Portugal, ${ }^{2}$ Centro de Investigação em Tecnologias e Serviços de Saúde, Faculdade de Medicina da Universidade do Porto, CINTESIS / FMUP, Portugal; Centro Hospitalar do Porto - Hospital de São João, Portugal, ${ }^{3}$ Centro de Investigação em Tecnologias e Serviços de Saúde, Faculdade de Medicina da Universidade do Porto,
CINTESIS / FMUP, Portugal; Departamento de Neurociências Clínicas e Saúde Mental, Faculdade de Medicina da Universidade do Porto, DNC\&SM / FMUP, Portugal, ${ }^{4}$ Centro de Investigação em Tecnologias e Serviços de Saúde, Faculdade de Medicina da Universidade do Porto, CINTESIS / FMUP, Portugal; Departamento de Medicina da Comunidade, Informação e Decisão em Saúde, Faculdade de Medicina da Universidade do Porto, MEDCIDS / FMUP, Portugal, ${ }^{5}$ Centro de Investigação em Tecnologias e Serviços de Saúde, Faculdade de Medicina da Universidade do Porto, CINTESIS / FMUP, Portugal; Departamento de Neurociências Clínicas e Saúde Mental, Faculdade de Medicina da Universidade do Porto, DNC\&SM / FMUP, Portugal; Clínica de Psiquiatria e Saúde Mental do Centro Hospitalar do Porto - Hospital de São João, Portugal, ${ }^{6}$ Centro de Investigação em Tecnologias e Serviços de Saúde, Faculdade de Medicina da Universidade do Porto, CINTESIS / FMUP, Portugal; Departamento de Medicina, Faculdade de Medicina da Universidade do Porto, FMUP, Portugal; Serviço de Cardiologia, Centro Hospitalar do Porto - Hospital de São João, Portugal, ${ }^{7}$ Centro de Investigação em Tecnologias e Serviços de Saúde, Faculdade de Medicina da Universidade do Porto, CINTESIS / FMUP, Portugal; Faculdade de Ciências da Nutrição e Alimentação da Universidade do Porto, FCNAUP, Portugal

Introduction: Frailty is prevalent among heart failure (HF) patients. This work intends to describe the frailty profiles in younger and older HF patients.

Methods: Patients were recruited from an outpatient clinic in a University Hospital in Portugal. New York Heart Association (NYHA) HF classes were registered. Frailty was assessed using Fried phenotype. Mid-arm muscular circumference (MAMC) was estimated from mid-acromial-radial circumference and triceps skinfold.

Results: Overall 86 patients were included: age $57 \pm 13$ (24-81 years), 66\% men, 34\% older than 65 years. Regarding NYHA class, 30,53 and $17 \%$ were respectively at class I, II and III. Overall, $58 \%$ were pre-frail and $14 \%$ frail. Frailty was more frequent among NYHA III patients $(\mathrm{p}<0.001)$ and in men at the lowest MAMC tertile $(\mathrm{p}<0.001)$. While frailty was more prevalent at older ages $(58 \%, \mathrm{p}<0.05), 54 \%$ and $9 \%$ of younger patients were pre-frail and frail, respectively. Exhaustion (37\%), low physical activity (35\%) and weakness $(35 \%)$ were the most frequent overall criteria (and for those aged $<65$ ), whereas among older patients, weakness was the most determinant criterion $(62 \%$ vs. $21 \%, \mathrm{p}<0.001)$. NYHA classification was not associated with age.

Conclusions: As expected, frailty in HF patients is associated with older age and more severe NYHA class. Nevertheless, a considerable proportion of younger patients are frail/pre-frail and this syndrome shows different profiles between ages.

Acknowledgements: Supported by ERDF through POCI-01-0145FEDER-007746, funded by COMPETE2020, National Funds through FCT, within CINTESIS (UID/IC/4255/2013), and Project "NORTE01-0145-FEDER-000026- Symbiotic technology for societal efficiency gains: Deus ex Machina", financed by NORTE2020 under PORTUGAL2020.

\section{P-211}

\section{Frailty in EHPAD in Tunisia}

Dorsaf Abbes ${ }^{1}$, Olfa Hizem ${ }^{1}$, Saoussen zrour $^{2}$

${ }^{1}$ CHU Monastir, Monastir, Tunisia, ${ }^{2}$ Department of Rheumatology, Monastir, Tunisia

Introduction: Fragility is a reversible geriatric clinical syndrome. The objective of this study was to assess the prevalence of frailty of elderly residents living with long-term care, to identify associated 
factors and to propose effective interventions to prevent the loss of autonomy.

Materials and methods: This is a descriptive and analytical study in January 2017 in an EHPAD in Tunisia. Fragility data were collected using a pre-established questionnaire on resident social data, the EGS using screening scales, and the frailty level by the SEGA-A score. According to this score, patients were classified into 2 groups according to their degree of fragility: not fragile, versus fragile and very fragile.

Results: 53 residents were included with an average age of $77.42 \pm 8.3$ years. The average SEGA score was $9.36 \pm 4.17$. The prevalence of frailty in this EHPAD was $52.8 \%$ for fragile and very fragile vs $47.2 \%$ non-fragile. Fragility was higher among women. The modifiable factors associated with frailty, in our study, as in the literature, were poly-pathology, malnutrition according to the MNA score, depression, loss of autonomy, high risk of falling and dementia. In stepwise multivariable regression analysis, the presence of polypathology, dementia and undernutrition were independently associated with hypertension.

Conclusion: The prevalence of frailty in EHPAD is important. These associated factors present a vicious circle which should lead to screening to set up a personalized plan of care and avoid loss of autonomy.

\section{P-212}

The evaluation of daily functioning as part of the operational definition of frailty: a systematic review

Axelle Costenoble ${ }^{1}$, Veerle Knoop ${ }^{1}$, Aziz Debain ${ }^{1}$, Sofie Vermeiren ${ }^{1}$, Roberta Azzopardi Vella ${ }^{1}$, Ivan Bautmans ${ }^{2}$, Ellen Gorus ${ }^{2}$, Dominique Verté ${ }^{3}$, Mirko Petrovic ${ }^{4}$, Ingo Beyer ${ }^{5}$,

Patricia De Vriendt ${ }^{6}$, on behalf of the Gerontopole Brussels Study Group

${ }^{1}$ Frailty in Ageing Research Group and Gerontology Department, Vrije Universiteit Brussel, Belgium, ${ }^{2}$ Frailty in Ageing Research Group and Gerontology Department, Vrije Universiteit Brussel, Belgium \& Geriatrics Department, Universitair Ziekenhuis Brussel, Belgium, ${ }^{3}$ Frailty in Ageing Research Group and Gerontology Department, Vrije Universiteit Brussel, Belgium \& Belgian Ageing Studies Research Group, Vrije Universiteit Brussel, Belgium, ${ }^{4}$ ReFrail, UGhent, Belgium, ${ }^{5}$ Geriatrics Department, Universitair Ziekenhuis Brussel, Belgium, ${ }^{6}$ Frailty in Ageing Research Group and Gerontology Department, Vrije Universiteit Brussel, Belgium \& Artevelde Hogeschool, Ghent, Belgium

Background: The population is ageing, not all older adults are healthy and vital, a considerable part of them are frail. Disability in activities of daily living (ADL) can play multiple roles in frailty. Definitional confusion arises between frailty and disability due to the similarity of associated outcomes and the frequency of co-occurrence between the two. This systematic review aims to identify which frailty instruments incorporate ADL.

Method: A systematic search was performed in PubMed, Web of Knowledge and PsycINFO with the terms ("Aged"[Mesh] OR "Frail Elderly"[Mesh] OR “Aged, 80 and over"[Mesh]) AND Frailty AND ("Diagnosis"[Mesh] OR "Risk Assessment"[Mesh] OR "Classification"[Mesh]). The identified instruments were analysed and categorised in basic- (b-), instrumental- (i-) and advanced- (a-) ADL. Results: In total 144 articles described 149 frailty instruments. A distinction can be made between a physical $(n=47)$, multidomain $(\mathrm{n}=98)$, comorbidity $(\mathrm{n}=1)$, and biomarker model $(\mathrm{n}=3)$. At least one ADL was present in $77.9 \%$ of the instruments: $71.1 \%$ concerned b-ADL, $55.3 \%$ i-ADL and $33.6 \%$ a-ADL. Not all included ADL have the same aim, e.g. measuring physical activity, weakness, slowness etc. If these were excluded, the results would decrease to $53.0 \%, 50.3$, $36.2 \%$, and $10.1 \%$ respectively.

Conclusion: ADL is present in the assessment tools. Circular reasoning can occur as disability in ADL can be seen as a predictor, a characteristic, or an outcome of frailty, but also as a predictor of negative health outcomes in the frail. Also, the meaningful concept of the included ADL across various instruments can be interpreted differently.

\section{P-213}

Peculiarities of sarcopenia measured with bioimpedance in patients with diabetes mellitus type 2 in hospital settings

\section{Yulia Onuchina $^{1,2}$, Irina Gurieva ${ }^{1}$, Inna Begma ${ }^{1}$}

${ }^{1}$ Federal Bureau of Medical and Social Expertise. Department of Endocrinology, Moscow, Russian Federation, ${ }^{2}$ Pirogov Russian National Medical University, The Russian Clinical Research Center for Gerontology, Moscow, Russian Federation

Introduction: Persons who have diabetes mellitus tends to have accelerated aging process that places patients at greater risk for sarcopenia at earlier age. Purpose of this crossectional study was to describe features of sarcopenia and evaluate body composition with bioimpedance system in patients with type 2 diabetes mellitus (DM2) over 60 years old in hospital settings.

Methods: The study included 76 DM2 women at age of 72 [67; 77] years. Patients were examined with evaluation of muscle mass, muscle strength and muscle function. Muscle strength was assessed using carpal dynamometer. The muscle function was examined with special clinical tests constituting Short Physical Performance Battery (SPPB). Body composition was evaluated with bioimpedance analyzer with subsequent calculation of skeletal muscle mass index (SMMI $\left.\left(\mathrm{kg} / \mathrm{m}^{2}\right)\right)$. Patients with decrease of skeletal muscle mass index and/or muscle strength and/or muscle function were diagnosed as sarcopenic - « S+» group. Peripheral neuropathy was studied with calculation of NDS $(\max$ points $=8$ ) and NIS-LL scales ( $\max$ points $=96$ ).

Results: According the results of the examination patients were divided into 2 groups: the $\mathrm{S}+$ group $(\mathrm{n}=29)$ was with sarcopenia and the $\mathrm{S}-$ group $(\mathrm{n}=47)$ was with its absence. $\mathrm{S}+$ patients demonstrated higher points of SARC-F than $\mathrm{S}-(2[2 ; 4]$ vs $1[0 ; 3]$ points, $\mathrm{p}=0.048)$. Patients $\mathrm{S}+$ had smaller BMI vs $\mathrm{S}-(25.2$ [23.4; 27.9$]$ vs $\left.31.6[29.7 ; 34.25] \mathrm{kg} / \mathrm{m}^{2}, \mathrm{p}<0.001\right)$, smaller circumference of waist vs $\mathrm{S}-(94[87 ; 99]$ vs $104[100.5 ; 111] \mathrm{cm}, \mathrm{p}<0.001)$; smaller circumference of neck vs $\mathrm{S}-(37[34 ; 38]$ vs $39[37 ; 41] \mathrm{cm}, \mathrm{p}<0.001)$. Points of SPPB were lower in $\mathrm{S}+$ than in $\mathrm{S}-(9[8 ; 10]$ vs $10[9,11]$, $\mathrm{p}=0.006)$. Walking speed at $4 \mathrm{~m}$ distance was lower in group $\mathrm{S}+$ then in group $\mathrm{S}-(0.76[0.67 ; 0.8]$ vs $1[0.8 ; 1.33], \mathrm{p}<0.001)$. Muscle strength didn't differ between groups $\mathrm{S}+\mathrm{vs} \mathrm{S}-(15[10 ; 19]$ $\mathrm{kg}$ vs $15[12.5 ; 20] \mathrm{kg}, \mathrm{p}=0.09)$. According bioimpedance measurement, patients with sarcopenia characterized with more pronounced decrease of skeletal muscle mass vs $S-(14.3$ [13.5; 16.2] vs $18.5[17.1 ; 19.9] \mathrm{kg}, \mathrm{p}<0.001)$; decrease of fat mass vs $\mathrm{S}-(21.6$ $[19.3 ; 24.7]$ vs $31.5[27.4 ; 38.1] \mathrm{kg}, \mathrm{p}=0.007)$; decrease of mineral mass of bones vs $\mathrm{S}-(1.77[1.63 ; 1.85]$ vs $2.15[2.04 ; 2.3] \mathrm{kg}$, $\mathrm{p}<0.001$ ). Diabetic neuropathy (NDSm, NIS-LL) was severe in S+ group (NDSm: 5[3;6] vs $\mathrm{S}-$ group 2 [2; 3], p = 0.000; NISLL: 12 [8; $16]$ vs $S-$ group 6 [4.8], p < 0.001). S+ patients noted more often frequency of falls $(66 \%)$ and fractures $(72 \%)$ in comparison with $\mathrm{S}-$ group $(36 \%, \mathrm{p}=0.002)$ and $(38 \%, \mathrm{p}=0.004)$. Patients in $\mathrm{S}+$ group received metformin less frequently $(52 \%)$ vs $\mathrm{S}-$ group $(77 \%)$ $(\mathrm{p}=0.01)$. 
Conclusion: Sarcopenia revealed in $38 \%$ of women with DM2. Sarcopenia was more often detected in patients with more severe peripheral neuropathy. Combination of severe stage of neuropathy with decreased muscle mass in $\mathrm{S}+$ increases the risk of falls and fractures. S+ patients demonstrated decrease of skeletal muscle mass and fat mass. S+ patients characterized with more severe changes in the body composition not only in the skeletal muss, but also in the amount of fat mass and bone mineral density.

\section{P-214}

\section{The screening of frailty syndrome in different settings-the} FRAILTOOLS study background

Anna Kańtoch ${ }^{1}$, Barbara Gryglewska ${ }^{1}$, Tomasz Grodzicki ${ }^{1}$, Agnieszka Parnicka ${ }^{1}$, Barbara Wizner ${ }^{1}$, Marta Checa ${ }^{2}$, Leocadio Rodriguez-Mañas ${ }^{2,3}$, on behalf of Frailtools Team

${ }^{1}$ Department of Internal Medicine and Gerontology, Faculty of Medicine, Jagiellonian University Medical College, Poland,

${ }^{2}$ Fundación para la Investigación Biomédica del Hospital

Universitario de Getafe, Spain, ${ }^{3}$ Servicio Madrileño de Salud, Spain

Introduction: The frailty is a common geriatric syndrome but still there is a discussion how it should be measured in clinical and social settings. The FRAILTOOLS project was designed to evaluate the usefulness of selected frailty scales and establish which scale has the highest predictive value. Moreover, is to build frailty detection algorithms. In the context of FRAILTOOLS study arise this abstract. The main objective of this abstract is to review academic articles to determine which instruments are preferred by clinicians and researchers to detect frailty in different settings in the last decade.

Methods: Keywords (frailty, frailty assessment tools, frailty assessment instruments, frailty screening) were extracted from articles $(\mathrm{n}=147500)$ from different disciplines, retrieved from Google Scholar, from 2008 to May 2018. Articles that have been doubled have been excluded from the analysis.

Results: At Google Scholar keywords: 'frailty' appeared 76000 times; 'frailty assessment tools'-25900, 'frailty assessment instruments'-17700 and 'frailty screening'-22800. The most common used scales were: Fried's criteria $(n=57700)$, FRAIL scale (41 400), Clinical Frailty Scale (17600), Frailty Trait Scale (17400), 35-Items Rockwood Frailty Index (8660), SHARE-FI (518) and The Gérontopôle Frailty Screening Tool (423).

Conclusion: The review of the Google Scholar database revealed that screening of frailty syndrome is not unified and at least 7 different scales are widely used to detect frailty in different settings and countries. Many studies have demonstrated the utility of certain assessment tools to evaluate frailty in populations, however the individual risk for disability has not been properly evaluated, which is the main interest in the FRAILTOOLS project. This abstract is part of the project "662887 / FRAILTOOLS" which has received funding from European Union's Health Programme (2014-2020).

\section{P-215}

Profiling trends in clinical frailty scale, serum vitamin D levels and bone turnover markers: a prospective cohort of frail older adults in the West of Ireland

S. Gorey ${ }^{1}$, T. P. Griffin ${ }^{2}$, R. Murphy ${ }^{1}$, E. Mannion ${ }^{1}$, E. Loughlin ${ }^{3}$, P. $\mathrm{O}^{\prime} \mathrm{Shea}^{4}$, E. Mulkerrin ${ }^{1}$, S. Robinson ${ }^{1}$

${ }^{1}$ Department of Geriatric and Stroke Medicine, GUH, Galway, Ireland, ${ }^{2}$ Centre for Endocrinology Diabetes and Metabolism, GUH,
Galway, Ireland, ${ }^{3}$ Department of Geriatric and Stroke Medicine, GUH, Galway Ireland, ${ }^{4}$ Department of Clinical Biochemistry, Galway Ireland

Background: Frail older adults are at 3.6 fold increased risk of falls; appropriate bone protection and monitoring strategies are a priority. Methods: Consecutive frail older adults were included. Each underwent comprehensive geriatric assessment and measurement of serum vitamin D, calcium, PTH, CTX-1, P1NP. Vitamin D deficiency was defined as $<50 \mathrm{nmol} / \mathrm{L}$. Correlation was sought between clinical frailty scale (CFS), serum vitamin D, vitamin D supplementation (VDS), bone protection medication (BPM) and bone turnover markers (BTM). Research ethics approved.

Results: We assessed 130 patients: average age 84 years, average CFS 5.5. BPM or VDS were not prescribed in 67 (52\%). Of BPM prescribed: bisphosphonates in $17(13 \%)$, denosumab in 9 (7\%), VDS in $37(28 \%)$, combination BPM/VDS in $14(10 \%)$. Vitamin D deficiency was present in $38 \%$. Vitamin D levels in those not taking BPM or VDS were lower than those who were $(40.6 \mathrm{nmol} / \mathrm{L}$ vs $81.8 \mathrm{nmol} /$ $\mathrm{L}, 95 \%$ CI -51.99 to $-30.41, \mathrm{p}<0.0001)$. Those with CFS score $>5$ were more likely to be vitamin D deficient than CFS $<5$ $(30.1 \%$ vs $9.1 \%$ p $<0.0063)$. VDS was associated with lower CTX-1 and higher vitamin D. Similarly, CTX-1 levels were lower in those on BPM compared with those who were not.

Conclusion: We demonstrate a high incidence of vitamin D deficiency in this frail cohort. We found VDS and BPM suppress CTX-1 and P1NP. Vitamin D and BTM appear to correlate with CFS. Further study is needed to assess if BTM apply clinically to monitor treatment effect and potentially as a biomarker for frailty.

\section{P-216}

\section{Relationship between frailty and inflammation}

Rana Tuna Dogrul ${ }^{1}$, Hacer Doğan Varan ${ }^{1}$, Muhammet Cemal Kizilarslanoglu ${ }^{1}$, Mustafa Kemal Kilic ${ }^{1}$, Fatih Sumer ${ }^{1}$, Gunes Arik ${ }^{1}$, Ozgur Kara $^{1}$, Meltem Halil ${ }^{1}$, Mustafa Cankurtaran ${ }^{1}$, Burcu Balam Yavuz $^{1}$

${ }^{1}$ Hacettepe University, Faculty of Medicine, Department of Internal Medicine, Division of Geriatrics, Ankara, Turkey

Background: It has been suggested that inflammation plays a role in the pathogenesis of frailty and many studies have been carried out to understand the underlying mechanism until now. In this study, the relationship between frailty and inflammation was examined.

Methods: 817 patients over 65 years of age who were referred to our clinic were included in the study. Comprehensive geriatric assessment was performed to all patients and Freid Criteria were used to assess physical frailty. Neutrophil count, lymphocyte count, erythrocyte sedimentation rate (ESR), C-reactive protein (CRP), albumin, CRP/ albumin ratio (CAR), and neutrophil/lymphocyte ratio (NLR) were used as inflammatory markers.

Results: The median age of the patients was 73 years (min-max: 65-94), 61.9\% was female (n: 506) and $10.8 \%$ was frail (n:88). Median CRP was $0.41 \mathrm{mg} / \mathrm{L}$ (min-max: $0.10-2.62$ ) in the non-frail group and $0.49 \mathrm{mg} / \mathrm{L}$ (min-max: $0.10-7.67$ ) in the frail group (p: $0.167)$. The CAR was higher in the frail group but this correlation was not significant (p: 0.07). The median NLR was 2.17 (min-max: $0.21-10.17$ ) in the non-frail group and 2.41 (min-max: 0.62-18.20) in the frail group and the difference between the two groups was significant (p: 0.014). ESR was significantly higher in the frail group $(\mathrm{p}<0.001)$. In multivariate analysis, when models with independently related factors created, it was found that ESR was related to frailty with statistical significance (OR: 1.026, \%95 CI: 1.005-1.047, p: 0.015). 
Conclusion: It has been shown that there may be a relationship between frailty and inflammation in our study.

\section{P-217}

\section{Validation of frail scale in Turkish older adults}

Benazir Hymabaccus ${ }^{1}$, Burcu Balam Yavuz ${ }^{1}$, Rana Tuna Dogrul ${ }^{1}$, Cafer Balci ${ }^{1}$, Cemile Ozsurekci ${ }^{1}$, Hatice Caliskan ${ }^{1}$, Fatih Sumer ${ }^{1}$, Erdem Karabulut $^{2}$, Meltem Halil ${ }^{1}$, Mustafa Cankurtaran ${ }^{1}$

${ }^{1}$ Hacettepe University Geriatric Medicine, Ankara, Turkey, ${ }^{2}$ Hacettepe University Biostatistics, Ankara, Turkey

Background and aim: Frailty is a geriatric syndrome characterized by decreased physical activity, decreased gait speed, weight loss, muscle power loss which are caused by cumulative decline in many physiological systems. Frail older adults suffer from increased mortality, morbidity and health expenditures when exposed to stress factors. In order to determine the frailty in patients, practical, validated and reliable scales are needed. The purpose of this study is to determine whether the FRAIL scale is an effective screening tool of frailty in the Turkish geriatric population.

Method: FRAIL scale, Fried index, FIND scale, comprehensive geriatric assessment, Time up and go test, and handgrip dynamometer were performed on 85 outpatients aged 65 years and older without acute diseases or severe dementia. Scale was translated into Turkish by forward-backward method. Construct validity, test-retest reliability, inter-rater reliability and correlation between scales were performed.

Results: FRAIL scale was strongly correlated to FIND scale and Fried index (correlation coefficient of 0.956 and 0.934 respectively). It was also found to be related to ADL (activities of daily living), IADL (Instrumental activities of daily living), MMSE, Yesavage geriatric depression scale, MNA-short form, Clock-drawing test, handgrip grip strength, and Time up and go test $(\mathrm{p}<0.05)$. Interrater and test-retest compliance were $100 \%$ (full compliance, Cronbach alpha coefficient 1.00).

Conclusion: In this study, the FRAIL scale has been proven to be a reliable and valid screening tool in the assessment of frailty in Turkish older adults.

\section{P-218}

The pain patterns in frail sarcopenic and osteosarcopenic subjects

Vincenza Valentina Angileri ${ }^{1}$, Yari Longobucco ${ }^{1}$, Fulvio Lauretani ${ }^{2}$, Elisa Adorni ${ }^{1}$, Chiara Benedetti ${ }^{1}$, Michele Pessina ${ }^{1}$, Sara Tagliaferri ${ }^{1}$, Marcello Giuseppe Maggio ${ }^{3}$

${ }^{1}$ Department of Medicine and Surgery, University of Parma, Parma, Italy, ${ }^{2}$ Department of Medicine and Surgery, University of Parma, Parma, Italy, ${ }^{3}$ Department of Geriatric Rehabilitation, Internal Medicine and Critical Subacute Care Unit, Cognitive and Motoric Ambula, Parma, Italy

Background: Increasing age is associated with a parallel increase of geriatric syndromes such as frailty, a condition of decreased functional reserve that precedes disability, and can be associated with sarcopenia and osteosarcopenia (combination of osteoporosis and sarcopenia). Daily pain is prevalent among frail subjects but its role in sarcopenia and osteosarcopenia has not been fully investigated.

Methods: We enrolled 70 subjects community-dwellers aged $\geq 70$ (40 sarcopenics, 30 osteosarcopenics). Sarcopenia was determined by DXA (FNIH criteria); osteosarcopenia was defined as the presence of sarcopenia and osteoporosis (T-Score $\leq-2.5$ ). To assess frailty we used SPPB $(3 \leq x \leq 9)$ and hand-grip strength $(<30 \mathrm{~kg}$ men, $<20 \mathrm{~kg}$ women) and sites, duration and level of pain was investigated by NRS.

Results: All osteosarcopenics (OS) and $85 \%$ of sarcopenics (S) reported pain. Greater, but not statistically significant, intensive pain (NRS $=7.12 \pm 0.33$ ) was observed in S than in OS (NRS $=6.7 \pm$ $0.31),(p=0.18)$. No significant difference was found in number of sites between $\mathrm{S}(2.94 \pm 0.36)$ and OS $(3.07 \pm 0.33),(\mathrm{p}=0.4)$. Osteosarcopenics were more likely to have prolonged pain ( $\geq 1$ year) $(78 \%)$ than $\mathrm{S}(58 \%),(\mathrm{p}=0.09)$. In OS, stratified by muscle-strength, NRS was significantly different $(\mathrm{NRS}=7.33 \pm 0.45$ weak vs $\mathrm{NRS}=6.07 \pm 0.37$ no-weak, $\mathrm{p}=0.02$ ). A significant association emerges in $\mathrm{S}$ between NRS and gender: men had lower levels $(\mathrm{NRS}=5.93 \pm 0.53) \quad$ than $\quad$ women $\quad(\mathrm{NRS}=8.05 \pm 0: 26)$, $(\mathrm{p}<0.001)$

Conclusion: Prevalence and pain intensity were high in both groups, without difference regarding the number of seats. In OS, was observed a longer duration of symptoms and an inverse association between muscle-strength and NRS. Only in S, men showed lower intensity of pain than women.

\section{P-219}

Glomerular filtration rate (GFR) and its association with frailty in geriatric patients

Olgun Deniz ${ }^{1}$, Suheyla Coteli ${ }^{1}$, Ozant Helvaci ${ }^{2}$, Rana Tuna Dogrul $^{1}$, Cagatay Cavusoglu ${ }^{1}$, Ali Oncul ${ }^{1}$, Hacer Dogan Varan ${ }^{1}$, Muhammet Cemal Kizilarslanoglu ${ }^{1}$, Berna Goker ${ }^{1}$

${ }^{1}$ Gazi University School of Medicine, Department of Internal Medicine, Division of Geriatrics, Ankara, Turkey, ${ }^{2}$ Gazi University School of Medicine, Department of Internal Medicine, Division of Nephrology, Ankara, Turkey

Background: Frailty is a novel concept in geriatric population and the related factors have still been investigated by the researchers to find out the pathological factors underlying frailty. In this study, we aimed to investigate the relationship between GFR and frailty status of geriatric patients.

Methods: 447 elderly patients whose frailty status and GFR were known were included for the study. Frailty status of the patients was evaluated by Fried Frailty Scale and grouped as frail, pre-frail and robust. The patients were divided into four groups as quartile $1(\mathrm{Q} 1)(\mathrm{n}=112)$, quartile $2(\mathrm{Q} 2)(\mathrm{n}=112)$, quartile $3(\mathrm{Q} 3)(\mathrm{n}=112)$ and quartile $4(\mathrm{Q} 4)(\mathrm{n}=111)$. GFR was $88-111 \mathrm{~mL} / \mathrm{min}$ in $\mathrm{Q} 1,77-88 \mathrm{~mL} /$ min in Q2, $60-77 \mathrm{~mL} / \mathrm{min}$ in Q3 and $7-59 \mathrm{~mL} / \mathrm{min}$ in Q4.

Results: The median age was 75 years (min-max: 60-97) and 274 $(61.3 \%)$ patients were female. The median GFR level was significantly lower in frail group [66 $\mathrm{mL} / \mathrm{min}$ (min-max: 7-109)] than both pre-frail $[79 \mathrm{~mL} / \mathrm{min}$ (min-max: $13-111)]$ and robust $[81 \mathrm{~mL} / \mathrm{min}$ (min-max: 26-102)] groups $(\mathrm{p}<0.001)$. Frailty frequency was detected to be significantly higher in Q4 $(43.2 \%)$ than other quartiles, Q3 (21.4\%), Q2 (17.9\%) and Q1 $(19.6 \%)$, ( $<<0.001)$. In the multivariate analysis model, GFR lower than $60 \mathrm{~mL} / \mathrm{min}$ was found to be significantly and independently associated factor for frailty (OR: $2.598,95 \%$ CI $1.527-4.421, \mathrm{p}<0.001$ ). Age (OR: $1.105,95 \%$ CI $1.062-1.149, \mathrm{p}<0.001$ ), female gender (OR: 2.691, 95\% CI 1.555-4.657, $\mathrm{p}<0.001$ ), diabetes mellitus (OR: $1.747,95 \%$ CI 1.007-3.031, $\mathrm{p}=0.047)$ and dementia (OR: 6.216, 95\% CI $2.916-13.252, \mathrm{p}<0.001)$ were also found to be independently associated factors for frailty in multivariate analysis.

Conclusion: In this study, we have found that GFR might be one of the independently associated factors for frailty. Further studies 
designed prospectively are needed to carry out exact relationship between these two entities.

\section{P-220}

DECI project: analysis of cognitive scales and its relation with frailty in patients with mild cognitive impairment

Myriam Oviedo Briones ${ }^{1}$, Rodrígo Pérez-Rodríguez ${ }^{1,2}$, Pedro Moreno $^{1,2}$, Myriam Valdés-Aragonós ${ }^{1,2,3}$, Mercedes Montenegro ${ }^{4}$, David Prada ${ }^{4}$, Andrea López Diez-Picazo ${ }^{1}$, Stefan Walter ${ }^{1}$, Leocadio Rodríguez-Mañas ${ }^{1,2,3,5}$

${ }^{1}$ Fundación de Investigación Biomédica del Hospital Universitario de Getafe, ${ }^{2}$ Centro de Tecnología Biomédica Universidad Politécnica de Madrid, ${ }^{3}$ Hospital Universitario de Getafe, ${ }^{4}$ Centro de Prevenciœn de Deterioro Cognitivo, ${ }^{5}$ Centro de Investigación Biomédica en Red Fragilidad y Envejecimiento Saludable

Introduction: DECI Project (Digital Environment for Cognitive Inclusion) is a multi-center, interventional study, designed for elderly with mild cognitive impairment to evolve traditional care organization models, through the support of digital technologies. Some studies suggest that frailty increases the risk of cognitive impairment and vice versa, interacting within a cycle of decline associated with ageing. The aim of this study was to analyze the relationship between cognition and frailty in elderly patients with mild cognitive impairment based on different cognitive scales.

Methods: 111 patients were included, $\geq 60$ years old, with diagnosis of mild and very mild cognitive impairment in the Centro de Deterioro Cognitivo in Madrid. The presence of frailty was assessed with Fried Frailty Criteria and the cognitive tests evaluated were: Clock Drawing Test (CDT), semantic fluency, phonologic fluency and Clinical Dementia Rating (CDR). $\chi 2$-distribution was used to analyze categorical variables.

Results: Among the patients with mild cognitive impairment $<15$ $(5 \%)$ were frail, $69(62 \%)$ prefrail and $37(33 \%)$ were robust. The mean age was 78 years. The CDT was similar between the three groups, $\mathrm{p}=0.93$. The majority of the sample had a CDR on 0.5 , $\mathrm{p}=0.41$. The semantic fluency test was 7.8 on frails, 7.36 on prefrails and 9.35 on robusts, $p=0.26$. The phonological fluency test showed a significant difference on frails (12.8 points), prefrails (13.3 points), and robusts (19.61 points), $\mathrm{p}=0.015$.

Conclusions: The phonological fluency test showed a significant difference in patients with mild cognitive impairment among frails, prefrails and robusts.

\section{P-221}

Evaluation of frailty in a Long Term Care and Convalescence unit at an Intermediate Hospital

Elizabeth Suárez S. ${ }^{1}$, Dolors Quera A. ${ }^{1}$, Lourdes Ferré ${ }^{1}$, Neus Saiz ${ }^{1}$

${ }^{1}$ Hospital Sociosanitario Mutuam Güell, Barcelona, Spain

Introduction: Frailty is a state of vulnerability to stressors of high prevalence in our population. Patients who are admitted to intermediate hospital have a high rate of multimorbidity and chronicity, which is difficult to measure, and correlate with mortality.

Methods: Prospective observational study of patients entered LTC and CU of a Intermediate Hospital during the period September 2017 April 2018. To evaluate the degree of frailty of patients whom are admitted at a long-term care (LTCU) and convalescence (CU) unit by using a new tool: Index fragil-VGI*, based on several items of the integral geriatric evaluation as well as its relationship with the diagnoses of admission and unit in which they are admitted. Family interview, the frailty questionnaire "fragile-VGI index" is applied, asking the patient's previous status (1 month before the current event), recording the data obtained in an Excel database.

Results: We included 274 patients, 149 at the LTCU (58.18\%) and $125(41.81 \%)$ al CU. In the LTCU the average frailty index was severe (0.56) and at the CU was mild fragility (0.26). The diagnosis of dementia and/or delirium is the most related with moderate frailty. Trauma patients obtained mild frailty.

Conclusions: AT the LTCU the sever frailty is more common, related to more elderly patients. The mild frailty index confirms the objective of enhancing autonomy and a need of personalized medicine. Planning a one-year follow-up in order to establish association between frailty index and mortality/number of hospital admissions.*AmblàsNovellas J, et al. En busca de respuestas al reto de la complejidad clínica en el siglo xxi: a propósito de los índices de fragilidad. Rev Esp Geriatr Gerontol. 2016. http://dx.doi.org/10.1016/j.regg. 2016.07.005

\section{P-222}

Prevalence of frailty in primary care: the influence of different hand grip strength cut-offs

João Tavares ${ }^{1,2,3}$, Pedro Sá-Couto ${ }^{4}$, Inês Machado ${ }^{5}$

${ }^{1}$ UCSP Soure, Coimbra, Portugal, ${ }^{2}$ The Health Sciences Research Unit: Nursing - Nursing School of Coimbra, Coimbra, Portugal, ${ }^{3}$ School of Education - Escola Superior de Educação de Coimbra, Coimbra, Portugal, ${ }^{4}$ Center for Research and Development in Mathematics and Applications (CIDMA), Department of Mathematics, University of Aveiro, Portugal, ${ }^{5}$ Department of Medical Sciences (DCM), University of Aveiro, Portugal

Introduction: Identify frail older adults in primary care is crucial to developed a geriatric care plan to prevent adverse outcomes. Frailty phenotype is considered a gold standard to recognize this geriatric syndrome and included five dimension: unintentional weight loss, hand grip strength (HGS), weakness, exhaustion, slow gait and low physical activity. Different cut-off has been propose to use in the HGS. Aim: Analyze the influence of three different HGS cut-offs in the prevalence of robust, pre-frail and frail older adults in primary care.

Methods: A quantitative, observational and correlational study was conducted. It was developed in primary health care. The non-random sampling of convenience constituted by 136 older adults. Fragility was assessed using the phenotypic approach. For the dimension HGS, three cut-offs values (A, B, and C) were tested: A: $<18 \mathrm{~kg}$ (women) and $<31 \mathrm{~kg}$ (man); $\mathrm{B}:<20 \mathrm{~kg}$ (women) and $<30 \mathrm{~kg}$ (man); $\mathrm{C}:<16 \mathrm{~kg}$ (women) and $<26 \mathrm{~kg}$ (man).

Results: Seventy (51.5\%) of participants was women, the mean age average was $74 \pm 6.2$ years and more than a half report a good selfassessment of health (?\%). The prevalence of robust, pre-frail and frail older adults was similar between the cut-off A and B, $18.4 \%$ vs $17.1 \%, 55.1 \%$ vs $54.8 \%$ and $26.5 \%$ vs $38.1 \%$, respectively. With cutoff $\mathrm{C}$ less older adults was considered frail $(19.3 \%)$ and more are classified was per-frail $(60 \%)$ and robust $(20.7 \%)$.

Conclusion: There is no universal consensus about the best HGS cutoff value. However, this single dimension in the frailty phenotype have a significant role in the percentage of frailty in primary care and should be considered according to the purpose of the study or intervention. 


\section{P-223}

The identification of frailty in primary care: Portuguese Prisma-7 analysis

João Tavares ${ }^{1,2,3}$, Pedro Sá-Couto ${ }^{4}$, Inês Machado ${ }^{5}$

${ }^{1}$ UCSP Soure, Coimbra, Portugal, ${ }^{2}$ The Health Sciences Research Unit: Nursing - Nursing School of Coimbra, Coimbra, Portugal, ${ }^{3}$ School of Education - Escola Superior de Educação de Coimbra, Coimbra, Portugal, ${ }^{4}$ Center for Research and Development in Mathematics and Applications (CIDMA), Department of Mathematics, University of Aveiro, Portugal, ${ }^{5}$ Department of Medical Sciences (DCM), University of Aveiro, Portugal

Introduction: In order to respond to the needs that fragile older adults care requires, the inclusion of an assessment of fragility in primary health care is crucial. There are several tools designed to assess fragility. However, they require significant amounts of time related to the instruments complexity. There is a need for a simple tool to quickly and easily identify fragile older people. The Prisma7 represents one of these instruments.

Aim: Examine the sensitivity, specificity of the Portuguese version of the Prisma7 when considering the Frailty Phenotype (FP) as the gold standard.

Methods: A quantitative, observational and correlational study was conducted. It was developed in primary health care. The non-random sampling of convenience constituted by 136 older adults. To assess the fraity two tools were used: Prisma7 and FP. The sensibility and specificity of Prisma7 threshold cut-off of $\geq 2$ and $\geq 3$ was analyzed. Result: Seventy $(51.5 \%)$ of participants was women, the mean age average was $74 \pm 6.2$ years and more than a half report a good selfassessment of health $(60 \%)$. The cut-off $\geq 2$, showed a specificity, sensibility, positive and negative predicted values of 53, 58, 30 and $77 \%$, respectively. The cut-off $\geq 3$, showed a specificity, sensibility, positive and negative predicted values of $97,19.4,70$ and $77 \%$, respectively.

Conclusion: The results are not considered optimal. The PRISMA7 threshold (cutoff $\geq 2$ ) presented a better compromise between sensitivity and specificity when compared to cutoff $\geq 3$ (lower sensitivity and higher specificity). Other study of validation are need to determine the best threshold of Prisma7.

\section{P-224}

Relations between frailty, multimorbidity and disability in specific activities in daily living in community-dwelling women: a cross-sectional study

Andrius Apsega $^{1}$, Asta Mastaviciute ${ }^{1}$, Marija Tamulaitiene ${ }^{1}$, Vidmantas Alekna ${ }^{1}$

${ }^{1}$ Vilnius University, Faculty of Medicine, Vilnius, Lithuania

Introduction: The purpose of this study was to investigate relations between frailty, multimorbidity and disability in elderly women.

Methods: A cross-sectional study in community-dwelling women aged 60 years and more was conducted. Frailty was defined using the frailty phenotype according to Fried et al. criteria. Multimorbidity was defined as the presence of two or more self-reported chronic diseases. Disability in specific activities of daily living (ADL) was defined as needing help or being unable to perform one or more basic or instrumental ADL assessed using Katz and Lawton scales. Statistical analysis was performed using Chi square test and Fisher exact test.

Results: The data of 131 women was analysed. Mean age of all participants was $71.1 \pm 7.11$ years. Overall, $55.7 \%$ women were classified as robust, $16.8 \%$ prefrail and $27.5 \%$ frail. Among all women, $24.5 \%$ women had multimorbidity. Multimorbidity was identified in $15.1 \%$ of robust, $31.8 \%$ of prefrail and $38.9 \%$ of frail women. Those who were frail had higher rates of multimorbidity than prefrail and robust women $(\mathrm{p}=0.017)$. Rate of ADL disability of frail women was $11.1 \%$ and no statistically significant differences were found between groups. Frailty was associated with higher rates of IADL disability $(p=0.001)$. We found that $25 \%$ of those who were frail reported difficulty in IADL. Difficulties in shopping and transportation were the most prevalent (33.3\% and $41.7 \%$, respectively). Key conclusions: Higher rates of multimorbidity and IADL disability were found in frail women compared to prefrail and robust women.

\section{P-225}

Application of the STOPPfrail criteria to the frailest nursing home residents (NHRs): a COME-ON sub-study

Fournier Anne ${ }^{1}$, Anrys Pauline ${ }^{2}$, Beuscart Jean-Baptiste ${ }^{3}$, Dalleur Olivia $^{1}$, Henrard Severine ${ }^{4}$, Foulon Veerle ${ }^{5}$, Spinewine Anne ${ }^{6}$

${ }^{1}$ Louvain Drug Research Institute, Clinical Pharmacy Research Group, Université Catholique de Louvain, Brussels, Belgium, Cliniques Universitaires Saint-Luc, Pharmacy Department, Université Catholique de Louvain, Brussels, Belgium, ${ }^{2}$ Louvain Drug Res

Background: The STOPPFrail criteria were recently developed to assist physicians in identifying inappropriate prescribing in frail older adults with a poor 1-year survival prognosis.

Aim: To describe the prevalence of potentially inappropriate medication (PIM) in frail nursing home residents (NHRs), using the STOPPFrail criteria.

Materials and methods: We performed a post-hoc analysis of data from the COME-ON study, a multicentric cluster-controlled trial that evaluated the effect of a complex intervention on the appropriateness of prescribing, performed in 1804 NHRs over a 15-month period. For the present work, frail NHRs with a poor 1-year survival prognosis were identified using data on functional status, comorbidities, level of care and survival data. Twenty-one STOPPfrail criteria were applied to measure PIMs at baseline.

Results: Three-hundred-six frail NHRs were included. Median age was 87 years $(83 ; 92), 76 \%$ were fully dependent and dement, $63 \%$ died over the study period. In total, 189 NHRs $(61.8 \%)$ had at least 1 PIM (median 1 PIM per resident, interquartile range 0-2, range 0-7). Among the 309 PIMs identified, the three most prevalent were: proton pump inhibitor at full dosage $\geq 8$ weeks (18\%), calcium supplementation (16.3\%) and anti-platelet therapy for primary cardiovascular prevention (15.7\%). At least one of these three PIMs was found in 129 NHRs $(42.2 \%)$.

Conclusion: A large proportion of frail NHRs with poor survival prognosis had at least one PIM. Unnecessary drugs as well as drugs with unfavorable risk-benefit balance should be thoroughly reassessed and stopped in such a context in order to prioritize symptom control management.

\section{P-226}

\section{Frailty and lipid profile in elderly hospitalized patients}

Giuliana Ciancio ${ }^{1}$, Daniela Brischetto ${ }^{1}$, Angela Alibrandi ${ }^{2}$, Alberto Sardella ${ }^{1}$, Nunziata Morabito ${ }^{1}$, Antonino Catalano ${ }^{1}$, Francesco Corica ${ }^{1}$, Antonino Lasco ${ }^{1}$, Giorgio Basile ${ }^{1}$

${ }^{1}$ Unit and School of Geriatrics-Department of Clinical and Experimental Medicine, Messina, Italy, ${ }^{2}$ Unit of Statistical and 
Mathematical Sciences-Department of Economics, University of Messina, Messina, Italy

Introduction: The relation between lipid profile and cardiovascular disease in frail and advanced-aged subjects is still debated. The main purpose of our study is to verify the correlation between lipid profile, frailty and mortality in a sample of elderly inpatients.

Methods: We evaluated 131 subjects, 73 women (mean age: $81.3 \pm 6.4$ years) consecutively admitted at our Geriatrics Unit. A Frailty Index (FI) was computed taking into account 46 potential deficits collected as part of standard clinical and laboratory parameters.

Results: Lipid profile appears significantly different between discharged patients (n. 118) and those who died during hospitalization (n. 13), respectively: total cholesterol $149 \pm 40 \mathrm{mg} / \mathrm{dl}$ vs $126 \pm 43 \mathrm{mg} / \mathrm{dl} \quad(\mathrm{p}=0.003)$, HDL cholesterol $42 \pm 16 \mathrm{mg} / \mathrm{dl}$ vs $31 \pm 14 \mathrm{mg} / \mathrm{dl} \quad(\mathrm{p}=0.001), \quad$ LDL cholesterol $88 \pm 32 \mathrm{mg} / \mathrm{dl}$ vs $74 \pm 35 \mathrm{mg} / \mathrm{dl} \quad(\mathrm{p}=0.02)$. Significant negative correlations were observed between frailty at admission and: total cholesterol level $(\mathrm{r}=-390, \mathrm{p}=0.001)$, HDL cholesterol $(\mathrm{r}=-359, \mathrm{p}<0.001)$ and LDL cholesterol $(r=-314, p=0.001)$. Logistic regression model showed that in-hospital mortality was significantly associated with both frailty at admission $(\mathrm{p}=0.02)$ and total cholesterol level $(\mathrm{p}=0.017)$. Moreover, univariate analysis showed that in-hospital mortality was significantly associated with total cholesterol level regardless frailty $(\mathrm{p}=0.001)$.

Key conclusions: Our findings confirm the negative correlation between frailty and lipid profile, even in a sample of elderly hospitalized individuals. Specifically, frail elderly patients show low total, low HDL and low LDL cholesterol levels; therefore, this evidence better represents a general health biomarker rather than a cardiovascular risk factor in this specific range of subjects.

\section{P-227}

Dementia as a factor of worse status and bad outcome in hip fracture patients. A study with 1-year follow-up

Isabel Rodríguez-Sánchez ${ }^{1}$, Silvia Lozoya-Moreno ${ }^{2}$, Martha Choquehuanca $^{1}$, Rocío Menéndez-Colino ${ }^{1}$, Isabel Martín-Maestre ${ }^{1}$, Teresa Alarcon ${ }^{1}$, Juan Ignacio González-Montalvo ${ }^{1}$

${ }^{1}$ Geriatrics Department Hospital Universitario La Paz, Madrid, Spain, ${ }^{2}$ Geriatrics department Hospital Universitario de Albacete, Spain

Introduction: The number of demented patients with hip fracture (HF) is increasing as the population ages. The aim of this study was to examine the outcomes in demented HF patients, compared with those patients without dementia.

Methods: All patients admitted consecutively with a frail HF during 1 year in an orthogeriatric unit at a university hospital were assessed. Baseline and admission demographic, clinical, functional, analytical and body-composition variables were collected at admission. One year after the fracture, patients or their carers were contacted. Patients were divided into two groups according to presence of dementia, which was defined as previous diagnosis or baseline Red Cross Mental Scale Score $\geq 2$. The differences between HF with and without dementia was evaluated with bivariate analyses.

Results: A total of 509 patients were included. Mean age was 85.6 $( \pm 6.9)$ years and $79.2 \%$ were female. Dementia patients $(n=171$, $33.5 \%$ ) were older (mean age $85.6 \pm 6.9$ vs $84.6 \pm 6.96$ years, $\mathrm{p}=<0.001)$, had worse baseline Barthel Index $(57.13 \pm 25.9$ vs $87.75 \pm 14.99, \mathrm{p}<0.001)$ and Functional Assessment Categories scores $(3.23 \pm 1.46$ vs $4.41 \pm 0.89, \mathrm{p}<0.001)$, received surgery less frequently ( $93 \%$ vs $98.2 \%, \mathrm{p}<0.05$ ), had a higher risk of nursing home placement $(42.7 \%$ vs $18.5 \%, \mathrm{p}<0.001)$, less access to a rehabilitation unit $(29.2 \%$ vs $49.4 \%, \mathrm{p}<0.001)$ and had higher rate of 1 -year mortality $(34.5 \%$ vs $17.3 \%, \mathrm{p}<0.001)$.

Key conclusions: HF dementia patients are older, receive less surgery, had less chances to came back home and more probability of 1 -year mortality. The presence of dementia should be monitored to emphasize care and optimize health outcomes.

\section{P-228}

The prevalence and associated factors of sarcopenic obesity are not the same as those of sarcopenia in older patients

Ploy Ruengsinpinya ${ }^{1}$, Prof. Prasert Assantachai ${ }^{1}$

${ }^{1}$ Siriraj Hospital, Mahidol University, Bangkok, Thailand

Background: The coexistence of sarcopenia and high accumulation of fat mass, termed sarcopenic obesity, has been under focused recently. Since both conditions synergistically cause even more adverse health outcomes than each condition does, e.g. increase risk of disability, morbidity and mortality. Therefore, the prevalence of sarcopenic obesity and its associated factors are essential for policy planning and implication in daily clinical practice.

Objective: To determine the prevalence and associated factors of sarcopenic obesity among older patients. The differences of prevalence and associated factors of sarcopenia and sarcopenic obesity are also evaluated.

Materials and methods: This cross-sectional study recruited 186 patients aged over 60 years old who were followed up at the outpatient department, Siriraj Hospital. Appendicular muscle mass and body fat mass were evaluated by bioelectrical impedance analysis. Hand grip strength and usual gait speed were also assessed. Sarcopenic obesity was defined as sarcopenia based on the consensus of Asian Working Group on Sarcopenia plus obesity based on body fat mass by WHO criteria.

Results: The prevalence of sarcopenic obesity was $5.4 \%$, while that of sarcopenia was $7.5 \%$. The associated factors of sarcopenic obesity were: increasing age, low education, high co-morbidity index and low physical activity. Meanwhile, the associated factors of sarcopenia were: increasing age, low BMI, poor nutrition status, high co-morbidity index and number of medications.

Conclusion: The prevalence of sarcopenia was higher than sarcopenic obesity. Their prevalences and associated factors are different. Both conditions should be screened in clinical practice for older patients.

\section{P-229}

Frailty status and mild-chronic hyponatremia in nursing home fallers admitted to emergency geriatric medicine unit (MUPA Unit)

Boyer Sophie $^{1}$, Gayot Caroline ${ }^{2}$, Bimou Charlotte ${ }^{1}$, Dantoine Thierry ${ }^{2}$, Tchalla Achille ${ }^{2}$

${ }^{1}$ Limoges University, IFR 145 GEIST; EA 6310 HAVAE (Disability, Activity, Aging, Autonomy and Environment), Limoges, France,

${ }^{2}$ Geriatric Medicine Department, CHU Limoges, Limoges, 87025, France

Introduction: Hyponatremia is the most common electrolyte disorder in older adults in nursing home. Studies have found that it increases morbidity and mortality. Approximately one in three older adults falls each year. Mild-chronic hyponatremia may predispose to falls and fractures which lead to dependency. Frailty status is a risk factor of 
dependency. Little is known of the association between mild-chronic hyponatremia at Emergency Department (ED), fall prevalence and frailty status in elderly. Therefore we are investigating the link between frailty status, mild-chronic hyponatremia and risk of falls in nursing home patients admitted to MUPA Unit.

Methods: We conducted a cross sectional study during four months including patients older than 75 years living in nursing home, admitted to MUPA Unit (Limoges CHU, France). Socio-demographic factors, falls event, Comorbidities, Medications, sodium levels were studied (hyponatremia was considered $\mathrm{Na}+<136 \mathrm{mmol} / \mathrm{l}$ ) and the short-CGA variables including the frailty score SEGA : not frail $($ SEGA $\leq 8)$, frail $(8<$ SEGA $\leq 11)$ and very frail (SEGA $>11)$ status. Results: Of 132 cases recruited, the mean age was $89 \pm 5.2$ and $72.2 \%$ were women. The prevalence of mild-chronic hyponatremia was $16.7 \%$ and the prevalence of falls was $25 \%$ in frail group. The prevalence of mild-chronic hyponatremia was $11.7 \%$ and the prevalence of falls was $18 \%$ in the group of very frail patients.

Conclusion: Given that mild-chronic hyponatremia could be considered a risk factor for falls, the inclusion of the determination of sodium level at ED would be an indicator of frailty status of elderly admitted to MUPA Unit.

\section{P-230}

\section{Handgrip strength predicts 1-year functional recovery} and mortality in hip fracture patients

Patricia Pérez-Rodríguez ${ }^{1}$, Laura Rabes ${ }^{1}$, Carmen Sáez-Nieto ${ }^{1}$, Fernando Ly-Yang ${ }^{1}$, Rocío Menéndez-Colino ${ }^{1}$, Juan Ignacio González-Montalvo ${ }^{1}$, Teresa Alarcon ${ }^{1}$

${ }^{1}$ Geriatrics Department Hospital Universitario La Paz, Madrid, Spain

Introduction: Muscle strength is a possible predictor of adverse events. It could have a prognostic value in patients with hip fracture (HF). The aim of this study was to determine if handgrip strength values are associated with functional impairment, readmissions, and mortality at 1 year in elderly patients with HF.

Materials and methods: A prospective observational study was carried out. It included a cohort of patients aged 65 years or older with a diagnosis of fragility HF, consecutively hospitalized from January 2013 to February 2014 and followed up at 1 year. Clinical and functional outcomes, analytical parameters and anthropometric measurements were collected. Statistical analysis was performed using SPSS v21 software.

Results: Five hundred and nine patients with a mean age of $85.4 \pm 0.3$ years were included, of which $403(79.2 \%)$ were women. Of the total sample, $339(66.6 \%)$ had low handgrip strength, and those patients were older, more frequently institutionalized, had worse functional and cognitive status, higher comorbidity, higher surgical risk, lower body mass index and a greater intra-hospital mortality (all $\mathrm{p}<0.01$ ). At 1 year, patients with low strength had greater impairment of their walking capacity $(32.7 \%$ vs. $10.9 \%, \mathrm{p}<0.001)$ and higher mortality $(30.4 \%$ vs. $8.8 \%, \mathrm{p}<0.001)$. There were no differences in the number of readmissions.

Key conclusions: A low handgrip strength in elderly patients with $\mathrm{HF}$ predicts greater functional disability and higher long-term mortality.

\section{P-231}

Is dysphagia associated with hand grip strength and fat-free mass at the hospital in older adults?

Sumru Savas ${ }^{1}$, Merve Y1lmaz ${ }^{2}$, Fulden Sarac ${ }^{1}$, Fehmi Akcicek ${ }^{1}$
${ }^{1}$ Section of Geriatrics, Department of Internal Medicine, School of Medicine, Ege University, Bornova, Turkey, ${ }^{2}$ Department of Internal Medicine, School of Medicine, Ege University, Bornova, Turkey

Aim: Oropharyngeal dysphagia is a geriatric syndrome associated with increased morbidity. Pharyngeal peak pressure has been shown to be significantly lower in healthy adult aspirators versus nonaspirators, and handgrip strength (HGS) to be correlated with posterior tongue strength in previous studies. In this study, we aimed to evaluate HGS, fat-free mass (FFM), and dysphagia in hospitalized elderly patients and to evaluate the associations between them.

Material methods: Sixty-one patients ( $\geq 60$ years) hospitalized in the Department of Internal Medicine were included in the study. Swallowing evaluation was performed with Yale-swallow protocol. Nutritional status, anthropometric measurements, HGS, serum biochemical parameters, FFM via BIA, and hospitalizations in last one year were recorded.

Results: Mean age was 69.7 $( \pm 8.8)$ years. Mean fasting blood glucose, total cholesterol levels, and body mass index, HGS, FFM values were $128.9( \pm 58.5) \mathrm{mg} / \mathrm{dL}, 179.1( \pm 34.8) \mathrm{mg} / \mathrm{dL}, 27.5( \pm 6.7) \mathrm{kg} /$ $\mathrm{m}^{2}, 15.4( \pm 7.8) \mathrm{kg}, 47.9( \pm 11.0) \mathrm{kg}$. Dysphagia and nutritional risk (as at risk plus malnutrition) were present in $19.7 \%$ and $88.5 \%$ of the patients. There was no relationship between the number of hospitalizations, nutritional status and gender with dysphagia $(r=-0.223$, $\mathrm{P}=0.08 ; \mathrm{r}=0.178, \mathrm{P}=0.16 ; \mathrm{r}=0.049, \mathrm{P}=0.70$, respectively). HGS and FFM values were similar between dysphagia group and the successful group $(\mathrm{P}=0.57$ and $\mathrm{P}=0.35)$.

Conclusion: We were unable to show associations of dysphagia with HGS and FFM. This may be because of the limited number of participants.

\section{P-232}

Determination of hand grip strength in healthy adults in Izmir in the context of medical school education

Sumru Savas ${ }^{1}$, Mehmet Cengiz Isik ${ }^{2}$, Dogukan Kilic ${ }^{2}$, Yusuf Can Koker ${ }^{2}$, Duygu Ozcelik ${ }^{2}$, Nur Beril Ozden ${ }^{2}$, Serkan Ozkan ${ }^{2}$, Hazal Zanclar ${ }^{2}$, Fehmi Akcicek ${ }^{1}$

${ }^{1}$ Section of Geriatrics, Department of Internal Medicine, School of Medicine, Ege University, Izmir, Turkey, ${ }^{2}$ Special Working Module, School of Medicine, Ege University, Izmir, Turkey

Aim: Sarcopenia is a worldwide problem. For Turkey, cut-off values to define low handgrip strength (HGS) have been defined. In this study, we aimed to evaluate healthy young adults between the ages of 20-45 with regard to HGS in Izmir and to compare the results with those studies.

Materials and methods: This research was made in the context of Special Working Module of 'Let's protect our muscles' of Ege University Medical School. The researcher trained the students $($ no $=7$ ) about sarcopenia. Volunteers between the ages of $18-44$ were included. HGS was measured by Takei Digital Hand Grip Dynamometer standing. Three measures were performed with the dominant hand.

Results: A total of 249 volunteers were taken into the study. Overall, 62.7 of the participants were males, and 37.3 were females. Mean (minimum-maximum) age, body mass index (BMI), HGS values were $21.7 \pm 5.4(18-44)$ years, $22.7 \pm 3.3(16.4-36.3) \mathrm{kg} / \mathrm{m}^{2}$, and $29.8 \pm 6.5(17.5-50.5) \mathrm{kg}$. Low HGS was defined by gender-specific T-scores of less than $-2(<22.5$ and $<16.6)$, and values of $\leq 25$ percentile and cut off values (30.5 and 22.5) for males and females, respectively.

Conclusion: A standardized method is needed to enable more consistent measurement of grip strength and better assessment of 
sarcopenia together with standardized cut off values for specific groups. The results will be discussed with the other studies of low HGS values for Turkish population.

\section{P-233}

\section{Frailty in nursing homes. An indicator of seniors' longevity?}

Haÿ Paul-Emile ${ }^{1}$, Armaingaud Didier ${ }^{1}$, Josseran Loïc ${ }^{2}$

${ }^{1}$ Korian SA, Saclay, France, ${ }^{2}$ APHP Paris-Saclay, France

Introduction: Korian is a private group specialized in medical accommodation for elderly and dependent people. Its professional data warehouse hosts all residents' data, including the residents' care narratives $(\mathrm{CN})$ fed on a daily basis. As residents grow older, they face multiple age-related syndromes. Among them frailty, a multifactorial process that seems undoubtedly linked to the end of life. However, does frailty really shorten life or is it just associated to seniors' longevity?

Methods: Through nursing $\mathrm{CN}$ extraction, using multi-step learning and text mining we built 28 syndromes including frailty, afflicting our residents. In this study, we chose to extract the complete syndromic residents' life course of residents, entered in one of 125 nursing homes $(\mathrm{NH})$ over [11/01/2010-05/28/2017], with at least one transmission date, either with a frailty syndrome or without. The idea here was to check what differentiates the firsts from the lasts, comparing their socio-demographic profiles and survival using Cox proportional hazards modelization.

Results: Splitting the 42,389 residents between frail and not frail ((6182 versus 36,207$)$, dead and alive (18,695 versus 23,694$)$, men and women $(13,529$ versus 28,860$)$, age and autonomy levels at $\mathrm{NH}$ entry gave us the opportunity to study each covariate effect on the survival analysis. Whereas age at entry was a bad prognostic factor, frailty, sex and autonomy at entry improved the survival time. By keeping all four covariates in the Cox modelization, we still found that frailty was a good prognostic factor.

Conclusion: Frailty seems to happen only late in life expressing residents' resilience.

\section{P-234}

\section{A quick and easy frailty detection tool with the potential} to identify distinct frailty profiles

Marina Kotsani ${ }^{1}$, Olivier Aromatario ${ }^{2}$, Carlos Labat ${ }^{3}$, Guy Vançon ${ }^{4}$, Jonathan Epstein ${ }^{1,3,5,6}$, Athanase Benetos ${ }^{1,6}$

${ }^{1}$ CHRU Nancy, France, ${ }^{2}$ Autonomous National Social Security Fund in the Mines of East, France, ${ }^{3}$ Inserm U1116, France, ${ }^{4}$ Office of Social Hygiene of Meurthe-et-Moselle, France, ${ }^{5} \mathrm{CIC} 1433$ Clinical Epidemiology, Nancy, France, ${ }^{6}$ University of Lorraine, France

Objective: To propose a simple and quick tool for early frailty detection in populations $>70$ years old, that would also be suitable for wide-scale administration by non-experts.

Methods: A 10 items' screening scale investigating social isolation, physical and surroundings' negligence, inappropriate behavior, weight loss, iterative hospitalizations, falls, activities' reduction, sensory deficiency, unsuitable housing and polymedication was administered in 591 people ( $80.1 \pm 5.4$ years old, $58 \%$ women) in primary health care settings. Comprehensive geriatric assessment (CGA) was performed to 106 of those, as a standard means to identify frailty.
Results: By applying multicomponent analysis and hierarchical clustering, 7 out of 10 items were considered pertinent, whereas those about unsuitable housing, sensory deficiency and polymedication were eliminated. Five distinct groups emerged from the answers' pattern, which could be attributed to specific frailty profiles. People found to belong to the "low risk of frailty" profile, were those who answered negatively to all 5 questions about lack of social support, negligence, weight loss, hospitalizations' and falls. Whereas this first group could be considered as "normal" $(68.9 \%)$, three other clinical profiles were highlighted, a "physical frailty" profile (12.7\%) mostly presenting falls and weight loss, a "social/cognitive/affective frailty" profile (14.7\%), by merging 2 groups derived from the multicomponent analysis and a "hospitalizations" profile $(3.7 \%)$, suggesting a rather established physical frailty status.

Key conclusions: Further validation studies are required. However, this quick and simple frailty screening tool presents promising properties in distinguishing early frailty profiles and seems to be eligible for a step-wise approach of frailty detection and referral to CGA.

\section{P-235}

Frailty and cognitive impairment in heart failure patients: preliminary findings from the Deus Ex Machina study

Emilia Moreira $^{1}$, Rui Valdiviesso ${ }^{1}$, Sónia Martins ${ }^{2}$, Rosário Ataíde ${ }^{3}$, Luís Filipe Azevedo ${ }^{4}$, Nuno Borges ${ }^{5}$, José Silva-Cardoso ${ }^{6}$, Lia Fernandes $^{7}$

${ }^{1}$ Centro de Investigação em Tecnologias e Serviços de Saúde, Faculdade de Medicina da Universidade do Porto CINTESIS/FMUP, Portugal, ${ }^{2}$ Centro de Investigação em Tecnologias e Serviços de Saúde, Faculdade de Medicina da Universidade do Porto CINTESIS/F

Introduction: Frailty and cognitive impairment (CI) are common in Heart Failure(HF), being associated with vulnerability to stressors and loss of physiologic and cognitive reserve. This work aims to analyse the association between Frailty and CI in HF outpatients.

Methods: Patients were recruited from an outpatient clinic in a University Hospital. Sociodemographic data and NYHA class were registered. Frailty was assessed using Fried phenotype. CI was defined as MoCA $<22$. A logistic regression model, with coexisting $\mathrm{CI}$ and frailty/pre-frailty as dependent variable, entered as covariates age, education and NYHA class.

Results: A sample of 86 patients was included: age $57 \pm 13[34 \% \geq 65$ years] and $66 \%$ men. Overall 30,53 and $17 \%$ of patients were respectively at NYHA class I/II/III. Generally $71 \%$ of patients were frail/pre-frail and $47 \%$ showed CI (54\% among frail patients; $\left.\mathrm{X}^{2}=4.497 ; \mathrm{p}=0.034\right)$. In 33 patients $(39 \%)$ CI coexisted with frailty/pre-frailty. The majority was $\geq 65$ years $(70 \%$; $\left.\mathrm{X}^{2}=16.838 ; \mathrm{p}<0.001\right)$, had $\leq 4$ years of education $(63 \%$; $\left.\mathrm{X}^{2}=17.097 ; \mathrm{p}<0.001\right)$, and was in NHYA class II-III $(84 \%$; $\left.\mathrm{X}^{2}=5.470 ; \mathrm{p}=0.027\right)$. In the regression model, age $(\mathrm{p}=0.003)$, education $(\mathrm{p}=0.041)$ and NYHA class showed $(\mathrm{p}=0.051)$ independent association with coexisting CI and frailty/pre-frailty.

Conclusions: Coexisting frailty/pre-frailty is associated with older age, less education, and more severe symptoms of HF. These preliminary results identified an especially vulnerable group of $\mathrm{HF}$ patients. This may be further studied to better understand the interaction of $\mathrm{CI}$ and frailty and its impact on HF prognosis.

Acknowledgements: Supported by ERDF through POCI-01-0145FEDER-007746, funded by COMPETE2020, National Funds through FCT, within CINTESIS(UID/IC/4255/2013), and Project "NORTE01-0145-FEDER-000026-Symbiotic Technology for Societal Efficiency Gains:Deus ex Machina", financed by NORTE2020 under PORTUGAL2020. 


\section{P-236}

\section{Frailty and hospitalization}

Ana Maria Canelas ${ }^{1}$, Rita Amorim Costa $^{1}$, Pedro Oliveira ${ }^{1}$, Pedro Salvador ${ }^{1}$, Fábio Murteira ${ }^{2}$, Fatima Leal Seabra ${ }^{1}$, Rafaela Veríssimo ${ }^{1}$, Agripino Oliveira ${ }^{1}$

${ }^{1}$ Centro Hospitalar de Vila Nova de Gaia/Espinho, Portugal, ${ }^{2}$ Centro Hospitalar de Vila Nova de Gaia/Espinho, Portugal

Introduction: Frailty has enormous impact on acute hospital care and has been shown to be a more effective predictor type of hospitalization and mortality than conventional clinical measures.

Aim: Association of type of hospitalization and mortality by frailty in tertiary hospital.

Methods: Hospitalized patients admitted at a teaching public hospital were studied to determine the prevalence of frailty over a period of 12 months. We used clinical frailty scale (CFS) to stratified frailty. We used binary logistic regressions to evaluate the type of hospitalization, adjusted for age, internal medicine and hospital mortality. We considered a $95 \%$ confidence interval, and tests with a p-value $<0.05$ were considered to be statistically significant.

Results: The records of 10656 patients, $1 / 2$ of hospitalization episodes, were reviewed. There were $54.1 \%$ females and median age of 66 years. The age group $\geq 65$ years was $53.2 \%$, and with age $\geq 75$ was $33 \%$. Emergency hospitalizations were present in $58.5 \%$ of the episodes, whereas $33.2 \%$ had frailty $(\mathrm{RR}=4.55)$. Internal medicine (IM) accounted for $17.5 \%$ of the episodes, whereas $57.5 \%$ had frailty $(\mathrm{RR}=3.95)$. Frailty had $\mathrm{OR}=6.12$ for hospital mortality, $\mathrm{OR}=4.77$ for internal medicine and $\mathrm{OR}=3.23$ for emergency hospitalizations. Conclusion: Our study reported that frailty was an independent predictor of emergency hospitalizations and higher hospital mortality. And, is associated an internal medicine inpatient. Impact: Frailty is a geriatric condition managed by Internal Medicine in our country. The internist must be able to manage these patients as a whole without fragmentation of care. This is especially true of the frailty patients.

\section{P-237}

Frailty assessment by two different methods in acute hospitalised elderly patients

Iñaki Echeverria ${ }^{1}$, Maria Amasene ${ }^{2}$, Miriam Urquiza ${ }^{1}$, Ane Errasti ${ }^{1}$, Cristina Bermudez-Ampudia ${ }^{3}$, Juan Jose Villareal ${ }^{4}$, Jon Irazusta ${ }^{1}$, Ana Rodriguez ${ }^{1}$, Ariadna Besga ${ }^{5}$

${ }^{1}$ Department of Physiology. Faculty of Medicine and Dentistry, University of the Basque Country (UPV/EHU), Leioa, Vizcaya, Spain, ${ }^{2}$ Department of Pharmacy and Food Science. Faculty of Pharmacy. University of the Basque Country (UPV/EHU), VitoriaGasteiz, ${ }^{3}$ Bioaraba Health Research Institute. Methodology and Statistics Unit, Vitoria-Gasteiz, Spain, ${ }^{4}$ Department of Internal Medicine. OSI Araba University Hospital, Vitoria-Gasteiz, Spain, ${ }^{5}$ Department of Internal Medicine. OSI Araba University Hospital, Vitoria-Gasteiz. Institute of Mental Health Research, CIBERSAM, Vitoria, Spain

Objective: The objectives of this study were to determine: (1) the prevalence of frailty using Fried's phenotype method and the Short Performance Physical Battery (SPPB) and (2) the concordance between both frailty assessment methods.

Method: 391 community-dwelling elderly patients aged $79.0 \pm 8.4$ years $(67 \%$ women and $33 \%$ men), were recruited at internal medicine ward of the University Hospital of Vitoria. Comprehensive geriatric assessment, Fried frailty phenotype component evaluation and SPPB were performed in all patients.
Results: According to Fried's phenotype method, the prevalence of frailty and pre-frailty was $75.45 \%$ and $24.55 \%$, respectively. According to the SPPB, the prevalence of frailty was $(44.7 \%+38.02 \%)$ and prefrail $(19.9 \%+10.39 \%)$, suggesting that both methods of frailty diagnosis are moderately concordant $\left(\mathrm{Chi}^{2}=82.278 ; \mathrm{p}<0.001\right)$.

Conclusions: This study showed that SPPB and FRIED tests can be reliably collected in hospitalized older patients to identify older persons at high risk of lower body functional limitations who would benefit most from preventive interventions.

\section{P-238}

Secular change in grip strength among older Europeans: preliminary findings from SHARE

Richard Dodds ${ }^{1}$, Eduwin Pakpahan ${ }^{1}$, Antoneta Granic ${ }^{1}$, Karen Davies ${ }^{1}$, Avan Sayer ${ }^{1}$

${ }^{1}$ AGE Research Group, Institute of Neuroscience, Newcastle University, Newcastle upon Tyne, UK

Introduction: Weaker grip strength is associated with worse health outcomes and is a key component of sarcopenia and frailty. Few studies have examined secular changes in grip: such changes, if present, could have implications for the planning of clinical services. Our aim was to investigate if there is evidence of secular change in grip in the Survey of Health, Ageing and Retirement in Europe (SHARE).

Methods: For this study we used data for participants aged 50-90 years from 10 European countries participating in wave 1 (2004-2005) and wave 6 (2015) of SHARE. Grip was measured with a Smedley dynamometer. We used the maximum from two attempts in each hand. We converted grip values to Z-scores using age- and gender-stratified British normative data. We grouped countries into northern, central and southern regions. We used linear regression to calculate mean differences in Z-scores between waves in each region, including after adjustment for height.

Results: We used a total of 60,551 measurements of grip from the two waves. There was no significant change in mean grip Z-score between wave 1 and wave 6 in the central region. The northern and southern regions showed mean increases in Z-score of 0.17 (95\% CI $0.13,0.21)$ and $0.12(95 \%$ CI $0.09,0.15)$ standard deviations, respectively. These increases attenuated but remained after adjustment for height.

Conclusions: We have shown secular increases in grip in two European regions, independent of secular increases in height. Further work will include investigation of other participant characteristics that may explain these changes.

\section{P-239}

\section{Osteosarcopenia and mortality in older Chileans}

Cecilia Albala ${ }^{1}$, Carlos Marquez ${ }^{1}$, Lydia Lera ${ }^{1}$, Barbara Angel ${ }^{1}$, Rodrigo Saguez ${ }^{1}$, Patricia Arroyo ${ }^{2}$

${ }^{1}$ INTA, University of Chile, Chile, ${ }^{2}$ Clinic Hospital, University of Chile, Chile

Introduction: Sarcopenia and osteoporosis are among the main causes of physical disability in older people. When both coexist, the consequences can be devastating. The objective of this study is to describe the prevalence of osteo-sarcopenia and its association with mortality in Older Chileans. 
Methods: Follow up of ALEXANDROS cohorts designed to study disability associated with obesity in community-dwelling people $60 \mathrm{y}$ and older living in Santiago/Chile. At baseline 1119 (68.5\% women, mean age 72 years \pm 6.7 ) from 2372 participants had DEXA scan and the measurements for the diagnosis of sarcopenia. WHO standards for Bone Mineral Density (BMD) classified them in normal, osteopenia and osteoporosis. Sarcopenia was identified using the algorithm from the EWGS validated for Chile. Information about deaths was available for the 1119 subjects.

Results: At baseline, Sarcopenia was identified in 19.5\%, osteoporosis in $23.2 \%$ and osteo-sarcopenia in $8 \%$ of the total sample. Osteo-sarcopenia increased with age reaching $17.5 \%$ in $\geq 80$ years. Osteo-sarcopenia was found in $34.4 \%$ of osteoporotic people and $40.8 \%$ of the people with sarcopenia. After 5640 person/years of follow-up 86 people were died (osteo-sarcopenia $20.2 \%$, osteopeniasarcopenia 11.7 and $6.1 \%$ of the people without these conditions). After adjusted Cox Regression analysis, the HR for death in people with osteo-sarcopenia was 2.16 (95\% CI 1.18-3.94).

Conclusions: Although the prevalence of osteo-sarcopenia is $8 \%$, when one of the conditions are present, the risk of osteo-sarcopenia increase to $34-40 \%$. Considering mortality is more than twice in people with both conditions, Screening for the second condition, at least when one is present should be the rule at primary care.

\section{P-240}

\section{Mitochondrial respiratory chain function and content are preserved in the skeletal muscle of healthy very old men and women}

Richard Dodds ${ }^{1}$, Karen Davies ${ }^{1}$, Antoneta Granic ${ }^{1}$, Kieren Hollingsworth ${ }^{2}$, Charlotte Warren ${ }^{3}$, Grainne Gorman ${ }^{3}$, Douglass Turnbull ${ }^{3}$, Avan Sayer ${ }^{1}$

${ }^{1}$ AGE Research Group, Institute of Neuroscience, Newcastle University, Newcastle upon Tyne, UK, ${ }^{2}$ Newcastle Magnetic Resonance Centre, Institute of Cellular Medicine, Newcastle University, Newcastle-upon-Tyne, UK, ${ }^{3}$ Wellcome Trust Centre for Mitochondrial, Newcastle-upon-Tyne, UK

Introduction: The loss of mitochondrial function and content have been implicated in sarcopenia although they have been little studied in the very old, the group in which sarcopenia is most common. Our aim was to determine if mitochondrial function and content are preserved among healthy 85 -year-olds.

Methods: We recruited 19 participants (11 female) through their general practitioner and assessed their medical history and self-reported physical activity. We identified sarcopenia using grip strength, Timed Up-and-Go and bioimpedance analysis. We assessed mitochondrial function using phosphorous magnetic resonance spectroscopy, estimating tau1/2 $\mathrm{PCr}$, the recovery half-time of phosphocreatine in the calf muscles following aerobic exercise. We performed a biopsy of the vastus lateralis muscle and assessed mitochondrial content by measuring levels of subunits of complex I and IV of the respiratory chain, expressed as Z-scores relative to that in young controls.

Results: Participants had a median (IQR) of $2(1,3)$ long-term conditions, reported regular aerobic physical activity, and one participant $(5.3 \%)$ had sarcopenia. Sixteen participants completed the magnetic resonance protocol and the mean (SD) tau1/2 $\mathrm{PCr}$ of 35.6 (11.3) seconds was in keeping with preserved mitochondrial function. Seven participants underwent muscle biopsy and the mean fibre Z-scores were $-0.3(0.4)$ and $-0.7(0.7)$ for complexes I and IV, respectively, suggesting preserved content of mitochondrial respiratory chain enzymes.
Conclusion: Muscle mitochondrial function and content are preserved in a sample of healthy, well-functioning 85-year-olds, among whom sarcopenia was uncommon. The results from this study will inform future work examining the association between mitochondrial deficiency and sarcopenia.

\section{P-241}

Systematic review on the pharmacotherapy of type 2 diabetes mellitus in functionally impaired elderly-sub-project of the Medication and Quality of Life in frail older persons (MedQoL) Research Group

Claudia Bollig ${ }^{1}$, Gabriel Torbahn ${ }^{1}$, Viktoria Mühlbauer ${ }^{2}$, Simone Brefka ${ }^{2}$, Dhayana Dallmeier ${ }^{2}$, Jürgen M. Bauer ${ }^{3}$, Walter E. Haefeli ${ }^{4}$, Michael Denkinger ${ }^{2}$, Sebastian Voigt-Radloff ${ }^{1}$

${ }^{1}$ Institute for Evidence in Medicine (for Cochrane Germany Foundation), Medical Center University of Freiburg, Faculty of Medicine, University of Freiburg, Freiburg, Germany,

${ }^{2}$ AGAPLESION Bethesda Clinic Ulm, Geriatric Research Unit, Ulm University and Geriatric Center Ulm/Alb-Donau, Ulm, Germany,

${ }^{3}$ Center for Geriatric Medicine, University of Heidelberg and AGAPLESION Bethanien Hospital, Heidelberg, Germany,

${ }^{4}$ Department of Clinical Pharmacology and Pharmacoepidemiology, University of Heidelberg, Heidelberg, Germany

Introduction: The benefit-risk balance of pharmacological therapies may depend on the functional status of older patients. Functionality is considered to be a more appropriate predictor for health status than chronological age. The EMA recently recommended a list of assessment instruments on functional health to be used for baseline characterization of older populations in clinical trials [1].

Objectives: To conduct a systematic review to investigate the effects of anti-diabetic agents in older patients with type 2 diabetes mellitus and at least moderately impaired functional status. An additional aim was to compare different blood sugar targets (as defined by study authors) in this population.

Methods: We systematically searched for randomised controlled trials (RCTs) and prospective non-randomised controlled trials (nonRCTs). In addition to MEDLINE, Embase and Central we also searched reference lists of included studies. Possible comparisons were antidiabetics versus placebo, no medication or versus another pharmacotherapy or comparisons of different blood sugar targets. Study selection, data extraction, and risk of bias assessment of the studies were conducted by two reviewers independently. Conflicts were resolved through discussion or a third person.

Results: The searches identified 12,955 results on RCTs and 3933 results on non-RCTs. Screening, data extraction and risk of bias assessment will be completed in summer 2018 and results will be presented at the conference.

Conclusion: Research on antidiabetic therapy in impaired older persons identified by functional status is currently an area in progress. This systematic review will summarize results of RCTs and prospective non-RCTs including an at least moderately functional impaired population.

\section{Reference:}

1. European Medicines Agency: Physical frailty: instruments for baseline characterisation of older populations in clinical trials (2018). Available from: http://www.ema.europa.eu/ema/index.jsp?curl=pages/ regulation/clinical_general/general_content_001232.jsp\&mid=WC0b01 ac0580032ec4 


\section{P-242}

Comparing the predictive accuracy of comorbidity and disability for frailty in patients hospitalized in a tertiary hospital

Pedro Oliveira ${ }^{1}$, Ana Maria Canelas ${ }^{1}$, Rita Amorim Costa $^{1}$, Pedro Salvador $^{1}$, Rafaela Verissimo ${ }^{1}$, Agripino Oliveira ${ }^{1}$

${ }^{1}$ Centro Hospitalar de Vila Nova de Gaia/Espinho, Portugal

Background: Frailty predicts inpatient mortality and length of stay, but its link to functional trajectories and comorbidity is under researched.

Objective: Evaluate and compare multimorbidity and disability with frailty.

Methods: Retrospective observational study in a tertiary Portuguese hospital. We analyzed all inpatients in the year 2016. Data was extracted from the hospital's information systems. Patients were classified as no frail with clinical frailty scale [CFS 1-4], and frail [CFS 5-8]. Disability was retrospectively measured with the instrument of basic activities of daily living [ADL-Katz] at preadmission. We used binary logistic regressions to evaluate frailty, adjusted for age, disability and multimorbidity. We considered a $95 \%$ confidence interval, and tests with a p-value $<0.05$ were considered to be statistically significant.

Results: The records of 10656 patients, $1 / 2$ of hospitalization episodes, were reviewed. There were $54.1 \%$ females and median age of 66 years. The age group $\geq 65$ years was $53.2 \%$, and with age $\geq 75$ was $33 \%$. Disability were present in $26.4 \%$ of the episodes, whereas $87.6 \%$ had frailty $(\mathrm{RR}=19.7)$. multimorbidity accounted for $73.2 \%$ of the episodes, whereas $95 \%$ had frailty $(\mathrm{RR}=6.91)$. Frailty had $\mathrm{OR}=47.19$ for disability and $\mathrm{OR}=2.22$ for multimorbidity.

Conclusion: In this study frailty is linked to disability and multimorbidity Prospective work is needed to confirm these trajectories and understand how to influence them.

\section{P-243}

Characteristics of sarcopenic patients admitted for hip fracture

MN Vaquero-Pinto ${ }^{1}$, C Sánchez-Castellano ${ }^{1}$, A Merello-De Miguel ${ }^{1}$, C Miret-Corchado ${ }^{1}$, AJ Cruz-Jentoft ${ }^{1}$

${ }^{1}$ Hospital Universitario Ramón y Cajal, Madrid, Spain

Introduction: This is a substudy of an ongoing study that aims to identify biological markers for the early diagnosis of sarcopenia. The aim was to assess the prevalence of sarcopenia in elderly patients admitted for hip fracture to an Orthogeriatric unit and their characteristics.

Methods: Patients admitted for hip fracture who agreed to participate in the study were included, excluding those carrying a pacemaker and those who have undergone urgent surgery. Muscle mass was assessed preoperatively using bioimpedance analysis, Janssen's (J) and Masanés (M) Spanish reference cutoff-points were used to define low muscle mass. Strength was assessed with handgrip strength (Jamar's dynamometer). Assessment included socio-demographic data, cognitive status (GDS-Reisberg), functional status (Barthel, FAC), nutrition (MNA-SF, BMI), number of falls, medications.

Results: $\mathrm{N}=150$. Mean age: $87.6 \pm 4.9$. Women: $78.7 \%$. Sarcopenia prevalence varied from $11.5 \%(\mathrm{~J})$ to $34.9 \%(\mathrm{M}) .67 \%$ had mild dependence, $18 \%$ moderate-severe dependence. $78 \%$ had independent ambulation before the fracture, $88 \%$ without technical aids or a cane, $40 \%$ reported two or more previous falls. $22 \%$ had mild dementia and $16 \%$ moderate-severe. $85 \%$ were on 4 or more drugs before admission. $13 \%$ presented malnutrition $13 \%$ and $42 \%$ overweight or obesity. In multivariate analysis, sarcopenic patients (M) had a lower body mass index ( 18.6 vs $24.3, \mathrm{p}=0.003$ ), and no differences were found in any of the others variables.

Conclusions: A third of the elderly patients admitted for hip fracture presented sarcopenia (according to the national Masanés cut-off points). Sarcopenic patients are similar to non-sarcopenic patients, except for a lower BMI

\section{P-244}

Low serum creatinine values overestimate renal function: the paradoxical relationship between mortality and eGFR in orthogeriatrics

Antonella Giordano ${ }^{1}$, Giulia Rivasi ${ }^{1}$, Virginia Tortù ${ }^{1}$, Daniela Balzi ${ }^{2}$, Iacopo Iacomelli ${ }^{3}$, Enrico Mossello ${ }^{1}$, Maria Flora D'Andria ${ }^{1}$, Lorella Lambertucci $^{1}$, Carlo Rostagno ${ }^{1}$, Alessandro Cartei ${ }^{1}$, Andrea Ungar ${ }^{1}$

${ }^{1}$ Careggi University Hospital-University of Florence, Florence, Italy, ${ }^{2}$ Health Company of Florence-Epidemiology Department, , Florence, Italy, "Santa Maria alla Gruccia" Hospital, Montevarchi (AR), Italy

Introduction: Serum creatinine is strictly related to muscle mass. Our study aimed at investigating the relationship between mortality and renal function as estimated by glomerular filtration rate formulas (eGFR) in the orthogeriatric setting, where frailty and sarcopenia are highly prevalent.

Methods: This is a prospective study carried out in the Orthogeriatric Unit of Careggi Hospital, including 569 consecutive patients aged 65 or older, with bone fractures requiring surgical treatment. Serum creatinine and cystatin $\mathrm{C}$ were determined at admission and GFR was estimated according to different formulas (CKD-EPIcr, CKD-EPIcrcysC, CKD-EPIcysC, BIS-1, BIS-2). Mortality was analyzed using data from regional registers.

Results: The study population had a mean age of $82.7 \pm 8.2$ years (77\% female). The $75 \%$ was admitted for hip fracture. At admission mean serum creatinine and cystatin C were $0.93 \mathrm{mg} / \mathrm{dL}$ and $1.48 \mathrm{mg} /$ dL, respectively. During a 4.5-year follow-up, all-cause mortality was $42.7 \%(\mathrm{n}=243$ patients). A preliminary analysis using Kaplan-Meier plots showed that participants with CKD-EPIcr eGFR $>80 \mathrm{~mL} / \mathrm{min} /$ $1.73 \mathrm{~m}^{2}$ had higher mortality than those with CKD-EPIcr eGFR $60-79 \mathrm{~mL} / \mathrm{min}$ all over the follow-up duration. At approximately 2 years of follow-up, the curves referring to eGFR $>80 \mathrm{~mL} / \mathrm{min} / \mathrm{m}^{2}$ and eGFR $<44 \mathrm{~mL} / \mathrm{min} / 1.73 \mathrm{~m}^{2}$ overlapped.

Key conclusions: All-cause mortality was similar in patients with eGFR $>80 \mathrm{~mL} / \mathrm{min} / \mathrm{m}^{2}$ and eGFR $<44 \mathrm{~mL} / \mathrm{min} / 1.73 \mathrm{~m}^{2}$, after 2 years of follow-up. These data suggest that higher eGFRs probably result from low serum creatinine in patients with sarcopenia. Cystatin $\mathrm{C}$ may be a useful alternative renal marker, in the orthogeriatric setting.

\section{P-245}

Prevalence of frailty and malnutrition in older people attending a health literacy event in Lisbon

Sofia Duque ${ }^{1}$, Paulo Almeida ${ }^{1}$, Marco Ribeiro Narciso ${ }^{1}$, João Gorjão Clara ${ }^{1}$, Luís Campos ${ }^{2}$

${ }^{1}$ Geriatrics Study Group (GERMI)-Portuguese Society of Internal Medicine, Lisbon, Portugal, ${ }^{2}$ Portuguese Society of Internal Medicine, Lisbon, Portugal

Introduction: Health literacy and patients empowerment are important interventions to achieve a healthy status, an active and healthy ageing and quality of life. The Portuguese Society of Internal Medicine (SPMI) held in July 2017, the "Health Party" in Belém gardens, 
targeted to citizens, to promote healthy lifestyle behaviors, to screen for chronic diseases and to educate about its management. The Geriatrics Study Group of the SPMI (GERMI) set up a booth targeted to citizens 65 years-old or more to screen for malnutrition and frailty and to educate about their prevention. OBJECTIVES: To identify sociodemographic characteristics of citizens who attended the GERMI booth and the prevalence of malnutrition and frailty.

Methods: Prospective study for 2 days. Screening and counseling was performed by doctors, medical students and physiotherapists. Malnutrition was screened applying Mini Nutritional Assessmentshort form (MNA-SF) and Frailty by gait speed $(<0.8 \mathrm{~m} / \mathrm{s})$, timedup-and-go test (TUGT) time ( $>10 \mathrm{~s}$ ) and PRISMA 7 questionnaire ( $>3$ points).

Results: 145 citizens visited GERMI booth, 66.2\% women. According MNA-SF: $1.4 \%$ malnourished, $17.2 \%$ at risk of malnutrition, $79.3 \%$ well nourished. Average gait speed $1.04 \mathrm{~m} / \mathrm{s}, 14.5 \%$ gait speed $<0.8 \mathrm{~m} / \mathrm{s}$. Average TUGT time $10.8 \mathrm{~s}, 42 \%$ TUGT time $>10 \mathrm{~s}$. Average PRISMA 7 score 1.04 points, $4.1 \%>3$ points. Conclusion: Physical frailty screening tests yielded different results (gait speed $14.5 \%$ vs TUGT 42\%). According PRISMA 7, social frailty is not a prevalent condition. If TUGT results are considered, physical frailty may be common in the community, although malnutrition was rarely found.

\section{P-246}

Treating blood pressure: what matters in later life?

Andrew Clegg ${ }^{1}$, Mary Godfrey ${ }^{1}$, Chris Gale ${ }^{1}$, Chris Wilkinson ${ }^{1}$, Marlous Hall ${ }^{1}$

${ }^{1}$ University of Leeds, Leeds, UK

Current international guidelines do not include advice for clinicians on the management of blood pressure for older people with frailty. From both trial and observational literature and from our own qualitative research with older people, we summarise evidence to determine whether blood pressure should be managed differently in the context of frailty. We performed a meta-analysis of eight cohort studies comprising 161,081 older adults. This showed that for older people with frailty, lower blood pressure was associated with higher mortality. However, a systematic review of two randomised control trials measuring frailty showed that low blood pressure reduced mortality. We also report the findings of 20 qualitative interviews exploring factors influencing blood pressure treatment in later life with older people themselves. These findings demonstrate different representations of frailty: in the trial and observational literature; between physicians and older people themselves; and between older people with one another. We discuss these challenges, and consider how to develop better evidence to manage blood pressure in later life.

\section{P-247}

The relationship between hemoglobin $\mathrm{A1C}(\mathrm{HbA1c})$ variability and frailty in elderly outpatients

Olgun Deniz ${ }^{1}$, Suheyla Coteli ${ }^{1}$, Hacer Dogan Varan ${ }^{1}$, Rana Tuna Dogrul ${ }^{1}$, Cagatay Cavusoglu ${ }^{1}$, Ali Oncul ${ }^{1}$, Muhammet Cemal Kizilarslanoglu ${ }^{1}$, Berna Goker ${ }^{1}$

${ }^{1}$ Gazi University School of Medicine, Department of Internal Medicine, Division of Geriatrics, Ankara, Turkey

Introduction: Frailty is a geriatric syndrome that significantly affects morbidity and mortality in elderly patients. The frequency of chronic comorbidities such as diabetes mellitus (DM) can be seen more in frail elderly than not. There is a new area of interest in the literature in studies of the relationship between frailty and insulin resistance and DM. In this study, we aimed to investigate the relationship between HbA1c-variability and frailty.

Methods: In a period of one year,452 elderly patients whose frailty status were known were included for the study. All patients underwent comprehensive geriatric assessment. Frailty status of the patients was evaluated by Fried Frailty Scale and grouped as frail, pre-frail and robust. 151(33.4\%) of the patients had DM. The retrospective HbAlc values of these patients were reached. The relationship between frailty and $\mathrm{HbA1c}$ variability was evaluated in 76 patients with at least three HbA1c measurements. The following parameters were calculated for $\mathrm{HbA1c}$ variability: HbA1c mean (HbA1c-M), $\mathrm{HbA1c}$ standard deviation (HbA1c-SD) and HbA1c variation coefficient $(\mathrm{HbA} 1 \mathrm{c}-\mathrm{VC})$.

Results: The mean age of the patients was $76 \pm 6$ years and 46 $(60.5 \%)$ patients were female. The most frequent concomitant comorbidity was hypertension with $86.8 \%$. The rate of frail patients was $19.7 \%$. Median HbA1c-M [7.93 (min-max: 6.61-9.60) vs 6.92 (min-max: 5.68-11.40)], median HbAlc-SD [0.93 (min-max: $0.19-2.69)$ vs. 0.58 (min-max: 0.17-2.69)] and median HbA1c-VC [0.13 (min-max: 0.03-0.36) vs. 0.09 (min-max: 0.03-0.36)] were found to be significantly higher in frail patients than in non-frail patients $(\mathrm{p}=0.010, \mathrm{p}=0.016, \mathrm{p}=0.026$, respectively). Conclusion: In this study, it has been shown that there may be relationship between $\mathrm{HbA1C}$ variability and frailty. Large prospective studies are needed to support these results.

\section{P-248}

Osteoprotegerin as a novel marker of prognosis in elder patients: a cohort study

VG, Vincenzo Gianturco ${ }^{1}$, VMS, Vincenzo Maria Saraceni ${ }^{2}$, LG, Luigi Gianturco ${ }^{3}$

${ }^{1}$ Filippo Turati Foundation, Zagarolo, Rome, ${ }^{2}$ Complex Unit of Physical Medicine and Rehabilitation, Policlinico Umberto I Hospital, "Sapienza" University of Rome, Rome, Italy, ${ }^{3}$ ICBM, Vigevano, Pavia, Italy

Osteoprotegerin (OPG) is a cytokine in the tumour necrosis factor receptor superfamily. OPG has been linked to cardiovascular disease and to the development of heart failure after myocardial infarction. In patients with chronic heart failure, OPG levels are associated with mortality in patients with systolic heart failure of any cause. We aimed to elucidate in detail the role of OPG as a marker of mortality and CV risk in elder subjects suffered from heart failure. Therefore, we explored the possible link between OPG, sarcopenia and malnutrition. This is a cohort, multicentre study. Elder patients ( $>65$ years) were collected and followed up for 3 years. Participants were separately divided into quartiles according to their baseline OPG levels and descriptive analyses of baseline characteristics were performed. Cumulative event rates (mortality, CV events) were calculated across quartiles of OPG with the Kaplan-Meier method and compared by use of a trend test. Cumulative event rates were also calculated stratifying patients on the basis of OPG levels and diagnosis of sarcopenia and/or malnutrition. Models were adjusted for the following clinical risk factors: age, sex, drugs, history of hypertension, history of diabetes mellitus, tobacco use, prior $\mathrm{CV}$ events, left ventricular ejection fraction, cognitive function. Among the 324 participants with a baseline measurement, the median level of OPG was $6.9 \mathrm{pMol} / \mathrm{L}$. Sarcopenia and malnutrition showed to be significantly associated with elevation of serum OPG. Before and after adjustment for traditional clinical risk factors, elevated concentrations of OPG (4th 
quartile group) remained independently associated with an increased risk of mortality or CV events (especially MI). Elevated serum OPG seems to be associated with an increased mortality and CV events. Further studies needed to explain the specific role of this protein in this particular type of patient.

\section{P-249}

\section{Hip fracture and $25(\mathrm{OH}) \mathrm{D}$ is connected, but there is more}

Sigrun Skuladottir ${ }^{1,2,3}$, Ingibjorg Hjaltadottir ${ }^{1}$, Lenore Launer ${ }^{4}$, Tamari Harris ${ }^{4}$, Paolo Caserotti ${ }^{5}$, Mary Cotch ${ }^{6}$, Thomas Lang ${ }^{7}$, Gudny Eiriksdottir ${ }^{8}$, Kristin Siggeirsdottir ${ }^{8}$, Vilmundur Gudnason ${ }^{3,8}$, Gunnar Sigurdsson $^{3,8}$, Laufey Steingrimsdottir ${ }^{2}$, Thorhallur Halldorsson ${ }^{2}$

${ }^{1}$ The Icelandic Gerontological Research Institute, Iceland, ${ }^{2}$ Faculty of Food Science and Nutrition, University of Iceland, Iceland,

${ }^{3}$ Landspitali National University Hospital of Iceland, Iceland,

${ }^{4}$ National Institute on Aging, Intramural Research Program, Laboratory of Epidemiology and Population Sciences, Bethesda, MD USA, ${ }^{5}$ Department of Sports Science and Clinical Biomechanics, University of Southern, Denmark, Odense, Denmark, ${ }^{6}$ Division of Epidemiology and Clinical Applications, National Eye Institute, National Institutes of Health, Bethesda, MD, USA, ${ }^{7}$ Department of Radiology and Biomedical Imaging, University of California, San Francisco, CA, USA, ${ }^{8}$ Icelandic Heart Association Research Institute, Kopavogur, Iceland

Introduction: Hip fractures is one of the most serious fracture that elderly may experience. Serum level of 25-hydroxy vitamin D $25(\mathrm{OH}) \mathrm{D}$ has been shown to be associated with risk of hip fracture. Other risk factors associated with hip fracture also tend to cluster among subjects with poor serum 25OHD status.

Aim: To examine other risk factors than serum $25(\mathrm{OH}) \mathrm{D}$ that may predict the risk of hip fractures independent of serum 25(OH)D.

Methods: Longitudinal study of 5764 participants (mean age 77 years at baseline) from the Age, Gene/Environment SusceptibilityReykjavik Study (AGES-Reykjavik) 2002-2006. Extensive clinical measurements including CT-scans, functional and leaning tests and history of past and present health was recorded.

Results: During follow-up of 9.7 years, were 486 hip-fractures (144 occurred in men) here of where 121 cases who had serum $25(\mathrm{OH}) \mathrm{D}$ lower than $30 \mathrm{nmol} / \mathrm{L}$. Men and women who experienced hip fractures were significantly ( $\mathrm{p}<0.001)$ older $(80 \mathrm{vs} 77$ years) and had lower serum 25(OH)D (48 vs $54 \mathrm{nmol} / \mathrm{L}, \mathrm{p}<0.001)$. After adjusting for age, gender and serum vitamin $\mathrm{D}$, on unit higher BMI was associated with a hazard ratio (HR) $0.92(95 \%$ CI $0.90,0.95)$ lower risk of fracture. The corresponding estimate for fat mass ( $\mathrm{kg}$ ), was 0.96 (95\% CI 0.94, 0.98). For leg strength the HR was 0.995 (95\% CI 0.994, 0.997) of each 1-N increase in strength; but 0.98 (95\% CI $0.97,0.98)$ for each $1-\mathrm{cm}^{2}$ increase in thigh muscle area. For each 1 -second increase in a Time up and go test risk increased by 1.08 (95\% CI 1.06, 1.10). Similarly, the better balance was inversely associated with hip fracture risk.

Key conclusions: After adjusting for age, gender and 25(OH)D, many factors affect the risk of hip fracture among elderly.

\section{P-250}

Prevalence of sarcopenia and fragility in the older adults with high comorbidity

Vincenzo Malafarina ${ }^{1}$, Letizia Suescun Puerta ${ }^{2}$, Igone $\operatorname{Imaz}^{1}$, Francisco Uriz Otano ${ }^{3}$, Iñaki Artaza Artabe ${ }^{4}$
${ }^{1}$ Complejo Hospitalario de Navarra, Pamplona, Spain, ${ }^{2}$ Hospital Reina Sofia de Tudela, Spain, ${ }^{3}$ Hospital San Juan de Dios de Pamplona, Spain, ${ }^{4}$ Igurco Unbe Functional recovery unit, IMQ group, Bilbao, Spain

Introduction: The aim of this study is to know the prevalence of sarcopenia and frailty in older adults with high comorbidity.

Methods: Prospective observational study (August 1-October 31, 2017). Subjects $>65$ years who attended the pluripathologic chronic unit. Those subjects who came with an ongoing acute process have been excluded. The SPPB and the Barthel index have been registered. For the study of sarcopenia, the criteria proposed by the EWGSOP (4 meters gait speed, grip strength and bioimpedance analysis, to calculate the skeletal mass index SMI) have been used. The anthropometric indexes have been calculated. For frailty we have used the FRAIL scale.

Results: 199 subjects were included (49\% women), $84.1 \pm 6.1$ years, Barthel 85 (65-95). Subjects with sarcopenia (n98, 48\%) were older $(85 \pm 5.9$ vs $83.7 \pm 5.8, \mathrm{p}=0.118)$ and had a significantly lower BMI than non-sarcopenic patients $\left(24.5 \pm 3.4 \mathrm{vs} 28.6 \pm 4.9 \mathrm{~kg} / \mathrm{m}^{2}\right.$, $\mathrm{p}<0.001)$. The prevalence of sarcopenia has been higher in men than in women $(69.8 \%$ vs $34.7 \%, \mathrm{p}<0.001)$. Walking speed was lower in 151 subjects $(76 \%)$, being older $(85.1 \pm 5.4$ vs $81.1 \pm 7.3$ years, $\mathrm{p}<0.001$ ) and with lower values of muscle mass (SMI $8.34 \pm 1.92$ vs $9.69 \pm 2.14 \mathrm{~kg} / \mathrm{m}^{2}$ ) with respect to subjects with normal gait speed. Of these, 139 subjects had reduced grip strength, and severe sarcopenia could be diagnosed in 73 subjects. We observed a prevalence of frailty of $51 \%$ (n 89), $85.7 \pm 4.9$ years vs $82.4 \pm 7.1$ of the non-frail group $(\mathrm{p}<0.001)$. The Barthel of the frail patients was $70(60-85)$ vs $95(85-100)$ of the non-frail patients $(p<0.001)$. Conclusions: Sarcopenia and frailty are very prevalent in the older adults with high comorbidity. Sarcopenia is associated with impaired nutritional status and frailty with greater functional dependence.

\section{Area: Geriatric education}

\section{P-251}

Prescriptive appropriateness-pharmacological deprescribing in elderly subjects

Tiziana Candiani ${ }^{1}$, Ilario Stefani ${ }^{2}$, Antonino Mazzone ${ }^{2}$

${ }^{1}$ General Medicine-Geriatric Hospital Cuggiono ASST WEST Milanese, Italy, ${ }^{2}$ General Medicine Hospital Legnano ASST WEST Milanese, Italy

The problem of poly-pharmacotherapy $(11 \%)$ in the elderly patient with multimorbidity is now recognized. It is also known that such subjects take on average at least five or more active ingredients per day, with the risk of drug interactions and adverse reactions (remembering that the aging process is accompanied by pharmacodynamic and pharmacokinetic modifications able to modify the risk ratio-benefication of pharmacological treatment). Recent studies show that inadequate prescriptions in the elderly subject, poor therapeutic adherence (46\%), cognitive impairment, fragility and rehospitalization can significantly and negatively influence the quality of life and the state of health. Therefore, the possibility of suspending an inappropriate active substance (deprescribing), in order to reduce the risks compared to the expected benefits, is still the subject of studies of international interest. In this perspective the integration between medical specialists Geriatrics/Internists/Pharmacologists, hospital/outpatient and local physicians specialists plays a fundamental role in the preparation of individual-personalized therapeutic pathways. To this end, a training course has been designed with a business - territorial value, repeated in different editions, aimed at (1) to improve the quality of the prescription that must be appropriate and 
safe (2) to verify the therapeutic adhesion by periodically reviewing the active ingredients taken by the patient (3) to evaluate the economic benefits of deprescribing

\section{P-252}

Prevalence and predictors of sleep disorders among institutionalized elderlies: a cross sectional study

\author{
Mohamad Itani ${ }^{1}$, Nabil Naja ${ }^{2}$, Ghassan Hamadeh ${ }^{3}$ \\ ${ }^{1}$ Nicosia University, Nicosia, Cyprus, ${ }^{2}$ Dar Al-Ajaza Alislamia \\ Hospital, Beirut, Lebanon, ${ }^{3}$ Americam University Hospital of Beirut, \\ Beirut, Lebanon
}

Background: Aging is a worldwide problem with increasing number of elderly people requiring institutionalized care in developing countries. This is a cross section observational study, which aims to identify the prevalence of sleep problems/disturbances in an institutionalized population of elderlies living in a philanthropic chronic care center with primary care hospital facilities located in an upper middle-income country-Lebanon.

Methods: The study involved 192 institutionalized elderly persons who have been living in the institution for more than 1 month. After filling an informed consent form, the participants were required to complete the Pittsburgh Sleep Quality Index (PSQI) questionnaires either on their own or by the nurses who take care of them. Our dependent variable was participants with a global PSQI score of five or more who were considered to have a sleep problem. The independent variables were demographic data, life style habits, current diseases and medications that were collected by the primary investigator from the participants' medical files. Statistically analysis was performed using the SPSS statistical analysis software.

Results: Participants were between 60 to 102 years old with a mean age of 77.4 years ( $\mathrm{SD}=8.7$ years). According to the global PSQI scores, the figure of $75 \%$ of the participants were found to have sleep problems (95\% CI of 69-81\%) with equal male to female distribution, in comparison to $27.6 \%$ of the participants who reported subjective sleep problems. Decreased activity and poor performance on memory tests were the only demographic and life style variables that had statistical association with sleep problems (OR 2.85, 95\% CI 1.41-5.68 and OR 4.21, 95\% CI 1.90-9.33 respectively). Alzheimer disease and schizophrenia were the only medical conditions that had statistical association with sleep problems. Alzheimer disease had a positive association (OR 2.28, 95\% CI 1.05-4.94), while schizophrenia had a negative association (OR 0.46, 95\% CI $0.23-0.92$ ). Among the medications that were studied proton pump inhibitors were the only drugs that approached significant association with sleep problems on statistical analysis (OR 1.85, 95\% CI $0.96-3.59)$.

Conclusion: Sleep problems is a major issue in elderly persons present in $75 \%$ of persons living in an elderly chronic care center in Lebanon. Its prevalence is underestimated by the nursing staff and under reported by the patients. For that reason, it needs to be frequently assessed by using a standardized sleep assessment tool like the PSQI questionnaire. Our study found an association between sleep problems and decreased activity, and the failure to pass either mini mental state examination or clock drawing memory tests. Alzheimer disease was positively associated with sleeping problems, while schizophrenia was negatively associated with them. The chronic intake of proton pump inhibitors was the only drugs that approached statistically significant association with sleep problems in our analysis. Further randomized control studies are required to confirm our findings.
P-253

Challenges of geriatrics and gerontology education in the eastern mediterranean region: reflection on the collaboration of MEAMA and ATG

Abdulrazak Abyad ${ }^{1}$, Sonia Hammami ${ }^{2}$

${ }^{1}$ Middle East Academy for Medicine of Ageing, Tunis, Tunisia, ${ }^{2} \mathrm{CHU}$ F Bourguiba Monastir-Tunis, Tunisia

As we enter the 21 st century, and increasing number of older adults will need more services and medical attention. Assuming the majority of medical care for this population will be provided by primary care practitioners. Therefore, training in Geriatric care must become an integral part of the curriculum for internal and family medicine residency programs. The changing demographics of the population in the region have created imperative for medical schools to increase their emphasis on the education and training of medical students and residents in the care of the elderly. In attempt to answer the above problem and challenges The Middle-East Academy of Medicine of Aging (MEAMA) was started in 2002. So far the Academy had organized five postgraduate courses each with 4 sessions in a 2 year cycle. The organizers of the Middle-East Academy for Medicine of Ageing organise this course with support of many regional, government and international organizations. The Tunisian Geriatric Association (ATG) in Collaboration with MEAMA has launched the first intensive postgraduate course in geriatric medicine stimulating interest and knowledge in geriatrics. The course is made of 4 sessions, that last 2 days each and will be finished within a year. The course is geared toward physicians mainly internists and family physicians. The topics of the four sessions will cover the major issues of geriatrics practice and issues including First session: metabolic disorders Second session: osteoporosis, Vitamin D, Falls Assessment and Prevention, rehabilitation, sarcopenia. Third session: Cognitive disorders; Diagnosis and Management of Dementia, Delirium and Depression, Fourth session: Comprehend the major geriatric syndromes The last session took place in May 2017 in Monastir Tunisia. A total of 42 participants graduated from the course. Evaluation of the course will be presented. The same cycle will be repeated next year and will be opened to people coming from outside Tunis.

\section{P-254}

What things in life are most important to older adults? And what do they know about geriatricians?

S. V. Hope ${ }^{1}$, H. Kerminen ${ }^{2}$, A. Koutsouri ${ }^{3}$, S. Marien ${ }^{4}$, M. R. Mellingsæeter ${ }^{5}$, H. M. Roitto ${ }^{6}$, B. Saka ${ }^{7}$,

F. J. Tarazona-Santabalbina ${ }^{8}$, K. Singler ${ }^{9}$

${ }^{1}$ University of Exeter \& Royal Devon \& Exeter NHS Trust, Exeter, UK, ${ }^{2}$ University of Tampere \& Tampere University Hospital,

Tampere, Finland, ${ }^{3}$ Henry Dunant Hospital Center, Athens, Greece, ${ }^{4}$ Geriatric Medicine, Cliniques Universitaires Saint-Luc, Brussels, Belgium, ${ }^{5}$ Department of Geriatric Medicine, Akershus University Hospital, Norway, ${ }^{6}$ Department of General Practice and Unit of Primary Health Care, University of Helsinki \& Department of Social Services and Health Care, Helsinki Hospital, Helsinki, Finland, ${ }^{7}$ Istanbul Medical School, Department of Internal Medicine, Division of Geriatrics, Istanbul University, Istanbul, Turkey, ${ }^{8}$ Geriatric Medicine Department, Hospital Universitario de la Ribera, Alzira, Valencia, Spain \& Faculty of Medicine, Catholic University of Valencia, San Vicente Màrtir, Spain, ${ }^{9}$ Institute for Biomedicine of Ageing, Friedrich-Alexander-University Erlangen-Nürnberg \& 
Geriatric Medicine, Klinikum Nürnberg, Paracelsus Private Medical University Nürnberg, Germany

Background: As 2017/18 European Academy for the Medicine of Ageing (EAMA) participants preparing a short video to promote geriatrics to the older public for dissemination across Europe, we wished to understand what is important to our older people as they get older and what they already know about geriatricians.

Methods: Eight geriatricians from Belgium, Finland, Greece, Norway, Spain, Turkey and UK developed an 8-question survey with which to interview a random selection of our inpatients, outpatients and non-patients in April 2017.

Results: 91 older adults (5-39 per country) were asked. Knowledge about geriatricians was fairly limited: "...taking care of those with dementia?" (Norway), “...look after geriatrics - i.e. patients who can't get to the loo" (UK). Things considered important as getting older were consistent both between and within countries: family and friends, maintaining functional independence, general good health. "My family, my health, enough money, being happy" (Turkey), "laugh and enjoy my hobbies" (Spain). Health-related aspects considered important were also consistent: mobility, not falling, no pain, sight and hearing, memory. "Walking - being able to read - avoid depression due to losses" (Greece), “...water aerobics, healthy nutrition... correct medication" (Finland). Conclusions: This was a small survey, but it gave interesting pan-European insights, re-emphasising the importance of holistic care of older people. Despite increasing awareness of an ageing population, it seems that the potential role of geriatricians is not currently well-communicated to older people, supporting the justification for making a video (translated into many languages) to promote geriatrics across Europe.

\section{P-255}

Improving access to geriatric medicine training for core medical trainees through the new joint royal of physicians training board internal medicine curriculum

\section{L. Clifford Lisk ${ }^{1}$, E. C. Elizabeth Carty ${ }^{2}$ \\ ${ }^{1}$ BH Barnet Hospital, UK, ${ }^{2}$ WC Whipps Cross Hospital, UK}

The UK population is ageing, and it is critical that doctors in training have exposure to and training in Geriatric Medicine. A new Internal Medicine (IM) has recently been approved by the General Medical Council. Stage 1 Training in IM comprise the first 3 years post Foundation training. Mandatory items in stage 1 training comprises Acute Medicine, Acute take, outpatient, Geriatric Medicine, critical care and simulation experience. This study aims to review the progress made at incorporating Geriatric Medicine within the first 2 years of IM training in Northeast and Northcentral London programmes comprising 200 trainees. An IM project Group was convened in 2016 comprising the heads and deputy heads of school, lead Training programme directors (TPDS) and project managers. Two IM workshops were held in 2017 to facilitate discussion of rotation alterations and compulsory curriculum requirements. Subsequently lead TPDS with support from local TPDS, trainers, trainee representatives, Clinical and Medical directors and head of school leads created new rotations to meet IM curricular requirements. $40 \%$ of CMT rotations did not meet IM curriculum requirements for Geriatric Medicine. 52 new IM ready rotations with 46/52 (89\%) comprising Geriatric Medicine were created to commence in August 2018 providing training for between 4 and 6 trainees ahead of the first IM intake in August 2019. The successful integration of Geriatric Medicine into the majority of IM ready Northeast and Northcentral London Training programmes demonstrates that it is possible for Curriculum to influence change within a sector with regional and local cooperation.

\section{P-256}

G4J connect: a model for European geriatricians to educate and inspire tomorrow's geriatricians?

\author{
P. Brock ${ }^{1}$, N. Saxton ${ }^{1}$, V. Gibson ${ }^{1}$, E. Fisken ${ }^{2}$, C. Taylor ${ }^{3}$, \\ M. Garside ${ }^{1}$, J. Fisher ${ }^{1}$
}

${ }^{1}$ Northumbria Healthcare NHS Foundation Trust, Newcastle Upon Tyne, UK, ${ }^{2}$ Newcastle Upon Tyne Hospitals NHS Foundation Trust, Newcastle Upon Tyne, UK, ${ }^{3}$ North Cumbria University Hospitals NHS Trust, Carlisle, UK

Introduction: There is wide variability in postgraduate geriatric medicine training across Europe [1] with some countries not recognising geriatrics as a standalone specialty [2]. There have been calls to harmonise post-graduate training across the continent [3]. Against this backdrop we share an innovative educational approach, with potential for up-scaling across Europe, which encourages interest in the speciality and learning about core geriatric medicine competencies.

Methods: The Association for Elderly Medicine Education (AEME) was established to encourage recruitment and education in geriatrics in the United Kingdom (UK) [4]. To increase engagement with doctors prior to specialisation AEME invited 'grassroots' UK geriatricians to organise regional educational events entitled Geriatrics for Juniors (G4J) Connect. Typical events provided practical advice on managing older patients and sessions designed to overcome negative perceptions of the specialty. Registration, advertising and feedback were co-ordinated through AEME's website. Social media was used for promotion. The events were free to attend through sponsorship, voluntary speakers and free venues.

Results: Since 2014 there have been 21 G4 J Connect events in 15 UK cities. Mean number of delegates attending each is 49 (range 25-109). Modal event length is $3 \mathrm{~h}$ (range 2.5-6.5 h). Events typically took place in the evening in a city centre hospital. Feedback from delegates has been consistently positive, including decisions made to pursue a career in geriatrics following attendance.

Key conclusions: G4 J Connect events provide a model for 'grassroots' geriatricians to engage healthcare professionals with geriatrics. We invite European geriatricians to collaborate with AEME and organise an event in their region.

References:

1. Stuck AE, Jonsdottir AB, Singler K, Roller RE, Holm EA, Masud T (2016) The length of postgraduate training for geriatric medicine in European countries: an update for the year 2015. Aging Clin Exp Res 28(1):169-170

2. Singler K, Holm EA, Jackson T, Robertson G, Muller-Eggenberger E, Roller RE (2015) European postgraduate training in geriatric medicine: data of a systematic international survey. Aging Clin Exp Res 27(5):741-750

3. Fisher JM, Masud T, Holm EA, Roller-Wirnsberger RE, Stuck AE, Gordon A et al (2017) New horizons in geriatric medicine education and training: the need for pan-European education and training standards. Eur Geriatr Med 8(5-6):467-473

4. Brock P, Gibson V, Garside M, Hunt K, Fisher J (2017) New ways to teach new doctors about older people. Clin Teach 14(4):289-291.

\section{P-257}

Oncology healthcare providers' knowledge, beliefs, and communication behaviors on Lesbian, gay, bisexual and transgender patient healthcare: an exploration

Koshy Alexander ${ }^{1}$, Smita Banerjee ${ }^{1}$, Chasity Walters ${ }^{1}$, Jessica Staley ${ }^{1}$, Patricia Parker ${ }^{1}$ 
${ }^{1}$ Memorial Sloan Kettering Cancer Center, New York, USA

Introduction: Cancer is diagnosed at a higher rate (53\%), accounts for a higher percentage of survivors $(59 \%)$ and results in more deaths among individuals 65 years and older $(68 \%)$ compared with younger adults. The population at risk is growing rapidly. 5.2 to 9.5 million adults in the United States identify as Lesbian, Gay, Bisexual, and Transgender (LGBT). Scarcity of Health Care Providers (HCP) competent in LGBT health contributes to healthcare underutilization by LGBT populations. In this study, we explored knowledge, beliefs and communication behaviors of HCPs regarding LGBT patient healthcare.

Methods: Using a cross-sectional design, an anonymous survey was emailed to HCPs at a comprehensive cancer center in the United States. The survey included the following measures: demographic data, LGBT healthcare knowledge, beliefs about LGBT patients, and communication with LGBT patients. Data were analyzed using descriptive and stratified analyses.

Results: Of the $3627 \mathrm{HCPs}$ emailed, 1253 (35\%) completed the survey. Only 50 participants (5\%) correctly answered all 7 knowledge items, and about half the respondents answered 3 (out of 7) items correctly. Stratified analyses demonstrated differences in study measures by demographic measures such as profession, religious affiliation, sexual orientation, and having LGBT friends/family.

Key conclusions: The results demonstrated an overall lack of medical knowledge about LGBT healthcare among oncology HCPs. Demographic differences in HCP respondents were associated with more favorable perceptions and higher open communication behaviors with LGBT patients. Overall, the study highlighted the need for targeted LGBT sensitivity training for oncology HCPs.

\section{P-258}

Educational initiative to improve understanding of geriatric syndromes in minority community caregivers

Koshy Alexander ${ }^{1}$, Ruth Manna ${ }^{1}$, Natalie Gangai ${ }^{1}$, Rosario Costas-Muniz ${ }^{1}$, Beatriz Korc-Grodzicki ${ }^{1}$

${ }^{1}$ Memorial Sloan Kettering Cancer Center, New York, USA

Introduction: Caregivers play a vital role in the care of older adults. In 2008, 34.2 million Americans provided unpaid care to an adult over 50 years; 15.7 million cared for someone with dementia. $57 \%$ report that their daily lives are impacted by their caregiving responsibilities. Caregivers often lack knowledge and skills to ease their burden; those in underserved groups face additional barriers in accessing resources.

Methods: In collaboration with community organizations and a Federal government grant through the Health Resources and Services Administration (HRSA), the Geriatric Resource Interprofessional Program (GRIP) was set up at Memorial Sloan Kettering Cancer Center (MSKCC). It spearheaded an educational program in South Asian communities in Queens. A focus group identified four areas of need. Culturally-competent, practical-skills oriented educational workshops on safe patient handling, cognitive impairment, caregiver burden and medication management were developed and implemented.

Results: In 2016-2017, 134 caregivers attended 12 sessions at 5 locations. $88 \%$ were women; the average age was 62 . Participants spoke 12 different languages and 76\% were born in India. Knowledge increased significantly for the Cognitive Impairment $[\mathrm{t}(35)=-3.55$, p.02] and the Safe Patient Handling workshops [ $t(9)=-3.74, \mathrm{p} .05]$. Caring for the Caregiver and Medication Management sessions did not yield significant results.
Key conclusions: The assessments showed improvement in knowledge about Cognitive Impairment and Safe Patient Handling. Qualitative data showed improved understanding, plans to share knowledge and to change behaviors. Challenges to the initiative included differences in literacy, multiple cultures and languages. Successes and barriers faced in implementing the initiative will be presented.

\section{P-259}

Technology and social media: friend or foe

Geraldine Donnelly ${ }^{1}$, Rebecca Cruise ${ }^{1}$, Ray Keelan ${ }^{1}$

${ }^{1}$ Wythenshawe Hospital, Manchester University Hospital Trust, UK

Keywords: Methodological issue Personalized medicine Prevention and health systems.

Introduction: Pressure on our Older Age department is growing. Community services require specialist input however resources are limited and consultant time is valuable. Social media and technology could increase efficiency and optimise patient care however we are concerned that guidance around the topic is not clear.

Methods: Anonymous polls used to question doctors and medical students. Questions included using messenger services to discuss patient care, send photographs to acquire advice and use social media to share cases for education. Finally, we asked about exposure to professional guidance and teaching on the topic.

Results: There were 150 respondents, $57 \%$ of which were aware of existing professional guidance and $38 \%$ had received formal education on the topic. $45 \%$ thought it acceptable to send an ECG and $30 \%$ a photo of a body part, to a colleague for the purpose of gaining advice. $56 \%$ would share an anonymized X-ray on Facebook for educational purposes and $13 \%$ use WhatsApp to handover patients. $11 \%$ of those questioned admitted that they don't always act appropriately on social media. There was limited variation across the grades.

Conclusions: Despite guidance from the General Medical Council, the British Medical Association, the Medical Defence Union and the Royal Colleges of Physicians, practice is variable. There remains uncertainty on the appropriate use of social media and technology in the workplace potentially leaving clinicians vulnerable to criticism and prosecution. A national consensus should be reached on how to embrace technology to help improve education and aid patient care whilst avoiding governance issues.

\section{P-260}

Do old adults know the purpose of their medication? A survey among community-dwelling people

Hoisnard Léa ${ }^{1}$, Brigitte Santos-Eggimmann ${ }^{2}$, Pauline Chauvin ${ }^{3}$, Anne Hiance-Delahaye ${ }^{1,4}$, Marie Herr ${ }^{1,4}$

${ }^{1}$ INSERM U1168 Aging And Chronic Diseases: Epidemiological And Public Health Approaches, Université Versailles Saint-Quentin-enYvelines, France, ${ }^{2}$ Institute Of Social And Preventive Medicine, Lausanne University Hospital, Switzerland, ${ }^{3}$ Laboratoire Interdisciplinaire de Recherche Appliquée en Economie de la Santé, EA4470, Université Paris Descartes, France, ${ }^{4}$ Hôpitaux Universitaires Paris Ile-de-France Ouest, AP-HP, France

Introduction: This study assessed the level of knowledge of the purpose of medication among community-dwelling old people and examined the characteristics influencing the level of knowledge, 
focusing on drugs frequently used and/or often involved in iatrogenic events.

Methods: A cross-sectional survey was conducted in the canton of Vaud, Switzerland. Participants: 2690 community-dwelling older adults aged 68 years and over who reported at least one drug of interest. Measurements: Participants reported drugs currently taken and what they thought their purpose was. A good level of knowledge was defined as knowledge of the exact purpose or identification of the anatomical system or organ targeted by the drug. A multivariate logistic regression analysis was performed to identify the factors associated with a good level of knowledge of all the drugs of interest taken. Sample weights were used to provide representative estimates. Results: On average, $80.6 \%$ of the drugs were well-known. The most known were the non-steroidal anti-inflammatory drugs, antidiabetics, analgesics, and endocrinological drugs. The least well-known were platelet aggregation inhibitors, minerals, anticoagulants, and other narrow therapeutic index drugs. Overall, $66 \%$ of the participants had a good of knowledge of the purpose of all the drugs of interest. Polypharmacy and receiving help with drug management were negatively associated with a good knowledge (aOR4-5 drugs $0.45 ; 95 \%$ CI $0.29-0.71$, aOR $>6$ drugs 0.20 ; 95\% CI $0.13-0.31$ and aORhelp 0.42 ; 95\% CI 0.18-0.99).

Conclusions: This study shows a need for therapeutic education among patients receiving multiple drugs and specifically anticoagulants and antiplatelet inhibitors.

\section{P-261}

Perceptions regarding old age and geriatric institutions in the Lebanese society

Amine Wehbe ${ }^{1}$, Haitham Abdel Rahman ${ }^{1}$, Sani Hlais ${ }^{1}$, Antoine Aoun $^{2}$, Lana El Osta ${ }^{1}$

${ }^{1}$ Faculty of Medicine, Saint-Joseph University of Beirut, Beirut, Lebanon, ${ }^{2}$ Faculty of Nursing and Health Sciences, Notre Dame University, Zouk Mosbeh, Lebanon

Introduction: In Lebanon, there is a lack of research on the perception of ageing. Our study aimed to explore the perceptions, beliefs and attitudes of Lebanese people regarding ageism and geriatric institutions.

Methods: An observational cross-sectional study was conducted among a representative sample of the population in Beirut city, Lebanon. Data were collected through an anonymous structured selfadministered questionnaire written in Arabic, and exploring the demographics and participant characteristics, their perceptions regarding ageism and geriatric institutions, as well as their fear from ageism.

Results: A total of 400 participants completed the survey: 50.3\% were male, and only $7 \%$ were 65 years and more. Participants considered that old age starts from $64.23 \pm 8.45$ years. Most of them have negative perceptions of ageing: old age means diseases (28\%), retirement $(14.5 \%)$, solitude $(12.9 \%)$, need and loss of autonomy $(8.2 \%)$, and disability (7.5\%). Our population seems to grow old with some concern, mainly about health problems (38.4\%) and loss of autonomy $(23.7 \%)$. Only $11.0 \%$ were not at all worried. Regarding the facilities for the elderly, almost half of respondents $(51.6 \%)$ have a more positive opinion of these institutions, however $3.5 \%$ have entrusted elderly relatives to these facilities. The multivariate analysis showed a positive association between favorable perceptions of old age and higher respondents' socio economic status ( $\mathrm{p}$-value $<0.05$ ).
Conclusions: Our survey emphasized the importance of creating a positive image of old age, where it is perceived as a period of life, not a disease.

\section{P-262}

Elderly women and their fears: a pilot investigation

Carlo Cristini ${ }^{1}$

${ }^{1}$ Univresity of Brescia, Owensboro, USA

Objectives: In old age many fears may appear, linked to personal biography and to what future life may envisage. The forgotten anxieties of childhood, the unresolved conflicts of adolescence, the uncertainties along all the life-span are expressed in old age that also confronts the possible emerging issues related to age. The aim of this study was to know the most frequent fears in elderly women.

Methods: A pilot sample of 30 women, aged 60-79 years, living in Milan, who reported to be in good psychophysical health, belonging to a cultural club, participated in the investigation. An interview, which was designed for the study, was administered together with the participants completing a measure of anxiety (SAS) and of depression (GDS).

Results: The average age of the sample was 68.75 years. The majority had a tertiary level of education. The main fears were: dementia, loss of control, loneliness, existential void, difficulty cooping, disability, assaults, unforeseen events, pain and depression. The interviewees were concerned above all else that their worries will increase in the future; with respect to overall worries, they report that men are more afraid than women, older people are more worried than younger people, and immigrants more than the locals. They wanted "fearful" people to be helped and understood. The SAS and the GDS were in the normal range for all participants.

Conclusion: The women expressed that they have fears linked to the years to come, but despite this they had a positive attitude to deal with these worries.

\section{P-263}

Complementary and alternative medicine use by older adults in Turkey: literature review

Özge Uzun ${ }^{1}$

${ }^{1}$ Near East University, Faculty of Nursing, Nicosia-TRNC, Cyprus

Introduction: The use of complementary and alternative medicine (CAM) appears to be on the rise in the elderly population. CAM modalities are often used as self-care to enhance well-being, to support medical treatments, to prevent and to cure illnesses. Many herbal and biologic preparations offer promise, but they are largely of unproven benefit. The aim of this literature review was to investigate the use of CAM among older adults in Turkey.

Methods: This literature search (1997-2018) was carried out, using MEDLINE, PubMed, AMED, EBSCOhost, Science Direct, Google Scholar, Turkish ULAKBİM EKUAL databases. Key words included "complementary and alternative medicines", "complementary and alternative therapies"; "elderly", "older adults", "Turkey".

Results: In studies carried out on Turkish older adults, the rate of CAM use was varying from $54 \%$ and $59 \%$. The most frequently used CAM therapies were use of herbal products (range 55.2-62.1\%) and non-herbal supplements (range 43-53.5\%). Other mainly used CAM types included prayer, exercise, massage, music, and thermal spring. Common reasons for use included improving physical and emotional 
well-being, protecting from disease, increasing immunity, relieving pain, praise, supporting medical treatments. Most of the older adults who used CAM methods believed that herbal products were not harmful, and they did not need to discuss these with their healthcare providers.

Conclusions: According to the results of limited studies, CAM modalities have been frequently used by older adults in Turkey, but they generally do not inform healthcare providers that they have used these methods. Healthcare providers should give information and assist to older adults to choose CAM modalities safely and appropriately.

\section{P-264}

Timed up and go test with a dual task in elderly, a tool for learning to practise by practising

\author{
J. L. Amorone Leiva ${ }^{1}$, R. A. Irigoyen ${ }^{1}$, D. M. Agüero ${ }^{2}$ \\ ${ }^{1}$ Instituto Superior Amit, Trujui, Argentina, ${ }^{2}$ Hospital Mariano y \\ Luciano de la Vega, Buenos Aires, Argentina
}

Introduction: The Timed Up and Go Test (TUG), the TUG with a cognitive dual task (TUGcog), and the TUG with a manual dual task (TUGman) are easy, inexpensive, quick and widely used clinical tool as a component of a multifactorial fall risk assessment. Objective: to determine scores of TUG, TUGcog and TUGman in communitydwelling older adults administered by nurse students and to evaluate their motivation, satisfaction and learning perception.

Methods: Prospective study. Inclusion criteria: $\geq 60$ years, living at home, able to: walk alone without assistive devices, understand simple instructions, without cognitive limitations, carry a glass of water and count numbers. A survey was conducted to evaluate their learning perception.

Results: Participants: 51. Mean TUG: 14.78; TUGman: 15.88; TUGcog: 21.19. Male (n: 17); 60-69 (n: 7): Mean TUG: 12.69; TUGman: 14.84; TUGcog: 17.26. 70-79 (n: 10): Mean TUG: 13.23; TUGman: 14.97; TUGcog: 24.49. Female (n: 34). 60-69 (n: 10): Mean TUG: 11.42; TUGman: 13.17; TUGcog: 21.55. 70-79 (n: 19): Mean TUG: 16.90; TUGman: 16.18; TUGcog: 26.75. 80-90 (n: 5): Mean TUG: 18.41; TUGman: 19.80; TUGcog: 36.35. Students: 51 (female: 46 years male: 5). Scores: motivation (mean: 8.25), satisfaction (mean: 9.12) and learning perception (mean: 8.87).

Conclusions: Direct relationship between age and tests duration. No significant differences between TUG and TUGman. This could be explained by learning curve for students and patients. Perception by students: very positive. This report stresses the importance of 'learning by doing', which locates medical education within a triad of mutual benefit, comprising skilled practitioner, patient, and student practitioner.

\section{P-265}

\section{From family physicians' perspective to the geriatric patient}

\section{Esra Karabıyık $^{1}$, Zeynel Abidin Öztürk ${ }^{2}$}

${ }^{1}$ Gaziantep University, Faculty of Medicine, Department of Internal Medicine, Gaziantep, Turkey, ${ }^{2}$ Gaziantep University, Faculty of Medicine, Department of Internal Medicine, Division of Geriatrics, Gaziantep, Turkey

Aim: Geriatric patients need to a comprehensive geriatric evaluation. The aim of present study is to evaluate the approach of primary care physicians to geriatric patients.
Methods: 214 volunteer family physicians were participated in the study, between May 2017 and August 2017. Participants were asked to fill out a questionnaire about their demographic situations and approaches to geriatric patients. The data obtained was evaluated by using the SPSS 22.0 statistical program.

Results: The 214 physicians between the ages of $24-65$ were participated to the research. The ratio of physicians over 50 years of age was $\% 52.5$ and $\% 69$ of participants were male. The number of people living together with individuals aged 65 years and over was 33, and $57.6 \%$ of them were directing patients to a geriatric department when needed. While the proportion of physicians who had geriatric education was $31 \%$, the ratio was $62.6 \%$ for the physicians under 50 years old. Having a geriatric education is more effective for questioning polypharmacy and especially in reduction of medication and directing patients to a geriatrics center. Physicians who are married and have children have been found to be more susceptible to vaccination of elderly individuals.

Conclusions: According to present study, being older than 50 years and female were found more sensitive and willing in most of preventive medicine practices. Successful implementation of prevention medicine practices will provide reduction of morbidity of geriatric age group due to diseases, so it will decrease the need for hospitalization and cost of health care.

\section{P-266}

\section{Integrated care through training_-joint GP/geriatric trainee} clinics

Towhid Imam ${ }^{1}$, Owen Ingram ${ }^{1}$, Tom Wilson ${ }^{1}$, Daniel Mullarkey ${ }^{1}$

${ }^{1}$ Croydon University Hospital, Croydon, UK

Introduction: The challenges of an ageing population with increasing frailty, complex co-morbidity and poly-pharmacy, are well documented. A model of care was developed that retains the benefits of access and continuity that primary care provides, whilst also providing time for a more comprehensive assessment and specialist input. In addition, an opportunity is provided for trainees from different specialties to work together, facilitating joint working and learning.

Methods: We developed a pilot joint clinic model, in which a Geriatric Specialist Trainee and GP ran a specialist geriatric clinic within primary care. Suitable patients with complex geriatric need were identified by GP colleagues and referred into the joint clinic. Clinic appointments were extended to $30 \mathrm{~min}$ to enable comprehensive geriatric assessment. Patient management plans were fed back to referring clinicians in the practice to enable whole practice learning. Results: We completed 4 clinics in 2 pairs of clinicians and saw 35 patients with an average age of 78 . Common themes included cognitive impairment, urinary incontinence, poly-pharmacy and movement disorders. Evaluation was by qualitative structured interview with Geriatric Trainee, GP and Consultant supervisor. The feedback demonstrated learning in clinical knowledge, service structure and collaborative approaches to patient care.

Key conclusions: The themes of learning from the structured interviews in this pilot study mirrored the learning in the Paediatric model, which had demonstrated a cost effectiveness and quantitative improvement in healthcare. This pilot project therefore provides an exciting template to improve both training of GPs and Geriatricians, facilitating closer working between primary and secondary care. 
P-267

GeriSim-a multidisciplinary approach to geriatric simulation

Towhid Imam $^{1}$, Vaughan Holm ${ }^{1}$

${ }^{1}$ Croydon University Hospital, Croydon, UK

Introduction: Despite an increasingly aging population and the increasing age of patients in hospitals, there is a lack of simulation based learning to address managing this patient group. A multidisciplinary approach to this is a cornerstone of elderly care medicine. However, frequent rotations can create a lack of cohesion and understanding within teams.

Methods: Junior doctors, physiotherapists, occupational therapists and nursing staff learnt about managing the elderly through simulated scenarios, case discussions and use of a unique geriatric simulation suit. This suit allowed the wearers to feel a variety of impairments that may limit their treatment of the older generation.

Results: The feedback from the session demonstrated that geriatric simulation based learning is uncommon in undergraduate training across all disciplines. Undertaken after graduation it was found to be beneficial and applicable to daily practice. The participants' fed back that this format is needed to develop the skills to manage elderly patients as a multidisciplinary team.

Key conclusions: As training is not organised across disciplines this format allowed the team to gain a greater understanding of each other's perspectives in managing the elderly. Simulation has a key role to play in developing teams to manage complex geriatric patients who commonly present to hospital in the UK. It is hoped that a greater understanding of the patient and each other in the team will increase the quality of care delivered to the older population.

\section{P-268}

What the Frax? Junior doctors' perspective of bone health assessment

Stephanie Louise Jordan ${ }^{1}$, Sujitha Selvarajah ${ }^{1}$, Aimee Harrison ${ }^{1}$, Tomas Welsh ${ }^{2}$

${ }^{1}$ Royal United Hospital, Bath, UK, ${ }^{2}$ Royal United Hospital Bath, University of Bristol, Research Institute for the Care of Older People, Bath, UK

Introduction: Well-established, evidence based, treatments for fracture risk reduction exist but remain underutilised. The first step in initiating treatment lies in assessment of fracture risk. Guidelines at both national and international levels stress the importance of using established tools, such as FRAX, to do so. However, this relies on healthcare professionals being aware of and confident in using such tools. We carried out a survey of junior doctors to assess awareness and use of FRAX as part of a wider quality improvement process.

Methods: Junior doctors based at a district general hospital completed a questionnaire. Participants were asked about their awareness and confidence in using FRAX, when fracture risk assessment was indicated and their knowledge of common medications for osteoporosis. Participants remained anonymous.

Results: Responses were obtained from 38 junior doctors. 27 (71.1\%) had never used FRAX and $14(36.8 \%)$ did not know how to access FRAX. $27(71 \%)$ stated time was a barrier to calculating FRAX and $23(60.5 \%)$ felt they did not have enough experience to use FRAX. 11 (29\%) reported no confidence in prescribing bisphosphonates. 6 $(15.8 \%)$ identified falls as a reason to assess bone health.

Conclusions: Use of FRAX and confidence in initiating treatment was low. There was a lack of awareness of when fracture risk should be assessed. Unless understanding and confidence in using fracture risk assessment tools improves, rates of treatment are unlikely to increase. These data highlight an important gap in knowledge and support the development of specific educational interventions.

\section{P-269}

Anticholinergic burden awareness within the older person's unit

Priya Deol ${ }^{1}$, Ellie Green ${ }^{1}$, Victoria Neilson ${ }^{1}$, Tomas Welsh ${ }^{2}$

${ }^{1}$ Royal United Hospital Bath, UK, ${ }^{2}$ Royal United Hospital Bath, University of Bristol, Research Institute for the Care of Older People, Bath, UK

Introduction: Many medications used in everyday clinical practice have anticholinergic properties. Cumulative anticholinergic exposure (anticholinergic burden) has been linked to adverse outcomes in older people including falls and hospitalisation. Interventions to reduce anticholinergic burden may therefore provide benefit but any attempt at reduction relies on medical practitioners being aware of the concept and being able to identify appropriate medications. This project, as part of a larger quality improvement project, assessed the awareness, understanding and use of anticholinergic burden amongst medical practitioners working with older inpatients.

Methods: Staff attending the Older Persons' Unit (OPU) departmental meeting were asked to complete a questionnaire asking about awareness, understanding and use of the concept of anticholinergic burden. Staff were then asked to identify 5 medications with anticholinergic properties out of 10 frequently used medications. Responses were anonymous. Descriptive statistics were then used to summarise these responses.

Results: 26 members of clinical staff (doctors of all grades and medical nurse practitioners) completed the questionnaire. $20(77 \%)$ reported being aware of the concept, $16(62 \%)$ reported understanding the concept, $2(8 \%)$ reported using anticholinergic burden scales. 0 correctly identified 5 medications with anticholinergic properties.

Key conclusions: Understanding of cumulative anticholinergic burden was low and very few practitioners routinely used it in clinical practice. Unless understanding and awareness of the concept increases attempts at systematic reduction of anticholinergic burden will be challenging. These findings highlight an important gap in knowledge, even within a specialist unit, and support the development of specific educational interventions.

\section{P-270}

Designing a blended course in pregraduate geriatric medicine through backwards design

\section{Susanne M. S Hernes ${ }^{1}$, Anette Hylen Ranhoff ${ }^{2}$}

${ }^{1}$ Associate professor, Department of Clinical Science, University of Bergen, Norway, ${ }^{2}$ Professor, Department of Clinical Science,

University of Bergen, Norway

Introduction: Transferring from the traditional monologue to active educational strategies can be daunting. This abstract describes our experience of this pedagogical transfer while developing a two-week elective blended course "The Acute Ill Older Patient" for 3rd year medical students.

Methods: Initially, the learning outcomes of the whole course as well as each specific day were determined. A single core geriatric topic, such as delirium or dementia, was tutored each day. Week one utilized blended learning techniques with short daily pre-class learning tasks posted online in advance. Each day consisted of a mixture of 
group-based and individual active learning activities, short lectures (by either students or teacher), questions and reflection. Week two had a more traditional structure with a combination of student's and teacher's lectures, student-led case demonstrations, questions and reflection. In addition, a communication course were held during the second week. Student responses were measured by online feedback immediate after course cessation, individual reflection papers and the Faculty of Medicine official evaluation.

Results: Overall, the students were enthusiastic with week one and no negative feedback was received on pre-class preparation. The students expressed great satisfaction in discovering the challenges and rewards associated with geriatric medicine. The student activities in week one and communication course in week two were highlighted as the most valuable parts of the course. For the educators, the pre-course workload were high but class time more inspiring during the blended week.

Key conclusions: The transition for traditional lectures to student active teaching is time achieving, but rewarding.

\section{P-271}

Role of gerontological centers (out institutional care)-Croatian model of successful practice for the elderly

Spomenka Tomek-Roksandić ${ }^{1}$, Branko Kolaric ${ }^{1}$, Tanja Ćorić ${ }^{1}$, Sanja Predavec ${ }^{1}$, Nada Tomasović Mrčela ${ }^{1}$, Zvonimir Šostar ${ }^{1}$, Dalma Sajko ${ }^{1}$, Marica Lukić ${ }^{1}$, Stela Mravak ${ }^{1}$, Manuela Maltarić ${ }^{1}$, Romana Galić ${ }^{1}$, Dorotea Franceković ${ }^{1}$

${ }^{1}$ Department of Health Gerontology, Andrija Stampar Teaching Institute of Public Health, Reference Center for Health Care of the Elderly of the Ministry of Health, Republic of Croatia, Zagreb

The main goal is to keep the old person in his home in his local community as long as possible and to ensure his active lifestyle and thus active and healthy aging. Croatia faces an accelerated aging population-older than 65 and over was $17.70 \%$ of the population in 2011, while in the 2016 population estimates, the proportion of those over the age of 65 is $19.41 \%$. (http://www.stampar.hr/gerontologija) So it's important to offer the elderly population a lot of opportunities for active and healthy ageing at the Gerontological centers. Role of Gerontological center is in providing multifunctional immediate care for the elderly in their local community, enables European and at the same time Croatian gerontological approach to implement integral programme in the health care of the elderly. Development of gerontological centers, promotion of out institutional care for the elderly in their local community and infrastructure connection with old age homes allows resistance to the present passivity and social isolation (loneliness) of the elderly to healthy active and productive ageing of Croatian old population. Zagreb started in 2004 with 3 Gerontological centers. In the meanwhile there are, because of the big success and demand for it, 12 Gerontological centers in Zagreb and in the entire Croatia there are 116 Gerontological centers.

\section{P-272}

\section{Monitoring nutritional status in the elderly via NRS 2002/GEROS/CEZIH web service}

Tajana Pavić ${ }^{1}$, Nina Smolej Narančić ${ }^{1}$, Spomenka TomekRoksandić $^{1}$, Branko Kolarić ${ }^{1}$, Tanja Corić ${ }^{1}$, Sanja Predavec ${ }^{1}$, Zvonimir Šstar ${ }^{1}$, Manuela Maltarić ${ }^{1}$, Veronika Laušin ${ }^{1}$, Suzana Hlatki-Matijević ${ }^{1}$, Tatjana Škarić-Jurić ${ }^{1}$, Dalma
${ }^{1}$ Department of Health Gerontology, Andrija Stampar Teaching Institute of Public Health, Reference Center for Health Care of the Elderly of the Ministry of Health, Republic of Croatia, Zagreb

Inappropriate nutritional status, especially over 65 , is considered to be aggravated by their health and functional abilities, as well as increased mortality and geriatric consumption. Therefore, the NRS 2002 network service is implemented in the GeroS/CEZIH information system; Network Services NRS 2002 includes focused gerontology—-public health indicators such as age, gender, primary and secondary diagnosis in geriatric patients, basic anthropometric measurements, functional abilities etc. Functional ability (physically and mentally) older people is one of the key determinants of assessment, monitoring and analysis of health care for the elderly. The innovative Internet service program NRS 2002/GeroS/CEZIH is used by doctors and other healthcare professionals in hospitals, family medicine teams, geriatric nurses in homes of elderly and nurses at the home of primary health care. The Center for Gerontology Public Health focuses on the analysis of monitoring through the NRS 2001/GeroS/CEZIH Internet Service (March 1, 2015 - May 4, 2018, Croatia) for persons over the age of $65(\mathrm{~N}=422$ geriatric and gerontological insured persons) based on physical mobility and mental independence towards the entities/place of nutrition. Using network services NRS 2002/GeroS/CEZIH establishes food risks in persons over the age of 65 , monitoring defined and target determinants important for individualized access to gerontology and monitoring of therapeutic outcomes indicators for persons of inappropriate nutritional status and over 65 years of age.

\section{P-273}

Gerontologic public health indicators via e-system GeroS/CEZIH

Spomenka Tomek-Roksandić ${ }^{1}$, Branko Kolaric ${ }^{1}$, Tanja Ćoric ${ }^{1}$, Sanja Predavec ${ }^{1}$, Zvonimir Šostar ${ }^{1}$, Nada Tomasović-Mrčela ${ }^{1}$, Dalma Sajko ${ }^{1}$, Marica Lukić $^{1}$, Stela Mravak ${ }^{1}$, Manuela Maltarić ${ }^{1}$, Antonija Balenović ${ }^{1}$, Tomislav Benjak ${ }^{1}$

${ }^{1}$ Department of Health Gerontology, Andrija Stampar Teaching Institute of Public Health, Reference Center for Health Care of the Elderly of the Ministry of Health, Republic of Croatia, Zagreb

On their initiative, the Reference Center for Health Care of the Elderly of the Ministry of Health of the Republic of Croatia, Department of Health Gerontology, Teaching Unit of Dr Andrija Stampar Institute of Public Health and the Croatian Health Insurance Fund have set their common goals in the field of computerization of the gerontologic and geriatric health care at all three health care levels, from primary health care for the elderly in old people's homes and geriatric nursing documentation on geriatric health care of geriatric insured persons to long-term treatment, gerontologic care providers and geriatric dental services, along with the system connection to CEZIH (Central Croatian Health Information System) via GeroS link. The ultimate goal of the project is computerization of the complete health care provided to the elderly and the geriatric patients irrespective of the ownership of the institutions where they are accommodated, as well as the gerontologic insured persons and geriatric patients on long-term treatment in hospitals, palliative geriatric and psychogeriatric care, in order to have all these data at one place, in the central health care system, integrating it with the CEZIH via GeroS. Computerization of geriatric health care will cover health care services provided at the three health care levels, from general/family medicine and geriatric health care at old people's homes through longterm treatment of geriatric patients and visiting nursing services, home care and palliative geriatric care through services provided by Gerontologic Centers as extra-institutional care for geriatric insured 
persons, geriatric dental services and geriatric nursing services. All this information will be collected in GEROS, a central system for gerontology, where overall health care services provided to gerontologic and geriatric patients will be recorded. The system will be connected to CEZIH, while the most relevant health care segments will be recorded in the e-Card. This will make the whole health care system efficient, appropriate, accessible and properly regulated, along with evaluation of the health care provided to geriatric insured persons and rationality of the growing geriatric health care utilization.

\section{P-274}

\section{Pilot study of aging game set up with medical students}

Ketz Flora ${ }^{1}$, Nghiem Duy ${ }^{2}$, Mavoungou Sandra ${ }^{3}$, Lastennet Diane ${ }^{3}$, Lafuente Carmelo ${ }^{2}$, Pautas Eric ${ }^{1}$, Kinugawa Kiyoka ${ }^{4}$

${ }^{1}$ Polyvalent Geriatric Center, Pitié-Salpétriere Hospital Group, Charles Foix Center, Ivry Sur Seine, Assistance Publique Hôpitaux de Paris (APHP), France, ${ }^{2}$ Department of Cardiology and Neurology Geriatry, Pitié-Salpétriere Hospital Group, Charles Foix Center, Ivry Sur Seine, Assistance Publique Hôpitaux de Paris (APHP), France, ${ }^{3}$ Department of Biostatistic, Public Health and Medical Information, Pitiõ-Salpétriere Hospital Group, Assistance Publique Hôpitaux de Paris (APHP), Paris, France, ${ }^{4}$ Functional Exploration Unit of older patients, Pitié-Salpétriére Hospital Groupe, Charles Foix Center, Ivry-sur-Seine, Assistance Publique Hôpitaux de Paris (AP-HP), France

Background: With the aging of the population, future physicians will be faced with caring of older patients. Some feelings towards older people seem negative during group discussions. Few studies have already been conducted to evaluate the effect of "aging game", on attitudes, empathy and knowledge towards elderly. We led this pilot study to make students aware of physiological changes, and to improve their empathy towards elderly.

Methods: Intervention-based pilot study led in a geriatric hospital, to medical student, during an internship in a geriatric ward. They filled self-report questionnaires including sociodemographic data, basic knowledge in geriatrics, empathy and attitude towards the elderly before and after experiencing aging game. Data were analyzed using McNemar test, paired sample student test and Wilcoxon test.

Results: A total of 67 students were assessed. 60 students $(90 \%)$ reported that aging game help them to realize difficulties of older patients. 43 students (64\%) thought it was fully helpful. 18 students (27\%) thought it was partially helpful. 55 students (82\%) thought it has to be maintained in the teaching course and 5 students $(7 \%)$ thought it should be continued but adapted. The analysis of modification about knowledge, attitude and empathy is still in progress. Conclusion: The set up of this Aging Game, should be able to improve knowledge, attitude and empathy of the future doctors towards older persons. This pilot study is the first step of educational strategy, and we are thinking about making an extension of this aging game to a whole class of medical student

\section{P-275}

Documentation of medication management in diabetic patients with dementia

Haroon Ahmed ${ }^{1}$, Hanadi Khamis ${ }^{2}$, Amin Abdelghany ${ }^{2}$, Shafi Hashmath $^{2}$, Ahmed Fouaad ${ }^{2}$, Marwa Awad ${ }^{2}$, Essa Mubarak ${ }^{2}$, Mani Chandran ${ }^{2}$

${ }^{1}$ Hamad Medical Corporation, Doha, Qatar, ${ }^{2} \mathrm{HMC}$, Doha, Qatar
Introduction: The management of diabetes in adults with cognitive dysfunction presents many challenges for patients, caregivers, and medical providers. It is important to be aware of the complexity of daily self-care tasks required of people living with diabetes and to recognize the ways in which cognitive impairment can interfere with these tasks. A team approach involving the patient, family, and caregivers and including the use of allied health professionals and assistive devices, as well as simplification of medication regimens with a focus on avoiding hypoglycemia and symptomatic hyperglycemia, is needed for adults with moderate-to-severe cognitive impairment. Currently only $10 \%$ of Diabetic Patient with Cognitive impairment attending Geriatric Clinic were assessed and advised regarding their medication management capacities and documented. This may lead to costly and life-threatening hypo or hyper- glycemic emergencies that will negatively impact the diabetic patient quality of life. A quality improvement project was carried out from September 2015 to December 2015.

Aim: To Improve the physician documentation of assessment and advice on medication management in Geriatric Clinics for Diabetic patients with Cognitive impairment from 10 to $50 \%$.

Methodology: - Data collection and analysis- Education sessions to the physicians- Assessment tools, as 1st Intervention.

Results: By the end of December 2017, after implementation of action plans, the compliance rate increased from 10 to $100 \%$.

\section{P-276}

Innovations in geriatric medicine: Social media and the UKs first trainee-led research collaborative

Lauren Mccluskey ${ }^{1}$

${ }^{1}$ Queen Elizabeth Hospital, Birmingham, UK

Introduction: Social media and online networking provides an innovative approach to development and promotion of a research collaborative. Using this model we set up the UKs first trainee-led geriatric medicine research collaborative (GeMRC) to improve trainees experience in research and patient care.

Primary aim: 1 . To set up the first UK trainee led geriatric medicine research collaborative.

Secondary aims: 1 . To enable conduct of audit and research projects with direct impact on patient care. 2 . To enable geriatric trainees to develop research skills.

Methods: Using social media and a trainee-led "grass-roots" approach GeMRC has representatives from 14 of the 15 regions in the UK. We created national and regional websites to disseminate project information, and utilised a combination of e-mail communication, social networking, word-of-mouth, and dissemination through the British Geriatrics Society. Project ideas are generated by trainees at regular meetings and communicated to regional representatives. ResultsWe have successfully completed three national audit projects, and are refining the protocols for two Randomised Controlled Trials (RCTs), four abstracts were presented at the BGS national conference, one journal article and more than 100 trainees have been involved.

Conclusions: The Geriatric Medicine Research Collaborative (GeMRC) offers an innovative approach to research. It facilitates the opportunity for trainees with limited research experience to participate in research that has early impact upon patient care. The use of social media and online networking allows rapid dissemination of project ideas which facilitates collection of much larger datasets and enhances the scientific validity of project outcomes. We welcome collaboration with European sites in the future. 


\section{P-277}

Competencies in geriatrics of internal medicine residents: a crosssectional study of Mexican trainees

\section{Sara Gloria Aguilar-Navarro ${ }^{1}$, José Alberto Ávila-Funes ${ }^{1}$ \\ ${ }^{1}$ National Institute of Medical Sciences and Nutrition Salvador Zubirán, Mexico}

Educational curricula for medical students and residents has not been updated at the same rhythm as the demographic and epidemiological transitions require it. Internal medicine residents (IMR) rely in their experience and teachers during residency to adapt their knowledge to attend the needs of older adults. Not all IMR are exposed to geriatric courses or geriatric teams, which limits their education. The objective was to stablish if there is an association between the degree of exposure to geriatric medicine and the fulfillment of competencies in geriatric medicine for IMR. An anonymous survey was sent to IMR in Mexico City, asking to rate themselves on the fulfillment of the 26 competencies in geriatric medicine as well as the exposure they have to geriatric medicine. We analyzed de frequencies for each competence and made comparisons for level of training, hospitals, Medical School and degree of exposure to geriatric medicine.91 IMR responded, $65 \%$ of the sample were men, $47.7 \%$ do not have any kind of geriatric medicine exposure. There was no association between the degree of exposure to geriatric medicine and the fulfillment of competencies $(\mathrm{P}>0.095)$, with Medical School $(\mathrm{P}=0.277)$ or with hospitals $(P=0.07)$. We found an association between the year of residency and the fulfillment of competencies $(\mathrm{P}=0.0005)$. IMR are not educated enough in geriatric medicine in Mexico City, even though it seems this might not have an influence in the acquisition of competencies. There still need to improve the performance in these competencies which are the minimum required for IMR.

\section{P-278}

Adapted physical activity's service: a support process for active ageing and empowerment in Genoa Socio-Sanitary Districts

\author{
Emilia Tasso ${ }^{1}$, Francesca Vitali ${ }^{2}$, Loredana Minetti ${ }^{3}$ \\ ${ }^{1}$ ASL3 Genovese and University of Genoa, Genoa, Italy, ${ }^{2}$ University \\ of Verona, Verona, Italy, ${ }^{3}$ ASL3 Genovese, Genoa, Italy
}

Introductions: The Non-communicable disease's, musculoskeletal conditions with other risk factors (insufficient physical activity, social deprivation, mental and neurological conditions), changed the health maps of the Nations. In Genoa the Socio-Sanitary Districts approach a virtuous process of Primary Care and information for old people, to improve an active lifestyle using an Adapted Physical Activity (APA) process to contrast frailty and to prevent comorbidity and diseases. AimThe main goal for public health systems, is to create a new collaborative leadership and empowerment for a new health pro-active deal with many partners (social and private stakeholders together with citizens).

Method: The study investigates: the APA process, based on the PDCA quality approach (Plan, Do, Check, Act), to support and to help the elderly with frailty and disabilities to socialize and enhance their own active life style the data analysis physical performances (balance, strength, and walking speed), and psychosocial dimensions (motivation and collaboration) are in progress. Preliminary study involves $\mathrm{N}=316$ elders $(\mathrm{F}=80.1 \% ; \mathrm{M}$ age $=71.4 \pm 7.2$ years; overweight $\mathrm{T} 0=40.8 \% ; \mathrm{M}$ drugs a day $\mathrm{T} 0=3$ ) participated to an APA program. Results: Starting statistical analyses show significant results on physical autonomy (Barthel Index, SPPBT), reduction of pain and use of drugs. Moreover, significant improvement on physical performance (balance, strength, and walking speed), and on psychosocial dimensions (motivation and collaboration) were found. The study confirms scientific literature and indicates some directions to promote health and wellness for elders.

\section{P-279}

Time to wake up: healthcare professionals' knowledge of insomnia management

Safa G. Attar ${ }^{1}$, Raunak Singh ${ }^{1}$, Ashish Patel $^{1}$

${ }^{1}$ University Hospitals of Leicester, UK

Introduction: Insomnia causes significant distress and impaired functional state. Insomnia is very common: $37 \%$ of elderly inpatients have insomnia [1]. It is imperative that all healthcare professionals (HCP) in hospitals have a high-level of knowledge in all aspects of insomnia management.

Methods: The multidisciplinary team (MDT) members within an acute medical hospital were surveyed on their knowledge in nonpharmacological and pharmacological management of insomnia. The MDT members included doctors, nurses, physiotherapists, occupational therapists, a pharmacist, Healthcare Assistants and a medical student. Wards across Medical, Orthopaedics and the Emergency Department were surveyed. The results amongst the MDT groups were compared.

Results: 39 Questionnaires in total (16 Doctors, 14 Nurses and 9 other MDT members). All MDT members lacked knowledge in both non-pharmacological and pharmacological management. Nurses were marginally more knowledge in different parameters of non-pharmacological management (such as lifestyle and diet).

Key conclusions: Knowledge across the MDT in non-pharmacological and pharmacological management of insomnia was lacking. Nurses were slightly more knowledgeable compared to other MDT members. As this was not of a high standard no significant trends can be drawn between the MDT groups. To better patient care, guidelines are being developed for our acute medical hospital. It is time to wake up to the fact that education and training of HCP needs improvement in the management of insomnia.

Reference:

1. Isaia $\mathrm{G}$ et al (2010) Insomnia among hospitalized elderly patients: prevalence, clinical characteristics and risk factors. Arch Gerontol Geriatr 52(2):133-137

\section{P-280}

Fostering academic geriatric medicine-the science forum of geriatrics in Germany

Manfred Gogol ${ }^{1}$, Albert Lukas ${ }^{2}$, Maria Cristina Polidori ${ }^{3}$, Adrian Rosada ${ }^{4}$

${ }^{1}$ Institute of Gerontology, University of Heidelberg, Germany, ${ }^{2}$ Centre for Geriatric Medicine, Malteser Hospital Seliger Gerhard, Von-Hompesch-Str. 1, 53123 Bonn, Germany, ${ }^{3}$ Ageing Clinical Research Department, Department of Internal Medicine, University Cologne, Kerpener Str. 62, 50937 Cologne, Germany, ${ }^{4}$ Department of Geriatrics, Charité University Medicine Berlin, Hindenburgdamm 30, 12200 Berlin, Germany

Introduction: Much efforts were done in the past years to develop scientific geriatric medicine but in Germany geriatric medicine is today not represented at every medical faculty. 
Methods: With the goal to foster the development of scientific geriatric medicine in 2014 some colleagues founded the Science Forum of Geriatrics.

Results: The Science Forum of Geriatrics nowadays has around 30 members including representatives from most university departments. Since his foundation it hosts an annual meeting on January with a mixture of presentations of senior and junior researchers as well as external keynote speakers. One major task is to promote networking, build research consortia, stimulate junior researcher to attend (including some travel funds) and to discuss research proposals. The Science Forum of Geriatrics has a close cooperation with the German Society of Geriatrics (DGG) and presented its work at the annual DGG conferences. During the first years the Science Forum of Geriatrics received financial funding for its work from the Robert Bosch Foundation.

Conclusions: Since 5 years the Science Forum of Geriatrics established and developed various activities to foster scientific networks within geriatric academics and starts to attract young researchers.

\section{P-281}

\section{Geriatric patient knowledge and responsibility about their regular medication}

Pablo Enrique Solla Suárez ${ }^{1}$, Javier Montero Muñoz ${ }^{1}$, Elena Valle Calonge $^{1}$, Eva María López Álvarez ${ }^{1}$, José Gutiérrez Rodríguez ${ }^{1}$, Juan José Solano Jaurrieta ${ }^{1}$

${ }^{1}$ Hospital Monte Naranco, Oviedo, Spain

Objectives: To assess knowledge and responsibility about regular medication in geriatric patients.

Materials and methods: Descriptive cross-sectional study, in April/ 2018. Included admitted patients from an Acute Geriatric Unit. Exclusion criteria was cognitive impairment or no collaboration. Variables: age, sex, Barthel Index, education level, drugs number, system used for remembering and dispensing drugs. Responsibility level. Person that assumes responsibility: characteristics.

Results: n:66. Female: 63.6\%. Age $88 \pm 4$. Barthel Index: 79.4. Prescribed drugs: average: 7; median: 4 . Education level: $18.2 \%$ nonscholarized, $74.2 \%$ basic, $3 \%$ university. $39.4 \%$ remembered the medication by memory, $27.3 \%$ written, $16.7 \%$ delegated to the nursing home. Dispensing system: pillbox: $33.3 \%$, medication box: $30.3 \%$. 36.4\% took the medication by their own and $34.8 \%$ just if it was previously dispensed. $28.8 \%$ didn't take the medication for themselves (physical disability). In these cases, the caregiver $(29 \%$ private caregiver, $25.8 \%$ daughter, $22.6 \%$ wife; age 59.5, female $81.9 \%$, 65\% domestic partnering) assumed responsibility about medication. $74.2 \%$ consumed $\geq 5$ drugs. Most used drugs: PPIs: 71.2\%, diuretics: $66.7 \%$, ACEI-ARBs: $45.5 \%$, OAC:42.4\%, benzodiazepines: $39.4 \%$. Among those who consumed the medication by themselves knew the indication: $64.7 \%$ PPI, $75 \%$ diuretics, $77.8 \%$ ACEI-ARBs, $88.9 \%$ OAC, $87.5 \%$ benzodiazepines. $34.8 \%$ were not interested to learn about their medication (indication, dosage, etc.). Conclusions: Most of elderly patients depend on other people, to take their medication, being most frequently a female family member. Pillbox is the most used dispensing system. A non eligible percentage of the patients don't know the indication about their regular medication. Approximately one third weren't interested to learn about their medication.

\section{P-282}

Do elderly patients take responsibility about his medication?

Javier Montero Muñoz ${ }^{1}$, Elena Valle Calonge ${ }^{1}$, Pablo Enrique Solla Suárez ${ }^{1}$, Eva María López Álvarez ${ }^{1}$, Cristina Guirola García-Pendes ${ }^{1}$, Juan José Solano Jaurrieta ${ }^{1}$

${ }^{1}$ Hospital Monte Naranco, Oviedo, Spain

Objectives: To suggest possible factors that influence responsibility about medication in elderly patients.

Materials and methods: Descriptive cross-sectional study that includes 66 patients from an Acute Geriatric Unit at Monte Naranco Hospital. This study includes two data samples taken in April/2018. Exclusion criteria was cognitive impairment, dementia, confusional syndrome or absence of collaboration. Variables were age, sex, Barthel Index, education level, responsibility, number of prescribed drugs, system used for remembering medical treatment and dispensing system. For analysis, Chi squared test was used.

Results: Among the patients who didn't assume responsibility of the medication $(\mathrm{n}=42,63.6 \%), 88.1 \%$ were polymedicated $(\geq 5$ drugs) $(\mathrm{p}=0.001)$ and medication was dispensed in a pillbox by $78 \%$ $(p=0.003)$. No significant differences were found between responsibility and gender, age or level of functional dependency. An association was found between poor education level and the nonresponsibility $(p=0.041)$. Regarding the system to remember the medication, those who assumed responsibility used mainly by memory $(26.8 \%)$, followed by writing $(25 \%)$, pillboxes $(14.6 \%)$ or other means $(4.2 \%)(\mathrm{p}=0.033) .58 .3 \%$ took medication directly from the box/blister $(\mathrm{p}=0.003)$. No relationship found between responsibility and interest to get more information about the treatment.

Conclusions: In our study, polymedication and absence of scholarization were related to non-responsibility. No relationship found between responsibility and gender, age, or functional dependence. The system most frequently related to responsibility was memory.

\section{P-283}

The postgraduate certificate in geriatric medicine is important to disseminate geriatric competence among different health care professionals in different settings

Giovanni Ricevuti ${ }^{1}$, Luigina de Carlo $^{2}$, Giulia Ionita ${ }^{3}$, Valentina Martello $^{2}$, Vitali Biffi ${ }^{4}$, Simone Cernesi ${ }^{5}$, Jacopo Sala ${ }^{6}$

${ }^{1}$ University of Pavia, Department of Internal Medicine, Master Degree in Geriatrics, Pavia, Italy, ${ }^{2}$ University of Pavia, Italy, Postgraduate Course in Geriatrics, Pavia, Italy, ${ }^{3}$ University of Pavia, Postgraduate Course in Geriatrics, Nursing Home Physician,

Ondazione Benefat, Pavia, Italy

The progressive aging of the population and the increase of frailty and disability in elderly people require geriatric preparation of health workers to ensure quality, safety care and respect of elderly human right. The University of Pavia organizes an annual multidisciplinary based MSci level Course of Geriatrics to provide general and specific skills in territorial geriatrics and the management of nursing home. It aims to develop in-depth knowledge of geriatrics for the professionals in geriatric care. Applicants must be graduated in Medicine, Psychology, Pharmacy, Economics, Nursing Sciences. The trainee will become expert in elderly multidimensional assessment with community oriented approach, with medical-legal-administrative knowledge, management and leadership skills. The professional figure trained in the MSci programme are: (1) General Practitioner (GP) expert in geriatric medicine (2) Geriatric coordinator in a GP association (3) Director/Health coordinator of nursing home (4) 
Psychologist/Doctor/Nursing operator in public and private clinics (5) Clinical Pharmacy in nursing home The Educational Structure consist in 6/7 modules: (1) Epidemiology, legislation and organizative models (2) Geriatrics and gerontology (3) Clinical care and assessment of Pharmacotherapy (4) Geriatric diseases of international and specialist interest (5) Emergencies and Urgencies (6) Medical-legal problems (7) Palliative Care in advanced dementia and/or end stage disease. It is important to organize a multi-centric European Geriatric Medicine Course to disseminate geriatric competence among different healthcare professionals in different settings. We are working to establish a European network with Universities who shared this vision and to gain experience by international collaboration.

\section{Area: Geriatric rehabilitation}

\section{P-284}

Prediction of geriatric rehabilitation outcomes: comparison between three cognitive screening tools

Noemi Heyman ${ }^{1}$, Tatyana Tsirulnicov ${ }^{1}$

${ }^{1}$ Department of Geriatrics, Shoham Medical Center, Pardes Hana, Israel, affiliated with the Rappaport Faculty of Medicine, TechnionIsrael Institute of Technology

Introduction: Several cognitive assessment tools are used to evaluate patients' cognitive status. However, it remains unclear which tool best predicts the functional rehabilitation outcomes of patients in geriatric rehabilitation. The present study compared between the ability of three cognitive screening tools-Mini Mental State Examination (MMSE), Montreal Cognitive Assessment (MoCA), and Informant Questionnaire on Cognitive Decline in the Elderly (IQCODE)- to predict functional rehabilitation outcomes at a department of geriatric rehabilitation.

Methods: This prospective study included 212 patients aged 65 and older admitted to rehabilitation departments at a geriatric facility in central Israel, from April 2016 to October 2016. The cognitive functioning of each patient was assessed using the MMSE, MoCA, and IQCODE. Upon discharge, rehabilitation outcomes were examined using the Functional Independence Measure (FIM), cognitive FIM, delta FIM ( $\Delta$ FIM), and ADL.

Results: Cognitive impairment was found to interfere with the rehabilitation process. The MMSE was the best predictor of functional rehabilitation outcomes at discharge, compared to the IQCODE, while the MoCA did not predict these measures. In addition, when distinguishing between patients by ethnicity (Jewish versus Arab), the MMSE and the IQCODE predicted FIM upon discharge among Jewish patients, while only the IQCODE predicted FIM upon discharge among Arab patients.

Key conclusions: The research findings show that cognitive assessment upon admission for rehabilitation-MMSE among Jewish patients and IQCODE among Arab patients-can help predict functional rehabilitation outcomes and make the appropriate adaptations in the rehabilitation program.

\section{P-285}

The effect of dual task training on static and dynamic balance of older adults having institutionalized living: randomised trial

Begüm Sarıpınarl ${ }^{1}$, H. Serap İnal ${ }^{2}$

${ }^{1}$ Okan University, Istanbul, Turkey, ${ }^{2}$ Bahcesehir University, Istanbul, Turkey
Introduction: Balance is one of the most complex functions of mankind and is carried out in the presence of a secondary or multitask rather than alone in a daily living activity. Therefore, in the presented study it was aimed to observe the effects of dual-task on static and dynamic balance and to present if static and dynamic balance training under dual-task performance effect the static and dynamic balance ability positively among the older adults having an institutional living.

Methods: The study presented is a randomized controlled clinical study. Fifty volunteer individuals (72.02 \pm 6.60 years of age, ranging between 64 and $91 ; 12 \%$ female, $n=6,88 \%$ male, $n=44$ ) took part in this study. The assessments as Barthel Index (BI), Berg Balance Scale (BBS), and Berg Balance Scale Under Dual-Task Performance (BBS-DTP were performed. All volunteers were above 65 years old and living in the T.C Darulaceze Presidency. The participants were divided into two groups by computer-generated randomization table: Group A ( $\mathrm{n}=26$, number of sessions $=5)$ and Group B $(\mathrm{n}=24$, number of sessions $=7$ ). After static and dynamic balance training, given under dual-task performance, BBS and BBS-DTP scores were recorded again and compared with statistical analyzes both using the group and between the groups.

Results: According to the outcomes, the balance score was not affected by dual-task performance, and no differences were found between groups $(p>0.05)$. For both groups, the BBS score and the BBS-DTP score showed positive improvement after training sessions $(p=0.00)$. When sub-parameters were analyzed for BBS-DTP, Group B had more task significance compared to Group A. Due to BI classification of functional independence, both before and after training sessions, positive improvements were found for BBS and BBS-DTP scores $(\mathrm{p}=0.00)$.

Conclusion: Training had positive effects at every level of functional independence according to outcomes of BI. For both groups, significant progress was found in BBS and BBS-DTP scores, but not affected by the session numbers.

Key words: Health Promotion, Dual Tasking, Balance, Elderly

\section{P-286}

Pain in older patients with COPD indicated for post-acute pulmonary rehabilitation

Eléonore F. van Dam van Isselt ${ }^{1}$, Karin H. Groenewegen-Sipkema ${ }^{2}$, Monica van Eijk ${ }^{1}$, Niels H. Chavannes ${ }^{1}$, Wilco P. Achterberg ${ }^{1}$

${ }^{1}$ Department of Public Health and Primary Care, Leiden University Medical Center, The Netherlands, ${ }^{2}$ Deventer Hospital, Pulmonary Department, Deventer, The Netherlands

Introduction: Recent literature shows that pain is a significant problem in stable chronic obstructive pulmonary disease (COPD) and is associated with worse health status and lower exercise capacity. The relation between pain and physical activity is important, since lifelong adherence to physical activity is essential to improve HRQoL and prognosis in COPD. Not much is known about pain in in relation to acute exacerbations (AECOPD) and post-acute pulmonary rehabilitation (PR). The primary aim of the present study is to investigate prevalence and characteristics of pain in older patients with COPD hospitalized for AECOPD and indicated for post-acute PR. Secondary aim is to investigate the relationship between pain and other symptoms and between pain and functional status.

Methods: This cross-sectional observational study included 149 patients (mean age $70.8( \pm 7.9)$ years, $49 \%$ male, mean FEV1\% predicted: $35.3( \pm 12.6)$ ). Pain was assessed using the Brief Pain Inventory. 
Results: Pain prevalence was $39.6 \%$ with moderate intensity and interference. Thirty-six percent of the patients with pain had no analgesic prescription and $40.7 \%$ reported no or only mild pain relief by pain treatment. In patients with pain, total symptom burden was higher and health status was more impaired due to more impaired experienced functional status.

Key conclusions: Prevalence of pain in patients hospitalized for AECOPD and indicated for post-acute PR was $39.6 \%$. Pain intensity and interference were moderate but pain treatment was suboptimal. Incorporation of standard pain assessment during AECOPD and postacute PR is recommended.

\section{P-287}

Effect of a strength training program with TheraBands on cognitive and functional parameters in frail elderly over 90 years. controlled randomized pilot study

Irimia Mollinedo-Cardalda ${ }^{1}$, José C. Millán-Calenti ${ }^{2}$, David Facal ${ }^{3}$, Adriana López ${ }^{1}$, José María Cancela ${ }^{1}$

${ }^{1}$ University of Vigo, Research group HealthyFit, Institute of Health Research Galicia Sur (IISGS), Hospital University Complex of Pontevedra (CHOP), SERGAS, Pontevedra, Spain, ${ }^{2}$ University of A Corupa, Gerontology Research Group, Biomedical Research Institute of A Coruña (INIBIC), Hospital University Complex of A Coruña (CHUAC), SERGAS, A Corupa, Spain, ${ }^{3}$ University of Santiago de Compostela, Departament of Evolutionary Psychology and Education, Santiago de Compostela, Spain

Introduction: Fragility is defined as a state of vulnerability that carries a higher risk of adverse results (Clegg et al., 2013). Because of this, the benefits of physical exercise on aging and especially on frailty have been the subject of recent scientific research. The aim of this study is to verify the effect of a strength training program with Therabands on cognitive status, functionality and general health in frailty institutionalized elderly population.

Methods: After random selection, 17 frailty institutionalized elderly were allocated to an intervention group with therabands (TG) and a control group $(\mathrm{CG})$. The TG $(\mathrm{n}=9,66.66 \%$ women, age $=92.56$ \pm 2.65 ) done 2 non-continuous weekly sessions of $1 \mathrm{~h}$ duration, for 12 weeks, of a strength training program with Therabands. The CG $(\mathrm{n}=8,87.50 \%$ women, age $=94.00 \pm 3.63)$ done activities that the center required for them, but not related to physical exercise. A cognitive (Mini-mental and Pfeiffer test), functional (Barthel index, Five sit to stand test, and Fall Index), and health (12-SF) assessments was carried out, pre and post intervention.

Results: After completing the intervention, significant intergroup differences were found in the variables of health (Mental health and mental component) and functionality (Five sit to stand and fall index) of improvement of TG over CG.

Conclusion: A strength training program with Therabands in frail elderly people over 90 years old, brings about benefits in the functionality and cognitive health of these people.

\section{P-288}

Are we over treating the blood pressure and making our elderly patients fall?

\author{
Sai Hyne ${ }^{1}$, Malathi Suppiah ${ }^{1}$, Kali Kodavali ${ }^{1}$, Bharath Lakkappa ${ }^{1}$ \\ ${ }^{1}$ Community Hospitals, Northamptonshire Healthcare NHS \\ Foundation Trust (NHFT), UK
}

Introduction: Hypertension is one of the most preventable causes of premature morbidity and mortality. It is a major risk factor for ischaemic and haemorrhagic stroke, myocardial infarction, heart failure, chronic kidney disease, cognitive decline and premature death. The NICE guideline recommends lowering of blood pressure to below $150 / 80$ in elderly patients over the age of 80 and $140 / 90 \mathrm{~mm}$ $\mathrm{Hg}$ for all others. Symptoms of postural hypotension are to be looked out for (NICE, 2016). Low blood pressure (systolic below 110), in the very elderly is associated with increased mortality (Ravindrarajah et al., 2017). In this audit, prevalence of low blood pressure in our community hospital patients was reviewed.

Methods: All inpatient's above 80 years of age in two community hospitals, on a particular day in July 2017 were included. Blood pressure measured a day after admission and falls history was obtained.

Results: There were 40 inpatients above 80 years of age, 18 were male and 22 female. Low blood pressure (systolic below 110) was found in $25 \%(10 / 40)$ of the patients. 9 out of these 10 patients $(90 \%)$ were on anti-hypertensive medication. $77 \%$ (31/40) were admitted with a fall.

Conclusions: Treatment for hypertension is beneficial in reducing morbidity and mortality from cardiovascular disease. However, overtreatment is common in the very elderly and this could increase the falls risk. The history of falls in patients with low blood pressure, on treatment with anti-hypertensives was stark. Therefore, we should be cautious in lowering the blood pressure excessively in the very elderly.

\section{P-289}

The effect of empowerment programs on depressive symptoms of older adults in day care rehabilitation centers

Shirin Golkarami ${ }^{1}$, Mohammad Kamali ${ }^{1}$, Hossein Mobaraki ${ }^{1}$, Fatemeh Farhudi $^{2}$

${ }^{1}$ Faculty of Rehabilitation Sciences, Iran University of Medical Sciences, Tehran, Iran, ${ }^{2}$ Vice-chancellor for Rehabilitation. Welfare Organization of Iran, Khorramabad, Iran

Introduction: Variation in the presentation of depressive illness among older adults may complicate assessment of depression, especially among those with multiple medical comorbidities and functional disabilities. The aim of this study was to evaluate the effect of empowerment programs on depressive symptoms of older adults in day care rehabilitation centers of Khorramabad, Iran.

Methods: This is a cross-sectional and case-control study. The study sample was selected among older adults referred to day care rehabilitation centers of Khorramabad, through the convenient sampling. The sample was consisted of 40 elderly participants who randomly allocated to the experimental and control groups, each included 20 (8 men and 12 women). The research instruments included Beck Depression Inventory-II (BDI-II), and a questionnaire for gathering the demographic data. Empowerment program included lifestyle education, rehabilitation services, support and cultural services, sports and arts, tourism and leisure services. Empowerment programs was performed for 40 days and $3 \mathrm{~h}$ in a day.

Results: The mean age of experimental and control group was $65.25 \pm 5$ and $64.95 \pm 4.79$, respectively. The results of t-test showed that there was a significant difference between mean score of BDI-II before and after intervention $(\mathrm{P}<0.047)$.

Key conclusion: Findings of this study suggest that empowerment program have a positive impact on the elders' depressive symptoms, and it can be considered as an efficient intervention. Future research is needed to determine if such an empowerment program could be used with newly institutionalized elders to speed as well as enhance their adjustment to nursing home life. 


\section{P-291}

Towards better geriatric rehabilitation by structural measuring and evaluation of patient outcomes

Arno Doornebosch ${ }^{1}$, Monique Caljouw ${ }^{1}$, Romke van Balen ${ }^{1}$, Wilco Achterberg ${ }^{1}$

${ }^{1}$ Leiden University Medical Center, Leiden, The Netherlands

Introduction: Geriatric Rehabilitation (GR) aims to restore functioning and participation in older persons after an acute functional deterioration. Our academic network (UNC-ZH) initiated a roadmap for a core set of generic measurement instruments to evaluate outcomes and enhance GR quality. These are the first results. Objectives The aims: (1) structural and unambiguous use and evaluation of patient outcomes, (2) benchmark between GR departments and (3) scientific research.

Methods: Development of (1) the core set by literature search, inventory of used instruments and discussions with experts to reach consensus; (2) digital application linked to patient file. (3) a pilot implementation study to evaluate user-friendliness and application in daily practice.

Results: Literature review found no basis for a valid core set of instruments, but our full procedure (1) resulted in a set with valid measures for comorbidity (FCI), functioning and participation (Premorbid BI, USER, COPM, NPI-Q), and quality of life (EQ-5D-5L). The set is completed with additional information; age, gender, living environment, nutritional status, hospitalisation, therapy intensity and ICD-10 diagnoses. The set covers all domains of the International Classification of Functioning, Disability and Health. In the pilot, professionals were positive regarding the usefulness of the instruments, but some practical concerns about the digital application were revealed.

Conclusion: The need for benchmarking, research and quality improvement in GR is evident- however, evidence is scarce. These are first steps in implementation and validation of a core set of generic instruments, in which the need for European cooperation is evident.

\section{P-292}

Assessment strategies in early, ward-based rehabilitation in vulnerable, multimorbid geriatric patients admitted to acute medical care: a systematic review

Patrick Heldmann ${ }^{1}$, Nacera Belala ${ }^{1}$, Jürgen M. Bauer ${ }^{2}$, Klaus Hauer ${ }^{2}$

${ }^{1}$ Network Aging Research (NAR), University of Heidelberg,

Germany, ${ }^{2}$ Agaplesion Bethanien Hospital, Geriatric Center at the University of Heidelberg, Germany

Introduction: The selection of assessment instruments to assess outcomes of early ward-based rehabilitation in vulnerable, multimorbid, geriatric patients with and without cognitive impairment poses specific challenges and may have caused ambiguous results in previous intervention trails.

Objectives: To identify assessment strategies as used in previous intervention trails and analyse them with respect to: $\bullet$ Match to study intervention, study design and phases of rehabilitation process including analyses of psychometric properties - Sub-group analyses of people with cognitive impairment (PWCI).

Methods: Established databases (PubMED, Cochrane CENTRAL, CINAHL, PEDro) were systematically searched. Inclusion criteria: (1) RCT; (2) mean age > 65 years; (3) acute medical ward; (4) exercise-based interventions (optional as part of complex interventions). Two reviewers independently extracted data.

Results: The intervention program of 14 studies (34\%) showed a high match to study outcomes. Physical performance tests were mainly used $(63 \%)$ in the early acute rehabilitation phase, with a number of tests suggesting a risk of floor effects. Functional status instruments were mainly used $(80 \%)$ at the end of the acute phase, with a tendency to show an inadequate responsiveness to change within the limited timeframe of early rehabilitation. Cognitive status of patients were reported in $\mathrm{n}=24(58 \%)$ of the studies and a specific cognitive intervention approach could not be found.

Key conclusion: Identified assessments showed substantial limitations with respect to their match to the study interventions and different rehabilitation phases. Although PWCI represent a major subgroup in early rehabilitation, specific assessment strategies are hardly recognizable as part of the treatment.

\section{P-293}

Cognitively frailty patients can be rehabilitated, preventing premature 24 hour care admissions

Geraldine Donnelly ${ }^{1}$, Gary Jevons ${ }^{1}$, Joanna Williams ${ }^{1}$, Lauren Wentworth ${ }^{1}$

${ }^{1}$ Wythenshawe Hospital, Manchester University Hospital Trust, UK

Key words: Personalized medicine- Prevention and communities and environments- Prevention in vulnerable population (oldest old, minorities, women, etc.).

Introduction: National Audit Office UK (2015) stated 2.7 million bed days were occupied by patients no longer requiring an acute bed. Intermediate Care (IMC) rehabilitation supports those with reduced physical functioning, however often declines those with cognitive frailty. This creates prolonged deconditioning hospital stays, patient flow issues and endorses premature admission to 24-h care. We created a specialized MDT led rehabilitation unit for those declined by IMC.

Methods: Retrospective analysis of 6 months of data, comparing functional scores from admission to discharge. We calculated the percentage of new 24-h care admissions in those over 80 entering hospital in comparison to the same period the year before.

Results: 177 patients, $63 \%$ female and a median age of $85.34 \%$ of admissions were from non geriatric wards, whom otherwise wouldn't have received a comprehensive geriatric assessment. $32 \%$ were admitted from acute care, demonstrating deflection of admissions into the main hospital. $67 \%$ of patients increased in weight, on average by $1 \mathrm{~kg}$. Barthel (functional) index increased in 38\% and the $6 \mathrm{~m}$ walking speed increased on average by $0.2 \mathrm{~s} .74 \%$ patients returned to their own home. Despite a $6.6 \%$ increase in hospital admissions in those over 80 , we contributed to creating a $11 \%$ decrease in new $24-\mathrm{h}$ care placements ( $\mathrm{p}$-value 0.0486).

Key conclusions: We have established a service for patients who were previously felt non-rehabable with subsequent prolonged hospital stays. In the appropriate environment their independence can improve reducing unnecessary long-term care admissions and support hospital flow. Both outcomes have positive health economic implications. [1]GREAT BRITAIN. Department of Health, Audit Commission for House of Commons. National Audit Office. (2016) Discharging older patients from hospital. London: Stationery Office 


\section{P-294}

Knowledge translation into practice: development of the Geriatric Activation Program Pellenberg, aimed to improve functional performance of geriatric inpatients

Margaretha van Dijk ${ }^{1}$, Patsy Allegaert ${ }^{1}$, Jos Tournoy ${ }^{1}$, Geert Verheyden ${ }^{2}$, Johan Flamaing ${ }^{3}$

${ }^{1}$ UZ Leuven, Campus Pellenberg, Belgium, ${ }^{2} \mathrm{KU}$ Leuven, Belgium, ${ }^{3}$ UZ Leuven, Belgium

Background: A hospitalisation in older persons mostly results in functional decline, but concurrently a basic level of functional performance is warranted for a discharge home. From a physical perspective, key components to perform activities of daily living (ADL) in a safe way include muscle strength and power to stand up or move objects, balance to prevent falls, speed and endurance to perform and complete ADL in real life. Multicomponent exercise programs to prevent falls and strength training to improve functional performance in the older community dwelling population have been proven effective. However, literature on multicomponent exercise programs for geriatric inpatients with multimorbidity is lacking. Aim: To develop a week program specifically designed for geriatric inpatients, with the intention of working efficiently within the given physical therapy time and achieving a higher level of functional performance and thus independence.

Result: Geriatric Activation Program Pellenberg (GAPP); each weekday, 45 min a day, we focus on a different aspect of functional performance: Monday speed and endurance, Tuesday functional training, Wednesday balance, Thursday strength and Friday either evaluation by the Berg balance scale or a group session where all core elements come together. For each day we have proximally ten standard exercises adjustable to patients' capacity. To further increase the effect, all exercises are performed at high intensities according to existing guidelines.

Discussion: GAPP has been implemented for over 2 years now and suits proximally $80 \%$ of our geriatric inpatient population. An observational study is ongoing to objectify patients' progress.

\section{P-295}

Motor performance in the transition between inpatient geriatric rehabilitation and home environment in cognitively impaired patients after hip or pelvic fracture

Bastian Abel ${ }^{1}$, Rebekka Pomiersky ${ }^{1}$, Jürgen M. Bauer ${ }^{1}$, Martina Schäufele ${ }^{2}$, Klaus Pfeiffer ${ }^{3}$, Klaus Hauer ${ }^{1}$

${ }^{1}$ AGAPLESION Bethanien Hospital Heidelberg, Geriatric Center at the University of Heidelberg, Heidelberg, Germany, ${ }^{2}$ University of Applied Sciences Mannheim, Faculty of Social Work, Mannheim, Germany, ${ }^{3}$ Robert-Bosch-Hospital, Department of Clinical Gerontology, Stuttgart, Germany

Background: Geriatric patients after hip/pelvic fracture (HPF) have reduced motor performance and are at risk of losing their autonomy during the transition from inpatient rehabilitation to their home environment. This risk increases with cognitive impairment (CI).

Aim of this study: The investigation of the change of motor performance in geriatric patients with $\mathrm{CI}$ and HPF in the transition between inpatient rehabilitation and home environment.

Methods: In 239 geriatric patients $(85 \pm 6$ years, Barthel Index: $78 \pm 13$ ) with CI (Mini-Mental State Examination: $22.8 \pm 2.7$ ) and HPF, motor performance (Short Physical Performance Battery (SPPB), habitual gait speed, absolute duration of the SPPB ChairStand-Test) was assessed at the end of rehabilitation and after discharge to the home environment ( $29 \pm 15$ days). An accelerometer-based motion sensor recorded detailed Sit-to-Stand/Stand-to-Sit transfer parameters. Dependent t-tests and effect sizes (Cohen's $\mathrm{d} ;<0.5=$ small, $0.5-0.8=$ medium, $>0.8=$ large) were calculated . Results: Due to nursing home admission $(\mathrm{n}=18)$, death $(\mathrm{n}=7)$, and other reasons $(n=23), 48$ vulnerable patients could not be further investigated. Motor performance at follow-up revealed following significant (all $\mathrm{p} \leq 0.001$ ) improvements: SPPB: Total-Score: $4.2 \pm 1.9$ vs. $5.0 \pm 2.2, \mathrm{~d}=0.43$; Balance-Score: $2.6 \pm 1.0 \mathrm{vs}$. $2.8 \pm 1.0, \mathrm{~d}=0.23$; Gait-Score: $1.3 \pm 0.6$ vs. $1.5 \pm 0.8, \mathrm{~d}=0.38$; Chair-Stand-Score: $1.4 \pm 0.8$ vs. $1.8 \pm 1.0, \mathrm{~d}=0.50$; Habitual gait speed: $0.32 \pm 0.16 \mathrm{~m} / \mathrm{s}$ vs. $0.38 \pm 0.18 \mathrm{~m} / \mathrm{s}, \quad \mathrm{d}=0.35$; Absolute duration Chair-Stand-Test: $22.24 \pm 8.99 \mathrm{~s} \quad$ vs. $17.81 \pm 5.82 \mathrm{~s}$, $\mathrm{d}=0.49$; Average duration Sit-to-Stand: $2.16 \pm 0.75 \mathrm{~s}$ vs. $1.74 \pm 0.57 \mathrm{~s}, \quad \mathrm{~d}=0.55 ; \quad$ Average duration Stand-to-Sit: $2.28 \pm 0.86 \mathrm{~s}$ vs. $1.92 \pm 0.59 \mathrm{~s}, \mathrm{~d}=0.41$.

Conclusion: The results show a moderate improvement of motor performance within a comparatively short post-rehabilitation period in the majority of geriatric patients with CI after HPF. However, a relevant vulnerable subgroup shows a negative course.

\section{P-296}

Factors associated with an improvement of quality of life (QoL) according to the Nottingham Health Profile (NHP) in a Geriatric Rehabilitation Day Hospital (GRDH)

Elisabet de Jaime ${ }^{1}$, Anna Renom ${ }^{1}$, Silvia Burcet ${ }^{1}$, MAngels Vidal ${ }^{1}$, MCarmen Delgado ${ }^{1}$, Montserrat Garcia ${ }^{1}$, MLuz Rodriguez ${ }^{1}$, Eulalia Sevilla $^{1}$, Olga Vazquez ${ }^{1}$, Ramon Miralles ${ }^{1}$

${ }^{1}$ Geriatric Service Centre Forum Parc de Salut Mar, Barcelona, Spain

Introduction: The aim of this study was to identify which factors are associated with QoL improvement among the patients attending a GRDH.

Methods: Longitudinal study including those patients that attended the GRDH for a minimum of seven sessions between January 2007 and December 2011. We registered the following variables at baseline and at the end of the rehabilitation program: sociodemographic variables, NHP questionnaire (global score and subareas), Barthel Index and Lawton Index for activities of daily living, Timed Up and Go and Tinetti for mobility, and Mini-Mental Folstein and GDSYesavage for mental status. We considered QoL improvement if a decrease of the NHP score occurred with an effect size of $\geq 0.50$. We performed bivariate and multivariate analyses.

Results: Of the 139 patients included (mean age $75.6 \pm 13.0,57.5 \%$ women), forty-seven experienced QoL improvement. In the bivariate analysis, being a woman and having attended a higher number of sessions were associated with an improvement of the QoL subarea emotion $(p=0.036)$ and social isolation $(p=0.019)$, respectively. In the multivariate analysis, a decrease in the Timed Up and Go score of $\geq 10 \mathrm{~s}$ was associated with QoL improvement [global QoL (OR 3.11, IC:1.28-8.93); subarea social isolation (OR 3.50, IC: 1.37-8.93)], as well as an increase of the Barthel Index score of $\geq 10$ [subarea physical mobility (OR 2.41, IC: $1.03-5.65$ ); subarea emotion (OR 2.61, IC: 0.98-6.90)].

Key conclusions: Those patients attending a GRDH who are women, attend a higher number of sessions and experience a greater functional improvement may perceive greater improvements of their QoL. 


\section{P-297}

Trans-sectional intervention program to improve geriatric care in Regensburg [TIGER]: the transitional care model applied in Germany

Freiberger Ellen ${ }^{1}$, Rimmele Martina ${ }^{1}$, Wingenfeld Klaus ${ }^{2}$

${ }^{1}$ Institute for Biomedicine of Aging-FAU Nürnberg-Erlangen, Germany, ${ }^{2}$ Institute of Nursing Science at the University of Bielefeld, Bielefeld, Germany

Background: Demographic change will bring us an increase in hospitalizations due to more older persons with comorbidities. As this group is vulnerable to stressors, hospitalizations promote loss of function, of quality of life, of independence as well as a higher infection rate leading to readmissions. Internationally an effective reduction of readmission rates in this population was demonstrated administering nurse support of the Transitional Care Model (TCM). Goal: The primary objective of the trans-sectional intervention project TIGER for geriatric patients is the reduction of readmission rates. Secondary outcomes are the increase in functional health status, quality of life and wound healing, and a decrease of risk of malnutrition.

Method: TIGER is an RCT, the control group receiving usual care. In the intervention group the geriatric patients and their care givers will be supported by special nurses according to TCM — called "Pfadfinder". The Pfadfinder contact the geriatric patients already in the hospital and accompany them over 12 months in the ambulatory setting, integrating different modules of support. The target number of participants is 400 . Inclusion criteria are 70 years and older, cognitive status measured by MMSE $\geq 22$, living in the vicinity of $\leq 50 \mathrm{~km}$, membership in a specific health insurance; being discharged to home. The "First patient in" was randomized in April 2018. The project will continue till August 2020.

Perspectives: The TIGER project addresses the current transitional health care gap in Germany in the geriatric hospital population. The presentation will address recruiting aspects and present data of included patients.

\section{P-298}

\section{Geriatric rehabilitation in older patients with cardiovascular} disease, a feasibility study

Eleonore F. van Dam van Isselt ${ }^{1}$, Jan van Wijngaarden ${ }^{2}$, Dirk J. A. Lok ${ }^{2}$, Wilco P. Achterberg ${ }^{1}$

${ }^{1}$ Department of Public Health and Primary Care, Leiden University Medical Centre, Leiden, The Netherlands, ${ }^{2}$ Department of Cardiology, Deventer Ziekenhuis, Deventer, The Netherlands

Introduction: Considering the worldwide ageing populations and growing numbers of older patients with cardiovascular disease (CVD), studies on development, implementation and outcomes of specific geriatric rehabilitation (GR) programs for patients with CVD are needed. We developed and implemented a GR program for older patients with significant functional decline after hospital admission because of CVD: 'the GR-cardio program'. Aim of the program is to restore functional capacity to such an extent that discharge back home is possible, improve quality of life and prevent hospital re-admissions. The primary goal of the present study is to investigate feasibility of the GR-cardio program.

Methods: This is a real life observational study with a follow-up period of 6 months in patients admitted to the GR-cardio program. We collected data on patient- and disease characteristics and course of functional status and quality of life. Six month after discharge data on re-admissions and mortality were collected.

Results: In total 58 patients (mean age $78.8( \pm 9.8)$ years) were included in the study. Co-morbidities were frequent and functional status and quality of life were severely impaired on admission but showed clinically relevant improvement during the program. Eightythree percent of all patients were discharged back home after a mean length of stay 38 days.

Key conclusions: This study indicates that GR for patients with CVD is feasible and can probably offer substantial benefits. More research is needed and should focus on (cost)-effectiveness of the program and identifying patient characteristics that can predict which older patients with CVD are most likely to benefit.

\section{P-299}

Pain as the main determinant of fall-associated self-efficacy after hip/pelvic fracture in geriatric rehabilitation

Rebekka Pomiersky ${ }^{1}$, Bastian $\mathrm{Abel}^{2}$, Anja Dautel ${ }^{3}$, Martina Schäufele $^{4}$, Klaus Pfeiffer ${ }^{3}$, Jürgen M. Bauer ${ }^{2}$, Klaus Hauer ${ }^{2}$

${ }^{1}$ Agaplesion Bethanien Hospital Heidelberg, Geriatric Center at the University of Heidelberg, Heidelberg, Germany and University of Applied Sciences Mannheim, Faculty of Social Work, Mannheim, Germany, ${ }^{2}$ Agaplesion Bethanien Hospital Heidelberg, Geriatric Center, Heidelberg, Germany

Introduction: Low falls-related self-efficacy (FSE) might negatively affect rehabilitation outcome. Aim of the study was to determine established (age, sex, motor performance and depressive symptoms) and hitherto neglected determinants (pain) of FSE in geriatric patients after hip/pelvic fracture.

Methods: In a cross-sectional design, 40 patients $(82.1 \pm 6.7$ years $)$ with hip/pelvic fracture without cognitive impairment (Mini-Mental State Examination: $28.3 \pm 1.1$ ) were assessed for demographic data, FSE (Short Falls Efficacy Scale International), motor performance (Short Physical Performance Battery), depressive symptoms (Montgomery-Åsberg Depression Rating Scale), and pain (Western Ontario and McMaster Universities Osteoarthritis Index, subscale pain). After univariate evaluation of correlations between FSE and established parameters, variables $(p \leq .100)$ were included in a first multiple linear regression model with FSE as dependent variable. In a second model, the variable list has been extended by the parameter pain. The parameters were interpreted on the basis of significance $(p \leq .05)$, standardized regression coefficients $\beta$, and determination coefficients $\mathrm{R}^{2}$.

Results: In the first model, the included significant determinants motor performance $(\beta=-.310, \mathrm{p}=.034)$, age $(\beta=.309, \mathrm{p}=.032)$, and depressive symptoms $(\beta=.358, \mathrm{p}=.013)$ elucidated $39.4 \%$ of variance $\left(\mathrm{R}^{2}=.394\right)$ for the FSE. In the second model, the parameters age $(\beta=.317, \mathrm{p}=.020)$ and pain $(\beta=.338, \mathrm{p}=.022)$ improved the explanation of variance up to $48.5 \%\left(\mathrm{R}^{2}=.485\right)$. The parameters motor performance $(\beta=-.249, \mathrm{p}=.072)$ and depressive symptoms $(\beta=.227, \mathrm{p}=.111)$ made no significant contribution.

Key conclusions: These results provide first indications that the FSE, as an important parameter for positive geriatric rehabilitation outcome, is significantly influenced by pain and age in vulnerable patients after a hip/pelvic fracture. 


\section{P-300}

Choice, voice and co-production in intermediate care: exploring the geriatric patients and their relatives perspectives on patient participation

M.Sc. Linda $\mathrm{Kvæl}^{1}$, Dr. Jonas Debesay ${ }^{2}$, Dr. Anne Langaas ${ }^{1}$, Dr. Asta Bye ${ }^{3}$, Prof. Astrid Bergland ${ }^{1}$

${ }^{1}$ Oslo Metropolitan University, Department of Physical Therapy, Norway, ${ }^{2}$ Oslo Metropolitan University, Department of Nursing and Health Promotion, Norway, ${ }^{3}$ Oslo Metropolitan University, Department of Nursing and Health Promotion/Oslo University, Norway

Introduction: Despite the knowledge that patient participation in intermediate care (IC) contributes to enhanced quality of care, elderly frail patients often do not feel involved in their own rehabilitation process. This study explore geriatric patients and their relatives' experiences and preferences of patient participation in IC, identifies types of patient participation and their potential empowering or disempowering effect. The study aims to expand the knowledge on how health professionals can contribute in tailoring good interventions in IC, and to improve the understanding of underlying structures.

Methods: Using a qualitative approach underpinned by critical realism and the European framework of Dent \& Pahor (2015), we explored the experiences and preferences of patient participation in IC of 15 patients and 12 relatives using semi-structured interviews. Thematic analysis based on Clarke and Braune (2006) were used to analyze the data.

Results: The analysis disclosed a lack of choice in a predetermined pathway. Being deserving means to be sick enough and is associated with the compliant patient that fit the system. Some main additional features are the need of a rehabilitation perspective and patient engagement to enable co-production as well as the important contribution of relatives as advocates and allies. Finally, the results show the patients vulnerable voice in the meeting with experts' views.

Key conclusions: All though most of the patients benefited from IC, patient participation in this context could indeed be structured in a more empowering manner. Patient participation in IC is influenced by underlying structures such as market, bureaucracy and psychology.

\section{P-301}

Life-space mobility among multimorbid older persons with multiple impairments following rehabilitation

Phoebe Ullrich $^{1}$, Tobias Eckert ${ }^{1}$, Martin Bongartz ${ }^{1}$, Christian Werner ${ }^{1}$, Rainer Kiss ${ }^{2}$, Jürgen M. Bauer ${ }^{1}$, Klaus Hauer ${ }^{1}$

${ }^{1}$ Department of Geriatric Research, AGAPLESION Bethanien Hospital Heidelberg, Geriatric Center at the Heidelberg University, Heidelberg, Germany, ${ }^{2}$ Department of Health and Social Affairs, FHM Bielefeld, University of Applied Science, Bielefeld, Germany

Introduction: Studies on life-space mobility (LSM) and its determinants have previously been conducted in older communitydwelling persons, but are lacking in older persons with cognitive impairment $(\mathrm{CI})$ following discharge from geriatric rehabilitation. The study objective was to describe LSM and identify sample-specific determinants in this high risk group for activity limitations.

Methods: The cross-sectional study included 118 older communitydwelling, former rehab patients with mild to moderate CI. LSM was evaluated by the validated Life-Space Assessment in Persons with Cognitive Impairment (LSA-CI). Associations between LSM and physical, cognitive, psychosocial, environmental, financial and demographic participant characteristics, selected following a theoretical framework on LSM mobility, were calculated and then analyzed using linear regression analysis. Parameters were assessed by internationally established motor performance tests, sensor-based measures resp. standardized interviews.

Results: Analysis revealed overall limited LSM in the group of multimorbid older persons (age: $82.3 \pm 6.0$ years) with CI (MiniMental State Examination score: $23.3 \pm 2.4$ ) with a wide range of restrictions. More than $30 \%$ of participants were confined to the neighborhood and half of all patients could not leave the bedroom without equipment or assistance. Physical performance, social activities, physical activity and gender were identified as independent determinants of life-space mobility and explained $42.4 \%$ (adjusted $r^{2}$ ) of the LSA-CI's variance in the regression model.

Key conclusions: This study documents the highly restricted LSM of a vulnerable study population. Identified determinants may represent potential targets for future life-space interventions. A gender specific approach may help to address more advanced restrictions in women.

\section{P-302}

Adherence to a home-based mixed training and motivation intervention in geriatric patients with cognitive impairment following inpatient rehabilitation

Tobias Eckert ${ }^{1}$, Martin Bongartz ${ }^{1}$, Phoebe Ullrich ${ }^{1}$, Rainer Kiss ${ }^{2}$, Jürgen M. Bauer ${ }^{1}$, Klaus Hauer ${ }^{1}$

${ }^{1}$ Department of Geriatric Research, AGAPLESION Bethanien Hospital Heidelberg, Geriatric Center at the Heidelberg University, Heidelberg, Germany, ${ }^{2}$ Department of Health and Social Affairs, FHM Bielefeld, University of Applied Sciences, Bielefeld, Germany

Objectives: Though adherence is a strong determinant for success of home-based exercise interventions among geriatric patients with cognitive impairment (CI), effectiveness of motivational strategies to increase adherence to regular exercise and physical activity (PA) has rarely been tested. Aim of the current study is to describe adherence to a 12-week home-based mixed training and motivation intervention among patients with CI after discharge from geriatric inpatient rehabilitation.

Methods: Secondary data analysis of a randomized-controlled intervention trial. Data from cognitively impaired (Mini-Mental Examination score: $23.5 \pm 2.5)$ geriatric patients $(81.5 \pm 5.9$ years $)$, recently discharged from inpatient rehabilitation, in intervention group $(n=54)$ were analyzed. To achieve daily execution of functional training (strength, balance) and increase of PA, motivational strategies (outdoor activity, goal achievement, monitoring by pedometers) were implemented. Adherence to training and motivational strategies was documented on base of exercise logs (\% of maximal sessions). To test for differences between week 2 and 12, Wilcoxon tests were conducted.

Results: At week 2 adherence to training was $74.5 \%$, outdoor activity $61.0 \%$, goal achievement $43.4 \%$ and monitoring by pedometer $66.7 \%$. At week 12 adherence rates to training $(51.5 \%)$, outdoor activity $(46.5 \%)$ and monitoring by pedometers $(51.5 \%)$ were significantly lower $(\mathrm{p}=0.001-0.022)$ than in week 2 , except for goal achievement $(34.5 \% ; \mathrm{p}=0.170)$.

Conclusions: The moderate to high adherence to our home-based mixed training and motivation intervention proved feasibility of this innovative motivational approach among geriatric patients with CI. Decreasing adherence indicated the potential need of more successive supervision to achieve long-term adherence and increased PA. 


\section{P-303}

Pressure ulcer risk and mortality in patients discharged to skilled nursing facilities

\author{
Paul Takahashi ${ }^{1}$ \\ ${ }^{1}$ Mayo Clinic, Rochester, USA
}

Introduction: Elderly patients discharged to skilled nursing facilities (SNF) for post-acute care are a high risk population. The Braden score, calculated to determine risk of developing pressure ulcers, includes elements that indicate frailty and functional dependence [1]. We conducted this analysis to evaluate the association between risk of pressure ulcers during hospitalization and mortality in this population. Methods: This was a retrospective analysis of 30-day, 6-month and 1-year mortality in patients discharged to SNF following hospitalization between January 1, 2009 and June 30, 2014. Braden scores, demographic, comorbidity, and mortality data obtained from clinical and administrative databases were analyzed to compare mortality rates in patients with Braden scores in the at-risk category during hospital stay to those not at risk.

Results: There were 8616 discharges from Mayo Clinic, Rochester hospitals to ten area SNF that were served by its long-term care practice during the study period. 5385 had Braden scores in the atrisk category while hospitalized. The 30-day, 6-month, and 1-year mortality rates were higher $(6.7 \%, 20.7 \%$ and $28.2 \%$ respectively) in patients with at-risk Braden score compared to those not at risk (1\%, $5.5 \%$ and $8 \%$ respectively) $(\mathrm{p}<0.001)$.

Conclusion: Patients at risk of pressures ulcers had persistently higher mortality rates suggesting that Braden scores during hospitalization may serve to identify high-risk patients at SNF admission. Reference:

1. Nancy Bergstrom et al (1987) The Braden score for predicting pressure sore risk. Nurs Res. 36(4)

\section{P-304}

Comparison of two fall risk screenings and fall prevention interventions in older in-hospital patients: an health economic evaluation

\section{Lilli Neumann ${ }^{1}$}

${ }^{1}$ Albertinen-Haus, Geriatrics Centre, Scientific Department at the University of Hamburg, Hamburg, Germany

Introduction: In-hospital falls are reported with 1.3-8.9 falls/1000 hospital days. They are associated with prolonged hospital stay, worsened immobility, disability, institutionalisation and cause added use of monetary resources (e.g. 4233 USD per patient). Effective fallprevention is based on fall-risk screening and multifactorial interventions. The LUCAS fall-risk screening was developed and validated based on the widely used STRATIFY screening. However, LUCAS consists of fewer fall-risk factors and is more suitable to define a dedicated high-risk group. The rationale was to perform an economic evaluation of a multifactorial fall-risk prevention concept in a German geriatric clinic using either the LUCAS or the STRATIFY fall-risk screening.

Methods: Two clinical data sets (patients aged $\geq 65$ years), were used for the analyses: (DS1) data-set 2004-2006 ( $\mathrm{n}=4735)$ and (DS2) 2010-2011 ( $\mathrm{n}=2402)$. From a hospital perspective, analyses included a cost-cost analysis (DS1) and a cost-effectiveness-analysis (STRATIFY: DS1, LUCAS: DS2). Sensitivity analyses examined robustness. Questionnaires were used to calculate costs for personnel and materials necessary for the fall prevention intervention concept. The outcome was defined as rate of fallers.
Results: Cost-cost-analysis: incremental costs LUCAS - 82.23 EUR/pat.; cost-effectiveness-analysis: incremental costs - 200.88 EUR; incremental outcome/pat. 0.02; ICER - 11,973.75 EUR. Differences were explained by changed patients' fall-risk structure and reduced length of hospital stay. Robustness of LUCAS' advantage was confirmed except the minimum-variation of the cost-cost analysis.

Key-conclusions: From a health economic view, LUCAS was the favourable screening alternative. It produced an economic benefit as applied in an identical patients' fall-risk structure.

\section{P-305}

The effects of decongestive physiotherapy on pain and sleep quality in elderly women with breast cancer related lymphedema

Orçin Telli Atalay ${ }^{1}$, Atiye $\mathrm{Kaş}^{2}$, Sevda Yılmaz ${ }^{2}$, Nesrin Yağc1 ${ }^{2}$

${ }^{1}$ Pamukkale University, Denizli, Turkey, ${ }^{2}$ Pamukkale University, Denizli, Turkey

Introduction: The incidence of lymphedema is increasing among elderly breast cancer survivors. Complex decongestive physiotherapy (CDP) is commonly used as a primary treatment. The aim of this study was to investigate the effects of CDP on pain and sleep quality in elderly breast cancer related lymphedema patients.

Methods: Twenty-one women with breast cancer related lymphedema were assigned as two groups; $\operatorname{CDP}(\mathrm{n}=13$, mean age $=65.16 \pm 6.23)$ and control group $(n=8$, mean age $=64.91 \pm$ 8.12). The CDP group received manual lymph drainage, compressive bandages and exercise therapy (5/week for 4 weeks). The subjects in the control group were given self-massage and exercise therapy as home program. The upper extremity level of pain were assessed with visual analog scale. The Pittsburgh Sleep Quality Index was used for the assessment of sleep quality. The circumference measurements were done for the assessment of lymphedema. All the subjects were assessed at baseline and after CDP and home progam.

Results: The level of pain and sleep quality in CDP group improved significantly $(\mathrm{p}=0.04, \mathrm{p}=0.001$ respectively).The extremity circumference also decreased in CDP group $(\mathrm{p}=0.012)$. There was also a significant decrease in the extremity circumference of control group but there was not any change in terms of pain level and sleep quality $(p>0.05)$. Significant differences were found between the two groups in terms of the decrease in edema, pain level and sleep quality $(\mathrm{p}=0.042, \mathrm{p}=0.001, \mathrm{p}=0.029)$.

Key conclusions: The CDP affects the level of edema, severity of pain and sleep quality positively in elderly women with breast cancer lymphedema.

\section{P-306}

The Relationship between respiratory function and functional performance in elderly patients with COPD

Orçin Telli Atalay ${ }^{1}$, Harun Taşkın ${ }^{1}$, Ali Yalman ${ }^{1}$, Ayşenur Yılmaz ${ }^{1}$, Erhan Kızmaz ${ }^{1}$

${ }^{1}$ Pamukkale University, School of Physical Therapy and Rehabilitation. Denizli, Turkey

Introduction: In patients with COPD, respiratory dysfunction causes a decrease in extremity function, exercise performance, reduced skeletal muscle strength, and severe limitations in physical functioning. Further deterioration in pulmonary function is the factor that affects the worsening of physical performance. The aim of this study 
was to investigate the relationship between respiratory functions and functional performance in elderly COPD patients.

Methods: Forty-four patients who were referred to the cardiopulmonary rehabilitation unit of the Physical Therapy and Rehabilitation of Pamukkale University were included in this study. Respiratory function tests of the subjects were performed with a portable spirometer according to the ATS-ERS guidelines. The Turkish version of Functional Performance Scale (FPS) was used in the evaluation of functional performance.

Results: Thirty-one (70.45\%) male 13 (29.55\%) female subjects with a mean age of $68.08 \pm 11.15$ were included in the study. The average of total score of FPS was $68.81 \pm 34.20$, Modified Medical Research Council dyspnea score was $1.78 \pm 0.96$ and the average results of Pulmonary function test were as $\% \mathrm{FEV} 1=62.68 \pm 17.21$, $\% \mathrm{FVC}=68.74 \pm 11.14, \quad \% \mathrm{FEV} 1 / \mathrm{FVC}=89.12 \pm 22.76, \quad \% \mathrm{PEF}=$ $64.22 \pm 19.45, \%$ MEF $25-75=57.42 \pm 16.71$. When the relation between respiratory functions and FPS was examined, there was a significant correlation between FEV1, FVC, MEF25-75 and FPS total score $(\mathrm{p}<0.05, \mathrm{r}=0.63)$.

Key conclusions: As a result of our study, it was found that in elderly COPD patients, there was a relationship between respiratory functions, dyspnea level and functional performance. Elderly patients with COPD have limitations in their physical exercise and body-care activities as their respiratory functions and dyspnea deteriorate.

\section{P-307}

Benefits of geriatric day hospital in patients with hip fracture

Paulo Almeida ${ }^{1}$, Raquel Sosa ${ }^{2}$, Pilar Cabezas ${ }^{2}$, Arturo Sevillano ${ }^{2}$, Sofia Duque $^{3}$, Carlos Verdejo ${ }^{2}$

${ }^{1} \mathrm{CHBV}$, Aveiro, Portugal, ${ }^{2} \mathrm{HCSC}$, Madrid, Spain, ${ }^{3} \mathrm{CHLO}$, Lisboa, Portugal

Introduction: Geriatric Day Hospital (GDH) is an interdisciplinary hospital care model, which main objective is functional rehabilitation of geriatric patients. The study aim is to analyse demographic and clinical data of patients submitted to hip fracture (HF) surgery and investigate their functional rehabilitation in GDH after hospital discharge.

Methods: Observational and longitudinal study by process analysis and observation of patients submitted to HF surgery, followed in GDH for 8 months. Demographic, clinical, and laboratorial variables were measured; functional assessment scores were calculated before $\mathrm{HF}$, at admission and discharge of GDH. Statistical analysis in SPSS. Results: 32 patients (mean age $86.2 \pm 4.8$ years and $78 \%$ women) were included. $56.3 \%$ came from the Convalescence Unit (CU). Most frequent comorbidities: osteoarthrosis $(84.0 \%)$, visual impairment $(46.9 \%)$ and depression (34.4\%). There was a functional decline between the period before HF and admission to GDH [mean Barthel's Scale (BS) value from $89.9 \pm 13.1$ to $69.8 \pm 14.3$ ] and improvement of functional status between admission and discharge of GDH (mean BS value from $69.8 \pm 14.3$ to $85.5 \pm 11.6$ ). The use of physical aids for gait decreased (from 87.5 to $77.0 \%$ ) and polypharmacy was reduced (mean of drugs from $10.6 \pm 2.9$ to $8.5 \pm 2.9$ ). The MannWhitney test showed improved Short Physical Performance Battery values in patients from CU. A positive association was found between Timed Up and Go test values improvement and vitamin D higher levels (Spearman's Rho $(17)=0.528$ ).

Conclusions: Follow-up in GDH and multidisciplinary approach in patients submitted to HF surgery are associated with improved functional rehabilitation and reduced polypharmacy.

\section{P-308}

Improving the management of asymptomatic bacteriuria in older patients undergoing elective orthopaedic surgery

Aine Goggins ${ }^{1}$, Vivien Nebo ${ }^{1}$, Cathryn Mainwaring ${ }^{1}$, Jugdeep Dhesi ${ }^{1}$

${ }^{1}$ Guy's \& St. Thomas Hospital, London, UK

Introduction: Asymptomatic bacteriuria (ASB) is common in older patients undergoing elective joint arthroplasty (EJA) (1). Testing for ASB is frequently undertaken at preoperative assessment (POA) due to historical concerns over possible spread to the prosthesis. This leads to unnecessary testing, delays in surgery \& antimicrobial overuse. A systematic review carried out at our centre found no evidence to support routine MSU/urinalysis in the perioperative setting.

Methods: 1st stage: Patients undergoing EJA from both the pre-operative assessment unit (POAU) and Proactive care of older people undergoing surgery (POPS) clinics at our centre were analysed. Of 180 patients, a total of 93 pre-operative MSUs were sent, 67 of which were in asymptomatic patients with no clear indication for testing documented. 2nd stage: We developed and implemented a MSU testing decision tool, based on NICE and SIGN guidance. All stakeholders approved the tool. Staff education and posters were used to facilitate this practice change. Rolling data was collected over 3 months to assess adherence to the decision-support tool in both POPS and POA unit.

Conclusion: Of 49 patients undergoing EJA in this time period only 2 MSUs were sent preoperatively; 1 appropriately and 1 inappropriately, resulting in $98 \%$ adherence to the decision support tool. Our simple decision flow chart has almost eliminated inappropriate MSU testing of ASB in the POA of older orthopaedic patients undergoing PJI. It is clear that the implementation of similar tools in other orthopaedic outpatient settings would lead to reduced costs, cancellations and improved antibiotic stewardship.

\section{P-309}

We must give the elderly a chance-it is our responsibility: homebased rehabilitation

Roni Gagin ${ }^{1}$, Neta HaGani ${ }^{1}$, Itay Levy ${ }^{1}$, Moriya Gozlan ${ }^{1}$, Doron Norman ${ }^{1}$

${ }^{1}$ Social Work Department, Rambam Health Care Campus, Haifa, Israel

Introduction: The elderly is the primary target population for rehabilitation services. Elderly people are at risk for a significant decrease in function during hospitalization. The return to their natural and familiar environment has a positive influence on rehabilitation pace and outcomes as long as they have supportive families and communities.

Methods: In a survey conducted by the social work department, we examined the overall satisfaction from home-based rehabilitation program. Telephone interviews were conducted among elderly orthopedic patients. The questionnaire included: waiting times for the program, satisfaction from the rehabilitation services and unmet needs.

Findings: The overall satisfaction from rehabilitation outcomes was high among most participants (73\%). However, participants reported on a long waiting time (Mean $=4.58, \mathrm{SD}=4.67$ days) from the hospital discharge date, and that was associated with lower satisfaction. Most participants reported they didn't receive nursing services (76\%) and doctor visits (46\%). Participants reported on gaps in the 
program like: accessibility at home, emotional difficulties, loneliness, lack of response to the needs of caregivers and long term follow-up. Conclusions: As the world's elderly population continues to grow, there is a need to establish guidelines and to promote a home-based rehabilitation "basket", suitable for patients' and families' needs that will allow reduction in hospitalization days and in medications. The challenge health professionals are facing today is how to define the home-based rehabilitation "basket" and how to have it approved and implemented by policy makers.

\section{P-310}

Associations of motor performance and qualitative/quantitative physical activity behavior in older persons with cognitive impairment after discharge from geriatric rehabilitation

Martin Bongartz ${ }^{1}$, Phoebe Ullrich ${ }^{1}$, Tobias Eckert ${ }^{1}$, Christian Werner $^{1}$, Rainer Kiss ${ }^{2}$, Bastian Abel ${ }^{1}$, Sabato Melone ${ }^{3}$, Jürgen M. Bauer $^{1}$, Klaus Hauer ${ }^{1}$

${ }^{1}$ Department of Geriatric Research Agaplesion Bethanien-Hospital, Geriatric Centre at the University of Heidelberg, Germany,

${ }^{2}$ Department of Health and Social Affairs, FHM Bielefeld, University of Applied Sciences, Germany, ${ }^{3}$ Department of Electrical, Electronic, and Information Engineering, University of Bologna, Italy

Introduction: Geriatric patients with cognitive impairment (CI) show deficits in motor performance and decreased physical activity (PA) behavior in everyday life. Aims of this cross-sectional study were the analysis of associations between motor performance, habitual PA and habitual gait behavior and the identification of modifiable, motor determinants of PA behavior in older persons with CI after discharge from geriatric rehabilitation.

Methods: In multi-morbid, geriatric patients with CI motor performance was measured using the Short Physical Performance Battery (SPPB). A newly developed and validated activity monitor (uSense) captured innovative, qualitative parameters of gait behavior and quantitative parameters of gait and general habitual PA. Bivariate associations were calculated using Spearman correlations (rho). Parameters of motor performance and qualitative gait behavior with significant correlations were included in a linear regression model to identify motor determinants of PA behavior.

Results: Motor performance showed high correlations with PA behavior in 110 older persons with CI (age $=82.3 \pm 5.9$ years, MiniMental State Examination $=23.3 \pm 2.4$ ). Qualitative gait characteristics during straight walking and turns showed moderate to high correlation with PA behavior. In the linear regression model mean turning velocity, gait symmetry, cadence and SPPB explained $45 \%$ of the variance $\left(\mathrm{R}^{2}=0.45\right)$ of PA behavior.

Conclusions: The results showed a high relevance of motor performance for PA behavior in geriatric, multi-morbid patients with CI. Besides established motor performance variables (SPPB), innovative qualitative parameters of habitual gait behavior may be taken in consideration of rehabilitation or activity promotion programs respectively.

\section{P-311}

Cognitive function in older adults admitted to a geriatric rehabilitation: does it really matter for functional recovery? Results from the FRAIL-BCN study

Alicia Calle ${ }^{1}$, Diana Lelli ${ }^{2}$, Laura Mónica Pérez ${ }^{3}$, Cristina Udina ${ }^{3}$, Neus $\mathrm{Gual}^{3}$, Mónica Mesas ${ }^{3}$, Nuria Torrents ${ }^{3}$, Miriam Colominas ${ }^{3}$, Marco Inzitari ${ }^{3}$

${ }^{1}$ Parc de Salut Mar. Hospital de la Esperanza, Barcelona, Spain,

${ }^{2}$ Università Campus Bio-Medico di Roma, Rome, Italy, ${ }^{3}$ Parc Sanitari Pere Virgili, Barcelona, Spain

Introduction: Cognitive impairment is associated with poorer functional outcomes (FO) in elderly undergoing rehabilitation; however its role on rehabilitation is controversial.

Objectives: To assess the association between cognitive function and FO in older adults admitted to a geriatric rehabilitation unit after hip fracture or stroke. To evaluate differences on functional recovery prediction between Symbol Digit Modalities Test (SDMT) and MiniMental State Examination (MMSE).

Methods: Prospective cohort study with patients $\geq 65$ years old admitted to a geriatric rehabilitation unit after hip fracture or stroke. FO were: absolute functional gain $>20$ (AFG, discharge or 6 monthsadmission Barthel index) and walking recovery (WR, discharge or 6 months-admission walking Barthel index item). The association was assessed with Relative Risk (RR, 95\% CI). Analyses were then stratified for diagnosis at admission. The discriminative capacity of SDMT and MMSE was assessed by comparing their AUC.

Results: 202 patients were included [mean age (SD) $83.4( \pm 6.7)$ years, $70 \%$ female]. Patients with $\mathrm{MMSE} \geq 20$ had a $\mathrm{RR}=2.22$ (95\% CI 1.33-3.72) of AFG $>20$ at discharge, results remained stable at 6 months $(\mathrm{RR}=1.70,95 \%$ CI $1.15-2.52)$. These results were confirmed only for hip fracture patients (Discharge: $\mathrm{RR}=3.74$, 95\% CI 1.30-10.72; 6 months: $\mathrm{RR}=2.55,95 \%$ CI $1.09-5.99)$. WR at discharge and after 6 months was not related with baseline cognitive function; these results were confirmed also after diagnosis stratification. Comparing SDMT and MMSE, there was no difference in their functional recovery prediction.

Conclusion: WR was not affected by cognitive function in older patients admitted to a rehabilitation unit. Furthermore, the effectiveness of rehabilitation program is maintained over 6 months after discharge. Therefore, rehabilitation should be considered in all patients, independently from their cognitive status.

\section{P-312}

The safety, efficacy and acceptability of non-pharmacologic treatments for orthostatic hypotension in older people: a mixedmethods study

Lisa J. Robinson ${ }^{1}$, Julia L. Newton ${ }^{2}$, Ruth M. Pearce ${ }^{2}$, Jake R Gibbon ${ }^{1}$, James Frith ${ }^{2}$

${ }^{1}$ Newcastle upon Tyne Hospitals NHS Trust, Newcastle upon Tyne, UK, ${ }^{2}$ Institute of Ageing, Newcastle University, Newcastle upon Tyne, UK

Introduction: Older people with $\mathrm{OH}$ are frequently prescribed nonpharmacologic therapies. However the evidence supporting their use is limited, moreover uptake and adherence can be low.

Methods: An exact, single-stage, phase 2 study was performed, with $80 \%$ power to demonstrate a $30 \%$ response rate and a $95 \%$ chance of rejecting interventions with response rates $\leq 10 \%$. This was complimented with semi-structured qualitative interviews, interpreted using thematic analysis. 25 participants were recruited from a Falls 
and Syncope Service. Participants rested supine for $10 \mathrm{~min}$ before standing upright for $3 \mathrm{~min}$ with beat-to-beat $\mathrm{BP}$ monitoring. Responses to bolus water drinking, grade 2 full leg compression stockings, abdominal compression, leg and abdominal compression, physical-counter manoeuvres (PCMs) were evaluated.

Results: Participants were aged 60-92 (median 74). Water drinking was efficacious in $56 \%$ (95\% CI 34.9, 75.6). This was generally considered acceptable, but with concerns around urinary frequency. PCMs were efficacious in $44 \%(24.4,65.1)$ and were considered the most acceptable as they are discreet and no equipment is required. There were mixed views on the acceptability of abdominal compression which was efficacious in $52 \%(31.3,72.2)$. Stockings were considered unacceptable due to difficult application/removal and were the least efficacious, $32 \%(15,53.5)$. Leg and abdominal compression were efficacious in $52 \%(31.3,72.2)$. There were no adverse events. Conclusions: Bolus water drinking and PCMs are safe, efficacious and acceptable. Stockings were unacceptable and the least efficacious and should be disregarded as first-line therapy. There is no additional benefit gained by adding leg compression to abdominal compression.

\section{P-313}

The management of complications of type 2 diabetes mellitus in a geriatric Rehabilitation Hospital in Malta

\section{S. Cauchi ${ }^{1}$, F. Farrugia ${ }^{1}$, M. A. Vassallo ${ }^{2}$}

${ }^{1}$ Mater Dei Hospital, Msida, Malta, ${ }^{2}$ Rehabilitation Hospital Karin Grech, Guardamangia, Malta

Introduction: The aims of this study were to compare the current management of ophthalmic and podology complications of type 2 diabetes mellitus (T2DM) in a Geriatric Rehabilitation Hospital in Malta to the National Institute for Health and Care Excellence Guidelines [1, 2].

Methods: All the patients with T2DM admitted in all the wards in the Geriatric Rehabilitation Hospital over a period of 2 weeks were included in the study. The data collection included basic demographic data, the physical examination findings in the patient's file, the documented referral to ophthalmic and podology services and the most recent level of glycated haemoglobin (HbAlc).

Results: A total of 78 patients had T2DM; 69\% were prescribed oral hypoglycaemic agents (OHAs), $17 \%$ had insulin and $14 \%$ of patients were prescribed both OHAs and insulin. $50 \%$ of patients did not have documented physical findings of peripheral vascular disease or ophthalmic complications. $14 \%$ of patients had ulcers and sores on physical examination, $9 \%$ of patients had documented cataracts and 9\% had no complications of note. $29.5 \%$ of patients had both an ophthalmic and podology review, 19\% had a documented podology review and $11.5 \%$ had a documented ophthalmic review within the previous year. $76 \%$ of patients had an HbA1c level taken within the previous 6 months.

Key conclusions: Health care professionals should ensure that referrals and follow-up appointments for podology and ophthalmology are offered to type 2 diabetic patients.

\section{References:}

1. National Institute for Health and Care Excellence (2015) Type 2 diabetes in adults: management. NICE guideline [NG28]

2. National Institute for Health and Care Excellence (2015) Diabetic foot problems: prevention and management [NG19].

\section{P-314}

Level of physical activity in men and women with chronic stroke.

\author{
Karin Hellström ${ }^{1}$, Annika Bring ${ }^{1}$ \\ ${ }^{1}$ Institute for Neuroscience, Physiotherapy, Uppsala University, \\ Uppsala, Sweden
}

Introduction: Community-dwelling stroke survivors generally show low levels of physical activity. An improved understanding of the factors influencing participation in physical activity after stroke is imperative to improve levels of physical activity. Furthermore, gender differences in physical activity have received little attention in stroke research. The objective of this study was to examine gender differences in physical activity, physical functioning and psychological factors and the association between these factors and physical activity in men and women 1-3 years post-stroke.

Materials and methods: A total of 187 community-dwelling individuals with stroke (65-85 years old, $29 \%$ women) were included in a secondary analysis based on data from a cross-sectional study. The exclusion criteria were severe cognitive or language dysfunction or dementia. The level of physical activity was measured by the Physical Activity Scale for the Elderly (PASE). Physical function included balance, walking speed and mobility. Psychological factors included depression, health-related quality of life and fall-related self-efficacy. Falls and fear of falling were each measured with a single question. Results: There were no significant differences in physical activity levels between men and women. In multiple regression analyses, walking speed $(\mathrm{p}<0.001)$ was associated with physical activity in men, and balance ( $\mathrm{p}=0.038)$ was associated with physical activity in women.

Conclusions: The results indicate that strategies to increase physical activity levels 1-3 years post-stroke could be improved by considering gender-specific factors. Published in Physiotherapy, Theory and Practice, April 2018.

\section{P-315}

Management of chronic constipation in geriatric patients: 5 years prospective study

Maria Cristina Neri ${ }^{1}$, Tassi Anna Paola ${ }^{1}$, Antoniotti Nadia ${ }^{1}$, Bergamaschini Luigi ${ }^{2}$

${ }^{1}$ ASP-IMMeS Pio Albergo Trivulzio, Geriatric Institute, Milan, Italy, ${ }^{2}$ Department of Internal Medicine, University of Milan, Italy

Chronic constipation (CC) is one of the most frequent intestinal disorders in daily medical practice and is strongly influenced by life and nutrition conditions. In the general population of industrialized countries, the prevalence varies from 10 to $20 \%$ and increases with age, to more than $70 \%$ in the Nursing Home Residents. Two pathophysiological conditions are recognized: Slow Transit Constipation (SRT) characterized by prolonged transit time of stools through the colon and reduction of evacuations less than three per week, and Dyssynergic Defecation (DD) due to difficult or unsatisfactory expulsion of stool from the anorectum, that may result from a lack of coordination between abdominal muscles contraction and pelvic floor muscle relaxation. Literature data suggest an important role in rehabilitative therapy, ie anorectal biofeedback, especially in DD. In this study we have reported our data on the management of elderly patients in a geriatric clinic dedicated to the treatment of chronic constipation. From June 2012 to June 2017, 908 patients with constipation (mean age $61 \pm 2.7$ years, $\mathrm{M} \pm \mathrm{ES}, 337 \mathrm{M}, 571 \mathrm{~F}$ ) have 
been evaluated according to the criteria of Rome III, the Bristol scale (constipation $=$ stool type $1-3$ ) and the Wexner scale (constipation $=>5 / 30$ ). After exclusion of secondary constipation, hygienic/ dietary requirements have been suggested for all patients, and prescribed osmotic fibers and/or laxatives (lactulose, macrogol) therapy and short-term, on-demand irritable laxatives (senna, bisacodile).All patients were asked to keep a daily diary on stool characteristics and were re-evaluated after 1, 3, 12 months. Patients who did not report treatment benefits after 3 months were treated with second-level drugs (prucalopride and/or linaclodide) and subsequently re-evaluate through functional tests included intestinal transit time and anorectal manometry, and, if indicated, initiated for rehabilitation treatment. The majority of patients reported benefit from therapy (Bristol scale type 3-4 with stools more frequent and soft, defecation and associated abdominal symptoms improved), with a reduction of laxative therapy. At the follow-up of 3 months, 336/908 (37\%) patients did not benefit from dietary and pharmacological treatment; 156 were evaluated with intestinal transit time and anorectal manometry. In 218 patients was

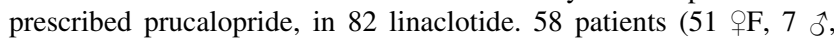
mean age $67.7 \pm 14.2$ years, $\mathrm{M} \pm \mathrm{ES}$ ) with SRT and/or $\mathrm{DD}$, not responding to therapy, performed pelvic floor rehabilitation with anorectal bio-feedback and physiotherapy with Kegel exercises for 20 sessions. In $47(81.03 \%)$ patients, there was considerable benefit, objectivable by reduced abdominal symptoms, macrogol assumed, improvement of items detected through the Wexner scale, and sometimes through normalization of anorectal manometry. In our case, $63 \%$ of patients with chronic constipation benefited from the dietetic and laxative therapy associated with toilette training. In nonresponders with DD isolated or associated with SRT, biofeedback treatment has allowed a significant improvement in intestinal disorders. Rehabilitation of the pelvic floor by bio-feedback confirms an effective procedure in the treatment of refractory constipation.

\section{P-317}

GR-HIP: geriatric rehabilitation of patients after HIP fracture; a study design

G. F. Mattiazzo ${ }^{1}$, M. van Eijk ${ }^{1}$, M. P. L. van der Sijp ${ }^{1}$, W. M. Ravensbergen ${ }^{1}$, W. P. Achterberg ${ }^{1}$, R. van Balen ${ }^{1}$

${ }^{1}$ Leiden University Medical Centre, Leiden, The Netherlands

Introduction: After the acute hospital phase, hip fracture patients are either discharged home or to inpatient geriatric rehabilitation, where they are treated by a multidisciplinary team. The effect of quality and intensity of treatment as well as important (age-related) characteristics on functional outcome and quality of life are poorly studied. As such, no clear evidence-based guidelines determining type, quality, or intensity of training during rehabilitation admission exist.

Method: The study has a mixed-methods approach with a large inception cohort and qualitative data-collection from elderly care physicians, physical therapists, patients, and primary caregivers. Patients are included after hospital admission at Bronovo Hospital, the Hague, with a hip fracture. They will be followed-up at 6 weeks, 3 months and 12 months in the outpatient clinic or place of residence during home-visits. Data collection includes information on patient and caregiver characteristics, comorbidity, functional abilities, cognitive abilities, quality of life, measures on sarcopenia, healthcareuse, psychological measures on coping and (proxy) fear of falling, and caregiver burden.

Results and Key conclusions: In this study, we aim to determine which variables predict successful as well as non-successful rehabilitation and explore effective treatment programmes with the purpose of developing good practice care-pathways. In order to develop these care-pathways, it is important to gather information on patient-related factors, healthcare use during and after admission to postacute GR wards as well as caregiver burden and quality of life.

\section{P-318}

Effects of nutritional interventions on nutritional and functional outcomes in geriatric rehabilitation patients: a systematic review with meta-analyses

Janneke van Wijngaarden ${ }^{1}$, Julia Wojzischke ${ }^{2}$, Claudia van den Berg $^{1}$, Rebecca Diekmann ${ }^{2}$, Jürgen Bauer ${ }^{3}$, Yvette Luiking ${ }^{1}$

${ }^{1}$ Danone Nutricia Research, Nutricia Advanced Medical Nutrition, Utrecht, The Netherlands, ${ }^{2}$ Carl von Ossietzky Universität Oldenburg, Oldenburg, Germany, ${ }^{3}$ Center for Geriatric Medicine, University Heidelberg, Germany

Background: It is increasingly recognised that nutritional interventions could play a role in improving nutritional and functional outcomes. This systematic review aims to summarise the evidence on the effects of nutritional interventions on nutritional and functional outcomes in patients in geriatric rehabilitation.

Methods: Eight electronic databases were searched to identify relevant nutritional intervention studies in patients aged $\geq 60$ years, admitted to a geriatric rehabilitation setting. A meta-analysis was performed on the intervention effect on Barthel Index (BI) and serum albumin.

Results: Of the 1441 studies screened, 14 eligible intervention studies were identified: 3 nutritional interventions (supplements with 20-30 g protein/day), 6 nutritional interventions (vitamin D, supplements with 10-32 g protein/day) combined with a physical exercise program, 1 study on timing of protein provision, 2 interventions providing dietary advice combined with exercise sessions, and 2 interventions with nutrition-related nursing care. Studies were heterogeneous in outcomes measured. Nutritional supplements that contain protein increased protein intake. The effect on physical function (BI, functional independence measure (FIM), hand grip strength, mobility, muscle strength) was limited, however, some individual studies showed positive effects. Meta-analyses showed no significant intervention effect on serum albumin (mean difference $0.45 \mathrm{~g} / \mathrm{L}, 95 \% \mathrm{CI}$ - 0.62:1.52; 3 studies) and BI (mean difference 2.25 points, $95 \% \mathrm{CI}$ - 3.90:8.40; 4 studies).

Conclusion: Nutritional interventions increase protein intake. Effects on physical function are limited. However, due to heterogeneity in interventions and outcome measures it is not possible to draw a clear conclusion on the effect of nutritional interventions in geriatric rehabilitation.

\section{P-319}

Effects of a new computerized, motor-cognitive memory training in older persons

\section{Klaus Hauer $^{1}$, Michaela Guenther-Lange ${ }^{1}$, Christian Werner ${ }^{1}$ \\ ${ }^{1}$ Agaplesion Bethanien Hospital Heidelberg, Geriatric Center at the University of Heidelberg, Heidelberg, Germany}

Introduction: Effect of supported (by procedural learning potentially affecting procedural memory) and non-supported exergame training using simultaneous motor and cognitive tasks have not been studied before. 
Objective: To test training- and long-term effects of a standardized exergame training program on dual task-related memory performance (immediate recall) in older persons.

Methods: RCT in $\mathrm{n}=60$ older persons (age 78.6 years). Intervention: supervised game-based training of motor-cognitive performance (dynamic postural control \& memory training), 10 training sessions vs. unspecific strength/functional training. Sum score of achieved performance levels (scores) and duration/movement time (s) tested at different performance levels (2-4) represented study outcomes derived from internal exergame data stream for 2 test conditions (unsupported vs. procedural support).

Results: Unsupported training increased total score $(p>0.002)$ as well as duration of task in level 3 ( $p>0.001)$ with a trend for level 2 $(\mathrm{p}=0.09)$ and level $4(\mathrm{p}=0.08)$, while procedural support did not further improve training gains compared to unsupported test (total score: $\mathrm{p}=0.241$; level 2: $\mathrm{p}<0.001$; level 3: $\mathrm{p}=0.151)$. Results were sustained during a 3 months follow up for both conditions.

Conclusion: Exergame training increased complex motor cognitive performances related to dynamic balance and memory performance in cognitively intact older persons independent from procedural support with training gains mostly sustained 3 months after training cessation. Test challenges may be sensitive to detect early memory deficits during dual tasking.

\section{P-320}

Criteria for referral to geriatric rehabilitation, a scoping review

\section{A. J. de Groot $^{1}$, L. M. Wattel ${ }^{1}$, J. C. van der Wouden ${ }^{1}$, R. van Balen ${ }^{2}$ \\ ${ }^{1}$ VUmc Amsterdam, Amsterdam, The Netherlands, ${ }^{2}$ LUmc Leiden,} Leiden, The Netherlands

Introduction: In rehabilitation medicine a patients rehabilitation potential is assessed before admission. In the growing field of geriatric rehabilitation (GR), we need to find criteria and methods that target (triage) eligible GR clients by predicting their readjustment. Our aim is to examine the breadth of literature to find criteria, measures or methods that help select GR patients.

Method: We searched PubMed, Embase, CINAHL, PsycINFO and the Cochrane Library for articles published from January 2000 to present, using a search string on 'geriatric patients', 'rehabilitation', 'referral/triage' and 'in-hospital'. Two reviewers independently screened abstracts, a third researcher was consulted when necessary. We sorted the abstracts according to geriatric rehabilitation diagnosis, study population (i.e. patients or professionals) and focus of the research (e.g. prognostic measure, patients experience). Through purposive sampling in each category we reached the full text phase and charted results.

Results: Out of 10,520 we included 1049 abstracts. GR diagnoses were stroke, other neurology, hip fracture, other traumatology, planned orthopaedic surgery, delirium and dementia, amputation, pulmonary rehabilitation, cardiac rehabilitation, cardiovascular disease, oncological disease and other medical diagnoses. The majority were patient studies of clinical and prognostic criteria.

Conclusion: In this scoping review we found a variety of factors like age(ism), frailty, gender, cognitive impairment, functional status, comorbidity, family support and patients preferences related to GR referral. We will present a comprehensive overview of results and the foundation it offers for triage methods.

\section{P-321}

The prevalence of malnutrition in a german geriatric rehabilitation population

Julia Wojzischke ${ }^{1}$, Sarah Egert ${ }^{2}$, Andreas Hein ${ }^{1}$, Jürgen M. Bauer ${ }^{3}$, Rebecca Diekmann ${ }^{1}$

${ }^{1}$ Carl von Ossietzky University Oldenburg, Oldenburg, Germany, ${ }^{2}$ Rheinische Friedrich-Wilhelms-University Bonn, Bonn, Germany, ${ }^{3}$ Center for Geriatric Medicine, University Heidelberg, Heidelberg, Germany

Introduction: Malnutrition may negatively affect functional outcomes during geriatric rehabilitation, but data on the prevalence of malnutrition in this setting are limited. The aim of this study is to investigate the prevalence of malnutrition in a geriatric rehabilitation population.

Methods: For this observational study, participants were consecutively recruited from September 2015 to September 2016 in a geriatric rehabilitation center in Oldenburg, Germany. At admission the Mini Nutritional Assessment-Short Form (MNA-SF) was applied, and malnutrition was diagnosed by the new ESPEN classification, which includes a parameter for body composition (fat free mass).

Results: 122 patients [70\% women, mean (SD) age 81.5 years (5.6)] were included; for all of them MNA-SF was completed, the ESPEN classification was applied in 102 patients, because of missing information on fat free mass index, e.g. due to a pacemaker. According to MNA-SF, the prevalence of malnutrition was $31 \%(\mathrm{n}=38) ; 60 \%$ $(n=73)$ were at risk of malnutrition; $9 \%(n=9)$ were malnourished according to the ESPEN classification. Eight out of nine malnourished patients (ESPEN criteria) were also malnourished according to MNASF. $72 \%(n=24)$ of the patients identified as malnourished by MNASF were not diagnosed as being malnourished by the ESPEN criteria. Key conclusions: The prevalence of malnutrition in a geriatric rehabilitation population differs according to method of diagnosis, higher with MNA-SF $(31 \%)$ than ESPEN (9\%), the availability of body composition data limited the use of ESPEN diagnosis in this study. As functional items are included in MNA-SF, this tool has a broader scope than the ESPEN classification for malnutrition.

\section{P-322}

Sarcopenia and malnutrition in a german geriatric rehabilitation population

Julia Wojzischke ${ }^{1}$, Sarah Egert ${ }^{2}$, Andreas Hein ${ }^{1}$, Jürgen M. Bauer ${ }^{3}$, Rebecca Diekmann ${ }^{1}$

${ }^{1}$ Carl von Ossietzky University Oldenburg, Oldenburg, Germany, ${ }^{2}$ Rheinische Friedrich-Wilhelms University Bonn, Bonn, Germany, ${ }^{3}$ Center for Geriatric Medicine, University Heidelberg, Heidelberg, Germany

Introduction: The presence of malnutrition and sarcopenia could negatively affect functional outcomes during rehabilitation. The aim of this study is to investigate the prevalence of these syndromes, and their potential overlap in a geriatric rehabilitation population.

Methods: For this observational study, participants were consecutively recruited from September 2015 to September 2016 in a geriatric rehabilitation center in Oldenburg, Germany. At admission, malnutrition was assessed by Mini Nutritional Assessment-Short Form (MNA-SF) and by the new ESPEN classification. Sarcopenia was diagnosed according to EWGSOP definition.

Results: 122 patients [70\% women, mean (SD) age 81.5 years (5.6)] were included. In all participants MNA-SF was assessed, 102 patients were classified according to ESPEN and EWGSOP sarcopenia 
definition ( $\mathrm{n}=20$ missing body composition data, due to e.g. presence of a pacemaker): $31 \% \quad(n=38)$ and $9 \% \quad(n=9)$ were malnourished according to MNA-SF and ESPEN criteria, respectively and $26 \%(n=26)$ were sarcopenic. Thirty-four per cent $(n=11)$ of the patients who were malnourished (MNA-SF) were also sarcopenic, and $42 \%(n=11)$ of the patients who were sarcopenic were also malnourished (MNA-SF); 89\% $(n=8)$ of the patients who were malnourished (ESPEN) were sarcopenic, and $31 \%(n=8)$ of the patients who were sarcopenic were also malnourished (ESPEN). 4 patients who were both sarcopenic and malnourished according to MNA-SF were not identified with the ESPEN criteria.

Key conclusions: Around a quarter of patients is sarcopenic in this German geriatric rehabilitation population. There is significant overlap between malnutrition and sarcopenia, however, the overlap differs based on the diagnostic tool for malnutrition that is applied.

\section{P-323}

Associations between nutritional and functional status of a german geriatric rehabilitation population

Julia Wojzischke ${ }^{1}$, Sarah Egert ${ }^{2}$, Andreas Hein ${ }^{1}$, Jürgen M. Bauer ${ }^{3}$, Rebecca Diekmann ${ }^{1}$

${ }^{1}$ Carl von Ossietzky University Oldenburg, Oldenburg, Germany, ${ }^{2}$ Rheinische Friedrich-Wilhelms University Bonn, Bonn, Germany, ${ }^{3}$ Center for Geriatric Medicine, University Heidelberg, Heidelberg, Germany

Introduction: Nutritional status is closely linked to physical function. This study aims to identify nutritional status, physical function and their inter-relationship in a geriatric rehabilitation population.

Methods: For this observational study, participants were consecutively recruited from September 2015 to September 2016 in a geriatric rehabilitation center in Oldenburg, Germany. At admission, dietary intake was assessed by a 3-day dietary record and fasting serum 25-hydroxy-vitamin D levels were measured. Physical function was measured by Short Physical Performance Battery (SPPB), Barthel Index (BI), handgrip strength (HGS) and timed-up-and-go (TUG). Muscle mass was measured with BIA. Statistical analyses were performed with $t$ test and ANOVA ( $p$ level $<0.05$ ).

Results: 122 patients [70\% women, mean (SD) age 81.5 years (5.6)] were included; for 116 and 95 data were available for dietary intake and vitamin D level. Prevalences were: low protein intake $[<0.8 \mathrm{~g} / \mathrm{kg}$ body weight (bw)] 35\% ( $\mathrm{n}=40),(0.8$ to $<1.0 \mathrm{~g} / \mathrm{kg}$ bw) $33 \%$ $(\mathrm{n}=38)$; low energy intake $(<25 \mathrm{kcal} / \mathrm{kg}$ bw) $55 \%(\mathrm{n}=64)$; vitamin D levels: $(<25 \mathrm{nmol} / \mathrm{l}) 61 \% \quad(\mathrm{n}=58), \quad(25-<50 \mathrm{nmol} / \mathrm{l}) \quad 18 \%$ $(\mathrm{n}=17)$. SPPB, BI, TUG, muscle mass did not differ significantly between subgroups of protein and energy intake, and vitamin D level. HGS was significantly lower when vitamin D was $<25 \mathrm{nmol} / \mathrm{l}$ vs. 25 to $<50 \mathrm{nmol} / \mathrm{l}(\mathrm{p}=0.026)$.

Key conclusions: Nutritional intake and vitamin D status are compromised in a majority of these geriatric rehabilitation patients. While parameters of physical function were not significantly different between groups of protein and energy intake, HGS varied with vitamin D status. However, nutritional intake already might be compromised earlier, during acute hospital treatment.

\section{P-324}

Hip fracture geriatric rehabilitation: a mixed-method crosssectional observational study of patient characteristics and current practices in nutrition

Laura den Boeft ${ }^{1}$, Lisette de Groot $^{2}$, Hans Hobbelen ${ }^{3}$, Gert-Jan van der Putten ${ }^{4}$

${ }^{1}$ Zorggroep Amaris, Laren, The Netherlands, ${ }^{2}$ Division Human Nutrition, Wageningen University, Wageningen, The Netherlands, ${ }^{3}$ Division Healthy Lifestyle, Aging and Health Care,

Hanzehogeschool, Groningen, The Netherlands, ${ }^{4}$ Zorggroep Amaris, Laren, The Netherlands

Introduction: In older hip fracture patients protein-energy malnutrition is associated with worse functional recovery. The current recommendation regarding daily protein intake is $>1.2 \mathrm{~g} / \mathrm{kg} / \mathrm{bw}$ in individuals with acute diseases, including hip fracture. Nutritional care in geriatric rehabilitation (GR) needs a multidisciplinary approach. The purpose of the present study was to gain insight into patient characteristics and the current situation regarding nutritional care in GR (protocols, communication, intervention).

Methods: First, data including physical functioning, muscle mass, comorbidities, nutritional status and protein intake was gathered from 40 hip fracture patients on admission to two GR units. Protein intake measurements were repeated after three weeks to detect possible changes over time. Secondly, semi-structured interviews were performed to gain insight in the given usual care [physical therapists $(n=4)$, dietitians $(n=3)$, physicians $(n=2)$, nursing staff $(n=4)]$. Results: Of the study population $74 \%$ were women. Mean age was 81.4 (SD 8 years). $11 \%$ of patients, were classified as undernourished and a further $59 \%$ as at risk of malnourishment. $95 \%$ of patients did not meet the recommendation for daily protein intake and intake did not significantly change over time. Data from the semi-structured interviews showed that the involved care-providers are aware of the importance of adequate nutrition in rehabilitation, but this knowledge is not implemented.

Conclusion: Malnourishment and insufficient protein intake are highly prevalent in older hip fracture patients visiting GR's. These results point to a compelling need to improve the organization of nutritional care.

\section{P-325}

first functional testing results of a new soft modular biomimetic lower-limb exoskeleton (XoSoft) in geriatric patients with moderate gait disorders

\section{Samuel Schülein ${ }^{1}$}

${ }^{1}$ Geriatrie-Zentrum Erlangen Germany, Malteser Waldkrankenhaus Erlangen gGmbH, Erlangen, Germany

Background: Walking is one of the most complicated and common motor activity of daily life. With increasing age, the automatism no longer functions as it does in young age. Yet, the desire for a prolonged self-determined life and social participation grows. An important prerequisite for this is good mobility and independence in everyday life. In this context, assistive devices for improving independence play an important role. The aim of our work is to outline the first results of the functionality of a modular soft and flexible lowerlimb exoskeleton (XoSoft) in its penultimate and final stage of development.

Methods/design: The objective is to gain an overview of the XoSoft's basic functionality. The testing design corresponds to the basic functionality with different tasks and conditions which are 
subdivided into exercises without and with an activated device. Patients are questioned about the respective load in the legs and classified by a scoring system. A survey on pressure nuisances is carried out and determined precisely. The functionality of XoSoft as well as the acceptance of the users will be written and documented. Kinematics are recorded, analyzed and evaluated by motion analysis. Geriatric patients will be recruited at the Geriatrics Centre Erlangen. Discussion: Wearable exoskeletons require extensive technical expertise innovation. Still the most innovative systems cannot be successful should they fail to be accepted by their intended users. This research provides an important step into the exploration of wearable soft biomimetic lower-limb exoskeletons as assistive devices from a user's perspective and its impact on common locomotion tasks in daily life.

Project Partners: Fondazione Instituto Italiano di Tecnologia, IT (coordinator) accelopment AG, $\mathrm{CH}$ Consejo Superior de Investigaciones Científicas, ES Geriatrie-Zentrum Erlangen, DE Össur hf, IS Roessingh Research and Development, NL Saxion University of Applied Science, NL University of Limerick, IE Zurich University of Applied Sciences, $\mathrm{CH}$.

\section{P-326}

\section{Physical training as a useful tool in brain fitness}

Cristian Romeo Revnic ${ }^{1}$, Floarea Revnic ${ }^{2}$, Silviu Voinea ${ }^{1}$

${ }^{1}$ UMF Carol Davila, Bucharest Romania, ${ }^{2}$ NIGG Ana Aslan, Bucharest Romania

Geriatric depression amplifies dysfunction and physical/somatic complains. Symptoms of anxiety are present in almost $90 \%$ elderly depressed patients who often are treated for depression not for anxiety.

Objective: To assess the effectiveness of a 24 weeks physical training program of moderate intensity in elderly men with depression. Patient selection: 42 patients admitted for post-traumatic pathologies in Rehabilitation Clinic, aged 60-84, divided into two groups of 21 patients each: A with physical activity (controls) and B sedentary depressed patients able to perform a 45 min physical exercise program 5 times/week including:weight lifting, for skeletal muscle motor units training performed with EMG Scwartzer-Picker 2000, 2 min exercise at ergometric bicycle $200 \mathrm{~W}$, as well as an aerobic component. Evaluation of cardio-circulatory parameters (heart rate, systolic and dyastolic blood pressure) and endocrine parameters (testosteron, HGH, Cortisol, HTSH) have been done before and after training. GDS was used to evaluate depression.

Results: Our data pointed out the positive effects of physical training upon depression scores with a positive impact upon sleep quality and upon physical ability to perform movements with a better neuromuscular coordination increasing the patients self confidence and the ability to self support. There is an increase following training in Testosterone and HGH secretion and a decrease in Cortisol secretion with a positive effect upon mental health increasing the sense of well being, the confidence and optimism about life.

Conclusion: Psychological changes that occur in elderly are associated with altered pharmacokinetics and physical training is an alternative for drug treatment of depression, stimulating brain function, enhancing the sleep and contributing to the increase in quality of life.

\section{P-327}

Can person characteristics predict engagement in physical activity? Experience from the promoting activity, independence and stability in early dementia (PrAISED) intervention

Jennie Hancox ${ }^{1}$, Rupinder Bajwa ${ }^{1}$, Clare Burgon ${ }^{1}$, Pip Logan ${ }^{1}$, Rowan H. Harwood ${ }^{2}$

${ }^{1}$ School of Medicine, University of Nottingham, Nottingham, UK, ${ }^{2}$ School of Health Sciences, University of Nottingham, Nottingham, UK

Introduction: Physical activity has multiple benefits in people with dementia but encouraging people to be more active can be difficult. The randomised controlled PrAISED feasibility study tested two interventions with different intensities ( 9 and 50 sessions) aimed to increase physical activity in people with Mild Cognitive Impairment (MCI) and early dementia. The aim of this analysis was to explore if physical activity at follow-up could be predicted by emotional, cognitive or physiological measures at baseline.

Methods: The 12 months follow-up assessments of the intervention groups included 39 participants with an average age of 76 years (33\% female) diagnosed with MCI or dementia. Pearson's correlations examined if age, fear of falling, frailty, cognition, depression, balance, walking speed, carer burden or activities of daily living (DAD) at baseline were related to physical activity (International Physical Activity Questionnaire scores) at follow-up.

Results: There was no significant difference between the moderate and high intensity intervention groups in physical activity scores at follow-up. Physical activity at 12 months was not significantly related to any of the baseline measurements. Only DAD baseline scores showed a trend towards a significant relationship with physical activity follow-up scores $(\mathrm{r}=0.36 ; \mathrm{n}=27 ; \mathrm{p}=0.07)$.

Conclusion: The psychological and physiological measures used in this study at baseline were unable to predict engagement in physical activity following the PrAISED intervention. Other parameters such as personality traits or health beliefs should be explored to investigate characteristics of people with MCI or early dementia to predict engagement in physical activity.

\section{P-328}

\#Loseapad: An innovative idea to prevent deconditioning in hospitalized elderly patients-a quality improvement exercise in a senior adult medical services (SAMS) department in a UK Hospital.

R Mahmood ${ }^{1}, \mathrm{G}$ O'shea ${ }^{1}, \mathrm{~S} \mathrm{Hardy}^{1}, \mathrm{P}$ Enwere $^{1}, \mathrm{~K}$ Yeong $^{1}, \mathrm{R} \mathrm{Lisk}^{1}$

${ }^{1}$ Ashford and St Peters NHS Foundation Trust

Introduction: Deconditioning leads to loss of muscle mass and strength, thus patient's ability to perform physical activities of daily living. Enabling independence and preventing deconditioning of older people in hospital is essential to prevent morbidity and unnecessary burden on health economy.

Innovation: The idea came from one of our matrons, who gave the story how she was surprised to see her cognitively intact, continent father given a continence pad when he was admitted to hospital; hence \#lose a pad was born. A poster was made to create awareness and to educate the staff to use fewer pads and thus promote patient independence. Project was started on three SAMS wards (Swift and Holly ward). Cost savings, length of stay (LOS) was measured before and after intervention.

Results: Pre-intervention phase: (August 17-October 17) total cost of pads used on 3 wards: $£ 6,538.98$ average length of stay: 11.93 days 
post-intervention phase (November 17- January 18) total cost of pads used on 3 wards: $£ 2,087.81$ average length of stay: 11.06 days total savings: $£ 4,454.17$, projected annual savings of $£ 17,816.68$.

Conclusion: Our intervention resulted in significant cost savings with reduction in length of stay despite winter pressure. Preventing deconditioning in older people is challenging but not impossible. Persistent efforts with innovation and continuous staff education are key to success. We plan to hold further training sessions including improvement days in an attempt to change the culture and methods we use to deliver care to our older population thus preventing deconditioning.

\section{P-329}

Prognostic predictors of rehabilitation in patients aged 75 and older with ischemic stroke

Marc Paccalin ${ }^{1}$, Nadege Guillard ${ }^{1}$, Adrien Julian ${ }^{2}$, Marie-Laure Bureau $^{1}$, Arnaud Caupenne ${ }^{1}$, Amelie Jamet ${ }^{1}$, Evelyne Liuu ${ }^{1}$

${ }^{1}$ Pôle de gériatrie, CHU Poitiers, 2 rue de la Milétrie, 86000 Poitiers, France, ${ }^{2}$ Service de neurologie, CHU Poitiers, 2 rue de la Milétrie, 86000 Poitiers, France

Introduction: Management of ischemic stroke in elderly is challenging, because of its growing incidence and its consequences on mortality and functional prognosis. Optimization of rehabilitation care in geriatric neurology would improve the chance of recovery. In this heterogeneous population, the identification of factors associated with prognosis is needed, to identify vulnerable patients.

Objectives: to assess factors associated to adverse outcomes, such as death, aggravation of neurological (increasing value of NIHSSNational Institute of Health Stroke Score) and functional status (decreasing value of GIR-Groupes Iso Ressources).

Methods: Observational retrospective study in Poitiers University Hospital, France, from May 1st 2015 to May 1st 2017, including patients $\geq 75$ years, hospitalized in the geriatric neurology rehabilitation unit for ischemic stroke. Variables of interest: sociodemographic data, geriatric and neurologic parameters, comorbidities and medication.

Results: 100 patients were included $(53 \%$ men, mean age $85 \pm 6$ years, mean stay $44 \pm 29$ days). Mortality rate at 3 months were $20 \%$, and $28 \%$ of patients had adverse outcomes according to our criteria. In univariate then multivariate analyses, a bad prognosis was significantly associated with high modified Charlson score $(\mathrm{p}=0.01), \quad$ high NIHSS $(\mathrm{p}=0.02), \quad$ cardio-embolic cause $(\mathrm{p}<0.001)$, and early complications such as epilepsy $(\mathrm{p}=0.002)$ and swallowing disorders $(\mathrm{p}=0.02)$.

Conclusion: This study showed that after an ischemic stroke, optimal management in elderly should include an early identification of comorbidities, notably an atrial fibrillation, and skills in detection of epilepsy and swallowing disorders during the hospitalization.

\section{P-330}

Short physical performance battery: study of content validity to Portuguese

João Tavares ${ }^{1,2,3}$, João Fonseca ${ }^{4}$, Benilde Barbosa ${ }^{4}$, Helder Esperto ${ }^{4}$, Ana Ferreira $^{4}$, Helena Loureiro ${ }^{4}$, Manuel Veríssimo ${ }^{4}$

${ }^{1}$ UCSP Soure, Coimbra, Portugal, ${ }^{2}$ The Health Sciences Research Unit: Nursing - Nursing School of Coimbra, Coimbra, Portugal, ${ }^{3}$ School of Education - Escola Superior de Educação de Coimbra, Coimbra, Portugal, ${ }^{4}$ Geriatric consultation, Coimbra Hospital and Universitary Centre, Coimbra, Portugal
Introduction: The Short Physical Performance Battery (SPPB) is well known and used worldwide to assess physical performance and functional status. These measures are crucial to develop a geriatric care plan. This test includes three components: the balance stand in three ways; gait speed and time to rise from a chair five time.

Methods: This study's aims were: (i) translate, adapt and validate the SPPB for the Portuguese population; (ii) evaluate content validity index (CVI) using a panel of expert. The first steps were translation and back translation with the help bilingual health professionals. Six experts in geriatric, sports science and nursing evaluated the final translation. The analysis of content validity included CVI, probability of a chance occurrence (pc) and modified Kappa (MK). SPSS software, version 20, was used for the statistical analysis.

Results: In this process three assessment was necessary to obtain a consensus in the validation process. In the first and second evaluation more than $13.3 \%$ of the item showed a CVI $<0.8$. After reviewing these items, in the third evaluation, all had come to present a CVI $=1$, and the average value of the IVC was $100 \%$ and the universal agreement of the IVC $=1$ and pc value was 0.0625 . MK was 1 , which was considered excellent

Key conclusions: The SPPB is used in different clinical contexts (hospital and community) and demonstrated good clinimetric properties. The study showed high levels of CVI, pc and MK in the Portuguese version of SPPB protocol. These results support the validity of the content of this tool. Additional criterion and construct validation studies are need to explore the SPPB in Portuguese context.

\section{P-331}

Predictor factors of increased length of stay of older patients with hip fracture in an Orthogeriatric Unit

Filipa Bianchi-de-Aguiar ${ }^{1}$, Sofia Duque ${ }^{2}$, Cristina CarvalhoGouveia $^{2}$, André Ferreira ${ }^{2}$, Inês Miguéis-Ferreira ${ }^{2}$, Joana Lopes ${ }^{2}$, Mafalda Sequeira ${ }^{2}$, Ana Lopes-dos-Santos ${ }^{2}$, Sara Trevas ${ }^{2}$, Ana Pedroso $^{2}$, José Guimarães-Consciência ${ }^{2}$

${ }^{1}$ Centro Hospitalar Lisboa Ocidental, Hospital de S Francisco Xavier, Lisbon, Portugal, ${ }^{2}$ Centro Hospitalar de Lisboa Ocidental, Hospital de S. Francisco Xavier, Lisbon, Portugal

Introduction: Orthogeriatric Units provide a multidisciplinary approach to the care of older patients with hip fracture, enhancing functional rehabilitation, minimizing complications and mortality, reducing time to surgery and length of stay (LOS), as already demonstrated in our unit. The analysis of factors related to increased LOS for these patients is important, since it may allow further optimization of the care provided.

Objectives: To identify sociodemographic characteristics, global geriatric assessment indexes, or other factors associated with hospitalization that may be associated with an increase in length of stay, in patients over 64 years-old with hip fracture, admitted to an Orthogeriatric Unit. METHODS: Retrospective study between June 2016 and March 2018 carried out by the consultation of the electronic processes. Comparative analysis of the characteristics of two groups of patients, divided in less than 13 days VERSUS equal to or greater than 13 days (median of LOS). Binary logistic regression performed for predictors of prolonged hospitalization.

Results: Of the 271 patients assisted, 131 (48.7\%) had hospitalization lasting 13 or more days. In the group with increased hospitalization time, there were significantly more men $[29.8 \%(\mathrm{n}=39)$ vs $18.1 \%$ $(\mathrm{n}=25), \mathrm{p}=0.025]$ and they presented a longer time-to-surgery (6.96 vs 2.6 days, $p=0.001$ ). Even not yet statistically significant, this group was also more dependent (average Barthel score 82.4 vs $79.1 / 100, p=0.072)$. There were no other independent factors 
predicting prolonged LOS, namely age, place of residence before hospitalization, educational level, marital status, type of femoral fracture, destination after discharge, the presence of delirium, autonomy for walking at discharge, index of comorbidities (Comorbidities Illness Rating Scale-Geriatrics), cognitive impairment, polypharmacy, or nutrition status.

Conclusion: Male gender and a longer time to surgery seem to be related with increased LOS. While measures to improve the modifiable factors may be implemented, a bigger and more detailed prospective study may help to identify other predictors, aiming to improve care and allow a further decrease hospitalization time.

\section{P-332}

Predictor factors of increased time to surgery of older patients with hip fracture in an Orthogeriatric Unit

Filipa Bianchi-de-Aguiar ${ }^{1}$, Sofia Duque ${ }^{1}$, Cristina CarvalhoGouveia $^{1}$, André Ferreira ${ }^{1}$, Inês Miguéis-Ferreira ${ }^{1}$, Joana Lopes ${ }^{1}$, Mafalda Sequeira ${ }^{1}$, Ana Lopes-dos-Santos ${ }^{1}$, Sara Trevas ${ }^{1}$, Ana Pedroso $^{1}$, José Guimarães-Consciência ${ }^{1}$

${ }^{1}$ Centro Hospitalar Lisboa Ocidental, Hospital de S Francisco Xavier, Lisbon, Portugal

Introduction: Orthogeriatric Units have improved the care of the elderly patient with hip fracture, minimizing complications due to a multidisciplinary approach. Some authors have showed that Orthogeriatric Units approach reduced Time To Surgery (TTS) as well as mortality and length of stay (LOS). The analysis of factors related to increased TTS is still important, since it may allow further optimization of the care provided.

Objectives: To identify sociodemographic characteristics, global geriatric assessment indexes, or other factors associated with hospitalization that may be related to an increase in TTS, in patients over 64 years-old with hip fracture, admitted to an Orthogeriatric Unit.

Methods: Retrospective study between June 2016 and March 2018 carried out by the consultation of the electronic processes. Comparative analysis of the characteristics of two groups of patients, divided in TTS till 4 days VERSUS greater than 4 days (median of TTS in this sample). Binary logistic regression performed for predictors of prolonged time to surgery.

Results: Of the 271 patients assisted, 135 (49.8\%) had a time to surgery of more than 4 days. In the group with higher TTS, there were significantly less patients with adequate nutritional status [14 (10.3\%) vs $17(12.6 \%), \mathrm{p}=0.11]$, and lower prevalences of cognitive impairment [42 (30.9\%) vs $64(47.4 \%), p=0.034]$. Even not yet statistically significant, this group was also more dependent (average Barthel score 81.47 vs $79.7 / 100, p=0.088$ ) and took more drugs previously to admission (6.92 vs $6.64, \mathrm{p}=0.062)$. There were no other independent factors predicting increased time to surgery, namely age, gender, place of residence before hospitalization, years of schooling, marital status, type of femoral fracture, destination after discharge, the presence of delirium, autonomy for walking at discharge, index of comorbidities (Comorbidities Illness Rating ScaleGeriatrics).

Conclusion: Variables related to patients' physical frailty, such as malnourishment, polypharmacy and dependency seem to be related to an increased TTS. However, cognitive frailty didn't seem to have an impact on delay of surgery. While measures to improve the modifiable factors may be implemented, a bigger and more detailed prospective study may help to identify other predictors, aiming to improve care.
P-333

ACANTO project: short physical performance battery evaluation after intervention with a friendly robotic walker (FriWalk) during admission in Acute Care Unit and Orthogeriatrics Unit.

Myriam Valdés Aragonés ${ }^{1}$, Rodrigo Pérez Rodríguez ${ }^{2}$, Myriam Oviedo Briones $^{2}$, Pedro Antonio Moreno ${ }^{2}$, Stefan Walter ${ }^{2}$, Nuria García Grossocordon $^{3}$, Leocadio Rodríguez Mañas ${ }^{4}$

${ }^{1}$ Getafe University Hospital, Madrid, Spain, ${ }^{2}$ Biomedical Research Foundation, Getafe University Hospital, Madrid, Spain, ${ }^{3}$ Getafe University Hospital, Madrid, Spain, ${ }^{4}$ Getafe University HospitalMadrid, Spain

Introduction: ACANTO is an European project funded by the EU Horizon 2020 program aimed at improving physical performance in elderly people through a robotic friendly walker (FriWalk). The aim of the project is to compare the evolution of the participants in terms of physical performance during admission measured by Short Physical Performance Battery (SPPB) at admission and at discharge.

Methods: A total of 22 patients aged +75 admitted in the Acute Care and Orthogeriatrics units of the Getafe University Hospital (Madrid) were analysed. Participants were randomized into one of the study groups: an intervention group where participants received an individualized multicomponent physical exercise program provided by the FriWalk during hospitalization; and a control group where patients received the usual care, also during hospitalization. A linear mixed model was used to evaluate the change in SPPB attributable to being randomized to the intervention group. Analyses were adjusted by age and sex and a $\mathrm{p}$ value threshold of 0.05 was considered statistically significant.

Results: Physical performance, measured by SPPB, at discharge was significantly improved in the intervention group compared to the control group. These patients improved 1.03 points in the SPPB score [95\% CI $(0.31-1.77), \mathrm{p}=0.02]$. There were no significant differences in mean baseline SPPB score.

Conclusions: Results indicate that patients who have used the FriWalk during hospitalization in the Acute Care and Orthogeriatric Units have a better evolution in terms of physical performance measured by SPPB.

\section{P-334}

ACANTO project: recruitment description on elderly admitted into Geriatrics departments through the use of a robotic friendly walker

Myriam Valdés Aragonés ${ }^{1}$, Pedro Antonio Moreno ${ }^{2}$, Rodrigo Pérez Rodríguez ${ }^{3}$, Myriam Oviedo Briones ${ }^{3}$, Stefan Walter ${ }^{3}$, Nuria García Grossocordon ${ }^{4}$, Leocadio Rodríguez Mañas ${ }^{1}$

${ }^{1}$ Getafe University Hospital, Madrid, Spain, ${ }^{2}$ Biomedical Research Foundation, Getafe University Hospital, Madrid, Spain, ${ }^{3}$ Biomedical Research Foundation, Getafe University Hospital, Madrid, Spain,

${ }^{4}$ Getafe University Hospital, Madrid, Spain

Introduction: ACANTO is an European project funded by the EU Horizon 2020 program aimed at improving physical performance in elderly people through a robotic friendly walker (FriWalk). A pilot study undertaken at Getafe Hospital (Madrid) compares the effectiveness of FriWalk. In the intervention group, the FriWalk provided a means to carry out physical tasks during the hospitalization in the Acute care and Orthogeriatric units. In the control group, usual care was being delivered. The aim of the project was to verify that the randomization was correctly performed and to know the characteristics of the sample. 
Methods: A total of 25 patients aged $75+$ were included while they were hospitalized in the Acute Care and Orthogeriatric. Participants were randomized into the intervention or the control group. A transversal analysis has been performed to evaluate the collected data. Results: 25 patients were analysed. In the control group 14 patients were recruited: $35.7 \%$ men, mean age of $88.14,85.7 \%$ frail, 14.3 prefrail, mean gait speed in $6 \mathrm{~m}$ of $0.48 \mathrm{~m} / \mathrm{s}$ and mean Short Physical Performance Battery (SPPB) of 4.0. In the intervention group, 11 patients were recruited: $45.5 \%$ men, mean age of $89.18,81.9 \%$ were frail, $18.2 \%$ were prefrail, mean gait speed in $6 \mathrm{~m}$ of $0.42 \mathrm{~m} / \mathrm{s}$ and mean SPPB of 3.73. No significant differences were identified.

Conclusion: The randomization was properly performed, so the different groups are comparable. In both groups, most of the participants were frail according to Fried Frailty Criteria, with a low SPPB and slow gait speed.

\section{P-335}

Predictors of 30-days walking recovery in older people with hip fracture: results from Gruppo Italiano Di Ortogeriatria (GIOG) National Registry

B. Govoni ${ }^{1}$, G. Mantovani ${ }^{1}$, S. Volpato ${ }^{2}$, P. Rapazzini ${ }^{3}$, A. Zurlo ${ }^{1}$, C. Mussi ${ }^{4}$, M. Corsi ${ }^{5}$, ML Lunardelli ${ }^{6}$, A. Andreano ${ }^{7}$, G. Castoldi ${ }^{8}$, P. Floris ${ }^{9}$, M. Pizzonia ${ }^{10}$, A. Barone ${ }^{11}$, A. March ${ }^{12}$, A. Ungar ${ }^{13}$, V. Galmarini ${ }^{14}$, S. Franzoni ${ }^{15}$, P. Ranieri ${ }^{16}$, G. Bellelli ${ }^{17}$

${ }^{1}$ Orthogeriatric Unit, Arcispedale S Anna, Ferrara University, Ferrara, Italy, ${ }^{2}$ Department of Medical Sciences, Ferrara University, Ferrara, Italy, ${ }^{3}$ Orthogeriatric Unit, Circolo Hospital, Varese, Italy,

${ }^{4}$ Orthogeriatric Unit, University of Modena and Reggio Emilia, Modena, Italy, ${ }^{5}$ Orthogeriatric Unit, S Gerardo hospital, Monza, Italy, ${ }^{6}$ Orthogeriatric Unit, S.Orsola Malpighi, Bologna, Italy, ${ }^{7}$ School of Medicine and Surgery, Milano-Bicocca University, Milan, Italy, ${ }^{8}$ Orthopaedic Unit, ASST Vimercate, Carate Brianza hospital, Vimercate (MB), Italy, ${ }^{9}$ Orthogeriatric Unit, Hospital of SondrioASST VAL, Sondrio, Italy, ${ }^{10}$ Orthogeriatric Unit, San Martino hospital, Genova, Italy, ${ }^{11}$ Orthogeriatric Unit, Galliera hospital, Genova, Italy, ${ }^{12}$ Orthogeriatric Unit, Bolzano hospital, Bolzano, Italy, ${ }^{13}$ Geriatrics and Intensive Care Unit, Florence, Italy, ${ }^{14}$ Orthopedic Unit, ASST Fatebenefratelli Sacco, Milano, Italy, ${ }^{15}$ Orthopedic Unit, Poliambulanza hospital, Brescia, Italy, ${ }^{16}$ Department of Medicine and Rehabilitation, S. Anna hospital, Brescia, Italy, ${ }^{17}$ School of Medicine and Surgery, Milano-Bicocca University, Milan, Italy; Orthogeriatric Unit, S Gerardo hospital, Monza, Italy

Introduction: In older people, hip fractures often lead to catastrophic disability and loss of independence. We investigated clinical predictors of walking recovery using data from the Gruppo Italiano OrtoGeriatria study.

Methods: Prospective multicenter observational study of Italian patients aged $\geq 65$ undergoing surgery for hip fracture. $\mathrm{N}=2570$ patients were recruited in 14 Italian hospitals from 2016 to 2018. A telephonic 30-day follow-up was performed in a subgroup of 595 $(23 \%)$. Of those, $\mathrm{n}=587(99 \%)$ were able to walk before the fracture (97\% in the whole sample). In these patients, we assessed the association between selected pre-fracture clinical features and walking recovery, defined as the ability to walk with or without aids.

Results: Walking recovery occurred in $83.5 \%$ patients and $19 \%$ had a full recovery or a better walking performance as compared to baseline. In a multivariable logistic regression model, walking recovery was significantly associated with younger age (OR 1.05 per year, 95\% CI 1.01-1.10), pre-operative cognitive impairment (SPMSQ $>3$ errors, OR $0.34,95 \%$ CI $0.17-0.67)$, independent pre-operative walking ability (OR 3.2, 95\% CI 1.5-6.4), need for transfusion (OR
$0.44,95 \%$ CI $0.20-0.97$ ), partial or full bone loading (OR 9.6, 95\% CI 4.65-19.7), lower ASA score (OR 4.3, 95\% CI 1.34-5.90), and postdischarge rehabilitation program (OR 2.82, 95\% CI 1.35-5.90).

Conclusion: 30 days walking recovery was associated with younger age, lower level of multimorbidity and disability before the fracture and post-operative physiotherapy program. This information may help geriatrician in prognosis definition and tailoring post-surgery management.

\section{P-336}

Introduction of discharge to assess on an elderly care ward: a quality improvement project

Tim Pattison ${ }^{1}$, Arturo Vilches-Moraga ${ }^{1}$, Karen Smith ${ }^{1}$, Imogen Lyons ${ }^{1}$, Laura Hammond ${ }^{1}$

${ }^{1}$ Salford Royal NHS Foundation Trust, Salford, UK

Introduction: Increased waiting time for social care and therapy cause significant delays in transfer of care in the UK. The Discharge to Assess model examines ongoing care needs in the home to deliver a personalised support plan. This study aims to determine the impact of embedding HomeSafe to the multidisciplinary team of an acute elderly care unit.

Methods: Retrospective study of consecutive discharges from a geriatric ward between 1st January and 20th May 2018. Tests of change started on 5th April 2018, integrating the community team onto the ward to improve communication and understanding of discharge to assess process, expedite assessment by community team and allow early contact with relatives.

Results: We included 247 patients with a mean age of 85 years and female predominance $(70 \%)$. Overall discharge rate increased from 25 to 57 patients per month. Median length of stay was 7 days, however, HomeSafe patients median LOS was 11 days. Seven-day readmission was reduced from baseline. The rate-limiting step identified was the capacity of HomeSafe to take on the volume of new referrals, which was shown to triple.

Conclusions: Communication between hospital and community health professionals is integral for the success of a discharge to assess team. The service increased the number of ward discharges with no increase in readmission or mortality rates. More data is required to streamline and determine the impact of this service.

\section{P-337}

Physical activity and functional performance in persons over 70 years

Andrejus Cernovas $^{1}$, Egle Vikertaite ${ }^{1}$, Asta Mastaviciute ${ }^{1}$, Marija Tamulaitiene ${ }^{1}$, Rimantas Stukas ${ }^{1}$, Vidmantas Alekna ${ }^{1}$

${ }^{1}$ Faculty of Medicine, Vilnius University, Vilnius, Lithuania

Introduction: Age-related changes decline physical activity and decrease functional performance that has been related with chronical diseases, morbidity and mortality. Many evidences suggest that functional performance is the key for health and functional independence in later life. The aim of this study was to compare physical activity and functional performance in 70-79 years and 80+ years age groups of elderly.

Methods: The cross-sectional study was performed on communitydwelling ambulatory people. Inclusion criteria were: age 70 years and more, MMSE $\geq 10$ points. Participants were divided into two groups according age: group I (70-79 years, $\mathrm{n}=33)$ and group II (80+ years, 
$\mathrm{n}=15$ ). Physical activity was assessed by Physical Activity Scale for the Elderly (PASE). Functional performance was assessed by Short Physical Performance Battery (SPPB). The SPPB was calculated from three components: (A) ability to stand for up in three feet positions; (B) time to complete 4-m walk; (C) time to rise from a chair five times. We used JAMAR dynamometer (Patterson Medical, Great Britain) to measure handgrip strength (HGS, kg). HGS measurement was performed three times for each arm, only maximum handgrip result was used for further analysis. The statistical analysis were made with software SPSS for Windows (version 18.0). Mann-Whitney U test is used to compare differences between two independent groups, $\mathrm{p}<0.05$ determined as significant.

Results: The study sample consisted of 48 persons -40 women and 8 men. Mean age of them was $76.38 \pm 4.96$ years. The mean values in questionnaire scores were: PASE-202.84 \pm 55.73 , SPPB$10.83 \pm 1.56$, HGS $-29.92 \pm 8.12$. SPPB total score was statistically significant different between group I and group II age groups $(11.12 \pm 1.36$ and $9.78 \pm 1.86, \mathrm{p}=0.03)$. Further analysis also revealed that coordination and balance score was statistically significant different between this two age groups $(3.98 \pm 0.09$ and $3.56 \pm 0.53, p=0.002$ ). No statistically significant differences of physical activity wasn't found in observed age groups.

Conclusion: Our study showed that coordination and balance were better in 70-79 years age group compared with persons aged 80 years and older.

\section{P-338}

Survival following discharge from a UK intermediate care facility

\author{
M. Taylor ${ }^{1}$ \\ ${ }^{1}$ Blackpool Teaching Hospitals, Blackpool, UK
}

Introduction: Elderly hospitalised patients have reduced life expectancy; one study showed 1 year mortality of $45.6 \%$ for patients aged over 85 years. Clifton Hospital is a UK intermediate care facility, where patients are transferred following an acute hospital stay, either for rehabilitation or assessment for $24 \mathrm{~h}$ care. Little was known about how long people survived following discharge from Clifton.

Methods: All patients discharged from June 2013 onwards were identified, basic demographic data collected (age, sex, length of stay (LOS) and how long they survived until death). Kaplan-Meier curves were created to assess survival differences. Statistical significance was tested using the log ranked statistic.

Results: Data were collected for 3173 patients (1793 female, 1380 male) with an age range of 19-104 years (median 84, mean 83, standard deviation 9.85). Survival at 12 months was $64.6 \%$, 24 months $52.8 \%$ and 36 months $46.9 \%$. There was no survival difference between females and males $(\mathrm{p}=0.069)$. Being aged 85 years or more was associated with lower survival $(57.3 \%$ at 12 months aged $85+$ years vs $71.1 \%$ below 85 years, $\left.p=1.21 \times 10^{-13}\right)$. LOS greater than 2 weeks was associated with lower survival (73.4\% for LOS up to 2 week, $63.6 \%$ for LOS greater than 2 weeks, $p=5.52 \times 10^{-23}$ ). Conclusion: Age of $85+$ years was associated with clinically and statistically significantly higher mortality following discharge from intermediate care. LOS over 2 weeks was also associated with increased mortality. This would suggest that both age and prolonged LOS should trigger conversations around advanced care planning at discharge.

\section{P-339}

The associations between loneliness and hedonic well-being in elderly people

Andrejus Cernovas ${ }^{1}$, Indre Marija Kavaliauskaite ${ }^{1}$, Rimantas Stukas ${ }^{1}$, Gene Surkiene $^{1}$, Marija Tamulaitiene ${ }^{1}$, Vidmantas Alekna ${ }^{1}$

${ }^{1}$ Faculty of Medicine, Vilnius University, Vilnius, Lithuania

Introduction: Loneliness is a common, emotionally distressing experience and is associated with adverse physical, mental health and well-being in older people. The aim this of study is to investigate the associations between loneliness and hedonic well-being in community-dwelling persons aged 65 years and older.

Methods: This cross-sectional study involved community-dwelling ambulatory people aged 65 years and over; were cognitively competent with MMSE $\geq 10$ points. Loneliness was valued by Revised University of California of Los Angeles Loneliness Scale (R-UCLA). Hedonic well-being was measured using three questionnaires: (1) the Positive and Negative Affect Schedule (PANAS) that consists of two scales to measure both positive affect (PA) and negative affect (NA); (2) Geriatric Depression Scale (GDS-SF); (3) satisfaction with life scale (SWLS). Data was analyzed using Statistical Package for Social Sciences IBM software (version 18.0). Correlation analysis was performed using Spearman's coefficient, $\mathrm{p}<0.05$ was determined as significant.

Results: The study population consisted of 61 people -25 (41\%) men and $36(59 \%)$ women aged from 65 to 83 years (mean age $73.7 \pm 5.2$ years). Mean and standard deviation of explored values were: $\quad$ R-UCLA $-42.66 \pm 16.63, \quad$ PANAS-PA $-27.63 \pm 8.47$, PANAS-NA-22.32 $\pm 7.8, \quad$ GDS-SF-6.37 $\pm 4.19, \quad$ SWLS$22.6 \pm 6.11$. Our study showed that R-UCLA score negatively moderately correlated with PANAS-PA $(\mathrm{r}=-0.5, \mathrm{p}<0.001)$ and SWLS $(r=-0.44, p<0.001)$ scores. Further correlation analysis revealed positive R-UCLA score association with PANAS-NA $(\mathrm{r}=0.43, \mathrm{p}<0.001)$ and GDS-SF $(\mathrm{r}=0.59, \mathrm{p}<0.001)$ scores.

Conclusion: In people age and 60 years and older loneliness were associations positive emotions, depression and satisfaction with life.

\section{P-340}

Muscle strength is associated with course of mobility among older persons after acute hospitalization: the Hospital ADL study

J. J. Aarden ${ }^{1,2,3}$, M. Van der Schaaf ${ }^{1,2}$, Van der Esch Martin ${ }^{4}$, L. A. Reichardt ${ }^{5}$, Van Seben Rosanne ${ }^{5}$, J. A. Bosch ${ }^{6}$, J. W. R. Twisk ${ }^{7}$, B. M. Buurman ${ }^{2,5}$, R. H. H Engelbert ${ }^{1,2}$

${ }^{1}$ Amsterdam UMC, University of Amsterdam, Department of Rehabilitation, Amsterdam Movement Sciences, Meibergdreef 9, Amsterdam, Netherlands, ${ }^{2}$ Amsterdam Center for Innovative Health Practice (ACHIEVE), Faculty of Health, Amsterdam University of Applied Sciences, Amsterdam, Netherlands, ${ }^{3}$ European School of Physiotherapy, Faculty of Health, Amsterdam University of Applied Sciences, Amsterdam, Netherlands, ${ }^{4}$ Reade, Center for Rehabilitation and Rheumatology/Amsterdam Rehabilitation Research Center, Amsterdam, Netherlands, ${ }^{5}$ Amsterdam UMC, University of Amsterdam, Department of Internal Medicine, Section of Geriatric Medicine, Amsterdam, Netherlands, ${ }^{6}$ Department of Clinical Psychology, University of Amsterdam, Amsterdam, Netherlands,

${ }^{7}$ Amsterdam UMC, Vrije Universiteit Amsterdam, Department of Epidemiology and Biostatistics, Boelelaan 1117, Amsterdam, Netherlands

Objective: The objectives of this study were to determine (i) the course of mobility of acute hospitalized older persons and (ii) the 
association between muscle strength and the course of mobility over time including influencing factors.

Methods: A multicenter, prospective, observational cohort study on older persons who were acutely admitted to one of the 6 participating hospitals was conducted by a multidisciplinary team. Mobility was assessed by the De Morton Mobility Index (DEMMI) and muscle strength by the JAMAR. Measurements were taken at admission, discharge, 1 and 3 months post-discharge. The longitudinal association between muscle strength and mobility was analysed with a linear mixed model and controlled for potential confounders such as age, gender, cognitive impairment, fear of falling and depressive symptoms

Results: A total of 401 older persons were included with a mean (SD) age of 79.6 (6.7) years. Mobility improved significantly from 57 points at admission up to 68 points at 3 months post-discharge. No improvement was observed from one-up to 3 months post-discharge. Even after controlling for potential demographic and psychological confounders, muscle strength was associated with the course of mobility $(\beta=0.64 ; p<0.01)$. Age, cognitive impairment, fear of falling and depressive symptoms were identified as confounders and gender as an effect modifier.

Conclusion: For the development of an intervention to improve mobility in acute hospitalized older persons, a multifactorial approach (muscle strength in combination with factors such as fear of falling and depressive symptoms) focusing on the first month following hospital discharge is warranted.

\section{P-341}

Alcoholism and fractures in women in a functional recovery unit

Lucia Barandiaran ${ }^{1}$, Maria Urien ${ }^{2}$, Nerea Alberdi ${ }^{2}$, Naiara Fernandez ${ }^{2}$, Vincenzo Malafarina ${ }^{3}$

${ }^{1}$ Igurco Unbe Functional Recovery Unit, ${ }^{2}$ Igurco Unbe Functional Recovery Unit, ${ }^{3}$ Servicio de Geriatría Hospital de Navarra

Introduction: Chronic excessive alcohol consumption compromises the health of the bones and increases the risk of osteoporosis.

Objective: To determine the clinical characteristics of women with alcoholism to income in a unit of functional recovery.

Methods: Prospective observational study of a cohort of women with alcoholism admitted for rehabilitation of a fracture during the year 2017. We analyze Socio-demographic data and the cognitive, affective and nutritional situation and the hepatic function at admission to the unit.

Results: During 2017, 168 patients (96 women) were admitted in our functional recovery unit. Six women, who were admitted for rehabilitation of a fracture (Hip fracture 2, pelvis fracture 2, vertebral fracture and rib fractures 1 and 5th metatarsal diaphyseal fracture 1 had a history of alcoholic dependence and recurrent falls. The average age was 74.3 years (63-83), 7.3 years younger than the mean of the unit. Three had university studies, and the $83.3 \%$ had a high economic level. Two patients had cognitive impairment and all had depressive symptomatology and had scheduled at least 2 psychodrugs. All patients had sings of malnutrition: Albumin mean $3.03 \mathrm{~g} / \mathrm{dL}$ (2.6-3.4), Prealbumin mean $15.6 \mathrm{mg} / \mathrm{dl}(11-18)$ and $83.3 \%$ had high Gamma-glutamyltransferase (GGT), mean of 124 U/1 (45-268).

Conclusions: (1) Alcoholic dependence is a cause of repeated falls and, consequently, of fractures in the women. (2) Women with chronic alcoholism are younger than the average, have a high purchasing power and a higher level of education. (3) There's always a depressive symptoms, a high consumption of psychotropic drugs, malnutrition and liver involvement.
Area: Geriatrics in organ disease

\section{P-342}

\section{Systemic lupus erythematosus of the elderly}

Sayhi Sameh ${ }^{1}$, Boussetta Najeh ${ }^{2}$, Arfaoui Bilel ${ }^{2}$, Guedich Nour ${ }^{2}$, Ben Abdelhafidh Nedia ${ }^{2}$, Ajili Faida ${ }^{2}$, Louzir Bassem ${ }^{2}$

${ }^{1}$ Hospital Military of Tunis, Tunis, Tunisia, ${ }^{2}$ Hospital Military of Tunis, Tunis, Tunisia

Introduction: Systemic lupus erythematosus (SLE) is an autoimmune disease that affects mainly the young woman, rarely the elderly clinical-biological characteristics in this population is important to know in order to avoid delayed diagnosis and therapeutic errors.

Patients and methods: A retrospective descriptive study performed during a period of 17 years including patients with an age greater than or equal to 65 years were included in whom the diagnosis of LES was retained according to the criteria of the ARA.

Results: Eight elderly patients had SLE among 97 lupus patients, including 3 men and 5 women (sex ratio $\mathrm{H} / \mathrm{F}$ at 0.6 ). The average age was 69 years old. Extra-renal disease was frequently found with rheumatic disease in 6 patients ( 5 cases of arthritis, 2 cases of myalgia, one case of myositis and one case of isolated arthralgia). Mucocutaneous involvement was observed in 5 patients. It is detailed. Cardiac involvement was present in 4 patients, renal involvement was observed in 5 patients. Hematological involvement was observed in 5 patients. Seven patients had received corticosteroid treatment. The other treatments used are detailed. The treatment with synthetic antimalarials was stopped in a patient with the retinal toxicity that caused it.

Discussion: Systemic lupus erythematosus is very rare after 65 years with an estimated frequency of $5 \%$ or less.

Conclusion: The diagnosis is difficult to make because of the nonspecificity of many clinical lesions and the constant entanglement with comorbidities.

\section{P-343}

Functional and cognitive status of patients after a transcatheter aortic valve implantation

Cristina Roqueta ${ }^{1}$, Marta Herrero ${ }^{1}$, María Isabel MartínezFernández ${ }^{1}$, Andrea Sánchez-Carpintero ${ }^{2}$, Nuria Farre ${ }^{2}$, Beatriz Vaquerizo $^{2}$, Silvia Burcet ${ }^{3}$, Olga Vázquez ${ }^{1}$

${ }^{1}$ Geriatric Medicine Department, Parc de Salut Mar, Centro Fórum, Hospital del Mar, Barcelona, Spain, ${ }^{2}$ Department of Cardiology, Parc de Salut Mar, Hospital del Mar, Barcelona, Spain, ${ }^{3}$ Geriatric Medicine Department, Parc de Salut Mar, Centro Fórum, Hospital de

Introduction: Aim of the present study was to assess functional and cognitive status of patients 3 months after a transcatheter aortic valve implantation (TAVI).

Methods: Prospective, descriptive and unicentric study of patients with symptomatic severe aortic stenosis were evaluated by a geriatrician from April 2016 to December 2017. An interdisciplinary team decided the treatment for each patient [medical treatment, TAVI and aortic valve replacement (AVR)]. Socio-demographic variables (age, sex) were registered. Functional and cognitive status were evaluated before and 3 months after TAVI. Functional status was performed by Barthel index (BI), Lawton index (LI) and Short Physical Performance Battery (SPPB). Short Physical Performance Battery values the balance (4 points), gait speed (4 points) and muscle strength of the lower extremities (chair stand test) (4 points). Cognitive status was performed by Mini-Mental of Folstein (MMSE). 
Results: Fifty-nine patients were evaluated ( $55.93 \%$ women); mean aged $81.55 \pm 6.24$ years. Twenty-nine patients received medical treatment, 23 TAVI and 7 AVR. Three patients who received TAVI treatment refused follow-up by geriatrician. Mean of the parameters evaluated before TAVI: LI:5.80 $\pm 1.90 ; \quad$ BI:98.35 \pm 4.92 ; SPPB:8.30 $\pm 2.53 ; \quad$ SPPB (balance):3.35 $\pm 0.81 ; \quad$ SPPB (gait speed):3.05 $\pm 0.88 ; \quad$ SPPB (chair stand test): $1.90 \pm 1.16$; MMSE: $27.00 \pm 2.92$. Mean of the parameters evaluated 3 months after TAVI: LI:5.85 $\pm 2.00,(\mathrm{p}=0.84)$; BI:95.90 $\pm 8.76,(\mathrm{p}=0.09)$; SPPB:8.35 $\pm 2.45, \quad(\mathrm{p}=0.91) ; \quad$ SPPB $\quad$ (balance):3.35 \pm 0.74, $(\mathrm{p}=1.00)$; SPPB (gait speed):3.10 $\pm 0.85,(\mathrm{p}=0.81)$; SPPB (chair stand test):2.05 $\pm 1.31,(\mathrm{p}=0.54)$; MMSE:27.40 $\pm 3.11,(\mathrm{p}=0.49)$. Conclusions: Patients with severe symptomatic aortic stenosis evaluated by a geriatrician have maintained their previous cognitive and functional status (basic and instrumental activities of daily living, and a physical performance test) 3 months after TAVI.

\section{P-344}

\section{Assessment of non motor symptoms in geriatric medicine led} Parkinson's disease clinic

\section{J. Challberg ${ }^{1}$, Z. Tait ${ }^{2}$, G. Stannard ${ }^{2}$, S. Thompson ${ }^{1}$}

${ }^{1}$ Department of Clinical Geratology, John Radcliffe Hospital, Oxford University Hospitals Foundation Trust, Oxford, UK, ${ }^{2}$ Oxford University Medical School, Oxford, UK

Introduction: Parkinson's disease (PD) is thought of primarily as a movement disorder with the main symptoms: tremor, bradykinesia, rigidity and postural instability. In practice, this more complex disease is associated with a broad spectrum of non motor symptoms (NMS). These are well recognised features of both early and advanced PD and have a significant impact on patients' quality of life. Nevertheless, non-motor symptoms are often overlooked in clinical practice. The UK National guidelines suggest that clinicians in Movement Disorder clinics should ask about non motor symptoms at least once per year as part of a routine review. This survey looked at our service performance, as our participation in the National Parkinson's Disease Audit highlighted insufficient detection of NMS. Methods: After obtaining verbal consent, 50 patients were asked to complete a validated questionnaire of non-motor symptoms in Parkinson's disease before their clinic appointment. Thirty questions covered 10 domains of non-motor symptoms frequently experienced by patients with Parkinson's disease.

Results and discussion: The administration of a self-completed questionnaire significantly improved the rate of detection of NMS. The most common NMS were identified as excess salivation, gastrointestinal symptoms and sleep disturbances. Once symptoms were identified, appropriate interventions could be taken, depending on the individual case and problem. This survey had shown that simple intervention had improved detection rate as well as appropriate intervention and ultimately quality of care of older patients with PD.

\section{P-345}

Safety of low-dose methotrexate for the treatment of bullous pemphigoid in older adults with and without chronic renal impairment

Alexandra Fisch ${ }^{1}$, Lucas Morin ${ }^{2}$, Toomas Talme ${ }^{3}$, Kristina Johnell ${ }^{2}$, Irène Gallais Sérézal ${ }^{3}$

${ }^{1}$ Gävle Hospital, ${ }^{2}$ Karolinska Institutet, Solna Municipality, Sweden, ${ }^{3}$ Karolinska University Hospital, Solna Municipality, Sweden
Aims: Bullous pemphigoid is an autoimmune skin disease which affects primarily older adults. Low-dose methotrexate is a common alternative to systemic corticosteroids. However, there is little evidence about the safety of methotrexate, especially among patients with impaired renal function.

Methods: We included older adults ( $\geq 70$ years) diagnosed with bullous pemphigoid between 2006 and 2015 at a single university hospital in Sweden and treated with low-dose methotrexate. Data was collected in electronic medical records. Renal function was estimated with the CKD EPI equation for glomerular filtration rate (eGFR). Multivariate Cox proportional hazard modelling was used to estimate the risk of adverse outcomes during follow-up.

Results: A total of 232 older patients were included. Mean age at time of diagnosis was 84.6 years (SD 6.7), $52.6 \%$ of patients were women, $41.6 \%$ lived in nursing homes, $31 \%$ had $\geq 3$ chronic comorbidities, and $37 \%$ had dementia. Median prescribed dose of methotrexate was $5 \mathrm{mg} /$ week. During the period of exposure to methotrexate (including 90-day washout period after treatment discontinuation), overall mortality was 26.3 (95\% CI 21.9-31.5) per 100 person-years. After adjustment for relevant confounders, we found no statistically significant difference in the risk of death when comparing individuals with eGFR $>60 \mathrm{~mL} / \mathrm{min}$ at baseline, eGFR $>45-60$ $\mathrm{mL} / \mathrm{min}$, and eGFR $<45 \mathrm{~mL} / \mathrm{min}$ (log-rank $\mathrm{p}=0.11$ ). There was also no association between eGFR at baseline and unplanned hospitalization for acute renal failure, liver failure, bone fracture, infectious disease, haematological event, or gastrointestinal event. Finally, clinician-reported outcomes (dose-decrease and treatment switch for clinical event, renal impairment or other biological adverse event) did not vary significantly according to eGFR at baseline, although older patients with eGFR $<45 \mathrm{~mL} / \mathrm{min}$ experienced higher rates of treatment switch or adverse clinical or biological events.

Conclusion: In the absence of robust evidence from randomized clinical trials, our observational study suggests that low-dose methotrexate is safe for the treatment of bullous pemphigoid in older adults with impaired renal function. Regular clinical monitoring is warranted to prevent adverse events and adapt treatment regimen if necessary.

\section{P-346}

Psychosocial assessment in patients with Congestive heart failure in an acute geriatric care unit

Barbara Peña Mella ${ }^{1}$, Elena Valle Calonge ${ }^{1}$, Eva Lopez Alvarez ${ }^{1}$, Gloria Ordoñez Estebañez ${ }^{1}$, Lucia Saenz de Santa Maria Benedet ${ }^{1}$, Andrea Fernandez Soneira ${ }^{1}$, Rocio Garcia Ronderos ${ }^{1}$, Margarita Garcia ${ }^{1}$, Juan Jose Solano Jaurrieta ${ }^{1}$, Jo

${ }^{1}$ Geriatric's Clinical management section, Monte Naranco Hospital, Oviedo, Spain

Objectives: To identify psychosocial characteristics of patients admitted in an acute Geriatric unit with decompensated heart failure in relation to mortality and admission at 6 months of discharge.

Method: Descriptive, prospective study of patients admitted to an acute geriatric unit with decompensated heart failure. Variables: age, sex, Inpatient-mortality, morbid history, medications, laboratory assessment, previous echocardiogram, New-York-Heart-AssociationFunctional-Classification, Charlson-Comorbidity-Index, Barthel-Index (BI), Short-Physical-Performance-Battery (SPPB), MiniNutritional-Assessment, Global-Deterioration-Scale (GDS), Yesavage Depression Scale, Pfeiffer Test (SPMSQ), Gijon's-socialfamilial-evaluation-scale (SFES), Minnesota-Living-with-Heart-Failure-Questionnaire (MLHFQ) and admission at 6-month.

Results: $\mathrm{N}=110,62 \%$ women, age: $87.8 \pm 4.5$, average stay $9.7 \pm 4.4$ days. Inpatient mortality: $10.9 \%$. $21.6 \%$ were readmitted 
within 1 month of discharge and 39\% at 6-month. Yesavage $<5$ : $54.5 \%$, altered values were related to SPMSQ $(\mathrm{r}=-0.25)$, MLHFQ $(\mathrm{r}=0.57)$, higher previous BI $(\mathrm{P}<0.05)$ and inpatient mortality $(\mathrm{p}<0.05)$. No cognitive impairment (GDS): $45.5 \%$ and mild Cognitive impairment: $30.9 \%$. GDS correlated with SPMSQ $(\mathrm{r}=0.6)$, Weight $(\mathrm{r}=-0.6)$ and previous IB $(\mathrm{r}=-0.3)$. SPMSQ was $<$ $3: 66.3 \%$. Patients with an impaired quality of life (MLHFQ) were more likely to take digoxin $(\mathrm{p}<0.05)$ and to have Dementia diagnose $(\mathrm{p}<0.05)$. The mean SFES was $6.6 \pm 3.4$ and correlated with Pfeiffer test $(r=0.3)$, Yesavage $(r=0.32)$ and MLHFQ $(r=0.38)$. Patients with worst quality of life (MLFQ), lower SPPB and lower heart rate were more likely to be admitted at 6-month.

Conclusions: Most of our patients had mild or no cognitive decline, neither depression symptoms. Those with worst social support (SFES) were more depressed and had worst quality of life. Psychosocial assessment could be a predictor of mortality and admission at 6 months. We didn't find a relationship among the rest of the other variables.

\section{P-347}

Delirium in an elderly patient and a different kind of white matter

L. Dreessen ${ }^{1}$, N. Maréchal ${ }^{1}$, A. De Becker ${ }^{2}$, A. Meuwissen ${ }^{3}$, I. Beyer ${ }^{1}$

${ }^{1}$ Department of Geriatric Medicine, Universitair Ziekenhuis Brussel, Vrije Universiteit Brussel (VUB), Laarbeeklaan 101, 1090 Brussels, Belgium, ${ }^{2}$ Department of Hematology, Universitair Ziekenhuis Brussel, Vrije Universiteit Brussel (VUB), Laarbeeklaa, Jette, Belgium

Background: Hematological malignancies can spread to the central nervous system (CNS) either as a focal lesion or leptomeningeal carcinomatosis. Marginal zone lymphoma (MZL) is a low grade nonHodgkin's lymphoma and is generally an indolent disease. This case illustrates CNS invasion by MZL presenting as a delirium without B symptoms or any abnormalities on radiologic examinations.

Case report: An 80 year old patient is described, presenting a subacute, progressive confusion. MMSE score was 21/30. The patient's medical history included monoclonal B-cell lymphocytosis (MBL) with a clone indicative for a MZL. After excluding most common etiologies through classical work-up including a normal head magnetic resonance imaging, a lumbar puncture (LP) was performed, showing an elevated protein and lymphocyte count. Beta-amyloid and tau protein levels in CSF were normal. Immunophenotyping of the lymphocytes confirmed CNS invasion by the MZL clone. Staging revealed mild splenomegaly. Prednisolone, intrathecal and systemic chemotherapy was initiated, leading to quick cognitive improvement with MMSE score of $28 / 30$.

Discussion: To the best of our knowledge a delirium in an older patient due to leptomeningeal carcinomatosis by a MZL has never been described. MZL is an indolent disease that rarely spreads to the CNS. To date, reports of CNS invasion by MZL describe focal intracranial lesions. After exclusion of common etiologies, physicians should remain vigilant when confronted with elderly presenting a confusional state with a prior history of MBL. This case demonstrates that the threshold for a LP should be low also for patients with normal imaging studies.

\section{P-348}

Differences in time to stability and 1-year mortality among elderly patients with community-acquired pneumonia

Isabel Arnau-Barrés ${ }^{1}$, Manuel Baca ${ }^{1}$, Olga Vázquez ${ }^{1}$, Ramón Miralles ${ }^{1}$, Robert Güerri-Fernández ${ }^{2}$

${ }^{1}$ Geriatrics Department, Hospital del Mar, Barcelona, Spain, ${ }^{2}$ Infectious Diseases, Hospital del Mar Research Institute, IMIM, Barcelona, Spain

Introduction: Community-acquired pneumonia (CAP) continues to have a significant impact on elderly individuals, who are affected more frequently and with more severe consequences than younger populations.

Methods: The principal aim was to assess the differences in the time to clinical stability (defined as normalization of heart rate, systolic blood pressure, respiratory rate, temperature, oxygenation status, ability to eat and mental status) and mortality (30 days) between both groups. As secondary objectives we analyze differences among the different therapies and analyze prognosis factors in clinical outcomes (performance at discharge, 1-year post episode mortality). Retrospective analysis of electronic charts from January to June 2017. Two groups defined: elderly ( $>65$ years-old) (EP) and non-elderly $(<65$ years-old) (non-EP).

Results: One hundred and thirty six episodes data of patients admitted throughout the 6-month study period. Elderly $82(60 \%)$ and 54 non-EP 54 (40\%).EP mean age EP 79 (SD 6) vs. non-EP 51 (SD 10). No differences were found in the clinical presentation, inflammatory-markers or empirical antimicrobial treatment. Time to stability was higher among EP mean: 6 days (SD 5) vs. non-EP 4 days (SD 3); $\mathrm{p}=0.05$. In a multivariable regression model the significant independent factors to time to stability were: being part of EP group [beta coeff 2.03 (95\% CI 0.073-4.14); $\mathrm{p}=0.049$ ] and receiving Amoxicilin-Clavulanate [beta coeff -2.4 (95\% CI -4.6 to $-0.13) ; p=0.03]$. No differences in 30-day mortality were found $(2(2.5 \%)$ in the EP group). However, mortality at 1-year after the CAP episode was higher among EP $(13(16 \%)$ vs $2(3 \%) ; p=0.02)$. In the elderly group the factors associated with 1-year post CAP mortality were 6-month prior hospital admission (OR $6.2695 \% \mathrm{CI}$ $1.6-23.1 ; \mathrm{p}=0.006)$.

Conclusions: In conclusion, in our series time to stability in CAP was higher among EP, and lower in those receiving amoxicillin-clavulanate. A prior hospital admission was strongly correlated with 1-year post episode mortality in the elderly group.

\section{P-349}

A proposal for the ex post identification and categorization of older people with functional impairments in scientific studies: Recommendations of the Medication and Quality of Life in frail older persons (MedQoL) Research Group

Simone Brefka ${ }^{1}$, Dhayana Dallmeier ${ }^{1}$, Viktoria Mühlbauer $^{1}$, Christine A.F. von Arnim ${ }^{2}$, Claudia Bollig ${ }^{3}$, Graziano Onder ${ }^{4}$, Mirko Petrovic ${ }^{5}$, Carlos Schönfeldt-Lecuona ${ }^{6}$, Moritz Seibert ${ }^{2}$, Gabriel Torbahn ${ }^{7}$, Sebastian Voigt-Radloff ${ }^{3}$, Walter E. Haefeli ${ }^{8}$, Jürgen M. Bauer ${ }^{9}$, Michael D. Denkinger ${ }^{1}$, for the Medication and Quality of Life Research Group

${ }^{1}$ AGAPLESION Bethesda Clinic, Geriatric Research Unit Ulm University, Geriatric Center Ulm/Alb-Donau, ${ }^{2}$ University Clinic Ulm, Departments of Neurology, Ulm, Germany, ${ }^{3}$ Center for Geriatric Medicine and Gerontology and Medical Center, University of Freiburg, Cochrane Germany, Freiburg, Germany, ${ }^{4}$ Department of 
Geriatrics, Neurosciences and Orthopaedics, A. Gemelli University Hospital, Universitá Cattolica del Sacro Cuore, Rome, Italy, ${ }^{5}$ Department of Internal Medicine (Geriatrics), Ghent University, Ghent, Belgium, ${ }^{6}$ University Clinic Ulm, Department of Psychiatry and Psychotherapy III and Geriatric Center Ulm/Alb-Donau, Ulm, Germany, ${ }^{7}$ Institute for Biomedicine of Aging, Friedrich-AlexanderUniversity Erlangen-Nürnberg and Medical Center, University of Freiburg, Cochrane Germany, Freiburg, Germany, ${ }^{8}$ Department of Clinical Pharmacology and Pharmacoepidemiology, University of Heidelberg, Heidelberg, Germany, ${ }^{9}$ Center for Geriatric Medicine, University of Heidelberg and Agaplesion Bethanien Hospital,

Heidelberg, Germany

*For the Medication and Quality of Life research Group: Christine A.F. von Arnim, Jürgen M. Bauer, Claudia Bollig, Simone Brefka, Dhayana Dallmeier, Michael D. Denkinger, Annette Eidam (9), Walter E. Haefeli, Anette Lampert (8), Viktoria Mühlbauer, Carlos Schönfeldt-Lecuona, Hanna M. Seidling (8), Sebastian Voigt-Radloff, Gabriel Torbahn.

Background: When treating older adults, a main factor to consider is functional status. Because appropriate assessments in clinical trials are frequently lacking, critical appraisal of treatment evidence with respect to functional status is challenging. Our aim was to identify and categorize assessments for functional status in clinical trials to allow for a retrospective characterization and indirect comparison of treatment evidence.

Method: We conducted four different systematic reviews of randomized and non-randomized controlled clinical trials in older people with hypertension, diabetes, depression and dementia. All identified assessments that reflected functional status were analyzed. Assessments were categorized into four pre-defined different "functional status levels". If available from the literature, cut-offs were extracted. If not, they were defined by our expert group comprised of geriatricians, pharmacists, pharmacologists, neurologists, psychiatrists.

Results: We identified 51 instruments for assessment that included measures of functional status. While some had clearly defined cut-offs across our predefined categories, many others did not. In most cases, no cut-offs existed for slightly impaired or severely impaired older adults. Missing cut-offs or values to adjust were determined by our expert group using a patient-centred approach and will be presented as described.

Conclusion: All categorized functional status assessments are now suitable for a retrospective characterization of functional status in primary studies (e.g. RCTs and observational studies). Allocated categories only serve as approximations and should be head-to-head validated in future studies. Moreover, upcoming studies involving older adults should include and explicitly report functional status as a baseline characteristic of all participants enrolled.

\section{P-350}

Functional and cognitive status of patients after an aortic valve replacement

Cristina Roqueta ${ }^{1}$, Nuria Farre ${ }^{2}$, Maria Isabel Martínez-Fernández ${ }^{3}$, Sandra Urbano ${ }^{3}$, Andrea Sánchez-Carpintero ${ }^{2}$, Mercè Cladellas ${ }^{2}$, Beatriz Vaquerizo $^{2}$, Olga Vázquez ${ }^{3}$

${ }^{1}$ Geriatric Medicine Department. Parc de Salut Mar, Centre Fórum, Hospital del Mar. Barcelona, Spain, ${ }^{2}$ Department of Cardiology, Parc de Salut Mar, Hospital del Mar, Barcelona, Spain, ${ }^{3}$ Geriatric

Medicine Department, Parc de Salut Mar, Centro Fórum, Hospital d

Introduction: Aim of the present study was to assess functional and cognitive status of patients 3 months after an aortic valve replacement.
Patients and methods: Prospective, descriptive and unicentric study of patients with symptomatic severe aortic stenosis were evaluated by a geriatrician from April 2016 to December 2017. An interdisciplinary team decided the treatment for each patient [medical treatment, TAVI and aortic valve replacement (AVR)]. Socio-demographic variables (age, sex) were registered. Functional and cognitive status were evaluated before and 3 months after AVR. Functional status was performed by Barthel index (BI), Lawton index (LI) and Short Physical Performance Battery (SPPB). Short Physical Performance Battery values the balance (4 points), gait speed (4 points) and muscle strength of the lower extremities (chair stand test) (4 points). Cognitive status was performed by Mini-Mental of Folstein (MMSE).

Results: Fifty-nine patients were evaluated (55.93\% women); mean aged $81.55 \pm 6.24$ years. Twenty-nine patients received medical treatment, 23 TAVI and 7 AVR. All the patients who received aortic valve replacement were followed-up by a geriatrician. Mean of the parameters evaluated before AVR: LI:7.14 \pm 1.46 ; BI:97.14 \pm 4.87 ; SPPB:9.28 $\pm 1.38 ; \quad$ SPPB (balance):3.28 $\pm 0.75 ; \quad$ SPPB (gait speed):3.42 $\pm 0.78 ; \quad$ SPPB (chair stand test):2.42 \pm 0.78 ; MMSE: $27.71 \pm 1.97$. Mean of the parameters evaluated 3 months after AVR: LI:6.57 $\pm 1.27,(\mathrm{p}=0.10)$; BI:96.14 $\pm 6.89,(\mathrm{p}=0.35)$; SPPB:8.70 $\pm 0.75, \quad(p=0.27) ; \quad$ SPPB $\quad$ (balance):3.42 \pm 0.53 $(\mathrm{p}=0.60)$; SPPB (gait speed):2.83 $\pm 0.75,(\mathrm{p}=0.10) ;$ SPPB (chair stand test):2.14 $\pm 0.37,(\mathrm{p}=0.35)$; MMSE:28.57 $\pm 1.51,(\mathrm{p}=0.30)$. Conclusions: Patients with severe symptomatic aortic stenosis evaluated by a geriatrician have maintained their previous cognitive and functional status (basic and instrumental activities of daily living, and a physical performance test) 3 months after AVR.

\section{P-351}

Human papillomavirus infection and coronary artery disease in postmenopausal women

Leonardo Victor Galvão-Moreira ${ }^{1}$, Luciane Maria Oliveira Brito ${ }^{1}$, Haissa Oliveira Brito ${ }^{1}$, Rita da Graça Carvalhal Frazão Corrêa ${ }^{1}$, Clariano Pires de Oliveira Neto ${ }^{1}$, Joyce Pinheiro Leal Costa ${ }^{1}$, Sally Cristina Moutinho Monteiro ${ }^{1}$, Flávia Ca

${ }^{1}$ Federal University of Maranhão, São Luís, Brazil, ${ }^{2}$ Portuguese Institute of Oncology, Lisbon, Portugal, ${ }^{3}$ Federal University of São Paulo, São Paulo, Brazil

Cardiovascular disease (CVD) is a leading cause of death among adults and elderly. Additionally, recent studies suggest that some viruses, including the human papillomavirus (HPV), may play an important role in CVD pathogenesis by affecting atheromatous coronary arteries. Nevertheless, a potential association between HPV infection and coronary artery disease (CAD) in postmenopausal women remains unclear.

Objective: To investigate a possible relation between HPV infection and CAD risk among postmenopausal women.

Methods: The presence of both CAD and cervical HPV DNA was evaluated in 54 postmenopausal women. Sociodemographic variables and metabolic profiles were also obtained.

Results: A total of 27 patients presented with CAD, 16 of whom were positive for HPV DNA, whereas 11 were negative. The presence of cervical HPV infection was strongly associated with CAD when women with and without HPV DNA were compared [odds ratio $(\mathrm{OR})=3.74 ; 95 \%$ confidence interval $(\mathrm{CI})$ 1.16-11.96], after controlling for demographics, health/sex behaviors, medical comorbidities and CVD risk factors. This association was even higher when comparing women with cancer-associated HPV types to HPVnegative women $(\mathrm{OR}=4.90 ; 95 \% \mathrm{CI} 1.26-19.08)$. 
Conclusion: These findings support the hypothesis that cervical HPV infection might be associated with an increased risk of developing CAD among postmenopausal women. Population-based longitudinal studies are thus necessary towards further investigating this relationship.

\section{P-352}

Vitamin D deficiency and risk of helicobacter pylori infection in older adults: a cross-sectional study

Deniz Mut Surmeli ${ }^{1}$, Zeki Gokhan Surmeli ${ }^{2}$, Remzi Bahsi ${ }^{1}$, Tugba Turgut ${ }^{1}$, Hande Selvi Oztorun ${ }^{1}$, Volkan Atmis ${ }^{3}$, Murat Varli ${ }^{1}$, Sevgi Aras ${ }^{1}$

${ }^{1}$ Department of Geriatrics, Ankara University School of Medicine, Ankara, Turkey, ${ }^{2}$ Department of Medical Oncology, Ankara Medical Park Hospital, Ankara, Turkey, ${ }^{3}$ Department of Geriatrics, Bursa Şevket Yılmaz Training and Research Hospital, Bursa, Turkey

Introduction: Vitamin D deficiency is known to cause increased predisposition to various infectious diseases and addition of vitamin $\mathrm{D}$ to antimicrobial treatment may improve treatment responses. However, the relationship between vitamin D and Helicobacter pylori $(H$. pylori) remains to be determined. In this study, we assessed the association between vitamin $\mathrm{D}$ deficiency and $H$. pylori infection.

Methods: This cross-sectional study included patients aged 65 and over, who underwent gastroscopy and had gastric biopsy performed between 2010 and 2017. Of the 441 patients, 254 had available 25-hydroxyvitamin D level results and were included in the analyses. Patients were categorized into $\mathrm{H}$. pylori $(+)$ and $\mathrm{H}$. pylori $(-)$ groups, according to histopathological examination results of gastric biopsies. Serum $25(\mathrm{OH})$ vitamin D levels less than $20 \mathrm{ng} / \mathrm{mL}$ were defined as vitamin $\mathrm{D}$ deficiency.

Results: Of all patients, 43 were $H$. pylori (+) and 211 were $H$. pylori $(-)$. More patients had vitamin D deficiency $(<20 \mathrm{ng} / \mathrm{mL})$ in the $H$. pylori (+) group than the H. pylori (-) group (86 vs $67.3 \%$, $\mathrm{p}=0.014)$. The proportion of $\mathrm{H}$. pylori (+) patients decreased across increasing quartiles of $25(\mathrm{OH})$ vitamin $\mathrm{D}$ levels ( $\mathrm{p}$ for trend $=0.010$ ). In multivariable logistic regression analysis, vitamin $\mathrm{D}$ deficiency was associated with increased odds of $H$. pylori infection after adjustment for age, gender, and Charlson comorbidity index $(\mathrm{OR}=3.02,95 \% \mathrm{CI}$ $1.19-7.69, \mathrm{p}=0.020$ ).

Conclusion: Vitamin D deficiency can be associated with increased risk of $H$. pylori infection. The potential protective effect of vitamin D against $H$. pylori infection and its possible role in the treatment of $H$. pylori should be evaluated in prospective trials.

\section{P-353}

Is reverse dipping pattern a risk factor for falls in hypertensive older adults?

Deniz Mut Surmeli ${ }^{1}$, Tugba Turgut ${ }^{1}$, Remzi Bahsi ${ }^{1}$, Hande Selvi Oztorun ${ }^{1}$, Sevgi Aras ${ }^{1}$, Murat Varli ${ }^{1}$

${ }^{1}$ Department of Geriatrics, Ankara University School of Medicine, Ankara, Turkey

Introduction: Falls are one of the preventable geriatric syndromes which can lead to significant disabilities in older adults. In this study we aimed to assess whether nocturnal blood pressure (BP) changes are associated with falls.

Methods: In this cross-sectional study, we included 199 hypertensive patients aged over 60 years. All patients underwent 24-h ambulatory blood pressure monitoring. Patients were classified according to changes in their nocturnal systolic BP from daytime systolic BP as follows: dipper (D) $>10 \%$ decrease, non-dipper (ND) $0-10 \%$ decrease, reverse-dipper (RD) increase to any extent. Falls were defined as any fall history in the recent 1 year.

Results: Out of 199 patients (61.3\% female; median age 76.6, range $61-100), 47$ (23.6\%) had a history of fall, and $12.6 \%, 49.7 \%, 37.7 \%$ of patients had D, ND and RD BP patterns, respectively. Age, gender distribution, body mass index, Charlson comorbidity index, handgrip strength, gait speed, mini-mental state examination and geriatric depression scores were similar among BP dipping pattern groups. However, Mini Nutritional Assessment (median RD: 12, ND: 13, D: 13; p: 0.003) and Lawton instrumental activities of daily living scores (median RD: 12, ND: 15, D: 15; p: 0.039) were significantly lower in the reverse dipper group. Falls were significantly more common in the RD group (RD: $35 \%$, ND: 16\%, D: $20 \%$; p: 0.016). In the multivariate logistic regression analysis after adjustment for age and gender, presence of RD pattern as opposed to ND pattern was significantly associated with increased risk of falls (Odds ratio: 3.0, 95\% CI 1.4-6.3; p: 0.003).

Conclusions: Presence of a reverse dipping pattern of nocturnal BP can be associated with increased risk of falls in older adults.

\section{P-354}

Use of IDPP4 in elderly patients with diabetes and heart failure

Ana Merello de Miguel ${ }^{1}$, Raúl Ruiz Ortega ${ }^{1}$,

Genoveva López Castellanos ${ }^{1}$, Maria Pumares ${ }^{1}$, Miriam Moralejo ${ }^{1}$, Luis Manzano Espinosa ${ }^{1}$

${ }^{1}$ Hospital Ramón y Cajal Madrid, Spain

Objectives: To describe a sample of diabetic patients older than 75 years with heart failure (HF) and the influence of IDPP4 in our sample.

Methods: Preliminary case-control study of diabetic patients, over than 75 years, with a protocolized follow-up in the Heart Failure and Vascular Risk Unit of Internal Medicine Service, recruited consecutively from January 2014 to April 2017. The minimum follow-up was 1 year. We collected basal characteristics, functional situation, comorbidities, drugs, lab tests in the first visit, left ventricular ejection fraction (LVEF) and frequence of emergency visits and hospitalizations due to $\mathrm{HF}$ and other cardiovascular events (CVD).

Results: We included 153 patients (65\% women, average age 83, within 75-98), 94\% had hypertension, 78\% dyslipemia, 66\% atrial fibrillation, $23 \%$ previous stroke, $24 \%$ previous heart attack, $13 \%$ COPD, 75\% NYHA class II-III, 85\% Barthel $>60,83 \%$ LVEF > $50 \%, 54 \%$ Glomerular filtration rate (GFR) $<45 \mathrm{ml} / \mathrm{min}$ and they took an average of $13 \pm 4$ drugs. The median of the laboratory test results were: BNP $435 \pm 658 \mathrm{pg} / \mathrm{ml}$, glycosylated hemoglobin (HbA1c) $6.9 \pm 1.2 \%$, GFR $52.72 \pm 21.96 \mathrm{ml} / \mathrm{min}, 28 \%$ use IDPP4, presenting higher HbAlc $(7.3 \pm 1.2 \% \mathrm{p}=0.001)$ and lower GFR $(46.9 \pm 20.5 \mathrm{ml} / \mathrm{min} \mathrm{p}=0.049)$. The most used IDPP4 is linagliptina $56 \%$. In 1-year follow-up, there were no significant differences in emergency visits for HF $(p=0.73)$ or CVD $(p=0.40)$, nor in hospitalizations for HF $(p=0.73)$ or CVD $(p=0.48)$.

Conclusions: In our preliminary analysis there were no difference in emergency visits or hospital admissions due to HF and CVD in association with the use of IDPP4 in elderly diabetic patients with HF. 


\section{P-355}

Cognitive function after transcatheter aortic valve implantation (TAVI)

Tora Torgersen Engstad ${ }^{1}$, Eva Cecilie Knudsen ${ }^{2}$, Per Steinar Halvorsen ${ }^{2}$, Siri Rostoft ${ }^{3}$

${ }^{1}$ University of Oslo, Norway, ${ }^{2}$ Oslo University Hospital, Norway,

${ }^{3}$ University of Oslo and Oslo University Hospital, Oslo, Norway

Introduction: Aortic stenosis (AS) is prevalent in older adults, and can be treated by surgical aortic valve replacement. TAVI may be more appropriate in high-risk surgical patients. Clinically apparent stroke is detected in $5-17 \%$ of TAVI patients [1,2], while cerebral microembolization seems to occur in over $90 \%$ [2]. The cognitive impact of stroke and cerebral microembolization after TAVI is uncertain, but may accelerate cognitive decline. The primary aim of this study is to identify predictors of cognitive decline at 3 and 12 months after TAVI.

Methods: This is a prospective observational study. Patients are recruited from Oslo university hospital at admittance for TAVI. Study participants are examined pre-procedure with cognitive tests sensitive to cerebrovascular pathology; MoCA (Montreal Cognitive assessment), trail-making test $\mathrm{A}$ and $\mathrm{B}$, and 10-words recall. Physical performance and frailty, functional status, quality of life, anxiety/ depression, and comorbidity are also tested. Post-procedure we register delirium and stroke until discharge. The test battery performed pre-TAVI is repeated at 3 and 12 months.

Results and Key conclusions: Inclusion was started December 2017. So far 37 patients are included. Frailty and cognitive impairment is detected in some before TAVI. Stroke and permanent pacemaker are complications observed post-procedure. Baseline data from the first 37 patients and follow-up data for patients who have been assessed by August 2018 will be presented at the conference.

References:

1. Lansky. Am J Cardiol 2016

2. Messe. JAMA 2016

\section{P-356}

Mortality in hospitalized older adults associated with Clostridium difficile infection

Cyprien Arlaud ${ }^{1}$, Claire Chenesseau ${ }^{1}$, Florence Lieutier ${ }^{2}$, Thierry Fosse ${ }^{1}$, Veronique Mondain ${ }^{1}$, Olivier Guerin ${ }^{1}$

${ }^{1}$ Medicine, ${ }^{2}$ Pharmacy

Introduction: Clostridium difficile infection (CDI) is most common cause hospital acquired infectious diarrhea. The frail elderly is vulnerable and is associated with significant morbi-mortality. The objective of the study is to define risk factors for mortality in patients over 75 years old with CDI.

Material: A prospective observational cohort study was conducted from January 1, 2016 to December 31, 2017 in a University Hospital Center. The included patients had a diagnosis of CDI defined by diarrhea with stool culture positive for CDI confirmed A / B toxin by immunohistochemistry.

Results: 66 patients had been included, with an average age of 85 . 8 years $(75-98)$. More than $80 \%(n=53)$ were women, $65 \%(n=43)$ had a loss of autonomy and more than $75 \%(\mathrm{n}=50)$ with malnutrition. Almost $32 \%(n=21)$ patients had solid or hematologic cancer treated (12) or not (9). Metronidazole treatment is prescribed for $17.5 \%(\mathrm{n}=12)$, vancomycin $77 \%(\mathrm{n}=51)$ and fidaxomycin $5.5 \%$ $(\mathrm{n}=3)$. Three-month mortality rate was $35 \%(\mathrm{n}=23)$. Among them, $57 \%(\mathrm{n}=12)$ patients solid tumors and hematologic malignancy, treated or not $(\mathrm{p}=0.04)$. In multivariate analysis, predicting 3-month mortality among patients were: male sex $[\mathrm{OR}=5.457(1.205-24.706)$ $\mathrm{p}=0.028]$, solid tumors and hematologic malignancy, treated or not $[\mathrm{OR}=4.686(1.193-18.403) \mathrm{p}=0.027]$ and hypoalbuminum $[\mathrm{OR}=$ $0.855(0.748-0.977) \mathrm{p}=0.021]$.

Conclusion: CDI is predicting 3-month mortality among patients with solid tumors and hematologic malignancy, treated or not.

\section{P-357}

Mood disorders and chronic pain in older people with gonarthrosis

G. I. Prada ${ }^{1}$, I. D. Alexa ${ }^{2}$, R. M. Nacu ${ }^{3}$, A. C. $\mathrm{Ilie}^{2}$, A. I. Pislaru ${ }^{2}$, A. G. Prada ${ }^{3}$, A. Kozma ${ }^{4}$, R. Stefaniu ${ }^{2}$, I. A. Sandu ${ }^{2}$, C. Nuta ${ }^{5}$, O. L. Bajenaru ${ }^{1}$, A. M. Herghelegiu ${ }^{3}$

1"Ana Aslan" National Institute of Gerontology and Geriatrics, Bucharest, Romania, ${ }^{2 "}$ "Gr.T. Popa" University of Medicine and Pharmacy, Iasi, Romania, "Carol Davila" University of Medicine and Pharmacy, Bucharest, Romania, ""Fr.I.Rainer"

Anthro, Bucharest, Romania

Introduction: Gonarthrosis or osteoarthritis of the knee is a frequent, chronic and invalidating condition in elderly. Study aimed at identifying mood disorders associated with chronic pain in these patients. Material and methods: A total of 187 subjects, age-range 50-95 years, were included. They were divided into two groups: 92 adults (50-64 years), 95 elderly (75-96 years).

Results: Gonarthrosis was significantly more prevalent in urban area irrespective of age $(\mathrm{p}<0.05)$. In rural area it was more prevalent in men $(\mathrm{p}<0.05)$, but in urban area was more prevalent in women $(p<0.01)$. One in four patients presented diabetes mellitus, higher prevalence in men over 75 years $(p<0.05)$. Almost all patients presented chronic pain, with a higher intensity and longer duration in older women. Prevalence of mood disorders was highest in older women $(\mathrm{p}<0.001)$. Age at menopause younger than 45 years was more prevalent in elderly women with depression $($ chi square $=$ $9.762, \mathrm{p}<0.05)$. In general sample depression was significantly more prevalent than anxiety $(\mathrm{p}<0.001)$. Majority of older women had combined mood disorders. Older patients in urban area without a family support network had significantly higher prevalence of depression $(p<0.01)$, irrespective of gender. Older women had significantly higher prevalence of somatization as compared to other gender and age-group $(\mathrm{p}<0.01)$. Interestingly, over $75 \%$ of all patients reported favorable effects of medication for chronic pain. Conclusions: Recognizing and treating chronic pain due to gonarthrosis has complex effects including on mood in elderly. Nevertheless, pain is not the only factor involved in mood disorders in elderly.

\section{P-358}

Patterns and complications of arterial hypotension in older people

G. I. Prada ${ }^{1}$, R. M. Nacu ${ }^{2}$, A. C. $\mathrm{Ilie}^{3}$, A. I. Pislaru ${ }^{3}$, A. G. Prada ${ }^{2}$ I. D. Alexa ${ }^{3}$, A. Kozma ${ }^{4}$, R. Stefaniu ${ }^{3}$, I. A. Sandu ${ }^{3}$, C. Nuta ${ }^{1}$, O. L. Bajenaru ${ }^{1}$, A. M. Herghelegiu ${ }^{1}$

1"Ana Aslan" National Institute of Gerontology and Geriatrics, Bucharest, Romania, " "Carol Davila" University of Medicine and Pharmacy, Bucharest, Romania, ${ }^{3 "}$ Gr.T. Popa" University of Medicine and Pharmacy, Iasi, Romania, "'Fr.I.Rainer" Anthro, Bucharest, Romania 
Introduction: Older people often present arterial hypotension and it has significant consequences: falls, syncope, myocardial infarction, stroke and even death. Study objective was to identify patterns and risk factors of this condition in elderly.

Material and methods: A total of 387 subjects, age-range 50-95 years, were included. They were divided into two groups, both presenting arterial hypotension: 197 adults (50-64 years) and 190 elderly (75-93 years).

Results: We identified three types of arterial hypotension in older people: orthostatic hypotension, post-prandial, post-exercise hypotension. In our sample, most cases had orthostatic hypotension, other variants had less than $4 \%$ prevalence. Parkinson disease was more often seen in older patients $(\mathrm{p}<0.05)$. Type 2 diabetes mellitus and chronic renal disease were also more prevalent in elderly $(\mathrm{p}<0.05)$. Smoking was more prevalent in adult males. Three groups of medicines were involved: psychoactive, anti-parkinsonian, vasodilator drugs. Age of menopause younger than 45 years was more prevalent in elderly women with arterial hypotension (chi square $=$ 9.762, $\mathrm{p}<0.05$ ). Anemia was significantly more prevalent in elderly $(\mathrm{p}<0.001)$. Stroke and myocardial infarction was significantly more prevalent in elderly patients $(\mathrm{p}<0.01)$. Vertigo and syncope occurred as complications of arterial hypotension more often in elderly ( $p<0.01$ ), as well as falls, fractures and ischemic heart disease. Orthostatic hypotension was identified in elderly more than twice in adults. Headache and asthenia occurred more often in elderly $(\mathrm{p}<0.05)$.

Conclusions: Recognizing arterial hypotension, its forms and addressing some of its most important risk factors, could improve standing and prevent falls and other complications in older people.

\section{P-359}

Frailty index in elderly patients with severe aortic stenosis referred for transcatheter aortic valve implantation (TAVI)

Cristina Roqueta ${ }^{1}$, Marta Herrero ${ }^{1}$, María Isabel MartínezFernández ${ }^{1}$, Sandra Urbano ${ }^{1}$, Silvia Burcet ${ }^{1}$

${ }^{1}$ Geriatric Medicine Department, Parc de Salut Mar, Centro Fórum, Hospital del Mar, Barcelona, Spain

Introduction: Aim of the present study was to assess frailty of patients with severe aortic stenosis referred for transcatheter aortic valve implantation (TAVI).

Patients and methods: Prospective, descriptive and unicentric study of elderly patients with symptomatic severe aortic stenosis were evaluated by a geriatrician from April 2016 to March 2018. Sociodemographic variables (age, sex) were registered. Frailty was performed by Gait Speed Test $(<0.8 \mathrm{~m} / \mathrm{s})$; Short Physical Performance Battery (SPPB) $(0-12$ points) $(<10$ points), Clinical Frailty Scale (CFS) (very fit, well, managing well, vulnerable, mildly frail, moderately frail, severely frail, very severely frail, terminally ill) and Essential Frailty Toolset (EFT) (0-5 points) ( $\geq 1$ points).

Results: Eighty-eight patients were evaluated (60.23\% women); mean aged $82.29 \pm 5.98$ years. Mean value of gait speed test: $0.65 \pm 0.23 \mathrm{~m} / \mathrm{s}$; SPPB: $7.20 \pm 3.03$ points and EFT: $1.52 \pm 1.10$ points. Prevalence of frailty assessed by: Gait Speed Test $(<0.8 \mathrm{~m} / \mathrm{s})$ : 59 (67.05\%; Short Physical Performance Battery (SPPB) $(<10$ points): $62(70.79 \%)$ [(0-3 points: $10(11.36 \%$; 4-6 points: 25 (28.41\%); 7-9 points: $27(30.68 \%)$; 10-12 points: $26(29.55 \%)]$; Clinical Frailty Scale (CFS): $0(0 \%)$ very Fit, $0(0 \%)$ well, 18 $(20.45 \%)$ managing well, $20(22.73 \%)$ vulnerable, $30(34.09 \%)$ mildly frail, $18(20.45 \%)$ moderately frail, $2(2.27 \%)$ severely frail, 0 $(0 \%)$ very severely frail, $0(0 \%)$ terminally ill); Essential Frailty Toolset (EFT) ( $\geq 1$ points): $71(80.68 \%)$.
Conclusions: Prevalence of frailty in elderly patients with severe aortic stenosis referred for TAVI was high. EFT has showed the highest percentage of frail patients.

\section{P-360}

Biological and therapeutic factors determining bone mineral density in multiple myeloma patients over 65 years old

Mouanaa Hamza ${ }^{1}$, Zrour Saoussen ${ }^{1}$, Hammami Sonia ${ }^{2}$, Hachfi Haifa $^{3}$, Jguirim Mahbouba ${ }^{1}$, Bejia Ismaiel ${ }^{1}$, Touzi Mongi ${ }^{1}$, Youness Mohamed ${ }^{3}$, Bergaoui Nacer ${ }^{1}$

${ }^{1}$ Rheumatology Department of Monastir University Hospital, Monastir, Tunisia, ${ }^{2}$ Geriatric Unit of Monastir University Hospital, Monastir, Tunisia, ${ }^{3}$ Rheumatology Department of Mahdia University Hospital, Mahdia, Tunisia

Background: Multiple myeloma (MM) is usually characterized by an aggressive skeletal disorder. Our Objectives are to determine bone mineral density profile of MM patients over 65 years old and associated factors to bone density decrease.

Methods: This is a cross-sectional study of patients over 65 years old with MM (diagnostic criteria of the International Myeloma Working Group 2014), followed between 2011 and 2016. Bone densitometry (BMD) was measured by Lunar Prodigy DEXA at spine and femoral neck.

Results: Ten patients ( 7 men and 3 women) were collected. The average age was $77 \pm 4.5$ years (72-86). According to Durie and Salmon classification, patients were in stage III in $70 \%$ and stage II in $10 \%$ of cases. The T-score less than $-2.5 \mathrm{SD}$, was observed in $60 \%$ of cases. The average cumulative dose of corticosteroid received per patient was significantly associated with spine T-score $(\mathrm{p}<0.011)$ and femoral neck $\mathrm{T}$-score $(\mathrm{p}<0.012)$. Low bone density (T-score less than $-2.5 \mathrm{SD}$ ), at the spine, was significantly associated with age over 65 years old $(\mathrm{p}<0.024)$, increase of $24 \mathrm{~h}$ proteinuria $(\mathrm{p}<0.001)$ and at femoral neck, with Durie and Salmon classification advanced stage $(\mathrm{p}<0.005)$, high bone marrow cell infiltration ( $\mathrm{p}<0.004)$, IgA chain type $(\mathrm{p}<0.04)$ and double monoclonal peak in protein electrophoresis $(\mathrm{p}<0.05)$.

Conclusions: In multiple myeloma, low bone density was observed in $60 \%$ of cases. Bone density decrease was associated age, to stage III of Durie and Salmon classification, heavy chain type Ig A and high dose corticosteroids.

\section{P-361}

Steinbrocker score and the Health Assessment Questionnaire for rheumatoid arthritis over 65 years old

Zrour Saoussen ${ }^{1}$, Mouanaa Hamza ${ }^{1}$, Hammami Sonia ${ }^{2}$, Bergaoui Nacer ${ }^{1}$

${ }^{1}$ Rheumatology Department of Monastir University Hospital, Monastir, Tunisia, ${ }^{2}$ Geriatric Unit of Monastir University Hospital, Monastir, Tunisia

Despite recent therapeutic advances, rheumatoid arthritis (RA) continues to induce significant disabilities and mortality. Our objectives are to evaluate the functional status and its determinants in RA patients over 65 years old.

Patients et methodes: This is a monocentric, cross-sectional study of 21 consecutive RA over 65 years old, according to the ACR 1987 conducted at the Rheumatology Department, 2016-2017. The 
functional evaluation was based on Steinbrocker functional score and Tunisian Health Assessment Questionnaire (HAQ).

Results: The mean age of patients was 72 years \pm 4 (65-78). Females accounted for $83 \%$. In more than half of the patients, RA was moderately active. RA remission was noted in $10 \%$ of cases. The mean HAQ was $1.22 \pm 0.73(0-2.88)$ compared to $1.6 \pm 0.7$ for all ages. Thirty per cent of the patients had low handicap, $41 \%$ moderate handicap and $29 \%$ severe handicap. There was no disability in $11 \%$ of cases. The most important scores (most difficult tasks) were found for items 3 (eating) and 5 (holding). Mean Steinbrocker score was $2.14 \pm 0.75$. Low handicap was noted in $20 \%$ of cases, moderate handicap in $47 \%$ and severe handicap in $34 \%$ of cases. HAQ was statistiquely associated to DAS $28(\mathrm{p}<0.041)$, advanced age $(p<0.025)$. Logistic regression noted that HAQ determinants were RA disease activity and duration over 10 years.

Conclusion: Nowadays, RA still results in moderate functional handicap in almost half of the cases. This functional disability does not depend on the age of the patient.

\section{P-362}

Effects of coronary artery disease on quality of life in older adults

G. I. Prada ${ }^{1}$, O. L. Bajenaru ${ }^{2}$, R. M. Nacu ${ }^{1}$, I. D. Alexa ${ }^{3}$, I. A. Sandu ${ }^{3}$, R. Stefanoiu ${ }^{3}$, A. Kozma ${ }^{4}$, A. G. Prada ${ }^{1}$, A. I. Pislaru ${ }^{3}$, A. C. Ilie $^{3}$, C. $\mathrm{Nuta}^{2}$, A. M. Herghelegiu ${ }^{1}$

1"Carol Davila" University of Medicine and Pharmacy, Bucharest, Romania, "Ana Aslan" National Institute of Gerontology and Geriatrics, Bucharest, Romania, ${ }^{3}$ "Gr.T. Popa" University of Medicine and Pharmacy, Iasi, Romania, "'Fr.I.Rainer" Anthro, Bucharest, Romania

Introduction: Coronary artery disease (CAD) is often encountered in elderly and induces changes in life-style, inter-personal relationships, self-perception. Study objective was to evaluate impact of this condition on quality of life in elderly.

Material and methods: We analyzed 537 older patients, $26 \%$ men, $74 \%$ women, women mean-age 72.35 , men 74.24 years; $73 \%$ subjects from urban area; $67 \%$ with income above poverty level. Health-related quality of life (HRQOL) was evaluated with Seattle Angina Questionnaire (SAQ). Cardiovascular comorbidities were considered. HRQOL divided in 4 classes of severity: 4-mild/absent; 3-average; 2-severe; 1-very severe.

Results: In CAD older than 10 years most patients (46.67\%) were in class 3 for angina frequency; in disease less than 10 years old, $50 \%$ of patients were in class $4(\mathrm{p}<0.05)$. Physical limitation was more severe in urban patients and with disease less than 10 years old (26.7\% class 1) as compared to patients with disease older than 10 years $(13.34 \%$ class 1$)(\mathrm{p}<0.01$; CI $2.367-0.245)$. Angina stability was influenced by comorbidities $(p<0.01)$. Treatment satisfaction was not influenced by comorbidities $(\mathrm{p}<0.5)$. Angina perception was influenced by disease duration (higher for longer disease, $\mathrm{p}<0.05$ ), comorbidities and income level. Physical limitation, disease perception were more affected in women $(\mathrm{p}<0.01 \%)$; frequency of angina more affected in men $(p<0.05)$.

Conclusions: Frequency of angina symptoms, physical limitation and disease stability were influenced by length of disease and comorbidities. Treatment satisfaction was not influenced by comorbidities and gender. Disease perception was influenced by comorbidities, length of disease and income level. A gender difference was noticed.

\section{P-363}

The associations between gastroesophageal reflux disease and the central obesity of the elderly

Eirie $\mathrm{Cho}^{1}$, Jae Hak Kim ${ }^{1}$

${ }^{1}$ Dongguk University College of Medicine, Seoul, South Korea

Introduction: Central adipose tissue not only has metabolic effects but also increase intra-abdominal pressure, which is associated with the development of gastroesophageal reflux disease (GERD). Abdominal visceral adipose tissue volume is associated with an increased risk of GERD. The aim of this study was to investigate the associations between erosive esophagtis (ERD) and minimal change esophagitis (MCD) in terms of central obesity.

Methods: The subjects aged over 65 years who underwent abdomen computerized tomography and upper endoscopy for routine checkup at the same day were collected from Feb 2007 to October 2016. 24 subjects with ERD and 18 subjects with MCD were enrolled. The abdominal obesity was evaluated by measuring visceral adipose tissue (VAT), subcutaneous adipose tissue (SAT), ratio of VAT to SAT, total adipose tissue (TAT), body mass index (BMI).

Results: Male was significantly frequent in ERD than MCD (83.3 vs. $50.0 \%, \mathrm{p}=0.04)$. No significant differences were observed between groups in terms of $H$. pylori infection and presence of hiatal hernia. BMI $(24.4 \pm 2.7$ vs. $24.7 \pm 2.2, \mathrm{p}=0.78)$, VFA $(64.8 \pm 26.1$ vs. $69.7 \pm 26.1, \quad \mathrm{p}=0.55), \quad$ SAT $\quad(75.7 \pm 26.5 \quad$ vs. $\quad 90.6 \pm 36.0$, $\mathrm{p}=0.13)$, ratio of VAT to SAT $(0.90 \pm 0.42$ vs. $0.85 \pm 0.41$, $\mathrm{p}=0.71)$, and TAT $(140.5 \pm 40.1$ vs. $160.3 \pm 50.9, \mathrm{p}=0.17)$ were not significantly different between two groups.

Key conclusions: In elderly, male sex was more affected ERD. However, central obesity was not different between ERD and MCD.

\section{P-364}

Adrenal insufficiency among elderly: a retrospective study from tertiary level care hospital in Qatar

Sameer Acharath Valappil ${ }^{1}$, Hanadi Alhamad ${ }^{1}$, Navas Naddukkandiyil ${ }^{1}$, Abdul Rahiman Kammadath ${ }^{1}$

${ }^{1} \mathrm{HMC}$

Background: Adrenal insufficiency (AI), a medical emergency disorder, usually starts with symptoms and signs of tiredness, fever, gastrointestinal discomfort, and may progress to adrenal crisis with severe electrolyte imbalance. The study results are important to provide insights since it is being conducted among a seldom-studied population, i.e., elderly Gulf Arabs, and comparing broad variety of possible clinical correlates in statistical testing.

Methods: A retrospective review of electronic Cerner data used to identify reported cases on the prevalence of adrenal insufficiency in cohorts of at least 117 individuals aged 65 years and living in Qatar, together with criteria used to define AI. The purposes of the current study are to measure the point prevalence of adrenal insufficiency based on analysis hospitalized elderly people (age $>65$ years) in Qatar. The data was collected for a number of comorbidities.

Results: Of the 117 people in the sample, 51 (43.59\%) were diagnosed with adrenal insufficiency. The point estimate of the prevalence of AI was 0.436 as noted. The prevalence of AI among the Qatari population aged above 65 is between 34.470 and $52.71 \%$.

Conclusion: It was found that the women were 1.98 times more likely than men to be adrenally insufficient. Finally, cerebrovascular disease (CVA) is a significant predictor of adrenal insufficiency $(\mathrm{p}=0.051$ ), as someone with CVA is 2.19 times more likely than without CVA to 
suffer from adrenal insufficiency. The prevalence of AI among the Qatari population is between 34.470 and $52.71 \%$.

Keywords: Adrenal Insufficiency, geriatrics, prevalence, comorbidities

\section{P-365}

Fivefold recurrent Tako-Tsubo cardiomyopathy in a patient with left anterior descending artery myocardial bridging: a case report

Giulia Rivasi ${ }^{1}$, Valerio Vanni ${ }^{1}$, Chiara Lorenzi ${ }^{1}$, Martina Rafanelli ${ }^{1}$, Paolo Valoti ${ }^{1}$, Andrea Ungar ${ }^{1}$

${ }^{1}$ Geriatric Intensive Care Unit, Department of Geriatrics, University of Florence and Azienda Ospedaliero-Universitaria Careggi,

Florence, Italy

Introduction: Tako-Tsubo cardiomyopathy (TTC) is an acute reversible left ventricular dysfunction, which occurs in the absence of obstructive coronary artery disease. Its onset is frequently related to physical or emotional stress; the pathogenesis of this condition is unknown, but some authors hypothesized a catecholamine-induced myocardial stunning or a coronary spasm. Recurrence is uncommon, occurring in $3-10 \%$ of patients.

Methods: We describe a fivefold recurrent TTC in a geriatric patient with left anterior descending (LAD) artery myocardial bridge.

Results: A 70-year old patient was hospitalized in 2015 for chest pain presenting at rest, ST elevation and mild troponin I increase; the echocardiogram revealed a mild depression of left ventricular function and apical akinesis, suggesting TTC. Coronary angiography showed patent coronary arteries, confirming the diagnosis; a LAD myocardial bridge was detected, with no evidence of obstruction. During the following 3 years, the patient experienced four TTC recurrences, all with benign course and treated with medical therapy (beta-blocker and calcium-antagonist). For each episode, no physical or emotional stressor could be identified as a trigger.

Conclusions: This is the first case report of fivefold recurrent TTC, presenting with two additional unusual features: the absence of triggering stress and a LAD myocardial bridge. The lack of triggers could support the hypothesis of a non-catecholaminergic etiology and suggest a role of myocardial bridging as potential substrate in the pathogenesis of TTC. Coronary spasm related to bridging may be supposed, in agreement with some studies reporting a higher prevalence of this finding in patients with TTC.

\section{P-366}

\section{Prostate and lung corpora amylacea in the elderly}

Kyosuke Kanenawa ${ }^{1}$, Mitsuharu Ueda ${ }^{1}$, Aito Isoguchi ${ }^{1}$, Yukimoto Tsuda ${ }^{1}$, Toshiya Nomura ${ }^{1}$, Yasuteru Inoue ${ }^{1}$, Teruaki Masuda ${ }^{1}$, Yohei Misumi ${ }^{1}$, Taro Yamashita ${ }^{1}$, Yukio Ando ${ }^{1}$

${ }^{1}$ Department of Neurology, Graduate School of Medical Science, Kumamoto University, Kumamoto, Japan

Introduction: Corpora amylacea (CA) are intraluminal contents frequently found in the elderly. However, histological and biochemical features of CA are still not fully understood. The aim of this study was to elucidate histobiochemical features of CA in the elderly.

Methods: To determine the occurrence of CA, we performed Congo red staining in 50 prostate and 81 lung samples obtained from postmortem patients administered at Kumamoto University Hospital from 2007 to 2017. To identify the precursor protein of prostatic CA, we performed mass spectrometry for prostatic CA isolated from prostatic glands by means of laser microdissection. We also analyzed histobiochemical features of CA by means of immunohistochemical staining and immunoblotting with an anti-lactoferrin antibody.

Results: We found prostatic CA in $43(86 \%)$ of 50 , and lung CA in 2 (2\%) of 81 autopsied patients. Those CA contained amyloid deposits which were Congo red positive and showed apple-green birefringence under polarized light. Mass spectrometric analysis and immunoblotting revealed that full-length lactoferrin was the major component of CA. Immunohistochemical staining revealed that prostatic and lung CA were strongly stained with an anti-lactoferrin antibody.

Conclusions: Prostatic and lung corpora amylacea contain amyloid deposits. Lactoferrin may be the major component of prostatic and lung corpora amylacea.

\section{P-367}

\section{Antihypertensive choice in older diabetics: an overview} from geriatric perspective

Remzi Bahsi ${ }^{1}$, Deniz Mut Sürmeli ${ }^{1}$, Tugba Turgut ${ }^{1}$, Hande Selvi Oztorun ${ }^{1}$, Volkan Atmis ${ }^{2}$, Ahmet Emre Yalcin ${ }^{3}$, Sevgi Aras ${ }^{1}$, Murat Varli ${ }^{1}$

${ }^{1}$ Department of Geriatrics, Ankara University School of Medicine, Ibn-i Sina Hospital, Ankara, Turkey, ${ }^{2}$ Department of Geriatrics, Bursa Education and Research Hospital, Bursa, Turkey, ${ }^{3}$ Department of Geriatrics, Ankara Atatürk Education and Research Hospital, Ankara, Turkey

Introduction: Diabetes Mellitus and hypertension are two common diseases that often occur together. Cognitive status and physical performance may be affected by antihypertensive drugs. In this study, we aimed to investigate how antihypertensive agents affect cognitive and physical status in older diabetics.

Methods: The study was conducted retrospectively and 453 (311 female vs. 142 male) diabetic patients were included. Patients were divided into groups according to the antihypertensive drugs they were using, groups were compared with independent-T test in terms of arithmetic means of mini-mental state examination (MMSE), geriatric depression scale (GDS), grip strength and gait speed. Risk analysis was done by logistic regression analysis. $\mathrm{p}<0.05$ was considered statistically significant.

Results: The average age of participants were $73.12 \pm 6.18$. MMSE arithmetic means were found to be higher among patients using ACE inhibitors and beta-blockers than those who did not use these drugs $(25.49 \pm 4.11$ vs. $24.27 \pm 4.72, \quad$ p: $0.025 ; 25.30 \pm 4.35$ vs. $24.07 \pm 4.73$, p: 0.006 ; respectively). ACE inhibitors and betablockers were found to reduce cognitive impairment risk (OR: 4.12, p: 0.042 ; OR: 4.38, p: 0.036; respectively). Also, calcium channel blocker users were found to have a slower gait speed than those who did not use $(0.63 \pm 0.13$ vs. $0.66 \pm 0.14$, p: 0.034$)$.

Conclusions: We recommend that ACE inhibitors and beta-blockers should be preferred in diabetic patients with cardiovascular disease and/or dementia. We also suggest that ACE inhibitors should be preferred to angiotensin receptor blockers (ARBs), because in our study ARBs found no positive effect on MMSE. Additionally, it may be considered to avoid the use of calcium channel blockers to preserve functional status. 


\section{P-368}

Incidental diagnosis of polycystic kidney disease (PKD) in a patient in his ninth decade; a case report

\section{Atef Michael ${ }^{1}$ \\ ${ }^{1}$ Russells Hall Hospital, Dudley, Turkey}

An 83 years old male was admitted with a fall resulting in fracture right neck of femur and right distal radius. His past medical history included hypertension and a stroke with slight residual right-sided weakness. There was no significant family history. Heart, chest and abdominal examination was unremarkable. Chest X-ray showed cardiomegaly. On admission his $\mathrm{Na}$ and $\mathrm{K}$ were normal. Urea and creatinine were slightly elevated at $9.4 \mathrm{mmol} / \mathrm{L}$ and $144 \mu \mathrm{mol} / \mathrm{L}$ respectively with eGFR of 41 . With intravenous fluids the eGFR improved to 59. His albumin creatinine ratio was $2.96 \mathrm{mg} / \mathrm{mmol}(\mathrm{N}$ 0-2.5). His $\mathrm{Ca}, \mathrm{PO}_{4}$ and liver functions were normal. His haemoglobin was $111 \mathrm{~g} / \mathrm{L}$. He underwent right hip hemiarthroplasty. Post-operatively he had more right sided weakness. An urgent CT showed a small amount of intraventricular fresh blood. CT angiogram showed no evidence of any aneurysm or arteriovenous malformation. Subsequently, he developed urinary tract infection. Abdominal ultrasound showed multiple large cysts within both kidneys with virtually no renal tissue identified consistent with polycystic kidney disease. Right kidney length was $18 \mathrm{~cm}$ and the left $19 \mathrm{~cm}$. There was normal appearance of the liver. The main types of cystic kidney disease in adults are: Polycystic kidney disease (PKD) either autosomal dominant $(90 \%)$ or recessive $(10 \%)$, Medullary cystic kidney disease (MCKD) which results in shrinkage of the kidneys, Medullary Sponge Kidney (MSK); a congenital disorder and Acquired Cystic Kidney Disease which occurs in patients who have CKD or ESKD that requires a kidney transplant or dialysis. In our patient, the presence of bilateral kidney enlargement with multiple cysts, without symptoms, signs or evidence of other cystic kidney disease was highly suggestive of PKD, even in the absence of family history. Genetic testing was not required especially that it would not affect the management, however the family could have preferred to do the test. The case highlight the variable expressivity of the genes, and it seems that some PKD cases are not diagnosed or not discovered. However new diagnosis of PKD in patients older than 80 years is rare. Interestingly, 4 years later, at the age of 87 years, $\mathrm{Na}$ and $\mathrm{K}$ remained normal, Kidney function and Haemoglobin remained stable with $\mathrm{U}$ $12.6 \mathrm{mmol} / \mathrm{L}$, Cr 112 umol/L, eGFR 51 and $\mathrm{Hb} 110 \mathrm{gm} / \mathrm{L}$

\section{P-369}

The effect of ultrasound on thromboembolic model of brain stroke in rat

Masoumeh Mohamadi ${ }^{1}$, Farnaz Etesam ${ }^{2}$, Zahra Vahabi ${ }^{2}$, Alireza Namazi Shabestari ${ }^{2}$, Mohammad Bodaghabadi ${ }^{2}$, Alireza Shabanzadeh $^{3}$

${ }^{1}$ Department of Dermatology, Iran University of Medical Sciences, Tehran, Iran, ${ }^{2}$ Geriatric Department, Ziaeian Hospital, Tehran University of Medical Sciences (TUMS), Tehran, Iran, ${ }^{3}$ Neuroscience Center, University of Alberta, Edmonton, Canada

Background: Ultrasound (US) has been used in neuroprotection after cerebral ischemia; however, its use is controversial. Application of US in combination with fibrinolytic agents may improve fibrinolytic effects. In this study the effects of US, alone or in combination with tissue plasminogen activator (tPA), on brain ischemic injury were examined.
Methods: We studied whether US alone or in combination with tPA is neuroprotective in thromboembolic stroke. To induce focal cerebral ischemia, a clot was formed in a catheter. Once the clot had formed, the catheter was advanced $17 \mathrm{~mm}$ in the internal carotid artery until its tip was 1-2 mm away from the origin of the middle cerebral artery (MCA). The preformed clot in the catheter was then injected, and the catheter was removed. The wound was then closed and the infarction volume, edema and neurological deficits were measured after MCA occlusion.

Results: In control, US + low tPA, low tPA, US + high tPA and, high tPA groups, the infarct volume $(\%)$ was $34.56 \pm 4.16$, $17.09 \pm 6.72, \quad 21.25 \pm 7.8, \quad 13.5 \pm 10.72$ and $20.61 \pm 6.17$ (mean $\pm \mathrm{SD}$ ) at $48 \mathrm{~h}$ after MCA occlusion, respectively. The results indicate that US alone reduces the infarct volume by $30 \%$ compared to that of the control group $(\mathrm{P}<0.05)$. US improved neurological deficits and reduced brain edema significantly $(\mathrm{p}<0.05)$.

Key conclusions: This study indicates that US appears to have a protective effect, alone and in combination with tPA, in an embolic model of stroke.

\section{P-370}

Repeated transsphenoidal surgery or gamma knife radiosurgery in recurrent cushing disease after transsphenoidal surgery in elderly

Mohammad Bodaghabadi ${ }^{1}$, Masoumeh Mohamadi ${ }^{2}$, Mohamad Ali Bitaraf $^{3}$, Fatemeh Mirzadeh ${ }^{1}$, Alireza Namazi Shabestari ${ }^{4}$

${ }^{1}$ Geriatric Department, Ziaeian Hospital, Tehran University of Medical Sciences (TUMS), Tehran, Iran, ${ }^{2}$ Department of Dermatology, Iran University of Medical Sciences, Tehran, Iran,

${ }^{3}$ Neurosurgery Department, Emam Khomeini Hospital, Tehran University of Medical Sciences (TUMS), Tehran, Iran

Background: This study compared Gamma knife radiosurgery (GKRS) and repeated transsphenoidal adenomectomy (TSA) to find the best approach for recurrence of Cushing disease (CD) after unsuccessful first TSA in elderly.

Material and methods: Thirty-eight old age patients with relapse of CD after TSA were enrolled and randomly underwent a second surgery or GKRS as the next therapeutic approach. They were followed for a mean period of $3.05 \pm 0.8$ years by physical examination and hormone measurement as well as magnetic resonance imaging.

Results: Patients admitted in Neurosurgery department for operation and stay in hospital for mean 4 days, but in Gamma Knife Center, they admitted in morning and Discharged at evening without any generalized anesthesia. No significant difference was observed in sex ratio, mean age, adenoma type, follow-up duration, and initial hormone level between the two groups. No significant relationship was found between preoperative 24-h free urine cortisol and disease-free months or tumor volume among both groups. There is significant less complications in GKRS group with less length of hospital stay and no delirium report after gamma knife procedure. Our statistical analysis showed higher recurrence-free interval in the GKRS group compared with TSA group.

Conclusion: With longer recurrence-free interval, GKRS could be considered a good and safe treatment alternative to repeated TSA in recurrent $\mathrm{CD}$ especially in older community with less morbidity and complications. 


\section{P-371}

Searching for orthostatic hypotension in the elderly: getting up from a chair or from a bed is not the same

Cristiano Donadio ${ }^{1}$, Witold Jarzebowski ${ }^{2}$, Lynda Sawadogo ${ }^{3}$, Lyiale Alonge $^{3}$, Emmanuelle Magny ${ }^{3}$, Florian Maronnat ${ }^{3}$, Carmelo Lafuente $^{4}$, Joël Belmin ${ }^{4}$

${ }^{1}$ Hôpitaux Universitaires Pitié Salpêtrière, Charles Foix, Paris, France, ${ }^{2}$ Hôpitaux Universitaires PSL-CFX, Paris, France, ${ }^{3}$ Hôpitaux Universitaires PSL-CFX, Paris, France, ${ }^{4}$ Hôpitaux Universitaires Pitié Salpêtrière-Charles Foix, UPMC Faculté de Médecine, Paris, France

Introduction: Testing for orthostatic hypotension $(\mathrm{OH})$ is widely recommended in frail elderly subjects, but guidelines differ substantially about which is the best test for diagnosing $\mathrm{OH}$. We aimed to investigate the value of sitting-to-standing orthostatic testing for the diagnosis of $\mathrm{OH}$, as compared to supine-to-standing as reference testing.

Methods: One-centre cross-sectional study conducted in consecutive patients admitted to a French geriatric hospital ward. Blood pressure (BP) and heart rate were measured during supine-to-standing orthostatic testing (used as the reference test) and sitting-to-standing orthostatic testing done within a $1-\mathrm{h}$ period using an automatized BP monitor.

Results: 197 patients aged $83.5+/ 6.3$ years were included. They comprised 69 males (35\%) and 128 females (65\%). $\mathrm{OH}$ according to the reference diagnosis was found in 71 patients $(37 \%)$ and was significantly associated with male gender, a history of falls, a greater Charlson comorbidity score and with higher systolic and diastolic supine BP. Using solely sitting-tostanding testing, 44 patients $(23 \%)$ fitted the criteria for $\mathrm{OH}$, and 57 patients (29\%) would have been misdiagnosed for $\mathrm{OH}$. The concordance between supine-to-standing and sittingto-standing testing for the $\mathrm{OH}$ diagnosis was poor $($ kappa $=0.361)$.

Key conclusions: In the elderly, $\mathrm{OH}$ should be diagnosed by classic supine-to-standing testing, since sitting-to-standing testing leads to misdiagnosis of this condition.

\section{P-372}

Epidemiological, clinical and therapeutic feature of systemic sclerosis in the elderly

Fatma Saïd $^{1}$, Hakim Oueslati ${ }^{2}$, Monia Khanfir ${ }^{3}$, Mounir Lamloum ${ }^{4}$, Mohamed Habib Houman ${ }^{5}$

${ }^{1}$ University Hospital La Rabta-Tunis University of Medicine of Tunis-University Tunis-El Manar, Tunis, Tunisia, ${ }^{2}$ University Hospital La Rabta-Tunis University of Medicine of Tunis-University Tunis-El Manar, Tunis, Tunisia, ${ }^{3}$ University Hospital La Rabta-Tunis University of Medicine, Tunis, Tunisia

Background: Systemic sclerosis (SSc) is a rare connective tissue disease. Its occurrence at the extreme ages of life is exceptional. There is little available data describing the particularities of SSc of the elderly subject. The aim of our work was to determine the frequency, clinical, paraclinical and prognostic features of late onset SSc.

Methods: This is a retrospective, descriptive and comparative study of patients with SSc, followed in the internal medicine department of the la Rabta University Hospital Center over a period of 29 years. These patients were divided according to their age in 2 groups: the group 1 composed of the patients aged at least 65 years and the group 2 including the patients aged less than 65 years old. The epidemiological, clinical, para clinical, therapeutic and evolutionary features of the 2 groups were then compared.

Results: Group 1 consisted of 16 patients $(12.8 \%)$, with a mean age at diagnosis of 69.5 years and a $\mathrm{F} / \mathrm{H}$ sex ratio of 1.66 . This female predominance was significantly higher in younger than in older subjects $(p=0.001)$. The time to diagnosis was comparable between the two groups. Raynaud's syndrome was the main revealing sign of the disease in both groups $(62.5$ vs. $78.9 \%)$ with no significant difference. The comparison between the 2 groups of frequencies of cutaneous, rheumatologic, pulmonary (both PID and PAH), cardiac and renal manifestations and those of different antibodies specific of SSc showed no significant difference. The frequencies of gastrointestinal manifestations were comparable except esophagitis, which was significantly more frequent in the elderly $(37.5$ vs $11 \%$, $\mathrm{p}=0.003)$. The frequency of the use of different treatments and of different outcome modalities was also comparable between the 2 groups.

Conclusion: Our study showed that late-onset SCS is characterized by a lower female predominance. Moreover, no other epidemiological, clinical, paraclinical, therapeutic or evolutionary specificity had been found.

\section{P-373}

Effect of age on 24-h ECG Holter monitoring in patients with prior stroke and arterial hypertension

Małgorzata Sobieszczańska ${ }^{1}$, Ewa Trzmielewska ${ }^{2}$, Paweł Gać ${ }^{3}$, Małgorzata Poręba ${ }^{3}$, Grzegorz Mazur ${ }^{3}$, Rafał Poręba ${ }^{3}$

${ }^{1}$ Wroclaw Medical University, Wroclaw, Poland, ${ }^{2}$ Wroclaw Medial University, Wroclaw, Poland, ${ }^{3}$ Wroclaw Medical University, Wroclaw, Poland

Previous stroke and presence of arterial hypertension increase a risk of further cardiovascular episodes in elderly patients. Aim of the study was to determine the influence of patients' age on indices of 24-h ECG Holter monitoring, including time domain heart rate variability (HRV). Out of the study group $88.1 \%$ had ischemic stroke, and $11.9 \%$ hemorrhagic stroke. The group was divided to 2 subgroups: A—over 65 years $(n=40)$, and B-under 65 years $(n=19)$. Results: In subgroup A, as compared with subgroup B, there were significantly higher values of maximal heart rate (HRmax): $120.0 \pm 16.0$ vs. $107.0 \pm 15.5 ; \mathrm{p}<0.05$; in turn, SDANN marker of time domain HRV were significantly lower, i.e., A: $57.6 \pm 16.1$ vs. B: $74.2 \pm 22.9 ; \mathrm{p}<0.05$. In multivariable stepwise backward regression analysis, it was shown that in the study group, the more advanced age represents the independent risk factor for the higher HR and for the reduced HRV, expressed as lower SDANN values. Additionally, higher BMI and higher blood glucose concentration constituted the independent risk factors for higher HR. The use of beta blockers was the independent protective factor against higher HR mean and decreased HRV, and moreover, the normalization of blood pressure, the use of anti-platelet drugs and anticoagulant drugs also were protective factors against higher HR mean in 24-h ECG Holter monitoring.

Conclusion: In patients with arterial hypertension and prior stroke, the advanced age constitutes the independent risk factor for the negative cardiovascular indices on 24-h ECG Holter monitoring. 


\section{P-374}

Carbon disulfide exposure estimate and prevalence of chronic disease after carbon disulfide poisoning-related occupational diseases

Hweemin Chung ${ }^{1}$, Kanwoo Youn ${ }^{1}$, Yanggyun $\mathrm{Lee}^{2}$, Chooyon $\mathrm{Cho}^{2}$ ${ }^{1}$ Green Hospital, ${ }^{2} \mathrm{SCH}$ University Hospital, Seoul, South Korea

Introduction: In Korea, carbon disulfide (CS2) toxicity was an important social problem from the late 1980s to the early 1990s but there have been few large-scale studies examining the prevalence of diseases after CS2 exposure discontinuance. So we investigated past working exposure to CS2 characteristics from surviving ex-workers of a rayon manufacturing plant including cumulative CS2 exposure index. Furthermore, we studied prevalence of their chronic diseases recently after many years.

Methods: We interviewed 633 ex-workers identified as CS2 poisoning-related occupational diseases to determine demographic and occupational characteristics and reviewed their medical records. The work environment measurement data from 1992 was used as a reference. Based on the interviews and foreign measurement documents, weights were assigned to the reference concentrations followed by calculation of individual exposure index, the sum of the portion of each time period multiplied by the concentrations of CS2 during that period.

Results: The cumulative exposure index was $128.2 \mathrm{ppm}$ on average. Workers from the spinning, electrical equipment repair, and motor repair departments were exposed to high concentrations of $\geq 10$ $\mathrm{ppm}$. Workers from the maintenance of the ejector, manufacturing of CS2 post-process, refining, maintenance and manufacturing of viscose departments were exposed to low concentrations below $10 \mathrm{ppm}$. The prevalence for hypertension, coronary artery disease, cerebrovascular disease, diabetes, arrhythmia, psychoneurotic disorder, disorders of the nervous system and sensory organ were 69.2, 13.9, $24.8,24.5,1.3,65.7,72.4 \%$ respectively.

Key conclusions: We estimated the individual cumulative CS2 exposure based on interviews and foreign measurement documents, and work environment measurement data. Comparing the work environment measurement data from 1992, these values were similar to them. After identified as CS2 poisoning, there are subjects over 70 years of average age with disorders of the nervous system and sensory organs, hypertension, psychoneurotic disorder, cerebrovascular disease, diabetes, coronary artery disease, and arrhythmia. Because among ex-workers of the rayon manufacturing plant, only 633 survivors recognized as CS2 poisoning were studied, the others not identified as CS2 poisoning should also be investigated in the future.

\section{P-375}

Assessment of functional status and cognition in elderly patients before cardiac surgery

Pavlina Capova $^{1}$, Jan Matejka ${ }^{1}$, Vojtech Mezera ${ }^{2}$

${ }^{1}$ Department of Cardiology, Pardubice Hospital, Pardubice, Czech Republic, ${ }^{2}$ Geriatric Center, Pardubice Hospital, Pardubice, Czech Republic

Introduction: With the expanding aging population, there is an increasing number of patients with ischemic heart disease and/or valvular heart disease. The risk of cardiac surgery must be considered together with potential benefit for patient's outcome. We compared several geriatric scales against postoperative outcomes.
Methods: Forty-six patients from Department of Cardiology, Pardubice Hospital, were referred to cardiac surgery from June to September 2017, and assessed by means of Activities of daily living (ADL), Mini-mental state examination (MMSE), Edmonton frail scale (EFS) and Reported Edmonton frail scale (REFS). These scores were compared to Euroscore II, a standardized risk scale in cardiac surgery, and to the incidence of perioperative complications and the length of hospital stay. The complications were further divided into arrythmias, neurological, renal and other.

Results: In 6 patients, the surgery was not performed; these patients had lower ADL and MMSE and higher EFS and REFS scales $(p<0.05)$ that those operated. Of the 40 patients operated, all survived the 6 months follow-up. Patients with poor ADL and Euroscore II had increased risk of overall perioperative complications $(\mathrm{p}<0.05)$. Patients with poor EFS had increased risk of other complications $(p<0.05)$. REFS and Euroscore II had a trend to predict length of hospital stay $(0.05<\mathrm{p}<0.1)$. The effects of ADL and EFS were independent on age and Euroscore II.

Key conclusions: ADL revealed patients with increased risk of overall complications and EFS those with increased risk of other perioperative complications independently on age and Euroscore II. These scales might be beneficial in preoperative risk assessment.

\section{P-376}

Inclusion of older patients with cancer in clinical trials: the SAGE prospective multicenter cohort survey

Canouï-Poitrine Florence ${ }^{1,2}$, Lievre Astrid ${ }^{3}$, Dayde Florent ${ }^{1}$, Lopez-Trabada-Ataz Daniel ${ }^{4}$, Baumgaertner Isabelle ${ }^{1}$, Dubreuil Olivier ${ }^{5}$, Brunetti Francesco ${ }^{1}$, Coriat Romain ${ }^{6}$, Maley Karin ${ }^{7}$, Pernot Simon ${ }^{8}$, Tournigand Christophe ${ }^{1}$, Aparicio Thomas ${ }^{9}$, Paillaud Elena ${ }^{2,8}$, Bastuji-Garin Sylvie ${ }^{1,2}$

${ }^{1}$ APHP-Hôpital Henri-Mondor, Créteil, France, ${ }^{2}$ UPEC, CEpiA EA 7376, Créteil, France, ${ }^{3} \mathrm{CHU}$ Pontchaillou, Rennes, France, ${ }^{4}$ APHPHöpital Saint-Antoine, Paris, France, ${ }^{5}$ APHP-La Pitié-Salpétriére, Paris, France, ${ }^{6}$ APHP-Hôpital Cochin, Paris, France, ${ }^{7}$ Hôpital Les Diaconnesses, Paris, France, ${ }^{8}$ APHP-HEGP, Paris, France, ${ }^{9}$ APHPHôpital Saint-Louis, Paris, France

Purpose: We aimed to address the proportion of older patients with colorectal cancer (CRC) who were eligible and invited to participate and finally enrolled in a clinical trial. The secondary objective was to assess reasons and factors associated with non-eligibility, non-invitation and non-inclusion in a clinical trial.

Patients and methods: The SAGE multicenter prospective cohort was established in 7 centers between 2012 and 2016. All patients aged 65 years or more with CRC were studied. The endpoints were the patient's eligibility, invitation and enrollment in a clinical trial.

Results: We included 577 patients [mean age: 75.6 years (SD 7); 56\% men; $41 \%$ metastasis]; 37 trials were ongoing (phase I/II: $60 \%$; chemotherapy: $76 \%$; one dedicated to older patients). Overall, 71 $(12 \%)$ patients were enrolled in a trial [30 (42\%) aged 65-69 years; 20 (28\%) 70-75 years; 17 (24\%) 75-79 years and $4(6 \%) 80$ years or older; $\mathrm{p}<0.001]$. For patients with at least one available trial for their cancer stage and location $(\mathrm{N}=474), 127(27 \%)$ were eligible; $88(19 \%)$ were invited for inclusion and $71(15 \%)$ agreed to participate. By multivariate analysis, non-invitation was associated with increased age [adjusted odds ratio $(\mathrm{aOR}) 70-75$ vs $65-69$ years $=$ 0.23 (95\% confidence interval $(95 \% \mathrm{CI})(0.07-0.83)$; aOR $75-80$ vs $65-69$ years $=0.28(0.07-1.11) ;$ aOR $\geq 80$ vs $65-69$ years $=0.05$ $(0.01-0.34) ; \mathrm{p}=0.016]$. Main reasons for non-invitation in trials were related to general status and comorbidities. 
Conclusion: The inclusion of older patients in CRC trials, especially those aged 80 years or older, remains low. Three-quarters of CRC patients were not enrolled because of ineligibility. One-third of eligible patients are not invited to participate. Few trials are dedicated to older patients.

\section{P-377}

Influence on cardiac function of adriamicin in the treatment of malignant lymphoma

Kazuhiko Natori ${ }^{1}$, Daisuke Nagase ${ }^{1}$, Akiko Shibuya ${ }^{1}$, Yurika Mitsui ${ }^{1}$, Hirofumi Irita $^{2}$, Ai Hirota ${ }^{1}$, Haruka Fujino ${ }^{1}$

${ }^{1}$ Hematology and Oncology, Toho University Medical Center Oomori Hospital, Tokyo, Japan, ${ }^{2}$ Hematology and Oncology, Toho University Medical Center, Tokyo, Japan

Background: The standard therapies of NHL are R-CHOP therapy. Key drug of CHOP therapy is adriamicin. In adriamicin to heart is toxic. The adriamicin has the limit of the dose. Sometimes cardiac function may decrease with in the limit. Even if treatment is successful, cardiac function may limit activity of daily life.

Methods: In the case that malignant lymphoma was diagnosed in our hospital in elderly (more than 65 years old) by 2013 from 2014. 92 cases were eligible to our study. We intended for 92 cases that were diagnosed non-Hodgkin's lymphoma (NHL) 87 cases, Hodgkin lymphoma 5 cases. We investigated ejection fraction (EF) in NHL cases that were received chemotherapy included adriamicine.

Result: In 92 cases, 51 cases were received RCHOP therapy or RCHOP like regimen that adriamicine is included chemotherapy, 11 cases $\mathrm{CHOP}$ therapy or CHOP therapy like regimen Of 62 cases, we examined 46 cases that measured $\mathrm{EF}$ in before and after chemotherapy. Median age was 68 years ranged from 65 years to 86 years, gender ratio was male 19 cases, female 27 cases. Cumulative dose were $320 \mathrm{mg} / \mathrm{m}^{2} /$ body (original rejimen $400 \mathrm{mg} / \mathrm{m}^{2}$ ). This dose was within permitting dose. The case treated more than an allowable dose had nobody. It was 31 cases $(67.4 \%)$ that EF had decreased in before and after therapy. There was not the case that performance status (PS) decreased by EF decrease.

Conclusions: About $67 \%$ of cases that EF decreases are present. The EF observation after the treatment is necessary.

\section{P-378}

\section{Tuberculosis of the elderly: about 19 cases}

Sayhi Sameh ${ }^{1}$, Abid Rim ${ }^{1}$, Arfaoui Bilel ${ }^{2}$, Ben Abdalhfidh Nedia ${ }^{2}$, Batikh Riadh $^{2}$, Ajili Faida ${ }^{2}$, Louzir Bassem ${ }^{2}$

${ }^{1}$ Hospital Military of Tunis, Tunis, Tunisia, ${ }^{2}$ Hospital Military of Tunis, Tunis, Tunisia

Tuberculosis is a pathology characterized by the multiplicity of clinical forms. This is especially noted in the elderly with a higher frequency of extra-pulmonary forms and multifocal forms to the detriment of the classic pulmonary forms. This is a retrospective descriptive study of the records of patients aged 65 years or older who were diagnosed with tuberculosis. Nineteen patients were included in our study. There were 11 men and 8 women with a sex ratio $\mathrm{F} / \mathrm{H}$ at 1.37. The average age of our patients was 71.2 years (extremes: 65-82 years). The chest X-ray showed the presence of pulmonary nodules in 7 cases, pleurisy in 1 case and miliary tuberculosis in 1 case. Multifocal tuberculosis was noted in 5 of our patients. 11 patients had an extra-pulmonary localization of tuberculosis associated in 3 cases with pulmonary tuberculosis. The intradermal reaction was contributive to the diagnosis in 6 cases. The diagnosis of tuberculosis was retained on pathological arguments in 6 cases, after isolation of $\mathrm{BK}$ in 3 cases and it was a diagnosis of presumption in the rest of the cases. The average duration of antituberculous treatment was 7.1 months. Corticotherapy was associated in 7 . Hepatic cytolysis with anti-tuberculosis treatment was noted in 4 cases and hepatic cholestasis in 3 cases. The evolution under treatment was favorable in 13 cases. In a geriatric environment, in the presence of an unexplained infectious symptomatology, the diagnosis of tuberculosis must be evoked even in the absence of favoring factors.

\section{P-379}

\section{Ogilvie syndrome and strok in elderly}

Sayhi Sameh ${ }^{1}$, Boussetta Najeh ${ }^{1}$, Arfaoui Bilel ${ }^{1}$, Guediche Nour ${ }^{1}$, Ben Abdelhafidh Nour ${ }^{1}$, Ajili Faida ${ }^{1}$, Louzir Bassem ${ }^{1}$

${ }^{1}$ Hospital Military of Tunis, Tunis, Tunisia

Introduction: Ogilvie's syndrome is an important dilatation of the colon without mechanical obstacle. This colectasia is due to an attack of the vegetative nervous system.

Observation: 76 years man, hypertensive, with a history of complete arrhythmia by atrial fibrillation which has been admitted for heaviness of the left hemicorps brutal installation. The examination objectified a patient with left hemiplegia. The brain scan showed an ischemic stroke in the right Sylvian territory. At day 25, the patient had severe abdominal bloating associated with nausea and withdrawal. The examination finds a very distended abdomen sensitive in its entirety. The abdominal CT showed significant colonic distention without mechanical obstacle. In front of this table, an Ogilvie syndrome was strongly suspected and was confirmed by a colonoscopy which showed a colectasis without focal lesions and allowed exsufflation relieving the patient. Treatment with Prostigmine and antispasmodic has been associated with favorable evolution.

Discussion: The diagnosis of ogilvie syndrome should be evoked in case of any acute colic dilation in a particular clinical condition: metabolic disorders, heart failure, renal failure, neurological disease, retroperitoneal metastatic cancer, orthopedic, retroperitoneal or pelvic surgery. Its treatment is mainly based on neostigmine, possibly supplemented by endoscopic exsufflation and/or water-soluble enema. Colectomy are reserved for the rare failures of conservative treatments.

Conclusion: Oglivie syndrome is a serious complication of cerebrovascular accidents, it must be known and treated early because of the evolutionary risk of cecal perforation (mortality $40 \%$ ).

\section{Area: Longevity and prevention}

\section{P-380}

Study on atherogenic indices and overall cardiovascular risk in elderly patients

Opris Simona ${ }^{1}$, Draghici Rozeta ${ }^{1}$, Avadanei Alexandra ${ }^{1}$, Pena Catalina ${ }^{1}$

${ }^{1}$ National Institute of Gerontology and Geriatrics “Ana Aslan”, 
Bucharest, Romania

Introduction: Cardiovascular disease (CVD) is a multifactorial condition and it is essential that all risk factors and determinants of cardiovascular be treated both individually and socially. It is also a major cause of disability and loss of quality of life.

Methods: The study aims to identify atherogenic risk from atherogenic indices (Atherogenic coefficient, Castelli risk I, Castelli risk II, Atherogenic index) and quantifying the effectiveness of overall cardiovascular risk in elderly patients.

Results: From the data of 378 patients, aged over 55 years, were obtained as follows: $68.51 \%$ of patients experience cardiovascular disease, $55.02 \%$ locomotor diseases, $29.36 \%$ psychological disorders, $15.34 \%$ diabetes mellitus and $8.46 \%$ thyroid diseases. We found a substantial increase in overweight and obesity in the target population. The prevalence of overweight is 39.68 and $39.94 \%$ obesity. Also there is a high risk atherogenic index at $62.69 \%$ and high-risk HeartScore at $15.34 \%$ patients. In patients with a low or medium risk, this may mask a very high risk and thus require a quantification of all risk factors and their share to evaluate global cardiovascular risk.

Key conclusions: It is necessary the development of a clear and effective strategy that combine individual attitudes with populational one, with the aim of reducing CVD morbidity and mortality. Prevention applicability extends throughout life, as the most effective way to reduce the risk of CVD. The burden of established cardiovascular disease can be reduced by early diagnosis, appropriate disease management, rehabilitation and prevention, including counseling about lifestyle organization.

\section{P-381}

Role of impulse oscillometry in assessment of pulmonary functions among elderly

Mohamed Mohamed ${ }^{1}$, Hala Sweed ${ }^{2}$, Nermine Riad $^{3}$, Hind Taha ${ }^{4}$

${ }^{1}$ Assistant Lecterer of Geriatric and Gerontology Ain Shams University Hospitals (ASUHs), Cairo, Egypt, ${ }^{2}$ Professor and Section Head of Geriatric and Gerontology Department Ain Shams University Hospitals (ASUHs), Cairo, Egypt, ${ }^{3}$ Associate Professor of Chest Diseases Ain Shams, Cairo, Egypt

Introduction: The world's population is not only growing larger, it is also becoming older. Early diagnosis of Chronic Obstructive Pulmonary Disease (COPD) has a large impact on quality and longevity of life for elderly. Diagnosis is sometimes missed as patients learn to limit their physical activities to escape the gradually emerging dyspnea on exertion. Spirometry is a standard, objective lung function test for diagnosis of airflow limitation but it is highly dependent on the performance technique and patient cooperation in contrast to Impulse Oscillometry (IOS) that can especially be recommended to elderly patients who have poor pulmonary functions with physical and mental limitations facing difficulty in caring out spirometry properly. Methods: We include 100 Elderly patients 60 years and above with no acute or chronic lung airways problems performed both spirometry and IOS.

Results: The results appeared that IOSR5 is more sensitive than spirometry in an early detect patient with mild obstructive symptoms. There was a significant high agreement between obstruction diagnosis by IOSR5 and FEV1/FVC in spirometry. Linear logistic analysis done for factors affecting FEV1 and IOSR5 revealed that only age and BMI are significantly associated with both of FEV1 and IOSR5.

Conclusion: IOS was found to be reliable in early detection of airway disease in elderly because IOS needs a lower dependency on cooperation during tidal breathing especially in those having difficulty in caring out the spirometry properly especially patient with cognitive impairment. Age and BMI are significantly associated with both of FEV1 and IOSR5.

\section{P-382}

Functional fitness influencing bicycle riding in community dwelling older adults

Ryuichi Hasegawa $^{1}$, Fumihiko Goto ${ }^{2}$, Hidehiro Watanabe ${ }^{2}$, Misaki Sato ${ }^{2}$, Akihiro Yoshida ${ }^{2}$, Nanae Kato ${ }^{2}$, Haruhi Umeki ${ }^{2}$, Manami Mizukoshi ${ }^{2}$, Hisanori Ido $^{2}$, Naoki Okayama ${ }^{2}$

${ }^{1}$ Chubu University, Kasugai, Japan, ${ }^{2}$ Tokai Memorial Hospital, Kasugai, Japan

Introduction: Bicycling can be good transportation for people of all ages; it is a form of physical activity that effectively taxes the cardiorespiratory and metabolic functions of the whole body in a wide range of intensities, lending itself to many potential health benefits. However, seniors have few extra factors to consider beyond basic bicycle safety. As we age, the level of physical activity inevitably declines. This study aimed to examine the relationship between functional fitness and the ability to ride a bicycle in community dwelling older adults.

Methods: Forty-eight Japanese community dwelling older adults (13 males, 35 females; mean age $74.1 \pm 4.7$ years) were divided into a riding group $(n=34)$ that could ride a bicycle and a control group $(n=14)$ that could not ride a bicycle. A binomial logistic regression analysis was conducted with riding a bicycle as the dependent variable and Arm Curl Test, Chair Stand Test, Back Scratch Test, Sit and Reach Test, Functional Reach Test, One-Leg Stand Test, Four Square Step Test, 5-Meter Walk Test, and Timed Up and Go Test (TUG) as independent variables.

Results: The binomial logistic regression analysis showed that TUG (odds ratio: $0.29 ; 95 \%$ CI $0.12-0.68$ ) was a significant variable that affected riding a bicycle.

Key conclusion: Our results suggested that riding a bicycle is associated with TUG scores in community dwelling older adults. These results indicated that TUG scores are expected to be useful for determining the ability to ride a bicycle and for setting treatment goals.

\section{P-383}

Role of impulse oscillometry in assessment of pulmonary functions among elderly

Mohamed Mohamed ${ }^{1}$, Hala Sweed ${ }^{2}$, Hind Taha ${ }^{3}$, Nermine Riad ${ }^{4}$

${ }^{1}$ Assistant Lecterer of Geriatric and gerontology Ain Shams University Hospitals (ASUHs), Cairo, Egypt, ${ }^{2}$ Professor and Section Head of Geriatric and Gerontology Department Ain Shams University Hospitals (ASUHs), Cairo, Egypt, ${ }^{3}$ Associate Professor of Geriatric and Gerontology, Cairo, Egypt

Introduction: The world's population is not only growing larger, it is also becoming older. Early diagnosis of Chronic Obstructive Pulmonary Disease (COPD) has a large impact on quality of life for patients. Diagnosis is sometimes missed as patients learn to limit their physical activities to escape the gradually emerging dyspnea on exertion. Spirometry is a standard, objective lung function test for diagnosis of airflow limitation but it is highly dependent on the performance technique and patient cooperation in contrast to Impulse Oscillometry (IOS) that can especially be recommended to elderly 
patients who have poor pulmonary function with physical and mental limitations facing difficulty in caring out the spirometry properly.

Methods: We include 100 Elderly patients 60 years and above (both males and females) admitted to Ain Shams University Hospitals with no acute or chronic lung airways problems.

Results: The results appeared that IOSR5 is more sensitive than spirometry to an early detect patient with mild obstructive symptoms. There was a significant high agreement between obstruction diagnosis by IOSR5 and FEV1/FVC in spirometry with the significant negative correlation between them. Linear logistic analysis done for factors affecting FEV1 and IOSR5 revealed that only age and BMI are significantly associated with both of FEV1 and IOSR5.

Conclusion: IOS was found to be reliable in early detection of airway disease in elderly because IOS needs a lower dependency on cooperation during tidal breathing especially in those having difficulty in caring out the spirometry properly. Age and BMI are significantly associated with both of FEV1 and IOSR5.

\section{P-384}

Homocysteine levels and increase thiolactonase activities in elderly patients at high risk of cardiovascular disease

Sonia Hammami ${ }^{1}$, Nadia Hammami ${ }^{1}$, Amel Nakbi ${ }^{1}$, Kaldoun Ben Hamda ${ }^{2}$, Mohamed Hammami ${ }^{1}$

${ }^{1}$ Biochemistry Laboratory, LR12ES05 LR-NAFS 'NutritionFunctional Food \& Vascular Health' Faculty of Medicine-University of Monastir (Tunisia), Tunis, Tunisia, ${ }^{2}$ Department of Cardiology, CHU F Bourguiba, Monastir, Tunisia

Background: Elevated blood homocysteine is a risk factor for cardiovascular disease. A $5-\mathrm{mmol} / \mathrm{L}$ increase is associated with an approximately $70 \%$ increase in relative risk of cardiovascular disease in adults. For patients with established risk factors, this risk is likely even greater. Thiolactonase (HTLase); the antioxidant enzymatic component of HDL plays a crucial role in metabolizing homocysteine thiolactone and reducing homocysteine endothelial damages.

Objective: Was to evaluate plasma homocysteine levels (tHcy); thiolactonase activity (HTase) according to diabetes in coronary elderly patients.

Methods: A total of 53 elderly coronary artery disease patient were involved in this preliminary study. They were divided into diabetic patient $(n=27$; age $=68.3$ years $)$ and non-diabetics $(n=26$; age $=$ 68.7 years). Plasmatic total homocysteine (tHcy) were determined by a validated highly sensitive and accurate capillary gas chromatography mass spectrometry method HTLase activity was estimated by a commercially available kit assay.

Results: Significantly higher median tHcy levels were found in diabetic patients as compared to the non diabetic ones $[18.5 \mu \mathrm{mol} / \mathrm{L}$ (15.4-24.8) vs. $15.8(14.3-17.2) ; \mathrm{p}=0.057]$ associated to lower HTase activities [266.8 U/L (162-365.2) vs. 327.4 (240.3-406.8); $\mathrm{p}=0.058]$. A negative association between tHcy levels and thiolactonase activities was found in the two studied subgroups: $(\mathrm{r}=-0.637 ; \mathrm{p} \leq 0.00$ for the diabetic ones and $\mathrm{r}=-0.759$; $\mathrm{p}=0.00$ for the non diabetic coronary artery disease patients.

Conclusion: Elevated homocysteine in diabetic patients may partly be explained by the diminished thiolactonase activities and could be considered as an additional risk factor for cardiovascular events.

\section{P-385}

How do hundred-year-old people die? Results from a populationlevel study of centenarians in Sweden

Lucas Morin $^{1}$, Jonas Wastesson ${ }^{1}$, Stefan Fors ${ }^{1}$, Kristina Johnell ${ }^{1}$

${ }^{1}$ Karolinska Institutet, Solna, Sweden

Background: A growing proportion of older adults live beyond the age of 100 years. Little is known about how they die. We aimed to describe the patterns of dying in a large cohort of centenarians, and to investigate their healthcare utilization during the last month of life. Methods: Nationwide, register-based, longitudinal cohort study in Sweden. All people aged $\geq 100$ years who died in 2013-2015 were identified in the National Cause of Death Register. Data were then linked to the National Patient Register, the Social Services Register, the Swedish Prescribed Drugs Register, and several other registries with national coverage in Sweden. The likelihood to have experienced the different outcomes of interest was calculated by the mean of adjusted logistic regression models. Odds ratios (OR) are reported with $95 \%$ confidence intervals.

Results: A total of 2763 deceased centenarians were included. Mean age was 101.3 years (SD 1.6), $82 \%$ were women, and $96.7 \%$ had no living spouse. The main reported causes of death were dementia $(23.7 \%)$, ischaemic heart diseases $(15 \%)$, heart failure $(10 \%)$, cerebrovascular diseases $(7 \%)$, hypertensive diseases $(7 \%)$, influenza and pneumonia (6\%), and cancer (4\%). 217 (8\%) died from conditions indicative of a sudden death (e.g. sepsis). On average, decedents had 4.5 diagnosed chronic diseases (SD 2.7). 74\% died in nursing homes, $14 \%$ in hospitals, and $11 \%$ at home. During the last 3 months of life, $11 \%$ had a fall-related injury, $16 \%$ were hospitalized for more than 7 days, and 35\% experienced at least one unplanned hospitalization. Only 1 patient was admitted in an inpatient palliative care unit. While adjusting for possible confounders, we found that centenarians with organ failure were the most likely to be hospitalized in emergency (OR $=1.99,95 \%$ CI 1.65-2.40 compared with dementia patients). During the final month before death, the median number of prescribed drugs was 7 (IQR 4-10). Drug utilization was fuelled not only by opioids (44\%) and non-opioid analgesics (59\%), but also by the continuation of antihypertensives (46\%), antiplatelet agents (35\%), and calcium supplements (8\%).

Conclusion: Centenarians have a considerable burden of morbidity near the end of life, and live most often in nursing homes. Healthcare utilization should be optimized to avoid unplanned hospital admissions and reduce the prescription of unnecessary drugs. Better access to specialist palliative care is warranted for this particularly frail population.

\section{P-386}

Blood pressure and long term mortality in the elderly: results of the Fiesole Misurata follow-up study

G. Turrin ${ }^{1}$, M. Bulgaresi ${ }^{1}$, E. Lucenteforte ${ }^{2,3}$, G. Rivasi $^{1}$, N. Nesti ${ }^{1}$, A. Giordano ${ }^{1}$, L. Martella ${ }^{1}$, C. Lorenzi ${ }^{1}$, M. F. D Andria ${ }^{1}$, V. Tortú ${ }^{1}$, A. Vannacci ${ }^{1}$, A. Mugelli ${ }^{1}$, A. Ungar ${ }^{1}$

${ }^{1}$ Referral Centre for Hypertension in the Elderly, Department of Geriatrics and Geriatric Intensive Care Unit, Careggi Hospital and University of Florence, Italy, ${ }^{2}$ Department of Neuroscience, Psychology, Pharmacology \& Children Health (NEUROFARBA), University of Florence, Florence, Italy, ${ }^{3}$ Department of Clinical and Experimental Medicine, University of Pisa, Pisa, Italy

Background: Optimal blood pressure (BP) control is an important health care challenge, but target values are still controversial, 
especially in older patients with comorbidity and disability. The aim is to evaluate mortality according to BP values in a cohort of elderly enrolled in the Fiesole Misurata Study, after a 6-year follow-up.

Methods: Vital status at December 31st 2016 was obtained in 385 subjects participating to the Fiesole Misurata study; patients characteristics were analysed to detect predictors of mortality. At baseline all patients had undergone office BP measurement and a geriatric multidimensional assessment.

Results: After a 6-year follow-up, 97 participants had died (25.2\%). Mortality was higher in underweight $(\mathrm{p}=0.04)$ and obese patients $(p=0.04)$, and in patients treated with ACE-inhibitors $(p=0.003)$ or diuretics $(\mathrm{p}<0.0001)$. Patients with systolic blood pressure $(\mathrm{SBP})<120 \mathrm{mmHg}$ had the highest mortality rate; mortality was significantly lower in patients with SBP $140-159 \mathrm{mmHg}$ as compared with SBP $120-139 \mathrm{mmHg}$ (HR: 0.54), particularly in those aged 75 or older (HR: 0.49), with disability (HR: 0.36) or taking antihypertensive drugs (HR: 0.49).

Conclusions: After a 6-year follow-up, patients with SBP $140-159 \mathrm{mmHg}$ had lower mortality as compared with SBP $120-139 \mathrm{mmHg}$, particularly those aged 75 or more, with disability or taking anti-hypertensive treatment. Conversely, SBP $<120 \mathrm{mmHg}$ was associated with a higher mortality rate. Overweight showed a protective value in elderly patients.

\section{P-387}

Promoting positive perceptions of aging among young adults by means of age simulation suits. An explorative pilot study

Sylvana Fink ${ }^{1}$, Danny Rüffert ${ }^{1}$, Patrick Brzoska ${ }^{1}$

${ }^{1}$ Chemnitz University of Technology, Chemnitz, Germany

Background: Individuals' perceptions of aging may influence their health in later life, with positive perceptions being associated with better health and more advantageous health behavior. Promoting positive perceptions in young adults may therefore be a valuable intervention into improving their future health. This experimental pilot study aims to explore how an age simulation suit can contribute to promoting positive perceptions of aging in this population group. Method: A total of 50 individuals was randomized into an intervention $(n=23)$ and a control group $(n=27)$. Individuals in the intervention group were asked to perform a standardized set of everyday activities while wearing the suit. Before (T0) and 4 weeks after the intervention (T1), an online questionnaire comprising several standardized instruments assessing, amongst others, four dimensions of perceptions of aging was administered. Additionally, qualitative interviews on the feasibility of the experimental set-up were conducted with 4 participants of each group.

Results: Study participants had a mean age of 23 years. $38 \%$ were females. Individuals in the intervention group tended to have more positive perceptions of aging after the intervention (depending on the dimensions studied, Cohen's d ranged between 0.1 and 0.6). Differences, however, were not significant. Interviews supported the feasibility of the experimental set-up.

Discussion: This pilot study suggests that age simulation suits may be a useful measure to modify individuals' perceptions of aging. The study may serve as a blueprint for a comprehensive randomized controlled trial thoroughly evaluating the benefits of age simulation suits on promoting positive perceptions of aging.

\section{P-388}

Effects of a falls prevention programme on health-related quality of life in older home care recipients: a randomised controlled trial

Maria Bjerk $^{1}$, Therese Brovold ${ }^{1}$, Dawn A Skelton ${ }^{2}$,

Teresa Liu-Ambrose ${ }^{3}$, Astrid Bergland ${ }^{1}$

${ }^{1}$ Faculty of Health Sciences, Oslo Metropolitan University, Norway, ${ }^{2}$ School of Health and Life Sciences, Glasgow Caledonian University, UK, ${ }^{3}$ Djavad Mowafaghian Centre for Brain Health, Centre for Hip Health and Mobility, Vancouver Coastal Health Research Institute, University of British Columbia, Canada

Background: Falls have serious consequences for quality of life (QOL) and contribute substantially to the global burden of disease [1, 2]. Home care is an important arena to address falls prevention and QOL, but this vulnerable group is underrepresented in health research [3]. This study explores the effects of a falls prevention programme on health-related quality of life (HRQOL), physical function and falls efficacy in older fallers receiving home care.

Methods: The design is a parallel-group randomised controlled trial, where the intervention group performed a falls prevention programme based on the Otago Exercise Programme (OEP). The control group received usual care. 155 participants were recruited from primary health care in six Norwegian municipalities. The primary outcome, HRQOL, was measured with the Short Form 36 Health Survey (SF36). Secondary outcomes were Bergs Balance Scale (BBS), Sit to Stand (STS), 4-m Walk Test, Instrumental Activities of Daily Living, Falls Efficacy Scale International and Mini Nutritional Assessment. Results: 136 participants completed the trial. Intention-to-treat analysis showed that, compared to the control group, the intervention group improved on SF-36's Physical Component Summary (3.8; CI $1.5,6.2)$ as well as BBS scores $(2.4$; CI 0.1, 4.7). However, the intervention group also demonstrated a decline in the Mental Health subscale $(-6.8$; CI $-11.7,-1.9)$ of SF-36. Per protocol analyses showed significant improvements in all physical subscales of SF-36, STS and BBS scores in the intervention group.

Conclusion: A falls prevention programme based on OEP significantly improved physical HRQOL and balance in older adults receiving home care.

Keywords: Primary care, Prevention in vulnerable population References:

1. Vieira ER, Palmer RC, Chaves PH (2016) Prevention of falls in older people living in the community. BMJ, 353(1):1419

[2] Hay SI, Abajobir AA, Abate KH, Abbafati C, Abbas KM, AbdAllah F, Aboyans V (2017) Global, regional, and national disabilityadjusted life-years (DALYs) for 333 diseases and injuries and healthy life expectancy (HALE) for 195 countries and territories, 1990-2016: a systematic analysis for the Global Burden of Disease Study 2016. The Lancet, 390(10100):1260-1344

[3] Genet N, Boerma WG, Kringos DS, Bouman A, Francke AL, Fagerström C, Devillé W (2011) Home care in Europe: a systematic literature review. BMC Health Serv Res 11(1):207

\section{P-389}

Survival in community dwelling people with young onset dementia: a six-year cohort study: the NeedYD-study

Adrie A.J. Gerritsen ${ }^{1}$, Christian Bakker ${ }^{2}$, Frans R.J. Verhey ${ }^{3}$, Yolande A.L. Pijnenburg ${ }^{4}$, K. Millenaar Joany ${ }^{3}$, Marjolein E. de Vugt ${ }^{5}$, Raymond T.C.M. Koopmans ${ }^{6}$

${ }^{1}$ De Wever, Centre for Elderly Care, Tilburg, The Netherlands. Department Of Primary And Community Care, Nijmegen, 
Radboudumc, The Netherlands, ${ }^{2}$ Department of Primary and Community Care, Nijmegen, Radboudumc, The Netherlands. Florence, Mariahoeve, Centre for Specialized Care In Young-onset Dementia, Den Haag, The Netherlands. Radboudumc Alzheimer Centre, Nijmegen, The Netherlands, ${ }^{3}$ School For Mental Health And Neuroscience, Alzheimer Centre Limburg, Maastricht-umc, Maastricht, The Netherlands, ${ }^{4}$ Department Of Neurology And Alzheimer Centre, VU-umc, Amsterdam, The Netherlands, ${ }^{5}$ School For Mental Health And Neuroscience, Alzheimer Centre Limburg, Maastricht-umc, Maastricht, The Netherlands, ${ }^{6}$ Department of Primary and Community Care, Nijmegen, Radboudumc, The Netherlands. Radboudumc Alzheimer Centre, Nijmegen, The Netherlands. Joachim En Anna, Centre For Specialized Geriatric Care, Nijmegen, The Netherlands

Introduction: More insight into survival and associated characteristics is necessary to better understand the prognosis of young onset neurodegenerative diseases, and for planning of specific services. This study investigated the survival rates of people with Young-Onset Dementia (YOD) and the relationship with age, gender, dementia subtype and comorbidity.

Methods: Survival was examined in 198 community-dwelling participants in the Needs in Young-onset Dementia (NeedYD) study. People with Alzheimer's dementia (AD), vascular dementia (VaD) and frontotemporal dementia (FTD) were included. The primary outcomes were survival after date of symptom onset and date of diagnosis. The relationship between survival and age at symptom onset or diagnosis, gender, dementia subtype and comorbidity were explored using Cox Proportional Hazard models.

Results: The mean survival time after symptom onset was 208 months and 120 months after diagnosis. Survival was associated with dementia subtype, with AD participants having a statistically significant lower survival rate compared to $\mathrm{VaD}$ participants. Furthermore, a younger age at diagnosis was associated with higher survival rates. Gender and comorbidity showed no association with both survival outcomes.

Key conclusion: It is relevant to take into account the subtype of YOD when discussing the future perspectives of people diagnosed with YOD and their families. The uncertainty about prognosis and life expectancy, may be reduced by using our study outcomes when informing YOD persons and families about prognosis. Furthermore, intensive care support, including advanced care, is needed for a prolonged time.

\section{P-390}

Turkish validation of a new scale from older people perspectives: older people's quality of life (OPQOL-brief)

Hatice Caliskan ${ }^{1}$, Gozde Sengul Aycicek ${ }^{1}$, Cemile Ozsurekci ${ }^{1}$, Rana Tuna Dogrul ${ }^{2}$, Cafer Balci ${ }^{2}$, Fatih Sumer ${ }^{2}$, Münevver Ozcan ${ }^{2}$, Erdem Karabulut $^{3}$, Meltem Halil ${ }^{2}$, Mustafa Cankurtaran ${ }^{2}$, Burcu Balam Yavuz ${ }^{2}$

${ }^{1}$ Section of Geriatrics and Gerontology, Department of Internal Medicine, Hacettepe University Medical Faculty, Ankara, Turkey, ${ }^{2}$ Division of Geriatrics and Gerontology, Department of Internal Medicine, Hacettepe University Medical Faculty, Ankara, Turkey, ${ }^{3}$ Hacettepe University, Ankara, Turkey

Introduction: There are various 'quality of life' scales developed for older people. Although quality of life is a subjective concept, most of these scales are based on expert opinions rather than perspectives of older people. The aim of this study is to evaluate validity and reliability of Older People's Quality of Life-Brief Scale (OPQOL-Brief), which is based on perspectives of older people, in Turkish population.
Methods: Total number of 168 patients who speak in Turkish fluently were recruited. Comprehensive geriatric assessment was performed to all participants. Exclusion criteria were malignancy, surgery or hospitalization anamnesis in the last month, acute infection or disease, and immobility. Language validation was performed by forwardbackward translation method. Another quality of life scale validated in Turkish population, CASP-19 (Control, Autonomy, Self-Realisation, Pleasure), was applied to all individuals together with OPQOLBrief scale. OPQOL-Brief scale was re-applied to 30 patients after 7-10 days for test-retest reliability.

Results: Mean age of study population was $73.3 \pm 5.9$ years. Female participants were $64.9 \%(n=109)$. Internal consistency was assessed by Cronbach $\alpha$ coefficient. OPQOL-Brief scale demonstrated high internal consistency (Cronbach $\alpha=0.876$ ). Test-retest reliability was assessed by interclass correlation coefficient (ICC) and showed high reliability ( $\mathrm{ICC}=0.98,95 \%$ CI $0.96-0.99, \mathrm{p}<0.001$ ). Strong and significant correlation was detected between OPQOL-Brief and CASP-19 scales $(\mathrm{r}=0.763, \mathrm{p}<0.001)$.

Conclusions: Turkish version of OPQOL-Brief is valid and reliable in Turkish older people. It is clinically more practical than other quality of life scales due to its short and simple structure. Furthermore, it is the first scale depending on older people's perspectives.

\section{P-391}

Development and feasibility of a portable, multifactorial testbattery intended for fall risk prediction in community-dwelling older adults in a non-clinical setting

Gustav Valentin Blichfeldt Sørensen ${ }^{1}$, Martin Grønbech Jørgensen ${ }^{1}$, Jesper Ryg ${ }^{2,3}$, Tahir Masud ${ }^{3}$, Stig Andersen ${ }^{1,5}$

${ }^{1}$ Department of Geriatric and Internal Medicine, Aalborg University Hospital, Denmark, ${ }^{2}$ Department of Geriatric Medicine, Odense University Hospital, Denmark, ${ }^{3}$ Institute of Clinical Research, University of Southern Denmark, Denmark, ${ }^{4}$ Nottingham University Hospitals NHS Trust, England, ${ }^{5}$ Department of Clinical Medicine, Aalborg University, Denmark

Introduction: Falls are common in older people with serious consequences. Accurate fall prediction is important in targeting prevention.

Objective: To develop and evaluate the feasibility of a portable, multifactorial test-battery intended for fall risk prediction in community-dwelling older adults within a set time-frame.

Methods and results: A consensus meeting with multi-disciplinary healthcare professionals was held to choose candidate predictors to be tested within a 30-min time-frame. Eight participants (+75 years) were recruited during preventive-home-visits and at senior activity centres. Tests were performed by carers at recruitment-sites. Predictors included measures of strength, reaction time and balance using a Nintendo Wii Balance Board (NWBB), arrhythmias using continuous heart-rate monitoring (ePatch), vision using tablet-based software (King Devick Technologies, inc.), cognition using Stroop test, Trailmaking-test and Orientation-Memory-Concentration-test (OMC), gait speed, sit-to-stand-test, and home-hazard evaluations. Timeconsumption was recorded. Afterwards, participants and carers were interviewed about their experiences with the tests to inform a second consensus meeting, where a modified nominal group technique was used. A pre-meeting survey was completed by attendees prioritising each predictor based on scientific value, time-consumption, participant- and carer evaluations. Priorities were ranked and assembled into a final prioritised list of predictors. Cumulative time-consumption was used to make a cut-off of predictors based on the overall time-frame. 
Conclusion: The modified nominal group consensus technique successfully selected a group of candidate predictors for a portable, multifactorial test-battery intended for fall risk prediction in a nonclinical setting. The final test-battery consisted of gait speed, NWBB measures (strength, reaction time, and balance), ePatch, and OMC.

Keywords: Prevention and communities and environments; Big data, e-health and technologies

\section{P-392}

SAPREMO: Safe ageing-patient centered precision medicine and interprofessional education to prevent risks from polypharmacy and guidelines' cocktails

\section{Ursula Wolf ${ }^{1}$}

${ }^{1}$ Pharmacotherapy Management, University Hospital Halle (Saale), Halle, Germany

Worldwide public health's challenge esp. concerning manifestation of cognitive dysfunction and falls and fractures in the elderly imperatively requires prevention instead of the overall most expensive attempt to iron out manifestations of undetected thus disregarded drug related problems. Demographic ageing and working as (1) general practitioner, (2) caregiver or (3) pharmacist means being involved in management of polypharmacy in geriatric patients. Within this vulnerable patient group drug safety requires more educated and interprofessional engagement of all health professionals. To obtain intense and precise cooperation, communication and networking with regard to polypharmacy SAPREMO (Safe Ageing-Prevention and Demography at Centre Stage Respecting Polypharmacy) was designed addressing all three health professions equally. Operating as a team is the fundamental idea of this interprofessional project involving the three groups treating the same patient at confluencing stages. The innovative aspect is to identify new symptoms that might result from drug effects or side effects and adapt as early as possible. Interprofessional round table educating workshops are implemented throughout the German federal state Saxony Anhalt. SAPREMO aims to leave the most critically universalized step up treatment with further drug therapy to cope with drug induced new symptoms as concerningly observed by the author's own synoptic internal medicine and clinical pharmacological reviews of more than 10,000 geriatric medication lists. Cooperation partners are the Medical Council, Pharmacists" Chamber, General Practitioners" Association and Association of Statutory Health Insurance Saxony Anhalt. The challenging project has started with great resonance striking a significant chord. Funded by the Robert Bosch Foundation.

\section{P-393}

Serum $\beta$-carotene levels, Alzheimer's disease and peripheral telomerase activity in old age subjects

Virginia Boccardi ${ }^{1}$, Beatrice Arosio ${ }^{2}$, Luigi Cari ${ }^{1}$, Martina Casati ${ }^{2}$, Evelyn Ferri ${ }^{2}$, Cristina Gussago ${ }^{2}$, Michela Scamosci ${ }^{1}$, Patrizia Bastiani ${ }^{1}$, Matteo Cesari ${ }^{2}$, Paolo Dionigi Rossi ${ }^{2}$, Patrizia Mecocci ${ }^{1}$

${ }^{1}$ University of Perugia, Perugia, Italy, ${ }^{2}$ University of Milan, Milan, Italy

Introduction: Advancing age represents the strongest risk factor for Alzheimer's disease (AD) and the identification of biomarkers able to define what characterizes physiological aging from $\mathrm{AD}$ may represent a potential starting point for novel preventive strategies. Among these biomarkers telomeres seem promising targets. Interestingly, high intake of carotenoid-rich food may play a role in protecting telomeres. Accordingly, low serum $\beta$-carotene concentrations have been found in $\mathrm{AD}$ subjects when compared with controls. We aim at investigating the hypothesis that the lower $\beta$-carotene in $\mathrm{AD}$ might be associated with markers of accelerated cellular aging, including telomerase activity and shortened telomere length.

Methods: The study was conducted in 93 old age subjects, $53 \mathrm{AD}$ and 40 sex-and age-matched healthy controls. Telomerase activity in PBMC has been evaluated by a PCR-ELISA protocol. The $\beta$-carotene levels were obtained by HPLC and Apolipoprotein E (ApoE) genotype by RFL-PCR.

Results: Subject affected by $\mathrm{AD}$ had significantly lower plasmatic levels of $\beta$-carotene $(448 \pm 66 \mathrm{mg} / \mathrm{ml})$ as compared with healthy controls $(497 \pm 59 \mathrm{mg} / \mathrm{ml}, \mathrm{p}<0.0001)$. In all population $\beta$-carotene significantly and positively correlated with telomerase activity controlling for gender $(r=0.280, p=0.029)$. The association between $\beta$ carotene and AD risk (OR: 1.012, 95\% CI 1.004-1.020, p = 0.004) was independent of age, gender, smoking habit and ApoE genotype. A final model having telomerase activity variability as the dependent variable while age, gender, smoking habit and $\beta$-carotene as independent variables, showed that $\beta$-carotene was independently associated with telomerase variability $(\beta=0.286, \mathrm{p}=0.035)$.

Conclusion: Our data show that in this cohort lower plasmatic $\beta$ carotene levels are associated with lower peripheral telomerase activity and $\mathrm{AD}$ risk.

\section{P-394}

Imperative focus on prevention: drug induced and drug aggravated dysphagia in the elderly patient

Sandra Gregor ${ }^{1}$, Grit Walter ${ }^{2}$, Christina Naumann ${ }^{2}$, Andreas Wienke ${ }^{3}$, Stefan Plontke ${ }^{4}$, Sylva Bartel ${ }^{4}$, Ursula Wolf ${ }^{1}$

${ }^{1}$ Pharmacotherapy Management, University Hospital Halle (Saale), Halle, Germany, ${ }^{2}$ Geriatric Department, Diakonie-Hospital Halle, Halle, Germany, ${ }^{3}$ Institute for Medical Epidemiology, Biometrics and Informatics, Faculty of Medicine, Martin-Luther-University HalleWittenberg, Halle, Germany

To analyse the causes of oropharyngeal dysphagia in the elderly patient with focus on polypharmacy is the aim of this investigation. Methods: In a cross-sectional study based on a random sample of 200 patients admitted to a geriatric hospital comprehensive data were included besides the detailed list of drugs and an intensely clinical investigation of swallowing according to Stanschus as a screening method for oropharyngeal dysphagia performed in all 200 patients.

Results: Within the 200 patients, mean age $84 \pm 6.5$ years, $65 \%$ female, prevalence of dysphagia is $29 \%(\mathrm{n}=58)$. Compared to women $(21 \%)$ men reveal a higher rate $(45 \%)$. Dysphagia is more often in nursing home residents (36\%) versus own households $(27 \%)$. With regard to concomitant diseases as diabetes mellitus, renal dysfunction, COPD and upper gastrointestinal illnesses there are no effects. Patients with dysphagia present 1.5 fold risk of pneumonia (95\% CI 0.65; 3.58). Central nervous system diseases (CNS-D) pose a 6.9 fold sign. Risk of dysphagia $(95 \%$ CI $3.7 ; 14.8)$. Another sign. Risk is associated with neuroleptics OR $=2.5$ (95\% CI 1.2; 5.04)adjusted for CNS-D (adj): OR = 1.9 (95\% CI 0.88; 4.31); antiParkinson drugs: $\mathrm{OR}=2.8(95 \%$ CI $1.19 ; 6.73)$-adj.: $\mathrm{OR}=1.3$ (95\% CI 0.52; 3.51); benzodiazepines: $\mathrm{OR}=4.4$ (95\% CI 1.01 ; 18.93)—adj.: OR 2 (95\% CI 0.42; 9.5). With antidepressants we find a 2.1 fold risk for dysphagia $(95 \%$ CI $0.96 ; 4.72)$, not affected by CNS-D-adj.

Preliminary conclusion: As well as in patients with central nervous system diseases and without we can identify specific drug groups 
associated with risk of aggravating and even inducing dysphagia respectively. Restricted indication for neuroleptics, benzodiazepines and antidepressants might be a preventative contribution and requires implementation in future dysphagia guidelines.

\section{P-395}

Increased risk of self-reported asthma in older, competing, crosscountry skiers

\author{
Anette Hylen Ranhoff ${ }^{1}$ \\ ${ }^{1}$ Department of Clincal Science, University of Bergen, \\ Diakonhjemmet Hospital, and National Institute of Public Health, \\ Oslo, Norway
}

Introduction: Regular physical activity is beneficial for health and longevity in all age groups. The effects of long-term, high levels of physical activity in old age are less known. The prevalence of asthma in competing cross-country skiers have been subject to several discussions. We wanted to study the prevalence of asthma in older, still competing, cross-country skiers.

Methods: The Birkebeiner Aging Study recorded self-seported asthma and smoking habits for 555 participants in the $54 \mathrm{~km}$ Birkebeiner skirace in 2009, aged 65-90 years. The findings were compared to an age- and sex-matched cohort from the Tromso-6 population study. Validated and identical questionnaires regarding asthma, leisure-time physical activity, and smoking habits were used in both surveys. Chi square and $\mathrm{T}$ tests were used when appropriate. Results: The skiers had participated in the Birkebeiner race for a median of 14 times, indicating long-term, engagement in crosscountry skiing. $205(38 \%)$ of the skiers reported the highest level of regular, leisure-time physical activity, wereas only $1(0.2 \%)$ of the controls. The prevalence of self-reported asthma was $51(9 \%)$ and $44(8 \%)$ among the skiers and controls respectively $(\mathrm{p}=0.5)$. A history of ever-smoking was reported for $199(36 \%)$ and $392(71 \%)$ respectively $(\mathrm{p}<0.001)$. Mean age of onset of astma was 45 and 46 yrs $(p=0.8)$ and there were no sex differences $(p=0.2)$. Key conclusion: long-term, competing, cross-country skiers have the same prevalence of self-reported asthma as the general population, despite a significantly lower exposure to sigarette smoke. Long-term, crosscountry skiing might be associated to an increased risk of asthma independent of smoking history.

\section{P-396}

Risk factors associated with single and recurrent falls among community-dwelling elderly in Canada

\author{
Edris Haghir ${ }^{1}$, John Moraros ${ }^{1}$, Cindy Feng ${ }^{1}$, Yelena Bird ${ }^{1}$ \\ ${ }^{1}$ School of Public Health, University of Saskatchewan, Saskatoon, \\ Canada
}

Introduction: Falls are a major public health concern among community-dwelling elderly in Canada.

Objective: To identify and compare the risk factors associated with single and recurrent falls in elderly Canadians.

Methods: This is a cross-sectional study that uses data from the Canadian Community Health Survey-Healthy Aging. It included 16,369 individuals aged 65 years and older. Data related to the incidence of single (one fall) and recurrent falls ( $\geq 2$ falls) in the previous 12 months, as well as associated factors from selected variables (demographics, general health and functional condition, chronic conditions, medication use, and fall history) were examined by univariate analysis and multinomial logistic regression modeling. Results: This study found that $19.8 \%$ of the participants had experienced falls in the past year with $7.2 \%$ of them being recurrent fallers. The multinomial logistic regression modeling showed that participants who were males (OR 1.4; 95\% CI 1.1-2.0) and perceived their health as being poor to fair (OR 1.4; 95\% CI 1.1-1.8) were more likely to experience recurrent falls when compared to single falls. Relative to single fallers, recurrent fallers were also more likely to have arthritis (OR 1.3; 95\% CI 1.0-1.7), urinary incontinence (OR $1.4 ; 95 \%$ CI $1.1-1.9)$, polypharmacy (OR $1.6 ; 95 \%$ CI $1.2-2.1$ ) and fear of falling (OR 1.6; 95\% CI 1.2-2.1).

Conclusion: Findings of this study suggest that fall prevention efforts should consider a variety of risk factors in order to ensure the most effective interventions for this rapidly increasing and vulnerable population.

\section{P-397}

Is CAIDE score an efficient tool in the prevention of Alzheimer's disease in general population?

Anca Iuliana Pislaru ${ }^{1}$, Irina Mihaela Abdulan ${ }^{1}$, Ioana Dana Alexa ${ }^{1}$, Gabriel Ioan Prada ${ }^{2}$, Anna Marie Herghelegiu ${ }^{2}$

${ }^{1}$ University of Medicine and Pharmacy "Grigore T Popa", Iasi, Romania, ${ }^{2}$ University of Medicine and Pharmacy "Carol Davila", Bucharest, Romania

Introduction: Alzheimer's disease is one of the most devastating and costly condition worldwide. Due to the increase in life expectancy and to the population ageing it is estimated that the number of people with dementia will double in the next 30 years. In Romania there are about 300,000 people suffering from Alzheimer's dementia so far and it is estimated that the number will triple by 2025 . Unfortunately, about $20 \%$ of this global number are diagnosed in the advanced stages of the disease, when even the best therapeutic plans are associated with modest results, institutionalization and very high costs. This is why early identification of people at risk of developing Alzheimer's disease becomes an absolute priority.

Methods: CAIDE score is designed for general adult population as a tool that estimates the risk of Alzheimer's dementia in the next 20 years. Age, gender, education, systolic blood pressure, body mass index (BMI), cholesterol, and physical activity are used to count the score. An abnormal score should be the trigger to start prevention of chronic diseases in order to achieve a healthy ageing.

Results: We present the partial outcomes of a prospective study that evaluates the predictive capacity of CAIDE score in the general adult population, and whether it can be improved by taking into account other mid-life risk factors. The evaluation of the cognitive status would be performed each year to monitor if there are changes in the normal evolution according to the ageing. If alterations of the cognitive status should occur, the identification of the responsable factor(s) should trigger specific prevention.

\section{P-398}

Physical activity, cardiovascular risk and employment status of 60- to 65-year-old subjects

Bartłomiej Sołtysik ${ }^{1}$, Joanna Kostka ${ }^{1}$, Kamil Karolczak ${ }^{1}$, Cezary Watała $^{1}$, Tomasz Kostka ${ }^{1}$

${ }^{1}$ Medical University of Łódź, Łódź, Poland 
Objectives: The aim of the study was to investigate if cardiovascular risk factors may differ according to workload, and if physical activity (PA) connected to health related behaviours and energy expenditure may produce the impact on cardiovascular risk factors profile in the elderly with different workload.

Methods: 300 subjects aged 60-65 were divided into three equal groups of intellectual, manual workers and unemployed subjects. 50\% were women. The subjects were examined for the presence of cardiometabolic diseases and major cardiovascular risk factors such as smoking, anthropometric indices, blood pressure, lipid profile, glucose, uric acid, homocysteine and metabolic syndrome.

Result: Manual workers were more obese, had higher blood pressure and higher PA indices in comparison with other two group. PA connected to health related behaviours had a positive impact on body mass indices, lipids, glucose or uric acid, with no such an impact observed for PA-related energy expenditure.

Conclusion: Higher cardiovascular risk may be observed in the group of manual workers. PA connected to health related behaviours has a positive impact on global cardiovascular risk and may decrease the risk of metabolic syndrome among seniors. No impact was observed for PA-related energy expenditure. Thus, employment status may play important role in how PA influences cardiovascular risk.

\section{P-399}

\section{Fear of falling in older adults in rural northern Greece}

Vaitsa Giannouli ${ }^{1}$, Nikolaos Syrmos ${ }^{1}$

${ }^{1}$ School of Medicine, Aristotle University of Thessaloniki, Thessaloniki, Greece

Introduction: Fear of falling is a major problem among elderly people and can lead to activity restriction. The aim of this study is to identify if old persons can develop a subsequent fear of falling, and whether this fear of falling is associated with cognitive, psychological and functional parameters.

Methods: Eighty-seven older adults ( 52 females) from rural northern areas in Greece participated voluntarily in this research. Their cognitive function was assessed with MMSE, their daily functioning with FRSSD and FUCAS, and their fear of falling was assessed using FESI-I. The mean age of the participants was 74.65 years $(\mathrm{SD}=6.75$, range $66-89)$, their level of education 8.60 years $(S D=3.92)$, and their mean MMSE score was 26.50 ( $\mathrm{SD}=3.99$ ).

Results: Results revealed that $9 \%$ of the participants expressed no fear of falling, $26 \%$ expressed a little concern about falling, and the rest $65 \%$ expressed a great concern. Fear of falling was not associated with age $(r=0.065, p=0.555)$ or cognitive function $(r=0.184$, $\mathrm{p}=0.091)$.

Conclusions: The fear of falling seems not to be related with factors, such as the advancing age, gender or even slight deterioration in cognitive functions. Future studies should clarify the possible factors that affect and differentiate fear of falling in older adults.

\section{P-400}

Relationship between muscular performance trainability and other physical performances, and characteristics in resistance exercise among community-dwelling older adults

Naoki Tomiyama ${ }^{1}$, Ryuichi Hasegawa ${ }^{2}$

${ }^{1}$ Seijoh University, Tokai, Japan, ${ }^{2}$ Chubu University, Kasugai, Japan
Introduction: Resistance exercise has been reported to be more effective for older adults with low muscular performance. Therefore, trainability might be affected by pre-intervention characteristics. The purpose of this study was to determine the factors influencing trainability for muscular performance in resistance exercise among older adults.

Methods: The participants were attended a 9-week elastic band-based resistance exercise class. Pre- and post-class physical performance tests were performed by 281 participants. Muscular performance was evaluated using an arm curl test (AC) and 30-second chair stand test (CS). Balance performance was assessed using the timed up and go test (TUG) and four square step test. Flexibility performance was measured using the back scratch test and sit and reach test (SR). Other variables, including age, sex, height, weight, exercise habits, hypertension and diabetes mellitus, were examined. Multiple linear regression analysis was performed to examine whether trainability for muscular performance was relevant to other pre-intervention physical performances or characteristics.

Results: AC results of trainability were associated with TUG ( $\beta=-$ $0.368, P<0.000)$, exercise habits $(\beta=0.173, P=0.001)$ and weight $(\beta=0.128, P=0.019)$ adjusted for the pre-intervention AC. CS results of trainability were also associated with TUG $(\beta=-0.283$, $\mathrm{P}<0.000)$, exercise habits $(\beta=0.185, \quad \mathrm{P}=0.001)$ and $\mathrm{SR}$ $(\beta=0.158, P=0.007)$ adjusted for the pre-intervention CS.

Conclusion: These results suggest that trainability for muscular performance is affected by pre-intervention characteristics, especially agility and exercise habits, among community-dwelling older adults.

\section{P-401}

Relationships between functional physical fitness, variables cardiovascular and quality of life in elderly

Daniel Pietko Da Cunha ${ }^{1}$

${ }^{1}$ Universidade de Coimbra, Coimbra, Portugal

Introduction: The performance of daily activities is crucial to the quality of life in old age, and physical fitness gets significant impact in this context. Cardiovascular variables are very important for morbidity and mortality in the general population, with particular relevance to the geriatric age group. The challenge nowadays is to understand the factors that influence the quality of life, for the human being to live longer without losing autonomy.

Objectives: To study the relationship between functional fitness, anthropometric, hemodynamic and blood variables and quality of life. Furthermore, to investigate how these variables predict quality of life of elderly people.

Materials and methods: Twenty participants over 60 years old from the geriatric ambulatory of city of Cachoeirinha, in Brazil, had physical fitness tests for elderly people and had blood pressure (BP) and body mass index (BMI) measured. They had blood test to determinate lipid and glucose profiles. The quality of life was assessed with the SF-36v2. Multivariate analysis of techniques of variance and line arregression were also used.

Results: Participants with normal glucose levels had lower BMI $(\mathrm{p}<0.05)$ than pre-diabetics and diabetics participants. The older adults with normal triglycerides levels showed lower systolic and diastolic blood pressure $(\mathrm{p}<0.05)$. Age, sex and BMI explained $31.4 \%$ of the SF-36v2. Including systolic blood pressure in the model explanation raised to $43.6 \%$ the R2, increasing progressively to $53.8 \%$ when lower limbs strength was considered and to $64.7 \%$ with variable aerobic resistance.

Conclusion: BMI and BP, particularly systolic, cause great impact on general and cardiovascular morbidity, as well as in the quality of life 
for seniors. The lower limb strength and aerobic endurance affect decisively their quality of life. The maintenance and optimization of these physical parameters positively impact the functional autonomy, and the level of independence of the elderly population.

\section{P-402}

Prevalence and vascular risk factors of basal ganglia calcifications in a cerebrovascular cohort

E. J. M. de Brouwer Esther ${ }^{1}$, P. A. de Jong Pim ${ }^{1}$,

R. Kockelkoren Remko ${ }^{1}$, J. B. De Vis Jill ${ }^{1}$,

J. W. Dankbaar Jan Willem ${ }^{1}$, R. A. P. Takx Richard ${ }^{1}$,

M. H. Emmelot-Vonk Marielle ${ }^{1}$, A. de Jonghe Annemarieke ${ }^{2}$,

H. L. Koek Huiberdina ${ }^{1}$

${ }^{1}$ UMC Utrecht, Utrecht, The Netherlands, ${ }^{2}$ Tergooi Hospital Hilversum, Hilversum, The Netherlands

Introduction: Risk factors for and meaning of basal ganglia calcifications are poorly understood. Studies in patients with Fahr syndrome who often have neurologic, psychiatric and cognitive symptoms identified calcifications in the basal ganglia within (tunica media) and around the vessel walls. We aimed to assess the prevalence of basal ganglia calcifications and the association with vascular risk factors.

Methods: 1133 patients with suspected stroke from the Dutch acute stroke (DUST) study who underwent thin-slice non-contrast enhanced brain CT were analyzed. Basal ganglia calcifications were scored bilaterally as absent, mild (dot), moderate (multiple dots or single artery), severe (confluent). Multivariable logistic regression analysis was used to determine risk factors for basal ganglia calcifications. Cardiovascular disease (CVD) risk factors were entered in the model as independent variables, including age, gender, history of stroke, smoking, hypertension, diabetes mellitus, hyperlipidemia, body mass index, renal function and family history of cardiovascular disease under 60 years.

Results: The mean age was 67.4 years (SD: 13.8 ) and $56.8 \%$ was male. Basal ganglia calcifications were present in $337(29.7 \%)$ patients, of which $196(58 \%)$ were mild, $103(31 \%)$ moderate and 38 $(11 \%)$ severe. In multivariable logistic regression analysis, age (OR: $1.03, \mathrm{p}<0.01$ ) and family history (OR: $0.78, \mathrm{p} 0.04$ ) were significantly associated with the presence of basal ganglia calcifications. The other cardiovascular risk factors were not significantly associated with the presence of basal ganglia calcifications.

Conclusions: In this cohort of Dutch patients suspected for acute ischemic stroke, basal ganglia calcifications are common and significantly associated with older age and inversely associated with family history of cardiovascular disease. We found no significant association with cardiovascular risk factors.

\section{P-403}

Prescribing practice of proton pump inhibitors in nursing homes: results of a secondary data analysis

Martina Wegener ${ }^{1}$, Almuth Berg ${ }^{1}$, Steffen Fleischer ${ }^{1}$, Gabriele Meyer, On behalf of the EPCentCare Consortium ${ }^{1}$, Ursula Wolf ${ }^{2}$

${ }^{1}$ Institute of Health and Nursing Science, Medical Faculty, Martin Luther University Halle-Wittenberg, Halle, Germany,

${ }^{2}$ Pharmacotherapy Management, University Hospital Halle (Saale), Halle, Germany

Background: Despite substantial risks Proton pump inhibitors (PPI) are often prescribed without indication and/or with too high dosages. In this context we analysed the current situation in nursing home residents.
Methods: From baseline data of the cluster-randomized, controlled EPCentCare study (BMBF grant) a secondary data analysis was performed to investigate adequate prescription of PPI concerning (1) indication as well as, (2) correct dosages with regard to all latest valid (a) guideline-recommendations and (b) drug informations.

Results: Within 437 residents of 37 nursing homes in Saxony-Anhalt, North Rhine Westphalia and Schleswig Holstein, mean age $83 \pm 9.2$ years, $71.9 \%$ female, prevalence of PPI-prescription is $44.2 \%(\mathrm{n}=193)$. In $138(71.5 \%)$ of these residents there is an adequate indication for PPI-prescription and in $52(26.9 \%)$ there is no indication (plus $1.6 \%$ ambiguous cases). Yet, 54 participants (28\%) with adequately indicated prescription reveal faulty PPI-overdoses. In less than one third of adequate prescription the indications are according to the PPIs' approvals, most are off label indications corresponding to guideline recommendations. Risk of inadequate PPIprescription without indication is associated with total number of prescribed drugs $(\mathrm{OR}) 1.32$; 95\% CI 1.18-1.62 ( $\mathrm{p}=0.013)$. Number of chronic diseases reveal an elevated risk OR 1.9; 95 CI 0.16-1.14 (not statistically significant). There is no significant association with age, level of care, dementia, prescription of psychotropic drugs, physicians' consultation rates and local region.

Conclusion: Despite partly generous and liberal guideline-recommendations there is a "Germany-wide" high prevalence of false PPIprescription in $>55 \%$ - either inadequate or overdosed-in nursing home residents. Ambulatory adequate PPI-prescription as implemented in our clinical setting is an urgently trans-sectorial imperative.

\section{P-404}

Hyperkyphosis in geriatric outpatients: which kyphosis measurement method associates best with physical performance and fall risk?

M. C. Koelé ${ }^{1}$, J. P. van Campen ${ }^{2}$, S. A. E. van Haelst ${ }^{3}$, N. van der Velde ${ }^{4}$, H. C. Willems ${ }^{4}$

${ }^{1}$ Amsterdam Public Health, Department of Internal Medicine, Division of Geriatrics, Academic Medical Centre Amsterdam, Meibergdreef 9, 1105 AZ Amsterdam, The Netherlands, ${ }^{2}$ Department of Geriatrics, MC Slotervaart, Louwesweg 6, 1066 EC Amsterdam, The Netherlands

Introduction: Hyperkyphosis is common in older adults and is associated with lower physical performance and a doubled fall risk [1, 2]. Several kyphosis measurement methods have been developed. However, the current gold standard kyphosis measurement- the Cobb angle-is poorly validated [3-5]. We aimed to investigate which of three kyphosis measurement methods associates best with physical performance and falls in geriatric outpatients.

Methods: We measured kyphosis with the Cobb angle, blocks method and occiput-to-wall distance (OWD). Hyperkyphosis was defined as a Cobb angle $\geq 50^{\circ}, \geq 3.4 \mathrm{~cm}$ of blocks and an OWD of $\geq 5.5 \mathrm{~cm}$. Participants reported fall-incidents monthly during 6 months. Physical performance was assessed through Timed Up and Go Test (TUG), Short Physical Performance Battery (SPPB), and grip strength. Through multifactorial regression analysis, we controlled for potential confounding.

Results: The mean age of the 337 included patients was $80.0 \pm 7.9$ years. Hyperkyphosis prevalence ranged from 41 to $73 \%$, depending on the measurement method. In the interim analyses (May 2018), hyperkyphosis, measured with the Cobb angle, was neither associated with physical performance nor with falls. Hyperkyphosis, measured with blocks and OWD, was significantly associated with TUG [OR 1.16, 95\% CI 1.01-1.42 (blocks); OR 1.23, 95\% CI 
1.06-1.67 (OWD)], and not with SPPB, grip strength and falls (follow-up complete August 2018).

Conclusion: Hyperkyphosis, measured with the Cobb angle, was not associated with clinically relevant outcome measures, in contrast to hyperkyphosis measured with the blocks method and OWD. Future studies should focus on finding an appropriate gold standard kyphosis measurement, to standardize hyperkyphosis research.

References:

1. Katzman WB et al (2011) Increasing kyphosis predicts worsening mobility in older community-dwelling women: a prospective cohort study. J Am Geriatr Soc 59(1):96-100

2. van der Jagt-Willems HC et al (2015) Associations between vertebral fractures, increased thoracic kyphosis, a flexed posture and falls in older adults: a prospective cohort study. BMC Geriatr 15:34

3. Carman DL, Browne RH, Birch JG (1990) Measurement of scoliosis and kyphosis radiographs. Intraobserver and interobserver variation. J Bone Jt Surg Am 72(3):328-33

4. Stotts AK et al (2002) Measurement of spinal kyphosis: implications for the management of Scheuermann's kyphosis. Spine (Phila Pa 1976) 27(19):2143-2146

5. McDaniels-Davidson C et al (2017) Kyphosis and incident falls among community-dwelling older adults. Osteoporos Int

\section{P-405}

Does heart failure with preserved ejection fraction progress through stages: a 3-year outcome study mapping the departure from pre-clinical diastolic dysfunction to increasing clinical events

R. O. Ross Obukofe ${ }^{1}$, W. T. William Thomson ${ }^{1}$, N. D. Nimai Desai ${ }^{1}$, N. A. K. Nayaab Abdul Kader ${ }^{1}$, J. B. Joshua Burke ${ }^{1}$, P. B. Prithwish Banerjee $^{1,2}$

${ }^{1}$ Warwick Medical School, Gibbet Hill Road, Coventry CV4 7AL, United Kingdom, ${ }^{2}$ Department of Cardiology, University Hospital Coventry and Warwickshire, Coventry CV2 2DX, United Kingdom

Introduction: Heart failure with preserved ejection fraction (HFpEF) is now a substantial and growing contributor the leading cause of mortality globally; cardiovascular disease. Despite this, its understanding remains poor.

Aim: The objective of this study was to further elucidate a clinical state before HFpEF; described as pre-HFpEF by Banerjee et al, 2016. Methods: This was a retrospective cohort study over three years. Patients were recruited based on primary attendance to a community heart failure (HF) clinic with: NYHA Class 2 breathlessness, elevated NT-proBNP, left ventricular ejection fraction $\geq 45 \%$ and no signs of HF. Total cardiovascular and all-cause clinical events (deaths, admissions, and length of hospital stay) were reviewed at 36 months and compared against increasing left ventricular filling pressure (E/e').

Results: 175 patients were included from 1294 screened. The mean age was 77 years. Hypertension was the most common comorbidity followed by lipid disorders then valvular heart disease. Almost two thirds of patients took ACEi's, half took $\beta$-Blockers or diuretics, one third took antiplatelets or calcium blockers. Results show a significant association between E/e' and emergency cardiac and total cardiac admissions and length of cardiac admissions $(\mathrm{P}<0.05)$. Patients with $\mathrm{E} / \mathrm{e}^{\prime} \geq 16$ had more frequent and longer inpatient admission. There was no difference in 3-year survival.

Conclusions: Increasing E/e' is directly related to adverse cardiac clinical outcomes; those with $\mathrm{E} / \mathrm{e}^{\prime} \geq 16$ are significantly worse. Elucidation of a pre-HFpEF stage provides a potential target for earlier identification and intervention through more aggressive management of risk factors and associated comorbidities.

\section{P-406}

Anthropometric and physical changes in normal weight, overweight and obese community dwelling older adults during a 12 week resistance exercise program

Milan Chang ${ }^{1}$, Olof Gudny Geirsdottir ${ }^{2}$, Palmi V. Jonsson ${ }^{3}$, Inga Thorsdottir $^{2}$, Alfons Ramel ${ }^{2}$

${ }^{1}$ Faculty of Health promotion, Sport, and Leisure studies, University of Iceland, Reykjavik, Iceland, ${ }^{2}$ Faculty of Food Science and Nutrition, University of Iceland, Reykjavik, Iceland, ${ }^{3}$ Department of Geriatrics, National University Hospital of Iceland, Reykjavik, Iceland

Resistance exercise is important for the prevention of sarcopenia and physical dependence of old adults. However, little is known whether overweight/obesity affect the outcomes of a resistance exercise program. Community-dwelling Icelandic old adults $(\mathrm{N}=236$, $73.7 \pm 5.7$ years) participated in a 12 -week resistance exercise program. Anthropometrics, muscular strength and physical function were measured at baseline and endpoint. Group was defined retrospectively as normal, overweight and obese BMI level according to international cut off values. Statistical analyses were corrected for age and gender. Of the participants, $22.0 \%$ were normal, $41.4 \%$ were overweight and $36.6 \%$ were obese. BMI categories were neither related to drop-out $(11.9 \%)$ nor to attendance $(88.4 \%)$. All groups experienced improvements in outcome measurements, but improvements in individuals with obese BMI were less pronounced compared to normal BMI individuals. There was similar weight gain in the groups $(+0.48 \mathrm{~kg}, \mathrm{P}<0.001)$, however, normal BMI group gained more lean mass $(+0.70 \mathrm{~kg}, \mathrm{P}=0.015)$, appendicular muscle mass $(+0.42 \mathrm{~kg}$, $\mathrm{P}=0.007)$ but lost more fat mass $(-0.75 \mathrm{~kg}, \mathrm{P}=0.081)$ as compared to obese BMI group. This resulted in a greater change in body fat percent in subjects with normal BMI as compared to obese $(-1.5 \%$, $\mathrm{P}=0.014)$. Absolute gains in quadriceps strength were similar between groups, but relative to body weight, normal BMI group gained more than obese BMI group $(+0.31 \mathrm{~N} / \mathrm{kg}, \mathrm{P}=0.017)$. This translated into a greater improvement in 6-minutes-walk-for-distance $(+24 \mathrm{~m}, \mathrm{P}<0.001)$ in normal BMI group compared to obese BMI group. Interestingly, grip strength increased more in obese BMI group (2.4 lb., $\mathrm{P}=0.020$ ). Timed-up-and-go improved similarly in all groups $(-0.64 \mathrm{~s}, \mathrm{P}<0.001)$. Independently from their BMI status, older adults benefited from a resistance exercise program in terms of body composition and physical function. However, obese individuals improved significantly less than normal weight participants although there was similar attendance to the exercise classes. Great care has to be taken of obese elderly in order to maintain their physical independence as long as possible.

\section{$\mathbf{P}-407$}

Medication-related harm due to non-adherence may explain the relationship between polypharmacy and mortality

Nikesh Parekh ${ }^{1}$, Khalid $\mathrm{Ali}^{2}$, Jennifer Stevenson ${ }^{3}$, Graham Davies ${ }^{3}$, Elisaveta Krasteva ${ }^{4}$, Rebekah Schiff ${ }^{5}$, Greg Scutt ${ }^{6}$, Jatinder Harchowal $^{7}$, Tischa Van der Cammen ${ }^{8}$, Chakravarthi Rajkumar ${ }^{1}$

${ }^{1}$ Department of Elderly Medicine, Brighton and Sussex Medical School, Brighton UK, ${ }^{2}$ Medication-related harm due to nonadherence may explain the relationship between polypharmacy and 
mortality, Brighton UK, ${ }^{3}$ Institute Of Pharmaceutical Science, King's College, London, UK

Introduction: Strong evidence exists for a relationship between polypharmacy and mortality [1], independent of comorbidity. The mechanisms underlying this relationship are unclear. Medication-related harm (MRH) may occur due to non-adherence or adverse drug reactions. We sought to determine if MRH due to non-adherence or adverse drug reactions may explain the association between polypharmacy and mortality.

Methods: The PRIME study recruited 1280 older adults at hospital discharge from 5 hospitals in England between 2013 and 2015 [2]. Patients were followed up in the community for 8-week by senior pharmacists to identify MRH using data from hospital readmissions, GP records and patient interviews. Mortality data at 12 months postdischarge were obtained from hospital records. Non-adherence was determined using a modified version of a validated questionnaire [3]. Adverse drug reactions were assessed using the Naranjo algorithm [4]. Adjusted logistic regression models were used to investigate the relationship between (1) number of medicines and MRH, (2) MRH and mortality.

Results: 1116 out of 1280 patients completed follow-up (median age 82 years, range $65-103$ years, 58\% female). Patients were discharged with a median of 9 medicines (range 0-27). A higher number of medicines was strongly associated with MRH due to non-adherence $(\mathrm{p}<0.01)$ and adverse drug reactions $(\mathrm{p}<0.001)$. In multivariable analysis, MRH due to non-adherence was associated with 1-year allcause mortality (OR 1.80, 95\% CI 1.08-2.99, p = 0.02), however MRH due to ADR was not (OR 1.20, 95\% CI 0.86-1.68, $\mathrm{p}=0.28$ ). Key conclusions: Harm from non-adherence to medications may explain the relationship between polypharmacy and mortality.

References:

1. Leelakanok N, Holcombe AL, Lund BC, Gu X, Schweizer ML (2017) Association between polypharmacy and death: a systematic review and meta-analysis. J Am Pharm Assoc 57:729-738

2. Stevenson J, Parekh N, Ali K, Timeyin J, Bremner S, Van Der Cammen $\mathrm{T}$ et al (2016) Protocol for a prospective (P) study to develop a model to stratify the risk (RI) of medication (M) related harm in hospitalized elderly (E) patients in the UK (The PRIME study). BMC Geriatr 16:22. https://doi.org/10.1186/s12877-0160191-8

3. Morisky DE, Green LW, Levine DM (1986) Concurrent and predictive validity of a self-reported measure of medication adherence. Med Care 24:67-74

4 Naranjo CA, Busto U, Sellers EM, Sandor P, Ruiz I, Roberts EA et al (1987) A method for estimating the probability of adverse drug reactions. Clin Pharmacol Ther 30:239-45.

\section{P-408}

Oropharyngeal dysphagia in an Irish nursing home: screening, prevalence, and remediation

Deirdre McCartan ${ }^{1}$, Professor Tara Coughlan ${ }^{1}$, Professor Desmond O'Neill $^{2}$

${ }^{1}$ Tallaght University Hospital, Dublin, Ireland, ${ }^{2}$ Tallaght Hospital, Dublin, Ireland

Introduction: Oropharyngeal dysphagia (OPD) is a significant cause of morbidity in the Nursing Home $(\mathrm{NH})$ population with adverse associations including aspiration pneumonia and malnutrition. OPD has many aetiologies including stroke, dementia and COPD. A lack of guidelines persists in detecting and defining the presence and severity of OPD as well as in defining and managing optimal treatment in this vulnerable group.
Methods: Medical and nursing notes, as well as Speech and Language Therapist (SLT) directives to kitchen and care staff on recommendations for food and fluid consistencies, were reviewed for evidence of OPD for each of the 42 residents of an Irish $\mathrm{NH}$ facility. Nursing care plans outlining "swallowing difficulty" of 7 types, SLT documentation of swallow assessment and of food and fluid recommendations were reviewed for content and consistency.

Results: There was evidence of OPD in 18/42 (42\%) residents, of whom $12 / 18$ (67\%) were women: dementia was a diagnosis in $8 / 18$ (44\%). Four patients expressed a preference not to adhere to safe swallow recommendations, one of whom received thickener despite a care plan to the contrary. Two patients had less stringent kitchen guidelines than SLT recommendations. "Coughing/choking" $6 / 18(33 \%)$ was the swallowing difficulty most commonly observed by nursing staff. Bedside review, mealtime observation and chart review formed the bulk of swallow assessments.

Key conclusions: Screening for OPD should be a key consideration in the care of NH residents. Early objective SLT review is essential to provide clear swallow recommendations to nursing, care and kitchen staff thus eliminating the subjectivity of observational records.

\section{P-409}

\section{Dietary sodium restriction increases long-term risk of fall among middle-aged and older adults}

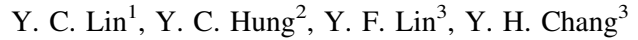

${ }^{1}$ Chinese Medicine Department, China Medical University Hospital, Taichung, Taiwan, ${ }^{2}$ Institute of Population Health Science, Taichung, Taiwan, ${ }^{3}$ Department of Public Health, China Medical University, Taichung, Taiwan

Introduction: Dietary sodium restriction (DSR) is widely suggested by clinicians to reduce risk of cardiovascular events which mainly occur in middle-aged and older adults. Falling events in older people can lead to serious consequence. It was little known about the association between DSR and falls in later life, which this study aims to understand.

Methods: The data was based on the Taiwan Longitudinal Study in Aging (TLSA) from 1999 to 2007. There were 5131 participants aged 50 and over, where participants without primary study variables had been excluded. Low sodium diet was evaluated in 1999 by asking whether participants are required to reduce sodium intake from food due to any illness. Fall experiences during past year were assessed in each survey. General estimating equation models were performed to examine the association between low sodium diet and falling experience with adjustment for covariates including age, sex, education, marital status, smoking, alcohol drinking, exercise frequency, appetite, incontinence and some chronic diseases.

Results: Totaling 16481 person-times was in three follow-up surveys, which left 3880 person-times from of observations after exclusion. The prevalence rates of fall experience were $25.7 \%$ in DSR and $17.8 \%$ in non-DSR groups. After adjusted for covariates, DSR compared to non-DSR, were at a higher risk of falls [adjusted odds ratio $(\mathrm{AOR})=1.34,95 \%$ confidence interval $(\mathrm{CI})$ 1.02-1.77].

Conclusions: DSR was associated with an increased risk of falling among middle-aged and older adults; the associations were independent of demographic, health behavioral factors and related chronic diseases. 


\section{P-410}

The impact of long bout sedentary time on cardiometabolic risk factors in a stroke population

Katinka Nordheim Alme ${ }^{1}$, Halvor Naess ${ }^{2}$, Anne-Brita Knapskog ${ }^{3}$, Mala Naik ${ }^{1}$, Torunn Askim ${ }^{4}$

${ }^{1}$ Haraldsplass Deaconess Hospital, Bergen, Norway, ${ }^{2}$ Haukeland University Hospital, Bergen, Norway, ${ }^{3}$ Oslo University Hospital, Oslo, Norway, ${ }^{4}$ Norwegian University of Science and Technology, Trondheim, Norway

Introduction: Sedentary behavior is a risk factor for vascular disease, and the focus has changed from total sedentary time to sedentary bouts and breaks. In this study we investigate the impact of long bouts of sedentary behavior in a stroke population.

Methods: We included 423 patients as part of the Norwegian Cognitive Impairment After Stroke-study (NorCOAST) 3 months after acute ischemic stroke. Sedentary behavior was measured by registering position transition by a single thigh worn sensor (ActivPal ${ }^{\circledR}$ ) for at least four consecutive days. Matlab was used to extract categories of sedentary time; (1) < $30 \mathrm{~min}$, (2) 30-60 min, (3) 60-90 min and (4) $>90 \mathrm{~min}$. The relationship between time spent in different categories and cholesterol, HbA1c and blood pressure was analyzed. Results: In total, 423 participants $(58 \%$ men; mean age 72.7 years, SD 11.2) were included. Sedentary behavior in total $(\mathrm{p}=0.003)$ and in bouts of more than $30(p=0.006)$ and $60 \mathrm{~min}(\mathrm{p}=0.013)$ was associated with a higher HbAlc in patients with diabetes mellitus, after adjusting for age, BMI and anti-diabetic drugs. The effect was comparable to the effect of oral antidiabetic medications. We found a negative relationship between blood pressure and sedentary behavior. Conclusion: Our finding supports our hypothesis that long bout sitting is more harmful than short bout sitting and that the impact of sedentary behavior varies between patient groups. The relationship between blood pressure and sedentary time might be explained by the coexistence of lower blood pressure with a low activity level in the frail patients.

\section{P-412}

Stroke, major bleeding, and mortality outcomes in frail old anticoagulant users with atrial fibrillation and chronic kidney disease

Yoko Yamada ${ }^{1}$, Taro Kojima ${ }^{2}$, Yumi Kameyama ${ }^{2}$, Sumito Ogawa ${ }^{2}$, Masato Eto $^{2}$, Masahiro Akishita ${ }^{2}$

${ }^{1}$ Department of Cardiology, Kitasato University Kitasato Institute Hospital, Tokyo, Japan, ${ }^{2}$ Department of Geriatric Medicine, Graduate School of Medicine, The University of Tokyo, Tokyo, Japan

Purpose: Preventing stroke is important since it decreases activity of daily living and quality of life in old people. However, the balance between stroke reduction and increased bleeding associated with oral anticoagulant (OAC) therapy among frail old patients with atrial fibrillation (af) and chronic kidney disease (CKD) is controversial.

Methods: 1173 patients aged $\geq 75$ who were admitted to the geriatric ward of the University of Tokyo Hospital between 2012 and 2017 were enrolled. From 175 af patients (men 47\%, mean age 85.5 years), we identified 94 (men 48\%, mean age 87 years) patients with CKD. We followed the patients for 1-5 years after baseline. Stroke, major bleeding and all-cause mortality was investigated for outcome.

Results: Among them, $53.2 \%$ were taking OAC therapy. The incidence of stroke in those taking OACs were higher than those not taking OACs $(\mathrm{HR}, 5.85 ; 95 \% \mathrm{CI} 0.01-0.89 ; \mathrm{P}=0.03)$ and major bleeding was marginally higher but not statistically significant (HR
4.58; 95\% CI $0.84-85.0 ; \mathrm{P}=0.08)$. The incidence of mortality in those taking $\mathrm{OACs}$ were marginally lower but not statistically significant (HR 0.53; 95\% CI 0.23-1.21; $\mathrm{P}=0.13$ ).

Conclusions: Present study shows that frail old OAC users with af and CKD might have less benefit than younger users. Further studies are needed to clarify the medical appropriateness of the use of OACs.

\section{P-413}

Fear of falling? Interventions to prevent or reduce fear of falling in community dwelling elderly: a systematic review

Andrea Fink ${ }^{1}$, Daniela Schober ${ }^{2}$

${ }^{1}$ Medical University of Graz, Graz, Austria, ${ }^{2}$ Institut für Pflegewissenschaft, Graz, Austria

Introduction: At least every fifth person over 65 years of age is scared of falling and fall-related consequences. Particularly for community-dwelling elderly, a fall can cause a sudden change in their previous independence. Consequently, older people restrict their physical activity and become socially isolated, which further increases their risk of falling. Little attention has been paid to interventions in only few systematic reviews. The aim of this systematic review is to identify and summarize effective interventions to prevent or reduce fear of falling among community-dwelling older people.

Method: A systematic literature search was performed using PubMed, CINAHL, EMBASE and the Cochrane Central Register of Controlled Trials and different search engines and reference lists were scanned for additional studies. Only English and German articles between 2005 and 2015 were included, which focus on people over 65 years and who were living at home during the trial. Two independent reviewers critically assessed the risk of bias in the included studies by using the "risk of bias tool" from the Cochrane Collaboration. If the interventions and diagnostic instruments were similar, the data were pooled in a meta-analysis.

Results: A total of 18 studies were identified for inclusion in this review. In 14 studies, the fear of falling of community-dwelling elderly was significantly prevented or reduced by the following interventions: seven exercise trainings, one education program and six multicomponent interventions.

Discussion: The results of this systematic literature review show the effectiveness of interventions with different components for the prevention or reduction of the fear of falling. Due to the variety of definitions of fear of falling, the different instruments for measurement, as well as differing study populations, the comparability of the included studies is questionable. In addition, the results have to be regarded with care, due to the included studies limited methodological quality. Further studies, especially from the nursing science perspective, with a special focus on certain high risk groups are required.

\section{P-414}

\section{Recruitment to the PrAISED feasibility study}

Rupinder Kaur Bajwa ${ }^{1}$, Veronika Van der Wardt ${ }^{1}$, Jennie Hancox ${ }^{1}$, Sarah Goldberg ${ }^{2}$, Clare Burgon ${ }^{1}$, Rowan Harwood ${ }^{3}$

${ }^{1}$ Division of Rehabilitation and ageing, University of Nottingham, Nottingham, UK, ${ }^{2}$ School of Health Sciences, University of Nottingham, Nottingham, UK, ${ }^{3}$ School of Health Sciences, University of Nottingham, Nottingham, UK, Nottingham University Hospitals, Nottingham, UK 
Introduction: Promoting Activity, Independence and Stability in Early Dementia (PrAISED) is a 12-month, multi-component physical exercise programme in people with mild cognitive impairment (MCI) or early dementia. This study examined the feasibility of utilising a mixed recruitment pathway and explored participants' reasons for taking part.

Method: Participants were recruited via Memory Assessment Service (MAS) clinics and join dementia research (JDR; a national research register developed by the National Institute for Health Research). Participants and their carers were recruited from MAS and JDR. Semi structured interviews were conducted with a subsample of participants $(n=20)$ and their carers to explore the reasons for participation in the trial.

Results: 60 participants ( 34 male, 26 female, mean age $=75.78, \mathrm{SD}$ 6.87) were recruited over a 6 month period. $70 \%(n=42)$ of participants were recruited through MAS and 30\% $(\mathrm{n}=18)$ of participants were recruited through JDR. Main reasons for participating were, wanting to help others and help future generations, to learn more about dementia, to stay active and for the potential cognitive and health benefits.

Conclusions: The mixed recruitment pathway was successful in recruiting to time and target. Futures studies should look at ways to adopt the use of a mixed pathway to optimise recruitment. Participating in clinical research is a way to learn more about dementia and find new ways to benefit general health. Based on findings from the interviews, future research could tailor recruitment materials, to emphasis the potential individual benefits of participating (learning more about the condition, achieving personal goals and helping others)

\section{P-415}

Relationship between health behaviors and marital adjustment and marital intimacy in multicultural old aged couples.

Jungyoon Lee ${ }^{1}$, Jongsung $\mathrm{Kim}^{2}$, Sungsoo Kim ${ }^{2}$, Jinkyu Jeong ${ }^{2}$, Seokjun Yoon ${ }^{2}$, Sunjin Kim ${ }^{2}$, Sami Lee ${ }^{2}$

${ }^{1}$ Department of Healthcare Center, The Catholic of Korea Daejeon St. Mary's Hospital, Daejeon, South Korea, ${ }^{2}$ Department of Family Medicine, Chungnam National University Hospital, Daejeon, South Korea

Background: This study analyzed the relationship between health behaviors and marital adjustment in multicultural old aged couples to evaluate their health status.

Methods: Married couples (70 Korean husbands and their immigrant wives) completed a structured interview on health behaviors and sociodemographic factors, the Revised Dyadic Adjustment Scale (RDAS), and the Marital Intimacy Scale (MIS). Based on the cut-off value of the RDAS, respondents were classified into two groups: high/ low dyadic adaptation groups. The collected data were compared with health behavior regarding smoking, alcohol consumption, exercise, and weight.

Results: The odds ratio (OR) [95\% confidence interval (CI)] by logistic regression with adjustment for age, education, career, occupation, length of residence in Korea, nationality, religion, age difference between couple, number of children, monthly income, and proficiency in Korean was 1.279 (1.113-1.492) for unhealthy exercise and 1.732 (1.604-1.887) for unhealthy body weight in female immigrants with low marital adjustment. In Korean husbands with low marital adjustment, the OR (95\% CI) was 1.625 (1.232-2.142) for smoking and $1.327(1.174-1.585)$ for unhealthy exercise. No significant relationship was found between marital intimacy and health behaviors in female immigrants or Korean husbands.
Conclusion: More desirable health behaviors were observed in old aged couples who were highly adapted. Therefore, family physicians should be concerned with marital adjustment and other associative factors to evaluate and improve multicultural old aged couples' health status.

\section{P-416}

The objective evaluation of hand dexterity using wearable fingertip sensors

Hitomi Oigawa ${ }^{1}$, Tomohiro Umeda ${ }^{1}$

${ }^{1}$ Nara Medical University, Kashihara, Japan

Introduction: Hand dexterity decreases with age. However, maintaining and promoting dexterity is important for extending healthy life expectancy. We used fingertip sensors to analyze and objectively evaluate the dexterous movement of the hands.

Methods: Fingertip motion was measured and used to determine how many times a patient closed their hand in a 10 -second test. We measured the movement of each fingertip using a wearable sensor (HapLog) that can measure the three-axis acceleration and contact pressure of the fingertip. The sampling frequency was $200 \mathrm{~Hz}$ and the acceleration range was $4 \mathrm{G}$. From the obtained time series data, we calculated the maximum and minimum values, compared the data of each finger and compared the frequency, and extracted the feature quantity.

Results: It was found that it is possible to calculate the number of hand closures by analyzing the $\mathrm{z}$ axis acceleration data from the 10 -second test. Moreover, by comparing the time series data of each finger, the features of the opening and closing motion of each finger could be detected. Finally, it was shown that it is possible to evaluate the awkwardness of opening and closing by frequency analysis.

Conclusions: By using wearable sensors, it is suggested that analysis and evaluation of three-dimensional finger movement can be performed. In the future, in order to improve the evaluation accuracy, we collect more data of the 10-second test and the evaluation should be performed by machine learning.

\section{P-417}

Process evaluation of an osteoarthritis disease management programme for older people with knee and hip osteoarthritis

Christopher Sammut ${ }^{1}$, Suzanne Timmons ${ }^{2}$, Sheena $\mathrm{McHugh}^{3}$

${ }^{1}$ University College Cork, Cork, Ireland, ${ }^{2}$ Centre for Gerontology and Rehabilitation, School of Medicine, UCC, Cork, Ireland, ${ }^{3}$ School of Public Health, UCC, Cork, Ireland

Introduction: Exercise plays a crucial role in osteoarthritis management in older people. Group-based interventions are effective at reducing pain and improving physical function and quality of life. Health professionals frequently encounter difficulties implementing such programmes, particularly ensuring high levels of patient attendance and participation.

Objective: To conduct a process evaluation alongside the implementation of a new disease management programme for older people with knee and hip osteoarthritis to examine programme uptake, fidelity, barriers and facilitators to implementation.

Methods: A mixed method design was used. Quantitative data were collected through routine monitoring of the service, including attendance records and outcome assessments. A fidelity checklist was used 
during site-visit observations. Interviews were conducted with the intervention staff and purposefully sampled programme participants. Results: Fifteen participant interviews, four physiotherapist interviews and three site-visits were conducted. Facilitators of attendance included the development of a physiotherapist-patient rapport, participants' positive perception of exercise and previous experience of physiotherapy. Barriers to implementation included the intervention's vague structure and staff turnover.

Conclusions: A group-based osteoarthritis intervention should consist of a well-structured and scheduled programme with emphasis on education aimed at improving participants' perception of exercise and developing a physiotherapist-patient rapport. This may help to maximise participation in osteoarthritis patients and ensure greater implementation success.

\section{P-418}

Profiles of elderly people participating in prevention programmes of the 'Centre for Memory and Mobility' in Luxembourg

Elisabeth Bourkel ${ }^{1}$, Carine Federspiel ${ }^{1}$, Neguine Rastegar ${ }^{2}$, Jean-Paul Steinmetz ${ }^{1}$

${ }^{1}$ Centre for Memory and Mobility, ZithaSenior, Luxembourg,

${ }^{2}$ Seniorie Saint Jean de la Croix, ZithaSenior, Luxembourg

The 'Centre for Memory and Mobility'constitutes a new offer in the provision of prevention programmes focusing age-associated mobility and cognitive declines. The Centre promotes general health and allows maintaining autonomy and independence in everyday activities by providing highly structured and evidence based cognitive and mobility training programmes. The aim of the present study is to identify the profiles of the population participating in the Centre's preventive measures by analyzing their cognitive physical, medical and social characteristics. To date, a total of 162 patients (age mean 77.4 years, SD 8.09) have visited the Center. Each patient's body mass index, grip strength, self evaluated state of health, general cognitive capacities and concentration capacities are measured on their first visit. Furthermore, each patient's social and medical characteristics are assessed. Using cluster analysis, the following three groups are identified: (1) "Healthy patients", (2) "Patients with cognitive deficits", and (3) "Patients with physical deficits". Moreover, we describe the social and medical characteristics for each group of patients, using a descriptive analysis. By October 2018, approximately 200 patients will have attended the Centre. The additional patients will be included in the cluster analysis. The discussion of the findings focuses on the characteristics of patients willing to partake in preventive programs which allow maintaining their autonomy and independence in everyday life. Furthermore, we discuss how the results help adapting preventive strategies to the individual needs of those patients.

\section{P-419}

Introduction of an acute care navigation service and its impact on hospital admissions in the vulnerable population

\author{
Joe Street ${ }^{1}$, Asa Bancroft ${ }^{1}$, Mathew Lewis ${ }^{1}$, Amy Green ${ }^{2}$, \\ Eleanor Ginbey ${ }^{2}$, Lauren Wentworth ${ }^{2}$, Mike Channon ${ }^{1}$ \\ ${ }^{1}$ Acute Care Navigator Service, LSO, Manchester, UK, \\ ${ }^{2}$ Wythenshawe Hospital, MFT, Manchester, UK
}

Introduction: The Acute Care Navigation service was introduced to Wythenshawe Hospital, Manchester Foundation Trust in January
2018, with the aim to reduce hospital attendances and admissions. Through signposting patients to local services and regular contact by the team ensures their individual needs are met. The service identifies patients who are vulnerable in the community, the majority of whom are elderly. It is a non-clinical service comprising of three care navigators who liaise and share information between acute and community-based health services, social and third sector groups.

Methods: To evaluate the services' impact on the number of ED attendances and non-elective inpatient admissions through comparing data 3 months before, and 3 months post referral.

Results: In the 3 months prior to referral the 71 patients seen by the Care Navigators had 136 ED attendances with a resultant 106 inpatient admissions. In the following 3 months ED attendances reduced to 57 , a reduction of $58 \%$ and admissions by 64 , a reduction of $60.4 \%$. The preservice admission data accounted for 612 bed days (length of stay plus one), which reduced to 312 for subsequent admissions, a $49 \%$ reduction.

Key conclusions: 3 months post referral there was a significant reduction in $\mathrm{ED}$ attendances and hospital admissions with a huge improvement in inpatient stay. The service has supported those who frequently attend ED either through frailty or lifestyles crisis. This highlights the potential of the service to help prevent unnecessary admissions amongst the vulnerable population and free up hospital capacity with economic benefit.

\section{$\mathbf{P}-420$}

Loss of independence trajectories of older adults living in community: results of a cohort study

Charlotte Bimou ${ }^{1}$, Michel Harel ${ }^{2}$, Cécile Laubarie-Mouret ${ }^{3}$, Noëlle Cardinaud ${ }^{4}$, Justine Trimouillas ${ }^{5}$, Nassima Toumi ${ }^{6}$, Caroline Gayot ${ }^{6}$, Thierry Dantoine ${ }^{7}$, Achille Tchalla ${ }^{8}$

${ }^{1}$ EA 6310 HAVAE, Doctoral School Biological Sciences and Health, Limoges University, Limoges, France, ${ }^{2}$ Toulouse Institute of Mathematics, Higher School of Teaching and Education at Limoges University, Limoges, France, ${ }^{3}$ Geriatric Medicine Service, University Hospital Center Dupuytren, Limoges, Limoges, France, ${ }^{4}$ Department of Geriatric Medicine, Unit for Prevention of Monitoring and Analysis of Aging, University Hospital Center Dupuytren, Limoges, France, ${ }^{5}$ Clinical Research Associate, Unit for Prevention of Monitoring and Analysis of Aging, ${ }^{6}$ Clinical Research Associate, Clinical Research and Geriatrics Innovation Unit, ${ }^{7}$ EA 6310 HAVAE, Limoges University, Limoges, France, ${ }^{8}$ EA 6310 HAVAE, Geriatrics Clinical Research and Innovation Unit, Limoges Dupuytren Teaching Hospital, University Hospital Center of Clinical Gerontology, Department of Geriatric Medicine, Unit for Prevention of Monitoring and Analysis of Aging, Limoges, France

Background: The loss independence is the inability for a person to decide, ensure some activities of daily life. The independence's loss depends on physicals, psychologicals, biological and socio-economic factors. Its risks factors knowledge may help to plan appropriate preventive measures to maintain independence.

Objective: To identify of loss of independence trajectories and risk factors associated.

Method: Independence assessing criterion used for this study was the SMAF (Système de Mesure de l'Autonomie Fonctionelle). Three methods: k-means, hierarchical ascending classification and GroupBased Trajectory Modeling was used to classify subjects in homogenous groups and performing independence trajectory. Multinomial logistic regression was used to identify the predictors of independence loss. 
Results: 221 subjects were evaluated between 2011 and 2014. The average of age was $86.1( \pm 5)$. Women represented 64\%. Polypathology and poly-medication prevalence rate were respectively $88 \%$, $77 \%$. Falls prevalence rate was $62 \% .53 \%$ of population were dependent for ADL and $62 \%$ for IADL. Three distinct profiles of independence were identified: independent (68\%), moderately independent (24\%) and dependent $(8 \%)$. Predictors of moderately independent were age. Predictors of the dependence were school level, lifestyle, family support, number of daily medications, urinary incontinence, cognitive disorders, nutrition, physical performance and frailty.

Conclusion: Half of the population was independent. A quarter had moderate to severe dependence. To our knowledge, such a study has not yet been the subject of research. The results of this study indicate that some sociodemographic and physiological criteria are good predictors of moderate to severe independence loss.

\section{$\mathbf{P - 4 2 1}$}

Discriminative ability of standard clinical and instrumented measures of physical performance in distinguishing between different levels of functional status in high-functioning people aged 61-70 years

Alice Coni ${ }^{1}$, Jeanine M. van Ancum ${ }^{2}$, Nini H. Jonkman ${ }^{2}$, Ronny Bergquist ${ }^{3}$, Stefanie Mikolaizak ${ }^{4}$, Andrea B. Maier ${ }^{5}$, Sabato Mellone ${ }^{1}$, Lorenzo Chiari ${ }^{6}$, Mirjam Pijnappels ${ }^{2}$

${ }^{1}$ Department of Electrical, Electronic and Information Engineering "Guglielmo Marconi" (DEI), University of Bologna, Bologna, Italy, ${ }^{2}$ Department of Human Movement Sciences, @ AgeAmsterdam, Faculty of Behavioural and Movement Sciences, Vrije University, Amsterdam, The Netherlands

Introduction: Physical performance is traditionally measured by clinical tests such as Repeated Chair Stand (CST) test and Timed-Upand-Go (TUG) test. Inertial sensors are rapidly emerging to measure physical performance, since the instrumented measures obtain more details. Our aim is to compare the outcome of the standard clinical with instrumented measures of physical performance in distinguish between High and Very High Functional Status (HFS, VHFS) in community-dwelling adults aged $61-70$ years.

Methods: Data is from the baseline assessment of the Prevent IT study. The Late Life Function and Disability Instrument (LLFDI) was used as a measure of functional status (median score as cut-off for HFS and VHFS). Participants performed the CST and TUG wearing a smartphone at L5. The number of CST repetitions and the total TUG duration were recorded. Instrumented measures were computed from the smartphone embedded sensors. The area Under the Receiver Operating Curve (AUC) was calculated and compared using the DeLong test.

Results: We included 189 participants $(66.3 \pm 2.5$ years, 92 females, LLFDI score $146 \pm 15$, median 151). Standard clinical and instrumented measures of CST showed moderate discriminative ability, AUC of 0.68 (95\% CI $0.60-0.76$ ) and 0.72 (95\% CI $0.65-0.80$ ) respectively, p value 0.19 . Similarly, for TUG: AUC of $0.68,(95 \%$ CI $0.60-0.77)$ and $0.65(95 \%$ CI $0.56-0.73)$ respectively, $p$ value 0.27 . Conclusions: In a relatively fit and healthy population of adults aged 61-70 years, standard clinical and instrumented measures distinguish, with moderate discriminative ability, between HFS and VHFS. This finding supports the hypothesis that an early identification of risk of age-related functional decline can be achieved.

\section{P-422}

The association between hyperkyphosis and fall risk in older adults: a prospective cohort study

M. C. Koelé ${ }^{1}$, I. M. Harmsen ${ }^{1}$, K. M. A. Swart ${ }^{2}$, S. C. van Dijk ${ }^{3}$, A. W. Enneman ${ }^{3}$, J. P. van Wijngaarden ${ }^{4}$, E. M. Brouwer-Brolsma ${ }^{4}$, A. C. $\mathrm{Ham}^{3}$, P. T. A. Lips ${ }^{2}$, A. G. Uitterlinden ${ }^{4}$,

L. C. P. G. M. de Groot ${ }^{4}$, N. M. van Schoor ${ }^{2}$, T. J. M. van der

${ }^{1}$ Amsterdam Public Health, Department of Internal Medicine, Division of Geriatrics, Academic Medical Centre Amsterdam, Meibergdreef 9, 1105 AZ Amsterdam, The Netherlands, ${ }^{2}$ Amsterdam Public Health, Department of Epidemiology and Biostatistics, Amsterdam, The Netherlands

Introduction: Hyperkyphosis is present in $20-40 \%$ of communitydwelling older adults $[1,2]$. Studies investigating the association with fall risk show conflicting results, and only two small cohort studies have a prospective design [3, 4]. Furthermore, several kyphosis measurement methods have been applied in research [5]. We aimed to investigate the association between hyperkyphosis and fall risk in community-dwelling older adults prospectively, using the current gold standard kyphosis measurement- the Cobb angle [5].

Methods: The Cobb angle was measured on DXA-based Instant Vertebral Assessments. Participants reported falls monthly during 2-3 years. Through multifactorial regression analysis, we controlled for potential confounding.

Results: Almost half of the 1220 participants (mean age $72.9 \pm 5.7$ years) fell at least once during follow-up. Neither number of falls nor time-to-first-fall was statistically significant associated with hyperkyphosis in multifactorial analyses, in which we added age, gender, smoking, alcohol use and use of a walking aid (IRR 1.01, $95 \%$ CI $0.82-1.25$, p 0.915; HR $1.02,95 \%$ CI 0.81-1.27, p 0.892). In the Cox regression analysis, an interaction over time was found. However, unlike two small prospective cohort studies $[3,4]$, we did not find an association in the first year of follow-up.

Discussion: In contrast to previous studies [3, 4], hyperkyphosis was not statistically significant associated with fall incidence in the multifactorial models in this large cohort of community-dwelling older adults. This difference may be due to the healthier population of this large cohort study and to variation in kyphosis measurement methods. In future studies, the correlation and validity of the various kyphosis measurement methods should be investigated in geriatric patients.

\section{References:}

1. Ryan SD, Fried LP (1997) The impact of kyphosis on daily functioning. J Am Geriatr Soc 45(12):1479-862

2. Kado DM et al (2004) Hyperkyphotic posture predicts mortality in older community-dwelling men and women: a prospective study. J Am Geriatr Soc 52(10):1662-73

3. van der Jagt-Willems HC et al (2015) Associations between vertebral fractures, increased thoracic kyphosis, a flexed posture and falls in older adults: a prospective cohort study. BMC Geriatr 15:344

4. McDaniels-Davidson $C$ et al (2018) Kyphosis and incident falls among community-dwelling older adults. Osteoporos Int 29(1):163-1695

5. Katzman WB et al (2010) Age-related hyperkyphosis: it's causes, consequences and management. J Orth Sport Phys Ther 40(6):352-360

\section{P-423}

Relationship between falls, medication usage and chronic diseases in elderly women

Dovydas Vitkus ${ }^{1}$, Inga Tamulaityte-Morozoviene ${ }^{1}$, Vidmantas Alekna $^{1}$, Marija Tamulaitiene ${ }^{1}$, Jolanta Dadoniene ${ }^{1}$ 
${ }^{1}$ Faculty of Medicine, Vilnius University, Vilnius, Lithuania

Introduction: The purpose of this study was to investigate the association between falls, usage of medication and presence of chronic diseases.

Methods: Community-dwelling ambulatory women aged 65 years and older who had fallen in previous 12 months were included in this study. Data on fall history, medication and chronic diseases were collected. Statistical analysis was performed using independent samples T-test, Chi-Square test and Pearson correlation criterion.

Results: Overall, data of 310 women were analyzed. Average age was $72.7 \pm 4.95$ years. Of all women, $87.1 \%(\mathrm{n}=270)$ were taking medications, most common were antihypertensives $(50.3 \%, \mathrm{n}=156)$. The average amount of medications consumed was 1.24 ( $\min .0$, max. 4). Majority $(74.8 \%)$ of women had reported one or more chronic diseases, most common was hypertension $(51 \%, \mathrm{n}=158)$. Average number of chronic diseases per person was $1.08 \pm 0.84$. We have found that women who were consuming medications had more falls in previous 12 months $(1.7 \pm 1.3, \mathrm{n}=270)$ compared with women who weren't using any medications $(1.3 \pm 0.7, n=40 ; \mathrm{p}=0.015)$. There was a very weak correlation between amount of medications and number of falls in previous year $(\mathrm{r}=0.12 ; \mathrm{p}<0.05)$. No statistically significant correlation was found between number of diseases and number of falls.

Key conclusions: Medication usage was related to falling in investigated elderly women. There was very weak correlation between amount of medication used and number of falls.

\section{Area: Metabolism and nutrition P-424}

Validation in Spanish of the Mini Nutritional Assessment (MNA) questionnaire for the evaluation of nutritional status in elderly patients

Maria del Carmen Arias-Blanco', Belén Muñoz-Díaz ${ }^{1}$, G. Molina-Recio ${ }^{2}$, M. Romero-Saldaña ${ }^{3}$, Jorge Martinez de la Iglesia $^{4}$, Cristina Aguado-Taberné ${ }^{5}$, Juana Redondo-Sánchez ${ }^{6}$

${ }^{1}$ Médico de Familia, UGC La Sierra, Córdoba, Spain, ${ }^{2}$ Enfermeria UCO, Córdoba, Spain, ${ }^{3}$ Seguridad y Salud laboral, Ayuntamiento de Córdoba, Córdoba, Spain, ${ }^{4}$ Medico de Familia UGC Lucano,

Córdoba, Spain, ${ }^{5}$ Médico de Familia UGC Sta. Rosa, Córdoba, Spain, ${ }^{6}$ Medico de Familia, Lucano, Córdoba, Spain

Background: The MNA is a nutritional survey used to detect malnutrition and the risk of malnutrition in the elderly. However, as yet, it has not been completely validated in the Spanish language.

Aim: To validate the MNA in a Spanish population over 65 years of age and calculate its reliability (internal consistency and concordance), as well as the validity of its criterion.

Design: This validation study used the Chang nutritional assessment method as a reference test. Participants: 248 subjects $(75.4 \%$ female and $24.6 \%$ male), with a mean age of 83 , completed the study, out of an original total of 255 .

Setting: Three health centres and three residential care homes situated in Cordoba (Spain). The subjects were divided into three groups: (a) autonomous patients who were able to take part in activities outside their home; (b) patients living at home who were unable to go out; (c) patients living in a care home.

Results: The Kappa index readings for intra-observer and inter-observer agreement were 0.870 and 0.784 , respectively. The intraobserver ICC was 0.874 and the inter-observer ICC 0.789 . Cronbach's alpha for all items was 0.778 , which went up to 0.810 after four items were eliminated. The sensitivity and specificity readings for the diagnostic accuracy of MNA were 63.2 and $72.9 \%$, respectively. The area under the curve was 0.726 . When the MNA was given a new cutoff point of 25 , the sensitivity improved to $75 \%$.

Conclusion: The results for the reliability of the survey were excellent, and its internal consistency was acceptable. The diagnostic accuracy, as measured by the sensitivity and specificity readings, was lower than that obtained with the original survey, although it is likely that the variations were influenced by the reference test used.

Keywords: Malnutrition, Elderly, MNA

\section{P-425}

Factors associated with poor nutritional status in patients over 65 years of age

Belén Muñoz-Díaz ${ }^{1}$, Maria del Carmen Arias-Blanco ${ }^{2}$, Maria LoboMarín $^{3}$, Jorge Martinez de la Iglesia ${ }^{4}$, G. Molina-Recio ${ }^{5}$, Juana Redondo-Sánchez ${ }^{6}$, Cristina Aguado-Taberné ${ }^{7}, \mathrm{M}_{\text {Romero-Saldaña }}{ }^{8}$

${ }^{1}$ Medico de Familia, UGC L Sierra, Córdoba, Spain, ${ }^{2}$ Médico de Familia, UGC La Sierra, Córdoba, Spain, ${ }^{3}$ Médico residente UGC Lucano, Córdoba, Spain, ${ }^{4}$ Médico de Familia UGC, Lucano,

Córdoba, Spain, ${ }^{5}$ Departamento de enfermería, UCO, Córdoba, Spain, ${ }^{6}$ Médico de Familia UGC Lucano, Córdoba, Spain

Objective: To analyze the nutritional status (NS) of patients older than 65 years and establish their relationship with sociodemographic and health variables.

Method: Cross-sectional observational study in patients over 65 years of age from 3 health centers. Sample of 255 ambulatory patients (PI), with home care (PD) and institutionalized (PR). The Chang method was applied to determine the NS. Sociodemographic, anthropometric, dependency, mood, cognitive and analytical were collected. The associations were analyzed through the application of chi-square and analysis of variance. The prevalence ratio (PR) for malnutrition was calculated and a multivariate model (binary logistic regression) was applied. Significance was considered for $\mathrm{p}<0.05$.

Results: The average age was 81.3 years (SD 7.4), $72.0 \%$ were women. $48.9 \%$ were PI, $26.8 \%$ PD and $24.2 \%$ PR. $29.6 \%$ (95\% CI 23.9-35.8) presented malnutrition. Greater malnutrition was established in relation to living in a residence or precise home care (PR 5.3), age over 85 years (PR 4.9), presenting a moderate or higher dependence for activities of basic daily life (PR 3.9) and instrumental (PR 3.3), need help for mobility (PR 2.9) and present moderate/severe cognitive impairment (PR 2.1). The determinants of malnutrition in the multivariate model were more than 85 years and were PR or PD. Conclusions: Special emphasis should be placed on the response to NS in patients older than 85 years of age who live in a residence or a precise home care, since they are the groups at highest risk of malnutrition.

Keywords: Geriatric population, Malnutrition, Risk of malnutrition 


\section{P-426}

What older adults in Lithuania think about nutritional support, specifically oral nutritional supplements

Jurgita Knašiene ${ }^{1}$, Rita Grimalauskaite ${ }^{1}$, Gytė Damulevičienè ${ }^{1}$, Vita Lesauskaité ${ }^{1}$, Daiva Leskauskaité ${ }^{2}$, Milda Keršiené ${ }^{2}$

${ }^{1}$ Geriatric Department, Lithuanian University of Health Sciences, Kaunas, Lithuania, ${ }^{2}$ Department of Food Science and Technology, Kaunas University of Technology, Kaunas, Lithuania

Introduction: Oropharyngeal dysphagia (OD) prevalence is about $60 \%$ among frail, neurological and institutionalized patients. One of OD correction methods is modified food and thick liquids. The patients with OD must receive special oral nutritional supplements (ONS) for malnutrition prevention. One of the aims of the research project supported by the LSMU and KTU Science fund (grant SV50171/BN17-95) "Design and evaluation of fluid food products for geriatric patients with swallowing disorders" was to determine the knowledge about nutritional support possibilities among older adults and their opinion about the usage of ONS.

Methods: 148 patients ( $\geq 60$ years): 62 patients of Geriatric Department and 86 older adults living in the local community were interviewed.

Results: The mean age of respondents was $77.9 \pm 7.8$ years, $71.8 \%$ were female. Actually $31.8 \%$ respondents had completed higher education and $53.4 \%$ secondary education. $61.1 \%$ of respondents were informed on malnutrition problems, $75.2 \%$ on food fortification, $57.7 \%$ on ONS, $53.7 \%$ on enteral nutrition, and $35.6 \%$ on parenteral nutrition. It has been found that $44.3 \%$ respondents would like to consume only $200 \mathrm{ml}$ of ONS per day, $60.8 \%$ prefer nectar-thick liquids. $34.9 \%$ prefer bouillon-, $32.9 \%$ strawberry-, $26.8 \%$ mushroom- and $25.5 \%$ chocolate-flavoured ONS. $74.3 \%$ respondents would be able to afford ONS for 1.5-2 Eur per $200 \mathrm{ml}$. $91.2 \%$ of respondents would prefer to use ONS produced in Lithuania.

Key conclusions: Majority of older adults would be willing to consume only $200 \mathrm{ml}$ of bouillon-, strawberry- or mushroom-flavoured, nectar-thick liquid, which would cost approximately 1.5-2 Eur and would be produced in Lithuania.

\section{$\mathbf{P}-427$}

Association of vitamin D deficiency and falls risk among geriatric patients at Geriatric Department of Kaunas Clinical Hospital

Jurgita Knašiene $^{1}$, Indrè Zubačiūte ${ }^{1}$, Juozas Cèpla ${ }^{1}$

${ }^{1}$ Lithuanian University of Health Sciences, Kaunas, Lithuania

Introduction: There is a growing number of studies about vitamin D deficiency association with the risk of falls among hospitalized elderly patients.

Methods: In a prospective study, the risk of falls of 53 patients older than 60 years of Kaunas Clinical Hospital Geriatric Department between 2017 April and September was evaluated. The 'Get Up and Go' and 'Romberg' tests were performed to assess the risk of fall. In the study, vitamin D deficiency was defined as less than $75 \mathrm{nmol} / \mathrm{l}$. Results: The study analyzed 53 individuals, $22.6 \%(\mathrm{n}=12)$ of men and $77.4 \%(\mathrm{n}=41)$ of women, an average age of the subjects $79.25 \pm 8.036$. Increased risk of falls was found to $86.8 \%(n=46)$ of patients. Statistically significantly more often female than men had increased risk of falls $(92.7$ vs. $66.7 \%, p=0.036) .92 .5 \%(n=49)$ of patients had vitamin D deficiency. There was no statistically significant difference between age and vitamin $\mathrm{D}$ deficiency $(\mathrm{F}=0.234$, $\mathrm{df}=1, \mathrm{p}=0.631$ ). The risk of falls has the relationship with serum vitamin $\mathrm{D}(\mathrm{F}=8.277, \mathrm{df}=3, \mathrm{p}=0.06)$. Vitamin $\mathrm{D}$ deficiency was more commonly observed among patients at risk of falls $95.7 \%$ $(\mathrm{p}<0.05)$. Compared to those who had normal serum vitamin $\mathrm{D}$, patients who had vitamin D deficiency had 8.8 times higher odds to have increased risk of falls (95\% 1.008-76.863).

Key conclusion: The increased risk of falls was associated with vitamin D. Compared to those who had normal serum vitamin D, patients who had vitamin D deficiency had 8.8 times higher odds to have increased risk of falls.

\section{P-428}

Effect of serum gamma-glutamyltransferase on incidence of diabetes mellitus in korean elderly people: a 12 year retrospective longitudinal study

Semi Park ${ }^{1}$, Kyunam Kim ${ }^{1}$

${ }^{1}$ Ajou University School of Medicine, Suwon, South Korea

Serum gamma-glutamyltransferase (GGT) is a novel type 2 diabetes risk factor, but little is known about the GGT on the development of diabetes in Korean elderly populations. We evaluated 257 women and 337 men, more than 65 years of age, without baseline diabetes. From the baseline health screening to the follow-up examination, the development of diabetes, based on changes in GGT quartile levels, was analyzed. The development of diabetes gradually increased with an increase in the circulating levels of GGT. After adjusting for confounders, for the highest quartile of GGT, hazard ratios of diabetes compared with the lowest quartile were 3.54 (95\% confidence interval: $1.21-10.31, \mathrm{P}=0.021)$ in women and 5.08 (95\% confidence interval: $2.39-10.80, \mathrm{P}=0.009)$ in men. These findings in Korean elderly populations suggest potential utility of GGT as an additional biomarker in predicting the development of diabetes.

\section{P-429}

Nutritional status of the elderly after discharge from the acute geriatric unit: a pilot study

Berglind Blöndal ${ }^{1}$

${ }^{1}$ University of Iceland, Reykjavik, Iceland

Nutritional status of hospitalized old adults is often inadequate after discharge. The aim of the study was to assess dietary intake, food security and nutritional status of old adults after discharge. In this pilot study community-dwelling old adults ( $\mathrm{N}=13$; $87.7 \pm 5.6$ years; MMSE $\geq 20$; no catabolic diseases) discharged from the Acute Geriatric Unit of the National University Hospital of Iceland were included. Anthropometrics, dietary intake, food security and quality of life (QoL) were assessed at discharge, 1 week (home) and 2 weeks later (home).Baseline BMI was $24.7 \pm 5.1 \mathrm{~kg} / \mathrm{m}^{2}$ and there was significant weight loss during the 2 weeks period in participants $(-2.6 \mathrm{~kg}, \mathrm{P}=0.0001)$ resulting in an endpoint BMI of $23.8 \pm 4.7 \mathrm{~kg} / \mathrm{m}^{2}$. Actual daily energy- $(759.0 \pm 183.4 \mathrm{kcal})$ and protein intake $(35.1 \pm 7.5 \mathrm{~g})$ were significantly lower (both $\mathrm{P}<0.001)$ than the corresponding estimated requirements (2061.6 kcal; $82.4 \mathrm{~g})$. Kitchen assessment revealed that $33 \%$ of all foods were expired and $24 \%$ of all foods had visible mold. Of the participants, $75 \%$ experienced loneliness and QoL $(31.5 \pm 8.6)$ was significantly lower than the age and gender dependent reference values of 50. Loneliness, malnutrition, inadequate dietary intake and food insecurity are serious problems in discharged old adults in Iceland. There is a great need for individualized nutritional therapy, during and after hospital stays to ensure proper dietary intake with the 
aim to reduce malnutrition and re-admissions as well as to increase the quality of life of old adults.

\section{P-430}

E-mail alerts about rapid weight loss and ... increased mortality?

Nir Tsabar ${ }^{1}$, Yan Press ${ }^{1}$, Yonatan Grossman ${ }^{1}$, Maya Vainshtein-Tal ${ }^{1}$, Bracha Klein ${ }^{1}$, Johanna Rotman ${ }^{1}$, Sophia Eilat-Tsanani ${ }^{1}$

${ }^{1}$ Clalit Health Services, Tel Aviv, Israel

Background: The benefit of alerting clinical staff to rapid weight loss in patients aged $75+$ years to survival is uncertain.

Methods: In a randomized, controlled trial, 362 patients with bodymass index $(\mathrm{BMI})<23 \mathrm{~kg} / \mathrm{m}^{2}$ that dropped $\geq 2 \mathrm{~kg} / \mathrm{m}^{2}$ during previous 2 years were assigned to have an alert electronic mail sent to their physician, and 344 were assigned to receive usual clinical care (control-group). The primary outcome of the trial was death from any cause.

Results: In the following year, dietitian visits were recorded only for 22 patients $(6 \%)$ in the alert-group and 14 patients $(4 \%)$ in the control-group (odds ratio $1.5 ; 95 \% \mathrm{CI} 0.78-2.9 ; \mathrm{P}=0.24$ ). However, 77 patients $(21 \%)$ died in the alert-group and 46 patients (14\%) died in the control-group [hazard ratio $1.59 ; 95 \%$ CI $1.14-2.22 ; \mathrm{P}=0.007$; number needed to harm $(\mathrm{NNH})=12.7]$. In subgroup analysis, baseline vascular heart or brain diseases were associated with a significantly higher mortality rate in the alert-group than in the control-group [27 vs. 13\%; hazard ratio (HR) 1.9; 95\% CI 1.2-3.0; $\mathrm{P}=0.004 ; \mathrm{NNH}=8]$, while having none of these diseases at baseline was associated with no effect of the E-mail alert on mortality [14\% for both; HR 1.01 (0.6-1.7); $\mathrm{P}=0.97]$.

Conclusions: In this trial, alerting clinical staff to rapid weight loss in patients aged $75+$ years was not associated with higher dietitian visit rates but was associated with a significantly higher death than was usual clinical care.

\section{P-431}

Nutritional evaluation of elderly patients before and after hospital admission in an internal medicine ward

Diana Oliveira ${ }^{1}$, Francisca Mendes ${ }^{2}$, Adélia Simão ${ }^{1}$, Manuel Veríssimo ${ }^{1}$, Armando Carvalho ${ }^{1}$

${ }^{1}$ Centro Hospitalar e Universitário de Coimbra, Coimbra, Portugal, ${ }^{2}$ Faculdade de Medicina da Universidade de Coimbra, Coimbra, Portugal

Malnutrition is highly prevalent in geriatric patients and is a challenge for health professionals.

Objectives: To know the impact of hospitalization on the nutritional status of the elderly. An exploratory, descriptive and inferential study was performed, using a sample of geriatric patients with full motor skills hospitalized in an internal medicine ward of a terciary hospital, in January and February/2017. Data were obtained through a questionnaire that included economic, sociodemographic and serial measurements of bioimpedance parameters in the Body $720^{\circledR}$ machine, collected at the beginning and end of hospitalization. Statistical analysis was performed on IBM SPSS Statistics $24{ }^{\circledR} 0.70$ elderly (53 male, 17 female) with median age of 78 years (minimum of 65, maximum 93) and mean hospital stay of 8 days were selected. During hospitalization were found: normalization of the blood pressure, decrease in body mass index (BMI) (from 26.1 to $25.8 \mathrm{~kg} / \mathrm{m}^{2}$ ), body fat mass (BFM) (from 24.6 to $23.7 \%$ ), muscle mass (MM) (from
35.6 to $34.5 \%$ ) and body edema; 56 elderly patients lived in their own homes and 14 lived in residential units. At the admission those who lived in residential units presented BMI (24.6 vs $\left.26.4 \mathrm{~kg} / \mathrm{m}^{2}\right)$, BFM ( 23 vs $24.9 \%$ ) and MM (32.6 vs $35.9 \%$ ) lower than those who lived in their own homes. Patients with specific restriction in the diet presented a better control of the standards. Hospitalization contributed to a significant improvement in almost all nutritional parameters evaluated. The elderly in residential units had lower BMI, BFM and MM values than those who lived in their own homes.

\section{P-432}

Mediterranean diet in elderly patients at high risk of cardiovascular disease

Sonia Hammami ${ }^{1}$, Nadia Koubaa ${ }^{2}$, Amel Nakbi ${ }^{2}$, Kaldoun Ben Hamda ${ }^{3}$, Mohamed Hammami ${ }^{2}$

${ }^{1}$ CHU F Bourguiba, Biochemistry Laboratory, LR12ES05 LR-NAFS 'Nutrition-Functional Food \& Vascular Health' Faculty of MedicineUniversity of Monastir (Tunisia), Tunis, Tunisia, ${ }^{2}$ Biochemistry Laboratory, LR12ES05 LR-NAFS 'Nutrition-Functional Food and Vascular Health, Tunis, Tunisia

Background: Food items might have a synergistic and antagonistic effect on health. The Mediterranean diet has long been associated with lower incidence of cardiovascular disease and cancer.

Objective: Our objective was to evaluate the adherence to a Mediterranean diet (MD) according to diabetes in elderly coronary patients.

Methods: A total of 53 elderly coronary patients were involved in this study. They were divided into diabetic patient $(\mathrm{n}=27$; age $=$ 68.3 years $)$ and non-diabetics $(n=26$; age $=68.7$ years $)$. The dietary habits of the patients were evaluated using 1-week diet recalls. 11 main components of the MD (non refined cereals, fruits, vegetables, potatoes, legumes, olive oil, fish, red meat, poultry, full fat dairy products and alcohol) were used. A total score $(0-55)$ was calculated. Higher values of this diet score indicate greater adherence to the MD. Results: Compared to non-diabetics, individuals who developed diabetes had significantly higher mean values for, fasting glucose, triglyceride and urea levels. No significant differences between the nutrient intakes of the two subgroups. Nevertheless the diabetic patients group showed significantly higher magnesium intakes associated with higher vitamin $\mathrm{C}$ and folate intakes but lower vitamin $\mathrm{E}$ intakes the value of the MD score is about 28.53 for the non diabetic elderly CAD patients and 28 for the diabetic ones. There is a negative correlation between the MD score, diastolic and systolic blood pressure in non diabetic group.

Conclusion: Adherence to MD could be the first efficient step to prevent these complications.

\section{$\mathbf{P}-433$}

The acceptability of offering fortified foods to older people whilst in hospital: a pilot study

Kinda Ibrahim ${ }^{1}$, Lisa Methven ${ }^{2}$, Colette Fagan ${ }^{2}$, Sian Robinson $^{3}$, Margot Gosney ${ }^{4}$, Helen C. Roberts ${ }^{5}$

${ }^{1}$ Academic Geriatric Medicine; University of Southampton, Southampton UK; NIHR CLAHRC, University of Southampton, Wessex, UK, ${ }^{2}$ Department of Food and Nutritional Sciences, University of Reading, Reading, UK, ${ }^{3}$ MRC Lifecourse Epidemiology Unit, University of Southampton, Southampton, UK 
Introduction: Malnutrition is a significant problem amongst hospitalized older people, and can impede recovery. Oral nutritional supplements (ONS) may be poorly tolerated; food fortification could be a better alternative. We aimed to establish the acceptability of fortified foods to older inpatients including those who have dementia and frailty.

Methods: The intervention involved offering older patients in two UK hospitals between-meal fortified foods (enhanced with protein and energy) three times a day for 6 days, over 2 weeks. Fortified foods included biscuits, soup, cake, and ice-cream, providing on average $210 \mathrm{kcal}$ and $5 \mathrm{~g}$ protein/serving. The type and frequency of fortified foods ordered by patients was recorded. Patients' views were captured by a 10-point likeability scale. Interviews were conducted with 15 staff members to explore their views.

Results: 169 patients ordered 500 portions of fortified foods. Ice cream was the most frequently requested food $(199,40 \%)$, followed by cakes $(120,24 \%)$ and soups $(125,25 \%)$ and least biscuits $(56$, $11 \%)$. Patients' likeability scores were high $(>7 / 10)$ for all products. The median energy and protein intake per fortified food portion for men and women was $195 \mathrm{kcal}$ and $145 \mathrm{kcal}$ and 5.5 and $4.2 \mathrm{~g}$, respectively. Staff reported a number of potential advantages of fortified foods: easy to eat, suitable for patients with dementia/modified diet, mitigate against sliding into frailty, and improve engagement with therapy. Few reported concerns, including missing main meals. Conclusions: This study revealed high likeability and acceptability of fortified foods in older inpatients. The results will inform the design of a definitive clinical trial.

\section{P-434}

Protein intake characteristics of community-dwelling older adults with a low versus high protein intake

Linda M. Hengeveld ${ }^{1}$, Anouk D. A. Pelgröm ${ }^{2}$, Marjolein Visser ${ }^{1}$, Jolanda M. A. Boer ${ }^{2}$, Annemien Haveman-Nies ${ }^{3}$, Hanneke A. H. Wijnhoven ${ }^{1}$

${ }^{1}$ Department of Health Sciences, Faculty of Science, Amsterdam Public Health research institute, Vrije Universiteit, Amsterdam, the Netherlands, ${ }^{2}$ Division of Human Nutrition, Wageningen University \& Research, Wageningen, the Netherlands, ${ }^{3}$ Centre for Nutrition, Prevention and Health Services, National Institute for Public Health and the Environment (RIVM), Bilthoven, the Netherlands

Introduction: Adequate protein intake is essential for muscle health in old age, but low protein intake is common in older adults. This study aimed to compare community-dwelling older adults with low and high protein intake with regard to protein intake per day and per meal moment, and food sources of protein.

Methods: This study is conducted within the PROMISS project. Data were used from 727 community-dwelling older adults aged $\geq 70$ years from the Dutch National Food Consumption Survey Older Adults 2010-2012. Protein intake was measured with two non-consecutive $24-\mathrm{h}$ recalls by trained dieticians. Low protein intake was defined as below the Recommended Dietary Allowance of $0.8 \mathrm{~g}$ protein per kg adjusted body weight per day. Differences in the relative contribution of food groups to total protein intake between those with low and high protein intake were assessed with the MannWhitney U test.

Results: Low protein intake was present in $15 \%$ of the participants. Mean total protein intake was $51.9 \pm 10.7$ and $80.9 \pm 17.3 \mathrm{~g} /$ day in the low and high protein group, respectively. Protein intake was substantially lower in the low compared to the high protein group at all meal moments (breakfast 8.6 vs $12.0 \mathrm{~g}$; lunch 14.3 vs $25.6 \mathrm{~g}$; dinner 21.7 vs $32.0 \mathrm{~g}$; all $\mathrm{P}<0.001$ ). In both the low and high protein group, most protein was obtained from meat (12.1 vs $22.9 \mathrm{~g}$ ), dairy (13.0 vs $18.9 \mathrm{~g}$ ) and cereals (11.7 vs $16.2 \mathrm{~g})$. However, the low protein group obtained less protein relative to their total protein intake from meat ( $22.1 \mathrm{vs} 27.8 \%$ in the high protein group), but more from cereals $(22.4$ vs $20.1 \%$ ) (all $\mathrm{P}<0.01)$.

Conclusions: Dutch community-dwelling older adults with a low protein intake $(\sim 15 \%)$ consistently ate less protein at all meal moments and obtained relatively less protein from meat, but more from cereals than those with a high protein intake.

\section{$\mathbf{P}-435$}

Prevalence of low protein intake in older persons: a multi-cohort approach within the PROMISS project

Linda M. Hengeveld ${ }^{1}$, Jolanda M. A. Boer ${ }^{2}$, Pierrette Gaudreau ${ }^{3}$, Tamara B. Harris ${ }^{4}$, Martijn W. Heymans ${ }^{5}$, Carol Jagger ${ }^{6}$, Nuno Mendonça ${ }^{7}$, Marga C. Ocké ${ }^{2}$, Nancy Presse ${ }^{8}$, Stefania Sette ${ }^{9}$, Heli Tapanainen ${ }^{10}$, Aida Turrini ${ }^{9}$, Suvi M. Virtanen ${ }^{11}$,

Hanneke A. H. Wijnhoven ${ }^{1}$, Marjolein Visser $^{1}$

${ }^{1}$ Department of Health Sciences, Faculty of Science, Amsterdam Public Health research institute, Vrije Universiteit, Amsterdam, the Netherlands, ${ }^{2}$ Centre for Nutrition, Prevention and Health Services, National Institute for Public Health and the Environment (RIVM), Bilthoven, the Netherlands, ${ }^{3}$ Department of Medicine, University of Montreal, Montreal, Quebec, Canada; Research Centre, Hospital of the University of Montreal, Montreal, Quebec, Canada, ${ }^{4}$ Laboratory of Epidemiology and Population Sciences, Intramural Research Program, National Institute of Aging, Bethesda, Maryland,

${ }^{5}$ Department of Epidemiology and Biostatistics, Amsterdam Public Health research institute, VU Medical Center, Amsterdam, the Netherlands, ${ }^{6}$ Newcastle University Institute for Ageing, Newcastle University, Newcastle upon Tyne, UK; Institute of Health and Society, Newcastle University, Newcastle upon Tyne, UK,

${ }^{7}$ Newcastle University Institute for Ageing, Newcastle University, Newcastle upon Tyne, UK; School of Agriculture Food and Rural Development, Newcastle University, Newcastle upon Tyne, UK; Human Nutrition Research Centre, Newcastle University, Newcastle upon Tyne, UK, ${ }^{8}$ Research Centre, University Institute of Geriatrics of Montreal, Montreal, Quebec, Canada; Department of Nutrition, University of Montreal, Montreal, Quebec, Canada, ${ }^{9}$ Council for Agricultural Research and Economics, National Research Centre for Food and Nutrition, Rome, Italy, ${ }^{10}$ Unit of Nutrition, Department of Health, National Institute for Health and Welfare, Helsinki, Finland, ${ }^{11}$ Unit of Nutrition, Department of Health, National Institute for Health and Welfare, Helsinki, Finland; School of Health Sciences, Faculty of Social Sciences, University of Tampere, Tampere, Finland; Centre for Child Health Research, University of Tampere and Tampere University Hospital, Tampere, Finland; The Science Centre of Pirkanmaa Hospital District, Tampere, Finland

Introduction: Low protein intake in older adults was found to be associated with sarcopenia, poor physical functioning, and incident mobility limitations. This multi-country study aimed to assess the variation in the prevalence of low protein intake in older adults across multiple cohorts and national surveys.

Methods: As part of the PRevention Of Malnutrition In Senior Subjects in the EU (PROMISS) project, dietary information of community-dwelling men and women aged $\geq 55$ years was obtained from four cohorts (Longitudinal Aging Study Amsterdam, LASA, The Netherlands, 2014-2015; Newcastle 85 + study, United Kingdom, 2006-2007; Quebec Longitudinal Study on Nutrition and Aging, NuAge, Canada, 2007-2008; Health, Aging and Body Composition Study, HABC, USA, 1998-1999) and four national surveys 
(Dutch National Food Consumption Survey, DNFCS, 2010-2012; National FINDIET Survey, Finland, 2007 and 2012; Italian National Food Consumption Survey INRAN-SCAI, 2005-2006). Individuals with poor cognitive status and very high reported energy intakes (women $>3500 \mathrm{kcal} /$ day; men $>4000 \mathrm{kcal} /$ day) were excluded. Low protein intake was defined as below the Recommended Dietary Allowance (RDA) of $0.8 \mathrm{~g}$ protein per $\mathrm{kg}$ body weight per day ( $\mathrm{g} /$ $\mathrm{kg}$ /day).

Results: The analytic sample comprised 8103 older people. Mean protein intake ranged from 64.3 (SD 22.3) (Newcastle $85+$ ) to 80.6 (23.7) g/day (LASA), or when related to body weight from $0.90(0.38)$ (HABC) to $1.13(0.31) \mathrm{g} / \mathrm{kg} /$ day (INRAN-SCAI). The prevalence of low protein intake ranged from 13.2 to $45.0 \%$ in all older adults (9.0-45.0\% in men; $15.9-47.0 \%$ in women).

Conclusions: Based on this large-scale, multi-country study, the prevalence of a low protein intake in older people varies between countries but is substantial, about $13-45 \%$. New strategies must be developed to encourage older people to increase their protein intake, especially in light of the current debate on increasing the RDA to $1.0-1.2 \mathrm{~g} / \mathrm{kg} / \mathrm{day}$.

\section{P-436}

Malnutrition and malnutrition risk can be associated with systolic orthostatic hypotension in older adults

Suleyman Emre Kocyigit ${ }^{1}$, Pinar Soysal ${ }^{2}$, Esra Ates Bulut ${ }^{1}$, Ahmet Turan Isik ${ }^{1}$

${ }^{1}$ Department of Geriatric Medicine, Faculty of Medicine, Dokuz Eylul University, Izmir, Turkey, ${ }^{2}$ Geriatric Center, Kayseri Education and Research Hospital, Kayseri, Turkey

Background: Malnutrition and orthostatic hypotension $(\mathrm{OH})$ are the two important geriatric syndromes, which have similar negative outcomes such as falls. The aim of the study is to detect whether there is any relation between malnutrition and $\mathrm{OH}$.

Methods: 862 geriatric patients, who had undergone comprehensive geriatric assessment (CGA), were included in the retrospective study. $\mathrm{OH}$ was identified as $20 \mathrm{and} / \mathrm{or} 10 \mathrm{mmHg}$ dropped for systolic and/or diastolic blood pressures with the active standing test when patients got up from supine to standing position. Nutritional status was checked according to Mini Nutritional Assessment-Short Form (MNA-SF).

Results: The mean age of the patients was $74+8.05$, and $66.3 \%$ of them were female. The revalence of malnutrition, malnutrition-risk and $\mathrm{OH}$ were detected as 7.7, 26.9 and $21.2 \%$, respectively. When $\mathrm{OH}$, systolic $\mathrm{OH}$, diastolic $\mathrm{OH}$ and control group were compared with CGA parameters and the effects of age and gender were removed, the frequency of falls and Timed-Up and Go Test were higher, activity daily living indexes and TINETTI-Balance scores were lower in systolic $\mathrm{OH}$ than without it $(\mathrm{p}<0.05)$. Systolic $\mathrm{OH}$ was more frequent in malnutrition-risk and malnutrition group than control group ( $p<0.002$ and $\mathrm{p}<0.05$, respectively). Diastolic $\mathrm{OH}$ was not associated with nutritional status $(\mathrm{p}>0.05)$. $\mathrm{OH}$ was only higher in malnutrition-risk group than robust $(\mathrm{p}<0.05)$.

Conclusion: Our findings suggest that not only malnutrition but also malnutrition-risk may be associated with systolic $\mathrm{OH}$, which leads to many negative outcomes in older adults. Therefore, nutritional status should be checked during the evaluation of $\mathrm{OH}$ patients.

\section{P-437}

Cardiovascular risk prediction according to anthropometric indices in Spanish elderly of rural areas

Sergio Rico-Martin ${ }^{1}$, Mariana Martinez-Alvarez ${ }^{2}$, Sergio CordovillaGuardia $^{1}$, Esperanza Santano-Mogena ${ }^{1}$, Cristina Franco-Antonio ${ }^{1}$, David Conde-Caballero ${ }^{1}$, Lorenzo Mariano-Juarez ${ }^{1}$, Julian F. Calderon-Garcia ${ }^{1}$

${ }^{1}$ Nursing and Occupational Therapy College, University of Extremadura, Badajoz, Spain, ${ }^{2}$ Faculty of Medicine, University of Extremadura, Badajoz, Spain

Background and objective: Several anthropometric indices can be used to estimate obesity. The objective was to determine the best anthropometric index to identify elderly patients with moderate or high cardiovascular risk.

Methods: This cross-sectional study investigated a consecutive sample of 301 elderly patients (38.9\% males) without previous cardiovascular event, who had lived in rural areas of Cáceres (Spain). Seven obesity indices, including the body mass index (BMI), waist circumference (WC), Clínica Universidad de Navarra-body adiposity estimation (CUN-BAE), conicity index (CI), body roundness index (BRI) body shape index (ABSI) and waist-to-height ratio (WHtR) were investigated. The cardiovascular risk was evaluated by the 10-year coronary event risk (Framhingam Risk Score). Receiver operating characteristic (ROC) curve analyses were used to evaluate the predictive ability of the obesity indices for cardiovascular risk.

Results: Of the whole population, 213 (70.8\%) participants had a risk score higher than $10 \%$. Those who suffered medium or high CHD risk were more likely to have higher mean anthropometric indices, being statistically significant $(\mathrm{p}<0.05) \mathrm{WC}, \mathrm{CUN}-\mathrm{BAE}, \mathrm{CI}$ BRI ABSI and WHtR. According to the ROC analyses, CI provided the largest area under the curve $(\mathrm{AUC}=0.670 ; \mathrm{p}<0.001)$, and BMI showed the lowest value (AUC $=0.508 ; \mathrm{p}=0.828$ ).

Conclusions: CI was the best anthropometric index for estimating cardiovascular risk in elderly analyzed.

Funding: This work was supported by the 4 IE project (0045-4 IE-4$\mathrm{P})$ funded by the Interreg V-A España-Portugal (POCTEP) 2014-2020 program and IB16218 funded by Consejeria de Economía, Competitividad e Innovación del Gobierno de Extremadura (Spain).

\section{P-438}

Individually tailored dietary counseling among old home care clients: effects on nutrient intake

Tarja Kaipainen ${ }^{1}$, Miia Tiihonen ${ }^{1}$, Sirpa Hartikainen ${ }^{1}$, Irma Nykänen ${ }^{2}$

${ }^{1}$ Kuopio Research Centre of Geriatric Care, School of Pharmacy, Faculty of Health Sciences, University of Eastern Finland, Kuopio, Finland, ${ }^{2}$ Kuopio Research Centre of Geriatric Care, School of Pharmacy, Faculty of Health Sciences, University of Eastern Finland, Kuopio, Finland

Introduction: Prevalence of malnutrition and risk of it is $49-86 \%$ among home care clients. In this study we evaluate the effect of individually tailored dietary counseling on protein and nutrients intake among home care clients aged 75 or older.

Methods: This is a part of larger Nutrition, Oral Health and Medication (NutOrMed) study in Finland. The nutrients intake were assessed from 24-h dietary recall at the baseline and after 6-month intervention. The study population consisted of persons who had at baseline protein-energy malnutrition (PEM) or risk of PEM (intervention group $n=112$, control group $n=87$ ). PEM was defined by 
Mini Nutritional Assessment score $<24$ and/or plasma albu$\min <35 \mathrm{~g} / \mathrm{L}$.

Results: The mean age was 84.3 in both groups. The intervention was effective and increased significantly the mean intake of protein per bodyweight $(\mathrm{g} / \mathrm{kg}) 0.04 \mathrm{~g}(95 \% \mathrm{CI} 0.03-0.2)$, fiber $1.1 \mathrm{~g}(95 \% \mathrm{CI}$ $0.2-4.1)$, vitamin D $1.4 \mu \mathrm{g}(95 \%$ CI $0.4-3.8)$, vitamin E $0.1 \mathrm{mg}(95 \%$ CI $0.3-1.9)$, iron $0.5 \mathrm{mg}$ (95\% CI 0.4-2.1) and zinc $0.7 \mathrm{mg}(95 \% \mathrm{CI}$ $0.4-1.9$ ), adjustment for baseline value, age, gender and dementia.

Conclusions: Individually tailored dietary counseling improve protein and nutrients intake among older home care clients.

\section{P-439}

Nutritional status assessment in older patients with cancer: a national cross-sectional Survey (NutriAgeCancer)

Elena Paillaud ${ }^{1}$, Claudia Martinez-Tapia ${ }^{1}$, Anne-Laure Couderc ${ }^{2}$, Mathilde Gisselbrecht ${ }^{3}$, Evelyne Liuu ${ }^{4}$, Frédéric Pamoukdjian ${ }^{5}$, Laure De Decker ${ }^{6}$, Marie-Odile Hager ${ }^{7}$, Jing Remigereau ${ }^{8}$, Berengere Beauplet $^{9}$, Catherine Catenoz Catherine ${ }^{10}$, Pascaline Boudou-Rouquette ${ }^{11}$, Galdric Orvoen ${ }^{12}$ Olivier Guillem ${ }^{13}$, Cherifa Taleb ${ }^{14}$, Cedric Gaxatte ${ }^{15}$, Virginie Fossey-Diaz ${ }^{16}$, Tristan Cudennec ${ }^{17}$, Karin Maley ${ }^{18}$, Capucine Baldini ${ }^{19}$, Gilles Albrand ${ }^{20}$, Florence Rollot-Trad ${ }^{21}$, Damien Heitz ${ }^{22}$, Anne-Laure Scain ${ }^{23}$

${ }^{1}$ DHU A-TVB, IMRB-EA 7376 CEpiA (Clinical Epidemiology and Ageing Unit, Universite Paris-Est, UPEC, 94010, Creteil, ${ }^{2}$ UCOG PACA OUEST, Hopital Sainte Marguerite, Marseille, ${ }^{3}$ UCOG Paris Ouest, Hopital Europeen Georges Pompidou, Paris, 75015, ${ }^{4}$ UCOG Poitou-Charentes, CHU Poitiers, ${ }^{5}$ UCOG Paris Seine Saint Denis, CHU Avicenne, 93 000, ${ }^{6}$ UCOG Pays de la Loire, CHU Nantes, ${ }^{7}$ AOG-Auvergne, Centre Jean Perrin, Clermont-Ferrand, ${ }^{8}$ UCOG Paris Seine Saint Denis, Grand Hôpital de l'Est Francilien - Site de Meaux, ${ }^{9}$ UCOG Normandie; CHU Caen, ${ }^{10}$ UCOG Bretagne, CHU Rennes, ${ }^{11}$ UCOG Paris Ouest, Hopital Cochin, Paris, ${ }^{12}$ UCOG Paris Ouest, Hopital Broca, Paris, ${ }^{13}$ UCOG PACA Est, Centre hospitalier intercommunal des Alpes du Sud, ${ }^{14}$ UCOG Paris Seine Saint Denis; Hopital Rene Muret, Sevran, ${ }^{15}$ UCOG Nord de France CHRU Lilles, ${ }^{16}$ UCOG Paris Nord, hôpital Bretonneau, Paris, ${ }^{17}$ UCOG Paris Ouest, Hopital Amboise Pare, Boulogne-Billancourt, ${ }^{18}$ UCOG Paris Est, Hopital Diaconesses Croix Saint Simon, Paris, ${ }^{19}$ UCOG Paris Sud, Institut Gustave Roussy, Villejuive, ${ }^{20} \mathrm{UCOG}$ Rhodanienne ; CH Lyon Sud, Lyon, ${ }^{21}$ UCOG Paris ouest, Institut Curie, Paris, ${ }^{22}$ UCOG PACA Est, Hôpital de Cimiez, Nice, 06000, ${ }^{23}$ UCOG Strasbourg, Hopital de Hautepierre, strasbourg, 67000, ${ }^{24}$ UCOG Paris SUD, Hopital Henri Mondor, Creteil, 94010

Introduction: Weight loss, an indicator of nutritional status, is common in older patients with cancer and strongly associated with poor outcomes. We aim to assess weight loss in older patients with cancer and identify its associated factors.

Methods: A national cross-sectional survey (44 geriatric oncology clinics, France) of older cancer patients aged $\geq 70$ years (inclusion period: November-December 2017). Data collection: demographic and clinical data and nutritional assessment performed by a geriatrician.

Primary outcome: Weight loss within the last 6 months precedent cancer treatment. Patients were classified into three groups according to the percentage of weight loss (WL): minimal $(\leq 5 \%)$; moderate $(>5$ and $<10 \%)$; and severe $(\geq 10 \%)$.

Results: A total of 571 patients were included in the study median age 83 years (interquartile range 79-87); 50\% males; $43 \%$ with metastasis. The main cancer types were breast $(17 \%)$, colorectal (14\%) and upper digestive tract/pancreas (10\%). In total, 124 patients
(22\%) had moderate WL (mean $5.2 \mathrm{~kg}$; range 2.5-11), and 146 (26\%), had severe WL (mean $12.1 \mathrm{~kg}$; range 5-35). In univariate analysis, patients with moderate or severe weight loss were more likely to have comorbidities $(\mathrm{P}=0.018)$, upper digestive tract/pancreas and lung cancer $(\mathrm{P}=0.001)$, metastasis $(\mathrm{P}=0.016)$, depressive mood $(\mathrm{P}=0.005)$, cognitive impairment $(\mathrm{P}=0.038)$, dependency on Activities of Daily Living, altered mobility and sarcopenia ( $\mathrm{P}<0.0001$ each), when compared to patients with no or minimal weight loss.

Conclusion: Half of the study population have lost more than 5\% of body weight in the last 6 months. Oncologic and geriatric factors are associated with moderate or severe weight loss.

\section{P-440}

Leisure time physical activity is not associated with higher 25OHD in obese community dwelling Icelandic old adults

Ramel Alfons ${ }^{1}$, G. Geirsdottir Olof ${ }^{1}$, Chang Milan ${ }^{1}$, V. Jonsson Palmi ${ }^{1}$, Thorsdottir Inga ${ }^{1}$

${ }^{1}$ University of Iceland, Reykjavik, Iceland

Introduction: In several studies, obesity has been associated with low levels of 25-hydroxy-vitamin D (25OHD). The detailed causes of hypovitaminosis $\mathrm{D}$ in obese individuals have not been clarified. The aim of the present work was to compare $25 \mathrm{OHD}$ in obese and normal-/overweight subjects with particular consideration of physical activity and fish oil intake.

Methods: Community dwelling subjects from the Reykjavik area in Iceland $(\mathrm{N}=229,73.7 \pm 5.7$ years, $58.2 \%$ female $)$ participated in this cross-sectional study. Leisure time physical activity (LTPA), dietary intake, body composition and background variables were assessed. 25OHD was measured in fasting blood samples.

Results: Mean LTPA was $5.7 \pm 5.6 \mathrm{~h} /$ week and the most common activities were walking and gardening. Mean concentration of $25 \mathrm{OHD}$ was $66.7 \pm 28.1 \mathrm{nmol} / \mathrm{L}$ and $8.5 / 21.2 \%$ were below 30 and $50 \mathrm{nmol} / \mathrm{L}$, respectively. Obese participants $(\mathrm{n}=84)$ had lower 25OHD $(-11.0 \pm 3.8 \mathrm{nmol} / \mathrm{L}, \mathrm{P}<0.001)$ and lower LTPA ($2.5+0.8 \mathrm{~h} /$ week, $\mathrm{P}=0.001)$ than normal-/overweight subjects $(\mathrm{n}=145)$. According to linear models corrected for various confounders and stratified by obesity status we found that LTPA (h/week) was associated with higher $25 \mathrm{OHD}$ in normal-/overweight participants only $(1.6 \mathrm{nmol} / \mathrm{L}, \mathrm{P}<0.001)$ but not in obese $(-0.5 \mathrm{nmol}$, $\mathrm{P}=0.394)$. On the other hand, fish oil intake was associated with higher 25OHD both in normal-/overweight $(13.9 \pm 4.5 \mathrm{nmol} / \mathrm{L}$, $\mathrm{P}=0.003)$ and obese subjects $(16.2 \pm 5.3 \mathrm{nmol} / \mathrm{L}, \mathrm{P}=0.003)$.

Key conclusions: Our study shows that obese community dwelling adults in Iceland have lower 25OHD than their normal-/overweight counterparts. We also found that LTPA was associated with higher 25OHD in normal-/overweight, but not in obese participants. Fish oil was associated with higher $25 \mathrm{OHD}$ independently of obesity status and is therefore especially important for obese old adults.

\section{P-441}

Prevalence and risk factors of malnutrition in older hospitalized patients: an observational Middle Eastern study

Nada El Osta ${ }^{1}$, Haissam El Arab ${ }^{2}$, Robert Saad ${ }^{2}$, Antoine Aoun ${ }^{3}$, Amine Wehbe ${ }^{2}$, Lana El Osta ${ }^{2}$

${ }^{1}$ Department of Prosthetic Dentistry, School of Dentistry, SaintJoseph University, Beirut, Lebanon, ${ }^{2}$ Department of Public Health, School of Medicine, Saint-Joseph University, Beirut, Lebanon, 
${ }^{3}$ Faculty of Nursing and Health Sciences, Notre Dame University, Beirut, Lebanon

Introduction: Malnutrition in hospitalized patients is known to be associated with many adverse clinical outcomes and increased healthcare costs. Our study aimed to describe the nutritional status of older hospitalized patients and to evaluate the associated risk factors. Methods: This was an observational cross-sectional study conducted at a major tertiary teaching hospital in Beirut city. During a 7 months period, patients aged 60 and over and admitted in the medical and surgical departments of the hospital were invited to participate in the study. Data were collected by means of a questionnaire including sociodemographic and medical characteristics, the Arabic version of the Mini Nutritional Assessment, the Activity of Daily Living (ADL) scale, and the American Society of Anesthesiologists (ASA) score.

Results: 171 participants aged $73.15 \pm 8.06$ years were included in the study. $52.0 \%$ of them were at risk of malnutrition and $13.5 \%$ were malnourished. Patients hospitalized in medical departments $(16.2 \%)$ presented a high prevalence of malnutrition compared to those hospitalized in surgical departments $(10.5 \%) \quad(\mathrm{p}$ value $=0.003)$. Moreover, low education level, high age, extended hospital stay, number of medical comorbidities, polymedication, high ASA score and low ADL score were significantly associated with malnutrition ( $\mathrm{p}$ value $<0.05)$

Conclusions: Malnutrition is prevalent in senior hospitalized patients and is associated with several specific risk factors. Screening and management of malnutrition should be considered a priority in order to improve the overall medical status of the elderly and to ensure a better quality of life.

\section{P-442}

Associations between BMI, physical function and bone health in community dwelling old adults

Olof Gudny Geirsdottir ${ }^{1}$, Alfons Ramel ${ }^{2}$, Milan Chang ${ }^{3}$, Palmi V Jonsson $^{4}$, Inga Thorsdottir ${ }^{5}$

${ }^{1}$ The Icelandic Gerontological Research Center, Reykjavik, Iceland \& Faculty of Food Science and Nutrition, University of Iceland, Reykjavik, Iceland, ${ }^{2}$ Faculty of Food Science and Nutrition, University of Iceland, Reykjavik, Iceland \& The Icelandic, Reykjavik, Iceland

Background: The associations between BMI, physical function and bone health were investigated in a group of apparently healthy, physically active, community dwelling old adults.

Methods: This was a cross-sectional study including 236 community dwelling old people (age range $65-92$ years, $58.2 \%$ female). Bone mineral density (DXA), timed-up-and-go (TUG), six-minute-walkfor-distance (6MWD), anthropometrics, quadriceps strength and clinical blood variables were assessed.

Results: Mean age was $73.7 \pm 5.7$ years. According to linear models corrected for age, smoking and gender, higher obesity was related to lower quadriceps strength relative to body weight, to lower physical function, i.e., lower gait speed and longer TUG time, as well as to lower 25OHD but to higher PTH. Of the participants, only $1 \%$ of men and $4 \%$ of women had osteoporosis, the corresponding numbers for osteopenia were 35 and $45 \%$. Obesity was related to high bone mineral density (T-score) in femur, lumbar and total.

Conclusions: In this highly functional cohort of old adults, obesity was related to poorer strength and less physical function in older adults which should make obese individuals more prone for falls and fractures. However, although obesity was also related to a poorer hormonal profile, obesity was related to higher BMD. Future studies need to determine the net effects of higher BMI and fall and fracture risk of obese elderly.

\section{P-443}

Determination of malnutrition prevalence in older inpatients by the new ESPEN criteria

\author{
Merve Yllmaz ${ }^{1}$, Sumru Savas ${ }^{2}$, Fulden Sarac ${ }^{2}$, Fehmi Akcicek ${ }^{2}$ \\ ${ }^{1}$ Internal Medicine Department, School of Medicine, Ege University, \\ Izmir, Turkey, ${ }^{2}$ Geriatrics Section, İnternal Medicine Department, \\ School of Medicine, Ege University, Izmir, Turkey
}

Aim: The European Society for Clinical Nutrition and Metabolism (ESPEN) has recently published new criteria for the diagnosis of malnutrition. We also aimed at this retrospective study to determine the prevalence of malnutrition by using the new ESPEN criteria.

Methods: We retrospectively evaluated 52 patients aged 60 years or older hospitalized in the Internal Medicine clinic. Anthropometric measurements and BIA analyses for fat mass (FM), fat-free mass (FFM) were performed. FFMI were calculated by FFM/height ${ }^{2}$. Mini nutritional assessment (MNA-SF) was applied and the patients who were at risk of malnutrition, reassessed with the ESPEN new criteria. Albumin, hs-CRP and total protein values were recorded retrospectively from the hospital records.

Results: The mean age of the study population was $72.4 \pm 8.2$ years and $58 \%$ of the patients were female. The mean weight $(\mathrm{kg})$, body mass index (BMI) $\left(\mathrm{kg} / \mathrm{m}^{2}\right), \mathrm{FM}(\mathrm{kg}), \mathrm{FFM}(\mathrm{kg}), \mathrm{CC}(\mathrm{cm})$ were respectively $67.6 \pm 15.0,25.4 \pm 5.7, \quad 19.7 \pm 11.5, \quad 49.2 \pm 10.9$, $32.8 \pm$ 4.6. Mean levels of the parameters were; albumin: $3.7 \pm 0.5$ g/dL, CRP: $2.3 \pm 3.1 \mathrm{mg} / \mathrm{dL}$, total protein: $7.4 \pm 6.1 \mathrm{~g} / \mathrm{dL}$, respectively. Overall, $30.8 \%(\mathrm{n}=16)$ of patients had malnutrition and $59.6 \%(\mathrm{n}=31)$ had risk of malnutrition with MNA-SF. Patients' malnutrition prevalence was $40.3 \%(n=21)$ with the new ESPEN criteria.

Conclusion: According to ESPEN criteria $40.3 \%$ of patients had malnutrition. PS: Presented in International Izmir Elderly Symposium 2018.

\section{P-444}

Morus nigra $L$. leaf extract for climateric syndrome: A randomized, double-blind, placebo-controlled trial

Leonardo Victor Galvão-Moreira ${ }^{1}$, Joyce Pinheiro Leal Costa ${ }^{1}$, Luiz Gustavo Oliveira Brito ${ }^{2}$, Lucia Helena Simões Costa-Paiva ${ }^{2}$, Haíssa Oliveira Brito ${ }^{1}$, Luciane Maria Oliveira Brito ${ }^{1}$

${ }^{1}$ Federal University of Maranhão (UFMA), São Luís, Brazil, ${ }^{2}$ University of Campinas (UNICAMP), Campinas, Brazil

Objective: To evaluate the efficacy of Morus nigra L. (black mulberry) leaf extract in comparison with hormone replacement therapy (HRT) and placebo for the treatment of climacteric syndrome.

Methods: A randomized, double-blind, placebo-controlled trial was carried out and included a total of 62 climacteric women. Primary endpoints adopted were the severity of climacteric symptoms detected by the Blatt-Kupperman Index (BKI) and quality of life evaluated by the SF-36 scores. Secondary endpoints included biochemical tests, colposcopy and oncotic colpocitology findings, and adverse effects. Patients were randomly assigned into three experimental groups: Black mulberry ( $\mathrm{n}=20$; Morus nigra $L$. capsules $250 \mathrm{mg}$ /day); HRT $(n=20$; capsules of estradiol $1 \mathrm{mg} /$ day with or without norethisterone 
acetate $0.5 \mathrm{mg} /$ day); Placebo $(\mathrm{n}=22$; placebo capsules $250 \mathrm{mg} /$ day $)$. After a 60-day treatment period, patients were reassessed.

Results: IBK scores were decreased in both Black mulberry and HRT groups $(\mathrm{p}<0.05)$, but not in Placebo, after the experimental period. Four domains of SF-36 were improved in the Black mulberry group after treatment, compared to only one domain in TRH and three domains in Placebo $(\mathrm{p}<0.05)$. Biochemical profiles, oncotic colpocitology and colposcopy findings were similar between all groups $(\mathrm{p}>$ $0.05)$. Mastalgia and spotting occurred only among women exposed to HRT.

Conclusion: The effects of Morus nigra $L$. leaf extract in climacteric symptoms were similar to HRT and superior to placebo. An additional improvement in quality of life and less side effects were also shown in patients treated with Morus nigra $L$.

\section{$\mathbf{P}-445$}

The effect of dark chocolate flavanols on cognition in older adults: a randomized controlled trial (FlaSeCo)

Salmenius-Suominen Heli ${ }^{2}$, Jyväkorpi Satu ${ }^{1}$, Pitkãlä Kaisu ${ }^{1}$, Hongisto Sanna-Maria ${ }^{3}$, Tuukkanen Kristiina ${ }^{3}$, Suominen Merja ${ }^{1,2}$

${ }^{1}$ Department of General Practice and Primary Health Care, University of Helsinki, Finland, ${ }^{2}$ Society for Gerontological Nutrition in Finland, ${ }^{3}$ Fazer Group

Background: Cocoa flavanols may have positive effects on cognition, blood lipid levels and glucose metabolism.

Methods: Older adults $65-75$ years without chronic diseases were recruited to 8-week randomized, double-blind controlled trial to investigate effectiveness of cocoa flavanols on cognitive functions. Intervention group received dark chocolate $50 \mathrm{~g}$ containing $410 \mathrm{mg}$ flavanols per day and control group $50 \mathrm{~g} / 86 \mathrm{mg}$ respectively. Cognition was assessed with SLUMS, Verbal Flow (VF) and Trail Making Test (TMT). Food and nutrient intake, quality of life (15D) and depression (GDS30) were assessed. Blood lipids and glucose were measured.

Results: 100 (63\% women) mean 69 years participated. They were highly educated mean 14.9 years (SD 3.6) and their health-related quality of life was good. Total cholesterol in whole group at baseline was 5.6 (SD 0.7) and after intervention 5.6 (SD 0.6). There were no statistically significant differences in changes in cognition between groups. Mean change ( \pm SEs) in the time complete TMT A and B in intervention group was $-4.6(-7.1$ to -2.1$) \mathrm{s}$ and $-16.1(-29.1$ to $-3.1) \mathrm{s}$ and in controls $-4.4(-7.0$ to -1.9$) \mathrm{s}$ and $-12.5(-22.8$ to -2.1 ) s. Blood lipids, glucose levels, quality of life, depression and body weight remain unchanged.

Conclusions: There was no effect of dark chocolate flavanols on cognition among healthy older adults in this study. Study also indicates that inclusion of dark chocolate daily in the diet had no harmful effects on blood lipids, glucose and body weight.

\section{P-446}

Fractures related to OSteoporosis in Umbria: FROST-Umbria study

Maria Lapenna ${ }^{1}$, Marta Baroni ${ }^{1}$, Valentina Prenni ${ }^{1}$, Riccardo Bogini ${ }^{2}$, Rita Valecchi ${ }^{2}$, Giuliana Duranti ${ }^{2}$, Virginia Boccardi ${ }^{1}$, Patrizia Mecocci $^{1}$, Carmelinda Ruggiero ${ }^{1}$

${ }^{1}$ Geriatric and Gerontological Section, S. Maria della Misericordia Teaching Hospital, University of Perugia, Perugia, Italy, ${ }^{2}$ General Medicine, ASL Umbria 1, Umbria Region, Perugia, Italy
Introduction: Standardized algorithms for estimation of fracture risk show low sensibility and specificity and remain unknown to general practitioners (GPs). This study aims to describe the prevalence of low bone mineral density (BMD), major fragility fractures, clinical risk factors and antifracture treatments in the general population visiting the GPs in Umbria region.

Materials and methods: This is a prospective observational study conducted in persons aged 30 or more years, randomly selected from the database of GPs with a comprehensive evaluation including fracture risk, fracture history, diseases, drugs, functional status and lifestyle.

Results: 1356 participants, mainly women $(n=756,55 \%)$ with $63 \%$ $(\mathrm{n}=847)$ of them aged more than 50 years. Low BMD was detected in $10 \%(n=139)$ and history of fragility fractures in $9 \%(n=109)$. Major clinical risk factors were female gender, family history, gait disorders and falls. Disease-causing bone disorders were detected in $5 \%(\mathrm{n}=7)$ and $9 \%(\mathrm{n}=10)$, drug affecting bone metabolism in $9 \%$ $(\mathrm{n}=13)$ and $9 \%(\mathrm{n}=10)$ of persons with low BMD and previous fragility fractures. $56 \%(\mathrm{n}=62)$ persons with previous fragility fractures missed the diagnosis of low bone strength. Antifracture drugs and vitamin D are prescribed to $34 \%$ and $43 \%$ of persons with low BMD, but to $24 \%$ and $22 \%$ of those with previous fragility fractures and a combination therapy in $14 \%$ of persons with low BMD and $9 \%$ of those with a fragility fracture.

Conclusions: Older adults are confirmed as high risk of fractures. The GPs should maximize the clinical judgment while using available algorithms.

\section{P-447}

\section{Fibrinolytic activity in metabolic syndrome}

Sercan Savci ${ }^{1}$, Gizem Ayan ${ }^{2}$, Hakan Yavuzer ${ }^{3}$, Suna Avci ${ }^{3}$, Serdar Ozkok $^{3}$, Rabia Bag Soytas ${ }^{3}$, Deniz Suna Erdincler ${ }^{3}$, Alper Doventas ${ }^{3}$

${ }^{1}$ Istanbul University Cerrahpasa Medical Faculty, Internal Medicine, Istanbul, Turkey, ${ }^{2}$ Health Sciences University, Okmeydani Research and Traşnşng Hospital, Internal Medicine, Istanbul, Turkey,

${ }^{3}$ Department of Geriatric, Istanbul University Cerrahpasa Medical Faculty, Internal Medicine, Istanbul, Turkey

Background: To investigate the relation between the serum levels that have role in inflammation of Peroxisome Proliferator-Activating Receptor Alfa (PPAR- $\alpha$ ), Fibrinogen, Plasminogen Activator Inhibitor (PAI-1), Tissue-Type Plasminogen Activator (t-PA), t-PA/PAI-1 and fibrinolysis in elderly patients with Metabolic syndrome (MetS). Patients and method: 86 patients over 60 years of age, including 43 patients with MetS and 43 healthy controls, were included in the study. The patients who have abdominal obesity and two of these criteria simultaneously: diabetes mellitus or impaired glucose tolerance, hypertension (blood pressure systolic $>130$, diastolic $>85 \mathrm{mmHg}$ or using anti-hypertensive medication), triglyceride level $>150 \mathrm{mg} / \mathrm{dl}$ or $\mathrm{HDL}$ level $<40 \mathrm{mg} / \mathrm{dl}$ in men and $<50 \mathrm{mg} / \mathrm{dl}$ in women were accepted as MetS. Patients' biochemical parameters of follow up visits in the last month, were evaluated. Patient/control group were determined during 12 months. The serum of patients were stored at $-800{ }^{\circ} \mathrm{C}$. PPAR $\alpha$, t-PA, PAI-1, tPA/PAI-1 complex and fibrinogen parameters were studied concurrently with ELISA technique.

Results: No significant correlation was found between PPAR-alpha and fibrinolytic parameters in patients with MetS $(p=0.183)$. Significant associations were found between MetS and PAI-1, tPA, tPA/ PAI-1 complex and fibrinogen $(p=0.009 / 0.001 / 0.011 / 0.022$, respectively). t-PA, t-PA/PAI-1 complex and PAI-1 can be used as markers (AUC:0.753, 0.717, 0.679 respectively) for evaluation of 
fibrinolytic activity in MetS. Fibrinogen and PPAR-alpha have low specificity and sensitivity.

Conclusion: Fibrinolytic activity is increased in MetS, and t-PA, t-PA/PAI-1 complex and PAI-1 are more powerful predictors of this activity than others. Fibrinogen and PPAR-alpha weren't available as valid markers for this evaluation.

\section{P-448}

Prevalence and predictors of subclinical micronutrient deficiency in German older adults: results from the population-based KORA-Age study

Romy Conzade ${ }^{1}$, Wolfgang Koenig ${ }^{2}$, Margit Heier ${ }^{1}$, Andrea Schneider $^{1}$, Eva Grill ${ }^{3}$, Annette Peters ${ }^{1}$, Barbara Thorand ${ }^{1}$

${ }^{1}$ Helmholtz Zentrum München, German Research Center for Environmental Health $(\mathrm{GmbH})$, Munich, Germany, ${ }^{2}$ German Heart Centre Munich, Technical University of Munich, Munich, Germany, ${ }^{3}$ Institute for Medical Information Processing, Biometrics and Epidemiology, Ludwig-Maximilians-University, Munich, Germany

Introduction: Subclinical micronutrient deficiency in older adults is associated with chronic age-related diseases and adverse functional outcomes. In Germany, the older population is at risk of insufficient micronutrient intake, but representative studies on micronutrient status in old and very old adults are scarce.

Objectives: We estimated the prevalence of subclinical vitamin D, folate, vitamin B12 and iron deficiencies among older adults, aged 65-93 years, from the KORA-Age study in Augsburg, Germany $(\mathrm{n}=1079)$, and determined associated predictors.

Methods: Serum concentrations of 25-hydroxyvitamin D (25OHD), folate, vitamin B12, and iron were analyzed and compared to selected cut-offs for subclinical micronutrient deficiency. Predictors of subclinical deficiency were determined using multiple logistic regression analysis.

Results: The prevalence of subclinical vitamin D and vitamin B12 deficiencies were high, with 52.0 and $27.3 \%$ of individuals having low 25OHD $(<50 \mathrm{nmol} / \mathrm{L})$ and low vitamin B12 concentrations $(<221 \mathrm{pmol} / \mathrm{L})$, respectively. Furthermore, $11.0 \%$ had low iron $(\mathrm{men}<11.6 \mu \mathrm{mol} / \mathrm{L}$, women $<9.0 \mu \mathrm{mol} / \mathrm{L})$ and $8.7 \%$ had low folate levels $(<13.6 \mathrm{nmol} / \mathrm{L})$. Common predictors associated with subclinical micronutrient deficiency included very old age, physical inactivity, frailty and no/irregular use of supplements.

Conclusion: Subclinical micronutrient deficiency is a public health concern among older German adults, especially for vitamins D and B12. The predictors identified provide further rationale for screening high-risk subgroups and developing targeted public health interventions to tackle prevailing micronutrient inadequacies among older adults.

\section{P-449}

Exploring the views and dietary practices of older people at risk of malnutrition and their carers: the nutrition in later life study

Christina Avgerinou ${ }^{1}$, Cini Bhanu ${ }^{1}$, Kate Walters ${ }^{1}$, Jennifer Rea ${ }^{1}$, Ann Liljas ${ }^{1}$, Yehudit Bauernfreund ${ }^{1}$, Helen Croker $^{2}$, Kalpa Kharicha ${ }^{1}$

${ }^{1}$ Primary Care and Population Health, University College London, London, UK, ${ }^{2}$ Health Behaviour Research Centre, University College London, London, UK

Background: Malnutrition is an important cause of morbidity and mortality in later life, however it is under-recognised in general practice. There is little evidence on the views of older people and their carers regarding the management of malnutrition in the community. Aims: To explore the views and dietary practices of older people at risk of malnutrition and their carers, to identify gaps in knowledge, barriers/facilitators to eating, and to explore potential interventions to support nutrition in the community.

Methods: Up to 40 semi-structured interviews with communitydwelling people aged $\geq 75$ years from general practices, identified as malnourished/at risk of malnutrition, and their carers. Thematic analysis is being used to identify key emergent themes and their meaning, with input from the research team including lay members. Findings: Early interview findings $(n=27)$ suggest that 'healthy eating' beliefs established in earlier life are maintained in later life. Some participants were aware of recent weight loss, reduced appetite or eating smaller portions, although they did not always understand why. Some reported reduced appetite and low energy which they considered part of normal ageing, whereas others attributed weight loss to acute illness, incomplete recovery following discharge from hospital, mental health issues and other conditions. Although only a few of them had discussed weight loss with their GP, many liked the idea of a nutritional intervention delivered in primary care. Some felt that the doctor was best placed to provide that support, whereas others would welcome advice from a nurse, dietitian or other trained professional.

\section{P-450}

The importance of measuring nutritional status among elderly patients with previous cardiovascular disease in north-eastern of Romania

Ioana Dana Alexa ${ }^{1}$, Adina Carmen $\mathrm{Ilie}^{2}$, Ramona Stefaniu ${ }^{2}$, Ioana Alexandra Sandu ${ }^{2}$, Irina Mihaela Abdulan ${ }^{2}$

${ }^{1}$ University of Medicine and Pharmacy "Grigore T Popa" Iasi, Iasi, Romania, ${ }^{2}$ University of Medicine and Pharmacy "Grigore T Popa" Iasi, Iasi, Romania

Introduction: Of all the morbid conditions affecting the elderly, cardiovascular disease is the most common cause of mortality over which the subclinical nutritional deficiency often overlaps.

Material and methods: The study group consisted of 481 elderly patients with a history of cardiovascular disease, divided into two groups, 319 patients with rhythm disorders and 162 without rhythm disorders, from the Geriatrics Department, "Dr. C.I.Parhon “, Iasi. All patients were applied a questionnaire Mini assessment nutrition (MNA), validated screening malnutrition.

Results and discussions: Age group shows a significantly higher percentage of people with malnutrition at the age of 75 years $(76 \%$; $\mathrm{p}=0.011$ ) and from $24 \%$ of people with normal nutritional status, $41.2 \%$ associate different degrees of obesity $(p=0,001)$.In the group with rhythm disorders, $59.2 \%$ of patients are at risk of malnutrition and $15.7 \%$ are malnourished. Similar percentages were also recorded in the group without rhythm disorders - $60.5 \%$, at risk of malnutrition and $15.4 \%$ malnutrition. Of those malnourished, $66.7 \%$ had rhythm disorders and $66.8 \%$ of patients with malnutrition risk had rhythm disorders $(\mathrm{p}=0.995)$.

Conclusions: Malnutrition and the risk of malnutrition among geriatric patients is a cardiovascular risk factor. Patients declared with a normal nutritional status through MNA and with obesity degrees require additional exploration to detect sarcopenic obesity. The absence of statistically significant percentage differences between the two studied groups allows us to affirm that malnutrition is not a direct factor for rhythm disorders. 


\section{P-451}

Relationship of nutrients intake to functional and cognitive status in community-dwelling and institutionalized older people: a casecontrol study

Agnieszka Guligowska ${ }^{1}$, Małgorzata Pigłowska ${ }^{1}$, Tomasz Kostka ${ }^{1}$

${ }^{1}$ Department of Geriatrics, Medical University of Lodz, Lodz, Poland

Objectives: The study is a case-control analysis of the relationship between nutrient intake, nutritional status and physical and cognitive functioning in two groups of elderly: community-dwelling (CD) and institutionalized $(\mathrm{NH})$.

Patients and methods: A total of 200 older subjects (aged $73 \pm 10$ years) from Lodz, Poland, participated in the study: 100 from nursing homes ( 31 men and 69 women) and 100 age- and sexmatched community-dwelling controls. Nutritional and functional status we assessed with Mini Nutritional Assessment (MNA), MiniMental State Examination (MMSE), activities of daily living (ADL) and Timed "Up \& Go" test (TUG). The pattern of consumption of various nutrients was analyzed in detail.

Results: There were no differences in energy intake but the NH group consumed significantly less animal protein, long chain poly-unsaturated fatty acids, dietary fiber, vitamins and minerals and more sucrose. According to MNA, only $19 \%$ had normal nutritional status (vs. 78\% of CD group). Higher intake of sucrose and lower intake of protein, vitamins C, B6, B2, B3, folates, magnesium, iron, copper, phosphorus and potassium was associated with worse results in the ADL, TUG and MMSE tests.

Conclusion: This analysis shows deeply unsatisfactory nutritional status and enormous deficiencies in the diet of institutionalized elderly. The observed relationships indicate the importance of intake of an appropriate level of protein, vitamins B and minerals, and limitation of sucrose intake. Stronger correlations in the CD group are likely associated with a greater diversity of their diet.

\section{$\mathbf{P}-452$}

A dangerous combination in geriatrics; obesity and malnutrition

İbrahim Halil Türkbeyler ${ }^{1}$, Zeynel Abidin Öztürk² ${ }^{2}$ Mehmet Göl ${ }^{3}$, Azer Abiyev ${ }^{4}$

${ }^{1}$ Ersin Aslan Education and Research Hospital, Gaziantep, Turkey, ${ }^{2}$ Department of Geriatrics, Faculty of Medicine, Gaziantep University, Gaziantep, Turkey, ${ }^{3}$ Department of Physiology, Faculty of Medicine, Gaziantep University, Gaziantep, Turkey, ${ }^{4}$ Faculty of Medicine, Gaziantep University, Gaziantep, Turkey

Aim: All societies, including Turkish society, are experiencing a dramatic growth in proportion of elderly individuals. Geriatric malnutrition and obesity are substantial health problems. The aim of this study is to investigate the prevalence of malnutrition and obesity in elderly individuals attending a geriatric outpatient clinic in Turkey.

Methods: This study included 520 elderly patients. All patients underwent Mini Nutritional Assessment-Short Form (MNA-SF) test via face to face interview. The participants were divided into four groups, as follows: underweight group (BMI $<22.0 \mathrm{~kg} / \mathrm{m}^{2}$ ), normal weight group (BMI $22.0-24.9 \mathrm{~kg} / \mathrm{m}^{2}$ ), overweight group (BMI $25.0-29.9 \mathrm{~kg} / \mathrm{m}^{2}$ ) and obese group (BMI $\geq 30 \mathrm{~kg} / \mathrm{m}^{2}$ ).

Results: The mean age was $71.8 \pm 5.8$ and 285 patients $(54.8 \%)$ were female. $11(2.1 \%)$ of them were underweight, $91(17.5 \%)$ were normal, $193(37.1 \%)$ were overweight and $225(43.3 \%)$ were obese in conformity with BMI measurements. According to MNA-SF, 37 $(7.1 \%)$ were diagnosed with malnutrition, $165(31.7 \%)$ had a risk of malnutrition, and $318(61.2 \%)$ displayed a good nutritional status.
$68.7 \%$ of them were obese or at risk of malnutrition and $33.7 \%$ were both obese and at the risk of malnutrition.

Conclusion: At least two of every three elderly patients exhibited malnutrition or obesity as a consequence of a disequilibrium in nutrition. To prevent malnutriton and obesity in old age, elderly patients should be evaluated comprehensively through several domains including assessment of medical, nutritional, or social statues, novel strategies should be found to promote physical activity through out the society and to raise awareness about the nutrition.

\section{P-453}

Does adequate energy intake 2 weeks after discharge prevent weight loss and preserve ADL function eight weeks after discharge?

Jette Lindegaard Pedersen ${ }^{1}$, Else Marie Damsgaard ${ }^{1}$, Preben Ulrich Pedersen ${ }^{2}$

${ }^{1}$ Department of Geriatrics, Aarhus University Hospital, Aarhus, Denmark, ${ }^{2}$ Aalborg University, Aalborg, Denmark

Background: Disease-related malnutrition leads to poor appetite and decreased food intake. This affects the convalescence negatively in older adults after discharge from hospital.

Objective: To compare the effect of adequate and inadequate energy intake 2 week after discharge on weight loss and ADL function when measured 8 weeks after discharge.

Method: The study sample comprises two intervention groups from an RCT.

Inclusion: Malnourishment or risk of malnutrition, 75+ years, home dwelling and living alone.

Exclusion: Terminal illness, cognitive impairment and nursing home residency. At discharge, all patients received a diet plan covering their specific daily energy needs. Individualised nutritional counselling was given 1, 2 and 4 weeks after discharge. Based on patient reported data on daily food intake at week two after discharge, the patients were grouped into two groups (1) participants who had adequate energy intake (AQ) and (2) participants who had inadequate intake (IAQ). Weight loss and ADL was analysed using $\chi^{2}$-test.

Results: In our study, 111 patients were included 2 weeks after discharge $(\mathrm{AQ}=48, \mathrm{IAQ}=63)$. Both groups had lost weight $(\mathrm{AQ}=$ mean $0.5 \mathrm{~kg}$, IAQ = mean $1.6 \mathrm{~kg})$, but in the IAQ group more patients had $(\mathrm{p}=0.03)$. Mean ADL improved in both groups, but there was no significant difference between groups $(p=0.21)$. ADL deteriorated in $6 \%$ of AQ patients and $17 \%$ of IAQ patients $(\mathrm{p}=0.06)$.

Conclusion: Adequate energy intake 2 weeks after discharge from hospital may reduce weight loss. A trend towards maintained ADL was seen 8 weeks after discharge of patients who had adequate energy intake at week two.

\section{P-454}

Systematic review of non-pharmacological interventions to prevent or treat malnutrition in older people. The SENATOR (ONTOP series) and MaNuEL Knowledge Hub project

Andrea Correa-Pérez ${ }^{1}$, Iosef Abraha ${ }^{2}$, Antonio Cherubini ${ }^{2}$, Avril Collinson ${ }^{3}$, Dominique Dardevet ${ }^{4}$, Lisette CPGM de Groot ${ }^{5}$, Marian A. E. de van der Schueren ${ }^{6}$, Antje Hebestreit ${ }^{7}$, Mary Hickson ${ }^{3}$, Javier Jaramillo-Hidalgo ${ }^{8}$, 
Isabel Lozano-Montoya ${ }^{1,9}$, Denis O’Mahony ${ }^{10}$, Roy L. Soiza ${ }^{11}$, Marjolein Visser ${ }^{12}$, Dorothee Volkert ${ }^{13}$, Maike olters ${ }^{7}$,

Alfonso J Cuz Jentoft ${ }^{1}$

${ }^{1}$ Servicio de Geriatría. Hospital Universitario Ramón y Cajal (IRYCIS), Madrid, Spain, ${ }^{2}$ Geriatria, Accettazione geriatrica e Centro di ricerca per l'invecchiamento, Italian National Research Center on Aging (IRCCS-INRCA), Ancona, Italy, ${ }^{3}$ Institute of Health and Community, University of Plymouth. United Kingdom, ${ }^{4}$ Université Clermont Auvergne, INRA, UNH, Centre de Recherche en Nutrition Humaine (CRNH). Clermont-Ferrand, France, ${ }^{5}$ Division of Human Nutrition and Health, Wageningen University. Wageningen,

Netherlands, ${ }^{6}$ Department of nutrition and health, HAN University of Applied Sciences, Nijmegen, The Netherlands, and department of nutrition and dietetics, VU University Medical Center, Amsterdam, the Netherlands, ${ }^{7}$ Leibniz Institute for Prevention Research and Epidemiology - BIPS, Bremen, Germany, ${ }^{8}$ Servicio de Geriatría, Hospital Central de la Cruz Roja San José y Santa Adela, Madrid, Spain, ${ }^{9}$ Servicio de Medicina Interna, Hospital El Escorial, Madrid, Spain, ${ }^{10}$ Department of Geriatric Medicine, Cork University Hospital, Cork, Ireland, ${ }^{11}$ Department of Medicine for the Elderly, NHS Grampian, Aberdeen, United Kingdom, ${ }^{12}$ Department of Health Sciences, Vrije Universiteit Amsterdam, The Netherlands, ${ }^{13}$ Institute for Biomedicine of Aging, Friedrich-Alexander-Universität ErlangenNörnberg, Nuremberg, Germany

Introduction: Systematic reviews (SRs) on malnutrition suggest that energy and protein intake can be improved, but results for functional or clinical outcomes have been inconclusive. Following the ONTOP methodology, we aimed to perform a review of SRs of non-pharmacological interventions in older patients with well-defined malnutrition using relevant outcomes agreed by a broad panel of experts.

Method: SPubMed, Cochrane, EMBASE, and CINHAL databases were searched for SRs. Primary studies from those SRs, in any setting, were included. Quality assessment was made using Cochrane and GRADE criteria.

Results: Nineteen primary studies from seventeen SRs were included. The most frequent intervention was oral nutritional supplementation (ONS) compared with usual care (11 RCTs). Meta-analysis was only feasible for six studies assessing changes in body weight (BW) and two studies for body mass index (BMI) at follow-up. Neither showed beneficial effects of the intervention [mean difference (95\% CI) BW $0.59(-0.08,1.96)$; BMI $0.31(-0.17,0.79)]$. No beneficial effects of ONS treatment in MNA scores, muscle strength, activities of daily living, timed Up\&Go, quality of life and mortality were found. Other intervention studies (dietary counselling and ONS, ONS combined with exercise, nutrition delivery systems) could not be pooled and results of individual studies were inconsistent. The overall quality of the evidence was very low due to risk of bias and small sample size. Conclusions: This review does not show beneficial effects of ONS or other non-pharmacological interventions versus usual care on nutritional, functional or other health outcomes in older malnourished patients. High quality research studies are urgently needed in this area.

\section{$\mathbf{P}-455$}

Survey on nutrition in older patients before and after hospital admission: a multicentric study

Francisco J. Tarazona-Santabalbina ${ }^{1}$, Katrien Cobbaert ${ }^{2}$, Natassa Koutsouri ${ }^{3}$, Hanadi Al-Hamad ${ }^{4}$, Bülent Saka ${ }^{5}$, Thomas Münzer ${ }^{6}$

${ }^{1}$ Department of Geriatric Medicine, University Hospital of La Ribera, Alzira, Valencia, Spain; Catholic University of Valencia, Sant Vicent
Màrtir, Valencia, Spain, ${ }^{2}$ AZ Delta, Roeselare, Belgium, ${ }^{3}$ Department of Internal Medicine, Erricos Dunant Hospital, Athens, Greece

Introduction: Adequate nutritional intake is a serious problem in older adults following acute hospital admission. Awareness of malnutrition and inadequate food intake is crucial in providing appropriate care and implementing actions to increase food intake during hospitalization.

Methods: Multicenter survey to assess the prevalence of nutrition before hospital admission and during the hospital stay in Belgium, Greece, Qatar, Turkey, Spain and Switzerland. Data included anthropometric, nutritional assessment, medical history and medication variables.

Results: We analyzed 277 patients, mean $( \pm$ SD) age 81.4 years (8.5) years, 56\% women. Half of the patients consumed 3/4-1/4 of the amount food of the week prior to admission. Daily requirements and the effective input differed by $28 \%$. During the hospital stay $41 \%$ required assistance to eat. 53\%, underwent routine nutrition screening, $45 \%$ rated the hospital food as satisfying. $89 \%$ underweight patients were identified as malnourished and $67 \%$ of those received oral protein supplements. $56 \%$ of underweight patients ate less than $1 / 4$ of a normal amount the week prior to hospital admission compared with $12 \%$ of the remaining patients $(\mathrm{p}=0.003)$.

Conclusions: This survey highlights the necessity of routine nutrition assessment in geriatric patients. Underweight seems an indicator for poor nutrition at home. Given that malnutrition is a risk factor for poor hospital outcome and mortality, nutrition screening should be part of ambulatory care. Likewise, future studies on nutritional interventions in hospitals to improve this observed situation are necessary.

\section{P-456}

Relationship of adherencie to mediterranean diet with new anthropometric indices in Spanish elderly of rural areas

Sergio Rico-Martín ${ }^{1}$, Sergio Cordovilla Guardia ${ }^{1}$, Cristina Franco Antonio ${ }^{1}$, Esperanza Santano Mogena ${ }^{1}$, David Conde Caballero ${ }^{1}$, Lorenzo Mariano Juárez ${ }^{1}$, Mariana Martínez Álvarez ${ }^{2}$, Jorge De Nicolás Jiménez ${ }^{1}$, Julián Fernando Calderón-G

${ }^{1}$ Nursing and Occupational Therapy College, University of Extremadura, Badajoz, Spain, ${ }^{2}$ Faculty of Medicine, University of Extremadura, Badajoz, Spain

Background and objective: Several anthropometric indices can be used to estimate obesity. The objective was to relate the level of adherence to the Mediterranean diet with new anthropometric indices to predict obesity in Spanish elderly of rural areas.

Methods: This cross-sectional study investigated a consecutive sample of 266 healthy elderly (96 males and 170 females; age mean $72 \pm 6$ ), who had lived in rural areas of Cáceres (Spain). Seven obesity indices, including the body mass index (BMI), waist circumference (WC), Clínica Universidad de Navarra-body adiposity estimation (CUN-BAE), conicity index (CI), body roundness index (BRI) body shape index (ABSI) and waist-to-height ratio (WHtR) were investigated. A validated 14-item questionnaire (PREDIMED: PREvención con DIeta MEDiterránea) was used for the assessment of adherence to the Mediterranean diet. The questionnaire considers low adherence to the Mediterranean diet at scores $\leq 9$.

Results: Of the whole population, 141 (53\%) participants had low adherence to the Mediterranean diet $($ score $\leq 9)$. We found significant differences between the groups low and high adherence to the Mediterranean diet with respect to BMI $(31 \pm 5$ vs $27 \pm 4$; $\mathrm{p}<0.001)$, CUN-BAE (42 \pm 7 vs $38 \pm 7 ; \mathrm{p}<0.001)$, BRI $(7 \pm 3$ vs $6 \pm 2 ; \mathrm{p}=0.013)$ and $\mathrm{WHtR}(0.64 \pm 0.10$ vs $0.61 \pm 0.08$; 
$p=0.012$ ). After adjusting by age, sex, smoking and level of physical activity, these differences are maintained.

Conclusions: BMI, CUN-BAE, BRI and WHtR have been related to adherence to the Mediterranean diet. Subjects with low adherence to the Mediterranean diet obtained higher values.

Funding: This work was supported by the 4 IE project (0045-4 IE-4P) funded by the Interreg V-A España-Portugal (POCTEP) 2014-2020 program.

\section{P-457}

Decreased rehospitalization risk in elderly patients with iron deficiency (ID) hospitalized in a Geriatric Recuperative Care and Rehabilitation Unit (GRCRU) who received intravenous iron (IVI) supplementation: a case-control study

Catherine Legué ${ }^{1}$, Pierre Levy ${ }^{2}$, Sylvie Meaume ${ }^{1}$, Michel Vaubourdolle ${ }^{3}$, Christine Forasassi ${ }^{1}$

${ }^{1}$ Service de Gériatrie, Hopital Rothschild, APHP, 75012, Paris, France, ${ }^{2}$ Unité de Santé Publique, Hopital Tenon, APHP, 75020, Paris, France, ${ }^{3}$ Pôle Biologie médicale et Pathologie, Hopital Saint Antoine, APHP, 75012, Paris, France

Introduction: ID and rehospitalization are frequent, deleterious and associated with a poor prognosis in elderly patients. Main purpose of this study was to determine if IVI supplementation in patients with ID hospitalized in a geriatric RCRU can reduce their risk of rehospitalization within 90 days of hospital discharge. Secondary objective is to evaluate the association between ID and risk of 90-day readmission. Methods: A case-control study among patients with ID hospitalized in a GRCRU. Cases were 30 patients who were readmitted to the hospital within 90 days of discharge. The 30 control patients (not readmitted) were matched to the case group by 9 factors (anemia, inflammation, undernutrition, polypharmacy, functional dependence, cognitive disorders, comorbidity, risk of rehospitalization and fall). We used $\chi^{2}$ test to search a significant difference in the number of patients who received IVI between the 2 groups. Secondary, we selected 35 patients rehospitalized versus 35 non-rehospitalized with individual matching on the same 9 factors and also IVI administrations or not. We used $\chi^{2}$ test to search a significant difference in the presence of ID between the 2 groups.

Results: ID supplementation with IVI in GRCRU patients reduced significantly the risk of 90-day rehospitalization after discharge from hospital $(\mathrm{p}=0.0008)$. There is no significant difference in ID prevalence among the 2 groups $(\mathrm{p}=0.23)$.

Conclusions: The diagnosis of ID and its supplementation with IVI improve the prognosis of elderly hospitalized patients. It would be interesting to develop, standardize and systematize this care.

\section{P-458}

Association of polypharmacy with nutritional status and daily living function in older outpatients

Kaori Kinoshita $^{1}$, Shosuke Satake ${ }^{1}$, Shuji Kawashima ${ }^{1}$, Keiji Nishihara ${ }^{1}$, Hidetoshi Endo ${ }^{1}$, Hidenori Arai $^{1}$

${ }^{1}$ National Center for Geriatrics and Gerontology, Obu, Japan

Introduction: To determine the association of polypharmacy with malnutrition and functional ability in daily lives among older people. Methods: Subjects were 396 independent outpatients of National Center for Geriatrics and Gerontology, Japan, aged $\geq 65$ years who had not been diagnosed as dementia. We assessed nutritional status using the short form of mini nutritional assessment (MNA-SF) and evaluated daily living function with Kihon-Checklist (KCL). Polypharmacy status was categorized as non-polypharmacy $(0-5$ drugs), polypharmacy (6+ drugs). We used ANCOVA and Logistic regression model to analyze the impact of polypharmacy on MNA-SF and KCL.

Results: We identified 157 patients as polypharmacy. The MNA-SF was significantly lower in the polypharmacy group than in the nonpolypharmacy group $(9.8 \pm 2.9$ vs $10.6 \pm 2.6$, respectively; $\mathrm{P}=0.005$ ). Polypharmacy was significantly associated with unintentional weight loss ( $\geq 2 \mathrm{~kg} / 6$ months) by logistic regression analysis [odds ratio (OR) 1.6, 95\% confidence interval (CI) 1.0-2.4]. Polypharmacy and daily living function were significantly associated with low endurance (OR 2.1, 95\% CI 1.2-2.9), fear of falling (OR 2.2 95\% CI 1.3-3.8), dry mouth (OR 1.9, 95\% CI 1.2-2.9), and low activity (OR $1.6,95 \%$ CI 1.0-2.4).

Key conclusions: There was a significant relationship between polypharmacy and unintentional weight loss. Polypharmacy was also significantly associated with several daily living functions which could affect components of physical frailty.

\section{P-459}

Fluid balance problems among elderly people

Nurhan Bayraktar ${ }^{1}$, Umran Dal Yilmaz ${ }^{1}$

${ }^{1}$ Near East University Faculty of Nursing, Nicosia, Cyprus

Introduction: Water is vital for biochemical processes to activity of the human body. Ageing is associated with many changes in the homeostatic systems involved in the regulation of water and electrolyte balance. This review provides an overview to relevant literature on fluid balance problems of older people.

Methods: A literature search (2000-2018) was carried out, using MEDLINE, Pub Med, Science direct, Scopus, Cochrane library, Turkish Medical Index and related intuitional websites. All references including researches or reviews were included in this search.

Results: In the relevant literature, there are many references addressing fluid and electrolyte balance problems of the elderly people. Age-related physiological changes predispose elderly people to fluid imbalances which can cause morbidity and mortality with causes ranging from physical disability restricting access to fluid intake to iatrogenic causes including polypharmacy and unmonitored diuretic usage. Dehydration is the most common fluid and electrolyte problem among the elderly. Older adults are also susceptible to water retention and related electrolyte abnormalities.

Key conclusions: As fluid imbalances in the elderly are associated with poor health outcomes, including increased risk of disability and mortality, prevention may improve health, functional status, and quality of life. Staff in institutions should be careful about the hydration status of any elderly people. Improved awareness, diagnosing and managing are important among health care providers to prevent fluid balance problems in elderly people.

\section{P-460}

A social network intervention to promote diet quality in older adults: a pilot study

Ilse Bloom ${ }^{1}$, Lindsay Welch ${ }^{2}$, Ivaylo Vassilev ${ }^{3}$, Anne Rogers ${ }^{4}$, Karen Jameson ${ }^{5}$, Janis Baird ${ }^{1}$, Cyrus Cooper ${ }^{6}$, Sian Robinson ${ }^{1}$

${ }^{1}$ MRC Lifecourse Epidemiology Unit, University of Southampton, Southampton, UK, ${ }^{2}$ NIHR Southampton Biomedical Research Centre, 
University of Southampton and University Hospital Southampton NHS Foundation Trust, Southampton, UK, ${ }^{3}$ Faculty of Health Southampton, Southampton, UK

Introduction: Higher diet quality (greater consumption of fruits, vegetables, wholegrain cereals) in older people is associated with better health. Social factors, such as social support, social networks and participation in activities, have been linked with higher diet quality in older people. This pilot study describes the implementation and evaluation of a social networking tool (GENIE-Generating Engagement in Network Involvement), which facilitates engagement with local support resources and activities. Using a randomised control trial design, the study aimed to assess the impact of GENIE on diet quality in a group of older community-dwelling adults with Chronic Obstructive Pulmonary Disease (COPD) and to compare changes with those in a control group.

Methods: Twenty-two men and women [mean (SD) age 71.7 (6.7) years] were recruited from a local COPD Service. Diet was assessed by administered food frequency questionnaire; diet scores were calculated to describe diet quality at baseline and follow-up [median follow-up time in months (IQR) 3.2, (3.0-4.4)]. Change in diet scores were expressed per month, from baseline to follow-up.

Results: There was no change in diet quality in the intervention group over the period of follow-up [median change per month (IQR): 0.03 , $(-0.24-0.07)]$; whereas an overall fall in diet quality was observed in the control group $[-0.15,(-0.24$ to 0.03$)]$. The difference between groups was not statistically significant $(P=0.26)$.

Conclusions: These data indicate potential for beneficial effects of GENIE on quality of diet among older adults. It is not clear why diet quality declined among control participants during the study. Further evaluation in a larger group is needed.

\section{P-461}

Cross-sectional study on prevalence of functional dyspepsia in community-dwelling older people

Jurgita Knasiene ${ }^{1}$, Gertruda Rudaityte ${ }^{2}$, Vaiva Tiskute ${ }^{2}$

${ }^{1}$ Geriatric Department, Lithuanian University of Health Sciences, Kaunas Clinical Hospital, Kaunas, Lithuania, ${ }^{2}$ Lithuanian University of Health Sciences, Kaunas, Lithuania

Introduction: Chronic unexplained epigastric pain or burning, or postprandial fullness or early satiety, labeled as functional dyspepsia (FD), is a common disorder in older people which adversely impacts the quality of life.

Methods: In cross-sectional study 300 community-dwelling older adults were questioned. We analyzed the following variables: gender, age, food, drugs, satisfaction with health status, most common symptoms of FD and SODA (severity of dyspepsia assessment) questionnaire.

Results: FD symptoms were identified in 96 (31.9\%) cases. There is no statistically significant difference in prevalence of dyspepsia between male and female $(\mathrm{p}=0.128)$ and no correlation between age and FD symptoms $(p=0.276)$. Our findings show that there is relationship between FD symptoms and satisfaction with health status. Statistically significant is the satisfaction with its current state comparing healthy $(8.68 \pm 0.23)$ and FD people $(5.99 \pm 0.22)$ $(p<0.01)$. Patients with FD are more likely to feel slightly unwell than healthy subjects $(\mathrm{p}<0.01) .18(18.8 \%)$ of FD patients think that their symptoms are related to drug use. Patients with FD more often experienced symptoms related to drug use than healthy subjects $(\mathrm{p}<0.05)$. Also $80(83.3 \%)$ respondents of FD patients think that their symptoms are related to consumed food. The observed difference is statistically significant between healthy and FD patients $(\mathrm{p}<0.01)$.

Conclusions: Prevalence of dyspepsia was recorded for one third of older adults. There is no statistically significant difference in prevalence of dyspepsia between gender and age. And there is statistically significant relationship between FD symptoms and food, drugs use, satisfaction with health status.

\section{P-462}

The prevalence of metabolic syndrome and lifestyle factors among community living older adults in iceland: cross-sectional study

Milan Chang ${ }^{1}$, Alfons Ramel ${ }^{2}$, Palmi V. Jonsson ${ }^{3}$, Inga Thorsdottir ${ }^{2}$, Olof Gudny Geirsdottir ${ }^{2}$

${ }^{1}$ Faculty of Health Promotion, Sport, and Leisure Studies, University of Iceland, Reykjavik, Iceland, ${ }^{2}$ Faculty of Food Science and Nutrition, University of Iceland, Reykjavik, Iceland, ${ }^{3}$ Faculty of Medicine, University of Iceland, Reykjavik, Iceland

One of the strong indicators of increased risk for cardiovascular disease and diabetes is Metabolic syndrome (MetS). Epidemiological studies have shown that lifestyle, such as physical activity (PA) and body mass index (BMI), is associated with increased prevalence of MetS. The prevalences of MetS, inactivity, and obesity are particularly higher among older adults. The purpose of the study was to investigate the joint association between MetS and combination of two lifestyle factors, PA and BMI, among healthy communitydwelling older adults. Participants were 237 older adults (73.7 \pm 5.7 years, $58.2 \%$ female). The main outcome was the prevalence of MetS which is defined according to NIH criteria. Participants with 3 or more risk factors (waistline $>=102 \mathrm{~cm}$ for men and waistline $>=88 \mathrm{~cm}$ for women, triglycerides $>=150 \mathrm{mg} / \mathrm{dL}$, HDL cholesterol $<50 \mathrm{mg} / \mathrm{dL}$ for women and $<40 \mathrm{mg} / \mathrm{dL}$ for men, a blood pressure $>=130 / 85 \mathrm{mmHg}$, and fasting glucose $>=100 \mathrm{mg} /$ $\mathrm{dL})$ were defined as having MetS. The study participants were divided into 4 groups according to the level of PA (low 50\% vs. high 50\%) and BMI [normal and high (overweight/obese)]. The association was examined using logistic regression, adjusted for age, gender and cognitive function. Those with normal BMI and high PA level had no MetS and were excluded from the analysis. Compared with people who have normal BMI and low PA, those who had high BMI and low PA had a significantly higher likelihood of having MetS [odds ratio (OR); 15.0, 95\% confidence interval (CI) 3.4-66.1, $\mathrm{P}<0.0001]$. Those with high BMI and high PA level had attenuated but still significantly higher likelihood of having MetS (OR 10.0, 95\% CI 2.2-44.9, $\mathrm{P}=0.003$ ). Those with high BMI had increased risk of having MetS compared with those who had normal BMI. Although BMI plays a critical role in the definition of MetS, higher PA level showed that it may reduce the risk of having MetS among older adults for those with high BMI.

\section{P-463}

A comparison of the safety of starch vs. gum based thickening products for patients with post stroke dysphagia

\section{G. Aldridge ${ }^{1}$, K. Nagaratnam ${ }^{1}$}

${ }^{1}$ Royal Berkshire Hospital, Reading, UK

Introduction: Dysphagia is estimated to affect $50-70 \%$ of acute stroke patients [1]. In the acute phase, dysphagia management 
commonly centres on compensatory measures such as diet and fluid modification [2]. Fluid thickener is one such compensatory measure. Two varieties of thickening products are available-starch-based and gum-based. The gum-based product has seen growing use secondary to reported shortcomings of starch-based products, such as poor stability, product becoming too thick over time or becoming thin on contact with salivary amylase, and ambiguous instructions [3].

Method: Starch-based and gum-based thickening products were trialled on the acute stroke unit at two different time periods. During each period, drinks available to patients (at their bedside) were rated by a qualified SLT for their compliance with specific fluid consistency recommendation.

Results: Total of 22 assessments were carried out. Compliance of the starch-based product was found to be poor; only $11 \%$ of drinks rated as appropriate to patient's specific SLT recommendation. The gumbased product was found to have improved compliance with $92 \%$ of drinks rated as appropriate.

Conclusion: This audit demonstrates there is high incidence of patients with dysphagia receiving inappropriate fluid consistency due to poor stability of starch-based thickening products and gum-based product is likely to improve the compliance of recommended fluid consistency.

\section{References:}

1. Martino R (2005) Dysphagia after stroke: incidence, diagnosis, and pulmonary complications. Stroke 36(12):2756-63

2. Singh S (2006) Dysphagia in stroke patients. Postgrad Med J $82: 383-391$

3. Hanson B (2012) The effect of saliva on the viscosity of thickened drinks. Dysphagia. 27:10-19

\section{P-464}

\section{A nutrition-focused program targeting at risk for malnutrition} community-dwellers reduces hospitalization rates

Katie Riley ${ }^{1}$, Suela Sulo ${ }^{2}$, Firas Dabbous ${ }^{1}$, Jamie Partridge ${ }^{2}$, Sarah Kozmic ${ }^{1}$, Wendy Landow ${ }^{1}$, Gretchen VanDerBosch ${ }^{1}$, Mary Kay Falson ${ }^{1}$, Krishnan Sriram ${ }^{1}$

${ }^{1}$ Advocate Health Care, Downers Grove, IL, USA, ${ }^{2}$ Abbott Nutrition, Columbus, OH, USA

Introduction: Malnourished, especially undernourished, patients transitioning through different healthcare settings experience different adverse events when their nutrition status remains unaddressed. This is especially true for at-risk/malnourished older adult patients receiving home health care services. We evaluated the impact of a nutrition-focused Quality Improvement Program (QIP) on hospitalization risk of at-risk/malnourished patients receiving home health care and especially older adults.

Methods: Data from 1546 QIP patients and 7413 pre-QIP historical controls were analyzed. Patients were categorized based on admission source [hospital $(n=5072)$, skilled nursing facility $(\mathrm{SNF})(\mathrm{n}=2332)$, and outpatient office $(n=925)]$ and age $[<65(n=2083), \geq 65$ $(n=6876)]$. QIP patients were screened for malnutrition upon admission to home health. Nutrition education and oral nutritional supplements were administered to at-risk/malnourished patients.

Results: Hospitalization rates at 30, 60, and 90 days reduced significantly for all QIP patients compared with pre-QIP historical controls [relative risk reductions (RRRs): $24.3,22.6$, and $18 \%$, respectively; $\mathrm{p}$ values $<0.001]$. The highest impact was observed for outpatients (RRRs: $38.9,48.7$, and $44.7 \%$, respectively; $p$ values $<0.05$ ), followed by SNF [RRRs of 39.3, 31.7, 30.6\% (p values $<0.01$ )] and hospital [RRRs of $16.3,12.4$, and $7.1 \%$ (p values $>0.05$ )] patients. Notably, hospitalization rate reduction was statistically significant for $\geq 65$ patient population (RRRs: $26.3,24.5$, and $20.1 \%$, respectively, p values $<0.001$ ).

Conclusions: Unplanned hospitalizations were significantly decreased among at-risk/malnourished home health patients following implementation of the nutrition-focused QIP. These results highlight the importance of similar QIPs for all home health patients but most importantly for older adults, who are more likely to experience malnutrition and poor health outcomes.

\section{P-465}

Dietary protein and muscle in aging people: the potential role of the gut microbiome and its metabolome

\section{Mary Ni Lochlainn ${ }^{1}$, Ruth C. E. Bowyer ${ }^{1}$, Claire J. Steves ${ }^{1}$ \\ ${ }^{1}$ The Department of Twin Research, Kings College London, 3-4th Floor South Wing Block D, St Thomas' Hospital, Westminster Bridge Road, London, SE1 7EH, UK}

Muscle mass, strength and physical function are known to decline with age. This is associated with the development of geriatric syndromes including sarcopenia and frailty. These conditions are associated with disability, falls, longer hospital stay, institutionalisation, and death. Moreover, they are associated with reduced quality of life, as well as substantial costs to health services. Dietary protein is essential for skeletal muscle function. Older adults have shown evidence of anabolic resistance, where greater amounts of protein are required to stimulate muscle protein synthesis. Research shows that resistance exercise has the most beneficial effect on preserving skeletal muscle. A synergistic effect has been noted when this is combined with dietary protein, yet studies in this area lack consistency. This is due, in part, to variation that exists within dietary protein, in terms of dose, quality, source, amino acid composition and timing. Research has targeted participants that are replete in dietary protein with negative results. Inconsistent measures of muscle mass, muscle function, physical activity and diet are used. The plasticity and diversity of the gut microbiome and its metabolome represent exciting future prospects for personalised medicine. The gut microbiome is substantially altered with ageing and the development of frailty. It remains an unexplored filter between diet and host physiology which may contribute to anabolic resistance. Research is needed on the role of dietary protein in muscle ageing and investigate the influence of the gut microbiome in relation to anabolic resistance, skeletal muscle mass and function. This review attempts to summarise these issues.

\section{P-466}

Nutritional status among hip fracture unit patients in a Singapore's Hospital

Chew Sia Tan ${ }^{1}$, Rui Yin $\operatorname{Tan}^{1}$, Chee Hong Toh ${ }^{1}$, Pek Yee Chow ${ }^{1}$, $\mathrm{Su} \mathrm{Su}^{1}$, Ai Ling Hong ${ }^{1}$, Priscilla $\mathrm{Ng}^{1}$, Mallya Ullal Jagadish ${ }^{1}$

${ }^{1}$ Khoo Teck Puat Hospital, Yishun, Singapore

Introduction: The detrimental effects of malnutrition on hip fracture patients are well known. The aims of this study were to evaluate the prevalence of malnutrition and its risk among hip fracture patients admitted to Khoo Teck Puat Hospital (KTPH).

Methods: Nutritional status of patients admitted to Hip Fracture Unit (HFU), KTPH from mid-March to April 2018 were included. Nutrition assessment includes subjective Global Assessment (SGA) and premorbid diet history was performed by a dietitian. Patients' Body Mass Index (BMI), serum 25-Hydroxy Vitamin D level, clinical 
frailty score (CFS), bone mineral density (BMD) and swallowing function assessment were retrieved from medical record.

Results: 38 patients with mean age of $81 \pm 10$ years were studied. $65.8 \%(\mathrm{n}=25)$ was well-nourished, $26.3 \%(\mathrm{n}=10)$ was mild-tomoderate malnourished and $7.9 \%(\mathrm{n}=3)$ was severely malnourished. The mean BMI was $21.74 \pm 3.30 \mathrm{~kg} / \mathrm{m}^{2}$ with $34.2 \%(\mathrm{n}=13)$ was found to have BMI $<20 \mathrm{~kg} / \mathrm{m}^{2}$. The premorbid energy and protein intake were $1347 \pm 359 \mathrm{kcal} /$ day $(27 \pm 6 \mathrm{kcal} /$ day $)$ and $42.2 \pm 18.6 \mathrm{~g} / \mathrm{day} \quad(0.8 \pm 0.3 \mathrm{~g} / \mathrm{kg} /$ day $)$ respectively which were lower than the recommended energy and protein requirement which were $30 \mathrm{kcal} / \mathrm{kg} / \mathrm{day}$ and $1.0 \mathrm{~g} / \mathrm{kg} / \mathrm{day}$. The mean serum 25 -Hydroxy vitamin D level was $24.63 \pm 9.92 \mu \mathrm{g} / \mathrm{L}$ with $73.7 \%(\mathrm{n}=28)$ had low serum 25-Hydroxy Vitamin D level $(<30 \mu \mathrm{g} / \mathrm{L}) .63 .2 \%(\mathrm{n}=24)$ had osteoporosis and $10.5 \%(n=8)$ had osteopenia. The mean CFS was $5.03 \pm 1.16$ (mildly frail). $34 \%$ developed dysphagia after surgery.

Key conclusions: One in three patients admitted to HFU was malnourished. One in three HFU patients developed dysphagia after surgery. High prevalence of osteoporosis and low serum vitamin D level were found among HFU patients. Comprehensive nutrition assessment and intervention, and routine swallowing assessment were warranted to optimize the recovery journey.

\section{P-467}

Effect of oral nutritional supplementation in malnourished older adults in nursing-homes. PROT-e-GER study

Vincenzo Malafarina ${ }^{1}$, José Antonio Serra Rexach ${ }^{2}$, Ferran Masanes ${ }^{3}$, Alfonso J. Cruz-Jentoft ${ }^{4}$

${ }^{1}$ Departamento de Ciencias de la Alimentación y Fisiología, Facultada de Farmacia y Nutrición, Universidad de Navarra, Pamplona, Spain, ${ }^{2}$ Servicio de Geriatría, Hospital Universitario Gregorio Marañón de Madrid, Spain, ${ }^{3}$ Servicio de Medicina Internal, Madrid, Spain

Introduction: To assess if oral nutritional supplements (ONS) improves the nutritional and functional status of malnourished residents.

Methods: Prospective, multicenter observational study of people $>65$ years, who live in nursing-homes, in whom treatment is initiated with ONS (Fortimel Complete). Anthropometric (weight, height and BMI), nutritional scales (MNA) and geriatric assessment (Barthel, grip strength, MMSE) were recorded at the beginning and at 12 weeks.

Results: We included 282 residents $(85.4 \pm 7.1$ years, $67 \%$ women, MMSE 12 (5-20). One hundred eighty-two participants were frail $(65 \%)$; these were older ( $86.2 \pm 7$ vs $83.4 \pm 7, \mathrm{p}=0.002)$, more dependent (Barthel 32 vs 70, p $<0.001$ ) and had a worse cognitive situation (MMSE 10 vs $14, \mathrm{p}=0.016$ ). The results show that after 12 weeks of treatment there is an improvement in nutritional status (BMI basal $20.2 \pm 3.0 \mathrm{~kg} / \mathrm{m}^{2}$ vs 12 weeks $21.2 \pm 2.7 \mathrm{~kg} / \mathrm{m}^{2}$, $\mathrm{p}<0.001)$ in functional situation [Barthel index basal 45 (15-70) vs 12 weeks $55(20-80) \quad \mathrm{p}<0.001]$ (basal hand grip strength $11.9 \pm 7.2 \mathrm{~kg} \quad$ vs 12 weeks $14.0 \pm 7.2 \mathrm{~kg}, \quad \mathrm{p}<0.001)$. The improvement in nutritional status is most evident in subjects in the lowest quartile of BMI $\left(\leq 18.3\right.$ vs $\geq 24.4 \mathrm{~kg} / \mathrm{m}^{2}$ ) (delta-BMI $1.3 \pm 1.2 \mathrm{~kg} / \mathrm{m}^{2}$ in lower quartile, vs $0.0 \pm 2.0 \mathrm{~kg} / \mathrm{m}^{2}$ in the highest quartile), while hand grip strength improves uniformly throughout the sample (delta HG-strength $1.3 \pm 2.3 \mathrm{~kg}$ in lowest quartile vs $1.5 \pm 7.4 \mathrm{~kg}$ in highest quartile).

Key conclusions: ONS is associated with an improvement in nutritional and functional status at 12 weeks in malnourished residents, as shown by the BMI, Bathel and hand grip strength improvements.

\section{P-468}

The ESPEN 2015 malnutrition consensus criteria for elderly people how effective screening at malnutrition?

Gulistan Bahat ${ }^{1}$, Banu Ozulu Turkmen ${ }^{1}$, Cihan Kilic ${ }^{1}$, Mehmet Akif Karan ${ }^{1}$, Ozlem Yilmaz ${ }^{1}$

${ }^{1}$ Istanbul University Istanbul Medical Faculty, Istanbul, Turkey

Introduction: "Mini Nutritional Assessment" (MNA) is gold standard for screening of malnutrition in elderly. In ESPEN consensus statement (2015), new criteria was suggested for screening of malnutrition. The aim of our study was to compare ESPEN 2015 criteria with MNA, for success on detection of malnutrition, in patients from our outpatient geriatric clinic.

Methods: November 2015-January 2018 were included patients in to the study. MNA, ESPEN test screening, hand grip strength (HGS), age, gender, weight were recorded. Body mass index (BMI) $<18.5$ $\mathrm{kg} / \mathrm{m}^{2}$ and fat free mass index $<15 \mathrm{~kg} / \mathrm{m}^{2}$ for female and $<17 \mathrm{~kg} /$ $\mathrm{m}^{2}$ for male with involuntary weight loss or BMI values $<20 \mathrm{~kg} / \mathrm{m}^{2}$ ( $<70$ years old) and $<22 \mathrm{~kg} / \mathrm{m}^{2}$ ( $>70$ years old) are accepted as malnutrition (ESPEN) $<11$ point for short-form MNA and $<23.5$ point for long-form MNA was accepted as malnutrition.

Findings: 438 geriatric patients were included in our study; $34.5 \%$ was male, and $65.5 \%$ female. Mean age was $75.0 \pm 6.2$ (65-97). Mean BMI was $29.4 \pm 5.4$. According to MNA, sensitivity and specificity of ESPEN criteria was 64.3 and $80.4 \%$. Positive predictive value (PPV) and negative predictive value (NPV) of ESPEN criteria was found 9.8 and $98.6 \%$ respectively. HGS in malnourished group according to MNA test was statistically low $(\mathrm{p}=0.004)$. BMI was significantly lower in malnourished group according to both MNA and ESPEN criteria $(\mathrm{p}<0.001)$.

Conclusion: Due to the low sensitivity and PPV of ESPEN 2015 criteria, it is considered that use of ESPEN 2015 criteria for screening of malnutrition may not be appropriate. However, ESPEN 2015 is found to be very effective for determination of elderly patients without malnutrition.

\section{Area: Oral and dental health}

\section{P-469}

Oral health and falls in geriatric patients: an interdisciplinary approach

Anna Greta Barbe ${ }^{1}$, Michael Bussmann ${ }^{2}$, Pia Schmidt ${ }^{3}$

${ }^{1}$ Department of Operative Dentistry and Periodontology, Centre of Dental Medicine, University of Cologne, Cologne, Germany,

${ }^{2}$ Department of Orthogeriatrics and Surgery, Evangelisches Krankenhaus Köln Kalk, Cologne, Germany, ${ }^{3}$ Health Science, University of Bremen, Bremen, Germany

Introduction: Prevalence of falls is rising with age. Sarcopenic loss of muscles is a risk factor for falls in aged people. Sarcopenia is also acknowledged to be associated with dysphagia and dysfunctional chewing ability. Until now, however, it has remained unknown, whether impaired oral health might be associated with an increased risk of falling in geriatric patients.

Methods: An interdisciplinary (geriatrician, surgeon, dentist) clinical controlled trial was performed on 40 geriatric in-patients [28 orthogeriatric patients with fall history versus 12 geriatric non-fallers (women $73 \%$, mean age $83 \pm 7$ )]. Study parameters comprised geriatric assessment results, parameters of oral health and quality of life as well as laboratory parameters of red blood, liver, kidney and thyroid gland. 
Results: Several oral health parameters revealed differences between groups: dysphagia $(\mathrm{p}=0.043)$, impaired tasting abilities $(\mathrm{p}=0.006)$, and dysphagia because of dry mouth problems $(\mathrm{p}=0.013)$. Patients with fall history showed associations between subjective dry mouth and dysphagia (Spearman's rho $=0.5, \mathrm{p}<0.05$ ), unstimulated salivation rate and dysphagia $(r=0.4, p<0.05)$, and between stimulated salivation rate and prostheses hygiene $(r=5, \mathrm{p}<0.05)$.

Key conclusions: Oral health parameters such as dysphagia, tasting abilities, dry mouth and oral hygiene have an impact on fall probability among geriatric patients. Our results may help to raise awareness for occurring problems in the other disciplines and help to establish new interdisciplinary risk assessments in order to achieve and maintain the best possible quality of life for geriatric patients.

\section{P-470}

Informal carers' experiences of identifying and managing oral pain and discomfort in community dwelling older people living with dementia

Paul Newton ${ }^{1}$

${ }^{1}$ University of Greenwich, London, UK

Introduction: Increased prevalence of dementia and poor oral health in older people is associated with an increased number of people living with dementia who experience oral pain and discomfort. Little is known about how oral pain impacts on people living with dementia in the community and their carers. This study aimed to explore informal carers' experiences of identifying and managing oral pain and discomfort in people living with dementia, and barriers and enablers they encountered.

Methods: Focus groups with of informal carers of people living with dementia were conducted, transcribed verbatim, and analysed using thematic analysis.

Results: Carers' accounts suggested that day-to-day contact was required to identify oral pain and discomfort, and a symptomology of the signs and symptoms was developed. Carers' accounts also highlighted problems in maintaining oral health, difficulties in accessing the mouth, managing dentures, competing demands, and difficulties in accessing treatment due to health service-, behavioural- and treatment- related barriers. Enablers included informal carers' pivotal role in the identifying and managing oral pain and discomfort in people living with dementia.

Conclusions: Partnership work with dental professionals and carers, and clearer care pathways are needed to meet the oral health needs of people living with dementia who experience oral pain.

\section{P-471}

An oral health screening and intervention program for older patients in acute care

Tzvi Dwolatzky ${ }^{1}$, Sonia Chorubczyk ${ }^{1}$, Mona Khaskia ${ }^{1}$, Yael Carlin ${ }^{1}$, Dani Noy ${ }^{1}$, Jacob Horwitz ${ }^{1}$, Irina Sabin ${ }^{1}$

${ }^{1}$ Rambam Health Care Campus, Haifa, Israel

Introduction: Poor oral hygiene among older people has a marked effect on general health and wellbeing. Older age is often associated with an increased prevalence of dental caries, periodontal disease, dry mouth, fungal infections, ulceration and oral cancer [1]. The admission of an older person to an acute care hospital is an important point of care for enabling health screening. The aim of our study was to institute an oral health screening program by volunteer oral hygienists as part of a comprehensive care program.

Methods: As part of the Hospital Elder Life Program [2], frail older patients at risk for delirium and functional decline are provided volunteer support to maintain cognitive function, nutrition and hydration, and mobility. As an addition to this program, volunteer oral hygienists visited the patients, administered an oral health questionnaire, performed an oral examination and cleaning of dentures, and advised patients regarding ongoing oral health care.

Results: A total of 100 patients over the age of 65 years hospitalized in acute care internal medicine departments at the Rambam Health Care Campus were visited by volunteer oral hygienists. Clinical findings included a coated tongue $(30 \%)$, redness of the mucosa (20\%), gingival swelling (18\%), plaque accumulation (48\%), and poorly-maintained dentures (34\%). Staff and patient satisfaction with the visit and advice of the oral hygienist was high.

Key conclusions: Oral hygiene screening of frail older patients in acute care has clear potential benefits and should be investigated further.

\section{References:}

1. Gil-Montoya JA, Ferreira de Mello AL, Barrios R, Gonzalez-Moles M, Bravo M (2015) Oral health in the elderly patient and its impact on general well-being: a nonsystematic review. (2015) Clin Interv Aging 10:461-467

2. http://www.hospitalelderlifeprogram.org

\section{P-472}

Multidimensional Prognostic Index (MPI) and oral health: design and preliminary results of the MPIOH study

Noetzel Nicolas ${ }^{1}$, Pickert Lena ${ }^{1}$, Rarek Marcel ${ }^{1}$, Meyer Anna ${ }^{1}$, Pilotto Alberto ${ }^{2}$, Benzing Thomas ${ }^{3}$, Polidori M. Cristina ${ }^{1}$

${ }^{1}$ Ageing Clinical Research, Dpt. II for Internal Medicine, University Hospital of Cologne, Cologne, Germany and Nephrology, Rheumatology, Diabetology and Internal Medicine, Dpt. II for Internal Medicine, University Hospital of Cologne, Cologne, Germany

Introduction: It is well known that there is an association between oral health and geriatric syndromes including malnutrition as well as chronic systemic illnesses. The Multidimensional Prognostic Index (MPI) predicts short- and long-term mortality in older subjects and has been validated for several acute and chronic diseases. The aim of this study is to identify associations between MPI and oral health.

Methods: This study begun December 2017 and will include about 100 older subjects aged 65 or older hospitalized in a large German hospital. Besides a comprehensive geriatric assessment (CGA) and prognosis evaluation by means of MPI, an initial assessment of oral health is performed. To identify the oral health quality of life the Geriatric/General Oral Health Assessment Index (GOHAI) is used. To assess the current oral health status the Kayser-Jones Brief Oral Health Status Examination (BOHSE) and a specifically designed questionnaire including "Decayed, Missing, and Filled Teeth- Index" (DMFT) are used. After 6 and 12 months a reevaluation of dental diseases and use of dental treatment will be performed.

Results: Until now, 34 patients $(22 \mathrm{M}, 77.8 \pm 6.0$ years, $12 \mathrm{~F}$, $73.2 \pm 6.2$ years) have been recruited. First results show a slightly positive correlation of MPI values to DMFT Index $(\rho=0.36$, $p>0.05)$ and a negative correlation to GOHAI Sum Score $(\rho=$ $-0.55, \mathrm{p}<0.05)$. More results are expected in July/August 2018.

Conclusions: MPI might correlate to oral health status and oral health quality of life. 


\section{P-473}

\section{Halitosis in elderly people}

Tamer Yilmaz ${ }^{1}$, Umran Dal Yilmaz ${ }^{2}$

${ }^{1}$ Near East University Dental Faculty, Nicosia, Cyprus, ${ }^{2}$ Near East University Faculty of Nursing, Nicosia, Cyprus

Introduction: The impact of oral and dental health on general health status of the elderly people is too big to be ignored. In elderly individuals, tooth loss, abrasions, periodontal diseases, tooth decay, oral mucosal lesions and various systemic diseases cause oral malfunction and malodor.

Methods: A literature search (2000-2018) was carried out, using MEDLINE, Pub Med, Science direct, Scopus, Cochrane library, Turkish Medical Index and related intuitional websites. Key words included "Malodorous", "Halitosis", "Elderly", "Older".

Results: Geriatric orofacial changes occur in both hard and soft tissues. In either case, one of the inevitable consequences is oral malodor. Not only bacteria are the cause of bad smell noticed in the mouth. The destruction of proteins and sulfur-containing compounds on the tonsils and tongue surface can also cause bad odor in the mouth. One of the common complaints in the elderly is the mouth dryness (xerostomia) and it is an important source of oral malodor. Burning mouth syndrome is another cause of oral malodor in elderly individuals. The significantly relation of oral malodor and the accumulation of bacterial plaque on the tongue, oral dryness, burning mouth, and overnight denture wear is shown by Nalcaci and Baran (2008). Incorrect restorations, and implants, are another important cause of oral bad breath in the elderly.

Key conclusions: Periodic intraoral and restorative controls should be done frequently and carefully in the elderly. In the elderly, oral malodor can be prevented by carefully controlling with physician and dentist.

\section{P-474}

Viewpoint of dental care and mealtime care accompanying dementia progression.

Ayako Edahiro $^{1}$, Hirohiko Hirano ${ }^{2}$, Shuichi Awata ${ }^{1}$

${ }^{1}$ Tokyo Metropolitan Institute of Gerontology, Tokyo, Japan, ${ }^{2}$ Tokyo Metropolitan Geriatric Hospital, Tokyo, Japan

Objectives: Eating and swallowing dysfunctions are caused by progression of dementia. We examined the eating and swallowing dysfunctions in elderly peoples with Alzheimer's disease (AD) on the basis of FAST.

Methods: Subjects included 175 elderly AD patients requiring care in Japan. The subjects' average age was $86.6 \pm 6.1$, and included 29 males and 146 females. Subjects underwent vital function tests (selffeeding assessment, screening test for swallowing dysfunctions, oralfunction assessment was included), cognitive assessment (ex. FAST), and a basic information survey. All data was classified by FAST and analyzed with SPSS ver.22 (IBM) for eating and swallowing dysfunctions. The protocol for the present study was designed in accordance with the Declaration of Helsinki.

Results: Self-feeding assessment showed that eating difficulty occurred from the FAST6e stage $(\mathrm{p}<0.001)$. Signs of dysphagia, became pronounced especially after FAST7a $(\mathrm{p}=0.042)$, and the dysphagia screening test with a stethoscope showed choking and/or wet hoarseness sounds in the pharynges increased after FAST6e $(\mathrm{p}=0.022)$. Prominent difficulty in rinsing became apparent after FAST7b $(p<0.001)$, and difficulty in gargling was seen after FAST6e $(\mathrm{p}<0.001)$. However, meal time lengthened (over $40 \mathrm{~min}$ ) after FAST6b and was further lengthened (over $60 \mathrm{~min}$ ) after FAST7b $(\mathrm{p}=0.021)$.

Conclusion: Eating and swallowing dysfunctions were greater with increasing severity of AD. These results showed that the decline in oral functions occurred after finding self-feeding dysfunction, difficulty in gargling and signs of dysphagia. It is necessary to offer predictive support for elderly patients with $\mathrm{AD}$. We developed a viewpoint of dental care and mealtime care for Alzheimer's disease (AD).

\section{P-475}

Oral hygiene status and blood biomarkers of cardiovascular and metabolic risk in elderly patients

Leonardo Victor Galvão-Moreira ${ }^{1}$, Milena Maria Trovão ${ }^{1}$, Fernanda Ferreira Lopes ${ }^{1}$, Consuelo Penha Castro Marques ${ }^{1}$, Flávia Lopes Carvalho ${ }^{1}$, Maria Carmen Fontoura Nogueira da Cruz ${ }^{1}$

${ }^{1}$ Federal University of Maranhão, São Luís, Brazil

Objective: To investigate whether there is any correlation between the oral hygiene status and some blood biomarkers for cardiovascular and metabolic risk among elderly patients.

Methods: A total of 201 individuals, of both genders and aged 60 years or over, were selected from an elderly community sample in São Luís, Brazil, and included in this study. A structured interview was carried out with the participants, followed by the visual evaluation of the oral cavity. The Simplified Oral Hygiene Index (OHI-S) scores were obtained and then categorized as follows: $0-1.2=$ adequate; $1.3-3.0=$ regular; $3.1-6.0=$ inadequate. The cardiovascular/ metabolic risk biomarkers analyzed included total serum cholesterol, fasted glucose and triglyceride levels. A descriptive statistical analysis was used and the Pearson's correlation coefficients were calculated.

Results: Most of the participants $(78.1 \%)$ had not utilized dental services in the last year. Moreover, only $16.4 \%$ of them presented with adequate OHI-S scores. Still, a negative correlation between the number of years of study and OHI-S scores was shown $(\mathrm{p}<0.05)$. There was no statistically significant correlation between OHI-S scores and the values of total serum cholesterol, fasted glucose or triglyceride levels $(\mathrm{p}>0.05)$.

Conclusion: In the present study, a group of elderly individuals presented with regular oral hygiene, which was correlated with their educational level. Cardiovascular and metabolic risk biomarkers were not correlated with the oral hygiene status.

\section{Area: Organisation of care and gerotechnology}

\section{P-477}

The elderly benefit when they join a social network

Peter Zaki ${ }^{1}$

${ }^{1}$ Penn State College of Medicine, Hershey, USA

Introduction: Programs of All-Inclusive Care for the Elderly (PACE) have existed for years but there remains no strong evidence for their effectiveness. The objective of this study was to assess the perceptions of members at a PACE.

Methods: A quality assessment and improvement study was conducted to assess the perceptions of members at a PACE. Information was collected on demographics, satisfaction, access to medical care, quality of medical care, strengths, and areas of improvements. Data was analyzed using paired 2-tail t tests. 
Results: The average age of participants was $74 \pm 9$. There were 8 males and 21 females. There were 20 Caucasian (includes Hispanic), 4 African American, and 5 unanswered. 14 participants lived at home by themselves and 15 lived with a family member. There was significant improvement in overall satisfaction after joining the PACE $\mathrm{p}<0.001$ (living alone: $\mathrm{p}<0.001$; living with family: $\mathrm{p}<0.01$ ). There was significant improvement in access to a nurse $\mathrm{p}<0.001$ (living alone: $p<0.001$; living with family: $p<0.001$ ) and primary care physician $(\mathrm{PCP}) \mathrm{p}<0.01$ (living alone: $\mathrm{p}<0.01$; living with family: $p<0.05)$. There was significant improvement in quality of medical care $\mathrm{p}<0.01$ (living alone: $\mathrm{p}<0.01$; living with family: $\mathrm{p}<0.05$ ). The most common strength was friendly and caring community, while the most common area of improvement was the transportation schedule.

Key conclusions: Members are significantly more satisfied overall, more satisfied with access to medical care, and more satisfied with the quality of medical care since joining the PACE. Living situation was an effect modifier for overall satisfaction, access to PCP, and quality of medical care.

\section{P-478}

Vulnerability of Lithuanian older adults: potential users of gerontechnologies

Vita Lesauskaite ${ }^{1}$, Jurgita Knašiene ${ }^{1}$, Gyte Damulevičiene ${ }^{1}$, Egidijus Kazanavičius $^{2}$, Audrone Janavičiūte ${ }^{3}$, Vygintas Kazanavičius ${ }^{3}$

${ }^{1}$ Geriatric Department, Lithuanian University of Health Sciences, Kaunas, Lithuania, ${ }^{2}$ Centre of Real Time Computer Systems, Kaunas University of Technology, Lithuania, Kaunas, Lithuania, ${ }^{3}$ Centre of Real Time Computer Systems, Kaunas University of Technology, Kaunas, Lithuania

Introduction: The integration of older adults into society is encumbered by their vulnerability. A new type of services, gerontechnology, decreases vulnerability and permits to age in place. Presented study aimed at identifying the main vulnerability factors of older adults and their use of technologies.

Methods: 517 older adults, 265 geriatric in-patients and 252 community-dwelling, were questioned about vulnerability factors and usage of technologies.

Results: Vulnerability factors were prevalent. From physiological factors the most prevalent were weakness, decreased mobility and balance, poor vision; from environmental-unsafe home environment. About $80 \%$ of study subjects were complaining on poor memory. 56.3\% reported falls during last 6 months. Majority of those who sustained a fall reported post-fall syndrome. Most of older adults were using common household equipment. Only minority were using more sophisticated electronic devices such as computers with internet connection, but on the other hand, over 80 percent used cell phones. Majority of the persons living in the community expressed interest in trying new technologies, while only minority of geriatric in-patients expressed such interest. $24.2 \%$ of geriatric in-patients and $59.5 \%$ of those living in the community knew about existence of gerontechnologies.

Conclusion: The study showed high prevalence of vulnerability factors among older adults. Opinion of older adults about technologies was positive. The obtained data prove that most of them would use gerontechnologies if proposed.

Acknowledgement: This research was supported by the Research Council of Lithuania (grant No. SEN-06/2016, research project „Smart Gerontechnology for Healthy Ageing “).

\section{P-479}

The assessment of the level of independence and geriatric patients' needs for home care after their discharge from a nursing unit

Lina Urbiete $^{1}$, Vita Lesauskaite ${ }^{1}$, Jurate Macijauskiene ${ }^{1}$

${ }^{1}$ Geriatric Department, Lithuanian University of Health Sciences, Kaunas, Lithuania

Introduction: According to statistical projections in 2060 each third (37\%) Lithuanian will be an older adult. Demographic transition requires urgent solutions adjusting national health care system to the needs of an aging society. The quality and continuity of medical and social care should be ensured.

Methods: The quantitative survey design was employed. Patients aged $>60$ years who stayed in a nursing unit for 90 days and longer participated in the study. The assessment was carried out on the day before their discharge. The interRAI Home Care Assessment System was applied for the assessment of the patients' independence level and care needs. In total, 100 patients were assessed.

Results: Almost half of the discharged older patients (48\%) had very weak cognitive abilities and $30 \%$-moderately weak. The majority of patients $(87 \%)$ did not have the abilities for meal preparation, $88 \%$ dish washing, house keeping and $89 \%$ managing of finances. $85 \%$ of patients on the discharge from the nursing unit were fully dependent on the nursing staff during bathing, $32 \%$ were in need of sufficient assistance during personal hygiene and $48 \%$ were bedridden.

Conclusions: Low level of independence observed in older patients and their limited abilities for personal and daily living activities at the moment of discharge from the nursing unit evidence the need for home care service.

\section{P-480}

\section{SHOP-75: Safer handover for older people 75+}

A. Pedersen ${ }^{1}$, C. Earnshaw ${ }^{1}$, A. Howe ${ }^{1}$, S. Akbar ${ }^{1}$, W. Chmiel ${ }^{1}$, H. Choonara ${ }^{1}$, H. Jalil ${ }^{1}$, G. Logue ${ }^{1}$, J. Evans ${ }^{1}$, O. Gaillemin ${ }^{1}$, A. Vilches-Moraga ${ }^{1}$

${ }^{1}$ Salford Royal Hospital, Salford, UK

Introduction: Accurate discharge summary documentation plays a crucial role in the continuing health care of patients discharged from hospital. The Academy of Medical Royal Colleges provides guidance on the structure and content of discharge summaries. Content required includes a social and functional assessment, a list of new diagnoses, and details of the patient's past medical history (PMH).

Methods: We performed a baseline audit to assess Trust-wide performance, and then designed a series of measures (including FY1 year-wide teaching, and induction training for doctors during each rotation) designed to improve the quality of discharge summaries. We presented the project to the different specialties involved at their respective clinical governance meetings. We then designed a novel live-feedback system to help facilitate the improvements. This allows us to analyse discharge summaries on a monthly basis using a Plan-Do-Study-Act (PDSA) approach, and feed the results back directly to the doctors completing the summaries.

Results: We audited 482 discharge summaries across 7 wards. Several aspects of were recorded poorly. Specifically, these were: social assessment (included in only 23\%), PMH (50\%), and a list of diagnoses (59\%). Our first PDSA cycle was completed in February 2018. Subsequent cycles have seen our documented social assessments improve to $47 \%$, and have shown improvements in the $\mathrm{PMH}$ and diagnoses documentation (to 82 and $65 \%$ respectively). 
Key conclusions: We identified several areas that are not being included in discharge documentation as required. We have designed a novel live-feedback system, performed monthly, that aims to improve the quality of this documentation.

\section{P-481}

Hospital at home care for older patients with cognitive impairment: a protocol for a randomised controlled feasibility trial

Maaike A. Pouw ${ }^{1}$, Agneta H. Calf ${ }^{1}$, Barbara C. van Munster ${ }^{2}$, Jan C. ter Maaten ${ }^{3}$, Nynke Smidt ${ }^{4}$, Sophia E. de Rooij ${ }^{1}$

${ }^{1}$ Department of Geriatrics, University Medical Center Groningen, University of Groningen, Groningen, The Netherlands, ${ }^{2}$ Department of Geriatrics, Gelre Hospitals, Apeldoorn, The Netherlands, ${ }^{3}$ Department of Internal Medicine, Emergency Department, University of Groningen, Groningen, The Netherlands

Introduction: An acute hospital admission is a stressful life event for older people, particularly for those with cognitive impairment. The hospitalisation is often complicated by hospital-associated geriatric syndromes, including delirium and functional loss, leading to functional decline and nursing home admission. Hospital at Home care aims to avoid hospitalisation-associated adverse outcomes in older patients with cognitive impairment by providing hospital care in the patient's own environment.

Methods and analysis: This randomised, non-blinded feasibility trial aims to assess the feasibility of conducting a randomised controlled trial in terms of the recruitment, use and acceptability of Hospital at Home care for older patients with cognitive impairment. The quality of care will be evaluated and the advantages and disadvantages of the Hospital at Home care programme compared with usual hospital care. Eligible patients will be randomised either to Hospital at Home care in their own environment or usual hospital care. The intervention consists of hospital level care provided at patients' homes, including visits from healthcare professionals, diagnostics (laboratory tests, blood cultures) and treatment. The control group will receive usual hospital care. Measurements will be conducted at baseline, during admission, at discharge and at 3 and 6 months after the baseline assessment.

Conclusion: The study findings will contribute to knowledge on the implementation of Hospital at Home care for older patients with cognitive disorders. The results will be used to inform and support strategies to deliver eligible care to older patients with cognitive impairment.

\section{P-482}

SHOP-75: lessons learned from the development of our interdisciplinary project

\author{
A. Pedersen ${ }^{1}$, A. Howe ${ }^{1}$, C. Earnshaw ${ }^{1}$, S. Akbar ${ }^{1}$, W. Chmiel ${ }^{1}$, \\ H. Choonara ${ }^{1}$, H. Jalil ${ }^{1}$, G. Logue ${ }^{1}$, J. Evans ${ }^{1}$, O. Gaillemin ${ }^{1}$, \\ A. Vilches-Moraga ${ }^{1}$ \\ ${ }^{1}$ Salford Royal Hospital, Salford, UK
}

Introduction: Accurate discharge documentation is critical in ensuring the safe handover of care from hospital to primary care clinicians. Our geriatric medicine department has a target of improving the quality of discharge documentation in our hospital. We have created a taskforce to overcome this challenge, and have learned some important lessons.
Methods: Our first priority was to perform a baseline audit, and we successfully analysed 482 discharge summaries. We realised we could impact every discharged patient, and so we expanded our aims to include acute physicians and orthogeriatric surgeons within the remit. We designed a data collection tool that is simple to understand and collects 38 individual data points about each summary. We performed a survey of senior clinicians to identify the main areas to include. We designed a novel live-feedback system that allows us to provide direct feedback to the authors of the discharge summaries on a monthly basis. We designed a poster that allows us to identify required improvements in each clinical area.

Results: We have seen an improvement in the quality of our discharge documentation, with an average of $65 \%$ of summaries containing required information (up from $44.2 \%$ ). Having a multidisciplinary team, and involving our local Quality Improvement service, has allowed us to make more changes than we could individually.

Key conclusions: The live-feedback system has proven useful in getting junior doctors engaged with the project. The local Quality Improvement service has allowed our project's data collection and analysis to improve beyond what we could initially perform.

Keywords: Methodological Issue, Big Data, e-Health and Technologies

\section{P-483}

Can home care for homebound patients with chronic heart failure reduce hospitalizations and costs?

Boris Punchik ${ }^{1}$, Roman Komarov ${ }^{2}$, Dmitry Gavrikov ${ }^{2}$, Anna Semenov $^{2}$, Tamar Freud ${ }^{3}$, Ella Kagan ${ }^{1}$, Yury Goldberg ${ }^{2}$, Yan Press ${ }^{1}$

${ }^{1}$ Unit for Community Geriatrics, Ben-Gurion University of the Negev, Beer-Sheva, Israel, ${ }^{2}$ Home Care Unit, Clalit Health Services, Beer-Sheva, Israel, ${ }^{3}$ Sial Family Medicine and Primary Care Research Center, Ben-Gurion University of the Negev, Beer-Sheva, Israel

Introduction: Congestive heart failure (CHF) is associated with multiple hospitalizations, high mortality rates and high costs. In the present study we evaluate whether home care for elderly homebound patients with $\mathrm{CHF}$ reduces healthcare service utilization and overall costs.

Methods: A retrospective study of healthcare utilization among homebound patients who received home care for CHF from 2012-1015. The outcome measures were a mean number of hospital admissions per month, total number of hospitalization days and days for CHF only, emergency room department visits, and overall costs. A comparison was conducted between the 6-month period prior to the initiation of home care and the time in home care.

Results: Over the study period 196 patients were treated by home care for CHF with a mean age of $79.4 \pm 9.5$ years. $113(57.7 \%)$ were women. Compared to the 6 months prior to home care, there were statistically significant decreases in hospitalizations $(46.3 \%)$, in the number of total in-hospital days $(28.7 \%)$, in the number of in-hospital days for CHF (66.7\%), in Emergency Department visits (47\%), and in overall costs $(23.9 \%)$.

Conclusion: Home care for homebound adults with CHF can reduce healthcare utilization and healthcare costs. 


\section{P-484}

Inadequate nutritional care and nutritional documentation in medical records of older patients

Christine Hillestad Hestevik ${ }^{1}$, Lisa Mari Gaustadnes ${ }^{2}$, Mari Hval Høyem ${ }^{2}$, Marianne Molin ${ }^{3}$, Asta Bye ${ }^{4}$

${ }^{1}$ Department of Physiotherapy, Faculty of Health Sciences, Oslo Metropolitan University, Oslo, Norway, ${ }^{2}$ Department of Nursing and Health Promotion, Faculty of Health Sciences, Oslo Metropolitan University, Oslo, Norway, ${ }^{3}$ Department of Nursing and Health Promotion, Oslo Metropolitan University, Oslo, Norway

Introduction: Undernutrition is common among older hospitalized patients and nutritional status often declines during the stay. Undernourishment has serious negative implications for health and quality of life. Thus, nutritional support during hospital stay and follow-up care post-discharge is essential. Unfortunately, poor communication and coordination of nutritional information and care in transition between different levels of care is common. This study aimed to explore older patients' experiences of nutritional care during hospital stay and post-discharge and to investigate documentation of nutritional information in medical records.

Methods: Semi-structured interviews with 15 older patients were conducted post-discharge. Inclusion criteria were documented nutritional risk or undernourishment (Mini Nutritional AssessmentMNA), age above 65 years and discharge to home care services. The interview guide focused on experiences of food, meals and nutritional care. Data were analyzed thematically. Eight hospital medical records were reviewed for nutritional documentation using a record investigation form.

Results: Most of the patients were unaware their nutritional situation. They experienced lack of nutritional counseling and involvement in nutritional care both in hospital and at home. Not knowing the people from the care services made interaction with carers challenging. Unstructured and inadequate documentation of information in the medical records made it difficult to get an overview over the patients" nutritional situation.

Conclusion: The results indicate lack of continuity of care and inadequate communication and involvement of elderly patients in their own nutritional health and care planning. Nutritional information was insufficiently documented in the medical records.

\section{P-485}

Integrated new model of care in a Buckinghamshire care home

Unoma Okoli $^{1}$, S. Chimahu ${ }^{1}$, Beatrix Nagyova ${ }^{2}$, Theresa Frang ${ }^{2}$, Rizvana Ali ${ }^{1}$

${ }^{1}$ Buckinghamshire Clinical Commissioning Group, Amersham, UK, ${ }^{2}$ Buckinghamshire Healthcare NHS Trust, Amersham, UK

Integrated New Model of Care in a Buckinghamshire Care Home. Okoli, U. 1, Chimhau, S.1, Nagyova, B. 2, Frang, T. 2, Rizvana, A.1 Buckinghamshire CCG 1, Buckinghamshire Healthcare NHS Trust 2, United Kingdom. One of the key drivers for the focus on medicines optimisation for older person is an ageing population. By 2030 in Buckinghamshire the Office of the National Statistics projections indicates the number of $65+$ years is expected to form over a quarter of the population [1]. $36 \%$ of over 75 take 4 or more prescribed medicines, with increased risk of adverse reactions [1].

Methods: A new interdisciplinary model of care was delivered in 60 bed care Buckinghamshire based care home between March-December 2017. Clinical Commissioning Group pharmacist, general practitioner and pharmacy technician reviewed medication for all residents with geriatrician review of the most complex individuals.

Results: Overall 56 medications were stopped for 32 residents, with 35 interventions to reduce falls risk. Total cost savings on medicines optimisation, waste and non-elective admission prevented is $£ 20808$. Residents care plans were updated to reflect best practice standards. Conclusion: Future direction of this project focus on system wide improvements to promote interdisciplinary healthcare professionals work in care homes. This new integrated model of care was recognised by NHS England and will be shared on the Future NHS platform as an exemplar for Care Homes Pharmacy Services.

Reference:

1. Medicines and older people National Service Framework (2001) Implementing medicines related aspect of the NSF for older people

\section{P-486}

Education, late life disability level and care receiving status among community dwelling older adults in Iceland

Olof Gudny Geirsdottir ${ }^{1}$, Alfons Ramel ${ }^{2}$, Milan Chang ${ }^{3}$, Ingemar Kåreholt ${ }^{4}$, Sigurveig H. Sigurdardottir ${ }^{5}$

${ }^{1}$ The Icelandic Gerontological Research Center, National University Hospital of Iceland \& Faculty of Medicine, Reykjavik, Iceland \& Unit for Nutrition Research, National University Hospital of Iceland \& Faculty of Food Science and Nutrition, University, Reykjavik, Iceland

Older adults in Iceland have a good access to the health care and social services that support elderly to maintain independent living. However, receiving informal care is common among older adults living in Iceland. The aim of this study is to analyze whether education is associated with the use of formal and informal care among older adults in Iceland. A survey of Icelandic older people was conducted including a national sample of 721 persons aged 65 years and older living in Iceland. Older adults living in nursing homes were excluded from the survey. The telephone survey included questions on socioeconomic status (education and occupation), social network, health status, activities of daily living, and the status of received help either from the community elderly care system and/or from close family members and friends. Among the total sample, $372(52 \%)$ people reported to receive either type of care. Among those who received care, 197 (53\%) people reported receiving informal care only. The status of receiving informal care had shown that people with higher education had a significantly lower risk to receive informal care compared with people with lower education [odds ratio (OR) $0.67,95 \%$ CI 0.47-0.97, $\mathrm{p}=0.031$ ], however formal care was not significantly associated with education level. Informal care which is provided from the social network and family members are an important source for the elderly care system in Iceland. The contribution of informal care/help should be recognized when preparing the care of older people. After attending this session, participants will know about formal and informal care in Icelandic old adults. After attending this session, participants will know that receiving care is associated with education in Icelandic old adults.

\section{P-487}

Prevalence and predictors of sleep disorders among institutionalized elderlies: a cross sectional study

Mohamad Itani ${ }^{1}$, Ghassan Hamadeh ${ }^{2}$, Nabil Naja ${ }^{3}$ 
${ }^{1}$ Nicosia University, Nicosia, Cyprus, ${ }^{2}$ American University Medical Center of Beirut, Beirut, Lebanon, ${ }^{3}$ Dar Al-Ajaza Al-Islamia Hospital, Beirut, Lebanon

Background: This is a cross section observational study, which aims to identify the prevalence of sleep problems/disturbances in an institutionalized population of elderlies living in a philanthropic chronic care center located in an upper middle-income country.

Methods: The study involved 192 institutionalized elderly persons who have been living in the institution for more than 1 month. After filling an informed consent form, the participants were required to complete the Pittsburgh Sleep Quality Index (PSQI) questionnaire either on their own or by the nurses who take care of them. Our dependent variable was participants with a global PSQI score of five or more who were considered to have a sleep problem. The independent variables were demographic data, life style habits, current medical conditions and medications that were collected from the participants' medical files. Statistical analysis was performed using the SPSS software.

Results: Participants were between 60 to 102 years old with a mean age of 77.4 years (SD 8.7 years). According to the global PSQI scores, $75 \%$ of the participants were found to have sleep problems (95\% CI 69-81\%) with equal male to female distribution, in comparison to $27.6 \%$ of the participants who reported subjective sleep problems. Decreased activity and poor performance on memory tests were the only demographic and life style variables that had statistical association with sleep problems (OR 2.85, 95\% CI 1.41-5.68 and OR $4.21,95 \%$ CI $1.90-9.33$ respectively). From the current medical conditions, Alzheimer disease had a positive association with sleep problems (OR 2.28, 95\% CI 1.05-4.94), while schizophrenia had a negative association (OR 0.46, 95\% CI 0.23-0.92). Among the medications that were studied proton pump inhibitors were the only drugs that approached significant association with sleep problems on statistical analysis (OR 1.85, 95\% CI 0.96-3.59).

Conclusion: Sleep problems is a major issue in elderly persons present in $75 \%$ of persons living in an elderly chronic care center in Lebanon. Its prevalence is underestimated by the nursing staff and under reported by the patients. Our study found an association between sleep problems and decreased level of activity, and the failure to pass either mini mental state examination or clock drawing memory tests. Alzheimer disease was positively associated with sleep problems, while schizophrenia was negatively associated with them. The chronic intake of proton pump inhibitors was the only drugs that approached statistically significant association with sleep problems in our analysis. Further randomized control studies are required to confirm our findings.

\section{P-488}

The introduction of a parenteral fluid prescription chart in St Vincent De Paul Hospital, a geriatric long-term care Hospital in Malta

Claire Callus ${ }^{1}$, Francesca Camilleri ${ }^{1}$, Svetlana Brincat ${ }^{1}$, Marco Grech ${ }^{1}$, Antoine Vella ${ }^{1}$

${ }^{1}$ Department of Geriatric Medicine, University of Malta, Msida, Malta

Introduction: Parenteral fluid administration requires clinical and biochemical monitoring to guide its correct prescription. St Vincent De Paul Hospital (SVPR), the largest long-term care hospital in Malta, lacked a formal procedure and prescription chart for administration of parenteral fluids. The aim of the full cycle audit was to introduce a standard operating protocol for fluid prescription and a parenteral fluid prescription chart.
Methods: An audit was carried out at SVPR on patients over 65 years old who were administered fluids over a five day period. Documented information related to their fluid prescription was collected. A fluid prescription chart and its Standard Operating Procedure (SOP) document was then created by a focus group of doctors and nurses. Following staff education, the chart was piloted for 2 months on randomly selected wards. A re-audit was performed, discussions with stakeholders held, and final amendments made. The finalized protocol and chart were implemented in a staggered method until all wards of SVPR were included. A re-audit was then conducted.

Results: The initial audit proved that there was barely any documentation on fluid prescription and monitoring. After implementation of the SOP and fluid prescription chart this improved dramatically. The focus group was instrumental in the creation of the SOP and chart, especially so in educating and promoting a culture change in the staff. Key conclusions: The importance of a fluid prescription SOP and chart to ensure safe fluid administration was made evident. The need for education and culture change when introducing a new concept to the hospital was also highlighted.

\section{P-489}

Determining heart rate variability in elderly oncological patients using wrist-worn pulse wave monitor

Nicolae Hariton Costin ${ }^{1}$, Cristian Rotariu ${ }^{1}$, Gabriel Ioan Prada ${ }^{2}$, Ioana Dana Alexa ${ }^{1}$, Andrei Kozma ${ }^{3}$

${ }^{1}$ University of Medicine and Pharmacy "Grigore T. Popa", Iasi, Romania, ${ }^{2}$ University of Medicine and Pharmacy "Carol Davila", Bucharest Iasi, Romania, ${ }^{3}$ Romanian Academy of Scientist, Bucharest Romania

Introduction: Heart rate variability (HRV) has become an important marker for prognosis not only in cardiovascular, diabetic or renal patients, but also in oncological patients. Cancer is one of the most frequent disease in elderly and the need for proper treatment and care is increasing. HRV as an indicator of autonomic nervous system dysfunction is associated with cancer progression, cancer-related-pain intensity, cancer-related fatigue and even quality of life. This paper aims to determine an easy, noninvasive, low-cost method for determining HRV in elderly oncological patients.

Methods: In this study we included patients over 65 years old with an established previous diagnose of cancer. To determine HRV using an easy, non-invasive, inexpensive method we compared a wrist-worn photoplethysmography (WWPPG) device to the classical ECG Holter. Also we wanted to determine the relationship between cancer progression and HRV and confirm HRV as a prognostic factor in oncological elderly patients.

Results: Data obtained revealed that HRV parameters obtained using ECG Holter are comparable with HRV parameters obtained using WWPPG. WWPPG device can be used in elderly oncological patients in order to determine HRV. HRV can be, in certain circumstances, a prognostic factor in elderly patients with oncological disease.

Conclusions: WWPPG is a new, easy to use, inexpensive, effective method in determine HRV in elderly oncological patients. HRV can be a parameter in establishing the prognostic in elderly oncological patients. 


\section{$\mathbf{P}-490$}

Monitoring of malnutrition incidence among elderly according to age group through web-service NRS-2002/GeroS/CEZIH

Nada Tomasović Mrčela ${ }^{1}$, Branko Kolarić ${ }^{1}$, Spomenka Tomek-Roksandić ${ }^{1}$, Tanja Ćorić ${ }^{1}$

${ }^{1}$ Andrija Stampar Teaching Institute of Public Health, Zagreb, Croatia

Introduction: Malnutrition in the population over 65 years of age is associated with worsening of general health status and functional ability. For this reason, gerontology information system GeroS/ CEZIH was implemented with web-service Nutritional Risk Screening 2002 (NRS-2002) that is able to provide quick screening of nutritive status focused on gerontologic-public health indicators such as age group, sex, functional ability, main and accompanying diagnoses of geriatric patients, basic anthropometric measurements and similar.

Methods: Gerontology-oriented public health analysis of specific determinants (1) in different age groups of elderly through NRS-2002/ GeroS/CEZIH web-service was performed. A total of 187 patients from the database were randomly included in the analysis during the period from March to May 2016 and were stratified in three groups; younger-elderly (65-74 years), middle-age“-elderly (75-84 years) and old-elderly ( $>85$ years). Patients were analyzed in terms of location and entities in which their nutritional screening was performed.

Results: According to obtained body mass index (BMI) values of all 187 patients, $1.6 \%(\mathrm{~N}=3)$ were malnourished and these patients belonged to $75-84$ years $(\mathrm{N}=2)$ and $65-74$ years age group $(\mathrm{N}=1)$, while $3.2 \%(\mathrm{~N}=6)$ of patients had severe malnourishment. Severe malnourishment was most pronounced in the 75-84 years age group in which 5 cases were recorded. Among the oldest patients ( $>85$ years of age) only one patient was severely malnourished.

Conclusion: The incidence of malnourishment was more common in older age groups of elderly patients. The use of NRS-2002/GeroS/ CEZIH web-service enables prompt evaluation and aggregation of important gerontologic-public health indices in the elderly population. Keywords: NRS-2002; GeroS; malnourishment

\section{P-491}

CT scan injected by mistake and contrast-induced nephropathy: how avoid these errors?

Riffard Cindy ${ }^{1}$, Grevy Armance ${ }^{1}$, Gibert Prudence ${ }^{1}$, Lopez Clara ${ }^{2}$, Mitha Nathalie $^{2}$, Zerhouni Nabil ${ }^{2}$, Bedouch Pierrick ${ }^{3}$, Gavazzi Gaetan $^{4}$

${ }^{1}$ Pôle pharmacie, CHU Grenoble Alpes, 38000, Paris, France, ${ }^{2}$ Service Universitaire de Médecine Gériatrique, Pôle pluridisciplinaire de Médecine, CHU Grenoble Alpes, 38000, Paris, France, ${ }^{3}$ Pôle pharmacie, CHU Grenoble Alpes; Univerersité Grenoble Alpes; ThEMAS, CNRS/UGA TIM, Alpes, France

Background and Objective: Contrast-induced nephropathy is one of the leading causes of hospital-acquired acute renal failure (ARF). In less than 1 month two patients developed an ARF related to inappropriate administration of iodinated contrast media (ICM) in our acute geriatric unit. The objective of this case report is to analyse causes of these errors.

Design: The 1st patient (81 years old) was hospitalized for a LightChain amyloidosis. During the hospitalization a CT scan of the chest/ abdomen/pelvis (CAP) was performed. An ICM have been injected by radiologist despite the creatinine clearance $(\mathrm{ClCr})$ of $49 \mathrm{~mL} / \mathrm{mn}$ and amyloidosis. Two days after the serum creatinine (SC) doubled and reached a peak of $456 \mu \mathrm{mol} / \mathrm{L}$ at day 4 . Few days later the patient died of $E$. coli sepsis from a urinary tract infection. The 2 nd patient (90 years old) was hospitalized for a pyelonephritis. Following lower gastrointestinal bleeding and alertness disorders a scanner of the CAP was requested. This $\mathrm{CT}$ was injected with ICM despite chronic renal failure worsening the known ARF (SC: $190 \mu \mathrm{mol} / \mathrm{L}$ day 1 to $237 \mu \mathrm{mol} / \mathrm{L}$ day 3 ). The patient died at day 5 because of a heart failure induced by the hyperhydration protocol established after the exam.

Results: On both CT scan requests it was not specified not to inject ICM but the $\mathrm{SC}$ and $\mathrm{CrCl}$ were notified. For the 1 st patient the $\mathrm{CrCl}$ was not excessively low $(49 \mathrm{~mL} / \mathrm{mn})$ so ICM injection could be possible; however amyloidosis mentioned on the submission was a contraindication. About the 2 nd patient the $\mathrm{CrCl}$ of $18 \mathrm{~mL} / \mathrm{mn}$ was sufficient to contraindicate ICM administration.

Conclusion: Benefit/risk ratio and alternative imaging need to be discussed between radiologists and prescribers when patient profile is at risk especially in geriatric population. Safety is the main priority; yet, process of care should be reexamined in a multidisciplinary way. We modified scan requests by adding a box to let the possibility to prescribers to inform that ICM is not wanted.

\section{P-492}

Evaluation of motor abilities with aging using HD-sEMG and IMU data

Loubna Imrani ${ }^{1}$, Sofiane Boudaoud ${ }^{1}$, Kiyoka Kinugawa Bourron ${ }^{2}$, Kevin Lepetit ${ }^{1}$, Jeremy Laforet ${ }^{1}$, Frédéric Marin ${ }^{1}$

${ }^{1}$ Sorbonne Universités, Université de technologie de Compiègne, UMR CNRS 7338, Compiègne, France, ${ }^{2}$ Functional Exploration Unit, Hôpitaux universitaires Pitié-Salpêtrière-Charles Foix. DHU FAST, Assistance Publique Hôpitaux de Paris (APHP), Paris, France

Introduction: Preliminary study obtained in older patients [1] by using the HD-sEMG and IMU techniques have shown that HD-sEMG descriptors related to muscular activity and the trunk maximum acceleration are discriminant with the age. The aim of this study is to characterize the motor abilities with aging, including middle-aged subjects and using new descriptors, by combining both techniques, embedded in a recent ambulatory portable device.

Materials and methods: Nine subjects participated in the STS test. Three were aged $25 \pm 2$ years, four were aged $34.75 \pm 3.09$ years, and two were aged $57 \pm 7.07$ years. All of them performed sit-tostand motion 3 times at spontaneous pace. New features, were also tested in addition to previous descriptors [1], in order to check their efficiency. For this purpose, $4 \times 8 \mathrm{HD}$-sEMG grids were placed on the quadriceps muscle. Simultaneously, the trunk maximum acceleration was also measured. Thus, the results for the three age categories were statistically evaluated. The results showed that the statistical and also previous descriptors, tested on the three categories with ANOVA test, have a significant sensitivity to the motor efficiency with aging in the STS test $\left(\mathrm{p}<0.001^{* * *}\right)$ in agreement with [1].

Conclusion: The obtained results demonstrated the potential of statistical and previous descriptors from HD-sEMG and IMU data recorded by an ambulatory device for evaluating functional motor abilities with aging. Acknowledgements: This work has received support from EIT Health BP2018. [1] EUGMS 2017 Congress, "Evaluation of Motor Abilities in young and older subjects using HDsEMG and IMU data", IMRANI et al. 


\section{P-493}

Decision making affecting care of older people with cancer: improvement of general practitioners decision-making criteria (Lorraine, France)

Athanasios Benetos ${ }^{1}$, Christine Perret-Guillaume ${ }^{1}$, Frederique Claudot ${ }^{2}$, Phi Linh Nguyen-Thi ${ }^{1}$, Hubert Rousselot ${ }^{3}$, Jean-Marie Hubert

${ }^{1}$ CHRU Nancy, Vandœuvre-lès-Nancy, France, ${ }^{2}$ Université de Lorraine, Vandœuvre-lès-Nancy, France, ${ }^{3}$ Institut de Cancérologie de Lorraine, Vandœuvre-lès-Nancy, France, ${ }^{4}$ Spincourt

Multidisciplinary MSP, Vandœuvre-lès-Nancy, France

Objective: The objective of this study was to identify changes in the decision-making criteria of general practitioners (GPs) concerning the care of elderly cancer patients after 1 year of corrective measures for care practices in the Lorraine region, France.

Materials and methods: In 2014, a postal mail questionnaire was sent to all GPs in the Lorraine region. This questionnaire was designed to identify GPs' decision-making criteria. It was based on the results of a literature review and on existing guidelines. During 1 year, corrective measures were implemented to improve practices, especially training sessions for physicians and production of specific tools, including a guide to the accepted ideas in geriatric oncology. In 2015 , the same questionnaire was resent to all GPs to compare the answers.

Results: In 2014, 430 questionnaires were returned out of 2,048 sent, and in 2015, 378 questionnaires were returned out of 2,066 sent. Our results show for the first time that there exists a significant difference in the overall decision criteria between the two survey periods. This difference mainly concerns criteria related to the cancerous diseases. Physicians tend to consider the principal decision criteria to be less important after the training period. GPs express the importance of accessibility to specialists for additional advice in both 2014 and 2015; the distance between the patient's home and an adapted care facility and the interval before care begins are viewed as similarly important.

Conclusion: Training and information sessions for physicians remain the most important tool for improving care practices. Such training strategies are more effective when carried out at the geographical scale at which the cancer professionals practice, allowing them to exploit their local organizational structure. The analysis of our data makes it possible to further integrate the patient into the care path, which remains a public health issue in terms of cost and organization.

\section{P-494}

\section{Cost-effectiveness analysis of a continuity care unit}

Gabriel Ariza Zafra ${ }^{1}$, Matilde León Ortiz ${ }^{1}$, BEnedicta SánchezGonzález $^{1}$, Beatriz Martínez-García ${ }^{1}$, Silvia Lozoya Moreno ${ }^{1}$, Laura Plaza Carmona ${ }^{1}$, Borja Gil García ${ }^{1}$, Ana Pérez Fernández-Rius ${ }^{1}$, Melisa López-Utiel ${ }^{1}$, Isabel Huedo Róden

${ }^{1}$ Complejo Hospitalario de Albacete, Albacete, Spain

Introduction: The high healthcare resources use, and the adverse effects associated with hospitalization in older adults urge for the development of new assistance strategies in order to improve health outcomes, quality of life and associated costs. These strategies must be based on coordination, continuity of care and integrated care.

Methods: Retrospective study of the first 320 patients attended by the Geriatrics Continuity Care Unit (GCCU) of the Albacete Healthcare Area during the first year of implementation. Main diagnosis, Charlson index, Barthel index, Reisberg GDS, referral service, and number of visits were collected. 30-day visits to the Emergency Department (ED) and hospitalizations were recorded, as well as reduction of hospital length of stay. We calculated the hospitalization costs $(€)$ saved per patient, associated to hospitalization avoidance or reduction in the length of stay.

Results: Age 87.3 (SD 5.6). 179 women (55.9\%). 185 from geriatrics ward $(57.8 \%$ ). Barthel 44 (SD 31), Charlson 3.8 (SD 2.3), GDS 3.9 (SD 1.9). Average number of visits/patient 1.8 (SD 0.8). Within 30 days after the intervention, 70 patients needed ED visit $(21.9 \%)$, 50 were hospitalized $(15.6 \%)$ and 21 died $(6.6 \%)$. The average saving/patient was $€ 2,271$ (SD $€ 1,998)$ and total savings were $€ 726,925 /$ year. In 50 patients $(15.6 \%)$ there were no savings. Average cost reduction was $€ 2,874 / \mathrm{ED}$ patient and $€ 1,801 /$ hospitalized patient. Average cost reduction was $€ 2,807 € /$ heart failure patients, $2,256 € /$ respiratory infection patients, and 2,107€/patient with other diagnosis. Key conclusions: The GCCU reduced hospitalizations, shortened hospital stays and reduced visits to the ED, with a cost saving of $€ 726,925 /$ year.

\section{P-495}

Design for autonomous ageing: interchange between industrial design engineering and health care

Tischa van der Cammen ${ }^{1}$

${ }^{1}$ Delft University of Technology, Delft, The Netherlands

Introduction: The world is ageing rapidly. Between 2000 and 2050, the number of people aged $\geq 65$ will double as a proportion of the global population, from 7 to $16 \%$, respectively. By 2050, for the first time in human history, there will be more older people than children in the population. More distinctive is the tremendous increase in the oldest old aged $\geq 85$. This challenges society to adapt, in order to maximise the health and functional capacity of older people as well as their social participation and security. The World Report on Ageing and Health has outlined a framework for action to foster Healthy Ageing built around the new concept of functional ability.

Methods: Literature search on applications of design and technology to improve autonomy and functional ability of older people.

Results: Design thinking has embraced ageing as a topic where it can add to public health interventions. Applications of design and technology can contribute to 'autonomous ageing', i.e., independent living and life style support, and can compensate for functional deficits associated with ageing. The focus is on supporting and reinforcing the reduced physical, mental, social and functional capacities of older people by applying innovative design inclusive engineering methods, always starting with a human-centered integrated approach. Examples include design for falls prevention, dementia care and integrated care.

Key conclusions: Applications of design and technology can contribute to 'autonomous ageing'. The establishment of collaborative networks between clinicians and designers, academia and industry is required to advance design for autonomous ageing.

\section{P-496}

New technologies in the health field: what is the opinion of European Geriatricians?

Sebastien Piccard ${ }^{1}$, Antoine Piau ${ }^{2}$, Jean Petermans ${ }^{3}$

${ }^{1}$ Geriatric Department, University of Liege, Liège, Belgium,

${ }^{2}$ Department of Geriatric Medicine, University Hospital of Toulouse, 
Toulouse, France, ${ }^{3}$ Geriatric Department, CHU Liege, Liège, Belgium

Introduction: There is currently a growing development of gerontechnologies, particularly in the health field. These technologies aim to meet various objectives and are intended for seniors but also for healthcare professionals. In this context, it seemed important to gather the opinion of geriatricians.

Methods: We conducted an online survey (on the behalf of the SIG on gerontechnologies of the EuGMS), sent to the geriatricians in Europe through their national societies, to obtain their opinion about technologies and aging and to better know their involvement and perspectives in this area.

Results: 179 geriatricians responded. They perceive gerontechnologies as useful for alarm, follow-up, prevention, treatment and adherence support. However, alarm technologies are perceived as significantly more useful than others $(\mathrm{p}<0.01)$. These technologies are also considered adequate for mobility, social link and cognitive, vital and psychic functions. It appears that mobility technologies are perceived as significantly more useful $(\mathrm{p}<0.01)$, while those for psychic functions are considered less relevant $(\mathrm{p}<0.01)$. Moreover, even if geriatricians perceive gerontechnologies as useful, their concrete involvement in the use is still weak. Indeed, $48 \%$ of respondents say they are not involved in their use. However, $78 \%$ think that new technologies can positively change the organization of care and $90 \%$ say that it would be necessary to address this topic in medical courses. Conclusion: Geriatricians are aware of the value of new technologies in the health field. However, these are still little used in practice. It seems necessary to integrate the question of technologies into the training of future professionals.

\section{P-497}

\section{Analysis of the relationship between repeated hospitalizations and the occurrence of new hospitalization in the elderly using models of frailty in survival}

Babykina Genia $^{1}$, Lamer Antoine ${ }^{2}$, Ficheur Gregoire ${ }^{1}$, Chazard Emmanuel $^{1}$, Puisieux Francois ${ }^{3}$, Beuscart Jean-Baptiste ${ }^{2}$

${ }^{1}$ CERIM EA2694, Lille, France, ${ }^{2}$ CERIM EA2694, Lille, France, ${ }^{3}$ LILLE Hospital, Lille, France

Introduction: Older people are at higher risk of re-hospitalization than other patients because of a higher frequency of polypathology, geriatric syndromes or social problems, but the association between repeated hospitalizations and the risk of occurrence of a new hospitalization has been less studied in this population. The objective was to analyze the relationship between repeated hospitalizations and the risk of new hospitalization in people aged 75 and over, after first hospitalization in medicine for an acute event.

Method: Analysis by weakened survival models. The fragility approach is a statistical modeling concept that aims to represent heterogeneity in a study population caused by unmeasured covariates. In survival analyzes, which take into account the time elapsed before the occurrence of an event, these models make it possible to take into account repeated events over time. SNIIRAM-PMSI chain data on all persons aged 75 and over in the Valenciennois-Quercitain territory from 2014 to 2016 were used. These data contain all the information on hospitalizations (dates, durations, location, diagnoses) and deaths (hospital or home via the cross with CEPI-death).

Results: Between April 01, 2014 and June 30, 2016, 28,496 patients were followed. The proportion of patients with at least one hospitalization is $73 \%$. The use of specific models for the analysis of recurrent events, here successive rehospitalizations, demonstrate that the risk of re-hospitalization increased according to the number of previous hospitalizations.

Conclusion: The risk of hospitalization is related to the presence of previous hospitalizations. The analysis of hospitalization by more concrete statistical models, allows a reliable approach and closer to reality. These models are necessary for the analysis of large databases.

\section{P-498}

Elderly and technologies: between myths and reality

Sebastien Piccard ${ }^{1}$, Pierre Pagacz ${ }^{2}$, Christelle Boulanger ${ }^{3}$, Pierre Leclercq ${ }^{3}$, Murielle Lona ${ }^{4}$, Alex Peltier ${ }^{5}$, Fabien Delcourt ${ }^{5}$, Pierre Cools ${ }^{6}$, Jerome Vrancken ${ }^{6}$, Thibault Mestre ${ }^{6}$, Mohamed Boukhebouze ${ }^{7}$, Jean Petermans ${ }^{8}$

${ }^{1}$ University of Liege - Geriatric department, Liege, Belgium, ${ }^{2}$ University of Namur - Research Centre in Information, Law and Society - CRIDS, Namur, Belgium, ${ }^{3}$ University of Liege - Lab for User Cognition \& Innovative Design - LUCID, Liege, Belgium, ${ }^{4}$ Union Nationale des Mutualités Libres, Belgium, ${ }^{5}$ Alliance Nationale des Mutualites Chretiennes - ANMC, Belgium, ${ }^{6}$ Union Nationale des Mutualites Socialistes - Solidaris, Belgium, ${ }^{7}$ Centre of excellence in information and communication technologies - CETIC, Belgium, ${ }^{8} \mathrm{CHU}$ Liege - Geriatric department, Liege, Belgium

Introduction: Currently, the picture of an old person using technology is still very negative. Indeed, it is often said that old people are not interested in technologies, can't understand their utility or have not the physical capacity to use them. These stereotypes can negatively influence the adoption of technologies with aging. Indeed, despite the increasing availability of new technologies for the benefit of old people, their use is currently extremely low.

Methods: We therefore wanted to explore, through an online survey widely distributed in Wallonia (Belgium) (11,579 respondents), the perception and use of new technologies by the olders.

Results: With regard to the use of technologies, analyses indicate that older people use the smartphone and the tablet less than younger. However, results show that this lower use does not appear to be related to an inability, but rather to the fact that people do not perceive it as useful $(\mathrm{p}<0.00)$. Furthermore, we note that the difficulties in using these technologies are relatively similar in the different age groups $(41-50,51-60,61-70,71-80,81-90$ years old). The difficulties that can increase with age are generally related to the ergonomics of the device and not to a problem of sight (readability of the screen) or fine motor skills (writing with the touch keyboard). Conclusion: These data therefore seem to confirm that older people have the physical abilities to use new technologies and that they can be interested in them if they are useful and adapted to their needs.

\section{P-499}

Impact of antibiotic stewardship program: a prospective audit and feedback study in a elderly's hospital

Cyprien Arlaud ${ }^{1}$, Marina Orloff ${ }^{2}$, Katia Muller ${ }^{2}$, Remy Collomp ${ }^{2}$, Veronique Mondain ${ }^{1}$, Olivier Guerin ${ }^{1}$

${ }^{1}$ Medicine, ${ }^{2}$ Pharmacy

Introduction: Older patients generally have greater susceptibility to infections but inappropriate use of antibiotics is one of the most important factors contributing to the emergence of drug resistant pathogens. The purpose of this study was to measure the clinical 
impact of antimicrobial stewardship programme about reassessment of antibiotic therapy by the infectio-geriatrician (IG) and the clinical pharmacist (CP).

Methods: A prospective audit with intervention and feedback began between 48 and $72 \mathrm{~h}$ after treatment initiation in november 2017 to subjects aged 75 years and older. 2 months during Data collected included clinical and demographic data; use of antimicrobials measured by defined congruence with guidelines in the use of antibiotics. Results: Overall, 160 interventions were made the IG and CP team (3.5 prescriptions per day) with an average acceptance rate of $93.8 \%$ among. The main antibiotics were amoxicillin-clavulanic acid $38.8 \%$, cefotaxime $15.8 \%$ and amoxicillin $14.8 \%$. The most common etiological diagnosis were respiratory diseases $(36.4 \%)$, urinary $(26.6 \%)$ and bacteremia (14.8\%). Average compliance rates to guidelines were $36.1 \%$. In multivariate analysis, referent intervention improves correct use of $\mathrm{AB}$, OR $8.7 \mathrm{p} \leq 0.005$ (3.9-19.3) 95\% CI.

Conclusion: Our prospective audit was associated with positive impact on antimicrobial use. The synergy between infectio-geriatrician and pharmacist makes it possible to reinforce the good use of $\mathrm{AB}$. Limit the duration of treatments $\mathrm{AB}$ remains a priority action to reduce $\mathrm{AB}$ consumption too important in France.

\section{P-500}

Integration of translational aged care research into practice: experience within an Australian aged care organisation

Tracy Aylen ${ }^{1}$, Rajna Ogrin ${ }^{2}$, Claudia Meyer ${ }^{2}$, Judy Lowthian ${ }^{2}$, Elizabeth Cyarto ${ }^{2}$

${ }^{1}$ Bolton Clarke, Care Innovation Team, Melbourne Australia, ${ }^{2}$ Bolton Clarke Research Institute, Melbourne Australia

Introduction: Quality care relies on identification of issues and moving current research into practice to address these issues through knowledge translation and implementation, to ensure people in the health and aged care system benefit promptly from the latest evidence. We describe the approach of a research institute, embedded in a large Australian health and aged care service provider, designed to underpin quality care of all older people using their service, and to build a knowledge base in this cohort.

Method/process: Using a collaborative approach, older people, front line care staff, management and researchers work together to: identify and prioritise pressing issues; scope current evidence to address these issues; co-design interventions to be addressed with key stakeholders, building on the identified current evidence. The intervention is then piloted and evaluated, with adaptations made to the learnings before full evaluation.

Results: A selection of current and past projects are presented: optimising diabetes management of older community-dwelling adults using remote consultancy, improving medication safety for home nursing clients through visiting pharmacist review, barriers to accessing services for older women living alone, and piloting a diabetes foot App. We discuss strategies for translating recommendations arising from the research into clinical practice, including the importance of relationships between the researchers, internal and external stakeholders.

Key conclusions: Integration of researchers and research capacity into a health and aged care service provider, together with an inclusive co-design approach, has ongoing benefits for clients and residents, the staff, and more broadly, the health and aged care sector.

\section{P-501}

An EuGMS survey: who are the main medical care providers for European nursing home residents?

Fitzpatrick Donal $^{1}$, Olafur Samuelsson ${ }^{2}$, Iva Holmerova ${ }^{3}$, Martin Finbar ${ }^{4}$

${ }^{1}$ Mater Misericordiae University Hospital, Dublin, Ireland, ${ }^{2}$ National University Hospital of Iceland, Reykjavik, Iceland, ${ }^{3}$ Charles University, Prague, Czech Republic, ${ }^{4}$ Guy's and St. Thomas' NHS Foundation, London, UK

Introduction: Nursing home residents are a complex, vulnerable and historically neglected cohort. The EuGMS is committed to improving care for these individuals: it established formal standards of medical care for nursing home residents in 2015 , and is currently developing a curriculum of core competencies [1]. Integral to these efforts is identifying the physicians, for whom, education in these competencies needs to be directed.

Method: A survey was distributed to EuGMS members representing national geriatrician societies across Europe. We asked members their perceptions of what proportion of nursing home care was delivered by various physician specialties, and what were the main functions carried out in nursing homes in their countries.

Results: We received 22 replies from 33 countries. The vast majority of medical care in nursing homes is delivered by general practitioners, over $75 \%$ in 17 of 22 countries, rather than by geriatricians or physicians specifically qualified in nursing home medicine. The primary function of nursing homes is residential care. However, in most countries, nursing homes play an important role in rehabilitation, respite and palliative care-almost a quarter of nursing home activity. Conclusion: The majority of medical care in nursing homes is delivered by general practitioners. While the primary function of nursing homes is residential care, rehabilitation, respite and palliative care are important components of nursing home activities. Education and training structures to achieve the highest standards of medical care must be designed for general practitioners without specialist training in geriatric medicine.

\section{Reference:}

1. Briggs R, Holmerová I, Martin FC, O’Neill D, McGee H, O’Neill $\mathrm{D}$ et al (2015) Towards standards of medical care for physicians in nursing homes. Eur Geriatr Med [Internet]. 6(4):401-413. http:// linkinghub.elsevier.com/retrieve/pii/S187876491500087X. Accessed 2 Jun 2017

\section{P-502}

The experience of Pietro Thouar Unit, an example of intermediate care for older persons in the framework of tuscany health system

Matteo Bulgaresi ${ }^{1}$, Anna Maria Mello ${ }^{1}$, Federica Sgrilli ${ }^{1}$, Riccardo Barucci ${ }^{1}$, Ilaria Del Lungo ${ }^{1}$, Sante Giardini ${ }^{1}$, Salvatore Gangemi ${ }^{1}$, Marina Cappugi ${ }^{1}$, Antonella Notarelli ${ }^{1}$, Stefania Bandinelli ${ }^{1}$, Enrico Benvenuti ${ }^{1}$

${ }^{1}$ SOC Geriatria Firenze-Empoli, Azienda USL Toscana Centro, Pistoia, Italy

Introduction: In Italian health services, Intermediate Care setting (IC) is growing, like in most of European countries. The aim of IC is to improve the transition from the hospital to territorial primary care, reducing in frail older institutionalization and delaying disability.

Methods: This retrospective study included all patients admitted to Geriatric IC Unit Pietro Thouar in Florence, from January 2017 to May 2018. Clinical, functional and social data were collected from medical records at discharge. 
Results: 409 subjects, mean age 81 , were included. $65 \%$ had previous disability ( $22 \%$ Barthel Index score $<40), 38 \%$ had dementia, $62 \%$ showed social distress. $54 \%$ of subjects was bedridden, $54 \%$ had important comorbidities, $13 \%$ active infections and $72 \%$ took more than 5 drugs. Delirium was present in $11 \%$ of sample at admission, while incident delirium was $12 \% .70 \%$ of the sample underwent some kind of complications during stay; falls were $4 \%$. Mean duration of recovery was 18 days; $56 \%$ of subjects was discharged at home while $28 \%$ was institutionalized; $8 \%$ was moved to ED and $0.2 \%$ died. At discharge $8 \%$ of patients shown behavioural and psychological symptoms of dementia (BPSD), $58 \%$ was able to walk, $32 \%$ had severe disability.

Key conclusions: The population hospitalized in Geriatric IC is an example of society aging. Disability, comorbidities, dementia and social distress were highly prevalent and represent a challenge for health care. In these patients at discharge BPSD improved, one third of subjects regained walking ability. Our experience could help to improve strategy for disability's contraction and optimization of health care.

\section{P-503}

Evaluating the benefits of connect house: an innovative step-down facility for patients awaiting care home placements

\section{Z. Vettasseri ${ }^{1}$, J. Pattinson ${ }^{1}$, J. Chapman ${ }^{1}$, R. Machin ${ }^{1}$, A. Blundell ${ }^{1}$ \\ ${ }^{1}$ Nottingham University Hospitals NHS Trust, Nottingham, UK}

Introduction: An innovative step-down facility (Connect House), under the shared ownership of community providers and local hospitals, was opened to address delays discharging patients awaiting care home placements. Comprehensive geriatric assessment (CGA) is provided by a multi-disciplinary team (MDT) including an onsite medical team and visiting Consultant Geriatricians.

Methods: A retrospective case note review of 91 residents was mapped against metrics including MDT input, admission/ discharge Rockwood score, healthcare-associated morbidity, re-admission rates, advanced care plan (ACP) initiation and discharge destination.

Results: All patients received a medical review. 83 (92\%) patients saw physiotherapists, $82(90 \%)$ social workers and 67 (74\%) occupational therapists. At discharge, 17 (19\%) patients improved their Rockwood score. 27 (30\%) patients experienced delirium, 29 (32\%) developed infections and there were 23 recorded falls. 16 of the 25 patients with pressure damage on arrival fully healed by discharge. A hospital re-admission was avoided on 95 separate occasions. ACPs were created for $21(23 \%)$ patients. $9(10 \%)$ patients were initiated on palliative care. $20(22 \%)$ patients returned home, $50(55 \%)$ were discharged to care homes, 17 (19\%) were re-admitted and 4 (4\%) died in Connect House. The mean length of stay (LOS) was 39.5 days.

Conclusions: The coordinated CGA approach at Connect House led to patients improving functionally and returning home despite being highlighted in hospital as requiring long-term nursing care. The onsite medical team prevented re-admissions by addressing healthcare-associated morbidity, managing prolonged delirium and creating appropriate ACPs. Further work is required to identify factors that can reduce the LOS.

\section{P-504}

Process evaluation of a complex intervention for optimizing appropriateness of prescribing in the nursing home setting (COME-ON study): focus on the interdisciplinary case conferences

Anrys Pauline ${ }^{1}$, Strauven Goedele ${ }^{2}$, Roussel Sandrine ${ }^{1}$, Vande Ginste Marie ${ }^{2}$, De Lepeleire Jan ${ }^{3}$, Foulon* Veerle ${ }^{2}$, Spinewine* Anne ${ }^{4}$

${ }^{1}$ Université Catholique de Louvain, Louvain Drug Research Institute, Clinical Pharmacy Research Group, Brussels, Belgium, ${ }^{2} \mathrm{KU}$ Leuven, Department of Pharmaceutical and Pharmacological Sciences, Leuven, Belgium, ${ }^{3} \mathrm{KU}$ Leuven, Department Public Health, Brussels, Belgium

Introduction: The COME-ON study (Collaborative approach to Optimize Medication use for Older people in Nursing homes) assessed the impact of a complex intervention consisting of interdisciplinary case conferences (ICC, i.e. face-to-face medication reviews performed by an interdisciplinary team: general practitioner, pharmacist and nurse) supported by training and local concentrations [1]. Participants were requested to conduct three ICCs per nursing home resident (NHR) over a 12-month period. The process evaluation aimed to explore the implementation, mechanisms of impact and contextual factors that influenced the intervention [2].

Methods: Quantitative data on implementation were collected throughout the study period. Qualitative data were collected through multidisciplinary focus groups in 11 nursing homes (NHs).

Results: 1675 ICCs were registered for 681 NHRs [median (P25P75): 3 (2-3) per NHR]. Overall, healthcare professionals were satisfied with ICCs and perceived benefits for themselves. Their perception of the impact on NHRs varied from limited impact to positive impact in terms of number of medications or cost. Several barriers and facilitators to the implementation and the outcomes of the ICCs were identified. They related to: (a) the intervention (e.g. the face-to-face and interdisciplinary aspects contributed to the success of ICCs, despite organizational constraints); (b) HCPs (e.g. GP's attitude); (c) organizational level (e.g. presence of a leader); (d) external context (e.g. financial incentives).

Key conclusions: Quantitative as well as qualitative data on experiences and satisfaction were essential to generate recommendations for Belgian policymakers on the future implementation of ICCs in $\mathrm{NHs,}$ for example in terms of frequency, format, prerequisites

\section{References:}

1. Anrys $P$ et al (2016) Collaborative approach to optimise medication use for older people in nursing homes (COME-ON): study protocol of a cluster controlled trial. Implement Sci 11(1)

2. Moore GF et al (2015) Process evaluation of complex interventions: medical research council guidance. BMJ 350:h1258

\section{P-505}

Measurement of quality of care in elderly in Turkish Hospitals using LPZ tool

Bulent Saka ${ }^{1}$, Sibel Akin ${ }^{2}$, Celalettin Kocatürk ${ }^{4}$, Ilker Tasci ${ }^{4}$, Sumru Savas ${ }^{5}$, Teslime Atli ${ }^{6}$, Pinar Tosun ${ }^{7}$, Cafer Balci ${ }^{8}$, Hakan Yavuzer ${ }^{9}$, Murat Varli ${ }^{10}$, Asli Tufan ${ }^{11}$, Zeynel Abidin Ozturk ${ }^{12}$, Gulbuz Sezgin $^{13}$, Meftun Firtina ${ }^{14}$, Mehmet Akif karan ${ }^{1}$

${ }^{1}$ Istanbul University, Istanbul School of Medicine, Div. Geriatrics, Istanbul, Turkey, ${ }^{2}$ Erciyes University School of Medicine, Div. Geriatrics, Kayseri, Turkey, ${ }^{3}$ Health Sciences University, Dep. Surgery, Yedikule Hospital, Istanbul, Turkey, ${ }^{4}$ Health Sciences 
University, Gülhane Faculty of Medicine, Dep. Internal Medicine, Ankara, Turkey, ${ }^{5}$ Ege University School of Medicine, Div Geriatrics, Izmir, Turkey, ${ }^{6}$ Guven Hospital, Div Geriatrics, Ankara, Turkey, ${ }^{7}$ Erzurum Teaching Hospital, Div. Internal Medicine, Erzurum, Turkey, ${ }^{8}$ Hacettepe University School of Medicine, Div. Geriatrics, Ankara, Turkey, ${ }^{9}$ Istanbul University, Cerrahpasa School of Medicine, Dep. Internal Medicine, Istanbul, Turkey, ${ }^{10}$ Ankara University School of Medicine, Div. Geriatrics, Ankara, Turkey,

${ }^{11}$ Marmara University School of Medicine, Div. Geriatrics, Istanbul, Turkey, ${ }^{12}$ Gaziantep University School of Medicine, Div. Geriatrics, Gaziantep, Turkey, ${ }^{13}$ Maltepe University School of Medicine, Dep. Internal Medicine, Istanbul, Turkey, ${ }^{14}$ Nutricia Istanbul, Turkey

LPZ is an annual international multicenter cross-sectional prevalence measurement of care problems on institution, department and patient level.

Materials and methods: Measurement was done on November 2017 in 13 hospitals of Turkey. Patients $\geq 65$ years old were evaluated. This study was supported by Nutricia Turkey.

Results: 298 patients were taken into the study (mean age $76.8 \pm 7.9$ years) from 13 hospitals. National/international guidelines were used for pressure ulcer (PU) in $100 \%$, for urinary incontinence (UI) in $45.7 \%$, for malnutrition (MN) in $91.6 \%$, for falls in $100 \%$, for restrictions in $57.5 \%$, for pain in $87.1 \%$ and for delirium in $45.2 \%$ of the patients. PU risk was $73.5 \%, 12.8 \%$ stage $\geq 1 \mathrm{PU}$ and $6.4 \%$ nosocomial PU. Main interventions were active/reactive support surface, repositioning and moisturiser/barrier cream usage. $40.3 \%$ of the patients had UI. Main interventions were urinary catheters, medications, bladder training and inlay pads/underslips. $7.4 \%$ of the patients showed incontinence associated dermatitis. MN risk was $30.2 \%$ and 76 patients had dysphagia. Main interventions were energy/protein rich snacks, enteral feeding $(12.8 \%)$ and parenteral nutrition $(12.1 \%)$. Fall prevalence was $26.2 \%$ (nosocomial falls $9.1 \%$ ) and restraints were used in 59 patients (53 mechanical, 14 physical and 16 pharmacological). 158 patients (53\%) had pain. $47.3 \%$ received pharmacological treatment (paracetamol: $36.6 \%$, NSAII: $10.1 \%$, opioids: $11.4 \%$, pregabaline: $5.7 \%$ ). Delirium was diagnosed in $8.1 \%$ of the patients.

Conclusion: Annual measurement of risk/prevalence, preventive measures and treatment interventions will provide better hospital care plans for elderly.

\section{P-506}

\section{Measurement of quality of care in elderly in Turkish Nursing Homes using LPZ tool}

Bulent Saka ${ }^{1}$, Hafize Dogan ${ }^{2}$, Sevnaz Sahin ${ }^{3}$, Teslime Atli ${ }^{4}$, Meftun Firtina $^{5}$, Mehmet Akif Karan ${ }^{1}$

${ }^{1}$ Istanbul University, Istanbul School of Medicine, Div. Geriatrics, Istanbul, Turkey, ${ }^{2}$ Istanbul Municipality, Istanbul Darulaceze

Kayisdagi Nursing Home, Istanbul, Turkey, ${ }^{3}$ Ege University, School of Medicine, Div. Geriatrics, Izmir, Turkey, ${ }^{4}$ Guven Nursing Home, Ankara, Turkey, ${ }^{5}$ Nutricia Istanbul, Turkey

LPZ is an annual international multicenter cross-sectional prevalence measurement of care problems on institution, department and patient level. Materials and methods: Measurement was done on November 2017 in 5 nursing homes of 3 big cities in Turkey. Patients $\geq 65$ years old were evaluated. This study was supported by Nutricia Turkey.

Results: 680 residents were taken into the study (mean age $78.6 \pm 8.0$ years). National/international guidelines were available for only pressure ulcer (PU) in $100 \%$, for malnutrition (MN) in $82.2 \%$ and for falls in $73.7 \%$ of the patients. PU risk was $55.3 \%, 2.2 \%$ stage $\geq 1 \mathrm{PU}$ and $1.9 \%$ nosocomial PU. Main interventions were active/reactive support surface, repositioning and moisturiser/barrier cream usage and heel suspension. $54.0 \%$ of the patients had UI and 26 patients $(3.8 \%)$ had urinary catheter. $8.4 \%$ of the patients showed incontinence associated dermatitis. $\mathrm{MN}$ prevalence and $\mathrm{MN}$ risk were $14.6 \%$ and $17.6 \% .93$ patients $(13.7 \%)$ had dysphagia. Main interventions were energy/protein rich diet, enteral nutrition $(9.3 \%)$, parenteral nutrition $(0.4 \%)$ and adjustment of meal consistency (18.4\%). Fall prevalence was $14.3 \%$ (nosocomial falls $7.1 \%$ ) and restraints were used in 280 patients (258 mechanical, 15 physical and 44 pharmacological, 28 psychological and 57 one-to-one supervision). 113 patients (16.6\%) had pain. $19.1 \%$ received pharmacological treatment (paracetamol: $15.3 \%$, NSAII: $3.4 \%$, opioids: $0.7 \%$, pregabaline: $2.5 \%$ ). Sarcopenia was diagnosed in 445 patients $(65.4 \%)$ according to SARC-F. Delirium was diagnosed in $1.2 \%$ of the patients $(n=8)$.

Conclusion: Annual measurement of risk/prevalence, preventive measures and treatment interventions will provide better care plans for elderly.

\section{P-507}

Effective communication between ward, pharmacy and patient is required to enable reliable flow through the discharge lounge

\author{
Hassan Khan ${ }^{1}$, Arturo Vilches-Moraga ${ }^{2}$, Alison Blair ${ }^{2}$ \\ ${ }^{1}$ Manchester Medical School, Manchester, UK, ${ }^{2}$ Salford Royal NHS \\ Foundation Trust, Salford, UK
}

Introduction: The purpose of a hospital discharge lounge is to provide a safe environment for patients to await medication and transport. With acute hospital bed occupancy in the English NHS running $>90 \%$ and Emergency Departments consistently failing the $95 \% 4 \mathrm{~h}$ standard, pressure to maintain "flow" has concentrated focus on maximising discharge lounge use risking deterioration in patient care. Our aim was to understand the breakdown of time spent in the discharge lounge to optimise future development.

Methods: Cross-sectional study of 88 patients attending the discharge lounge at Salford Royal Hospital during a week in April 2018. The patients were split into $\geq 75$ years and $<75$ years groups. Time for the various determinants facilitating discharge (prescribing and dispensing of medication, completion of discharge summary, awaiting transport etc) was measured.

Results: 40 patients were aged $\geq 75$ years and 44 were $<75$ years. Mean time spent for patients $\geq 75$ years is 115.7 min with a standard deviation of $66.7 \mathrm{~min}$; mean time spent for $<75$ years is $94.6 \mathrm{~min}$ with a standard deviation of 61.4. Mean time taken for pharmacy to be alerted to prescribed medication for dispensing is $67.7 \mathrm{~min}$ with a standard deviation of 97.3. Average wait for patients in the discharge lounge was $113 \mathrm{~min}$ if medication was prescribed before patient arrival, and $143 \mathrm{~min}$ if it was incomplete.

Conclusions: The high variance of time spent in the discharge lounge supports completion of the discharge summary and improved communication between ward and pharmacy prior to patients' transfer to the discharge lounge.

\section{P-508}

Prevention of acute admission by digital health. Preliminary experiences from the implementation of a GERI-toolbox and a cross-sectorial generic telemedicine platform in four Danish municipalities

\section{Karen Andersen-Ranberg}

${ }^{1}$ Department of Geriatrics, Odense University Hospital, Odense, Denmark 
Introduction: Europe is challenged by a significant rise in $80+-$ year olds as well as expansion of morbidity in the coming decades. Yet, during the last decades hospital beds in most European countries have been reduced, and further reductions are anticipated. In Denmark, this mismatch is expected to be alleviated by digital health, and the introduction of acute community nurses (ACN). ACNs may act as 'frontline officers' for early identification in-home of older citizens at risk of acute admission. In collaboration with primary care physicians (PCPs) timely treatment may be initiated thereby potentially reducing acute hospital admission.

Methods: The "GERI-toolbox" is a portable toolbox containing Point of Care Testing (POCT) tools, including blood testing, for inhome clinical assessment. Values are uploaded via mobile network $(3 \mathrm{G} / 4 \mathrm{G})$ to a generic digital platform (GTP), accessible to treating PCP, ACN, and hospital physicians.

Results: The GERI-toolbox was implemented March 1, 2018 in four Danish municipalities and is ongoing. Implementation has been challenging as it involves organizational changes and overcoming professional barriers. Yet, the GERI-toolbox has already become a success. Trained ACNs feel that their competences are appreciated, and the PCPs receive nurse reports of higher quality. Preliminary results are promising with respect of reducing acute admissions. The study ends December 31, 2019.

Key conclusions: The GERI-toolbox likely supports early clinical decision-making and has the potential to prevent acute hospital admission of older citizens. The concept may be adapted to other health care systems in Europe and should be further evaluated in a future cross-national multicentre study.

\section{P-509}

\section{Choosing wisely: the French approach}

Corvol Aline ${ }^{1}$, Jarno Pascal ${ }^{2}$, Cario Thomas ${ }^{2}$, Veillard David ${ }^{2}$, Menand Emilie ${ }^{2}$, Passadori Yves ${ }^{3}$, Prud'homm Joaquim ${ }^{4}$, Somme Dominique $^{5}$

${ }^{1} \mathrm{CHU}$ Rennes, ARENES, Rennes, France, ${ }^{2} \mathrm{CHU}$ Rennes, Rennes, France, ${ }^{3} \mathrm{CH}$ Mulhouse, Mulhouse, France, ${ }^{4} \mathrm{CHU}$ Rennes, Universite Rennes 1, Rennes, France, ${ }^{5}$ Université Rennes 1, CHU Rennes, ARENES, Rennes, France

Introduction: "Choosing Wisely" is an international campaign led by scientific societies. It aims to reduce waste overuse in health care and avoid unnecessary tests and procedures by promoting patientphysician dialogue. Every scientific society drafts and communicates on 5 short recommendations to improve the appropriateness of care. Method: We will present the approach of the "Choisir avec soins" working group of the French Society for Geriatrics and Gerontology. We choose to work closely with a patients group and to operationalize directly our recommendations through a campaign addressed to geriatric wards to allow an evaluation of their practice.

Result: The redaction of one recommendation has been given to the patient group, who decides to promote patient-physician dialogue on the level of care. Other recommendations concern screening for urinary tract infections, long term prescription of benzodiazepine, and antipsychotics for persons living with dementia. A recommendation on prescription review is currently being rewritten for more accuracy. We conducted two campaigns in 20 different wards, collecting data on 1036 patients. The results will be presented.

Conclusion: Our dynamic approach allowed a strong collaboration with patients, a fast operationalization of recommendations, and a continuous adjustment to the context of care.

\section{P-510}

Long-term validation of the TIAGo social robot in care for older persons living in the community

Slawomir Tobis ${ }^{1}$, Joanna Piasek ${ }^{1}$, Sylwia Kropinska ${ }^{1}$, Katarzyna Stachnik $^{1}$, Katarzyna Wieczorowska-Tobis ${ }^{1}$

${ }^{1}$ Poznan University of Medical Sciences, Poznań, Poland

The ENRICHME project was directed at development and evaluation of the impact of new solutions (including social robots) on supporting older people with mild cognitive impairment. The paper aims to present the results of a long-term (10 weeks) validation of the TIAGo robot (PAL Robotics, Spain) in the living environments of older persons in Poland. The validation was done with four persons (three women aged 66, 83 and 84 years, and one man aged 83 years); all of them had an ethnographic observation of qualitative parameters performed. The study also had a control arm (four subjects with standard care).All participants accepted the robot right after its presentation. They approached it with confidence, considered it safe and believed they could handle it with support from the ENRICHME team. They pointed out that (with the robot) there was someone to talk to at home, it was easier to cope with loneliness, and the house was more friendly. During the validation, participants were interested and engaged in various functions of the system. Their favourite option was "cognitive games". The reminders of medicines and appointments were also used on a regular basis, much like the dietary advice and healthy tips. The project confirmed that the TIAGo robot was real support for the participants, i.a. by increasing cognitive, physical and social activity. It also showed that it was essential to observe and monitor validations in the long term to realistically evaluate human-robot interactions, because of the confusion caused by the introduction of the robot.

\section{P-511}

The role of volunteers in preventing hospital-associated deconditioning among older people: a feasibility and acceptability study

Stephen Lim $^{1}$, Kinda Ibrahim ${ }^{2}$, Richard Dodds ${ }^{3}$, Gayle Strike ${ }^{4}$, Mark Baxter $^{4}$, Anne Rogers ${ }^{5}$, Avan Aihie Sayer ${ }^{6}$, Helen Roberts ${ }^{7}$

${ }^{1}$ Academic Geriatric Medicine, University of Southampton, Southampton, UK, NIHR CLAHRC Wessex, University of Southampton, Southampton, UK, ${ }^{2}$ Academic Geriatric Medicine, University of Southampton, Southampton, UK; NIHR CLAHRC Wessex, University of Southampton, Southampton, UK, ${ }^{3}$ NIHR Newcastle Biome, Southampton, UK

Introduction: Deconditioning is the physiological change associated with prolonged inactivity and bedrest. Low mobility levels among older inpatients is associated with increased risk of functional decline, institutionalisation and death. This study aimed to explore the feasibility and acceptability of training volunteers to encourage older inpatients to be more active.

Methods: This pre-post study was conducted on acute medical wards for older people, using a mixed methods approach. Inclusion criteria included patients aged $\geq 70$ years who were mobile prior to admission. Two physical activity measures were used: the StepWatch Activity Monitor and GENEActiv. Volunteers were trained to deliver twice daily activity sessions which consisted of mobility and/or bedside exercises. Nurses, therapists, volunteers and patients were interviewed to determine the acceptability of the intervention.

Results: 50 participants were recruited pre-intervention (mean age 87 years, SD 4.6), with a median daily step count of 626 (IQR 298-1468) and mean daily acceleration of 9.1 milligravity (SD 3.3). 
16 volunteers were trained to deliver the intervention. 310 activity sessions were offered and $230(74 \%)$ delivered. 50 participants (mean age 86.2, SD 5.1) received the intervention, with a daily step count of 912 (IQR 295-1824) and mean daily acceleration of 9.1 milligravity (SD 3.3). The intervention was well-received by patients. Staff members valued the help of volunteers in promoting increased physical activity among older inpatients.

Key conclusions: Volunteers can be trained to deliver mobility and exercise interventions for older inpatients. A future controlled study is required to determine the impact of the intervention on patient outcomes.

\section{P-512}

Intermediate Care in the framework of health care services for older persons: data from an Italian experience

Anna Maria Mello ${ }^{1}$, Matteo Bulgaresi ${ }^{1}$, Riccardo Barucci ${ }^{1}$, Federica Sgrilli $^{1}$, Sante Giardini ${ }^{1}$, Ilaria Del Lungo ${ }^{1}$, Marina Cappugi ${ }^{1}$, Salvatore Gangemi ${ }^{1}$, Stefania Bandinelli ${ }^{1}$, Antonella Notarelli ${ }^{1}$, Enrico Benvenuti ${ }^{1}$

${ }^{1}$ SOC Geriatria Firenze-Empoli, Azienda USL Toscana Centro, Pistoia, Italy

Introduction: Intermediate Care (IC) is an emerging concept in Italian health services for frail olders that has been developed to facilitate the transition from hospital to primary care, reducing disability and istitutionalization.

Methods: This retrospective study included all patients admitted to the Geriatric IC Unit in San Miniato (Pisa), from April 2017 to May 2018. ClinicaI, functional and social data were collected from medical records at discharge.

Results: 205 subjects, mean age 79 and 55\% women, were included. $96 \%$ of patients had previous disability (47\% showing a Barthel Index score $<40$ ), $50 \%$ had dementia, $55 \%$ showed social distress. $84 \%$ of subjects was bedridden, $94 \%$ had important comorbidities, $65 \%$ active infections and $90 \%$ took polipharmacy. Delirium was present in $21 \%$ of sample at admission, while incident delirium was $5 \% .25 \%$ of patients required artificial nutrition and $32 \%$ of the sample underwent some kind of complications during stay; falls were $2 \%$. Mean duration of recovery was 11.5 days; $54 \%$ of subjects was discharged at home while $24 \%$ was istitutionalized; $10 \%$ was moved to Emergency Department and $6 \%$ died. At discharge $5 \%$ of patients showed behavioural and psychological symptoms of dementia (BPSD), 34\% was able to walk, $81 \%$ had severe disability.

Key conclusions: Between these older patients admitted to a Geriatric IC Unit, severe comorbidities, previous disability, polipharmacy, dementia and social distress were highly prevalent. At discharge, BPSD improved, one third of subjects regained walking ability but serious disability remained. This "real word" data could be useful to improve trajectories of public health planning.

\section{P-513}

Potentially inappropriate prescriptions of antibiotics in older hospitalized patients: French experts' explicit definitions (a qualitative study)

Nicolas Baclet ${ }^{1}$, Matthieu Calafiore ${ }^{2}$, Celine Fregnac ${ }^{2}$, Gaetan Gavazzi $^{3}$, Emmanuel Forestier ${ }^{4}$, Claire Roubaud-Baudron ${ }^{5}$, Thibaut Fraisse $^{6}$, Serge Alfandari ${ }^{7}$, Eric Senneville ${ }^{7}$, Jean-Baptiste Beuscart ${ }^{2}$

${ }^{1}$ Univ. Lille, EA 2694-Santé publique: épidémiologie et qualité des soins, 59000, Lille, France. Lille Catholic Hospitals, Department of
Infectious Diseases, 59160, Lille, France, ${ }^{2}$ Univ. Lille, EA 2694-Santé publique: épidémiologie et qualité, Lille, France

Context: Explicit definitions of potentially inappropriate prescriptions of antibiotics (antibiotic-PIPs) could provide an innovative approach to tackle antimicrobial resistance. In the field of geriatric medicine, it has been shown that explicit criteria for PIPs increase the appropriateness of prescriptions. A recent systematic review showed that explicit definitions of antibiotic-PIPs in older people have not previously been listed. Our objective was to develop explicit definitions of antibiotic-PIPs for older hospitalized patients.

Method: We performed a qualitative study in accordance with the COREQ criteria (Consolidated criteria for reporting qualitative research), through focus groups involving French geriatricians and infectiologists. Audio recordings were transcribed then analyzed by two reviewers. Mentions related to explicit definitions were extracted and rephrased in definitions, which were classified according to the type of inappropriateness. The resulting list was corrected by an independent expert group and then validated by the whole focus groups participants.

Results: Four focus groups involved 28 stakeholders; 22 (78.6\%) had an antimicrobial stewardship activity. The analysis identified 96 explicit definitions: $63(65.6 \%)$ corresponded to inappropriate choice of antibiotic, $17(17.7 \%)$ to inappropriate use and $16(16.7 \%)$ to overuse of antibiotics.

Conclusion: Our study provides explicit definitions of antibioticPIPs, especially for older patients and public health challenges. This list will be refined through a Delphi survey to reach a consensus, which could deliver key messages for prescribers and open up new perspectives for the analysis of electronic healthcare databases to reduce inappropriate antibiotic prescriptions.

\section{P-514}

Resource based active and healthy ageing as a product to finance long term community based care provision

\section{Stella I. Tsartsara ${ }^{1}$}

${ }^{1}$ VUB, Ixelles, Belgium

There is a need for healthcare policy coordination for various reasons. First, to scale up on-going innovative solutions adapted to a regional context to make sure as many people as possible can benefit from high value care services. Second, to reduce budgetary costs for LongTerm-Care (LTC) for elders at both regional and local level. This could get shaped by a sustainable funding model of social care whereby the finance of it is cut all over Europe, anchored to the organizational model at community scale, to assure the delivery of care in a sustainable manner, integrated into the administrative and institutional LTC setting of a region. This study proposal is inspired by the study "Greece 2020" suggesting a Greek National Strategy for Integrated Elderly LTC in Greece as opposed to in-hospital episodebased care, one of the 6 Rising Stars for Gross Domestic Product rise until the year 2020. This, however, is a challenge for most of the European Regions today specially in remote areas and in those EU countries with continuous cuts in social care.

The research question: The primary research question at hand is: what is the potential of an innovative funding of "Design-for-All Integrated LTC model", to replace state and public LTC funding in EU Regions under economic crisis? The secondary research question is: How responsive is such a model to induce change in planning, organizing and funding LTC, in one pilot area of Greece? This pilot area is characterized by a reverse availability ratio of budgetary resources to the rising needs of elderly care in a co-morbidity context of an ageing population and a directly relevant ratio to the availability 
of resources to allow the necessary income to fund this LTC model. The current study will investigate what resources dispose some of the regions of Greece to create robust silver tourism services and products to fund a LTC model according to the needs of the pilot areas and what are the changes they should adopt, in organizational and LTC financing terms, to allow for cashable savings that would guarantee Return on Investment (RoI) and sustainability of the LTC provision at community level. A large part of this exercise concerns the mentality shift induced to the local actors to understand and endorse innovative funding in long term care run by own resources and not state funded. Study objectives: The main study objective is to assess the assets and needs for LTC in the selected Greek region and to construct a model that could sustainably finance LTC provision in the pilot area, via income generation from local economic growth, reinvested to fund LTC provision. The second objective is to observe the responsiveness of this model and how change in healthcare organization and funding is perceived by local authorities and how susceptible they are to that change. The specific objectives of this study are: (1) to map resources for local economic growth and the needs for elderly LTC of the selected municipality (pilot area); (2) to assess the costs and resources for the elderly LTC services integration in the area, the human and other resource capacity, the skills and needs for the LTC provision in relation to products or services (i.e. tourism) that would generate income from that model and guarantee the cashable savings of the investment return to sustain LTC provision. (3) Observation of the responsiveness of the model throughout the data collection and analysis and model preparation.

Results: Initiating a innovative LTC financing model through Social Impact Investment in LMIC, requires the cooperation of all local actors and a consensus in revealing, valuating, and putting the income generated from the local resource at the service of public health through non profit trade and investment activities. The main obstacle was unawareness of innovation procedures in health financing that created reservation to express interest in the model of the present study, combined with a generally low developmental capacity of the municipal actors so as to exercise control over the assets of their community. Where this was the case, the local governor was not stronger from private interests that preferred to see the resource procured for profit making actions away from redistribution of the RoI at the service of public health, in this case long term care. On the contrary, where the municipality had prior experience from innovation procedures and had concluded successfully in the past such operations and business activities around the new models of care, the response was better and the commitment in the new learning process higher. In addition the cooperation with the private sector was managed in an inclusive way that streamlined RoI to public health purposes through the proposed model of this study. Education and awareness of LGAs in innovative LTC financing and organization procedures of new care models, are key for the local authorities to adopt change, since chronic care is primarily organized at community based level.

\section{Area: Acute care \\ P-515}

Gastrointestinal senile amyloidosis: a rare but relevant geriatric condition

Frank Mickley ${ }^{1}$

${ }^{1}$ Klinikum Sankt Georg gGmbH, Department of Geriatric Medicine, Erlangen, Germany

Introduction: Isolated gastrointestinal amyloidosis is a rare (Rowe et al. 2017) but clinically relevant disease in geriatric patients.
Methods: We present 2 female patients (age 82 and 88 years), who were admitted to our center for evaluation of chronic anemia with iron deficiency. One patient suffered from a previous endoscopic colonic perforation and was now readmitted for worsening anemia under oral anticoagulation due to atrial fibrillation. The second had unexplained iron deficiency and anemia for $>1$ year.

Results: In both women on bidirectional endoscopy including capsule endoscopy no bleeding stigmata were identified. Iron resorption was normal. Endoscopic biopsies from the upper and lower gastrointestinal tract did show typical Congo-red staining in the perivascular submucosa. Further testing did not demonstrate any other typical organ involvement from amyloidosis. Laboratory analysis ruled out plasma cell disease, connective tissue disease and chronic inflammation. Symptomatic therapy is restricted to blood transfusion and iron supplementation. While amyloid deposits in the gastrointestinal tract lead to occult bleeding anticoagulation was stopped in the first patient.

Conclusions: Although a rare manifestation of amyloidosis, staining for amyloid should be considered in elder patients undergoing gastrointestinal biopsy who have unexplained iron deficiency with anemia. Further diagnostic examination should focus on the type of amyloidosis. Therapy directs against the underlying cause or remains symptomatic in case of senile ATTR-Amyloid. Once a definitive diagnosis is made no repeat endoscopic exams should be performed given the high perforation risk in this patient cohort. Oral anticoagulation is relatively contraindicated.

\section{P-516}

Parvovirus B19 infection in an 88-year-old woman: a case report

Frank Mickley ${ }^{1}$

${ }^{1}$ Klinikum Sankt Georg gGmbH, Department of Geriatric Medicine, Erlangen, Germany

Introduction: Most cases of human parvovirus B19 infection are asymptomatic and occur in childhood. In Germany approximately $75 \%$ of all people at the age of 65 years are seropositive. Reports of parvovirus B19 infection in the geriatric population are rare. There are descriptions of lupus-like presentation (Federici et al. 2007).

Methods: We present a 88-year-old, home-dwelling women, who was admitted for jaundice, severe hemolytic anemia (haptoglobine $<0.01 \mathrm{~g} / \mathrm{l}, \mathrm{Hk} 0.25$ ) and huge hematomas of her upper limbs and her thighs without previous trauma. After exclusion of autoimmune and drug-induced hemolysis we found a primary infection with parvovirus B19 (positive IgM). Jaundice and hemolysis disappeared spontaneously. The hematomas were explained by an overdosing of phenprocoumon, subscribed for atrial fibrillation 3 years ago, following a higher absorption due to previous fasting and inappetence. Phenprocoumon was stopped. After IV application of vitamin K, PPSB and red blood cell transfusion the patient slowly recovered.

Conclusion: In geriatric patients with acute onset of hemolytic anemia with ineffective erythropoiesis a primary infection with parvovirus B19 should be excluded. Multimorbidity and medication can worsen symptoms and outcome. An interdisciplinary approach may be helpful. 


\section{P-517}

\section{Enhancing early ambulatory rate of patients after abdominal} surgery

\author{
Hsuan-ju Huang ${ }^{1}$ \\ ${ }^{1}$ Chi Mei Medical Center, Tainan, Taiwan
}

Background: Early ambulation can promote physical recovery of a patient, and reduce the occurrence of postoperative complication and shorten hospital stay. From January to March 2015, the patients of the hospital who ambulate within $48 \mathrm{~h}$ after abdominal operation accounted for $50.9 \%$.

Purpose: The percentage of patient ambulation within $48 \mathrm{~h}$ after abdominal operation was expected to increase to $76.8 \%$.

Solutions: Through detailed status quo investigation and verification by the quality control circle, it has been found that there are six factors affecting early ambulation of patients: wound pain, weak feet, many tubes after operation, no instruction by physicians on the permission of ambulation, the idea of having more rest after operation by patients, and lack of supporting nursing staff. According to literature, brainstorm of the members of the circle, and vote, the following three solutions had been concluded: integration measures to "strengthen the awareness of early ambulation after operation", adjustment of the work content of functional nursing staff, and design of tube hanger on an IV infusion set. "Strengthening the awareness of early ambulation after operation" include: apply "clinical care guidelines for postoperative pain" to pain care unit; establish simple ambulation steps for processes. Department heads shall lead doctors to inform patients the necessity of early ambulation after operation. The TV in ward shall have health education channels.

Results: The satisfaction of patients for intervention measures was $96.6-100 \%$. The ambulation rate increased to $83.3 \%$.

Conclusions: Through the improvement of early ambulation, this unit not only shortened hospital stay by 1.7 days, but also reduced patients' cost by NTD 1,733/day. The early ambulation rate has become a special indicator of this unit

\section{P-518}

\section{Fall in the elderly: devastating outcome}

J. E. Mateus ${ }^{1}$, C. Silva ${ }^{1}$, M. Gonçalves ${ }^{1}$, M. Ferreira ${ }^{1}$, J. Porto ${ }^{1}$, I. Fonseca ${ }^{1}$, M. T. Veríssimo ${ }^{1}$

${ }^{1}$ Internal Medicine Department, Centro Hospitalar e Universitário de Coimbra, Coimbra, Portugal

Introduction: Although trauma remains a leading cause of morbimortality across all ages, elder patients experience higher risk of severe disability and higher mortality than younger people since they are more susceptible to injury and more likely to take multiple medications, some of which may blunt their response to the physiologic stress of trauma.

Case description: A 84 year old man presented to the hospital after a fall at home. He had a history of arterial hypertension and Parkinson disease and was treated with acetylsalicylic acid, levodopa and carbidopa. He had a Glasgow coma scale 15 and a nasal trauma with active bleeding and bone exposure. Cervical X-ray showed C4-C5 luxation and fracture of $\mathrm{C} 5-\mathrm{C} 6$ spinous processes, confirmed by CT scan. Four hours later the patient suddenly developed dyspnea and stridor progressing to cardiac arrest due to airway obstruction with blood. After $3 \mathrm{~min}$ cardiopulmonary resuscitation the patient was stabilized and admitted to the intensive care unit. One week later he underwent spinal surgery, after which he developed Acinetobacter baumannii pneumonia, successfully treated with trimethoprim- sulfamethoxazole. He was discharged 2 months later with a rehabilitation program. On follow-up evaluation 6 months after the fall the patient has quadriplegia and dysphagia.

Key conclusions: Clinicians need to routinely assess older patients for fall risk and address modifiable underlying risk factors (physical conditions and environmental situations). Medication should be reviewed and unnecessary drugs discontinued. Multiple strategies for fall prevention have been evaluated in different settings, it's time to apply them.

\section{P-519}

\section{Subdural empyema: rare but serious condition}

J. E. Mateus ${ }^{1}$, C. Silva ${ }^{1}$, P. Marques ${ }^{2}$, M. Gonçalves ${ }^{1}$, M. Ferreira ${ }^{1}$, J. Porto ${ }^{1}$, I. Fonseca ${ }^{1}$, M. T. Veríssimo ${ }^{1}$

${ }^{1}$ Internal Medicine Department, Centro Hospitalar e Universitário de Coimbra, Coimbra, Portugal, ${ }^{2}$ Neurology Department, Centro Hospitalar e Universitário de Coimbra, Coimbra, Portugal

Introduction: Spinal epidural empyema is a rare infection of the central nervous system which may result from hematogenous infection. This condition requires prompt recognition and management since it can progress rapidly leading to coma and death if untreated. Case description: A 65 year old man presented with a 1 week history of rapidly progressing 4 limb weakness and voiding difficulty. He had a history of lumbar spinal stenosis with complaints of chronic lower back pain. On physical examination the patient was confused and feverish, had flaccid tetraparesis (motor power grade: leg II; arm III), algic hypoesthesia with socks distribution and warm and swollen left knee. Laboratory investigations showed leucocytosis and elevated C-reactive protein. Cervical CT scan showed a hypodense fluid collection in the anterior epidural space with compression of the spinal cord, extending from $\mathrm{C} 1$ to $\mathrm{C} 4$, consistent with epidural empyema. Surgical drainage of the epidural empyema was performed as well as knee arthrocentesis. The patient was given vancomicin and gentamicin for 6 weeks with clinical improvement and was discharged 3 months later. Methicillin-sensitive Staphylococcus aureus was isolated from three blood cultures, knee pus and epidural pus culture. Key conclusions: Successful treatment of subdural empyema usually requires a drainage procedure and antibiotic therapy for $6-8$ weeks. With aging there is a progressive alteration of the immune system and its responses, resulting in immunodepression. This case illustrates the need to consider this rare but serious condition, even in the absence of a contiguous focus of infection or trauma/neurosurgery.

\section{P-520}

A case of severe HSV gingivostomatitis in a previously immunocompetent septic shock elderly patient

Rachna Kapoor $^{1}$, Gigi Diamond ${ }^{1}$, Sunil Sapru ${ }^{1}$

${ }^{1}$ Saint Barnabas Medical Center, Livingston, USA

Introduction: Immunocompromised patients with hematologic malignancies or organ transplant need prophylactic antiviral medication against herpesvirus if seropositive for HSV $1 \mathrm{gG}[1,2]$. In the ICU setting, risk factors for reactivation of HSV are: septic shock, Acute Respiratory Distress Syndrome (ARDS), used high dose steroids and abdominal infections [3]. Past studies have demonstrated that critically ill patients on high dose steroids are susceptible to developing viremia with HSV [3]. 
Case presentation: A 80-year-old male admitted with septic shock secondary to cholecystitis. He received high dose steroids for 9 days. On physical exam, he developed vesiculo-ulcerative lesions; extremely tender, hemorrhagic. The lesions were around perioral cutaneous tissue, vermillion border, buccal mucosa, gingiva, soft/hard palate, pharynx and tongue. Serum HSV-1 IgG antibody was $>58$ index and HSV-1 DNA level was 5043 copies $/ \mathrm{mL}$. He was managed with two weeks of IV acyclovir. As a result of a poor appetite due to excruciating pain in the oropharynx he required a gastrostomy tube feeding. The patient was discharged from hospital after clinical improvement.

Discussion: Immunocompromised patients usually have atypical presentations [4]: more aggressive, extensive, slower healing painful lesions and prolonged infection leading to dissemination. Severe HSV gingivostomatitis leads to significant morbidity [4], perhaps increased mortality in a previously immunocompetent elderly patient. To date no data studying use of prophylactic acyclovir in critically ill immunocompetent elderly patients (ie: comparing acyclovir prophylaxis vs wait-and-see strategy) [3].

Conclusion: Elderly patients with a critical illness who are seropositive for HSV might benefit from pre-emptive acyclovir.

References:

1. Tomblyn M, Chiller T, Einsele H, Gress R, Sepkowitz K, Storek J, Boeckh MA (2009) Guidelines for preventing infectious complications among hematopoietic cell transplantation recipients: a global perspective. Biol Blood Marrow Transplant 15(10):1143-1238. https://doi.org/10.1016/j.bbmt.2009.06.0192.

2. Arduino P, Porter S (2006) Oral and perioral herpes simplex virus type 1 (HSV-1) infection: Review of its management. Oral Dis 12(3):254-270. https://doi.org/10.1111/j.1601-0825.2006.01202.x3

3. Ong DS, Bonten MJ, Spitoni C, Lunel FM, Frencken JF, Horn J, Cremer OL (2017) Epidemiology of multiple herpes viremia in previously immunocompetent patients with septic shock. Clin Infect Dis 64(9):1204-1210. https://doi.org/10.1093/cid/cix1204

4. Arduino PG, Porter SR (2007) Herpes Simplex virus type 1 infection: overview on relevant clinico-pathological features. J Oral Pathol Med 37(2):107-121. https://doi.org/10.1111/j.16000714.2007.00586.x

\section{P-521}

\section{Adherence to geriatric Emergency Department guidelines in routine care}

L. C. Blomaard ${ }^{1}$, A. M. Booijen ${ }^{2}$, J. A. Lucke ${ }^{2}$, J. de Gelder ${ }^{1}$, Prof. J. Gussekloo ${ }^{1}$, Dr. B. de Groot ${ }^{2}$, Dr. S. P. Mooijaart ${ }^{1}$

${ }^{1}$ L.U.M.C. Department of Geriatrics, Leiden, The Netherlands, ${ }^{2}$ L.U.M.C. Emergency Department, Leiden, The Netherlands

Introduction: There is growing interest in the complex health care needs of older people presenting to Emergency Departments (EDs). Geriatric Emergency Department (GED) guidelines provide recommendations on how to improve care for these patients. The aim of this study was to describe adherence to GED guidelines for older ED patients.

Methodology: A prospective observational cohort study including ED patients $\geq 70$ years, during 2 months from 8 am till $11 \mathrm{pm}$. The following recommendations of the ACEP GED Guidelines were observed as a proxy for guideline adherence: use of urinary catheters, family presence, use of hospital bed instead of ED gurney and provision of food. The degree of a stressful environment was measured by counting the number of involved care providers and the number of door movements of the treatment room.
Results: In total 998 older patients visited the ED, of which 605 $(60.6 \%)$ were observed during their ED stay. Urinary catheters were used in $6.8 \%$ of all older patients. For $88.8 \%$ of patients family was present, $35.6 \%$ of patients were nursed on a bed and $7.4 \%$ of patients received food during their ED visit. The mean number of involved care providers was 8 (SD 3.7) and the median number of door movements was 41 (IQR 24-62).

Conclusions: Geriatric Emergency Department Guidelines adherence is low. The use of urinary catheters and presence of family in the ED seems good, but there is room for improvement of hospital bed use, presence of food and stressful environmental factors. To make sure that routine care follows guidelines, interventions such as education programs and environmental changes seem necessary. During the conference this data will be compared with data after implementation of a system improvement program.

\section{P-522}

\section{Older patients' satisfaction with Emergency Department Care}

L. C. Blomaard ${ }^{1}$, F. E. H. P. van Baarle ${ }^{2}$, J. A. Lucke ${ }^{2}$, J. de Gelder ${ }^{1}$, Prof. J. Gussekloo ${ }^{1}$, Dr. B. de Groot ${ }^{2}$, Dr. S. P. Mooijaart ${ }^{1}$

${ }^{1}$ L.U.M.C. Department of Geriatrics, Leiden, The Netherlands, ${ }^{2}$ L.U.M.C. Emergency Department, Leiden, The Netherlands

Introduction: Patient-centred care is especially important for the growing numbers of older Emergency Department (ED) patients, because their complex health care needs may require other outcomes than usually investigated. We therefore investigated satisfaction of older patients with ED care as a starting point to improve care for this patient group.

Methodology: A prospective observational cohort study in ED patients $\geq 70$ years. Within 7 days after their visit patients received the Picker Patient Experience questionnaire (PPE-15) with additional questions specific for ED care and older patients. Patients were asked to grade their ED visit on a scale from 0 to 10, 10 representing $100 \%$ satisfaction. The other questions were grouped into eight domains and coded as a dichotomous 'problem score', indicating the presence or absence of a problem.

Results: In total 869 questionnaires were sent of which $374(43.0 \%)$ were returned. Patients graded their ED visit with a mean of 8.6 (SD 1.1). Domains with the highest problem scores were "physical comfort", "shared decision making" and "transition of care". Topics of worst evaluated questions were "presence of food", "shared decision making in use of additional tests" and "attention for living situation prior to discharge", in which $69.0,36.1$ and $32.6 \%$ of the patients indicated the presence of problems, respectively.

Conclusions: Overall, older patients are satisfied with their ED visit. But in order to meet the complex health care needs of older ED patients, care providers need to focus more on physical comfort, shared decision making and transition of care future studies should investigate if results will improve after generating this awareness. During the conference this data will be compared with data after implementation of a system improvement program.

\section{P-523}

Vital signs in elder patients, urgent need to develop "GEWS" (= Geriatric Early Warning System)

Baeyens Hilde $^{1}$, Dekoninck Julien ${ }^{1}$, Baeyens Jean-Pierre ${ }^{1}$, Vanhecke Kyri ${ }^{1}$

${ }^{1}$ AZ ALMA, Eeklo, Belgium 
Introduction: Accreditation programs in Belgium forces geriatricians to use MEWS in elder persons. In 2015, Churpek et al. demonstrated that the risk for cardia arrest increases with age but that the accuracy of MEWS is not sufficient. After all, vital signs (blood pressure, pulse rate, oxygen saturation, respiratory rate, consciousness and pain) in older and frail persons differ from adults. Physiopathology of ageing can help us to explain this difference and determine which cut-off values to use to develop a GEWS (= Geriatric Early Warning System).

Methods: A literature study on 'vital signs' in the elder population was used to determine new cutoff values, based on the available data. A stress test was made by scoring all elder patients before and after relocation to the new hospital by MEWS and GEWS. Refinements were made on GEWS, especially on pulse rate and blood pressure cutoff values. In the 3-month winter period 2017-2018, a retrospective study of patients was made. On 541 acute geriatric hospital stays, 42 patients died. Of them, 14 died unexpectedly.

Results: A specific tool, GEWS, was developed and evaluated in a pilot study. Cutoff values are experienced by nurses to be closer to reality. In a small, retrospective pilot study, GEWS score is more sensitive than MEWS for detecting acute deterioration.

Conclusion: There is an urgent need for developing an accurate Early Warning System for elder patients. The GEWS seems to have added clinical value against MEWS. A multicentric study is needed to determine the accuracy.

\section{P-524}

Improving the safety of intravenous fluid prescriptions in older adult inpatients on medical wards through a structured knowledge based approach using established best clinical practice guideline NICE CG 174

Olugbenro Akintade ${ }^{1}$, Cynthia Dzingina ${ }^{1}$, Hannah Cobley ${ }^{1}$, Prachi Prachi ${ }^{1}$, Gajendra Pun ${ }^{1}$, Madhavi Ratnayake ${ }^{1}$

${ }^{1}$ North West Anglia NHS Foundation Trust, Peterborough, UK

Introduction: Daily intravenous fluid prescribing (IVF) is an essential task for older patients on medical wards. This task is often delegated to junior health care professionals despite the inherent risk of 1 in 5 patients suffering complications and morbidity from inappropriate fluid prescriptions. NICE (National Institute for Health \& Care Excellence) clinical guideline 174 aims to address deficit in knowledge and deliver an essential practical tool for safer prescribing. Method: We completed an audit cycle in 2015 \& 2016 respectively based on recommendations in NICE guidance 174. An initial audit reviewed the clinical notes of 80 patients administered intravenous fluids lasting more than $20 \mathrm{~h}$ to determine compliance with core principles of safe prescribing. These include providing indications for prescribing and monitoring prescriptions (daily evaluation of need for intravenous fluids and fluid status, reassessment of electrolytes, urea, $\&$ twice weekly weight monitoring). Departmental medical education was undertaken based on these principles. A re audit of 100 patients was conducted for the same parameters in the initial audit a year later. Results: The documentation for indication of IVF prescription improved from 58 to $85 \% \%$ on using a clinical guide. Clinical record of fluid balance chart recording improved from 43 to $59 \%$. Recorded data for monitoring of electrolytes \& urea following prescription was $75 \& 83 \%$ respectively. There was also improvement in twice weekly weight monitoring from 0 to $25 \%$ in the follow up audit. Mean age for both audits was 75 years old.

Conclusion: Continuing education of the medical workforce of an acute hospital is required to safeguard the safety of older adult inpatients prescribed intravenous fluids. The use of a consensus guideline like the NICE CG 174 is a good first step in this regard. This will promote appropriate prescribing to avoid complications \& morbidity. Indications for IVFs must be provided, appropriate monitoring in place. Institution of a clinical guide like the NICE CG 174 will improve the overall quality of prescriptions for older people in hospitals.

\section{P-525}

A community based innovation prevents hospital admissions and facilitates early discharge from hospital

A. Chaudhry ${ }^{1}$, G. Donnelly ${ }^{1}$, R. S Cruise ${ }^{1}$, E. Ryan ${ }^{1}$, M. Channon ${ }^{1}$, R. Keelan ${ }^{1}$

${ }^{1}$ Wythenshawe Hospital, Manchester University NHS Foundation Trust, Manchester, UK

Keywords: Prevention and communities and environments, Prevention in vulnerable population

Introduction: The population of the UK is growing and people are living longer bringing extra strain on the health and social care system [1]. The NHS 5 years forward view and the Kings fund outlined the need to look at safe and appropriate alternative options to provide care for patients who otherwise would require hospitalisation [2]. In recognition of the problem, the Community Urgent Care Team in South Manchester was created to provide medical and social crisis care at home. Referrals are accepted from the community, A\&E and hospital wards. This follow up study analyses the effectiveness of this intervention.

Methods: All patients reviewed by the service in April to July 2017 were included in the analysis, which compared the number of admissions 6 months before and 6 months after the intervention.

Results: 129 patients were reviewed in the 4 months. $78 \%$ of referrals were from the community therefore avoided patients presenting to A\&E. Admission rates were reduced for $31 \%$ of people comparing 6 months before and after the intervention. Overall $65 \%$ of patients reviewed had no admissions in the 6 months post intervention, showing that patients stayed out of hospital rather than presenting at a later date. In addition, the service saved an estimated 18.5 bed days per month.

Key conclusions: The intervention reduced hospital admissions and acute bed days by providing an effective alternative to hospital admission for patients assessed to be safe at home with additional support. The service benefits patients and reduces demands on acute hospital services.

\section{References:}

1. Office for national statisticshttps://www.ons.gov.uk/peoplepopulationandcommunity/populationandmigration/populationestimates/articles/overviewoftheukpopulation/july2017. Accessed 15 Nov 2017

2. Five year forward year https://www.england.nhs.uk/wp-content/ uploads/2014/10/5yfv-web.pdf. Accessed 15 Nov 2017

\section{P-526}

Hospital-acquired hyperkalemia events in older patients: a frequent and multifactorial adverse drug reactions

Laurine Robert ${ }^{1}$, Grégoire Ficheur ${ }^{1}$, Bertrand Decaudin ${ }^{2}$, Juliette Gellens ${ }^{1}$, Michel Luyckx ${ }^{3}$, Renaud Perichon ${ }^{1}$, Sophie Gautier ${ }^{4}$, Francois Puisieux ${ }^{5}$, Emmanuel Chazard ${ }^{1}$, Jean-Baptiste Beuscart ${ }^{1}$

${ }^{1}$ Univ. Lille, EA 2694-Santé Publique: épidémiologie et qualité des soins, 59000, Lille, France, ${ }^{2}$ Department of Pharmacy, CHU Lille, 
59000, Lille, France, ${ }^{3}$ Department of Pharmacy, CH Denain, 59220, Denain, France, ${ }^{4}$ Department of Pharmacy, CHU Lille, Lille, France

Introduction: Hyperkalemia is a frequent and potentially adverse drug reaction (ADR) among older patients, but its preventability has been insufficiently studied. Moreover, drugs and other risk factors were assessed separately. The aim of our study was to use electronic health records to identify hyperkalemia induced by drugs and to analyze the association between drugs and precipitating factors among hospital-acquired hyperkalemia events in older patients.

Methods: We conducted a retrospective, mono-centric cohort in a general hospital over a four-year period. We automatically identified hyperkalemia events $(\mathrm{K}+\geq 5.4 \mathrm{mmol} / \mathrm{l})$ occurring 3 days or more after admission, amongst 9339 eligible inpatient stays of patients aged 75 or more. Two independent experts reviewed all hyperkalemia events to adjudicate ADR (Kramer's algorithm), identify inappropriate prescriptions (STOPP criteria), evaluate avoidability of the event (Hallas criteria), and identify other factors known as at-risk of hyperkalemia in the previous days.

Results: The expert review confirmed 471 hyperkalemia events in 421 hospital stays (4.5\% of hospital stays) and concluded that 379 $(80.5 \%)$ of them were induced by drugs. The cause was multifactorial (i.e. at least one drug with a precipitating factor or with another drug) in $350(92.3 \%)$ hyperkalemia induced by drugs. Most of hyperkalemia induced by drugs were avoidable $(79.9 \%)$, mainly in relation with a multifactorial cause. Only $19.5 \%$ of prescriptions involved in hyperkalemia induced by drugs were inappropriate.

Conclusion: Hospital-acquired hyperkalemia events among older patients are most often multifactorial and preventable ADRs. Prevention of hyperkalemia induced by drugs need to focus more on precipitating factors.

\section{P-527}

Current status of an acute care unit in geriatrics in Colombia: application of an European successful model

Diego Andrés Chavarro-Carvajal ${ }^{1}$

${ }^{1}$ Pontificia Universidad Javeriana, Bogotá, Colombia

Objective: Describe the characteristics of the state of health, as well as the outcomes in patients hospitalized by the geriatric service of the San Ignacio University Hospital.

Methods: Observational, descriptive, retrospective analysis that seeks to characterize the elderly population attended by the geriatric acute care service in the period from January 1, 2016 to December 31, 2017. Results: In total, 1599 subjects were studied, the average age of the population was 85.34 years. The main reason for hospitalization was derived from infectious diseases and $49.91 \%$ had $4-6$ comorbidities. Hospital functional deterioration was found in $35.48 \%$ of the patients and this was related to the nutritional alteration and delirium; $1 / 4$ of the patients did not have an adequate support network. The average hospital stay was 6.46 days and $13.34 \%$ of the patients attended corresponded to readmissions and suffered $9.26 \%$ complications.

Conclusions: Encouraging the creation of geriatric services connected in a network that provides prevention and acute management of comorbidities can contribute to having an elderly population more independent and functional, in addition to saving health costs that allow the system a better distribution of resources. Our results are good when compared to the world literature and other centers-It then becomes an option to take into account in other institutions of the country that face a similar problem.

Keywords: Aged, hospitalization, Acute Care, Hospital, Geriatrics

\section{P-528}

\section{Influence of health behaviors in the incidence of frailty}

Mario Ulises Pérez-Zepeda ${ }^{1}$, Carlos Alberto Cano-Gutierrez ${ }^{2}$

${ }^{1}$ Instituto Nacional de Geriatría, Mexico, Mexico, ${ }^{2}$ Pontificia Universidad Javeriana, Bogotá, Colombia

Introduction: Frailty is a clinical state defined as an increase in an individual's vulnerability for developing adverse health-related outcomes. We propose that healthy activities could prevent the incidence of frailty.

Methods: The Mexican Health and Aging Study is a nationally representative longitudinal study of Mexican adults 50 years or older with four waves of data collected so far. For the purpose of this manuscript, only the 2012 and 2015 waves are used. This study analyzes the association between health behaviors and incidence of frailty. Frailty was determined using a 39-item frailty index. Only respondents classified as non-frail at baseline (2012) were included in the analyses $(n=6087)$. Logistic regression models were used to assess the odds of incident frailty given the following health behaviors: exercise, vaccination, non-smoking, and screening activities. Confounding variables were included in the regression models.

Results: At baseline, $55.2 \%$ of subjects were male and the mean age was $62.2(\mathrm{SD} \pm 8.5)$ years, the incidence of frailty was $37.8 \%$. Older adults that reported exercising in 2012 had a lower incidence of frailty in 2015 (48.9 vs. $42.2 \%, \mathrm{p}<0.0001$ ). From the activities assessed in the adjusted multivariate models, exercise was the only variable independently associated with lower odds of incident frailty (odds ratio of $0.79 ; 95 \%$ confidence interval $0.71-0.88 ; p<0.001$ ).

Conclusions: Older adults that exercised had a lower 3-year incidence of frailty, even when adjusted for confounding variables. This could be a strategy to reduce the incidence of frailty and its consequences.

\section{P-529}

Acute stroke patients needing timely vascular interventions: a quality improvement project

Ijeoma Tonia Obi ${ }^{1}$, Hina Butt ${ }^{1}$, Karuna Subba ${ }^{1}$, Tanyah Ewen ${ }^{2}$, Fiona Miller ${ }^{2}$, Santosh Subramonian ${ }^{2}$, Peter Owusu-Agyei ${ }^{2}$

${ }^{1}$ North West Anglia NHS Foundation Trust, Peterborough, UK, ${ }^{2}$ Orth West Anglia NHS Foundation Trust, Peterborough, UK

Introduction: Evidence has shown that Carotid endarterectomy reduces the 5 years absolute risk of ipsilateral ischemic stroke by $16.0 \%$ in patients with $70-99 \%$ carotid artery stenosis, and by $4.6 \%$ in patient with $50-69 \%$ stenosis [1]. We aim to refer symptomatic stroke patients with $50 \%$ or more carotid artery stenosis to the vascular surgery team within 1 week of symptom onset as recommended by the stroke guideline [2].

Methods: A clinical audit and re-audit was done over 2 years in a district hospital. Data were collected over specific period of time. The re-audit findings were compared to that of the initial.

Results: A total of 220 patients were audited initially and 200 patients for the re-audit. There was $20 \%$ increase of carotid doppler scans done in $24 \mathrm{~h}, 4 \%$ increase in the quality of requests and $19 \%$ increase in compliance with time to referral to vascular surgeons within 1 week for stroke patients with significant carotid stenosis. $80 \%$ of referred stroke patients had carotid scans done within a week of symptom(s) onset as opposed to $69 \%$ from the initial audit.

Conclusions: The results showed an improvement in time of carotid doppler scan testing and appropriate referral to the vascular surgeons within 1 week of acute stroke. We were able to achieve this by 
education and modifying the electronic request form reminding test requesters to supply relevant information, and indicating suitability for vascular intervention. Following the re-audit, the trust's vascular lab department has dedicated 5 days service of $1 \mathrm{~h}$ each for carotid doppler scan tests for stroke patients.

References:

1. Rothwell PM, Eliasziw M, Gutnikov SA, Fox AJ et al (2003a) Analysis of pooled data from the randomised controlled trials of endarterectomy for symptomatic carotid stenosis. Lancet, 361, $107-116$

2. (2016) Royal college of Physicians National Clinical Guideline for Stroke, 5th edn

\section{P-530}

Identification of vulnerable older patients in the Emergency Department: How about the gut feeling?

A. H. Calf ${ }^{1}$, S. Lubbers ${ }^{2}$, A. A. van den Berg ${ }^{2}$, E. van den Berg ${ }^{2}$, C. J. Jansen ${ }^{3}$, B. C. van Munster ${ }^{4}$, S. E. de Rooij ${ }^{3}$, J. C. ter Maaten ${ }^{5}$

${ }^{1}$ Department of Geriatric Medicine, University of Groningen,

University Medical Center Groningen, Groningen, The Netherlands, ${ }^{2}$ Department of Emergency Medicine, University of Groningen,

University Medical Center Groningen, Groningen, The Netherlands,

${ }^{3}$ University of Groningen, Groningen, The Netherlands

Introduction: Timely identification of vulnerable older patients in the Emergency Department (ED), followed by patient-tailored interventions, decreases the risk of adverse health outcomes in these patients. Clinical impression of vulnerability ('gut feeling') assessed by physicians and nurses in the ED might be a useful first step for deciding to introduce a more comprehensive geriatric assessment in the ED setting.

Methods: In this prospective observational study, patients aged $\geq 65$ years, and their responsible physicians and nurses in the ED were eligible during 2 weeks. Patients were assessed for vulnerability with a Dutch screening tool for vulnerability (considered as gold standard in this study). Clinical impression was qualified with the question 'Do you consider this patient to be vulnerable?', answered by both attending physician and nurse.

Results: We included 196 patients, and their physicians and nurses. Physicians' clinical impression was $81.6 \%$ sensitive and $53.8 \%$ specific, and was associated with higher patient age (OR 1.13, 95\% CI 1.06-1.21), polypharmacy (OR 2.73, 95\% CI 1.27-5.85), higher risk of malnutrition (OR 3.57, 95\% CI 1.61-7.92) and functional impairment (OR 11.48, 95\% CI 2.48-53.06). Sensitivity and specificity of nurses' clinical impression was 74.8 and $52.7 \%$ respectively, and was associated with higher patient age, presence of polypharmacy, higher risk of malnutrition and functional impairment, and with female gender of the patient.

Key conclusions: In this evidence-based medicine era, the clinical impression of vulnerability has additional value and can be integrated in daily practice as a first screening to identify vulnerable older patients in the ED.

\section{P-531}

Patients with dementia who call out repetitively when in an acute hospital: characterization, staff and family responses

J. Beaver ${ }^{1}$, R. H. Harwood ${ }^{1}$, S. E. Goldberg ${ }^{1}$, A. Edgley ${ }^{1}$

${ }^{1}$ Nottingham University, Nottingham, UK
Introduction: Some patients with dementia 'call out' repetitively whilst in acute hospitals. This persistent and disruptive vocal behaviour is widely believed to indicate distress or unmet need, but there is little empirical evidence about this problem in this setting. This study aimed to characterise the phenomenon, follow its natural history, and explore staff and family views on its causes and management.

Methods: A mixed research methods case-series was undertaken of 30 older people who called out, across seven wards at two hospitals. We recorded baseline and ongoing quantitative measures of physical and mental health, undertook non-participant observations, family and staff interviews, and examined nursing documentation.

Results: Participants had severe cognitive impairment $(70 \%$ MMSE $<10$ ), poor functional ability, and $85 \%$ had delirium. There were high levels of disinhibition, anxiety, depression and pain. Mean length of stay was 29 days (compared with national age-standardised mean of 12 days). Within 3 months of recruitment, a third of participants had died, and more than half were readmitted. Staff stated that calling out indicated distress, and believed they could identify when there was a treatable cause. But they also believed that some cases were intractable, leading to avoidance of the patient, and potentially increasing the risk that 'real' needs will be neglected.

Conclusions: Calling out is associated with severe cognitive and functional impairment and delirium. Health and nursing needs are high, and prognosis poor. Staff understand the theory of unmet needs, but may overestimate their ability to identify a cause.

\section{P-532}

Acute geriatric daily board ward round (DBWR): a quality improvement activity (QIA) in a Tertiary Hospital, Singapore

Surendra Doraiswamy Varman ${ }^{1}$, A. W. B. Tan ${ }^{2}$, Diraviyam Balasubramanian ${ }^{2}$, G. K. Sidhu' ${ }^{2}$, S. P. W. Lim² ${ }^{2}$, F. Y. Li ${ }^{2}$, S. L. Long 2

${ }^{1}$ Changi General Hospital, Singapore, Singapore, ${ }^{2} \mathrm{CGH}$, Singapore, Singapore

Introduction: Inter-professional communication is the core of healthcare delivery and a key to an efficient in-patient care. Effective discharge encompasses efficient communication and teamwork amongst doctors, nurses and allied healthcare teams. A preliminary analysis of discharges from Geriatric Wards (GRM) showed that our readmission rates were high compared to non-GRM wards, along with a low "before 1130 am" discharges, (hospital wide initiative aims for discharges before $1130 \mathrm{am}$ ), primarily as a result of ineffective communication amongst teams. Methods Hence, a QIA visioning a safe, patient-centric discharge, to improve overall communication amongst teams; with a primary aim to improve discharges before 11.30 am and 30-day readmission rate, without an increase in mortality and average length of stay (ALOS), was conceived and implemented in our GRM ward; aided by Daily board ward round (DBWR), check-lists and educational training to relevant stakeholders.

Results: Discharges pre \& post DBWR implementation, 4 months, were 348 vs post-board 318. ALOS 12.1 vs 11.8 ; mortality $20(5.6 \%)$ vs 23 (6.5\%); 30-day re-admission 63 (26.2\%) vs 41 (18.2\%); Early ward discharges $(<11.30 \mathrm{am})$ were pre -board $36(11.1 \%)$ vs 56 $(19.1 \%)$.

Conclusion: ALOS remained same; however noteworthy improvements in early discharges $(<11.30 \mathrm{am})$ and 30 day re-admission rates, with a stable mortality rate have mandated us in sustaining DBWR as ongoing QIA. We believe, with appropriate training, supervision and participation, DBWR has contributed significantly to improve communication, understanding and consolidation of day-to- 
day ward tasks amongst healthcare staffs; necessitating future refinements including electronic versions of board to improve userfriendliness and adoption all across Hospital.

\section{P-533}

\section{Predictors of recurrent Emergency Department visits of seniors}

\author{
Ksenija Slankamenac ${ }^{1}$, Kathrin Krähenmann ${ }^{1}$, Dagmar I. Keller ${ }^{1}$ \\ ${ }^{1}$ Emergency Department, University Hospital Zurich, Zurich, \\ Switzerland
}

Background: Senior patients are responsible for an increasing proportion of recurrent Emergency Department (ED) visits. To our knowledge, only few risk factors for recurrent ED visits in seniors have been identified. Therefore, the aim was to investigate which predictors were associated with recurrent ED visits of seniors.

Methods: We enrolled consecutively seniors ( $\geq 70$ years.) visiting the ED of a tertiary care hospital at least four times in 2015. Recurrent ED senior patients were grouped in frequent visitors presenting with different disorders and in repeated visitors with the same disorder for each ED visit. We assessed the prevalence of recurrent ED senior patients and investigated predictors associated with recurrent ED visits.

Results: Of 27,998 ED patients, 65 ED senior patients (0.2\%) performed 316 recurrent ED visits. Of these, 40 frequent ED seniors presented 195 times whereas 25 repeated ED seniors were responsible for 121 ED visits. In a stepwise backward regression analysis, recurrent ED visits by seniors were strongly associated with weekend presentations $(\mathrm{p}<0.001)$. Predictors of frequent ED visits were increased age ( $>75$ years) $(\mathrm{p}=0.004)$ and chronic kidney failure $(p=0.021)$. Repeated ED visits were associated with female gender $(\mathrm{p}=0.016)$ and underlying coronary heart disease $(\mathrm{p}=0.046)$, underlying malignant disease showed a trend to an increased risk $(\mathrm{p}=0.081)$.

Conclusions: Based on the predictors of recurrent ED visits by senior patients, novel models for outpatient medical care and long-term follow-up strategies are needed allowing reduction of recurrent ED visits in the future and improvement of seniors' quality of life.

\section{P-534}

Behind a back pain: case report

Mariana Gonçalves ${ }^{1}$, José Mateus ${ }^{1}$, Elsa Gaspar ${ }^{1}$, Lèlita Santos ${ }^{1}$, Manuel Veríssimo ${ }^{1}$, Armando Carvalho ${ }^{1}$

${ }^{1}$ Centro Hospitalar e Universitário de Coimbra, Coimbra, Portugal

Introduction: Back pain is a common cause to attend the Emergency Department in elderly people. The differential diagnosis is varied, with a spectrum of pathologies of major and minor severity.

Case report: A 68-year-old men was admitted due to two days history of constant left back pain, with increasing intensity and irradiation to the left upper quadrant of the abdomen. On physical examination he had no fever, was hemodynamically stable, with pain at the deep palpation of the left hypochondrium. Blood workup revealed leukocytosis $\left(15.7 \times 10^{4} \mathrm{G} / \mathrm{L}\right)$ and lactate dehydrogenase elevation $(585 \mathrm{U} / \mathrm{L})$. Chest X-ray showed left pulmonary base hypotransparency. Abdominal ultrasound revealed a splenic nodular mass, heterogeneous, with $10 \mathrm{~cm}$ and, left pleural effusion. Several complementary diagnostic tests were performed, including a thoracoabdominal-pelvic CT scan showing multiple celiac and lomboaortic adenophaties, the largest with $9.5 \mathrm{~cm}$ and, a splenic mass of $13 \mathrm{~cm}$.
The biopsy of the splenic mass was inconclusive. Due to the patient's clinical worsening and mass growth, a splenectomy was performed. Histological exam revealed a diffuse large $B$ cell lymphoma (DLBCL). He started treatment with rituximab-cyclophosphamide, doxorubicin, vincristine and prednisone, with clinical improvement.

Conclusion: DLBCL is the most common histologic subtype of nonHodgkin lymphoma (25\%). There is a male predominance $(55 \%)$, with median age at presentation of 64 years. Patients typically present with a rapidly enlarging symptomatic mass. DLBCL is curable in approximately half of cases with current therapy, however advanced age, poor performance status, and lower socioeconomic status are associated with worse outcomes.

\section{P-535}

\section{A rare cause of hemolysis}

Marc Johnsen $^{1}$

${ }^{1}$ St. Marien-Hospital Köln, Cologne, Germany

Anaemia is a common finding in hospitalised elderly patients. Apart from frequent causes like gastrointestinal bleeding, major surgery and primary or secondary bone marrow dysfunction, hemolysis is one of the reasons that should be excluded. Here, we present an 83-year old woman that presented with increasing anaemia after recent hip surgery. Surprisingly, her laboratory results showed evidence of ongoing hemolysis. Further diagnostic work-up revealed acute hypothyroidism and ultrasound showed signs of thyroid inflammation consistent with Hashimoto thyroiditis. After excluding alternative causes we treated the patient successfully with steroids and thyroid hormones. In conclusion, this case emphasises the need to thoroughly assess the often multifactorial aetiology of anaemia in elderly patients and highlights that autoimmune disease may manifests for the first time in this age group. In general, it has to be considered that the prevalence of anaemia is high and that manifest thyroid dysfunction is rare, as shown in our patients in an arbitrarily chosen month.

\section{P-536}

Sedative-hypnotic initiation and renewal at discharge in hospitalized older patients: an observational study

Elsa Bourcier ${ }^{1}$, Adrien Borowik ${ }^{2}$, Lucas Zerbib ${ }^{2}$, Dominique BonnetZamponi $^{3}$, Florence Tubach ${ }^{4}$, Christine Fernandez ${ }^{5}$, Patrick Hindlet ${ }^{6}$

${ }^{1}$ Sorbonne Université, INSERM, Institut Pierre Louis

d'épidémiologie et de Santé Publique, IPLESP UMR-S1136, 75012, Paris, France; APHP, Hôpital Saint-Antoine, Service de Pharmacie, 75012, Paris, France; Université Paris-Sud, Faculté de Pharmacie, Paris, France

Introduction: Sedative-hypnotics (SHs) are widely used in France but there are no available data addressing their prescription specifically in hospitalized older patients. Our objective was to determine the proportion of $\mathrm{SH}$ treatments initialized during a hospital stay of older patients, the proportion of SH renewal at discharge and to study associated risk factors.

Methods: We conducted a retrospective observational study in six internal medicine units and six acute geriatric units from eight hospitals (France). We included inpatients aged 65 and older without $\mathrm{SH}$ treatment prior to hospitalization. Primary outcome was the rate of $\mathrm{SH}$ initiation. Secondary outcomes were the rate of $\mathrm{SH}$ renewal at discharge, risk factors for $\mathrm{SH}$ initiation and renewal at discharge 
(patient characteristics, hospital organization; mixed effects logistic regression model).

Results: Among the 1194 patients included, SH initiation occurred in $15.5 \%(\mathrm{n}=185)$ of participants. SH renewal at discharge occurred in $44.9 \%(\mathrm{n}=83)$ of patients with SH initiation during their stay. Risk factors for SH initiation were: a length of stay over 7 days [OR 1.70, 95\% CI (1.12-2.58)] and more treatments prior to hospitalization [OR $1.05,95 \%$ CI (1.00-1.10)]. Being discharged to a rehabilitation structure was a risk factor for SH renewal at discharge [OR 2.69, 95\% CI (1.30-5.57)].

Conclusions: Hospitalization is a period at risk for SH initiation in older people, especially those hospitalized more than a week and those with polypharmacy. Nearly half of these prescriptions are maintained at discharge. To reduce the use of $\mathrm{SH}$, interventions focusing on in-hospital $\mathrm{SH}$ initiation and their renewal at discharge are needed.

\section{P-538}

Treatment escalation plan in Buckinghamshire Community Hospital: avoiding inappropriate hospital admissions

\author{
Akhil Khera $^{1}$, Beatrix Nagyova ${ }^{1}$ \\ ${ }^{1}$ Buckinghamshire Healthcare NHS Trust, Amersham, UK
}

Introduction: The care of older patients with multi-morbidities is a major challenge faced by the health service. The complexity of these individuals makes it often difficult for healthcare professionals to make challenging decisions at the point of hospital admission. Treatment Escalation Plan (TEP) is a Buckinghamshire Healthcare NHS Trust developed document which provides information on the appropriate level of management for a patient in the event of clinical deterioration. The aim of this project was to ensure that patients in Buckinghamshire community hospital had valid TEPs within $72 \mathrm{~h}$ of admission in order to share appropriate level of treatment plan for each frail individual with general practitioners, foundation and general practice trainees, nurses, families and patients themselves.

Methods: We undertook two cycles of data collection in August and October 2017. 45 patient records were reviewed on both occasions. All sections of patient's notes were checked for a presence of TEP. Results: $71 \%$ of patients had a TEP completed by the community hospital team. The completion rate was increased to $87 \%$ during second data collection. The number of uncompleted TEPs decreased from 18 to $9 \%$ during second cycle. The staff felt more supported in decisions making when TEPs were in place.

Conclusions: Implementation of TEP in community hospital setting showed more complete information exchange between healthcare professionals and patients. The project supported development of 'an organisation wide task group to enrol TEP to other clinical areas including surgical and orthopaedics wards.

\section{P-539}

Adverse drug reaction among community-acquired acute kidney injury in older patients: a multifactorial and preventable event

Laurine Robert ${ }^{1}$, Grégoire Ficheur ${ }^{1}$, Bertrand Decaudin ${ }^{2}$, Alexandre Servais $^{1}$, Michel Luyckx ${ }^{3}$, Renaud Perichon ${ }^{1}$, Sophie Gautier ${ }^{4}$, Francois Puisieux ${ }^{5}$, Emmanuel Chazard ${ }^{1}$, Jean-Baptiste Beuscart ${ }^{1}$

${ }^{1}$ Univ. Lille, EA 2694-Santé publique: épidémiologie et qualité des soins, 59000, Lille, France, ${ }^{2}$ Department of Pharmacy, CHU Lille, 59000, Lille, France, ${ }^{3}$ Department of Pharmacy, CH Denain, 59220, Denain, France, ${ }^{4}$ Department of Pharmacy, CHU Lille, Lille, France
Background: Community-acquired acute kidney injury (CA-AKI) is a frequent and severe adverse drug reactions among older patients. Prevention of CA-AKI is insufficiently studied. Drugs are the main risk factors but association with other risks factors was scarcely evaluated.

Objective: The aim of our study was to accurately identify CA-AKI induced by drugs in older patients, and their association with other risks factors in order to assess the preventability of CA-AKI. Methods: We conducted a retrospective, mono-centric study in a general hospital over a 2-year period. An automated detection identified CAAKI according to KDIGO criteria, amongst 4,767 eligible hospital stays by patients aged 75 years or older. Two independent experts reviewed all CA-AKI events to adjudicate drug involvement (Naranjo scale), identify inappropriate prescriptions (STOPP criteria), evaluate avoidability (Hallas criteria) and identify associated risk factors.

Results: Expert review confirmed 713 CA-AKI (15.0\% of hospital stays). A drug was deemed responsible of CA-AKI in 434 (60.9\%) cases. A multifactorial cause (i.e. an association of at least one drug with a precipitating factor or with another drug) was found in $83.4 \%$ of CA-AKI induced by drugs. Expert review considered $67.7 \%$ of CA-AKI induced by drugs as avoidable, mainly in relation with a multifactorial cause. Among drugs responsible of CA-AKI, only 67 (9.7\%) prescriptions were rated as inappropriate.

Conclusion: CA-AKI among older patients are most often multifactorial and preventable. Prevention of CA-AKI need to focus more on intercurrent illness during drug therapy rather than on inappropriateness.

\section{P-540}

A joint Wythenshawe Hospital and red cross innovation reduces reattendance and readmissions

R. S. Cruise ${ }^{1}$, L. Wentworth ${ }^{1}$, J. Williams ${ }^{1}$, A. Allcutt ${ }^{1}$, L. Middleton ${ }^{1}$, A. Palin ${ }^{2}$

${ }^{1}$ Wythenshawe Hospital, Manchester Foundation Trust, Manchester, UK, ${ }^{2}$ British Red Cross, London, UK

Keywords: Prevention and health system, Prevention and communities and environments, Prevention in vulnerable population (oldest old, minorities, women...)

Introduction: The NHS has faced increasing operational and financial pressures over the last decade culminating in what the British Red Cross (BRC) last year described as a 'Humanitarian Crisis'. Vulnerable patients are often admitted to acute hospital beds awaiting social support as there is no alternative to allow them to return home safely. Methods: A combined initiative with BRC and Wythenshawe Hospital was designed to promote independence and assist recovery for patients who are medically optimised, but require practical and emotional support to return home safely. A BRC donor is funding the service for 3 years, which operates Monday-Friday 11 am to $7 \mathrm{pm}$, differing from similar schemes elsewhere as support can continue for up to 6 weeks after discharge. Services on the day of discharge include transport home and necessary care to allow patients to settle safely at home. Ongoing support may include phone calls and further home visits.

Results: There were 300 patient contacts between August 2017 to January 2018. The 3 months before being reviewed by the service was compared to 3 months after and showed a $44 \%$ reduction in Emergency Department attendances and 38\% reduction in hospital admissions.

Key conclusions: This innovation proved economically and socially valuable in providing effective support to our most vulnerable older patients. The positive results have resulted in further donation to 
extend the operational hours of the service and an additional donation from a separate donor to provide a weekend admissions prevention service for patients who attend regularly due to loneliness and isolation.

\section{P-541}

Safely reducing admissions in frail older people presenting to Emergency Department (ED)

Lara Whitmore ${ }^{1}$, Bettina Wan ${ }^{2}$

${ }^{1}$ UCL, London, England, ${ }^{2}$ UCLH, London, England

Introduction: Older people have higher rates of attendance to ED and are more likely to be admitted. An interface service that safely reduces admissions in this group could alleviate pressures in hospitals and ensure that the right care is delivered in the right place. To develop this service we need to quantify the patients with health and social needs that could be met in the community.

Aim: To assess the number of frail older people presenting to ED who could be safely discharged.

Method: Two independent clinicians from the geriatric multi-disciplinary team screened 447 patients aged $\geq 75$ who presented to ED over 19 days. 256 of those who could be assessed were deemed frail based on a Clinical Frailty Score $\geq 5$. A joint decision was made to see whether patients could safely be discharged with alternatives for admission to hospital. This showed the proportion of avoidable admissions.

Results: $67 \%$ of patients $\geq 75$ admitted from ED were found to be frail. $65 \%$ of these patients were admitted. $23 \%$ of these admissions were potentially avoidable if urgent medical follow up, intermediate care units and/or community support were available.

Discussion: This study highlights the prevalence of frailty among elderly ED attendees. It also suggests that admissions can be avoided by the involvement of specialised clinicians, using admissions alternatives. This data, along with other data we have collected on the health and social characteristics this group of patients, has guided a 50 patient pilot study of an acute geriatric service in ED.

\section{P-542}

\section{Geriatric population features in a stroke hospitalization context}

M. Fieldes ${ }^{1}$, M. Richard ${ }^{1}$, S. Toubal ${ }^{1}$, S. Bouly ${ }^{2}$, J. M. Kinowski ${ }^{1}$, L. Salomon ${ }^{1}$, G. Leguelinel-Blache ${ }^{1}$

${ }^{1}$ Department of Pharmacy, Nîmes University Hospital, Nîmes, France, ${ }^{2}$ Department of Neurology, Nîmes University Hospital, Nîmes, France

Introduction: Stroke is the leading cause of acquired disability and third cause of death in France. Close to three quarters of the 150, 000 cases occur at elderly age with an earlier onset for men [1].

Methods: Our monocentric observational prospective study includes a 1 year consecutive cohort of patients hospitalized for stroke in neurology department (2015-2016). Home-based treatment was determined by at least 3 various corroborative sources. Medication compliance was scored by simplified Morisky Medication Adherence Questionnaire. The main purpose of this work was to highlight geriatric population characteristics in acute cerebral episode context. Results: 400 patients were included. Men were more numerous (gender ratio: 1.6) and stroke seems to occur earlier in male population (70 vs 73.4 years, $\mathrm{p}=0.0015$ ). Moreover, strokes were more frequent in 65 year olds and over $(71.5$ vs $28.5 \%, \mathrm{p}<0.0001)$.
Average drug consumption was significantly higher in geriatric population $(6.5$ vs $3.8, \mathrm{p}<0.0001)$, but no tendency was found according to sex or stroke subtype. Elderly people were more compliant $(\mathrm{p}=0.0237)$. Nevertheless no difference on medical benefit of drugs was seen. Length of hospital stay was longer in geriatric population $(p=0.0029)$ notably those who had a hemorrhagic stroke $(\mathrm{p}=0.0480)$.

Conclusion: Elderly people hospitalized for stroke appear to be overmedicated and more compliant for home-based treatment.

Keywords: Stroke, Elderly people, Prevention in vulnerable population, Health promotion

Reference:

1. Fery-Lemonnier, La prévention et la prise en charge des accidents vasculaires cérébraux en France (2009) http://solidarites-sante.gouv.fr/IMG/pdf/AVC-rapport_final-vf.pdf

\section{P-543}

Diagnosis and treatment of catheter-related bloodstream infection in a geriatric hospital

Miguel Araújo Ordóñez ${ }^{1}$, Manuela Martínez Camacho², Encarna Rodríguez Jiménez ${ }^{3}$, Romeo Rivas Espinoza ${ }^{1}$

${ }^{1}$ Servicio Geriatría Hospital Virgen del Valle, Toledo, Spain,

${ }^{2}$ Servicio Farmacia Hospital Virgen del Valle, Toledo, Spain,

${ }^{3}$ Servicio de Farmacia Hospital Virgen del Valle, Toledo, Spain

Introduction: The Total Parenteral Nutrition (TPN) is an invasive method to provide the fundamental nutrients to assure a correct nutritional balance and ensure the energy requests according to clinical situation. Sometimes the venous accesses for its administration can suffer local or systemic infections identified like CatheterRelated Bloodstream Infection (CRBSI), constituting an important cause of hospital-acquired infection associated with an increase morbidity, mortality, and cost.

Methods and objective: Determine the valuation of CRBSI in geriatrics patients hospitalized with TPN. To analyze the possible relations between CRBSI and the type of venous access [Central venous catheters (CVCs) or peripheral venous catheters (PVCs)], the place of canalization, the duration and its care.

Design: An observational prospective study of $>75$ years old patients admitted with TPN between January 2016 and December 2017 in a geriatric hospital. Demographic factors, diagnosis and management of suppurative thrombophlebitis and local complications, indication of TPN, type of venous access, duration of venous catheter and laboratory pathogen results was evaluated.

Results: 125 participants, 79\% women. Middle aged 86.22 years (95\% confidence interval (CI) 73-98). Venous access: 63\% PVC. Duration of venous catheter: 6.62 days in PVC and 9 days in CVC. Cases of CRBSI: 24 of 984 days of catheter. Valuation of CRBSI: 24.39\%. More frequent Pathogen: Staphylococcus epidermidis and Candida spp. Significant differences were not detected in the infection risk according to the type of venous access ( $p=0.643$ ).

Key conclusions: The CRBSI valuation in geriatric patients is upper than intensive units care series, probably related to the biggest vulnerability to endure infections in older people. Similar valuations have been described in not critical patients and general population, being this register pioneering. It is necessary to implant measurements of prevention of the CRBSI based on the progress of the care of the venous accesses destined for TPN. 


\section{P-544}

The management of blunt chest trauma in a geriatric population

Bethan Pincher ${ }^{1}$, Conor Rainey ${ }^{2}$, Sunil Auplish ${ }^{2}$, Syed Bokhari ${ }^{1}$

${ }^{1}$ Pinderfields General Hospital, Wakefield, UK, ${ }^{2}$ York Hospital, York, UK

Introduction: Blunt chest injury is thought to be an underdiagnosed condition in elderly patients presenting following minor trauma. Low energy mechanisms of injury can often lead to significant rib fractures and respiratory compromise in the geriatric population. Mortality from blunt chest trauma can be as high as $60 \%$, with elderly patients known to have poorer outcomes. The aim of this review was to analyse the types of thoracic injuries being admitted to our unit and to identify predictors of poor prognosis and mortality.

Methods: Data collected retrospectively on all patients over the age of 70 admitted with blunt chest trauma between January 2008 and August 2017. Details recorded on patient demographics, mechanism of injury, injury severity score, interventions and 30 day mortality. Data analysis was performed to identify those in need of invasive interventions and the risk factors for mortality.

Results: 137 patients admitted during this time period with blunt chest trauma. The average age was 82.7 years (70-98.6) and the injury severity scores ranged from 1-34 (mean 9.8). The majority of thoracic injuries were caused by falls from less than 2 metres $(72.3 \%)$. 30 day mortality was calculated at $8.0 \%$. The presence of 3 or more rib fractures would appear to be an indicator of poor prognosis.

Conclusions: A significant proportion of these thoracic injuries were caused by falls from less than 2 metres in this elderly population. These low energy injuries should not be underestimated, and a thorough evaluation of rib fractures seems vital in predicting adverse outcomes.

\section{P-545}

A clinical evaluation of the diagnosis and management of urinary tract infections in secondary care

Krishanthi Sathanandan ${ }^{1}$, Sadia Saber ${ }^{1}$, Devesh Shah $^{2}$, Thomas Ainge ${ }^{2}$, James Noble Johnston ${ }^{1}$

${ }^{1}$ Queens Hospital, Romford, UK, ${ }^{2}$ Queens, Romford, UK

Background: Urinary tract infection (UTI) is the leading cause of hospitalisation in the UK. Multimorbid patients with chronic urogenital symptoms, asymptomatic bacteriuria, long-term catheters and atypical presentations, represents a diagnostic challenge to secondary care. Inconsistent trust practices led to a collaborative clinical audit between Microbiology and Geriatric departments aimed at evaluating and improving diagnosis and management of UTI.

Methods: Prospective cross-sectional data was collected across a sample of emergency, acute and inpatient medical wards across two sites. Quantitative analysis of data categories was undertaken with an expected audit standard of $80 \%$ set.

Results: UTI diagnosis was made by clinicians in 42 patients within study period. Over $50 \%$ diagnosed had $<1$ clinical feature attributable to UTI, with non-specific symptoms most frequent. Urine dipstick was frequently used inappropriately in those over 65 years and with long term catheters. Improper antibiotic usage was evident in over $50 \%$. Overall expected standards of practice were not met in the diagnosis, investigation or treatment of UTI, with less than $50 \%$ adherence in subgroup analysis of lower UTI and those over 65 years. Conclusion: Poor trust practice standards, particularly in the elderly, leads to inappropriate treatment, subsequent risks of antibiotic resistance, as well as unnecessary cost. Evidence based trust guidelines have been developed and implemented for diagnosis of UTI in three distinct patient groups: under 65 years, over 65 years and those with urinary catheter in situ. Multimodality dissemination techniques are currently being undertaken with plans to re-evaluate trust practice thereafter.

\section{P-546}

Bleeding Dieulafoy lesion: an uncommon case report of haematemesis in a nonagenarian man

Oriol Miralles ${ }^{1}$, Diana Ariza ${ }^{1}$, Xavier Ariza ${ }^{2}$, Benito Fontecha ${ }^{1}$

${ }^{1}$ Department of Geriatrics and Palliative Medicine, Sant Joan Despí Moisès Broggi Hospital-Consorci Sanitari Integral, Barcelona, Spain, ${ }^{2}$ Department of Gastroenterology, Sant Joan Despí Moisès Broggi Hospital-Consorci Sanitari Integral, Barcelona, Spain

Introduction: A Dieulafoy lesion (DL) is a congenital, abnormally large and winding submucosal artery that has the potential to bleed through a small mucosal erosion $(1-5 \mathrm{~mm})$. It typically represents less than $2 \%$ of upper gastrointestinal bleeds, normally found in the lesser curvature of the stomach. Hematemesis and melena were the most common presenting symptoms. Gastroscopy being the main diagnostic and therapeutic procedure. Some case series have shown an increased incidence in men aged between 60 to 80 years.

Case report: A 94 years old man, admitted to an acute geriatric unit with moderate-severe disability affecting activity of daily living, diabetic with retinopathy, iron-deficiency anemia since 2009 with normal endoscopic study, atrial fibrillation without anticoagulation therapy and taking $100 \mathrm{mg}$ acetylsalicylic acid per day. Presented to hospital because of 3 episodes of haematemesis, tachycardia, hypotension and hemoglobin of $8.9 \mathrm{~g} / \mathrm{dL}$ (regular of $11 \mathrm{~g} / \mathrm{dL}$ ) that required one blood transfusion. A gastroscopy was performed and showed a $5 \mathrm{~mm}$ vascular lesion without bleeding on the greater curvature. The patient received a combined therapy of 3 hemoclips hemostasis and adrenaline injection that was successful, $48 \mathrm{~h}$ later he started oral tolerance with no incidences.

Conclusion: DL should be part of differential diagnosis in chronic iron-deficiency anemia and gastrointestinal bleeding with no-lesion endoscopic studio. Identifying the site of bleeding by endoscopy may be difficult, because of this it is not easy to visualize low dimension of mucosa break in the context of a large amount of blood or if the bleeding stops. Non-pathogenetic association between NSAIDs and DL has been identified in literature. Endoscopy combined therapy (pharmacological and hemoclips) could be the most effective method for the stopping of bleeding.

\section{P-547}

Ensuring a safe transition from hospital to home: the critical role of the ANP in running a virtual clinic

Polly May ${ }^{1}$

${ }^{1}$ Poole Hospital NHS Foundation Trust, Poole, UK

Introduction: Advanced practice roles for nurses provide opportunities to fill gaps in the medical workforce, meet service demands and improve clinical continuity [1]. The unique role of the Advanced Nurse Practitioner (ANP) blends autonomous medical ability with senior nursing skills to simplify transitional care for a defined population of vulnerable patients.

Method: A virtual clinic has been introduced on a Short Stay Older Persons Unit. A Masters level educated ANP has been employed to 
identify patients for early discharge, suitable for follow-up once home. The virtual clinic presents an opportunity to provide personalised medicine for the less acute/more able patient, involving a biopsychosocial telephone consultation with remote review of test results, thus removing pressures from the GP and community services.

Results: It has been reported that a large number of re-admissions occur during the week after discharge, often attributed to inadequate communication between Primary and Secondary Care [2]. The ANP overcomes this issue by personally following the known, more able/less acute patient from admission to a safe transition home; this has contributed to a reduction in length of stay and a more efficient discharge rate.

Key conclusions: The inclusion of an ANP within the medical team for older people services can improve quality of care at both the Secondary and Primary arenas. It can deliver quality personalised medicine to a vulnerable population, avoiding unwarranted readmission by utilising the holistic decision making ability of an ANP in a virtual clinic.

\section{References:}

1. Imison C et al (2016) Reshaping the workforce to deliver the care patients need. Nuffield Trust

2. Deschodt $\mathrm{M}$ et al (2015) Characteristics of older adults admitted to the Emergency Department (ED) and their risk factors for ED readmission based on comprehensive geriatric assessment: a prospective cohort study. BMC Geriatr 15:54

\section{P-548}

Analysis based on age of characteristics and immediate outcomes of older adults presenting to Spanish Emergency Departments after a fall

Francisco Javier Martín- Sánchez ${ }^{1}$, Oscar Mirò ${ }^{2}$, Karina Liz Quiñones Huayna $^{1}$, Berenice Nayla Brizzi ${ }^{1}$, Javier Jacob Rodríguez ${ }^{3}$, Pere LLorens Soriano $^{4}$, Pablo Herrero Puente ${ }^{5}$, Esteban Toral Valdivieso ${ }^{1}$, María Redondo Martín ${ }^{1}$

${ }^{1}$ Hospital Clínico San Carlos, Madrid, Spain, ${ }^{2}$ Hospital Clínic, Barcelona, Spain, ${ }^{3}$ Hospital Universitari de Bellvitge, L'Hospitalet de Llobregat, Barcelona, Spain, ${ }^{4}$ Hospital Universitario de Alicante, Alicante, Spain, ${ }^{5}$ Hospital Universitario central de Asturias, Oviedo, Spain, ${ }^{6}$ Hospital Clínico San Carlos, Madrid, Spain

Objectives: To study patient profile, fall-related characteristics and immediate outcomes according to age among older adults presenting to the ED after a fall.

Methodology: Cross-sectional analysis of the FALL-ER register that included patients aged $\geq 65$ older who presented to 5 Spanish ED after a fall. Patients were classified into three age categories and demographic, comorbidity, medical treatment, fall-related characteristics, immediate outcomes and cost data were analyzed.

Results: We included 1610 patients, 541 (28\%) were 65-74 years old, $647(40.2 \%)$ were $75-85$ years old and $512(31.8 \%)$ were 85 years or older. According to age, there was an increase of medications associated with falls and severe consequences of fall, indoors falls, without witness and during the night, because of non identified cause, with more probability of suffering physical, functional and psychology consequences and healthcare costs. Age was in independent factor associated with the probability of presenting severe injury (adjusted OR 1.02; 95\% CI 1.01-1.04; $\mathrm{p}=0.004$ ), fear of falling (adjusted OR 1.02; 95\% CI 1.01-1.04; $p=0.010$ ), acute functional impairment (adjusted OR 1.02; 95\% CI 1.00-1.04; $\mathrm{p}=0.024)$.
Conclusions: Older adults presented to Spanish ED after a fall have important comorbidity, geriatric syndromes and potentially inappropriate medication use. According to age, probability of presenting severe injury, fear of falling and acute functional impairment increases.

\section{P-549}

Profile of elderly patients treated for falls in the emergency room (FALL-ER Registry): magnitude of the problem and possibilities for improvement in the Emergency Departments

Francisco Javier Martín Sánchez ${ }^{1}$, Oscar Mirò², Esteban Toral Valdivieso $^{1}$, Berenice Nayla Brizzi ${ }^{1}$, Javier Jacob Rodríguez ${ }^{3}$, Pere Llorens Soriano ${ }^{4}$, Pablo Herrero Puente ${ }^{5}$, Karina Liz Quiñones Huayna ${ }^{1}$, Natalia Vázquez Alvarado

${ }^{1}$ Hospital Clínico San Carlos, Madrid, Spain, ${ }^{2}$ Hospital Clinic, Barcelona, Spain, ${ }^{3}$ Hospital Universitari de Bellvitge, L'Hospitalet de Llobregat, Barcelona, Spain, ${ }^{4}$ Hospital Universitario de Alicante, Alicante, Spain, ${ }^{5}$ Hospital Universita central de Asturias, Oviedo, Spain

Objective: To study the profile of the patients, the characteristics related to fall and the grade and factors associated with make recommendations about prevention of falls (RPF) among patients aged $\geq 65$ years attended for fall in Emergency Departments (ED). Methodology: FALL-ER is a multipurpose prospective cohort study with a systematic sampling that included all patients aged $\geq 65$ years attended with a fall in 5 EDs during 52 days a year. We collected 68 variables. Patients were classified in function of receiving or not RPF (any of the following: exercise, education about prevention of falls, referral to a specialist and modification of drugs related to falls).

Results: We included 1507 (93.6\%) of 1610 patients. Patients were very old and had a high level of comorbidity, polypharmacy and previous geriatric syndromes. The fall usually occurred at home, and half of them without witness. Out of total a $48 \%$ had fear of falling, a $22 \%$ had acute functional impairment, a $16 \%$ was admitted and a $0.6 \%$ died during the hospitalization. $509(33.8 \%)$ patients were made RPF. The arthrosis, decreased hearing acuity, self-reported cognitive impairment, medical care in the place of the fall, fear of falling, acute functional impairment and hospitalization were associated with a higher risk of RPF, and the decreased visual acuity with a lower likelihood.

Conclusions: Only three of ten older patients attended for fall in ED received RPF although several characteristics related to the patient and the fall are associated with a higher probability of receiving it.

\section{P-550}

Nurses' awareness on hospital acquired infection risks of the geriatric patients

Ibrahim S. S. Abumettleq ${ }^{1}$, Nurhan Bayraktar ${ }^{1}$

${ }^{1}$ Near East University Faculty of Nursing, Nicosia, Cyprus

Introduction: A hospital-acquired infection (HAI) is an infection that is acquired in a hospital or other healthcare facility. There is need to increase awareness of nurses on HAI risks and prevention. The main aim of the study was determination of nurses' awareness of hospitalacquired infection risks of the geriatric patients.

Methods: The study was performed on the registered nurses who work in a university hospital of North Cyprus. A total of 168 voluntary nurses who work in adult care clinics was composed the sample of the study. After the ethical approval, data were collected 
using a questionnaire in September and October 2017 using selfcompletion method. The methods used to analyze the data included descriptive statistics and Pearson Chi-Square test.

Results: Results of the study showed high level knowledge of nurses only on general knowledge on HAI of the geriatric patients. Whereas nurses had inadequate knowledge on HAI risks of the geriatric patients. Comparison of the nurses' some descriptive characteristics with their knowledge on hospital acquired infection risks of the geriatric patients showed that, there weren't statistically significant differences in terms of majority of the items. Regarding the educational degree of the nurses, bachelor's degree nurses' correct knowledge rates were higher than nurses graduated from the health care vocational high school in terms of some items.

Key conclusions: Based on the results of the study implementations of comprehensive, systematic, and continuous educational programs in order to enhance the knowledge and practices of the nurses on HAI was recommended.

\section{P-551}

Safety and efficacy of treatment with hypertonic serum in patients with refractory $\mathrm{CHF}$ of the chronic ill geriatric complex program in a day hospital

\section{Marta Isabel Tomé Guerreiro ${ }^{1}$ \\ ${ }^{1}$ Hospital General de Granollers, Barcelona, Spain}

Refractory heart failure (HF) is a prevalent entity with difficult clinical management. Different therapeutic alternatives have been promulgated, among which the use of furosemide stands out intravenous (EV) at high doses with hypertonic serum (SH).

Objectives: To describe our experience with the use of EV furosemide at high doses $(125-250 \mathrm{mg})$ and $\mathrm{SH}$ in a population of geriatric patients.

Methodology: Prospective and descriptive study of all treatments performed with furosemide $\mathrm{EV}$ and $\mathrm{SH}$ in the program of chronic geriatric patients from October, 2017 to January 2018. The concentration of SH was variable according to the patient's sodium (Na) (Na $<125$ mm/L: $230 \mathrm{cc} \mathrm{SH} 3 \%$, Na 126-135 mm/L: $150 \mathrm{cc} \mathrm{SH} 3 \%$ and $\mathrm{Na}>135 \mathrm{~mm} / \mathrm{L}$ : $75 \mathrm{cc} \mathrm{SH}$ with $75 \mathrm{cc}$ physiological saline $0.9 \%)$.

Results: 360 patients were reviewed: $317(88 \%)$ had a diagnosis of HF, 93 (29.3\%) are NYHA III-IV and 56 treatments were performed in 15 patients.

Characteristics: $61 \%$ men, average 83.93 years (SD 4.86), $40 \%$ cognitive impairment, $86 \%$ autonomous ambulation, $100 \%$ lived at home.

Previous treatments: $45 \% \mathrm{~K}$-sparing, $48 \%$ ACEI, $53 \%$ beta-blockers, medium dose furosemide $170 \mathrm{mg} /$ day. Mean dose of furosemide EV: $241 \mathrm{mg} /$ treatment. Weight loss/treatment: $0.55 \mathrm{~kg}$ (SD 1.0). No significant differences were found between glomerular filtration, $\mathrm{Na}$ and systolic blood pressure and diastolic pre and post treatment. No adverse effects were described (dizziness, arterial hypotension or general discomfort) during the procedure nor were there any admissions or deaths during the study.

Conclusions: Treatment with furosemide at high doses EV and $\mathrm{SH}$ in a population of geriatric patients is effective and does not present relevant adverse effects.

\section{P-553}

What is the effect of time of day of Emergency Department attendance on length of stay, readmission rates and mortality in Parkinson's disease?

\section{Craig Kirk ${ }^{1}$, David Ahearn ${ }^{2}$}

${ }^{1}$ University of Manchester, Manchester, UK, ${ }^{2}$ Manchester University NHS Foundation Trust, Manchester, UK

An 'out of hours' effect, where admission to hospital is associated with poorer outcomes during evenings, nights and weekends, has been demonstrated in the literature. However, there is no substantial research on the subject for Parkinson's disease (PD). Controlling for demographics, a retrospective analysis of three outcomes, length of stay (LOS), mortality and 30-day readmission, at University Hospital of South Manchester (UHSM) was performed for 288 Emergency Department admissions of patients with and without PD in four time windows across the day: 02.00-07.59, 08.00-13.59, 14.00-19.59 and 20.00-01.59. These three outcomes were compared within control groups across these time windows in order to observe any baseline effect, then compared within time windows between patients with and without PD. This revealed that it is unlikely that time of day of admission has any impact on LOS, mortality and 30-day readmission rates for control patients, nor any extra impact for patients with PD. Disregarding time of admission, patients with PD overall had a statistically significant increase in LOS of 2 days on average (median) compared to patients without, and also had a relative increase in mortality of $40 \%$, although this was not statistically significant. There was no clear effect of a PD diagnosis on 30-day readmission. These findings are consistent with previous literature that demonstrates increased LOS and mortality for patients with PD. However, as this is a service evaluation at a single centre, UHSM, and due to various other limitations on this study, these conclusions should be considered with caution.

\section{P-554}

Using point-of-care C-reactive protein to guide antibiotic prescribing for respiratory tract infections in elderly nursing home residents (UPCARE): study design of a cluster randomized controlled trial

T. M. Boere ${ }^{1}$, L. W. van Buul ${ }^{1}$, J. W. L. Cals ${ }^{2}$, R. M. Hopstaken ${ }^{2}$, M. W. van Tulder ${ }^{3}$, R. B. Veenhuizen ${ }^{1}$, T. J. M. Verheij ${ }^{4}$,

C. M. P. M. Hertogh ${ }^{1}$

${ }^{1}$ Department of General Practice \& Elderly Care Medicine, Amsterdam Public Health research institute, VU University Medical Center, Amsterdam, The Netherlands, ${ }^{2}$ Department of General Practice, Maastricht University, Maastricht, The Netherlands, ${ }^{3}$ Department of Health Sciences, VU University, Amsterdam, The Netherlands, ${ }^{4}$ Department of General Practice, Julius Centrum, University Medical Center Utrecht, Utrecht, The Netherlands

Introduction: Antibiotics are over-prescribed for lower respiratory tract infection (LRTI) in nursing homes $(\mathrm{NH})$ due to diagnostic uncertainty. Inappropriate antibiotic use is undesirable both on patient level, considering their exposure to side-effects and drug interactions, and on societal level, given the potential development of antibiotic resistance. The diagnosis of LRTI is challenging in NHs, because of limited availability and applicability of diagnostic tools, and because $\mathrm{NH}$ residents often lack typical symptoms. In addition, cognitive disabilities can impede communication of experienced complaints. C-reactive protein (CRP) point-of-care testing (POCT) may be a 
promising diagnostic tool to reduce unnecessary antibiotic use for LRTI in NHs.

Methods study design: A cluster Randomized Controlled Trial (cRCT) will be conducted in twelve NHs in The Netherlands between September 2018 and March 2020. NHs in the control group will provide usual care, and $\mathrm{NHs}$ in the intervention group will provide usual care including CRP POCT for NH residents with (suspected) LRTI.

Study population: NH residents with a new diagnosis 'suspected LRTI' can participate in the cRCT. Patients are excluded if they reside at hospice/palliative wards, if they have a different type of infection, if they do not wish to be treated with antibiotics, or if they are already taking antibiotics.

Main outcomes: (1) Antibiotic prescribing for suspected LRTI at index consultation (yes/no). (2) Associations between CRP POCT values and (a) signs and symptoms in $\mathrm{NH}$ patients with suspected LRTI, (b) antibiotic treatment. (3) Cost-effectiveness and cost-benefit of the use of CRP POCT.

Results/conclusions: Expected in 2020.

\section{P-555}

The characteristics of head trauma from low-energy falls in the oldest-old patients

Han Sung Choi ${ }^{1}$, Seo Kyong Lee ${ }^{2}$, Sung Kyu Kim ${ }^{1}$, Joonghyun Kim ${ }^{1}$, Songyi An ${ }^{1}$, Jeongmin $\mathrm{Choi}^{1}$, Hyunbyul Choi ${ }^{1}$

${ }^{1}$ Department of Emergency Medicine, Kyung Hee University Hospital, ${ }^{2}$ Department Neuropsychiatry, Rosen Hospital

Background: The population of oldest-old (aged $\geq 85$ years) is growing rapidly in Korea. The head trauma from low-energy falls is common in this population. A better understanding of head trauma from low-energy falls in oldest-old person is thus of increasing national and global importance. We therefore aimed to investigate the differences of head trauma between oldest-old patients who were injured by low-energy falls and young-to-middle-old (aged 65-84 years) patients or young adult patients.

Methods: This was a single-center retrospective study. The medical records of head trauma patients from the Emergency Department after low-energy falls that occurred between November 2015 and December 2017 were analyzed. Patients were divided into an older adult group (aged $\geq 65$ years) and a young adult group (aged 18-64 years); the older adult group was subdivided into an oldest-old group and a young-to-middle-old group.

Results: The underlying diseases (such as DM, HTN, dementia, Parkinson disease, malignancy, cerebrovascular accident), antithrombotic agent medication, and traumatic intracranial hemorrhage (TICH) were more in older adult group $(\mathrm{p}<0.001)$, but alcohol ingestion were more in young adult group $(\mathrm{p}=0.016)$. Furthermore, more cases of TICH were found in the oldest-old group than in the young-to-middle-old group $(\mathrm{p}=0.021)$. In the oldest-old group, TICH was significantly correlated with older age, hypertension, cardiac diseases, anticoagulant regimen, and other chronic conditions $(\mathrm{p}=0.037, \mathrm{p}=0.033, \mathrm{p}=0.025, \mathrm{p}=0.029$, and $\mathrm{p}=0.024$, respectively), whereas the correlation with sex, drinking status, and other underlying conditions was not statistically significant. Similarly, a binary logistic regression analysis of the variables considered significant in the univariate analysis was conducted to examine the relationship between TICH and older age. Of these variables, only 'very old age' was found to be independently significant $(\mathrm{p}=0.025)$. Conclusion: The characteristics of head trauma from low-energy falls in the oldest-old patients are different from young adult patients or young-to-middle old patients. Especially, the risk of TICH from low- energy falls in the oldest-old patients was higher than in the young-tomiddle old patients. Therefore, physicians need to pay particular attention to the oldest-old patients, even to those with mental integrity and without neurological deficits.

\section{P-556}

Five-year evolution of antihypertensive treatment after reduction of this treatment during hospitalisation in an acute geriatric unit

Anna Kearney-Schwartz ${ }^{1}$, Axel Schumacher ${ }^{1}$, Elisabeth Steyer ${ }^{2}$, Pierre Gillet $^{3}$, Athanase Benetos ${ }^{1}$

${ }^{1}$ Geriatrics Department Nancy University Hospital, Vandœuvre-lèsNancy, France, ${ }^{2}$ General Practice Department Nancy University Hospital, Vandœuvre-lès-Nancy, France, ${ }^{3}$ Pharmacology Department Nancy University Hospital, Vandœuvre-lès-Nancy, France

Objective: To evaluate the short and long term evolution of antihypertensive medication after reduction of this treatment in polymedicated older subjects during acute care hospitalization.

Methods: We analyzed the medication data of patients who had a reduction of the anti-hypertensive drugs during hospitalization in acute geriatric unit (T0: admission; T1: discharge). These patients were followed-up at 3 months (T3 M) and 5 years (T5Y) after discharge, or untildeath. The data collected were: number and class of antihypertensive drugs, blood pressure (BP), the reason for a possible increase of treatment and, if necessary, the date of death.

Results: During the hospitalization, 59 out of 318 hospitalized patients had a reduction in the anti-hypertensive medications (mean age $86 \pm 6$ years). The number of drugs in the different times of the follow-up was: T0: $9.1 \pm 3.2 / 2.1 \pm 0.9$ (total number/antihypertensive drugs) $\mathrm{T} 1: 8.1 \pm 2.7 / 0.9 \pm 0.9 \mathrm{~T} 3 \mathrm{M}: 8.2 \pm 3.0 / 1.3 \pm 1.0 \mathrm{~T} 5 \mathrm{Y}:$ $8.0 \pm 2.89 / 1.2 \pm 1.0$ Calcium antagonists and ARA2 were more frequently stopped, whereas ACE inhibitors more frequently initiated during hospitalization. Among the 42 patients who reached the T3 M visit, $40 \mathrm{kept}$ the same number or had a reduction of the number of antihypertensive drugs during 5-year follow up.

Conclusion: Reduction of the treatment operated during acute hospitalisation is largely sustainable in the short and long term. Review of the medical prescription in older patients during hospitalization could lead to a long term reduction of the poly-medication.

\section{P-557}

Elderly patients with nosocomial pneumonia in an internal medicine department

Margarida Torres ${ }^{1}$, Marilia Fernandes ${ }^{1}$, Diogo Borges ${ }^{1}$, Rita Alves ${ }^{1}$, Inês Figueiredo $^{1}$, Sara Castro ${ }^{1}$, Filipa Lourenço ${ }^{1}$, Heidi Gruner ${ }^{1}$, António Panarra ${ }^{1}$

${ }^{1}$ Medicina Interna 7.2, Hospital Curry Cabral, Centro Hospitalar Lisboa Central, Lisbon, Portugal

Introduction: Nosocomial pneumonia (NP) is the second most frequent nosocomial infection. It's associated with an increase in length of hospitalization and a high mortality rate, especially in the elderly. Objectives: To compare elder patients with and without NP, regarding the presence of risk factors and outcomes.

Methods: A retrospective one-year study was conducted at an Internal Medicine department of a Tertiary Hospital. We compared a group of elder patients with (wNP) and without (nNP) nosocomial pneumonia regarding: age, gender, Katz $<3$ and presence of the following risk factors: Charlson score, MUST score, number of 
medications, use of proton pump inhibitors (PPI's) and chronic obstructive pulmonary disease (COPD). The outcomes were: average length of stay, Intensive Care Unit admittance (ICU) and mortality. Results: The patients included were nNP:284 (153 male)/wNP:17 (9 male); average age was nNP: $81.29 / \mathrm{wNP}: 81.82$ years; patients with a Katz $<3$ were nNP:141/wNP:9; average Charlson score was nNP:3.3/wNP:3.5.

Concerning risk factors: Presence of nutritional risk according to the MUST score was nNP:84/wNP:3; average number of medication was nNP:6.89/wNP:6.94; patients who used PPI's were nNP:131/wNP:10; patients with COPD were nNP:51/wNP:3.The outcomes were as follows: average length of stay was nNP:9.85/wNP:19.23 days; patients admitted to the ICU were nNP:18/wNP:4; and mortality was nNP:35/wNP:6 patients.

Discussion: As expected, the small wNP group of patients showed no difference concerning gender, age or nutritional status. However, these patients were more dependent, had a higher average Charlson score and used more medications, particularly PPI's. All of the outcomes, namely average length of stay, ICU admittance (23.5 vs $6.3 \%$ ) and mortality ( $35 \mathrm{vs} 12 \%$ ) were either higher or more prevalent in the patients with nosocomial pneumonia.

\section{P-558}

The role of echo doppler method in treatment and prevention of cerebrovascular disease in non diabetic and diabetic population with macroangiopathy

Cristian Romeo Revnic ${ }^{1}$, Floarea Revnic ${ }^{2}$, Silviu Voinea ${ }^{3}$

${ }^{1}$ UMF Carol Davila, Bucharest Romaniac, ${ }^{2}$ NIGG Ana Aslan, Bucharest, Romania, ${ }^{3}$ UMF Carol Davila, Bucharest, Romania

Diabetes mellitus is one of the main risk factors of cerebrovascular disease (CVD).

Aim: Echo Doppler evaluation of arteries in diabetic and non-diabetic elderly patients. We selected 112 patients admitted in Neurology Clinic of Vlad Voiculescu Cerebrovascular Institute: 63 non diabetics mean age 63 years with acute ischemic stroke (CT scan confirmed) in carotid territory or a TIA and 59 diabetic patients mean age 62.8 included into this study, independently from the presence or absence of acute ischemic stroke investigated by CT/MRI. The carotid EchoDoppler examination applying a $4 \mathrm{MHz}$ transducer for extracranial and transcranial vascular Doppler investigation performed in both groups of patients revealed that simple carotid plaques, bilateral carotid stenoses (CS), and unilateral CS associated with contralateral carotid occlusion (CO) occurred with higher frequency in the nondiabetic patients, than in diabetic ones. On the other hand, the remaining other findings, such as unilateral $\mathrm{CS}$, unilateral $\mathrm{CO}, \mathrm{S} / \mathrm{O}$ of intracranial carotid artery (ICA) and vertebral/subclavian $\mathrm{S}$ were more frequent in the diabetic group. The findings allow us to conclude that the correct and early treatment of diabetes as well as a possible lowering of the risks for cerebrovascular disease are obligatory steps in the primary and secondary prevention of the cerebral ischemic events in diabetic patients with carotid atheromatous lesions. This consideration may help the physicians to have a deeper understanding of the pathophysiology, and to implement the necessary treatment and prevention of CVD in the diabetic population of high-risk.

\section{P-559}

Predictors of long lenght of stay in a cohort of real elderly patients hospitalized for acute heart failure

Alessandra Pratesi ${ }^{1}$, Francesco Orso ${ }^{2}$, Anna Chiara Baroncini ${ }^{3}$, Aldo Lo Forte ${ }^{3}$, Giulia Biagioni ${ }^{4}$, Francesca Caramelli ${ }^{3}$, Giulia Lucarelli ${ }^{3}$, Andrea Herbst ${ }^{3}$, Silvia Parlapiano ${ }^{4}$, Camilla Ghiara ${ }^{3}$, Mauro Di Bari ${ }^{3}$, Niccolò Marchion

${ }^{1}$ Department of Experimental and Clinical Medicine, Careggi Univeristy Hospital, Florence, Italy, ${ }^{2}$ Geriatrics Unit, Careggi University Hospital, Florence, Italy, ${ }^{3}$ Department of Experimental and Clinical Medicine, Careggi University Hospital, Florence, Italy, ${ }^{4}$ Univer

Introduction: Heart failure (HF) clinical registries and clinical trials are known to have some selection bias, often including patients admitted only to cardiology units. "Real world" administrative data show that only few patients (about a quarter) are hospitalized in cardiology units, while most of them are admitted to internal medicine and geriatrics wards. Aim of our study was to identify the independent predictors of long length of stay of elderly patients hospitalized for acute HF in different clinical settings of care: cardiology, internal medicine and geriatrics.

Methods: Data derived from the ATHENA (AcuTe Heart faliurE in AdvaNced Age) retrospective observational study which included elderly patients ( $\geq 65$ years) admitted with diagnosis of AHF to a tertiary University teaching-hospital in the period 01.12.201401.12.2015.

Results: 401 patients were enrolled, $14.7 \%$ hospitalized in geriatrics, $15.2 \%$ in cardiology and $70.1 \%$ in internal medicine. Mean age was 83.5 years and was higher in geriatrics (86.9 years) than in internal medicine (83.4 years) and in cardiology (81.0 years), $\mathrm{p}=0.001$. Females were $52.6: 55.7 \%$ in cardiology, $52.7 \%$ in internal medicine and $49.2 \%$ in geriatrics $(\mathrm{p}=0.770)$. Of those alive at discharge, mean length of stay was 9.0 days; then, we considered long length of stay (LLS) a bed rest $>9$ days. LLS occurred in $108(29,6 \%)$ participants, and was longer in geriatrics (13.7 days) than in cardiology (9.9 days) and internal medicine ( 8.0 days), $\mathrm{p}<0.001$. In multivariate analysis, adjusted for age and sex, resulted independent predictors of LLS: NTproBNP in Emergency Department (ED)—subsequently expressed as logarithm with base 10 (OR 3.78, 95\% CI 1.89-7.54, p < 0.001); Chronic Kidney Disease (OR 3.08, 95\% CI 1.38-6.90, p = 0.006) and eGFR at ED (OR 1.02, 95\% CI 1.00-1.03, p = 0.047); Charlson Comorbidity Index (CCI) (OR 0.76, 95\% CI 0.65-0.81, p < 0.001); cognitive impairment (OR 2.10; 95\% CI 1.01-4.36; $\mathrm{p}=0.047$ ).

Conclusion: NT-proBNP in ED, CCI renal function and cognitive impairment are independent predictors of long length of stay in a large cohort of real elderly patients hospitalized for acute heart failure in different clinical setting of care.

\section{P-560}

Improving sepsis management in long term care

Shirmila Syamala ${ }^{1}$, Hanadi Khamis Al Hamad ${ }^{1}$, Irshad Badarudeen ${ }^{1}$, Haroon Saleh ${ }^{1}$, Mahmoud Refaee ${ }^{1}$, Wasim Akram ${ }^{1}$, Jonathan Sumaway ${ }^{1}$

${ }^{1}$ Hamad Medical Corporation, Doha, Qatar

Introduction: Sepsis is a life threatening condition. It accounted for $31 \%$ of deaths in Qatar in 2016. According to international guidelines, sepsis management bundle should be initiated within the first hour of suspected sepsis. Rumailah Hospital is a multi-specialty hospital that provides medical, surgical and long-term care to the population of 
Qatar. It is part of Hamad Medical Corporation (HMC) of Qatar. In long-term care units in Rumailah hospital, we observed that the administration of initial dose of antibiotics was delayed beyond $1 \mathrm{~h}$ in more than $70 \%$ of suspected sepsis patients, leading to poor clinical outcomes such as increase in mortality and morbidity rates, transfer of patients to critical care facilities, and increase in the length of stay. A quality improvement project was carried out from May 2015 to June 2017. AIMTo be able to administer the 1st dose of antibiotic within $1 \mathrm{~h}$, and to increase compliance to Sepsis-Six bundle in at least $70 \%$ of suspected sepsis patients in long-term care units, by 2017 end.

Process: The following measures were implemented: Suspected Sepsis Checklist Card New tool for monitoring administration of first dose of antibiotic within $1 \mathrm{~h}$ : Awareness sessions: Audit and feedback.

Result: After intervention, the time to administration of initial dose of antibiotics reduced from $4.5 \mathrm{~h}$ to within $1.5 \mathrm{~h}$. Sepsis death-to-case ratio reduced from $12.5 \%$ to $4.8 \%$ : compliance to sepsis bundle increased from $53 \%$ to consistently over $80 \%$ : patient transfers reduced from 50 to $27.6 \%$

\section{P-561}

Effective safety netting for older people after acute care: the 'Silver Phone'

Samantha Sargent ${ }^{1}$, Emma Bridle ${ }^{1}$, Kerry Porter $^{1}$, Susan Greenwood $^{1}$, Julie Tuck ${ }^{1}$, Jenna Chandler ${ }^{1}$

${ }^{1}$ Poole Hospital, Poole, UK

Ambulatory Emergency care for Older People (AEC) promotes same day assessment, diagnosis and treatment which reduces the risk of inappropriate hospital admissions and discharge delays for vulnerable frail patients. This pathway for older people has become firmly embedded in a 600 acute care hospital in the South of England which can see up to 200 patients per month. With so many interventions and potential changes to treatment taking place over the course of 1 day, it is highlighted that patient communication has to be paramount (NHS England 2014). Older patients can get lost in the ether between secondary and primary correspondence delays resulting in crisis events for these patients or even unnecessary readmissions to an inpatient bed. The Silver phone line was developed as a means of supporting these patients during the acute period after their discharge from AEC. It is a phone line manned by skilled Advanced Nurse Practitioners for Older People, operative 7 days per week. Its purpose is twofold; to provide a point of contact for older patients should they require clinical advice, signposting or just reassurance. Advanced Nurse Practitioners also undertake agreed virtual telephone consultations monitoring patient's ongoing symptoms, to impart outstanding test results and to ensure the patient is confident about being at home. The Silver Phone line ensures older patients have the appropriate safety netting in place after discharge from a fast paced patient pathway such as AEC. There has been an overwhelmingly positive response from older patients who feel that 'somebody cares'.

\section{P-562}

Postural hypotension contributing to falls: a project to improve the practice of falls assessments in the elderly

Nisha Rajcoomar ${ }^{1}$, Ahmed Abdhalla ${ }^{2}$

${ }^{1}$ Princess Alexandra Hospital, Harlow, UK, ${ }^{2}$ North Devon District Hospital, Harlow, UK
Introduction: Falls are a common presenting complaint in patients aged over 65. Multiple factors contribute to falls in the elderly. Postural hypotension is common and often secondary to medication. NICE recommend an examination and medication review in falls assessments. Lying and standing blood pressure (BP) assessments and medication reviews were documented poorly in our hospital. Our project reviewed current practice, aiming to improve falls assessments.

Methods: 30 patients notes, aged over 65, were reviewed. Current falls assessments including lying standing BP and medications review, within $48 \mathrm{~h}$, was audited. Round 1 , the initial findings were presented to Doctors, who received teaching in the components of falls assessments. Round 2, a falls sticker was introduced to improve falls assessments. Completing the cycle, falls assessment practice was re-audited 6 months later.

Results: Initial results showed 5/30 (17\%) had lying standing BP and $6 / 30$ (20\%) medication reviews. Round 1 improved to 20/30 (67\%) lying standing BP and 18/30 (60\%) medication reviews. Round 2 showed 22/30 (73\%) lying standing BP and 24/30 (80\%) medication reviews documented. Round 3 after 6 months, 23/30 (77\%) had lying standing BP and 23/30 (77\%) medication reviews documented.

Key conclusions: Current practice showed lying standing BP and medication reviews in falls assessment was poorly documented. After 2 rounds, lying standing BP improved from 17 to $73 \%$, and medication reviews from 20 to $80 \%$. Feeding back audit results, education and a falls sticker contributed to sustained improved practice. Recommendations include continued use of the falls sticker and education to maintain good practice.

\section{P-563}

Long-term prognosis according to setting of care in elderly patients hospitalized for acute heart failure: data from the Athena Registry

Anna Chiara Baroncini ${ }^{1}$, Francesco Orso ${ }^{1}$, Alessandra Pratesi ${ }^{1}$, Giulia Biagioni $^{2}$, Andrea Herbst ${ }^{1}$, Silvia Parlapiano ${ }^{2}$, Filippo Fedeli ${ }^{2}$, Aldo Lo Forte ${ }^{1}$, Camilla Ghiara ${ }^{1}$, Emanuele Carrassi ${ }^{2}$, Mauro Di Bari ${ }^{1}$, Andrea Ungar ${ }^{1}$, Samuele Baldasseroni ${ }^{1}$

${ }^{1}$ Careggi University Hospital (AOUC), Department of medicine and geriatrics, Florence, Italy, ${ }^{2}$ University of Florence, Florence, Italy

Introduction: Acute heart failure (AHF) is a clinical syndrome that typically affects elderly. Our purpose was to evaluate long-term prognosis and predictors of mortality of elderly patients hospitalized for AHF in different settings of care (Cardiology, Internal Medicine and Geriatric wards).

Methods: Data derived from the ATHENA retrospective observational study which included patients aged 65 years or more, admitted for AHF to the Emergency department (ED) of a tertiary University teaching-hospital and transferred to the above described settings of care between 12.2014-12.2015. A telephone interview to evaluate vital status and functional level was conducted.

Results: 401 patients were enrolled: mean age was 83.5 years, females were $51 \%$. Telephone interviews were conducted among patients discharged alive (365 patients, $91 \%$ ). One-year mortality was $33.2 \%$, significantly higher for patients discharged from Geriatrics (45.8\%) and Internal Medicine (32.5\%) compared to Cardiology (17.3\%), $\mathrm{p}=0.009$. During the total FU (median 21 months), mortality rate was $49.7 \%$, significantly higher in Geriatric $(60.4 \%)$ and Internal Medicine (52.0\%) wards compared to Cardiology $(32.7 \%), p=0.013$. By multivariable analysis, independent predictors of long-term mortality were NT-pro BNP level in ED (OR 1.77, CI $1.03-3.04, \mathrm{p}=0.039$ ) and Charlson Comorbidity Index (OR 1.30, CI 
$1.13-1.50, \mathrm{p}=0.001)$, with a protective effect of Beta-Blockers therapy at discharge (OR $0.53, \mathrm{CI} 0.29-0.95, \mathrm{p}=0.033$ ) and functional status evaluated through Barthel Index (OR .99, CI 0.98-1.0, $\mathrm{p}=0.005$ ).

Conclusions: In elderly patients hospitalized for AHF, long term prognosis varies according to the different setting of care. Long-term mortality is high and is influenced by geriatric and cardiological variables.

\section{P-564}

Acute ambulatory care in the community: processes of care and outcomes for a consecutive patient cohort from an Emergency Multidisciplinary Unit

Tania C. N. Elias ${ }^{1}$, Jordan S. T. Bowen ${ }^{1}$, Roya Hassanzadeh ${ }^{2}$, Sarah T. Pendlebury ${ }^{3}$, Daniel S. Lasserson ${ }^{4}$

${ }^{1}$ Department of Geratology, Oxford University Hospitals NHS Foundation Trust, Oxford, UK; Emergency Multidisciplinary Unit, Abingdon Hospital, Oxford Health NHS Foundation Trust, Abingon, UK, ${ }^{2}$ Department of Geratology, Oxford University Hospitals NHS, Oxford, UK

Introduction: Impetus exists for acute care without hospital admission. But little information exists regarding care processes, patient suitability, and outcomes, especially for older adults. We describe those receiving ambulatory care in an Emergency Multidisciplinary Unit, outcomes, and factors associated with hospital admission.

Methods: Consecutive patients were included in a prospective service evaluation. Data was acquired as part of standard care, including demographics, comorbidities, presenting problems, diagnoses, clinical status, diagnostics, interventions, 30-day ambulatory status and mortality. Relative risks and two-sample t-tests were calculated for associates of admission vs ambulatory care.

Results: 533 new patients were assessed (August-December 2015). Median age was 81 (IQR 68-87), median Charlson Comorbidity Index was 5 (IQR 3-6), $283(53 \%)$ received personal and domestic care, and $71(13 \%)$ residential care. The commonest presentations were: reduced mobility $(256 ; 48 \%)$, increased care needs $(230 ; 43 \%)$, dyspnoea $(198 ; 37 \%)$, and falls $(106 ; 20 \%) .381(71 \%)$ were initially managed as ambulatory. Of these, $56(15 \%)$ required unplanned hospital admission within 30 days, and 208 (55\%) underwent $\geq 1$ planned subsequent review for the index problem. 364 (68\%) of all patients had new outpatient appointments arranged. Age, needing transport, receiving care, Charlson Comorbidity, sensory impairment, polypharmacy, previous falls, clinical scores (MUST, Braden, Barthel, Modified Rankin, NEWS, AMTS), delirium, dehydration, and intravenous treatment were all significantly associated with admission $(\mathrm{p}<0.001)$

Conclusions: Although selected older, multimorbid patients can receive successful ambulatory care, this frequently involves multiple assessments. Further research is needed to determine which clinical features can help identify patients who can be successfully treated along ambulatory pathways.

\section{P-565}

\section{Oxygen prescription in acute geriatric medicine}

Atinuke Akinmolayan ${ }^{1}$, Edward Williams ${ }^{1}$, Olubayode Adewole ${ }^{1}$

${ }^{1}$ Barking Havering and Redbridge Hospital Trust, London, UK
Introduction: Oxygen, used to treat hypoxia, is a commonly used drug and is often poorly prescribed. There have been instances whereby patient care has been delayed due to lack of or invalid oxygen prescriptions. It's British Thoracic Society's guidelines for all patients to have target saturations documented and it is our trust policy for all patients to have an oxygen prescription. The safety issues surrounding the prescription of oxygen are well-recognised and incorrect prescription in patients who are at risk of hypercapnia can be fatal.

Methods: An audit was carried out looking at oxygen prescriptions and their validity across 4 acute geriatric medicine wards. Following the first data collection, educational interventions such as presentations, posters and emails were used. The prescriptions were then reaudited after the intervention.

Results: 60 inpatient prescriptions were reviewed in each cycle. Age range 51-95, male-to-female ratio 7:8. Our first cycle revealed that $73 \%$ of patients did not have oxygen prescribed. Of the patients that had oxygen prescribed, $75 \%$ were appropriate for the patient, and only $13 \%$ of prescriptions were checked daily by the nursing team. Following our interventions these figures improved. We found that $30 \%$ of patients had an oxygen prescription and $89 \%$ of these were appropriate for the patient.

Conclusions: We have demonstrated that oxygen prescription is inadequate and remains so after interventions. Educational interventions, such as posters, have not proven to be very effective in changing clinical practice. Further work needs to be done to provide a long-lasting change to improve patient care.

\section{P-566}

Retrospective study of harms due to omitted doses of medications for Idiopathic Parkinson's Disease (IPD) in medical inpatients

Gregson $\mathrm{E}^{1}$

${ }^{1}$ Blackpool Teaching Hospitals, Blackpool, UK

Introduction: Although it's frequently stated that it's dangerous to omit medications for IPD there is surprisingly little data published on the effect of missed dosed on medical wards. Various harms are assumed (neuropsychiatric, declining mobility, dysphagia, prolonged length of stay, rigidity, neuroleptic malignant syndrome and death). Methods: Notes were retrospectively obtained for all patients who were either coded for IPD on the hospital discharge summary, or who had been dispensed medicines that were indicated for IPD, over a 3 months period at a medium sized general hospital. The notes were reviewed to identify patients who had IPD (vascular Parkinsonism, drug induced Parkinsonism, Parkinson's plus syndromes were excluded). Omitted doses were identified and the notes reviewed separately for potential harms. Harms were compared between patients who had missed doses, those who received all doses and those in who it was unclear if doses were missed, using Fisher's exact test.

Results: 29 medical inpatients were identified with IPD (30 admissions). Seven patients died. The ages of the patients ranged from 67-89 years (mean 79.6). There were 18 males and 11 females. 21 patients missed at least one dose, 5 received all doses and in 4 admissions it was unclear. There were 7 deaths, all had missed doses $(\mathrm{p}=0.19)$. There were 16 admissions associated with potential harms (including death), all had missed doses $(\mathrm{p}=0.0001)$.

Conclusions: Although the number of patients studied was small, the data suggest that there is a strong association of missed doses of medications for IPD and harms including death. 


\section{P-567}

Outcomes in cervical and upper thoracic vertebral fractures

Katherine Horgan ${ }^{1}$, Jane Edmondson ${ }^{2}$

${ }^{1}$ Manchester University Hospitals NHS Foundation Trust, Manchester, UK, ${ }^{2}$ Manchester University Hospitals NHS Foundation Trust, Manchester, UK

Introduction: Cervical spinal fractures are common in the elderly with an incidence of approximately 2 in 10,000 yearly at the level of $\mathrm{C} 2$ alone [1]. These can have a devastating impact with some studies quoting mortality rates of $35.1 \%$ at 1 -year post injury [1].

Method: We examined case notes for patients admitted over the age of sixty with suspected cervical or upper thoracic vertebral fractures over the course of 2017.

Results: 12 sets of notes were reviewed for the purposes of this audit. 6 males and 6 females were studied with a mean age of 79.3 (ranging from 60-94 years-old) As well as outcome data based on NICE guidelines, we found practicalities involving discussion with tertiary services to be time consuming and that $33 \%$ of our patients had a decline in their previous level of function. This emphasises the devastating impact of these injuries on an elderly cohort of patients, and the length of stay would corroborate this further being almost 3 weeks on average which leads to huge costs for the National Health Service at a time when it is under more pressure than ever.

Conclusion: These results demonstrate that even for spinal column injuries with no cord involvement there can be significant input on patient morbidity and functional baseline, with $5(42 \%)$ of our patients declining in their level of function to the point where 2 required 24-h care on discharge from hospital and 3 required intermediate care. This can also impact on National Health Service in general.

\section{Reference:}

1. Leaden M, Nye C et al (2017) A five-year retrospective analysis of the management of elderly patients with an upper cervical spine fracture. Br J Neurosurg 31(2): 120-121.

\section{P-568}

Acute heart failure un the elderly: which is the treatment of choice at discharge? Real world data from the ATHENA registry

Aldo Lo Forte ${ }^{1}$, Francesco Orso ${ }^{1}$, Alessandra Pratesi ${ }^{1}$, Andrea Herbst ${ }^{1}$, Silvia Parlapiano ${ }^{1}$, Giulia Biagioni ${ }^{1}$, F. Fedeli ${ }^{1}$, Camilla Ghiara ${ }^{1}$, Anna Chiara Baroncini ${ }^{1}$, F. Bacci ${ }^{2}$, G. Ciuti ${ }^{2}$, Mauro Di Bari ${ }^{1}$, Niccolò Marchionni

${ }^{1}$ Department of medicine and geriatrics, Careggi University Hospital (AOUC), Florence, Italy, ${ }^{2}$ Department of emergency and Internal Medicine, Careggi University Hospital (AOUC), Florence, Italy, ${ }^{3}$ Careggi University Hospital (AOUC), Cardiothoracova, Florence, Italy

Introduction: The prognosis of patients with $\mathrm{HF}$ with reduced $\mathrm{EF}$ has improved in recent years thanks to the availability of evidence based treatments. Currently, equally effective treatments are not available for patients with HFpEF (preserved) and for those with HFmrEF (mid-range), the new category of patients with HF.

Methods: Data derived from the ATHENA (AcuTe Heart failurE in advaNced Age) retrospective observational study which included elderly patients ( $\geq 65$ years) admitted with diagnosis of AHF (worsening or de novo) to the Emergency Department (ED) of a tertiary University teaching-hospital in the period
01.12.2014-01.12.2015. Inclusion criteria were: mean age of three group of patients was respectively $84.2,84.3$ and 80.3 years, $\mathrm{p}<0.001$. Pharmacological treatments at discharge in the three HF groups are shown in the attached table. BBs and MRAs were the only two classes of drugs that had a statistically different prescription rate across the three HF groups: patients with HFmrEF received BBs in similar percentage to patients with HFrEF. Instead MRAs prescription rate in HFmrEF was more similar to the one of patients with HFpEF. Key conclusion: Elderly patients with AHF differ in terms of clinical characteristics and in-hospital prognosis according to the different settings of care. In this population in-hospital mortality seems to be correlated with clinical variables already known to impact prognosis, but also with geriatric variables such as functional status and cognitive impairment and with setting of care of assignment after the Emergency Department, with a protective effect of management in cardiological settings.

\section{P-569}

\section{European geriatric emergency medicine: priority research questions}

Lisa S. van Tol ${ }^{1}$, Christian H. Nickel ${ }^{2}$, Jacinta A. Lucke ${ }^{1}$, Jay Banerjee ${ }^{3}$, Bianca M. Buurman ${ }^{4}$, F. Javier Martin-Sãnchez ${ }^{5}$, Parabath W. B. Nanayakkara ${ }^{4}$, James D. van Oppen ${ }^{3}$, Yvonne Schoon $^{6}$, Marije van der Velde ${ }^{7}$, Simon P. Mooijaart ${ }^{1}$

${ }^{1}$ Leiden University Medical Center, Leiden, the Netherlands, ${ }^{2}$ University of Basel, Basel, Switserland, ${ }^{3}$ University of Leicester, Leicester, United Kingdom, ${ }^{4}$ Amsterdam University Medical Center, Amsterdam, The Netherlands, ${ }^{5}$ San Carlos Clinical Hospital, Madrid, Spain, ${ }^{6}$ Radboud University Medical Center, Nijmegen, The Netherlands, ${ }^{7}$ Utrecht University Medical Center, Utrecht, The Netherlands

Introduction: With the ageing European society, there is an increasing demand for geriatric emergency medicine. More research to acquire knowledge in this field is needed. Therefore, the Geriatric Emergency Medicine (GeriEM) taskforce aims to develop a European research agenda on GeriEM by collecting and prioritising research questions.

Methods: A two-round Delphi study will be conducted. A broad scope of European national healthcare associations will be approached to invite emergency care physicians, geriatricians, nurses, general practitioners, and physiotherapists to fill in their research questions in our online survey (free-text responses). Furthermore, the survey was spread via the GeriEM website and social media. After quality check and categorization, respondents will be asked for inputs on priorities in a second round. By calculating mean priority ratings, the final top priority research questions in the field of geriatric emergency medicine will be listed.

Results: The first round survey will be closed early summer 2018 . Input will be quality checked and categorised. Expectedly, input of the second round will be finished in the fall. During EuGMS 2018, we will be able to present preliminary results.

Conclusions: The list of priority research questions can guide researchers in the field of geriatric emergency medicine in the coming years. 
P-570

A snapshot from the older patient's Emergency Department visit: design and preliminary results of the MPI (Multidimensional Prognostic Index)—HOPE (Influence of the MPI on the Hospitalisation of Older Patients admitted to the EMERGENCY DEPARTMENT) study

Marcel Rarek ${ }^{1,2,3}$, Anna Maria Meyer ${ }^{1,2}$, Lena Pickert ${ }^{1,2}$, Volker Burst $^{2,3}$, Alberto Pilotto ${ }^{4}$, Thomas Benzing ${ }^{2,3}$, Maria Cristina Polidori ${ }^{1,2}$

${ }^{1}$ Ageing Clinical Research, Dpt. II for Internal Medicine, University Hospital of Cologne, Cologne, Germany, ${ }^{2}$ Nephrology, Rheumatology, Diabetology and Internal Medicine, Dpt. II for Internal Medicine, University Hospital of Cologne, Cologne, Germany, ${ }^{3}$ Emergency Department, University Hospital of Cologne, Cologne, Germany, ${ }^{4}$ Department of Geriatric Care, Orthogeriatrics and Rehabilitation, Frailty Area, E.O. Galliera, Genova, Italy

Background: The Emergency Department (ED) is usually the first contact of older patients with the hospital system and an essential checkpoint to influence the patients' further trajectories. At this early stage of treatment, a comprehensive geriatric assessment (CGA)based identification of patients' prognosis and needs may improve clinical decision and patients' quality of life (QoL) by reducing the length of hospital stay and rehospitalization.

Methods: Patients aged 75 years and older $(75+)$ admitted to the ED of the University Hospital of Cologne were consecutively screened over a 3-month period for enrolment in the study. On admission, patients underwent physical and instrumental examination as well as CGA-based calculation of the Multidimensional Prognostic Index (MPI) and a questionnaire on QoL (EQ5D). Healthcare indicators were collected at baseline as well as at a phone-follow up interview three and 6 months after discharge.

Results: Of the 119175 + patients admitted from October 2017 to January 2018 , 175 were willing/able to participate and to undergo MPI completion. 80\% was transferred to another ward, one fifth being discharged home. "Feeling uncomfortable" was the main symptom in $9 \%$ of the cases, with hypertension and heart diseases as main diagnoses. Forty-six $9 \%$ of the patients belonged to MPI-1 (low mortality risk at 1 month and 1 year), $45.1 \%$ to MPI-2 (medium risk) and $8 \%$ to MPI-3 (high risk) groups. Further analyses including follow-up results are ongoing.

Conclusions: A structured CGA-based prognosis assessment in the ED might further improve clinical decision making and substantially influence healthcare resources allocation.

\section{P-571}

High need of geriatric awareness in the Emergency Department: a Danish population-based cohort study

\section{Jesper Ryg ${ }^{1}$, Jens-Ulrik Rosholm ${ }^{2}$, Annmarie Lassen ${ }^{3}$ \\ ${ }^{1}$ Department of Geriatric Medicine, Odense University Hospital, Odense, Denmark, ${ }^{2}$ Department of Geriatric Medicine, Odense University Hospital, Odense, Denmark, ${ }^{3}$ Department of Emergency Medicine, Odense University Hospital, Odense, Denmark}

Objectives: The objective was to describe the prevalence of geriatric target areas among acute older medical patients in the Emergency Department (ED) and the association between geriatric target areas and admission, length of admission, in-hospital mortality, 30 days post discharge mortality, 30 days hospital re-attendance, and 360 days loss of independency.

Setting: ED of a large university hospital.
Participants: All medical patients $\geq 65$ years of age from a single municipality with a first attendance at the ED during a 1 year period (November 2013 to November 2014). Primary and secondary outcome measures: Based on information from healthcare registers we defined prevalence of geriatric target areas as existence of impairment, recently increased impairment, polypharmacy, or comorbidity. Outcomes measured were admission, length of admission, post-discharge mortality, hospital re-attendance, and home care dependency 0-360 days following ED contact.

Results: Totally, 3775 patients (55\% women) were included, age 78 (71-85) years [median (IQR)]. Follow-up was complete. Prevalence of $0-4$ geriatric target areas were $14.9,27.3,25.2,22.3$, and $10.3 \%$, respectively. Number of target areas was significantly associated to hospital admission, length of admission, 30 day mortality, and hospital re-attendance after discharge. Among patients with no target areas $70 \%$ lived independent all 360 days after discharge, whereas all patients with $\geq 3$ target areas had some dependency or were dead within 360 days following discharge.

Conclusion: Among older medical ED patients $50 \%$ had two or more geriatric target areas which were associated with poor outcome. This highlights the need of geriatric awareness and competences in the ED.

\section{P-572}

Multidimensional Prognostic Index (MPI) predicts non-invasivemechanical-ventilation (NIV) success and mortality in older people with acute hypoxemic respiratory failure (AHRF)

Federica Gandolfo ${ }^{1}$, Francesco Corradi ${ }^{1}$, Romina Custureri ${ }^{1}$, Francesca Tricerri ${ }^{1}$, Davide Gonella ${ }^{1}$, Ilaria Indiano ${ }^{1}$, Roberta Pienovi ${ }^{1}$, Daniela Maloberti ${ }^{1}$, Marco Lattuada ${ }^{1}$, Alberto Pilotto ${ }^{1}$, Claudia Brusasco ${ }^{1}$

${ }^{1}$ E.O Galliera, Geneva, Italy

Background: AHRF is a frequent cause of hospitalization in older subjects. NIV may represent a possible treatment strategy in older patients, but no studies explored the usefulness of the Multidimensional Prognostic Index (MPI) in predicting the NIV outcomes.

Aim: Evaluate the accuracy of CGA-based MPI to predict NIV outcome Methods: we enrolled patients older than 75 years, admitted to an Acute Geriatrics Unit for AHRF $\left(\mathrm{PaO}_{2} / \mathrm{FiO}_{2}\right.$ ratio $\left.<300\right)$ and treated with NIV plus standard medical therapy. MPI was assessed at admission according to standard methods. The primary outcome was 1 year-mortality rate; secondary outcomes were NIV success, i.e. improving the oxygenation index $\left(\mathrm{OI}, \mathrm{PO}_{2} / \mathrm{FiO}_{2}\right.$ ratio $\left.\geq 300\right)$ and the days of OI improvement. Receiver operator characteristics (ROC) analysis was used to identify the best MPI cut-off to predict mortality in this population (MPI $\geq 0.78$ ).

Results: We enrolled 15 patients $(10$ females, mean age $86.7 \pm 5.2$ years, mean MPI $0.75 \pm$ SD), 8 with MPI $\geq 0.78$ and 7 with MPI $<0.78$. At baseline, no differences in arterial blood parameters were observed. MPI values $\geq 0.78(\mathrm{p}<0.05)$ and NIV failure $(\mathrm{p}=0.001)$ were significantly associated with 1 -year mortality. NIV success was significantly higher in patients with lower MPI $(\mathrm{p}<0.05)$, regardless of basal $\mathrm{PaO}_{2} / \mathrm{FiO}_{2}$ ratio. In NIV success group patients with MPI $<0.78$ improved OI in fewer days (4 vs 8 days, $\mathrm{p}=0.06)$.

Conclusion: In frail older patients with AHRF, MPI predicts 1-year mortality and NIV success, in terms of OI improvement and the time of OI improvement. Only in patients with lower MPI, NIV could reduce the mortality risk. 


\section{P-573}

What is the proportion of viral origin of infectious respiratory syndromes presented by elderly people hospitalized during the winter of 2017-2018?

Sophie-Charlotte Riboux ${ }^{1}$, Alice Gioanni ${ }^{2}$, Caroline Hyernard ${ }^{3}$, Florent Guerville $^{1}$, David Kobeh ${ }^{1}$, Paul Gassie ${ }^{1}$, Laura Haw-Shing ${ }^{3}$, Hélène Domergue ${ }^{3}$, Isabelle Bourdel-Marchasson ${ }^{3}$, Claire RoubaudBaudron $^{3}$

${ }^{1}$ CHU Bordeaux, Pôle de gérontologie clinique, 33000, Bordeaux, France, ${ }^{2}$ Service de Gériatrie, Hôpital Pitié Salpêtrière, Paris, France, Université Pierre et Marie Curie Paris VI, Sorbonne Université., Bordeaux, France, ${ }^{3}$ Univ. Bordeaux, CHU Bordeaux, Pôle de gérontologie, Bordeaux, France

Introduction: Respiratory virus PCR may be helpful in management of patients with pulmonary symptoms. Our goal was to determine the proportion of infectious respiratory syndromes with a viral diagnosis in hospitalized elderly patients during last winter and to describe clinical and/or biological factors associated with viral diagnosis and morbi-mortality during hospitalization and after 3 months.

Method: Prospective non-interventional study. Multiplex viral PCR was used for all patients aged 75 and over hospitalized in a geriatric ward (Bordeaux and Paris) between 14/12/2017 and 27/4/2018 with a respiratory infectious syndrome. Demographic, clinical, biological and prognostic data were collected. A uni- and multivariate analyses according to PCR positivity determined factors associated with positive PCR.

Results: 182 patients ( $88 \pm 6$ years, $56 \%$ female) were included (mean ADL score $4.2 \pm 1.9$ and Charlson score $2.6 \pm 2.3$ ). Viral PCR was positive in 106 (58\%) patients: flu 67(63\%) patients A $(\mathrm{n}=23), \mathrm{B}(\mathrm{n}=43)$ and A + B $(\mathrm{n}=1)$, RSV $(\mathrm{n}=11)$, Rhinovirus $(n+18)$, Bocavirus $(n=1)$, Metapneumovirus $(n=10)$, Coronavirus $(\mathrm{n}=5)$ and Adenovirus $(\mathrm{n}=1)$. Factors independently associated with a positive PCR were presence of a cough (OR 3.2 CI 1.6-6.3, $\mathrm{p}=0.001$ ), wheezing (OR 3.0 IC 1.3-6.7, p = 0.007) and low eosinophil level (OR 0.057 CI $0.01-0.57, \mathrm{p}=0.006$ ). More than half of PCR positive patients received antibiotics $(56 \%)$. In-hospital mortality was not different (13\%). 3-Month morbi-mortality data are pending.

Conclusion: Viral diagnosis was present in more than half of patients whith suspicion of pulmonary infection. The proportion of non-influenza viruses is notable. Presence of wheezings, cough and eosinopenia is more frequently associated with viral pulmonary infections.

\section{P-574}

Intramuscular epinephrine as first-line treatment of anaphylaxis: still concerns about its safety in the elderly?

\section{Simone Cernesi ${ }^{1}$, Rosalba Buquicchio ${ }^{2}$, Maria Teresa Ventura ${ }^{3}$, Elisa Boni ${ }^{4}$ \\ ${ }^{1}$ General Practitioner and Nursing Home Physician Casa Serena Sassuolo, Rome, Italy, ${ }^{2}$ University of Bari Medical School, Policlinico, Bari, Italy, ${ }^{3}$ Department of Interdisciplinary Medicine, University of Bari, Italy, ${ }^{4}$ Allergy Unit, Hospital Santo Spirito, Rome, Italy}

Background: Anaphylaxis is a severe condition that can affect patients of all ages. Elderly patients must be considered particularly vulnerable to severe anaphylaxis due to many risk factors such as concomitant diseases and medications. Intramuscular administration of epinephrine is recommended as first line therapy for anaphylaxis
We present a revision of the literature concerning efficacy and safety of epinephrine administration in older patients suffering of anaphylaxis.

Method: We reviewed bibliographic data and guidelines on treatment of anaphylaxis published in the last decade.

Results: There are no absolute contraindication to the administration of epinephrine through intramuscular route in a patient experiencing anaphylaxis since benefits outweigh the risks in the elderly and in patients with preexisting cardiovascular disease. There are no absolute contraindications to the prescription of self-injectable adrenaline in older patients at risk of anaphylaxis.

Conclusion: Epinephrine autoinjectors should be prescribed for all patients with a history of anaphylaxis. Patients and their caregivers should be taught why, when, and how to inject adrenaline and should be equipped with a personalized written anaphylaxis emergency action plan. History of allergic reactions and anaphylaxis besides an emergency action plan should be noted in patient records in nonhospital care settings such as nursing homes and epinephrine should always be available. Further education of clinicians regarding the appropriate route of epinephrine administration in the management of anaphylaxis should be promoted to avoid adverse events. Its use should be promoted among clinicians in every setting.

\section{P-575}

Burden of influenza in older patients over the 2016-2017 winter season in France

Gaetan Gavazzi Gavazzi ${ }^{1}$, Marc Paccalin ${ }^{2}$, Benoit D. E. Wazieres ${ }^{3}$, Claire Roubaud $^{4}$, Louis Bernard ${ }^{5}$, Thibault Fraisse ${ }^{6}$, Laurence Legout $^{7}$, Jean Pierre Aquino ${ }^{8}$, Olivier Guerin ${ }^{9}$, Emmanuel Forestier $^{10}$

${ }^{1}$ Geriatric Department, University hospital of Grenoble-Alpes, Alpes, France, ${ }^{2}$ Chu-poitiers, Poitiers, France, ${ }^{3}$ Geriatric Department Chunimes, Poitiers, France, ${ }^{4}$ Geriatric Department, CHU Bordeaux, Bordeaux, France, ${ }^{5}$ Infectious Diseases Department, CHU Tours, Tours, France, ${ }^{6}$ Geriatric Department, $\mathrm{CH}$ Al, Alpes, France

Introduction: Flu is one of the most prevalent winter-season infections, with a poor prognosis in older populations. However, as viral diagnosis was difficult until the last years, available data on the burden of flu are based on mathematical models. We then aim to study the real impact of flu during the hospital stay in elderly populations.

Methods: French multicenter retrospective study carried out in Infectious Diseases (ID) and Geriatric wards through a group of interest on infections in elderly (GInGer) gathering French National Geriatric (SFGG) and ID Societies (SPILF). The unique inclusion criterion was to get a positive influenza PCR on nasopharyngal samples within the outbreak (15.12.2016-30.03.2017). Data on flu, oseltamivir, antibiotic, and outcome have been collected.

Results: 49 wards participated to the study; among 8814 patients, 515 patients had a positive PCR out of 1268 NP samples. $126(24.5 \%)$ were nosocomial; mean age was 86.7 years, mean ADL of 4.06. 77 (15\%) came from Nursing Home. Mean Charlson index was 2.9, but $62(12 \%)$ without comorbidity. Oseltamivir was used for $61 \%$ of cases during 5.08 days and antibiotic in $58.7 \%$ over 9.1 days. More than $50 \%$ patients presented a medical complication, death rate was $12.23 \%$. Mean length of stay was 16.1 days.

Conclusions: The real burden of flu seems high (nosocomial, antibiotic use, death rate, complications, length of stay), but flu seems to affect different subset of elderly patient. There is an urgent need to collect data on the burden of flu in different older populations to adopt preventive and therapeutic recommendations. 


\section{P-576}

Patients admitted directly home from Emergency Department may be more active and return faster to their activities of daily living

\section{Gregersen ${ }^{1}$}

${ }^{1}$ Department of Geriatric Medicine, AUH, Aarhus, Denmark

Introduction: Older patients are lying in bed $17 \mathrm{~h}$ per day which increases the risk of institutionalization, falls, poor quality of life, and death.

Aim: examine whether patients discharged directly to their home from the Emergency Department (ED) and followed-up by either a geriatric team or a general practitioner have a better functional capacity when measured 8 weeks after admission compared to those who were admitted to a geriatric ward.

Methods: Community-dwelling 75+ years old patients were consecutively enrolled on admission to the ED. A physiotherapist assessed the functional capacity by Functional Recovery Score (FRS) retrospectively 14 days before current illness and prospectively 8 weeks after discharge. Changes in functional capacity were compared in a logistic regression model.

Results: A total of 157 patients were included. Fifty-eight patients (36.9\%) were discharged directly to their homes and 99 (63.1\%) were admitted to a hospital ward. The two groups were comparable in gender, age, comorbidity, previous functional capacity, mental status. Fifty-four percent of the patients who were discharged directly to their homes had either maintained or improved their functional capacity compared to only $36 \%$ in the group admitted to a hospital ward. Adjusted for baseline characteristics: [OR 2.1 (95\% CI 1.05; 4.09)], $\mathrm{p}=0.036$.

Conclusion: Patients discharged directly to their homes from the ED may be more active and return sooner to their activities of daily living. It is important to aim at a physically active hospitalization to prevent in-hospital functional decline. And if possible, discharge the patients early and treat them at home.

\section{P-577}

\section{Comprehensive geriatric assessment and frailty screening in a sample of elderly patients with severe aortic stenosis considering transcatheter aortic valve implantation}

Leire Eguzkiza ${ }^{1}$, Rocío Menéndez-Colino ${ }^{2}$, Gabriela Guzmán ${ }^{3}$, Guillermo Galeote ${ }^{3}$, Isabel Martín-Maestre ${ }^{1}$, José Luis LópezSendón ${ }^{3}$, Juan Ignacio González-Montalvo ${ }^{4}$

${ }^{1}$ Hospital Universitario la Paz, Madrid, Spain, ${ }^{2}$ Geriatric, Hospital Universitario la Paz, Madrid, Spain, ${ }^{3}$ Cardiology, Hospital Universitario la Paz, Madrid, Spain, ${ }^{4}$ Geriatric, Hospital Universitario la Paz, Madrid, Spain

Background: Frailty is present in half of patients being screened for transcatheter aortic valve implantation (TAVI). The aim of this study was to describe characteristics of older people with severe aortic stenosis (SAS) considering to TAVI.

Methods: A prospective study of patients with SAS referred from Cardiology to a cardio-geriatric consult was carried out. Socio-demographic, clinical, functional status [Barthel index (BI) Functional ambulation category (FAC)], cognitive status [Red Cross Mental Scale (CRM), Short Portable Mental Status Questionnaire (SPMSQ)], analytical and nutritional variables [body mass index (BMI)] were registered. Frailty was considered when Short Physical Performance Battery $(\mathrm{SPPB})<9$, gait speed $<8 \mathrm{~m} / \mathrm{s}$ or hand grip strength $<13$ $\mathrm{kg}$ in women or $<23 \mathrm{~kg}$ in men.
Results: Ninety-nine patients were included. Mean age was $81.78 \pm 6.70$ years, $54.5 \%$ female. Mean values were: BI: $92.69 \pm 12.8$, FAC: $4.68 \pm 0.69$, CRM: $0.23 \pm 0.51$, SPMSQ: $1.25 \pm 1.65$, gait speed $0.67 \mathrm{~m} / \mathrm{s} \pm 0.24$, hand grip strength: $17.3 \pm 6.33 \mathrm{~kg}$ in women and $29.47 \pm 7.61 \mathrm{~kg}$ in men, SPPB: $8.54 \pm 3.09$, BMI: $28.82 \pm 4.94 \mathrm{~kg} / \mathrm{m}^{2}$, serum creatinine $1.14 \pm 0.52 \mathrm{mg} / \mathrm{dl}$. Comorbidities registered were diabetes: $34.9 \%$, chronic obstructive pulmonary disease: $18.1 \%$, cancer: $15.7 \%$, chronic renal failure: $15.7 \%$. Prevalence of frailty were $41.4,74.7$, $25.9,13.3 \%$ according to SPPB, gait speed and handgrip strength (women and men) criteria respectively.

Conclusion: Patients with SAS considering to TAVI were in a good functional and mental status. Frailty was very frequently detected, what can add important predictive power in a great number of these patients.

\section{P-578}

Frailty assessment tools help to discriminate patients with severe aortic stenosis undergoing transcatheter aortic valve implantation or medical treatment

Rocío Menéndez-Colino ${ }^{1}$, Leire Eguzkiza ${ }^{1}$, Gabriela Guzmán ${ }^{2}$, Guillermo Galeote ${ }^{2}$, Pilar Gotor ${ }^{3}$, José Luis López-Sendón ${ }^{2}$, Juan Ignacio González-Montalvo ${ }^{1}$

${ }^{1}$ Geriatric, Hospital Universitario la Paz, Madrid, Spain, ${ }^{2}$ Cardiology. Hospital Universitario la Paz, Madrid, Spain, ${ }^{3}$ Hospital Universitario la Paz, Madrid, Spain

Background: Frailty can be an essential component in older adults with aortic stenosis assessed for transcatheter aortic valve implantation (TAVI). The aim of this study was to analyze if frailty measures can differentiate patients with severe aortic stenosis (SAS) treated by TAVI or by medical treatment.

Methods: A prospective study of patients with symptomatic SAS referred from Cardiology to a cardio-geriatric consult was carried out. Socio-demographic (age and sex), clinical, functional status [Barthel index (BI) Functional ambulation category (FAC)], cognitive status [Red Cross Mental Scale (CRM), Short Portable Mental Status Questionnaire (SPMSQ)], analytical and nutritional variables [body mass index (BMI)] were registered. Frailty was considered when Short Physical Performance Battery (SPPB) $<9$, gait speed $<8 \mathrm{~m} / \mathrm{s}$ or hand grip strength $<13 \mathrm{~kg}$ in women or $<23 \mathrm{~kg}$ in men.

Results: Ninety nine patients were included. Mean age was $81.78 \pm 6.7$ years, $54.5 \%$ female. TAVI patients $(\mathrm{n}=75,75 \%)$ were younger (mean $80.8 \pm 7$ vs $84.6 \pm 4.79, \mathrm{p}=0.027$ ), had better BI $(95.07 \pm 6.55$ vs $85.8 \pm 22.78, \mathrm{p}=0.065)$ and FAC $(4.79 \pm 0.44$ vs $4.3 \pm 1.21, \mathrm{p}=0.039)$. Prevalence of frailty was $36 \%$ in TAVI and $55 \%$ in medical treatment patients according to SPPB $(\mathrm{p}=0.1), 69 \%$ in TAVI and $100 \%$ in medical treatment patients according to gait speed $(\mathrm{p}=0.005)$.

Conclusion: Among elderly patients with SAS, those who finally receive TAVI treatment were younger, in better functional and mental status and less frequently frail than those that underwent medical treatment. Geriatric assessment and frailty screening tools could be useful to identify patients that finally receive one type of treatment or another. 


\section{P-579}

Non specific dusease presentation on older patient in Emergency Department

Giovanni Ricevuti ${ }^{1}$, Andrea Ungar ${ }^{2}$, Luca Laghi ${ }^{3}$, Thomas Dreher-Hummel ${ }^{4}$, Laura Blomaard ${ }^{5}$, Ciro Paolillo ${ }^{6}$

${ }^{1}$ Postgraduate Course in Geriatrics, Dpt of Internal Medicine, University of Pavia, Pavia, Italy, ${ }^{2}$ Geriatrics, Syncope Unit, Hypertension Centre, Head division of Geriatrics and Intensive Care Unit, University of Florence and Careggi Hospital, V.le Pieracci,

Florence, Italy

For many older people the Emergency Department (ED) is the main portal of entry to urgent care. In the ED $12-43 \%$ of the patients are elderly. They visit the Emergency Department more frequently, with more urgent diseases, need more diagnostics and stay longer. Furthermore, they have a greater chance of being hospitalised and being misdiagnosed. The acute medical problems of older people are often similar to those of younger adults but the presentation can be atypical or there can be a number of co-existing problems that make diagnosis difficult. Further difficulties occur in frailer, older adults who continue to manage at home despite the effects of increasing age and multiple medical problems. In these patients an apparently minor illness can lead to deterioration in a non-specific manner leading to immobility, a fall, or acute confusion and they need a more specific triage and a screening program for older ED patients. The social circumstances and the availability of social support may be of greater importance than the management of the medical illness. In the elderly, many common conditions can exist without their characteristic features. Instead, the elderly may have more than one nonspecific geriatric syndromes (eg, delirium, dizziness, syncope, falling, weight loss, incontinence, abdominal pain). These syndromes result from multiple disorders and impairments; nonetheless, patients may improve when only some of the precipitating factors are corrected. An even better strategy is to identify risk factors for these syndromes and correct as many as possible.

\section{P-580}

Profile of the Emergency Department high frecquent user elderly patient

\section{T Mendez ${ }^{1}$}

${ }^{1}$ Geriatric Department, Hospital Universitario Nuestra Señora de La Candelaria, Tenerife, Spain

Introduction: High frequent users ( $\geq 4$ visits/year) of Emergency Departments (ED), are an important health problem. Defining the characteristics of these patients can facilitate the detection of risk factors and the development of specific interventions that decrease the frequency of visits.

Method: Population-based retrospective study. In 2017, 7599 visits at the ED of the hospital were patients $\geq 85$ years old, $31.64 \%$ were performed by 424 high-frequency patients. Demographic, functional, social and clinical data and characteristics of the ED visits have been collected. The data come from the computerized record and ED Medical History.

Results: The average age was 89.22 years, $64.15 \%$ were women. More than half come from the metropolitan area. Social, mental and functional state were registered only 50\%: most lived with family, had partial dependence on basic activities of daily living and cognitive impairment. The Charlson Index was 2.9. The average visits was 6 and the frequency was slightly higher in winter. Half of the patients attended on their own initiative. Medical pathology was the most frequent cause of visit. $2 / 3$ didn't require admission.

Conclusions: Age, being a woman, functional dependence, cognitive impairment, multimorbidity and living in the metropolitan area are frequent characteristics in the High-frequent user. Medical pathology, which doesn't require admission, and without having consulted in primary care, can be an intervention target. The heterogeneity of the data collection in ED, limit the results of this study. Collaboration with primary care physicians would help to better define the profile of these patients.

\section{P-581}

\section{Sarcopenia and related factors in hospitalized patients}

Ozlem Yilmaz ${ }^{1}$

${ }^{1}$ Geriatrics Division, Internal Medicine Department, Istanbul Medical Faculty, Istanbul University, Istanbul, Turkey

Introduction: Patients admitted to the hospital due to an acute illness may develop sarcopenia due to the acute illness itself or previous chronic diseases, decreased physical activity and nutrition and appetite problems. We aimed to investigate sarcopenia and associated factors in patients admitted to our inpatient clinic.

Materials and methods: Between April 2017-December 2017, prospectively with patients admitted to the study. Patients were evaluated with bioimpedance analysis (BIA), normal gait speed (NGS) and hand grip strength (HGS) within the first $48 \mathrm{~h}$ after admission and BIA, NGS and HGS measurements were repeated during discharging. Age, height, weight and gender data were also recorded.

Findings: A total of 143 patients admitted to the study were included. The mean age of the patients was $62.3 \pm 17.6$. The prevalence of sarcopenia was $24 \%$. There was a significant difference the HGS $(21.8 \pm 11.4$ at the time of admission and $23.9 \pm 10.4$ at the discharge) between admission and discharge. The difference between the mean values of the BIA muscle analysis $(47 \pm 9.2$ on admission and $44.8 \pm 7.4$ on exit) between admission and discharge was found to be statistically significant $(\mathrm{p}=0.02)$. There was a significant positive correlation between hand grip strength and BIA muscle analysis at admission and discharge. This suggests that patients recovering from acute illness may have improved muscle function, although there is no increase in muscle mass (Table 1).

Conclusion: In the study, it was determined that muscle function measured by HGS and NGS was better at the discharge. This suggests that recovery of acute disease can improve muscle function without changing muscle mass.

\section{P-582}

A pilot of a pharmacy acute care of the elderly team

Tonya Sammon ${ }^{1}$, Joanna Batcup ${ }^{1}$, Jane Vella-Brincat ${ }^{1}$

${ }^{1}$ Christchurch Hospital, Canterbury District Health Board, Christchurch, New Zealand

1 Pharmacy Department, Christchurch Hospital, Canterbury District Health Board, Christchurch, New Zealand.

Introduction: The addition of pharmacy services to a ward based Health Care of the Elderly (HCOE) team improves quality of care [1, 2]. A pilot ward based HCOE pharmacy service was set up to evaluate patient care benefits. 
Methods: A pharmacy team was attached to orthopaedic HCOE teams over 8 weeks in 2017. Activities included HCOE ward rounds, medicines histories/reconciliations and reviews. Interventions were analysed by activity then graded by significance to patient care using a pharmacy intervention tool. To examine improvements pre/post pilot data were collected from Decision Support. A survey of clinicians exposed to the pilot service was undertaken.

Results: A total of 148 patients were studied. $87 \%$ of patients had admission reconciliations and 59\%/28\% had discharge/transfer reconciliations. 1178 interventions were performed, $72 \%$ of these resulted in change. Addition of a medicine were $19 \%$ of these and reconciliation/history $25 \%$. Grading was: avoidance of catastrophic harm $0.5 \%$, of major harm $14 \%$, of moderate harm $23 \%$ and of minor harm 5\%. Pre/post pilot data showed a significant increase in the number of patients seen by a pharmacist from 50 to $76 \%$ (p < 0.0001) and the use of kiwicrush (laxative) from $1 \%$ of patients to $27 \%$ $(\mathrm{p}<0.0001)$. When surveyed $9 / 12$ clinicians thought the service met their expectations and saved them time.

Conclusions: The embedding of a pharmacy service into a geriatric team increased the number of patients exposed to pharmacy expertise, avoided harms from catastrophic to minor, increased the use of laxatives, met clinician expectations and saved other clinicians' time.

References:

1. Lee J, Slack M, Martin J et al (2013) Geriatric Patient Care by U.S Pharmacists in Healthcare Teams: systematic review and meta-analyses. J Am Geriatr Soc 61:1119-1127

2. Reilly T, Barile D, Reuben S (2012) Role of the Pharmacist on a General Medicine Acute Care for the Elderly Unit. Am J Geriatr Pharmacother 10(2):95-100

\section{Area: Cognition and dementia}

\section{P-583}

Borrelia burgdorferi infection as a risk factor for Alzheimer's dementia and mild cognitive impairment

A. Herrera-Landero ${ }^{1}$, F. Solórzano-Santos ${ }^{2}$, C. d'Hyver de las Deses ${ }^{3}$, L. E. Amaya-Sánchez ${ }^{4}$, M. G. Gordillo-Pérez ${ }^{5}$

${ }^{1}$ Departamento de Geriatría, Hospital de Traumatología y Ortopedia, "Lomas Verdes", Instituto Mexicano del Seguro Social, México,

${ }^{2}$ Hospital Infantil de México “Federico Gómez', Secretaria de Salud, México, ${ }^{3}$ Facultad de Medicina, Universidad Nacional Autónoma de México, México, ${ }^{4}$ Departamento de Neurología, Hospital de Especialidades, Centro Médico Nacional Siglo XXI, Instituto Mexicano del Seguro Social, México, ${ }^{5}$ Unidad de Investigación Médica de Enfermedades Infecciosas y Parasitarias, Hospital de Pediatría, Centro Médico Nacional Siglo XXI, Instituto Mexicano del Seguro Social, México

Introduction: An infectious participation in the etiology of Alzheimer's disease has been proposed. In cases of Borrelia burgdorferi infection, an OR 4.4 (CI 95\% 2.3-8.5) has been determined.

Objective: To determine the association of B. burgdorferi infection with Alzheimer's disease or mild cognitive impairment (MCI) in older adults.

Methods: Cases and controls study. Patients older than 60 years, both sexes were included. Three groups were created: with probable Alzheimer's disease (NINCDS-ADRDA criteria), MCI (Montreal Cognitive Assessment (MoCA) 19-25/30) and cognitively healthy controls (MoCA > 26/30). Western blot IgG against B. burgdorferi in serum was done in all the participants. Non-conditional logistic regression was applied to estimate the association of Alzheimer's disease or MCI and seropositive to B. burgdorferi.
Results: 38 patients with Alzheimer's disease, mean age of $75.6 \pm 3.4$ years, $69 \%$ were females, education $8.3 \pm 4.8$ years; 39 patients with MCI mean age of $72.2 \pm 6.8$ years, $85 \%$ were females, education $11.2 \pm 4.2$ years. We founded positive to B. burgdorferi 11/38 (29\%) with Alzheimer's disease, 9/39 (23\%) with MCI and $11 / 108$ (10\%) of controls. In patients with Alzheimer's disease, an OR 3.65 (CI 95\% 1.2-11.1) adjusted for education and a history of cerebrovascular disease (CVD) was estimated, and in patients with MCI an OR of 3.2 (CI 95\% 1.1-9.1) adjusted for a history of diabetes mellitus and CVD was estimated. There were tick bite in 2 cases.

Conclusions: There is an increased risk of Alzheimer's disease and $\mathrm{MCI}$ in seropositive IgG patients to B. burgdorferi.

\section{P-584}

How common are neurocognitive disorders in patients with diabetes and chronic kidney disease: results from a community cohort of patients in North Wales

Peter Hobson ${ }^{1}$, Mick Kumwenda ${ }^{1}$

${ }^{1}$ Academic Unit, Glan Clwyd Hospital, Betsi Cawaladr University Health Board North Wales, United Kingdom

Introduction: Chronic kidney disease (CKD) affects nearly $9 \%$ of global populations and is strongly associated with older age. Neurocognitive disorders (NCD) which include mild cognitive impairment (MCI) and dementia are rising as population's age throughout the world. This study aim is to report the crude prevalence of mild to major NCD in a clinical cohort of older adults with mild to moderate CKD (stages 3-4) and diabetes.

Methods: Cognitive function was assessed in 178 patients over aged 55 years with an estimated glomerular filtration rate $($ eGFR $)<60$ $>15 \mathrm{ml} / \mathrm{min} / 1.73 \mathrm{~m}^{2}$, attending a renal and diabetes outpatient clinic. The diagnosis of NCD was based upon patient and informant interview, case note review, neuropsychological assessment and application of Diagnostic and Statistical Manual of Mental disorders version 5 (DSM-V).

Results: Upon neuropsychological assessment and application of DSM-V criteria, 86/178 (48\%) of the cohort had a NCD ranging from mild $(\mathrm{n}=49)$ to major symptoms $(\mathrm{n}=37)$. Neuropsychological predictors for mild or major NCD entered into a stepwise regression analysis found that Fluency and Memory impairment accounted for over $66 \%$ of the variance, $\mathrm{p}<0.0001$.

Conclusions: To our knowledge this is the first study in the UK to report that cognitive changes occur in a significant number of older adults with CKD and concomitant diabetes. The unexpected finding was that prior to cognitive assessment, none of the cohort had a preexisting diagnosis of cognitive impairment, suggesting that the current prevalence and incidence rates of NCD in the general population are significantly underestimated.

\section{P-585}

Vital signs and impaired cognition in older Emergency Department patients: the APOP study

Jacinta Lucke ${ }^{1}$, Jelle de Gelder ${ }^{1}$, Laura Blomaard ${ }^{1}$, Christian Heringhaus ${ }^{1}$, Jelmer Alsma ${ }^{2}$, Stephanie Schuit ${ }^{2}$, Anniek Brink ${ }^{2}$, Sander Anten ${ }^{3}$, Gerard J Blauw ${ }^{1}$, Bas de Groot ${ }^{1}$, Simon P. Mooijaart ${ }^{1}$

${ }^{1}$ Leiden University Medical Center, Leiden, The Netherlands,

${ }^{2}$ Erasmus University Medical Center, Rotterdam, The Netherlands,

${ }^{3}$ Alrijne Hospital, Leiden, The Netherlands 
Background: Cognitive impairment is a frequent problem among older patients attending the Emergency Department (ED) which can be the result of pre-existing cognitive impairment, delirium, or neurologic disorders. Another cause can also be acute disturbance of brain perfusion and oxygenation, which may be reversible by optimal resuscitation. We therefore assessed the relationship between vital signs, as a measure of acute hemodynamic changes and cognitive impairment in older ED patients.

Methods: A prospective cohort study, performed in two tertiary care and two secondary care hospitals in The Netherlands. All consecutive ED patients aged 70-years and older were included. Vital signs were measured at the moment of ED arrival as part of routine clinical care. Cognition was measured using the Six-Item Cognitive Impairment Test (6-CIT).

Results: We included 2629 patients with a median age of 78 years (IQR 74-84). Cognitive impairment was present in 738 patients $(28.1 \%)$. When comparing lowest with highest quartiles, systolic BP (OR 1.37, 95\% CI 1.05-1.77) and diastolic BP (OR 1.32, 95\% CI $1.02-1.71)$ were associated with cognitive impairment. As were respiratory rate (OR 2.07 (95\% CI 1.55-2.77) and oxygen saturation (OR $1.63,95 \%$ CI $1.25-2.13)$.

Conclusion: Abnormal vital signs that associate with decreased brain perfusion and oxygenation also associate with cognitive impairment in older ED patients. Although this may partially reflect the association of disease severity with delirium, impaired cognition may also be caused by acute disturbance of brain perfusion and oxygenation. More research is needed to establish whether intervening and improving these vital signs will also acutely improve cognition.

\section{P-586}

Prevalence of cognitive impairment in diabetics and associated factors

\section{Z. $\operatorname{Lim}^{1}$, E. Khoo ${ }^{2}$, E. Koo ${ }^{3}$, R. A. Merchant ${ }^{1}$}

${ }^{1}$ Department of Geriatric Medicine, National University Hospital, Singapore, Singapore, ${ }^{2}$ Department of Endocrinology, National University Hospital, Singapore, Singapore, ${ }^{3}$ Yong Loo Lin School of Medicine, Singapore, Singapore

Introduction: Type 2 diabetes (T2DM) is an established risk factor for cognitive impairment (CI). With increasing prevalence of T2DM, identifying patients with $\mathrm{CI}$ is important as it impacts disease management and quality of life. This study seeks to establish that T2DM is associated with early-onset $\mathrm{CI}$ and its associated risk factors.

Methods: A cross-sectional study was conducted among patients ( $\geq 40$ years) who attended General Medicine and Endocrine outpatient clinics over 10 months. Cognition was assessed using the Montreal Cognitive Assessment Scale, with cut-off score of 26 to determine CI. Data for diabetes control, complications and management, and cardiovascular co-morbidities was analyzed using chisquare and two-sample T-test.

Results: A total of 210 diabetic and 97 non-diabetic subjects aged 40-88 years were enrolled. Prevalence of CI in diabetic vs non-diabetic patients was 41.0 vs $25.8 \%(\mathrm{P}=0.011)$ respectively, and $35.5 \mathrm{vs}$ $16.9 \%(\mathrm{P}=0.013)$ in patients aged $<60$ years. In patients $>60$ years, the difference in prevalence of $\mathrm{CI}$ between the two groups was not significant $(47.0 \%$ vs $39.5 \%, \mathrm{P}=0.450)$. In diabetics, CI was significantly associated with older age (61.8 vs 56.9 years), lower education levels (9.4 vs 12.1 years), longer T2DM duration (15.7 vs 12.6 years), higher glycated hemoglobin levels (8.4\% vs $7.9 \%$ ), insulin usage ( $49.5 \%$ vs $33.6 \%$ ), presence of nephropathy $(\mathrm{CrCl} 73.0$ vs $97.8 \mathrm{~mL} / \mathrm{min}$ ), retinopathy (52.1 vs $34.7 \%$ ) and hypertension $(44.8$ vs $27.7 \%$ ).
Conclusion: Patients with T2DM have higher prevalence of CI at a younger age compared to non-diabetics. Active case finding in this group may enable better compliance and disease control.

\section{P-587}

Prevalence of anticholinergic drug use in older adults with dementia in a large Tertiary Hospital in Singapore

Kaysar Mamun ${ }^{1}$

${ }^{1}$ Singapore General Hospital, Singapore, Singapore

Introduction: The use of anticholinergic drugs is controversial in patients diagnosed with dementia due to increased risk of cognitive impairment and psychosis in this population. Anticholinergic drugs are often involved in explicit criteria for inappropriate prescribing in older adults. However, the extent of anticholinergic drug use in Singapore General Hospital's patient population is unknown. This study aims to determine the prevalence of anticholinergic drug use in older patients with dementia and evaluate the association between its use with mortality and morbidity outcomes in these patients.

Methods: This is a retrospective cross-sectional analysis of patients aged 65 or older with dementia and at least one hospital admission in $2013(\mathrm{n}=460)$. Identified subjects were followed up prospectively for 1 year after first admission in 2013 for morbidity and mortality events. Data on exposure to anticholinergic drugs 3 months prior to admission were collected. Anticholinergic burden was determined using the Anticholinergic Risk Scale (ARS).

Results: Most patients aged 75 years old and above (77.8\%), with mean age, $80.8 \pm 8.4$ years. Majority were female $(60.4 \%)$ and Chinese $(84.1 \%)$. Overall proportion of patients prescribed with anticholinergic drug use based on ARS scale was $55.9 \%(n=257)$. ARS level 1 drugs were most commonly prescribed $(n=86)$, followed by ARS level 3 drugs $(n=63)$ and ARS level 2 drugs $(n=41)$. The top three ARS level 1 drugs prescribed were mirtazapine $(\mathrm{n}=36)$, quetiapine $(\mathrm{n}=17)$ and risperidone $(\mathrm{n}=12)$; ARS level 3 drugs were hydroxyzine $(\mathrm{n}=20)$, chlorpheniramine $(\mathrm{n}=11)$ and diphenhydramine $(\mathrm{n}=9)$; followed by ARS level 2 drugs, loratadine $(n=21)$, tolterodine $(n=7)$ and prochlorpherazine $(n=4)$.Increased use of ARS level 3 drugs was associated with more hospitalizations, increased in length of stay and emergency visits. Use of drugs with significant anticholinergic activity (ARS level 2 or 3 ) was found to have a significant association with morbidity outcomes but not mortality.

Conclusions: Anticholinergic drug use in patients with dementia is highly prevalent, especially in poly-medicated older adults. This may have contributed to increased morbidity for these patients. Efforts to increase awareness among health professionals regarding potential risks of anticholinergic drug usage may improve medication prescribing practice.

\section{P-588}

Cardiovascular outcomes of cholinesterase inhibitors in patients with dementia: A meta-analysis and systematic review

Ahmet Turan Isik ${ }^{1}$, Pinar Soysal ${ }^{2}$, Brendon Stubbs ${ }^{3}$, Marco Solmi ${ }^{4}$, Cristina Basso ${ }^{5}$, Stefania Maggi ${ }^{6}$, Patricia Schofield ${ }^{7}$, Nicola Veronese ${ }^{6}$, Christoph Mueller ${ }^{3}$

${ }^{1}$ Unit for Aging Brain and Dementia, Department of Geriatric Medicine, Faculty of Medicine, Dokuz Eylul University, Izmir, Turkey, ${ }^{2}$ Kayseri Education and Research Hospital, Geriatric Center, Kayseri, Turkey, ${ }^{3}$ Institute of Psychiatry, Psychology and 
Neuroscience, King's College London, London, UK, ${ }^{4}$ Department of Neurosciences, University of Padova, Padova, Italy, ${ }^{5}$ Azienda ULSS (UnitÁ Locale Socio Sanitaria) 3, "Serenissima", Dolo-Mirano District, Italy, ${ }^{6}$ National Research Council, Neuroscience Institute, Aging Branch, Padova, Italy, ${ }^{7}$ Health, Social Care and Education, Anglia Ruskin University, Chelmsford, United Kingdom

Introduction: To evaluate the cardiovascular effects of acetylcholinesterase inhibitors (AChEIs) in patients with dementia.

Methods: Two authors independently searched major electronic databases from inception until 06/17/2017 for longitudinal (without a control group) and cohort (with a control group) studies reporting cardiovascular $(\mathrm{CV})$ outcomes in relation to AChEIs. Randomized controlled trials were excluded due to the inclusion of relatively healthy subjects. Changes in CV parameters were summarized through standardized mean differences (SMD) with 95\% confidence intervals (CI). Event rate was used to assess the incidence of hypertension and bradycardia. The incidence of $\mathrm{CV}$ events vs. healthy controls were compared using hazard ratios (HRs).

Results: Out of 4,588 initial hits, 31 studies including 258,540 patients with dementia and 2,246,592 controls were analyzed. Across longitudinal and open-label studies, AChEIs were associated with a significant higher incidence of hypertension (1573 patients; 4\%; 95\% CI $\left.2-8 \%, \mathrm{I}^{2}=47 \%\right)$ and bradycardia (13,703 participants; $2 \% ; 95 \%$ CI $\left.1-6 \%, \mathrm{I}^{2}=98 \%\right)$. AChEIs were associated with a decrease in heart rate $\left(\mathrm{SMD}=-1.77 ; 95 \% \mathrm{CI}-3.58\right.$ to $\left.0.03, \mathrm{I}^{2}=78 \%\right)$ and a prolongation of the PR interval (SMD $=0.10 ; 95 \%$ CI $0.008-0.19$; $\mathrm{I}^{2}=3 \%$ ) compared to baseline. During a median follow-up time of 116 weeks, AChEIs were associated with a significantly lower risk of $\mathrm{CV}$ events (i.e. stroke, acute coronary syndrome and CV mortality) $\left(\mathrm{HR}=0.63 ; 95 \%\right.$ CI $\left.0.45-0.88 ; \mathrm{I}^{2}=18 \%\right)$, without a significant increased risk of bradycardic events (HR 1.40; 95\% CI 0.76-2.59; $I^{2}=98 \%$ ).

Key conclusions: AChEIs therapy may be associated with negative chronotropic and hypertensive effects, whilst their use may be associated with a reduced risk for $\mathrm{CV}$ events.

\section{P-589}

Falls among persons with Alzheimer disease: description, risk factors, and exercise interacting them

Niko Perttila ${ }^{1}$, Hanna Öhman ${ }^{1}$, Timo Strandberg ${ }^{1}$,

Hannu Kautiainen ${ }^{1}$, Minna Raivio ${ }^{1}$, Marja-Liisa Laakkonen ${ }^{1}$, Nina Savikko ${ }^{1}$, Reijo Tilvis ${ }^{1}$, Kaisu Pitkala ${ }^{1}$

${ }^{1}$ University of Helsinki, Department of General Practice and Unit of Primary Health Care, Helsinki University Hospital, Helsinki, Finland

Introduction: There is scarce of studies investigating fall-risk factors and especially their interaction with exercise among persons with dementia. The aim is to explore these features.

Methods: FINALEX (randomized-controlled-trial) included 194 Alzheimer disease (AD) persons, of which two-thirds participated in exercise intervention $1 \mathrm{~h}$ twice weekly for 12 months. We investigated fall-risk factors (diseases, fall-related-drugs, physical functioning) and possible interaction between exercise and fall-related-drugs.

Results: Of 355 falls (altogether) 123 led to injuries, 50 to Emergency Department visits, and 13 to fractures. Stumbling $(n=61)$ was the most common reason for falling. The persons with $1(n=34)$ or $\geq 2$ falls $(\mathrm{n}=57)$ were older and had more severe dementia than those without falls $(\mathrm{n}=103)$. Good nutritional status and physical functioning protected against falls whereas fall history, osteoarthritis, and diabetes increased fall-risk. We compared the fall-risk associated with fall-related-drugs between intervention and control groups: There was no difference in the number of falls among those without antihypertensives or psychotropics between intervention and control groups. In intervention group with antihypertensives, the IRR was 0.5 falls/ person-year (95\% CI 0.4-0.6); among controls the IRR was 1.5 falls/ person-year (95\% CI 1.2-1.8) ( $\mathrm{p}<0.001$ for group, $\mathrm{p}=0.067$ for medication, $\mathrm{p}<0.001$ for interaction). Among participants using psychotropics the intervention group had an IRR of 0.7 falls/personyear (95\% CI 0.6-0.9); the IRR for the control group was 2.0 falls/ person-year (95\% CI 1.6-2.5) $(\mathrm{p}<0.001$ for group, $\mathrm{p}=0.071$ for medication, $\mathrm{p}<0.001$ for interaction).

Key conclusions: We explored that exercise has a potential to decrease the risk for falls among people with $\mathrm{AD}$ using antihypertensives and psychotropics.

\section{P-590}

\section{EMDR and dementia}

Vaillant Ciszewicz Anne-Julie ${ }^{1}$, Quaderi André ${ }^{2}$, Robert Philippe ${ }^{3}$, Guerin Olivier $^{4}$

${ }^{1} \mathrm{CHU}$ EHPAD CIMIEZ, ${ }^{2}$ NICE université, ${ }^{3} \mathrm{CMRR}$ NICE-COBTEK LAB-NICE, ${ }^{4} \mathrm{CHU}$ NICE

More than 24 randomized controlled trials are evaluating the effect of EMDR therapy in traumatized patients. Alternate Bilateral Stimulations (ABS) induced by EMDR allow desensitization of traumatic information and reprocessing of associated memory. Currently, the EMDR does not have enough experimental data concerning the elderly person. Our research aims to establish a link between trauma symptomatology and behavioral symptomatology in neurocognitive pathologies. Verbal agitation and anxiety are found in both neurocognitive pathology [1] and also in PTSD. Amano et al. [2] are the pioneers of research in the field of EMDR and neurocognitive pathologies. According to the authors, behavioral disorders are directly related to past events that replay themselves here unless the psychic scene of the subject. Although the results are encouraging the study has only 3 subjects. We wish to demonstrate that it is possible to reduce verbal agitation by adapting the EMDR protocol.

Method: 8 subjects in nusery home with verbal agitation (DSMV criteria for major cognitive impairment). At first we will assess primary needs (first source of verbal agitation). We have chosen 2 groups which are randomized (EMDR and Control). We will analyze intensity of verbalizatoons using a sound level meter (fréquence duration and intensity) on two repeated sessions. E use some evaluations like NPIES, CMAI, ALGOPLUS, PITTSBURGH, MMSE and statistical analysis of the results.

Results: Current research indicates a reduction in verbal agitation for EMDR patients.

Key conclusion: According to our first results, we can say that performing ABS in eldery people with cognitive disorders does not induce a healing phenomenon as in PTSD but a soothing of the limbic system and therefore of verbal agitation.

\section{P-591}

Relationship of neuropsychiatric symptoms with falls in Alzheimer's disease: does exercise modify the risk? Secondary analysis of a randomized controlled trial

H. M. Roitto ${ }^{1}$, H. Kautiainen ${ }^{1}$, H. Öhman ${ }^{1}$, N. Savikko ${ }^{1}$, T. E. Strandberg ${ }^{1}$, M. Raivio ${ }^{1}$, M. L. Laakkonen ${ }^{1}$, K. H. Pitkälä ${ }^{1}$

${ }^{1}$ University of Helsinki, Helsinki, Finland 
Introduction: Alzheimer disease (AD) is associated with both neuropsychiatric symptoms (NPS) and falls. The aim of this study was to explore how NPS are associated with the number of falls and how exercise modifies the risk of falling among community-dwelling people with AD and NPS.

Methods: This study is a secondary analysis of a 12 month randomized controlled trial (FINALEX) among people with AD. The original trial examined home-based and group-based exercise groups and compared them to the control group receiving normal community care. Both exercise interventions were supervised by physiotherapists and comprised $1 \mathrm{~h}$ sessions twice a week for 12 months. In this secondary analysis we merged the home-based and group-based exercise groups and compared them to the control group. NPS were measured with the Neuropsychiatric Inventory (NPI) at baseline and falls were recorded in daily fall diaries by spousal caregivers during a 1-year follow-up.

Results: The number of falls increased linearly with NPI points. Fall rate was 1.48 (95\% CI 1.26-1.73) per person/year in the intervention group whereas it was 2.87 (95\% CI 2.43-3.35) in the control group. Adjusted for age, gender, MMSE, and SPPB, IRR was $0.48(95 \% \mathrm{Cl}$ $0.39-0.60)(\mathrm{p}<0.001)$. Main effects for fall rate were significant for group $(\mathrm{p}<0.001)$ and NPI total $(\mathrm{p}<0.024)$; interaction effect was also significant $(\mathrm{p}=0.009)$ (adjusted for gender, age, MMSE and SPPB, psychotropic medication use).

Conclusions: Regular, long term exercise decreases significantly the risk of falling in community dwelling AD patients with NPS.

\section{P-592}

Influence of the degree of dementia on the final parameters of static equilibrium in the female population over 80 years of age.

\section{Irimia Mollinedo-Cardalda ${ }^{1}$, Adriana López ${ }^{1}$, José María Cancela ${ }^{1}$}

${ }^{1}$ Universidade de Vigo, Grupo de Investigación HealthyFit. Instituto de Investigación Sanitaria Galicia Sur (IISGS), Complejo

Hospitalario Universitario de Pontevedra (CHOP), SERGAS,

Pontevedra, Spain

Introduction: Dementia is a chronic and progressive syndrome that presents with deterioration of cognitive function. This syndrome affects the region of the brain that controls movements, so that balance, the most important capacities for self-valence, could be affected it. The aim of this study is to show how the degree of dementia influences the static balance in women over 80 years of age.

Methods: A total of 54 institutionalized women of average age $86.47 \pm 6.12(16.7 \%$ without dementia (WD), $31.5 \%$ mild dementia (MLD), 25.9\% moderate dementia (MD), 25.9\% severe dementia (SD) The degree of dementia was assessed by means of the MiniMental test and the static balance with the pressure platform EPS-R1 of the LORAN-Engineering Company (Italy).

Results: Significant differences were found according to the degree of dementia (Mini-mental test: WD $27.56 \pm 1.94$, MLD $21.35 \pm 2.21$, MD $14.21 \pm 1.93$, SD $6.07 \pm 3.75)$ in the variables of speed of movement $(\mathrm{m} / \mathrm{s})$ and oscillation of the pressure centre. The speed of movement increased in the moderate stage, compared with the advanced stage, mild and with people without dementia (WD $4.72 \pm 2.09$, MLD $7.02 \pm 4.37$, MD $8.59 \pm 4.67$, SD $5.28 \pm 1.68$ $\mathrm{p}<0.05$ ), and the oscillation of the higher pressure center when the disease is more advanced (WD 23.61 \pm 10.49 , MLD $35.08 \pm 21.94$, MD $42.95 \pm 23.42$, SD $26.41 \pm 8.42, \mathrm{p}<0.05)$.

Conclusion: The degree of dementia significantly influences static balance parameters, inducing changes in the centre of body pressures, in terms of speed and range of motion, thus causing alterations in balance, and increasing the risk of falls the demented population.

\section{P-593}

Likelihood of institutionalization for demented patients: a lineal or a collateral story? (Study from the French National Alzheimer database)

Huvent-Grelle Dominique ${ }^{1}$, Ficheur Gregoire ${ }^{2}$, Beuscart Jean Baptiste $^{2}$, Boulanger Eric ${ }^{2}$, Demarle Sylvie ${ }^{3}$, Puisieux François ${ }^{2}$

${ }^{1}$ pôle de GERONTOLOGIE, CHU LILLE, Lille, France, ${ }^{2} \mathrm{CHU}$ LILLE, Lille, France, ${ }^{3}$ La Vie Active, ARRAS, Lille, France,

Background: The institutionalization of Alzheimer's disease and other dementias (ADOD) patient is a last resort measure decided by the family caregiver. The relationship with the caregiver could modify the likelihood of institutionalization.

Objective: To assess the association between institutionalization and the degree of kinship with their family caregiver among ADOD patients.

Methods: A cross-sectional study analysing the first visit in a Memory Center in France for each ADOD patient, over 75, between 2011 and 2014 (French National Alzheimer Database). A multivariate logistic regression was conducted to assess the association of interest adjusted by age, gender, MMSE, educational level, and type of dementia.

Results: 52874 old patients were included. The primary caregiver is mostly a child $(54.8 \%)$ or the spouse $(36.7 \%)$. Compared with the category of reference "spouse", all other categories are significantly at risk of institutionalization. The ORs (95\% CI) are 5.48 [4.93-6.09], and 4.68 [3.67-5.92] for respectively children and grandchildren and $8.76[7.15-10.70]$ and 8.93 [7.48-10.65] for respectively sibling, and niece-nephew .

Conclusion: Elderly institutionalization risk factor is modified according to the degree of kinship. Compared to the reference "spouse", this risk is increased for all type of caregivers, especially if the caregiver is not in direct line of descendants with the patient (eg: sibling, niece-nephew).

\section{P-594}

Cognitive screening instruments in vascular cognitive impairment: a systematic review

\section{Z. Ghafar ${ }^{1}$, H. N. Miptah ${ }^{1}$, S. Gorey ${ }^{1}$, R. O’Caoimh ${ }^{1}$}

${ }^{1}$ Galway University Hospital, Ireland

Background: The prevalence of cognitive impairment will continue to increase as society ages. Vascular dementia is the second most common type of dementia after Alzheimer's disease (AD) and is closely associated with stroke and cardiovascular disease. Detection of vascular cognitive impairment (VCI) is essential as controlling the risk factors may prevent progression. There is no consensus as to which cognitive screening instrument (CSI) is most suitable for VCI. Objectives: To systematically review the psychometric properties of brief CSIs across the spectrum of VCI from vascular mild cognitive impairment (VMCI) and vascular dementia $(\mathrm{VaD})$.

Method: A literature search for all available papers published from inception until 31st May 2018 was conducted using the scholarly databases. Bibliographies of all included studies were also screened for any related studies. The titles and abstract were screened and the studies that met the inclusion and exclusion criteria were thoroughly reviewed. Relevant data were extracted and summarised to produce clinically relevant inferences. Bias was assessed using the Quality in Prognosis Studies (QUIPS) tool.

Results: Fifteen papers were included in the final review. The data was reviewed in a narrative fashion as it was not possible to do a 
meta-analysis with the data obtained. CSIs were grouped according to their properties of differentiating between (1) VaD and normal cognition, (2) MCI and normal cognition, and (3) VCI and AD.

Conclusion: Not all studies reported the reliability and consistency measures for the CSIs. Of the ones that were reported, the Montreal Cognitive Assessment (MoCA) was most sensitive and accurate, both for differentiating $\mathrm{VaD}$ and VMCI from normal cognition.

\section{P-595}

A classic presentation of an uncommon disease: Binswanger disease

Rikitha Menezes ${ }^{1}$, Theresa Redling ${ }^{1}$

${ }^{1}$ Saint Barnabas Medical Center, Livingston, USA

Introduction: Binswanger disease is a subtype of vascular dementia $[1,4]$ that was first described in 1894 [2,3]. It affects men and women equally in the sixth to seventh decade of life with a $3-12 \%$ increasing incidence in elderly [1].

Case presentation: An 89 year-old woman with history of hypertension, hyperlipidemia, legal blindness, gait disturbances, urinary incontinence, presents with 1 year of short term memory impairment, suicidal ideations. On neuropsychiatric evaluation the patient demonstrated deficiencies in the following: executive: information processing speed, attention, working memory, learning, retention, orientation, semantic fluency, executive systems; MMSE 9/25; sensory: impaired visual acuity, bradykinesia, mild motor programming deficits, gait ataxia; psychiatric: generalized anxiety, depression.

Discussion: Binswanger Disease is a subtype of vascular dementia which is an underrecognized gradually progressive demyelinating disease resulting in subcortical arteriosclerotic encephalopathy. It overlaps with other neuropathologies thus history and imaging is essential $[1,3,6]$. The disease is associated with hypertension $(98 \%$ of cases) and lacunar infarctions [1]. CT brain demonstrates periventricular lucencies (hypodense); MRI brain shows hyperintense white matter abnormalities from fronto-temporal region to the centrum semiovale [1,3]. Treatment is primarily supportive: discussions on advanced directives such as goals of care, social support and antidepressant therapy, etc. Management of hypertension and aspirin prophylaxis may help slow progression.

Conclusion: Binswanger Disease is an underrecognized disease resulting in subcortical arteriosclerotic encephalopathy. It is important to recognize this entity as disease management will be different from other forms of neurodegenerative dementias.

References:

1. Olsen C, Clasen M (1998) Senile dementia of the Binswanger's Type. Am Fam Physician J 58(8):2068-2074

2. Binswanger O (1894) Die Abgrenzung der allgemeinen progressive Paralyse. Berl Klin Wochenschr. 31:1102-1105, 1137-1139, $1180-1186$

3. Pantoni L, Garcia JH (1995) The Significance of cerebral white matter abnormalities 100 years after binswangers report: a review. Stroke 26(7): 1293-1301. https://doi.org/10.1161/01.str.26.7.1293.

4. Binswanger's Disease Information Page. (2017, May 23). Retrieved April 18, 2018, from https://www.ninds.nih.gov/Disorders/ All-Disorders/Binswangers-Disease-Information-Page

5. Bennett D, Wilson R, Gilley D, Fox J (1990) Clinical diagnosis of Binswanger's disease. J Neurol Neurosurg Psychiatry 53:956-61.

6. Grossman RI, Yousem DM (2010) Neuroradiology: the requisites. Mosby, Philadelphia, p 238

\section{P-596}

Deprescribing in advanced dementia: where do we stand?

Renato Oliveira ${ }^{1}$, Tomás Teodoro ${ }^{1}$, Miguel Marques Ferreira ${ }^{1}$

\section{${ }^{1}$ NMS-FCM}

Keyword: Prevention in vulnerable population.

Introduction: Data suggest that older adults with dementia are commonly prescribed potentially unsafe medications. Deprescribing refers to supervised tapering or cessation of drugs, aiming to minimize inappropriate polypharmacy and improve patient outcomes. This study reviews the literature about deprescribing in advanced dementia, its benefits, safety and barriers.

Material and methods: Non-systematic review of literature using PubMed database using the following keywords: "advanced dementia", "deprescribing" and "discontinuing medication".

Results: Most research on potentially inappropriate prescribing is focused on the elderly in general rather than dementia specifically. However, studies are increasingly focusing on prescribing for people with advanced dementia. The PEACE program, in particular, has produced criteria that show promise in identifying potentially inappropriate medications in advanced dementia, including anticholinesterase inhibitors and lipid-lowering agents. Potential benefits of deprescribing are widespread, including health and quality of life benefits to patients and cost benefits to the health care system. Challenges to successfully discontinuing medications include patient-, clinician-, and system-related barriers. Limited life expectancy and cognitive impairment appear to be the most important factors driving deprescribing. Being an essential issue, deprescribing should be part of the medical curriculum and throughout the career of senior clinicians. Key conclusions: Evidence supporting the benefits and safety of deprescribing in the elderly continues to grow, strengthening the cause for greater integration of regular deprescribing into medical culture. More evidence about deprescribing in advanced dementia is needed. Advance care planning is the cornerstone of high-quality palliative care in advance dementia and deprescribing should be part of that process.

\section{P-597}

An Augmented Prescribed Exercise Programme (APEP) for frail older medical inpatients in the acute setting: a randomised controlled trial

Ruth McCullagh ${ }^{1}$, Eimear O'Connell ${ }^{2}$, Sarah O'Meara ${ }^{1}$, Darren Dahly ${ }^{1}$, N. Frances Horgan ${ }^{3}$, Suzanne Timmons ${ }^{1}$

${ }^{1}$ University College Cork, Cork, Ireland, ${ }^{2}$ Mercy University Hospital, Cork, Ireland, ${ }^{3}$ Royal College of Surgeons, Cork, Ireland

Aim: To measure the effects of an augmented prescribed exercise programme on physical performance, quality of life and healthcare utilisation for frail medical patients in the acute setting.

Methods: Within 2 days of admission, older medical inpatients with an anticipated length of stay $\geq 3$ days, needing assistance/aid to walk, were blindly randomly allocated to the intervention or control group. Until discharge, both groups received twice daily, Monday-toFriday half-hour assisted exercises. The intervention group completed tailored strengthening and balance exercises; the control group, stretching and relaxation exercises. Length of stay (primary outcome measure), readmissions, physical performance (Short Physical Performance Battery), and quality of life (EuroQOL-5D-5L) were measured at discharge and at 3 months. Time-to-event analysis was used for length of stay analysis, and linear regression models were used for physical performance and quality of life analysis. 
Results: Data from 190 patients (aged $80 \pm 7.5$ years) were analysed. Groups were comparable at baseline. Crude analysis showed no effect on length of stay [HR 1.09 (CI 0.77-1.56) p = 0.6], however strengthened slightly when patients transferred to rehabilitation were excluded [ $\mathrm{n}=125$, HR, 1.3 (CI 0.9-1.87) $\mathrm{p}=0.16$ ]. Physical performance was significantly better at discharge $[\beta=0.88$ (CI $0.20-1.57) \mathrm{p}=0.01]$, but lost at follow-up $[\beta=0.45$ (CI -0.43 to 1.33) $\mathrm{p}=0.3$. A small but significant improvement in quality of life was detected at follow-up in the intervention group $[\beta=0.28$ (CI 0.9 to 0.47$) \mathrm{p}=0.004$ ]

Conclusion: The physical performance and quality of life improvements suggest that this intervention is valuable to frail medical inpatients. Its effect on length of stay remains unclear.

\section{P-598}

The Montreal Cognitive Assessment is a better tool to screen for cognitive impairment in elderly patients with Diabetes Mellitus

J. J. Walgers ${ }^{1}$, S. Simsek ${ }^{2}$, F. Stam ${ }^{2}$, M. E. Popescu ${ }^{1}$, R. W. M. M. Jansen ${ }^{2}$

${ }^{1}$ Northwest Clinics, Alkmaar, The Netherlands, ${ }^{2}$ Northwest Clinics, Alkmaar, The Netherlands

Introduction: The aim of this study was to evaluate the prevalence of cognitive impairment $(\mathrm{CI})$ in elderly patients with diabetes mellitus (DM) compared with patients without DM. The Mini Mental State Examination (MMSE) may not be the appropriate test to evaluate CI in patients with DM.

Methods: We included 475 patients with a mean age of 79.6 years (standard deviation 6.6), who were evaluated at the fall and syncope day clinic (FSC) and who completed the MMSE and the Montreal Cognitive Assessment (MoCA). The prevalence of cardio-vascular diseases is high in all patients. We compared patients with DM with patients without DM. The main outcome was the prevalence of CI comparing MMSE with the MoCA. CI was defined as a MMSE score $<26$ or a MoCA score $<26$.

Results: There were 101 patients with DM and 374 patients without DM. The prevalence of CI was $21 \%$ (MMSE) versus $73 \%$ (MoCA) in the DM-group ( $\mathrm{P}=0.00)$ and 20\% (MMSE) versus 63\% (MoCA) in the Non-DM group $(\mathrm{P}=0.00)$. With the MoCA, there was a trend towards more $\mathrm{CI}$ in patients with DM compared with patients without $\mathrm{DM}(\mathrm{p}=0.06)$.

Key conclusions: CI was found in $73 \%$ in elderly diabetic patients, which is more than three times the prevalence reported in the general population aged $\geq 75$ years. CI seems to be more common in DM patients compared to non-DM patients at the FSC. These findings suggest that CI may be underdiagnosed in elderly patients with DM. We recommend using the MoCA for screening CI in these patients.

\section{P-599}

Atrial septal aneurysm as a rare cause of vascular dementia: a case report

Francesco Bolzetta $^{1}$, Marino Formilan ${ }^{1}$, Flavio Busonera ${ }^{1}$, Annalisa Tessari ${ }^{1}$, Nicola Veronese ${ }^{2}$, Alberto Cester ${ }^{1}$

${ }^{1}$ Medical Department, Geriatric Unit, Azienda ULSS3 Serenissima, Dolo-Mirano District, Padua, Italy, ${ }^{2}$ National Research Council, Neuroscience Institute, Aging Branch, Padua, Italy
An atrial septal aneurysm (ASA) is a congenital malformation characterized by a localized bulging of the atrial septum into atrium. ASA is a poor known putative risk factor for cardioembolism. Its prevalence in the general population is $2.2 \%$. We report a case of a 77 years old woman who came to our attention for progressive cognitive impairment. Symptoms were primarily loss of short-term memory and apathy, but was also reported an episode of walking disorder and one of misidentification of her husband. Medical history included only hypertension. Neurological examination was negative, while cardiac examination revealed a mesocardial heart murmur. Mini Mental State Examination score was $15.7 / 30$. Basal and instrumental activities of daily living were also inadequate. Carotids ultrasonography and electrocardiogram were negative. The patient underwent to a cerebral tomography that founded chronic cerebrovascular disease with evidence of little ischemic strokes. A subsequent echocardiogram revealed the presence of an ASA. Our diagnosis was of vascular dementia as a result of repeated ischemic stroke caused by ASA presence. The patient is waiting to perform a transesophageal echocardiogram. To best of our knowledge this is the second case reported in literature of vascular dementia as result of ASA presence. This medical condition should be kept in mind for a possible cause of vascular dementia, particularly in the presence of multi-infartual strokes with negative carotids ultrasonography and normal electrocardiogram or in case of unknown cardiac murmur.

\section{P-600}

The effect of selected factors on the quality of life of the elderly with early-stage dementia

Helena Kisvetrová ${ }^{1}$, Jana Bermellová ${ }^{1}$, Roman Herzig ${ }^{2}$, Martin Vališ ${ }^{2}$, Božena Jurašková ${ }^{2}$, Petra Krulová ${ }^{3}$, Kateřina Langová ${ }^{1}$, David Školoudík ${ }^{3}$

${ }^{1}$ Palacký University, Olomouc, Czech Republic, ${ }^{2}$ University Hospital Hradec, Králové, Czech Republic, ${ }^{3}$ University Hospital, Ostrava, Czech Republic

Introduction: The aim was to determine the factors affecting the quality of life (QoL) amongst the elderly with early-stage of dementia (EwESD), as assessed by the Quality of Life in Alzheimer's Disease (QOL-AD).

Methods: The questionnaire battery included the following questionnaires: QOL-AD, Geriatric Depression Scale, Mini-Mental State Examination, Patient Dignity Inventory, Short Physical Performance Battery, Bristol Activities of Daily Living Scale, and a set of sociodemographic data. Criteria for inclusion were: any type of dementia at an early stage; $>60$ years of age; living at home and having contact with a family caregiver at least once a week. EwESD were recruited from geriatric and neurologic outpatient departments of university hospitals in Hradec Králové and Ostrava in the Czech Republic. Pearson and Spearman correlation analysis, single-factor ANOVA, and multidimensional linear regression model were used for statistical processing.

Results: The sample consisted of 272 patient-proxy pairs (mean EwESD age $79.8 \pm 7.8$ years; mean MMSE score $22.6 \pm 1.7$ points). The results show that QoL assessed by the EwESD is significantly associated with dignity (beta $=-0.347$ ), self-sufficiency (beta $=0.265$ ), and depression (beta $=-0.266$ ). The QoL of the PwESD assessed by the caregivers correlated only with the level of self-sufficiency (beta $=0.571$ ) and depression (beta $=-0.138 ; \mathrm{P}<$ 0.001 in all cases).

Key conclusion: The results show that the elderly with early-stage dementia assess the quality of their life better when they suffer from less depression, are more self-sufficient in ADL, and have a greater 
sense of self-dignity. Study was supported by grant Nr. 16-28628A by Ministry of Health of the Czech Republic.

\section{P-601}

Promoting activity, independence and stability in early dementia and mild cognitive impairment: feasibility trial

RH Harwood ${ }^{1}, \mathrm{~V}$ Van der Wardt ${ }^{1}$, SE Goldberg ${ }^{1}$, T Masud $^{1}$, $\mathrm{P}_{\text {Logan }}{ }^{1}, \mathrm{~A}$ Brand ${ }^{2}, \mathrm{~V}$ Booth $^{1}$, Clare Burgon ${ }^{1}$, RK Bajwa ${ }^{1}$

${ }^{1}$ Nottingham University, Nottingham, UK, ${ }^{2}$ Bangor University, Bangor, UK

Introduction: People with mild dementia and Mild Cognitive Impairment are at high risk functional deterioration. We developed an exercise-based therapy intervention aiming to promote safe activity and reduce risk of falling.

Methods: We conducted a randomised feasibility trial, to test intervention delivery in practice and to prepare for a definitive trial. Sixty participants were recruited from Memory Clinics across two sites. Three randomisation arms compared intervention with high level supervision (50 professional visits), moderate supervision (nine visits), and control. We collected health status data at baseline and follow-up by interview with participants and family carers, and ascertained falls by diary.

Results: Mean age was 76. Mini Mental State Examination scores were $20-30$, mean 26 . We followed up $49(82 \%)$ after 12 months. One died, three moved to care homes. $81 \%$ of therapy sessions were delivered. $88 \%$ of diaries were returned. There were 19 reported adverse events, none related to the intervention. The Disability Assessment in Dementia primary outcome deteriorated over 12 months (mean -18 control, -17 moderate, -8 high). Berg Balance Score were better with active intervention $(-6.8,-0.5$ and +0.1 ). Incidental and Planned Activity scores, pedometer step count and timed up and go test improved in the high intensity supervision group.

Conclusions: We successfully recruited participants, delivered the intervention and collected data. The intervention resulted in improved balance, activities of daily living and physical activity, in underpowered comparisons, with greater benefits associated with higher levels of supervision. A full-scale multi-centred randomised controlled trial will commence in September 2018.

\section{P-602}

High mortality in patients with dementia admitted to psychiatric care

Mirjam Geerlings ${ }^{1}$, Irene van de Vorst ${ }^{1}$, Ilonca Vaartjes ${ }^{1}$, Annemarieke de Jonghe ${ }^{2}$, Michiel Bots ${ }^{1}$, Dineke Koek ${ }^{1}$

${ }^{1}$ University Medical Center Utrecht, Utrecht, The Netherlands,

${ }^{2}$ Tergooi, Blaricum, The Netherlands

Introduction/aim: To examine the mortality risk, and influencing factors, of patients with dementia admitted to psychiatric care.

Methods: We constructed a cohort of dementia patients through data linkage of four Dutch national registers: the Psychiatric Case Register Middle Netherlands (PCR-MN), hospital discharge register (HDR), population register (PR) and national cause of death register. All patients with dementia in PCR-MN aged between 60 and 100 years between 1 January 2000 and 31 December 2010 were included. Risk factors of mortality were investigated using Cox proportional hazard regression models with adjustment for possible confounders.
Results: 4895 patients were included in the cohort with a median age of 80 years. The 1-year, 3-year and 5-year mortality risks were $15.6 \%, 36.5 \%$ and $45.4 \%$ respectively. Determinants that increased the mortality risk were: male sex (Hazard ratio (HR) 1-year mortality: $1.52,95 \%$ confidence interval (CI) 1.29-1.79), higher age (HR 1.08, 95\% CI 1.07-1.09), living together/married (HR 1.25, 95\% CI 1.06-1.48), admitted for inpatient psychiatric care (HR $1.60,95 \% \mathrm{CI}$ 1.28-2.00), more somatic comorbidities (HR 1.68, 95\% CI 1.47-1.93) and specifically cardiovascular disease (HR 1.24, 95\% CI 1.09-1.40). Results for 3-year and 5-year mortality were comparable. Being of Dutch ethnicity increased the 3 and 5-year mortality. There were no differences in mortality according to type of psychiatric comorbidity. Conclusion: Mortality of patients with dementia admitted to psychiatric care was high. The results of the present study should raise awareness in clinicians about the unfavourable prognosis of patients with dementia in psychiatric care, particularly older inpatient men with more somatic comorbidity.

\section{P-603}

\section{Caregiver burden in Alzheimer's disease}

\section{Sonia Hammami ${ }^{1}$, Latifa Ghanmi ${ }^{2}$, Salem Bouomrani ${ }^{3}$}

${ }^{1}$ Biochemistry Laboratory, LR12ES05 LR-NAFS 'Nutrition-

Functional Food and Vascular Health' Faculty of MedicineUniversity of Monastir, Monastir, Tunisia, ${ }^{2}$ Gabes Hospital, Gabes, Tunisia, ${ }^{3}$ Hopital Militaire Gabes, Gabes, Tunisia

Problematic: Alzheimer disease (AD) imposes a severe burden upon patients and their caregivers. Although there is substantial evidence of the adverse impact of burden, considerably less is known about its specific correlates and potential causes.

Objectives: Assessment of the level of burden and frequency of depression among caregivers in $\mathrm{AD}$, and identification of the main factors associated with the burden in these caregivers.

Methods: We conducted a cross-sectional study at the psychiatric department. All caregivers who accompanied their relatives with AD to their appointments from 01 November 2016 to 15 January 2017 were invited to take part. We used the Zarit Burden Inventory to assess Beck's burden level in the caregiver.

Results: The caregiver was a woman (74\%) with a mean age of 46 and cohabiting with the patient in $68 \%$ of the cases. The mean score on the burden interview was 43 . The burden was perceived as high by $48 \%$ of caregivers. A high caregiver burden was significantly associated with the absence of recreational activity (57\% vs $83 \%$ ), being the primary caregiver of the patient (50\% vs $83 \%)$ and cohabiting with him/her (53\% vs $83 \%$ ). Regarding patient variables, only the level of dependence of the patient correlated with a high level of caregiver burden (38\% vs. $79 \%)$.

Conclusion: Dependency and patients' quality of life have stronger association with caregiver perception of burden than cognitive functioning. Combinations of pharmacological and family interventions targeting these two aspects of the disorder are likely to alleviate patient suffering but also mediate improved caregiver well-being.

\section{P-604}

Antiepileptic drug use and the risk of stroke among communitydwelling persons with Alzheimer's disease: a matched cohort study

Tatyana Sarycheva ${ }^{1}$, Piia Lavikainen ${ }^{1}$, Heidi Taipale ${ }^{1}$, Jari Tiihonen ${ }^{2}$, Antti Tanskanen ${ }^{2}$ 
${ }^{1}$ University of Eastern Finland, School of Pharmacy, Kuopio, Finland, ${ }^{2}$ Department of Clinical Neuroscience, Karolinska Institutet, Stockholm, Sweden, Department of Forensic Psychiatry, Niuvanniemi Hospital, University of Eastern Finland, Kuopio, Finland

Background: Persons with Alzheimer's disease (AD) are more predisposed to seizures than older people in general, and use of antiepileptic drugs (AEDs) is more frequent. AED use has been linked to higher risk of vascular events in general population, however, it is not evident whether the same association is in persons with $\mathrm{AD}$. We assessed the risk of stroke associated with incident AED use among persons with AD.

Methods: The MEDALZ cohort includes all Finnish persons who received a clinically verified AD diagnosis $(\mathrm{N}=70,718)$ in $2005-2011$. Persons with previous strokes were excluded. For each incident AED user ( $\mathrm{n}=5617$ ) one non-user was matched according to sex, age and time since $\mathrm{AD}$ diagnosis. Analyses were conducted with Cox proportional hazards models and inverse probability of treatment weighting (IPTW).

Results: Compared with non-use, AED use was associated with an increased risk of stroke (IPTW hazard ratio (HR) 1.37; 95\% CI 1.07-1.74). The risk was strongest during the first 90 days (adjusted HR 2.36, 95\% CI 1.25-4.47) of AED use. The association was more evident with ischemic strokes (IPTW HR 1.34, 95\% CI 1.00-1.79) than hemorrhagic ones (IPTW HR 1.44, 95\% CI 0.86-2.43). Use of older AED was associated with similar risk of stroke as newer AED use (adjusted HR 1.04, 95\% CI 0.71-1.53).

Conclusions: AED use was related to an increased risk of stroke, regardless of AED type. Our results highlight caution in AED use in this vulnerable population.

\section{P-605}

\section{The test of the watch drawing as a method of evaluation} of cognitive deterioration

\section{Maria Isabel Porras-Guerra ${ }^{1}$, Carlos Verdejo ${ }^{2}$, Germán Gómez-Porras ${ }^{3}$}

${ }^{1}$ Complejo Asistencial Universitario de León (CAULE), León, Spain, ${ }^{2}$ Hospital Clínico San Carlos, Madrid, Spain, ${ }^{3}$ Universidad de Salamanca (USAL) Salamanca, Spain

The clock drawing test is widely used in clinical practice because it is a fast and reliable method for assessing cognitive impairments.

Objective: To assess and compare the cognitive status of two groups of patients attending the hospital day care center.

Materials and methods: Observational, longitudinal, descriptive study. The clock drawing test was carried out to assess the cognitive status of two groups of patients attending the day care center during the months of November and December 2014. One group had functional recovery and the second had cognitive stimulation.

Variables: sex, age, domicile, pathological antecedents, reason to go to day care center and in the second group, the prescribed treatment for cognitive impairment. SPSS v.15 statistical treatment.

Results: $\mathrm{n}=3036.7 \%$ males. $63.3 \%$ females. Group 1 (functional recovery) $43.3 \%$. Group 2 (cognitive stimulation) $56.4 \%$. Average age: 81.2 years. Domicile: alone $24.5 \%$, with marriage $47.1 \%$, with family 28.4\%. Average pathology: 5.6. Hypertension, dyslipidemia, depressive disorder and diabetes more frequent pathologies. Group 1: derived to Day center by: hip fracture $69 \%$, vertebral fracture $7.7 \%$, stroke $7.7 \%$, discopathy $7.7 \%$, fall syndrome $7.7 \%$. Group 2: derived by: Degenerative dementia: $64.7 \%$, mixed dementia: $23.5 \%$, vascular Parkinsonism $5.9 \%$, stroke sequels $5.9 \%$. Test of the clock: $65.4 \%$ pathological. Group 1: pathological 50\%; Group 2: $17.6 \%$ pathological.

Conclusions: Increased cognitive impairment in women and in the functional recovery group. Worst functionality worst result of the clock drawing test. It is the pluripatology a protective factor for dementia or, pluripathological patients are less concerned about their memory? Nor are all who are, nor are all who are.

\section{P-606}

\section{Is there relationship between frailty fried criteria and cognitive} impairment?

Farshd Sharifi ${ }^{1}$, Hossein Fakhrzadeh ${ }^{1}$, Mahtab Alizadeh-Khoei ${ }^{2}$, Masoud Arzaghi ${ }^{1}$, Gita Shaffeiee ${ }^{3}$, Ramin Heshmat ${ }^{3}$, Iraj Nabipour ${ }^{4}$, Afshin Ostovar ${ }^{5}$, Bagher Larijani ${ }^{6}$, Reyhaneh Aminalroaya ${ }^{7}$, Fatemeh-Sadat Mirzadeh ${ }^{7}$, Seyyedeh Zahra Badrkhahan ${ }^{7}$

${ }^{1}$ Elderly Health Research Center, Endocrinology and Metabolism Population Sciences Institute, Tehran University of Medical Sciences,Tehran, Iran, ${ }^{2}$ Geriatric Medicine department, Medical school, Tehran University of Medical Sciences \& Ziyaiyan Hospital, Tehran-Iran \& Elderly Health Research Center, Endocrinology and Metabolism Population Sciences Institute, Tehran University of Medical Sciences, Tehran, Iran, ${ }^{3}$ Chronic Disease Research Center, Endocrinology and Metabolism Population Sciences Institute, Tehran University of Medical Sciences, Tehran, Iran, ${ }^{4}$ The Persian Gulf Biomedical Research Institute, Bushehr University of Medical Sciences, Bushehr, Iran, ${ }^{5}$ Osteoporosis Research Center, Endocrinology and Metabolism Clinical Sciences Institute, Tehran University of Medical Sciences, Tehran, Iran, ${ }^{6}$ Endocrinology and Metabolism Research Center, Endocrinology and Metabolism Clinical Sciences Institute, Tehran University of Medical Sciences, Tehran, Iran, ${ }^{7}$ Geriatric Medicine department, Medical school, Ziyaiyan Hospital,Tehran University of Medical Sciences, Tehran, Iran

Introduction: Since underlying pathophysiology of physical frailty and cognitive frailty might be similar; therefore, finding any association could guide clinicians, to recognize effective strategies for each type of frailty resulting to preventive approach.

Methods: This is a cross-sectional Bushehr elderly health survey in community setting. Frailty was assessed based on Fried's frailty phenotype criteria. Cognitive impairment was assessed by Mini Mental State Examination (MMSE), Mini Cog, Functional Assessment Staging Tool (FAST), and Category Fluency Test (CFT). The participants were categorized based on Fried's criteria and cognition tools. Multivariable logistic regression models were applied to determine association between frailty and cognitive impairment.

Results: Among people $\geq 60$ years $(\mathrm{N}=2392)$ women were $51.6 \%$. Mean age of the participants were 69.3 years old. The prevalence of prefrailty and frailty was 42.9 and $8.1 \%$, respectively and cognitive impairment was $21.7 \%$. The odds ratio of association between frailty and cognitive impairment was 2.51 (CI 95\%, 1.76-3.59) (adjusted for age, sex, and education levels). All components of Fried's frailty criteria, except unintentional weight loss were associated with cognitive impairment.

Conclusion: We observed strong association between frailty criteria and cognitive impairment, that cognitive decline is determined as a crucial factor to define frailty. Further studies are needed to discover common causes of physical frailty and cognitive frailty to make a better geriatric management.

Keywords: Physical frailty, Cognitive frailty, Cognitive impairment, Geriatric. 


\section{P-607}

The impact of activity-role participation on cognitive status in geriatric individuals

Ayşe Göktaş ${ }^{1}$, Hüseyin Kudret ${ }^{2}$, İrem $\mathrm{Kar}^{3}$, Murat Varlı
${ }^{4}$ Ankara University Vocational School of Health Services, Physiotherapy, Ankara, Turkey, ${ }^{2}$ Ankara University School of Medicine, Department of Physiotherapy and Rehabilitation, Ankara, Turkey, ${ }^{3}$ Ankara University School of Medicine, Department of Bioistatistics, Ankara, Turkey, ${ }^{4}$ Ankara University, Ankara, Turkey

Introduction: Cognitive decline increases with aging and also activity of daily living and quality of life of the older people with cognitive impairment decreases over time.

Materials and Methods: This study was performed in Ankara University School of Medicine, Division of Geriatrics. Ethical approval was taken from the local Ethics Committee, which is numbered 21-1310-17. The standardized Mini Mental State Examination (SMMT), Human Activity Role Scanning Tool (IARMTA), SF 36 Quality of Life and Life Satisfaction Scale were used in the study of 79 volunteers aged 65 years and older.

Results: The mean age of the patients was $76.72 \pm 7.26$ years, $46 \%$ were female and $33 \%$ were male. Of the patients examined, 34 $(43.0 \%)$ had mild, $28(35.5 \%)$ had severe dementia and 17 patients $(21.5 \%)$ had normal cognitive status. The weakest areas were found to be success expectancy, interest, choices, problem solving, organization and energy. Between the SMMT scores and Activity Role Pattern $(\mathrm{r}=-0.400, \mathrm{p}<0.001)$, Process Skills $(\mathrm{r}=-0.495, \mathrm{p}<0.001)$, Motor Skills $(\mathrm{r}=-0.364, \mathrm{p}<0.001)$, Environment $(\mathrm{r}=-0.3700$, $\mathrm{p}<0.001)$, IARMTA Total scores $(\mathrm{r}=-0.454, \mathrm{p}<0.001)$ were found statistically negative strong correlation. It was found a positive correlation between scores of IARMTA and SF 36 Scale $(\mathrm{p}<0.001)$. Conclusion: The low level of activity-role participation in the elderly was found to be an important risk factor for cognitive status, quality of life and satisfaction.

Keywords: Elderly, Cognitive status, Health promotion, ActivityRole

\section{References}

1. Naqvi R, Liberman D, Rosenberg J et al (2013) Preventing cognitive decline in healthy older adults. CMAJ 185:881-885

2. Salthouse T (2012) Consequences of age-related cognitive declines. Annu Rev Psychol 63:201-226

3. Kremen WS, Lachman ME, Pruessner JC et al (2012) Mechanisms of age-related cognitive change and targets for intervention: social interactions and stress. J Gerontol A Biol Sci Med Sci 67:760-765

4. Blasko I, Jungwirth S, Kemmler G, Weissgram S, Tragl KH, Fischer P (2014) Leisure time activities and cognitive functioning in middle European population-based study. Eur Geriatr Med 5(3):200-207

\section{P-608}

What do healthcare professionals do when the patient with dementia says 'no' to a healthcare procedure?

RH Harwood ${ }^{1}$, B O’Brien ${ }^{2}$, SE Goldberg ${ }^{2}$, A Pilnick ${ }^{2}$, S Beeke $^{3}, \mathrm{R}$ Allwood $^{2}$

${ }^{1}$ Nottingham Univerisity, Nottingham, UK, ${ }^{2}$ Nottingham University, Nottingham, UK, ${ }^{3} \mathrm{UCL}$, London, UK

Background: Poor communication is a barrier to processes essential for good treatment and care. People with dementia and those who work with them can experience difficulties in communicating. Much healthcare involves a professional requesting an action from a patient). People with dementia often initially refuse these requests for action. We aimed to identify communication skills used by experienced professionals when a request for action is refused by a patient with dementia.

Methods: We video-recorded 41 healthcare encounters between 26 professionals (eleven nurses, nine doctors and six therapists) and 26 people with dementia (16 women), comprising $378 \mathrm{~min}$. We used a socio-linguistic research method, called conversation analysis, to study patterns in the communication encounters.

Results: Twenty-eight (68\%) of our recordings contained refusals, often repeated. Requests that were made explicitly and directly (imperatives), with high entitlement (authority to ask), and which lowered the perceived difficulties with the task ('contingencies') were more likely to succeed. Requests that referred to the person living with dementia's ability or willingness to comply ('can you...?') were often refused. Highly entitled requests could take the form of announcing future action, proposals, or statements of need. Contingencies were lowered by using words that minimised the size or duration of the task, asking the person 'to try', by offering help, or proposing joint action.

Conclusion: How we frame requests to patients with dementia influences the likelihood of the patient granting that request. This study identified practical ways professionals can improve their communication with patients with dementia.

\section{P-609}

Development of the promoting activity, independence and stability in early dementia and mild cognitive impairment (PrAISED) intervention

RH Harwood ${ }^{1}, V_{\text {Booth }}{ }^{1}$, V Hood-Moore ${ }^{1}$, T Bramley $^{1}$, JE Hancox ${ }^{1}$, K Robertson ${ }^{1}$, J Hall ${ }^{1}$, V van der Wardt ${ }^{1}$, PA Logan ${ }^{1}$

${ }^{1}$ Nottingham University, Nottingham, UK

Introduction: Older adults with dementia are at a high risk of falls. Standard interventions have not been shown to be effective. An intervention is required that addresses the particular needs of older people with dementia in a community setting.

Methods: We followed guidelines for intervention development, which recommend a structured approach considering theory, evidence and practical issues. Data were included from literature reviews, clinician workshops, expert opinion meetings, patient and relative interviews, focus groups with people with dementia and clinicians, a cross-sectional survey of risk factors, a proof-of-concept intervention study, and case studies. Key findings from each source were synthesised using triangulation. A steering group comprising clinicians, researchers and patient carer and public involvement representatives reviewed and refined the triangulation matrix. Practical consideration of how an intervention could be delivered and implemented were considered from the outset.

Results: A multicomponent intervention was designed. The focus is on promoting safe activity rather than risk or prevention of falls. Many information sources were consistent in their recommendations and influenced final intervention. Core elements of the intervention included individually-tailored, dementia-appropriate, balance, strength and dualtask exercises, functional training, and activities aimed at improving environmental access and risk enablement. Delivery of the intervention is through a motivational approach to support communication, adherence and longer term continuation of activity.

Conclusion: We used a systematic process to develop a dementiaspecific intervention to promote activity and independence whilst reducing falls risk in older adults with mild dementia. The intervention is currently being feasibility tested. 


\section{P-610}

Factors associated with an improvement of quality of life (QoL) according to the Nottingham Health Profile (NHP) after a multicomponent cognitive stimulation program in a Geriatric Day Hospital (GDH)

Elisabet de Jaime ${ }^{1}$, Anna Renom ${ }^{1}$, Silvia Burcet ${ }^{1}$, M Angels Vidal ${ }^{1}$, M Carmen Delgado ${ }^{1}$, Montserrat Garcia ${ }^{1}$, M Luz Rodriguez $^{1}$, Eulalia Sevilla $^{1}$, Olga Vazquez ${ }^{1}$, Ramon Miralles ${ }^{1}$

${ }^{1}$ Geriatric Service, Centre Forum, Parc de Salut Mar, Barcelona

Introduction: The aim of this study was to identify which factors are associated with QoL improvement among the patients receiving a multicomponent cognitive stimulation program in a GDH.

Methods: Longitudinal study including those patients that attended the GDH for a minimum of seven sessions between January 2007 and December 2011. We registered the following variables at baseline and at the end of the rehabilitation program: sociodemographic variables, NHP questionnaire (global score and subareas), Barthel Index and Lawton Index for activities of daily living, Timed Up and Go and Tinetti for mobility, and Mini-Mental Folstein and GDS-Yesavage for cognitive status and depression, respectively. We considered QoL improvement if a decrease of the NHP score occurred with an effect size of $\geq 0.50$. We performed bivariate and multivariate analyses.

Results: Of the 107 patients included (mean age $78.5 \pm 5.5,60.7 \%$ women), thirty-five experienced QoL improvement. In the bivariate analysis, a lower baseline Yesavage score was associated with an improvement of all QoL subareas except for pain and physical mobility. In the multivariate analysis, a decrease in the Yesavage score of $\geq 1$ was associated with QoL improvement [global QoL (OR 4.66; IC 1.62-13.40); subarea energy (OR 5.37; IC 1.78-16.20)], as well as a decrease of the Timed Up and Go score of $\geq 2 \mathrm{~s}$ [global QoL (OR 4.11; IC 1.20-13.99), subarea social isolation (OR 6.33; IC 2.00-19.99)].

Key conclusions: Baseline depression and improvement of symptoms of depression may play an important role in the QoL improvement among patients receiving a multicomponent cognitive stimulation program.

\section{P-611}

Hospitalization after oral antibiotic initiation in Finnish community dwellers with and without Alzheimer's disease: retrospective register-based cohort study

Heli Järvinen ${ }^{1}$, Heidi Taipale ${ }^{1}$, Marjaana Koponen ${ }^{1}$, Antti Tanskanen ${ }^{2}$, Jari Tiihonen ${ }^{2}$, Anna-Maija Tolppanen ${ }^{1}$, Sirpa Hartikainen ${ }^{1}$

${ }^{1}$ University of Eastern Finland, Joensuu, Finland, ${ }^{2}$ Karolinska Institute, Solna, Sweden

Introduction: Persons with Alzheimer's disease (AD) are frequently hospitalized from infection-related causes. There are no previous studies investigating hospitalization associated with antibiotic initiation in persons with AD. The objective of this study was to investigate the frequency and risk of hospitalization associated with oral antibiotic initiation among community dwellers with and without AD.

Methods: We performed a retrospective register-based study utilizing register-based Medication Use and Alzheimer's disease (MEDALZ) cohort. It includes all community dwellers diagnosed with AD during 2005-2011 in Finland and their matched comparison persons without AD. Antibiotic use was initiated by 34,785 persons with and 36,428 without AD. Drug use data were collected from Prescription Register and comorbidities from Special Reimbursement and Hospital Care Registers. Infection diagnoses were collected from the Hospital Care
Register. Factors associated with hospitalization were estimated utilizing logistic regression models.

Results: Risk of hospitalization following antibiotic initiation was higher among antibiotic initiators with $\mathrm{AD}$ than without $\mathrm{AD}$ (adjusted odds ratio, aOR, 1.37, 95\% Cl 1.28-1.46). Strongest association with hospitalization was found for oral glucocorticoid use, aOR 1.41 (1.25-1.59); epilepsy, aOR 1.33 (1.10-1.63); active cancer, aOR 1.30 (1.14-1.49). Among initiators of cephalexin, pivmecillinam, amoxicillin/amoxicillin and enzyme inhibitor and doxycycline, persons with $\mathrm{AD}$ were more frequently hospitalized than persons without AD. A quarter of hospitalized antibiotic initiators had infection diagnosis in their hospital care records.

Key conclusions: Persons with AD initiating an antibiotic had a higher risk for hospitalization than antibiotic initiators without AD. Further research is needed to determine whether infection-related hospitalization could be reduced.

\section{P-612}

Hospital-treated pneumonia associated with opioid use among community dwellers with Alzheimer's disease: a nationwide matched retrospective cohort study

Aleksi Hamina ${ }^{1}$, Heidi Taipale ${ }^{1}$, Niina Karttunen ${ }^{1}$, Anna-Maija Tolppanen $^{1}$, Antti Tanskanen ${ }^{2}$, Jari Tiihonen ${ }^{2}$, Sirpa Hartikainen ${ }^{1}$

${ }^{1}$ University of Eastern Finland, Joensuu, Finland, ${ }^{2}$ Karolinska Institute, Solna, Sweden

Introduction: Pneumonia is a common cause for hospitalization and excess mortality among persons with Alzheimer's disease (AD), but there is little research to drug use as its risk factor. We aimed to investigate the association of opioid use and hospital-treated pneumonia among community-dwelling persons with AD.

Methods: This study was part of the Medication use and Alzheimer's (MEDALZ) cohort. We included all community-dwelling persons newly diagnosed with AD during 2010-2011 in Finland with new prescription opioid use $(n=5623)$ and matched nonusers $(n=5623)$. Data on drug use, modelled from pharmacy dispensings, and hospitalization for pneumonia were retrieved from nationwide registers. Patients with active cancer treatment were excluded.

Results: Use of opioids was associated with a higher risk of hospitalization for pneumonia compared to nonuse (adjusted HR, aHR 2.03 95\% CI 1.53-2.69). The risk was observed only during the first 2 months of use (aHR 3.65, 95\% CI 2.45-5.42) and became statistically non-significant after that. Compared to mild opioid use, buprenorphine use was associated with a higher risk of pneumonia (HR 1.47, 95\% CI 1.01-2.15), as was strong opioid use (HR 2.20, 95\% CI 1.38-3.51). Similarly, the risk was higher for those using $\geq 50$ morphine milligram equivalents (MME)/day, when compared to those using < $50 \mathrm{MME} /$ day (aHR $1.72,95 \%$ CI 1.29-2.29).

Key conclusions: Risk of pneumonia associated with opioid use needs to be considered in pharmacotherapy of pain among persons with $\mathrm{AD}$, especially when opioid therapy is introduced.

\section{P-613}

Do different medicine formulations and dysphagia effect the incidence of pneumonia amongst people with dementia?

Eleanor Dann-Reed ${ }^{1}$, David Wright ${ }^{2}$, George Savva ${ }^{3}$

${ }^{1}$ University of East Anglia (UEA), Norwich, UK, ${ }^{2}$ University of East Anglia (UEA), Norwich, UK), ${ }^{3}$ Quadram Institute, Norwich Research Park, UK 
Introduction: Dysphagia is experienced by people with late stage dementia [1] and is associated with aspiration pneumonia (AP), which is a common cause of death in dementia [2,3].Although diet and liquid intake are modified to minimise the risk of AP, appropriateness of medicine formulation is often not considered.

Aim: Determine whether medication formulation and dysphagia are associated with incident pneumonia among people living with dementia.

Methods: A case-controlled study design nested within those with dementia was conducting using the Clinical Practice Research Datalink, which includes primary care data of over 20 million UK patients. Cases had a diagnosis of pneumonia whereas controls did not. There were no age restrictions and patients with pneumonia diagnosis $<90$ days after dementia diagnosis were excluded. Conditional logistic regression was used for data analysis.

Results: The dataset contained 28,671 controls and 7259 cases. An increased risk of developing pneumonia was observed to be associated with dysphagia (odds ratios (OR) 3.57, 95\% confidence interval (CI) 3.20-3.98, $\mathrm{p}<0.001$ ) and liquid formulations (OR 2.37, CI $2.11-2.68, \mathrm{p}<0.001)$. Patients with a dysphagia diagnosis as well as prescribed liquids were associated with ((adjusted) OR 4.49, CI $3.38-5.95, \mathrm{p}<0.001)$.

Conclusions: Patients with dysphagia or prescribed liquid medicines are associated with a greater risk of developing pneumonia. Whilst the relationship between dysphagia and pneumonia is predictable, the relationship with liquid medicines is less intuitive. They may be a proxy for severity of dysphagia. Further work to explore this relationship is warranted.

\section{References:}

1. Horner J et al (1994) Swallowing in Alzheimer's disease. Alzheimer Dis Assoc Disord 8(3): 177-89

2. Brunnström HR, Englund EM (2009) Cause of death in patients with dementia disorders. Eur J Neurol 16(4):488-492

3. Loeb M et al (1999) Risk factors for pneumonia and other lower respiratory tract infections in elderly residents of long-term care facilities. Arch Intern Med 159(17):2058-2064

\section{P-614}

Characterising the structural and functional architecture of falls risk and response to exercise therapy in people with dementia: feasibility using Magnetic Resonance Imaging (MRI)

RH Harwood ${ }^{1}$, R Dineen ${ }^{1}, \mathrm{P}$ Wells ${ }^{1}, \mathrm{~V}$ van der Wardt $^{1}$, V Booth ${ }^{1}$, D Auer ${ }^{1}$

${ }^{1}$ Nottingham University, Nottingham, UK

Introduction: The Promoting Activity, Independence and Stability in Early Dementia (PrAISED) programme has designed and implemented a high-intensity, exercise-based therapy programme to promote safe activity and reduce falls risk among people with early dementia. We plan to investigate if MRI allows quantification of exercise-induced structural and functional plasticity in motor and postural control networks, and if quantitative imaging markers of motor and postural network integrity on pre-intervention MRI predicts treatment response.

Methods: We invited 23 people with diagnosed dementia who took part in the PrAISED study to undergo a battery of multi-modal MRI imaging on a 3.0 Tesla GE Discovery MR 750 scanner. We included functional imaging of a virtual reality-based, imagined, walking and obstacle avoidance tasks, and a novel postural instability experience (a stumble).

Results: 21 people agreed to take part, and 20 tolerated scanning. Mean age was 76 (range 67-92) years, mean Mini-Mental State
Examination was 26/30 (range 19-30). Participants agreed that tolerability was good, and all would be willing to be scanned again. We obtained structural T1-weighted, T2-weighted FLAIR, 3D ASL perfusion, neuromelanin-sensitive T1, Re-fMRI, Rs-fMRI and task fMRI images and diffusion tensor imaging to map white-matter tracts. Initial analysis included correlating fMRI images with MMSE, demonstrating positive correlations with deep brain structures, including those associated with memory, and negative correlations with visual association areas.

Conclusion: Multi-modal research MRI scanning is feasible and well tolerated. Functional imaging for imagined walking and obstacle avoidance tasks, and brain responses to a loss of balance.

\section{P-615}

Similar dementia risk in APOE- 84 carriers and non-carriers with high life-long cognitive reserve: a population-based cohort study

Serhiy Dekhtyar ${ }^{1}$, Anna Marseglia ${ }^{1}$, Weili Xu ${ }^{1}$, Alexander Darin-Mattsson ${ }^{1}$, Hui Xin Wang ${ }^{2}$, Laura Fratiglioni ${ }^{1}$

${ }^{1}$ Aging Research Center, Karolinska Institutet, Solna, Sweden, ${ }^{2}$ Stress Research Institute, Stockholm University, Stockholm, Sweden

Aspects of life experiences, such as education, occupational characteristics, or social activities have been linked with a reduced risk of dementia, potentially due to enhancing cognitive reserve. In this study, we collected four reserve-enhancing factors observed over the life course - education (early adulthood), substantive work complexity (midlife), size of the social network, and leisure activities (both late life) and considered their contribution to a latent indicator of cognitive reserve in a Structural Equation Modelling approach. Using data from the Swedish National Study on Aging and Care in Kungsholmen - a community-based longitudinal study of aging-we examined the association between the resulting latent indicator of cognitive reserve and clinically-ascertained incident dementia over a nine-year follow-up period, while also considering the interplay between latent reserve and APOE- $\varepsilon 4$ status. The best-fitting SEM model of cognitive reserve indicator based on four observed factors had a good fit $\left(\chi^{2}=2.04, p=0.153\right.$; RMSEA $=0.019$; $\left.\mathrm{CFI}=0.99\right)$. Predicted latent reserve was entered into a Cox proportional hazard model and a reduced risk of dementia was found in those with moderate and high levels of a latent cognitive reserve indicator (HR 0.65 and 0.35 , respectively; both $\mathrm{p}<0.01$ ). A significant interaction on an additive scale was found between latent reserve and genetic risk of dementia. Dementia risk reduction was similar in both carriers and non-carriers of APOE- $\varepsilon 4$ allele with high cognitive reserve (HR: 0.26 and 0.24 , respectively; $p<0.01$ for both). Not only is cognitive reserve protective of dementia, but it appears to override the detrimental effects of genetic risk.

\section{P-616}

How to prepare from caregiver turn to surrogate decision maker for people with dementia: A integrative review

$\mathrm{Ya} \mathrm{Su}^{1}$, Miku Izutsu ${ }^{1}$, Kengo Hirayama ${ }^{2}$, Michiko Yuki ${ }^{2}$

${ }^{1}$ Graduate School of Health Sciences, Hokkaido University, Sapporo, Japan, ${ }^{2}$ Faculty of Health Sciences, Hokkaido University, Sapporo, Japan

Background: There is increasing evidence that aging and impaired cognitive functions affect the decision-making capability of older 
patients that surrogate decision-making for loved ones is becoming more common, especially for dementia. However, it was unclear about how to prepare and support when family member from caregiver turn to surrogate decision maker for people with dementia.

Method: An integrative review design was used based on Whittemore and Knafl's study [1]. A review of English and Japanese language publications before 2018 March was conducted. Searched electronic databases included MEDLINE, CINANL, EmBase, PubMed and ICHUSHI, CiNii. Critical appraisal was performed by researchers used MMAT.

Results: 46 articles with 8 main types of surrogate decisions were included: Placement, Clinical treatment research, End-of-life, Feeding, Advance directive, Cardiac Implantable Electronic Devices, Hemodialysis and others. A total of 3486 surrogates were involved and more than half were women. 9 studies focused on Clinical treatment research shown that who participated was mainly spouse as caregivers, adult children were contrasted. The review conducted the preference, influence, process of surrogates with variety of decision types that explored the evidence for caregivers to preparation and identified the advice for healthcare providers about support and intervention.

Conclusions: Family members often care for people with dementia for several years, the more decision need the more burden with caregivers. However, many surrogates face the difficult decision with little professional support. Thus, it is urgently needing to support for caregiver turn to surrogate.

\section{Reference:}

1. Whittemore R, Knafl K (2005) The integrative review: updated methodology. J Adv Nurs 52(5):546-553

\section{P-617}

\section{Impact of hearing rehabilitation on cognitive functions}

Völter Christiane ${ }^{1}$, Götze Lisa ${ }^{2}$, Bruene Cohrs Ute ${ }^{3}$, Dazert Stefan ${ }^{1}$, Thomas Jan Peter ${ }^{1}$

${ }^{1}$ ENT Department, St. Elisabeth Hospital, Ruhr University, Bochum, Bochum, Germany, ${ }^{2}$ ENT Department, St. Elisabeth Ruhr University Bochum, Bochum, Germany, ${ }^{3}$ Department of Psychiatry Ruhr University Bochum, Bochum, Germany

The association between cognition and hearing is well known. With regard to the growing number of older persons and the incidence of demential illness the question arises whether hearing rehabilitation might counteract cognitive decline in aging. Since 2016 patients aged 50 or more suffering of severe to profound hearing loss and scheduled for cochlear implantation underwent a computer-based evaluation of neurocognitive functions prior to surgery. The multi modular computer-based test battery (ALAcog) composed of ten different subtests covering short- and long-term memory, processing speed, attention, working memory and inhibition. So far 80 patients (median: $67 \mathrm{yrs}$ ) were included. Out of these 33 patients were reassessed 6 months and 20 patients 12 months post implantation. After 6 months cognitive functions increased significantly regarding attention $(\mathrm{p}<.001)$, delayed recall $(\mathrm{p}=.03)$, inhibition $(\mathrm{p}=.04)$ and working memory $(2-$ back $\mathrm{p}=.0041$; OSPAN-test $\mathrm{p}=.0077)$. The benefit of the executive functions was the most remarkable. Results remained mostly stable after 12 months. Only working memory measured by the OSPAN additionally enhanced after one year $(\mathrm{p}<.001)$. In contrast, long-term memory did not improve earlier than after 12 months $(\mathrm{p}=.021)$. In general, improvement was statistically better for subjects with poor baseline results. Cochlear implantation has a positive impact on cognitive abilities mostly on executive functions even in patients with lower preoperative performance. Further studies have to show whether hearing restoration through technical devices has a long-term effect on cognition and might even prevent demential illness.

\section{P-618}

An assessment of cognitive screening in a population of geriatric patients at a local rehabilitation hospital in Malta

Maria Bonnici ${ }^{1}$, Valentina Fenech ${ }^{1}$, Gabriella Balzan ${ }^{1}$, Peter Ferry ${ }^{2}$

${ }^{1}$ Mater Dei Hospital, L-Imsida, Malta, ${ }^{2}$ Karin Grech Rehabilitation Hospital, Pieta, Malta

Introduction: This retrospective study aims to: (1) establish whether cognitive screening is carried out with validated tools of assessment. (2) Assess role of radiological investigations for cognitive impairment and if underlying aetiology was identified. (3) Evaluate if treatment was initiated or reviewed following diagnosis.

Method: Random selection of patients admitted to Karin Grech Hospital from different sources was carried out. Data was collected from patient files and electronic discharge summaries including: demographics; type of cognitive screening tools and documentation on discharge; brain imaging modality; attempts at diagnosis and review of treatment.

Results: A total of 85 patients, with an average age of 81.5 years. While $43 / 85(50.6 \%)$ patients had cognitive screening, this was only documented in the discharge summary of $23 / 85(27.1 \%)$. AMT4 was performed in 29/85 (34.1\%), MMSE in 21/85 (24.7\%) and RUDAS in $5 / 85(5.9 \%)$ with no patients having a MOCA or clock-drawing test. Cognitive impairment was detected in $8 / 29(27.6 \%)$ on AMT4, 18/21 (85.7\%) on MMSE and 4/5 (80\%) on RUDAS. Only 3/8 (37.5\%) patients with abnormal AMT4 scores were followed up by an MMSE. Computed tomography scan was the primary imagining modality used. Only $6 / 42(14.3 \%)$ were scanned as part of their cognitive assessment and a formal diagnosis of cognitive impairment was documented in only one case. 10/85 (11.8\%) patients were started on an acetylcholinesterase inhibitor (donepezil).

Conclusions: This audit highlights lacunae in cognitive screening that still need to be addressed on a local level while also emphasizing its importance in the delivery of patient-centred care.

\section{P-619}

Comparing cut-off values by age and educational level for short cognitive screens in Turkish older adults

Hacer Dogan Varan ${ }^{1}$, Muhammet Cemal Kizilarslanoglu ${ }^{2}$, Cafer Balci ${ }^{3}$, Olgun Deniz ${ }^{3}$, Suheyla Coteli ${ }^{3}$, Rana Tuna Dogrul ${ }^{2}$, Gozde Sengul Aycicek ${ }^{3}$, Mustafa Kemal Kilic ${ }^{3}$, Rónán O’Caoimh ${ }^{4}$, David William Molloy ${ }^{5}$, Meltem Halil ${ }^{3}$

${ }^{1}$ Hacettepe University Faculty of Medicine, Ankara, Turkey, ${ }^{2}$ Gazi University Faculty of Medicine, Ankara, Turkey, ${ }^{3}$ Hacettepe

University Faculty of Medicine, Ankara, Turkey, ${ }^{4}$ Clinical Sciences Institute, National University of Ireland, Galway, Galway, Republic of Ireland

Introduction: To compare three short cognitive screening impairments: the Quick Mild Cognitive Impairment screen-Turkish version (Qmci-TR), Mini-Mental State Examination (MMSE), and Montreal Cognitive Assessment (MoCA) by age and education levels in Turkish older adults.

Methods: Patients aged 60 years old and over, diagnosed with normal cognition, amnestic mild cognitive impairment (aMCI) or 
Alzheimer's disease (AD) were included in this study. Patients with major depression were excluded. Those with dementia were selected from previously diagnosed patients according to the NINCDSADRDA and DSM-V diagnostic criteria. Patients with aMCI were diagnosed using Petersen criteria. The Qmci-TR, MMSE, and MoCA were administered sequentially and alternated by geriatricians experienced in the application of all three tests. Interrater reliability was determined in advance. The Reisberg FAST scale was used to stage cognitive status. Education and age-specific cut-offs were determined for all three screening tests for the normal cognition, aMCI and $\mathrm{AD}$, and their discriminative values were compared. SPSS 18.0 and MedCalc programs were used for statistical analyses.

Results: A total of 321 patients (133 with normal cognition, 88 aMCI, $100 \mathrm{AD})$ were included. Median (min-max) age was 75 (60-98) years. One-quarter $(25.1 \%)$ had completed high school and above, $65 \%$ primary school or lower education levels. Half $(51.1 \%)$ of the patients were aged 75 years old and over. The Qmci-TR was statistically significantly better than the MoCA in distinguishing between $\mathrm{aMCI}$ and $\mathrm{AD}$ in the whole sample and for those attaining high school or higher levels of education (AUC 0.833; $0.759 \mathrm{p}=0.004$ and AUC $0.918 ; 0.752 \mathrm{p}=0.04$, respectively). In addition, the Qmci-TR and MMSE were superior to the MoCA in discriminating normal cognition from cognitive impairment in the whole sample and in individuals over 75 years of age.

Conclusion: This is the first study to compare the discriminative values of a broad selection of short cognitive screening tests in an older Turkish population. In distinguishing between MCI and AD, the Qmci-TR appears to be preferable to the MoCA; it is more sensitive for an older Turkish population and those with more education.

\section{P-620}

Persistent depressive symptoms are independently associated with lower processing speed in healthy older adults

\section{A Clark $^{1}, \mathrm{~N}$ Pendleton ${ }^{1}$ \\ ${ }^{1}$ University of Manchester}

Introduction: Age-related deterioration in processing speed may drive decline in cognitive abilities in healthy older adults. We therefore need to understand factors that affect processing speed. Persistent depressive symptoms have previously been associated with processing speed decline - this may relate to confounding by a common cause, rather than an aetiological link. This study aimed to establish whether the relationship between depressive symptoms and processing speed is accounted for by cerebrovascular disease or neuroticism.

Methods: Participants of the Manchester and Newcastle Study of Age and Cognitive Performance aged over 60 were included. Depressive symptoms were assessed on up to 4 occasions (using Beck Depression Inventory (BDI) and Geriatric Depression Scale). Processing speed was assessed using an alphabet coding task. Multiple linear regression was used to examine the relationship between depressive symptoms and processing speed adjusted for potential confounding factors.

Results: 2919 participants were included (mean age 67.49 years). Low levels of depressive symptoms were reported (mean baseline BDI score 7.15). Average depressive symptoms over mean 8.6 years were significantly associated with processing speed (beta $=-0.06$, $\mathrm{p}=0.02$ ). Neither neuroticism (beta $=0.024, \mathrm{p}=0.415$ ) nor cerebrovascular disease (beta $=-0.03, \mathrm{p}=0.32$ ) were associated with processing speed.

Conclusion: Persistent low-level depressive symptoms in healthy older adults are associated with lower processing speed at follow-up over a mean 8.6-year period. This relationship is not accounted for by neuroticism or cerebrovascular disease. Subclinical depressive symptoms may therefore represent a modifiable target for interventions to promote healthy cognitive ageing.

\section{P-621}

Correlations between dual-task performance and Alzheimer's disease cerebrospinal fluid biomarkers

Hanna Bozkurt Åhman ${ }^{1}$, Vilmantas Giedraitis ${ }^{1}$, Ylva Cedervall ${ }^{1}$, Lars Berglund $^{1}$, Kevin McKee $^{2}$, Lena Kilander ${ }^{1}$, Erik Rosendahl ${ }^{3}$, Martin Ingelsson $^{1}$, Anna Cristina Åberg ${ }^{4}$

${ }^{1}$ Department of Public Health and Caring Sciences, Geriatrics, Uppsala University, Uppsala, Sweden, ${ }^{2}$ School of Education, Health and Social Studies, Dalarna University, Falun, Sweden, ${ }^{3}$ Department of Community Medicine and Rehabilitation, Physiotherapy, Umeå University, Umeå, Sweden

Introduction: Studies have indicated that gait disturbances occur early in the development of dementia and that dual-task performance including mobility may predict the progression from mild cognitive impairment (MCI) to dementia [1]. Amyloid- $\beta-42$ (A $\beta 42)$, total tau (ttau) and phosphorylated tau (p-tau) in the cerebrospinal fluid (CSF) are acknowledged biomarkers for conversion from MCI to Alzheimer's disease (AD) [2].

Aim: To investigate correlations between CSF biomarkers and outcomes of I) the mobility test Timed Up-and-Go (TUG) combined with the verbal task of reciting animals (TUGdt), II) the Mini Mental State Examination (MMSE), and III) the Word Fluency Test (WFT).

Methods: Eighty-three patients at two memory clinics who had undergone lumbar puncture were included with mean age 71 years (range 50-84). Thirty-one had a dementia diagnosis (22 AD) and 52 had MCI. The number of animals recited during the performance of TUGdt was counted. Spearman's rank correlations adjusted for age, gender and education were used.

Results: Significant inverse $(p<0.05)$ correlations were shown between CSF tau concentrations and I.I) the number of recited animals/10 s during TUGdt ( $\mathrm{t}$-tau: $\mathrm{R}=-0.25$, $\mathrm{p}$-tau: $\mathrm{R}=-0.25)$, I.II) the total number of animals recited during TUGdt ( $\mathrm{t}$-tau: $\mathrm{R}=-0.26$, p-tau: $\mathrm{R}=-0.27)$, II) MMSE results (t-tau: $\mathrm{R}=-0.25$, $\mathrm{p}$-tau: $\mathrm{R}=-0.26$ ), III) WFT results ( $\mathrm{t}$-tau: $\mathrm{R}=-0.24$, $\mathrm{p}$-tau: non-significant). CSF A $\beta 42$ did not correlate with any of the test results.

Conclusions: Correlations between CSF tau and TUGdt performance were found at similar levels as between CSF tau and MMSE among patients with dementia or MCI. This may indicate that TUGdt results could be used as an aid in early identification of neurodegeneration. References:

1. Montero-Odasso MM et al (2017) Association of dual-task gait with incident dementia in mild cognitive impairment: results from the gait and brain study. JAMA Neurol 74(7), 857-865

2. Blennow K et al (2015) Clinical utility of cerebrospinal fluid biomarkers in the diagnosis of early Alzheimer's disease. Alzheimers Dement 11(1) 58-69

\section{P-622}

Assessment of the relationship between cognitive function and physical performance in older adults with challenging walk tests: the Figure of 8 Walk Test (F8WT). Pilot study

Cristina Udina ${ }^{1}$, Martina Paoli ${ }^{1}$, Jessie VanSwearingen ${ }^{2}$, Jennifer Brach $^{2}$, Marta Roqué $e^{3}$, Luis Soto ${ }^{1}$, María Verónica Robles ${ }^{1}$, Laura Mónica Pérez ${ }^{1}$, Marco Inzitari ${ }^{1}$ 
${ }^{1}$ Parc Sanitari Pere Virgili, Barcelona, Spain, ${ }^{2}$ University of Pittsburgh , Pittsburgh, USA, ${ }^{3}$ Fundació Salut i Envelliment de la Universitat Autònoma de Barcelona, Barcelona, Spain

Introduction: Current literature supports that cognitive function and physical performance are associated. Gait is more often evaluated on a straight walkway. F8WT, involving straight and curved walking to walk a figure-of- 8 around two cones, might challenge cognitive function. Our aim was to assess the relationship between physical and cognitive function (specifically executive function) using different physical performance tests in community-dwelling older adults with cognitive impairment.

Methods: Cross-sectional study of patients aged $\geq 65$ attending a Geriatric Day Hospital. Demographic, clinical and comprehensive geriatric assessment variables were collected. Cognition was assessed using Mini-Mental State Exam(MMSE), Montreal Cognitive Assessment(MoCA) and Symbol Digit Modalities Test(SDMT) and physical performance with Gait Speed(GS), Short Physical Performance Battery(SPPB), F8WT and Dual-Task(DT) [walking during verbal fluency].

Results: We enrolled 55 subjects $(69 \%$ women, mean age + SD $81.2 \pm 6.1$ years, baseline Barthel Index 92.27 \pm 12.46 , MMSE $22.27 \pm 3.17$, GS $0.84 \pm 0.24$ ). Linear regression models showed significant associations of: SDMT with GS $(\beta=22.10, p<0.001)$, SPPB $(\beta=1.31, \mathrm{p}<0.05)$, F8WT time $(\beta=-0.53, \mathrm{p}<0.05)$ and F8WT steps $(\beta=-0.59, \mathrm{p}<0.05)$; MoCA with SPPB $(\beta=0.58$, $\mathrm{p}<0.05)$ and DT-GS $(\beta=8.17, \mathrm{p}<0.05)$. Verbal fluency of MoCA (VF-MoCA) was associated with GS $(\beta=8.28, \mathrm{p}<0.01)$, SPPB $(\beta=0.57, p<0.01)$ and DT-GS $(\beta=10.45, p<0.001)$. No significant equation was found for MMSE. After adjusting for age, only associations of SDMT with GS, VF-MoCA with GS, and SPPB with DT-GS remained significant.

Key conclusions: Our findings support an association between executive function and GS, but traditional linear physical performance measures appear superior to more challenging tasks. We hypothesize that F8WT might be more influenced by gait impairments because of the curved pathway. Hence, we need future research with larger study samples to better investigate this aspect.

\section{P-623}

Reliability and validity of Rowland Universal Dementia Scale (RUDAS) in the diagnosis of mild dementia in Turkish population

Gizem Ayan ${ }^{1}$, Ceyda Afacan ${ }^{2}$, Burc Cagri Poyraz ${ }^{3}$, Onur Bilgic ${ }^{3}$, Suna $\mathrm{Avci}^{4}$, Hakan Yavuzer ${ }^{4}$, Mehmet Yuruyen ${ }^{5}$, Burak Ayan, Deniz Suna Erdincler ${ }^{4}$, Alper Doventas ${ }^{4}$

${ }^{1}$ Health Sciences University, Okmeydani Education and Research Hospital, Department of Internal Medicine, Istanbul, Turkey, ${ }^{2}$ Mimar Sinan Fine Arts University, Institute of Science and Technology, Department of Statistics, Istanbul, Turkey, ${ }^{3}$ Istanbul University Cerrahpasa Medical Faculty, Department of Psychiatry, Istanbul, Turkey, ${ }^{4}$ Istanbul University Cerrahpasa Medical Faculty, Department of Internal Medicine, Geriatric Medicine, Istanbul, Turkey, ${ }^{5}$ Health Sciences University, Bakirkoy Dr Sadi Konuk Education and Research Hospital, Department of Internal Medicine, Geriatric Medicine, Istanbul, Turkey, ${ }^{6}$ Istanbul Bilim University, Department of Anesthesiology, Istanbul, Turkey

Background: Novel screening tests are needed to top up lacking parts of current tests. Aim of our study is, to validate the Turkish version Rowland Universal Dementia Scale (RUDAS).

Methods: 140 patients over 65 years of age $(70$ control/70 mild dementia) were included. Power of cohort was over $80 \%$. Diagnosis was supported by DSM V criteria in all patients. In 79 patients neuropsychometric tests were applied. RUDAS, was translated into Turkish, applied by the researcher. RUDAS was applied in 2 days to determine test-retest reliability. Results were compared with MMSE; reliability, validity, statistical values, factors affecting the results were determined.

Results: Cohorts were distributed homogenously. Time validity was verified. MMSE was correlated wtih results of RUDAS with $45,3 \%$ in control, $73 \%$ in dementia group. Test was applied approximately in 5 min. Factor weights varied $0.44-0.81$ and construct was verified as 6 item scoring system. When 25 was cut-off point, sensitivity, specificity, PPV, NPV were as follows respectively, $92.86 \%, 92.86 \%$, $92.9 \% ; 92.9 \%$. Content validity index was determined by 7 specialists as $100 \%$. Cronbach's alpha was 0.692 , Test-retest reliability (ICC $=$ 0.987) was determined. Results were not affected by educational, immigrant status, language used; however age, gender have significant effect.

Conclusion: As a result of increased migration trend, screening tests became less reliable. Our study showed, Turkish version of RUDAS is valid, reliable that could be used in mild dementia patients from diverse populations while education, immigrant status, preferred language have no effect on results.

\section{P-624}

Roodkapje (Little red riding hood): the development of a gamified application as conversation facilitator for people with dementia and their informal carers

\section{Kiki Spanjers ${ }^{1}$, Sophia de Rooij ${ }^{1}$ \\ ${ }^{1}$ University Medical Center Groningen, University of Groningen, Groningen, The Netherlands}

Together with the diagnosis dementia, many things in daily life change or will change quickly. These changes might lead to issues you would like to discuss together to anticipate, but are sometimes difficult to discuss (e.g. cooking, personal hygiene, inheritance, and preferences for future care). In order to support people in having these conversations, the Dutch application Roodkapje (Little red riding hood) was developed. Roodkapje is a so called gamified application that is designed as a conversation facilitator for people with dementia and their informal carers. Grandmother comes across different topics, depicted in such a manner that players are encouraged to reflect and start the conversation on their own situation. Roodkapje was developed in collaboration with older persons and their relatives, both with and without dementia. The development process of applications as this is often complex, that is why we would like to share our learned lessons regarding content production, usability testing and app development. We used literature, surveys, and a focus group to collect the most important and difficult to discuss issues. During the development process older adults played prototypes of the game in order to provide us with feedback on user friendliness and use of the game as conversation facilitator. This feedback gave us insight on how to develop an application for older adults with dementia; we started the app as a serious game, but this did not fit the target audience. The current application is an interactive tool to facilitate conversations on changes due to dementia. 


\section{P-625}

Helicobacter pylori infection is associated with an increased incidence of dementia in a cohort population study of old farmers

Hugo von Campe ${ }^{1}$, Karine Peres ${ }^{2}$, Luc Letenneur ${ }^{3}$, Morgane Linard ${ }^{3}$, Catherine Helmer ${ }^{3}$, Philippe Lehours ${ }^{1}$, Francis Mégraud ${ }^{1}$, Jean-François Dartigues ${ }^{3}$, Claire Roubaud Baudron ${ }^{4}$

${ }^{1}$ INSERM U1053, University of Bordeaux, Bordeaux, France, ${ }^{2}$ ISPED, INSERM U1219, Bordeaux Population Health Research Center, University of Bordeaux, Bordeaux, France, ${ }^{3}$ ISPED, INSERM U1219, Bordeaux Population Health Research, University of Bordeaux, Bordeaux, France, ${ }^{4}$ INSERM 1053, Geriatrics Department, University of Bordeaux, Bordeaux, France

Context: Alzheimer's disease (AD) is a chronic neurodegenerative disease and the first cause of dementia in developed countries. A new theory has emerged and concerns the possible impact of infectious agents on AD course. Helicobacter pylori, responsible for a lifelong gastritis, may be involved in the pathogenesis of AD.

Objective: To determine whether $H$. pylori infection increases the risk of dementia (including AD), possibly by cerebrovascular lesions. Participants: Prospective community-based cohort AMI of 689 former farmers aged 65 and older, living in the southwest of France and followed from 2007 to 2017.

Measurements: A descriptive and comparative analysis was performed at baseline according to H. pylori status (serology). Then Cox proportional hazard model was done to determine factors associated with dementia incidence during 10 years. Brain MRI was performed in order to investigate cerebrovascular burden regarding infectious status in a subsample of 260 volunteers.

Results: At baseline, 200 (29.0\%) subjects were infected by H. pylori. They were more cognitively impaired compared to non-infected ones. After 10 years of follow-up, 90 incidents cases of dementia were diagnosed. After controlling for age, sex, educational level, Apolipoprotein E4 status, wine and cardiovascular risks factors, $\mathrm{H}$. pylori was associated with an increased incidence of dementia ( $\mathrm{HR}=1.70, \mathrm{P}=0.03)$. However, white matter hyperintensity (i.e. cerebrovascular burden) was not increased in case of infection.

Conclusion: This study provides more evidence of an association between $\mathrm{H}$. pylori and dementia, especially with AD. But our hypothesis that the underlying mechanism was based on brain vascular damages was not confirmed.

\section{P-626}

Influence of memory disorders perceptions on quality of life in people with memory complaints

Anaïck Besozzi ${ }^{1}$, Amélie Coquelet ${ }^{1}$, Adeline Dreyer ${ }^{1}$, Elisabeth Spitz ${ }^{2}$, Christine Perret-Guillaume ${ }^{1}$

${ }^{1}$ University Hospital of Nancy, Nancy, France, ${ }^{2}$ EA4360 APEMAC, University of Lorraine, Lorraine, France

The alteration of the quality of life (QoL) in people suffering from Alzheimer's disease is well documented in literature. It seems that QoL is also altered in mild cognitive impairment and subjective memory complaint. In the absence of loss of autonomy in these pathologies, the quality of life appears to be influenced by anxiety and depression. Little is known about QoL before diagnosis, at the beginning of care process. Our goal was to study the influence of memory problems perceptions on QoL in people expressing memory complaints before diagnosis (at the first visit at a Memory Clinic). We also control factors known to influence QoL: anxiety, depression and cognitive decline. One hundred fifty six persons expressing memory complaints were recruited at the Memory Clinic of the University Hospital of Nancy, France (mean age: 73 years; sex ratio: 99 female). Correlation Analysis, mean comparisons and regressions were carried out. Results show that, in a population expressing memory complaints without diagnosis, QoL does not seem to be influenced by anxiety, depression or cognitive decline. However, negative perceptions of memory disorders, such as a low personal control on memory loss, affect QoL, especially for social domains: if an individual thinks he cannot control his memory problems, then he will be less committed with his social life. Given the importance of social activities in maintaining cognitive abilities and quality of life, providing advises and mnemonic techniques in psychoeducational intervention could improve control on memory problems and reduce the risk of social withdrawal.

\section{P-627}

Good outpatient services delay nursing home admission of people with dementia

Dyntar Daniela ${ }^{1}$, Geschwindner Heike ${ }^{2}$, Meier Andreas ${ }^{3}$, Gmünder Regula $^{3}$, Negatsch Siegfried ${ }^{4}$, Schelling Hans Rudolf ${ }^{5}$, Theill Nathan ${ }^{6}$, Wettstein Albert ${ }^{7}$

${ }^{1}$ Research Assistant Medical Service of the City of Zurich, Switzerland, ${ }^{2}$ Clinical Research Assistant, Pflegezentren, Zürich, Switzerland, ${ }^{3}$ Senior Consultant Medical Service of the City of Zurich, Switzerland, ${ }^{4}$ RAI-NH Representative Pflegezentren, Zürich, Switzerland, ${ }^{5}$ Managing Director, Center

Introduction: The city of Zurich emphasizes outpatient services with diagnostics, treatment, medical and social care and counselling for patients with dementia and their formal or informal caregivers. There are three memory clinics, many day-care-centers offering respite care as well as increasing dementia specialisation of homecare services (Spitex). In 2008 the new service Home-visit SiL was launched. A team of specialised nurses provides a diagnostic assessment and specific counselling at the people's homes. They offer case-management and organize care for patients without a close social net. The goal of this service is to help people with dementia to stay longer at home. Appropriate outpatient services should delay the nursing home admission. This study measured the state of dementia and other data at patient's admission to two nursing homes in Zurich and compares it with data of RAI-NH MDS (Resident Assessment Instrument Nursing Home Minimum Data Set) collected at 354 Swiss nursing homes.

Methods: The data i.e. the scores of MMSE (Mini Mental State Examination) and CPS (Cognitive Performance Scale) at nursing home admission in the years 2009, 2010 and 2013 have been analysed retrospectively $(\mathrm{n}=375)$.

Results: The results show higher CPS ( $\mathrm{M}=2.88$ vs. 3.06$)$ and lower MMSE-Scores $(M=15.43$ vs. 12.67) in 2013 than in 2009 at the moment of admission. These results differ from other nursing homes in Switzerland $(\mathrm{N}=27,987)$ where CPS-scores decline. This shows a later admission.

Conclusion: Hence the appropriate outpatient services for people with dementia in Zurich seem to delay nursing home admission successfully.

\section{P-628}

Development of an integrated care pathway for people with dementia availing of acute care services

Siobhan M. Kennelly ${ }^{1}$, Susan O'Reilly ${ }^{1}$ 
${ }^{1}$ Connolly Hospital, Dublin, Republic of Ireland

Background: In Ireland, Persons living with dementia (PlwD) account for approximately $25 \%$ of acute hospital admissions. Acute care services are often fragmented with little integration between inhouse teams, ambulatory or community services, resulting in poorer care outcomes for PlwD, including deskilling and re-admissions. This project developed a person-centred integrated Dementia care pathway (IDCP) for PlwD availing of services. The pathway was developed over a 3-year period (2015-2017) and was governed by a consortium of key stakeholders.

Method: Using Quality Improvement methodology and PDSA cycles, project activities were supported by the development of a Memory Assessment and Support Hub, accessed from various entry points on the pathway. Activity evaluation consisted of data capture and review, interviews, focus groups and clinical audit.

Findings: Pathway activities focused on: dementia identification/diagnosis ( $\mathrm{n}=376$ new diagnosis), $(\mathrm{n}=1006$ assessments/reviews), ( $\mathrm{n}=1250$ signposting/post diagnostic supports), crisis intervention/ appropriate acute hospital avoidance $(\mathrm{n}=181)$, enhanced inpatient care ( $\mathrm{n}=1013$ assessment/reviews), $(\mathrm{n}=73$ Dementia/Delirium Care Bundle (DDCB)), supported discharge $(n=372)$, and targeted staff education $(\mathrm{n}=902)$. Evaluation indicates improvements in personcentred inpatient care delivery, timely access to diagnostics, crisis management and post diagnostic supports and enhanced hospital/community integration.

Conclusion: This flexible IDCP has different entry points for the PlwD depending on their individual needs at a given point in time. This acknowledges the complexity of person-centred care delivery for PlwD. Many challenges exist in sustaining the IDCP. Most importantly it requires a culture change at all levels of the organisation, for which educational initiatives and consortium governance is essential. Further targeted research is needed.

\section{P-629}

Neuropsychological performance in elderly people with diabetes mellitus type 2. DIABDEM Project: preliminar results of a Chilean sample

\author{
Agnieszka Bozanic Leal ${ }^{1}$, Francesc Formiga ${ }^{2}$, Pablo Toro ${ }^{3}$ \\ ${ }^{1} \mathrm{PhD}$ student, Medicine and Translational Research, Barcelona \\ University, Barcelona, Spain, ${ }^{2}$ Geriatric Department, Bellvitge \\ University Hospital, Barcelona University, Barcelona, Spain, \\ ${ }^{3}$ Department of Psychiatry, Faculty of Medicine, Pontificia \\ Universidad Católica de Chile, Santiago, Chile
}

Introduction: Cognitive disorders have been shown to be associated with type 2 diabetes mellitus (T2DM). This study will assess differences in neuropsychological performance in Chilean people with and without T2DM.

Methods: The sample compared 33 subjects with T2DM with 67 control participants aged $65-80$ years, community-dwelling, divided according years of education. The neuropsychological battery was designed to examine attention, verbal and visual memory, language, information processing speed, and executive functions.

Results: Mean age was 71.2 \pm 3.9 years, $66 \%$ women and a mean of $9.5 \pm 4.1$ years of education. T2DM subjects had significantly lower scores than subjects without T2DM in Addenbrooke Cognitive Examination Revised ( $p<0.0001)$, Pfeiffer test $(p<0.0003)$, Digit Span (Forward $p<0.0091$; Backward $p<0.0001$ ), Total Free Recall from the Free and Cue Selective Reminding Test ( $p<0.0101)$, ReyOsterrieth Complex Figure-copy $(\mathrm{p}<0.0001)$ and delayed reproduction $(\mathrm{p}<0.0004)$, Boston Naming Test $(\mathrm{p}<0.0001)$, Trailmaking Test A $(\mathrm{p}<0.0001)$, Trail-making Test $\mathrm{B}(\mathrm{p}<0.0001)$,
Semantic fluency (Animals) $(p<0.0001)$, Fonologic fluency (F) $(\mathrm{p}<0.0001)$, Digit-Symbol test $(\mathrm{p}<0.0001)$ and Similarities test $(\mathrm{p}<0.0001)$.

Key conclusions: Chilean subjects with T2DM presented cognitive dysfunction compared with the rest in screening cognitive performance, attention, language, verbal and visual memory, information processing speed and executive functions. This difference is even more significant when involve education years category.

\section{P-630}

Risk factors related to developing cognitive disorders in elderly people with diabetes mellitus type 2. DIABDEM Project: results of daily habits of a Chilean sample

Agnieszka Bozanic Leal ${ }^{1}$, Francesc Formiga ${ }^{2}$, Pablo Toro ${ }^{3}$

${ }^{1} \mathrm{PhD}$ student Medicine and Translational Research, Barcelona University, Barcelona, Spain, ${ }^{2}$ Geriatric Department, Bellvitge University Hospital, Barcelona University, Barcelona, Spain, ${ }^{3}$ Department of Psychiatry, Faculty of Medicine, Pontificia Universidad Católica de Chile, Santiago, Chile

Introduction: There is a well known association between type 2 diabetes mellitus and cognitive disorders. Daily habits (diet, sleep, physical activities, etc.) may have a decisive role in this interaction. This study will assess the daily habits in elderly Chilean people with and without T2DM.

Methods: The sample compared 33 subjects with T2DM with 67 control participants aged 65-80 years, community-dwelling, divided according years of education. The daily habits battery was designed to examine dietary, physical activity, perform in activities of daily living, sarcopenia, fragility, sleep, and depression.

Results: Mean age was $71.2 \pm 3.9$ years, $66 \%$ women and a mean of $9.5 \pm 4.1$ years of education. T2DM subjects had significantly lower scores than subjects without T2DM in proper dietary habits (Questionnaire of Mediterranean Diet Adherence $p<0.0072)$ and type of physical activities (Rapid Assessment of Physical Activities (RAPA) $\mathrm{p}<0.004)$.

Key conclusions: In this sample, subjects with T2DM presented different daily habits in comparison to subjects without T2DM. This could increase the risk of developing a cognitive disorder in future.

\section{P-631}

Alcohol use and abuse: old before your time

A. Medioli ${ }^{1}$, E. Palumbo ${ }^{1}$, W. Colosio ${ }^{1}$, L. Arcangeli ${ }^{1}$, L. Scaglia $^{1}$

${ }^{1}$ Geriatric Acute Care Unit F. Richiedei, Gussago, Italy

Introduction: The over 65 people with high alcohol consumption show a worsening of short and long term cognitive skills; alcohol intake in the elderly is associated with more frequent memory and encephalopathy deficits compared to the general population.

Methods: A comparative study was carried out by comparing the data related to over 65 inpatients of a alcohol rehab centre to patients admitted to a geriatric acute care unit at Fondazione Richiedei. We have considered scales related to cognitiveness (evaluating MMSE) and possible encephalopathies in the two patient clusters.

Results: The data refer to patients admitted from 10/1/10 to 28/2/18. Out of 1145 patients admitted for alcoholism, 76 (6.63\%) were over 65 . Of these $46(60.52 \%)$ had encephalopathy (6 vascular, 16 cortical atrophy, 24 mixed); 30 (39.47\%) cognitive impairment patients, 20 $(26.31 \%)$ cognitive impairment associated with encephalopathy. Of 
these 1145 patients (of all ages) $86(7.51 \%)$ had cognitive impairment. Out of 5149 patients admitted to the geriatric unit $65(1.26 \%)$ needed alcohol-based detoxification. Of these $64(98.46 \%)$ had cognitive deficits, 48 (73.84\%) had encephalopathy.

Keyconclusions: The data collected agree with the data of literature and show how the use of alcohol inflicts cognitive performance regardless of the personal abilities of each and levels the differences that, constitutionally, individuals can present. In alcoholics there is an anticipation of the reduced cognitive function that normally occurs with advancing age. Not only the elderly are more likely to develop cognitive deficiency or encephalopathies, but also non-over 65 alcoholics who will become the elderly of tomorrow.

\section{P-632}

The kynurenine pathway and cognitive performance in community-dwelling older adults. The Hordaland Health Study

Jan Erik Nordrehaug ${ }^{1}$, Grethe S. Tell ${ }^{2}$, Adrian McCann ${ }^{3}$, Per Magne Ueland $^{3}$, Oivind Midttun ${ }^{3}$, Klaus Meyer ${ }^{3}$, Christian A. Vedeler ${ }^{4}$, Dag Aarsland $^{5}$, Helga Refsum ${ }^{6}$, A. David Smith ${ }^{7}$, Lasse Melvaer Giil ${ }^{1}$, Ottar Nygård ${ }^{8}$

${ }^{1}$ Department of internal medicine, Haraldsplass Deaconess Hospital, Bergen, Norway, Institute of Clinical Science, University of Bergen, Norway, ${ }^{2}$ Division of Mental Health, Department of Global Public Health and Primary Care, University of Bergen, Norway

Introduction: Tryptophan, its downstream metabolites in the kynurenine pathway and neopterin have been associated with inflammation and dementia. We aimed to study the associations between plasma levels of these metabolites and cognitive function in community-dwelling, older adults.

Methods: This cross-sectional study included 2174 participants aged 70-72 years of the community-based Hordaland Health Study. Tryptophan, kynurenine, neopterin and eight downstream kynurenines were measured in plasma. Kendrick Object Learning Test (KOLT), Digit Symbol Test (DST) and the Controlled Oral Word Association Test (COWAT) were all outcomes in standardized Zellner's regression. The Wald test of a composite linear hypothesis of an association with each metabolite was adjusted by the Bonferroni method. Age, education, sex, glomerular filtration rate, smoking, diabetes, pyridoxal 5-phosphate, C-reactive protein and depressive symptoms were considered as potential confounders.

Results: Higher levels of the kynurenine-to-tryptophan ratio (KTR) and neopterin were significantly associated with poorer, overall cognitive performance $(\mathrm{p}<0.002)$. Specifically, KTR was negatively associated with $\operatorname{KOLT}(\beta-0.09, \mathrm{p}=0.001)$ and COWAT $(\beta-0.08$, $\mathrm{p}=0.024)$, but not with DST $(\beta-0.03, \mathrm{p}=0.139)$. This pattern was also seen for neopterin (KOLT: $\beta-0.08 ; \mathrm{p}=0.001$; COWAT: $\beta$ $-0.06, p=0.010$; DST: $\beta-0.01, p=0.709)$. The associations were not confounded by the examined variables. No significant associations were found between the eight downstream kynurenines and cognition.

Conclusion: Higher KTR and neopterin levels, biomarkers of monocyte activation, were associated with reduced cognitive performance, implying an association between the innate immune system, memory and language.
P-633

Intracerebral infection with Streptococcus pneumoniae does not influence medium-term spatial memory and learning in a mouse model of Alzheimer's disease

Sandra Schütze ${ }^{1,2}$, Benedikt Kellert ${ }^{1}$, Lena Rollwagen ${ }^{1}$, Stephanie Bunkowski ${ }^{1}$, Roland $\mathrm{Nau}^{1,3}$

${ }^{1}$ Institute of Neuropathology, University Medical Center Göttingen, Gottingen, Germany, ${ }^{2}$ Department of Geriatrics, AGAPLESION

Frankfurter Diakonie Kliniken, Frankfurt, Germany, ${ }^{3}$ Department of Geriatrics, Evangelisches Krankenhaus Göttingen-Weende, Germany

Introduction: In patients with Alzheimer's disease (AD), bacterial infections are often associated with a cognitive decline. To elucidate the underlying mechanisms for this clinical phenomenon, we assessed the effect of an intracerebral (i.c.) infection with Streptococcus pneumoniae type 3 (SP3) in a mouse model of AD.

Methods: Tg2576+/- mice (11-14 months) received an i.c. injection of either $1 \times 104$ CFU live SP3 $(n=47)$ or saline $(n=30)$, followed by antibiotic treatment with ceftriaxone starting $22 \mathrm{~h}$ post infection (p.i.) $2 \times /$ day for 5 days. Mice were monitored for 4 weeks p.i. for clinical status, weight, motor functions (rotarod and tight rope test) and neuropsychological status including spatial memory and spatial learning using the Morris water maze. $\beta$-Amyloid (A $\beta$ ) 1-40 and $1-42$ loads in mouse brains were quantified by ELISA.

Results: I.c. infection with SP3 followed by antibiotic treatment led to a high mortality of $\mathrm{Tg} 2576+/-$ mice $(53 \%)$, and a transient weight loss during the acute phase of the infection. Motor functions, spatial memory and learning as well as $\mathrm{A} \beta$ loads of surviving $\mathrm{Tg} 2576+1-$ mice were not affected by i.c. SP3 infection during 4 weeks p.i.

Key conclusions: In contrast to our previous study in $\mathrm{Tg} 2576+/-$ mice in which i.c. E. coli infection led to impaired spatial learning 4 weeks p.i., here, we did not detect any influence of i.c. SP3 infection on medium-term spatial memory and learning. This indicates that the disease-modulating effect of bacterial infections on cognition in $\mathrm{AD}$ is pathogen-specific and less pronounced when fast and adequate antibiotic treatment is performed.

\section{P-634}

Prevalence of cognitive impairment among elderly patients with and without memory complaints

O. N. Tkacheva ${ }^{1}$, N. N. Yakhno ${ }^{2}$, N. K. Runikhina ${ }^{1}$, E. A. Mkhitaryan ${ }^{1}$, V. S. Ostapenko ${ }^{1}$, N. V. Sharashkina ${ }^{1}$, M. A. Cherdak ${ }^{1}$

${ }^{1}$ Pirogov Russian National Research Medical University, Russian Clinical and Research of Gerontology Center, 16, 1st Leonova Str., Moscow, 129226, Russia, ${ }^{2}$ Scientific-Research Department of Neurology of I.M. Sechenov First Moscow State Medical University, Moscow, Russia

Introduction: Cognitive impairment (CI) is the most common neurological problem among elderlies. According to the present increase of the world population life expectancy, the number of elderly and senile people is increasing too, leading to the growth of the proportion of patients with dementia and non-dementia CI.

Methods: 258 patients (59 men (23\%) and 199 women (77\%); mean age $75.63 \pm 5.94$ years) were examined during their visit for whatever reason in the primary care settings. Patients underwent a neuropsychological examination, which included Montreal Cognitive Assessment Test (MoCA-test), Mini Mental State Examination (MMSE), Clock Drawing Test (CDT), Trail Making Test, part A 
(TMT-A), the 12th Word Recall Test and evaluation of specific memory complaints presence.

Results: $83.4 \%$ of the examined patients pointed a decline in memory functioning or mental performance. However, based on the results of neuropsychological examination among patients without specific memory complaints only $6.6 \%$ (17) had no CI. $22 \%$ of patients without specific complaints were diagnosed with subtle CI, $35 \%$ had mild cognitive impairment (MCI) and $8 \%$ had dementia. In general, cognitive disorders without dementia were detected in $76 \%$ of patients who did not complain on memory and mental functions.

Key conclusion: The study showed a rather high prevalence of $\mathrm{CI}$ among elderly patients. Specific memory complaints could be absent in a wide part of elderly population with no-dementia cognitive disorders, and therefore all elderly patients, especially at the age of 75+, should be routinely screened for CI.

\section{P-635}

Prevalence of cognitive impairment in the elderlies in primary care settings

O. N. Tkacheva ${ }^{1}$, N. N. Yakhno ${ }^{2}$, N. K. Runikhina ${ }^{1}$,

E. A. Mkhitaryan ${ }^{1}$, V. S. Ostapenko ${ }^{1}$, N. V. Sharashkina ${ }^{1}$, M. A. Cherdak

${ }^{1}$ Pirogov Russian National Research Medical University, Russian Gerontology Clinical Research Center, 16, 1st Leonova Str., Moscow, 129226, Russia, ${ }^{2}$ Scientific-Research Department of Neurology of I.M. Sechenov First Moscow State Medical University, Moscow, Russia

Introduction: Cognitive impairment (CI) is one of the most common types of geriatric syndromes. We investigated prevalence of the cognitive disorders in elderly and senile patients in primary care settings.

Methods: 258 patients (23\% men and $77 \%$ women; mean age $75.63 \pm 5.94$ yrs) were examined. Patients underwent a neuropsychological examination, which included Montreal Cognitive Assessment Test (MoCA-test), Mini Mental State Examination (MMSE), Clock Drawing Test (CDT), Trail Making Test, part A (TMT-A), the 12th Word Recall Test. Also patients were assessed for educational and emotional status (15-item Geriatric Depression Scale).

Results: Mean MoCa score was 23.64 \pm 3.62, MMSE 27.27 \pm 2.29 , CDT $1.7 \pm 8.18$, TMT-A-75.62 $\pm 44.24 \mathrm{~s}$. CI was absent only in $17.1 \%$ of patients. The major part of patients diagnosed with cognitive disorders (49.6\%) consisted of patients with mild cognitive impairment, additional $26 \%$ of patients had subtle cognitive impairment. In total, non-dementia CI were detected in $76 \%$ of cases. There was no difference in depression prevalence between patients with CI of any type and no-CI. Education status significantly $(\mathrm{p}<0.05)$ negatively correlated with CI severity.

Key conclusions: Results of our study suggest of the very high prevalence of non-dementia CI among elderly patients in general population, which challenges development of more intensive preventive measures for dementia. CI could not be explained by coexistence of emotional disorders.

\section{P-636}

Association of chronic pain, affective and cognitive disorders in elderly patients O. N. Tkacheva ${ }^{1}$, Y. V. Kotovskaya ${ }^{1}$, T. M. Manevich

E. A. Mkhitaryan

${ }^{1}$ Pirogov Russian National Research Medical University, Russian Gerontology Clinical Research Center, 16, 1 st Leonova str., Moscow, 129226, Russia

Introduction: Chronic pain syndromes in aged patients substantially deteriorate of their quality of life and foster of cognitive decline in later life. We analyzed the combination and comorbid cognitive and affective disturbances in patients of the in-patient neurologic department for 1-month period.

Methods: Study included 140 patients (123 women, 17 men, mean age $76 \pm 13.27$ years), hospitalized on chronic pain in neurologic department for 1-month period. The survey included analysis of complains, anamnesis, somatic and neurologic status, DN4 inventory, Mini-cog, clock drawing test, visual analogue scale (VAS) of pain, quantity of pain days measure and pain periods during of the day measurement and 15-item geriatric depression scale (GDS). Patients with cancer pain were excluded.

Results: 95 patients $(67.96 \%)$ have muscular-skeletal pain, 33 patients $(23.57 \%)$ have neuropathic pain, 12 patients $(8.57 \%)$ have mixed pain. 63 patients $(45 \%)$ have subtle cognitive impairment, 42 patients $(30 \%)$ have mild cognitive impairment, 10 patients $(7.1 \%)$ have mild dementia. 135 patients $(96,43 \%)$ have depressive disorders. Patients with cognitive decline and affective disorders had more pain days in the month and more pain duration.

Conclusion: comorbidity of chronic pain syndromes and affective and cognitive disorders can be associated with common pathophysiologic mechanisms. Chronic pain have influence on cognitive and affective disorders in aged patients, so the prompt pain treatment is essential.

\section{P-637}

Transferability and sustainability of effects of dual-task training in patients with dementia

Klaus Hauer ${ }^{1}$, Nele C. Lemke ${ }^{1}$, Christian Werner ${ }^{1}$, Stefanie Wiloth ${ }^{1}$

${ }^{1}$ Agaplesion Bethanien Hospital Heidelberg, Geriatric Center at the University of Heidelberg, Heidelberg, Germany

Introduction: Transferability of trained to untrained dual task (DT) performances has not been studied in persons with dementia (PWD). Objective: The study objective was to evaluate immediate and longterm transferability of DT performances in PWD.

Methods: Multi-morbid, older PWD $(\mathrm{n}=105)$ were included in a 10 -week randomized, controlled trial with the intervention group (IG) performing a DT-based training ('Walking and Counting') while the control group (CG) performed unspecific, low-intensity exercises. DT-performance was measured under 3 conditions: 'Walking and Counting' (trained), 'Walking and Verbal Fluency' (semi-trained) and 'Strength and Verbal Fluency' (untrained). Motor and cognitive performance was documented as single-task (ST) and DT at baseline (T1) after training (T2) and at follow up (T3).

Results: The trained DT 'Walking and Counting' was significantly improved for motor performances $(\mathrm{p}<0.001-0.011$, effect sizes as partial eta squared $\left.\eta^{2}=0.071-0.249\right)$ as well as cognitive performances $\left(p<0.001-0.047, \eta^{2}=0.044-0.295\right)$. Significant transfer 
effects were found for the semi-trained DT 'Walking and Verbal Fluency' in motor ( $\left.\mathrm{p}<0.001-0.016, \eta^{2}=0.068-0.150\right)$ and partly cognitive performances $\left(p=0.026, \quad \eta^{2}=0.059\right)$. No significant transfer effects were found for the completely untrained DT 'Strength and Verbal Fluency'. Effects sustained for the trained DT for most motor $\left(p=0.009-0.038, \eta^{2}=0.051-0.089\right)$ and partly cognitive outcomes $\left(\mathrm{p}<0.001, \eta^{2}=0.157\right)$. Transfer-effects could in parts be preserved for semi-trained DTs at follow up.

Conclusion: Study results document for the first time a transfer from trained to untrained DTs which partly sustained after follow up in patients with moderate stage dementia. With increasing difference in task conditions and time lap to intervention, transfer effects were reduced.

\section{P-638}

Evaluation of postural stability with clinical and laboratory tests in Alzheimer's disease patients

Hatice Caliskan ${ }^{1}$, Kezban Ulku Sahin ${ }^{2}$, Mine Baydan $^{3}$, Cemile Ozsurekci $^{1}$, Gozde Sengul Aycicek ${ }^{1}$, Arzu Demircioglu ${ }^{4}$, Pelin Unsal $^{1}$, Rana Tuna Dogrul ${ }^{1}$, Fatih Sumer ${ }^{1}$, Cafer Balci ${ }^{1}$, Mert Esme ${ }^{1}$, Songul Aksoy ${ }^{3}$, Nuray Kirdi ${ }^{2}$

${ }^{1}$ Hacettepe University, Faculty of Medicine, Department of Internal Medicine, Division of Geriatrics, Ankara, Turkey, ${ }^{2}$ Hacettepe University, Faculty of Health Sciences, Department of Physiotherapy and Rehabilitation, Ankara, Turkey, ${ }^{3}$ Hacettepe University, Ankara, Turkey,

Introduction: Gait and balance disturbances are seen more often in Alzheimer's disease patients, even in the early stage, than cognitively normal older people. We aimed to evaluate postural stability, gait and balance with clinical and laboratory balance tests in Global Deterioration Scale Stage 4 and 5 Alzheimer's disease patients and compare with cognitively normal patients.

Methods: We recruited 37 patients with Alzheimer's disease (AD) and 21 cognitively normal older people. Individuals with advanced stage dementia, hearing problems, severe arthritis, history of surgery in previous month, Parkinson's disease, stroke sequel, and major depression were excluded. Comprehensive geriatric assessment, Berg Balance Scale and Timed Up\&Go Test were performed to all participants. Fall history in previous year and blood tests results in the previous month were recorded. Walk across (WA), sit to stand (STS), tandem walk (TW) and step quick turn (SQT) tests were applied to all participants using computerized posturography, NeuroCom Balance Master (2011). SPSS 21.0 programme was used for statistical analysis. Results: Mean age was $76 \pm 5$ vs. $73 \pm 4$ years in $\mathrm{AD}$ and control groups, respectively. Twenty two of the AD patients and 12 of the controls were female. The walking speeds in WA and TW tests were slower in AD patients than the controls $(\mathrm{p}=0.005$ and $<0.001)$. WA-step width increased $(\mathrm{p}=0.034)$ and WA-step length decreased $(p=0.006)$ in the AD group. SQT-sway increased $(p=0.017)$ and SQT-time increased $(\mathrm{p}<0.001)$ in patients with AD. Regression analysis including age, vitamin B12, WA-step length, WA-step width, WA-speed, STS-sway velocity, SQT-turn sway, SQT-turn time, and TW-speed parameters showed that WA-step length $(\mathrm{OR}=1.05, \% 95$ CI $1.009-1.110, \mathrm{p}=0.02)$, SQT-turn sway $(\mathrm{OR}=0.95, \% 95 \mathrm{CI}$ $0.910-0.998, \quad \mathrm{p}=0.04)$, SQT-turn time $(\mathrm{OR}=0.33, \% 95 \mathrm{CI}$ $0.148-0.766, \mathrm{p}=0.01)$, STS-sway velocity $(\mathrm{OR}=0.45$, \%95 CI $0.219-0.963, \mathrm{p}=0.04)$, and $\mathrm{TW}$-speed $(\mathrm{OR}=1.14, \% 95 \mathrm{CI}$ $1.024-1.267, \mathrm{p}=0.01$ ) were independently associated with Alzheimer's disease.

Conclusion: Slower walking speed, higher turn sway and time show that patients with $\mathrm{AD}$ may have increased risk of falls risk even in early stages. The parameters associated with Alzheimer's disease independently from vitamin B12 levels and age may cause this outcome.

\section{P-639}

Association between day to day blood pressure variability and cognitive impairment in memory clinic patients AL Meendering ${ }^{1}$, RAA de Heus ${ }^{2}$, LM van den Ingh ${ }^{1}$, JAHR
Claassen $^{2}$

${ }^{1}$ Radboudumc, Department of Geriatric Medicine, Nijmegen, The Netherlands, ${ }^{2}$ Radboudumc, Department of Geriatric Medicine, Nijmegen, The Netherlands; Donders Institute for Brain, Cognition and Behaviour, Nijmegen, The Netherlands

Background: High blood pressure variability (BPV) might play a role in the association between hypertension and cognition. The main objective of this study is to gain more insight in the association between day-to-day BPV and cognitive impairment.

Design and method: In this cross-sectional study, 213 memory-clinic patients were included. They performed a duplo measurement of their blood pressure (BP) twice a day for 1 week. After averaging duplo measurements and removing day 1 the mean BP and coefficient of variation $(\mathrm{COV})$ were calculated. Patients were divided in three groups, based on clinical diagnosis by a geriatrician: group $1=\mathrm{pa}-$ tients without suspicion of neurodegenerative disease, group $2=$ mild cognitive impairment (MCI) or vascular cognitive impairment (VCI), group 3 = dementia (vascular or Alzheimer's disease). ANCOVA $(\mathrm{p}<0.05)$ was conducted to compare BPV $(\mathrm{COV})$ between the 3 groups, with correction for age and mean BP.

Results: COV was higher in patients with a diagnosis of dementia than in patients with MCI/VCI or without suspicion of neurodegenerative disease, $\mathrm{F}(2,210)=4198, \mathrm{p}=0.016$. However, after adjusting for age and mean systolic $\mathrm{BP}$, there was no significant difference in $\mathrm{COV}$ of systolic $\mathrm{BP}$ between the groups, $\mathrm{F}(2$, 208) $=1755, \mathrm{p}=0.175$.

Conclusion: In the current study we did not found evidence for an association between BPV and cognitive impairment. Follow-up of this population is interesting to explore the longitudinal association of BPV and cognition.

\section{P-640}

Tablet-based outpatient care for people with dementia: Guideline-based treatment planning, personalized disease management and network-based care

Sonia Lech ${ }^{1}$, Johanna Nordheim ${ }^{1}$, Paul Gellert ${ }^{1}$, Julia O SullivanSolte $^{1}$, Jan Voigt-Antons ${ }^{2}$

${ }^{1}$ Charité-Universitätsmedizin Berlin, Berlin, Germany, ${ }^{2}$ Technische Universität Berlin, Berlin, Germany

Background: The current S3 Dementia Guideline is often not enough implemented in the care practice. Accordingly, the outpatient care of people with dementia is often poor. Thus, integrated, cross-sectoral and guideline-oriented approaches are needed in order to improve the care and life quality of people with dementia. Information and communication technologies (ICTs) such as tablets have great potential in this context, since they can support GPs and specialists in patient-centered and guideline-oriented diagnostics and therapy. Further, ICTs enable patients, their caregivers and other carers to implement effective therapeutic approaches in the home environment 
and can contribute to the improvement of intra and intersectoral health care. The objectives of the presented project "DemTabTablet-based outpatient care of people with dementia: guidelinebased treatment planning, individual health management and networking" are the development and scientific evaluation of a tablet based intervention as an improvement of outpatient dementia care.

Method: Within the framework of DemTab, a qualitative feasibility study will be carried out to test a tablet-based intervention in everyday outpatient care. The feasibility study takes into account the needs of patients with dementia and their caregivers as well as GPs in order to develop, document and systematically evaluate a tablet-based intervention (mixed methods approach). In a second step, the developed tablet-based intervention will be tested. In a cluster randomized control study, GPs $(\mathrm{N}=20)$ and their patients with dementia together with their caregivers $(\mathrm{N}=204)$ will be randomly assigned to an intervention $(\mathrm{N}=102)$ and a control $(\mathrm{N}=102)$ group. Clinical outcomes will be measured at baseline, after 9-months and after 12 -months using standardized and validated measurements as well as continually using the tablet and medical records data.

Results: The study design of DemTab and the first results of the feasibility study will be presented. Based on the feasibility study, first contents and applications of the tablet will be presented and discussed.

\section{P-641}

Dietary intake of community-dwellers by cognitive performance cluster

Belina Rodrigues ${ }^{1}$, Carlos Portugal-Nunes ${ }^{1}$, Teresa Castanho ${ }^{1}$, Nuno Sousa ${ }^{1}$, Nadine Correia Santos ${ }^{1}$

${ }^{1}$ ICVS, ICVS/3B's, CCA, Guimarães, Portugal

Introduction: Ageing is a critical issue worldwide. As one ages, several physiological changes take place, being cognitive decline one of the most meaningful for older adults. Once cognitive decline is influenced by dietary variables, we explored the patterns of dietary intake between poor and good cognitive performers.

Methods: A group of 50 years or older community-dwellers from Northern Portugal were assessed in two moments (M1 and M2) 18 months apart. The dietary intake was evaluated through 24-h dietary recall, and nutrient density computed for macronutrients as percentage of total energy value and micronutrients as nutrient unit/ $1000 \mathrm{kcal}$. Cognitive performance clusters were derived from a principal component analysis (PCA) comprising scores of a battery of neuropsychological tests. Cross-sectional and longitudinal statistical analyses were carried out resorting to independent t-tests, MannWhitney U test, PCA, binomial logistic regressions, paired t-tests and Wilcoxon Signed Rank Test.

Results: In the cross-sectional analysis, subjects whose total fat, monosaturated fat and saturated fat intakes were higher were more likely to be good performers ( $\beta=0.62, \mathrm{p}=0.02$ ). In the longitudinal analysis, despite energy intake has not changed over time, a significant decrease was recorded on carbohydrates and PUFA intake. At M2, poor performers reported a lower PUFA and Vit B9 intake, whereas good performers recorded a significative reduction in calcium, Vit E and several B vitamins intake. Overall, good performers stated a larger number of significant decreases.

Conclusion: These findings suggest that food patterns might be a better predictive factor of the cognitive performance than a set of nutrients.

\section{P-642}

Concomitant use of benzodiazepines and opioids in communitydwelling older people with or without Alzheimer's disease: a nationwide register-based study in Finland

Niina Karttunen ${ }^{1}$, Heidi Taipale ${ }^{2}$, Aleksi Hamina ${ }^{1}$, Antti Tanskanen ${ }^{3}$, Jari Tiihonen $^{4}$, Anna-Maija Tolppanen ${ }^{5}$, Sirpa Hartikainen ${ }^{6}$

${ }^{1}$ Kuopio Research Centre of Geriatric Care, University of Eastern Finland, Kuopio, Finland, ${ }^{2}$ Kuopio Research Centre of Geriatric Care, University of Eastern Finland, Kuopio, Finland, ${ }^{3}$ Department of Clinical Neuroscience, Karolinska Institutet, Solna, Sweden

Introduction: The use of benzodiazepines and related drugs (BZDRs) and opioids has been associated with serious risks in older persons, especially in individuals with Alzheimer's disease (AD). We investigated the prevalence and associated factors of concomitant use of these drugs among older people with or without AD.

Methods: We utilized data from the register-based Medication Use and Alzheimer's disease (MEDALZ) study, including all communitydwelling residents of Finland who received a clinically verified AD diagnosis between 2005 and $2011(\mathrm{n}=70,718)$ and their matched comparison persons without AD. After exclusion of individuals who were hospitalized throughout the follow-up, 69,353 persons with and 69,353 without $A D$ were included in this study.

Results: BZDRs were used by 28,475 (41.1\%) of those with and 24,506 $(35.3 \%)$ of those without AD. Prolonged ( $\geq 90$ days) concomitant use of BZDRs and opioids was more common among BZDR users without $\operatorname{AD}(\mathrm{N}=3936 ; 16.1 \%)$ than among those with $\mathrm{AD}(\mathrm{N}=2963 ; 10.4 \%)$. A shorter duration of concomitant use (1-89 days) revealed similar results, $\mathrm{N}=3821 ; 15.6 \%$ and $\mathrm{N}=3008 ; 10.6 \%$, respectively. Prolonged concomitant use of BZDRs and opioids was associated with female sex, low socioeconomic position, most of the common comorbidities and history of substance abuse or long-term benzodiazepine use. The most commonly used combinations were Z-drug $(31.7 \%)$ or benzodiazepine (29.9\%) with a weak opioid.

Key conclusions: Despite the recommendations and risks, the prevalence of concomitant BZDR and opioid use was common in older persons with or without AD. It is important to develop strategies to reduce unnecessary concomitant use of these drugs.

\section{P-643}

Food intake and cognition in older adults: baseline findings in FlaSeCo-trial

Suominen $\mathrm{MH}^{1,2}$, Salmenius-Suominen $\mathrm{H}^{2}$, Torvi $\mathrm{A}^{3}$, Jyväkorpi $\mathrm{SK}^{1,2}$, Hongisto S-M ${ }^{4}$, Tuukkanen $\mathrm{K}^{4}$, Pitkälä $\mathrm{KH}^{1}$

${ }^{1}$ Department of General Practice and Primary Health Care, University of Helsinki, Finland, ${ }^{2}$ Society for Gerontological Nutrition in Finland, ${ }^{3}$ Department of Food and Environmental Sciences, University of Helsinki, Finland, ${ }^{4}$ Fazer Group

Background: Quality of diet, fish and vegetable consumption have been associated with cognition among older people.

Methods: Older adults 65-75 years without chronic diseases took part in FlaSeCo-trial. At baseline participants filled 3-day food records. Use of vegetables, meat, fish and nutrient intakes from their diet, quality of life (15D) and depression (GDS30) were assessed. Cognition was assessed with SLUMS and Trail Making Test A and B (TMT). Blood lipids were measured.

Results: 104 (63\% women), mean 69 years participated. They were highly educated, mean 14.9 years (SD 3.6) and their health-related quality of life was good. Their mean energy intake was $1890 \mathrm{kcal}$ (SD 455), protein intake $77.2 \mathrm{~g}$ (SD 23) and total cholesterol 5.64 (SD 
0.68). Participants were divided in three groups according to their fish consumption: group $\mathrm{I}, \mathrm{n}=35(0 \mathrm{~g}$ fish/day), group II, $\mathrm{n}=34$ (3.3-51.2 g/day), group III, $\mathrm{n}=35(53.3-200 \mathrm{~g} /$ day). Intake of veg-

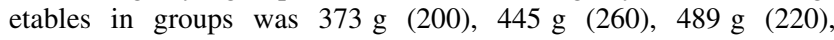
$\mathrm{p}=0.019$ and read meat 81 (72), 68 (94), 53 (45), $\mathrm{p}=0.072$. Intake of DHA in groups accordingly was $325 \mathrm{mg}$ (SD 437), $1172 \mathrm{mg}$ (1307), $3231 \mathrm{mg}$ (2337) $\mathrm{p}<0.001$ and EPA $868 \mathrm{mg}$ (2905), $2716 \mathrm{mg}(5246), 5956 \mathrm{mg}(5712) \mathrm{p}<0.001$. TMT B times in groups I, II and III were 100.9 (47.8), 96.1 (45.2), 82.8 (32.1), ( $\mathrm{p}=0.062)$ when adjusted for age, gender and education years.

Conclusions: There was positive association in fish consumption and cognition among healthy older adults with overall good quality of diet.

\section{P-644}

The promoting activity, independence and stability in early dementia (PrAISED) intervention: implementing an exercise programme in the voluntary sector and leisure industry

Martyn Harling ${ }^{1}$, Tahir Masud ${ }^{2}$, John Gladman ${ }^{3}$, Rowan Harwood ${ }^{4}$

${ }^{1}$ Division of Rehabilitation and Ageing, School of Medicine, The University of Nottingham, Nottingham, NG7 2UH, UK, ${ }^{2}$ Health Care of Older People, Nottingham University Hospitals NHS Trust, Queens Medical Centre, Nottingham, NG7 2UH, UK, Division of Rehabilitation and Ageing, School of Medicine, The University of Nottingham, Nottingham, NG7 2UH, UK, ${ }^{3}$ Division of Rehabilitation and Ageing, School of Medicine, The University of Nottingham, Nottingham, NG7 2UH, UK, Health Care of Older People,

Nottingham University Hospitals NHS Trust, Queens Medical Centre, Nottingham, NG7 2UH, UK, CLAHRC East Midlands, Nottingham, UK, ${ }^{4}$ Division of Rehabilitation and Ageing, School of Medicine, The University of Nottingham, Nottingham, NG7 2UH, UK

Introduction: The PrAISED intervention is a person-centred programme of exercise and daily activity aimed at maintaining independence and well-being for individuals with dementia. It was designed to be delivered by professionals in the UK National Health Service (NHS), but this approach is potentially expensive. This presentation focuses on a qualitative enquiry which considered if the intervention could be adopted in the voluntary or leisure industry sectors. Methods: The aim of the enquiry was to gain insights into whether factors linked to the 'inner setting' and 'individuals involved' sections of Damschroder's Consolidated Framework for Implementation Research (CFIR) might impact on delivering the PrAISED intervention outside the NHS. One-to-one interviews were conducted with three occupational therapists, two physiotherapists, a health care team manager and a local authority commissioning manager, all of whom had previous experience of working on projects involving the transition from service delivery in the NHS to the voluntary or leisure industry sectors.

Results: Whilst informants raised no absolute objections to delivering the PrAISED intervention outside the NHS, they felt that the challenge would be to provide an individualised programme, based on an assessment and supported by motivational strategies. This was considered to contrast with the group exercise and activity classes currently delivered in the voluntary or leisure sectors for individuals with dementia.

Key conclusions: The CFIR allowed the researchers to predict and plan for some of the issues which may surface when implementing PrAISED outside the constraints of the NHS or the requirements of a clinical trial.

\section{P-645}

Time course of changes in motor-cognitive exergame performance during task-specific training in patients with dementia: identification and predictors of early training response

C. Werner ${ }^{1}$, R. Rosner ${ }^{2}$, S. Wiloth ${ }^{3}$, N. C. Lemke ${ }^{4}$, J. M. Bauer ${ }^{1}$, K. Hauer $^{1}$

${ }^{1}$ Department of Geriatric Research, Agaplesion Bethanien Hospital Heidelberg, Geriatric Center at the Heidelberg University,

Heidelberg, Germany, ${ }^{2}$ Department of Radiological Diagnostics, Theresien Hospital Mannheim, Mannheim, Germany, ${ }^{3}$ Institute of Gerontology, Heidelberg University, Heidelberg, Germany, ${ }^{4}$ Network of Aging Research (NAR), Heidelberg University, Heidelberg, Germany

Introduction: Few studies suggested positive effects of exergaming on physical, cognitive, and psychological outcomes in patients with dementia (PWD). However, little is known about the training volume required to produce such positive effects. The study aim was to analyze the time course of changes in motor-cognitive exergame performances during a task-specific training program and to identify independent predictors of early training response (ETR) in PwD.

Methods: Fifty-six PwD participated in a 10-week, task-specific training program $(2 \times /$ week $)$ on an exergame-based balance training system (Physiomat ${ }^{\circledR}$ ), combining postural control tasks with cognitive tasks of a modified Trail-Making-Test. Physiomat ${ }^{\circledR}$ performance was assessed at baseline (T1), at training session 7 (TS7) and 14 (TS14), and after 20 training sessions (T2) as the time required to complete different Physiomat ${ }^{\circledR}$-Tasks (PT). Reliable change indices were used to identify early responders (T1-TS7). A multivariate logistic regression analysis was performed to determine predictors of ETR.

Results: For all PT, significant improvements were already observed from T1 to TS7 ( $\mathrm{p} \leq 0.001-0.006$ ). Performance in most PT did not significantly improve from TS7 to TS14 and from TS14 to T2. Across all PT, significant improvements ( $\mathrm{p} \leq 0.001-0.036)$ were found from TS7 to T2. Low baseline exergame performance, low performance in constructional abilities, and low dual-task performance predicted ETR. Conclusion: Substantial improvements in complex motor-cognitive exergame performances can be achieved within a surprisingly short task-specific intervention period in PwD. Patients with low baseline exergame performance and low visuospatial and divided attention abilities are more likely to improve early in the intervention.

\section{P-646}

Occurrence of newly diagnosed cognitive disorders during a systematic two-year follow-up after hip fracture

Roope Jaatinen ${ }^{1}$, Matti Viitanen ${ }^{2}$, Maria Nuotio ${ }^{1}$

${ }^{1}$ Seinäjoki Central Hospital, Seinäjoki, Finaland, ${ }^{2}$ Turku University Hospital, Turku, Finland

Introduction: Cognitive disorders are increasingly affecting the ageing populations with hip fractures another major concern. Cognitive disorders and dementia are common in hip fracture patients but little is known about the specific diagnoses of cognitive disorders emerging after hip fracture.

Methods: Consecutive $\geq 65$-year-old patients sustaining their first hip fracture between January 2010 and August $2015(\mathrm{n}=1165)$ in the Seinäjoki Central Hospital area (population 193,000) were systematically followed up for two years. Only patients without pre-fracture diagnosis of cognitive disorder were included $(n=831)$. The patients underwent a comprehensive geriatric outpatient assessment 
4-6 months post-hip fracture with diagnostic investigations of cognitive disorders based on the 2010 update of the National Care Guideline including internationally accepted diagnostic criteria. Data on diagnostic investigations and diagnoses were extracted manually from the electronic patient files by the research physician (RJ).

Results: Of the patients, $238(28.6 \%)$ had died before diagnostic investigation. A cognitive disorder was diagnosed in $184(22.1 \%)$ patients. Of these, Alzheimer's disease ( $A D, n=79,42.9 \%)$, vascular cognitive impairment (VCI, $\mathrm{n}=23,12.5 \%)$ and mixed type (AD + VCI, $\mathrm{n}=73,39.7 \%$ ) were the most common diagnoses. Less common cases were also identified (Lewy body disease, dementia with Parkinson's disease, alcohol-induced dementia, Fahr's disease, ALS dementia; $\mathrm{n}=9,4.9 \%$ ). At the two-year time-point diagnostic examinations were still ongoing in 52 patients $(6.3 \%)$.

Conclusions: Previously undiagnosed cognitive disorders are common among hip fracture patients. Specific diagnoses of cognitive disorders are important in planning the short- and long-term treatment scheme. A systematic follow-up is warranted to identify the new cases.

\section{P-647}

Low cognitive test score predicts 30-day mortality in hospitalised patients without known dementia

Filipa Quaresma ${ }^{1}$, Ana Sofia Pessoa ${ }^{1}$, Nicola Lovett ${ }^{2}$, Catherine Ashton $^{2}$, Sarah Smith ${ }^{2}$, Sarah T. Pendlebury ${ }^{1}$

${ }^{1}$ Centre for Prevention of Stroke and Dementia, Nuffield Department of Clinical Neurosciences, University of Oxford and Departments of Medicine and Geratology, John Radcliffe Hospital, Oxford, UK,

${ }^{2}$ Departments of Medicine and Geratology, John Radcliffe Hospital, Oxford, UK

Background: Delirium and undiagnosed dementia are prevalent in older patients and are associated with poor outcomes but diagnosis may be challenging in the acute hospital setting. In contrast, a short cognitive screening test is quick, identifies objective cognitive deficits and can be done by non-specialist staff. We therefore determined the predictive value of low cognitive test score for mortality in patients without known dementia.

Methods: Consecutive patients aged $>65$ years without known dementia admitted to a single acute medicine team over three 2-month periods $(2010,2012,2015)$ had on-admission abbreviated mental test score (AMTS, low score $<8$ ) and delirium screen with follow-up to 2 years using electronic medical records. Hazard ratios (HRs) for death were calculated by low versus normal cognitive score adjusted for age, sex, illness severity, and pre-morbid function.

Results: Among 495 patients (mean age/ $\mathrm{SD}=80.5 / 8.5$ years, 237 (47.9\%) male) 83 had pre-admission dementia diagnosis and 125 (25.2\%) had delirium. Among the 412 without dementia, 92 (22.3\%) had delirium and 106/286 (37.1\%) tested patients had low cognitive score. Adjusted HR for 30-day mortality was 6.5 (95\% confidence interval 1.3-13.4) for low cognitive score vs 3.0 (1.2-7.4) for delirium. Associations were non-significant thereafter on follow-up to 2 years after adjustment for confounders.

Conclusions: Low cognitive score is prevalent in older patients without pre-admission dementia diagnosis and is an independent predictor of 30-day mortality. Simple cognitive testing is more straightforward than dementia/delirium diagnosis and should be routine at first assessment to identify co-morbid cognitive disorder.

\section{P-648}

Evolution of oral-health and cognition in elderly patients with MCI

Dina Zekry ${ }^{1}$, Sabrina Maniewicz ${ }^{2}$, Martin Schimmel ${ }^{2,3}$, Murali Srinivasan $^{2}$, Elena Duvernay ${ }^{2}$, Gemma Gabriel $^{1}$, Chistophe Graf ${ }^{1}$, Gabriel Gold ${ }^{1}$, François R. Herrmann ${ }^{1}$, Frauke Müller ${ }^{1,2}$

${ }^{1}$ Dept of Internal Medicine, Rehabilitation and Geriatrics, Geneva University Hospitals, Geneva, Switzerland, ${ }^{2}$ Division of Gerodontology and Removable Prosthodontics, University Clinics of Dental Medicine, University of Geneva, Geneva, Switzerland, ${ }^{3}$ Division of Gerodontology, School of Dental Medicine, University of Bern, Bern, Switzerland

Objectives: to investigate the relationship between oral status and the conversion from Mild Cognitive Impairment (MCI) to dementia and to assess the evolution of dental status and chewing efficiency, as well its association with cognitive function.

Methods: prospective study with 5 years follow-up, amnestic MCI patients, $\geq 70$ years. Cognitive function was assessed using a standardized neuropsychological battery. Participants underwent an extensive clinical dental examination. Chewing efficiency was assessed using a two-color chewing gum mixing test; the results being expressed by the variance of hue. Outcomes were measured at baseline, and on an annual basis. Distribution was tested for normality; ANOVA and linear regression models with repeated measures adjusted for age and gender were used.

Results: 89 MCI were included $(\operatorname{men}=48$, women $=41$; age $=$ $74.4 \pm 6.6 \mathrm{y}$ ) and underwent a total of 347 evaluations. The conversion rate from MCI to dementia was $12.4 \%$ in 5 years of followup. The initial mean number of 21 teeth decreased significantly between visits but not when adjusted for age (ANOVA: $\mathrm{p}=0.0109$, page adjusted $=0.0748$ ), whereas chewing efficiency did not $(\mathrm{p}=0.5503)$. MMSE scores remained stable throughout the years and were associated with oral-health. The number of teeth present at any point in time could explain $5.8 \%$ of the variance in MMSE $(\mathrm{p}=0.0003$, page adjusted $=0.0085)$. Each tooth present granted 0.07 additional MMSE point.

Conclusions: The results confirm the association between number of teeth and cognitive function. The high number of natural teeth at baseline may have contributed to the rather stable cognitive and chewing function over time.

\section{P-649}

Examination of the effectiveness of a specialised cognitive rehabilitation programme for adults with dementia in the community in the Irish context

Elaine Harrington ${ }^{1}$, C. Keady ${ }^{1}$, A. M. Norris ${ }^{1}$, N. Mathlin ${ }^{1}$, A. O'Gorman ${ }^{1}$

${ }^{1}$ St. James's Hospital, James's Street, Dublin 8, Republic of Ireland

Introduction: Dementia is one of the three major diseases related to health care consumption [1-5] and it is a major cause of care burden and disability in the elderly. Therefore it is important to attempt to implement new effective and efficient health care interventions that increase independence and well-being of patients with dementia and decrease care giver burden resulting in a more efficient use of scarce health care resources (Baker et al. 2004). In response, a cognitive rehabilitation programme was developed to potentially improve quality of life, cognitive ability and functional performance of meaningful occupations and activities for patients with dementia 
called the Specialised Memory and Attention Rehabilitation Therapy (SMART) Programme.

Methods: A quantitative and qualitative approach was used to examine the effectiveness of a 5 week specialised cognitive rehabilitation group programme on patient's cognition, quality of life and function. The study used a convenient sample of patients who met the inclusion criteria.

Results: The Rivermead Behavioural Memory Test indicated a 169 percentage change from baseline post intervention and $277 \%$ change from baseline at 3 months post intervention. With regards the Canadian Occupational Performance Measure (COPM) Performance section, there was $45.3 \%$ change from baseline post intervention and $51.1 \%$ change from baseline at 3 month post intervention.

Key conclusions: The results indicated the beneficial impact of the specialised cognitive group programme on participant's cognition, quality of life and completion of meaningful and valuable occupations.

References:

1. Burgener SC, Bakas T, Murray C, Dunahee J, Tossey S (1998) Effective caregiving approaches for patients with Alzheimer's disease. Geriatr Nurs 19:121-152

2. Coen J (1998) Dementia and caregiving. J Health Gain 2:5-6

3. Jepson C, McCorkle R, Adler D, Nuamah I, Lusk E (1999) Effects of home care on caregivers' psychosocial status. Image J Nurs Sch 31:115-120

4. Luijpen MW, Scherder EJ, Van Someren EJ, Swaab DF, Sergeant JA (2003) Nonpharmacological interventions in cognitively impaired and demented patients - a comparison with cholinesterase inhibitors. Rev Neurosci 14:343-368

5. Wimo A, Winblad B, Aguero-Torres H, von Strauss E (2003) The magnitude of dementia occurrence in the world. Alzheimer Dis Assoc Disord 17:63-67

\section{P-650}

Carer strain is associated with activities of daily living in people with cognitive impairment

Clare Burgon ${ }^{1,2}$, Veronika Van der Wardt ${ }^{1}$, Sarah Goldberg ${ }^{2}$, Rupinder Bajwa ${ }^{1}$, Rowan H Harwood ${ }^{2,3}$

${ }^{1}$ Division of Rehabilitation and Ageing, University of Nottingham, UK, ${ }^{2}$ School of Health Sciences, University of Nottingham, UK,

${ }^{3}$ Nottingham University Hospitals, UK

Introduction: Carer strain is associated with increased risk of carer depression and social isolation and institutionalisation of the care recipient. Activities of daily living (ADL) are impaired across all stages of dementia, and advanced ADL are argued to be impaired in MCI. People with greater ADL impairment may require more support, which could increase carer strain. This study aimed to assess the relationship between $\mathrm{ADL}$ and carer strain in people with dementia and MCI.

Methods: Carers assessed caring-related strain and patient's ADL, as part of a baseline assessment of the Promoting Activity Independence and Stability in Dementia (PrAISED) study. The relationship between $\mathrm{ADL}$ and carer strain was explored using hierarchal linear regression. Carer gender and age, co-residency and spousal status, were entered in step 1 , as evidence suggests these factors are associated with carer strain. ADL was entered in step 2.

Results: 54 carers were recruited. 49 lived with the patient. 45 were spouses, and 9 were sons or daughters. Limitations in ADL was significantly associated with carer strain $\left(r^{2}=26 \%, \beta=-0.448\right)$ when controlling for carer age and gender, co-residency, and spousal status.
Key conclusions: Carer strain is significantly and independently associated with worse ADL in people with cognitive impairment. Causation cannot be established and results may not be representative. However, future research should examine whether improvements in ADL following interventions lead to reduced carer strain. Qualitative interviews with carers could shed light on this association.

\section{P-651}

\section{Cs audit: cognition, collateral and CT}

Hannah Smyth ${ }^{1}$, Lorna Kilbane ${ }^{1}$, Claire Noonan ${ }^{1}$, Helen O’Brien ${ }^{1}$, Diya Mary ${ }^{1}$, Hugh O'Sullivan ${ }^{1}$, Tara Coughlan ${ }^{1}$, Dan Ryan ${ }^{1}$, Ronan Collins ${ }^{1}$, Sean Kennelly ${ }^{1}$, Des O'Neill ${ }^{1}$

${ }^{1}$ Tallaght University Hospital, Dublin, Republic of Ireland

Introduction: Dementia and/or delirium are present in 30\% of admissions of older people to hospital but there is widespread concern that neither syndrome is appropriately assessed or managed. We reviewed whether appropriate assessment procedures were carried out to evaluate cognitive impairment in older patients referred for specialist geriatric assessment so as to develop appropriate educational interventions.

Method: An audit was carried out on consults with cognitive impairment to a geriatric medicine service in a university teaching hospital. The following data was collected from patients' records: (1) formal cognitive screen (2) collateral history (3) neuroimaging (4) screening for delirium (5) relevant laboratory tests (6) referral to relevant allied health professionals (AHPs).

Results: Of 75 consecutive consults, mean age 80.4 (49\% women), $70(93 \%)$ did not have a formal cognitive screen and $61(81 \%)$ a complete collateral history. Only $2(3 \%)$ had a formal delirium screening assessment and 19 (25\%) had documentation of possible features of delirium. Almost all referred, 71 (95\%) had neuroimaging (51\% CT brain, $44 \%$ MRI). Less than half, 31 (41\%) had thyroid function test, vitamin B12 and folate sent. Over half of patients were referred to AHPs.

Conclusion: Key diagnostic elements of assessing and managing dementia and delirium-cognitive screening, collateral history and delirium screening-were overlooked by referring teams to an alarming extent. Further education on these aspects of care among all medical and nursing staff in general hospitals are key to improving the care of older patients with cognitive impairment.

\section{P-652}

Mobility predictors of trajectories of cognitive decline in elderly subjects. The InCHIANTI study

Vieri Del Panta ${ }^{1}$, Marco Colpo ${ }^{1}$, Laura Casprini ${ }^{1}$, Samanta Corradi ${ }^{2}$, Stefania Bandinelli ${ }^{1}$

${ }^{1}$ Local Health Unit Tuscany Centre, Florence, Italy, ${ }^{2}$ Geriatria, Accettazione Geriatrica d'Urgenza e Centro di Ricerca per l'Invecchiamento IRCCS INRCA, Ancona, Italy

Introduction: Older adults exhibit different patterns of change in cognitive functioning over time. Results from previous studies indicate gait speed as relevant predictor of cognitive decline. This study was aimed to test different physical performance features as predictors of the Mini Mental State Examination (MMSE) score behaviour over a 15-year period separately for men and women.

Methods: We estimated the longitudinal trajectories of MMSE in the InCHIANTI sample with age $\geq 65$, MMSE $\geq 26$, no-loss ADLs and 
without neurological disease at baseline evaluation, characterized by Latent Class Growth Models (LCGM) and categorized as 5 different profiles by Bayesian Information Criterion (BIC) for both gender. Missing data of predictors were imputated by the Multivariate Imputation by Chained Equati (MICE) procedure and were identified by ordinal logistic regression with backward selection among age, Body Mass Index (BMI), number of medications, Short Physical Performance Battery (SPPB), $400 \mathrm{~m}$ speed $(400 \mathrm{~m} / \mathrm{s})$ and Handgrip (HG)).

Results: 621 participants $(292 \mathrm{M}, 71.7 \pm 5.1$ years and $329 \mathrm{~F}$, $72.8 \pm 6.0$ years) were included. Significant predictors of MMSE decline were BMI $(\mathrm{OR}=1.09)$, $\mathrm{HG}(\mathrm{OR}=1.03)$ and $400 \mathrm{~m} / \mathrm{s}$ $(\mathrm{OR}=6.50)$ for males, while for females were: age $(\mathrm{OR}=1.11), \mathrm{HG}$ $(\mathrm{OR}=1.03)$ and SPPB $(\mathrm{OR}=1.13)$.

Conclusion: The results support that decline in MMSE both men and women could be predicted by functional profile at baseline. The impact of grip strength is predictive for both gender but of particular interest is the relevance of the endurance test for men while the physical performance score for women. We confirm the relevance of mobility measurements to detect cognitive decline over a long period of time.

\section{P-653}

The role of standard physical exercise of moderate intensity in the management of depression in elderly female patients

\section{Cristian Romeo Revnic ${ }^{1}$, Floarea Revnic ${ }^{2}$, Silviu Voinea ${ }^{3}$ \\ ${ }^{1}$ UMF Carol Davila, Bucharest, Romania, ${ }^{2}$ NIGG Ana Aslan, Bucharest, Romania, ${ }^{3}$ UMF Carol Davila, Bucharest, Romania}

Introduction: Aging is associated with a decline in HPA axis. The decrease in hGH secretion and an increase in stress hormones such as Cortisol is associated with depression and cognitive decline which in part can be accounted for a reduction in blood flow in the brain.

Objective: Evaluation of a 24 weeks Standard Physical exercise program of moderate intensity(Geriatria,vol.XIV,nr.1 2002)including a series of stretching, toning and weight lifting exercises combined with an aerobic component, on depression and cognitive abilities as well as upon hGH and Cortisol secretion.

Material and method: 42 female patients aged between $56-76$ years old patients admitted in Rehabilitation Clinique for osteoarticular and post traumatic pathologies divided into two groups: group $\mathrm{A}(21$ active patients) and group B(21 sedentary depressed patients associated with obesity). Evaluation of depression has been done using Geriatric depression scale. hGH and Cortisol levels were estimated in serum before and after physical training with Eu+ labelled hGH and Cortisol kits purchased from Pharmacia using a 1234 Delfia Research Spectrofluorimeter. Following training there was an increase in $\mathrm{HGH}$ and a decrease in Cortisol levels with a positive impact upon cognitive and affective state in patients from group B without physical activity.

Conclusion: Our data have pointed out that while the depression gets ameliorated, the cognitive functions are improved and in the same time the compliance to the rehabilitation program Physical training can be accounted as an alternative for releasing the depressive states in the elderly taking into account the adverse reactions of different classes of antidepressants and may even help to prevent Alzheimers' disease.

\section{P-654}

Hypertension in dementia (HIND): the tolerability of $24 \mathrm{~h}$ ambulatory BP monitoring

Tomas Welsh ${ }^{1}$, Adam Gordon ${ }^{2}$, Simon Conroy ${ }^{3}$, John Gladman ${ }^{4}$

${ }^{1}$ Research Institute for the Care of Older People (RICE), Bath; Royal United Hospital Bath, University of Bristol, Bristol, UK, ${ }^{2}$ University of Nottingham; Derby Hospitals NHS Foundation Trust, Nottingham, UK, ${ }^{3}$ University of Leicester, Leicester, UK, ${ }^{4}$ University of Nottingham, Nottingham, UK, Collaboration

Introduction: Guidelines increasingly stress the role of ambulatory blood pressure monitoring (ABPM) in the identification and management of hypertension but its tolerability in people with dementia is uncertain. This small study aimed to evaluate the tolerability of $24 \mathrm{~h}$ blood pressure monitoring in people with diagnoses of dementia and hypertension.

Methods: A subgroup of participants in the Hypertension in Dementia study (a cohort of 180 participants followed over 6-months with diagnoses of dementia and hypertension monitored for adverse events) were recruited and underwent $24 \mathrm{~h}$ ABPM. A participant was considered tolerant if able to wear the monitor for $20 \mathrm{~h}$. Adequate measurement was assumed if at least 21 readings were taken.

Results: 24 (89\%) of 27 participants consented to participate, aged 83 (SD 5.5), 23 (96\%) female, MMSE 25 (4.7), Barthel Index 19 (17.5-20). Mean clinic BP 131/76 (SD 20/11), Mean ambulatory BP 124/66 (SD 11/6.6). 15 (63\%) wore the monitor for at least $20 \mathrm{~h}$, all of whom achieved at least 21 measurements. In the remaining 9 participants the monitor was removed because of 'unsure/found off' 4 (44\%), 'kept awake' 2 (22\%), 'painful' 2 (22\%), 'wanted to wash' 1 (11\%).

Key conclusions: ABPM was generally well tolerated in this group of people with dementia. However this group were only mildly cognitively impaired with a high Barthel Index. Further investigation into the tolerability of ABPM in people with more significant impairment is warranted.

\section{P-655}

Infections and other clinical complications among institutionalized elderly patients with mild dementia in a one year follow-up

Barbara Gryglewska ${ }^{1}$, Jadwiga Wojkowska-Mach ${ }^{2}$, Piotr Heczko ${ }^{2}$, Tomasz Grodzicki ${ }^{1}$

${ }^{1}$ Department of Internal Medicine and Gerontology, Jagiellonian University, Medical College, Kraków, Poland, ${ }^{2}$ Department of Microbiology, Jagiellonian University, Medical College, Kraków, Poland

Introduction: Medical complications and geriatric problems are frequent in nursing home residents with advanced dementia. Little is known about the clinical course of residents with mild dementia. The aim of the study was to investigate the incidence rate of infections and other clinical problems among institutionalized elderly patients with diagnosis of mild dementia during a one-year follow-up.

Methods: A study was performed among elderly residents in three long-term care facilities in Poland. The initial evaluation included analysis of medical documentation, performance of Abbreviated Mental Test Score (AMTS), Mini Nutritional Assessment Short-Form (MNA-SF) and Barthel Index. Episodes of infections or other acute illnesses, exacerbation of chronic diseases, hospitalizations and deaths were recorded during follow-up. The data were compared in two groups: patients with the diagnosis of mild dementia (group I) and residents without dementia (group II). 
Results: The sample consisted of 189 residents, $76.3 \pm 11.2$ years old, $38.6 \%$ men. Residents of group I $(\mathrm{n}=65)$ were older $(79.9 \pm 10.8$ vs $74.5 \pm 11.0$ years), suffered from more diseases $(4.7 \pm 1.6$ vs $3.7 \pm 1.8)$ and had lower scores of AMTS $(4.5 \pm 3.2$ vs $7.6 \pm 2.4)$, MNA $(8.8 \pm 2.8$ vs $11.3 \pm 2.7)$ and Barthel Index $(32.8 \pm 38.6$ vs $51.8 \pm 37.0)$ than group II $(\mathrm{n}=124)$. The frequency of deaths ( $21.5 \%$ vs $8.6 \%)$, number of hospitalizations $(33.8 \%$ vs $19.4 \%)$, rate of respiratory $(23.1 \%$ vs $11.3 \%)$ and urinary $(13.8 \%$ vs $2.4 \%)$ infections were higher in group I than II.

Key conclusions: Elderly residents with mild dementia revealed higher probability of death, hospitalizations and infections during 1 year follow-up.

\section{P-656}

Neuropathological changes associated with cognitive decline in patients with diabetes: a systematic review

Gina Hadley ${ }^{1}$, Jiali Zhang ${ }^{2}$, Zoi Alexopoulou ${ }^{3}$, Gabriele C DeLuca ${ }^{1}$, Sarah T Pendlebury ${ }^{1}$

${ }^{1}$ Nuffield Department of Clinical Neurosciences, University of Oxford, Oxford, UK, ${ }^{2}$ St Anne's College, University of Oxford, Oxford, UK, ${ }^{3}$ St Anne's College, University of Oxford, Oxford, UK

Introduction: Type 2 diabetes (T2DM) is a risk factor for cerebrovascular disease and cognitive dysfunction. Diabetic patients with post-stroke dementia exhibit cognitive decline over and above small vessel disease and stroke severity. This systematic review considers the neuropathological correlates of cognitive decline in diabetes.

Methods: Two authors screened titles and abstracts from EMBASE (1974-present) and MEDLINE (1946-present) using: (diabet* OR glucose OR "blood sugar" OR HbA1c) AND (dementia OR (cognitive* AND (impair* OR decline))) AND (neuropathol* OR immunohisto* OR MMSE OR "Mini mental status examination" OR "Mini-mental status examination" OR MOCA OR "Montreal Cognitive Assessment"). Included studies reported on diabetes and cognition and had neuropathological endpoints.

Results: Eleven studies containing 25 to $>1300$ subjects met the inclusion criteria from 3567 publications identified. There were seven cohorts of aging, three retrospective hospital series and one database of cohorts. Diabetes was recorded via self-reporting/hospital records. Cognition was assessed using a variety of scales. There was heterogeneity in neuropathological quantification. Diabetics demonstrated mainly cerebral infarction and lacunes but not Alzheimer pathology. A synergistic relationship emerged where those with diabetes and infarcts, or with diabetes and Alzheimer pathology, had lower cognitive scores than those with diabetes, infarcts or Alzheimer pathology alone.

Conclusion: Evidence suggests that diabetes augments the risk of cognitive decline which is mediated by vascular and not Alzheimer pathology. Future longitudinal studies should consider reliable recording of diabetes, consistent cognitive tests and standardised measures of neuropathology. This will allow better understanding of underlying mechanisms aid the acquisition of novel therapeutic strategies.

\section{P-657}

Reporting of weapon license in 69100 dementia patients in Swedish Dementia Registry SveDem

Dorota Religa ${ }^{1}$, Emilia Schwertner ${ }^{1}$, Juraj Secnik ${ }^{1}$, Hugo Söderström ${ }^{1}$, Björn Johansson ${ }^{1}$, Bengt Winblad ${ }^{1}$, Maria Eriksdotter ${ }^{1}$

${ }^{1}$ Karolinska Institutet, Stockholm, Sweden

Introduction: In Sweden there is more than 600,000 licensed firearm owners and approximately 170,000 patients with a dementia diagnosis. This study aims to explore properties of firearm ownership in a large population of Swedish dementia patients. The aim is to specify socio-demographic and clinical characteristics of dementia patients owning firearms, as well as factors influencing the decision to report a patient as non-suitable to possess firearms to authorities.

Methods: This was a registry-based cohort study. Sample consisted of patients diagnosed with dementia and registered in the Swedish Dementia Registry $(n=69,100)$. Eight subtypes of dementia were included the study: Alzheimer Disease (AD), Mixed Dementia (Mixed), Vascular dementia (VAD), Lewy body dementia (LBD), Frontotemporal dementia (FTD), Unspecified dementia (UNS) and Other types of dementia (Other). Logistic regression was used to estimate odds ratio (OR) of being reported as non-suitable to possess firearms to authorities in each dementia diagnoses with $\mathrm{AD}$ as a reference. Final model was adjusted for age, sex, Mini-Mental State Examination and, living arrangement and antipsychotics use.

Results: In total 1826 (3.4\%) patients had weapon licence and 347 $(10.2 \%)$ were reported to authorities. In adjusted models, VAD and FTD were associated with increased risk of being reported as nonsuitable to possess firearms comparing to $\mathrm{AD}$ patients $(\mathrm{OR}=1.41$ 95\% CI(1.01-1.97) and OR $=4.18$ (2.22-7.89), respectively).

Conclusions: Our results indicate that in the physicians' opinion, FTD and VAD patients may pose a greater risk of committing violent act. We recommend and this should be included in future guidelines.

\section{P-659}

The prevalence and severity of delirium and dementia related behavioural disturbances on medical wards

M. Z. Vettasseri ${ }^{1}$, C. Hewkins ${ }^{1}$, A. Mamarelis ${ }^{1}$, B. Baath ${ }^{1}$, R. H. Harwood ${ }^{1}$, A. Ali ${ }^{1}$

${ }^{1}$ Nottingham University Hospitals NHS Trust, Nottingham, UK

Introduction: Behavioural disturbances associated with delirium and dementia negatively affect patient outcomes. Our aim was to identify the range of behavioural disturbances encountered on medical wards. Methods: A point prevalence survey of 159 patients, aged over 65, across 5 elderly care and 4 general medical wards was conducted to identify the presence of dementia and delirium. The prevalence and severity of behavioural disturbances displayed by these patients and the management difficulties they presented were identified by interviewing nursing staff.

Results: 59 patients (37\%) had a pre-admission dementia diagnosis. On admission, 76 patients (48\%) displayed features suggesting delirium, with $49(31 \%)$ cases formally diagnosed. Nursing staff reported the following behavioural disturbances: sleep disturbances (49 patients $(31 \%))$, drowsiness $(49(31 \%))$, shouting out $(48(30 \%))$, refusing food (46 (29\%)), resisting care (46 (29\%)), aggression (27 $(17 \%))$ and wandering $(21(13 \%))$. The prevalence and severity of behavioural disturbances was higher on elderly care wards. $46(29 \%)$ patients required nursing in continuous supervision bays, $6(4 \%)$ required $1: 1$ supervision and $12(7.5 \%)$ received pharmacological 
sedation. Nurses identified $23(14.5 \%)$ patients with severe behavioural needs whose care would be enhanced in a Mental Health supported environment.

Conclusions: The high prevalence of behavioural issues across all wards has implications on our ability to provide effective care. It is essential staff receive training on the recognition and management of delirium and dementia and adequate resources must be allocated to appropriately manage the behavioural needs of patients. Creating dementia-friendly environments and increasing Mental Health Team support represent potential solutions.

\section{P-660}

Depression or early dementia: can we differentiate and does it matter?

Reena Tewari ${ }^{1}$, Hannah Keage ${ }^{1}$, Tobias Loetscher ${ }^{1}$
${ }^{1}$ University of South Australia, Adelaide, Australia

Introduction and aim: In day- to- day clinical practice of Geriatric Medicine, the dilemma whether we are dealing with clinical depression or early dementia is becoming very common. Based on currently available literature there is evidence of complex correlation between Depression and Dementia. None of the cognitive assessments are designed to help this situation. Management of both these conditions by itself is not easy and needs clear approach plan to intervene at the right time and regular follow- ups. Untreated Depression can be catastrophic in older people and similar is true for early dementia, which needs intervention at the right time Questions that need to be answered: (1) Is this differentiation important to establish or important in clinical practice? (2) Are Neuropsychiatric assessment tools helpful in answering this dilemma? (3) Is combination of Neuropsychiatric assessment along with radiological imaging will assist in differentiation. (4) What should be the further management protocol if differentiation is established or not.

Methods: (1) Review of available literature to find answers of above questions. (2) Analysis of available data from other relevant studies. (3) Designing a Cohort study to test the hypothesis.

Results: There is evidence that Neuropsychological assessment may be helpful in differentiating early dementia from depression. Additional imaging studies may confirm the diagnosis though there is not many studies examining this approach. Key-findings of literature review will be presented.

\section{P-661}

Wandering by acute akathisia in an old patient with severe dementia of Alzheimer's type

Luis María García Santos ${ }^{1}$, Antonio Blanco Orenes ${ }^{2}$, Elisa García-Tercero ${ }^{2}$, Rosa María García-Tercero ${ }^{3}$, Felipe Madruga Galán ${ }^{2}$, Paula Vicent Valverde ${ }^{4}$

${ }^{1}$ Department of Psychiatry, Hospital Virgen del Valle, Toledo, Spain, ${ }^{2}$ Department of geriatrics, Hospital del Valle, Toledo, Spain,

${ }^{3}$ Department of Neurology, University Hospital of Alicante, Alicante, Spain, ${ }^{4}$ Department of Geriatrics, University Hospital of Getafe, Madrid, Spain

Introduction: Acute akathisia is described as a state of subjective restlessness characterized by the impossibility of remaining still, with the need to move. It's usually induced by neuroleptics, in most cases after the first or second week or even more days after the start of treatment. It can also occur after a dose increase. A frequent accompanying clinical picture is insomnia. The treatment in the first instance consists of the withdrawal or reduction of the dose of the neuroleptic. The drugs more used for the treatment are propranolol and benzodiazepines.

Method: We present the case of an 83-year-old patient diagnosed with Alzheimer's dementia GDS6 in treatment with memantine $20 \mathrm{mg}$ : $1 / 24 \mathrm{~h}$, pregabalin $25 \mathrm{mg}$ : $1 / 12 \mathrm{~h}$ and quetiapine $100 \mathrm{mg}$ : $1 / 8 \mathrm{~h}$. His family reports that for several weeks and coinciding with a rise in the dose of quetiapine, the patient is more anxious and doesn't stop moving throughout the day. Clinical exploration highlights psychomotor restlessness, change of position and balance of both lower extremities. Given the medical, pharmacological and clinical examination history, the patient is diagnosed with acute akathisia due to neuroleptics. Quetiapine descent starts to take quetiapine $25 \mathrm{mg}$ : $1 / 2$ every $8 \mathrm{~h}$, increase of pregabalin to take pregabalin $100 \mathrm{mg}$ : $1 / 12 \mathrm{~h}$. Propanolol is added to the treatment $10 \mathrm{mg}: 1 / 2$ every $8 \mathrm{~h}$ and clonazepam 0.5: $1 / 2$ when he goes to sleep.

Results: After one month, he attended the review accompanied by his family, who reported an important improvement in the clinical picture. The patient remains calm throughout the day and without disorders of the sleep. Key conclusions: The dose adjustment of the neuroleptic with the treatment with propranolol was shown to be effective in a patient with acute akathisia due to neuroleptics. This same conclusion has been revealed by other authors in similar clinical cases.

\section{P-662}

A statement of the position for dental care and dental treatment guidelines for elderly people with dementia from The Japanese Society of Gerodontology (JSG)

\section{Hirohiko Hirano $^{1}$, Ayako Edahiro ${ }^{1}$, Tetsuo Itikawa ${ }^{2}$}

${ }^{1}$ Tokyo Metropolitan Geriatric Hospital and Institute of Gerontology, Tokyo, Japan, ${ }^{2}$ Guidelines Committee of The Japanese Society of Gerodontology, Tokyo, Japan

The rapidly aging society and increase of people with dementia seen in Japan is unprecedented in the world. The Japanese society of Gerodontology (JSG) has been proactively working in pioneering research regarding the association between dementia and dental care in elderly people. In 2015, the Ministry of Health, Labour and Welfare of Japan adopted a "Comprehensive Strategy to Accelerate Dementia Measures (called New Orange Plan)" as a new strategy to bolster measures against dementia. This plan documents the implementation of improvements in training for dementia support among dentists. Based on the plan, JSG made "A statement of position for dental care for the elderly peoples with dementia", 2015. Our mission is to draft the guidelines for dental care for elderly peoples with dementia based on evidence and to promote the maintenance of dignity and quality of life in dementia patients. Based on the presented background, the previously stated mission statement for dental care for elderly people with dementia is representative of the current position of JSG. Furthermore, JSG started to create the dental treatment guidelines for elderly peoples with dementia from 2016. The contents of the guidelines will be in accordance with the existing "Providing health care and long-term care services in a timely and appropriate manner as the stages of dementia progress" shown in the Japanese Dementia Strategy (New Orange Plan). This presentation will include the assertions made above, as well as information on current dental care and oral function management in elderly patients with dementia in Japan. 


\section{P-663}

Long-term Effect of masked hypertension management on cognitive functions: G-MASH-Cog MONITOR study

Cafer Balc1 ${ }^{1}$, Mert Eşme ${ }^{1}$, Fatih Sümer ${ }^{1}$, Serkan Asil ${ }^{2}$, Bünyamin Yavuz $^{3}$, Rana Tuna Doğrul ${ }^{1}$, Cemile Özsürekci ${ }^{1}$, Hatice Çalışkan ${ }^{1}$, Pelin Ünsal ${ }^{1}$, Gözde Şengül Ayçiçek ${ }^{1}$, Meltem Halil ${ }^{1}$, Mustafa Cankurtaran $^{1}$, Burcu Balam Yavuz ${ }^{1}$

${ }^{1}$ Hacettepe University Faculty of Medicine Department of Internal Medicine Division of Geriatrics, Ankara, Turkey, ${ }^{2}$ Ankara Gulhane Education and Research Hospital Department of Cardiology, Ankara, Turkey, ${ }^{3}$ Ankara Medical Park Hospital Department of Cardiology, Ankara, Turkey

Introduction: Eventhough masked hypertension $(\mathrm{MH})$ is associated with cognitive decline ${ }^{1}$, it's long-term effect is not known. In this study, the effect of the management of masked hypertension on cognitive functions was investigated.

Methods: Total of 102 participants in the index study ${ }^{1}$ were followed-up for 1-year, while 41 of them lost follow-up. In masked hypertensive individuals, lifestyle modification and/or antihypertensive treatment were initiated for blood pressure control. Mini Mental State Examination (MMSE), Montreal Cognitive Assessment Test (MOCA), Quick Mild Cognitive Impairment Screen Test (Q-MCI) and neuropsychiatric test battery were used to assess cognitive functions. Measurements of cognitive tests and ambulatory blood pressure monitoring at baseline and at 1-year follow-up were compared.

Results: A total of 61 patients ( 30 in the $\mathrm{MH}$ group; 31 in the control group) were included in the study. Mean age was $72.3 \pm 5.1$ and 36 (59\%) were female. Mean change in baseline blood pressure at the end of 1-year follow-up period was $6.1 \pm 9.4 \mathrm{mmHg}$ in all day systolic blood pressure and $2.8 \pm 5.5 \mathrm{mmHg}$ in diastolic blood pressure in the MH group (p: 0.001; p: 0.01, respectively). In masked hypertensive patients, the change in MMSE and MOCA was not statistically significant, while the increase in Q-MCI was statistically significant (baseline Q-MCI: $41.6 \pm 11.8$; end of 1 st year: $45.7 \pm 14.5$, p: 0.004).

Conclusion: This is the first study that evaluates the long-term effect of management of masked hypertension on cognitive functions in older adults. Blood pressure control in $\mathrm{MH}$ group was associated with improvement in cognitive functions. Masked hypertension should be kept in mind while assessing older people, and when detected, blood pressure must be controlled regarding the positive long-term effect on cognitive functions.

\section{Reference:}

1. Esme M, Yavuz BB, Yavuz B, Asil S, Tuna Dogrul R, Sumer F, et al. (2017).Masked Hypertension is associated with cognitive decline in geriatric age-geriatric masked hypertension and cognition (G-MASH-cog) study. J Gerontol Ser A 73(2):248-254.

\section{P-664}

Incorporating the $\mathbf{A} / \mathbf{T} / \mathbf{N}$ CSF biomarker pathological scheme into the diagnostic algorithm for Alzheimer's disease (AD) and neurodegeneration in an Irish Tertiary memory clinic setting

Deirdre McCartan ${ }^{1}$, Robert Briggs ${ }^{1}$, Cathy McHale ${ }^{1}$, Professor Sean Kennelly ${ }^{1}$

${ }^{1}$ Tallaght University Hospital, Dublin, Ireland

Background: The A/T/N classification scheme, reported in the 2018 NIA-AA review of the Alzheimer's disease (AD) continuum, incorporates CSF biomarkers into an unbiased descriptive classification scheme for $\mathrm{AD}$ and neurodegeneration. $\mathrm{A} / \mathrm{T} / \mathrm{N}$ is designed to provide a unifying binary biomarker classification scheme to permit consistent reporting of the pathophysiological status of individuals and to assist diagnosis in the context of the presenting cognitive syndrome be it normal cognition, mild cognitive impairment(MCI) or dementia.

Method: A review of the clinical diagnosis and subsequent CSF biomarker results using $\mathrm{A} / \mathrm{T} / \mathrm{N}$ criteria was performed for 44 patients attending a tertiary referral memory service between 2016 and 2018. The selected biomarkers were CSF Amyloid Aß42 (A), CSF Phosphorylated Tau $(\mathrm{T})$ and neuronal loss quantified by total CSF tau $(\mathrm{N})$, plus radiological investigations. We retrospectively compared the patients' $\mathrm{A} / \mathrm{T} / \mathrm{N}$ to the original consensus diagnosis made prior to availability of their biomarker results.

Results: Of 23 patients with initial diagnosis of MCI, 16 had CSF biomarkers consistent with the $\mathrm{A}+/ \mathrm{T}+\mathrm{N}+$ pattern of $\mathrm{AD} .4$ showed typical AD pathology requiring Amyloid PET for formal diagnosis. 1 was classified as "MCI unlikely due to AD", 2 were "undefined". 2 patients diagnosed with "language predominant dementia" showed biomarkers consistent with AD pathology.

Conclusion: This study demonstrates the incorporation of CSF biomarkers into the routine clinical diagnostic pathway of a tertiary memory referral centre. AD CSF biomarkers are central to clinical diagnosis of MCI, Alzheimer's Disease and Alzheimer's Dementia and give certainty to the underlying pathological process causing memory and/or neurodegenerative symptoms.

\section{P-665}

The risk of head trauma associated with antipsychotic use among community-dwellings with Alzheimer's disease

Vesa Tapianen ${ }^{1}$, Piia Lavikainen ${ }^{1}$, Marjaana Koponen ${ }^{1}$, Heidi Taipale $^{1}$, Antti Tanskanen ${ }^{2}$, Jari Tiihonen ${ }^{2}$, Sirpa Hartikainen ${ }^{1}$

${ }^{1}$ School of Pharmacy, University of Eastern Finland, Kuopio, Finland, ${ }^{2}$ Department of Clinical Neuroscience, Center for Psychiatry Research, Karolinska Institutet and Stockholm County Council, Stockholm, Sweden

Introduction: Antipsychotic use is associated with increased risk of falls in older population. However, there are no previous studies concerning antipsychotic use and risk of head traumas. We studied the association between antipsychotic use and head traumas among community-dwelling persons with Alzheimer's disease (AD).

Methods: A matched cohort study comparing new antipsychotic users with matched nonusers in the MEDALZ cohort include all Finnish community-dwellings with clinically verified $\mathrm{AD}$ in 2005-2011. Antipsychotic use was extracted from Prescription Register and one-year washout period were used to identify new users. Antipsychotic users were matched with nonusers by age, sex and time since $\mathrm{AD}$ diagnosis. The number of user-nonuser pairs was 21,795. Head traumas (ICD-10 S00-S09) and traumatic brain injuries (ICD-10 S06.0-S06.9), were extracted from Hospital Discharge and Causes of Death Registers. The association was investigated using Cox proportional hazard models. Comorbidities, medication use and socioeconomic position were taken into account as confounders.

Results: Antipsychotic use was associated with increased risk of head traumas [event rate per 100 person-years 1.69 (95\% CI 1.53-1.86) for users and $1.26(1.16-1.37)$ for nonusers, adjusted HR (aHR) 1.33 (1.16-1.51)] and TBIs [event rate per 100 person-years 0.91 $(0.80-1.04)$ for users and $0.73(0.65-0.81)$ for nonusers, aHR 1.24 (1.04-1.47). Quetiapine, compared to risperidone raised risk for TBIs [aHR 95\% Cl 1.39 (1.03-1.88)] but not for head traumas [aHR 1.14 (0.91-1.42)]. 
Conclusions: Antipsychotic use was associated with increased risk of head traumas and TBIs among community-dwellings with AD. Therefore, use of antipsychotics should be carefully considered in this vulnerable population.

\section{P-666}

Repetitive transcranial magnetic stimulation (rTMS): a potential therapy for cognitive disorders?

\section{Charlotte Nouhaud ${ }^{1}$, Philippe Charru ${ }^{2}$, Joël Belmin ${ }^{3}$}

${ }^{1}$ Service de gérontologie, hôpital Louis Mourier, Assistance publique-Hôpitaux de Paris, 92700 Colombes, France, ${ }^{2}$ Service de gérontologie, hôpital Louis Mourier, Assistance publique-Hôpitaux de Paris, 92700 Colombes, France, ${ }^{3}$ Service de Gériatrie, Colombes, France

Considering the limited effectiveness of drugs treatments in cognitive disorders, the emergence of non-invasive techniques could be of benefit to patients. Among these techniques, repetitive transcranial magnetic stimulation (rTMS) can modulate cortical excitability and have therapeutic effects on cognition and behavior. These effects are due to physiological modifications in the stimulated cortical tissue and their associated circuits, depending on the stimulation parameters. The objective is to present the current knowledge and efficacy of rTMS in cognitive disorders to perform more studies and offer rTMS as a routine therapy for cognitive dysfunction $[1,2]$. Previous studies found very encouraging results with significant improvement of higher brain functions. Some of rTMS are now associated with cognitive training exercises during the stimulation $\left(\right.$ NeuroAD $\left.{ }^{\circledR}\right)$. The rTMS effects last to 3 to 6 month and seem more to slowdown the symptoms of cognitive disorders after 15 consecutive daily sessions of stimulation. Limits have been found in studies like few patients enrolled, lack of brain imaging control of the stimulation, insufficiently formalized technique and cognitive tests. Despite the difficulties to realize them, studies are getting more and more accurate and controlled.

\section{References:}

1. Nouhaud et al (2017) Repetitive transcranial magnetic stimulation: a potential therapy for cognitive disorders? Rev Med Intern 38:188

2. Lefaucheur JP et al (2014) Evidence-based guidelines on the therapeutic use of repetitive transcranial magnetic stimulation (rTMS). Clin Neurophysiol 125:2150.

\section{P-667}

A reliable tool for assessing MCI and dementia: validation study of DemTect for Turkish population

Gozde Sengul Aycicek ${ }^{1}$, Hatice Caliskan ${ }^{1}$, Cemile Ozsurekci ${ }^{1}$, Pelin Unsal ${ }^{1}$, Josef Kessler ${ }^{2}$, Elke Kalbe ${ }^{3}$, Mert Esme ${ }^{1}$, Rana Tuna Dogrul ${ }^{1}$, Cafer Balc1 ${ }^{1}$, Umran Seven ${ }^{3}$, Erdem Karabulut ${ }^{4}$, Meltem Gülhan Halil ${ }^{1}$, Mustafa Cankurtaran ${ }^{1}$, Burcu Balam Yavuz ${ }^{1}$

${ }^{1}$ Hacettepe University Faculty of Medicine, Department of Internal Medicine, Division of Geriatrics, Ankara,Turkey, ${ }^{2}$ Department of Neurology, Neuropsychology, University Hospital Cologne, Cologne, Germany, ${ }^{3}$ Department of Medical Psychology I Neuropsychology and Gender Studies, University Hospital Cologne, Cologne, Germany, ${ }^{4}$ Hacettepe University Faculty of Medicine, Department of Biostatistics, Ankara,Turkey

Background and aim: MCI and dementia prevalence are expected to increase with aging. DemTect is very easy and quick tool to administer and recognize the early stages of dementia and MCI. In this study we aimed to evaluate the reliability and validity of Turkish version of DemTect.

Patients and methods: Fifty four patients with mild cognitive impairment (MCI), 55 with Alzheimer' s Disease (AD) and 91 with normal cognitive functions (NCF) were enrolled in the study. DemTect was translated into Turkish by forward-backward translation and compared with Mini Mental State Examination (MMSE), Quick Mild Cognitive Impairment (QMCI) and Montreal Cognitive Assessment (MOCA). In order to test interrater reliability, DemTect was administered to 11 patients, on the same day, by the 2 trained raters. To establish test-retest reliability the same rater scored the tool a second time on 11 patients within 2 weeks.

Results: Median age of patients was 73 (min-max: 65-90) years, $54.5 \%$ were female. We found a strong correlation of DemTect scores with MMSE, QMCI and MOCA $(r=0.725, r=0.816, r=0.821$, respectively; $\mathrm{p}<0.001$ ). In ROC analysis, cut-off point of DemTect to differentiate MCI from NCF was 11.5 with $92.6 \%$ sensitivity, $91.2 \%$ specificity, AUC 0.973 and the cut-off to differentiate AD from NCF was 9.5 with $96.4 \%$ sensitivity, $100 \%$ specificity, AUC 0.916 . Cronbach $\alpha$ was 0.823 . Intraclass correlation coefficient was $0.873(95 \%$ CI $0.598-0.964)$ for interrater reliability and $0.966(95 \% 0.777-0.982)$ for test-retest reliability (Cronbach $\alpha$ 0.932, 0.966 respectively).

Conclusion: DemTect is a very reliable tool to assess Turkish patients with MCI and dementia.

\section{P-668}

Measuring physical activity: results from the PrAISED feasibility study

Sarah Goldberg ${ }^{1}$, Clare Burgon ${ }^{2}$, Rupinder Bajwa ${ }^{2}$, Rowan H. Harwood ${ }^{1}$

${ }^{1}$ School of Health Sciences, University of Nottingham, Nottingham, UK, ${ }^{2}$ School of Medicine, University of Nottingham, Nottingham, UK

Introduction: Physical activity has multiple benefits in people with dementia including positive effects of functional abilities, mood, mobility and cognition. Measuring levels of physical activity can be challenging in this population. As part of the follow-up assessment of the Promoting Activity, Independence and Stability in Early Dementia (PrAISED) feasibility study, several methods of measuring physical activity were assessed.

Methods: The follow-up assessments of PrAISED included 49 participants with an average age of 78 years (35\% female) diagnosed with Mild Cognitive Impairment (MCI) or dementia. Physical activity was measured using accelerometers and two physical activity questionnaires, the International Physical Activity Questionnaire (IPAQ) and the LASA Physical Activity Questionnaire (LAPAQ), which was added at a later point.

Results: The completion rates were $78 \%$ for the pedometer and $83 \%$ for the IPAQ questionnaire. Correlation analyses between the different measurements showed very strong correlations between the IPAQ and LAPAQ questionnaires $(r=0.86 ; n=20 ; p=0.00)$ and the number of steps as measured with the accelerometer and the LAPAQ scores $(\mathrm{r}=0.83 ; \mathrm{n}=18 ; \mathrm{p}=0.00)$ and a moderate correlation between IPAQ scores and number of steps $(r=0.45 ; n=35$; $\mathrm{p}=0.01$.

Conclusion: The findings indicate that both questionnaires as well as the accelerometer are valid outcome measures for physical activity in people with MCI or dementia. The questions included in LAPAQ were shorter and seemed slightly easier to answer for the participants. The difference in questions regarding walking (type of walking and 
time frame to consider) might have led to the different correlations of these two questionnaires with accelerometer results.

\section{P-669}

DECI Project: exercise habits in patients with mild cognitive impairment and its relation with SPPB

Myriam Oviedo-Briones ${ }^{1}$, Pedro A. Moreno ${ }^{1,2}$, Rodrígo PérezRodríguez $^{1,2}$, Myriam Valdés-Aragonés ${ }^{1,2,3}$, David Prada ${ }^{4}$, Mercedes Montenegro ${ }^{4}$, Andrea López Diez-Picazo ${ }^{1}$, Stefan Walter ${ }^{1}$, Leocadio Rodríguez-Mañas ${ }^{1,2,3,5}$

${ }^{1}$ Fundación de Investigación Biomédica del Hospital Universitario de Getafe, ${ }^{2}$ Centro de Tecnología Biomédica - Universidad Politécnica de Madrid, ${ }^{3}$ Hospital Universitario de Getafe, ${ }^{4}$ Centro de Prevención de Deterioro Cognitivo, ${ }^{5}$ Centro de Investigación Biomédica en Red Fragilidad y Envejecimiento Saludable

Introduction: DECI Project (Digital Environment for Cognitive Inclusion) is a multi-center, interventional study, designed for elderly with mild and very mild cognitive impairment to evolve traditional care organization models, through the support of digital technologies. The Short Physical Performance Battery (SPPB) is an assessment tool for evaluating lower extremity functioning in older persons. This study was conducted to determine whether the SPPB is related to exercise habits in patients with mild cognitive impairment.

Methods: 110 patients were included, $\geq 60$ years old, with diagnosis of mild and very mild cognitive impairment from the Centro de Deterioro Cognitivo in Madrid. The variables studied were the performance of any physical activity, which varied from exercise while sitting to running, its frequency and its duration. Sex, age and educational level were analyzed as confusing factors.

Results: In relation to the duration of exercise, 85 (77\%) patients exercised $\geq 30 \mathrm{~min}$ and $74(68 \%)$ did any type of physical activity on a daily basis. The mean days of physical exercise was 6 . The majority of elders, 93 (84\%), had a high SPPB score (10-12 points). Men had less physical adherence than women. Older patients did less days of physical exercise compared to younger patients, these results were statistical significant according to the logistic regression analysis.

Conclusions: Most of the elders with mild cognitive impairment did any type of physical activity more than 30 min per day, 6 days a week. There was a statistical significant difference in exercise habits in men versus women and with the increasing aging.

\section{P-670}

Evaluation of a multicomponent intervention in a group of patients with dementia and their principal caregivers through perception of psychological and behavioral symptoms of dementia

María Soledad Chiriboga Lozada ${ }^{1}$, Elsa Nelly Baca Lopez ${ }^{2}$, María Elena Contreras ${ }^{3}$

${ }^{1}$ Ecuadorian Society of Geriatrics and Gerontology, Genoa, Italy, ${ }^{2}$ Ecuadorian Society of Psychiatry, Genoa, Italy, ${ }^{3}$ Ecuadorian Society of Rehabilitation Medicine, Genoa, Italy

Introduction: New challenges in health policy need for establishing new training strategies in situations of dependency and disability in which the care of the dementia elderly is run by a family who assumes the role of informal caregiver. This is a study to evaluate a multicomponent intervention in a group of patients with dementia and their principal caregivers through neuropsychiatric inventory (NPI), Cornell depression scale and Zarit test score (Zarit).
Materials and methods: Design: non-controlled, intervention study. Setting: Geriatric day hospital in Quito-Ecuador. Participants: patients with dementia and principal caregivers of patients with dementia. Interventions: Patients were evaluated by a geriatrician and psychiatrist. Caregivers received group education sessions provided by multidisciplinary groups composed of, psychologist, physical therapist, and occupational therapist. Measures: Disease stage. Perception of psychological and behavioral symptoms of dementia (BPSD). Caregiver burden. Tests were performed before and after the intervention.

Results: 252 patient with caregivers participated in the program, of which 200 completed the program. All the domains of NPI (frequency, intensity and distress of the caregiver) improved after the intervention. Caregivers' burden subjective evaluation through Zarit also improved after the intervention. The remaining variables studied, including disease stage, showed association with the scores obtained. Conclusions: our findings suggest that intervention which includes clinical interventions in patients with dementia at the same time as a caregiver education program can improve both the management of the behavioral disorder and the degree of overload of the caregiver. In this way improving the quality of life of the patient and caregiver can improve quality of life.

\section{P-671}

Patients with Lewy body disease: diagnosis criteria and evolution of the disease

Valentine Zirabe ${ }^{1}$, Annaick Besozzi ${ }^{2}$, Therese Jonveaux ${ }^{2}$, Christine Perret-Guillaume $^{1}$, Athanase Benetos ${ }^{1}$, Anna Kearney-Schwartz ${ }^{1}$

${ }^{1}$ Geriatrics Department Nancy University Hospital, Nancy, France, ${ }^{2}$ Memory Clinic Nancy University Hospital, Nancy, France

Introduction: The Lewy Body Disease (LBD) diagnosis is based on clinical, neuropsychological and neuroradiological criteria. The aim of this study was to describe the ambulatory follow-up of LBD patients diagnosed in the Memory Clinic of Nancy-Lorraine University Hospital (CMRR CHRU Nancy) between 2012 and 2017. Methods: This is a monocentric, descriptive, retrospective study. We used the "Banque Nationale de Alzheimer" (BNA) database to obtain the sociodemographic description of LBD patients. Throughout the medicals records, the clinical data: age, sex, hallucinations, parkinsonian syndrome, symptoms' fluctuation, falls, behavior disturbances and treatments were collected. The follow-up of the same medical events was obtained from General Practitioner (GP) and Nursing Home physicians through telephone survey.

Results: The GPs responded for 67 LBD patients out of 88 found in the BNA database. Mean age was 78.1 years (SD 7.4 years) [60; 91], $53 \%$ were males. Mean MMSE score was $17 / 30$ at the onset of diagnosis. Diagnostic delay between first symptoms and diagnosis was 734 days and there was no correlation between this delay and patients' age or MMSE score. Symptoms that lead the patient to the Memory Clinic were: cognitive disorders $(26.5 \%)$, hallucinations $(13.3 \%)$ and parkinsonian syndrome $(13.3 \%)$. At the baseline in whole population there was a negative correlation between positive DATSCAN imaging and neuroleptics administration $(\mathrm{p}<0.001)$. The comparison of two groups: alive at the time of the phone survey and deceased was done. Both groups were followed-up for about 3 years (902 days for still living and 980 days for deceased ones). Patients who deceased during the follow-up period had initially, more neuroleptics (60\% vs. $19 \%$ respectively; $\mathrm{p}=0.046)$.

Conclusion: In our population, the neuroradiological criteria permitted less neuroleptic prescription. This was confirmed by subgroup study where the alive patients had less neuroleptics. 


\section{P-674}

Prevalence and incidence rates of early-onset dementia in France: analysis of the French National Health Data System

Laure Carcaillon-Bentata ${ }^{1}$, Cécile Quintin ${ }^{1}$, Marjorie Boussac-Zarebska ${ }^{1}$, Alexis Elbaz ${ }^{2}$

${ }^{1}$ Santé publique France, Saint-Maurice, France, ${ }^{2}$ Université ParisSaclay, Univ. Paris-Sud, UVSQ, CESP, Inserm, Villejuif, France/ Santé publique France, Saint-Maurice, France

The onset of dementia in patients aged $<65$ years (early-onset dementia-EOD) has dramatic consequences. While the burden of EOD is likely to grow due to the ageing of the baby boomer generation, its epidemiology remains poorly characterized. We estimated prevalence and incidence rates of EOD in France. Using the French national health data system, we identified all dementia cases aged 40-64 years, affiliated to the main health insurance fund, and alive at the end of December 2016. Due to the low sensitivity of administrative data to identify EOD, criteria (anti-dementia drugs consumption, hospitalization or benefits for chronic diseases with an ICD10 dementia code) were searched over 5 years (2012-2016). Incident cases were those identified in 2016 but not in 2015. Age- and sex-specific prevalence and incidence rates of EOD were estimated; we compared age-adjusted rates by sex using Poisson regression. A total of 26,874 prevalent (prevalence $=159 / 100,000$ ) and 6521 incident (incidence $=38.6 / 100,000$ person-years) cases of EOD were identified. Both prevalence and incidence rates sharply increased with age between 40 and 65 years. Age-adjusted prevalence and incidence rates were lower in women than men (prevalence rate ratio $=0.70$, $95 \%$ CI $0.68-0.72$; incidence rate ratio $=0.65,95 \%$ CI $0.6-0.68$ ). Our findings are consistent with those from a small number of studies that provided data on EOD frequency in the general population. Previous work shows that the underestimation of dementia frequency using administrative databases is likely to be less important for EOD. Based on these figures, we extrapolate that there were about 37,000 cases of EOD in France in 2016, including 9000 incident cases.

\section{P-675}

A novel technique to collect cerebrospinal fluid in the freely behaving mice as a preclinical model for biomarker research

Tsuneo Nakajima ${ }^{1}$, Shuko Takeda ${ }^{2}$, Akane Oyama $^{1}$, Yuki Ito ${ }^{2}$, Youichi Takami ${ }^{1}$, Yasushi Takeya ${ }^{1}$, Ken Sugimoto ${ }^{1}$,

Kouichi Yamamoto ${ }^{1}$, Hiromi Rakugi ${ }^{1}$, Ryuichi Morishita ${ }^{2}$

${ }^{1}$ Department of Geriatric and General Medicine, Graduate School of Medicine, Osaka University, Suita, Japan, ${ }^{2}$ Department of Clinical

Gene Therapy, Graduate School of Medicine, Osaka University, Suita, Japan,

With the emergence of disease-modifying therapies for neurodegenerative disorders like Alzheimer's disease, there is an urgent need for the development of better biomarkers detecting early stage of neurodegeneration and predicting rate of progression. Cerebrospinal fluid (CSF) provides direct representation of pathophysiological changes occurring in the central nervous system, and CSF biomarkers have proven to be useful for the diagnosis and prognosis of neurodegenerative disorders. Preclinical work using mouse models would be useful to explore novel CSF biomarkers; however, detailed characterization of CSF proteins has been challenging due to the difficulty in collecting large amount of CSF from mice. Here, we developed a novel technique that allows consistent recovery of CSF in the awake, freely behaving mouse. A small incision was made on the posterior atlantooccipital membrane and a collecting tube was placed and fixed on the surface of the posterior atlantooccipital membrane that CSF can be drawn via the small hole. We were able to collect large volume of high-quality CSF from the same animal over time. Contamination of brain tissue or blood, which could potentially affect biomarker measurement, was carefully assessed using sensitive methods. This technique would provide the opportunity to identify novel CSF biomarkers using mouse models.

\section{P-676}

The impact of mental health liaisons services on the health care of elderly ward

Amy Hillarious ${ }^{1}$, Jagdish Sharma ${ }^{2}$, Nisha Sunwar ${ }^{1}$, Monique Patel ${ }^{1}$

${ }^{1}$ United Hospitals of Lincolnshire, Lincolnshire, UK, ${ }^{2}$ United

Hosptials of Lincolnshire, Lincolnshire, UK

Cognitive Impairment is the primary or contributing cause of admissions for elderly patients presenting either as behavioural changes of acute delirium or BPSD. Mental Health Liaison services (MHLS) services provide psychogeriatric support for inpatients with complex needs. Our aim is to audit the value of the input of MHLS into patient management and assess the follow up arranged by MHLS on discharge. 24 Patients who had been referred and reviewed by the mental health team were randomly selected over a fourth month period. Referral outcomes were graded as per the Framework for the routine outcome measurement in Liaison Psychiatry using the IRAC scale. Of those referred $66 \%$ were seen within $24 \mathrm{~h}$, reasons for referral were Behavioural issues (62\%) suspected cognitive impairment $(25 \%)$ and medication review (13\%). A diagnosis was made in $50 \%$ of patients, majority of these made by a doctor $(60 \%)$ vs specialist nurse. For behavioural issues pharmacological management was advised in $46 \%$. Community follow up was not arranged in any of the patients with suspected dementia. The MHLS achieved an IRAC score of fully achieved in $25 \%$. Training of the MHLS staff will ensure a diagnosis and management plan in each patient, with remote support from Consultants. Education of the doctors working on geriatric wards in the guidelines of managing delirium will minimise inappropriate referrals with the expectation that MHLS will advise appropriate pharmacological interventions when referred. All those with suspected dementia inpatient have to be referred to outpatient memory clinics to ensure continuity of care.

\section{P-677}

\section{Art and music therapy}

Florence Bonte ${ }^{1}$

${ }^{1}$ Hopital Sainte Marie Paris, Paris, France

Art therapy and music therapy are genuine care practices still too little known. Performed by trained therapists, they can be integrated into care plans for people with Alzheimer's disease or related disorders. Relying on scientific publications, the use of these artistic mediation engages the patient improves his verbal communication, and soothe his psychological and behavioral symptoms, too often impacting patients and caregivers' quality of life. We report our experience using these techniques in Rehabilitation Memory Day Care Hospital, with the modalities of organizing these interventions, in combination with other non-pharmacological interventions. Recommended by reference agencies, they should be more widely available and scientifically supported,their indications are wider than the field of dementia, but only if only performed by licensed therapists. 


\section{P-678}

Screening cognitive function of elderly patients assisted at a reference center in Northeast Brazil

Leonardo Victor Galvão-Moreira ${ }^{1}$, Maria de Fátima Carvalhal Martins ${ }^{1}$, Rita da Graça Carvalhal Frazão Correa ${ }^{1}$, Rodrigo de Souza Barcelos Barroqueiro $^{1}$, Luciane Maria Oliveira Brito ${ }^{1}$

${ }^{1}$ Federal University of Maranhão, São Luís, Brazil

Objective: To identify the cognitive profile of elderly outpatients seen at a reference center in São Luís, Brazil.

Methods: A cross-sectional study was carried out and included a total of 102 non-institutionalized elderly patients. A questionnaire with socio demographic variables and three screening tests for cognitive impairment detection were used. Data analysis included the Fisher exact, Chi squared and Mann-Whitney tests.

Results: The majority of patients were women (72\%), aged 60-64 years (33.3\%), married or living in consensual union $(43.1 \%)$, and had non-white skin color $(52.9 \%)$. The prevalence of cognitive impairment was $60.7 \%$ for the Mini-mental state examination, $23.5 \%$ for the Verbal Fluency test and 59.8\% for the Clock Drawing test. Regarding the presence of cognitive impairment in at least one test, a total of $83.3 \%$ was observed. There was an association between the occurrence of cognitive impairment with both marital status and selfperception of health status $(\mathrm{p}<0.05)$.

Conclusion: In the present study, the occurrence of cognitive impairment was considered high and might be associated with marital and health status.

\section{P-679}

\section{The impact of obesity and APOE4 gene on dementia}

Carlos Márquez ${ }^{1}$, Barbara Angel ${ }^{1}$, Lydia Lera ${ }^{1}$, Hugo Sanchez ${ }^{1}$, Cecilia Albala ${ }^{1}$

${ }^{1}$ Institute of Nutrition and Food Technology (INTA), University of Chile, Santiago, Chile

Obesity is one of the main risk factors for chronic non-communicable diseases. In older people (OP), Dementia may be the one with the greatest negative repercussions. Among the genes associated with dementia, Apolipoprotein E4 (ApoE4) is the most common. The objective of this research is to determine the impact of obesity and APOE4 on the development of dementia in Chilean OP.

Methods: Follow-up of ALEXANDROS cohorts. We followed $522(69,6 \%$ women) cognitively healthy OP at baseline for at least 5 years, Cognition was evaluated using a screening test validated for Chile consisting in Mini-Mental State Examination score $<22$ and a score $>5$ in the Pfeffer Activities Questionnaire. To assess Obesity we used the BMI cut-off of $\geq 30 \mathrm{~kg} / \mathrm{m}^{2}$. APOE4 was measured into a frozen basal sample. To determine ApoE4 genotypes, we used StepOneTM PCR.

Results: At baseline the Mean age was $67+4.9$ years, the $21.7 \%$ of the sample was obese, higher in women $(24.9 .9 \%)$ than men $(13.9 \%)$ $\mathrm{p}<0.05 .16 .3 \%$ of the sample were carriers of APOE4, $21.1 \%$ of them were obese. In the follow-up, we found 43 new dementia cases, of them $17.1 \%$ were obese at baseline and $25.6 \%$ were APOE4 carrier. After adjusting by gender, age, and education, the risk of dementia in Obese APOE4 carriers was OR $=6.3(95 \%$ CI $1.42-27.8)$, in Obese non-carrier $=0.61 ; 95 \%$ CI $0.13-2.81$ ) and in APOE4 carriers non-obese OR $=1.67(95 \%$ CI $0.55-5.04)$.

Conclusion: Dementia is a big problem for public health, to be APOE4 carrier and obese would favor the development of dementia in OP.

\section{P-680}

Intervention program to promote personal autonomy in mild and moderate cognitive impairment

Mireia Tofiño García ${ }^{1}$, Rocío Molás Robles ${ }^{2}$, Elena Cabrero Montes ${ }^{1}$, Enrique Pérez Sáez ${ }^{1}$, Myriel López Tatis ${ }^{3}$

${ }^{1}$ National Reference Centre for Alzheimer's and Dementia Care (CREA), Imserso, Madrid, Spain, ${ }^{2}$ National Reference Centre for Alzheimer's and Dementia Care (CREA), Imserso, Madrid, Spain, ${ }^{3}$ National Reference Centre for Alzheimer's and Dementia Care (CREA), Imserso, Madrid, Spain

Introduction: Dementia is a major cause of disability and burden of care. Occupational therapy interventions could be easily implemented in daily live to improve autonomy, cognitive and functional status.

Objective: to evaluate the effects of an intervention based on instrumental and advanced activities of daily living (ADL) in autonomy, cognition, behaviour and quality of life.

Methods: Setting, a day clinic specialized in dementia care. 29 patients aged $\geq 60$ with mild to moderate cognitive impairment living in the community, independent for ADL and independent for $\geq 2$ instrumental activities. The present study was prospective. The intervention was focused on instrumental and advanced ADL and training caregivers on coping behaviours and support autonomy at home. It was performed $30 \mathrm{~h}$ a week for 9 months. Participants were evaluated at baseline, at 9 months and 6 months follow up. Attendance to the sessions was $90 \%$.

Results: Mean age was $80.52 \pm 6.34$ years. Mean Mini-Mental Status Examination (MMSE), $21.66 \pm 2.94$, Barthel $98.10 \pm 4.31$, instrumental activities (IADL), $3.85 \pm 1.79$. Quality of life (QolAD) $33.93 \pm 4.22$, Neuropsychiatric Inventory (NPI-Q) severity $7.26 \pm 4.46, \quad$ NPI-Q distress $9.37 \pm 7.68$. MMSE remained stable $(\mathrm{p}=0.33)$, and Barthel was slightly lower( $\mathrm{p}=0.079)$.IADL, Qol-AD, NPI-Q severity and distress improved at 9 months, $(\mathrm{p}=0.042),(\mathrm{p}=0.000),(\mathrm{p}=0.001)$ and $(\mathrm{p}=0.007)$ respectively. $86 \%$ were living at home at 6 months follow up.

Conclusions: occupational therapy intervention improved IADL. Improvement in Neuropsychiatric symptoms and overall QoL was significant at 6 months. Most patients remained community dwellers.

\section{P-681}

Dementia care in nursing homes: results from the DEMDATA study

Iva Holmerová ${ }^{1}$, Stefanie Auer ${ }^{2}$, Anna Beránková ${ }^{1}$, Michal Steffl ${ }^{3}$

${ }^{1}$ Univerzita Karlova FHS CELLO, Prague, Czech Republic, ${ }^{2}$ Donau Universität Krems, ${ }^{3}$ Univerzita Karlova FHS CELLO a FTVS, Donau, Austria

Introduction: Dementia is among most frequent conditions in nursing homes. However it often remains unclear how many persons living in nursing homes are suffering from cognitive impairment and dementia and how many of them receive appropriate diagnosis and care.

Methods: The DEMDATA: cross sectional multi-centre mixed methods study focused on dementia prevalence and care in Austrian and Czech Nursing homes (2016-2018) - stratified and randomly drawn nursing homes in Austria and the Czech Republic provided data on: Resident, Care team, Relatives and Environmental Factors. 571 (Austria) and 514 (Czech Republic) randomly selected residents participated in the research. Resident's data include cognition and dementia, general health status, mobility, nutrition, behavioural symptoms, pain and quality of life [1]. 
Results: The average age of participants was $84.8 \pm 7.5$ years, $78.9 \%$ were female. Data analysis from the Czech Republic have shown that dementia diagnosis in the health care documentation often does not reflect the current cognitive status of residents: $16 \%$ of all residents had cognitive impairment which was not documented.

Conclusions: Timely and proper diagnosis of dementia and its cause is a necessary condition for good quality care. Therefore it is necessary to improve expertise in dementia-of physicians and all health staff who provide care in nursing homes.

Reference:

1. Auer S, Linsmayer E, Beránková A, Pascher P, Firlinger B, Prischl D, Ratajczak P, Span E, Holmerova I (2017) DEMDATA: the Austrian-Czech institutional long term care project-design and protocol of a two-centre cross sectional study. BMC Health Serv Res 17(1):296.

\section{P-682}

Dementia and Hospital Admission Post TIA and Stroke: Longitudinal Population-based Study

Aubretia McColl ${ }^{1}$, Ramon Luengo-Fernandez ${ }^{1}$, Linxin $\mathrm{Li}^{1}$, Peter M Rothwell ${ }^{1}$, Sarah T Pendlebury ${ }^{1}$

${ }^{1}$ Centre for Prevention of Stroke and Dementia, University of Oxford, UK

Introduction: Dementia after TIA/stroke is associated with age, lesion burden and neurodegenerative disease but systemic factors including inflammation/infection may also play a role. We therefore determined associations between TIA/stroke-associated dementia and hospitalisation in a longitudinal study.

Methods: In a population-based study of TIA and stroke (Oxford Vascular Study/2002-2012) multiple overlapping methods including face-to-face interview were used to ascertain dementia to 5 years follow-up. Frequency and characteristics of hospital admissions between index event and end follow-up were compared between patients with dementia versus those remaining dementia-free at 5 -years using hospital diagnostic coding data adjusted for age and sex. Results: Among 2305 patients (693 TIA/1478 ischaemic stroke/134 primary intracerebral haemorrhage), $657(28.5 \%)$ were identified as having dementia. Patients with dementia were older (mean/SD 80.8/ 8.6 vs $70.9 / 13.4, \mathrm{p}<0.001)$ and more likely to be female $(60.6 \%$ vs $46.7 \%, \mathrm{p}<0.001)$ than those without. During 5-year follow-up, there were 8861 admissions to the regional district hospital, of which 4157 (46.9\%) were unplanned with $2931(70.5 \%)$ of these being to medical specialities. Patients with dementia were more likely to have any hospital admission (adjusted $\mathrm{OR}=2.24$, 95\% CI 1.66-3.01, $\mathrm{p}<0.001$ ) or any unplanned hospital admission (adjusted $\mathrm{OR}=3.00$, $2.32-3.88, \mathrm{p}<0.001)$ compared to patients without dementia, particularly for infection related illness $(29.1 \%$ vs $14.2 \%$, adjusted $\mathrm{OR}=1.99,157-2.51, \mathrm{p}<0.001$ ).

Conclusions: Patients with previous TIA/stroke who develop dementia, have more hospitalisations including unplanned admissions for acute medical illness. Further studies are required to determine whether acute illness, and particularly infection, are independent risk factors for cognitive decline after TIA/stroke.

\section{P-683}

Evolution of cognitive function after transcatheter aortic valve implantation in octogenarians

El Nakada Badih ${ }^{1}$, Higuet Sandra ${ }^{1}$, Denewet Nathalie ${ }^{1}$
${ }^{1}$ CHU Charleroi, Charleroi, Belgium

Background: The transcatheter aortic valve implantation (TAVI) procedure is a good technique in octogenarian patients suffering from symptomatic aortic valve stenosis and high surgical risk. The evolution of cognitive function, a central clinical factor in geriatrics, remains uncertain after this procedure.

Objective: The aim of this prospective study is to assess cognitive function after TAVI.

Method: In a prospective cohort of patients $>80$ years, cognitive function was measured using MMSE and MOCA, before as well as one and 6 months after TAVI. Results: Eighteen patients undergoing TAVI have been enrolled between March 2017 and March 2018. The average age was $86 \pm 4.5$ years. The comparison of MMSE and MOCA over time did not show any significant change. We noted an improvement in the MOCA results performed on the first and sixth month $(\mathrm{p}=0.07)$. There was also a significant positive trend in some items such as orientation in MMSE $(\mathrm{p}=0.03)$, and abstraction $(p=0.001)$ and orientation $(p=0.014)$ in MOCA. Although TAVI is associated with more ischemic cerebral lesions, most studies show that cognitive function was preserved in the great majority of patients throughout the first 2 years after implantation. A cognitive improvement after TAVI had already been shown in patients with very severe aortic stenosis, related to hemodynamic improvements [1].

Conclusion: While it would be interesting to perform a global cognitive functions assessment and functional magnetic resonance imaging, our study already confirms cognitive function stability using both MMSE and MOCA.

\section{P-684}

Is dual task combined switching training an effective tool to improve cognitive functions?

Nina Trushkova ${ }^{1}$, Natalia Ermolina ${ }^{2}$, Ekaterina Trushkova $^{3}$, Giovanni Zelano ${ }^{4}$

${ }^{1}$ Rehabilitaition Research Center "Armonia”, Latina, Italy,

${ }^{2}$ Astrakhan State University, Astrakhan, Russia, ${ }^{3}$ Cognitive Education Center "Seneca", Murcia, Spain, ${ }^{4}$ Rehabilitaition Research Center "Armonia", Latina, Italy

Introduction: Several studies suggest that the human ability to rapidly and accurately switch between tasks multiple times employs working memory capacity This ability decreases with age and that is why older people have great difficulty in dual-task performance and Trail Making Test (TMT) part B test. Studies have indicated that the working memory capacity is trainable. The purpose of our study is to investigate the effect of Dual Task Combined Switching Training based on motor coordination tasks and working memory exercises in healthy adults.

Methods: We established dual tasks which require the ability to rapidly and accurately switch between tasks. All tasks of training vary from easiest to more difficult to avoid automatic. The study subjects were 27 healthy adults, 11 men and 16 women, aged $\geq 73$ years, cognitive status screened (MMSE $>25$ ). All of them had a poor TMT part B performance. We have proposed to them to perform Dual Task Combined Switching Training $1 \mathrm{~h}$ twice a week for 26 weeks. At the beginning and at the end of treatment, participants received a cognitive examination that included Mini-Mental State Examination (MMSE), Forward and Backward Digit Span Test, TMT-A, TMT-B. Results: The cognitive assessment showed statistically significant improvement in all the scores tested (t-test performed).

Key conclusions: Our findings suggest that Dual Task Combined Switching Training based on motor coordination tasks and working memory exercises has proven to be effective tool to improve 
cognitive functions in healthy adults. We also discussed several directions for future combined training studies.

\section{P-685}

People with young onset dementia: investigating their hospital healthcare utilisation and related palliative care support needs

Siobhan Fox ${ }^{1}$, Bryan Tan $^{1}$, Claire Kruger ${ }^{2}$, Marie Lynch ${ }^{3}$, Deirdre Shanagher ${ }^{3}$, Suzanne Timmons ${ }^{1}$

${ }^{1}$ University College Cork, Cork, Republic of Ireland, ${ }^{2}$ Mercy University Hospital, Cork, Republic of Ireland, ${ }^{3}$ Irish Hospice Foundation, Dublin, Republic of Ireland

Introduction: There is a knowledge gap about the specific needs of, and utilisation of healthcare services by, people with Young-Onset Dementia (YOD), defined as those diagnosed $<65$ years. People with YOD and their families face unique challenges compared to those with late-onset dementia (LOD), and these can be addressed with a palliative care approach. Palliative care for dementia has received increasing attention, however those with YOD have remained overlooked. The study aim was: To explore healthcare utilisation, including at end-of-life, of people with YOD, using hospital electronic records for case finding.

Method: We obtained Hospital In-Patient Enquiry data identifying all people with YOD admitted to three large urban hospitals between 2009 and 2016, and conducted a retrospective chart review. Information collected included demographics, medical and psychosocial history, functional capacity, last hospital admission, mortality and Advance Care Plan (ACP) details.

Results: Of the 121 patients, 50\% were male. The commonest dementia types were: Dementia secondary to Down's Syndrome (16\%), Vascular (14\%), Frontotemporal (13\%) and Alzheimer's Disease $(13 \%) .88 \%$ had $\geq 1$ comorbid disease, including neurological (55\%), cardiovascular (36\%), and mental health illnesses (29\%). Although $70 \%$ of People with YOD had an indication for an ACP (i.e. one or more markers of limited life expectancy), only $11 \%$ had any ACP recorded. $37 \%$ of patients had died, most commonly due to a complication of advanced dementia (e.g. aspiration pneumonia) rather than comorbid illness.

Conclusion: There were notable differences between this sample of people with YOD and LOD populations, regarding their diagnosis, comorbidities, diagnosing physician, etc, which has implications for multidisciplinary team input and post-diagnostic support. People with YOD most commonly die from complications of dementia, as opposed to people with LOD who often die with dementia. Advanced care planning appears to be suboptimal in people with YOD. More research is essential to inform future policies and services for this often neglected population.

\section{Area: Comorbidity and multimorbidity \\ P-686}

Commorbidity in chronic obstructive pulmonary disease

Tiziana Candiani ${ }^{1}$, Giuseppina Alessandro ${ }^{1}$, Simonetta Vernocchi ${ }^{2}$, Chiara Schena ${ }^{2}$

${ }^{1}$ U.O.C. General Medicine-Geriatrics ASST WEST Milanese, Legnano, Italy, ${ }^{2}$ U.O.C. General Medicine-Geriatrics ASST WEST Milanese, Legnano, Italy)

Many elderly patients with COPD have psychological problems such as anxiety and depression as an effect of the disease and represent the two least-treated comorbidities in the chapter of this pathology. The aim of the present study is to verify a possible correlation between COPD and depression in elderly outpatients.

Materials: To all patients suffering from COPD who came for specialized assessment with spirometry examination at the Pneumology Clinic of Cuggiono Hospital of ASST WEST Milanese in the period October 2016 - December 2017 self-administered the Geriatric Depression Scale_-J. Yesavage (GDS, score used in the geriatric field to highlight the mood tone deflection). The group consisted of nr. 162 subjects M: 98 F: 64 with a mean age 76, 2. The indices considered in this study were: FEV1-FEV1/fvc and GDS. We therefore wanted to verify a possible correlation between the above mentioned indices. The results were as follows: correlating the indices among them of all the subjects studied we obtained: FEV1 versus GDS 0.05524, FEV1/ fvc versus GDS 0.019986. We then divided the subjects in relation to the severity of the GDS in three groups. A first group consisting of 100 subjects with GDS less than 10, a second group composed of 39 subjects with GDS between 11-15 and a third group composed of 21 subjects with GDS greater than 15. By evaluating the possible correlations we obtained the following data: Group I: - 0.02752-0.138366, Group II: 0.00178-0.02204, Group III: $0.130583-0.322169$.

\section{P-687}

Behavioral pain scale (BPS) validity and reliability among chronically unconscious geriatric ventilated patients

Dani Kirshner ${ }^{1}$, Svetlana Zheludkov ${ }^{1}$, Nabil Gharra ${ }^{1}$, Irina Rahamimov ${ }^{2}$, Tanya Bogoslavsky ${ }^{1}$

${ }^{1}$ Shoham Geriatric Medical Center, Minestry of Health, PardesHanna, Israel, ${ }^{2}$ Dorot Geriatric Medical Center, Minestry of Health, Netanya, Israel

Introduction: The inability to express pain in unconscious, chronically ventilated geriatric patients is a significant barrier to assess pain intensity. This puts this population at risk for inappropriate pain management. BPS has been evaluated and validated as a tool for assessing pain among ventilated patients in intensive care units, but has not been tested among chronically unconscious ventilated patients. The aim of this study was to examine the reliability and validity of the BPS in this population.

Methods: This observational study enrolled chronically ventilated old patients, at two different geriatric long-term care facilities. Each patient was assessed for pain by two assessors using the BPS score. Pain was evaluated during repositioning, venous puncture and tracheal suction. Each assessor, independently, assessed the BPS score in three time points: before, during and after each one of the aforementioned procedures. Data regarding background morbidity, demography, cause and duration of ventilation, laboratory parameters and usage of analgesics was collected.

Results: 864 observations were conducted among 48 patients recruited. The mean $( \pm \mathrm{SD})$ age of the patients was $75.9 \pm 9.8$ years. 24 patients $(50.0 \%)$ were men. Total BPS score showed a good internal reliability for repositioning, venous puncture and tracheal suctioning (Cronbach $\alpha=0.885,0.868$ and 0.693 respectively). Validity was attained by demonstrating significant increase from baseline to painful levels of BPS score and significant decrease from painful levels to the post-procedure levels during all three procedures $(\mathrm{P}<0.001)$.

Conclusion: BPS is a valid and reliable pain assessment tool for chronically, unconscious ventilated old patients. 


\section{P-688}

Geriatric patients visits the Emergency Department at an Academic Tertiary Care Center in Jeddah, Saudi Arabia

Mohammed Basheikh ${ }^{1}$, Jameel T. Abualenain ${ }^{2}$, Daniah M. Kamfar ${ }^{1}$, Ekram S. Faden ${ }^{1}$

${ }^{1}$ King Abdulaziz University, Jeddah, Saudi Arabia, ${ }^{2}$ King AbdulAziz University, Jeddah, Saudi Arabia

Objectives: Geriatric patients aged more than 60 years are a vulnerable group of patients needing special care in the emergency department. This study aimed to determine the prevalence of geriatric patients visiting the emergency department and explore the various aspects of those visits.

Methods: A retrospective chart review of all emergency department visits from August 2016 to July 2017 was performed at King Abdulaziz University Hospital in Jeddah, Saudi Arabia.

Results: Of 34,127 emergency department visits by adults, 6533 (19.14\%) were made by geriatric patients (age range, $60-115$ years; average, 71.25 years), representing about 45 nationalities $(48.62 \%$ were Saudi). Triage priority using the "Canadian Triage and Acuity Scale" showed that $36.08 \%$ of the visits were emergencies, $49.35 \%$ were urgent, and $14.57 \%$ were non-urgent. Most $(40.47 \%)$ of the visits were made during the morning, almost double the number made at night $(23.30 \%)$. Geriatric patients presenting with a single complaint represented $62.47 \%$ of these visits, those presenting with two complaints represented $31.01 \%$, and those presenting three or more complaints, $6.52 \%$. The most common complaints were dyspnea (25.28\%), chest pain (13.16\%), and abdominal pain (10.82\%). In the final diagnoses, diseases of circulatory system were most frequent $(15.20 \%)$. The admission rate was $28.38 \%$, and the mortality rate was $1.94 \%$.

Conclusion: Geriatric patients visiting the emergency department represent one-fifth of all emergency department visits by adults. They commonly present with complex medical conditions, making the approach to their diagnoses more challenging. Therefore, we recommend paying greater attention using multidisciplinary services and follow up.

\section{P-689}

Association of renal function and falls risk among geriatric patients at Geriatric department of Kaunas Clinical Hospital

Jurgita Knašiene ${ }^{1}$, Indre Zubavičiūte ${ }^{1}$, Juozas Cépla ${ }^{1}$

${ }^{1}$ Geriatric Department, Lithuanian University of Health Sciences, Kaunas, Lithuania

Introduction: The increased risk of falls is associated with disability, increased risk of hospitalization or even death in elderly patients.

Methods: In a prospective study data of 103 patients $>60$ years age were analysed. The 'Get Up and Go' test, 'Romberg' tests were performed to assess the risk of falls. To evaluate renal function, GFR and electrolyte balance were estimated. RESULTS: Increased risk of falls was found in $84.5 \%(\mathrm{n}=87)$ of patients. Female patients had a greater risk of falls $89.3 \%$ than men $71.4 \%(p=0.023)$. Based on eGFR $90.3 \%(\mathrm{n}=93)$ of patients renal impairment was observed: mild $50.5 \%(\mathrm{n}=52)$, mild-moderate $36.9 \%(\mathrm{n}=38)$, moderate-severe $1 \%(n=1)$, severe $1.9 \%(n=2)$. Patients with renal impairment were statistically significantly older (mean age $80.14 \pm 6.54$ ) than patients with good renal function (mean age $73.70 \pm 8.616$ ) $(\mathrm{p}<0.05)$. Risk of falls has relationship with sodium level in serum $(\mathrm{F}=3.898 \mathrm{df}=1 \mathrm{p}=0.04)$. Hyponatremia was more commonly observed among patients with increased risk of falls $(19.3 \%)$, $\mathrm{p}=0.036$. The female patients had 3.35 times increased risk of falls $(\mathrm{OR}=3.35,95 \% 1.115-10.063)$. Every year the risk of falls for patients is rising by 1.17 times. $(\mathrm{OR}=1.169,95 \% 1.071-1.276)$. Compared to those who had normal serum sodium, patients who had hyponatremia had 7.03 times higher odds of having increased risk of falls ( $95 \% \mathrm{CI}=0.804$ to 61.375 ).

Key conclusions: The increased risk of falls was associated with age, female gender and hyponatremia, but was not found to be statistically significant in relation to renal function.

\section{P-690}

Impact of comorbidity and chronic diseases on 5-years mortality in the patients 90 years old and over in Moscow population

Yuliya Kotovskaya ${ }^{1}$, Olga Tkacheva ${ }^{1}$

${ }^{1}$ Pirogov Russian National Research Medical University, Russian Clinical and Research Center of Gerontology, Moscow, Russia

Background: the prevalence of chronic diseases and comorbidity increases with aging. However, influence of comorbidity and single chronic diseases on mortality risk in very old patients is still controversial.

Purpose: to evaluate impact of comorbidity and single chronic diseases on 5-years total mortality in very old patients in Moscow population.

Methods: seventy-two patients (19 men) aged 90-98 (mean $91.7 \pm 2.1$ ) years were included in the observational prospective study. We estimated the presence of any chronic diseases and calculated Charlson comorbidity index (CCI). The follow-up period was 5 years. Endpoint was all-cause mortality.Results: median of follow-up was 3.13 years ( $\min 40$ days, $\max 5.79$ years, IQR $1.85-4.08$ years). Thirty-six $(50 \%)$ patients died. CCI was ranged from 4 to 11 (median 6, IQR 5-7) points. Kaplan-Meier analysis didn't confirm that comorbidity (CCI $\geq 6$ points) influence 5 -years total mortality (Chi square $1.2 ; \mathrm{p}=0.27$ ). Also Kaplan-Meier analysis identified only one chronic disease which was associated with 5 -years mortality: atrial fibrillation (Chi square 27.0; $p<0.001$ ). Cox regression with age and sex adjustment showed that atrial fibrillation increases the 5-years mortality risk in 5, 6 times (HR 5.59; 95\% CI 2.72-11.49; $\mathrm{p}<0.001$ ) in very old patients.

Conclusions: Comorbidity (CCI $\geq 6$ points) is not associated with increase of 5-years total mortality risk in the very old Moscow population. Atrial fibrillation not included into the CCI is an independent predictor of 5-years total mortality in these patients and should also be considered for evaluation of life prognosis.

\section{P-691}

Analysis of white blood cell values in older patients: results of a cross sectional study on hematologic laboratory parameters among outpatients aged $\geq 60$ years

\section{Ingrid Becker $^{1}$, Kai Gutensohn ${ }^{2}$, Thomas Nebe ${ }^{3}$}

${ }^{1}$ Institute of Medical Statistics and Computational Biology, University Hospital Cologne, Germany, ${ }^{2}$ AescuLabor Hamburg $\mathrm{GmbH}$, Hamburg, Germany, ${ }^{3}$ Laboratory of Hematology, Mannheim, Germany

Introduction: Hematological abnormalities are frequently found in geriatric patients. However, little data is available on normal reference values for total blood counts in older people [1,2]. After having analyzed the normal values of erythrocytic blood parameters, our 
study group has now laid the focus on the analysis of normal values for white blood cell counts in aged persons $\geq 60$ years [3].

Methods: Cross sectional study of outpatient laboratory data of 2015 from a German countrywide working laboratory company; inclusion criteria were age $\geq 60$ years, normal CRP, transferrin saturation, reticulocytes, $\mathrm{LDH}$, haptoglobin and soluble transferrin receptor; exclusion criteria: GFR $<60 \mathrm{ml} / \mathrm{min}$, lack of inclusion criteria; primary objective was the assessment of the mean leucocyte count, secondary objectives were the assessments of the mean counts of lymphocytes, eosinophil, neutrophil and basophil leucocytes as well as platelets in hematologic healthy persons aged $\geq 60$ years.

Preliminary results/conclusions: Of 32532 patients(p) between 60-99 years 19496 met with inclusion criteria; age groups were 60-70, 71-80, 81-90 and >90 years; normal reference values were calculated based on 2.5 and 97.5 percentiles (all patients): platelets 144.000-374.00/ $\mu \mathrm{l}$; leucocytes $4.0-10.7 / \mu \mathrm{l}$; basophils $0.3-1.5 \%$; eosinophils $0.5-7.3 \%$; lymphocytes $16.8-44.9 \%$; monocytes 5.6-13.7\%; neutrophils 41.8-69.9\%; preliminary results suggest no age associated changes of total white blood count values and platelet values; completed data evaluation including age and gender specific analysis will be presented at EUGMS 2018.

\section{References:}

1 Starr JM, Deary IJ (2011) Sex differences in blood cell counts in the Lothian Birth Cohort 1921 between 79 and 87 years. Maturitas 69(4):373-376. https://doi.org/10.1016/j.maturitas.2011.05.007

(Epub 2011 Jun 8)

2. Troussard X, Vol S, Cornet E, Bardet V, Couaillac JP, Fossat C, Luce JC, Maldonado E, Siguret V, Tichet J, Lantieri O, Corberand J (2014) French-Speaking Cellular Hematology Group (Groupe Francophone d'Hématologie Cellulaire, GFHC). Full blood count normal reference values for adults in France. J Clin Pathol. 67(4):341-344. https://doi.org/10.1136/jclinpath-2013-201687 (Epub 2013 Oct 29)

3. Röhrig G, Becker I, Gutensohn K, Nebe T (2017) Red blood cell counts and indices in the elderly German population. J Lab Med. https://doi.org/10.1515/labmed-2017-0080

\section{P-692}

Necrotizing pneumonia vs Pulmonary tuberculous in the elderly patient: a case report

Carlos D. Silva ${ }^{1}$, José E. Mateus ${ }^{1}$, Bárbara Marques ${ }^{1}$, Mariana Brites ${ }^{1}$, Teresa Vaio ${ }^{1}$, Rui M. Santos ${ }^{1}$, Manuel T. Veríssimo ${ }^{1}$, Armando Carvalho ${ }^{1}$

${ }^{1}$ Centro Hospitalar e Universitário de Coimbra, Coimbra, Portugal

Necrotizing pneumonia is a rare complication of bacterial pneumonia that occurs on chronic and immunocompromised patients. There are inflammation and lung necrosis with formation of small cavitations. Streptococcus pneumoniae, Staphilococcus aureus and Klebsiella pneumoniae are the most frequent etiological agents. Desfavorable course to antibiotic therapy and CT-scan images make the diagnosis. However, pulmonary tuberculosis must be excluded. The elder patient has an atypical presentation. A 92-years-old institutionalized and dependent woman presented with fever, dyspnea, dry cough and anorexia at 3 days. She had dementia, cachexia, ischemic cardiopathy, and past-history of aspiration pneumonia 1-year before, and genital tuberculous 40-years before. She was diagnosed with rightbase pneumonia and started empiric antibiotherapy. Microbial studies were negative as well as interferon- $\gamma$ release assay. Due to persistence of symptoms the antibiotherapy was escalated without improvement. After naproxen fever resolved and sustained with linezolid/metronidazol. 25-days after she underwent thoracic computed tomography that was suggestive of necrotizing pneumonia. Bronchoscopy didn't aid morphologic information but the direct examination of bronchoalveolar lavage fluid shows mycobacterium tuberculosis. Done the diagnostic of pulmonary tuberculous (PT) she died 1 week after tuberculostatics onset. The incidence of tuberculosis in nursing home residents is three to four times higher than that of those living in the community. Comorbidities, immunosenescence, malnutrition, immunosuppressive therapy and unfavourable socioeconomic conditions, all contribute to the higher incidence of tuberculosis in the elderly patient. Advanced age is associated with PT atypical clinical presentations leading to delayed diagnosis and an increased rate of postmortem diagnosis.

\section{P-693}

Delirium and dysphagia are relevant risk factors for permanently increased degree of care

Barbara Hanussek $^{1}$, Hoell Christine ${ }^{1}$, Gaertner Thomas ${ }^{1}$, Ried Matthias ${ }^{1}$, Langhans Markus ${ }^{1}$, van Essen Joerg ${ }^{1}$

\section{${ }^{1}$ MDK HESSEN, Darmstadt, Germany}

Objective: How do hospitalised geriatric patients with initially comprehensive need of care (Barthel index $<35$ Points), with or without need for extra care complex measurements "PKM", differ from each other regarding their long term degree of care.

Method: Retrospective comparative analysis of 44 cases of geriatric inpatients ( 2 groups of $\mathrm{n} 22, \mathrm{n}=22$, each $\mathrm{f}=13, \mathrm{~m}=9$, age 70-97) according to the assessment practice of medical expert opinion of the health insurance "MDK", taking into account their degree of care certificate.

Results: Patients with PKM achieved only a minor functional improvement after geriatric intervention (stagnant Barthel Index at discharge $<35: 59 \%$ vs. $36 \%$ ). Patients with PKM were more likely to receive a first time or higher degree of care certificate $(68 \%$ vs. $50 \%$ ). The main complications in the PKM group were Delirium (64\%) and Dysphagia (50\%). Pre-existing cognitive impairment was equally common in both groups.

Discussion and conclusions: Delirium is known to be associated with an increased need of long-term care. Our data indicated this correlation also for dysphagia. Delirium and Dysphagia are associated with malnutrition and often cause a prolonged hospitalisation. Thus, it appears necessary to extend the geriatric basic assessment for patients with comprehensive need of care, in order to allow targeted indications for specific interventions, eg drug or nutritional therapy, in order to reduce the incidence of complications and inpatient readmissions, which would show positive effects on treatment costs and quality of life.

\section{P-694}

Home medicine today: the high prevalence of heart failure with preserved systolic function among home-bound residents

\section{Jun Chiong ${ }^{1}$, Cloie June Chiong ${ }^{2}$, Alan B. Miller ${ }^{3}$}

${ }^{1}$ MedEx Health Network, Inc., North York, Canada, ${ }^{2}$ University of California San Diego, San Diego, USA, ${ }^{3}$ University of Florida, Gainesville, Florida

Background: In the coming years, home medicine will comprise an increasing share of patient encounters, due to the unprecedented and continuing demographic changes. The primary aim of the study was to identify the diagnosis of the cohort to prepare training for in house providers. 
Methods: We analyzed data of consecutive patients visited at home due to mobility problems, vision problems, cognitive decline and in some cases, mental health issue in 2017. ICD-10 codes were extracted on all the primary encounter diagnosis from all the visits.

Results: There were a total of 3.408 patient home encounters during the study period. There were 2345 ICD codes extracted. Mean age is 81.9 years, The ICD by organ system are cardiovascular $(\mathrm{I})=1085$, endocrine $(E)=232$, Malignancy $C=30$, Respiratory $=179, \mathrm{M}-179$, Renal $\mathrm{N}=63$. The top $10 \mathrm{ICD}$ classification includes: I11.9: Hypertension heart disease without heart failure $(\mathrm{N}=288)$ I5032: Chronic diastolic heart failure $(n=239)$ E11.xx: Type 2 diabetes mellitus (all types) $(\mathrm{n}=153)$ I110: Hypertensive heart disease with heart failure $(n=149) I 48 . x x$ atrial fibrillation $(n=137) J 44 . x$ : Chronic obstructive pulmonary disease $(n=90)$ I2510: Athscl heart disease of native coronary artery $(\mathrm{n}=88) \mathrm{K} 21 . \mathrm{x}$ : Gastro-esophageal reflux disease $(n=52)$ M179: Osteoarthritis $(n=47) J 209$ : Acute bronchitis $(n=42)$.

Conclusions: Most home bound medical encounters of older adults were due to cardiovascular causes mostly from diastolic heart failure. The present findings clearly emphasize focus on specialized training in heart failure to providers dedicated to practicing home medicine.

\section{P-695}

Anemia in elderly patients is associated with high rather than low serum vitamin B12

\author{
JF Abrahamsen ${ }^{1,2}$, AL Bjørke Monsen ${ }^{3,4}$, M Fredbo ${ }^{1}$, K Kruger ${ }^{1}$, \\ Anette Hylen Ranhoff ${ }^{2,4}$ \\ ${ }^{1}$ Department of Nursing Home Medicine, Municipality of Bergen, \\ Norway, ${ }^{2}$ Kavli Research Centre for Geriatrics and Dementia, \\ Haraldsplass Deaconess Hospital, Bergen, Norway, ${ }^{3}$ Laboratory of \\ Clinical Biochemistry, Haukeland University Hospital and \\ Department of Clinical Science, University of Bergen, Bergen, \\ Norway, ${ }^{4}$ Department of Clinical Science, University of Bergen, \\ Bergen, Norway
}

Introduction: Evidence of an association between subnormal vitamin B12 and anemia is limited and inconclusive in older patients. To get more knowledge of this, blood tests and geriatric assessment were carried out in 829 home-dwelling older patients, who were transferred to nursing home after acute hospitalization.

Methods: Different parameters, including serum folate, plasma homocysteine, anemia $(\mathrm{Hb}<13$ in men, $<12$ in women), renal insufficiency (eGFR $<60 \mathrm{~mL} / \mathrm{min} / 1.73 \mathrm{~m} 2$ ), Mini- Mental Status Examination, sex and age were compared in patients with low $(<250 \mathrm{pmol} / \mathrm{L})$, normal $(250-650 \mathrm{pmol} / \mathrm{L})$ and high $(>650 \mathrm{pmol} / \mathrm{L})$ serum vitamin B12. In addition, iron deficiency (Transferrin receptor $>4.5 \mathrm{mg} / \mathrm{L}$ or Ferritin $<35 \mathrm{ug} / \mathrm{L}$ ) was included in multiple regression analysis to examine the odds ratio for anemia.

Results: Mean age was 84 years, 396 (52\%) had anemia. Seventyeight (9\%) had low, 465 (56\%) had normal and 286 (35\%) had high serum B12. Comparing patients with low and high versus normal serum $\mathrm{B} 12$, a significant lower $\mathrm{Hb}$ and higher incidence of anemia was demonstrated in patients with high serum B12, $\mathrm{n}=162(57 \%)$, $\mathrm{Hb} 11.9 \mathrm{~g} / \mathrm{dL},(\mathrm{p}<0.001)$, while no higher incidence of anemia was demonstrated in patients with low serum vitamin B12, $\mathrm{n}=36(46 \%)$, $\mathrm{Hb} 12.5 \mathrm{~g} / \mathrm{dL}$. No decrease in cognitive function was demonstrated in the patients with low B12. In multiple regression analysis, male sex $(p<0.001)$, renal failure $(p=0.001)$, and high serum B12 $(p=0.02)$ gave higher odds for anemia.

Key conclusions: B12 deficiency was uncommon and not associated with anemia, while high B12 were more common and associated with anemia.

\section{P-696}

\section{Ictus in patient with endocarditis related to ICD catheter}

Francesca Maria Nigro ${ }^{1}$, Irene Marozzi ${ }^{2}$, Marta Migliorini ${ }^{2}$, Simone Pupo $^{2}$, Serena Boni ${ }^{2}$, Stefano Fumagalli ${ }^{2}$

${ }^{1} \mathrm{AOU}-\mathrm{Careggi}$, University of Florence, Florence, Italy, ${ }^{2} \mathrm{AOU}-$ Careggi, University of Florence, Florence, Italy

Background: The management of complex patients affected by Atrial fibrillation and Heart Failure could lead difficulties in the choice of strategy therapy, due to the concomitant presence of implantable device.

Case: Man 69 years old diabetic, hypercholesterolemic, former smokers, affected by ischemic dilated cardiomyopathy (EF 20\%, ICD implant in 2016) due to inferior infarction (1996) and anterior ones (2007), both undergoing PCI and atrial fibrillation on rivaroxaban therapy, was hospitalized in Geriatric ICU because of septic shock and exacerbation of heart failure (pro-BNP: $19824 \mathrm{pg} / \mathrm{mL}$ ).

Results: Hospitalization was complicated by acute hepatic failure (ALT: 1042 U/L; INR: 15.1), that required subsequent modification of anticoagulant therapy with edoxaban. During the hospitalization surgery check of pacemaker was performed because of inflammation sign elevation, positive blood cultures and daptomicina was started, continued for 4 weeks. One month later, he was hospitalized due to recurrence of sepsis, therefore PET was performed with evidence of hypermetabolism in correspondence with PM and the removal of device was programmed. Three days later, Edoxaban was stopped, reintroduced for $24 \mathrm{~h}$ and stopped again to permit implantation of subcutaneous ICD. Discharge test with sinus rhythm restoration was performed. At the release, fall due to left hemiplegia occurred (NIHSS18). At the Angio-Tc evidences of occlusion of media cerebral artery appeared, therefore he underwent endarterectomy procedure with progressive full recovery. Finally we released him with anticoagulant therapy with dabigatran $150 \mathrm{mg} / \mathrm{bid}$.

Conclusion: In patient with comorbidity, personalized medicine could be no sufficient to avoid serious but inevitable complications.

\section{P-697}

\section{Hospital management of a complex case of geriatric cardiology}

Aldo Lo Forte ${ }^{1}$, Alessandra Pratesi ${ }^{1}$, Camilla Ghiara ${ }^{1}$, Emanuele Carrassi $^{1}$, Andrea Herbst ${ }^{1}$, Andrea Ungar ${ }^{1}$, Mauro Di Bari ${ }^{1}$, Francesco Orso ${ }^{1}$, Samuele Baldasseroni ${ }^{1}$

${ }^{1}$ Carreggi University Hospital, Florence, Italy

Man, 88, independent in B/IADL. Hypothyroidism, arterial hypertension, DM2, CRF, primary PTCA on LADA (2000), paroxysmal AF in OAC. Polypharmacy (15 drugs). In charge at Syncope Unit where he goes for a control. We found left conduction delay associated with ST segment alteration in the precordials (V1-V6) and overlap in aVR. The patient was asymptomatic. He then enters our Geriatric-ICU where we decided to perform a coronary angiography, preceded by CRRT, and PTCA (multiple DES implanted). The subsequent hospitalization was complicated by delirium episode requiring dexmedetomidine, substituted by midazolam for SAE (hypotension and bradycardia). IOT was required for ARF (inhalation following vomiting). The course was further complicated by the onset of ACS (NSTEMI) requiring PTCA with post-procedural hemodynamic instability treated with inotropes and AF. Once stabilized, the patient was transferred to an internal medicine department then to a cardiological rehabilitation center. At discharge OAC was not confirmed, to be reassessed in the follow-up on ischemic/hemorrhagic risk ratio. Three months after the acute event, the 
patient is again at home, independent in BADL and partly in the instrumental ones (he does not drive the car anymore).

Conclusions: Clinical trials published in the literature often do not include complex patients such as the case reported above, which instead represent daily life in our hospitals. The intra-hospital management of these patients must therefore take into account the relationship between the risks to which the patient is subjected and the benefit that is expected to be obtained.

\section{P-698}

\section{Breast cancer in elderly}

Sonia Hammami ${ }^{1}$, Olfa Zoukar $^{2}$, Imen Ghaddab ${ }^{2}$, Marwa Souissi ${ }^{2}$, Anis Haddad ${ }^{2}$, Raja Elfaleh ${ }^{3}$

${ }^{1}$ CHU F Bourguiba, LR-NAFS 'Nutrition-Functional Food \& Vascular Health', University Monastir, Monastir, Tunisia, ${ }^{2} \mathrm{CHU}$ F Bourguiba, Maternity Center, Monastir, Tunisia, ${ }^{3} \mathrm{CHU}$ F Bourguiba, Maternity center, University of Monastir, Monastir, Tunisia

Introduction: The number of elderly patients with breast cancer (BC) will increase substantially over the next decades. It is expected that by the year $2035,60 \%$ of all new breast cancer patients are 70 years or older.

Patients and methods: A retrospective cohort study of women aged 65 years and older diagnosed with breast cancer at the Maternity Center of Monastir University Tunisia between January 2010 and December 2017.Results:We report 92 postmenopausal women $(34.2 \%)$ over the age of 65 , including 77 women with $\mathrm{BC}(83.6 \%)$ and 15 women with benign breast diseases $(16.4 \%)$. The median age at diagnosis of $\mathrm{BC}$ was $72.5 \pm 6.6$ years. Tumors ranked $\mathrm{T} 2$ were more frequent $(54.5 \%)$ followed by those classified T4 (37.7\%). Metastases were found in $5.3 \%$ of the cases. Invasive ductal carcinoma was found in $85.7 \%$ of the patients. Hormonal receptors were positive for estrogens in $64.7 \%$ of cases and progesterone for $48.6 \%$ of cases. Surgery was performed in 65 women $(83.3 \%)$ and followed neoadjuvant chemotherapy for 8 patients in whom the tumor was diagnosed at an advanced stage. Adjuvant treatment was prescribed for 67 women $(86 \%)$. Among the patients who underwent surgery, the complication rate was $16.6 \%$.

Conclusion: Older women with BC present with more advanced disease requiring more aggressive treatment. Strategies aimed at earlier detection, improved access to care, and downstaging among older adults are greatly needed in Tunisia.

\section{P-699}

Family system and depression in the elderly, a cross-sectional study

Sonia Hammami ${ }^{1}$, Farhat Chelbi $^{2}$, Said Hajem ${ }^{3}$, Mohamed Hammami ${ }^{4}$

${ }^{1}$ CHU F Bourguiba, LR-NAFS 'Nutrition-Functional Food \& Vascular Health', University Monastir, Monastir, Tunisia, ${ }^{2} \mathrm{CHU}$ F Bourguiba, University Monastir, Monastir, Tunisia, ${ }^{3}$ Insitut National de santé Publique, Tunis, Tunisia, ${ }^{4}$ (CHU F Bourguiba, LR-NAFS

'Nutrition-Functional Food \& Vascular Health', University Monastir, Monastir, Tunisia

Background: The most common geriatric psychiatric disorder is depression. The role of family systems in depression among the elderly has not been studied extensively. We conducted this study in Monastir City, to determine the relationship between the type of family system and depression as well as correlation of depression with other important sociodemographic variables.

Methods: A community based sample of 598 non-institutionalized elderly (age $\geq 65$ years), was selected using probabilistic multistage cluster sampling. Questionnaire based interviews were conducted among the elderly people. Depression was assessed using the miniGeriatric Depression Scale.

Results: There was a predominance of female $(66 \%)$ and mean age was 72.3 years $( \pm 7.4)$.Of all the subjects, $40 \%$ were unmarried (single, divorced, widowed or separated) at the time of study and only $11 \%$ were educated. A large proportion $(59.9 \%)$ of males were unemployed or retired. Eight percent of the subjects were living alone. Out of the 598 subjects, $22.7 \%$ of thesubjects screened positive for depression on the mini-GDS. The elderly living in a nuclear family system were 2.5 times more likely to suffer from depression than those living in a joint family system (OR $=2.5 \quad[95 \%$ $\mathrm{CI}=1.3-4.5]$ )

Conclusion: Residing in a nuclear family system is a strongIndependent predictor of depression. The prevalence of depression in the elderlypopulation was moderately high and a cause of concern. The transition in familysystems towards nucleation may have a major deleterious effect on the physical and mental health of the elderly.

\section{P-700}

\section{Gynecologic problems in geriatric-aged women}

Olfa Zoukar ${ }^{1}$, Sonia Hammami ${ }^{2}$, Imen Ghadab ${ }^{3}$, Anis Haddad ${ }^{4}$, Raja Faleh ${ }^{4}$

${ }^{1}$ CHU F Bourguiba, maternity center, ${ }^{2}$ Biochemistry Laboratory, LR12ES05 LR-NAFS 'Nutrition-Functional Food \& Vascular Health' Faculty of Medicine-University of Monastir, Monastir, Tunisia, ${ }^{3} \mathrm{CHU}$ F Bourguiba, Maternity center, ${ }^{4} \mathrm{CHU}$ F Bourguiba, Maternity Center, Monastir, Tunisia

Introduction: Gynecologic concerns in postmenopausal women are common. Although various conditions may affect all women in this age group, the prevalence of certain disorders, and also diagnostic approaches and treatment options, may vary significantly when considering elderly women.

Objective: The focus of this study is to address several commonly gynecologic issues in postmenopausal women.

Methods: This is a retrospective descriptive study carried out at the Monastir Maternity Center over a period of 9 years. This study involved 269 women aged over 65.

Results: The average age of our patients was $70.6 \pm 5.6$ years. The average duration of hospitalization for elderly women was 17.2 days. Comorbidities were found in $72.5 \%$ of women, Cardiovascular disease, including hypertension, and diabetes mellitus were the most common associated medical problems. In our patients, the gynecological pathology of the elderly women was dominated by cancers (46.1\%) followed by benign conditions $(27.1 \%)$ and pelvic static disorders $(26.8 \%)$. Breast cancer was the most common, followed by those of the cervix and the body of the uterus. For the benign pathology, that of the uterus was the most frequent followed by that of the breast and ovaries.

Conclusion: In Tunisia the interest in gynecological pathology has been centered more on the active women than on the elderly. This population requires significant health care and services. It is important to be aware of common gynecologic concerns and give a particular attention to aspects that must be considered when caring for women in the geriatric age group. 


\section{P-701}

Loss of autonomy and comorbidity in the elderly living in nursing homes

Sonia Hammami ${ }^{1}$, Amel Barhoumi ${ }^{1}$, Said Hajem ${ }^{2}$, Mohamed Hammami ${ }^{1}$

${ }^{1}$ Biochemistry Laboratory, LR12ES05 LR-NAFS 'NutritionFunctional Food \& Vascular Health' Faculty of Medicine-University of Monastir, Monastir, Tunisia, ${ }^{2}$ Institut de santé publique, Brussels, Belgium

Background: Comorbidity and loss of autonomy are two consequences of the ageing. The objectives of this study were the screening of the loss of autonomy and the evaluation of comorbidities.

Population and methods: Descriptive and analytical study of 62 elderly living in a nursing home in Tunisia. The screening of the loss of autonomy was carried out by the AGGIR and KATZ - ADL scales. The evaluation of comorbidity was obtained by the CIRS-G scale.

Results: The average age was $76 \pm 7.7$ years. Men were predominant $(62.9 \%)$. The loss of autonomy according to AGGIR was $37 \%$. It was higher among women (52.2\% vs $28.2 \%$ ), among the single $(52.2 \%$ vs $21.2 \%)$, the undereducated $(82.6 \%$ vs $17.4 \%)$ and among elderly persons without children $(91.3 \%$ vs $11.1 \%)$. The loss of autonomy according to KATZ-ADL was $32.3 \%$. It was higher among women (55\% vs $23.1 \%$ ), among senior older than 75 years $(55.6 \%$ vs $23.1 \%$ ), among the single (70\% vs $18.2 \%)$, the workers $(61.8 \%$ vs $35.9 \%$ ) and elderly persons without children (90\% vs $11.1 \%$ ). A positive correlation has been found between AGGIR and KATZADL. The global average of CIRS-G scale was 9.91. Multiple logistic regression analysis revealed that the following were significant: living alone $(\mathrm{OR}=4.5)$, osteoarticular disorders $(\mathrm{OR}=8.5)$ and neuropsychiatric disorders $(\mathrm{OR}=12.7)$. A negative correlation has been found between AGGIR and CIRS-G scales.

Conclusion: An adequate screening of the loss of autonomy and the management of comorbidities constitute the best prevention against the loss of autonomy in old age.

\section{P-702}

Predictors of mortality in elderly patients admitted to an acute geriatric ward

Danielle Gommers ${ }^{1}$

${ }^{1}$ Department of Geriatrics, Rijnstate Hospital, Arnhem, The Netherlands

Background: Common characteristics of patients admitted to an acute geriatric ward are frailty and older age. The goal of this study was to assess the association between patient, health and functional characteristics and 12 month mortality after discharge. Knowledge of these predictors of mortality can support medical decision making.

Methods: Retrospective study conducted between December 2014 and September 2015 acute geriatric wards of a Dutch teaching hospital. Included were patients admitted for at least one overnight stay. Demographic characteristics and health-related patient factors, including frailty and disability were collected.

Results: 290 patients with a mean age of 84 years were included. The majority were women $(60 \%)$. Mortality 12 months after discharge was $40.3 \%$. Prevalence of disability, impaired mobility and cognitive decline were 81,84 and $67 \%$ respectively. Significant predictors of mortality after 12 months were occurrence of delirium (Odds ratio (OD) 2.4), Charlson Comorbidity Index $\geq 3$ (OR 2.1) and low BMI (OR 0.9) in the multivariate analyses. The occurrence of all these predictors simultaneously raised the risk of mortality 17 fold. Frailty characteristics were not associated with higher mortality.

Conclusion: in predominantly frail elderly patients 1 year mortality is associated with incident delirium, low body weight and concurrent comorbidity and not so much frailty features. Furthermore, the high mortality rate in these patients emphasizes the need for timely consultation of the patient and/or his family about the goal(s) of medical treatment in order to prevent interventions during hospital stay that are not beneficial for the patient.

\section{P-703}

\section{Vitamin D and comorbidity in hospitalized very older persons}

Marta Baroni ${ }^{1}$, Maria Lapenna ${ }^{1}$, Lorenzo Gaggi ${ }^{1}$, Valentina Prenni ${ }^{1}$, Sara Ercolani ${ }^{1}$, Patrizia Mecocci ${ }^{1}$, Virginia Boccardi ${ }^{1}$, Carmelinda Ruggiero $^{1}$

${ }^{1}$ Geriatric and Gerontological Section, S. Maria della Misericordia Teaching Hospital, University of Perugia, Perugia, Italy

Hypovitaminosis D is a highly prevalent condition in older subjects, with rates from 50 to $90 \%$ depending on the setting. It is well established that an inverse associations exists between $25(\mathrm{OH}) \mathrm{D}$ serum levels and cardiovascular, infectious and neurologic diseases, glucose metabolism disorders, inflammation, impaired physical functioning and all-cause mortality. Whether $25(\mathrm{OH}) \mathrm{D}$ is the cause or a marker of organ diseases is still under debate. In addition, the burden of comorbidities in this relationship between 25(OH)D remains unknown. The aim of this study is to investigate the relationship between comorbidity and $25(\mathrm{OH}) \mathrm{D}$ testing the hypothesis that comorbidity is inversely associated with $25(\mathrm{OH}) \mathrm{D}$ in hospitalized very old subjects.

Materials and methods: This is a retrospective study, including 308 subjects consecutively admitted to an acute care geriatric ward, with available data about $25(\mathrm{OH}) \mathrm{D}$ serum levels. $25(\mathrm{OH}) \mathrm{D}$ serum levels were defined according with the following cut-off: 50-30 (optimal range), 29-20 (insufficiency), 19-10 (deficiency), and $<10$ (severe deficiency). Comorbidity was assessed using the Cumulative Illness Rating Scale-Geriatric (CIRS-G). Two summary measures can be constructed: the illness Severity Index (CIRS-SI) and the Comorbidity Index (CIRS-CI).

Results: 157 (74.10\%) women and $55(25.90 \%)$ men with mean age of $86+6$ and $85+6$ years old, respectively. Overall, the burden of comorbidity was $1.89+1.33$ for CIRS-CI and $1.15+0.41$ for CIRSSI. $25(\mathrm{OH}) \mathrm{D}$ serum levels were $14.5+12.4 \mathrm{ng} / \mathrm{ml}$, with $90.9 \%$ of subjects having insufficiency and $46.1 \%$ with severe deficiency. An inverse correlation was found between $25(\mathrm{OH}) \mathrm{D}$ and both CIRS-SI $(\mathrm{r}$ $-0.325 ; \mathrm{p}<0.0001)$ and CIRS-CI ( $\mathrm{r}-0.317 ; \mathrm{p}<0.0001)$. Independent of age, gender, season, smoking habit and renal function, an inverse association between $25(\mathrm{OH}) \mathrm{D}$ and both CIRS-SI $(\mathrm{b}$ : -5.23 ; $\mathrm{p}=0.001)$ and CIRS-CI (b: $-1.70 ; \mathrm{p}<0.0001)$ was confirmed. Conclusion: These findings support the hypothesis that in hospitalized very old subjects comorbidity is associated with lower $25(\mathrm{OH}) \mathrm{D}$ serum levels.

\section{P-704}

Medical follow up of hip fracture patients after hospital discharge Atef Michael ${ }^{1}$, Mahboob Anwar ${ }^{1}$, Ziyanda Mabuza ${ }^{1}$

${ }^{1}$ Russells Hall Hospital, Dudley, UK 
Introduction: The medical complexity of hip fracture patients still exist and may increase after surgical hip repair, and despite recent improvement in care the mortality is still $30 \%$ within 1 year. Many medical aspects should be addressed as in- or outpatient. We aimed to identify the reasons and indications of postoperative medical follow up after discharge.

Methods: Analysis of 30 consecutive hip fracture patients' electronic records (retrospectively) and 12 patients' clinical notes and electronic records (prospectively).

Results: The reasons/indications for medical follow up of hip fracture patients include: (1) Follow up of postoperative complications e.g. delirium and the response of postoperative anaemia to iron therapy. (2) Review the results of investigations for the cause of falls e.g. CT scans, Electroencephalography, Nerve Conduction Studies, 24 tapes, echocardiography. (3) Review the results of investigations for other conditions incidentally diagnosed during admission e.g. thyroid function tests, endoscopies. (4) Review the results of investigations to rule out pathological fracture e.g. bone scan, bone biopsy, electrophoresis. (5) Osteoporosis management: to start the anti-resorptive medications following normalization of calcium and vitamin D levels, review the DXA scan results, and ensure compliance with the medications and absence of side effects. (6) Check whether some medications e.g. antihypertensives and anticoagulants need to be omitted or restarted. (7) Better and more realistic assessment of gait and risk of falls. (8) Assessment of postoperative functional recovery and the need for further rehabilitation. (9) Follow up of other chronic diseases and comorbidities e.g. Parkinson's disease, heart failure. (10) As a safety net to ensure action upon any unnoticed abnormal results, unintended omission of medications or the need to request further investigations.

Conclusion: Some hip fracture patients need to be reviewed following hospital discharge to follow up postoperative complications or results of investigations, start or monitor osteoporosis treatment, reinstate or adjust some medications, follow up of chronic diseases and assessment of gait and risk of falls.

\section{P-705}

Detection of possible chronic neuropathic pain among elderly using DN4 Questionnaire

Małgorzata Stompór ${ }^{1,2}$, Tomasz Grodzicki ${ }^{2}$, Tomasz Stompór ${ }^{3}$, Jerzy Wordliczek $^{4}$, Marzena Dubiel ${ }^{5}$, Iwona Kurowska ${ }^{6}$

${ }^{1}$ Department of Cardiology and Cardiac Surgery, Collegium Medicum, University of Warmia and Masuria, Olsztyn, Poland,

${ }^{2}$ Department of Internal Medicine and Gerontology, Collegium Medicum, Jagiellonian University, Cracow, Poland, ${ }^{3}$ Department of Internal Medicine, Collegium Medicum, University of Warmia and Masuria, Olsztyn, Poland, ${ }^{4}$ Department of Intensive Interdisciplinary Care, Collegium Medicum, Jagiellonian University, Cracow, Poland, ${ }^{5}$ Assisted Living Residence Na Wzgórzu, Głogoczów, Poland,

${ }^{6}$ Geriatric Outpatient Clinic Med-All, Cracow, Poland

Introduction: Prevalence of neuropathic pain in general population is estimated to be around $6.5-11.5 \%$, but its exact frequency in geriatric population is poorly investigated. We tried to assess the prevalence of neuropathic pain symptoms among elderly using DN4 (Douleur Neuropathique 4) Questionnaire.

Methods: 145 subjects older than 60 years (nursing home residents, or geriatric outpatient clinic patients) scored $\geq 7$ points in the AMTS (Abbreviated Mental Test Score) were included. Chronic pain was defined as lasting $>3$ months, possible neuropathic pain was assessed using DN4 Questionnaire.
Results: The mean age of patients equaled $76 \pm 9.68$ years, $78 \%$ reported chronic pain and $32 \%$ possible chronic neuropathic pain (DN4 score $\geq 4$ points). The most common complaints were: numbness, tingling, electric shocks, burning, hypoesthesia to touch and hypoesthesia to prick. Usually patients reported 4/10 complaints in DN4 questionaire. The most common localizations of possible neuropathic pain were feet, hands and lower limbs. It has been shown that chronic pain and possible neuropathic pain were more common among patients suffering from endocrine, nutritional and metabolic diseases, than with other diseases $(p=0.01)$. There was a higher prevalence of type 2 diabetes in patients with possible neuropathic pain $(\mathrm{p}=0.08)$.

Conclusions: The prevalence of chronic neuropathic pain in the elderly population seems to be higher than previously expected. Diabetes was the most common cause of possible neuropathic pain's symptoms. The problem requires further research and dissemination of knowledge on the diagnosis of neuropathic pain in the elderly among medical practitioners.

\section{P-706}

A paradigm shift in home medicine: the high prevalence of heart failure with preserved ejection fraction among home-bound residents

\section{Jun R. Chiong ${ }^{1}$}

${ }^{1}$ MedEx Health Network, Inc, North York, Canada

Background: The volume of patient encounters in home-bound individuals has recently shown unprecedented growth. The reasons for this are multifactorial but one aspect is the increasing age of these patients. Consequently it is important to identify disease mechanisms in this cohort in order to develop appropriate training for medical providers.

Methods: We analyzed data of consecutive patients visited at home due to mobility problems, vision problems, cognitive decline and in some cases, mental health issue in 2017 . ICD-10 codes were extracted on all the primary encounter diagnosis from all the visits.

Results: There were a total of 3.408 patient home encounters during the study period. There were 2345 ICD-10 codes extracted. Mean age is 81.9 years, The top ICD by organ system are cardiovascular $(n=1085)$, endocrine $(n=232)$, respiratory $(n=179)$, renal $(n=63)$, malignancy $(n=30)$. The top 10 ICD classification includes:ICD-10: description1. Heart Failure with preserved systolic function. I5032: Chronic diastolic heart failure. $\mathrm{n}=239$. I11.0: hypertensive heart disease with heart failure. $n=149$. Total $\mathrm{n}=3882$. I11.9: Hypertensive heart disease without heart failure. $\mathrm{n}=2883$. E11.xx: type 2 diabetes mellitus (all types). $\mathrm{n}=1534$. I48.xx atrial fibrillation. $\mathrm{n}=1375$. J44.x: chronic obstructive pulmonary disease. $n=906$. I2510: atherosclerotic heart disease of native coronary artery. $\mathrm{n}=887$. K21.x: gastro-esophageal reflux disease. $n=528$. M179: osteoarthritis. $n=479$. J209: acute bronchitis. $\mathrm{n}=4210$. R29.6: falls. $\mathrm{N}=30$

Conclusions: Most home bound medical encounters of older adults were due to cardiovascular related causes mostly from heart failure with preserved ejection fraction. With heart failure being one of the most common causes of hospitalization and readmission in the elderly population today. The present findings clearly emphasize focus on specialized training in heart failure to providers dedicated to practicing home medicine. 


\section{P-707}

Orthostatic hypotension and overall mortality in 1050 elderly patients of the outpatient comprehensive geriatric assessment unit

Yan Press ${ }^{1}$, Tamar Freud ${ }^{2}$, Ella Kagan ${ }^{1}$, Alex Barzak ${ }^{1}$, Boris Punchik ${ }^{1}$

${ }^{1}$ Unit for Community Geriatrics, Ben-Gurion University of the Negev, Beer-Sheva, Israel, ${ }^{2}$ Sial Research Center for Family Medicine and Primary Care, Faculty of Health Sciences, Ben-Gurion University of the Negev, Beer-Sheva, Israel

Introduction: Orthostatic hypotension $(\mathrm{OH})$ is a common problem in the elderly population, with prevalence between $6.9 \%$ and $55 \%$. In some studies, an association between $\mathrm{OH}$ and negative medical conditions, including falls, cardiac events, heart failure and stroke, have been shown. The association of $\mathrm{OH}$ with mortality is not clearcut. In our previous retrospective study of 571 frail elderly who underwent a comprehensive geriatric assessment (CGA) over the years 2005-2013 we did not find any association between $\mathrm{OH}$ and mortality. The aim of the present study was to assess possible associations between $\mathrm{OH}$ and mortality in a broader sample that included not only patients who underwent CGA, but also those who underwent a geriatric consultation (GC) in the same unit.

Methods: Individuals who were evaluated in the Outpatient Comprehensive Geriatric Assessment Unit between January 2005 and December 2015 and who had data on orthostatic hypotension were included in the study. The database included socio-demographic characteristics, body-mass index, functional and cognitive state, geriatric syndromes, co-morbidity and mortality data.

Results: The study sample included 1050 people of who 626 underwent comprehensive geriatric assessment and 424 underwent geriatric consultation. The mean age was $77.3 \pm 5.4$ years and $35.7 \%$ were males. Orthostatic hypotension was diagnosed in 294 patients $(28.0 \%)$. In univariate analysis orthostatic hypotension was associated with overall mortality only in patients aged $65-75$ years old (hazard ratio $=1.5 ; 95 \%$ CI $1.07-2.2$ ), but in the multivariate model this association disappeared.

Conclusions: In elderly frail patients orthostatic hypotension was not an independent risk factor for overall mortality.

\section{P-708}

\section{Accurate detection of arterial hypotension in elder}

Hilde Baeyens ${ }^{1}$, Eva Van Lerberge ${ }^{2}$, Julien Dekoninck ${ }^{1}$, Celine Vandevijver ${ }^{1}$, Jean-Pierre Baeyens ${ }^{1}$

${ }^{1}$ AZ Alma, Eeklo, Belgium, ${ }^{2}$ General Practioner, Oordegem, Belgium

Introduction: Orthostatic hypotension $(\mathrm{OH})$ and arterial hypotension $(\mathrm{AH})$ are common problems in frail older patients. There is a higher morbidity and mortality. The PARTAGE study (Benetos) showed that mortality rises if blood pressure (BP) is lower than $130 \mathrm{mmHg}$. Hypertension guidelines mention that the blood pressure should be measured in sitting position (BP S). However in this way, concomitant hypotension in standing position is often not detected. This occurs more in older patients with atherosclerotic, non-elastic arteries. Which method of measuring the blood pressure in frail elderly is more accurate to detect this problem?

Methods: Inclusion of 40 patients with clinical suspicion by geriatricians of blood pressure problems. Usual gait speed was $0.5 \mathrm{~m} / \mathrm{s}$. MMSE 24/30. Dementia occurred in 54\% of the patients. Measurements of BP S and BP S/S, 24-h ambulatory BD measurement. We defined that if $\geq 20 \%$ of the daytime $(06-22 \mathrm{~h})$ the systolic blood pressure is below $130 \mathrm{mmHg}$ there is a problem of arterial hypotension.

Results: The sensitivity of measuring the BP S/S is $69 \%$, the PPV $96 \%$, the sensitivity of BP S is only $28 \%$. The accuracy of the investigation BP S/S is $73 \%$ and BP S 7\%. But the specificity for BP S is $100 \%$.

Conclusion: In frail ambulatory geriatric patients measuring BP in supine/standing position is more accurate then BP in sitting position, to detect $\mathrm{AH}$ en $\mathrm{OH}$. In this way drug adjustment (more or less) can be made more safely in this fragile population.

\section{P-709}

Are very old patients with atrial fibrillation treated with new or classic anticoagulants different?

Luisa Alejandra Hernández Sánchez ${ }^{1}$, Génesis Estefanía Olaya Loor ${ }^{1}$, Jana Albéniz López ${ }^{1}$, Ana Merello de Miguel ${ }^{1}$, Paula Fernández Montalbán ${ }^{1}$, Beatriz Montero Errasquín ${ }^{1}$, Alfonso José Cruz Jentoft ${ }^{1}$

${ }^{1}$ Hospital Universitario Ramón y Cajal, Madrid, Spain

Objective: To compare baseline characteristics and risk of mortality using Multidimensional Prognostic Index (MPI) of older patients with atrial fibrillation $(\mathrm{AF})$ treated with coumadin versus new oral anticoagulants (NOACs) admitted for acute care.

Methods: Single-center, prospective, study of all patients with AF consecutively admitted to an acute geriatric unit during 6 months. The Multidimensional Prognostic Index (MPI) was used to define three groups according to the mortality risk: MPI-1 ( $\leq 0.33$, low risk), MPI-2 (0.34-0.65, moderate risk) and MPI-3 ( $\geq 0.66$, high risk). Anticoagulant therapy, CHA2DS2-VASc and HAS-BLED scores and in-hospital mortality were collected. Patients treated with coumadin and those with NOACs were compared.

Results: 62 patients included (69\% women, mean age $93 \pm 4$ years) most with high physical dependency and severe comorbidity. $63 \%$ were included in the high mortality risk group (MPI-3). In-hospital mortality was $13 \% .79 \%$ received anticoagulation therapy at admission $57.1 \%$ coumadin, $36.7 \%$ NOACs and $6.1 \%$ low molecular weight heparin (excluded from the analysis). Apixaban was the most used NOAC (44.4\%). $100 \%$ had CHA2DS2-VASc score $\geq 2$ and $58 \%$ HAS-BLED score $\geq 3$. No significant differences were found in sociodemographic, functional, nutritional, cognitive, comorbidity, number of medicines, MPI and in-hospital mortality between groups (coumadin vs NOACs). However, the coumadin group had a higher bleeding risk $(\mathrm{HAS}-\mathrm{BLED} \geq 3)$ than those with NOACs $(78 \%$ vs $44 \%, \mathrm{p}=0.018)$.

Conclusion: Older patients with AF treated with coumadin do not differ in baseline characteristics or mortality risk from those on NOACs, but have a higher bleeding risk.

\section{P-710}

The burden of disease associated with invasive extra-intestinal $E$. coli infections in adults: a systematic literature review

Stefan Gravenstein ${ }^{1}$, Anke Stuurman ${ }^{2}$, Georgalis Leonidas ${ }^{2}$, Marc Bonten ${ }^{3}$, James Johnson ${ }^{4}$, Patricia Ibarra de Palacios ${ }^{5}$, Jan Poolman ${ }^{6}$, Peter Hermans ${ }^{6}$, Thomas Verstraeten ${ }^{2}$

${ }^{1}$ Brown University and Providence Veterans Administration Hospital, Rhode Island 02912, USA., ${ }^{2}$ P95 Pharmacovigilance and Epidemiology Services, Leuven, Belgium, ${ }^{3}$ Julius Center, UMC Utrecht, Utrecht, Netherlands, ${ }^{4}$ University of Minnesota, Minneapolis, Minnesota 
Background: Infections due to extraintestinal pathogenic Escherichia coli (ExPEC) result in many hospitalizations and deaths. However, the burden of invasive ExPEC disease (IED), including bloodstream infection (BSI), is ill defined.

Methods: Pubmed, Embase, SciELO, and LILACS databases were reviewed systematically (period: January 2007-March 2017) using search terms ' $E$. coli' and 'invasive disease'. Total burden of $E$. coli BSI was estimated by multiplying age-specific pooled incidence and case-fatality rates by 2016 US and EU population estimates.

Results: Sixty-one studies reported E. coli BSI incidence rates, which (per 100,000 person-years) ranged from 28-74, with higher values in recent studies. Rates increased with age, especially after 60 (age range, specific rate range: age $15-29,9-18$; age 30-39, 12-24; age 40-49, 17-30; age 50-59, 24-114; age 60-69, 78-135; age 70-79, 118-349; age 80+, 309-586). Incidence was higher in women, especially among adults $<40$ years (female-to-male ratio, range 2.1-4.9). 30-day case fatality rates ranged from $8-18 \%$ overall (up to $37 \%$ in adults age $80+$ ). Accordingly, an estimated 107,000-246,000 E. coli BSIs and 8000-44,000 associated deaths could occur annually in the US, and 218,000-510,000 E. coli BSIs and 17,000-92,000 associated deaths in the EU.

Key conclusion: IED is associated with considerable morbidity and mortality, which is greatest in the elderly and when extrapolated to the US or EU adult population rivals the reported influenza burden [1]. Preventive measures are sorely needed.

Reference:

1. Paules CI (2018) N Engl J Med. 378:7-9

\section{P-711}

An innovative approach of multilevel collaboration to improve resident centred care in a Buckinghamshire Nursing Home

Jacqui Kent ${ }^{1}$, Beatrix Nagyova ${ }^{2}$, Nicola Turner ${ }^{1}$

${ }^{1}$ Buckinghamshire Clinical Commissioning Group, Aylesbury, UK, ${ }^{2}$ Buckinghamshire Healthcare NHS Trust, Aylesbury, UK

In Buckinghamshire, Office of National Statistics projects an increase of 65 years + population by $27 \%$ by 2030 [1]. Health Survey for England 2013 found that $70 \%$ of the UK population aged 75 take at least three prescribed medicines [1]. CHUMS study reports that the number of medication is 7.2 medicines per resident [2].

Methods: A new multidisciplinary specialist support service for vulnerable residents was piloted in a large nursing home in Buckinghamshire between November 2016 and August 2017. After reviewing medication for all residents prescribing pharmacist and general practitioner identified the most complex individuals for comprehensive assessment by geriatrician. The dietitian supported home with use of malnutrition screening tool and a food first approach.

Results: Overall 114 medications including eight high risk medicines were stopped for 55 residents. Training was delivered to improve on catheter care.Total cost savings on medicines optimisation, food-first approach to malnutrition, medicines waste and prevention of nonelective admission was calculated as $£ 17510$ during the study period. Conclusions: Buckinghamshire Healthcare NHS Trust succeeded as one of eight Integrated Care System Trust in England in 2017. Future directions of the project focus on county wide system improvements with aim to standardise best practice in medicines optimisation and promote interdisciplinary healthcare professionals training.

\section{References:}

1. https://digital.nhs.uk/data-and-information/publications/statistical/ health-survey-for-england/health-survey-for-england-2013[2]. CHUMS study https://www.birmingham.ac.uk/Documents/college-mds/haps/
projects/cfhep/psrp/finalreports/PS025CHUMS-FinalReportwithappen dices.pdf.

\section{P-712}

Complex diagnosis of transient loss of consciousness in older patients: a case report

Francesca Tesi ${ }^{1}$, Martina Rafanelli ${ }^{1}$, Alice Ceccofiglio ${ }^{1}$, Giulia Rivasi ${ }^{1}$, Flavia Carla Sacco ${ }^{1}$, Andrea Ungar ${ }^{1}$

${ }^{1}$ Syncope Unit, Cardiology and Geriatric Medicine, University of Florence and Azienda Ospedaliero-Universitaria Careggi, Florence, Italy

Introduction: The diagnostic evaluation of syncope in the elderly with transient loss of consciousness is often complex. The establishment of a Syncope Unit has certainly improved the diagnostictherapeutic approach to older patients with syncope.

Methods: V.M., female, 84 years old, fit elderly affected by hypertension, hypercholesterolemia, depressive syndrome. Since 2014 epileptic absences. On May 2015, an episode of transient global amnesia. First diagnosis: epilepsy. The patient started therapy with levetiracetam.

Results: Since 2015 several recurrent syncope, sometimes traumatic and preceded by chest pain, dyspnea and palpitations. On March 2017: the patient was referred to our Syncope Unit: the physical examination showed the presence of right carotid bruits and the Orthostatic Hypotension test was positive. Second diagnosis: orthostatic hypotension. An Echocardiogram showed mild aortic valve stenosis. The Carotid Doppler Ultrasound revealed mild carotid atherosclerosis without significant stenosis. The Carotid Sinus Massage was negative. On June 2017: two falls with consequent mild trauma and suspected presyncopal episodes with dizziness. An Implantable loop recorder was implanted. On the 19th of August 2017: dizziness episode after a prolonged orthostatic period, consequent fall and occipital trauma. The loop recorder described an episode of paroxysmal supraventricular tachycardia, causing presyncope and fall, treated with ablation. Third diagnosis: Paroxysmal supraventricular tachycardia.

Conclusion: The diagnostic evaluation of syncope in the elderly with transient loss of consciousness is often complex: more causes can coexist and sometimes there is an overlapping between pseudo syncope and syncope. It is necessary to follow an individualized and targeted diagnostic pathway to avoid unnecessary tests.

\section{P-713}

Multimorbidity and functional dependency in elderly who are assisted by home health care teams: a cross-sectional study

Paula Broeiro-Gonçalves ${ }^{1}$, Pedro Aguiar ${ }^{2}$, Isabel Loureiro ${ }^{3}$

${ }^{1}$ Family Physician, UCSP Olivais, Lisbon, Portugal; Prof Assistent Faculdade de Medicina de Lisboa, Lisbon, Portugal, ${ }^{2}$ Assistant Professor, Public Health Research Center, NOVA University of Lisbon, Lisbon, Portugal, ${ }^{3}$ Professor, Public Health Research Center, NOVA University of Lisbon, Lisbon, Portugal

Introduction: Multimorbidity and dependency are frequent among the elderly. The objectives of this study are to determine multimorbidity and dependence and verify the association between them in old people receiving home health care.

Methods: This is a cross-sectional study of the elderly who are assisted by home health care teams in the Lisbon and Tagus Valley 
region, from Portugal. After sample size calculation $(n=228,5 \%$ error, $92 \%$ prevalence and two design effect), cluster sampling was used. The analysis was completed via the generalised estimation equations using the IBM SPSS-v24.0 tool.

Results: The 230 seniors, distributed across 23 home health care teams, were divided almost equally by gender ( $54 \%$ women) and had a mean age of 84 . The average conditions per person were 9.5 and the Charlson index was 8.48; males scored higher on both of these measures. Multimorbidity was present in all participants. The Barthel's score average was higher in men (younger and less ADL dependent than women). Dementia $(\mathrm{OR}=10.873)$ and cerebrovascular disease $(\mathrm{OR}=3.013)$ are the medical conditions most associated with dependence. Obesity $(\mathrm{OR}=0.522)$ and arterial hypertension $(\mathrm{OR}=0.186)$ appear to be protective. Mobility activity dependency is associated with multimorbidity severity (Charlson $\geq 9$ ), but not with multimorbidity magnitude (counted conditions).

Discussion: Elderly dependence was only related to multimorbidity severity, not magnitude. The results confirm the neuropsychiatric pattern of dependency. The protective obesity effect highlights the obesity paradox. In the elderly, longitudinal studies are needed to understand the relationship between multimorbidity and dependence.

\section{P-714}

Diagnostic delay is longer in elderly patients with Amyotrophic Lateral Sclerosis

Francesca Palese ${ }^{1}$, Arianna Sartori ${ }^{2}$, Giancarlo Logroscino ${ }^{3}$, Federica Pisa ${ }^{4}$

${ }^{1}$ Medical Area Department, University of Udine, Udine, Italy, ${ }^{2}$ Department of Medical, Surgical and Health Sciences, Neurology Clinic, Azienda Sanitaria Universitaria Integrata di Trieste, Trieste, Italy, ${ }^{3}$ Neurodegenerative Diseases Unit, Department

Introduction: Older age has been associated with diagnostic delay in Amyotrophic Lateral Sclerosis (ALS). However, most studies did not account for type of onset (bulbar, spinal) and other patient characteristics. We assessed time-to-diagnosis and evaluated if age is an independent predictor of diagnostic delay.

Methods: Our population-based cohort included all patients with incident ALS in Friuli-Venezia Giulia, Italy, 2010-2014, admitted to one of the two regional University Hospitals. We compared Total Diagnostic Time (TDT) (interval from symptoms onset to ALS diagnosis) between groups of patients using Wilcoxon-Mann-Whitney test and identified predictors of a TDT $\geq 1$ year using multivariate logistic regression analysis.

Results: Among 134 patients with ALS, 79.5\% ( $\mathrm{N}=107)$ were aged $\geq 60$ years at disease onset, $51.5 \%$ were women and $64.9 \%$ had spinal onset. Median TDT was 11.5 months (interquartile range: $7.1-18.3$ ) and $48.5 \%$ had a TDT $\geq 1$ year. Diagnostic delay was significantly longer in patients aged $\geq 60$ years (12.4 vs. 8.1 months; $\mathrm{p}=0.0064)$ and in those with neurologic comorbidities (19.7 vs. 11.1 months, $p=0.0243$ ). Patients $\geq 60$ years at disease onset were 11.5 times more likely of having a TDT $>1$ year, when type of onset, sex, having undergone physical, rehabilitation or surgical therapy or first referral to a specialist non-neurologist were adjusted for.

Key conclusions: Despite it is known that ALS is an aging-related disease, older patients had higher adjusted risk of diagnostic delay, thus raising specific concerns on their opportunities for getting timely adequate healthcare, maybe because symptoms are wrongly attributed to other comorbidities.

\section{P-715}

CRP point of care (POCT) for airway infections (AI) in long term care facilities (LTCF) A location-clustered intervention pilot in two LTCF

Marianne Vos ${ }^{1}$, Tom Starmans ${ }^{2}$, Roland Wetzels ${ }^{3}$

${ }^{1}$ Opella, Radboud UMC Nijmegen, Netherlands, ${ }^{2}$ RSZK, Radboud UMC Nijmegen, Netherlands, ${ }^{3} \mathrm{ZZG}$, Radboud UMC Nijmegen,

Netherlands

Introduction:Patients in LTCF are often treated for AI based on clinical parameter. We researched the additional value of CRP POCT next to clinical evaluation. The aim of the study is to determine if implementation of CRP POCT reduces the use of antibiotics in LTCF. We also investigated a relationship between use of antibiotics and the CRP value.

Method:This is an exploratory location-clustered intervention study in Dutch LTCF. The intervention group consists of the patients staying in the main location in both facilities, the control group consists of the other patients. Included are patients suspected of an AI where the physician has doubts about prescribing antibiotics. Excluded are patients suffering dementia. The intervention is the addition of CRP POCT to regular best common practice.Primary endpoint is the difference in direct and total (after 7 days) antibiotic use between the groups. A secondary endpoint is to determine if there is a relationship between CRP values and direct and total antibiotic use.

Results:In this study, 19 included cases were analysed, of which eleven in the intervention group. No significant difference between use of antibiotics is found.

Key conclusion:In this study, not enough patients were included to find a significant difference in anbiotic use between intervention and control groups or in CRP categories. However, there are clues that the use of CRP POCT in suspected AI will help the physician start appropriate therapy earlier. More research in larger patientgroups in more LTCF is needed to conform this hypothesis.

\section{Reference:}

1. van Buul LW, van der Steen JT, Veenhuizen RB, e.a. Antibiotic Use and Resistance in Long Term Care Facilities. J Am Med Dir Assoc. 2012;13(6):568.e1-568.e13. doi:10.1016/j.jamda.2012.04.004 2. Daneman N, Bronskill SE, Gruneir A, e.a. Variability in Antibiotic Use Across Nursing Homes and the Risk of Antibiotic-Related Adverse Outcomes for Individual Residents. JAMA Intern Med. 2015;175(8):1331-1339. doi:10.1001/jamainternmed.2015.2770

3. van Buul LW, Veenhuizen RB, Achterberg WP, e.a. Antibiotic Prescribing In Dutch Nursing Homes: How Appropriate Is It? J Am Med Dir Assoc. 2015;16(3):229-237. doi:10.1016/j.jamda.2014. 10.003

4. Cals JWL, Schot MJC, de Jong SAM, Dinant G-J, Hopstaken RM. Point-of-care $\mathrm{C}$-reactive protein testing and antibiotic prescribing for respiratory tract infections: a randomized controlled trial. Ann Fam Med. 8(2):124-133. 2010 doi:10.1370/afm.1090

5. Verheij ThJM, Hopstaken RM, Prins JM, Salomé PhL, Bindels PJ, Ponsioen BP, Sachs APE, Thiadens HA VE. NHG-Standaard Acuut hoesten I NHG. Huisarts en weteschap. 2011;54:68-92. https://www. nhg.org/standaarden/volledig/nhg-standaard-acuut-hoesten. Geraadpl eegd maart 19, 2018

6. Snoeck-Stroband JB, Schermer TRJ, Van Schayck CP, Muris JW, Van der Molen T, In 't Veen JCCM, Chavannes NH, Broekhuizen BDL, Barnhoorn MJM, Smeele I, Geijer RMM TM. NHG-Standaard COPD | NHG. Huisarts Wet. 2015;58:198 -211. https://www. nhg.org/standaarden/volledig/nhg-standaard-copd. Geraadpleegd ma art 19, 2018

7. Berger MY, De Wit NJ, Vogelenzang R, Wetzels RV, Van Rijnvan Kortenhof NMM OW. NHG- Standaard Diverticulitis I NHG. 
Huisarts en wetenschap. https://www.nhg.org/standaarden/volle dig/nhg-standaard-diverticulitis. Published 2011. Geraadpleegd maart 19, 2018

8. Peters CM, Schouwenaars FM, Haagsma E, Evenhuis HM, Echteld MA. Antibiotic prescribing and C-reactive protein testing for pulmonary infections in patients with intellectual disabilities. Br J Gen Pract. 2013;63(610):e326-30. doi:10.3399/bjgp13X667187

9. Gong C, Wei D, Wang Y, e.a. A Meta-Analysis of C-Reactive Protein in Patients With Alzheimer's Disease. Am J Alzheimer's Dis Other Dementiasr. 2016;31(3):194-200. doi:10.1177/153331 7515602087

10. Beloosesky Y, Grinblat J, Pirotsky A, Weiss A, Hendel D. Different C-Reactive Protein Kinetics in Post-Operative Hip-Fractured Geriatric Patients with and without Complications. Gerontology. 2004;50(4):216-222. doi:10.1159/000078350

\section{P-716}

The orthogeriatric prevention service improves the automatic capture of hip fracture persons

Valentina Prenni ${ }^{1}$, Marta Baroni ${ }^{1}$, Luca Parretti ${ }^{2}$, Jacopo Penzo ${ }^{2}$, Valentina Bubba ${ }^{2}$, Giuseppe Rinonapoli ${ }^{3}$, Maurizio Massucci ${ }^{4}$, Auro Caraffa ${ }^{3}$, Patrizia Mecocci ${ }^{1}$, Carmelinda Ruggiero ${ }^{1}$

${ }^{1}$ Geriatrics, S.M. della Misericordia Hospital, University of Perugia, Perugia, Italy, ${ }^{2}$ Geristrics, S.M. della Misericordia Hospital, University of Perugia, , Perugia, Italy, ${ }^{3}$ Orthopaedics and Traumatology Ward, S.M. della Misericordia Hospital, University of Perugia, Perugia, Italy, ${ }^{4}$ Orthopaedics and Traumatology Ward, S.M. della Misericordia Hospital, University of Perugia, Perugia, Italy

Introduction: A gap exists between evidence-based recommendations for post-fracture care and actual clinical practice. Our purpose is to evaluate the effectiveness of an outpatient ortho-geriatric service in the prevention of re-fractures.

Materials and methods: 271 hip fracture older patients were assessed within 40 days from hip fracture surgery. Patients underwent clinical assessment and standardized questionnaires by a multidisciplinary team. They receive pharmacological and non-pharmacological indications for falls and fracture prevention. All participants receive follow-up evaluation at 6 and 12 months for adverse events, including falls and re-fractures.

Results: $79 \%$ women and $21 \%$ male with a mean age of 84 years, which preserved a good level of functional autonomy before the fracture, despite previous falls and fractures. More than half presents substantially preserved cognitive functions and received FKT program privately. Furthermore, patients receive a recommendation to change drug therapy, to promote the prevention and the appropriate management of ulcers from pressure. The anti-fracture treatment has been initiated in the $97 \%$ of cases with supplements of vitamin D and diet integration of calcium, while in the $66 \%$ of cases with most suitable drugs.

Conclusions: The ortho-geriatric outpatient service has the potential to identify and manage certain clinical and care needs of frail elderly who are at a high risk of falls, fractures, and adverse events.
P-717

Identifying loneliness and its predictors in the inpatient setting

Aneesha Chauhan ${ }^{1}$, Masanori Watanabe ${ }^{2}$, Adrian Wells ${ }^{3}$, Natalia Cotton ${ }^{4}$, Rebecca $\mathrm{Li}^{2}$, Sanja Thompson ${ }^{5}$

${ }^{1}$ Chelsea and Westminster NHS Trust, London, UK, ${ }^{2}$ Oxford

University Hospitals NHS Trust, London, UK, ${ }^{3}$ University of Oxford, Oxford, UK, ${ }^{4}$ London North West University Healthcare NHS Trust, London, UK, ${ }^{5}$ Oxford University Hospitals NHS Trust, London, UK

Introduction: Loneliness and social isolation adversely impact morbidity and mortality in older adults. This study aims to identify loneliness and its predictors within the hospital inpatient population and those suitable for intervention.

Methods: 193 patients (112 male, 81 female; average age 82.8 years) admitted to the acute general wards at the John Radcliffe Hospital, Oxford, were assessed for loneliness and its predictors using a questionnaire measuring self-reported perceived loneliness, and the UCLA 3-item loneliness scale. Inclusion criteria required consenting patients aged $\geq 75$ years old, who were not confused, and had been an inpatient for $\geq 3$ days.Answers were converted into numerical scores for statistical analyses.

Results: $20.2 \%$ of participants were lonely according to the UCLA score; $30.6 \%$ perceived themselves to be lonely at least some of the time in hospital. Perceived loneliness was significantly correlated to loneliness measured by the UCLA score $(r=0.41, p<1 e-8)$. Statistically-significant predictive factors of inpatient loneliness were: loneliness pre-admission $(r=0.72, r=0.48)$, depression $(r=0.52$, $r=0.43)$ and anxiety $(r=0.48,0.37)$. Those with better self-reported general health were less likely to feel lonely during inpatient admission $(r=-0.25, r=-0.18)$.Interestingly, a patient's social network did not predict loneliness.Perceived loneliness, living alone, and anxiety, were correlated with willingness to accept intervention. Conclusion: The predictive factors for loneliness in older inpatients are easily ascertained by both self-reporting questionnaires and the UCLA score. The inpatient setting could be an opportunity to recognise those who are feeling lonely both in and out of hospital, and offer intervention.

\section{P-718}

Functional decline, mortality and institutionalization, and associated factors in patients with multimorbidity at 18 months of follow-up: the FUNCIPLUR longitudinal study

Iñaki Martin Lesende ${ }^{1}$, Luis Ignacio Mendibil Crespo ${ }^{2}$, Anne Sophie Denise Otter ${ }^{3}$, Irati Garaizar Bilbao ${ }^{4}$, Jakobe Pisón Rodríguez ${ }^{3}$, Sonia Castaño Manzanares ${ }^{3}$, Ion Negrete Pérez ${ }^{5}$, Iraide Sarduy Azcoaga ${ }^{6}$,

María Jesús de la Rua Fernánde

${ }^{1}$ San Ignacio Health Centre, Bilbao-Basurto Integrated Healthcare Organisation (IHO), Basque Health Service (Osakidetza), Spain,

${ }^{2}$ Bizkaia Unit for Multiprofessional Training in Family and Community Care, Spain, ${ }^{3}$ Bilbao-Basurto IHO, Osakidetza, Spain

Objective: To analyse long-term (18-month period) functional decline and associated factors in multimorbidity patients aged $\geq 65$. Methods: Prospective multicentre study.Of the 241 patients $\geq 65$ years included randomly, 155 were already part of a multimorbidity programme (stratified by ACGs) and 86 newly included (Ollero's criteria and $\geq 1$ hospital admission in the previous year). Institutionalised or receiving dialysis patients were excluded. The primary outcome was the decrease in functional status category (Barthel Index or Lawton Scale), analysed independently and 
combined with data regarding patients who died or became institutionalized. Other variables: socio-demographic characteristics, comorbidity, medications, admissions and functional status on discharge.

Results: Patients (46.9\% women) had a median of 82 years (P75 86), 5 chronic conditions (IQR 4-6), 11 (IQR 9-14) regular medications, and $38.2 \%$ impaired function at baseline.Overall, 158 persons completed the follow-up (of these 81, 51.3\% 95\%CI 43.5-58.9, experienced functional decline), $47 \mathrm{died}$, and 11 institutionalized. $64.4 \%$ (IC95\% 57.8-70.4\%) had functional decline, died or were institutionalized, excluding other causes of lost; associated with older age (OR 1.1, 95\% CI 1.0-1.1) and having $\geq 1$ admission (OR 4.1, $95 \%$ CI $2.1-8.9 \%$ ). There were 234 admissions, with functional decline in $36.0 \%$ (95\% CI $27.3-45.8 \%$ ) of the 100 discharges in which this was assessed.

Key conclusions: High percentage of the patients $(64.4 \%)$ showed functional decline/loss of independence $(37.5 \%)$, died or were institutionalized in the period. These findings are important as functional decline and the increasing care needs are potentially predictable and modifiable. Age and hospitalisation were closely associated.Funding. This work was supported by a Bilbao-Basurto IHO grant (OSIBB17/ 002).The results will be compared with the short-term follow-up (8 months) [1].

\section{Reference:}

1. Martin-Lesende I, Mendibil L, Castaño S, et al (2018) Functional decline and associated factors in patients with multimorbidity at eight months of follow-up in primary care: the FUNCIPLUR longitudinal descriptive study. BMJ Open (in press)

\section{P-719}

The assessment of anemia rate, relation between anemia and antiaggregant -anticoagulant medication and other factors

Hatice Aksu' ${ }^{1}$ Gizem Ayan ${ }^{2}$, Rabia Bag Soytas ${ }^{3}$, Suna Avci ${ }^{3}$, Serdar Ozkok ${ }^{3}$, Hakan Yavuzer ${ }^{3}$, Deniz Suna Erdincler ${ }^{3}$, Alper Doventas ${ }^{3}$

${ }^{1}$ Istanbul University, Cerrahpasa Medical Faculty Department of Geriatric Medicine, Istanbul, Turkey, ${ }^{2}$ Health Sciences University, Okmeydani Research and Training Hospital, Internal Medicine, Istanbul, Turkey, ${ }^{3}$ Istanbul University, Cerrahpasa Medical Faculty, Internal Medicine, Department of Geriatric Medicine, , Istanbul, Turkey

Objective: The aim was to determine the frequency of anemia, the relationship between the use of antiagregants and anemia in patients over 65 years of age.

Materials and methods: This study is designed as retrospective, cross-sectional. The data of patients who admitted to the Istanbul University Cerrahpaşa Medical Faculty Geriatric Medicine Outpatient Clinic, were reviewed, the clinical, demographic data of anemia patients were evaluated, anemia types, related factors were determined.

Results: Totally 207 patients (72 males, 135 females) aged 65-97 years (mean $78.82 \pm 7.5$ years) were included. $92(44.4 \%)$ and $26(12.6 \%)$ of the patients were using at least one antiaggregant and anticoagulant medication respectively. Acetyl salicylic acid $(38.8 \%)$ was the most common antiaggregant while warfarin $(5.3 \%)$ was the most common anticoagulant. Hypertension, diabetes mellitus, and hyperlipidemia were the most frequent comorbidities, and at least 4 comorbidities were found in $70(33.38 \%)$ patients. Iron deficiency, megaloblastic, chronic illness and indeterminate anemia were found in patients as follows; 106 (51.2\%), 27 (13\%) 49 (23.7\%), 25 (12.1\%) respectively. By the age increases, the hemoglobin levels were found to be decreased $(r=-0.208, p=0.003)$. The frequency of patients on antiagregant treatment was higher in the unexplained anemia group $(\mathrm{p}<0.001)$.

Conclusion: The use of antiaggregant and anticoagulant drugs in geriatric patient population is quite frequent. This entity requires to be considered in the etiology of anemia. It should be remembered that the use of antiaggregant and anticoagulant drugs in the unexplained anemia may be a major risk factor for etiology, especially in elderly patients.

\section{P-720}

Diabetes mellitus is associated with vitamin D deficiency in older people

Cafer Balc1 ${ }^{1}$, Mert Eşme ${ }^{1}$, Pelin Ünsal ${ }^{1}$, Gözde Şengül Ayçiçek ${ }^{1}$, Burcu Balam Yavuz ${ }^{1}$, Meltem Halil ${ }^{1}$, Mustafa Cankurtaran ${ }^{1}{ }^{1}$ *

${ }^{1}$ Hacettepe University, Faculty of Medicine Department of Internal Medicine Division of Geriatrics, Ankara, Turkey, *Presenting Author

Introduction: Diabetes mellitus is common among older people and sufficient serum concentrations of vitamin D are required for bone health. The purpose of the current study was to determine whether diabetes is an independent risk factor for vitamin D deficiency in community-dwelling older people.

Methods: A retrospective cohort analysis was performed using health records of patients admitted to outpatient clinic of our department. Demographic characteristics, co-morbid conditions, fasting concentrations of serum 25-hydroxy vitamin D (25-OH-D), plasma parathyroid hormone $(\mathrm{PTH})$ and calcium were recorded. Vitamin D deficiency was defined as serum 25-OH-D concentrations $<20 \mathrm{ng} /$ $\mathrm{ml}$. Patients taking calcium and vitamin D supplementations, thiazide diuretics, bisphosphonates were excluded.

Results: A total of 399 patients over 65 years of age were included in the study. Mean age was $72 \pm 6.1$ and $62.2 \%(n=248)$ was female. Twenty four percent of the patients had diabetes mellitus. Among diabetic and non-diabetic patients, mean serum 25(OH) D concentrations were $17.4 \pm 14.2 \mathrm{ng} / \mathrm{ml}$ and $21.4 \pm 15.2 \mathrm{ng} / \mathrm{ml}$, respectively $(\mathrm{p}<0.001)$. In multivariate analyses, diabetes (adjusted odds ratio 2.4; 95\% confidence interval, 1.3-4.1) and female gender (adjusted odds ratio 2.1; $95 \%$ confidence interval, 1.3-3.2).

Conclusion: Our findings highlight the importance of diabetes mellitus in the development of the vitamin D deficiency in the older people. According to our results, patients with diabetes mellitus should be screened for vitamin D deficiency.

\section{P-721}

The Impact of respiratory syncytial virus infection on morbimortality in elderly patients

F. Salgueiro ${ }^{1}$, J. Fonseca ${ }^{1}$, J. Gomes ${ }^{1}$, B. Barbosa ${ }^{1}$, H. Pereira ${ }^{1}$, A. Carvalho ${ }^{1}$

${ }^{1} \mathrm{CHUC}$

Background: A better understanding of respiratory syncytial virus (RSV) infection in the elderly is necessary. The aim of this study was to analyze clinical outcomes and therapeutic management in elderly people with RSV infection.

Methods: A retrospective cohort study was conducted on elderly patients ( $\geq 65$ years) admitted to the emergency department of a Portuguese university hospital for suspected respiratory infection between November 2017 and March 2018. All patients with a positive 
RSV nasopharyngeal swab were included. Demographic information, comorbidities, clinical and laboratory data were recorded from each patient.

Results: RSV infection was identified in 126 patients, with a mean age of $79.5 \pm 7.8$ years $(62.7 \%$ female). Most patients were dependent on at least one activity of daily living $(61.1 \%)$ and had several comorbidities (Charlson comorbidity index mean 6.0). One hundred $(79.3 \%)$ were hospitalized with a mean length of stay of 10.6 days, and three were admitted in intensive care unit. Respiratory failure was the leading cause of admission (51.6\%); other complications as pneumonia $(23.8 \%)$; acute heart failure $(24.6 \%)$; chronic obstructive pulmonary disease exacerbation (18.2\%); acute kidney injury $(11.1 \%)$; delirium $(6.3 \%)$; and asthma exacerbation $(5.6 \%)$ were also prevalent. The treatment strategy included: nebulized bronchodilators $(81.0 \%)$, antibiotics $(75.4 \%)$, corticosteroids $(65.9 \%)$, oxygen therapy $(81.7 \%)$, noninvasive ventilation $(9.5 \%)$, and invasive mechanical ventilation $(1.6 \%)$. All-cause mortality was $10.3 \%$.

Conclusions: The impact of RSV on morbimortality among elderly was significant. Further studies are needed to evaluate the future importance of RSV screening in the elderly patient with respiratory infection, and the development of new therapeutic approaches.

\section{P-722}

Subclinical thyroid disease and cardiovascular, metabolic and cognitive comorbility in elderly admitted in long term care

Alba Malara ${ }^{1}$, Giuseppe Andrea De Biase ${ }^{1}$, Nadia Grillo ${ }^{2}$, Luana Putrino ${ }^{2}$, Francesco De Francesca ${ }^{3}$, Michele Garo ${ }^{2}$ Valentina Guerrieri ${ }^{2}$, Francesco Luciano ${ }^{2}$, Fausto Spadea ${ }^{4}$

${ }^{1}$ Scientific Committee of National Association of Third Age Residences (ANASTE), Calabria, Italy, ${ }^{2}$ Centro Residenziale e di Riabilitazione San Domenico, Lamezia Terme, Italy, ${ }^{3}$ RSA Villa Elisabetta, Cortale, Italy, ${ }^{4}$ RSA Casa Amica, Fossato Serralta

Introduction: The objective of this study was to evaluate the prevalence of Subclinical hypothyroidism (IPO Sub) and Subclinical hyperthyroidism (IPER Sub) in a cohort of elderly people and to correlate the thyroid dysfunctions with cardiovascular, metabolic and cognitive comorbidities.

Methods: A retrospective, observational study was conducted on a sample of 316 subjects admitted to LTC facilities. The analysis was carried out on the anamnestic data and multidimensional evaluation acquired on admission, and blood tests performed within the first week of recovery. MMSE and GDS were used to asses cognitive status and mood. IPO Sub and IPER Sub were set for values of $\mathrm{TSH}>4 \mathrm{uIU} / \mathrm{ml}$ and $\mathrm{TSH}<0.4 \mathrm{uIU} / \mathrm{ml}$ respectively with normal plasma concentration of FT4. Spearman correlation test $(\rho)$ was performed for the bivariate analysis.

Results: $59.8 \%$ of the sample was F (average age $83.40 \pm 9.68$ ) and 40.2\% M (mean age $78.09 \pm 12.39$ ). IPO Sub patients were $5.4 \%$ $(76.5 \% \mathrm{~F}$ and $23.5 \% \mathrm{M}) ; 58.8 \%$ had already been diagnosed on admission and were on levothyroxine therapy. IPER Sub patients were $10.1 \%(50.0 \% \mathrm{~F}$ and $50.0 \% \mathrm{M}) ; 9.4 \%$ had already been diagnosed and $12.5 \%$ were on Tiamazole therapy. We found a positive association between IPO Sub and diabetes $(\rho=0.008)$, hypertension $(\rho=0.011)$, stroke $(\rho=0.024)$ and between IPER Sub and heart failure $(\rho=0.036)$. No significant correlation was found between IPO Sub or IPER Sub and cognitive impairment and depressive symptoms. Conclusion: This study highlights a close association between subclinical thyroid dysfunction, cardiovascular comorbidity and diabetes but not with cognitive impairment.

\section{P-723}

Do current clinical guidelines in Germany and the US address geriatric issues properly?

Franziska Scherr ${ }^{1}$, Heinrich Burkhardt ${ }^{1}$

${ }^{1}$ Universitätsmedizin Mannheim-Geriatrisches Zentrum, Mannheim, germany

Background: Guidelines are significant tools in medicine. However, as risk-benefit ratios may often differ in the elderly and specific geriatric issues have to be taken in consideration, there is still some discussion about missing geriatric aspects in those guidelines, concerning properly clinical decision-making in the elderly.

Methods: A previously committed search strategy identified german guidelines (AWMF) and corresponding US-guidelines (National Guideline Clearing House) according to guideline titles. There was a wide range of clinical topics included in search strategy. These were chosen in advance either by an increasing prevalence in the elderly (e.g. diabetes, stroke, syncope, dementia, incontinentia) or by the topic itself if representing a specific geriatric issue. If possible pairs of guidelines (US and Germany) were analyzed. A previously built scoring matrix was applied to evaluate the guidelines (range 0-38). Within this matrix following issues were evaluated: mentioning elderly patients in general, locomotion problems, cognition, polypharmacia, multimorbidity.

Results: 30 guidelines were analyzed (13 german, 17 US). Only the US-guideline concerning diabetes (ADA) reached the highest category ( $75 \%$ and more of maximum possible score). 13 guidelines were placed in category II (50-75\% of maximum possible score), 11 in category III $(25-50 \%$ of maximum possible score) and 5 guidelines remained in the lowest category (below 25\% of maximum possible score). Out of the 5 issues applied polypharmacia was least addressed ( $0 \%$ of the possible maximum score in $41.1 \%$ of guidelines).

Discussion: There is a remarkable heterogeneity how geriatric issues are addressed in current clinical guidelines. Only few guidelines mention geriatric issues properly with a remarkable lack in the case of polypharmacia. On the other hand in most guidelines at least some geriatric aspects are mentioned. To improve this further a comprehensive effort is recommended in the guideline consenting process to address geriatric issues and provide special comments to clinical situations where those may influence recommendations.

\section{P-724}

Comorbidity and multimorbidity in older adults living in rural northern Greece

\author{
Vaitsa Giannouli ${ }^{1}$, Nikolaos Syrmos ${ }^{1}$ \\ ${ }^{1}$ School of Medicine, Aristotle University of Thessaloniki, \\ Thessaloniki, Greece
}

Introduction: Comorbidity and multimorbidity in older adults is generally accepted as having a major impact on healthcare resources and costs. The aim of this study is to examine comorbidity and multicomorbidity, by assessing which are the most prevalent health problems in a representative sample of older adults living in rural northern Greece.

Methods: Eighty-seven older adults from Northern Greece (52 females) participated voluntarily in this door-to-door study. The data were collected during 2017. The cognitive function of the participants was assessed with the MMSE, and their daily functioning with FRSSD and FUCAS. The participants with severe cognitive deficits were excluded from the study. The existence of chronic conditions was examined with a written closed-ended questionnaire. The mean 
age of the participants was 74.65 years $(\mathrm{SD}=6.75$, range $66-89$ ), their level of education 8.60 years $(\mathrm{SD}=3.92)$, and their mean MMSE score was $26.50(\mathrm{SD}=3.99)$.

Results: Results revealed that $21 \%$ did not experience comorbid health conditions, $22 \%$ of respondents reported simple comorbidity, and $57 \%$ reported three or more chronic conditions. The frequency of the 8 most reported conditions is in descending order: vision problems, hearing loss, high blood pressure, heart trouble, diabetes, arthritis-rheumatism, stroke, and lung diseases.

Conclusions: These preliminary findings support that older adults, coming from a little investigated geographical area, experience two or more chronic health conditions. Future research in larger samples should elucidate the factors that influence the initiation and progression of comorbidity and multicomorbidity.

\section{P-725}

Strong association between malnutrition, inflammation and depression in elderly patients, novel geriatric complex based on malnutrition; MID Complex?

Ibrahim Halil Turkbeyler ${ }^{1}$, Zeynel Abidin Öztürk ${ }^{2}$

${ }^{1}$ Ersin Aslan Education and Research Hospital, Gaziantep, Turkey, ${ }^{2}$ Gaziantep University, Faculty of Medicine, Department of Geriatrics, Gaziantep, Turkey

Objective: Many chronic diseases emerge in aging period. The aim of the present study was to investigate the frequency of the association of inflammation and depressive symptoms in elderly patients at risk for malnutrition and/or malnutrition.

Methods: 217 individuals aged 65 years and over admitted to the outpatient clinic of the Department of Internal Medicine, Division of Geriatric Medicine at Gaziantep University Hospital for routine medical care were enrolled in this cross-sectional study. All patients underwent comprehensive geriatric assessment tests via one-on-one interview method.

Results: The median age of patients was $72.48 \pm 5.98$ years and the age range was between 65 and 90 years. A total of 128 patients $(59 \%)$ were female and $89(41 \%)$ were male. According to mini-nutritional assessment short form (MNA-SF) 41 (18.9\%) were in malnutrition group, $82(37.8 \%)$ were in malnutrition risk group, and $94(43.3 \%)$ were in normal nutritioneal status. In malnutrition group, $51.2 \%$ had elevated CRP levels and $70.7 \%$ had depressive symptoms. In malnutrition risk group, $39.0 \%$ had elevated CRP levels and $70.7 \%$ had depressive symptoms. There was a statistically significant negative correlation between MNA-SF score with geriatric depression scale short form (GDS-SF) score and CRP levels (r: - 0,201, p: 0,003, r: $-0,495$, p: 0,000 , respectively). The incidence of association of inflammation-depression in elderly patients with malnutrition and malnutrition risk was $36.6 \%$ and $12.2 \%$, respectively.

Conclusion: Depression and inflammation can accompany each other at an important frequency in elderly malnutrition patients.

\section{P-726}

\section{Management of older patients with diabetes in primary care -} results from Slovenia

\author{
Marija Petek Šter ${ }^{1}$, Barbara Jemec Zalar ${ }^{1}$ \\ ${ }^{1}$ Department of Family Medicine, University of Ljubljana, Poljanski \\ nasip 58, Ljubljana, Slovenia
}

Introduction: Diabetes is one of the most prevalent chronical diseases in multimorbid elderly population. The aim of the research was to recognise the basic characteristics of elderly patients with diabetes, quality of process of care and outcomes in elderly patients with diabetes in Slovenia.

Methods: In a cross sectional study, family physicians from different regions of Slovenia included data for consecutive patients with diabetes aged 65 or over. Data about the comorbidities, process of care and outcomes were retrieved from patient's medical record.

Results: In the sample there were 226 patients; 115 female $(50.9 \%)$ and 111 male (49.1\%) from 65 to 93 years (mean 73.7, SD 5.6 years); were included. Depression was documented in $11.1 \%$ of patients. $18.2 \%$ were treated with diet only, $62.7 \%$ taking oral antidiabetics and $19.1 \%$ insulin. $36.5 \%$ of patients had micro or macrovascular complications of diabetes. The mean HbA1c was $7.0 \%$. Foot examination in the last year was made in $81.0 \%$ and eye examination in $70.2 \%$ of patients. $74.3 \%$ of patients reached individually determined value of $\mathrm{HbA} 1 \mathrm{c}, 68.0 \%$ had their blood pressure under the control and $54.5 \%$ reached the target values of LDL.

Conclusions: Patients with diabetes underwent interdisciplinary team management were carefully followed and in high percentages reached the target values of risk factors for cardiovascular disease. In the future more effort should be put in more individualised treatment plan for multimorbid patients with diabetes.

\section{P-727}

\section{Predictors of mortality in geriatric patients}

Siddarth Agrawal ${ }^{1}$, Mateusz Luc ${ }^{1}$, Filip Winkowski ${ }^{1}$, Karolina Lindner ${ }^{1}$, Anil Kumar Agrawal ${ }^{1}$, Marta Woźniak ${ }^{1}$, Małgorzata Sobieszczańska ${ }^{1}$

${ }^{1}$ Department and Clinic of Geriatrics, Wroclaw Medical University, Wroclaw, Poland

Early identification a risk of mortality is crucial to provide adequate interventions and healthcare resources in elderly patients. The aim was to determine the strongest predictors of all-cause mortality in 208 patients admitted to the geriatric ward in 2013 year. A retrospective analysis on the patients' status at hospital admission and a primary outcome of 4-year mortality were used. Results: The mortality rate was $26 \%$. Age, red blood and white blood cells count, as well levels of C-reactive protein, albumin and high-density lipoprotein cholesterol significantly correlated with mortality. The presence of some clinical symptoms, i.e., pressure ulcers and weakened consciousness was predictive of poor outcome. Multidimensional aspects of aging assessed in the Comprehensive Geriatric Assessment (Activities of Daily Living, Instrumental Activities of Daily Living, Barthel scale, Mini Mental State Examination, Clock Drawing Test) appeared to be strong predictors of 4 -year mortality. The probability of mortality based on the examined variables correctly classified nearly $85 \%$ of the analyzed cases.

Conclusion: Early detection of high-risk patients could enable to reach longer life-span in the elderly.

\section{P-728}

The association between age-related hyperkyphosis and depression

Gülçimen Soylu ${ }^{1}$, Yusuf Yalvaç ${ }^{2}$, Funda Datlı Yakaryılmaz ${ }^{1}$, Zeynel Abidin Öztürk ${ }^{1}$ 
${ }^{1}$ Gaziantep University, Faculty of Medicine, Department of Internal Medicine, Division of Geriatrics, Gaziantep, Turkey, ${ }^{2}$ Gaziantep University, Faculty of Medicine, Department of Internal Medicine, Gaziantep, Turkey

Aim: Hyperkyphosis commonly affects elderly people and closely related to morbidity and mortality. On the other hand, depression is an important disorder which is also prevalent among geriatric population. The relation between depression and physical illness is apparent and disturbs life quality of patients and their relatives. This study aims to investigate the relation between hyperkyphosis and depression.

Methods: The study included 142 participants who applied to our university's geriatric outstanding clinic. The kyphosis grade was measured using the block method. The participants were told to lie in a supine position on a radiology table. Blocks with thickness of $1.7 \mathrm{~cm}$ were placed under their heads (avoiding hyperextension or hyperflexion) to keep participants' heads in neutral position. The block of one and above was considered as hyperkyphosis. Yesavage Geriatric Depression Scale (YGDS) was used for diagnosis of depression. Patients assessed at five and above points in the scale were regarded as in depression.

Results: A total of 142 individuals were participated in the study. The mean age of the patients was $72.1 \pm 6.90$. Thirty-six of the participants were male $(25.3 \%)$ and 106 were female $(74.6 \%)$. Seventy-one of our participants were hyperkyphotic while the other 71 were normal. The depression frequency of the patients according to YGDS were significantly higher in the hyperkyphotic group than normal group (p: 0.03).

Conclusion: The depression scale of hyperkyphotic patients was statistically significant. This might be a result of functional restriction and pain related to hyperkyphosis.

\section{P-729}

\section{The relationship between hyperkyphosis and life quality}

Gülçimen Soylu ${ }^{1}$, Yusuf Yalvaç ${ }^{2}$, Funda Datlı Yakaryılmaz ${ }^{1}$, Zeynel Abidin Öztürk ${ }^{1}$

${ }^{1}$ Gaziantep University, Faculty of Medicine, Department of Internal Medicine, Division of Geriatrics, Gaziantep, Turkey, ${ }^{2}$ Gaziantep University, Faculty of Medicine, Department of Internal Medicine, Gaziantep, Turkey

Aim: Hyperkyphosis is one of the risk factors that disrupt the quality of life. There is no study concerning hyperkyphotic men in this respect. We aimed to evaluate the relationship between hyperkyphosis and quality of life.

Methods: The study included 107 patients applied to geriatric outpatient clinic. The hyperkyphosis of the individuals were determined by using Cobb's angle. The Cobb's angle was calculated blindly by two clinicians using the lateral graphs of standing individuals, and the angle greater than $40^{\circ}$ was accepted as hyperkyphosis. The SF-36 life quality scale was used to assess the life quality of the patients. High scores indicate a good level of health, while low scores indicate health deterioration.

Results: The mean age of participants was $72.1 \pm 6.90$, and $22.6 \%$ of the patients were male while $77.4 \%$ were female. According to the Cobb's angle, $44(41.1 \%)$ patients were hyperkyphotic and 63 $(58.9 \%)$ patients were normal. There were no significant statistical differences between the parameters of life quality of hyperkyphotic patients determined by Cobb's angle and healthy individuals. The scores of EVV (energy, vitality, viability), MH (mental health), SF (social functionality), and pain of hyperkyphotic group were lower than the healthy ones, nevertheless, it was not statistically insignificant.
Conclusion: This is the first study in the literature that reveals the relationship between age-related hyperkyphosis and life quality using SF-36 scale. Although there were no differences in terms of life quality between individuals with and without hyperkyphosis, it is important to conduct studies with large and fragile patient groups.

\section{P-730}

Predictive validity at 18 months of follow up of the VIDA questionnaire considering functional decline, institutionalization or death in multimorbidity patients

Iñaki Martin Lesende ${ }^{1}$, Luis Ignacio Mendibil Crespo ${ }^{2}$, Beatriz Porto Hormaza $^{3}$, Irune Martinez Blanco ${ }^{3}$, Batirtze Maray Gondra ${ }^{3}$, Nerea Aguirre Basaras ${ }^{4}$

${ }^{1}$ San Ignacio Health Centre, Bilbao-Basurto Integrated Healthcare Organisation (IHO), Basque Health Service (Osakidetza), Basque, Spain, ${ }^{2}$ Bizkaia Unit for Multiprofessional Training in Family and Community Care, Bilbao, Spain, ${ }^{3}$ Bilbao-Basurto IHO, Osakidetza, Basque, Spain

VIDA questionnaire, widely validated [1], assesses instrumental activities of daily living (IADL).OBJECTIVE. To analyze the predictive validity at 18 months of follow-up of the VIDA questionnaire in multimorbidity patients $\geq 65$ years included in an institutional programm, without significant functional decline (Barthel $\geq 60$ ), considering functional decline in basic activities (BADL, Barthel $<60$ ), institutionalization or death.

Methods: Prospective multicenter study for diagnostic test. 185 PATIENTS of the "FUNCIPLUR study" (which analyses functional decline in multimorbidity patients) who completed the follow-up, died or were institutionalized, were included. Variable: VIDA questionnaire, with 10 items (AIVD) and a Likert scale, and a maximum of 38 points. Result event: functional decline in BADL (Barthel $<60$ ), institutionalization, or death. Analysis. ROC curve and cut-off point with better sensitivity/specificity. Using IBM SPSS 23 software. Results: Considering the 185 patients, 57 (30.8\%) had the result event (32 died, 19 had functional decline, 6 institutionalized). Median of the questionnaire 27 (IQR 19.5-33) in those with event vs. 34 (30-36) patients without it $(\mathrm{p}<0.001)$.AUC $0.76, \leq 29$ being the best cut-off point (sensitivity $61.4 \%$-95\% CI $47.6-73.7$, specificity $76.6 \%$ 95\% CI 68.1-83.4, predictive positive rate-PPR-53.9, NPR 81.7). Odds ratio considering this cut-off point $2.9-95 \%$ CI 1.9-4.6.

Key conclusions: Good predictive validity of VIDA in patients and events considered, highlighting the high PPR. Potentiality for being used in the detection of frailty in this kind of complex patients. Results will be compared with others related to short-term (8 months), presented previously in another Congress.

\section{Reference:}

1. Martin-Lesende I, Vergara I, Vrotsou K, Lopetegui P, Nuñez J, Sánchez I, Bueno A, Diez AI (2015) Validation of "VIDA questionnaire" for assessing instrumental activities of daily living; aspects connecting to frailty. Eur Geriatr Med 6(Sup 1):S32-S156

\section{P-731}

Giant cell arteritis characteristics in patients older than 75 years

Lamloum Mounir ${ }^{1}$, Ben Salem Thouraya ${ }^{2}$, Ben Ghorbel Imed ${ }^{2}$, Houman Mohamed Habib ${ }^{2}$

${ }^{1}$ la Rabta Hospital, Faculte De Medecine, Tunis, Tunisia, ${ }^{2}$ La Rabta Hospital, Tunis, Tunisia 
Background: The aim of this study was to describe clinical, biological and pathological features of giant cell arteritis (GCA) in patients older than 75 years and to compare them to younger patients with GCA.

Methods: We conducted a retrospective and descriptive study in the department of internal medicine at la Rabta Hospital, over a 16-year period. Ninety six patients were diagnosed with GCA according to the American College of Rheumatology 1990 criteria for the classification of giant cell arteritis.

Results: Thirty five patients with CGA were aged more than 75. Sexratio $\mathrm{M} / \mathrm{W}$ was 1.69 . Mean age at diagnosis was $79.26 \pm 3.3$ years. Median delay to diagnosis was 1.75 months [extremes 0 and 24 months]. Disease was mostly revealed with headache $(65.7 \%)$. Constitutional symptoms and fever were noted in 54.3 and $31.4 \%$ of patients respectively. Headache was the most frequent sign (97.1\%). Jaw claudication and scalp tenderness were found in 74.3 et $42.8 \%$ of patients respectively. Polymyalgia rheumatica was diagnosed in $45.7 \%$ of patients. On physical examination, abnormal temporal artery was found in $91.4 \%$ patients. Thirteen patients (37.1\%) had ocular involvement (ischemic optic neuropathy in all of them). Laboratory markers of inflammation were positive in $94.2 \%$ of patients. Temporal artery biopsy showed compatible pathology report in $75 \%$ of cases. Jaw claudication $(\mathrm{p}=0.009)$ and high gammaglobulin rate $(\mathrm{p}=0.018)$ were significantly more frequent in patients older than 75 years.

Conclusion: GCA characteristics in patients older than 75 years were similar to other ethnic groups and to younger patients.

\section{P-732}

Predictive model for one year mortality in a geriatric acute unit

C. Elías de Molins Peña ${ }^{1}$, B. Gamboa Huarte ${ }^{1}$, F. Arribas Monzon ${ }^{2}$

${ }^{1}$ Servicio Geriatría, Hospital Real Nuestra Señora de Gracia, Zaragoza, España, ${ }^{2}$ Asesor técnico, Dirección General de Asistencia Sanitaria, Dpto Sanidad, Gobierno de Aragón, Spain

Objectives: The objective of this study is to determine, through a logistic model, factors that can be predictors of one-year mortality and identify a population with a greater risk, in inpatients in a geriatric acute care unit.

Methods: Collected data from June to November 2014, with one year mortality follow up after discharge. Variables: biographical, clinical and pathological characteristic, analytic parameters, cognitive status and functional index. Logistic regression (LR) was performed to assess the relationship between death and studied variables. In previous multivariate analysis, Chi square and ANOVA was used. SPSS. Results: $\quad 318$ inpatients. Model: $\operatorname{prob}($ exitus): $1 /$ $\left(1+\mathrm{e}^{\wedge}(-[-0.198+0.753\right.$ infection +1.396 barthel $<60+0.815$ polypharmacy -0.2175 surgery +0.792 anemia -0.801 albumin(quantitative)]).The most significant risks detected in the explanatory variables considered were: the infection history (OR 2.124, 95\% CI 1.089-4.166), Barthel's index < 60 (OR 4.04, 95\% CI 1.77-9.26), anemia (OR 2.26, 95\% CI 1.12-4.354), and polypharmacy (OR 2.26, 95\% CI 1.057-4.831). Surgery (OR 0.114, 95\% CI 0.03-0.410) and high levels of albumin (OR 0.049, 95\% CI $0.253-0.796)$ were related with better prognosis. The multivariate model correctly classified $74.2 \%$ of patients, showing a high specificity $(85.2 \%)$ and sensitivity $(52.6 \%)$. The discriminatory power of the model, according to ROC curve, was $84.6 \%$ of the maximum possible [AUC: $0.78995 \% \mathrm{CI}(0.733-0.846) \mathrm{P}=0.000$ ].

Conclusion: Geriatric syndromes, polypharmacy and functional loss, as well as the analytical parameters of our study, low values of hemoglobin and albumin, showed to be predictive factors of mortality at one year. The diagnostic and therapeutic adequacy is today the biggest challenge of a geriatrician. We have varied information on several factors that contribute to mortality, but we still don't have a gold standard.

\section{P-733}

The challenge of recruiting multimorbid elderly patients to a randomised controlled trial

\author{
Karol Biegus ${ }^{1}$, Richard Frobell ${ }^{2}$, Anne Ekdahl ${ }^{1}$
}

${ }^{1}$ Geriatric Medicine, Helsingborg Hospital, Sweden, Department of Clinical Sciences, Lund University, Lund, Sweden, ${ }^{2}$ Department of Clinical Sciences, Lund University, Lund, Sweden

Introduction: Research involving the multimorbid elderly is gaining momentum. However, little is known about how to plan a randomised controlled trial (RCT) involving this group of patients. Having an evidence based approach could guide researchers designing RCTs and prevent underpowered trials.

Method: We used baseline data from the GerMoT trial, a RCT comparing proactive outpatient care based on Comprehensive Geriatric Assessment (CGA) with usual care. Multimorbid elderly patients with high healthcare utilisation were recruited to the trial. We define the number of patients that need to be identified and the number of eligible patients that need to be invited in order to achieve the desired recruitment number.

Results: Of 1122 patients identified in a database as meeting inclusion criteria only 770 could be invited to participate in the trial. The rest had to be excluded or could not be reached for a variety of reasons despite considerable efforts made by our team of researchers. 420 of the invited patients agreed to participate with younger patients being more inclined to consent.

Key conclusions: When planning a RCT involving this cohort of patients one can anticipate that less than $70 \%$ of patients identified as meeting inclusion criteria can be invited. We have also established that of those that can be invited the consent rate for such a trial is $54 \%$ and it varies slightly with age. We believe this information is generalizable beyond RCTs and can be used to plan recruitment to new proactive healthcare interventions involving this vulnerable group of patients.

\section{P-734}

A New geriatric syndrome; hyperkyphosis? determining the frequency of hyperkyphosis and comparison of three methods

Gülçimen Soylu ${ }^{1}$, Zeynel Abidin Öztürk ${ }^{2}$, Yusuf Yalvaçç, Funda Datlı Yakaryılmaz

${ }^{1}$ Gaziantep University, Faculty of Medicine, Department of Internal Medicine, Division of Geriatrics, Gaziantep, Turkey, ${ }^{2}$ Gaziantep University, Faculty of Medicine, Department of Internal Medicine, Division of Geriatrics, Gaziantep, Turkey, ${ }^{3}$ Gaziantep University, Faculty of Medicine, Gaziantep, Turkey

Aim: Age-related hyperkyphosis (ARH) is an exaggerated anterior curvature of the thoracic spine. Though the prevalence of hyperkyphosis in older individuals is not clear, existing studies show that the prevalence is between 20 and $40 \%$. This study aimed to determine the frequency of ARH and to compare three methods used to evaluate hyperkyphosis in patients.

Method: Patients who applied to geriatric outpatient clinic were included in this study. Participants were evaluated by using the Cobb's angle, the kyphotic index, and the block method. Patients who have Cobb's angle of $40^{\circ}$ and above, the kyphotic index of 12 and 
above, or the block of one and above were considered as hyperkyphosis.

Results: The study included 161 participants comprised of 121 women $(75.2 \%)$ and 40 men $(24.8 \%)$, with an average age of $72.5 \pm 6.90 .66(41.0 \%)$ patients were hyperkyphotic by the Cobb's angle. In the kyphotic index measurements, $65(40.4 \%)$ patients were hyperkyphotic. According to the block method, 81 (50.3\%) patients were hyperkyphotic. There is a significant correlation between the Cobb's angle and the kyphotic index ( $\mathrm{rs}=0.30, \mathrm{p}$ : 0.002) and the kyphotic index and the block method ( $\mathrm{rs}=0.32, \mathrm{p}:<0.001)$ while the Cobb's angle and the block method is not significantly correlated ( $\mathrm{rs}=0.15$, p: 0.13)

Conclusion: Hyperkyphosis is one of the deformities that occur in musculoskeletal system with ageing. This is the first study that used three different methods concurrently to evaluate hyperkyphosis that is a clinical manifestation which would be discussed as a new geriatric syndrome imminently.

\section{P-735}

Fracture risk is under-recognised and under-treated in memory clinic attendees

Tomas Welsh ${ }^{1}$, Danielle Tingley ${ }^{2}$, Zoe Lampshire ${ }^{2}$, Aron Jarvis ${ }^{2}$, Cathy Wernham ${ }^{2}$, Julian Hughes ${ }^{3}$

${ }^{1}$ Royal United Hospital Bath, University of Bristol, Research Institute for the Care of Older People, Bath, UK, ${ }^{2}$ Research Institute for the Care of Older People, Bath, UK, ${ }^{3}$ Royal United Hospital Bath, University of Bristol, Bath, UK

Introduction: People with cognitive impairment are more likely to sustain a fracture than their cognitively intact peers. Those referred to the memory clinic with suspected dementia therefore represent a group where fracture risk assessment and risk factor modification should be prioritised. This project set out to evaluate the management of fracture risk in memory clinic attendees.

Methods: Memory clinic attendees from the 1st of February until the 12th of March 2018 had their fracture risk calculated by FRAX ${ }^{\circledR}$ as part of their routine appointment. Their 10-year-risk of fracture was calculated and the UK National Osteoporosis Guideline Group (NOGG) recommendations were documented. Treatment and use of DEXA scans were compared to that recommended.

Results: Data were available from 79 attendees with a mean age of 83 (SD5.9), 47 (59\%) female, 54 (68\%) with dementia, MMSE 21 (SD 4.7). $22(28 \%)$ reported falling at least once in the last year and sustained a total of 57 falls. Those reporting a fall fell a median of 2 (IQR 1-3) times. 26 (33\%) were taking calcium/vitamin D supplementation while $10(13 \%)$ were taking an antiresorptive. $9(32 \%)$ of those recommended to be treated were on treatment, while $4(13 \%)$ of those recommended a DEXA had had one.

Key conclusions: Despite being at high risk of fracture, this memory clinic population was under-treated compared to national guidelines. These findings highlight an important deficit in fracture risk assessment which will inform future interventions to help improve treatment rates.

\section{P-736}

\section{Loneliness as geriatric syndrome}

Kiryl Prashchayeu ${ }^{1}$

${ }^{1}$ Belarusian Republican Gerontological Public Association, Navapolatsk, Belarus
Introduction: Loneliness is one of the common psychological conditions in elderly which is experienced according to different sources $20 \%$ of all people in age 65 plus. The aim of the study was to reveal medical effects of loneliness and to consider it as a socio-medical geriatric syndrome.

Materials and methods: Study was performed in three stages, including the revealing of elders with acute feeling of loneliness $(\mathrm{n}=340)$ by D. Russel, M. Ferguson Questionary, comprehensive geriatric assessment of elders with acute feeling of loneliness $(n=69)$, working out the ways of overcoming of loneliness $(n=69)$. Results: In lonely elders were observed the decreasing of general nutritional status, loss of appetite and psychological problems which with unregular including of fruits and vegetables into the diet, decreased level of protein intake were correlated with decreasing of grip strength without changing of muscle mass. Elders with loneliness had the worst indicators of general health, mental health and physical functioning, more adherence to the pain, $p<0,05$. To overcome loneliness the special educational programmes for volunteers were worked consisted in 36 academic hours, after education volunteers had 6-months working with lonely elders, including visits, control of food consumption, creation of positive emotional environment, cognitive training. After such intervention we observed the positive dynamics of loneliness and increasing of functional ability, $\mathrm{p}<0.05$. Conclusion: Loneliness may be the factor of decreasing of intrinsic capacity and important geriatric syndrome with the decreasing of nutritional status as causative element of complex medical changes.

\section{P-737}

Patterns of multimorbidity in older medical patients ( $\geq 65$ years): and how they relate to mobility the first year after an acute admission

HG Juul-Larsen ${ }^{1}$, O Andersen ${ }^{1}$, AC Bodilsen ${ }^{2}$, LM Jørgensen ${ }^{3}$, TQ Bandholm ${ }^{1}$, J Petersen ${ }^{1}$

${ }^{1}$ Optimized Senior Patient Program (Optimed), Clinical Research Centre, Copenhagen University Hospital, Hvidovre, Denmark, ${ }^{2}$ Department of Exercise and Health, Roskilde Municipality, Roskilde, Denmark, ${ }^{3}$ The Emergency Department, Copenhagen University, Copenhagen, Denmark

Introduction: Multimorbidity is common among older people and may contribute to adverse health effects, such as functional decline. Identification of differences in mobility after an acute hospitalization among multimorbidity patterns may help stratify treatment and provide the basis for including patients in randomized controlled trials to study the effect of evidence-based multi-disciplinary rehabilitation strategies.

Aim: To describe older patients' mobility in relation to patterns of multimorbidity the first year after an acute medical hospitalization.

Methods: Prospective cohort study of 369 medical patients (77.9 years, 62\% women) acutely admitted to the emergency department. During the first $24 \mathrm{~h}$ of admission, 30 days and one year after discharge we assessed mobility level using the de Morton Mobility Index. Information about chronic conditions was collected by national registers. We used Latent Class Analysis to determine differences among patterns of multimorbidity based on 24 chronic conditions.

Results: We identified five different patterns of multimorbidity: (1) acutely hospitalized without chronic conditions (prevalence $37 \%$, expected number of chronic conditions 1.77); (2) sensory organs and osteoporosis $(19 \%, 4.25)$; (3) cardiovascular $(26 \%, 4.31)$; 4) Lifestyle diseases $(8 \%, 6.75)$; and (5) degenerative and metal disorders $(9 \%$, 6.98). Low mobility was associated with membership of pattern 4 
(lifestyle diseases) and 5 (degenerative and metal disorders) at all time points. No differences were found in mobility change between the patterns after admission.

Key conclusions: The results support that chronic conditions cluster together and that these patterns differ in mobility, which suggests a differentiated approach towards treatment and rehabilitation is needed.

\section{P-738}

Patterns of multimorbidity in older medical patients ( $\geq 65$ years): and how they relate to mobility the first year after an acute admission

HG Juul-Larsen ${ }^{1}$, O Andersen ${ }^{1}$, AC Bodilsen ${ }^{2}$, LM Jørgensen ${ }^{3}$, TQ Bandholm ${ }^{1}$, J Petersen ${ }^{1}$

${ }^{1}$ Optimized Senior Patient Program (Optimed), Clinical Research Centre, Copenhagen University Hospital, Hvidovre, Denmark, ${ }^{2}$ Department of Exercise and Health, Roskilde Municipality, Roskilde, Denmark, ${ }^{3}$ The Emergency Department, Copenhagen University, Copenhagen, Denmark

Introduction: Multimorbidity is common among older people and may contribute to adverse health effects, such as functional decline. Identification of differences in mobility after an acute hospitalization among multimorbidity patterns may help stratify treatment and provide the basis for including patients in randomized controlled trials to study the effect of evidence-based multi-disciplinary rehabilitation strategies.

Aim: To describe older patients' mobility in relation to patterns of multimorbidity the first year after an acute medical hospitalization.

Methods: Prospective cohort study of 369 medical patients (77.9 years, $62 \%$ women) acutely admitted to the emergency department. During the first $24 \mathrm{~h}$ of admission, 30 days and one year after discharge we assessed mobility level using the de Morton Mobility Index. Information about chronic conditions was collected by national registers. We used Latent Class Analysis to determine differences among patterns of multimorbidity based on 24 chronic conditions.

Results: We identified five different patterns of multimorbidity: (1) acutely hospitalized without chronic conditions (prevalence 37\%, expected number of chronic conditions 1.77); (2) sensory organs and osteoporosis $(19 \%, 4.25)$; (3) cardio vascular $(26 \%, 4.31)$; (4) lifestyle diseases $(8 \%, 6.75)$; and (5) degenerative and metal disorders $(9 \%$, 6.98). Low mobility was associated with membership of pattern 4 (lifestyle diseases) and 5 (degenerative and metal disorders) at all time points. No differences were found in mobility change between the patterns after admission.

Key conclusions: The results support that chronic conditions cluster together and that these patterns differ in mobility, which suggests a differentiated approach towards treatment and rehabilitation is needed.

\section{P-739}

\section{Rehabilitation geriatric day care in French Hospital}

Florence Bonte ${ }^{1}$

${ }^{1}$ Hopital Sainte Marie Paris, Paris, France

The management of patients in geriatric day care hospitals (DCH) is an addition to short-stay units with a predominant diagnostic activity: rehabilitation units are organized around weekly or multi-weekly rehabilitation interventions. The purpose of this study, was to draw up an inventory of the contents of stays among 50 rehabilitation day care hospitals in France. The method used was a multicenter retrospective study. The main items collected were:-data about the center (professionals, team, organization...) - goals of care; duration; number of acts performed; presence of an initial assessment, a therapeutic objective and a final assessment. The results showed an average duration of stay of 25-45 days, with 2 interventions per day; by at least 2 different professionals. The care team was different from one center to the other. The main pathologies found, motivating this care were dementia and falls, with improvement of these after care. In conclusion, this study gave us the opportunity to show that with objective care goals and experienced professionals, we now have a better knowledge of the place of day care hospital in the geriatric sector.

\section{P-740}

Factors determining self-rated health status and global quality of life perception in older adults

Marina Kotsani ${ }^{1,6}$, Thekla Chatziadamidou ${ }^{2}$, Carlos Labat $^{3}$, Georgios Soulis ${ }^{4}$, Ioannis Georgiopoulos ${ }^{4}$, Irini Tzanetakou ${ }^{4}$, Dimitrios Oikonomidis $^{5}$, Athanase Benetos ${ }^{6,7}$

${ }^{1}$ Aristotle University of Thessaloniki, Thessaloniki, Greece, ${ }^{2} \mathrm{Health}$ Center of Diabata, Greece, ${ }^{3}$ Inserm U1116, France, ${ }^{4}$ Hellenic Society for the Study and Research of Aging, Greece, ${ }^{5}$ Aristotle University of Thessaloniki, Greece, ${ }^{6} \mathrm{CHRU}$ Nancy, France, ${ }^{7}$ University of Lorraine, France

Introduction/objective: We aimed to investigate those factors that are correlated with self-rated health status (SRHS) and perceived global quality of life (QoL) by older adults.

Methods: A cross sectional study was conducted in 273 adults, 65 years and older, in primary health care settings, using clinical interviews and simple geriatric tests. SRHS and QoL were evaluated with two semi-quantitative questions derived from the SF-36 questionnaire. Medical conditions were self-reported.

Results: Neither sex nor age affected the SRHS and the QoL. Geriatric Depression Scale, Global Disability Scale, qualitative evaluation of the gait by the individual and by the physician, pain and anxiety were significantly related to both SRHS and QoL respectively (all $p<0.05)$, whereas time in Up and Go test $(p<0.001)$ and fear of falling $(\mathrm{p}<0.001)$ only affected SRHS. The presence of chronic conditions like hypertension, diabetes, heart failure, atrial fibrillation, stroke and arthritis, the number of medications or the presence of $\geq 1$ hospitalizations in the last year did not affect SRHS and QoL perception, whereas the number of comorbidities only marginally affected SRHS $(\mathrm{p}=0.048)$. Among several social factors explored, only leisure activities shared with the spouse were related to both SRHS and QoL (both $\mathrm{p}<0.01$ ), whereas loneliness only affected QoL $(\mathrm{p}<0.001)$.

Key conclusions: Perceived SRHS and QoL of older adults are not necessarily affected by "classical" morbidity but are often related to geriatric syndromes mostly expressing functional ability and psychological well-being.

\section{P-741}

Demographic transition and socio-demographic indicator in Monastir City

Saoussen Zrour ${ }^{1}$, Said Hajem ${ }^{2}$, Hammami Mohamed $^{3}$ 
${ }^{1}$ Department of Rhumatology CHU Monastir, Monastir, Tunisia,

${ }^{2}$ Public Health Institute, Tunis, Tunisia, ${ }^{3}$ LR-NAFS 'Nutrition-

Functional Food \& Vascular Health' Faculty of Medicine-University of Monastir, Monastir, Tunisia

The demographic transition in Tunisia is characterized by an increase in the prevalence of the elderly population and its related socioeconomic and health problems.

Materials and methods: Our study aimed at providing reliable information to assess quantitatively and qualitatively the health status, the actual needs in healthcare and social assistance among the elderly population (65 years and more) living at home in the governorate of Monastir.

Results: The study concern 598 people, the mean age is 72,3 years, with a predominance of women (66\% VS 34\%). Education shows that $77 \%$ have a lowest level of education. Most of our population are still married $(60 \%)$ and $60 \%$ are inactive. Widowhood concern more frequently women $53.5 \%$ than men $6.9 \%$.Subjects without occupation or having never worked represent $60 \%$ of cases. The perception of the state of health is good in $35 \%$ of the cases, moderate in $40 \%$ of the cases. The assessment of autonomy and quality of life revealed a dependency in $10 \%$. The most common morbidities are arterial Hypertension (52\%), diabetes (22\%) and polypharmacy $>3$ medications in $60 \%$. The prevalence of psychological disorders is $29 \%$ for depression according to mini GDS score and $3 \%$ in cognitive impairment according to MMSE adapted to Tunisian population.

Conclusion: The demographic transition in Tunisia is causing an increase in the prevalence of the elderly population, an increase in the chronic diseases and an impairment of the quality of life.

\section{P-742}

\section{Psychotropes is too much!}

Liliana Blaj $^{1}$, Pierre Lemaire ${ }^{2}$

${ }^{1} \mathrm{CHU}$ Mons Hainaut, Mons, Belgium, ${ }^{2}$ Service de Gériatrie, CHU Mons Hainaut, Mons, Belgium

Belgium is the European country that consumes the most psychotropic drugs, mainly benzodiazepines The side effects of these drugs are well known, including cognitive disorders and balance disorders favoring falls, with the cascade of multiple consequences, complications often resulting in prolonged hospitalization, loss of autonomy and institutionalization. Withdrawal these medication requires first the collaboration of the patient, his entourage and all the professionals of the first line: attending physician, nursing home staff, etc. This is what the RGH Mons Hainaut geriatrics team proposes: in the context of day hospitalization, one of the planned actions concerns cognitive revalidation and resocialization, which includes support for medicated withdrawal. In practice, after consultation with the patient, the family and the attending physician and the home help services, a care plan is drawn up by the multidisciplinary team. Than a program is set up, providing a day hospitalization and then, two to three times per week the patient is welcomed into the institution. During these days, workshops and group activities are organized (speech spaces, workshops led by occupational therapists, speech therapists and physiotherapists) and individual interviews with the psychologist and dietitian. The average duration of weaning is of the order of 3 months with 2-3 sessions per week with about 40 sessions. After a period of 6 months, the first results can be presented: improvement of the multidisciplinary evaluation (cognition, risk of falling, activities of the daily life and instrumental activities, etc.)

\section{P-743}

\section{Antihypertensive therapy effects on functional status markers} in patients with frailty

Nadezda Runikhina ${ }^{1}$, Anna Shchedrina ${ }^{1}$, Madina Balaeva ${ }^{1}$, Alexandra Luzina ${ }^{1}$, Olga Tkacheva ${ }^{1}$, Natalia Sharashkina ${ }^{1}$

${ }^{1}$ Pirogov Russian National Research Medical University-Russian Clinical and Research Center of Gerontology, Moscow, Russian Federation

Introduction: Frailty is a reversible condition of vulnerability towards disability and other outcomes in older adults. Goal. To assess the effect of antihypertensive therapy (AGT) on functional status markers in patients with frailty.

Materials and methods: We examined 200 patients with frailty, aged 60 to 91, both sexes suffering from arterial hypertension (AH) who underwent AGT for 6 months (month) with an assessment of the functional status markers on the 180th day of the study. The evaluation of the effectiveness, safety of the AGT, as well as its impact on the functional status markers was carried out.

Results: Based on the results of the study, the clinical efficacy and safety of AGT has been assessed. In patients with achieved target levels of arterial pressure (BP) $140 / 80-90 \mathrm{mmHg}$. improvement in the functional status markers was observed. Against the backdrop of AHT cases of falls and orthostatic hypotension was not recorded. In assessing the baseline daily activity index (Barthel Index), an easy $98.6 \pm 3.9$ score was observed initially and a significant increase in baseline activity on the 180th day was $99.1 \pm 2.86$ points. The decrease in instrumental activity assessed by the IADL scale was stable throughout the study and was $26.3 \pm 2.1$ and $26.4 \pm 2.13$ on the initial and on the 180th day, respectively. An improvement in walking markers, as measured by the "Stand Up and Go" test, was noted.

Conclusion: The results of the study proved clinical efficacy and safety of AGT, its beneficial effect on functional status markers.

\section{P-744}

Body composition of long-liver patients with coronary artery disease

Svetlana Topolyanskaya ${ }^{1}$, Irina Osipovskaya ${ }^{2}$, Olga Vakulenko ${ }^{2}$, Tatyana Eliseeva ${ }^{2}$, Natalya Balyasnikova ${ }^{2}$, Gennadyi Kalinin ${ }^{2}$, Leonid Dvoretsky ${ }^{3}$

${ }^{1}$ First Moscow State Medical University (Sechenov University), Department of hospital therapy N2, Moscow, Russia, ${ }^{2}$ War Veterans Hospital N3, Moscow, Russia, ${ }^{3}$ First Moscow State Medical University (Sechenov University), Department of hospital therapy N2, Moscow, Russia

The study purpose was investigation of body composition in longliver patients with coronary artery disease (CAD).

Methods: 190 patients with CAD (females-69.3\%, males-30.7\%) aged 90-106 years were enrolled in this study. Body composition were assessed by dual-energy X-ray absorptiometry.

Results: $70.4 \%$ of patients were overweight or obese. Mean body mass index was $27.6 \mathrm{~kg} / \mathrm{m}^{2}$. Women had more fat mass then men $(\mathrm{p}<0.0001)$. Overall bone mineral density (BMD) was $1005.9 \mathrm{mg} / \mathrm{cm}^{3}$, mean T-score: $-1.75 \mathrm{SD}$. The greatest BMD was in lower extremities $\left(1058.6 \mathrm{mg} / \mathrm{cm}^{3}\right)$, the lowest BMD-in ribs $\left(626.2 \mathrm{mg} / \mathrm{cm}^{3}\right)$. Female patients had lower BMD $(\mathrm{p}<0.0001)$. Significant correlation between fat and BMD was observed; the greatest significance-for correlation between trunk BMD and trunk fat $(r=0.61 ; p<0.0001)$. Significant positive correlation between BMD and learn mass was revealed; the 
greatest significance-for upper extremities $(\mathrm{r}=0.65$; $\mathrm{p}<0.0001)$. Positive correlation between $\mathrm{BMD}$ and handgrip strength was registered $(\mathrm{r}=0.48 ; \mathrm{p}<0.0001)$. BMD positively correlated with distance covered in the 6-min walk test $(r=0.35 ; p=0.002)$. Positive correlation between lean mass and handgrip strength was registered $(\mathrm{r}=0.53 ; \mathrm{p}<0.0001)$ as well as with distance in the 6-MWT test $(r=0.22 ; p=0.007)$. Negative correlation between BMD and frailty scale scores was observed $(\mathrm{r}=-0.45 ; \mathrm{p}<0.0001)$ as well as between lean mass and frailty scale score $(\mathrm{r}=-0.22, \mathrm{p}=0.003)$.

Conclusion: Study results demonstrated some features of body composition in patients with CAD aged 90 years or older. Significant associations between bone, fat and lean tissue as well as between $\mathrm{BMD}$, muscle strength and functional capacities were observed in the study population.

\section{P-745}

\section{Implementation of a geriatric program as support for primary} care

Javier Alonso $^{1}$, Antonia Solano ${ }^{1}$, Domingo De Guzman ${ }^{1}$, Carmen Alastuey $^{1}$, Fran Balea ${ }^{1}$, Nuria Herrera ${ }^{1}$, Ortzi Barasas ${ }^{1}$, Dacil Cabezas $^{1}$, Concha Peinado ${ }^{1}$

${ }^{1}$ Hospital Insular de Lanzarote, Arrecife, Spain

Introduction: In the national and autonomous framework of the approach to chronicity, support teams for primary care (PC) emerge. Given the need for comprehensive care of these patients, mainly geriatric, we have initiated a support program in our health area.

Methods: Descriptive observational study of patients attended from January to May 2018 by the support team of the HIL Geriatrics Service. We collected 24 variables: demographic, clinical, frailty (IF-VGI and Frail), functional: Barthel (IB), cognitive: Global Deterioration Scale (GDS), social, comorbidities (Charlson), geriatric syndromes, intervention and follow-up. Data analyzed with SPSS v25.

Results: Total of 85 patients of average age 86.37 years. Women $75.3 \%$. Main reason for consultation: medical symptoms (37.6\%), with dyspnea and behavioral disorders being the most frequent (37.5 and $31.3 \%$ ). $51.8 \%$ came from PC. IB of 44.18 points, a $69.4 \%$ with severe dependence. $45.2 \%$ with moderate-severe cognitive impairment. The IF-VGI, FRAIL and Charlson average of $0.44,3.4$ and 2.8 points. The main diagnosis was dementia and heart failure (23.5 and 18.8\%). The most performed actions were family intervention, therapeutic adjustment and polypharmacy control in $95.1 \%, 42.7 \%$ and $30.5 \%$.

Conclusions: The start-up of a unit represents a possible assistance challenge thanks to the support of the entire service. We are faced with a profile of a female patient, multi-pathological with moderate frailty. PC demands support in dementia and heart failure. The interventions are based on family orientation, polypharmacy control and therapeutic adjustment.

\section{P-746}

Impact of a complex intervention on the appropriateness of prescribing for nursing home residents (come-on study): results of a cluster-randomized controlled trial

Goedele Strauven ${ }^{1}$, Pauline Anrys ${ }^{2}$, Eline Vandael ${ }^{1,3}$, Séverine Henrard $^{2,4}$, Jan De Lepeleire ${ }^{5}$, Anne Spinewine ${ }^{2,6}$, Veerle Foulon ${ }^{1}$

${ }^{1}$ Department of Pharmaceutical and Pharmacological Sciences, KU Leuven, Leuven, Belgium, ${ }^{2}$ Louvain Drug Research Institute, Clinical Pharmacy Research Group, Université catholique de Louvain,
Brussels, Belgium, ${ }^{3}$ Sciensano Epidemiology and Surveillance, Brussels, Belgium, ${ }^{4}$ Institute of Health and Society, Université catholique de Louvain, Brussels, Belgium, ${ }^{5}$ Department Public Health and Primary Care, ACHG, KU Leuven, Leuven, Belgium, ${ }^{6}$ Pharmacy Department, CHU UCL Namur, Universitá catholique de Louvain, Yvoir, Belgium

Introduction: The COME-ON study (Collaborative approach to Optimize Medication use for Older people in Nursing homes) aimed to investigate the impact of a complex intervention on the appropriateness of prescribing for Belgian nursing home residents (NHRs) [1]. Methods: A multicenter, cluster-randomized controlled trial was set up. The complex intervention consisted of blended training, local concertation (discussion on the appropriate use of specific medication classes on the nursing home (NH) level) and repeated interdisciplinary case conferences (ICC) (involving the GP, the pharmacist and the nurse) to perform medication review for each NHR. Control NHs delivered usual care. The primary outcome measure was an improvement in appropriateness of prescribing from baseline to end of study, defined as solving at least one potentially inappropriate medication (PIM) or potentially prescribing omission (PPO) from baseline at end of study, and without a new PIM or PPO at end of study. Detection of PIM and PPO occurred via an automatic algorithm based on explicit tools (STOPP/START v2 and BEERS 2015) [2].

Results: Fifty-four NHs (24 intervention and 30 control NHs) and 1804 NHRs (847 intervention, 957 control) participated. The median percentage of NHRs for whom the intervention was successful in the intervention arm was $20.4 \%$ (IQR: 13.2-27.3) versus $13.0 \%$ (IQR: 6.8-20.4) in the control group. Using a three-level mixed effects model accounting for clustering of the data, a significant effect in favor of the intervention was observed (odds ratio 1.479 [95\% CI $1.062-2.059, \mathrm{p}=0.021])$.

Key conclusions: The complex intervention tested in the COME-ON study was successful in improving appropriateness of prescribing in NHs.

\section{References:}

1. Anrys P, Strauven G, Boland B, Dalleur O, Declercq A, Degryse J-M, et al (2016) Collaborative approach to Optimise MEdication use for Older people in Nursing homes (COME-ON): study protocol of a cluster controlled trial. Implement Sci 11.

2. Anrys P, Boland B, Degryse JM, De Lepeleire J, Petrovic M, Marien S, et al (2016) STOPP/START version 2-development of software applications: easier said than done? Age Ageing 45:590-593

\section{P-747}

\section{Falls in hospital: a special form for monitoring the phenomenon}

Giulia Mazzarolo ${ }^{1}$, Carla Destro ${ }^{2}$, Claudio Terranova ${ }^{2}$, Natalina Frison $^{2}$, Teresa Gelmini ${ }^{2}$, Maria Seriana Ferraresso ${ }^{2}$, Elisa Fattoretto $^{2}$, Stefano Vicari ${ }^{2}$, Patrizia Benini ${ }^{3}$, Michele Tonon ${ }^{2}$, Marianna Noale ${ }^{4}$, Vincenzo Baldo ${ }^{5}$

${ }^{1}$ Dipartimento di Scienze Cardiologiche, Toraciche e Vascolari, Scuola di Specializzazione in Igiene e Medicina Preventiva, Università di Padova, Padova, ${ }^{2}$ Direzione Medica Ospedaliera, Ospedale Immacolata Concezione, Piove di Sacco, AULSS 6 Euganea

Purpose of the study: (1) Analyze the most important risk factors for accidentals falls in hospitalized patients. 2. Verify the approach applied to prevent falls.

Methods: An observational study has been conducted (1.1.2014-30.4.2018) in 2 different hospitals in Veneto Region. Falls prevention and management in those hospitals follow NICE guidelines, with a multidisciplinary and multifactorial approach. Accidental 
falls of hospitalized patients have been reported through a specific tool that collects information about the patient, hospitalization, risk factors for fall, applied prevention strategies, consequences of the fall and treatments. ODDS Ratios $(95 \%)$ coefficients, multivariate analysis and logistic regression were performed.

Results: A total of 607 falls have been reported (2014: 77; 2017: 182; $+136,6 \%$ ); $76,9 \%$ of patients were over 65 years of age; $92 \%$ of falls occurred during ordinary hospitalization; $64,7 \%$ falls occurred in patients considered being at risk for falls. Multivariate analysis highlights that patients with a history of accidental falls and gait instability have a greater risk of fall with injury compared with patients without those risk factors $(\mathrm{OR}=5.61,95 \%$ CI (1.06-29.8); patients with gait instability have a minor risk of fall with injury compared to patients without previous history of fall and gait instability $(\mathrm{OR}=0,46,95 \% \mathrm{CI}(0.25-0.84)$.

Conclusions: The results of the study show an increased sensibility of healthcare workers in reporting falls. The risk for fall with injury is higher in patients with a previous history of fall and gait instability: this confirms the results of other published studies. Those risk factors must be carefully considered by healthcare workers in order to identify the most effective prevention strategy, the lack of which can have health and legal consequences; for these reasons it is extremely important to sensitize and train healthcare workers.

\section{P-748}

\section{Risk factors of iatrogenic disease in elderly}

Lamloum Mounir $^{1}$, Ben Kheder Messa ${ }^{2}$, Ben Salem Thouraya ${ }^{3}$, Ben Ghorbel Imed ${ }^{4}$, Houman Mohamed Habib ${ }^{5}$

${ }^{1}$ La Rabta Hospital, Faculte de Medecine de Tunis, Tunis, Tunisia, ${ }^{2}$ La Rabta Hospital, Tunis, Tunisia, ${ }^{3}$ La Rabta Hospital, Faculte de Medecine Tunis, Tunis, Tunisia, ${ }^{4}$ La Rabta Hospital, Faculte de Medecine de Tunis, Tunis, Tunisia, ${ }^{5}$ La Rabta Hospital, Faculte de Medecine de Tunis

Background: Drug iatrogenic disease has a very high human and economic cost in elderly. It is often serious and may be responsible for more than $10 \%$ of hospitalizations in this population. Our objectives were to describe iatrogenic disease features in elderly and to determine risk factors for iatrogenic disease in these patients.

Methods: We conducted a retrospective, descriptive and analytical study including patients aged of 65 years or older, who developed at least an iatrogenic drug effect. We compared demographic, clinical, paraclinical, therapeutic characteristics and outcome of elderly and non-elderly subjects.

Results: Fifty-eight elderly subjects presented an iatrogenic event. The sex-ratio (M/W) was 1.07. Mean age of our patients was $72.2 \pm 7.3$ years. Polypathology was present in $58.6 \%$ of patients and was statistically more common among older people. Arterial hypertension was the most common medical history in our patients (51.7\%), followed by thromboembolic disease (41.4\%). Arterial hypertension, stroke, rhythm disturbances and moderate renal failure were significantly more frequent in elderly. Cardiovascular drugs were significantly more prescribed in elderly, particularly anti-vitamin $\mathrm{K}(\mathrm{AVK})$. AVK overdose was the most noted iatrogenic drug effect in our series $(29.3 \%)$, followed by hematological impairment found in $17.2 \%$. AVK Overdose, renal failure and rhabdomyolysis were significantly more common in elderly. Incriminated drug was discontinued in 39 patients $(67.2 \%)$ and doses was decreased in ? patients. Improvement was noted in $74.1 \%$. No differences were noted between elderly and younger patients.
Conclusion: Older people had more polypathology and cardiovascular drugs. AVK Overdose, rhabdomyolysis and renal failure were the most common side effects in this group of patients.

\section{P-749}

\section{Prevalence of vitamin D deficiency in elderly patients} with osteoporosis and cardiovascular diseases

Zdoca Rucsandra Maria ${ }^{1}$, Enache Emanuela ${ }^{2}$, Aurelian Sorina Maria $^{3}$, Zamfirescu Andreea ${ }^{2}$, Ruxandra Dascalescu ${ }^{4}$, Capisizu Ana ${ }^{4}$

${ }^{1}$ University of Medicine and Pharmacy "Carol Davila", Bucharest, Romania, ${ }^{2}$ University of Medicine and Pharmacy "Carol Davila", Chronic disease "Sf Luca" Hospital, Bucharest, Romania,

${ }^{3}$ University of Medicine and Pharmacy "Carol Davila", Chronic Disease "Sf Luca" Hospital, Bucharest, Romania, ${ }^{4}$ University of Medicine and Pharmacy "Carol Davila", Chronic Disease "Sf Luca" Hospital, Bucharest, Romania

Introduction: Vitamin D deficiency is defined as a decrease in the level of 25 hydroxy vitamin $\mathrm{D}<20 \mathrm{ng} / \mathrm{ml}$. Meta-analysis studies have shown an association between vitamin D deficiency and the prognosis of patients with osteoporosis and cardiovascular diseases.

Methods: Transversal study on 100 patients, 99 women, mean age $83.71 \pm 7.6$ years with cardiovascular risk factors. Vitamin D was analyzed in all patients and comparative data were obtained. Vitamin D deficiency $<20 \mathrm{ng} / \mathrm{ml}$, insufficient $=20-30 \mathrm{ng} / \mathrm{ml}$ and opti$\mathrm{mal}=30-80 \mathrm{ng} / \mathrm{ml}$ was recorded. The presence of vertebral/ nonvertebral fractures, some biochemical data (glycemia, cholesterol, LDL, triglycerides), bone density by dual x-ray absorptiometry were observed. Descriptive analysis by SPSS statistical tools.

Results: 73 patients had Vitamin D insufficiency $(15.85 \pm 5.34$, $\mathrm{p}<0.001$ ), mean age $77.99 \pm 11.62$ years. $70-79$ years age group has the highest prevalence of osteoporosis $(42 \%)$. The high prevalence of comorbidities in the case of insufficient Vitamin D was: diabetes mellitus $(15.06 \%)$, high blood pressure $(69.86 \%)$ and ischemic heart disease $(30.13 \%)$. Mean cholesterol levels $(\mathrm{p}<0.572)$ and triglycerides $(\mathrm{p}<0.134)$ are elevated regardless of the vitamin $\mathrm{D}$ level and may suspect a correlation between dyslipidemia and osteoporosis. In patients with normal vitamin D were found low densitometry parameters: T-score hip $-2.3 \pm 0.9$ and T-score spine- $3.27 \pm 0.85$. Nonvertebral fracture is more common $(15.08 \%)$ than vertebral $(6.8 \%)$ in patients with insufficient vitamin D.

Key conclusions: Chronic cardiovascular diseases are associated with vitamin D insufficiency in the elderly population. Although vitamin D is within the normal range, $\mathrm{T}$ scores are low, showing the importance of screening for osteoporosis and early initiation of treatment. A follow of this study is to analyze in comparison vitamin D level after osteoporosis treatment.

\section{P-750}

Evaluation of life and sleep quality in elderly with metabolic syndrome

Funda Datli Yakaryılmaz ${ }^{1}$, Zeynel Abidin Öztürk ${ }^{1}$, Gülçimen Soylu ${ }^{1}$

${ }^{1}$ Gaziantep University, Faculty of Medicine, Department of Internal Medicine, Division of Geriatrics, Gaziantep, Turkey

Aim: Prevelance of metabolic syndrome (MS) increases with age. Chronic diseases, which are extensively seen in elderly, profoundly affect the quality of life and sleep. The aim of this study is to evaluate life and sleep quality in elderly patients who have MS. 
Methods: 345 patients who have registered to geriatric outpatient clinic were participated to study. 2001 NCEP ATP III criteria was used for diagnosis of MS. Pittsburgh sleep quality index was performed to assess sleep quality. Short Form of health survey-36 (SF36) was used to evaluate the patients' quality of life. Geriatric depression scale (GDS), mini-mental state examination (MMSE), Katz index of activities of daily living (ADL), Lawton Brody index of the instrumental activities of daily living (IADL), short form of the Mini Nutritional Assessment Tool (MNA-SF), Tinetti Gait and Balance Test were applied to all participants.

Results: 198 patients out of 345 were diagnosed with MS. The mean age of MS and non-MS were 71.5 and 72.6, respectively ( $\mathrm{p}=0.07$ ). The average number of drugs taken by MS patients and non-MS were 4.4 and 3.1 , respectively $(\mathrm{p}<0.001)$. There were no statistically significant differences in ADL, IADL, MMSE, GDS, MNA, falling risk, sleep quality and quality of life in elderly patients with and without MS.

Conclusion: Increasing chronic diseases with advancing age affect the quality of life in all fields. In our study; chronic diseases are common in geriatric population thus there was no significant difference in sleep and life quality among those who are diagnosed with or without MS

\section{P-751}

Morbidity indicators in Tunisian elderly: results of the Tunisian health examination survey

Zoghlami $\mathrm{N}^{1}$, Ben Mansour $\mathrm{N}^{2}$, Guezmir $\mathrm{K}^{3}$, Saidi Olfa ${ }^{4}$, Skhiri Afef ${ }^{5}$, Ben Slemal Fathi ${ }^{1}$, Lamloum $\mathrm{M}^{6}$, Hsairi $\mathrm{M}^{7}$, Skhiri1 H Aounallah ${ }^{8}$

${ }^{1}$ Institut National de la Santé, Tunisie INSP, Tunis, Tunisia, ${ }^{2}$ Institut National de la Santé, Tunisie INSP, Tunis, Tunisia, ${ }^{3}$ Laboratoire de recherche en épidémiologie et prévention des maladies cardiovasculaires, FMT, Tunis, Tunisia, ${ }^{5}$ Faculté de Médecine de Tunis, Université Tunis El Manar, Tunis, Tunisia

Introduction: Tunisia is in advanced stage of demographic transition; as a result, the country is facing great socio-economic challenges, including health concerns. The aim of our study is to update health indicators specific to the Tunisian elderly.

Materials and methods: Data were obtained from the household National Health Examination Survey (THES 2016), target population was aged 15 years and over. Data collection was performed using questionnaires and biological, functional and anthropometric measurements. The data analysis concerned elderly aged 65 and more, via the software R. version 3.3.2.

Results: People aged 65 and over represented $16.9 \%$ of the included population $(\mathrm{n}=1552)$, with a slight male predominance. Illiteracy rate was $68.5 \%$, with a female significant superiority $(84.1 \%)$. Subjects without occupation or having never worked represented $47.1 \%$ of subjects. Slightly more than one-quarter belonged to the lowest socio-economic quintile. Perceived health status was good in $30.7 \%$ of the cases, moderate in $42.7 \%$ and bad in $26.6 \%$ of the cases. Measurements revealed that most common morbidity was arterial Hypertension (72.2\%), followed by abdominal obesity $(51.9 \%)$, dyslipidemia (49.8\%), anemia (45.5\%), impaired vision $(42.6 \%)$, diabetes $(36.9 \%)$ and finally obesity $(30.2 \%)$. More than $15 \%$ of subjects combined hypertension, dyslipidemia and diabetes while $14 \%$ combined anemia and overweight, with a significant difference by gender.

Conclusion: The prevalence of a number of chronic conditions pose a significant and particular threats for elderly Tunisians. It is essential and urgent that health system make effective response to these current challenges, in addition to promoting universal prevention and healthy lifestyle for a healthy aging within future generations.

\section{P-752}

Prevalence of Comorbidities in a Large Representative Sample of Swiss Older Adults

FR Herrmann ${ }^{1}, \mathrm{C} \mathrm{Luthy}^{1}, \mathrm{C}$ Ludwig $^{1}$, AF Allaz ${ }^{1}, \mathrm{C}$ Cedraschi ${ }^{1}$, D Zekry ${ }^{1}$

${ }^{1}$ Geneva University Hospitals and University of Geneva, Geneva, Switzerland

Introduction: In older adults, comorbidities, the presence of concomitant diseases, largely contribute to individual differences in risks of frailty and reduced well-being. This paper quantifies the prevalence of comorbidities in an older community-dwelling population.

Methods: A cross-sectional study survey conducted between 2011 and 2013, in a large sample $(N=3073)$ of the Swiss population aged $\geq 65$. Comorbidities were assessed with the Geriatric Index of Comorbidity (GIC, R Rozzini et al. Age Ageing 2002) each of 15 clinical conditions are graded on a $0-4$ disease severity scale (DSG) $(0=$ absence to $4=$ most severe $)$. The GIC classifies patients into 5 classes of increasing somatic comorbidity (Class $0=$ None; Class 1 : $\geq 1$ conditions $+\mathrm{DSG} \leq 1$. Class $2: \geq 1$ condition $+\mathrm{DSG}=2$. Class 3: 1 condition $+\mathrm{DSG} \leq 3$, Class $4: \geq 2$ conditions + DSG $=3$ or $\geq 1$ condition + DSG $=4$ ). Multivariate ordered logistic regression was used to predict the severity of GIC classification with sex, age group, linguistic region and education level as predictors.

Results: Increasing age (ORage 65-69 $=1.0$; ORage 70-74 $=1.3$, 95\% CI 1.1-1.6; ORage $90=2.1,95 \%$ CI 1.6-2.7) and being a women $(\mathrm{OR}=1.3,95 \%$ CI $1.2-1.5)$ is associated with a higher GIC class. German speaking and participants with more years of education were less likely to belong to a higher GIC class.

Conclusions: Comorbidity is associated with increased adverse outcomes. Its study is essential for a comprehensive appraisal of health in the aged population.

\section{P-753}

Predictive markers of mortality at day-30 in patients over 75 years old with Clostridium difficile infection: the French survey CLOdi

Paccalin Marc ${ }^{1}$, CAUPENNE Arnaud ${ }^{1}$, ROUBAUD Claire ${ }^{2}$, FORESTIER Emmanuel $^{3}$, INGRAND Isabelle ${ }^{4}$, INGRAND Pierre ${ }^{5}$, BRUNET Thomas ${ }^{6}$, GAVAZZI Gaëtan ${ }^{7}$

${ }^{1}$ Pôle de Gériatrie CHU La Milétrie Poitiers, Poitiers, France, ${ }^{2}$ Département de Gériatrie CHU Bordeaux, Bordeaux, France, ${ }^{3}$ Service de Maladies Infectieuses CH Annecy, Metz-Tessy, France, ${ }^{4}$ Pôle BIOSPHARM CHU La Milétie Poitiers, Poitiers, France, ${ }^{5}$ Pôle BIOSPHARM, CHU La Milétrie, 86000 Poitiers, France

Introduction: To assess the in-hospital short term prognosis of Clostridium difficile infection (CDI) in the oldest patients.

Method: Observational prospective multicenter cohort study conducted by the French Infectious Diseases and Geriatrics societies network. Setting and Participants: Patients aged $\geq 75$ years hospitalized in French Geriatric or Infectious wards with confirmed diagnosis of Clostridium difficile infection (CDI) between March 2016 and May 2017. Medical history, and criteria at risk of severity and of recurrence were recorded as listed in the European guidance document. Severe comorbidity was defined with a cumulative illness 
rating scale comorbidity index $(\mathrm{CIRS}-\mathrm{CI}) \geq 3$. Prognosis was assessed at day-30 after CDI diagnosis.

Results: Two hundred and forty-seven patients were included; mean age was $87.2(\mathrm{SD}=5.4) ; 62(25.1 \%)$ patients presented with an organ failure and $182(73.7 \%)$ had a CIRS-CI $\geq 3$. Metronidazol was the main first intention treatment $(51.0 \%)$. At day 30, 36 recurrences $(14.6 \%)$ had occurred and 42 patients $(17.0 \%)$ had died. Multivariate analysis showed that severe comorbidity Hazard Ratio (HR) 2.75, 95\% confidence interval (CI) 1.10-8.47, antibiotic therapy in previous 3 months (HR 2.42, 95\% CI 1.21-4.90), occurrence of organ failure while CDI (HR 2.36, 95\% CI 1.16-4.74), were significantly associated with mortality.

Conclusion: European markers at risk of severity appear adequate in the oldest old. Comorbidities impact the short-term prognosis. Our results point out the lack of adequacy with current guidelines in this vulnerable population.

\section{P-754}

Chronic lung disease in nonagenarians and centenarians admitted to hospital: morbidity profiles of chronic obstructive pulmonary disease (COPD) patients

A. Santos Gutiérrez ${ }^{1}$, C. J. Romero Carrete ${ }^{1}$, J. Mascaró Lamarca ${ }^{1}$, E. Francia Santamaria ${ }^{1}$, M. Barceló Trías ${ }^{1}$, D. Ruiz Hidalgo ${ }^{1}$, J. Casademont I Pou ${ }^{1}$, O. H. Torres Bonafonte ${ }^{1}$

${ }^{1}$ Unidad de Geriatría. Servicio de Medicina Interna. Hospital de Sant Pau. Universitat Autònoma de Barcelona, Barcelona, Spain

Objectives: To evaluate chronic lung disease comorbidity in nonagenarians and centenarians admitted to hospital and to analyse morbidity profiles of COPD patients.

Methods: Prospective nested case-control study. All the centenarian admissions to our hospital from 2006 to 2016 were recorded and, for each one, 3 nonagenarian controls were included. Chronic lung disease history was retrospectively reviewed in detail. Lifetime diagnoses of COPD where classified in morbidity profiles: "survivors" (diagnosed $<80$ years), delayers (80-100 years and scapers ( $>100$ years or diagnosed at admission).

Results: We recruited 602 admissions, $74.75 \%$ female. The APACHE was $10.85 \pm 4.14$, Charlson Index $1.6 \pm 1.35$. Main reasons for admission: respiratory infections in $22.9 \%$, decompensated heart failure $14.5 \%$, urinary infection $5.1 \%$, stroke $3.3 \%$, and hip fracture $2.6 \%$. In 73 patients $(12.1 \%)$ chronic lung disease was recorded: 44 COPD (60.3\%), 13 asthma (17.8\%), 2 tuberculosis sequels (2.7\%), 2 repeated pulmonary aspiration $(2.7 \%), 2$ bronchiectasis $(2.7 \%), 1$ pulmonary fibrosis $(1,4 \%)$ and 1 obesity-hypoventilation syndrome (1.4\%). COPD patients were $94 \pm 3.24$ years and $65 \%$ males. The APACHE was $11.4 \pm 4.2$, Charlson Index $2.4 \pm 1.2$. According to the COPD diagnosis, 28 were delayers $(63.6 \%)$ and 16 survivors (36.4\%). Pulmonary function tests were recorded in 19 COPD patients (43.2\%): VEMS(\%) $60 \pm 12.1, \operatorname{CVF}(\%) 71.9 \pm 13.3$, and VEMS/CVF(\%) $56.1 \pm 10.7$.

Key conclusions: Although almost a quarter of very elderly patients were admitted because of lower respiratory tract infection, only $12 \%$ had chronic lung disease, being COPD the most frequent diagnosis. Patients were mainly delayers for COPD diagnosis and pulmonary function tests were recorded in less than half, showing moderate airflow obstruction.
P-755

Challenges associated with usage of BMI as an adiposity index in a typical African elderly population cohort: the need for better approaches

Kelvin Leshabari ${ }^{1}$, Wazir Ndonde ${ }^{2}$, Luqmaan Mbululo ${ }^{3}$, Godfrey Chale $^{4}$, Shadrack Mponzi ${ }^{5}$

${ }^{1}$ H3 Research Unit of I-Katch Technology Ltd, Dar Es Salaam, Tanzania, ${ }^{2}$ I-Katch Techonology Ltd, Dar Es Salaam, Tanzania, ${ }^{3}$ I-Katch Technology Ltd, Dar Es Salaam, Tanzania, ${ }^{4}$ Obstetrics \& Gynaecology Clinics-Alshifa Medical \& Dialysis Centre, Dar Es Salaam, Tanzania, ${ }^{5}$ H3 Research Unit - I-Katch Technology Ltd, Dar Es Salaam, Tanzania

Background: Sub-Saharan Africa is recording a surge in population with excess adiposity with time. However, population based screening programmes hardly assess utility of variables included in measurements during screening procedures. Objective: To assess the compare the association between different non-invasive adiposity indices among the elderly cohort in the CRISTA-programme.

Methods: A cross-sectional community based screening exercise was conducted at Mikocheni ward of Dar es Salaam, Tanzania during the national diabetes week (Nov 2017). Sitting systolic \& diastolic BP, fasting glycaemia, electrocardiographic screening, weight, height, hip circumference, waist circumference as well as neck circumference formed the non-invasive data. Continuous \& categorical data were summarized using median (IQR) and frequency (proportions) respectively. Main analysis employed generalized linear model after appropriate validation of model assumptions. Data analysis was done using SAS version 9.4. All participants were verbally consented to participate prior to inclusion into the screening process.

Results: We screened 227 self-reported healthy participants during the 2 days screening exercise. Median age was 67 (IQR 60-75.2) years. Out of 227 participants, 124 (54.6\%) had hyperglycaemia (FBG $>7 \mathrm{mmol} / \mathrm{L}), 168(74 \%)$ had systolic BP > $130 \mathrm{mmHg}, 183$ (80.6\%) had diastolic BP $>85 \mathrm{mmHg}, 121$ (53.3\%) were obese $\left(B M I \geq 30 \mathrm{~kg} / \mathrm{m}^{2}\right)$. There was marginal but significant correlation between waist-to-hip ratio and Framingham cardiometabolic risk score $(\eta=0.421, \quad P<0.01)$. Neck circumference $(\eta=0.65$, $P<0.01)$, waist-to-hip ratio $(\eta=0.73, P=0.002)$ was strongly and significantly associated with mean arterial BP.

Conclusion: BMI was found to be a weak predictor of adiposity in this study population.

\section{P-756}

Dysphagia Frequency of elderly residents in an institution with different tools

Sumru Savas ${ }^{1}$, Numan Demir ${ }^{2}$, Asli Kilavuz ${ }^{1}$, Fulden Sarac ${ }^{1}$, Fehmi Akcicek $^{1}$

${ }^{1}$ Section of Geriatrics, Department of Internal Medicine, School of Medicine, Ege University, Izmir, Turkey, ${ }^{2}$ Department of Physical Therapy and Rehabilitation, Faculty of Health Sciences, Hacettepe University, Ankara, Turkey

Aim: The Eating Assessment Tool-10 (EAT-10) is a self-administered questionnaire for dysphagia screening. Whether the EAT-10 is a suitable tool for assessment of swallowing for residents in institutions is debatable. Yale Swallow Protocol (YSP) is an easily administered, reliable, and validated swallow screening protocol. The purpose of this study was to assess dysphagia with EAT-10 in elderly individuals living in an institution and to compare the results with Yale Swallow Protocol (YSP). 
Materials and methods: Elderly ( $\geq 65$ years) residents without eating and cognitive problems were enrolled. EAT-10, YSP, MiniNutritional Assessment-Short Form (MNA-SF), body mass index (BMI), diet type (normal food, oral nutrition supplement, soft food), calf circumference $(\mathrm{CC})$, comorbidities were noted, $\geq 3$ points pointed out the risk of dysphagia with EAT-10. For YSP; the results were recorded as successful and unsuccessful.

Results: Ninety-nine residents were enrolled. The mean age, BMI, $\mathrm{CC}$, and current comorbidity number of patients were $76.2 \pm 7.7$ years, $27.1 \pm 4.9 \mathrm{~kg} / \mathrm{m}^{2}, 34.9 \pm 4.1 \mathrm{~cm}$, and $1.2 \pm 0.9$. The mean scores of MNA-SF and EAT-10 score were $10.9 \pm 2.0$ and $2.47 \pm 3.8$. The risk of dysphagia was $31.3 \%$ with EAT-10 and $18.2 \%$ with YSP $(p=0.023)$. There was an agreement between EAT10 and YSP $(\kappa=0.231, \mathrm{p}<0.014)$.

Conclusion: The risk of dysphagia in the elderly residents of the institution was high and variable with different tools. There was a weak agreement between EAT-10 and YSP. PS: Presented in Academic Geriatrics Congress.

\section{P-757}

Improving Hypoglycemia management by using a standard protocol at a Longterm Facility - A Quality Improvement Project

Al Anoud Ali H Z Al Fehaidi ${ }^{1}$, Asma Mirghani Sayed Abbas ${ }^{1}$, Lamia Rezgui $^{1}$, Hanadi Khamis Mubarak Alhamad ${ }^{1}$, Magda Attia Abdelkawy Aly Elmarakby ${ }^{1}$, Jacintha Lewis ${ }^{1}$, Mahvesh Qureshi Abdulwaris ${ }^{2}$, Wahila Alam ${ }^{1}$, Haroon Ahmed Saleh ${ }^{1}$

${ }^{1}$ Rumailah Hospital, Doha, Qatar, ${ }^{2}$ Hamad General Hospital, Doha, Qatar

Introduction: Long-term Care Facility in Qatar has residents with multiple co-morbidities. Approximately a third of residents have Diabetes Mellitus. Complications such as hypoglycemia can infrequently occur amongst them. Sub-standard management may lead to increased glucose variability which in turn increases the risk of mortality. An audit revealed only $12 \%$ of the staff followed hypoglycemia management as per a standard protocol. Aim was to increase the percentage of staff at the long-term care facility using a standard protocol from $12 \%$ to $100 \%$ over 12 months.

Methods: This is a Quality Improvement project which was carried out between January 2017 and March 2018. A multidisciplinary team was formed who used "Dartmouth Microsystem Quality Improvement Model". Baseline Process map with cause and effect diagram identified likely reasons for not using the standard protocol. Various interventions were carried out including simplifying a previously established protocol (First Intervention), educational training events (Second Intervention) for all the primary front-liners was provided, posters at the nursing stations and presentations at the television units at the nursing stations. Establishing Clinical Practice Guideline (CPG) on Hypoglycemia in Long-term Care Facility (Third Intervention).

Results: A run-chart was used to monitor the response following aforementioned interventions. First Intervention showed significant improvement in the use of protocol to $60 \%$ within 2 months and subsequently increased to $100 \%$. After establishing the CPG it remained at $100 \%$.

Conclusion: Dedicated multidisciplinary team utilizing a standard model of Quality Improvement produced significant change in the care provided during hypoglycemia management in a Long-term Care Facility in Qatar.

\section{P-758}

Disease related characteristics and geriatric syndromes in older adults with diabetes

Marina Kotsani ${ }^{1}$, Thekla Chatziadamidou ${ }^{2}$, Magda Gavana ${ }^{3}$, Eustathios Giannakopoulos ${ }^{4}$, George Soulis ${ }^{5}$, Irini Tzanetakou ${ }^{5}$, Ioannis Georgiopoulos ${ }^{5}$, Athanase Benetos ${ }^{6}$

${ }^{1}$ Aristotle University of Thessaloniki, Greece and Hellenic Society for the Study and Research of Aging, Thessaloniki, Greece, ${ }^{2}$ Health Center of Diabata, Thessaloniki, Greece, ${ }^{3}$ Health Center of Michaniona, Thessaloniki, Greece, ${ }^{4}$ Health Center of Michaniona, Thessaloniki, Greece, ${ }^{5}$ Hellenic Society, Thessaloniki, Greece

Objective: To identify the disease characteristics of diabetes that are related to geriatric syndromes (GS) in older adults.

Methods: A cross sectional study was conducted in 198 individuals with diabetes, using clinical interviews and common GS's screening scales. Syndromes investigated were the presence of falls in the last year by questionnaire, depressive symptoms by the Geriatric Depression Scale, disability by the Global Disability Scale, physical function by the Timed Up and Go (TUG) test and cognitive function by the Mini Mental State Examination.

Results: Several geriatric syndromes like diminished physical function (TUG) $(\mathrm{p}=0.038)$, falls $(\mathrm{p}=0.021)$, disability $(\mathrm{p}=0.001)$ and depressive symptoms $(\mathrm{p}=0.028)$ were related to diabetes macrovascular complications (presence of coronary, and/or carotid and/or lower limb artery disease), even though people with and without complications did not differ in terms of age or sex. Diabetes duration only showed a tendency for more prevalent disability $(p=0.076)$ and worst TUG performance $(p=0.095)$. Type of diabetes treatment, incidence of hypoglycemia and $\mathrm{HbAlc}$ were not correlated to any of the GS investigated. By contrast, disability (both $\mathrm{p}<0.001$ ), TUG (both $\mathrm{p}<0.05$ ), cognitive function (both $\mathrm{p}<0.05$ ) and depression (both $\mathrm{p}<0.001$ ) were related to self-rated health status and quality of life respectively.

Key conclusions: The prevalence of GS in older adults with diabetes is mostly related to macrovascular complications, irrespectively of age or sex, and correlates to health status and quality of life subjective perception.

\section{P-759}

Casuistics of a project in the field of geriatrics in a medical ward: a one year results

Pedro Marques ${ }^{1}$, Rita Martins ${ }^{1}$, Eduardo Doutel Haghighi ${ }^{1}$, José Barata

${ }^{1}$ Hospital de Vila franca de Xira, Vila Franca de Xira, Portugal

Introduction: The Portuguese population is becoming increasingly older and multiple geriatric syndromes are becoming more common in the hospital wards. A project in the field of Geriatrics was created and implemented in an internal medicine department in order to prove the benefits of a differential geriatrics approach.

Objectives: Compare the data and outcomes of the patients included in the project with the remaining patients admitted in the same ward. Methods: Comparing age, gender, admission provenience, mortality, discharge destination, length of stay and mean number of diagnosis per patient during 1 year (April 2017 to April 2018) between the 2 groups.

Results: During that period, there were 92 patients included in the project versus (vs) 5269 not included; characteristics of both groups were similar, with exception of mortality, which was lower in the project group ( $4.35 \%$ vs $13 \%$ in the rest of the population), medium 
age (83.5 years vs 73.65 years) and number of total diagnosis (13.79 vs 11.41). Average length of stay was slightly higher in the project. The patients on the project group were also more frequently sent to a hospital consultation for reevaluation.

Discussion: Geriatric patients are very complex (older and with more comorbidities) and need a particular approach when admitted on a hospital ward in order to reduce morbidity, mortality and length of stay. With a geriatric approach, we were able to reduce mortality in an older population with more comorbidities, with only a slight increase in stay length.

\section{P-760}

\section{A rare complication of Paget's disease}

Amel Farhat ${ }^{1}$, Saoussen Zrour $^{2}$, Sonia Hammami ${ }^{3}$, Mohamed Amine EL Achek ${ }^{4}$, Mahbouba Jguirim ${ }^{5}$, Ismaeil Bejia ${ }^{5}$, Naceur Bergaoui ${ }^{5}$

${ }^{1}$ Department of Rheumatology, Monastir, Tunisia, ${ }^{2}$ Department of rhumatologie, Monastir Tunisia, ${ }^{3}$ Department of Internal Medicine, Monastir, Tunisia, ${ }^{4}$ Department of Rheumatology, Monastir, Tunisia, ${ }^{5}$ Department of Rheumatology, Monastir, Tunisia

Introduction: Sarcomatous degeneration is the major complication of Paget's disease of bone (PDB). Its diagnosis is usually easy but the prognosis is quickly fatal. We report a case of sarcomatous degeneration of a PDB.

Observation: Mr. KA, a 69-year-old high-smoking men (40 PA), with a history of dyslipidemia and ischemic coronary artery disease, had consulted for hip and left thigh inflammatory pain (EVA 8/10) since two months with impaired general condition not improved by symptomatic treatment. The physical examination showed a painful mobilization of the left hip with flexion limitation at $75^{\circ}$. The biology had shown a biological inflammatory syndrome (ESR $=80$ in $\mathrm{H} 1$ ), a normal phospho-calcium balance except increased alkaline phosphatase at $284 \mathrm{IU} / \mathrm{L}$. Pelvic X-ray radiography showed hypertrophy, cortical thickening and heterogeneous lytic lesion of the left iliac wing. A pelvic CT scan showed significant reshaping of the left iliac wing with multiple osteolytic areas and erasure of the cortex. A bone scintigraphy showed heterogeneous hyperfixation of the left hemipelvis with a discrete hyperfixation of the left scapula. A pelvic MRI showed bone lysis of the left iliac bone with bilobed tissue mass at the expense of ipsilateral psoas and gluteal muscles enhancing after Gadolinium injection. A bone biopsy was performed showing a pagetoid appearance with undifferentiated sarcoma of the bone. Thoracic CT was performed as part of an extension assessment showing pulmonary metastasis. The patient had been infused with zoledronic acid (Aclasta $5 \mathrm{mg}$ ) and was referred for chemotherapy.

Conclusion: Although PDB appears nowadays less active and less severe, sarcomatous degeneration may be inaugural. It would be useful to start a multicentre study comparing the different treatments for sarcomatous degeneration in order to increase the median survival of patients.

\section{P-761}

Oto-rhino-laryngological manifestations of rheumatoid arthritis over 65 years old

Mouanaa Hamza ${ }^{1}$, Zrour Saoussen ${ }^{1}$, Hammami Sonia ${ }^{2}$, Kolsi Naourez ${ }^{3}$, Mahoua Jguirim ${ }^{1}$, bejia ismaiel ${ }^{1}$, Touzi Mongi ${ }^{1}$, Koubaa Jamel ${ }^{3}$, Bergaoui Nacer ${ }^{1}$

${ }^{1}$ Rheumatology Departement of Monastir University Hospital, Monastir, Tunisia, ${ }^{2}$ Geratric Unit of Monastir University Hospital,
Monastir, Tunisia, ${ }^{3}$ ENT Department of Monastir University Hospital, Monastir, Tunisia

Background: Rheumatoid arthritis (RA) is a chronic inflammatory disorder that can damage a wide variety of body systems. Ear, nose and throat (ENT) involvement is frequent but not often reported. Objectives of our work are to determine the prevalence of ENT involvement during RA over 65 years old and to evaluate its correlation with RA disease activity.

Methods: This is a cross-sectional study of 21 consecutive RA over 65 years old, followed at the Rheumatology department of Monastir Teaching Hospital in Tunisia, during 06 months (November 2016 to April 2017) and 46 matched volunteers. ENT clinical examination with tonal audiometry and thyroid tests (TSH, T4, anti-Thyroperoxidase $\mathrm{Ab}$ (Anti TPO $\mathrm{Ab}$ ) and Anti-Thyroglobulin $\mathrm{Ab}(\mathrm{Ab}$ anti $\mathrm{Tg}$ ) were performed.

Results: ENT involvement prevalence was $62 \%$. The most frequent functional signs were intermittent dysphonia in 52\% and dysphagia in $47 \%$ of cases. The neck examination revealed painful larynx mobilization in $47 \%$ cases and cervical lymph nodes in $5 \%$ of cases. Indirect laryngoscopy, performed in the $57 \%$ of symptomatic patients, noted inflammatory mucosa in $33 \%$ of cases anddecrease in vocal cord mobility in $19 \%$ of cases. Fifty two percent of patients had temporomandibular Joint (TMJ) involvement. Tonal audiometry revealed $52 \%$ of cases of deafness: $33 \%$ sensorineural deafness, $23 \%$ conductive deafness and $2 \%$ cases mixed hearing loss. The ENT manifestations significantly associated to RA compared to the witness group $(\mathrm{p}<0.01)$ were intermittent dysphonia, dysphagia, painful larynx mobilization, inflammatory nasal mucosa, painful TMJ and deafness. Active disease (DAS $28>3.2$ ) is statistically associated with deafness $(\mathrm{p} \leq 0.048)$ and TMJ involvement $(\mathrm{p} \leq 0.009)$. The ENT manifestations significantly associated to RA over 65 years old against RA under 65 years old were compared to the witness group $(\mathrm{p}<0.01)$ were intermittent dysphonia, painful TMJ and deafness. Logistic regression study shows that RA duration over 10 years was associated to laryngeal dyspnea $(\mathrm{OR}=4.4, \mathrm{p} \leq 0.012$, IC $(95 \%)$ $[1.377,14.134])$ and deafness $(\mathrm{OR}=3.8, \mathrm{p} \leq 0.03$, IC $(95 \%)[1.142$, $12.882])$. In the other hand, age over 65 years old is associated with ENT involvement $(\mathrm{OR}=0.123, \mathrm{p} \leq 0.016$, IC $(95 \%)[0.076,0.772])$ and deafness $(\mathrm{OR}=7.8, \mathrm{p} \leq 0.017, \mathrm{IC}(95 \%)[1.431,43.175])$.

Conclusions: ENT involvement is a very common, usually asymptomatic extraarticular manifestation during RA. It is, mainly, TMJ involvement, deafness and dysphonia. The main relevant determinants are age over 65 years old, RA disease activity and duration.

\section{P-762}

Coronary calcification and its relationship to cardiovascular risk factors in asymptomatic community-dwelling men

Berenice M Werle ${ }^{1}$, Neide M Bruscato ${ }^{1}$, Lilian Vivian ${ }^{1}$, Waldemar De Carli ${ }^{1}$, Protasio Lemos da Luz ${ }^{2}$, Emilio H Moriguchi ${ }^{3}$, Paulo R Schvartzman ${ }^{4}$

${ }^{1}$ Health Care Association of Veranópolis (AVAES), Rio Grande do Sul/Brazil, Brazil, ${ }^{2}$ Heart Institute (INCOR-SP) São Paulo/Brazil, Brazil, ${ }^{3}$ Porto Alegre Clinicas Hospital (HCPA), Federal University of Rio Grande do Sul (UFRGS) Rio Grande do Sul/Brazil, Brazil, ${ }^{4}$ Moinhos de Vento Hospital Rio Grande do Sul/Brazil, Brazil

Objectives: We evaluated coronary artery calcification (CAC) and its association with cardiovascular risk factors in asymptomatic community-dwelling men.

Methods: The sample consisted of 150 asymptomatic men aged 50-70 years, who underwent multidetector computed tomography examinations. We evaluated CAC using the Agatston score, 
classifying results as $\leq 10$ (no evidence and low CAC) and $>10$ (moderate and severe CAC). A multivariate Modified Poisson regression model was used to identify risk factors associated independently with moderate/severe CAC.

Results: We identified the presence of CAC (calcium score $>0$ ) in $59.3 \%$ of participants. Bivariate analysis revealed significant associations between moderate/severe CAC and $<150 \mathrm{~min} /$ week physical activity $(\mathrm{p}=0.041)$, family history $(\mathrm{FH})$ of coronary artery disease (CAD; $\mathrm{p}=0.033)$, hypertension $(\mathrm{p}=0.014)$, and elevated blood glucose level higher $\mathrm{p}=0.023$ ). In the multivariate analysis, moderate/severe CAC remained independently associated with $\mathrm{FH}$ of premature $\mathrm{CAD}(\mathrm{PR}=1.39 ; 95 \%$ confidence interval $[\mathrm{CI}], 1.03-1.88$, $\mathrm{p}=0.029)$ and $<150 \mathrm{~min} /$ week physical activity $(\mathrm{PR}=1.40 ; 95 \%$ CI, 1.01-1.93; $\mathrm{p}=0.045)$.

Conclusion: Cardiovascular risk factors such as $\mathrm{FH}$ of premature CAD and low physical activity were associated independently with moderate/severe CAC. Our results suggest that these risk factors should be considered more fully when evaluating global cardiovascular risk.

Keywords: Coronary artery calcification, Risk factor, Family history, Physical activity.

\section{P-763}

\section{Subclinical hypothyroidism and cardio-cerebro-vascular risk} in older people

G. I. Prada ${ }^{1}$, I. D. Alexa ${ }^{2}$, R. M. Nacu ${ }^{1}$, C. Nuta ${ }^{3}$, A. Kozma ${ }^{4}$, A. G. Prada ${ }^{1}$, R. Stefaniu ${ }^{2}$, I. A. Sandu ${ }^{2}$, O. L. Bajenaru ${ }^{3}$, A. C. $\mathrm{Ilie}^{2}$, A. I. Pislaru ${ }^{2}$, A. M. Herghelegiu ${ }^{1}$

1"Carol Davila" University of Medicine and Pharmacy, Bucharest, Romania, ${ }^{2 " G r . T . ~ P o p a " ~ U n i v e r s i t y ~ o f ~ M e d i c i n e ~ a n d ~ P h a r m a c y, ~ I a s i, ~}$ Romania, "3"Ana Aslan" National Institute of Gerontology and Geriatrics, Bucharest, Romania, "'Fr.I.Rainer" Anthropology, Bucharest, Romania

Introduction: Prevalence of subclinical hypothyroidism increases with age. It increases the risk of myocardial infarction two- to threefold in older women. Often is under-diagnosed since its clinical picture is silent and atypical. Aim of the study was to identify the effects of this disorder on cardiovascular conditions in older people as compared to adults.

Materials and methods: A retrospective study performed on 120 randomly selected patients previously diagnosed with subclinical hypothyroidism, divided into 2 equal groups: adults (50-64 years), elderly ( $\geq 75$ years), with equal number of women and men.

Results: Prevalence of patients from rural area was $19.17 \%$. Obesity was more prevalent in adults (54\%). Hashimoto thyroiditis in past medical history was almost four times more prevalent in women in the general sample, and mostly in adults. Arrhythmias were more prevalent in older men (23\%), but atrial fibrillation was significantly more prevalent in older women $(\mathrm{p}<0.01)$. Atherosclerosis had a high prevalence in both age-groups $(>70 \%)$, but significantly more prevalent in older group $(\mathrm{p}<0.01)$ suggesting the long-term effects of subclinical hypothyroidism. Ischemic heart disease was significantly more prevalent in older men $(\mathrm{p}<0.01)$, nearly twice as adult men. Stroke was twice as prevalent in older age group as compared to adults, and significantly more prevalent in men (0.001). Heart failure was significantly more prevalent in older women as compared to men of the same age group $(\mathrm{p}<0.05)$ and to adult group $(\mathrm{p}<0.001)$.

Conclusions: Subclinical hypothyroidism increases the prevalence of cardio-cerebro-vascular conditions in older people most probably by being an additional risk factor.

\section{P-764}

Predictive factors of postoperative mortality and beyond 6 month of femoral neck fracture (FNF) in patients over 75 years old

Leborgne Fanny ${ }^{1}$, Brunschwig François-Hubert ${ }^{2}$, Dakka Dalila ${ }^{3}$, Ferrer Emilie ${ }^{2}$, Arlaud Cyprien ${ }^{4}, \mathrm{Li}$ Vigni Déborah ${ }^{4}$ Sanchez Marine ${ }^{4}$, Champigny Noémie ${ }^{1}$, Capriz Françoise ${ }^{5}$, Mailland-Putegnat Véronique ${ }^{5}$, Guérin Olivier ${ }^{6}$, Bereder

${ }^{1}$ Mobile Geriatric Team, University Hospital of Nice, Nice, France, ${ }^{2}$ Rehabilitation Unit, University Hospital of Nice, Nice, France, ${ }^{3}$ Mobile Geriatric Team, University Hospital of Nice, Nice, France, ${ }^{4}$ Acute Geriatric Unit, University Hospital of Nice, Nice, France

Introduction: Despite a preventive policy, the incidence of the « FNF» still reaches $8 / 1000$ after 80 years old with a hospital mortality of 2 to $14 \%$ and a mortality at 1 year of 20 to $30 \%$. The opening of the ortho-geriatrics' concept allowed to improve the life and functional prognosis of the elderly patients. The goal of our study was to define the predictive factors of the mortality at 30 days, 6 months and the overall mortality after an operated $« \mathrm{FNF} »$.

Methods: In 2016, the physicians of the «MOBILE GERIATRIC TEAM» (EMG) from the University Hospital of Nice opened an ortho-geriatrics department (daily visit at the trauma services). Accompanied by a social worker, they interviewed and examined the elderly patients over 75 years old who usually living at home and receiving surgical treatment for an $\langle\mathrm{FNF} »$. An unicentric prospective cohort was created as well as a collection of pre-, per-, and postoperative data. A phone calling to the caregivers and/or the general practitioner in charge allowed to complete the collected data and each was call back beyond the 6 months of post-operation in order to collect the judgment criteria.

Results: During one year, 252 patients (median: 88,1 years old, $76,2 \%$ of women) were included. Being a man or having a chronic respiratory disorder were predictive factors for the mortality postoperation after 30 days (respectively OR $2.9(1.05-8.12)$ and OR 3.8 (1.32-10.5)). Regarding the mortality at 6 months, the postoperative confusion syndrome was a predictive factor of the mortality (OR 4.6 $(1.75-12.87))$, as well as the atrial fibrillation or the cancer as comorbidities (respectively OR $3.75(1.25-11.34)$ and OR 1.4 (1.36-10.21)). Regarding the overall mortality, the predictive factors were the postoperative confusion syndrome (OR 3.5 (1.62-8.07)), the age, the cancer or the chronic respiratory disorder as comorbidities (respectively OR $1.1(1.03-1.17)$, OR 3.9 (1.65-9.52) and OR 2.7 (1.01-7.20)).

Conclusion: Despite that the FNF is an independent risk factor for death for elderly patients, this study has highlighted the predictive factors of death which are changeable or unchangeable. Having identified these risk factors, the geriatricians have to pay special attention to these elderly patients with death risk.

\section{P-765}

\section{Osteoporotic bone fracture in the elderly}

Heidi Gruner ${ }^{1}$, Ines Rego Figueiredo Figueiredo ${ }^{1}$, Marilia Fernandes ${ }^{1}$, Rita Alves ${ }^{1}$, Diogo Drummond Borges ${ }^{1}$, Sara Castro $^{1}$, Diogo Batista $^{2}$ Debora Valente ${ }^{2}$, Isabel Silva ${ }^{2}$, Sofia Encarnação ${ }^{2}$, Antonio Panarra ${ }^{1}$

${ }^{1}$ S. Medicina 7.2 Hospital Curry Cabral, Centro Hospitalar Lisboa Central, Lisboa, Portugal, ${ }^{2}$ NOVA Medical School - Universidade Nova de Lisboa, Lisboa, Portugal

Introduction: Osteoporosis and osteoporosis-related fracture are more and more common in the elderly and the association with high costs, morbidity and mortality is well known. Anyhow it continues to 
be undervalued and as such diagnostic exams and antiosteoporosis drugs are not used.

Objective: Compare hospitalized elderly patients with and without previous osteoporosis-related fracture.

Methods: Retrospective study comparing elderly patients admitted during 2017 to an Internal Medicine Ward of a Tertiary Hospital with (wOF) and without (nOF) osteoporotic related fracture. Patients were characterized by age, gender, Katz score, FRAX evaluation, MUST score, modified Charlson score, average number of medication used, and use of antiosteoporotic treatment. Outcomes evaluated were average length of stay and mortality.

Results: The study included $50 \mathrm{wOF}$ patients and $253 \mathrm{nOF}$ patients. Comparing the two groups the patients wOF had an average age of 83.2 vs 80.9 years; female gender $29(58 \%)$ vs $132(52 \%)$ patients; Katz $<3$ of $6(12 \%)$ vs $116(45 \%)$ patients; nutritional risk according to MUST 18 (36\%) vs 69 (27.3\%); average FRAX score of 13.7 vs 7,68 ; average Charlson score 3.72 vs 3.27 ; average number of medications used 7,51 VS 6,77; of which calcium, D vitamin and, bisphosphonates only in 6 vs 13 patients; and concerning realization of DEXA 14 vs 29 patients. Fractures were mostly hip 18, lumbar spine 10 and Colles fracture 6 . Concerning the outcomes average length of stay 14.72 vs 10.1 days and mortality $20 \%$ VS $12.6 \%$.

Discussion: Despite of a high FRAX score, in these elderly hospitalized population, only a small number had done a DEXA exam and an even smaller number was taking antiosteoporosis drugs. In the sample, $1 / 4$ of the patients had a previous osteoporosis-related fracture they had a tendency for older age, female gender, higher nutritional risk, Charlson score and a higher average number of medications. They were less dependent, but had a higher average length of stay and mortality.

\section{P-766}

Effectiveness of an innovative kinesio-taping-based treatment in fourth-stage pressure ulcers. A pilot study

\section{Giorgio Badino $^{1}$, Alberto Cella ${ }^{1}$, Nicola Veronese ${ }^{1}$, Alberto Pilotto ${ }^{1}$}

${ }^{1}$ Dept. Geriatric Care, Orthogeriatrics and Rehabilitation, E.O. Galliera Hospital, Genova, Italy

Introduction: Over $90 \%$ of stage III and IV pressure ulcers take longer than 2 months to heal. As reduced tissue vascularity is one of the mechanisms preventing skin ulcer healing, treatments able to improve local circulation could accelerate their clinical resolution. Given that Kinesio taping (KT), which is used in various painful musculoskeletal conditions, can improve local blood circulation and lymphatic drainage, this study aims to determine whether the application of KT near serious pressure sores can improve their healing. Methods: Ten unselected elderly poly-pathological patients (4 men, 6 women) with non-infected fourth-stage sacral bed sores were treated at home by our home-care service team. KT was applied close to a portion of the ulcer, while the contralateral portion was treated according to the standard protocol ('control', C). The surface reduction of both portions was measured every 4 days after KT application (5 follow-up visits: V1-V5).

Results: On each visit, the mean percentage reduction was significantly greater in the KT-treated areas (KT-A) than in C: V1 $=21.9 \%$ vs $8.3 \%, \mathrm{p}<0.001 ; \mathrm{V} 2=38.3 \%$ vs. $17 \%, \mathrm{p}<0.001 ; \mathrm{V} 3=58.1 \%$ vs $25.8 \%, \mathrm{p}<0.001 ; \mathrm{V} 4=70.3 \%$ vs $36.9 \%, \mathrm{p}<0.001 ; \mathrm{V} 5=81.1 \%$ vs $46.3 \%, \mathrm{p}<0.001$, respectively (paired-sample t-test). Furthermore, starting from V2, the number of ulcers that halved their extension was significantly higher in KT-A, with the maximum difference on V4 (8 ulcers halved in KT-A vs 2 in C, p $=0.007$; Chi squared test).

Key conclusions: KT can be an effective, rapid and low-cost therapy in fourth-stage pressure ulcers in elderly patients.
Area: Comprehensive geriatric assessment

\section{P-767}

Acute care of the elderly: improving quality of care and reducing hospital length of stay through CGA in frail older patients

Shahul Sheriff ${ }^{1}$, Laurenny Guzman ${ }^{1}$, Sanjeev Vasishta ${ }^{1}$

${ }^{1}$ Royal Gwent Hospital, Newport, UK

The gold standard for the management of all older people accessing the urgent care should be routine assessment for frailty syndromes and the presence of one or more should trigger a comprehensive geriatric assessment (CGA). Acute care of the elderly unit (ACE) was set up with the aim to provide to frail older patients the best quality of care involving a daily, holistic and interdisciplinary assessment to coordinate safe, clinical efficient and cost-effective early discharges. All patients admitted under acute care of the elderly were included. The LOS was collected from IT Data, CWS (Clinical Work Station) and the readmissions were collected from GRAPE and CWS during the period between January 2016 and December 2016. A total of 622 elderly patients were admitted to ACE in 2016 of which 437 patients were discharged as ACE. The average length of stay (LOS) in ACE during the period analysed was 3.7 days, compared with the national average of 9 days for patients over 65 years. Patient readmission rate was $7.9 \%$ within 30 days, compared with the national average of $15.5 \%$ (age over 75) indicating that a more rapid decision making does not pose a higher readmission risk if patients are assessed by a multidisciplinary team through a comprehensive evaluation. The pressure for hospital beds in the elderly continues to grow. A rapid population ageing needs changes in our current models of care assessing frail older people admitted with urgent care needs. It is proven that a comprehensive geriatric assessment of older people leads to better outcomes and units focuses on those patients likely to be able to return home within the first $24-72 \mathrm{~h}$ to admission are proving being efficient and effective.

\section{P-768}

\section{Evaluation of caregiver burden of geriatric homecare patients}

Asli Tufan ${ }^{1}$, Pemra Ünalan ${ }^{2}$

${ }^{1}$ Marmara University Pendik Education Research Hospital, Internal Medicine Department, Geriatrics, Istanbul, Turkey, ${ }^{2}$ Marmara University Pendik Education Research Hospital, Family Medicine Department, Istanbul, Turkey

Background: Home care is a providing health care to the patients in process of care in their home environments, after the diagnosis and treatment. Ones who need home care are mostly the patients aged over 65 . This care is generally run by the relatives of the patients. Often one person is in charge of caring the patient and that one may be in difficulty most of the time. This research aim is to determine the difficulty experienced by the one who provides home care for the patients aged over 65 by using "Zarit Burden Interview".

Material-method: In this descriptive research, the ones who provide home care for the patients traced by Marmara University during the period of February-March 2016 were included. After taking approval of participants, Zarit Burden Interview of 22 questions, put into practice by asking the questions face to face. The result from Zarit Burden Interview can be minimum 0, maximum 88. Higher result shows higher experienced difficulty. 24 is considered to be cut-off score. Information including socio-demographic characteristics of caring ones and patients were recorded. Results were analysed by using SPSS Statistics 23.0 program and Mann Whitney $U$ and 
Kruskal-Wallis tests. For the research ethical committee approval was received from Marmara University.

Results: Totally 119 care givers (100 females, 19 males) participated in this study. In the study Zarit Burden Interview score was found out to be $49.5 \pm 14.73$ (12-79). One hundred and eight (\%90) care giver's score was higher than 24 . The associations between the burden of care giver were; the period of caring the patient, care giver's age, gender, education level, relationship to the patient and working condition. Between care giver's burden score and his or her relationship with the patient $(\mathrm{p}=0.038)$, patient's education level $(p=0.008)$, and caring period of the patient $(p=0.008)$ were found statistically significant.

Conclusion: Evaluation of care giver's burden and determining the problems might help to increase living qualities of both the care givers and the patients. This awareness might provide more beneficial caring for the patient.

\section{P-769}

Use of InterRAI CHA instrument in Comprehensive Geriatric Assessment in Lebanon

\author{
Abdulrazak Abyad ${ }^{1}$ \\ ${ }^{1}$ Abyad Medical Center, Tripoli, Lebanon
}

The elderly in the middle-East are growing rapidly. Most government in the region are ignoring the need of the elderly assuming that the family are still enough to handle their problem. Middle Eastern countries have certain cultural, social and economic characteristics in common with similar aspiration. The percentage of elderly in the Middle East is expected to increase with improvement of the health care delivery in the area. The region, like other developing countries, needs to define the policies and programs that will reduce the burden of aging populations on the society and its economy. The number of Alzheimer patient in the region is increasing rapidly. A number of studies were done using InterRai instrument (community Health Assessment)-CHA to determine the prevalence in the community. Initial report from the study in Lebanon will be presented.CHA, Community, Dementia.

\section{P-770}

\section{A community study on the elderly in Dubai/UAE to plan} for future geriatric services

\section{Abdulrazak Abyad \\ ${ }^{1}$ Abyad Medical Center, Tripoli, Lebanon}

The percentage of elderly in the Middle East is expected to increase as the youthful masses work their way up the population pyramid due to the improvements in health care. According to the WHO Department of Aging 2000 report, most Middle Eastern countries will be entering the window of opportunity during this decade, and the window is expected to last until midcentury. It is during this lucrative period that the groundwork for future geriatric services should be firmly established and nurtured. Therefore, this cross sectional study aims to assess the health needs of the elderly in order to deliver solid data for providing future geriatric services that can improve the quality of life of the elderly in the region. Information was gathered from elderly attending many health care and elderly facilities in Dubai, UAE. People who lived in the UAE and were 65 years old or above were included whilst those who were only visiting the UAE were not included. Overall, the total elderly population in Dubai is estimated to be 32,000 people. With a margin of error of $5 \%$ and a confidence level of $90 \%$, the recommended sample size turned out to be 269 people. The sample size included in the study consisted of 300 individuals. The interRAI organization's community health assessment instrument (interRAI CHA) was used as a tool for gathering information. After filling in all the questionnaires, the data was entered into the SPSS Statistics program for analysis. The results obtained delineated which diseases need our support and improved prevention or management. By filling the gaps in elderly health care and by recognizing which aspects of care need our attention, we can ensure a better quality of life. This research is part of Middle East effort to propagate InterRAI instrument in the region. It will help as promoting the research in the field of aging in the region. In addition to comparing the data between countries in the Middle East. CHA, Community, Assessment.

\section{P-771}

The impact of depressive symptoms on social participation in older adults: a Turkish sample

\author{
Nesrin Yagci ${ }^{1}$, Ugur Cavlak ${ }^{1}$, Emre Baskan ${ }^{2}$, Mucahit Oztop ${ }^{3}$ \\ ${ }^{1}$ Prof, ${ }^{2}$ Assist Prof., ${ }^{3} \mathrm{PT}$, Msc
}

Introduction: Social participation in the elderly is positively or negatively affected by personal, environmental and economic factors. Emotional factors play a decisive role in social participation. The aim of this study was to investigate the effect of depressive symptoms on social participation in Turkish older adults.

Methods: Five hundred thirty seven older adults (279 females; 258 males; mean age: $72.33 \pm 6.56$ years) included in the study. Depressive symptoms were evaluated using by Short Form of the Geriatric Depression Scale (GDS). The participants were divided into four groups according to the GDS scores: Group $1(n=263)$; no symptoms, 0-4 points from GDS, Group $2(n=165)$; mild depressive, 5-8 points from GDS, Group $3(\mathrm{n}=58)$; moderate depressive, 9-11 points from GDS, Group $4(\mathrm{n}=51)$; severe depressive, $12-15$ points from GDS. Social participation was evaluated with a total of five open-ended questions.

Results: $54.1 \%$ of the participants reported that they participated in leisure activities. $53.4 \%$ of these participants reported that they performed housekeeping and gardening. $60 \%$ of older adults participated in social activities. $48.4 \%$ of the elderly participating in outdoor social activities stated that they participated with their children or relatives. As the level of depressive symptoms increased in the older adults, their leisure activities $(\mathrm{p}=0.0001)$, housekeeping and gardening ( $p=0.0001$ ) has been found to decrease their indoor activity participation. Also, increased depressive symptoms affected outdoor social participation of the older adults negatively $(p=0.0001)$.

Key conclusions: The findings indicate that indoor and outdoor social participation of the elderly negatively affected by increased depressive symptoms.

\section{P-772}

The relationship between chronic pain, depression and disability among older adults

Małgorzata Stompór ${ }^{1}$, Tomasz Grodzicki ${ }^{2}$, Tomasz Stompór ${ }^{3}$, Jerzy Wordliczek ${ }^{4}$, Marzena Dubiel ${ }^{5}$

${ }^{1}$ Department of Cardiology and Cardiac Surgery, Medical Faculty, Collegium Medicum, University of Warmia and Masuria, Warszawska Str. 30, 10-082 Olsztyn, Poland, Department of Internal 
Medicine and Gerontology, Collegium Medicum, Jagiellonian Universit

Introduction: The prevalence of chronic pain as well as depression among older adults is high and may adversely impact their everyday functioning, although it is often unrecognized. We tried to assess the relationship between chronic pain presence, depression and functioning level.

Methods: 145 subjects older than 60 years (nursing home residents, or patients of outpatient geriatric clinic) were included. Information of pain presence, functional and mental status was obtained using a questionnaire. Chronic pain was defined as lasting $>3$ months, severity of pain was assessed using Numeric Rating Scale (NRS), functional ability using Activities of Daily Living tool (ADL) and mood/possible depression using Geriatric Depression Scale (GDS).

Results: The mean age of patients equaled $76 \pm 9.68$ years, $78 \%$ reported chronic pain. Patients with chronic pain more often presented low mood, lower satisfaction with life, with no differences according to ADL. Unlike in case of pain, suspected depression was connected with poor functional status, with the lowest mood among bed-bounded people. Answer for question: 'what is your biggest health problem?' surprisingly was not pain, even among people with high intensity of pain ( 49 patients with $>4$ points in NRS), but inability to move/walk.

Conclusions: Chronic pain in the elderly population is common, but even for patients with severe pain the biggest health problem and cause of depression is not pain, but lack of independence. That shows the continuum pain - depression - disability requires more holistic approach to improve quality of life of elderly patients on a daily basis.

\section{P-773}

\section{Relationship between mean platelet volume and depression}

Seval Ay Ay ${ }^{1}$, Bahattin Gokdemir ${ }^{1}$, Ozge Kayhan Kocak ${ }^{1}$, Sercan Sahutoglu ${ }^{1}$, Gul Ispirli ${ }^{1}$, Sevnaz $\operatorname{Sahin}^{1}$, Z. Fulden Sarac ${ }^{1}$, Fehmi Akcicek ${ }^{1}$

\section{${ }^{1}$ European Geriatric Medicine Society (EuGMS), Genoa, Italy}

Introduction: Depression with high rates of morbidity and a risk for mortality is a common mood disorder in the elderly; associated with a chronic low-grade inflammation, but the underlying mechanism remains unclear. Mean platelet volume (MPV) is widely used as a measure of platelet size and it is known to be a marker of platelet activity. Clinical trials have proven that MPV could be used as a biomarkers of inflammation in various diseases. The aim of this study is to show a correlation between depression status and MPV in elderly population.

Methods: 266 patients aged 65 years or above, followed in outpatient geriatric clinic in Ege University Hospital from November 2015 to December 2017 are included in this observational, cross-sectional, retrospective study. The study included patients aged 65 years and nondiabetic, non-malignant, normal cardiac and renal function. Patients were divided into 4 groups according to short form geriatric depression scale; normal: 0-4, mild depression: 5-8, moderate depression: 9-11; severe depression: 12-15. The data was transferred to SPSS 17 (Statistical Package for Social Science) program. Values are given as mean \pm standard deviation. The Mann Whitney U test was used to compare values between groups, and the Kruskal-Wallis $\mathrm{H}$ test was used to analyze multiple group values.

Results: The study involved 266 patients (178 female, 88 male); mean age, platelet(PLT) and MPV of all patients were 78.2 \pm 7.5 , $253,000 \pm 63,000$ and $10.6 \pm 0.9$, respectively. There was no significant difference between the mean age of patients, PLT number and MPV values according to sex (F: age 78.2 \pm 7.7 , Plt
$260,000 \pm 62,000$, MPV $10.6 \pm 0.96$, M: age 78.0 \pm 7.0, PLT $258,000 \pm 65,000, \quad$ MPV $10.6 \pm 1.0)$. According to geriatric depression score, $62 \%$ (n: 166) of the patients were normal, $22 \%$ (n: 59) mild, $8.6 \%$ (n: 23 ), middle and $7.5 \%$ Statistically significant differences were found between the MPV and PLT numbers among the patients with moderate to severe depression $(10.2 \pm 1.1 /$ $11 \pm 1 \mathrm{P}$ value: $0.041,292,000 \pm 66,000236,000 \pm 53,000, \mathrm{P}$ value: 0.024$)$.

Conclusions: In this study we found a significant increase MPV in patients with severe depression in compared to patients with moderate depression. There was a significant decrease in PLT count in moderate and severe depression group. Further investigations from different centers are needed whether the deterioration in the MPV induces depression or if it changes due to neurohumoral changes in the depression.

Keywords: mean platelet volume; depression; geriatrics

\section{P-774}

Cognitive status, depressive symptoms and health related quality of life in older adults: gender differences in Turkish sample

Nesrin Yagci ${ }^{1}$, Emine Aslan Telcil ${ }^{1}$, Guzin Kara ${ }^{1}$, Umut Eraslan ${ }^{1}$

${ }^{1}$ Pamukkale University, Denizli, Turkey

Introduction: Older adults seek not only a longer duration of life but also a better quality of life. Physical or mental health-related problems have been associated with quality of life worldwide. The aim of this study was to compare cognitive status, depressive symptoms and quality of life in older adults according to gender.

Methods: This study was included 862 older adults (65-97 years; 430 female; 432 male; mean age: $71.9 \pm 6.4$ years). Cognitive status (Mini Mental Test-MMT), depressive symptoms (Geriatric Depression Scale-GDS) and health related quality of life (CDC Health Related Quality of Life-4; CDC HRQOL) were assessed in all older adults.

Results: The mean MMT scores were $12.1 \pm 7.5$ and GDS scores were 23.5 \pm 5.4 . As a result of CDC HRQOL-4 scores, the mean physically (PUD) and mental unhealthy days (MUD) were $10.35 \pm 10.03$ and $6.84 \pm 9.04$ days, respectively. Besides the mean of activity limited days (ALD) were 6.84 \pm 9.04 days. While GDS, PUD, MUD and ALD scores were significantly greater in female gender geriatrics $(\mathrm{p}=0.0001)$, the MMT scores was greater in male gender $(\mathrm{p}=0.0001)$.

Key conclusion: The results of the study indicated that cognitive, psychological status and health related quality of life is more affected in female geriatrics, negatively.

\section{P-775}

IGRIMUP: development of the Turkish inappropriate medication use (TIME) criteria: a methodological report

\section{Gülistan Bahat ${ }^{1}$, Tugba Erdoğan ${ }^{1}$, Merve Oren ${ }^{2}$, M. Akif Karan ${ }^{1}$ \\ ${ }^{1}$ 2Istanbul Medical School, Department of Internal Medicine, Division of Geriatrics, Istanbul University, Capa, 34390, Istanbul, Turkey, ${ }^{2}$ Erzurum Provincial Health Directorate, Public Health Services Presidency, 25100, Erzurum, Turkey}

Objective: We planned to develop Turkish Inappropriate Medication Use (TIME)-Criteria set taking account the local practices and emerging evidences. In this study, we aimed to report the methodology of the TIME-Criteria. 
Methods: The first draft of the TIME-criteria will be based on combination of STOPP/START version 2 and CRIME-tools.

We will ask the panelist consisting of more than 40 experts from geriatrics and various specialties to review the Draft 1 in light of current guidelines, expert opinions, and their practice in terms of importance and accuracy. Then we will ask them to send back with additional recommendations to improve the structure and content of the existing criteria in Phase 1. Draft 2 will be set according to the panelists comments in terms of additional criteria, revision of current criteria and removal of the criteria in Phase 2. The evidence will be sought for the proposed criteria and comments in Draft 2 by the authors and at least 1 relevant panelist for every criterion face-to-face in Phase 3. Then Draft 3 will be set in terms of revisions and removals in Phase 4. Phase 5 and following phases will consist of Delphi rounds. Draft 3 TIME-criteria will be sent panelists by e-mail for assessing clinical relevance. We will ask the panelists to rate how appropriate they found inclusion of each criterion for the final TIMEcriteria by rounds of Delphi. A Likert scale (between 0 and 5)will be used in terms of "Criteria should be included in the list of TIME1 "for scoring. Median value and 75th percentile value for each criterion will be calculated. Items with both median value of 4-5 and 75th percentile value $\geq 4$ will be included in the in the final "TIMEcriteria-Set".

Conclusion: TIME-criteria will be the first national PIP list in Turkey. We presented the planned methodology of the TIME-criteria on development process. This report may a methodological reference for developing new tools.

\section{P-776}

Relationship between IL-1beta and cardiovascular disease in elderly patients

\section{G. I. Constantin ${ }^{1}$, S. Opris ${ }^{1}$ \\ ${ }^{1}$ National Institute of Gerontology and Geriatrics "Ana Aslan”,} Bucharest, Romania

Introduction: Cardiovascular disease (CVD) is a major cause of mortality at elderly age, and are mainly caused by atherosclerosis, a complicated process governed by several risk factors. The basis of atherosclerosis is the chronic vascular inflammation. Proinflammatory cytokines secreted by adipose tissue and other tissues play an important role in this process. Interleukin-1beta (IL-1 $\beta$ ), a major proinflammatory cytokine, play an integral role in increased migration of inflammatory cells and through proinflammatory, procoagulant nature in developing atherosclerotic plaques.

Methods: The aim of this study was to determine changes in serum levels of IL-1 $\beta$ in elderly patients (aged $69 \pm 7$ years) with cardiovascular disease compared to a control group. Quantitative determination of IL- $1 \beta$ was performed by an ELISA method.

Results: Our results showed an increase of IL- $1 \beta$ levels at group of patients with CVD compared with a control group (9.412 \pm 16.86 vs $4.672 \pm 10.89 \mathrm{pg} / \mathrm{ml}$ serum).

Key conclusion: In conclusion, serum IL-1 $\beta$ levels are elevated in individuals with CVD, indicating that IL- $1 \beta$, as a proinflammatory cytokine, plays an important role in the progression of atherosclerotic plaques and in the pathophysiology of cardiovascular disease.

\section{P-777}

Implementation of geriatric care models in Europe (imAGE.eu): a cross-sectional survey in 8 countries

M Deschodt ${ }^{1}$, B Boland ${ }^{2}$, CM Lund $^{3}$, K. Saks ${ }^{4}$, VS Velonika ${ }^{5}$, O Samuelsson ${ }^{6}$, S Kennelly ${ }^{7}$, MA Vassallo ${ }^{8}$, G Veninsek ${ }^{9}$, J Flamaing ${ }^{10}$

${ }^{1}$ University of Leuven, Belgium; University of Basel, Switzerland, ${ }^{2}$ Belgian Society for Gerontology and Geriatrics; UCLouvain, Belgium, ${ }^{3}$ Danish Geriatric Society; Copenhagen University Hospital, Denmark, ${ }^{4}$ Estonian Association of Gerontology and Geriatrics; University of Tartu, Estonia, ${ }^{5}$ Hellenic Association of Gerontology and Geriatrics, Greece, ${ }^{6}$ The Icelandic Geriatrics Society, ${ }^{7}$ Irish Society of Physicians in Geriatric Medicine; Tallaght University Hospital, Ireland, ${ }^{8}$ Geriatric Medicine Society of Malta; Rehabilitation Hospital Karin Grech, Malta, ${ }^{9}$ Slovene Geriatric Medicine Society, ${ }^{10}$ Belgian Society for Gerontology and Geriatrics; University Hospitals Leuven, Belgium

Introduction: Despite the beneficial impact of comprehensive geriatric assessment (CGA) for older patients in the hospital, it is not clear to what extent these models have been implemented in European hospitals.

Methods: A cross-sectional survey study was conducted in autumn 2017 to map the implementation status of CGA-based care models in general hospitals in Belgium $(n=69)$, Denmark $(n=22)$, Estonia $(n=13)$, Greece $(n=49)$, Iceland $(n=4)$, Ireland $(n=11)$, Malta $(\mathrm{n}=3)$ and Slovenia $(\mathrm{n}=9)$.

Results: An overall response rate of $50 \%(\mathrm{n}=180)$ was achieved. Geriatricians are employed in the majority of Irish (100\%), Belgian (98.6\%) and Danish (86.4\%) hospitals. Acute geriatric units are implemented in all of the Belgian and the majority (72.7\%) of Danish hospitals, but are scarce in Malta, Estonia, Slovenia and Greece. Geriatric rehabilitation units are most common in Ireland $(63.6 \%)$ and Iceland $(75 \%)$. Multidisciplinary geriatric consultation teams are widely implemented in Belgium (100\%), Ireland (72.7\%) and Denmark $(59.1 \%)$, but are rare in Malta, Iceland, Estonia and Greece. Geriatric co-management teams are most often implemented in Malta (100\%), Denmark (63.6\%) and Belgium (40.6\%). Transitional care programs to ensure continuity of care after discharge are present in $59.8 \%$ of all hospitals. The intention to implement any of the care models in the next 5 years ranges from $10.3 \%$ to $25.2 \%$ and is the highest for co-management $(36.2 \%)$, systematic screening of ED patients $(35.9 \%)$ and transitional care programs $(29.2 \%)$.

Conclusion: CGA-based care models are widely implemented in Belgium, Denmark and Ireland, while the uptake has been lower in the other surveyed countries.

\section{P-778}

\section{PAINT: preventive art intervention therapy in geriatric patients}

Katrin Singler ${ }^{1}$, Johanna Masuch ${ }^{2}$, Franziska Baierlein ${ }^{2}$, Claudia Helbig $^{2}$, Sonja Krammel ${ }^{2}$, Markus Gosch ${ }^{2}$

${ }^{1}$ Institute for Biomedicine of Ageing, Friedrich-Alexander University Erlangen-Nuremberg, Department for Geriatric Medicine, Klinikum Nürnberg, Paracelsus Medical University, Salzburg, Austria,

${ }^{2}$ Department Of Geriatric Medicine, Klinikum Nürnberg, Paracelsus Medical University, Salzburg, Austria

Background: Art therapy is implemented in several geriatric settings. Although it is part of interprofessional therapeutic concepts, so far there exists no scientific evidence. The aim of the study is to evaluate 
the influence of art therapy on physical and cognitive function as well as the well-being in patients of an acute geriatric day clinic.

Methods: PAINT is a monocentric, randomised long-term study (09/ 2017-08/2019). Included are patients $\geq 70$ years with either mild dementia, depression or chronic pain syndrome as principal diagnosis or comorbidity. In the intervention group patients take part in a standardized art therapy for $60 \mathrm{~min}$ twice a week. All patients receive a comprehensive geriatric assessment including scores for QoL and well-being. Three months after discharge all patients receive a followup by telephone in order to evaluate the sustainability of the project. Results: So far 84 patients are included in the study. The mean age was 82.5 years. The mean number of art intervention sessions during the hospital stay was 5 times. Patients feedback on the intervention is very positive. Although exact preliminary results will be evaluated in summer 2018 there is evidence towards a positive influence of art therapy on emotion and well-being of the patients (5-WHO Well being Index), but no significant changes in function and cognition compared with patients in the control group of the study.

Key conclusion: Art therapy is very well accepted by geriatric patients. Preliminary results suggest a measurable positive effect on emotion and well-being in patients of a geriatric day clinic.

\section{P-779}

Segmental mesenteric ischemia: multidisciplinary action in the face of acute abdomen in an elderly patient

Yanira Aranda Rubio ${ }^{1}$, Maria Caridad Arenas ${ }^{1}$, Ivon Ribera ${ }^{1}$, Alberto Socorro ${ }^{1}$, Mercedes Martín ${ }^{1}$

\section{${ }^{1}$ Hospital Cruz Roja, Seville, Spain}

Introduction: Abdominal pain is a frequent reason for consultation in a surgical emergency service. A multidisciplinary action with a geriatric doctor will facilitate the adequacy of the therapeutic effort. Methods and results: We present the clinical case of 87 -year-old patient with a personal history of hypertension, insulin-dependent type II diabetes mellitus, stage IIIa chronic kidney disease, permanent atrial fibrillation anticoagulated with Apixaban. A comprehensive geriatric assessment is performed to know their baseline situation in which a good cognitive state stands out, maintaining orientation in the three spheres (space, time and person) without amnestic failures in recent memory. Performs walking with a cane, being independent for the basic activities of daily life. She reported abdominal pain located in the left iliac fossa of three days of evolution and without referring other accompanying symptoms, is afebrile, hemodynamically stable and in the abdominal exploration she underlines pain with deep palpation in the left lower quadrant with signs of peritoneal irritation. The patient evolves with increased pain and analytical worsening, metabolic acidosis, leukocytosis and increase of lactic acid dehydrogenase (LDH).The CT scan suggestive of mesenteric ischemia. After the evaluation of the multidisciplinary team composed of geriatrics, surgery and anesthesia, and once commenting with the family, surgical treatment was decided with previous infusion of fresh frozen plasma. Under general anesthesia, diagnostic laparoscopy is peritonitis without massive mesenteric ischemia. The patient evolved favorably.

Conclusions: Comprehensive geriatric assessment is the best tool to personalize the appropriate treatment for the elderly patient.

\section{P-780}

A comparative study of models of geriatric assessment and the implementation of recommendations by primary care physicians

Yan Press ${ }^{1}$, Tamar Freud ${ }^{2}$, Roni Peleg ${ }^{2}$, Ella Kagan ${ }^{1}$, Boris Punchik ${ }^{1}$, Alex Barzak ${ }^{1}$

${ }^{1}$ Unit for Community Geriatrics, Ben-Gurion University of the Negev, Beer-Sheva, Israel, ${ }^{2}$ Sial Research Center for Family

Medicine and Primary Care, Ben-Gurion University of the Negev, Beer-Sheva, Israel

Introduction: The effectiveness of outpatient geriatric assessment has been the subject of many studies that had inconsistent outcomes. In addition to the classic model of the comprehensive geriatric assessment unite (CGAU) there are other models in which the geriatrician comes to the patient's clinic without the multidisciplinary team. These models require fewer resources, but their effectiveness is not clear. In the present study we compared the rate of implementation of geriatric recommendations by different assessment models.

Methods: We compared Model of Outpatient CGAU (OGAU) with no direct contact between the geriatrician and the primary care physician (PCP) and three models of in-clinic geriatric assessment: "Clinic A-2007" in which the PCP participated in the assessment, "Clinic A-2013" where there was no contact with the PCP, and "Clinics B-2013" where the PCP participated in a staff meeting with the geriatrician in the clinic.

Results: Model "OCGAU" included 240 patients, "Clinic A-2007"-107, "Clinic A-2013"-127, and "Clinics B-2013"-133. The patients in Model "OCGAU" were older (mean age $83.2 \pm 6.2$ years) than in "Clinic" models (mean age was $79.7 \pm 6.5,81.5 \pm 6.1$, and $80.7 \pm 6.5, \mathrm{P}<0.001)$. More recommendations were given per patient (6.4) in the Model OCGAU than in the "Clinic" models (range 1.9-3.9, P $<0.05$ ), but the implementation of recommendations by PCP was lower in Model OCGAU $(48.9 \%)$ than in "Clinic" models (range 56.9-71.8\%, P < 0.005).

Conclusions: Although more recommendations were made in the "OCGAU" unit, the implementation rate was lower. This indicates the need for organizational changes, in particular improving communication between the geriatric staff and PCP.

\section{P-781}

Which factors affect the implementation of geriatric recommendations by primary care physicians?

Yan Press ${ }^{1}$, Boris Punchik ${ }^{1}$, Ella Kagan ${ }^{1}$, Alex Barzak ${ }^{2}$, Tamar Freud ${ }^{3}$

${ }^{1}$ Unit for Community Geriatrics, Ben-Gurion University of the Negev, Beer-Sheva, Israel, ${ }^{2}$ Unit for Community Geriatrics, BenGurion University of the Negev, Beer-Sheva, Israel, ${ }^{3}$ Sial Research Center for Family Medicine and Primary Care, Ben-Gurion University of the Negev, Beer-Sheva, Israel

Introduction: The overall implementation rate for outpatient comprehensive geriatric assessment unit (OCGAU) recommendations ranges from 48.6 to $71 \%$. The purpose of the study was to identify factors that reduce the implementation rate of geriatric recommendations.

Methods: The medical records of patients who were assessed in the OCGAU over an 8-year study period were surveyed. The data included patient's characteristics (socio-demographic, functional, cognitive, and affective condition, co-morbidity), number of recommendations, the identity of the geriatrician, and data related to the 
primary physician (age, sex, seniority, number of patients referred for geriatric assessment).

Results: Three thousand four hundred thirty-four recommendations were made for 488 patients (mean age $83.6 \pm 0.6$ years) of which $1634(47.6 \%)$ were implemented by their primary physician. In univariate analyses patients with an implementation rate $<25 \%$, compared to patients with implementation rate $\geq 75 \%$, had a higher Charlson Comorbidity Index Total Score (CCITS) $(2.5 \pm 1.9$ vs. $1.8 \pm 1.7, \quad \mathrm{P}<0.05)$, a lower Barthel Index $(82.8 \pm 16.2$ vs. $87.0 \pm 15.3, \mathrm{P}<0.05)$, and a lower Instrumental Activity of Daily Living score $(7.2 \pm 3.5$ vs. $8.2 \pm 3.7, \mathrm{P}<0.05)$. In the multivariate analysis only higher CCITS was associated with a lower rate of recommendation implementation by primary physicians.

Conclusions: There is a need to increase the implementation rate by primary physicians by increasing and strengthening the link with them and by further training in the field of geriatrics medicine.

\section{P-782}

A novel oxidative stress marker in contrast induced nephropathy; can dynamic thiol/disulphide homeostasis be predictive marker for elderly patients?

Tuğba Turgut ${ }^{1}$, Remzi Bahşi ${ }^{1}$, Hande Selvi Öztorun ${ }^{1}$, Deniz Mut Sürmeli ${ }^{1}$, Emine Feyza Yurt ${ }^{2}$, Özcan Erel $^{2}$, Murat Varl1 ${ }^{1}$, Sevgi Aras ${ }^{1}$

${ }^{1}$ Ankara University, Ankara, Turkey, ${ }^{2}$ Yildirim Beyazit University, Ankara, Turkey

Aim: The aim of this study was to investigate a novel oxidative stress marker (thiol/disulphide homeostasis) in patients with contrast induced nephropathy in elderly patients for the first time in literature. Methods: A total of 39 patients who administered contrast media for any reason were included in the study; 9 developed contrast induced nephropathy after intervention and other 30 not developed; matched for age, gender and baseline- $48 \mathrm{~h}$ creatinine and native thiol, total thiol, disulphide values were recorded. Additionally, antioxidant parameters were compared with other routinely performed clinical parameters within comprehensive geriatric assessment.

Results: In contrast induced nephropathy group native and total thiol levels decreased and disulphide level increased at $48 \mathrm{~h}$ but it was not statistically significant. Patients with contrast induced nephropathy had significantly higher level of neutrophil-to-lymphocyte ratio $(\mathrm{p}<0.005)$ than non-contrast induced nephropathy patients. Serum albumin, folic asit, Katz Activities of Daily Living score and Mini Nutritional Assessment score levels correlated with serum native thiol values in all patients and a significant inverse correlation was found between native thiol and C-reactive protein and ferritin.

Conclusion: This study demonstrated that antioxidant reserve is reducing with acute inflammation and inducing with nutrition and functionality in elderly patients. In the light of this study correlation between antioxidant status and fraility can be examined in the elderly in further studies.

\section{P-783}

Bilateral vestibulopathy; an underdiagnosed condition in elderly with gait unsteadiness and dizziness: cases from a Danish geriatric fall clinic

\author{
Mette Lindhardt ${ }^{1}$, Hanne Elkjær Andersen ${ }^{1}$ \\ ${ }^{1}$ Geriatric Section, Medical Department, Glostrup, University \\ Hospital Hvidovre, Hvidovre, Denmark
}

Introduction: Bilateral vestibulopathy may be an underdiagnosed condition in elderly with falls, gait unsteadiness and dizziness. Previously it has been difficult to examine the vestibular system and there has been no tradition of including this examination in fall assessment in the elderly. The video head impulse test (vHIT) is a new, easy and cheap way to examine the vestibular system and thereby possibly diagnose vestibulopathy.

Cases: An 84-year-old woman, previously no medical history overall. Complained about slowly progressing unsteadiness of gait and dizziness. Worsening of symptoms in the dark or on uneven ground. No symptoms at rest. No episodes of falls or syncope. Multifactorial fall assessment identified risk factors in form of vision impairment and impairment of balance due to peripheral neuropathy. Supplementary vHIT showed bilateral vestibulopathy with pathological gain with subsequent saccades on both right and left side (Gain: 0.37 and 0.56 respectively). An 89-year-old woman, complained about unsteadiness and oscillopsia, emerging after abdominal surgery where she had received aminoglycosides. Multifactorial fall assessment identified risk factors in form of impairment of balance and muscle strength. Caloric test showed bilateral canal paresis with no nystagmus at all on both sides. Supplementary vHIT confirmed markedly bilateral vestibulopathy with pathological gain (Gain: 0.05 and 0.09 ). Conclusion: Gait unsteadiness and dizziness are often multifactorial in origin. Bilateral vestibulopathy may be an underestimated condition in elderly with gait unsteadiness and dizziness. vHIT may be suggested as a part of falls screening guidelines in elderly. Nevertheless further research on the subject is recommended.

\section{P-784}

Role of neurogeriatric assessment in a candidate for percutaneous aortic valve replacement: case report

Chiara Lorenzi ${ }^{1}$, Giulio Maria Mannarino ${ }^{1}$, Valentina Berti ${ }^{2}$, Maria Chiara Cavallini ${ }^{1}$, Caterina Baroncini ${ }^{1}$, Mauro Di Bari ${ }^{1}$, Enrico Mossello ${ }^{1}$, Andrea Ungar ${ }^{1}$

${ }^{1}$ Research Unit of Medicine of Ageing, Department of Experimental and Clinical Medicine, University of Florence and Azienda Ospedaliero Universitaria Careggi, Florence, Italy, ${ }^{2}$ Department of Experimental and Clinical Biomedical Science, University of Florence, Florence, Italy

Introduction: Greater availability of less invasive methods, such as percutaneous valve replacement techniques, offers the opportunity of treating a growing number of elderly people affected by valvulopathy. A multi-dimensional evaluation including neuro-geriatric skills can be valuable to select candidates undergoing these procedures.

Methods: A 70-year-old woman with a non-clear functional impairment was observed at the Cognitive Disorders Center of Geriatrics (Florence) for preliminary evaluation of percutaneous aortic valve replacement (TAVI) for severe aortic stenosis.

Results: Presence of mild cognitive impairment, bradykinesia and postural instability determining a rapidly progressive functional impairment were reported since 2 years: preserved BADL (Basic Activities of Daily Living) 2/6. Multiple deficits emerged in a global cognitive assessment, especially in executive functions. Neurological examination showed ideomotor apraxia of left upper limb and left emineglect. A magnetic resonance showed right parieto-occipital hyperintensity. On suspicion of neurodegenerative disease as corticobasal degeneration, a Positron Emission Tomography was performed showing right hemisphere cortical hypometabolism and metabolic reduction in the right caudate and thalamus. Considering the modest cardiological symptomatology limited to the effort, reduced functional capacity and prognosis of the neurodegenerative 
disease, indication to TAVI was excluded. After 6 months the patient remained stable on cardiovascular level, while a further deterioration of the cognitive-motor pattern with progression of functional dependence (BADL 0/6) emerged.

Key conclusions: Identifying the pathogenesis of the disorders in the present case allowed to formulate a prognosis and to modify the interventional approach to heart disease. Neurogeriatric evaluation can be useful for the Heart Team to schedule intervention strategies.

\section{P-785}

\section{Achieving Best Practice in Parkinson's Disease Clinic}

Rebecca Cole ${ }^{1}$, Michael Leeming ${ }^{2}$, Sanja Thompson ${ }^{1}$

${ }^{1}$ John Radcliffe Hospital, Oxford, London, ${ }^{2}$ Oxford University, Oxford, London

Introduction: Gold standard guidance on management of Parkinson's disease (PD) by NICE was updated in 2017[1]. This audit compared current outpatient management in the Geratology Department at John Radcliffe Hospital against this guidance.

Methods: Ninety-two closed questions were designed to enable paper and electronic clinic notes to be audited against the NICE guidance. After exclusion of new referrals, seven cases were randomly selected from Geratology PD clinics.

Results: Areas of good practice included communication with patients and carers (accessible point of contact and advice about driving given in $100 \%$ cases), clinical diagnosis based on UKPDS Brain Bank Criteria (100\%) and offering levodopa early when quality of life was impacted (100\%). However, our analysis suggested a need for better provision of oral and written information about side effects of dopaminergic therapy (impulse control disorder 33\%; psychotic symptoms $17 \%$ cases). Other areas for improvement included offering a cholinesterase inhibitor in mild-moderate PD dementia (33\%), early multidisciplinary team (MDT) involvement (physiotherapy 43\%; occupational therapy 29\%) and provision of information regarding palliative care (advance care planning 14\%).

Key conclusions: Recommendations were made in the departmental audit meeting across three key areas: documentation, pharmacological and MDT. We suggested using a specialist nurse proforma to both prompt and document the provision of key information to patients and carers. We also recommended offering a cholinesterase inhibitor in mild-moderate PD dementia and early assessment by PD-experienced MDT specialists. Limitations of our analysis include the small sample size and focus on Geratology clinics (Neurology PD clinics were not audited).

\section{Reference:}

1. https://www.nice.org.uk/guidance/ng71

\section{P-786}

Polypharmacy and frailty: case review in buckinghamshire care home

Unoma Okoli ${ }^{1}$, Beatrix Nagyova ${ }^{2}$, Bobbi Jacobs ${ }^{1}$

${ }^{1}$ Buckinghamshire Clinical Commissioning Group, Aylesbury, UK, ${ }^{2}$ Buckinghamshire Healthcare NHS Trust, Aylesbury, UK

Introduction: In Europe 8.6 million unplanned hospital admissions is caused by adverse drug events [1]. 50\% of which are preventable and $70 \%$ of these are in patients over 65 years of age and on 5 or more medicines [1].
Methods: A new multidisciplinary team review was carried out for a 76 year old Buckinghamshire nursing home resident 3 days post hospital discharge. Clinical Commissioning group pharmacist, general practitioner, geriatrician and care home nurse reviewed the patient. Medical complexity of resident included complex epilepsy with seizures occurring three times a week, brittle asthma, ischaemic heart disease, old strokes, type 2 diabetes mellitus on background of congenital tetraplegia and impaired immune system. The resident was able to use an electric wheelchair to mobilise and on occasions presented to staff with drowsiness and confusion. Prescribed 35 medications.

Results: 11 medications were stopped. Phenytoin dose was optimised, Fentanyl dose reduced. Total cost savings on medicines stopped was $£ 2677$. On follow up at 4 and 12 weeks seizures reduced from 12 to 1 per month. Patient was alert and referred for physiotherapy on request.

Conclusion: A holistic multidisciplinary review ensures patient treatment is optimised to achieve their preferred outcome with reduction in medication burden and risk of harm.

\section{References:}

1. Scottish Government Polypharmacy Model of Care Group. Polypharmacy Guidance, Realistic Prescribing 3rd Edition, 2018. Scottish Government

\section{P-787}

Health quality of life in elderly population in Lebanon using the short form 36 health survey (SF-36)

Nada El Osta ${ }^{1}$, Fatme Kanso ${ }^{2}$, Jihad Fakhouri ${ }^{1}$, Robert Saad $^{2}$, Amine Wehbe ${ }^{2}$, Lana El Osta ${ }^{2}$

${ }^{1}$ Faculty of Dentistry, Saint-Joseph University of Beirut, Beirut, Lebanon, ${ }^{2}$ Faculty of Medicine, Saint-Joseph University of Beirut, Beirut, Lebanon

Introduction: Measuring health status of elderly is essential for the prediction of their health care needs. Health research usually considers objective outcome measure; however there is a need to increase self-reported measures of health. The aim of our study was to assess the psychometric properties of the SF-36 among a group of Lebanese elderly.

Methods: It was a cross-sectional observational study. A systematic sample of elderly people aged 60 years and more was selected from dispensary, private and governmental hospitals randomly selected in Beirut city. Data were collected using face-to-face interview. The first part of the questionnaire consisted of sociodemographic characteristics. The second part consisted of the Arabic version of the SF-36. The third part consisted of Activity of Daily Living (ADL) scale, number and type of health problems and number of medications consumed per day.

Results: A total of 251 elderly people were included. The mean age was $70.69 \pm 7.70$ years. Cronbach alphas for all SF-36 scales exceeded 0.798. The intra-Class correlation coefficient varied between 0.675 (item 2) and 0.980 (items 14, 16 and 18) indicating good reliability. SF-36 was able to discriminate participants according to their sociodemographic characteristics and health problems: The quality of life $(\mathrm{QoL})$ of women was poorer than men ( $\mathrm{p}$ value $<$ $0.001)$. It was significantly lesser when the number of health problems ( $\mathrm{p}$ value $<0.001$ ) and medications ( $\mathrm{p}$ value $<0.001$ ) increased, and the ADL score ( $\mathrm{p}$ value $<0.05$ ) decreased.

Conclusions: SF-36 is a valid and reliable instrument for measuring QoL among Lebanese elderly and could be used for monitoring the QoL of this population. 


\section{P-788}

Physical function and the health related quality of life of the Polish elderly

Ewa Zasadzka ${ }^{1}$, Tomasz Trzmiel ${ }^{1}$, Anna Pieczyńska ${ }^{1}$, Mariola Pawlaczyk ${ }^{1}$

${ }^{1}$ Poznan University of Medical Sciences, Poznań, Poland

Objective: The increase of elderly population forces the need for assessing the state of health, quality of life in order to create strategies to minimize the negative impact of aging. The aim of the study was to assess the impact of physical function on the quality of life of older people.

Methods: The study was conducted on 88 women and 11 men, aged $>60$ years, from diverse environments, with functionality of at least one upper limb and the Mini-Mental State Examination test result $>10$. All subjects underwent the measurements of the hand grip strength, the SPPB (Short Physical Performance Battery) test and assessment of health-related quality of life using the SF-36 questionnaire.

Results: The mean dominant hand grip strength in the studied group was $1703.4 \mathrm{kG}(\mathrm{SD}=436.3)$. The mean $\mathrm{SF}-36$ results was 38.9 $(\mathrm{SD}=12)$ and $52.6(\mathrm{SD}=12.7)$ for the physical and mental domain, respectively. The mean result of the total SPPB test was $7.4(\mathrm{SD}=$ 3.8). The greater strength of the dominant hand's grip correlated with the higher scores in SF-36 both in the mental $(r=0.204, p=0.043)$ and in the physical domain $(r=0.201, p=0.046)$. The results of the SF-36 physical domain also correlated with the SPPB score $(\mathrm{r}=0.360, \mathrm{p}<0.001)$.

Conclusion: Greater hand grip strength, and overall better physical function measured by the SPPB test improve the health related quality of life of people over 60 .

\section{P-789}

Cancer management in European long-term care facilities: results from the SHELTER study

E. Allocca ${ }^{1}$, E. R. Villani ${ }^{1}$, G. F. Colloca ${ }^{1}$, D. Fusco ${ }^{1}$, V. Valentini ${ }^{1}$, R. Bernabei ${ }^{1}$, G. Onder ${ }^{1}$

${ }^{1}$ Università cattolica del sacro cuore, Rome, Italy

Introduction: Up to $26 \%$ residents in Nursing Home (NH) have cancer, and their care represents a challenge. The aim of this study is to establish the prevalence of older adults with cancer in Europe to better understand its impact.

Methods: Longitudinal multi-centre cohort study based on data from the Services and Health for Elderly in Long TERm care (SHELTER) study. Participants were assessed through the interRAI-LTCF tool including cancer assessment.

Results: Among 3698 residents, 428 (10.7\%) had cancer (mean age $86.4 \pm 7.2 ; 62.8 \%$ women). France was the country having the highest number of reported cancer cases $(17.6 \%)$ and Italy the fewest $(6.6 \%)$. The most frequent kind of cancer were pancreas, breast, colon, skin and lung. The most common cancer patients were white women older than 85 years. Excessive polypharmacy ( $\geq 10$ drugs) was higher in residents with cancer $(30.1 \%$ vs. $23.6 \% \mathrm{P}<0.001)$. Cognitive impairment was more prevalent among cancer patients $(47.4-38.7 \%$ $\mathrm{P}<0.001)$. Threw up, falls, dyspnea and pain had a higher prevalence in residents with cancer $(\mathrm{p}<0.05$ for all). $20.8 \%$ cancer residents experienced daily pain in last 3 days, and the $5.8 \%$ experienced breakthrough pain despite large use of painkillers $(\mathrm{p}<0.05)$. Conclusions: Cancer patients are prevalent in $\mathrm{NH}$ but supportive seems to be poorly provided, especially in terms of pain management.
$\mathrm{NH}$ residents with cancer represent a population that requires further study. Learning more about supportive care that improve quality of life among residents with cancer is important in improving nursing and physicians care.

\section{P-790}

Visual handicaps in elderly patients: what does the doctor find, but what does the patient see and feel, how to help in everyday life

Udo Hennighausen ${ }^{1}$, Fritz Dannheim ${ }^{2}$, Sonja Krupp ${ }^{3}$, Stefan Strotzka ${ }^{4}$, Dagmar Verlohr ${ }^{2}$

${ }^{1}$ Private Practice for Ophthalmology, Heide, Germany, ${ }^{2}$ Private Practice for Ophthalmology, Seevetal-Hittfeld, Germany, ${ }^{3}$ Research Group Geriatrics, Hospital Red Cross, Luebeck, Germany, ${ }^{4}$ Center for Gerontopsychiatry, Vienna, Austria

Introduction: The visual complaints of elderly patients are not always easily understood primarily; sometimes, on the first view, they might not harmonize with the doctor's findings.

Methods: From the experience collated in a private practice for ophthalmology low vision caused by age related macular degeneration (AMD), visual field defects caused by glaucoma or stroke and hallucinations in Charles Bonnet Syndrome (CBS) were chosen as important topics, the clock-test as an important tool for understanding elderly patients with visual handicaps: patients' complaints and clinical findings were analyzed and compared with each other.

Results: The understanding of a patient's visual disability and perception is the first step. Second, in AMD magnifying low vision aids and reading strategies, in visual field defects, especially hemianopic field defects, reading strategies and training of explorative saccades into the hemianopic field can support the coping with everyday tasks, in CBS especially behaviorial strategies. The clock test was found to be a very important tool to differentiate visual impairment caused by diseases of the eye or optic pathways up to the primary visual cortex from neglect or visual impairment caused by beginning dementia.

Key conclusions: Understanding of the variety of visual impairment in different diseases of elderly patients, basically, is very beneficial not only for ophthalmologists but also for geriatricians in everyday work, especially when performing the geriatric assessment.

\section{P-791}

Shortness of breath and sarcopenic obesity: are they connected? Case presentation

Adina Carmen Ilie ${ }^{1}$, Irina Mihaela Abdulan ${ }^{1}$, Gabriel Ioan Prada ${ }^{2}$, Anna Marie Herghelegiu ${ }^{2}$, Raluca $\mathrm{Nacu}^{3}$

${ }^{1}$ University of Medicine and Pharmacy "GRIGORE T POPA", Iasi, Romania, ${ }^{2}$ University of Medicine and Pharmacy "Carol Davila", Bucharest, Romania, ${ }^{3}$ University of Medicine and Pharmacy "Carol Davila", Bucharest, Romania

Introduction: Shortness of breath is usually related to cardiovascular, respiratory or metabolic disease. In elderly patients with multiple comorbidities one should take into account other rather rare causes.

Methods: We present the case of a 62-year-old obese woman, with personal history of angina, hypertension and diabetes who was admitted for persistent shortness of breath. Investigations revealed that she had no cardiovascular, respiratory, metabolic causes, or electrolyte imbalance to justify the symptoms. Widening the search for etiology, we found that the patient is, despite stage 2 obese, at high risk of malnutrition, sarcopenic and depressed. Nutritional status was 
evaluated by mini nutritional assessment questionnaire, Prognostic nutritional Index, Geriatric Nutritional Risk Index, muscle strengthdetermined by dynamometer, and muscle and fat mass by bioimpedance.

Results: We considered that the shortness of breath is due to sarcopenic obesity (SO), a well-known risk factor for negative progression of cardiovascular and metabolic diseases. The management of SO in such a patient requires a multidisciplinary team whose purpose is improving muscle mass and muscle strength with physical exercise, nutritional intervention to reduce fat tissue and also maintaining a normal glycemic status and applying no strain on the cardiovascular system.

Conclusion: Malnutrition is not limited to cachectic or anorexic patients but is also related to obesity. Preventing progression of the chronic diseases, maintaining high quality of life and high level of independence is directly connected to reversing the SO by a tailormade medical, nutritional and exercise plan for these patients.

\section{P-792}

\section{Factors affecting fall of fear in community-dwelling elderly}

Nesrin Yagci $^{1}$, Emine Aslan Telci $^{1}$, Serbay Sekeroz ${ }^{1}$, Mucahit Oztop ${ }^{1}$

${ }^{1}$ Pamukkale University School of Physical Therapy and Rehabilitation, Denizli, Turkey

Introduction: Each year, about one third of the population over 65 years of age experience at least one fall. There are many different reasons for falls in elderly. Risk factors that may lead to falls should be investigated in the elderly. The purpose of this study to investigate the factors related to fear of falling in community-dwelling elderly. Methods: A sample of 210 elderly (109 females; 101 males; mean age: $71.38 \pm 5.84$ years) included in the study. Fear of falling was assessed with Falls Efficacy Scale-International (FES-I). Physical activity level was measured with Physical Activity Scale for the Elderly (PASE) and mental status with Hodkinson's Abbreviated Mental Test (HAMT). The Timed Up and Go test (TUG) and FiveTime-Sit-to-Stand Test (FTSTS) was used to determine subjects' functional mobility. One-Leg Standing Test (OLST), Functional Reach Test (FRT) and Sharpened Romberg test (SR) was used to evaluate balance.

Results: As a result of the linear regression analysis, the variables affecting fear of falling at the $\mathrm{p}<0.05$ level were found as HAMT score (Std. $\beta$ : -0.594$)$, SR (Std. $\beta$ : -0.382 ), education year (Std. $\beta$ : -0.356 ), hearing aid use (Std. $\beta$ : 0.355 ), fall history in the last 12 months (Std. $\beta: 0.349$ ), FRT score (Std. $\beta$ : -0.343 ) and gender (Std. $\beta$ : 0.331) in favor of male respectively.

Key conclusions: The results of our study showed that fear of fall mostly affected from mental status, balance, education, hearing aid use, previous fall history and gender. We think that multidisciplinary approach is important for effective fall rehabilitation in elderly.

\section{P-793}

Fail to prepare, prepare to frail: predictors of hospital length of stay at admission

Edel Mannion ${ }^{1}$, Christine McCarthy ${ }^{2}$, Elaine Loughlin ${ }^{2}$, Robert Murphy ${ }^{2}$, Miriam Conry ${ }^{3}$, Stephanie Robinson ${ }^{2}$

${ }^{1}$ Department of Nursing, GUH, Galway, Ireland, ${ }^{2}$ Department of Stroke and Geriatric Medicine, GUH, Galway, Ireland, ${ }^{3}$ Department of Medical Social Work, GUH, Galway, Ireland
Introduction: Frailty has a known association with increased length of stay (LOS) in hospital. Specific predictors of LOS have not been measured within a frail older population. Our goal is to determine predictors of LOS to enhance timely discharge from hospital.

Method: Comprehensive geriatric assessment (CGA), potential predictors and LOS data were prospectively collected on all patients admitted to a specialist frailty service in an Irish University Teaching Hospital. Both univariate and multivariate linear regression were used to model the impact of predictors on LOS.

Results: We analysed 135 consecutive cases, 64\% were female, median age was 84 (IQR: $80-88$ ). The majority of patients $(93 \%)$ were community dwelling. The median Clinical Frailty Scale (CFS) at baseline was 5 (IQR: 4-6). The most common frailty syndromes at presentation included "off functional baseline" $85 \%(\mathrm{n}=115)$, "polypharmacy" 83\% (n = 113) "falls" 53\% (n=71), "confusion" $36 \%(\mathrm{n}=49)$ and "incontinence" $33 \%(\mathrm{n}=44)$. In relation to regression analysis none of: age, CFS, frailty syndrome type, depression, nutritional status or medication number significantly predicted LOS. Predictors that approached significance in the multivariate model were presence of care giver burden $(\mathrm{p}=0.066)$ and living alone $(\mathrm{p}=0.079)$.

Key conclusions: The frail inpatient cohort is a heterogeneous patient group. Ongoing study of this population is required to ascertain predictors of prolonged hospitalisation including caregiver burden. Predictors of LOS should be measured in conjunction with length of ageing in place. Further research of this relationship is required.

\section{P-794}

Elderly abuse: characterization of a population hospitalized in CHUC

Antony Soares Dionisio ${ }^{1}$, Manuel Teixeira Marques Veríssimo ${ }^{2}$, João Filipe de Ferreira Gomes ${ }^{3}$

${ }^{1}$ Faculty of Medicine, University of Coimbra, Coimbra, Portugal, ${ }^{2}$ Internal Medicine unit, Hospitais da Universidade de Coimbra, Centro Hospitalar e Universitário de Coimbra, Coimbra, Portugal, ${ }^{3}$ Internal Medicine Unit, Hospitais da Universidade de Coimbra, Coimbra, Portugal

Mistreatment in the elderly people represents an increasingly problem in today's society. This study intends to evaluate the signs of mistreatment in a population of elderly people admitted to a central Hospital in Portugal and relate them with risk factors. The data were obtained through a questionnaire response, with the participation of 100 elderly people admitted to the Internal Medicine A services of CHUC. The questionnaire was divided into 5 sections, allowing the collection of sociodemographic data, information on emotional status through the Yesavage scale, evaluation of the functional state using the Katz index, the prevalence of abuse indicators with the QEEA instrument and data on health status. The analysis of the results concludes that $36 \%$ of the elderly have submitted at least one indicator of abuse. Emotional abuse (29\%) and negligence (24\%) were more frequently detected, followed by financial abuse (12\%) and physical abuse $(5 \%)$. When relating the indicators of mistreatment with the other variables, it was concluded that there is an association between mistreatment indicators and individuals of the female gender, low educational level, depressive symptoms, increased functional dependence, number of medicine taken per day, number of emergency services visits and number of hospitalizations. This study alerts for the high number of elderly people with signals of mistreatment hospitalized in central hospitals in Portugal. It is important to remember of this global health issue and to develop intervention strategies in order to contribute to reduction or extinction of elderly abuse. 


\section{P-795}

Differences between the sitting and supine blood pressure values in subjects over $\mathbf{7 5}$ years old according to their frailty status

Sara Gabriele ${ }^{1}$, Carlos Labat ${ }^{2}$, Marina Kotsani ${ }^{2}$, Sylvie Gautier ${ }^{2}$, Francesco Fantin ${ }^{1}$, Athanasios Benetos ${ }^{2}$

${ }^{1}$ Department of Medicine, Section of Geriatric Medicine, University of Verona, Verona, Italy, ${ }^{2}$ Geriatric Department of University Hospital of Nancy (CHRU), Nancy, France

Introduction: Hypertension guidelines for adults state that similar blood pressure (BP) values are recorded in supine and sitting positions. We aimed to test if this statement is also valid in individuals aged 75 and more with various frailty status.

Methods: BP was measured in our geriatric department, in supine, sitting and upright positions sequentially (three times in each position) in 107 patients aged $>75$ years. Subjects were classified into 3 frailty groups using the SEGA score: low $(n=32)$, moderate $(n=33)$ and high $(\mathrm{n}=42)$. Sitting hypotension (SitHypo) or hypertension (SitHyper) were defined respectively as a decrease or increase of $\geq 20 \mathrm{mmHg}$ in systolic BP (SBP) from supine to the sitting position. A similar classification was used for defining orthostatic hypotension (OrthHypo) or hypertension (OrthHyper) i.e. differences in SBP between sitting and upright position of $\geq 20 \mathrm{mmHg}$.

Results: Among these patients, $27.1 \%$ showed significant changes between supine and sitting positions. The prevalence of SitHypo was $17.8 \%$ and of SitHyper $9.3 \%$. SitHyper increases proportionally to the frailty status: $0,9.4$ and $16.9 \%$ respectively (age- and sex-adjusted values; $\mathrm{p}<0.02$ ). The frailty status did not influence the SitHypo. OrthHypo was more frequent than OrthHyper (19.6 and $2.8 \%$ respectively) and was more prevalent in frail patients.

Conclusions: In older adults, especially those with pronounced frailty, sitting and supine SBP present major differences. Patients with pronounced frailty showed more important position-related variations in BP, with a transient SitHyper followed by OrthHypo. The clinical significance of these changes should be further evaluated.

\section{P-796}

Nutritional screening of older hospitalized people and its relation to other comprehensive geriatric assessment tools

Lukasz Kroc $^{1}$, Bartlomiej Soltysik ${ }^{1}$, Elizaveta Fife ${ }^{1}$, Krzysztof Socha ${ }^{1}$, Tomasz Kostka ${ }^{1}$

${ }^{1}$ Department of Geriatrics, Healthy Ageing Research Centre, Medical University of Lodz, Pieniny 30, Łódź, Poland

Background and aim: Malnutrition is one of the most important problems in hospitalized geriatric patients. $35-65 \%$ of these patients experience problems of inadequate nutrition. Non-expensive and valid screening for malnutrition is of utmost importance in this population. We compared nutritional screening tools - Nutrition Risk Screening 2002 (NRS 2002) and Subjective Global Assessment Form (SGA) with other comprehensive geriatric assessment tools.

Methods: The study included 622 (431 women) consecutive older people, aged $81.7 \pm 7.8$ years, who were hospitalized in the acute care Geriatric University Clinic, Central Veterans' Hospital in Lodz (Poland), and had complete data with all the assessment tools.

Results: The mean NRS score for this group was $1.6 \pm 1.2,85 \%$ had A (well-nourished) category of SGA. Women were older $(81.9 \pm 7.7 \mathrm{vs}$ $81.4 \pm 8)$, had lower ADL and IADL but higher GDS $(5.3 \pm 3.6 \mathrm{vs}$ $4.6 \pm 3.5)$ and VES-13 scores $(6.8 \pm 2.9$ vs $6.1 \pm 3)$. Significant correlations between NRS and ADL $(r=-0.28)$, IADL $(r=-0.26)$, Mini-Mental State Examination (MMSE) $(r=-0.26)$ and the Vulnerable Elders Survey-13 (VES-13) scale $(r=0.26)$ were found. Well-nourished subjects according to SGA had higher ADL, IADL and MMSE but lower VES-13.

Conclusion: Nutritional screening scales NRS and SGA have some moderate correlations with comprehensive geriatric assessment tools in hospitalized older subjects.

Keywords: Malnutrition, NRS, SGA, VES-13

\section{P-797}

\section{Bringing a multidimensional approach to geriatric patients in different clinical settings: essential consonances of the multidimensional prognostic index}

Anna Maria Meyer ${ }^{1}$, Lena Pickert ${ }^{1}$, Marcel Rarek ${ }^{1}$, Petra Arenz ${ }^{1}$, Dirk Hoffmann ${ }^{1}$, Giacomo Siri' ${ }^{2}$, Ingrid Becker ${ }^{3}$, Paul Brinkkötter ${ }^{4}$, Volker Burst ${ }^{4}$, Thomas Betz ${ }^{5}$, Ralf-Joachum Schulz ${ }^{6}$, Alberto Pilotto ${ }^{7}$, Thomas Benzing ${ }^{4}$, Maria Cristina Polidori ${ }^{1}$

${ }^{1}$ Ageing Clinical Research, Dpt. II for Internal Medicine, University Hospital of Cologne, Cologne, Germany, ${ }^{2}$ Institute of Medical Statistics and Computational Biology, University of Cologne, Cologne, Germany, ${ }^{3}$ Nephrology, Rheumatology, Diabetology and Internal Medicine, Dpt. II for Internal Medicine, University Hospital of Cologne, Cologne, Germany, ${ }^{4}$ UniReha $\mathrm{GmbH}$, Centre for Prevention and Rehabilitation of the University Hospital of Cologne, Cologne, Germany, ${ }^{5}$ Social Services, University Hospital of Cologne, Cologne, Germany, ${ }^{6}$ Department of Orthogeriatrics, Rehabilitation and Stabilization, Frailty Area, Galliera Hospital, Genova, Italy, ${ }^{7}$ Cluster of Excellence Cellular Stress Responses in AgingAssociated Diseases, University of Cologne, Cologne, Germany

Introduction: The Multidimensional Prognostic Index (MPI) is a validated sensitive and specific prognosis calculation tool based on a comprehensive geriatric assessment (CGA) which accurately predicts mortality in multimorbid patients with acute disease or relapse of chronic conditions. The aim of the present study was to evaluate whether the MPI predicts indicators of healthcare resources in older patients across different clinical settings.

Methods: Data from 723 patients aged 70 years and older, recruited in 4 prospective ongoing studies from general practice (GP), emergency department (ED), acute internal ward (IW) and geriatric ward (GW) were retrospectively analysed. All patients underwent a CGA-based MPI calculation upon first visit and were subdivided in low (MPI-1, score 0-0.33), moderate (MPI-2, score 0.34-0.66) and high (MPI-3, score 0.67-1)-risk of mortality. Indicators of healthcare resources, including length of hospital stay (LHS) and Grade of Care (GC) were recorded. Results: The MPI score was significantly related to age $(p<0.0001)$, gender $(p=0.000)$, setting of the recruitment $(p<0.0001)$, source of referral $(\mathrm{p}<0.0001)$, LHS $(\mathrm{p}<0.0001)$, dysphagia $(\mathrm{p}<0.0001)$, sensorial impairment $(\mathrm{p}<0.0001)$, incontinence $(\mathrm{p}<0.0001)$, type 2 diabetes $(\mathrm{p}=0.001)$, hospitalization and falls in the last 12 months $(p<0.0001)$, years of education $(p=0.027)$ and level of educational requirements $(\mathrm{p}=0.001)$ as well as GC $(\mathrm{p}<0.0001)$.

Conclusions: The CGA-based MPI values are highly significantly associated with healthcare indicators including GC, LHS and discharge destination independent of the medical settings. These findings suggest that MPI may be useful to resource planning in the care of older multimorbid patients across multiple healthcare sectors. 


\section{P-798}

The role of prognosis for tailored multidimensional interventions in older multimorbid patients: the multidimensional prognostic index

Lena Pickert ${ }^{1,3}$, Anna Maria Meyer ${ }^{1,3}$, Nicolas Nötzel ${ }^{1,3}$, Ingrid Becker $^{2}$, Paul Brinkkötter ${ }^{3}$, Ingrid Dammertz ${ }^{4}$, Janne Höncke ${ }^{4}$, Daniela Karg ${ }^{4}$, Barbara Königkamp ${ }^{4}$, Michael Müller ${ }^{4}$, Dennis $\mathrm{Nano}^{3}$, Susanne Ratte-Henn ${ }^{5}$, Alberto Pilotto ${ }^{6}$, Thomas Benzing ${ }^{3,7}$, M. Cristina Polidori ${ }^{1,3}$

${ }^{1}$ Ageing Clinical Research, Dpt. II for Internal Medicine, University Hospital of Cologne, Cologne, Germany, ${ }^{2}$ Institute of Medical Statistics and Computational Biology, University of Cologne, Cologne, Germany, ${ }^{3}$ Nephrology, Rheumatology, Diabetology and Internal Medicine, Dpt. II for Internal Medicine, University Hospital of Cologne, Cologne, Germany, ${ }^{4}$ UniReha $\mathrm{GmbH}$, Centre for Prevention and Rehabilitation of the University Hospital of Cologne, Cologne, Germany, ${ }^{5}$ Social Services, University Hospital of Cologne, Cologne, Germany, ${ }^{6}$ Department of Orthogeriatrics, Rehabilitation and Stabilization, Frailty Area, Galliera Hospital, Genova, Italy, ${ }^{7}$ Cluster of Excellence - Cellular Stress Responses in AgingAssociated Diseases, University of Cologne, Cologne, Germany

Background: Interdisciplinary interventions including the comprehensive geriatric assessment (CGA) through the geriatric team constitute the cornerstone of geriatric medicine, but its adequate implementation is often limited by several factors inducing a "know-do gap". Here, we report a retrospective analysis of the relationship between CGA-based prognosis evaluation and multidimensional interventions in a German non-geriatric ward of internal medicine.

Methods: A structured weekly CGA-based calculation of the Multidimensional Prognostic Index (MPI) was performed at admission and discharge in 40 older multimorbid patients ( $24 \mathrm{M} 77.4 \pm 4.7$ years; $16 \mathrm{~F}$ $80.3 \pm 6.3$ years) undergoing a goal-oriented pilot intervention program performed by a team of physiotherapists, occupational therapists, social workers, pharmacists, nurses and geriatricians begun 2016. The MPI predicts mortality after one month and one year in older multimorbid patients with acute disease or chronic conditions. Patients were subdivided in low (MPI-1, score 0-0.33), moderate (MPI-2, score 0.34-0.66) and high (MPI-3, score 0.67-1)-risk of mortality.

Results: Seven 5\% of the patients belonged to MPI-1, 67.5\% to MPI2 and $25 \%$ to MPI-3. Half of the patients were discharged home or nursing homes, one fourth to a geriatric rehabilitation unit. Half of the remaining fourth were discharged to other clinical settings and half died during hospitalisation. For similar MPI values at admission, there is a non-significant trend towards MPI score improvement in patients undergoing multidimensional intervention. The relatively low number and the high heterogeneity of the patient population require further analysis and sample improvement which is ongoing.

Conclusions: Multidimensional interventions in non-geriatric medical departments, albeit representing logistic challenges, might be feasible and successful. Further research is needed to clearly identify implementation gaps.

\section{P-799}

Identification of valvular heart disease in older nursing home residents: design of a feasibility study

Maria Cristina Polidori ${ }^{1}$, Anna Maria Meyer ${ }^{1}$, Annika Diedrich ${ }^{1}$, Christina Eßfeld ${ }^{1}$, Lisa Ulbrich ${ }^{2}$, Alberto Pilotto ${ }^{3}$, Thomas Benzing ${ }^{4}$, Stefania Maggi ${ }^{5}$, Andrea Ungar ${ }^{6}$, Roman Pfister ${ }^{2}$
${ }^{1}$ Ageing Clinical Research, Dpt. II for Internal Medicine, University Hospital of Cologne, Cologne, Germany, ${ }^{2}$ Cardiology, Dpt. III of Internal Medicine, University Hospital of Cologne, Cologne, Germany, ${ }^{3}$ Department of Geriatric Care

Introduction: Valvular heart disease (VHD) is frequent in older persons, but largely underdiagnosed because of decreased physical activity, presence of limiting comorbidities or reduced access to care. Methods: The higher aim of the study is to assess the prevalence of VHD in nursing home residents and examine associations with comprehensive geriatric assessment (CGA) and the prognostic impact of both. The recruitment phase of a prospective study in two nursing homes in Cologne begun in April 2018. MD and PhD students underwent structured cardiac auscultation training and subsequent examination of nursing home residents willing and able to consent was planned which additionally included assessment of the performance of a structured CGA. Based upon the functional, psychosocial and clinical CGA domains, a Multidimensional Prognostic Index (MPI) is calculated which allows the classification of patients according to low- (MPI-1, 0-0.33), middle- (MPI-2, 0.34-0.66) and high- (MPI-3, 0.67-1) risk of mortality. Using a stepwise approach, based on auscultatory suspicion of valve disease, patients will undergo confirmatory transthoracic echocardiography. MPI values will be compared from patients with confirmed versus those without valve disease and clinical decision making will be evaluated accordingly. Preliminary Observations and research outlook. Despite the careful ethical and logistic improvement process which led to a patient-friendly, uncomplicated study design, the response rate of both nursing homes and nursing home residents/caregivers was low. Strategies are currently implemented to improve the recruitment process in this study, which has a high potential of providing important information on the impact of VHD in nursing home residents.

\section{P-800}

Profile of treatments prescribed in base of a multidimensional protocol in the follow-up of hip fracture patients included in a Geriatric Fracture Liaison Service (G-FLS)

P. Matovelle ${ }^{1}$, D. Moral-Cuesta ${ }^{1,2}$, T. Alarcón ${ }^{1,2}$, R. Velasco ${ }^{1}$, R. Menéndez-Colino ${ }^{1,2}$, P. Gotor ${ }^{1}$, J. Díez-Sebastián ${ }^{3}$, Juan Ignacio González-Montalvo ${ }^{1,2}$

${ }^{1} 1$ Geriatrics Department, Hospital Universitario La Paz, Madrid, Spain, ${ }^{2}$ Instituto de Investigación del Hospital La Paz IdiPAZ, Madrid, Spain, ${ }^{3}$ Preventive Medicine Department, Universidad Autónoma, Madrid, Spain

Introduction: The importance of Fracture Liaison Services (FLS) is growing up, because they identify patients after suffering a fracture, start the specific treatment to prevent new ones and avoid functional deterioration, but little is known about treatments prescribed to geriatric patients including in them.

Methods: Hip Fracture (HF) patients consecutively admitted during 2 years in a co-managed orthogeriatric unit at a university hospital were studied. During hospital stay, in addition to the usual orthogeriatric treatment, the FONDA protocol (Function, Osteoporosis, Nutrition, Pain, Anaemia, in its Spanish acronym) for comprehensive hip fracture treatment was applied in all patients. After 3 months, some selected FONDA patients were revised at the Geriatric-FLS (G-FLS) in the outpatient clinic. Treatments in all areas were recorded.

Results: Two hundred and twenty three patients were scheduled, and $186(83.4 \%)$ came to the G-FLS clinic. Antiosteoporotic drugs were prescribed in $14 \%$ at baseline, $9.5 \%$ at discharge and $70 \%$ at follow-up. Vitamin D was prescribed in $11 \%$ at baseline, $92.4 \%$ at discharge and $92 \%$ at follow-up. Calcium was prescribed in $10 \%$ at baseline, $84.3 \%$ at discharge and $74 \%$ at follow-up. Nutritional supplements were 
prescribed in $87.9 \%$ at admission and dropped to $11.3 \%$ at the follow-up. In-bed, seated and stand-up specific exercises were performed in $98.7 \%$ of patients during hospitalization and maintained after discharge.

Conclusions: Few HF patients in the community have a previous anti-osteoporotic treatment. The treatment in a G-FLS include not only the adjustment of anti-osteoporotic drugs but also nutritional support and the performance of specific exercises.

\section{P-801}

Characteristics of patients attended at a Geriatric Fracture Liaison Service (G-FLS) after a fragility hip fracture

D. Moral-Cuesta ${ }^{1,2}$, P. Matovelle ${ }^{1}$, T. Alarcón ${ }^{1,2}$, R. Menéndez-Colino ${ }^{1,2}$, A. Gutiérrez Misis ${ }^{2,3}$, I. Martín-Maestre ${ }^{1}$, J. Díez-Sebastián ${ }^{4}$, Juan Ignacio González-Montalvo ${ }^{1,2}$

${ }^{1}$ Geriatrics Department, Hospital Universitario La Paz, Madrid, Spain, ${ }^{2}$ Instituto de Investigaciœn del Hospital La Paz IdiPAZ, Madrid, Spain, ${ }^{3}$ School of Medicine. Autonoma University, Madrid, Spain, ${ }^{4}$ Preventive Medicine Department, Universidad Autœnoma, Madrid, Spain

Introduction: Fracture Liaison Services identify patients after suffering a fragility fracture and establish the treatment to prevent new ones, but little is known about geriatric patients including in them.

Methods: Hip Fracture (HF) patients consecutively admitted during 2 years in a co-managed orthogeriatric unit at a university hospital were studied. During hospital stay, in addition to the usual orthogeriatric treatment, the FONDA protocol (Function, Osteoporosis, Nutrition, Pain, Anaemia, in its Spanish acronym) for comprehensive hip fracture treatment was applied in all patients. After 3 months, some selected FONDA patients were revised at the outpatient clinic and an exhaustive geriatric assessment, including the Short Physical Performance Battery (SPPB), handgrip strength, and analytical study was performed.

Results: A total of 223 patients discharged after a HF were scheduled and $186(83.4 \%)$ came to the outpatient clinic. At baseline $97.7 \%$ of patients were independent for walking but only $69.9 \%$ at follow-up. Average baseline Barthel Index was $94( \pm 8)$ and $86( \pm 17)$ at followup. SPPB score was less than $8 / 12$ in $71.9 \%$ of revised patients. Average grip strength was $29.8( \pm 7.1) \mathrm{kg}$ in male and $16.8( \pm 4.4) \mathrm{kg}$ in female. Prevalence of dementia was very low (6.3\%). Serum total proteins, albumin, hemoglobin and vitamin D level improved until a normal level at 3 months.

Conclusions: Patients included in a G-FLS are in a good functional and cognitive situation, but a high rate of frailty is detected in them. Functional and analytical status improve quickly in patients discharged from an orthogeriatric unit.

\section{P-802}

\section{Prevalence of loneliness in osteoporosis clinic patients}

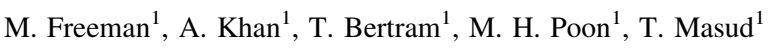 \\ ${ }^{1}$ Nottingham University Hospitals NHS Trust, Nottingham, UK
}

Introduction: Loneliness occurs when there is a mismatch between the number and quality of social relationships and connections that a person has and those that he/she would like. In the UK $5-16 \%$ of people aged $\geq 65$ years experience loneliness most or all of the time and a further $30 \%$ sometimes. Osteoporosis and resulting fractures cause much morbidity which may inhibit social interaction. The aim of this service improvement project was to assess the prevalence of loneliness in patients attending osteoporosis clinics.
Methods: A cross-sectional survey across 2 osteoporosis clinics was performed. The English Longitudinal Study of Ageing (ELSA) single item questionnaire ("How often do you feel lonely? - hardly ever or never, some of the time, or often"), and the De Jong Gierveld Loneliness Scale (DJGLS)(measures Emotional and Social loneliness) were used to assess loneliness. SPSS version-17 was employed to analyse the data.

Results: 108 patients, mean age 72.2 years $(\mathrm{SD}=9.3)$, (range $=$ 54-92), 95 female (88\%) were assessed. Using ELSA, proportion of patients reporting feeling lonely hardly ever (or never), some of the time, and often were $63.9 \%(\mathrm{n}=69), 34.3 \%(\mathrm{n}=37)$ and $1.9 \%$ $(\mathrm{n}=2)$ respectively. With DJGLS, $50.9 \%(\mathrm{n}=55)$ reported some degree of emotional loneliness, compared to $27.8 \%(\mathrm{n}=30)$ reporting social loneliness $\left[\mathrm{Chi}^{2}=16.9, \mathrm{p}<0.001\right]$.

Conclusion: Just over a third of osteoporosis clinic patients reported loneliness often or some of the time. Emotional loneliness was twice as common as social loneliness. Clinicians should be aware of this loneliness issue in osteoporosis. Further research is needed into factors leading to loneliness in this group.

\section{P-803}

Biomechanical characteristics of frail geriatric inpatient during chair-rising using four wheeled walker

Joao P. Batista $\mathrm{Jr}^{1}$, Laura Schmidt ${ }^{1}$, Thea Laurentius ${ }^{1}$, Bernd Markert $^{2}$, L.Cornelius Bollheimer ${ }^{1}$, Marion Mundt ${ }^{3}$

${ }^{1}$ Department of Geriatric Medicine, RWTH Aachen University Hospital, Aachen, Germany, ${ }^{2}$ Institute of General Mechanics, RWTH Aachen, Aachen, Germany, ${ }^{3}$ Institute of General Mechanics, RWTH Aachen University, Aachen, Germany

Introduction: Four wheeled walkers (FWW) are primary used by geriatric patients for walking but also for other functional activities, such as rising up from a chair. There are evidence in the literature about the biomechanics of chair rising, but the movement strategies of this task using a FWW have not yet been quantified. Aim: To analyze the biomechanical characteristics of rising up from a chair of geriatric patients using four wheeled walker.

Methods: Frail geriatric inpatients ( $\geq 70$ years) will be included in this study and divided into two sub-groups: FWW-Users and NonFWW-Users. The motion will be captured by ten infrared cameras with 42 reflective markers. Ground reaction forces will be measured with an integrated force plate. Non-FWW-Users rise up with the hands on the thighs. FWW-Users stand up with the hands on the FWW handles. All subjects have to place their feet in a pre-defined position on the force plate and will be instructed to stand up five times. Frailty will be assessed according to the phenotype of Fried.

Expected results and clinical relevance: Considering the results of a pilot study with four young healthy women (age \pm 27 years), we expect less trunk movement and acceleration, as well as decreased hip and knee joint angles by using a FWW during chair rising. Further, we expect less displacement of the center of pressure by FWW-Users. Our results might improve the recommendations and safety of standing up from a chair using a four wheeled walker.

\section{P-804}

Use of the Frailty Index in older persons with chronic kidney disease

Carlotta Vezza ${ }^{1}$, Simone Vettoretti ${ }^{2}$, Lara Caldiroli ${ }^{2}$, Luigi Bergamaschini ${ }^{3}$, Piergiorgio Messa ${ }^{2}$, Matteo Cesari ${ }^{3,4}$ 
${ }^{1} 1$ Geriatric Fellowship Program, University of Milan, Milan, Italy, ${ }^{2}$ Nephrology Dialysis and Kidney Transplantation Unit, Fondazione IRCCS Ca' Granda, Ospedale Maggiore Policlinico, Milan, Italy, ${ }^{3}$ Department of Clinical Sciences and Community, University of Milan, Milan, Italy, ${ }^{4}$ Geriatric Unit, Fondazione IRCCS Ca' Granda Ospedale Maggiore Policlinico, Milan, Italy

Chronic kidney disease (CKD) affects more than one third of people aged 65 and older. Many patients have renal impairment as one of the multiple deficits influencing their frailty status and prognosis. The routine assessment of frailty can be useful as prognostic marker as well as an instrument for designing personalized care interventions. We evaluated 115 nephrologic outpatients aged between 65 and 94 years with CKD (stage IV or V, but not yet undergoing dialytic procedures). Patients unable to collaborate and those with life expectancy $<6$ months were excluded. A 38 -item Frailty index (FI) using the cumulative deficit model was computed taking advantage of clinical and biological information. During the 12-month follow-up, hospitalisations, cardiovascular events, initiation of dialysis and death were recorded. The mean age of the sample was 80.2 years (standard deviation, SD 6.3); 30.3\% were women. The mean FI was 0.29 (SD 0.10 ); men 0.27, SD 0.09; women 0.35, SD 0.10). The FI increased with age for both sexes (Spearman $r=0.22$ ), consistently with the literature. It was also significantly higher in patients who experienced a hospitalisation $(\mathrm{p}=0.02)$, a cardiovascular event $(\mathrm{p}=0.02)$ or death $(p=0.003)$, independently of age and sex. The decision to start dialysis was predicted by the participant's glomerular filtration rate $(p=0.008)$, but not by the FI.FI is a strong predictor of negative outcomes in nephrology patients. Its use in the nephrological setting may support the identification of frail patients (independently of age), and promote the allocation of adapted care.

\section{P-805}

The risk of dysphagia is associated with malnutrition and poor functional outcomes in a large population of community-dwelling older individuals

Sara Tagliaferri ${ }^{1}$, Fulvio Lauretani ${ }^{2}$, Maria Giovanna Pelà ${ }^{1}$, Yari Longobucco ${ }^{1}$, Marcello Maggio ${ }^{3}$

${ }^{1}$ Department Medicine and Surgery, University of Parma, Parma, Italy, ${ }^{2}$ Frailty and Multimorbity Lab, Geriatric Clinic Unit, University-Hospital of Parma, Parma, Italy, ${ }^{3}$ Department Medicine and Surgery, University of Parma, Parma, Italy; Frailty and Multimor

Introduction: Oropharyngeal dysphagia (OD) is a widespread clinical condition among elderly. Although it represents a risk factor for metabolic and respiratory outcomes, its assessment and contribution to functional decline appears ignored. Our aim was to estimate the prevalence of OD in a large population of community-dwelling older people and to evaluate its relationship with malnutrition and physical function.

Methods: 10-item Eating Assessment Tool (EAT-10) and Mini Nutritional Assessment Short Form (MNA-SF) were used to identify the risk of dysphagia and malnutrition. Short Physical Performance Battery (SPPB) and handgrip strength were used as functional endpoints. The relationship between risk of dysphagia and functional outcomes was tested in a multivariate regression analysis adjusted for age and sex (Model 1) and for other confounders including Mini Mental State Examination (MMSE) and polypharmacy (Model 2).

Results: Mean age of 773 subjects (61.3\% females) was 81.97 years. $30.1 \%$ of participants was at risk of dysphagia (EAT $\geq 3$ ), $37.8 \%$ was malnourished (MNA-SF < 8), $46.2 \%$ was at risk of malnutrition $(8<$ MNA-SF $<11)$. EAT-10 was significantly and negatively associated to MNA-SF, SPBB and handgrip in both univariate and multivariate models $\quad(\beta=-0.28 \pm 0.07, \quad \mathrm{p}<0.0001$; $\beta=-0.25 \pm 0.05, \mathrm{p}<0.0001 ; \beta=-0.07 \pm 0.03, \mathrm{p}<0.0001$, respectively). After categorization of risk of dysphagia in at risk and not risk groups, MNA-SF, SPPB and handgrip were independently associated with higher risk of dysphagia $(\mathrm{OR}=0.91,95 \% \mathrm{CI}=$ $0.83-0.99, \mathrm{p}=0.03$; OR $=0.83,95 \% \mathrm{CI}=0.77-0.89, \mathrm{p}<0.0001$; $\mathrm{OR}=0.96,95 \% \mathrm{CI}=0.92-0.99, \mathrm{p}=0.02$, respectively).

Conclusion: In a large group of community-dwelling older individuals, we observed a significant negative association between risk of dysphagia and nutritional and physical performance, suggesting that the screening of OD, possibly supported by its assessment, should be implemented in the geriatric setting to potentially prevent the functional decline.

\section{P-806}

Does the Fried phenotype of frailty reflect muscle strength of the lower extremities? Consequences for mobility rehabilitation and fall prevention

Laura Schmidt $^{1}$, Thea Laurentius ${ }^{1}$, L. Cornelius Bollheimer ${ }^{1}$, Joao P. Batista $\mathrm{Jr}^{1}$

${ }^{1}$ Department of Geriatric Medicine, RWTH Aachen University Hospital, Aachen, Germany

Introduction: Handgrip strength is used as an overall surrogate for decreased muscle strength and is one criterion for physical phenotype of frailty according to Fried. Frailty is well reported as a predictor of falls but handgrip strength as well as gait velocity deliver only limited and indirect information about the muscle strength of the lower limbs which is very important in renowned tests to detect fall and functional mobility, such as the chair-rise(CR) and the timed up\&go test (TUG). Aim: To compare isometric knee extension strength in frail versus non-frail subjects with propensity to fall.

Methods: Geriatric patients ( $\geq 70$ years) will be included and divided into two sub-groups: Frail and non-frail subjects. To evaluate the propensity to fall the TUG and CR will be evaluated. Isometric knee extension strength will be investigated by a handheld dynamometer positioned at the front of the ankle with patient seated on bed with knee at $70^{\circ}$ flexion $\left(0^{\circ}=\right.$ max. extension $)$. Participants will be instructed to push the dynamometer as strong as possible for three times. Frailty will be measured based on the physical phenotype criteria provided by Fried.

Expected results and clinical relevance: We expect to find lower muscle strength of frail geriatric patients for upper and lower limbs as well as some relationships between Frailty scores and knee extension strength. Although handgrip is regarded to be accurate to measure muscle strength in frailty patients, little attention has been given to other muscle groups that would reflect closer dependency of the mobile capacity and fall prevention related tests.

\section{P-807}

Health for elderly: a physiotherapy assessment tool to assess physical and psychosocial skills and pain

Emilia Tasso ${ }^{1}$, Franca Spallarossa ${ }^{2}$, Loredana Minetti ${ }^{2}$, Lorenzo Sampietro ${ }^{2}$, Francesca Vitali ${ }^{3}$

${ }^{1}$ Asl3 Genovese and University of Genoa, Genoa, Italy, ${ }^{2}$ University of Genoa, Genoa, Italy, ${ }^{3}$ Università di Verona, Verona, Italy

Introduction: The assessment of physical and psychosocial skills and pain is central to support the medical and physiotherapy treatment 
with the aim to enhance autonomy and social participation in active life for elderly. Falls and physical inactivity prevention are the cornerstones of building a good perception on safety and promoting health and well-being of older people with disability or frailty. Ability to adapt, empowerment and reliability must be increased by an integrated socio-sanitary team, privileged point of connection and support.

Methods: A physiotherapy assessment tool (Svft_02) was used to assess physical and psychosocial skills and pain of elders who were patients and former patients of the physiotherapy Primary care unit in Genoa. This study aims at assessing physical and psychosocial skills linked to a physiotherapy treatment and to estimate correlations with pain.

Results: We investigated $\mathrm{N}=2.271$ elders. Pain correlates significantly and positively with balance (r: $0.125 ; \mathrm{p}<0.001$ ), movement skills (r: 0.162; $\mathrm{p}<0.001$ ), and posture changes (r: 0.157; $\mathrm{p}<0.001)$. A linear regression analysis show that posture changes $(\beta$ : $0.157 ; \mathrm{p}<0.001)$, motivation $\beta: 0.263 ; \mathrm{p}<0.001$ ), and self-efficacy $(\beta$ : $-0.130 ; p<0.001)$, were pain predictors. We are processing some socio-sanitary dimensions and we are monitoring the outcomes. The indications received allow us to enjoy with the elderly, the rehabilitation team and the social support context, the best solution for appropriateness, effectiveness and sustainability.

Key conclusions: Assessing physical and psychosocial skills linked to physiotherapy treatment is useful to predict pain and to support functional autonomy for elders.

\section{P-808}

Does preoperative comprehensive geriatric assessment and frailty predict postoperative morbidity, mortality and delirium?: a prospective study

Rana Tuna Dogrul ${ }^{1}$, Ahmet Bulent Dogrul ${ }^{2}$, Ali Konan ${ }^{2}$, Omur Caglar ${ }^{3}$, Fatih Sumer ${ }^{1}$, Hatice Caliskan ${ }^{1}$, Muhammet Cemal Kizilarslanoglu ${ }^{1}$, Mustafa Kemal Kilic ${ }^{1}$, Cafer Balci ${ }^{1}$, Gunes Arik ${ }^{1}$, Gözde Sengul Aycicek ${ }^{1}$, Cemile Ozsurekci ${ }^{1}$

${ }^{1}$ Hacettepe University, Faculty of Medicine, Department of Internal Medicine, Division of Geriatrics, Ankara, Turkey, ${ }^{2}$ Hacettepe University, Faculty of Medicine, Department of General Surgery, Ankara, Turkey, ${ }^{3}$ Hacettepe University, Faculty of Medicine, Department of Orthopedics and Traumatology, Ankara, Turkey

Background: The evidence for the fact that comprehensive geriatric assessment is part of the preoperative evaluation is rather limited. In this study, the influence of preoperative CGA and frailty on postoperative morbidity, mortality, and delirium was examined.

Methods: 108 geriatric patients for whom elective operation planned were evaluated. CGA and Freid Criteria were performed to patients. We used the Physiological and Operative Severity Score for the Enumeration of Mortality and Morbidity score(POSSUM), the American Society of Anesthesiologists Score(ASA), the British United Provident Association Score(BUPA) and the Charlson Comorbidity İndex $(\mathrm{CCI})$ to determine the risk of postoperative morbidity and mortality. Confusion Assessment Method (CAM) and Assessment Test for Delirium (4AT) were applied for detection of delirium. Patients were followed for postoperative delirium, morbidity and mortality.

Results: The median age was 71 years, $25 \%$ of the patients were frail. The mortality and morbidity rate were $0.9 \%$ and $28.7 \%$ respectively. The Instrumental Activities of Daily Living Scale(IADL) (p: 0.032), the Mini-Nutrition Assessment(MNA) (p: 0.01) and the Mini Mental State Examination(MMSE) score (p: 0.026) were found to be significantly lower in patients with morbidity. POSSUM physiology score (p: 0.005), operative score (p: 0.015) and CCI (p: 0.029) were significantly higher in the patients with morbidity. Patients who developed morbidity were found to be more frail $(p<0.001)$. Delirium developed in $3.7 \%$ of the patients. Patients who developed delirium were older (p: 0.039), all of them were frail, and 50\% had dementia. The patients with delirium were found to have lower IADL score (p: 0,049) and MMSE score (p: 0,004) and higher POSSUM physiology score (p: 0.005). As a result of multivariate analysis, it was found that frailty, POSSUM operative score and preoperative systolic blood pressure were found to be independently related factors in developing postoperative morbidity.

Conclusion: In our study, comprehensive geriatric assessment and frailty in the preoperative period indicated postoperative morbidity and delirium.

\section{P-809}

Falls in Older people in the community: should multi-factorial interventions extend beyond home?

\section{T. Obi ${ }^{1}$, H. Butt ${ }^{1}$, D. Dev ${ }^{1}$, S. Guptha ${ }^{1}$}

${ }^{1}$ North West Anglia NHS Foundation Trust, Peterborough, UK

Introduction: The National Institute for Health and Care Excellence recommends home hazard assessment and intervention in falls prevention in community dwelling older people. There are no specific recommendations in prevention of falls outside of home. We conducted a service evaluation project in a district hospital looking at falls presentation in older people attending the emergency department assessing the location of the fall and the immediate consequences.

Methods: We collected data of people aged 70 and above who had fallen and presented to the emergency department (ED) in the year 2017. We randomly selected 200 patients using simple randomisation methods of 1 in every 10 patients and exempted patients from care homes.

Results: The total number of visits to the ED of our district hospital was 105,116 for the year 2016-2017 across all age groups of which 21,243 were adults aged 70 years and above. A total of $2097(9.9 \%)$ patients aged 70 and above presented with a history of fall to the ED in the year 2017. Of the $200(9.5 \%)$ patients evaluated, the average age is 84 years, females $137(68.5 \%)$ and males $63(31.5 \%) .173$ $(87 \%)$ had fallen indoors at home, $20(10 \%)$ outdoors and $7(3 \%)$ not stated. $134(67 \%)$ in total were admitted, out of which $120(69 \%)$ had fallen indoors and $10(50 \%)$ outdoors. $43(21.5 \%)$ had significant injuries, consisting of $23(16.5 \%)$ fracture neck of femur, $18(9 \%)$ other fractures, and $5(2.5 \%)$ traumatic brain injury. Of these 43 patients, $42(98 \%)$ of them were admitted and $4(2 \%)$ of them had fallen outdoors. 4 (2\%) deaths were recorded.

Conclusion: Majority of the older people attending emergency department had fallen at home. While home based interventions are important in prevention of falls in older people, fall prevention advice outside of home should also be considered.

\section{P-810}

OPAL on the acute medical unit: positive impact on staff and patients

Katherine Horgan ${ }^{1}$, Kimberley Royle ${ }^{1}$

${ }^{1}$ Manchester University Hospitals NHS Foundation Trust, Manchester, UK 
Introduction: The Older Person Assessment and Liaison (OPAL) team was integrated on the Acute Medical Unit (AMU) in September 2015. This involves 14 beds on the 54-bedded AMU being ringfenced for frail elderly patients with complex needs with a seven-day service including multidisciplinary consultant geriatrician led ward rounds, frailty nurse input and close liaison with pharmacy and therapy staff. Initial impact: The impact of the service on patient outcomes has been positive, with a 55\% increase in discharges on Saturdays as well as a $95 \%$ increase in discharges of these patients on Sundays [1]. In addition to this, we have achieved double the amount of discharges in the morning [1].With regard to the over $90 \mathrm{~s}$, we have noted a $22 \%$ increase in discharges from the AMU rather than being admitted into the hospital as well as a reduction in 30-day readmission rates of $7.5 \%$ [1.

Staff survey: We undertook a written questionnaire survey of 20 Allied Health Professionals in 2018 including nursing staff, care support workers and junior doctors that demonstrated OPAL increased the confidence of these staff in working with complex elderly patients as well as the positive impact the service has had on patient care. It was also noted that those questioned would recommend the care received by patients to their family members and friends.

Conclusions: Embedding services on an Acute Medical Unit improves both outcomes with regard to patient flow as well as improving the understanding and confidence of healthcare professionals in undertaking comprehensive geriatric assessment.

\section{Reference:}

1. Wentworth L. OPAL Older Person Assessment and Liaison. Edinburgh SAM and RCPE 2016.

\section{P-811}

The role of bioelectrical impedance analysis (BIA) in identification of sarcopenia. Comparison among different classifications

Elena Colizzi ${ }^{1}$, Fulvio Lauretani ${ }^{2}$, Mario Pedrazzoni ${ }^{3}$, Giovanni Passeri $^{3}$, Sara Tagliaferri ${ }^{3}$, Marcello Maggio ${ }^{1,2,3}$

${ }^{1} 1$ Geriatric Clinic Unit, University-Hospital of Parma, Parma, Italy, ${ }^{2}$ Frailty and Multimorbity Lab, Geriatric Clinic Unit, UniversityHospital of Parma, Parma, Italy, ${ }^{3}$ Dept. Medicine and Surgery, University of Parma, Parma, Italy

Introduction: Sarcopenia is widespread clinical condition among elderly, characterized either by reduced muscle mass and muscle strength [1]. BIA has been used in identification of sarcopenia through skeletal muscle index (SMI). Different methods to estimate SMI are available $[2,3,4]$. The aims of the study were to evaluate the concordance between different SMIs and DXA in identification of sarcopenia and to examine the relationship between different sarcopenia criteria, in frail, community-dwelling older individuals.

Methods: DXA was the reference method [5] used to extrapolate appendicular lean mass (ALMcrude and ALM/BMI) and to identify sarcopenic subjects. Three different SMI definitions were considered: 1. SMI\% $=$ SMM/body weight $* 100$ and cut-offs (Janssen 2002), 2. SMI $\mathrm{kg} / \mathrm{m}^{2}=\mathrm{SMM} /$ body height and cut-offs (Janssen 2004) and 3. $\mathrm{SMIkg} / \mathrm{m}^{2}=\mathrm{SMM} /$ body height and cut-offs (Chien 2008). The statistical analysis was conducted by Pearson correlation and MantelHaenszel tests.

Results: The population was composed of 93 outpatients (62 women) age $\geq 70$. A significant correlation was observed between SMI\% and ALM/BMI $(r=0.57, \mathrm{p}<0.0001)$ and between SMIkg/m2 and ALMcrude $(r=0.37, p=0.0004)$. Sensitivity and specificity of different SMI definitions were extrapolate in low gait-speed subgroup
( $\mathrm{n}=31$, speed $<0.8 \mathrm{~m} / \mathrm{s}$ ) and were $85 \%$ and $33 \%$ for SMI $\%, 71 \%$ and $65 \%$ for SMIkg/m $\mathrm{m}^{2}$ (Janssen 2004), 36\% and 94\% for SMIkg/m² (Chien 2008), respectively.

Key conclusions: Our data showed good concordance between BIA (SMI) and DXA in muscle mass estimate of community-dwelling older individuals. Sensitivity and specificity varied along with different SMIs, indicating Janssen (2004), supported by EWGSOP, as the best estimate of sarcopenia at least in European population.

\section{References:}

1. Cruz-Jentoft AJ, Baeyens JP, Bauer JM, Boirie Y, Cederholm T, Landi F, Martin FC, Michel JP, Rolland Y, Schneider SM, Topinková E, Vandewoude M, Zamboni M; European Working Group on Sarcopenia in Older People (2010) Sarcopenia: European consensus on definition and diagnosis: report of the European Working Group on Sarcopenia in Older People. Age Ageing. 39:412-23.

2. Janssen I, Heymsfield SB, Ross R. (2010) Low relative skeletal muscle mass (sarcopenia) in older persons is associated with functional impairment and physical disability. J Am Geriatr Soc. 50: 889-896.

3. Janssen I, Baumgartner RN, Ross R, Rosenberg IH, Roubenoff R. (2004) Skeletal muscle cutpoints associated with elevated physical disability risk in older men and women. Am J Epidemiol 159:413-21. 4. Chien MY, Huang TY, Wu YT (2008) Prevalence of sarcopenia estimated using a bioelectrical impedance analysis prediction equation in community-dwelling elderly people in Taiwan. J Am Geriatr Soc 56:1710-1715

5. Studenski SA, Peters KW, Alley DE, Cawthon PM, McLean RR, Harris TB, Ferrucci L, Guralnik JM, Fragala MS, Kenny AM, Kiel DP, Kritchevsky SB, Shardell MD, Dam TT, Vassileva MT (2014) The FNIH sarcopenia project: rationale, study description, conference recommendations, and final estimates. J Gerontol A Biol Sci Med Sci 69(5):547-558

\section{P-812}

Analysis of comprehensive geriatrics assessment in a hemodialysis center

Florent Guerville ${ }^{1}$, Pauline Pinard ${ }^{1}$, Muriel Rainfray ${ }^{1}$, Aurelie Lafargue $^{1}$, Philippe Chauveau ${ }^{2}$, Catherine Lasseur ${ }^{2}$, Celine Nodimar ${ }^{2}$

${ }^{1}$ Bordeaux University Hospital-Clinical Gerontology Department, Talence, France, ${ }^{2}$ AURAD Aquitaine, Gradignan, France

Background: The role of comprehensive geriatric assessment (CGA) in older patients with end-stage renal disease needs to be defined. No screening tool predictive for an abnormal CGA is validated in this population. Our study aims were to describe CGA results in older patients on hemodialysis, to describe CGA-based interventions, and to determine factors associated with an abnormal CGA.

Methods: In this retrospective study, we included patients seen at the geriatric consultation implemented in a hemodialysis center from 2013 to 2017. We analyzed CGA results on 6 domains (dependency, cognition, locomotion, mood, nutrition and medications) and the CGA-based proposed interventions. Then we tested factors potentially associated with an abnormal CGA in at least 2 domains.

Results: 56 patients were included, mean age was 79 years (69-91). $57 \%$ had a loss of independence, $37 \%$ cognitive impairment, $26 \%$ mood disturbance, $57 \%$ gait or balance disturbance, $49 \%$ malnutrition and $64 \%$ potentially inappropriate medications. $75 \%$ had at least 2 abnormal CGA domains. These patients more often had a history of hypertension ( 88 vs $57 \%, \mathrm{p}=0.02$ ) and had lower mean albumin level ( 37.7 vs $35.8 \mathrm{~g} / \mathrm{l}, \mathrm{p}=0.03$ ). The most frequently proposed CGA-based interventions were to explore cognitive impairment with 
brain imaging, to add professional caregivers support and to modify inappropriate anti-hypertensive and psychotropic drugs.

Conclusion: The availability of a geriatric consultation in a hemodialysis center allows to identify frequent and multiple geriatric syndromes in older patients, especially those with a history of hypertension or an hypoalbuminemia, and to propose geriatric interventions.

\section{P-813}

Relation between hand grip strength and body composition in the elderly

Helena Loureiro ${ }^{1}$, Mário Monteiro ${ }^{1}$, Marlene Lajes ${ }^{1}$, Marco Silva ${ }^{2}$, Raul Martins ${ }^{2}$, Manuel Veríssimo ${ }^{3}$

${ }^{1}$ Coimbra Health School, Polytechnic Institute of Coimbra, Coimbra, Portugal, ${ }^{2}$ Faculty of Science of Sport and Physical Education of the University of Coimbra, Coimbra, Portugal, ${ }^{3}$ Hospital and University Center of Coimbra, Coimbra, Portugal

Introduction: The loss of muscle mass and, consequently, muscle strength occurs with age increase. Body mass and skeletal muscle strength are variables that contribute to the diagnosis of sarcopenia. This study aims to verify the relation between the Manual Hold Force (MHF) and the body composition.

Methods: The FPM of the right/left hand was evaluated by hand grip (Jamar, dynamometer). Dual Energy X-Ray Absorptiometry (DEXA, Lunar iDXA, General Electrics) was performed to elderly individuals to evaluate: total mass, fat mass, lean mass. Descriptive, correlational and inferential statistical analysis was performed.

Results: The sample was constituted by 100 individuals with balance body mass index (BMI): 67 women and 33 men, with a mean age of 69 and 71, respectively. Right and left MHF was higher in men than in women. Significant differences were founded between right and left MHF. Significant correlation was found for hands, total mass, and lean mass; and also for MHF and the arm, trunk; total right/left lean mass. No correlation was founded between fat mass and MHF of the dominant hand. For gynoid fat mass distribution the correlation was negative and non-existent for android fat distribution.

Key conclusions: The lack of correlation between fat mass and right/ left FPM refers to the reflection of sarcopenic obesity in individuals with android obesity. Considering these results a reflection about the negative correlation in gynoid obesity must be also made.

\section{P-814}

Bone mineral density and manual grip strength comparison in elderly

Helena Loureiro $^{1}$, Mário Monteiro ${ }^{1}$, Margarida Pocinho ${ }^{1}$, Marlene Lajes ${ }^{1}$, Marco Silva ${ }^{2}$, Raul Martins ${ }^{2}$, Manuel Veríssimo ${ }^{3}$

${ }^{1}$ Coimbra Health School, Polytechnic Institute of Coimbra Portugal, ${ }^{2}$ Faculty of Sport Science and Physical Education of the University of Coimbra Portugal, ${ }^{3}$ Hospital of University of Coimbra, Portugal

Introduction: The evaluation of the manual grip strength (MGS) allows the determination of the overall muscular strength on elderly. Individual characteristics such age, gender, body mass, height and dominance can influence MGS. Dual Energy X-Ray Absorptiometry (DEXA) is the gold standard image modality for body composition and bone mineral density (BMD) analysis. The aim of this study was to verify the relations between BMD and MGS.
Methods: The sample is probabilistic and random, consisting of 100 elderly. The MGS of the right / left hand was evaluated using a hand grip (Jamar, dynamometer). T-score and Z-score DEXA information was directly collect from the equipment (DEXA, Lunar iDXA, General Electrics). Descriptive, correlational and inferential statistical analysis was performed.

Results: The sample had homogeneous results for age, sex and body mass index (BMI). T-score revealed that $69.2 \%, 27.9 \%$ and $2.9 \%$ had normal, low and loss of BMD, respectively. Around $7 \%$ of the participants had results of bone pathology. T-score and Z-score combination indicate that $1.4 \%$ of the normal results when are evaluated by $\mathrm{T}$-score are considered bone pathology, $14 \%$ of those diagnosed with low BMD have osteopenia and $67 \%$ of those diagnosed with very low BMD have osteoporosis. For both hands the higher the bone loss revealed lower MGS.

Key conclusions: Z-score is fundamental to make the correct diagnosis, avoiding false negatives of BMD evaluation. The $\mathrm{T}$ Score determines fracture risk comparing with healthy individuals, but does not consider the variables gender, age, ethnicity.

\section{P-815}

Socio-economic determinants of anemia in polish elderly population: the results of PolSenior study

Arkadiusz Styszyński ${ }^{1}$, Małgorzata Mossakowska ${ }^{2}$, Jerzy Chudek ${ }^{3}$, Monika Puzianowska-Kuźnicka ${ }^{4}$, Alicja Klich-Raczka ${ }^{5}$, Agnieszka Neumann-Podczaska ${ }^{6}$, Aleksandra Szybalska ${ }^{2}$, Katarzyna Wieczorowska-Tobis ${ }^{1}$

${ }^{1}$ Laboratory for Geriatric Medicine, Department of Palliative Medicine, Poznan University of Medical Sciences, Poznan, Poland, ${ }^{2}$ International Institute of Molecular and Cell Biology, Warsaw, Poland, ${ }^{3}$ Department of Internal Medicine and Oncologic

Introduction: Anemia is a risk factor for functional decline and mortality among older adults. Since mild anemia is often under-diagnosed and ignored, its prevalence needs precise determination and recognition of predisposing factors. Our study identified the influence of socio-economic factors on the prevalence of anemia in the representative elderly population.

Method: PolSenior was a cross-sectional population-based study performed on the representative sample of Polish seniors. Complete blood count was assessed in 4003 respondents aged 65 years or above (1910 women) divided into six five-year cohorts and a reference group of 622 people aged 55-59 years (333 women). Anemia was defined based on WHO criteria: $\mathrm{Hb}<12.0 \mathrm{~g} / \mathrm{dL}$ in women and $\mathrm{Hb}<13.0 \mathrm{~g} / \mathrm{dL}$ in men. The following socio-economic factors were evaluated through the multiple logistic regression analysis: education level, marital status, place of residence, living arrangements and self-reported poverty.

Results: The prevalence of anemia in older persons standardized for the population was $10.8 \%$ (17.4\% of the study group) and was more frequent in men than in women $(20.8 \%$ vs $13.6 \%)$. The frequency of anemia progressed with age from $5.3 \%$ in the youngest to $37.7 \%$ in the oldest cohort, with stronger progression in men. We found the link between anemia and age in both genders, along with unmarried status and urban dwelling in men. When age was omitted, logistic regression showed the link between anemia and unmarried status, urban place of residence (both genders), and poor education (women only).

Conclusion: Oldest, poorly educated, unmarried and urbanites require intense screening for anemia. 


\section{P-816}

Validity of a set of smartphone-based measures to assess mobility in the elderly

Laura Casprini ${ }^{1}$, Marco Colpo ${ }^{1}$, Sabato Mellone ${ }^{2}$, Paola Tamburini ${ }^{2}$, Lorenzo Chiari $^{2}$, Stefania Bandinelli ${ }^{1}$

${ }^{1}$ Laboratory of Clinical Epidemiology InCHIANTI-Local Health Unit Tuscany Centre, Trondheim, Norway, ${ }^{2}$ Department of Electrical, Electronic and Information Engineering, University of Bologna, Bologna, Italy

Introduction: The study of human functional capacity requires a controlled environment and proper measurement systems which are usually expensive and time consuming. The use of smartphones in clinical research is progressively increasing with the availability of low cost/ freely available "apps" that could be used for functional assessment, and yet their clinical validity is unproven or unclear. The aim of this study is to verify the agreement between mobility measures derived from inertial sensors embedded into a smartphone and the measures obtained in a clinical movement analysis lab (gold standard). Four tests have been included: postural sway with different feet positions (stabilometric platform, Bertec), gait characteristics over a distance of $400 \mathrm{~m}$ (GAITrite-sensorised walkway), the TUG test, and the Five Times Sit-toStand Test (3D motion capture system, BTS).

Methods: We enrolled 96 healthy volunteers (age 20-80 years, 8 subjects for decade, $50 \%$ female). We adopted the InCHIANTI mobility assessment protocol. The SP was worn in a case waist belt placed on L5 in agreement with the FARSEEING project set up. A set of features is derived from both the smartphone and the gold standards.

Expected results: We expect to be able to select a subset of features with a good statistical agreement with those obtained in the movement analysis lab.

Conclusions: The identification of clinically valid smartphone-based mobility measures, along with a set of normative values, would allow the development of fast, affordable, and objective screening/assessment tests that can be administered in both supervised setting and in ecological settings.

\section{P-817}

Geriatric assessment and one-year mortality in older patients with cancer in the head and neck region, a cohort study

F. J. van Deudekom ${ }^{1}$, L. van der Velden ${ }^{2}$, W. H. Zijl ${ }^{1}$, A. S. Schimberg ${ }^{3}$, A. Langeveld ${ }^{4}$, M. Slingerland ${ }^{5}$, G. J. Blauw ${ }^{1}$, S. P. Mooijaart ${ }^{1,6}$

${ }^{1}$ Department of Internal Medicine, section of Gerontology and Geriatrics, Leiden University Medical Center, The Netherlands, ${ }^{2}$ Department of Head and Neck Surgery and Oncology, Netherlands Cancer Institute, Amsterdam, The Netherlands, ${ }^{3}$ Department of Otorhinolaryngology and Head and Neck Surgery, Radboud University Medical Center, Nijmegen, The Netherlands, ${ }^{4}$ Department of Head and Neck Surgery and Oncology, Leiden University Medical Center, The Netherlands, ${ }^{5}$ Department of Medical Oncology, Leiden University Medical Center, The Netherlands, ${ }^{6}$ Institute for Evidencebased Medicine in Old Age (IEMO), Leiden, The Netherlands

Introduction: Older patients with head and neck cancer (HNC) are at higher risk for adverse health outcomes after treatment, compared to younger patients. Careful selection could aid in optimizing treatment for individual patients. The aim is to describe the association of functional capacity and cognitive functioning with one-year mortality in older patients with cancer in the head and neck region.
Methods: We performed a cohort study in which all patients aged 70 years and older, or younger but with multiple comorbidities, diagnosed with stage III-IV HNC, or diagnosed with a lower stage but needing invasive treatment, received a geriatric screening prior to their treatment. Baseline assessment included patient characteristics, tumor staging and geriatric assessment in social, physical functioning and cognition domains. The main outcome was one-year mortality. Results: 102 patients were included. Median age was 78.7 years (interquartile range (IQR) 72.3-84.5). 65.6\% had stage III-IV HNC, $25 \%$ of the patients had cognitive impairment, $40 \%$ malnutrition, $40 \%$ were at risk for functional decline after hospitalization and $28.4 \%$ used a walking device. Overall, one-year mortality was $42.3 \%$. Male gender (hazard ratio (HR) 4.30; 95\% CI 1.35-13.67), (risk for) malnutrition (HR 2.55; 95\% CI 1.19-5.16) and the use of a walking device (HR 2.80; 95\% CI 1.13-6.93) were associated with mortality risk, independent of tumor stage and comorbidities.

Key conclusions: In older patient with head and neck cancer the mortality rates are high. Nutritional status and mobility are determinants of one-year mortality, independent of tumor stage, age and comorbidity.

\section{P-818}

Comprehensive geriatric assessment in nursing homes: what instruments fit?

\author{
S. Krupp ${ }^{1}$, J. Kasper ${ }^{1}$, F. Balck ${ }^{2}$, C. Ralf ${ }^{1}$, M. Willkomm ${ }^{1}$
}

${ }^{1}$ Research Group Geriatrics Lübeck, Hospital Red Cross Lübeck Geriatric Centre, Lübeck, Germany, ${ }^{2}$ Research Group Geriatrics Lübeck, Hospital Red Cross Lübeck Geriatric Centre, Germany, University Hospital Dresden, Technical University Dresden, Dresden, Germany

Introduction: In recent years the quality of care and additional interventions applied in nursing homes moves into focus. Using effects as quality indicators we have to struggle with three main difficulties: (1) Cognitive impairment reduces the reliability of information given by residents. (2). The view of the staff may be biased. (3) Residents in nursing homes are very vulnerable and the staff always under stress, so assessment has to be quick, easy and safe. Methods: By means of continuous practice research the Research Group Geriatrics Lübeck develops a multimodal program ("Lübeck Model Worlds of Movement") offered to seniors in need of long-term care. Evaluation of the effects must be based on profound knowledge about the situation in nursing homes. After modification of the target group by elimination of lack of walking ability as exclusion criterion we checked in what way we had to adapt the assessment battery.

Results: We found that ceiling effects occurred seldom, floor effects often. For some target parameters we could not find an instrument already established, so we modified existing tests or even developed new ones. In interviews simple language enhances cooperation-not only with the main target group but also with the staff.

Key conclusions: Monitoring effects via assessment calls for different instruments according to the functional level of the participants. We share our experiences with typical problems in assessment in nursing homes, show videos of those instruments that are yet unknown to most of the spectators and explain why we chose them. 


\section{P-819}

Relationship between skeletal muscle mass and left ventricular mass in older sarcopenic patients

Giovanna Pelà ${ }^{1}$, Felice Perrino ${ }^{1}$, Sara Tagliaferri ${ }^{1}$, Pietro Austoni ${ }^{1}$, Matteo Goldoni ${ }^{1}$, Fulvio Lauretani ${ }^{1}$, Mario Pedrazzoni ${ }^{1}$, Marcello Maggio $^{1}$

${ }^{1}$ Department of Medicine and Surgery, University of Parma, Parma, Italy

Background: Sarcopenia, defined as the loss of muscle mass and function, is a geriatric syndrome potentially reversible and often leading to frailty. Multiple factors positively influence and are affected by sarcopenia, including cardiac function. However, no study has fully addressed the relationship between skeletal muscle and cardiac muscle in older patients with sarcopenia and physical frailty. Methods: 100 sarcopenic and physical frail outpatients, 32 men (M), 68 women $(\mathrm{F})$, (mean age $79.8 \pm 5.1 \mathrm{M}, 78.6 \pm 5.3 \mathrm{~F}$; aLM $21.4 \pm 3.0 \mathrm{M}, 15.3 \pm 2.1 \mathrm{~F}$ ) with data available on appendicular lean mass (aLM) and left ventricular mass (LVM) were evaluated. $70 \%$ had hypertension. Each participant underwent cardiologic assessment and echocardiography to estimate LVM. Sarcopenia was evaluated with Dual X-ray absorptiometry basing on the values of aLM and was defined according to FNIH criteria. Physical frailty was defined on SPPB score between 3 and 9 .

Results: We found a positive and statistically significant correlation between aLM and LVM $(r=0.56 ; p<0.001)$ but also with other structural left ventricular parameters such as end-diastolic $(r=0.53$; $\mathrm{p}<0.001)$ and end-systolic volumes $(\mathrm{r}=0.47$; $\mathrm{p}<0.001)$, end-diastolic $(\mathrm{r}=0.49 ; \mathrm{p}<0.001)$ and end-systolic diameters $(\mathrm{r}=0.46$; $\mathrm{p}<0.001)$, septal $(\mathrm{r}=0.35 ; \mathrm{p}<0.001)$ and posterior wall thicknesses $(r=0.34 ; p<0.001)$ but not with functional left ventricular parameters.

Conclusions: In older people low skeletal muscle mass is associated with low LVM. The mechanisms underlying this relationship remain unclear; however physical activity, insulin resistance, inflammation may mediate this association.

\section{P-820}

Using gait speed as screening tool for frailty in elderly with severe aortic stenosis

Marc Paccalin ${ }^{1}$, Marie-Laure Bureau ${ }^{1}$, Evelyne Liuu ${ }^{1}$, Fabienne Bellarbre $^{1}$, Thomas Brunet ${ }^{1}$, Jean Mergy ${ }^{2}$, Luc Christiaens ${ }^{2}$

${ }^{1}$ Pôle de Gériatrie, Centre Hospitalier Universitaire de Poitiers, Université de Poitiers, Poitiers, France, ${ }^{2}$ Service de Cardiologie, Centre Hospitalier Universitaire de Poitiers, Université de Poitiers, Poitiers, France

Background: The increasing use of Transcatheter Aortic Valve Implantation (TAVI) in elderly and the usefulness of comprehensive geriatric assessment (CGA) warrant continued research to develop an accurate screening tool to identify older patients with severe aortic stenosis (AS) who would benefit the most from geriatric evaluation. Aim of this study was to assess the diagnostic performance of a tool combining short form of Mini Nutritional Assessment (MNA-SF) and gait speed.

Methods: Consecutive patients $\geq 75$ years referred for TAVI in Poitiers hospital between March 2013 and September 2017 were included. Geriatric evaluation and 6-minutes walking test (6MWT) were performed before the procedure. Gait speed was based on 6MWT. CGA was established as the reference test and included information on functional, cognitive and nutritional status; comorbidity; and medication. Two cohorts were established to develop and validate the tools.

Results: We included 280 patients $(46 \%$ women, mean age $85.5 \pm 4.2$ years), 139 in developmental cohort and 141 in validation cohort. CGA was impaired in $221(79 \%)$ patients. The screening tool combining MNA-SF and gait speed (cutoff, $0.83 \mathrm{~m} / \mathrm{s}$ ) showed good sensitivity (83 and $88 \%$, respectively) and specificity (71 and $77 \%$, respectively) in both cohorts. Positive likelihood ratio (LR) and negative LR were respectively 3.0 and 0.2 in first cohort and 3.6 and 0.2 in second cohort. The area under the curve was 0.8 in both cohorts.

Key conclusions: An easily performed screening tool that combines gait speed and MNA-SF could facilitate identification of elderly patients who should require evaluation by geriatrician before valvular therapy.

\section{P-821}

'Geriatric 8 Screening Tool' (G8) in cancer patients: an evidence map

Gabriel Torbahn ${ }^{1}$, Thomas Strau $\beta^{1}$, Cornel C Sieber ${ }^{1}$, Dorothee Volkert ${ }^{1}$, Eva Kiesswetter

${ }^{1}$ Institute for Biomedicine of Aging, Friedrich-Alexander-Universität Erlangen-Nürnberg, Erlangen, Germany

Introduction: The G8 screening tool was developed to identify older cancer patients who could benefit from comprehensive geriatric assessment (CGA). Our aim was to provide a systematic overview of the characteristics and results of primary studies using the G8 in cancer patients.

Methods: A systematic literature search was conducted in 4 databases and 3 study registries. Based on inclusion criteria (primary study with cancer patients, use of G8), titles/abstracts and full-texts were screened and data was extracted by 2 reviewers independently. Conflicts were resolved through discussion or a third person.

Results: Of 4683 identified references, 36 studies reported the use of G8 in cancer patients. Mean patients' age ranged from 72-83 years. Studies varied with regard to cancer type (e.g. specific types, mixed samples) and treatment modality (e.g. chemotherapy, surgery). Impairments in G8 ( $\leq 14$ of max. 17 points) were examined in 33 studies ranging from $26-91 \%$. In 28 studies, the G8 was compared with other (frailty) instruments (e.g. Vulnerable Elders Survey) or CGAs. The latter differed with regard to included instruments (e.g. Mini-Nutritional Assessment) and cut-offs for defining impairment. Prognostic relevance (e.g. mortality, functional decline) of G8 was investigated in 15 studies with varying results depending on outcome and cancer type.

Key conclusions: Since its development in 2012, G8 was used in numerous studies varying in purpose, patient groups, methods and results. More work in well-defined patient groups is needed to demonstrate the benefits of this tool.

Conflict of interest: The project was financially enabled by Nestec S.A.

\section{P-822}

There are no data about geriatric fracture liaison services. A literature review

D. Moral-Cuesta ${ }^{1,2}$, P. Matovelle ${ }^{1}$, T. Alarcón ${ }^{1,2}$, R. MenéndezColino $^{1,2}$, A. Gutiérrez Misis ${ }^{2,3}$, I. Martín-Maestre ${ }^{1}$, Juan Ignacio González-Montalvo $^{1,2}$ 
${ }^{1}$ Geriatrics Department, Hospital Universitario La Paz, Madrid, Spain, ${ }^{2}$ Instituto de Investigación del Hospital La Paz IdiPAZ, Madrid, Spain, ${ }^{3}$ School of Medicine. Autonoma University, Madrid, Spain

Introduction: Fragility hip fracture (HF) is an event in elderly people associated with high morbidity and mortality. Fracture Liaison Services (FLS) are developing one of the best practices in the follow-up of fracture patients in order to prevent new ones. The aim of this review was to know if geriatric aspects of patients and treatments have been collected in the published descriptions of FLS.

Methods: A literature database search was performed electronically via MEDLINE for papers from 2004 until 2018 combining the terms "fracture liaison service" with "hip fracture" and "osteoporosis" including patients aged $\geq 50$ years.

Results: Twenty three studies were included. Most of them were community studies and very heterogenic in number (including 400 to 151.00 patients). They categorized by type of fracture, type of antiosteoporotic treatment, re-fractures or economic matters. Ten studies included antiosteoporotic treatment. Most of them described a nonspecific bone health treatment before the fracture with a range between 7 to $20 \%$ of patients. The main fracture included was HF. Other fractures were wrist or spine. Three studies showed less risk of second fractures, three reported less mortality rates, and four were cost-effective programs. None of them reported the geriatric patients characteristics including functional, cognitive or social factors.

Conclusions: In the best of our knowledge none published studies have included samples of HF patients with data about their clinical, functional and mental characteristics, that are very important keys in order to appropriately manage geriatric patients. New studies are needed to solve this lack of data.

\section{$\mathbf{P - 8 2 3}$}

Functional status of geriatric patients admitted to an internal medicine and cardiology unit in an acute and planned setting

Elzbieta Kozak-Szkopek ${ }^{1}$, Judyta Samul ${ }^{2}$, Bartosz Blachucki ${ }^{3}$, Piotr Pruszczyk $^{2}$, Katarzyna Broczek ${ }^{1}$

${ }^{1}$ Department of Geriatrics, Medical University of Warsaw, Warsaw, Poland, ${ }^{2}$ Department of Internal Medicine and Cardiology, Medical University of Warsaw, Warsaw, Poland, ${ }^{3}$ Department of Geriatrics and Department of Internal Medicine and Cardiology, Medical University of Warsaw, Warsaw, Poland

Introduction: Functional status assessment is a useful component of medical examination of geriatric patients. The aim of the study was to evaluate functional status, comorbidities and hospitalization time in patients admitted in an acute and planned setting to the Department of Internal Medicine and Cardiology.

Methods: Observational single-center study. Individuals aged $\geq 60$ years, with VES-13 scale score $\geq 3$, hospitalized between December 2016 and December 2017 were included. Socio-demographic and medical data were collected at the baseline. The evaluation of functional status was performed by a geriatric team after initial treatment. Standardized functional assessment scales were used including ADL, IADL, Barthel and Tinetti scales. Results were analyzed in terms of planned and acute hospitalizations.

Results: 359 older adults were included, 59\% women. Mean age was 79 ( \pm 8 ) years (range: 60-98). Mean BMI was 27.5. Most admissions were due to cardiovascular causes, $59 \%$ were acute and the majority of patients had multimorbidities and polypharmacy. Mean hospitalization time was $8 \pm 7$ days (range: 1-42 days). The percentage of independent patients was $62 \%$ according to ADL (score 5-6) and 56\% according to Barthel scale (score $\geq 85$ ), patients with severe functional impairment comprised 25\% (ADL score $\leq 2$ ) and $18 \%$ (Barthel $\leq 20$ ), respectively. Differences in functional scores among patients admitted in acute and planned setting were observed, including risk of falls and a need for 24-hour care after discharge.

Conclusions: Incorporating functional assessment to general medical examination in internal medicine wards, especially in acute setting, may help to choose adequate treatment and disability prevention strategies.

\section{P-824}

Cognitive impairment and depressive symptoms in geriatric patients admitted to an internal medicine and cardiology unit

Katarzyna Broczek ${ }^{1}$, Monika Szymanska ${ }^{1}$, Bartosz Blachucki ${ }^{2}$, Judyta Samul ${ }^{3}$, Elzbieta Kozak-Szkopek ${ }^{1}$, Piotr Pruszczyk ${ }^{3}$

${ }^{1}$ Department of Geriatrics, Medical University of Warsaw, Warsaw, Poland, ${ }^{2}$ Department of Geriatrics and Department of Internal Medicine and Cardiology, Medical University of Warsaw, Warsaw, Poland, ${ }^{3}$ Department of Internal Medicine and Cardiology, Medical University of Warsaw, Warsaw, Poland

Introduction: In 2017, WHO recognized dementia and cognitive impairment as a public health priority. It is well known that cognitive impairment as well as mood disorders influence quality of life and adherence to treatment. The aim of the study was to assess cognitive status and depressive symptoms in geriatric patients hospitalized in the Department of Internal Medicine and Cardiology.

Methods: Observational single-center study. Patients aged $\geq 60$ years, hospitalized during 1 year period (2016-2017) were included. General medical data were collected. All subjects underwent Comprehensive Geriatric Assessment performed by a geriatric team. Evaluation of cognitive status, delirium and depressive symptoms was performed after initial treatment. Standardized assessment scales (MMSE, GDS-15) were used. The results were analyzed retrospectively in terms of functional status (ADL, IADL), hospitalization time and admission type (acute vs planned). The coexistence of depressive symptoms and cognitive decline was analyzed.

Results: 359 patients were examined (59\% women). Mean age was $79( \pm 8)$ years. Six in ten admissions were acute. Mean hospitalization time was $8 \pm 7$ days (range: $1-42$ ). Average MMSE score was 24.9. No cognitive impairment (MMSE 28-30) was diagnosed in $44 \%$ ( $\mathrm{n}=157)$ subjects, while $8 \%$ had severe cognitive impairment (MMSE 0-9). Delirium was observed in $12 \%(\mathrm{n}=25)$ of acute admissions. Depression screening was performed in 340 subjects: $21 \%$ presented mild and $3 \%$ severe depressive symptoms. Further relationship between mental and functional status was evaluated.

Conclusions: Cognitive impairment and depressive symptoms are prevalent in hospitalized older adults. Geriatric teams may help to evaluate mental status and formulate care plans.

\section{P-825}

Vision, hearing and the prevalence of ophthalmic diseases in the Polish population aged 65 years and above with relation to coexisted cardiovascular diseases
B. Wizner ${ }^{1}$, E. Klimek ${ }^{1}$, A. Skalska ${ }^{1}$, T. Grodzicki ${ }^{1}$, on behalf of PolSenior Study Group
${ }^{1}$ Department of Internal Medicine and Gerontology, Jagiellonian University Medical College, Krakow, Poland 
Introduction: Eyesight and hearing disorders are one of the main causes of disability in Poland. The aim of the study was to present data on sensory organ dysfunctions as well as ophthalmic diseases in older people.

Methods: The cross-sectional, population-based survey PolSenior (2007-2011) was performed on random sample of older people to assess medical and non-medical aspects of aging in Polish elderly. Finally 4979 subjects aged 65 years and more was included to the study. Based on standardized interview with respondents, the history of glaucoma, cataracts and macular degeneration were reported. Vision assessment involved examination using the Snellen tables, reading distance, watching television, counting fingers and assessing the sense of light. Hearing assessment was established during the interview and using whisper test.

Results: Mean age $( \pm \mathrm{SD})$ of the subjects was $78.8 \pm 8.4$ years, $51 \%$ were women. Visual impairment was found in nearly $50 \%$ of subjects-moderate visual impairment was most common, blindness was diagnosed in $2 \%$ of subjects. Hearing impairment was observed in $30 \%$ of respondents-total deafness or significant impairment of hearing was recognized in $1.3 \%$ of respondents. Overall, the prevalence of cataract, glaucoma and macular degeneration was respectively: $29 \%, 7 \%$ and $2 \%$. In subjects with history of hypertension or heart failure the hearing impairment occurred significantly often. Furthermore, in these patients ophthalmic disorders were more common.

Conclusions: The vision and hearing assessment should be routine procedure in geriatric evaluation of older patients, especially with coexisted cardiovascular diseases, for preserve the better functionality in the later life.

\section{P-826}

\section{Chronic pain and its effect on quality of life in elderly patients}

Suheyla Coteli ${ }^{1}$, Olgun Deniz ${ }^{1}$, Rana Tuna Dogrul ${ }^{1}$, Cagatay Cavusoglu ${ }^{1}$, Ali Oncul ${ }^{1}$, Muhammet Cemal Kizilarslanoglu ${ }^{1}$, Berna Goker $^{1}$

${ }^{1}$ Gazi University School of Medicine, Department of Internal Medicine, Division of Geriatrics, Ankara, Turkey,

Introduction: Chronic pain is the most commonly reported symptom in elderly patients. In this study, it was aimed to elucidate the features of chronic pain and its effect on quality of life, relation with comprehensive geriatric assessment and management in this age group.

Materials and methods: Ninety-two geriatric outpatients were taken into this study. A comprehensive geriatric assessment was performed. The short-form McGill Pain Questionnaire (SF-MPQ), The WongBaker FACES Pain Rating Scale (WBS) and Visual Analog Scale for Pain(VAS Pain) were used for the evaluation of pain intensity. Assessment of quality of life was performed by using the three-level version of the EuroQol-5 Dimension (EQ-5D-3L) and the Nottingham Health Profile (NHP) questionnaires.

Results: The median age of the patients was 71(min-max: 65-87) years and $56.5 \%$ of the patients was female. Chronic pain was observed in $63 \%$ of the patients. Katz Activities of Daily Living, Mini Mental State Examination, the Geriatric Depression scale-short form (GDS-SF), EQ-5D-3L and NHP scores in patients with chronic pain were worse than those without( $p<0.05$ for all parameters). Significant correlation was found between SF-MPQ and the clock drawing test $(r=-0.317)$, GDS-SF $(r=0.387)$, EQ-5D-3L $(r=0.487)$, emotional reaction $(r=0.464), \quad$ sleep $(r=0.453)$, social isolation $(r=0.331)$, physical abilities $(r=0.377)$ and energy $(r=0.377)$ subsections of NHP in patients with chronic pain $(\mathrm{p}<0.05$ for all parameters). It was observed that $93 \%$ of the patients received any pharmacotherapy and paracetamol $(67.3 \%)$ and non-steroid anti-inflammatory (NSAID) $(63.5 \%)$ drugs were the most preferred medical treatments. NSAID was the best-responding medication by near the half of the patients.

Conclusion: In this study, it was observed that chronic pain is an important health problem affecting cognitive functions, mood, functioning, sleep and social life in elderly patients. It is necessary to take precautions to reduce the frequency of use of NSAIDs, especially in geriatric patients, which have a potential for serious side effects.

\section{P-827}

Relationship between common geriatric syndromes and inappropriate medication use among older adults

Pinar Kucukdagli ${ }^{1}$, Gulistan Bahat ${ }^{1}$, Ilker Bay ${ }^{2}$, Ozlem Yilmaz ${ }^{1}$, Meryem Merve Oren ${ }^{3}$, Cihan Kilic ${ }^{1}$, Banu Ozulu Turkmen ${ }^{1}$, Mehmet Akif Karan ${ }^{1}$

${ }^{1}$ Istanbul University/Istanbul Faculty of Medicine/Division of Geriatrics, Istanbul, Turkey, ${ }^{2}$ Istanbul University/Istanbul Faculty of Medicine/Internal Medicine, Istanbul, Turkey, ${ }^{3}$ Istanbul University/ Istanbul Faculty of Medicine/Public Health, Istanbul, Turkey

Introduction: Optimizing drug therapy is an important part of caring for elder population. Proper prescribing is a dynamic process; including age-related metabolic changes (eg, renal and hepatic clearance),appropriate dosage for the patient's physiologic status, side effects and toxicity. We aimed to assess the relationship between inappropriate medication use and geriatric syndromes in our study.

Methods: Older patients(aged $\geq 65$ years) admitted to the outpatient clinic of a university hospital were retrospectively evaluated for PIM with Beers 2012. Age, sex, chronic diseases and number of drugs; functional, cognitive and nutritional statuses, presence of chronic pain, depression, falls, constipation, polypharmacy, urinary and fecal incontinence were studied with regression analysis as possible factors related to PIM.

Results: The study included 667 participants $(63.1 \%$ women, mean age $77.6 \pm 6.3$ years). The mean number of drugs was $6.1 \pm 3.4$. PIM prevalence was $33.3 \%$ detected by Beers 2012 criteria. There was no statistically significant difference in terms of sex $(p>0.05)$ or age ( $>0.05$ for both). Falls (OR 3.17, 95\% CI 2.12-4.74, $\mathrm{p}<0.001$ ), malnutrition (OR 0.66, 95\% CI 0.50-0.87, p = 0.003), constipation (OR $2.04,95 \%$ CI 1.20-3.47, p = 0.008), polypharmacy (OR 4.27, 95\% CI 2.82-6.45, p < 0.01) and depression (OR 2.58, $95 \%$ CI $1.70-3.92, \mathrm{p}<0.001)$ were independently associated with the use of at least one inappropriate medication use.

Conclusion: In our study; falls, malnutrition, constipation, polypharmacy and depression were independently associated with inappropriate medication use detected by Beers 2012. These conditions are associated with poor outcomes such as hospitalization, disability and mortality. Clinicians should examine and make an effort to reduce the PIM rates. Many inappropriate drug use-related problems can be prevent by proper prescribing.

\section{P-828}

Association between the impaired nutritional status and frailty in acute hospitalised elderly patients

Maria Amasene ${ }^{1}$, Iñaki Echeverria ${ }^{2}$, Miriam Urquiza $^{2}$, Ane Errasti ${ }^{2}$, Cristina Bermudez-Ampudia ${ }^{3}$, Mikel Aldamiz ${ }^{4}$, Idoia Labayen ${ }^{5}$, Ariadna Besga ${ }^{6}$

${ }^{1}$ Department of Pharmacy and Food Science. Faculty of Pharmacy, University of the Basque Country (UPV/EHU), Vitoria-Gasteiz, 
Spain, ${ }^{2}$ Department of Physiology, Faculty of Medicine and Dentistry, University of the Basque Country (UPV/EHU), Leioa, Spain, ${ }^{3}$ Bioaraba Health Research institute, Methodology and Statistics Unit, Vitoria-Gasteiz, Spain, ${ }^{4}$ Department of Internal Medicine, OSI Araba University Hospital, Vitoria-Gasteiz, Spain, ${ }^{5}$ Faculty of Health Science, Public University of Navarre (UPNA), Pamplona, Spain, ${ }^{6}$ Department of Internal Medicine, OSI Araba University Hospital, Vitoria-Gasteiz, Institute of Mental Health Research, CIBERSAM, Vitoria, Spain

Objective: This study aimed to explore the association between the impaired nutritional status and frailty in acute hospitalised elderly patients by using two tools, the MNA ${ }^{\circledR}$-SF (Mini Nutritional Assessment ${ }^{\circledR}$ short-form) and the FRIED frailty phenotype assessment.

Method: Acute hospitalised, community-dwelling elderly patients were recruited at internal medicine wards in Vitoria. All subjects underwent a standard protocol including: clinical, nutritional, functional and a cognitive evaluation. MNA-SF was used to assess malnutrition ( $<7$ points) and patients at risk of malnutrition ( 8 to 11 points). By using FRIED, subjects were classified as frail, pre-frail or robust. A factor analysis was applied to identify overlaps between the MNA $^{\circledR}$-SF and Fried items. Internal consistency of different dimensions was assessed by using Chi square test and logistic regression. Results: Of the total sample $21.4 \%$ were malnourished, whereas the $49 \%$ were at risk of malnutrition. In regard to frailty assessment, $74.12 \%$ and $25.87 \%$ of the total sample were found to be frail and prefrail, respectively. Among patients at risk of malnutrition $79.6 \%$ were frail and the $20.4 \%$ were prefrail. According to malnourished patients $95.2 \%$ were frail and $4.8 \%$ were prefrail.

Conclusions: The quickly and easily administered MNA-SF appears to be a good tool for predicting under-nutrition in elderly hospitalised people. This study underlines the association and the overlap between frailty and impaired nutritional status.

\section{P-829}

Geriatric assessment in decision-making process in older patients with breast cancer: the ELCAPA cohort study

Corsin Lola ${ }^{1}$, Boutin Emmanuelle ${ }^{2,3}$, Assaf Elias ${ }^{4}$, Belkacemi Yazid ${ }^{5}$, Bosc Romain $^{5}$, Bigorie Veronique ${ }^{6}$, Caillet Philippe ${ }^{2,3}$, Tournigand Christophe $^{4}$, Touboul Cyril ${ }^{7}$, Allain Myléne ${ }^{2}$, Rollot Trad Florence ${ }^{8}$, Laurent Marie ${ }^{2,3}$

${ }^{1}$ AP-HP, Hôpitaux universitaires Henri Mondor, Département de médecine interne et gériatrie, France, ${ }^{2}$ UPEC, DHU A-TVB, IMRB, EA 7376 CEpiA (Clinical Epidemiology And Ageing Unit), Créteil, F-94000, France, ${ }^{3}$ AP-HP, Hŏpitaux universitaires Henri Mondor Service de santé publique, France, ${ }^{4}$ AP-HP, Hŏpitaux universitaires Henri Mondor Service d'oncologie médicale, France, ${ }^{5}$ AP-HP, Hŏpitaux universitaires Henri Mondor, Service de radiothérapie, France, ${ }^{6}$ AP-HP Hŏpitaux universitaires Henri Mondor, Service de chirurgie plastique, France, ${ }^{7}$ Service de gynécologie obstétrique, Centre Hospitalier Intercommunal Créteil, ${ }^{8}$ Institut Curie, France

Introduction: Our objective was to assess the change of cancer treatment plan after geriatric assessment in older patient with breast cancer and factors associated.

Methods: Consecutive patients aged $\geq 70$ years with breast cancer referred to geriatricians for pre-therapeutic Comprehensive Geriatric Assessment (CGA) in two teaching hospitals were included (2014-2017). Main endpoint was change of cancer treatment in multidisciplinary meeting compared to initial proposal (increase, decrease, change of treatment modality). Multivariate logistic regression was used to assess factors associated with treatment change.

Results: 302 patients were included (Median age 81 [Quartile 1 (Q1): 76-Q3: 85] years, $86 \%$ with invasive breast cancer, $36 \%$ with metastasis. $48 \%$ had Activity of daily Living (ADL) $<6,28 \%$ Mini Mental Score (MMS) $<24$ and 33\%, malnutrition. Among them, $34.8 \%$ of patients had a change of cancer treatment decision $(22.6 \%$ decrease, $6.8 \%$ increase, $5.4 \%$ a switch of treatment modality) mainly driven by hormone therapy change. By multivariate analysis, increased age was associated with change of treatment decision, for surgery (OR 1.07 [1.02-1.11] p = 0.003) for chemotherapy (OR 1.08 [1.04-1.13] $\mathrm{p}=0.003)$, and for radiotherapy (OR 1.07[1.03-1.11] $\mathrm{p}=0.002)$. Mini Mental Status < $24($ OR 0.5[1.03-1.11] p = 0.03) was associated with treatment change for radiotherapy decision. Impaired functional status was associated with change of treatment decision for surgery $(1.54[1.07-1.18] \mathrm{p}=0.003)$ and for chemotherapy (OR $1.56[1.12-1.76](\mathrm{p}=0.01)$.

Conclusion: Age, functional and cognitive status were associated with cancer treatment change in older patients with breast cancer.

\section{P-830}

Comparison of the active standing test and head-up tilt test for orthostatic hypotension diagnosis in older adults

Ali Ekrem Aydin ${ }^{1}$, Suleyman Emre Kocyigit ${ }^{1}$, Pinar Soysal ${ }^{2}$, Ahmet Turan Isik ${ }^{1}$

${ }^{1}$ Center for Aging Brain and Dementia, Department of Geriatric Medicine, Faculty of Medicine, Dokuz Eylul University, Izmir, Turkey, ${ }^{2}$ Kayseri Education and Research Hospital, Geriatric Center, Kayseri, Turkey

Background: Correct evaluation of orthostatic hypotension $(\mathrm{OH})$ is crucial in geriatric practice, since $\mathrm{OH}$ is associated with mortality and morbidity. This study aimed to determine the accuracy of the active standing test (AST) for diagnosis of $\mathrm{OH}$ in older adults.

Methods: A comprehensive geriatric assessment (CGA) including AST and head-up tilt table test (HUT) was performed in 424 older adults. Blood pressure (BP) measurements performed with both the manual auscultatory technique and BP Monitor during AST and HUT. Orthostatic changes were assessed separately for 3rd minutes. HUT is used as the gold standard test.

Results: The prevalence of OH during HUT and AST was $19 \%$ and $15 \%$, respectively. In patients with OH during HUT, the frequency of balance disorder and the presence of symptoms during the test were higher $(\mathrm{P}<0.05)$. Comparison of the groups according to CGA measurements revealed significant differences in terms of balance function and up-go test in patients with $\mathrm{OH}$ only during HUT $(\mathrm{P}<0.05)$, but not during AST $(\mathrm{P}>0.05)$. The sensitivity, specificity, positive predictive value, and negative predictive values of AST were $32.5 \%, 89.5 \%$, $41.9 \%$, and $85.1 \%$ respectively, according to HUT.

Conclusion: It was demonstrated that the accuracy of evaluating orthostatic BP changes by AST has never reached that of HUT, and that manual auscultatory measurements have overdiagnosed the $\mathrm{OH}$ during both AST and HUT. Furthermore, the results suggested that orthostatic BP changes during HUT were higher clinical significance than that of AST. Therefore, it is important that HUT should be included in daily geriatric practice, to avoid overdiagnosis of $\mathrm{OH}$ in older adults. 


\section{P-831}

Evolution of functional status in older patients after transcatheter aortic valve implantation (TAVI)

Dominic Bertschi ${ }^{1}$, André Moser ${ }^{2}$, Stefan Stortecky ${ }^{3}$, Marcel Zwahlen ${ }^{4}$, Peter Wenaweser ${ }^{3}$, Stephan Windecker ${ }^{3}$, Thierry Carrel $^{5}$, Andreas Schoenenberger ${ }^{1}$, Andreas Stuck ${ }^{1}$

${ }^{1}$ Department of Geriatrics, Bern University Hospital, and University of Bern, Switzerland, ${ }^{2}$ Department of Geriatrics, Bern University Hospital, and University of Bern, Switzerland and Institute of Social and Preventive Medicine, University of Bern, Switzerland,

${ }^{3}$ Department of Cardiology, Bern University Hospital, and University of Bern, Switzerland, ${ }^{4}$ Institute of Social and Preventive Medicine, University of Bern, Switzerland, ${ }^{5}$ Department of Cardiovascular Surgery, Bern University Hospital, and University of Bern, Switzerland

Background: The aim of this study was to assess the course and prediction of functional status in older patients undergoing TAVI. Previous studies in TAVI patients used disease-specific instruments, such as the Kansas City Cardiomyopathy Questionnaire (KCCQ), which do not measure all-cause limitation of functional status.

Methods: In this prospective cohort study, functional status was measured with the Katz BADL (basic activities of daily living) index in 278 surviving patients $\geq 70$ years before and one year after TAVI. We used logistic regression models to investigate the association of baseline factors with BADL decline.

Results: Complete one-year follow-up data on BADL were available in all surviving patients. One year after TAVI, functional status declined in $28(10.1 \%)$ of the 278 surviving patients. In the subgroup of 32 patients with impaired BADL function at baseline, functional status improved in $17(53.1 \%)$ patients. Statistically significant baseline predictors of BADL deterioration were a Timed Get Up and Go Test (TUG) $\geq 20 \mathrm{~s}$ $(\mathrm{p}=0.01)$, and a Mini Mental State Exam (MMSE) $<26$ points $(p=0.01)$. Conventional cardiac risk scores (EuroSCORE, STS score) did not show an association with functional status decline.

Conclusions: To our knowledge, this is the first large cohort study to report on long-term follow-up of functional status in TAVI patients. Geriatric assessment parameters, but not conventional cardiac risk factors, predicted functional status course 1 year after TAVI. Early identification of at-risk patients may help to target interventions aimed at the prevention of functional status decline in these patients.

\section{P-832}

Comprehensive geriatric assessment in older patients undergoing cardiac surgery

Myriel López Tatis ${ }^{1}$, José María Gonzalez Santos ${ }^{1}$, Francisco Javier López Rodríguez ${ }^{1}$, María Elena Arnaíz García ${ }^{1}$, Ramón Adolfo Arévalo Abascal ${ }^{1}$, Ana María Barral Varela ${ }^{1}$, Marianna Javier González $^{2}$, Carlos Amorós Rivera ${ }^{1}$

${ }^{1}$ Hospital Clínico Universitario de Salamanca, Salamanca, Spain, ${ }^{2}$ Leeds Teaching Hospitals NHS Trust, Leeds, UK

Introduction: The rapid growing elderly population and the demand for cardiac surgery in this group of patients is a medical challenge. Objective: to describe the population of patients over 70 years old undergoing cardiac surgery at a tertiary hospital by using a comprehensive geriatric assessment (CGA) and evaluate the impact of frailty on their outcomes.

Methods: We performed a prospective analysis of data including 115 patients evaluated in the preoperative clinic for cardiac surgery from 10/2017-05/2018. All patients were assessed by a geriatrician.
Results: mean age was $76 \pm 2.3$ years, $62.3 \%$ were male, $74.9 \%$ had hypertension, $34.7 \%$ diabetes, $48.4 \%$ atrial fibrillation (AF) $52.8 \%$ were in New York Heart Association (NYHA) class II. Mean Euroscore II (ESII) was $3.56 \pm 3.2 \%$. Using the FRAIL scale $55.6 \%$ were frail, with Fried $43.2 \%$ were frail. There were significant differences, $\mathrm{p}(0.000)$ between the two frailty scales. Female sex, AF and NYHA were associated to frailty: $\mathrm{p}(0.001), \mathrm{p}(0.003)$ and $\mathrm{p}(0.01) .8 .2 \%$ had malnutrition, $25.6 \%$ depression, $98 \%$ were independent by Katz scale, mean Lawton scale was $6 \pm 1.45$ points, mean Montreal Cognitive Assessment (MOCA) $20.4 \pm 4.47$. The $56.53 \%$ of patients have undergone surgery. 30-day mortality is $5.7 \%$. ESII $\mathrm{p}(0.003)$, vitamin D $\mathrm{p}(0.03)$, serum albumin $\mathrm{p}(0.04)$, tricuspid valve replacement $\mathrm{p}(0.000)$, Fried $\mathrm{p}(0.03)$ and extracorporeal circulation time $\mathrm{p}(0.04)$ were mortality predictors. $50 \%$ of the frail patients who underwent surgery showed an improvement in their frailty status at follow-up. Conclusions: Frailty negatively influences mortality after surgery. Cardiac surgery improves frailty in survivors. Female and AF are strongly associated with frailty.

\section{P-833}

Time to surgery and multidimensional prognostic index (MPI) in older patients with hip fracture

C. Musacchio ${ }^{1}$, M. Razzano ${ }^{1}$, R. Raiteri ${ }^{1}$, A. Del Rio ${ }^{1}$, D. Torriglia ${ }^{1}$, M. Stella ${ }^{2}$, A. Barone ${ }^{1}$, M. Puntoni ${ }^{3}$, G. Siri ${ }^{3}$, A. Pilotto ${ }^{1}$

${ }^{1}$ Department of Geriatric Care, OrthoGeriatrics \& Rehabilitation, Frailty Area, E.O. Galliera Hospital, Genoa, Italy, ${ }^{2}$ Orthopedic Unit, Department of Geriatric Care, OrthoGeriatrics \& Rehabilitation, Frailty Area, E. O. Galliera Hospital, Genoa, Italy, ${ }^{3}$ Clinical Trial Unit, Scientific Directorate, E.O. Galliera Hospital, Genoa, Italy

Introduction: Time to surgery is one of the major modifiable risk factors influencing adverse outcomes in hip-fracture patients. National guidelines and recommendations have been introduced which advocate specific periods in which surgery should be performed i.e. within $24-48 \mathrm{~h}$. The aim of this study was to evaluate whether the Multidimensional Prognostic Index (MPI) is a predictor of time to surgery in older patients with hip fractures.

Methods: Patients admitted to the OrthoGeriatrics Unit for hip fracture of Genoa (Italy) underwent CGA-based MPI assessment, including functional (ADL,IADL), cognitive (SPMSQ), nutrition (MNA-SF), risk of pressure scores (Exton-Smith Scale), Comorbidity (CIRS), drugs and cohabitation and were divided in MPI class. The mean waiting time to surgery was 3 days. We adopted linear regression analysis using time to surgery (log-transformed) as dependent variable and MPI as predictor, adjusting for age, gender, delirium, anemia and infections.

Results: 247 hospitalized patients were recruited (mean age $85 \pm 6.9$ years, females $=84 \%$ ) and classified according to the MPI at admission: MPI-2 (moderate risk of mortality) $=103$ patients $(42 \%)$ and MPI-3 (severe risk of mortality) $=144$ patients $(58 \%)$. A $20 \%$ (95\% CI: $1.0-1.44)$ longer waiting time to surgery was detected in patients with severe MPI 3 vs. MPI $2(\mathrm{p}<0.05)$, adjusted for all factors considered. Furthermore, delay to surgery was significantly influenced by infections $(95 \%$ CI $1.19-1.71, \mathrm{p}<0.001)$ and delirium (95\% CI 0.98-1.49, p = 0.07).

Conclusion: This study suggests that MPI is an independent and significant predictor of the waiting time to surgery in older patients with hip fracture. 


\section{P-834}

Avoiding unplanned admission after hospital stay by a phone call

Peter Johnson ${ }^{1}$, Annica Rosenborg ${ }^{1}$

${ }^{1}$ Södertälje sjukhus, Södertälje, Sweden

Background: A large proportion of the healthcare resources in Stockholm County Council (SLL) are dedicated to emergency, unscheduled care ${ }^{1}$. Likewise, it is known that the risk of recurrent emergency hospital stays increases with increasing age. Older people have, to a greater extent, more hospital stays classified as avoidable than other patient groups. Good coordination and continuity is a prerequisite for these patients and their relatives to feel safe and involved. Research in the field shows that safe patients feel to a lesser extent that they are in urgent need. At the same time, statistics from 2015 show that 1 in 7 patients were unplanned admitted to hospital within 30 days, of which half occurred within 1 week. $30 \%$ had not had a care contact between discharge and readmission (2). Statistics also showed that Södertälje Hospital had a higher proportion of readmission than other emergency hospitals in SLL 2015. Within the geriatric sector, the proportion of readmissions fell within 30 days at $12.9 \%$. In the fall of 2015 , therefore, the geriatrics of STS, including three emergency care services with a total of 53 hospital beds, were decided to seek project funding to actively work to reduce the proportion of readmission through prevention work through the SVEA project, Combined Care through Uniform Work, initiated by Health and Safety health care administration within the Stockholm City Council.

Purpose: Through targeted phone follow-up to patients who are printed from geriatric hospital care, follow up on care and solve problems, thus optimizing the patient's situation and preventing readmission to hospital. In the healthcare team, visualize "the fragile patient" and increase knowledge and communication about factors that cause avoidable readmission, but not caught by our established trials estimates.

Method: Statistics from previous projects in the geriatrics show that it is neither meaningful nor workable to call all patients discharged from the geriatric care. Therefore, it was decided that all patients during their stay would be assessed according to a form developed at Brommageriatriken "Risk assessment for readmission". In the assessment form, the team answers yes or no whether the patient has the following; heart failure, COPD, pain, fall hazard or previous cases, more than two hospital stays in the past year, polypharmacy, anxiety and / or depression, cognitive impairment and risk medicines, such as Warfarin, NSAIDs, sedatives and / or neuroleptics. assessment is done by the entire care team to simultaneously visualize the fragile patient and increase knowledge about these. The patients who were considered to be fragile ( $\geq 4$ points) were booked for a telephone call with a doctor within 3-5 days after printing. The idea of the conversation is that it will focus on completing the planning done at the time before discharge and that the patient can handle his health condition as planned at home.

Results: The result shows that we have lowered the percentage of readmission from $12.9 \%$ to $10.1 \%$ between 2015 and 2016. This represents a decrease of about $22 \%$. Statistics show that all patients booked for follow-up call are called up of wich about $85 \%$ are dialed within 5 days of discharge. Approximately $39 \%$ of all patients who treated at hospital ward are called up, which corresponds to approximately 3-4 calls per department and doctor per week. A very small percentage of patients who are not followed up with telephone calls are reinstated, indicating that we capture the most fragile elderly in our assessment form. However, statistics show that our patients with dementia are to a small extent followed up by telephone calls, as they rarely reach up to 4 points, but at the same time have a higher readmission rate than other patients. Unfortunately, despite good results, the knowledge and communication in the healthcare team has not increased around these patients. The assessment is usually done by the doctor himself, without any remaining members of the team and the knowledge exchange and communication has not improved. Conclusions: By simple assessment, we can catch the "fragile elderly" patient group and with a follow-up phone call after discharge prevent readmission. On the other hand, we can see that the demented patient is not caught in the estimation and is still reintroduced to a greater extent. The challenge is to find a way to capture this patient group as well, and reduce the readmission rate. The assessment of the patient in connection with the round in the ward did not become the team-friendly task the project group hoped for. Because the doctor is the one who calls the patient after discharge, it also became the one who usually performed the assessment alone. In order to visualize "the fragile patients" in the care team and to increase knowledge and communication, the team probably needs support and resources in the introduction of new working methods.Application and dissemination of results. The result of the project has resulted in the fact that we now have telephone follow-up after geriatric end-care as a routine for our three geriatric care departments. The statistics for 2017 show the same low figures as 2016 .

\section{References:}

Förbättra livskvaliteten och minska antalet akuta inläggningar för de mest vårdtunga och utsatta patienterna inom SLL: Interimsrapport från AHS-projektet, 2010. Stockholm: Hälso- och sjukvårdsförvaltningen(HSF), Stockholms läns landsting; 2010.2.Stäck, P. (2012). Risken för akut återinläggning kan förutsägas. Läkartidningen11:27

\section{P-835}

\section{Polypharmacy and related factors}

\section{Pinar Kucukdagli ${ }^{1}$}

${ }^{1}$ Istanbul University/Istanbul Faculty of Medicine/Division of Geriatrics, Istanbul, Turkey

Introduction: Polypharmacy is also defined as the use of multiple medications and generally ranges from 5 to 10 medications. When older people compared with younger individuals, they tend to have more chronic diseases and more precriptions for these conditions.We aimed to assess the relationship between polypharmacy and other factors.

Methods: 1107 individuals $\geq 60$ years of age admitted to Istanbul Medical School Geriatrics outpatient clinic for the first time the period between 2013-2016 were enrolled to study. We used The International Association of Nutrition and Aging's FRAIL scale. Polypharmacy was defined as the use of five and more medications. Patients were asked about their falls, urinary incontinence, chronic pain, activities of daily living (ADL), instrumental activities of daily living (IADL), and assessed about their nutritional status by Mini Nutritional Assessment (MNA).

Results: 1107 patients were analyzed with a comprehensive geriatric assessment. The sample was composed of women (66.8\%) and men $(33.2 \%)$ with mean age of $78.5 \pm 5.7$ years. Prevalence of polypharmacy was $16 \% \quad(\mathrm{n}=179)$.Univariate and multivariate regression analysis were performed to investigate the association between polypharmacy and other factors. In multivariate analysis polypharmacy was found independently associated with urinary incontinence $(\mathrm{p}=0.022)$ and malnutrition $(\mathrm{p}=0.028)$.

Conclusions: Polypharmacy is a common problem among older adults; which is an independent risk factor for inappropriate medication use and adverse events. In our study malnutrition and urinary incontinence were found independently associated with 
polypharmacy. Clinicians should be aware of polypharmacy and prevent patients from poor outcomes associated with this condition.

\section{Area: Delirium \\ P-836 \\ The delirium recovery programme (DRP): evaluation of an innovative service providing cognitive enablement at home following hospital admission}

Tammy Angel ${ }^{1}$, Gemma Holland ${ }^{1}$, Adriana Vyse ${ }^{1}$, Adriana Jakupaj ${ }^{1}$, Mark Mandell ${ }^{2}$

${ }^{1}$ West Hertfordshire Hospitals NHS Trust, ${ }^{2}$ Hertfordshire Partnership University NHS Foundation Trust

Introduction: Delirium in acute hospitals can affect up to $60 \%$ of elderly in-patients. We have learnt from many studies how to reduce risk of delirium and yet this acute confusional state is common among s older adults in hospital. This study designed a 21 day pathway to provide cognitive enablement at home to support rapid recovery from delirium in a familiar home setting. Patients with delirium were identified and treated by consultant physician and psychiatrist. An individual care plan was produced after discussion with patient and family. Patients were discharged onto a daily virtual ward round, with a 24-hour live in carer and reviewed at day 4 by Occupational Therapist (OT) and day 7 by OT and social worker. Day 10 patients attended ambulatory care for joint review of physical and mental health. Patients received a financial assessment on discharge from the DRP.

Results: 105 patients have been discharged onto the DRP: all have improved functionally. 70 patients $(66 \%)$ have remained home: 52 with POC (74\%) and 18 no POC (26\%). Only 20 patients (19\%) have required placement. Only 4 patients $(4 \%)$ were readmitted.

Conclusion: Highly supported early discharge home from hospital for patients with delirium is effective and enables patients to regain cognitive function and to continue with good quality of life. It is cost effective to provide time-limited of $24 \mathrm{~h}$ care for people who would otherwise have required placement. Strategies to help regain cognitive function may be as important as preventative measures to support people who develop delirium.

\section{$\mathbf{P - 8 3 7}$}

CAM-ICU may not be the optimal screening tool for early delirium screening in older Emergency Department patients, a prospective cohort study

Jacinta Lucke ${ }^{1}$, Jelle de Gelder ${ }^{2}$, Laura Blomaard ${ }^{3}$, Jaap Fogteloo ${ }^{4}$, Jelmer Alsma ${ }^{5}$, Stephanie Schuit ${ }^{5}$, Anniek Brink ${ }^{5}$, Bas de Groot ${ }^{1}$, Gerard Jan Blauw ${ }^{3}$, Simon P. Mooijaart ${ }^{3}$

${ }^{1}$ Department of Emergency Medicine, Leiden University Medical Center, Leiden, The Netherlands, ${ }^{2}$ Department of Gerontology and Geriatrics, Leiden University Medical Center, Leiden, The Netherlands, ${ }^{3}$ Department of Gerontology and Geriatrics, Leiden, The Netherlands

Objective: Delirium is a frequent problem among older patients in the Emergency Department (ED) and early detection is important to prevent its associated adverse outcomes. Several screening tools for delirium have been proposed for the ED, such as the Confusion Assessment Method-Intensive Care Unit (CAM-ICU). Previous validation of this tool for use in the ED showed varying results, possibly because they were administered at different or unknown time points.
The aim was to study incidence of delirium in older ( $\geq 70$ years) ED patients using the CAM-ICU.

Methods: Prospective cohort study, in one tertiary care and one secondary care hospital in The Netherlands. Patients aged 70-years and older attending the ED were included. Delirium screening was performed within $1 \mathrm{~h}$ after ED registration using the CAM-ICU. The 6-Item Cognitive Impairment Test (6-CIT) was determined for comparison, using a cut-off point of $\geq 14$ points indicating possible delirium, which has previously associated with the presence of delirium using gold standard assessment.

Results: A total of 997 patients were included in the study, with a median age of 78 years (interquartile range $74-84$ ). Delirium as assessed with CAM-ICU was positive in only $13(1.3 \%)$ patients. 95 $(9.5 \%)$ patients had $6-\mathrm{CIT} \geq 14$.

Conclusions: We found a delirium incidence of $1.3 \%$ using the CAM-ICU, which was much lower than the expected incidence of around $10 \%$ as been frequently reported in literature and what we find when using the 6-CIT. Based on these results, caution is warranted to use the CAM-ICU for early screening in the ED.

\section{P-838}

The effect of the anticholinergic burden on duration and severity of delirium in older hip-surgery patients with and without haloperidol prophylaxis: a post-hoc analysis

M.P.H Tillemans ${ }^{1}$, M.H. Butterhoff - Terlingen ${ }^{2}$, R. Stuffken ${ }^{2}$, R. Vreeswijk ${ }^{3}$, T.C.G. Egberts ${ }^{4}$, K.J. Kalisvaart ${ }^{3}$

${ }^{1}$ Stichting Apotheek der Haarlemse Ziekenhuizen, Haarlem, The Netherlands, ${ }^{2}$ Ter Gooi Ziekenhuizen, Hilversum, The Netherlands, ${ }^{3}$ Spaarne Gasthuis, Haarlem, The Netherlands, ${ }^{4}$ University Medical Center, Utrecht, The Netherlands

Introduction: This post-hoc analysis was performed to assess the effect of the anticholinergic burden (ACB) on the duration and severity of delirium in older hip-surgery patients with or without haloperidol prophylaxis.

Methods: Older patients with a postoperative delirium following hipsurgery from a randomized controlled trial investigating the effects of haloperidol prophylaxis on delirium incidence were included in this study [1]. The ACB was quantified using two different tools, the Anticholinergic Drug Scale and an Expert Panel. Using linear regression, the association between the anticholinergic burden and delirium duration and severity was analyzed.

Results: Overall delirium duration and severity were not significantly associated with the ACB. Also, no statistical significant differences were found in delirium duration or severity between the placebo and haloperidol treatment groups for the anticholinergic burden groups. The protective effect of haloperidol on delirium duration and severity however tended to be present in the patients with no or a low ACB but not or to a lesser extent in patients with an intermediate to high ACB. Conclusions: The ACB was not significantly associated with delirium duration or severity. The use of haloperidol however tended to shorten delirium duration and decrease delirium severity in patients with no or a low ACB. Additional research with a larger study population and ACB quantification taking drug exposure into account is warranted to further assess the influence of anticholinergic drugs on delirium duration and severity and the effect of concomitant haloperidol use. Keywords: Anticholinergic burden, Delirium, Haloperidol Reference:

1. Kalisvaart KJ, De Jonghe JFM, Bogaards MJ, Vreeswijk R, Egberts TCG, Burger BJ, et al (20050 Haloperidol prophylaxis for elderly hipsurgery patients at risk for delirium: A randomized placebo-controlled study. J Am Geriatr Soc 53(10):1658-66 


\section{P-840}

Development of evidence-based clinical practice guidelines for delirium management in hospitalized older patients

\author{
H. W. Chang ${ }^{1}$, F. W. Hu ${ }^{1}$, Y. J. Chang ${ }^{1}$ \\ ${ }^{1}$ Dept. of Nursing, NCKUH, Tainan, Taiwan
}

Background: Delirium is a common neurocognitive disorder in hospitalized older patients. It has been shown to correlate with increased length of hospital stay, mortality, and admission to nursing home (Inouye, Westendorp, \& Saczynski, 2014; Siddiqi, House, \& Holmes, 2006; Vida et al., 2006). The purpose of this study was to develop an evidence-based practice guideline for delirium management in hospitalized older patients.

Method: A multidisciplinary task group at a medical center in southern Taiwan was established. Team members reviewed current delirium practice guidelines. An adapted practice guideline was developed by constructing foreground questions of key issues, systematical search, appraisal and synthesizing evidences. After a new draft of guidelines was established, the Delphi method was used to reach consensus among expert. Finally, the developed guideline was applied in a geriatric ward of a medical center to evaluate its applicability.

Results: The developed delirium guideline address 3 issues with 35 recommendations. The panel of clinical and methodological experts recommended that delirium guideline can be applied in practice. Most nurses in geriatric ward indicated that delirium guideline was feasible and easy to implement. However, few procedures difficult to be implemented were "provide a therapeutic environment", "promote good sleep patterns and sleep hygiene", and "consider psychotropic medication as a last resort for agitation".

Conclusion: The evidence-based delirium management guidelines for hospitalized older patients integrated recommendations from the best available evidence and obtained a high consensus among clinical experts. Thus, these guidelines are recommended for clinical application.

Keywords: Hospitalized Older Patients, Delirium, Clinical Practice Guidelines

\section{P-841}

\section{A cause of hallucinations in an octogenarian person}

Elisa García-Tercero ${ }^{1}$, Luis María García Santos ${ }^{2}$, Victoria Landaluce Pelligra $^{1}$, Maria Alonso Seco ${ }^{1}$, Clara Pedro Monfort ${ }^{1}$, Leonel A. Porta Gonzalez $^{1}$, Rosa María García-Tercero ${ }^{3}$, Patricia Fernández-Sotos ${ }^{4}$

${ }^{1}$ Servicio Geriatría, Complejo Hospitalario Toledo, Spain,

${ }^{2}$ Psiquiatría, Complejo Hospitalario Toledo, Toledo, Spain, ${ }^{3}$ Servicio Neurología, Complejo Hospitalario Alicante, Alicante, Spain,

${ }^{4}$ Psiquiatría, Hospital 12 de Octubre, Madrid, Spain

Objective: To describe an etiology of visual hallucinations in patients with sensory deficits.

Description: A 84-years-old man with hypertension (amlodipine $5 \mathrm{mg} / 24 \mathrm{~h}$ ), dyslipidemia (atorvastatin $20 \mathrm{mg} / 24 \mathrm{~h}$ ), chronic ischemic cardiopathy (AAS $100 \mathrm{mg} / 24 \mathrm{~h}$ )and bilateral open angle primary glaucoma greater in right eye (Bimatoprost). He went to the Emergency Department for presenting visual hallucinations at night for a month. He refers that in the night, he usually sees two men with cardboard masks. They disappear when he's trying to approach. Today, he has seen a gentleman who is into the closet. He knows that those objects don't exist but it generates quite anxiety. He doesn't hear any sounds. No infectious symptoms.
Physical exploration: A lucid exploration, calm and approachable, oriented, not depressive symptoms, no psychotic symptoms, no self or heterolesive ideas.

Complementary tests: PC: Attached laterCRANIAL-CT: Normalresults: Given the antecedents and ophthalmological exploration, the characteristics of the symptoms and the cognitive exploration, the patient was diagnosed of Charles-Bonnet syndrome. He has follow-up by Ophthalmology and Psychogeriatrics. His treatment was sertraline $100 \mathrm{mg} / 24 \mathrm{~h}$ and Risperdal Solution: $0.25 \mathrm{cc} / 24 \mathrm{~h}$.

Conclusions: Charles-Bonnet syndrome is a common condition in the elderly patients with visual impairment. It's imperative that the health workers know this entity to be able to reach an adequate diagnosis. It's essential to carry out a multidisciplinary approach of the patient to be able to treat it adequately and thus improve the quality of life of these patients, which they are usually very distressed with the clinic and with the confusion that they may be developing a dementia.

\section{P-842}

Non-pharmacological, multicomponent delirium interventions: Team-based approaches for the acute geriatric setting

\section{Claudia Eckstein ${ }^{1}$, Heinrich Burkhardt ${ }^{2}$}

${ }^{1}$ Network Aging Research (NAR), Heidelberg University, Heidelberg, Germany, ${ }^{2}$ Geriatric Centre, University Medicine Mannheim, Mannheim, Germany

Objective: Delirium is an often undiscovered and serious syndrome that often occurs in the geriatrics, and therefore requires delirium detection, prevention and management. Among these, non-pharmacological, multicomponent interventions have proven effective, which has been demonstrated primarily by selected, low-threshold and voluntarily provided prevention components. However, there are hardly any findings with regard to the service provision of the entire spectrum, which, as expected, requires the expertise and close cooperation of health care professionals. Therefore, the question of which team-based interventions are available was pursued.

Methods: A scoping review was performed; research strategies were generated based on predefined inclusion and exclusion criteria, followed by a systematic database search. Title, abstract and full text screening (3.809/77) by two independent reviewers led to the inclusion of 24 studies. The narrative presentation of the results was adapted from the PRISMA statement; the critical appraisal was made by means of the CReDECI 2 checklist and specific teamwork criteria. Results: $45.83 \%$ of intervention programs address detection/prevention; one management, while $50 \%$ address the entire spectrum. The number of identified intervention components varies from three to 18 , of which mobilisation and training $(83.33 \%)$ are most represented. Duration, frequency and type of interventions are described in different qualities; processes, procedures and areas of responsibility are hardly comprehensibly depicted. Patient outcomes (e.g. prevalence/ incidence) are usually reported in the studies, in contrast to teamassociated endpoints (e.g. adherence, education).

Conclusion: To date, few delirium intervention programs available cover the spectrum of detection/prevention/management for acute geriatric clients and adequately describe team-based approaches. 


\section{P-843}

Development of evidence-based clinical practice guidelines for delirium management in hospitalized older patients

\author{
H. W. Chang ${ }^{1}$, F. W. Hu ${ }^{1}$, Y. J. Chang ${ }^{1}$ \\ ${ }^{1}$ Dept. of Nursing, NCKUH, Tainan, Taiwan
}

Background: Delirium is a common neurocognitive disorder in hospitalized older patients. It has been shown to correlate with increased length of hospital stay, mortality, and admission to nursing home (Inouye, Westendorp, \& Saczynski, 2014; Siddiqi, House, \& Holmes, 2006; Vida et al., 2006). The purpose of this study was to develop an evidence-based practice guideline for delirium management in hospitalized older patients.

Method: A multidisciplinary task group at a medical center in southern Taiwan was established. Team members reviewed current delirium practice guidelines. An adapted practice guideline was developed by constructing foreground questions of key issues, systematical search, appraisal and synthesizing evidences. After a new draft of guidelines was established, the Delphi method was used to reach consensus among expert. Finally, the developed guideline was applied in a geriatric ward of a medical center to evaluate its applicability.

Results: The developed delirium guideline address 3 issues with 35 recommendations. The panel of clinical and methodological experts recommended that delirium guideline can be applied in practice. Most nurses in geriatric ward indicated that delirium guideline was feasible and easy to implement. However, few procedures difficult to be implemented were "provide a therapeutic environment", "promote good sleep patterns and sleep hygiene", and "consider psychotropic medication as a last resort for agitation".

Conclusion: The evidence-based delirium management guidelines for hospitalized older patients integrated recommendations from the best available evidence and obtained a high consensus among clinical experts. Thus, these guidelines are recommended for clinical application.Key words: Hospitalized Older Patients, Delirium, Clinical Practice Guidelines.

\section{P-844}

\section{Prevalence of delirium in patients 75 years or older admitted} to ten Norwegian hospitals

Marius Myrstad ${ }^{1}$, Sigurd Evensen ${ }^{2}$, Ingvild Saltvedt ${ }^{3}$, Anette Hylen Ranhoff $^{4}$, Christian Myrstad ${ }^{5}$, Marte Mellingsæter ${ }^{6}$, Marte Sofie Wang-Hansen ${ }^{7}$, Bjørn Erik Neerland ${ }^{8}$

${ }^{1}$ Bærum Hospital, Vestre Viken Hospital Trust, Gjettum, Norway, ${ }^{2}$ Norwegian University of Science and Technology (NTNU), Trondheim, Norway, ${ }^{3}$ St. Olavs hospital, Trondheim University Hospital, Trondheim, Norway, ${ }^{4}$ Diakonhjemmet Hospital, Oslo, Norway

Introduction: Delirium is characterized by a disturbance in attention, awareness and cognition, with a subacute or acute onset. Delirium is common in acutely admitted older patients, but the prevalence of delirium in the Emergency Departments of Norwegian Hospitals has not been investigated previously.

Methods: On World Delirium Awareness Day March 14th 2018, all patients aged $\geq 75$ years admitted to the Emergency Departments of ten Norwegian Hospitals between 8.00 a.m. and 10.00 p.m. were screened for delirium with $4 \mathrm{AT}$ by geriatricians. Delirium Motor Subtype was assessed using the Delirium Motor Subtype Scale. Age, sex and hospital department affiliation were registered. Informed written consents were obtained from the patients or close relatives.
Results: In total, 140 patients aged $\geq 75$ years (mean age 85.5 years, $54 \%$ women) were admitted during the study period, and $118(84 \%)$ of these were included. Delirium was diagnosed in $16(14 \%)$ of the patients. In addition, 37 patients $(31 \%)$ had signs of cognitive impairment. Hypoactive (50\%) was the most common delirium motor subtype, while $6 \%$ had hyperactive and $13 \%$ had mixed motor subtype. Patients with delirium or cognitive impairment were admitted to all hospitals and departments.

Conclusions: Cognitive impairment was prevalent in the Emergency Departments of all hospitals in the study. The prevalence of delirium was $14 \%$ and hypoactive delirium was the most common motor subtype. Screening with 4AT in the Emergency Department was feasible.

\section{P-845}

Prevalence of delirium and associated factors in convalescence units

Javier Santolaria ${ }^{1}$, Paula Ariana Bozín ${ }^{2}$, Alvaro José Betancourt ${ }^{3}$

${ }^{1}$ Hestia Duran i Reynals, , Llobregat, Spain, ${ }^{2}$ Consorci Corporació Sanitària Parc Taulí, Sabadell, Spain, ${ }^{3}$ Consorci Sanitari Integral, Llobregat, Spain

Introduction: Despite high frequency, rates of unrecognized delirium persist very high, particularly in convalescence units. Our objective was to determine the prevalence of the first episode of delirium in patients who were admitted in a post-acute convalescence unit and describing its principal associated factors, between January 1 to May 1 of 2018

Methods: Prospective and cross-sectional study. Patients $>65$ years old were recruited. Using a questionnaire, we studied probable predisposing factors (visual and hearing impairment, psychoactive drugs) and precipitating factors (bladder catheter, fever, poor control of pain and sleep disturbances). Additionally, demographic data, comorbidity, pre-existing cognitive impairment and physical function were recorded. Subjects with advanced dementia, diagnosis of any organic encephalopathy or evidence of delirium at the time of the admission were excluded. Delirium was defined according the Confusion Assessment Method.

Results: A total of 224 patients were recruited (mean 82.1 years old). Prevalence of delirium was $15.6 \%$ (95\% CI 11.5-21.0). Mean time for development of delirium was 16.7 days and its average hospitalization time was 37.1 days. Adjusted by age, the factors associated with delirium were: Pre-existing cognitive impairment $\mathrm{OR}=5.9(95 \% \mathrm{CI}$ 2.5-13.7) $(\mathrm{p}<0.001)$, previous delirium history $\mathrm{OR}=3.9(95 \% \mathrm{CI}$ 1.8-8.8) $(\mathrm{p}=0.001)$, total errors according Pfeiffer's Test $\mathrm{OR}=$ $1.3(95 \%$ CI $1.2-1.5)(\mathrm{p}<0.001)$ and type of hospital referral unit (emergency unit) $\mathrm{OR}=3.3(95 \%$ CI 1.2-9.1) $(\mathrm{p}=0.02)$. According to multivariable analysis, only cognitive impairment remained significative with $\mathrm{OR}=2.9(95 \%$ CI $1.1-8.2)(\mathrm{p}=0.04)$.

Key conclusions: Conditions related to cognitive impairment are strongly associated with delirium. Additionally being derived from emergency units also showed a significant association. 


\section{P-846}

Impact of the first episode of delirium on mortality and associated factors in a convalescence unit

\author{
Alvaro José Betancourt ${ }^{1}$, Javier Santolaria ${ }^{2}$, Paula Ariana Bozín ${ }^{3}$ \\ ${ }^{1}$ Consorci Sanitari Integral, Llobregat, Spain, ${ }^{2}$ Hestia Duran i \\ Reynals, Llobregat, Spain, ${ }^{3}$ Consorci Corporació Sanitària Parc Taulí, \\ Sabadell, Spain
}

Introduction: Our objective was to determine the incidence of delirium, describing its associated factors and impact on mortality in patients who were admitted in a post-acute convalescence unit between January 1 to April 1 of 2018.

Methods: Prospective study. Patients $>65$ years were recruited and followed until their hospital discharge. We studied probable predisposing and precipitating factors, demographic data, comorbidity, preexisting cognitive impairment and physical function. Additionally, date and reasons for hospital discharge were collected. Subjects with advanced dementia, diagnosis of any organic encephalopathy or evidence of delirium at the time of the admission were excluded. Delirium was defined according the Confusion Assessment Method. Results: A number of 180 patients were recruited (mean 82.1 years). A total of 44 cases were registered as first episode of delirium. Incidence was 66.8 per 1000 person-days (95\% CI 48.5-89.6). Meantime for development of delirium was 15 days and the average time for hospital discharge was 37.5 days. Adjusted by age, the factors associated with delirium were: Pre-existing cognitive impairment $\mathrm{OR}=3.9(95 \%$ CI $1.9-8.3) \quad(\mathrm{p}<0.001)$, previous delirium history $\mathrm{OR}=3.1 \quad(95 \%$ CI 1.4-6.7 $)(\mathrm{p}=0.006)$, total errors according Pfeiffer's Test OR $=1.4(95 \%$ CI $1.2-1.6)(\mathrm{p}<0.001)$ use of anticholinesterase agents $\mathrm{OR}=2.8(95 \% \mathrm{CI} 1.3-6.2)(\mathrm{p}=0.011)$ and Charlson comorbidity score OR $=1.2(95 \%$ CI $1.0-1.5)(\mathrm{p}=0.03)$. According survival analysis, delirium was strongly associated with mortality HR $=5.2(95 \%$ CI 1.3-21.1) $(\mathrm{p}=0.02)$. Risk adjustment was made for patient age and Charlson score.

Key conclusions: Conditions related to cognitive impairment are strongly associated with delirium. Additionally, adjusted by age and comorbidity, delirium is associated with mortality in convalescence units.

\section{P-847}

\section{Response to rivastigmine in elderly patients with delirium post} stroke

Elisa García-Tercero ${ }^{1}$, Luis María García Santos ${ }^{2}$, Rosa María García Tercero ${ }^{3}, \mathrm{M}^{\mathrm{a}}$ Dolores López Pizarro ${ }^{4}$, Ana Escolante Melich ${ }^{1}, \mathrm{M}^{\mathrm{a}}$ Esperanza Martín Correa ${ }^{1}$

${ }^{11}$ Department of geriatrics, Hospital del Valle,Toledo, Spain, ${ }^{2}$ Department of Psychiatry, Hospital Virgen del Valle,Toledo, Spain, ${ }^{3}$ Department of Neurology, University Hospital of Alicante, Alicante, Spain, ${ }^{4}$ Department of Radiology, Hospital Campo Arañuelo, Navalmoral de la Mata, Cáceres, Spain

Introduction: The presence of delirium after a stroke is a frequent clinical picture in elderly patients. The deficit of acetylcholine according to the different studies, would be involved in its etiopathogenesis. Based on studies conducted by some authors using acetylcholinesterase inhibitors in patients with cerebral vascular pathology, we describe the response to rivastigmine in 6 patients who present delirium.

Methods: We present 6 patients who are between 83 and 91 years, all of them with cardiovascular risk factors (HTA, DM, DL) who are admitted to the geriatric hospital with a recent diagnosis of stroke. All of them presented a hyperactive delirium with bad response to neuroleptics. After performing EKG, a treatment with rivastigmine patches of $4.6 \mathrm{mg}$ per day is started.

Results: After 3 and 6 days from the beginning of rivastigmine, the symptoms presented by the patients were re-evaluated. In 4 of the 6 cases, the symptoms were resolved after 3 days and the remaining 2 were resolved in the 4th and 6th days. All cases were resolved after 6 days.

Key conclusions: According to the authors, the treatment with rivastigmine could be useful in the delirium of elderly patients who have suffered a stroke as well as to reduce the duration of symptoms. Studies with a larger number of patients are needed to support these results.

\section{P-848}

Delirium in older adults "ergo" dementia, but if it was a brain tumor?

Ilaria Giannini ${ }^{1}$, Anna Chiara Baroncini ${ }^{1}$, Caterina Baroncini ${ }^{1}$, Ester Latini ${ }^{1}$, Veronica Nucci ${ }^{1}$, Enrico Mossello ${ }^{1}$, Andrea Ungar ${ }^{1}$, Mauro Di Bari ${ }^{1}$, Maria Chiara Cavallini ${ }^{1}$

${ }^{1}$ Dept. of Experimental and Clinical Medicine, Research Unit of Medicine of Aging, University of Florence and AOU Careggi,

Florence, Italy

Introduction: Delirium is common presentation of illness in elderly. The identification of underlying causes is essential to choose an appropriate treatment.

Methods: AL, 88 years, woman, until September 2017, presented functional independence despite recent reduction of memory. Then, rapidly, a severe deterioration with confusion and aggression emerged. She was discharged from Emergency Department with diagnosis "Behavioral and Psychological Symptoms of Dementia (BPSD)". A cranial Computer-Tomography (CT) scan demonstrated "tenuously hyperdense image of $8 \mathrm{~mm}$ at right temporal site with peripheral hypodense halo, compatible with cavernous angioma". After 1 week, she was admitted to General Ward; cranial CT shown "increase in size $(1.2 \mathrm{~cm})$ of the lesion previously highlighted, edema, signs of compression on ventricle". Patient refused other examinations, was addressed to Rehabilitation Center with same diagnosis and, after 40 days, without improving, she was discharged in a Nursing Home.

Results: In January 2018, in our Clinic, patient appeared confused, suffering, depressed; presented hyposthenia, impossibility to maintain upright and seat position. After anamnestic review, CT scan control was urgently planned and showed extensive expansive formation in right cerebral hemisphere (glioblastoma?), not surgically treatable. In consideration of progression, it was decided to undertake home palliative therapy.

Key conclusions: Brain tumors, in Europe, cause $2 \%$ of all cancerdeaths, incidence is increasing, more than doubled over 65 years, in which it begins with confusion and reduction of memory. The differential diagnosis between dementia and delirium is essential to choose an ethical approach, like as Palliative Care when appropriate, avoiding unnecessary, painful and expensive treatments. 


\section{P-849}

Delirium clinic: an outpatient follow up clinic to unmasked dementia

Sadia Saber ${ }^{1}$, Sophie Mitchell ${ }^{1}$, Ifeanyi Bernadette Bosah ${ }^{1}$, Shahriar Shafiq $^{1}$, Krishanthi Sathanandan ${ }^{1}$, Khalid Haque ${ }^{1}$

${ }^{1}$ Barking Havering and Redbridge Hospital Trust, London, UK

Introduction: Delirium is a serious and distressing medical condition in which a person suffers a severe and acute decline in memory. It is often caused by infections, acute illnesses or side-effects of prescription drugs. People with dementia are highly prone to delirium. Delirium is common, but it is under-diagnosed. It is known that 20 per cent of older people in hospital have delirium. Two-thirds of hospital patients with delirium also have dementia. In these cases the delirium seems to have 'unmasked' the dementia. At Barking Havering and Redbridge Trust provide an out patient follow up clinic. Patient assessed by geriatrician and psychiatrist of old age.

Methods: Retrospective study of one year carried out to confirm that delirium clinic adds the national initiative of improving early diagnosis of dementia, as delirium proven association with cognitive impairment.

Results: 82 patients reviewed by geriatrician in clinic over one year were analysed, median age 82.5, male and female ratio 35:47. Main source of referrals were from community followed by post hospital discharge. In clinic most people had investigations in keeping with national standards, patient's medication assessed particularly those with anticholinergic side effects, risk factors for vascular disease also identified, formal screening test for cognitive assessment carried out .During follow up delirium resolved in 44 patients, 18 patients were newly diagnosed with dementia and 10 with mild cognitive impairment.

Conclusions: Delirium is a common first presentation of an underlying dementing process, cognitive function periodically can be assessed in out-patient clinic even if delirium has resolved.

\section{P-850}

Delirium clinic: an outpatient follow up clinic to unmasked dementia

Sadia Saber ${ }^{1}$, Sophie Mitchell ${ }^{1}$, Ifeanyi Bernadette Bosah ${ }^{1}$, Shahriar Shafiq ${ }^{1}$, Krishanthi Sathanandan ${ }^{1}$, Khalid Haque ${ }^{1}$

${ }^{1}$ Barking Havering and Redbridge Hospital Trust, London, UK

Introduction: Delirium is a serious and distressing medical condition in which a person suffers a severe and acute decline in memory. It is often caused by infections, acute illnesses or side-effects of prescription drugs. People with dementia are highly prone to delirium. Delirium is common, but it is under-diagnosed. It is known that 20 per cent of older people in hospital have delirium. Two-thirds of hospital patients with delirium also have dementia. In these cases the delirium seems to have 'unmasked' the dementia. At Barking Havering and Redbridge Trust provide an out patient follow up clinic. Patient assessed by geriatrician and psychiatrist of old age.

Methods: Retrospective study of 1 year carried out to confirm that delirium clinic adds the national initiative of improving early diagnosis of dementia, as delirium proven association with cognitive impairment.

Results: 82 patients reviewed by geriatrician in clinic over 1 year were analysed, median age 82.5 , male and female ratio $35: 47$. Main source of referrals were from community followed by post hospital discharge. In clinic most people had investigations in keeping with national standards, patient's medication assessed particularly those with anticholinergic side effects, risk factors for vascular disease also identified, formal screening test for cognitive assessment carried out. During follow up delirium resolved in 44 patients, 18 patients were newly diagnosed with dementia and 10 with mild cognitive impairment.

Conclusions: Delirium is a common first presentation of an underlying dementing process, cognitive function periodically can be assessed in out-patient clinic even if delirium has resolved.

\section{P-851}

\section{Preventing delirium in the elderly population}

K. Alattar ${ }^{1}$, V. Amin ${ }^{1}$, B. Vijayakumar ${ }^{1}$

${ }^{1} \mathrm{KCL}$, London, UK

Background: Delirium is seen in $15-30 \%$ of secondary-care admissions [1]. It is associated with longer hospital stays, higher mortality and morbidity. Many causes of delirium are reversible; early recognition and correction can prevent adverse outcomes in vulnerable populations and ease cost arising from late diagnosis [2]. In 2017, the National Institute for Health and Care Excellence (NICE) [1]* published recommendations on the management of delirium, including screening of vulnerable groups. Despite this, local uptake has been poor. We investigated the feasibility of an educational intervention** as a means of improving uptake rate.

Method: Patients were identified using their Emergency Department $>75$ proforma at University Hospital Lewisham. Inclusion criteria: age $>65$, admitted due to acute or worsening confusion/delirium. Exclusion criteria: incomplete notes. Adherence to the NICE guidelines prior to and following our intervention was assessed.

Results: Despite some positive trends, no significant improvements were observed. Following our intervention, an increase in percentage of completed abbreviated mental test score (pre $=61 \%$; post $=95 \%$ ), mid-stream urine and imaging $* * *$ (pre $=47 \%$; post $=59 \%$ ) was observed. There was no improvement in completion of full confusion blood screen (pre $=50 \%$; post $=35 \%$ ). Overall, there was no change in percentage of patients receiving a complete confusion screen $($ pre $=17 \%$; post $=17 \%)$.

Discussion/conclusion: Education improved understanding of NICErecommendations for confusion screening, but alone was not successful in improving uptake. A multi-intervention approach aimed at adherence and engagement of the healthcare team may be more effective than education alone. This is imperative as the population of elderly patients increases, as will the need for elderly-focused care.

Footnotes/references: *NICE is the UK's national guidance which we audited against.

**Education intervention implemented involved an informative presentation followed by question and answer session.

***Imaging = chest $\mathrm{x}$-ray and $\mathrm{CT}$ head

1. Nice.org.uk. (2017). Delirium in adults I Guidance and guidelines I NICE. [online] https://www.nice.org.uk/guidance/qs63. Accessed 18 Jan 2018.

2. Saxena S, Lawley, D (2009) Delirium in the elderly: a clinical review. Postgrad Med J 85(1006), 405-413

\section{P-852}

Delirium progress and outcomes following hospital admission in adults: service evaluation

E Clayton $^{1}$, I. T Obi ${ }^{1}, \mathrm{~S}$ Guptha ${ }^{1}$

${ }^{1}$ North West Anglia NHS Foundation Trust, Peterborough, UK 
Introduction: Delirium is reported in $30 \%$ of older patients admitted to hospital medical wards. National Institute for Health and Care Excellence guidelines recommend tailored multi-component intervention in treating delirium. We conducted a service evaluation project to assess the prevalence and progress of various behavioural symptoms of delirium after admission and outcomes at discharge and 1 year following discharge from hospital.

Methods: We reviewed electronic case notes of patients coded with a diagnosis of delirium between August to December 2016. Data collected included age, sex, behavioural manifestations of delirium like agitation, aggression, hallucinations, and sleep disturbance at presentation and their progress and discharge outcomes following admission and 1 year after admission.

Results: A total of 49 patients were diagnosed with delirium during the period, 27 females and 22 males. Age range was 48 to 95 years, mean 82 years. Length of stay ranged from 1 to 86 days with a mean of 21 days. The most common symptoms of delirium in hospital stay were activity disturbance seen in $45(92 \%)$ patients, sleep disturbance in $41(84 \%)$ patients, affect in $36(73 \%)$ patients and paranoid/delusions in $33(63 \%)$ patients. The behavioural symptoms worsened in 25 (51\%) patients during the admission. $14(29 \%)$ had at least one recorded fall. The outcomes at discharge were $9(18 \%)$ deaths, 12 (24\%) patients to care home and $22(45 \%)$ patients returned to their own home. The outcomes at 1 year following discharge are 19 deaths (39\%) and 18 patients living in their own home (37\%).

Conclusions: Sleep and activity disturbances are common behavioural symptoms observed in adults admitted with delirium to the hospital. These behavioural symptoms show a worsening following admission resulting in possible increased risk of falls. Interventions aimed at improving sleep in patients admitted with delirium should be considered at admission.

\section{P-853}

Multidimensional prognostic index (MPI) predicts delirium in older patients with hip fracture who underwent surgical intervention

C. Musacchio ${ }^{1}$, M. Razzano $^{1}$, R. Raiteri ${ }^{1}$, A. Del Rio ${ }^{1}$, D. Torriglia ${ }^{1}$, M. Stella ${ }^{2}$, M. Puntoni ${ }^{3}$, A. Barone ${ }^{1}$, A. Pilotto ${ }^{1}$

${ }^{1}$ Department of Geriatric Care, OrthoGeriatrics \& Rehabilitation, Frailty Area, E.O. Galliera Hospital, Genoa, Italy, ${ }^{2}$ Orthopedic Unit, Department of Geriatric Care, OrthoGeriatrics \& Rehabilitation, Frailty Area, E.O. Galliera Hospital, Genoa, Italy, ${ }^{3}$ Clinical Trial Unit, Scientific Directorate, E.O. Galliera Hospital, Genoa, Italy

Introduction: Hip fractures precipitate several acute adverse outcomes in elderly people, thus leading to acute adverse outcomes. The aim of this study is to evaluate whether the multidimentional prognostic index (MPI) may predict delirium in older individuals admitted to the hospital for hip fracture who underwent a surgical intervention. Methods: This was a retrospective observational cohort study on older patients, admitted to the OrthoGeriatrics Unit for hip fracture. At baseline the MPI was calculated. According to previous cut-off analyses, MPI was expressed in three grades, i.e. MPI-1 (low-risk), MPI-2 (moderate-risk) and MPI-3 (high risk of mortality) Delirium was assessed by nurses and physicians during the hospitalization by means of 4 AT test. Covariates included age, sex, baseline mobility and functional status, preoperative cognitive impairment, and post operative complications were also assessed.

Results: 247 older patients (mean age $85 \pm 6.9$ years; Females $=$ $208,84,2 \%$ ) who underwent surgery for a hip fracture were included. 104 subjects $(41 \%)$ received a diagnosis of delirium. Patients with delirium showed higher pre-operative cognitive impairment $(\mathrm{p}=0.0001)$, lower baseline functional status $(\mathrm{p}=0.001)$ and were older than patients who did not experience delirium. Logistic regression analysis demonstrated a significant association between MPI grade and delirium $(\mathrm{p}=0.04)$. MPI score demonstrated a significant association with delirium $(\mathrm{p}<0.0001)$. Overall, the incidence of delirium during hospitalization was significantly higher in patients with more severe MPI score.

Conclusion: MPI predicts delirium in older patients with hip fractures who underwent surgical intervention.

\section{P-854}

Calf circumference as a correlate of delirium in hospitalized older people: data from the Italian delirium day 2017

Alessandro Morandi ${ }^{1}$, Simona Di Santo ${ }^{2}$, Enrico Mossello ${ }^{3}$, Antonio Cherubini $^{4}$, Mario Bo ${ }^{5}$, Alessandra Marengoni ${ }^{6}$, Emanuela Rossi ${ }^{7}$, Nicola Latronico ${ }^{8}$, Ciro Paolillo ${ }^{9}$, Giuseppe Bellelli ${ }^{7}$

${ }^{1}$ Department of Rehabilitation and Aged Care "Fondazione Camplani" Hospital, Cremona, Italy, ${ }^{2}$ Department of Clinical and Behavioral Neurology, Neuropsychiatry Laboratory, IRCCS Foundation S Lucia, Roma, Italy, ${ }^{3}$ Research Unit of Medicine of Ageing

Introduction: So far, there are no studies that assessed the relationship between calf circumference (CC), a marker of sarcopenia and perhaps of frailty in older people, and delirium. This study aims at exploring this potential association in a sample of older people acutely admitted to medical hospital wards.

Methods: The "Italian Delirium Day" is a multicenter study which is hold yearly in Italy to evaluate the delirium point-prevalence among older patients ( $\geq 65$ years) admitted to hospital wards and long-term cares facilities. Delirium is assessed on an index day according to 4AT score. Other variables are collected by the attending physician at each center, including demographics, comorbidity, functional status and drugs. CC is measured as the maximum horizontal distance around the left calf as the subject stood upright. Here we report data from the only medical units.

Results: Overall, among those who had full data (817), 191 (23.4\%) patients had delirium. Delirious patients were older (median 87 vs 83 yrs, p $<0.001$ ), more disabled (median NMS 2 vs $6,<0.001$ ) and demented $(59.2 \%$ vs $10.9 \%, \mathrm{p}<0.001)$ than counterpart. The median CC was $29 \mathrm{~cm}$ in delirious vs $31 \mathrm{~cm}$ in non-delirious, $<0.001)$. In a logistic regression model adjusted for covariates, $\mathrm{CC}$ was independent predictor of delirium (Odd Ratio (OR) 0.96, 95\% confidence intervals (CI) 0.92-0.99), along with age (OR 1.03, 95\% CI 1.00-1.06) and dementia (OR 10.49, 95\% CI 7.07-15.55).

Conclusions: $\mathrm{CC}$ is a correlate of delirium in acutely hospitalized older patients. Future studies are required to confirm this finding.

\section{P-855}

Visiting policy and delirium prevalence in older inpatients. Data from the delirium day 2016

Carlo Fumagalli ${ }^{1}$, Enrico Mossello ${ }^{2}$, Francesca Tesi ${ }^{2}$, Monica Torrini $^{2}$, Alessandro Morandi ${ }^{3}$, Simona Di Santo ${ }^{4}$, Antonio Cherubini $^{5}$, Mario Bo ${ }^{6}$, Alessandra Marengoni ${ }^{7}$, Andrea Mazzone ${ }^{8}$, Antonella Zambon ${ }^{9}$, Nicola Latronico ${ }^{10}$, Filippo Fimognari ${ }^{11}$ Raffaele Antonelli Incalzi ${ }^{12}$, Pietro Gareri ${ }^{13}$, Francesco Perticone ${ }^{14}$, Mauro Campanini ${ }^{15}$, Italo Penco ${ }^{16}$, Marco Montorsi ${ }^{17}$, Marco Trabucchi $^{18}$, Stefano Cappa ${ }^{19}$, Giuseppe Bellelli ${ }^{20}$

${ }^{1}$ Department of Experimental and Clinical Medicine, University of Florence and Azienda Ospedaliero-Universitaria Careggi, Firenze, 
Italy, ${ }^{2}$ Research Unit of Medicine of Ageing, Department of Experimental and Clinical Medicine, University of Florence and Azienda Ospedaliero-Universitaria Careggi, Firenze, Italy, ${ }^{3}$ Department of Rehabilitation and Aged Care "Fondazione Camplani" Hospital, Cremona, Italy, ${ }^{4}$ Department of Clinical and Behavioral Neurology, Neuropsychiatry Laboratory, IRCCS Foundation S Lucia, Roma, Italy, ${ }^{5}$ Geriatrics and Geriatric Emergency Care, IRCCS-INRCA, Ancona, Italy, ${ }^{6}$ Section of Geriatrics, Città della Salute e della Scienza - Molinette, Torino, Italy, ${ }^{7}$ Department of Clinical and Experimental Sciences, University of Brescia, Italy, 8 Redaelli Geriatric Institute, Milan, Italy, ${ }^{9}$ School of Medicine and Surgery, University of Milano-Bicocca, Italy,

${ }^{10}$ Specialitá medico-chirurgiche, scienze radiologiche e sanità pubblica, University of Brescia, Italy, ${ }^{11}$ Italian Society of Hospital s and Community Geriatrics (SIGOT), Italy, ${ }^{12}$ Gerontology Unit, University Hospital Campus Bio-Medico, Rome, Italy,

${ }^{13}$ Extrahospital Geriatric Association (AGE), Italy, ${ }^{14}$ Italian Society of Internal Medicin e, Rome, Italy, ${ }^{15}$ Federazione Italiana delle Associazioni Dirigenti Ospedalieri Internisti (FADOI), Italy,

${ }^{16}$ Societá italiana di Cure Palliative, Società italiana di Chirurgia, Tor Vergata Rome University Italy, ${ }^{17}$ Italian Society of Surgery, ${ }^{18}$ Tor Vergata, Rome University, Rome, Italy, ${ }^{19}$ Istituto Universitario di Studi Superiori di Pavia, NEtS Center, Pavia, Italy, ${ }^{20}$ Department of Health Sciences, University of Milano Bicocca and Acute Geriatric Unit, San Gerardo Hospital, Monza, Italy

Background and objectives: Extended visiting time (VT) was suggested as a protective factor for delirium in intensive care units, but its role in general acute hospital is still unclear. We performed an exploratory analysis on the association between VT and delirium in a national multi-centre point prevalence study.

Methods: A total of 2609 patients $>65$ years admitted to Medicine, Surgery, Geriatrics, Neurology and post-acute Rehabilitation wards during the Italian Delirium Day 2016 were included in the present analysis. Disability, history of dementia, multimorbidity (Charlson Comorbidity Index) and nutritional status were assessed. Delirium was defined with the 4-AT. Daily VT allowed in each ward was recorded.

Results: Delirium was detected in $22 \%(\mathrm{~N}=566)$ of patients. Patients with delirium were significantly older and had a higher prevalence of dementia, malnutrition, multimorbidity and disability. Daily VT was longer in wards hosting patients who experienced delirium ( 8.8 vs $7.7 \mathrm{~h}$ per day, $\mathrm{p}<0.001$ ) and was overall longest in Geriatric wards $(11 \mathrm{~h})$. However, the association between VT and delirium was not statistically significant after adjustment for age, dementia, disability, multimorbidity and malnutrition.

Conclusions: Older patients, with worse clinical and functional profile and higher prevalence of delirium, are more often admitted to wards with longer VT, often Geriatrics wards, in Italian hospitals. Whether longer VT mirrors the need to care for an already complex patient or if it can be an effective strategy to reduce incident delirium should be assessed in future longitudinal, intervention studies.

\section{P-856}

Delirium, frailty, and fast-track surgery in oncogeriatrics: is there a link?

\section{Fiammetta Monacelli ${ }^{1}$, Chiara Giannotti ${ }^{1}$}

${ }^{1}$ Hospital Policlinic San Martino, Section of Geriatric Medicine, Department of Internal Medicine and Medical Specialties (DIMI), University of Genoa, Genoa, Italy

Introduction: Postoperative delirium (POD) is more frequent in elderly patients undergoing major cancer surgery. The interplay between individual clinical vulnerability and a series of perioperative factors seems to play a relevant role. Surgery is the first-line treatment option for cancer, and fast-track surgery (FTS) has been documented to decrease postoperative complications. The study sought to assess, after comprehensive geriatric assessment (CGA) and frailty stratification (Rockwood 40 items index), which perioperative parameters were predictive of POD development in elderly patients undergoing FTS for colorectal cancer.

Methods: A total of 107 consecutive subjects admitted for elective colorectal FTS were enrolled. All patients underwent CGA, frailly stratification, Timed up \& go (TUG) test, 4AT test for delirium screening, anesthesiologists physical status classification, and DindoClavien classification.

Results: The incidence of POD was $12.3 \%$. Patients' prevalent clinical phenotype was pre-frail. The multivariate analysis indicated physical performance (TUG in seconds) as the most significant predictor of POD for each second of increase.

Conclusions: Only few procedure-specific studies have examined the impact of FTS for colorectal cancer on POD. This is the first study to investigate the risk factors for POD, in a vulnerable octogenarian oncogeriatric population submitted to FTS surgery and frailty stratification.

\section{P-857}

Nocturnal hypoxemia and delirium in hospitalized postoperative orthogeriatric patients with hip fracture surgery

Fella Tahmi ${ }^{1}$, Lucie Dourthe ${ }^{1}$, Lorraine Zerah $^{1,2}$, Helane Vallet ${ }^{1}$, Alice Gioanni ${ }^{1,2}$, Anthony Meziere ${ }^{3}$, Rebecca Haddad ${ }^{1}$, Mohamed Doulazmi $^{4}$, Judith Cohen-Bittan ${ }^{1}$, Jacques Boddaert ${ }^{1,2}$, Kiyoka Kinugawa $^{2,4,5}$

${ }^{1}$ Department of Geriatrics, Unit of Perioperative Geriatric care (UPOG), Groupe Hospitalier (GH) Pitié-Salpêtrière-Charles Foix, Assistance Publique-Hôpitaux de Paris (APHP), ${ }^{2}$ Sorbonne Université, Paris 06, ${ }^{3}$ Department of Rehabilitation, Groupe Hospitalier (GH) Pitié-Salpêtrière-Charles Foix, Assistance PubliqueHôpitaux de Paris (APHP), ${ }^{4}$ CNRS, UMR 8256 Biological Adaptation and Aging, Sorbonne Université, ${ }^{5}$ Functional Exploration Unit, Groupe Hospitalier (GH) Pitié-Salpêtrière-Charles Foix, Assistance Publique-Hôpitaux de Paris (APHP)

Introduction: Delirium is a common complication following hip fracture surgery (HFS) in older people. Our preliminary study of postoperative nocturnal hypoxia and intra-hospital complications including 46 patients, showed a significant association between the presence of nocturnal hypoxia and the occurrence of delirium (abstract 2017). To complete these results, the study's methodology was modified to control nocturnal oxymetry recording heterogeneity and also to collect daytime $\mathrm{SpO}_{2}$ with the hypothesis that the post-operative nocturnal hypoxia in patients with HSF is associated with the occurrence of delirium.

Methods: Patients hospitalized in orthogeriatric unit after HFS were monitored for nocturnal $\mathrm{SpO} 2$ with a pulse oximeter continuously up to $15 \mathrm{~h}$, covering from one to three night(s). In-hospital complications were recorded in all patients, especially the occurrence of delirium with the CAM scale.

Results: Interim analyzes are done on 38 additional patients included, with a mean age of 89,7 years, and a mean oxymetry recording of $601,73 \mathrm{~min} /$ night $(10 \mathrm{~h})$. The hypoxia vs non-hypoxia (time spent with $\mathrm{SpO}_{2}<90 \%$ more than $20 \%$ ) groups will be analyzed for clinical characteristics, comorbidities and medication use. We will look for statistical association between severe post-operative nocturnal hypoxemia and occurrence of delirium, and if its association is independent from daytime $\mathrm{SpO}_{2}$. 
Conclusion: The potential confirmation of an association between post-operative nocturnal hypoxemia in patients with HFS and the occurrence of delirium will lead us to reflect on a clinical trial testing the benefit of oxygen therapy in the prevention of post-operative delirium, to improve the medical care of orthogeriatric patients.

\section{P-858}

Prescription and administration of haloperidol in hospital admitted patients with an geriatrical approach

Pedro Marques ${ }^{1}$, Rita Martins ${ }^{1}$, Eduardo Doutel Haghighi ${ }^{1}$, Mariana Carvalho ${ }^{1}$

${ }^{1}$ Hospital de Vila Franca de Xira, Vila Franca de Xira, Portugal

Introduction: The frequency of delirium in the hospital admitted patient is very high in Portugal. Haloperidol is still used as first line therapy in these patients, unfortunately often in inadequate doses and routes of administration.

Objectives: To demonstrate that, when using a differentiated approach to elderly patients, that there is less need to use Haloperidol. Methods: In an internal medicine ward, 455 old patients $(>65)$ were admitted from January to September 2017; to 132 of them haloperidol was prescribed; the authors compared a 4 months period where no geriatric approach (GAP) was implemented with a 4 months period where selected patients (cognitive and/or recent functional impairment and/or falls) were submitted to a GAP, regarding to haloperidol prescription and administration.

Results: On the period where no GAP was implemented, from a total of 253 old patients, 71 were prescribed haloperidol whereas only 23 (from a total of 202) had haloperidol prescribed when GAP was performed. In the first group, 44 patients had haloperidol administered; in the second group, 12 patients had haloperidol administered. The patients with GAP also received lower doses and different routes (intramuscular or oral) comparing to others (mainly intravenous).

Discussion: - In the group where GAP was performed, haloperidol was less prescribed, less administered, in lower doses and different routes. The authors pretend to extend both groups to a bigger period to see if the results remain consistent. This work points out the crucial need of implementing a comprehensive geriatric assessment to old patients admitted in the hospital.

\section{P-859}

Association between anticholinergic load and delirium after hip fracture surgery in patients over 75 years

Rebecca Haddad ${ }^{1}$, Charlotte Tomeo ${ }^{1}$, Judith Cohen Bittan ${ }^{1}$, Alice Gioanni ${ }^{1}$, Cedric Villain ${ }^{1}$, Marc Verny ${ }^{1}$, Jacques Boddaert ${ }^{1}$

${ }^{1}$ Sorbonne Université, Université Pierre et Marie Curie (UPMC) Paris 06, DHU FAST, Unit of Peri-Operative Geriatric Care, Geriatric Department, AP-HP, Hôpitaux universitaires Pitié-Salpétriére-Charles Foix, 75013 Paris, France

Introduction: Delirium is common after hip fracture surgery(HFS), which could be explained by several factors, including drug utilization with anticholinergic effects. No study has evaluated the association between delirium and anticholinergic load (AL) after HFS. The objective of this study was to evaluate the association of AL measured by all existing scales with delirium in this population.

Methods: All patients admitted after HFS in the Geriatric Perioperative Unit (UPOG) were included. Delirium was diagnosed by a validated tool, the Confusion Assessment Method. AL was measured based on patients' computerized prescriptions and considered all treatments received during hospitalization. AL was evaluated by several scales: Drug Burden Index(DBI-Ach), Anticholinergic Drug Scale(ADS), Anticholinergic Risk Scale(ARS), Anticholinergic Cognitive Burden Scale(ACB), Chew's scale(Chew), Anticholinergic Activity Scale(AAS), Anticholinergic Load Scale(ALS), ClinicianRated Anticholinergic Scale(CrAS), Duran's scale(Duran) and Anticholinergic Burden Classification(ABC).

Results: On the 53 patients included (mean age $86.9 \pm 5.4$ years; $81.1 \%$ female), there was $39.6 \%$ of delirium. The mean \pm SD AL(scale) was: $0.72 \pm 0.40$ (DBI-Ach), $1.39 \pm 0.95$ (ACB), $0.28 \pm 1.26$ (ARS), $\quad 0.55 \pm 0.89$ (ADS), $\quad 0.73 \pm 1.30$ (ABC), $0.79 \pm 1.12$ (Chew), $1.39 \pm 0.74$ (Duran), $0.38 \pm 0.86$ (AAS), $0.72 \pm 0.95$ (ALS) and $1.45 \pm 1.08$ (CrAS). No significant association was found between AL and delirium.

Key conclusions: In patients after HFS managed in UPOG, no association between delirium and AL measured by the 10 existing scales was found. These preliminary results will be verified in a cohort of 764 patients

\section{P-860}

Cognition and delirium screening in older adults admitted acutely to hospital

M Randles ${ }^{1}$, M O' Callaghan ${ }^{1}$, B O’ Mahony ${ }^{1}$, D O’Mahony ${ }^{1}$

${ }^{1}$ Cork University Hospital (CUH)

Background: Delirium or acute confusional stare is a common clinical syndrome characterised by disturbed consciousness, cognitive function or perception, which has an acute onset and fluctuating course. Delirium can be associated with poorer outcomes for older patients [1]. The purpose of this study was to examine what, if any methods were used to assess cognition in older adults admitted acutely to an Irish hospital.

Methods: We conducted a retrospective review of a sample of acute medical admissions in patients over 75, between 25th February 2018-16th March 18 at Cork University Hospital. The type of cognitive assessment or delirium screening tools used were recorded.

Results: Among the 35 charts reviewed, 17 (49\%) had some form of cognitive screening or delirium assessment documented within $24 \mathrm{~h}$ of admission. 11 patients $(31 \%)$ were documented as "orientated to time, place, person", while $3(9 \%)$ had an Abbreviated Mental Test Score (AMTS), 1 patient had a 4AT, 1 patient was documented as "orientated" and 1 patient was assessed using the Mini Mental State Examination.

Conclusions: In our study only $49 \%$ of sampled patients had documentation of cognitive assessment within $24 \mathrm{~h}$ of admission. Our results suggest a need for improved documentation of cognitive status using a standardised approach to aid in identifying delirium and managing its risk factors.1.NICE CG 103 (delirium) https://www. nice.org.uk/guidance/cg103

\section{P-861}

\section{Delirium in the Emergency Depratment}

\section{Georgia Clarke ${ }^{1}$}

${ }^{1}$ University of Manchester, Manchester, Uk

Background: Delirium is a common condition yet it is poorly diagnosed within the Emergency Department. The Emergency Department introduced the 4 at tool to its admission document to 
increase the rate of screening. Clinicians were prompted to screen both those who are confused and all patients over 65 . Several initiatives have also been introduced to the department yet the screening rate of those over 65 remains below the target levels. Aims: This study aims to identify the reasons for the lack of screening.

Methods: Surveys were conducted within the emergency department with nurses, advanced practitioners and doctors who were identified as the clinicians who should be screening. Surveys were also conducted with health care assistants and porters to establish wider opinions around delirium. Screening rates were compared with the $4 \mathrm{~h}$ wait target and the number of patients attending to establish if departmental pressures were a factor.

Results: There is still a deficit in knowledge about delirium despite teaching on the subject. Clinicians also admitted to not screening all those over 65 due to doubts around the efficacy of screening over clinical judgement. Departmental pressures did not have a significant impact on screening rates.

Conclusion: Though it is clear that the new pathway for delirium and the teaching has increased screening it is apparent that this is not enough to sustain a high screening rate. Changing attitudes of clinicians may require new approaches to teaching.

\section{Area: Ethics and end of life care}

\section{P-862}

IAGG/IAGG GARN international survey of end-of-life care in nursing homes

D. L. Swagerty ${ }^{1}$

${ }^{1}$ Boonshoft SOM, Wright State University, Dayton, USA

Introduction: The findings of the IAGG/IAGG GARN survey on end-of-life (EOL) care in nursing homes of 18 long-term care (LTC) experts across 15 countries will be presented [1]. Although recommendations are that palliative care (PC) should be available to everyone with advanced illness, including those in nursing homes (NH), 10 of the 15 countries (67\%) reported not having access for $\mathrm{NH}$ residents.

Methods: The experts were chosen as a convenience-based sample of known LTC experts in each country. The survey had both open-ended responses for defining hospice care, PC, and EOL care, as well as a series of questions related to attitudes toward EOL care, current practices and EOL interventions, structure of care, and routine barriers.

Results: Overall, experts strongly agreed that hospice and PC should be available in NHs and that both are defined by holistic, interdisciplinary approaches using measures of comfort across domains. However, experts felt that in most countries the reality fell short of ideal care, as exemplified by the lower use of emotional and spiritual support, higher rates of antipsychotics, and ongoing use of restraints as an intervention. As a result, experts called for increased training, communication, and access to specialized EOL services within the NH.

Discussion: This survey further demonstrated ongoing inconsistency in access to EOL care in NHs worldwide, as well as the great variation of services in NHs in any given country, including prevalent barriers and inconsistent standards. Yet, attitudes about the importance of hospice and PC are overwhelmingly positive.

Reference:

1. Wallace CL, Swagerty DL, et al (2017) IAGG/IAGG GARN international survey of end-of-life care in nursing homes. JAMDA. $186,465-469$

\section{P-863}

\section{Gold standards framework}

Bushra Ghouri ${ }^{1}$

${ }^{1}$ Bradford Royal Infirmary, Bradford, UK

Introduction: The gold standards framework (GSF) is a national protocol which helps clinicians to recognise when a patient is approaching the end of life. The primary aim is to ensure that patients receive the best care at the end of life.

Aims: The aims of this closed loop audit and quality improvement project were to improve awareness of the GSF to elderly care department.

Methods: This was a closed loop audit and QIP. Two cycles were performed focused on patients from care homes from elderly wards. The data source was from patients' medical notes and SystemONE. The first cycle included 27 patients and the second loop had 53 patients.

Results: The results from the first loop showed that the patients who should have been on the GSF (20/27) only $525 \%$ of these patients were actually on GSF. The changes implements from the first cycle included; juniors teaching and posters advertised in elderly wards. The results showed that the patients who should have been on the GSF (33/53) now (11/33) 33\% were on it.

Conclusion: There has been an improvement in the number of patients on the GSF increased from $25 \%$ to $33 \%$. We have successfully achieved our audit aims and the changes implemented have aided positive correlation. For further improvement we have suggested; further teaching via coloured posters are put up on the acute and elderly wards. We also suggested discussion with palliative care for further improvement and to possibly generate a popup system on SystemONE so patients are not missed.

\section{P-864}

End of life program can benefit dying older patients in a geriatric step-down hospital

James Ka Hay Luk ${ }^{1}$

${ }^{1}$ Fung Yiu King Hospital, Sandy Bay, Hong Kong

Introduction: Fung Yiu King Hospital (FYKH) is a geriatric stepdown hospital in Hong Kong South. End of Life (EOL) Program in FYKH was established to foster dignified death. In the program, EOL Clinical Plan (EOL-CPi) was used and an 8 beds EOL ward was established.

Objectives: To evaluate the outcomes of EOL Program in enhancing dignified deaths.

Methods: Retrospective study with all inpatients (age $\geq 65$ ) in EOL Program who passed away in EOL ward between 24 August 2015 to 31 October 2017. Quality of Dying and Death Questionnaire (QODD) was used to assess family member satisfaction of the program.

Results: 235 patients with mean age of 87.9 were studied. Their chief diagnoses were advanced dementia $(50.2 \%)$, active cancers $(27.7 \%)$, organ failure $(13.2 \%)$, Stroke $(4.7 \%)$ and neurodegenerative diseases (2.6\%). In the $24 \mathrm{~h}$ before deaths, $99.2 \%$ of patients were pain free, not agitated and without excessive secretion. After activation of the EOL program, there were significant reductions (all p < 0.001) in intravenous broad spectrum antibiotics use, blood product transfusion, use of physical restraints, blood tests, haemoglucostix monitoring and cardiac monitoring. Number of regular medications dropped from 4.8 to 2.6 per patient $(\mathrm{p}<0.001)$. Patients received services from physiotherapists $(88.9 \%)$, occupational therapists $(87.2 \%)$, social workers $(86.4 \%)$ and chaplains (69\%). $90.6 \%$ family members stayed with 
patients outside hospital visiting hours and $87.7 \%$ could say goodbye to their dying relatives. All patients were not resuscitated when they died. $133(56.6 \%)$ QODD Questionnaire returned rated a total scores of $8.5 \pm 1.6$ (maximum score 10 ).

Conclusion: EOL Program composing of a clinical plan and a designated EOL ward could be useful to foster dignified deaths and comfort care among dying older patients in hospital.

\section{P-865}

\section{Advance Directives: Brazilian Reality}

\section{J. T. Casella ${ }^{1}$, V. G. Moreira ${ }^{2}$, E. M. Costa ${ }^{3}$}

${ }^{1}$ Hospital Adventista Sivestre - Unidade Integrada de Prevençao, Brazil, ${ }^{2}$ GeronLab - Laboratório de Pesquisa em Envelhecimento Humano - Faculdade de Ciências Médicas UERJ, Brazil, ${ }^{3}$ GeronLab - Laboratório de Pesquisa em Envelhecimento Humano - Faculdade de Ciências Médicas UERJ, Brazil

In recent decades technological advances in treatment and clinical propaedeutic brought dysthanasia as a side effect. It raised bioethical discussions on solving dilemmas. In this context, the advance directives are a proposal to ensure legal support to patients' rights. The objective of the present study is to analyze the statements of Federal Council of Medicine in Brazil concerning this subject. A review of articles published in Bioethics Journal of the Brazilian Federal Council of Medicine and it latest resolutions in the last ten years was carried out. It were identified nine articles and three key resolutions. The nine articles deal with the perception not only of the health professional but also of the patient on this matter, besides the legal reflexions in question. The three resolutions were published in 2006, 2009 and 2012, respectively. Resolution 1.805 deals with ethical aspects and the dignity of death. Then the new Code of Medical Ethics was created. And finally, the resolution 1.995/12 regulated the living will. It was observed that, in Brazil, advance directives have normative character among the medical class. Currently, its validity is directed through the current constitutional norms, among the principles of human dignity, autonomy, and the prohibition of inhumane treatment guaranteed in the Brazilian Federal Constitution of 1988. However, there is no specific bill about this subject in National Congress and the information consulted does not demonstrate the existence of specific laws on the subject.

\section{P-866}

Nurses' experiences of being confronted with tiredness of life in older persons: a qualitative study

\section{Let Dillen ${ }^{1}$, Ruth Piers ${ }^{1}$, Nele Van Den Noortgate ${ }^{1}$, Liesbeth Van Humbeeck $^{1}$ \\ ${ }^{1}$ Department of Geriatric Medicine, Ghent University Hospital, Ghent, Belgium}

Background: Nurses frequently encounter older persons who are tired of life (ToL). However, studies on the nurses' perspective are virtually non-existent. This study aimed to gain insight into nurses' (1) definition of, (2) attitude(s) towards, and ${ }^{3}$ ways of dealing with ToL.

Methods: A qualitative interview design, based on the principles of the Grounded Theory approach (Charmaz, 2014), was adopted. Both home care nurses (self-employed or working in an organization) and nursing home nurses were purposefully recruited. Interviewers (MM and EHP) had no previous relationship with the participants. The interview guide consisted of reflective and open-ended questions. To achieve credibility and confirmability, investigator triangulation was realized.

Results: A careful consideration indicative of an oscillation between three levels (e.g. behavioural, cognitive, and affective) is present. The nurse's actions created a continuum ranging from not discussing ToL to its open acknowledgment. This communicative behaviour is underpinned by a delicate deliberation on a cognitive and an emotional level, resulting in an attitude ranging from understanding to incomprehension. The cognitive component grounds the nurses' assessment of the validity of the presence of ToL. On the emotional plane, the participants stress the importance of being able to understand the patient's ToL or empathize with the ToL.

Conclusions: Present study shows the challenge to establish communication about ToL in both home care and nursing homes. Our findings indicate that nurses aim to provide good care, sensitive to the older person's needs when experiencing ToL, but this process is not without ambiguity.

\section{P-867}

Doctors' knowledge and attitudes regarding eating problems and enteral feeding in advanced dementia

Boris Punchik $^{1}$, Elena Komissarov ${ }^{2}$, Vladimir Zeldez ${ }^{3}$, Tamar Freud ${ }^{4}$, Tali Samson ${ }^{4}$, Yan Press ${ }^{1}$

${ }^{1}$ Unit for Community Geriatrics, Ben-Gurion University of the Negev, Beer-Sheva, Israel, ${ }^{2}$ Clalit Health Services, Beer-Sheva, Israel, ${ }^{3}$ Emergency Medicine Department, Soroka Medical Center, Beer-Sheva, Israel, ${ }^{4}$ Sial Research Center for Family Medicine, BeerSheva, Israel

Introduction: The use of feeding tubes (FT) in patients with advanced dementia does not yield positive health outcomes and can have a negative effect.

Methods: A cross-sectional study assessed the knowledge and attitudes of physicians on the use of FTs among patients with advanced dementia.

Results: 201 of 240 physicians (83.8\%) participated in the study; 89 of 110 hospital-based $(80.9 \%)$ and 112 of 130 community-based physicians (86.1\%).61.7\% of the physicians said that FTs intended to prevent aspirations, $51.7 \%$ - to prevent pneumonia, $38.8 \%$ to prevent weight. Almost one third (32.3\%) said that the decision to use FTs could be taken by a hospitalist or a primary physician (28.9\%). In the question about their preferences relating to insertion of a FT one hundred thirty-nine physicians $(69.2 \%)$ answered that they would prefer not to agree to FT if they would suffer from advanced dementia with eating problems. Only $7 \%$ would like that their close family members reach the decision and $15.4 \%$ would agree that the medical team decide.

Conclusion: In the present study there were large gaps in knowledge among physicians on the use of FTs and their consequences in patients with advanced dementia.

\section{P-868}

Development of a Japanese version of the 9-item shared decisionmaking questionnaire-physician version to visualize the quality of decision-making in a primary care setting

Yuko Goto ${ }^{1}$, Hisayuki Miura ${ }^{1}$, Kazuyoshi Senda ${ }^{1}$

${ }^{1}$ National Center for Geriatrics and Gerontology, Japan 
Introduction: In Japan, the number of elderly patients who need important medical decision-making is increasing every year. However, there is no scale to visualize the decision support in Japan. Shared Decision-Making Questionnaire-Physician Version (SDM-QDoc) can measure and visualize the degree of shared decision-making through a medical interview between patients and doctors. We aimed to develop a Japanese version of SDM-Q-Doc thorough Japanese translation and psychometric testing.

Methods: After obtaining approval from the copyright holder, we translated and back-translated between the original and Japanese versions of SDM-Q-Doc twice with the help of Japanese doctors. Participants were adult outpatients and their treating physicians who practiced family medicine. In total, 23 physicians rated their consultations with patients $(n=142)$ using SDM-Q-Doc at the first visit of each patient. Reliability and construct validity [exploratory and confirmatory factor analysis (EFA and CFA, respectively)] were assessed. For convergent validity, the Physicians' Confidence in the Medical Interview measure (PCMI) was used.

Results: Reliability was evaluated with internal consistency. The overall Cronbach's alpha was 0.87 , and the I-T correlation was high (0.84-0.87). EFA showed a one-component model similar to the original version. CFA yielded a good index of fit for the one-factor model. The total SDM-Q-Doc scores correlated with PCMI $(\mathrm{r}=0.53)$, as expected.

Key conclusion: The Japanese version of SDM-Q-Doc showed adequate reliability and acceptable clinical efficacy and may help visualize decision support and increase its quality in various settings, such as homes, where elderly patients desire to receive medical care in Japan.

\section{P-869}

identifying aims of advance care planning: a systematic literature review

N. Fleuren ${ }^{1}$, M. F. I. A. Depla ${ }^{1}$, M. Huisman ${ }^{2}$, D. J. A. Janssen ${ }^{3}$, C. M. P. M. Hertogh ${ }^{1}$

${ }^{1}$ Amsterdam UMC, Vrije Universiteit Amsterdam, General Practice \& Elderly Care Medicine, Amsterdam Public Health, De Boelelaan 1117, Amsterdam, The Netherlands, ${ }^{2}$ Amsterdam UMC, Vrije

Universiteit Amsterdam, Epidemiology and Biostatistics, Amsterdam Public Health, De Boelelaan 1117, Amsterdam, The Netherlands, ${ }^{3}$ MUMC+, Center of Expertise in Palliative Care, Maastricht, The Netherlands

Introduction: Since the introduction of the concept of advance care planning (ACP), many studies have been conducted exploring beneficial effects. However, the endpoints of those studies vary considerably, as do the goals that are pursued with ACP in clinical practice. Whilst originally introduced as a means of preserving patients' individual autonomy once they become incapacitated, nowadays ACP is also used for improving patient-clinician communication and preparing patients and their surrogates for future healthcare decision making.

Aim: To clarify which goals are pursued with ACP.

Methods: Systematic literature search in PubMed, EMBASE, PsychInfo, CINAHL and Cochrane Library, using various search terms for 'ACP' and 'ethics'. Articles on normative aspects of ACP were included, based on title and abstract. Due to the quantity of inclusions, of which many had similar content, purposive sampling was used to select articles for full-text analysis. Analysis stopped once saturation was reached. Sensitivity analysis was performed to guarantee that unfrequently mentioned goals were found as well.

Results: In total, 6497 unique articles were found of which 183 were included. Analysis of the purposive sample revealed a wide range of goals of ACP, either orientated at the patient, family, clinician or healthcare system.

Key conclusions: A variety of goals of ACP exists in medical literature. Defining the main aim of ACP should serve as a starting point when setting up ACP intervention research.

\section{P-870}

Progress in advance care planning among nursing home residents between 2003-2013 in Oulu, Finland

Tarja Konttila ${ }^{1}$, Harriet Finne Soveri ${ }^{2}$, Marja Liisa Laakkonen ${ }^{3}$, Riitta Antikainen ${ }^{4}$

${ }^{1}$ Kuusamo Public Health Care, Kuusamo, Finland, ${ }^{2}$ Social Services and Health Care, Helsinki, Finland, ${ }^{3}$ Social Services and Health Care, Helsinki, Finland, Department of General Practice, University of Helsinki, Unit of Primary Health Care, Helsinki, Finland,

Introduction: Advance care planning (ACP) is a process in which an individual reflects on and communicates his/her life values, goals and treatment preferences. ACP is considered essential in good palliative care among those with advanced dementia.

Methods: We conducted a retrospective study among patients with advanced dementia aged $\geq 65$ years who died between January 1st, 2003 and December 9th, 2013 in 18 Long-term care facilities in the city of Oulu, Finland. Data was collected from the Minimum Data Set and from the clinical and nursing records. We analysed separately the ACP data of residents who died between 2003-2009 (Group 1, $\mathrm{n}=212$ ) and between 2010-2013 (Group 2, $\mathrm{n}=192$ ). We studied whether the resident had a living will, a do-not-resuscitate order (DNR), an order to forego parenteral antibiotics or any antimicrobial therapy, an order to withdraw an artificial hydration or nutrition $(\mathrm{ANH})$, a do-not-hospitalize order $(\mathrm{DNH})$ and whether the treatment orders were discussed with the resident or proxy. Comparisons between proportions were performed using Pearson's Chi square test. Results: Practically all residents had a DNR order (group $198.1 \%$ vs group $2100 \%$ ). Residents who died earlier were less likely to have advance directive to forego life-sustaining antibiotic therapy $(38.1 \%$ vs $64.6 \%$; $p<0.001$, withdraw ANH $(40.0 \%$ vs $81.3 \% ; \mathrm{p}<0.001)$ and have a DNH order $(28.1 \%$ vs $69.6 \%$; p < 0.001$)$ compared to those who died later.

Key conclusions: Residents with advanced dementia who died between 2010-2013 were significantly more likely to have advance directives limiting aggressive care than those who died earlier.

\section{P-871}

Preferences for surrogate designation and decision-making process in older versus younger adults with cancer: a comparative cross-sectional study

Claudia Martinez-Tapia ${ }^{1}$, Florence Canoui-Poitrine ${ }^{2}$, Philippe Caillet $^{3}$, Sylvie Bastuji-Garin ${ }^{2}$, Christophe Tournigand ${ }^{4}$, Elias Assaf ${ }^{4}$, Gwénaëlle Varnier ${ }^{3}$, Frederic Pamoukdjian ${ }^{5}$, Etienne Brain ${ }^{6}$, Florence Rollot-Trad ${ }^{7}$, Marie Laure

${ }^{1}$ Paris-Est University, UPEC, DHU A-TVB, IMRB- EA 7376 CEpiA (Clinical Epidemiology and Ageing Unit), Créteil, France,

${ }^{2}$ Assistance Publique Hôpitaux de Paris (AP-HP), Henri-Mondor Hospital, Public Health Department, Créteil, France, ${ }^{3}$ AP-HP, Henri-

Background: Clinical communication to cancer patients plays a crucial role for understanding the care offered, quality of life and good patient-physician relationship. Our objective was to compare 
the preferences of older versus younger cancer patients regarding surrogate designation and decision making.

Methods: Cross-sectional survey of older ( $\geq 70$ years old) versus younger $(<70$ years old $)$ cancer patients. First group: older patients with solid cancer aged $\geq 70$ addressed for geriatric assessment, included in the ELCAPA cohort between January 2013 and June 2013; second group: adults with cancer, $<70$ years referred to the oncology unit for anti-cancer treatment decision and management between February 2013 and July 2014. Patient characteristics and information about surrogacy and involvement in decision making were collected. Associations between patient characteristics and preferences were examined.

Results: The study included 102 young patients (mean age 55 years) and 130 older patients (mean age 80 ). Factors independently associated with surrogate knowledge (66\%): young age, more children living nearby, high income; factors associated with having already designated a surrogate $(62 \%)$ : young age, decreased number of daily medications; factors associated with designating a surrogate after questionnaire administration (40\%): low education, metastasis. Patients requiring an informed consent for any intervention was associated with older age (adjusted OR $[\mathrm{aOR}]$ per year $=1.04[95 \%$ confidence interval 1.00-1.08]), not living alone $(\mathrm{aOR}=2.52$ [1.00-6.36]) and having children (aOR = 4.49 [1.13-17.81]).

Conclusion: All cancer patients, wanted to be fully informed and $72 \%$ wanted to be involved in medical decisions. Preferences for decision control vary between age groups, depending on family members' presence and living alone.Keywords: surrogate, medical information, medical decision-making, patient preference, cancer

\section{P-872}

\section{Advance care planning: what do patients want?}

Lucy Owen ${ }^{1}$

${ }^{1}$ Royal Free London NHS Foundation Trust, London, UK

Introduction: Advance care planning is the process by which patients can make decisions about their future healthcare and end of life wishes should they lose capacity. Such decisions are shown to improve quality of life and reduce institutionalization. A review of the literature has been carried out to ascertain the level of decision making patients would like, when conversations should take place and how clinicians should approach the topic.

Method: CINAHL, Medline, Embas and Pubmed were searched for journal articles on older patients' views on advance care planning. Key words included "elderly" "advance care planning" and "cardiopulmonary resuscitation".

Results: 64 abstracts were screened and 20 full text articles read. 11 articles were included in the final study. Patients will rarely initiate conversations themselves, often presuming that relatives and doctors already know their wishes. They value honest and open conversations; without which incorrect decisions about their care may occur. The majority of patients want conversations to be initiated opportunistically. Yet, a running theme throughout the literature is the degree of individual and cultural differences that need to be taken into account leading to differences in a patient's preferred timing of conversations and level of shared-decision making.

Conclusion: The level of shared decision-making that individuals personally want and the degree to which they would like relatives or carers to be involved should be established. Open and honest conversations should then be initiated at the earliest opportunity. However, research into an elderly, frail outpatient population is limited and this could guide future studies.

\section{P-873}

Early implementation and evaluation of a charter to improve medicines management and reduce inappropriate covert administration in care homes for older people

David Wright ${ }^{1}$, Frances Hollwey ${ }^{2}$, Rina Matala ${ }^{1}$

${ }^{1}$ University of East Anglia, ${ }^{2}$ The Patients Association

Background: Evidence in the UK suggests that residents with dysphagia in care homes for older people are more likely to experience medication errors and medicines are frequently covertly administered to residents without capacity in contravention of the Human Rights Act.

Aim: To pilot a charter to improve medicines administration to care home residents and develop strategies to enhance its implementation. Aim: To pilot a charter to improve medicines administration to care home residents and develop strategies to enhance its implementation. Methods: Expert working party developed a nine-point charter for care home staff and residents (http://www.carehomecharter.org). Care homes from England, Wales and Northern Ireland were recruited to pilot charter implementation. Centrally located evening workshops introduced the charter to senior and junior care home staff. Feedback was obtained one-month post workshop via an on-line survey.

Results: Twenty-two care homes attended the workshops. Eighteen respondents from 13 care homes completed the survey. Implementation was via staff bulletins, through care home meetings, provision of copies to staff and displaying in prominent locations. Sixteen $(88.9 \%)$ respondents were positive regarding the charter. All believed that the charter would improve detection and reporting of dysphagia, medicines administration, improve the quality of staff training and adherence by staff to the law when covertly administering medicines. Recommendations were to incorporate the charter into staff training and to involve the mutli-disciplinary team in the process.

Key conclusion: A staff-training package was developed for implementation within the home and an on-line assessment added to the website providing a certificate of commitment for those individuals who successfully completed it. Work is ongoing to assess the impact of the changes following piloting.

\section{P-874}

The palliative care patient ( $\mathrm{Z51.5}$ ): in a geriatric acute care unit

C Murillo Erazo ${ }^{1}, \mathrm{~J}_{\text {Corrales }}$ Cardenal $^{1}$, C Elías de Molins Peña ${ }^{1}$, B Gamboa Huarte ${ }^{1}$, MD Domingo Sánchez ${ }^{1}$

${ }^{1}$ Servicio Geriatría. Hospital Nuestra Señora de Gracia, Zaragoza, Spain

Introduction: Know the characteristics of the patients admitted in a geriatric acute care unit (GACU) with the diagnosis of palliative care at discharge.

Method: Descriptive, retrospective and transversal study. Included all the patients with the diagnosis of palliative care (Z51.5 code) at discharge, January 2016-September 2017.Variables: sociodemographic, previous diagnosis of palliative care patient, comorbidities, Barthel index, Short Portable Mental Status Questionnaire (SPMSQ), Walter index, main diagnosis at discharge, polypharmacy and extreme pharmacy and use of opioids. Statistical analysis: SPSS.

Results: 313 patients, mean age 87.3 years, $55.3 \%$ women. $80.2 \%$ were admitted through an emergency service of those, $79.6 \%$ had no previous diagnosis of palliative care. Polypharmacy: $81.5 \%$ on admission, $27.7 \%$ at discharge $(\mathrm{p}=0.00)$. Extreme pharmacy: $25.6 \%$ on admission, $4,2 \%$ at discharge $(\mathrm{p}=0.013)$. Previously prescribed 
opioids: $17.3 \%$ on admission and $14.4 \%$ at discharge. Characteristics most related to the need for palliative care in our sample were: severe dependence-Barthel index $<40$ (74.7\%), severe cognitive impairmentSPMSQ $\geq 6(69.4 \%)$, Walter index $\geq 7(62.5 \%)$, infections $33,5 \%$, neoplasm $(16.3 \%)$ and sequelae of a stroke (11.3\%). Hospital admission mortality: 55.8\%. At February 28th 2018 global mortality was $91.3 \%$.

Conclusions: The main demand for palliative care comes from chronic diseases, not from oncological processes. Our patients is an octogenarian women, severe cognitive impairment and clinical deterioration, who is admitted trough an emergency service in a GACU with an infectious disease of different etiology and an unfavorable prognostic index. There is a high tendency to desprescription. This could mean an adecuacy of treatment on this kind of patients.

\section{P-875}

Palliative and end-of-life care conversations in chronic obstructive pulmonary disease in Croatia

Petra Lukinac $^{1}$, Sanja Thompson ${ }^{2}$, Sanja Popović-Grle ${ }^{3}$, Vladimir Fijačko ${ }^{1}$

${ }^{1}$ Department of Pulmonology, University Hospital Center Osijek, Osijek, Croatia; Faculty of Medicine, J.J. Strossmayer University of Osijek,Osijek, Croatia, ${ }^{2}$ Department of Clinical Geratology,

University of Oxford, UK, ${ }^{3}$ Clinical Center for Pulmona

Introduction: Chronic obstructive pulmonary disease (COPD) is one of the leading causes of morbidity and mortality, estimated by 2020 to be the third cause of death worldwide. Despite the progressive nature of the disease and association of high morbidity and mortality with severe COPD, many older patients receive inadequate palliative care, not having timely end-of-life care (EOLC) discussions with healthcare professionals.

Methods: The aim of this study is evaluation of the practice of Croatian Pulmonologists in EOLC communication/palliative care in older COPD patients, as such data are unknown. We looked into the reasons behind the poor communication about EOLC. The study is designed as a cross-sectional research. Two groups of examinees, COPD patients $>65$ years and healthcare professionals were interviewed through an anonymous survey.

Results: Our analysis shows that in the majority of cases, there is no advance care planning, including any kind of patient-clinician communication about EOLC. Current care practices do not facilitate satisfactory conversation about palliative care in the older patients with severe COPD and healthcare professionals. Almost all patients reported that they have not had EOLC discussions with healthcare professionals, while many patients would like these conversations. Conclusions: Due to the progressive nature of the disease, EOLC discussion with the older patients suffering from advanced COPD should improve quality of life, by timely anticipating patients' likely future needs, and also providing proactive support in accordance to patients' preferences. We identified patients and clinicians attitude and obstacles towards such discussions, as well as present practice.

\section{P-876}

How do hospital-based nurses and physicians identify the start of the palliative phase and what difficulties exist? A qualitative study

Isabelle Flierman ${ }^{1}$, Ineke C. Nugteren ${ }^{2}$, Marjon van Rijn ${ }^{3}$, Bianca M. Buurman-van Es ${ }^{4}$, Dick L. Willems ${ }^{5}$

${ }^{1}$ 1. Department of General Practice, Section of Medical Ethics, Academic Medical Center, Amsterdam, The Netherlands 2. Department of Internal Medicine, Section of Geriatric Medicine, Academic Medical Center Amsterdam, The Netherlands, ${ }^{2} 1$ Department

Introduction: Early start of palliative care improves the quality of life of patients and their relatives. However, patients who could benefit from palliative care are often not identified timely by hospital-based health care professionals (HCPs). In this study we assessed how nurses and physicians identify the palliative phase and what difficulties exist. Methods: Semi-structured interviews were held with ten nurses and 18 physicians working in seven hospitals. The interviews were taperecorded, transcribed verbatim and analysed using thematic analysis. Results: HCPs feel insecure about how to define the palliative phase and identification is not something respondents reported doing structured or actively. A variety of ways to identify the palliative phase were described: (1) Prognostication, (2) Treatment trade-off and (3) Patients' needs. Within prognostication great variation existed in what was considered a limited life expectancy and was thought to be more difficult in non-cancer patients. When the benefits of treatment no longer outweighed the negatives, this was thought to be a transition point to the palliative phase. However, this was thought to result in late identification. Patients' needs and preferences were also important in identification. Some respondents wait for patients to vocalize preferences themselves, but many actively start these discussions themselves, but found this difficult.

Conclusion: Identification seems to be a non-structured process. Difficulties in identification occurred because of variance in definitions, unpredictability of non-oncological diseases, focus on treatment and difficulties in communication. These results contribute to the development of a training for HCPs to timely identify patients in the palliative phase.

\section{P-877}

The PalliSupport care pathway, a transitional care pathway for older patients in the last year of life: study protocol of a pragmatic stepped-wedge clustered randomised controlled trial

Isabelle Flierman ${ }^{1}$, Marjon van Rijn ${ }^{1}$, Dick L. Willems ${ }^{2}$, Bianca M. Buurman-van Es ${ }^{3}$

${ }^{1} 1$ Department of General Practice, Section of Medical Ethics, Academic Medical Center, Amsterdam, The Netherlands 2 Department of Internal Medicine, Section of Geriatric Medicine, Academic Medical Center Amsterdam, The Netherlands, ${ }^{2}$ Department of $\mathrm{G}$

Introduction: Most older patients wish to spend their last months at home. However, around 50\% is hospitalised in the last 3 months of life. Timely and well-organised palliative care could prevent these hospitalisations. However, multiple barriers exist, such as palliative needs not being identified, suboptimal collaboration between care settings and fragmented palliative care. The PalliSupport project aims to overcome these barriers through a transitional palliative care pathway for older patients. 
Methods/design: Five regions, each consisting of one hospital and multiple primary care organisations, will participate in this pragmatic stepped-wedge clustered randomised controlled trial. 600 patients aged $\geq 65$ years, acutely hospitalised and positive scores on vulnerability scales will participate. The intervention starts when patients are identified with palliative care needs using the Surprise Question and $\mathrm{SPICT}^{\mathrm{TM}}$. Advance care planning and a comprehensive palliative assessment is performed by a newly-formed transitional palliative care team (TPCT). Patients are discussed in an interdisciplinary team meeting, with both primary and secondary care professionals. A personalised palliative care plan is formalised and handed over. After discharge the TCPT can be consulted and visit patients at home. The control group will receive 'care as usual'. The primary outcome is unplanned hospitalisation within 6 months after discharge. Secondary outcomes are death at the place of patient's preference, quality of life, symptom burden, caregivers' burden and health care utilisation. Furthermore, a process-evaluation and cost-effectiveness analysis will be performed.

Discussion: This study will provide knowledge on the effectiveness of a transitional care pathway for older patient with palliative care needs.

\section{P-878}

Survival in patients aged 70 years and older with and without cognitive disorders following PEG placement, ethical concerns and considerations

Rozemarijn van Bruchem-Visser ${ }^{1}$, Inez de Beaufort ${ }^{2}$, Francesco Mattace Raso ${ }^{1}$, Ernst Kuipers ${ }^{1}$

${ }^{1}$ Erasmus Medical Center, Rotterdam, The Netherlands, ${ }^{2}$ Erasmus Medical Center, Rotterdam, The Netherlands

Introduction: In this study we investigated survival in patients with and without cognitive disorders, who received a PEG-tube, in four different hospitals in The Netherlands. Furthermore, we conducted a literature search, exploring the ethical concerns and considerations that might play in role in the decision whether or not to insert a PEG tube in a patient with severe dementia.

Results: 303 patients were included, mean age of 77.4 years. 42 patients had cognitive disorders. In analyses adjusted for age and sex, patients with cognitive disorders had a $49 \%$ increase on risk of mortality (HR 1.49, 95\% CI 1.01-2.19). A statistically significant difference was found for survival after PEG placement between the groups with and without cognitive disorders, there was no statistical difference in short-term complications.

Discussion: We found higher mortality rates after PEG placement in patients with cognitive disorders when compared with patients without cognitive disorders. There are several possible mechanisms to explain this difference: the incurable nature of dementia, the increase of long term complications such as pressure ulcers and the increased need to use restraints to keep the tube in place. Ethical concerns and considerations: In the studied literature, it is shown that multiple ethical concerns and considerations play a role, both medical and nonmedical, such as: beliefs regarding the benefits of a PEG tube, a lack of knowledge about the natural course of dementia in both professionals and family of patients, and a fear of letting the patient die hungry.

Discussion: We found higher mortality rates after PEG placement in patients with cognitive disorders when compared with patients without cognitive disorders. There are several possible mechanisms to explain this difference: the incurable nature of dementia, the increase of long term complications such as pressure ulcers and the increased need to use restraints to keep the tube in place. Ethical concerns and considerations: In the studied literature, it is shown that multiple ethical concerns and considerations play a role, both medical and nonmedical, such as: beliefs regarding the benefits of a PEG tube, a lack of knowledge about the natural course of dementia in both professionals and family of patients, and a fear of letting the patient die hungry.

\section{P-879}

Organ donation after euthanasia, morally acceptable under strict procedural safeguards

Rozemarijn van Bruchem-Visser ${ }^{1}$, Gert van Dijk ${ }^{1}$, Inez de Beaufort ${ }^{1}$

${ }^{1}$ Erasmus Medical Center, Rotterdam, The Netherlands

In this presentation, we will present a case of organ donation after active euthanasia (ODE) in The Netherlands from a patient who had his life ended at his explicit and voluntary request. The form of ODE we describe here concerns patients who are not unconscious and on life support, but who are conscious and want to have their life ended because of their hopeless and unbearable suffering, for instance due to a terminal illness such as Amyotrophic Lateral Sclerosis (ALS) or Multiple Sclerosis (MS). This form of ODE is of course only possible in jurisdictions where euthanasia is allowed. In these jurisdictions, organ donation after euthanasia is an option that may be considered. We believe ODE is worthwhile to pursue, as it can strengthen patient autonomy, can give meaning to the inevitable death of the patient, and be an extra source of much needed donor organs. To ensure voluntariness of both euthanasia and organ donation and avoid conflict of interest by physicians, ODE does need strict procedural safeguards however. The most important safeguard is a strict separation between the two procedures. The paper on whitch this abstract is based discusses several ethical issues such as who should broach the subject of organ donation and who should perform the euthanasia, and how a conflict of interest can be avoided.

\section{P-880}

Ethical reflections in hospice care: a new validated tool applicable to requests for sedation and/or euthanasia by senior patients!

Leners Jean-Claude ${ }^{1}$

\section{${ }^{1}$ OMEGA; LTCF}

Introduction: The unique hospice in our country has 15 beds . The average of admissions is around 130 persons per year; mean age 76,5 years and mean length of stay 44 days. Regularly our senior patients are requesting either sedation (in a temporary or terminal way) or as well euthanasia (the national law exists since 2009).

Method: In these specific situations we always meet us with the whole team (nurses, psychologists, supervisor) in order to evaluate ethical aspects of the requests. We have opted for a new standardized instrument in order to have an equal assessment. This validated tool is called: Ethical reasoning in difficult situations for Elderly. The method will be explained in details: aspects include the patient in first, the relatives, the team's view and societal aspects for equity.

Results: As we meet us monthly for evaluation of difficult situations, these sessions are used for ethical debate and in urgent situations we meet within $24 \mathrm{~h}$. The conclusions or consensus drawn out of this reflections are transmitted to the patient by the physician. Euthanasia requests are still rare: 15 for 2017 , whereas the wish for sedation are nearly a weekly decision. Two examples will illustrate the values, the consequences and the consensus. 
Key conclusions: As our senior patients should have access to the highest quality of end-of-life care, we need standardized assessment methods for our ethical reflections. This tool, in use regularly in another country, allows us to take into consideration all relevant aspects concerning autonomy, beneficience and equity for difficult clinical situations and the focus is specifically put on elder patients.

\section{P-881}

Electronic communication tool to support, record, share process of advance care planning (ACP) with adopting frailty evaluation axis in inter-disciplinary transitional care at the view of the patient in Japan

Tadashi Wada ${ }^{1}$, Shosuke Satake ${ }^{1}$, Kaori Kinoshita ${ }^{1}$, Sanae Takanashi $^{1}$, Yasumoto Matsui ${ }^{1}$, Hisayuki Miura ${ }^{1}$, Hidenori Arai ${ }^{1}$

${ }^{1}$ National Center for Geriatrics and Gerontology

Introduction: In unprecedented and the most aged society, Japan, high-quality transitional care (TC) based on continuous process of advance care planning (ACP) is essential for frail older people's sense of secure. The inter-disciplinary care team at the TC ward, National Center for Geriatrics and Gerontology (NCGG), needed electronic communication tool (ECT) for supporting older patients continuously and consistently, with supporting decision-making and sharing the information of process of ACP and medical problems including frailty status.

Methods: Organizing-committee for "Preventing, Avoiding, Postponing or Reducing Frailty Program" and "Respecting View of the Patient, Integrated Community Care System Planning Association/ Assembly" at NCGG, developed ECT to support, record, transfer, and share process of ACP correctly and effectively among different care settings.

Results: We had seminars to promote inter-disciplinary TC standing at the view of the patient for frail older people for once in 2014, 3 times in 2015, 21 in 2016, 16 in 2017. A total of 2856 health and long-term care professionals at local communities learned about TC to share process of ACP with frailty evaluation axis. Prototype of ECT, adopting Clinical Frailty Scale by Rockwood with concrete illustration, were shown and got favorable feedback at our TC seminars in NCGG, 2018.

Conclusion: ECT to share process of communication for ACP is fundamental for high-quality TC system at local communities to provide sense of secure. We promote Japanese inter-disciplinary TC with our ECT to share process of communication for ACP with frailty evaluation axis, which enables true person-centered care.

\section{P-882}

\section{The terminology of 'risk feeding': a help or hindrance?}

Dharinee Hansjee ${ }^{1}$, David G Smithard ${ }^{1}$

${ }^{1}$ Lewisham and Greenwich NHS Trust

Introduction: For individuals with advanced dementia who have swallowing impairments, the preferred option to maintain nutrition is to continue eating and drinking despite the risk of developing aspiration pneumonia $[1 ; 2]$. This choice is referred to as 'risk feeding'. Although addressing end of life care, the terminology has wider application within a diverse caseload which has led to an ongoing debate on the appropriateness of the term. Conformity in language will aid communication and understanding between professionals and individuals/family members. This study was undertaken to ascertain professionals' views on the terminology of 'risk feeding'.

Methods: In October 2017, a multidisciplinary consultation regarding the term 'risk feeding' was undertaken via Survey Monkey. The survey was cascaded to speech and language therapists (SLTs), consultant geriatricians, physiotherapists, dietitians, nurses and GPs. Results: The survey yielded 367 responses, of which $91 \%$ were SLTs. 'Risk feeding 'was used as a consistent term by $39 \%$ of the participants. Of all the respondents, $43 \%$ were positive about the term whilst $29 \%$ were negative and $28 \%$ remained neutral. The results raised key factors which influenced opinion on the term 'risk feeding'. These included the service setting, the use of the word 'risk', the impact of the term on the individual and family, the ambiguity of the term.

Conclusion: The survey has highlighted both enablers and barriers to the term risk feeding. Locally agreed terminology with an emphasis on a model of care to support discussions should be the way forward until national consensus is agreed.

References:

[1] RCP (2010) Guidelines on oral feeding difficulties and dilemmas. Available at:https://www.rcplondon.ac.uk/projects/outputs/oralfeeding-difficulties-and-dilemmas Accessed on 05/02/18].[2] Smith, H.A., Kindell, J., Baldwin, R.C., Waterman, D. and Makin, A.J. (2009). Swallowing problems and dementia in acute hospital settings: practical guidance for the management of dysphagia. Clinical Medicine, 9(6), pp. 544-548.

\section{P-883}

Pain and sedation monitoring using pupillometry at the end-oflife in palliative care practice : preliminary observations

Elyn Antoine ${ }^{1}$, Larson Merlin ${ }^{2}$, Mazerolles Michel ${ }^{3}$, Morin Lucas ${ }^{4}$, Saffon Nicolas ${ }^{1}$, Bongard Vanina ${ }^{5}$, Rolland Yves ${ }^{6}$

${ }^{1}$ palliative Care, Geriatric And Internal Medicine, University Hospital of Toulouse, Toulouse, France, ${ }^{2}$ Anesthesia and Perioperative Care, UCSF University Hospital of San Francisco, San Francisco, United States of America, ${ }^{3}$ Anesthesia and Resusc

Introduction: At the end of life, deep and continuous sedation practice require improvement in the monitoring of sedation and pain, in order to avoid the burden of unethical awake phases. Developed in anesthesiology, portable pupillometry is a nociception monitoring technique based on sympathetic and parasympathetic balance through pupil dynamic recording. It has never been used or evaluated in the palliative care complex framework near the end-of-life. We aimed to report clinical examples to provide primary evidence of the possible range of usefulness in end-of-life and palliative care practice.

Methods: Case series using narrative clinical aspects and descriptive statistics.

Results: We report 11 patients from a french palliative care unit. Age ranges from 22 to 97 years old. There are suffering complex health conditions and are exposed to multiple drugs to help symptom management. Light reflex might be progressively altered during sedation. Pain assessment might be possible using pupil dilatation recording during specific procedure. We observed a progressive alteration of light reflex until death. Intracerebral pathology might influence pupil size and dynamic (eg. vascular injury, septic embols for endocarditis, grade 4 glioblastoma). Instead of traditional scopolamine, butylbromure-scopolamine might not alter light reflex. Low dose infusion of ketamine might not influence pupil dynamic.

Conclusion: Pupillometry seems to provide useful information about awakeness state, pain experience and pharmacological mecanism. It might be an effective technique to monitor pain and sedation in palliative care practice. However, our cases illustrate the need to 
properly define variability of pupil dynamic among palliative care patients in regards to complexity of physiological aspects of end-oflife, progressive organ failure and pharmacological influence.

\section{P-884}

Functioning, quality of life, and end of life care of patients with huntington's disease living in long-term care facilities: the advanced HD study design

\section{MR Ekkel ${ }^{1}$, EML Verschuur ${ }^{2}$, MFIA Depla ${ }^{1}$, RB Veenhuizen ${ }^{3}$, BD Onwuteaka-Philipsen ${ }^{4}$, CMPH Hertogh ${ }^{1}$}

${ }^{1}$ Department of General Practice and Elderly Care Medicine, Amsterdam Public Health Research Institute, VU University Medical Center, Amsterdam, The Netherlands, ${ }^{2}$ HAN University of Applied Sciences, Nijmegen, The Netherlands, ${ }^{3}$ University Network of

Introduction: Huntington's disease (HD) is an inherited neurodegenerative disease, characterized by movement disorders, psychiatric symptoms, and cognitive decline. Care for HD patients in long-term care facilities (LTCFs) is complex and practice-based, consisting of interventions developed through years of experience. Little is known about the functioning, quality of life, and end of life care of these patients. Aims: - Gain insight in motor, psychiatric, and cognitive symptoms, functional ability, and quality of life of HD patients living in LTCFs.- Gain insight in perceptions of HD patients concerning their future and end-of-life, and whether these perceptions change over time.- Gain insight in views of elderly care physicians on advance care planning and palliative care for HD patients.

Aims: Gain insight in motor, psychiatric, and cognitive symptoms, functional ability, and quality of life of HD patients living in LTCFs.Gain insight in perceptions of HD patients concerning their future and end-of-life, and whether these perceptions change over time.- Gain insight in views of elderly care physicians on advance care planning and palliative care for HD patients.

Methods: This study consists of a cross-sectional, descriptive study, and a qualitative study.

Results: Data of the cross-sectional study are collected from September 2017 until May 2019. We aim to include 250 HD patients living in 9 specialized LTCFs in The Netherlands. The data will be obtained using observation scales and questionnaires. Data of the qualitative study are obtained by conducting multiple in-depth interviews every 6 months for the period of 2-2.5 years with 10-15 HD patients, and one in-depth interview with elderly care physicians. Results are expected in 2021.

Key conclusions: This study will provide valuable information on the functional status, quality of life and end-of-life care of HD patients living in LTCFs. Results may provide an evidence base to develop specific guidelines for care of institutionalized HD patients.

\section{P-885}

The 4-dimensional model applied to palliative care in nursing home, a cost-effective example of geriatric comprehensive assessment

Simone Cernesi ${ }^{1}$, Francesca Mele ${ }^{2}$, Daniela De Nadai ${ }^{3}$, Alessandra Taglini ${ }^{4}$, Giacomo Bernabei ${ }^{5}$

${ }^{1}$ General Practictioner, Nursing Home physician "Casa Serena" Palliative Care Consultant, Sassuolo, Italy, ${ }^{2}$ Nurse Coordinator "Casa Serena" Sassuolo, ${ }^{3}$ Nurse Expert in Cerebrolesion Management

"Casa Serena, ${ }^{4}$ Nurse in Palliative Care Mobile team
Background: Nursing Care Homes for will become the place of death of the elderly and fragile people. According to the study by A. Bone by 2040.Our Nursing home has developed a specific protocol of Palliative Care in Patient with advanced dementia. We Integrated the 4-Dimensions Model developed by Prof. Murray a multidimensional approach divided into 4-Dimensions: (1) Physical (2) Social (3) Psychological (4) Spiritual.

Method: Discussion in Equipe the 4 areas of the model were reproposed, applying them in the Advanced Care Plan and as a result the possible areas of intervention. (1) Physical Dimension: identification of the target patient (Gold Standard Framework / SIAARTI Document, use of KIS (key Information Summary). Adoption of KPS, ADL, IADL, PPI, PPS, CDR, MPI, NPI, 4-AT, PAINAD, ESAS, Dyspnea scales. End of life (EoL) discussion. PRN therapies Special write consent to avoid unnecessary hospitalization (2) Psychological: explain the trajectories of the disease were introduced and the SPIKES in family conference. were offered for mourning, addressed to local support groups. Psychological support discussion with Palliative Care Mobile Unit. (3) Social Extended visit times. Environment Intervention. (4) Spiritual Dimension: In the ACP of the end of life the spiritual dimension is explored and part of the assessment, pastoral support is available.

Results: In process of elaboration. Preliminary data show a significant increase in the ACP and increase of early palliative care, the care project failures were extremely rare. Equipe AUDIT following every critical case.Very low rate of Artificial Nutrition.

Conclusions: Preliminary data confirm that almost all the formalized end-of-life projects were concluded in Nursing home setting. Good feedback from family members with verbal and written acknowledgments. We involved students and other visiting professionals to enhance our palliative care experience in the elderly care.

\section{P-886}

Increases in agreement on opinion regarding "good death" between in-training physicians and older patients, trend of changes toward aging society in Thailand

Manchumad Manjavong ${ }^{1}$, Varalak Srinonprasert ${ }^{1}$

${ }^{1}$ Division of Geriatric medicine, Siriraj hospital, Mahidol University, Thailand

Background: In order to carry out qualitative care for older patients toward end-of-life (EOL), understanding of their wishes regarding good death is crucial. Some differences between physicians and older adults in component of good-death were apparent from previous study in Thailand. An educational programs have been implemented over few years. We explored the differences opinions between physicians and older people and the trend of change over 7-year period.

Methods: We conduct a cross sectional study using 13-situationbased questionnaire which developed from literature reviews to explore perspectives on 'good-death'. Participants were older patients and physicians at a university hospital. The results were compared between 2 groups and to previous study and also compared the physicians' preferences for their EOL and what they would do for patients.

Results: Three hundred fifty-two patients and 293 physicians were recruited. General concepts such as wishing to know the truth regarding prognosis, to treat suffering symptoms were important for most participants. Compared to previous study, higher proportion of physicians wished to tell all the truths about illnesses to patient $(96.9 \%$ vs $88.1 \%, \mathrm{p}<0.001)$ and agreed with patients regarding not to prolong suffering $(83.2 \%$ vs $71.0 \%, \mathrm{p}<0.001)$. When elicited opinion for their own EOL, higher proportion of physicians agree for most 
components in the questionnaire, particularly on not prolong suffering.

Conclusions: Some difference in opinions regarding good death between patients and physicians remains. Nevertheless, the gap has been narrow over the past 7 year. Current scheme to promote knowledge regarding palliative care appears to be successful.

\section{P-887}

A comparison of health care utilization and end of life care provided for cancer and non-cancer older patients at Department of Medicine Siriraj hospital

Tanchanok Chattaris ${ }^{1}$, Varalak Srinonprasert ${ }^{1}$, Sutisa Pitiyarn ${ }^{1}$, Patumporn Suraarunsumrit ${ }^{1}$

${ }^{1}$ Division of Geriatric Medicine, Faculty of Medicine, Siriraj Hospital, Mahidol University, Thailand

Background: Advance chronic diseases and cancers are major causes of death among older patients. Appropriate palliative and end of life care would enhance death without suffering and reduce healthcare cost. Study that compared between cancer and non-cancer Thai patients regarding care for them are limited.

Methods: A retrospective cohort of patients at least 60 years old admitted and discharged alive in 2013 was investigated. They were classified into cancer and non-cancer group, according to the study criteria. Healthcare utilization and advance directives were collected. Results were analyzed.

Results: There were 306 older patients (130 of metastatic cancer and 176 of advance stage of non-cancer cases). Non-cancer patients were older and had more comorbidities than those in cancer group. Advance directives before admission were conducted in $38.6 \%$ of non-cancer and $10.5 \%$ of cancer patients $(\mathrm{p}<0.001)$. The median length of hospitalization of non-cancer group and the other group were 11 and 7 days, respectively $(\mathrm{p}<0.001)$. In non-cancer patients, rate of invasive procedures such as endotracheal intubation were higher $(\mathrm{p}<0.001)$. Health care cost $(\mathrm{USD} /$ patient/year) were 6790 and 3900 in non-cancer and cancer, respectively, $\mathrm{p}<0.001$ ). Time interval to dead from last discharge date in 2013 was significantly longer in the non-cancer group (476.9 VS 180.3 days, $\mathrm{p}<0.001$ ).

Conclusion: In non-cancer group, health care utilization was higher than the other one. Palliative and end of life care should be considered in routine practice for caring of older patients with advance stage of illnesses, for the benefits of peaceful death and reduction in healthcare utilization. Keywords: palliative care, advance care planning, end of life care, cancer and non-cancer, older patients

\section{P-888}

The 4-dimensional model applied to palliative care in nursing home, a cost- effective example of geriatric comprehensive assessment

Simone Cernesi ${ }^{1}$, Francesca Mele ${ }^{2}$, Daniela De Nadai ${ }^{3}$, Alessandra Taglini $^{4}$, Giacomo Bernabei ${ }^{5}$

${ }^{1}$ General Practictioner, Nursing Home Physician "Casa Serena" Palliative Care Consultant, ${ }^{2}$ Nurse Coordinator "Casa Serena" Sassuolo, ${ }^{3}$ Nurse Expert in Cerebrolesion Management "Casa Serena”, ${ }^{4}$ Nurse in Palliative Care Mobile team Sassuolo, ${ }^{5} \mathrm{Nu}$

Background: Nursing Care Homes for will become the place of death of the elderly and fragile people. According to the study by A. Bone by 2040. Our Nursing home has developed a specific protocol of
Palliative Care in Patient with advanced dementia. We Integrated the 4-Dimensions Model developed by Prof. Murray a multidimensional approach divided into 4-Dimensions: (1) Physical (2) Social (3) Psychological (4) Spiritual.

Method: Discussion in Equipe the 4 areas of the model were reproposed, applying them in the Advanced Care Plan and as a result the possible areas of intervention. (1) Physical Dimension: identification of the target patient (Gold Standard Framework / SIAARTI Document, use of KIS (key Information Summary). Adoption of KPS, ADL, IADL, PPI, PPS, CDR, MPI, NPI, 4-AT, PAINAD, ESAS, Dyspnea scales. End of life (EoL) discussion. PRN therapies Special write consent to avoid unnecessary hospitalization (2) Psychological: explain the trajectories of the disease were introduced and the SPIKES in family conference. were offered for mourning, addressed to local support groups. Psychological support discussion with $\mathrm{Pal}-$ liative Care Mobile Unit. (3) Social Extended visit times. Environment Intervention. (4) Spiritual Dimension: In the ACP of the end of life the spiritual dimension is explored and part of the assessment, pastoral support is available.

Results: In process of elaboration. Preliminary data show a significant increase in the ACP and increase of early palliative care, the care project failures were extremely rare. Equipe AUDIT following every critical case. Very low rate of Artificial Nutrition.

Conclusions: Preliminary data confirm that almost all the formalized end-of-life projects were concluded in Nursing home setting. Good feedback from family members with verbal and written acknowledgments. We involved students and other visiting professionals to enhance our palliative care experience in the elderly care.

Area: Psychiatric symptoms and illnesses

P-889

The cause of death in geriatric schizophrenia patients

Marin Takasou ${ }^{1}$, Masahito Hitosugi ${ }^{2}$

${ }^{1}$ Shiga University of Medical Sciece, ${ }^{2}$ Shiga University of Medical Science

Introduction: The treatment of schizophrenia is improvement. Some doctors think schizophrenia patients can live until mean age if they do not choose suicide death.

Methods: We picked diagnosed schizophrenia deceased in Osaka medical examiner office and examined their cause of death for 3 years.

Results: The deceased more than 65 years old were 132 people ( 70 men average 56.1 years, 62 females average 57.5 years). 67 people were died due to diseases; 50 were heart disease, 11 were pneumonia and three were cerebral infarction, liver cirrhosis, malignant tumor respectively. In suicide, the average age were about 10 year younger than the deceased due to disease. The breakdown of the suicide were nine hanging, five jumping. In extrinsic death, ten deceased were accidental ingestion/asphyxia. We thought the result was associated with pica and side effect of drugs.

Key conclusions: We showed case reports which couldn't be decided whether suicide or not in case of drug intoxication and drowning. 


\section{P-890}

Religion and depression/distress symptomatology among community-dwelling elderly persons cancer

\section{Jeremy M Jacobs ${ }^{1}$, Lea Baider ${ }^{2}$, Gil Goldzweig ${ }^{3}$}

${ }^{1}$ The Jerusalem Institute of Aging Research, Hadassah-Hebrew University Medical Center Mount Scopus, and Hebrew UniversityHadassah Medical School, Mount Scopus, Jerusalem, Israel, ${ }^{2}$ Assuta Medical Center, Oncology Institute, Tel-aviv, Israel., ${ }^{3} \mathrm{Sc}$

Background: Religion may have an important role as a resource for coping with the negative emotional reaction to the cancer experience. The aim of this study is to examine the relation between self-declared levels of religiosity (religious, traditional and secular) and depression / distress symptomatology among community-dwelling elderly persons with cancer.

Methods: The current study included 243 cancer patients, aged 65-97 years old who have active cancer disease. Patients who had cognitive impairment, had hospice care or needed help in basic ADL activity were excluded from the study. Depressive symptomatology is measured using the 5-item GDS (Geriatric Depressive Scale) and distress by the Distress Thermometer.

Results: The findings indicate that the highest levels of depressive symptoms and distress were seen among cancer patients in the traditional group ( $2.24 \pm 1.82$ and $5.01 \pm 3.50$, respectively), among patients in the secular group lower levels of depressive and distress symptoms were reported $(1.78 \pm 1.87$ and $4.36 \pm 3.36$, respectively), while patients in the religious group had the lowest level of depression and distress $(1.26 \pm 1.45$ and $3.34 \pm 3.14$, respectively).ConclusionThe association between religion and depressive symptoms and distress in elderly adults with cancer is not linear. Understanding the complex mechanisms whereby religion may affect depressive symptoms and distress among elderly cancer patients remains an important research priority.

Conclusion: The association between religion and depressive symptoms and distress in elderly adults with cancer is not linear. Understanding the complex mechanisms whereby religion may affect depressive symptoms and distress among elderly cancer patients remains an important research priority.

\section{P-891}

The evaluation and design of a short depression screening tool in Turkish older adults

Ozge Dokuzlar ${ }^{1}$, Pinar Soysal ${ }^{2}$, Cansu Usarel ${ }^{3}$, Ahmet Turan Isik ${ }^{3}$

${ }^{1}$ Department of Geriatric Medicine, Dokuz Eylul University, Faculty of Medicine, Izmir, Turkey, ${ }^{2}$ Kayseri Education and Research Hospital, Geriatric Center, Kayseri, Turkey, ${ }^{3}$ Unit for Aging Brain and Dementia, Department of Geriatric Medicine, Doku

Introduction: Depression is a common and serious health care problem for older adults. This study aimed to determine validity and reliability of GDS-4 and GDS-5 in Turkish, and to establish a new short-form geriatric depression scale for our population, and also determine the superiority of each short scale to another.

Methods: A total of 437 outpatients were enrolled in the study. A researcher evaluated all cases according to the DSM-5 diagnostic criteria, and then another researcher applied GDS-15 to all cases. We obtained the answers of short GDS forms, examined in this study, from GDS-15 forms. After Cohen's kappa analysis, we compared the diagnostic value of each question for geriatric depression according to their kappa values and, developed three (TGDS-3), four (TGDS-4), five (TGDS-5) and, six (TGDS-6) question-scales to screen geriatric depression in the Turkish population.

Results: A total of 437 participants were assessed. The mean age (SD) of the patients was 72.95 (7.37). Cronbach's alpha values of GDS-4 and GDS-5 were $<0.70$. TGDS-3, TGDS-4, TGDS-5, TGDS6's Cronbach's alpha values were $>0.70$. The best cuf-off values were $\geq 5$ for GDS-15, GDS-5, and $\geq 1$ for others.

Discussion: GDS-15 is the most powerful screening scale for geriatric depression. GDS-4 and GDS-5 are not eligible for depression screening in Turkish older adults. All new short scales are valid and reliable, and the TGDS- 4 is a practical, less time-consuming option for daily practice.

\section{P-892}

Mental health inequalities among older adults in Iran: Findings from a population-based study (Urban HEART-2)

Vahid Rashedi ${ }^{1}$, Mohsen Asadi-Lari ${ }^{2}$, Mahshid Foroughan ${ }^{3}$, Reza Fadayevatan $^{3}$, Ahmad Delbari ${ }^{3}$

${ }^{1}$ Tehran Psychiatric Institute, School of Behavioral Sciences and Mental Health, Iran University of Medical Sciences, Tehran, Iran, ${ }^{2}$ Department of Epidemiology, School of Public Health,

Oncopathology Research Center, Iran University of Medical Science

Introduction: There is a widespread consensus that socioeconomic inequalities have remarkable implications for health of people, and the implications are even more prominent for mental health. Little is known about the impacts of socioeconomic inequalities on mental health of older people in Iran. This study aimed to analyze the mental health inequalities among older population of the Metropolitan Tehran.

Methods: This paper uses data from the second round of Urban Health Equity Assessment and Response Tool (Urban HEART-2) survey. In this large population-based study, 5326 individuals aged 60 years and over were selected randomly based on a multistage cluster sampling in Tehran, Iran, in 2011. Mental health was measured through General Health Questionnaire-28. Principal component analysis on assets was used to measure participants' socioeconomic status. Socioeconomic inequality in mental health status was measured by Concentration Index (CI).

Results: The mean age of the participants was $68.92 \pm 7.03$ years and $54.8 \%$ of participants were female. Overall, CI estimate was negative for the older population as a whole $(\mathrm{CI}=0.0325)$, as well as for two disaggregated groups of male $(\mathrm{CI}=0.0317)$ and female $(\mathrm{CI}=0.0207)$. Other indicators, such as gender, ethnicity, disability, and occupation were significantly associated with mental health in the older adults.

Key conclusions: This study found that there is socioeconomic inequalities in mental health among Iranian elders, favoring those of higher socioeconomic status. This finding contributes to identification of socioeconomic factors as important determinants of mental health that can lead to recommendations to close the gap in mental health between people. 


\section{P-893}

Clinical outcomes of elderly patients who had attempted deliberate self-poisoning in emergency department

Seon Hee Woo ${ }^{1}$

${ }^{1}$ Department of Emergency medicine, Incheon St. Mary's hospital, College of Medicine, the Catholic University of Korea, Incheon, Republic of Korea

Background: Many elderly patients present to the emergency department (ED) complaining of deliberate self-poisoning (DSP). The aim of this study was to determine some characteristics of elderly patients who committed DSP.

Methods: A retrospective review was performed on 1329 patients ( $>15$ years) who were treated with poisoning in two EDs between January 2010 and December 2016. Of these patients, we classified into two groups depending on age (elderly group $\geq 65$, adult group). Information regarding age, gender, cause, time of ingestion, type of drug, history of attempting suicide, and outcome, among other characteristics, was collected.

Results: The $242(18.2 \%)$ patients were included in elderly group. The $211(86.9 \%)$ patients in the elderly group were first suicide attempt ( $<<0.001) .186(77.2 \%)$ patients of elderly group visited in ED by 119 EMS Ambulances ( $<<0.001$ ). Regarding GCS $<13$ in visiting of ED, 95(39.7\%) of elderly group and 211(19.6\%) of adult group showed $(\mathrm{p}<0.001)$. In elderly group, admission to the ICU (43.8\% vs $25.5 \%)$ and endotracheal intubation ( $16.1 \%$ vs $4.9 \%)$ was found more frequently than adult group $(\mathrm{p}<0.001)$. The distribution of poisoning severity score showed more poor outcomes (score 3-4) in elderly $(\mathrm{p}<0.001)$. There were no differences in past psychiatric history between elderly and adult patients.

Conclusion: In elderly poisoning patients who visited in ED, first suicide attempt was found more frequently. Decreased mental status and poor outcome is a more common in elderly poisoning patients. Emergency physicians should be consider poor progression of elderly poisoning patients in ED.

\section{P-894}

Relationship between sleep duration and coronary heart disease in older adults

Pelin Ünsal ${ }^{1}$, Mert Eşme ${ }^{1}$, Cafer Balc1 ${ }^{1}$, Gözde Şengül Ayçiçek ${ }^{1}$, Burcu Balam Yavuz ${ }^{1}$, Meltem Halil ${ }^{1}$, Mustafa Cankurtaran ${ }^{1}$

${ }^{1}$ Hacettepe University Faculty of Medicine, Department of Internal Medicine, Division of Geriatric Medicine, Ankara, Turkey

Background and aim: Insomnia, a frequent geriatric syndrome, is thought to be linked with coronary artery disease and increased mortality. The aim of our study was to examine the relationship between sleep duration and coronary heart disease (CHD) and cardiovascular risk factors.

Patients and method: In this study, patients who applied to geriatric medicine outpatient clinic for comprehensive geriatric assessment were retrospectively studied. Optimal sleep duration was assumed to be $6 \mathrm{~h}$ and patients were divided into two groups; sleeping under $6 \mathrm{~h}$ and sleeping $6 \mathrm{~h}$ or more.

Results: Total number of 2255 patients (age $72 \pm 6$ years, $63.3 \%$ female) were included in this study. Patients who were sleeping less than $6 \mathrm{~h}$ were older $(14.4 \%$ vs. $10.3 \%$; $\mathrm{p}=0.005)$ and female sex was higher $(69.5 \%$ vs. $60.2 \%$; $<<001)$. The frequency of CHD $(31.9 \%$ vs. $25.9 \% ; \mathrm{p}=0.003)$, falls $(31.8 \%$ vs. $25.3 \% ; \mathrm{p}=0.002)$, and depression $(25.7 \%$ vs. $21.3 \%$; $=0.022)$ were higher, hypertension $(68.5 \%$ vs. $72.5 \% ; p=0.049$ ) was lower in the short sleep duration group. In the regression analysis, independent from other parameters, CHD (OR: $1.39 ; 95 \%$ CI: $1.14-1.7 ; \mathrm{p}=0.001)$, age (OR: $1.02 ; 95 \% \mathrm{CI}$ : 1.01-1.04; $\mathrm{p}=0.001)$, male gender (OR: 0.63 ; 95\% CI: $0.51-0.76$; $\mathrm{p}<0.001$ ), hypertension (OR: 0.74; \%95 CI: 0.6-0.9; $\mathrm{p}=0.003$ ), and falls history (OR: $1.25 ; 95 \%$ CI: $1.02-1.5 ; \mathrm{p}=0.026$ ) were found to be associated with short sleep duration.

Conclusion: Similar to the literature in our study, CHD was frequently observed in patients who had less than $6 \mathrm{~h}$ of sleep. Considering this relationship between short sleep duration and CHD, it is important to question sleep duration in comprehensive geriatric assessment.

\section{P-895}

Cross-sectional observational study of prevalence and factors associated with depression in patients admitted to a convalescence unit

Eva Martinez Suarez ${ }^{1}$, Elisabet Palomeras Fanegas ${ }^{1}$, Jose Antonio Martos Gutierrez ${ }^{1}$, Ramon Cristofol Allue ${ }^{1}$

${ }^{1}$ Hospital de Mataró

Introduction: Depression is frequent psychiatric disorder and leads to serious consequences [1]. They could developed in previous stages and persist or develop late. It's associated with comorbidities, functional deterioration, use of healthcare resources and increased mortality $[2,3]$. Depression in the geriatric patient is underdiagnosed and treated incorrectly [5,6,7].

Methods: Cross-sectional observational study took place in Hospital Sant Jaume (Barcelona), patients recruited from February-2017 until February-2018. Patients included were attended in the convalescent service who have signed informed consent. Patients excluded were: not accept assessment, not understand test questions, have dementia, Mini-mental state examination (MMSE) $<24$ points.

Results: 100 patients were analyzed, 62 women and 38 men, between $58-99$ years $(\mathrm{y})$ and an average age of $78.60 \mathrm{y}(+/-10.95)$.Origin of the patients $47 \%$ of community(alone), $47 \%$ community(living with relative),3\% community(living with family worker) and $3 \%$ institutionalized. Diagnosis: $45 \%$ functional deterioration in relation to new diagnosis, $7 \%$ of which to acute cerebrovascular accident; $25 \%$ femur fracture; $16 \%$ exacerbation of chronic disease; $6 \%$ acute arterial ischemia with amputation; $4 \%$ due to ulcer healing; $1 \%$ due to insufficient social support. The prevalence of depression according to the clinical history was $31 \%$. When screening: two question screener positive $82 \%$, Yesavage scale positive $57 \%$. Diagnosis with Hamilton scale it positive $61 \%$. Use of psychotropic drugs present in $44 \%$ patients: $31 \% 1$ drug, 19\% 2 drugs, 3\% 3 drugs and $1 \% 4$ drugs. Type of psychotropic drugs used $31 \%$ antidepressants, $28 \%$ benzodiazepines, $6 \%$ antipsychotics, $7 \%$ antiepileptic.

Key conclusions: Elderly patients have high prevalence of depression detected at screening or diagnostic and more frequently associated with greater social risk.

\section{P-896}

\section{Evaluation of sleep disorders and accompanying factors}

Banu Ozulu Turkmen ${ }^{1}$

${ }^{1}$ Istanbul University Istanbul Faculty of Medicine/ Department of Geriatrics Division of Geriatrics

Introduction: Sleep is a normal, transient, periodic and psychophysiological status that is caused by the decline in nervous 
sensation and voluntary muscle movements of organic activities. Sleep problems are highly prevalent in those over the age of 65 , with up to $50-70 \%$ in some studies. In elderly population sleep disorders have been related with poor quality of health, falls, inappropriate medication use and higher rates of morbidity and mortality. In our study, we aimed to evaluate the frequency of sleep problems and their relationship between the possible factors in our outpatient clinic.

Material and method: A retrospective review of medical reports of 295 patients, admitted (November 2013-November 2016) was conducted and the relationen sleep problems and age, gender, falls, fraility, restless leg syndrome (RLS) were evaluated.

Results: 295 geriatric patients were included in the study. 66\% $(\mathrm{n}=196)$ of the patients was female and, remaining $34 \%(\mathrm{n}=99)$ male. Mean age was $75.6 \pm 6.8$. Prevalence of sleep problems was $47.8 \%$. In bivariate analysis, the relation between insomina and age, polypharmacy, RLS was significant. There was no statistically significant difference between both genders. In regression analysis, the association between sleep problems and age, polypharmacy and RLS was found independent.

Conclusions: Sleep disorders in elderly is a major health problem affecting quality of life negatively and should be questioned during polyclinic visits. It should be kept in mind that the sleep problems may be related with important geriatric syndromes as polypharmacy.

\section{P-897}

Depression In later life is associated with blood pressure dependent frontal lobe hypoperfusion. Data from TILDA

\author{
Robert Briggs $^{1}$, Daniel Carey ${ }^{1}$, Sean Kennelly ${ }^{1}$, Rose Anne Kenny ${ }^{1}$ \\ ${ }^{1}$ The Irish Longitudinal Study on Ageing, Trinity College Dublin, \\ Ireland
}

Background: Frontal lobe white matter disease has been implicated in late life depression (LLD) and hypoperfusion has been suggested as a potential mechanism for this. The aim of this study is to examine the association between depression and frontal lobe perfusion during orthostasis in a large cohort of community-dwelling older people.

Methods: Over 2500 participants aged $\geq 50$ years were included and underwent measurement of orthostatic blood pressure (BP) by finometry and frontal lobe perfusion by near-infrared spectroscopy (NIRS). Depression was assessed by the 8-item CES-D. Real-time frontal lobe cerebral oxygenation was measured by the Portalite System, detecting changes in frontal lobe perfusion and reporting a \% Tissue Saturation Index (TSI).

Results: Almost 8\% (209/2616) met criteria for depression. Multilevel models demonstrated significantly lower TSI in participants with depression compared to the non-depressed group at both 60 and $90 \mathrm{~s}$ post-stand with coefficients of -0.43 (95\% CI -0.63 to $-0.22)$ and -0.37 (95\% CI -0.57 to -0.16$)$ respectively. Controlling for covariates, such as cardiovascular disease, stroke, antidepressant/antihypertensive use, did not significantly attenuate these associations. After addition of systolic BP this association was no longer significant however, suggesting lower BP may modify this relationship.

Conclusion: This study demonstrates that depression is associated with lower frontal lobe perfusion after standing in a cohort of community-dwelling older people and that this association is BP dependent. This finding is important because, given the established longitudinal association between hypotension and incident LLD, cerebral hypoperfusion may represent an underlying mechanism for a causative relationship and therefore a potential therapeutic target.

\section{P-898}

How does knowledge in diagnosis and management of insomnia differ between an acute medical hospital and a mental health hospital?

Safa G Attar ${ }^{1}$, Raunak Singh ${ }^{1}$, Sharada Abhilash ${ }^{2}$, Erik VanDiepen ${ }^{3}$, Ashish Patel ${ }^{1}$

${ }^{1}$ University Hospitals of Leicester, United Kingdom, ${ }^{2}$ Leicestershire Partnership NHS Trust, United Kingdom, ${ }^{3}$ University Hospitals of Leicester, Leicestersgire Partnership NHS Tust, United Kingdom

Introduction: Insomnia is a common complaint, particularly amongst elderly inpatients. It causes a significant negative impact on quality of life and daily functioning. Despite this, it is often poorly managed. The inappropriate first line prescription of sedating medication leads to adverse secondary consequences. To manage patients with insomnia effectively, it is key that healthcare professionals across all specialities have knowledge and understanding of insomnia diagnosis and management.

Method: We surveyed staff on their knowledge in diagnosis and management of insomnia within an acute medical hospital (AMH) and a mental health hospital (MHH). The wards included: Geriatrics, Stroke, Diabetes, Orthopaedics, Elderly Frailty Unit within Emergency Department, Old Age and Adult Mental Health. The results between the two hospitals were compared.

Results: 72 Questionnaires in total (39 at the $\mathrm{AMH}$ and 33 at the $\mathrm{MHH}$. 93\% had not received any training in insomnia. All the staff lacked knowledge in diagnosis and pharmacological management. $85 \%$ of the staff at the MHH were aware that non-pharmacological measures (for instance lifestyle and diet) are the first line management versus $54 \%$ at the $\mathrm{AMH}$.

Key conclusions: There is insufficient knowledge across both hospitals in diagnosis and pharmacological management. The increased knowledge of non-pharmacological management aspects within the MHH can be explained by increased awareness and emphasis of insomnia management as a factor in mental wellbeing and the part it plays in psychiatric illnesses. The overall gap in knowledge highlights the need for education and guidelines on insomnia to deliver the best appropriate care for our patients.

\section{P-899}

\section{Psychogenic falls in an elderly patient}

Atef Michael ${ }^{1}$, George Tadros ${ }^{2}$

${ }^{1}$ Russells Hall Hospital, Dudley, UK, ${ }^{2}$ Birmingham Heartlands Hospital, UK

An 86 years male attended the falls clinic with recurrent falls for more than 15 years and many "stumbles". Past medical history included hypertension, permanent pacemaker, Meniere's disease and anxiety. He was on Amlodipine, Citalopram and Betahistine. He was mobile with a stick and independent for ADLs.The nurse during preparation commented that while taking his observations he fell "strangely". Heart, chest, abdominal and full neurological exam was normal. After the consultation he stood and collapsed slowly in the chair, then he stood again steadily, walked outside the room without support. Approaching a chair he staggered, walked a step backwards and supported himself onto the wall until help came. Haematological and biochemical profile was normal. ECG showed paced rhythm and tilt table test revealed normal response. He had physiotherapy for 12 weeks. The therapist observed that he had an exaggerated response when balancing. For the subsequent 8 months he continued to fall three to four times daily but mostly managed to land safely on a near 
seat or against a wall. In the follow up clinics he displayed the same findings on standing and walking. His mild vestibular hypofunction did not explain the severity and extent of his symptomology. The pattern of falls, the frequency and duration without soft tissue or bone injury, with lack of signs or investigation result that can explain the falls supported a "psychogenic "cause. This was sensitively discussed with the patient but initially he resisted the concept. After reflection, 4 month later, he accepted the diagnosis. He was referred to and had eighteen sessions with a psychologist. When reviewed 10 months later he was definite that the frequency of falls had become less since the intervention. He had no falls during the next 15 months; but continued to stagger. In the clinics he was still observed to have staggering following standing or starting to walk, and if not supported he would adjust himself immediately or support himself against a wall. In one of the clinics he staggered on standing, a nurse ran to help him. His partner commented "the less people there are the more steady he will be". Then the patient started to fall again. Over the subsequent 3 years he was admitted seven times for a total of 63 days; with several investigations, courses of physiotherapy and addition of medications. When he was 92 years, he fell and fractured his neck of femur. It was difficult to diagnose the cause of his recurrent falls. Fear of falling is a well-known disabling consequence of falling. In our case there was another psychogenic element as well. The patient's falls were most likely due to conversion disorder; a functional neurological disorder. The patient was unlikely to be malingerer or factitious. The case demonstrated a rare cause for falls in older people; a conversion disorder. It showed how a psychogenic cause of falls could be difficult to diagnose, resistant to treat, protracted and costly with a serious devastating outcome. It is essential to consider and address the psychological element.

\section{P-900}

Fear of falling and activity avoidance is associated with life space in geriatric patients with cognitive impairment

\section{Klaus Hauer ${ }^{1}$, Phoebe Ulrich ${ }^{1}$, Martin Bongartz ${ }^{1}$, Tobias Eckert ${ }^{1}$ \\ ${ }^{1}$ Agaplesion Bethanien Hospital Heidelberg, Geriatric Center at the University of Heidelberg, Heidelberg, Germany}

Background: Analysis of fear of falling (FOF)-related determinants for life space (LS) in vulnerable, multi-morbid patients with cognitive impairment $(\mathrm{CI})$ and acute motor impairment following discharge from geriatric rehabilitation are lacking.

Objective: To identify association between LS and fall-related selfefficacy, activity avoidance and LS.

Objective: To identify association between LS and fall-related selfefficacy, activity avoidance and LS.

Methods: All relevant parameters were assessed by established validated methods: Fall-related self-efficacy (Falls Efficacy ScaleInternational, FES-I), activity avoidance (Fear of Falling Avoidance Behavior Questionnaire, FFABQ) and LS (Life-space Assessment in Persons with Cognitive Impairment, LSA-CI), while associations were analyzed by Spearman correlation in 117 home-dwelling patients with CI following discharge from geriatric rehabilitation.

Results: FES-I and FFABQ were significantly associated (all $\mathrm{p}<0.01$ ) with LSA-CI total-score (FES-I: (rho): - 0.024, FFABQ:.38 ) but also with LSA sub-scores documenting impact of personal as well as technical support: (FES-I: LSA-CI-I: - 0.25; FFABQ: LSACI-E: - 0.35; LSA-CI-I: - 0.44)

Conclusion: Fall related self-efficacy as well as fall-related activity avoidance was significantly associated with LS in a vulnerable, high risk group for FOF and activity restriction, highly relevant for quality of life in old age.

\section{P-901}

Depressive symptom profiles and survival in older patients with cancer: latent class analysis of the ELCAPA cohort study

Canouï-Poitrine Florence ${ }^{1}$, Gouraud Clément ${ }^{2}$, Martinez-Tapia Claudia $^{3}$, Segaux Lauriane ${ }^{2}$, Reinald Nicoleta ${ }^{2}$, Hoertel Nicolas ${ }^{4}$, Gisselbrecht Mathilde ${ }^{4}$, Mercadier Elise ${ }^{4}$, Boudou-Rouquette Pascaline ${ }^{5}$, Chahwakilian Anne ${ }^{5}$, Bastuji-G

${ }^{1}$ Hôpital Henri-Mondor, ${ }^{2}$ APHP-Hôpital Henri-Mondor, ${ }^{3}$ Université Paris Est Creteil, ${ }^{4}$ APHP-HEGP, ${ }^{5}$ APHP-Cochin, ${ }^{6}$ APHP-HenriMondor, ${ }^{7} \mathrm{APHP}-$ Corentin Celton

Introduction: The objective was to identify depressive symptoms profiles in older patients with cancer, describe the associated factors and assess the prognostic value of the profiles regarding survival.

Methods: Patients $\geq 70$ years old referred to geriatric oncology clinics were prospectively included. Depressive symptoms were used as indicators in a latent class analysis. Multinomial multivariable logistic regression and Cox models examined the association of each class with baseline characteristics and mortality.

Results: For the 847 complete-case patients included (median age 79 years; women, $47.9 \%$ ), we identified 5 depressive-symptom classes: "somatic only" (38.8\%), "pauci-symptomatic" (26.4\%), "severe depression" (20\%), "mild depression" (11.8\%) and "demoralization" (3\%). Compared to the "pauci-symptomatic" class, the "somatic only" and "severe depression" classes were characterized by more frequent comorbidities with poorer functional status and higher levels of inflammation. "Severe" and "mild" depression also featured poorer nutritional status, more medications and more frequent falls. "Severe depression" was associated with poor social support, inpatient status and increased risk of mortality at 1 year (adjusted hazard ratio $1.62,95 \%$ confidence interval $1.06-2.48)$ and 3 years (1.49; 1.06-2.10).

Conclusions: A data-driven approach based on depressive symptoms identified 5 different depressive symptom profiles, including demoralization, in older patients with cancer. Major depression was independently and substantially associated with poor survival.

\section{P-902}

"I knew they were not there", a case of Charles Bonnet syndrome Timothy Miggiani ${ }^{1}$

${ }^{1}$ St. Vincent De Paule residence-Malta

This case report describes a case of Charles Bonnet syndrome. A 71-year-old gentleman who is a known case of Parkinson's disease, diabetes mellitus and depression was admitted to a long-term facility as his son could not keep up with his increasing need of care due to his deteriorating mobility. His main complaints were decreased mobility and nocturnal hallucinations for which he was recently started on olanzapine after a psychiatric review. History revealed full insight with hallucinations consisting of small animals and children playing which the patient did not believe to be true. They only happened at night in poor lighting. Examination revealed rigidity with resting tremor and difficulty mobilising. Both MMSE and Addenbrooke's cognitive assessment were done and failed to indicate any significant cognitive impairment. Formal ophthalmology review was done in view of longstanding diabetes which revealed background diabetic retinopathy in both eyes. Olanzapine was tailed down as diagnosis of psychosis was reconsidered and rigidity improved with no further instances of hallucinations experienced during his stay at the admission ward. The patient continued to improve in terms of mobility and independence as olanzapine was eventually stopped. At 
this point, several diagnoses were entertained including worsening of parkinsonian symptoms due to antipsychotic use in addition to release hallucinations. A formal psychiatric review was done which revealed a probable mixed aetiology for the hallucinations including Charles Bonnet syndrome, hypnopompic hallucinations and organic hallucinations. It was advised by the psychiatrist to keep off olanzapine and consider stopping nortriptyline. Patient was reassured.Patient continued to improve and eventually started walking independently, playing cards and fully independent in basic ADLs.

\section{P-903}

Profile of depressive symptoms in community dwelling older adults in Poland

Katarzyna Broczek ${ }^{1}$, Aleksandra Szybalska ${ }^{2}$, Przemyslaw Slusarczyk $^{2}$, Malgorzata Mossakowska ${ }^{2}$

${ }^{1}$ Department of Geriatrics, Medical University of Warsaw,

${ }^{2}$ International Institute of Molecular and Cell Biology in Warsaw

Introduction: Mood and anxiety disorders are major psychological health disturbances in older individuals. Profile of depressive symptoms in older age is multidementional, may be less specific than in younger generations, and usually is complicated by multimorbidity. The aim of the study was to analyze profile of depressive symptoms in relation to their severity in community dwelling older adults, participants of the PolSenior project - the largest cross-sectional study of the aging population conducted between 2007 and 2011 in Poland.

Materials and methods: The study group consisted of 4001 people aged $\geq 65$ years. Depressive symptoms were assessed with the 15-Item Geriatric Depression Scale (GDS-15) administered by trained nurses. Individuals with moderate or severe dementia were excluded on the basis of Mini-Mental State Examination Test.

Results: No depression (GDS score $<5$ ) was diagnosed in $69.6 \%$, moderate depressive symptoms (GDS score 6-10) in 25\% and severe depressive symptoms (GDS score $>10$ ) in $5.4 \%$. Depressive symptoms were more prevalent in women than men $(36.9 \%$ vs $25.1 \%)$, and augmented with age. The least specific symptoms for depression were decline of activities, anxiety and preference to stay home, while the most specific symptoms for severe depression was: "Do you think it is wonderful to be alive now?".

Conclusions: The profile of depressive symptoms may indicate severity of depression and may be useful in the assessment of older adults living in the community.

Area: Urology and continence management

\section{P-904}

Antibiotic prescribing and non-prescribing in nursing home residents with signs and symptoms ascribed to urinary tract infection (ANNA)

Jeanine Rutten ${ }^{1}$, Laura van Buul ${ }^{1}$, Martin Smalbrugge ${ }^{1}$, Suzanne Geerlings $^{2}$, Debby Gerritsen ${ }^{3}$, Stephanie Natsch ${ }^{3}$, Ruth Veenhuizen ${ }^{1}$, Hans van der Wouden ${ }^{1}$, Cees Hertogh ${ }^{1}$, Philip Sloane ${ }^{4}$

${ }^{1} \mathrm{VUmc},{ }^{2} \mathrm{AMC},{ }^{3}$ Radboudumc, ${ }^{4} \mathrm{UNC}$

Introduction: lmost $60 \%$ of antibiotic $(\mathrm{AB})$ prescriptions in Dutch nursing homes $(\mathrm{NHs})$ are for treatment of suspected urinary tract infections (UTI). A third of these $\mathrm{AB}$ prescriptions for UTI are not (yet) required. Inadequate $A B$ use is associated with an increased risk of exposure to side-effects and drug interactions and plays an important role in the development of AB resistance. Recently, a UTI treatment decision tool for frail elderly was developed with the aim to reduce inadequate $\mathrm{AB}$ prescriptions in nursing NHs.

Objectives: (1) to evaluate whether the use of the UTI treatment decision tool results in an increase in appropriate $\mathrm{AB}$ prescriptions for $\mathrm{NH}$ residents with suspected UTI (2) to investigate the degree, quality, facilitators and barriers of the use and implementation of the UTI treatment decision tool (process evaluation)

Methods: Study design: 18-month pretest-posttest cluster randomized controlled trial (cRCT) with NHs as the unit of randomization Setting: $16 \mathrm{NHs}$ in The Netherlands. Study population: NH residents with a new diagnosis 'suspected UTI'. Exclusion criteria: - Recent antibiotic use (past 7 days) for a different type of infection;- A treatment policy indicating that the resident wishes not to be treated with $\mathrm{AB}$ in case of a UTIIntervention: UTI treatment decision toolControl: care as usual Main outcomes: (1) Inadequate AB prescribing for suspected UTI at index consultation (yes/no). (2) Facilitators and barriers for implementing and using the UTI treatment decision tool.

Results/conclusions: Expected in 2020.

Area: Urology and continence management P-905

Management of constipation: retrospective notes review

Darrien Henry ${ }^{1}$

${ }^{1}$ King's College Hospiatl

Constipation affects $14 \%$ of the global population. Older people are prone to constipation due to poor fluid intake, immobility, over use of laxatives and medication. Constipation may present as delirium, anorexia, food refusal, vomiting, faecal incontinence and urinary retention. The usual management is to prescribe oral laxatives, suppositories or enemas. Guidelines are available for the management of chronic constipation, but there appears to be little advice/ guidance on management in the acute setting.

Methods: The medical records of 81 people admitted to an acute frailty ward were reviewed. Information regarding prescribing of laxatives (prior to admission or on admission), whether there was a duration of prescription documented, diagnosis of constipation (rectal examination or abdominal radiograph) and type of stool at the time for review.

Results: 34 were male and 47 female; median age 87 years (50-95 years). On admission, 19 were taking previously prescribed laxatives. 66 were prescribed laxatives at the time of admission, 60/66 (90.9\%) did not have an abdominal X-ray and 61/66 (92.4\%) did not have a rectal examination $\left(\chi^{2}=14.07, \mathrm{p}<0.01\right)$. There was no difference between age, nor stool Bristol stool type and laxative prescription. Only $7(10.6 \%)$ had a review date for the prescription documented.

Conclusions: The management of constipation is a common clinical problem. This study has found that prescription of laxative medication is often undertaken with no documented indication nor duration of treatment. Prescription of laxatives is not without potential consequences (e.g. electrolyte imbalance, dehydration) and prescription should be time limited and indication documented. 


\section{P-906}

Improving rational prescribing for UTI in frail elderly (ImpresU)

Drs. Maarten de Jong ${ }^{1}$, Prof. dr. Cees Hertogh ${ }^{1}$, Dr Anna Monnier ${ }^{1}$, Prof. dr. Theo Verheij ${ }^{2}$, Dr Alma van de $\mathrm{Pol}^{2}$

${ }^{1}$ Department of General Practice and Elderly Care Medicine, Amsterdam Public Health research institute, VU University Medical Center, Amsterdam, The Netherlands, ${ }^{2}$ Julius Center for Health Sciences and Primary Care, University Medical Center Utrecht, Ut

Background: Antimicrobial resistance is a growing problem in long term care which calls for antibiotic stewardship interventions (ASI) in home care and institutional care settings for frail elderly. The study in this European project aims to reduce antibiotic (AB) overuse for urinary tract infections (UTI) through implementation of a new algorithm to support clinical decision making.Objectives:1) To design a tailored intervention to reduce inappropriate $\mathrm{AB}$ prescribing and usage of $\mathrm{AB}$ for UTI by implementing an algorithm that encourages 'watchful waiting' in case of non-specific S\&S, combined with a bundle of actions selected on the basis of a conceptual model of factors that influence prescription decisions and with use of a toolbox of interventions to support rational and restrictive prescribing of $A B$ in UTI.2) To conduct and evaluate a RCT of this multifaceted intervention in frail elderly in institutional and community care settings in the four participating countries.

Objectives: (1) To design a tailored intervention to reduce inappropriate $\mathrm{AB}$ prescribing and usage of $\mathrm{AB}$ for UTI by implementing an algorithm that encourages 'watchful waiting' in case of non-specific S\&S, combined with a bundle of actions selected on the basis of a conceptual model of factors that influence prescription decisions and with use of a toolbox of interventions to support rational and restrictive prescribing of $\mathrm{AB}$ in UTI. (2) To conduct and evaluate a $\mathrm{RCT}$ of this multifaceted intervention in frail elderly in institutional and community care settings in the four participating countries.

Methods: Study design: A cluster randomized controlled trial, using a modified community-based participatory action research (PAR) approach to be conducted in GP practices and care facilities in The Netherlands, Norway, Poland and Sweden. Study population: Older persons ( $\geq 70$ years) with physical and/or mental disabilities and ADL dependency requiring home care or institutional (intermediate or nursing home) care. Patients are excluded if they are already using $\mathrm{AB}$, receiving hospice care or have limited life expectancy ( $\leq$ 1 month).

Main outcomes: (1) AB use for suspected UTI at index consultation (2) Course of symptoms after the index consultation, alternative diagnosis after assessment, changes in treatment policy, hospitalization, mortality and complications. Data collection: Between April 2019 and September 2020

Results/conclusions: Expected in 2020.

\section{P-907}

\section{Is Urinary Incontinence Destiny of Elderly People?}

Umran Dal Yilmaz ${ }^{1}$, Nurhan Bayraktar ${ }^{1}$

${ }^{1}$ Near East University Faculty of Nursing

Introduction: Urinary incontinence is common condition in the elderly and is a disturbing condition. Advance age, female sex, cognitive impairment, genitourinary surgery, obesity, and impaired mobility are risk factors for urinary incontinence. Bladder dysfunction decreases the quality of life by reducing social relations and activities, affecting emotional and psychological well-being and affecting sexual relations. UI can cause skin lesions, maceration and irritation, pressure ulcers, and recurrent urinary tract infections.

Methods: A literature search (2000-2018) was carried out, using MEDLINE, Pub Med, Science direct, Scopus, Cochrane library, Turkish Medical Index and related intuitional websites. Key words included "Urinary incontinence", "Elderly", "Older adults".

Results: In studies aiming in the world-wide of elderly people living in the community, UI prevalence ranging from $8 \%$ to $34 \%$ have been reported, this ratio reaches up to $70 \%$ in the elderly nursing home residents. In the study of Ateskan et al. (2000), the prevalence of urinary incontinence in the Turkish population was $44.2 \%$. In the study of Leiros et al. (2017), the prevalence of urinary incontinence in the Spanish population was found to be $15 \%$ in women and $11.6 \%$ in men.

Key conclusions: The incontinence problem should be identified for elderly people living in hospital and at residents or nursing homes. Various evaluation forms can be used for this. The incontinence problems experienced by elderly individuals should be detected; it is thought that the living problems can be reduced and the quality of life can be increased.

\section{P-908}

Experiencing and managing urinary incontinence: a qualitative study

Arzu Aybek ${ }^{1}$

${ }^{1}$ RN, public Health Nurse Master Degre

Urinary incontinence is a complex problem from which millions of people are affected worldwide. How the affected persons experience and organize their day-to-day lives has hardly been studied. It is important to understand that the people who are involved in the process of business, In Strauss and Corbin (NOC), the conceptual framework and thirty-two problem-centered interviews were conducted with 18 women and 14 men, which were then analyzed using the grounded theory method, after Strauss and Corbin. The experience of urinary incontinence is dependent on the incontinence and on the episode of incontinence. Incontinence itself influences social, cultural, and partner relationships. The results show that persons with incontinence use different strategies that are prepared for the next episode of episode of incontinence and searching for possible therapiesto regain control. If the experiences of the incontinence with elderly, public health nurses can help them by using NIC and NOC methods.

\section{P-909}

To increase the success of removing the urinary catheter in elderly patients with recurrent urinary retention

Rambaud Cyrielle $^{1}$, Gonfrier Sebastien ${ }^{2}$, Livigni Deborah ${ }^{2}$, Mbeutcha Aurélie $^{3}$, Arlaud Cyprien ${ }^{4}$, Leborgne Fanny ${ }^{5}$, Durand Matthieu ${ }^{6}$, Guerin Olivier $^{2}$

${ }^{1}$ Geriatrics, CHU NICE, ${ }^{2}$ Geriatrics, CHU Nice, ${ }^{3}$ Urology, CHU NICE, ${ }^{4}$ geriatrics, CHU Nice, ${ }^{5}$ Geriatrics, CHU NIce, ${ }^{6}$ Urology, CHU Nice

Introduction: The indwelling urinary catheter (IUC) is overused in the elderly. We aim to analyze the success rate of alternative treatments (AT) to the IUC at 12-month follow-up after multidisciplinary decision-making.

Methods: Decision analysis was used to structure a decision process that ensured the best choice through consensus building among 
hospital decision makers: urologist, geriatrician. All the patients over 70 yo with failed removal of IUC were offered a board's decision. We assessed the success of AT (defined by the lack of urinary retention at 7 days, 1, 3, 6 and 12 months) and overall death rate and its risk factor by univariate and multivariate analyzes.

Results: 99 patients were enrolled with a mean age of 86.7 yo (men:66\%). Alternative technics were offered in 63.6\% $(n=63)$ of patients, including 30 catheter withdrawals, 15 urethral stents, 17 photovaporizations of the prostate (PVP) and 1 prostatic artery embolizations. Overall, the success rate was $88.9 \%$ at 7 days and $92.1 \%$ at $1,3,6$ and 12-month follow-up. The IUC group patients were significantly older $(89.3$ vs $85.2 \mathrm{y}, \mathrm{p}=0.001)$, very dependent (ADL $<2,72.2 \%$ vs $39.7 \%, \mathrm{p}=0.002$ ) and with neurologic comorbidities $(77.8 \%$ vs $50.8 \%, \mathrm{p}=0.007)$. The global rate of death of the cohort were at $1,3,6$ and 12 months: $6.1 \%(n=6), 23.2 \%(n=23)$,
$35.4 \%(\mathrm{n}=35)$ and $44.4 \%(\mathrm{n}=44)$. At 12 months, the rate of death of patiente with IUC was higher $(72.1 \%$ vs $27.9 \%, \mathrm{p}<0.001)$. In univariate analyzes, predictive factors of a 12-months death were neurologic comorbidities (HR: 3.1[1,3-7.4] $\mathrm{p}=0.001$ ), dependence $(\mathrm{ADL}<2)(\mathrm{HR}: 4.2$ [1.8-9.8],p = 0.001), aging (HR: 1,1 [1.1-1.2], $\mathrm{p}=0.001)$ and the IUC (HR: 8.5 [3.4-21.2] $\mathrm{p}<0.001)$. In multivariate analyzes, the factors were the IUC (HR: 7.3 [2.7-19.9], $\mathrm{p}<0.001)$ and aging (HR: 1.1 [1.1-1.2], $\mathrm{p}=0.018$ ).

Conclusion: Our type of decision analysis model may be a useful consensus building to offer a better chance to deal with IUC in elderly people with a global success rate of $92.1 \%$ catheter withdrawals at 12 months. The elevated rate of death in IUC group highlights the frailty of dependent patients and data is needed to report the relation with IUC. 


\section{Author index}

Aarden, J. J. P-340

Aarsland, Dag O-123, P-632

Abascal, Ramón Adolfo Arévalo P-832

Abbes, Dorsaf P-211

Abdalhfidh Nedia, Ben P-342, P-378

Abdel Rahman, Haitham P-261

Abdelghany, Amin P-275

Abdelhafidh Nour, Ben P-379

Abdeljalil Anne-Bahia, A. B. P-36

Abdhalla, Ahmed P-562

Abdulan, Irina Mihaela P-397, P-450, P-791

Abel, Bastian P-295, P-299, P-310

Abhilash, Sharada P-898

Abiyev, Azer P-178, P-452

Abizanda, Pedro O-22

Abizanda-Sol, Pedro P-198

Abraha, Iosef P-454

Abrahamsen, J. F. P-695

Abualenain, Jameel T. P-688

Abumettleq, Ibrahim S. S. P-550

Abyad, Abdulrazak P-253, P-769, P-770

Achille, Tchalla P-229, P-420

Achterberg, W. P. P-317

Achterberg, Wilco P. P-286, P-298, P-291

Acín-Gericó, María Teresa P-15

Adeline, Jullien P-39

Adeline, Roux O-83

Adewole, Olubayode P-565

Admant, L. P-208

Adorni, Elisa P-218

Afacan, Ceyda P-623

Afef, Skhiri P-751

Agahi, Neda O-120

Agnes, Lievre P-376

Agrawal, Siddarth P-727

Aguado-Taberné, Cristina P-424, P-425

Agüero, D. M. P-264

Aguiar, Pedro P-713

Aguilar-Navarro, Sara Gloria P-277

Ahearn, David O-49, P-553

Ahmad, Ali P-119

Åhman, Hanna Bozkurt P-621

Ahmed Saleh, Haroon P-757

Ahmed, Haroon P-275

Ainge, Thomas P-545

Airaghi, Victoria Garay P-102, P-103, P-104

Akbar, S. P-480, P-482

Akcicek, Fehmi P-231, P-232, P-443, P-756, P-773

Akerman, J. P-54

Akif Karan, M. P-775

Akif Karan, Mehmet P-35, P-147, P-157, P-158, P-468, P-506, P-827

Akin, Sibel P-505

Akinmolayan, Atinuke P-565

Akintade, Olugbenro P-524

Akishita, Masahiro P-45, P-159, P-412

Akram, Wasim P-560

Aksoy, Songul P-638

Aksu, Hatice P-719

Al Fehaidi, Al Anoud Ali H Z P-757

AL Hamad, Hanadi P-117

AL Husami, Mohammed P-117

Al Refae, Mahmoud P-117

Alaburic, Srdan O-81
Alam, Wahila P-757

Alarcon, Teresa P-227, P-230, P-800, P-801, P-822

Alastuey, Carmen P-745

Alattar, K. P-851

Alba, Iratxe Molinuevo P-66

Albala, Cecilia P-239, P-679

Alberdi, Nerea P-341

Albert, Wettstein P-627

Alberto, Pilotto P-472

Albrand, Gilles O-107

Albrecht, Diana O-17

Albuisson, Eliane P-126

Aldamiz, Mikel P-828

Aldridge, C. G. P-463

Alekna, Vidmantas P-224, P-337, P-339, P-423

Alessandro, Giuseppina P-686

Alexa, I. D. O-124, P-357, P-358, P-362, P-763

Alexa, Ioana Dana P-80, P-81, P-93, P-397, P-450, P-489

Alexa, Ovidiu P-81, P-93

Alexander, Aneeka P-131, P-132

Alexander, Koshy P-257, P-258

Alexandra, Avadanei P-380

Alexopoulou, Zoi P-656

Alfandari, Serge P-513

Alfons, Ramel P-440

Alhamad, Hanadi P-364

Al-Hamad, Hanadi P-455

Ali, A. P-659

Ali, Khalid O-103, O-105, P-48, P-127, P-407

Ali, Rizvana P-485

Ali, Talalah P-48

Alibrandi, Angela P-226

Aline, Corvol P-509

Alizadeh-Khoei, Mahtab P-151, P-606

Allain, Mylène O-107, O-58

Allaz, A. F. P-752

Allcutt, A. P-540

Allegaert, Patsy P-294

Allocca, E. P-789

Allue, Ramon Cristofol P-895

Allwood, R. P-608

Alme, Katinka Nordheim P-410

Almeida, Paulo P-245, P-307

Al-Mugheed, Khalid P-92

Alonge, Lyiale P-371

Alonso, Esther Lueje P-103

Alonso, Javier P-745

Alsma, Jelmer P-585, P-837

Altena, J. A. M. P-31

Alvarado, Natalia Vázquez P-549

Álvarez-Gómez, Esther P-86

Alves, R. V. P-68

Alves, Rita P-557, P-765

Alzner, Rainhard P-140

Amasene, Maria P-237, P-828

Amaya-Sánchez, L. E. P-583

Amin, V. P-851

Aminalroaya, Reyhaneh P-151, P-606

Amorone Leiva, J. L. P-264

An, Songyi P-555

Ana, Capisizu P-749

Andersen, Günnar P-197

Andersen, O. O-113, P-737, P-738

Andersen, Stig P-391

Andersen-Ranberg, Karen P-508

Ando, Yukio P-23, P-366 
Andrea De Biase, Giuseppe P-722

Andreano, A. P-98, P-335

Andreano, Anita O-10

Andreas, Meier P-627

Andreea, Zamfirescu P-749

Andres-Lacueva, C. P-130

Andreu, Oscar Miró I . P-548, P-549

Andrews, Tom O-87

Andrieu, Sandrine O-102

Ang, Y. H. P-76

Angel, Barbara P-239, P-679

Angel, Tammy P-836

Angileri, Vincenza Valentina P-218

Anik, Giguère P-70

Ankri, Joel O-102

Anna Paola, Tassi P-315

Anna, Meyer P-472

Anne, Chahwakilian P-901

Anne, Fournier P-225

Anne, Spinewine P-225, P-504

Anne-Laure, Couderc P-439

Anrys, Pauline P-746

Anten, Sander P-585

Antikainen, Riitta P-870

Antoine, Lamer P-497

Antón-Nieto, Esperança P-87

Anwar, Mahboob P-704

Aoun, Antoine P-261, P-441

Aounallah, Skhiri H. P-751

Apa P-376

Appay, Victor P-129

Apsega, Andrius P-224

Aquino, Jean Pierre P-575

Araghi, Sadaf P-25

Aragonés, Myriam Valdés P-333, P-334

Arai, Hidenori O-60, P-12, P-190, P-194, P-458, P-881

Arana, Lucía Fernández O-11, P-102, P-103, P-104

Aranda, Yanira P-779

Aranda-Martinez, Agustin O-65

Aras, Sevgi P-168, P-352, P-353, P-367, P-782

Arcangeli, L. P-631

Arenas, Maria Caridad P-779

Arenz, Petra P-797

Arévalo, Manuel P-71

Ariana Bozín, Paula P-845, P-846

Arias-Blanco, Maria del Carmen P-424

Arik, Gunes P-216, P-808

Ariza, Diana P-546

Ariza, Xavier P-546

Armance, Grevy P-60, P-61, P-62, P-63, P-491

Arnau-Barrés, Isabel P-348, P-94

Arnaud, Adrait $\mathrm{O}-83$

Arnaud, Pagès P-39, P-40

Arnaud, Potier P-65

Aromatario, Olivier P-234

Arora, S. P-179, P-181

Arosio, Beatrice P-393

Arribas Monzon, F. P-732

Arroyo, Patricia P-239

Artabe, Iñaki Artaza P-250

Arzaghi, Masoud P-151, P-606

Asadi-Lari, Mohsen P-892

Ascencio, Shirley Soledad Alamo O-101

Asgassou, Sanaa P-24

Ashton, Catherine O-96, P-647

Asil, Serkan O-41, P-663
Askim, Torunn P-410

Aslan Telci, Emine P-792, P-774

Asmar, Roland P-125

Assaf, Elias P-871

Assantachai, Prasert P-228

Ataíde, Rosário P-210, P-235

Atalay, Orçin Telli P-305, P-306

Ates Bulut, Esra P-436

Athanase, Benetos P-65

Atli, Teslime P-505, P-506

Atmiş, Volkan P-168, P-352, P-367

Attar, Safa G. P-279, P-898

Audureau, Etienne O-58

Auer, D. P-614

Auer, Stefanie P-681

Auñon, Angel Flores O-101

Auplish, Sunil P-544

Aurélie, Mbeutcha P-909

Aurucci, Maria Luigia P-58

Austoni, Pietro P-819

Avci, Suna P-447, P-623, P-719

Avgerinou, Christina P-175, P-449

Ávila-Funes, José Alberto P-277

Aviv, Abraham O-106

Awad, Marwa P-275

Awata, Shuichi P-474

Ay, Seval Ay P-773

Ay, Seval P-20

Ayan, Burak P-623

Ayan, Gizem P-447, P-623, P-719

Aybek, Arzu P-908

Aycicek, Gozde Sengul P-667

Aydin, Ali Ekrem P-830

Aydin, Kubra P-157

Aylen, Tracy P-500

Azevedo, Luís Filipe P-210, P-235

Baath, B. P-659

Baca, Manuel P-348

Bacci, F. P-568

Bacci, Francesca O-78

Baclet, Nicolas P-513

Badarudeen, Irshad P-560

Badih, El Nakada P-683

Badino, Giorgio P-766

Badrkhahan, Seyyedeh Zahra P-606

Baeyens, Hilde P-708

Baeyens, Jean-Pierre P-708

Bahat, Gulistan P-35, P-147, P-157, P-158, P-468, P-775, P-827

Bahşi, Remzi P-168, P-352, P-353, P-367, P-782

Baider, Lea P-890

Baierlein, Franziska P-778

Baird, Janis P-460

Bajenaru, O. L. O-124, P-357, P-358, P-362, P-763

Bajwa, R. K. P-601

Bajwa, Rupinder Kaur P-414

Bajwa, Rupinder P-327, P-650, P-668

Bakka, Arne Olav P-82

Bakrania, Prijay O-105, P-127

Balaeva, Madina P-743

Balasubramanian, Diraviyam P-532

Balci, Cafer O-41, P-217, P-390, P-505, P-619, P-638, P-667, P-808, P-663, P-720

Balck, F. O-33, P-818

Baldasseroni, Samuele O-78, P-697 
Baldo, Vincenzo P-747

Balea, Fran P-745

Balenović, Antonija P-273

Balzan, Gabriella P-618

Balzi, Daniela O-95, P-244

Bancroft, Asa P-419

Bandholm, TQ P-737, P-738

Bandinelli, Stefania O-28, P-502, P-512, P-652, P-816

Banerjee, Jay P-569

Banerjee, Prithwish P-405

Banerjee, Smita P-257

Bar, A. P-98

Barandiaran, Lucia P-341

Barasas, Ortzi P-745

Barata, José P-759

Barbe, Anna Greta P-469

Barbosa, B. P-721

Barbosa, Benilde P-330

Barca, Maria O-85

Barca, Maria Lage O-86

Barceló Trías, M. P-754

Bárcena Goitiandia, L. P-174

Barhoumi, Amel P-701

Baroncini, Anna Chiara P-563

Baroncini, Caterina P-784, P-848

Barone, A. P-335, P-833, P-853

Barone, Antonella O-10

Baroni, Marta P-185, P-446, P-703, P-716

Bartel, Sylva P-394

Barucci, Riccardo P-502, P-512

Barzak, Alex P-707, P-781, P-780

Basaras, Nerea Aguirre P-730

Basheikh, Mohammed P-688

Basile, Giorgio P-226

Baskan, Emre P-771

Bassem, louzir P-342, P-378, P-379

Basso, Cristina P-588

Bastiani, Patrizia P-393

Bastuji-G P-901

Bastuji-Garin, Sylvie O-107, O-58, P-871

Batcup, Joanna P-582

Batista Jr., Joao P. P-803, P-806

Batista, Diogo P-765

Baudry, Stéphane P-142

Bauer, J. M. O-68, P-645

Bauer, Jürgen M. O-16, O-56, P-18, P-241, P-292, P-295, P-299,

P-301, P-302, P-310, P-318, P-321, P-322, P-323, P-142

Bauernfreund, Yehudit P-449

Bautmans, Ivan O-24, P-212

Baxter, Mark P-511

Bay, Ilker P-827

Bayard, Charles P-129

Baydan, Mine P-638

Bayraktar, Nurhan P-92, P-459, P-550, P-907

Bazelmans, Ellen O-52

Be, Athanase P-125

Béatrice, Demoré P-65

Beaudart, Charlotte O-24, P-143, P-160

Beauvois, Nicola P-77

Beaver, J. P-531

Becker, Clemens O-17

Becker, Ingrid P-691, P-797, P-798

Beckwée, David P-142

Beeke, S. P-608

Beenakker, Karel O-104

Begma, Inna P-213
Bejia, Ismaeil P-360, P-760, P-761

Belala, Nacera P-292

Bell, J. Simon O-111, P-33

Bell, Simon P-29

Bellarbre, Fabienne P-820

Bellelli, Giuseppe O-10, P-854

Bellera (equally), Carine O-107

Belmin, Joël P-371, P-666

Benedetti, Chiara P-218

Bénétos, A. P-208

Benetos, Athanase O-106, P-126, P-234, P-493, P-556, P-671, P-740,

P-758, P-795

Benini, Patrizia P-747

Benítez González, Carmen P-99

Benjak, Tomislav P-273

Benjamin, Landré O-25, O-102

Benoit, Florence P-156

Benvenuti, Enrico O-95, P-502, P-512

Benzing, Thomas P-570, P-797, P-799

Beom Park, Wan P-107

Beránková, Anna P-681

Bereder P-764

Berengere, Beauplet P-439

Berg, Almuth P-403

Bergamaschini, C. P-804

Bergamaschini, L.C. O-77

Bergaoui, Naceur P-760

Bergland, Astrid O-64, P-300, P-388

Berglund, Lars P-621

Bergquist, Ronny P-421

Bergström, Ulrica O-84

Beril Ozden, Nur P-232

Bermellová, Jana P-600

Bermudez-Ampudia, Cristina P-237, P-828

Bernabei, Giacomo P-885, P-888

Bernabei, R. P-789

Bernabei, Roberto O-59, P-139

Bernal, Gabriela P-71

Bernard, Louis P-575

Bernhardt, Gerwin P-83

Bertelsen, Ann Sophia P-170

Berti, Valentina P-784

Bertram, T. P-802

Bertschi, Dominic P-831

Besga, Ariadna P-237, P-828

Besozzi, Anaïck P-626

Besozzi, Annaick P-671

Betancourt, Alvaro José P-846

Bettarini, Francesco O-94

Betz, Thomas P-797

Beuscart, Jean-Baptiste P-513, P-526, P-539

Beyer, I. P-347

Bhanu, Cini P-449

$\mathrm{Bi}$, Ken

Biagioni, Giulia O-78, P-559, P-563, P-568

Bianca, Dario P-58

Bianchi-De-Aguiar, Filipa O-115, P-331, P-332

Biegus, Karol P-733

Biffi, Vitali P-283

Bilbao, Irati Garaizar P-718

Bilel, Arfaoui P-342, P-378, P-379

Bilgic, Onur P-623

Bimou, Charlotte P-420

Bin Kim, Hong P-107

Bird, Yelena P-396

Bitaraf, Mohamad Ali P-370 
Bittan, Judith Cohen O-13

Bjerk, Maria P-388

Bjørke Monsen, A.L. P-695

Blachucki, Bartosz P-823, P-824

Blair, Alison P-507

Blaj, Liliana P-742

Blanco Orenes, Antonio P-661

Blanco, Irune Martinez P-730

Blauw, G.J. P-817

Blauw, Gerard J. P-585

Blomaard, L.C. O-73, P-521, P-522, P-579, P-585, P-837

Blöndal, Berglind P-429

Bloom, Ilse P-460

Blundell, A. P-503

Bo, Mario P-58, P-854, P-855

Boccardi, Virginia P-393, P-446, P-703

Bodaghabadi, Mohammad P-369, P-370

Boddaert, Jacques O-13, O-79, P-84, P-85, P-129, P-857, P-859

Bodilsen, A. C. P-737, P-738

Boer, Jolanda M. A. P-434, P-435

Boere, T. M. P-554

Boersma, Froukje P-34

Bogini, Riccardo P-446

Bogoslavsky, Tanya P-687

Böhme, Miriam P-49

Boietti, Edoardo P-58

Boj, Monica Tudorita P-87

Bojesen, Rasmus Dahlin O-99

Bokhari, Syed P-544

Boland, B. P-777

Bollheimer, Cornelius O-35

Bollheimer, L. Cornelius P-803

Bollig, Claudia P-241, P-349

Bolzetta, Francesco P-599

Bongartz, Martin O-16, O-56, P-301, P-302, P-310, P-900

Boni, Elisa P-574

Boni, Serena P-696

Bonnet-Zamponi, Dominique P-536

Bonnici, Maria P-618

Bonte, Florence P-677, P-739

Bonten, Marc P-710

Booijen, A. M. P-521

Booth, V. P-601, P-609, P-614

Borda, Miguel O-39

Borges, D. D. P-68

Borges, Diogo P-557

Borges, Nuno P-210, P-235

Borkar, V. O-14

Borowik, Adrien P-536

Bortolazzi, Elena O-116

Bosah, Ifeanyi Bernadette P-849, P-850

Bosch, J. A. P-340

Bosman, Sjanne P-52

Bosone, D. P-9

Bosseray, Annick O-71

Boström, A. M. O-21

Boström, Anne-Marie P-201

Bothe, M. K. P-152

Bots, Michiel P-602

Boudaoud, Sofiane P-492

Boudou-Rouquette, P. P-439

Boudou-Rouquette, Pascaline O-58

Boukhebouze, Mohamed P-498

Boulanger, Christelle P-498

Boulanger, Eric P-593

Bouly, S. P-542
Boumendil, Ariane O-79

Bouomrani, Salem P-603

Bourcier, Elsa P-536

Bourdaire, V. P-208

Bourdel-Marchasson, Isabelle P-573

Bourgeois, J. P-55

Bourkel, Elisabeth P-418

Bourron, Kiyoka Kinugawa P-492

Boussac-Zarebska, Marjorie P-674

Bouvy, Marcel P-57

Bowen, Jordan S. T. P-564

Bowman, Kirsty O-92

Bowyer, Ruth C. E. P-465

Boyle, Gerard O-66

Brach, Jennifer P-622

Brain, Etienne O-107, P-871

Bramble, Joanne O-76

Bramley, T. P-609

Brand, A. P-601

Braun, Larissa Maria P-19

Brazil, Kevin O-87

Brefka, Simone P-241, P-349

Bridle, Emma P-561

Briggs, Robert O-62, P-664, P-897

Brincat, Svetlana P-488

Bring, Annika P-314

Bringuier, Michael O-107

Brink, Anniek P-585, P-837

Brinkkötter, Paul P-797, P-798

Brischetto, Daniela P-226

Brites, Mariana P-692

Brito, Luciane Maria Oliveira P-351, P-444, P-678

Brito, Luis Gustavo Oliveira P-444

Brizzi, Berenice Nayla P-548, P-549

Brockmöller, Jürgen P-49

Brock, P. P-256

Broczek, Katarzyna P-823, P-824, P-903

Broeiro-Gonçalves, Paula P-713

Brogi, Michela P-183

Brouwer-Brolsma, E.M. P-422

Brovold, Therese P-388

Brunet, Thomas P-753, P-820

Brunetti, Enrico P-58

Brunie, Vanida P-67

Brusasco, Claudia P-572

Bruscato, Neide M. P-762

Bruyère, Olivier O-24, P-143, P-160, P-161, P-715

Brzoska, Patrick P-387

$\mathrm{Bu}$, Nari O-15

Bubba, Valentina P-185, P-716

Buckinx, Fanny P-160, P-161

Buckley, Catherine O-87

Bulent Dogrul, Ahmet P-808

Bulgaresi, M. P-386

Bulgaresi, Matteo P-502, P-512

Bulubas, I. P-208

Bulut, Esra Ates P-133

Bunkowski, Stephanie P-633

Buondonno, Ilaria O-8

Buquicchio, Rosalba P-574

Burcet, Silvia P-296, P-343, P-610, P-359

Bureau, Marie-Laure P-329, P-820

Burger, Huibert O-114, P-43

Burgon, Clare P-327, P-414, P-601, P-650, P-668

Burke, Joshua P-405

Burkhardt, Heinrich P-10, P-184, P-723, P-842 
Burst, Volker P-570, P-797

Busonera, Flavio P-599

Bussmann, Michael P-469

Butt, H. P-809

Butt, Hina P-529

Butterhoff-Terlingen, M.H. P-838

Buurman, B. M. P-340

Buurman, Bianca M. P-569

Buurman-van Es, Bianca M. P-876, P-877

Bye, Asta O-64, P-300, P-484

Ca, Flávia P-351

Carla, Lamberti P-182

Caballero-Mora, Maria Angeles P-139

Cabezas, Dacil P-745

Cabezas, Pilar P-307

Cabrero Montes, Elena P-680

Caglar, Omur P-808

Caillet, Philippe O-107, O-58, P-871

Calafiore, Matthieu P-513

Calderon-Garcia, Julian F. P-437

Calderon-Larranaga, A. P-177

Calderón-Larrañaga, Amaia O-43

Caldiroli, L. P-804

Calf, A.H. P-530

Calf, Agneta H. P-481

Calhoun, Shawna R. P-112

Caliskan, Hatice P-217, P-390, P-638, P-663, P-667, P-808

Caljouw, Monique P-291

Calle, Alicia O-18, P-311

Callus, Claire P-488

Calonge, Elena Valle P-66

Calpena, Carmen Perez O-101

Cals, J. W. L. P-554

Calvo, Jesús Seco P-99

Camacho, Manuela Martínez P-543

Camilleri, Francesca P-488

Campos, Luís O-115, P-245

Cancela, José María P-287, P-592

Canchucaja, Lizzeth P-94

Candiani, Tiziana P-251, P-686

Canelas, Ana Maria P-236

Cankurtar, Mustafa P-667

Cankurtaran, Mustafa O-41, P-216, P-217, P-390, P-663, P-720,

P-894

Cano-Gutierrez, Carlos O-39

Cano-Gutierrez, Carlos Alberto P-528

Canouï-Poitrine, Florence O-107, P-871

Canta-López, Elena P-87

Capova Pavlina P-375

Capan, Nalan P-147

Caporale, Tessa O-111

Cappa, Giorgetta P-58

Cappugi, Marina P-502, P-512

Caraffa, Auro P-185, P-716

Caramelli, Francesca P-559

Carcaillon-Bentata, Laure P-674

Cardenal, J. Corrales O-75

Cardinaud, Noëlle P-420

Cardoso, José Silva P-210

Carey, Daniel O-62, P-897

Cari, Luigi P-393

Carignano, Giulia O-8

Carl, Neuerburg O-23

Carla, Lamberti P-165
Carlin, Yael P-471

Carmelo, Lafuente P-274

Carmen Ilie, Adina P-450

Carmona, Laura Plaza O-22

Carnicero, J. A. P-204

Carolina Ruiz-Grao, Marta P-198

Caroline, Gayot P-229, P-420

Caroline, Sirois O-45

Carrassi, Emanuele P-563, P-697

Carrel, Thierry P-831

Carreño, Jose Antonio Carnicero O-101

Cartei, Alessandro P-244

Carvalho, A. P-721

Carvalho, Armando P-431, P-534, P-692

Carvalho, Flávia Lopes P-475

Carvalho, Mariana P-858

Carvalho-Gouveia, Cristina P-331, P-332

Casademont I Pou, J. P-754

Casas-Herrero, Álvaro P-15

Casati, Martina P-393

Casella, J. T. P-865

Caserotti, P. P-249

Casini, Giulia O-40

Casprini, Laura P-652, P-816

Castanho, Teresa P-641

Castelino, Ronald P-3

Castoldi, G. P-98, P-335

Castro, Sara P-557, P-765

Catalano, Antonino P-226

Catalina, Pena P-380

Catherine, Bioteau P-109

Catherine, Catenoz P-439

Cattelani, Luca O-28

Cauchi, S. P-313

Caulin, Héctor P-197

Caupenne, Arnaud P-329, P-753

Cavalier, Etienne P-161

Cavallini, M. Chiara O-95

Cavallini, Maria Chiara P-784, P-848

Cavlak, Ugur P-771

Cavusoglu, Cagatay P-176, P-219, P-247, P-826

Ceccofiglio, A. P-98

Ceccofiglio, Alice P-712

Cécile, Cantet P-39

Cécile, Laubarie-Mouret P-420

Cécile, McCambridge P-39, P-40

Cecília Soares Santos, Anna P-32

Cecilie Knudsen, Eva P-355

Cederholm, T. O-21

Cederholm, Tommy O-26, P-201

Cedervall, Ylva P-621

Cedraschi, C. P-752

Cella, Alberto O-70, P-766

Cengiz Isik, Mehmet P-232

Cépla, Juozas P-427, P-689

Ceravolo, Francesco O-94

Cereda, Emanuele P-141

Cernesi, Simone P-283, P-574, P-885, P-888

Cernovas, Andrejus P-337, P-339

Cesari, M. O-77, P-804

Cesari, Matteo O-38, O-39, P-145, P-160, P-393

Cester, Alberto P-599

Chahine, Mirna P-125

Chahwakilian, Anne O-58

Chale, Godfrey P-755

Challberg, J. P-344 
Challberg, Jennifer O-50

Chalvatzaki, G. O-68

Chandler, Jenna P-561

Chandran, Mani P-275

Chang, H. W. P-840, P-843

Chang, Milan O-5, P-169, P-209, P-406, P-442, P-462, P-486

Chang, Y. H. P-409

Chang, Y. J. P-840, P-843

Channon, M. P-525

Channon, Mike P-419

Chapman, J. P-503

Charles, Alexia P-161

Charles, Petitot O-83

Charlotte, Bimou P-229

Charru, Philippe P-666

Chattaris, Tanchanok P-887

Chatziadamidou, Thekla P-740, P-758

Chaudhry, A. P-525

Chauhan, Aneesha P-717

Chauveau, Philippe P-812

Chauvin, Pauline P-260

Chavannes, Niels H. P-286

Chavarro-Carvajal, Diego Andrés P-527

Chazard, Emmanuel P-526, P-539

Checa, Marta P-204, P-214

Chelbi, Farhat P-699

Chen, Esa O-111

Chen, Sijia O-104

Chen, Y. D. P-91

Cheng, Biao P-163

Cherdak, M. A. P-634, P-635

Cherubini, A. P-130

Cherubini, Antonio O-80, P-454, P-854, P-855

Chiara Baroncini, Anna O-78, P-559, P-568, P-848

Chiari, Lorenzo P-421, P-816

Chikusu, Clarence O-65

Chimahu, S. P-485

Chiong, Cloie June P-694

Chiong, Jun P-694

Chiong, Jun R. P-706

Chmiel, W. P-480, P-482

Cho, Chooyon P-374

Cho, Eirie P-363

Choi, Han Sung P-555

Choi, Hyunbyul P-555

Choi, Jeongmin P-555

Choi, Jung-Yeon P-107, P-144

Choonara, H. P-480, P-482

Choquehuanca, Martha P-227

Chorubczyk, Sonia P-471

Chotkan, S. P-72

Chow, Pek Yee P-466

Christensen, L.D. O-113

Christiaens, Luc P-820

Christian, Bakker P-389

Christian, Rochefort O-125

Christiane, Völter P-617

Christine, Hoell P-693

Christine, Perret-Guillaume P-65

Christophe, Arbus P-36

Christophe, Graf O-55

Christophe, Tournigand P-376, P-829

Chudek, Jerzy O-97, P-815

Chung, Hweemin P-374

Ciancio, Giuliana P-226

Cindy, Riffard P-60, P-61, P-62, P-63, P-491
Ciuti, G. P-568

Ciuti, Gabriele O-78

Claassen, Jahr O-82, P-639

Cladellas, Mercè P-350

Claire, Chenesseau P-356

Clara, Lopez P-60, P-61, P-62, P-63, P-491

Clark, A. P-620

Clarke, Darren O-66

Clarke, Georgia P-861

Claudia, Bollig O-19

Claudia, Martinez-Tapia P-439, P-901

Claudot, Frederique P-493

Clayton, E. P-852

Clegg, Andrew P-246

Clément, Gouraud P-901

Clemente, G. Jimenez O-75

Clementz, A. P-38

Clifford Lisk, C. L. P-69

Cobbaert, Katrien P-455

Cobley, Hannah P-524

Coffey, Alice O-87

Cohen Bittan, Judith P-84, P-85, P-129, P-857, P-859

Cohrs Ute, Bruene P-617

Cole, Rebecca P-785

Colizzi, Elena P-811

Collerton, J. O-44

Collignon, Marion P-67

Collins, Ronan P-651

Collinson, Avril P-454

Colloca, G.F. P-789

Colominas, Miriam O-18, P-311

Colosio, W. P-631

Colpo, Marco O-28

Colpo, Marco P-652, P-816

Comans, Tracy O-111

Concepcion, Peinado-Gallego P-171

Conde Caballero, David P-437, P-456

Coni, Alice P-421

Conroy, S. P. P-179

Conroy, Simon P-654

Conry, Miriam P-793

Consciência, José Guimarães O-115

Consonni, Dario O-38

Constantin, G. I. P-776

Contreras, María Elena P-670

Contreras-Escámez, Beatriz P-15

Conzade, Romy P-448

Cools, Pierre P-498

Cooper, Cyrus O-24, P-460, P-715

Cooper, Tina P-33

Copelli, Sergio O-69

Coquelet, Amélie P-626

Cordovilla Guardia, Sergio P-437, P-456

Ćorić, Tanja P-271, P-272, P-273, P-490

Corica, Francesco P-226

Corlis, Megan O-111

Cornally, Nicola O-87

Cornelius Bollheimer, L. P-806

Corner, L. O-44

Corominas, Miriam P-71

Corradi, Francesco P-572

Corradi, Samanta P-652

Corrales Cardenal, J. P-874

Correa-Pérez, Andrea P-454

Corsi, M. P-335, P-98

Corsi, Maurizio O-10 
Corvol, Aline O-47

Cossiolo, Denise Camilios P-594

Costa, A. P-9

Costa, E. P-865

Costa, Joyce Pinheiro Leal P-116, P-351, P-444

Costa, Rita Amorim P-236, P-242

Costa, S. P-108

Costas-Muniz, Rosario P-258

Costas-Paivas, Lucia Helena Simões P-444

Costenoble, Axelle P-212

Costin, Nicolae Hariton P-489

Cotch, Mary P-249

Cotelli, Suheyla P-176, P-219, P-247, P-619, P-826

CottaRamusino, M. P-9

Cotton, Natalia P-717

Coughlan, Tara P-408, P-651

Cowan, K. O-44

Craig, D. O-44

Crespo, Luis Ignacio Mendibil P-718, P-730

Cri, Maria P-797

Cristina Åberg, Anna P-621

Cristina Polidori, Maria P-570

Cristina, Polidori M. P-472

Cristini, Carlo P-262

Croker, Helen P-449

Cruise, R. S. P-525, P-540

Cruise, Rebecca P-259

Cruz-Jentoft, A. J. P-95, P-243

Cruz-Jentoft, Alfonso J. P-467

Curtin, Denis O-63

Custureri, Romina P-572

Cyarto, Elizabeth P-500

Cyprien, Arlaud O-72, P-118, P-356, P-499, P-764, P-909

Cyrielle, Rambaud O-72, P-909

Cyril, Touboul P-829

d'Hyver de las Deses, C. P-583

D'Amelio, Patrizia O-8

D'Andria, M.F. P-386

D'Andria, Maria Flora P-244

D'Angelo, A. P-9

da Costa Chein, Maria Bethânia P-116

da Cruz, Maria Carmen Fontoura Nogueira P-475

da Graça Carvalhal Frazão, Rita P-351, P-678

da Luz, Protasio Lemos P-762

Da Silva, Cristiane França P-594

Da Silva, Sofia P-24

Dabbous, Firas P-464

Dacil, Cabezas-Jaen P-171

Dadoniene, Jolanta P-423

Dahly, Darren P-597

Dal Yilmaz, Umran P-459, P-473, P-907

Dalila, Dakka P-764

Dallmeier, Dhayana P-156, P-241, P-349

Dalma P-272

Damanti, Sarah O-38

Dammertz, Ingrid P-798

Damsgaard, Else Marie P-203, P-453

Damulevičiené, Gyte P-426, P-478

D'Andria, Maria Flora O-40

Daniel, Lopez-Trabada-Ataz P-376

Daniela, Dyntar P-627

Dankbaar Jan Willem, J.W. P-402

Dannheim, Fritz P-790

Dann-Reed, Eleanor P-613
Dapp, Ulrike O-29

Dardevet, Dominique P-454

Darin-Mattsson, Alexander P-615

Dartigues, Jean-François P-625

Dascalescu, Ruxandra P-749

Datlı Yakaryılmaz, Funda P-728, P-729, P-734

Dauba, Jérôme O-107

Dautel, Anja P-299

David Smith, A. P-632

David, Jean-Philippe P-67

David, Veillard P-509

Davies, Graham O-103, O-105, P-48, P-127, P-407

Davies, Karen P-238, P-240

De Angelis, Nicola P-79

de Asteasu, Mikel Lopez P-199

de Beaufort, Inez P-878, P-879

De Becker, A. P-347

De Biase, Giuseppe Andrea O-94

de Boer, Anthonius P-57

de Brouwer Esther, E. J. M. P-402

De Carli, Waldemar P-762

De Carlo, Luigina P-283

De Caro, Anna O-116

De Cock, Anne-Marie P-142

De Costa, Jean Marcos P-594

De Costa, Jhayanne Brunna P-594

De Decker, Laure P-79, P-439

de Fátima Carvalhal Martins, Maria P-678

de Ferreira Gomes, João Filipe P-794

De Francesca, Francesco P-722

de Francisco Murga, E. Martín P-174

De Freitas, Yolanda Parada P-102, P-103

de Gelder, J. O-73, P-521, P-522, P-585, P-837

de Gomensoro, Eduardo P-106

de Groot, A. J. P-320

de Groot, B. O-73, P-521, P-522

de Groot, Bas P-585, P-837

de Groot, L.C.P.G.M. P-422

de Groot, Lisette C.P.G.M. P-454

de Groot, Lisette P-25, P-324

De Guzman, Domingo P-745

de Heus, R. A. A. O-82, P-639

de Jaegere, P. P. T. P-72

de Jaime, Elisabet P-296, P-610

de Jong Pim, P. A. P-402

de Jong, Maarten P-906

de Jonghe Annemarieke, A. P-402

de Jonghe, Annemarieke P-602

de la Iglesia, Jorge Martinez P-424, P-425

de la Rua, María Jesús P-718

De Lepeleire, Jan P-504, P-746

De Luca, Alice O-70

De Michieli, Lorenzo O-70

de Miguel, Ana Merello P-354

de Molíns Peña, C. Elías O-75, P-732, P-874

De Nadai, Daniela P-885, P-888

De Nicolás Jiménez, Jorge P-456

de Oliveira Neto, Clariano Pires P-351

de Palacios, Patricia Ibarra P-710

de Ronde-Tillmans, M. J. A. G. P-72

de Rooij, S. E. P-530

de Rooij, Sophia E. P-52, P-481

de Rooij, Sophia P-624

de Santa Maria, Lucia Saenz P-346

De Souto Barreto, Philip O-121

de Souza Barcelos Barroqueiro, Rodrigo P-678 
De Souza Orlandi, Fabiana O-24

de van der Schueren, Marian P-454

de Vet, Henrica C. W. O-3

De Vis Jill, J. B. P-402

de Visser, Marieke O-52

De Vriendt, Patricia P-212

de Vugt, Marjolein E. P-389

de Wazieres, Benoit P-575

Debain, Aziz P-212

Debesay, Jonas O-64, P-300

Déborah, Li Vigni P-764

Deborah, Livigni O-72, P-909

de Bruin, Eling D. P-150

Decaudin, Bertrand P-526, P-539

Deeg, Dorly J. H. P-137

Degerickx, Robin O-20

Dekhtyar, Serhiy O-43, P-615

Dekoninck, Julien P-708

del Carmen Arias-Blanco, Maria P-425

Del Giudice, Giuseppe P-106

Del Lungo, Ilaria P-502, P-512

Del Panta, Vieri O-28, P-652

Del Rio, A. P-833, P-853

Del Rosario Evangelista Cabrera, Lourdes O-11, P-104, P-115

Del Rosario García Martín, María P-115

Del Rosario Oviedo Briones, Myriam O-101

Dela, Flemming P-155

Delbari, Ahmad P-892

Delcourt, Fabien P-498

Delgado, MCarmen P-296, P-610

Delgado-Silvela, E. P-95

DeLuca, Gabriele C. P-656

Demarle, Sylvie P-593

Demir, Numan P-756

Demircioglu, Arzu P-638

den Boeft, Laura P-324

Denis, Angelique P-79

Deniz, Olgun P-176, P-219, P-247, P-619, P-826

Denkinger, Michael D. O-28, P-49, P-241

Depla, M. F. I. A. P-869, P-884

Derijks, H. J. P-31

Desai, Nimai P-405

Deschodt, M. P-777

Deskur-Śmielecka, Ewa O-122

Destro, Carla P-747

Dev, D. P-809

Dhayana, Dallmeier O-19

Dhesi, Jugdeep P-308

Di Bari, Mauro O-78, O-95, P-559, P-563, P-568, P-697, P-784, P-848

Di Cello, Serena O-94

Di Santo, Simona P-854, P-855

Diamond, Gigi P-520

Diane, Lastennet P-274

Dib, Laila P-114

Didier, Armaingaud P-233

Diedrich, Annika P-799

Diekmann, Rebecca P-318, P-321, P-322, P-323

Diez-Picazo, Laura López P-220

Díez-Ruiz, Ana O-100

Díez-Sebastián, Jesus P-800, P-801

Dillen, Let O-119, P-866

Dina, Zekry O-55

Dineen, R. P-614

Ding, Y. Y. P-134

Dionisio, Antony Soares P-794
Dipanda, Mélanie P-24

Djukic, Marija P-19

do Desterro Soares Brandão, Maria P-116

Dodds, Richard P-238, P-240, P-511

Dogan, Hafize P-506

Dogrul, Rana Tuna P-176, P-216, P-217, P-219, P-247, P-390, P-619,

P-638, P-663, P-808, P-826

Dokuzlar, Ozge P-133, P-891

Dolores López Pizarro, Mª . P-847

Dolors Quera, A. P-221

Domergue, Hélène P-573

Domingo Sánchez, M. D. P-874

Dominguez-Villoria, Luis O-66

Dominique, Fletcher P-79

Dominique, Somme P-509

Donadio, Cristiano P-371

Donal, Fitzpatrick P-501

Donnelly, G. P-525

Donnelly, Geraldine P-259, P-293

Doornebosch, Arno P-291

Dorothee, Florio P-109

Doudinskaya, Ekaterina P-120

Douglas, Miller P-138

Doulazmi, Mohamed P-857

Dourthe, Lucie P-857

Doutel Haghighi, Eduardo P-759, P-858

Doventas, Alper P-447, P-623, P-719

Dovjak, Peter P-140

Dreessen, L. P-347

Dreher-Hummel, Thomas P-579

Drennan, Jonathan O-87

Drennan, Vari P-175

Drevet, Sabine O-74

Drey, Michael P-154, P-195

Dreyer, Adeline P-626

Druiven, Lars P-7, P-8

Drummond Borges, Diogo P-765

Dubiel, Marzena P-705, P-772

Dudinskaya, Ekaterina P-26

Duems, O. P-191

Dukelow, Tim O-63

Duncan, R. P-181

Duque, Sofia O-115, P-245, P-307, P-331, P-332

Duranti, Giuliana P-446

Dutto, Francesca O-8

Duy, Nghiem P-274

Dwolatzky, Tzvi P-471

Dyks, Derek P-7, P-8

Dziewas, R. P-135

Dzingina, Cynthia P-524

Earnshaw, Alan O-76

Earnshaw, C. P-480, P-482

Easton, Jonathan P-145

Echeverria, Iñaki P-237, P-828

Eckert, Tobias O-16, O-17, O-56, P-301, P-302, P-310, P-900

Eckstein, Claudia P-842

Edahiro, Ayako P-474, P-662

Edeltraut, Kröger O-45, P-53, P-70

Edgley, A. P-531

Edith Pisa, Federica O-109, P-28

Edmondson, Jane P-567

Edwards, Susan O-111

Egberts, Angelique O-91

Egberts, T.C.G. P-838 
Egert, Sarah P-321, P-322, P-323

Eglseer, Doris P-83

Eguzkiza, Leire P-577, P-578

Eidam, Annette P-18

Eilat-Tsanani, Sophia P-430

Eiriksdottir, Gudny P-249

Eisert, Albrecht O-35

Ekdahl, Anne P-733

Ekkel, M.R. P-884

Ekrem Aydin, Ali P-133

EL Achek, Mohamed Amine P-760

El Arab, Haissam P-441

El Faquir, N. P-72

El Osta, Lana P-261, P-441, P-787

El Osta, Nada P-441, P-787

Elaswarapu, Rekha P-175

Elbaz, Alexis P-674

Eldholm, Rannveig O-85, O-86

Elena, Colizzi P-183

Elena, Duvernay P-648

Elena, Voronska P-165, P-182

Elexpuru Estomba, Jaione P-199

Elfaleh, Raja P-698

Elias, Assaf P-829

Elias, Tania C. N. P-564

Elise, Mercadier P-901

Elizabeth Carty, E.C. P-255

Elizabeth, Suárez S. P-221

Elkjær Andersen, Hanne P-783

Ellen, Freiberger P-297

Ellis, Graham O-126

Ellul, Y. O-53

Elmarakby, Magda Attia Abdelkawy Aly P-757

Elseviers, Monique P-29

Elstgeest, Liset E. O-3

Elyn, Antoine O-121, P-883

Emanuela, Enache P-749

Emilie, Ferrer P-764

Emilie, Menand P-509

Emily Sedano Gómez, Gianina P-114

Emily, Reeve O-45

Eminovic, Sandra P-83

Emmanuel, Barange O-98

Emmanuel, Chazard P-497

Emmanuelle, Boutin P-829

Emmelot-Vonk Marielle, M.H. P-402

Emre Kocyigit, Suleyman P-133, P-830

Emre Yalcin, Ahmet P-367

Encarnação, Sofia P-765

Endo, Hidetoshi P-12, P-196, P-458

Engberink, Mariëlle F. P-164

Engedal, Knut O-85, O-86

Engelbert, R. H. H. P-340

Engstad, Tora Torgersen P-355

Enneman, A. W. P-422

Ensink, Anne P-34

Enwere, P. P-328

Enwere, Peter O-65

Epaulard, Olivier O-71

Eppenga, W. L. P-31

Epstein, Jonathan P-234

Eraslan, Umut P-774

Erazo, C. Murillo P-874

Ercolani, Sara P-703

Erdincler, Deniz Suna P-447, P-623, P-719
Erdogan, Onur P-35

Erdogan, Tugba P-35

Erdoğan, Tugba P-775

Erel, Özcan P-782

Eric, Pautas P-44, P-274

Eriksdotter, Maria O-88, P-657

Eriksson, Johan G. O-27

Ermolina, Natalia P-684

Errasquín, Beatriz Montero O-24

Errasti, Ane P-237, P-828

Esbrí-Víctor, Mariano P-197, P-198

Escolante Melich, Ana P-847

Esme, Mert O-41, P-638, P-667, P-663, P-720, P-894

Esperanza Martín Correa, $\mathrm{M}^{\mathrm{a}}$. P-847

Esperto, Helder P-330

Espinosa, Luis Manzano P-354

Espinoza, Romeo Rivas P-543

Eßfeld, Christina P-799

Estefanía Olaya Loor, Génesis P-709

Etesam, Farnaz P-369

Eto, Masato P-412

Evans, J. P-480, P-482

Evelyne, Liuu P-439

Evensen, Sigurd P-844

Ewen, Tanyah P-529

Fabianowska-Majewska, Krystyna P-136

Facal, David P-287

Facchinetti, Roberto O-116

Fadayevatan, Reza P-892

Faden, Ekram S. P-688

Fagan, Colette P-433

Faida, Ajili P-342, P-378, P-379

Fakhouri, Jihad P-787

Fakhrzadeh, Hossein P-151, P-606

Falcone, Yolanda P-58

Faleh, Raja P-700

Fanny, Leborgne O-72, P-764, P-909

Fantin, Francesco O-116, P-795

Farhat, Amel P-760

Farhudi, Fatemeh P-289

Farre, Nuria P-343, P-350

Farrugia, F. P-313

Fastbom, Johan P-29

Fatemeh- P-606

Fatma, Said P-46

Fatoumata, Tall P-44

Fattoretto, Elisa P-747

Faxén, Gerd P-201

Faxén-Irving, G. O-21

Fazi, Andrea O-80

Fedeli, F. P-568

Fedeli, Filippo O-78, P-563

Federspiel, Carine P-418

Feeney, AnnMarie O-49

Feighan, Jennifer O-66

Feinberg, Leo P-78

Fenech, Valentina P-618

Feng, Cindy P-396

Ferarri, Uta P-195

Fereshtehnejad, Mohammad O-88

Fernandes, Lia P-210, P-235

Fernandes, M. A. P-68

Fernandes, Marilia P-557, P-765

Fernandez Soneira, Andrea P-346 
Fernandez, Christine P-536

Fernández, Jesús Mora O-11, P-102, P-103, P-104

Fernandez, Naiara P-341

Fernández, S. Solsona O-75

Fernández-Rius, Ana Pérez O-22

Fernández-Sotos, Patricia P-841

Fernando Calderón-G, Julián P-456

Ferracci, Marika P-185

Ferraresso, Maria Seriana P-747

Ferrari, Aline Garcia P-594

Ferrari, Uta O-23, P-154

Ferrat, Emilie O-58

Ferré, Lourdes P-221

Ferreira Lopes, Fernanda P-475

Ferreira, Ana P-330

Ferreira, André O-115, P-331, P-332

Ferreira, Inês Miguéis O-115

Ferreira, M. P-518, P-519

Ferreira, Miguel Marques P-148, P-596

Ferreri, Cinzia O-8

Ferri, Evelyn P-393

Ferry, Peter P-618

Feyza Yurt, Emine P-782

Ficheur, Grégoire P-526, P-539

Fieldes, M. P-542

Fife, Elizaveta P-136, P-796

Figueiredo, I. R. P-68

Figueiredo, Inês P-557

Figueiredo, Ines Rego Figueiredo P-765

Fijačko, Vladimir P-875

Finbar, Martin P-501

Fink, Andrea P-413

Fink, Sylvana P-387

Finne Soveri, Harriet O-59, O-118, P-870

Firtına, Meftun P-505, P-506

Fisch, Alexandra P-345

Fisher, J. P-256

Fisken, E. P-256

Fladby, Tormod O-123

Flamaing, J. P-777

Flamaing, Johan P-22, P-294

Flechtner-Mors, Marion O-1

Fleischer, Steffen P-403

Fleuren, N. P-869

Flierman, Isabelle P-876, P-877

Flora, Ketz P-274

Florence, Canouï-Poitrine P-376, P-901

Florence, Lieutier P-356

Florence, Rollot Trad P-829

Florent, Dayde P-376

Florent, Guerville O-98

Flores Ruano, Teresa O-22

Floris, P. P-335, P-98

Floris, Patrizia O-10

Fogari, R. P-9

Fogteloo, Jaap P-837

Fonck, Marianne O-107

Fonseca, I. P-518, P-519

Fonseca, J. P-721

Fonseca, João P-330

Fonte, Gianfranco P-58

Fontecha, Benito P-546

Foong, Y. L. P-76

Forasassi, Christine P-457

Forestier, Emmanuel P-513, P-575, P-753

Formiga, Francesc P-629, P-630
Formilan, Marino P-599

Formosa, Matthew P-6

Foroughan, Mahshid P-892

Fors, Stefan P-385

Forte, Aldo Lo P-568, P-697

Fouaad, Ahmed P-275

Fougère, Bertrand P-160

Foulon, Veerle P-746

Fourrière, M.J. P-208

Fox, Jenny O-12, P-90, P-96, P-97

Fox, Siobhan P-685

Fraisse, Thibault P-513, P-575

Franceković, Dorotea P-271

Frances Horgan, N. P-597

Francesco, Brunetti P-376

Francia Santamaria, E. P-754

Francisco Javier, Balea-Fernandez P-171

Franco Antonio, Cristina P-456, P-437

Francois, Herrmann O-55, P-648

François, Patrice O-74

Françoise, Capriz P-764

François-Hubert, Brunschwig P-764

Frang, Theresa P-485

Franzén, E. O-21

Franzén, Erika P-201

Franzon, Kristin O-26

Fratiglioni, L. P-177

Fratiglioni, Laura O-43, P-615

Frauke, Müller P-648

Fredbo, M. P-695

Frédéric, Eyvrard P-40

Frederic, Pamoukdjian P-439

Frederic, Prate P-118

Freeman, M. P-802

Fregnac, Celine P-513

Freiberger, Ellen O-3

Freitag, Mathias O-35

Freud, Tamar P-483, P-707, P-780, P-781, P-867

Friis, Marie O-99

Frison, Natalina P-747

Frith, J. O-44

Frith, James P-312

Frobell, Richard P-733

Frohnhofen, Helmut P-10

Frost, Rachael P-175

Fujino, Haruka P-377

Fukui, Kento O-37

Fulvio, Lauretani P-183

Fumagalli, Carlo P-855

Fumagalli, Stefano P-696

Fürst, Tomas P-27

Fusco, D. P-789

Gabbani, Luciano O-95

Gabriel, Gold P-648

Gabriel, Torbahn O-19

Gabriele Meyer on behalf of the EPCentCare Consortium P-403

Gabriele, Sara P-795

Gabrovec, Branko P-139

Gać, Paweł P-373

Gaëtan, Gavazzi O-71, O-74, P-60, P-61, P-62, P-63, P-109, P-491

Gaggi, Lorenzo P-703

Gagin, Roni P-309

Gaillemin, O. P-480, P-482

Gale, Chris P-246 
Galeote, Guillermo P-577, P-578

Galić, Romana P-271

Gallagher, Paul O-63, P-56

Galmarini, V. P-335

Galmarini, Valter O-10

Galvão-Moreira, Leonardo Victor P-116, P-351, P-444, P-475, P-678

Gamboa Huarte, B. P-732, P-874

Gan, Jasmine O-50

Gandolfo, Federica P-572

Gangai, Natalie P-258

Gangemi, Salvatore P-502, P-512

Garay Airaghi, Victoria O-11

García Tercero, Rosa María P-847

García, Borja Gil O-22

Garcia, Margarita P-346

García, María Elena Arnaíz P-832

García, Mireia Tofiño P-680

Garcia, Montserrat P-296, P-610

García-Molina, Rafael P-198

García-Sánchez, Inés P-139

García-Tercero, Elisa P-661, P-841, P-847

García-Tercero, Rosa María P-661, P-841

Gardner, Benjamin P-175

Garfinkel, Doron O-110

Garo, Michele O-94, P-722

Garofalo, Valentina O-70

Garside, M. P-256

Gąsowski, Jerzy O-97

Gaspar, Elsa P-534

Gasperini, Beatrice O-80

Gassie, Paul P-573

Gattazzo, Stefano O-116

Gaudreau, Pierrette P-435

Gaustadnes, Lisa Mari P-484

Gauthier, Julien P-24

Gautier, Juliette O-71

Gautier, S. P-208

Gautier, Sophie P-526, P-539

Gautier, Sylvie O-106, P-125, P-795

Gavana, Magda P-758

Gavazzi, Gaetan Gavazzi P-575

Gavazzi, Gaetan P-513

Gavazzi, Gaëtan P-753

Gavrikov, Dmitry P-483

Geerinck, Anton O-24

Geerlings, Mirjam P-602

Geerlings, Suzanne P-904

Gegout-Petit, Anne P-126

Geirsdottir, Olof G. P-169

Geirsdottir, Olof Gudny O-5, P-442, P-486

Gellens, Juliette P-526

Gellert, Paul P-640

Gelmini, Teresa P-747

Gemma, Gabriel P-648

Genia, Babykina P-497

Gennadyi, Kalinin P-744

Georgiopoulos, Ioannis P-740, P-758

Germain, Daphné P-126

Gerritsen, Adrie A. J. P-389

Gerritsen, Debby P-904

Ghadab, Imen P-698, P-700

Ghafar, M. Z. P-594

Ghanmi, Latifa P-603

Gharra, Nabil P-687

Gheorghevici, Teodor Stefan P-80
Ghiara, Camilla O-78, P-559, P-563, P-568, P-697

Ghorbel Imed, Ben P-46, P-731, P-748

Ghouri, Bushra P-863

Giannakopoulos, Eustathios P-758

Giannini, Ilaria O-95, P-848

Giannotti, Chiara P-100, P-101, P-856

Giannouli, Vaitsa P-399, P-724

Gianturco, Luigi P-248

Gianturco, Vincenzo P-248

Giardini, Sante P-502, P-512

Gibbon, Jake R. P-312

Gibello, Maddalena P-58

Gibson, V. P-256

Giedraitis, Vilmantas P-621

Giil, Lasse Melvaer P-632

Gil García, Borja P-494

Gil, Javier Idoate P-99, P-105

Gil-García, Borja P-197

Gillet, Pierre P-556

Ginbey, Eleanor P-419

Gineste, Yves O-89

Ginste Marie, Vande P-504

Gioanni, Alice O-13, P-573, P-857, P-859

Giordano, A. P-386

Giordano, Antonella O-40, P-244

Giorgeschi, Angela O-70

Gladman, John P-644, P-654

Godfrey, Mary P-246

Goedele, Strauven P-504

Gögenur, Ismail O-99

Goggins, Aine P-308

Goisser, Sabine O-1, P-18

Gokdemir, Bahattin P-773

Goker, Berna P-176, P-219, P-247, P-826

Göktaş, Ayşe P-607

Göl, Mehmet P-452

Goldberg, S. E. P-531, P-601, P-608

Goldberg, Sarah P-414, P-650, P-668

Goldberg, Yury P-483

Goldoni, Matteo P-819

Goldsby-West, Robert O-76

Goldzweig, Gil P-890

Golkarami, Shirin P-289

Gomes, J. P-721

Gomez, Armando Pardo O-101

Gomez-Arteaga, Ronald Camilo O-39

Gómez-Porras, Germán P-605

Gomez-Quintanilla, Alex O-12, P-90, P-96, P-97

Gómez-Valent, Mònica P-87

Gommers, Danielle P-702

Gonçalves, M. P-518, P-519

Gonçalves, Mariana P-534

Gonella, Davide P-572

Gonzales, Jimmy P-204

Gonzalez Santos, José María P-832

González, Marianna Javier P-832

González-Ávila, Bàrbara P-87

González-Montalvo, Juan Ignacio P-227, P-230, P-577, P-578, P-800, P-801, P-822

Goodman, Claire P-175

Gordillo-Pérez, M. G. P-583

Gordon, Adam P-654

Gorey, S. P-215, P-594

Gorjão Clara, João P-245

Gorman, Grainne P-240

Gorus, Ellen P-212 
Gosch, Markus P-778

Gosney, Margot P-433

Goto, Fumihiko P-382

Goto, Yuko P-868

Gotor, Pilar P-578, P-800

Goudzwaard, J. A. P-72

Gouveia, Cristina Carvalho O-115

Govoni, B. P-98, P-335

Gozlan, Moriya P-309

Grande, G. P-177

Granic, Antoneta P-238, P-240

Gravenstein, Stefan P-710

Grech, Marco P-488

Green, Amy P-419

Green, Ellie P-269

Greenwood, Susan P-561

Gregersen, M. P-576

Gregersen, Merete P-203

Gregoire, Ficheur P-497, P-593

Gregor, Sandra P-394

Gregson, E. P-566

Grellety, Thomas O-107

Griffin, T. P. P-215

Grifoni, Stefano O-95

Grill, Eva P-448

Grillo, Nadia P-722

Grimalauskaitè, Rita P-426

Grodzicki, Tomasz O-97, P-205, P-214, P-655, P-705, P-772, P-825

Groenewegen-Sipkema, Karin H. P-286

Grönstedt, H. O-21

Grönstedt, Helena P-201

Grossman, Yonatan P-430

Grossocordon, Nuria García P-333, P-334

Gruner, H. P-68

Gruner, Heidi P-557, P-765

Gryglewska, Barbara P-204, P-214, P-655

Gual, Neus P-311, P-71

Gudnason, Vilmundur P-249

Gudny Geirsdottir, Olof P-209, P-406, P-462

Güell, Carolina O-100, P-207

Guenther-Lange, Michaela P-319

Guerin, Olivier P-575

Guerra, Chiara O-116

Guerre, Ismael Dale Cotrim P-116

Guerreiro, Marta Isabel Tomé P-551

Guerrero, Katerin Leslie Quispe O-70

Guerrieri, Valentina P-722

Güerri-Fernández, Robert P-348

Guerville, Florent P-573, P-812

Guevara Guevara, Tania O-101

Guevara, Tania P-204

Guezmir, K. P-751

Guglielmetti, S. P-130

Guidet, Bertrand O-79

Guillard, Nadege P-329

Guimarães-Consciência, José P-331, P-332

Guirola García-Pendes, Cristina P-282

Gülhan Halil, Meltem P-667

Guligowska, Agnieszka P-136, P-451

Gungor, Funda P-147

Guptha, S. P-809, P-852

Gurieva, Irina P-213

Gussago, Cristina P-393

Gussekloo, J. O-73, P-521, P-522

Gustafson, Yngve O-84

Gutensohn, Kai P-691
Gutiérrez Misis, Alicia P-801

Gutiérrez Rodríguez, José P-281

Gutierrez, Jose Antonio Martos P-895

Gutiérrez-Misis, Alicia P-822

Gutiérrez-Valencia, Marta P-15

Guzmán, Gabriela P-577, P-578

Guzman, Laurenny P-767

Guzzardi, Maria A. O-27

Gzael, Imen P-146

Haapanen, Markus J. O-27

Haddad, Anis P-698, P-700

Haddad, Rebecca O-13, P-857, P-859

Hadley, Gina P-656

Haefeli, Walter E. O-19, P-241

Hafizah, N. P-134

HaGani, Neta P-309

Hagberg, Guri O-81

Hägg, S. P-30

Haghir, Edris P-396

Haifa, Hachfi P-360

Hajaoui, Manon P-143

Hajem, Said P-699, P-701, P-741

Halil Türkbeyler, İbrahim P-178

Halil, Meltem O-41, P-216, P-217, P-390, P-619, P-663, P-720, P-894

Hall, J. P-609

Hall, Marlous P-246

Halld, Thorhallur P-249

Halvorsen, Per Steinar P-355

Ham, A. C. P-422

Hamadeh, Ghassan P-252, P-487

Hamda, Kaldoun Ben P-384, P-432

Hamina, Aleksi P-612, P-642

Hammami, Mohamed P-146, P-384, P-432, P-699, P-701

Hammami, Nadia P-384

Hammami, Sonia P-146, P-253, P-360, P-361, P-384, P-432, P-603,

P-698, P-699, P-700, P-701, P-760, P-761

Hammond, Laura P-336

Hamza, Mouanaa P-360, P-761, P-361

Hancox, J. E. P-609

Hancox, Jennie P-327, P-414

Hannek P-435

Hansen, Stinus P-167

Hansjee, Dharinee P-882

Hanussek, Barbara P-693

Haque, Khalid P-849, P-850

Harchowal, Jatinder O-103, P-407

Hardel, Adrien P-24

Hardy, S. P-328

Harling, Martyn P-644

Harmsen, I.M. P-422

Harrington, Elaine P-649

Harris, Tamara B. P-435

Harris, Tamari P-249

Harrison, Aimee P-268

Hartigan, Irene O-87

Hartikainen, Sirpa O-112, P-438, P-611, P-612, P-642, P-665

Harvey, Nick P-715

Harwood, R. H. P-531, P-601, P-608, P-609, P-614, P-659

Harwood, Rowan H. P-327, P-650, P-668

Harwood, Rowan P-414, P-644

Hasegawa, Ryuichi P-382, P-400

Hashemi, Nima O-76

Hashmath, Shafi P-275

Hassanzadeh, Roya P-564 
Hauer, K. O-68, P-645

Hauer, Klaus O-16, O-17, O-56, P-292, P-295, P-299, P-301, P-302, P-310, P-319, P-637, P-900

Haug, Ulrike O-109, P-14

Haveman-Nies, Annemien P-434

Hawk, Emma O-50

Haw-Shing, Laura P-573

Hazen, Josje O-86

Hebestreit, Antje O-1, P-454

Heczko, Piotr P-655

Heier, Margit P-448

Heike, Geschwindner P-627

Hein, Andreas P-321, P-322, P-323

Heitz, Damien O-107

Hek, Karin O-114, P-43

Helbig, Claudia P-778

Helbostad, Jorunn L. O-28

Heli, Salmenius-Suominen P-445

Heldmann, Patrick P-292

Hellström, Karin P-314

Helmer, Catherine P-625

Helvaci, Ozant P-219

Heman, Daniëlle P-164

Hendrix, Ivanka P-33

Hengeveld, Linda M. P-434, P-435

Henkel, Michaela P-195

Hennighausen, Udo P-790

Henrard, Séverine P-746

Henry, Darrien P-905

Herbst, Andrea O-78, P-559, P-563, P-568, P-697

Herghelegiu, A. M. O-124, P-357, P-358, P-362, P-763

Heringhaus, Christian P-585

Heritt, Carolina Wilhems P-594

Hermans, Peter P-710

Hermes, A. O-33

Hernández Sánchez, Luisa Alejandra P-709

Hernández, María Jesús Molina P-115

Hernandez-Sanchez, L. A. P-95

Hernes, Susanne M. S. P-270

Herr, Marie P-260

Herrera Abian, M. P-174

Herrera, Nuria P-745

Herrera-Landero, A. P-583

Herrero, Marta P-343, P-359

Herrmann, F. R. P-752

Hertogh, C. M. P. H. P-884

Hertogh, C. M. P. M. P-554, P-869

Hertogh, Cees P-906, P-904

Herzig, Roman P-600

Heshmat, Ramin P-151, P-606

Hestevik, Christine Hillestad P-484

Hewkins, C. P-659

Heyman, Noemi P-284

Heymans, Martijn W. O-28

Hiance-Delahaye, Anne P-260

Hias, Julie P-21, P-22

Hickson, Mary P-454

Higuet, S. P-13, P-55

Hilde, Baeyens P-523

Hillarious, Amy P-676

Hilmer, Sarah O-111

Hindlet, Patrick P-536

Hintze, Stefan O-23

Hirano, Hirohiko P-474, P-662

Hirayama, Kengo O-34, P-616

Hirota, Ai P-377
Hitosugi, Masahito P-889

Hizem, Olfa P-211

Hjaltadottir, Ingibjorg P-249

Hlais, Sani P-261

Hlatki-Matijević, Suzana P-272

Hobbelen, Hans P-142, P-324

Hoedemakers, R.M.J. P-31

Hoekstra, Trynke O-28

Hoffmann, Dirk P-797

Hofmeister, Fabian O-23

Hogan, Michelle O-111

Holland, Gemma P-836

Hollingsworth, Kieren P-240

Hollwey, Frances P-873

Holm, Vaughan P-267

Holmerova, Iva P-128, P-501, P-681

Holvast, Floor O-114, P-43

Höncke, Janne P-798

Honda, Miwako O-89

Hong Toh, Chee P-466

Hongisto, Sanna-Maria P-643

Hood-Moore, V. P-609

Hoogendijk, Emiel O-22, O. P-137

Hope, S. V. P-254

Hope, Suzy P-156

Hopstaken, R. M. P-554

Horgan, Katherine P-567, P-810

Hortobagyi, Tibor O-61

Horwitz, Jacob P-471

Houman, Mohamed Habib P-372

Howe, A. P-480, P-482

Howson, Fiona F. A. P-149

Høyem, Mari Hval P-484

Hsairi, M. P-751

Hu, F. W. P-840, P-843

Huang, Allen P-7, P-8

Huang, Hsuan-Ju P-517

Huayna, Karina Liz Quiñones O-11, P-104, P-548, P-549

Hubert, Jean-Marie P-493

Huedo Róden, Isabel P-494

Huedo-Rodenas, Isabel P-198

Hughes, Julian P-735

Huisman, M. P-869

Huisman, Martijn P-137

Hung, Y. C. P-409

Hussein, Zeinab O-50

Huvent-Grelle, Dominique P-593

Hyernard, Caroline P-573

Hylen Ranhoff, Anette P-270, P-695, P-844

Hymabaccus, Benazir P-217

Hyne, Sai P-288

Iacomelli, Iacopo P-244

Ibrahim, Kinda P-149, P-433, P-511

Ido, Hisanori P-382

Iglseder, Bernhard P-140

Iijima, Katsuya P-159

Ilie, A. C. O-124, P-357, P-358, P-362, P-763

Ilie, Adina Carmen P-791

Iliffe, Steve P-175

Ilomäki, Jenni O-111

Imam, Towhid O-76, P-266, P-267

Imaz, Igone P-250

Imrani, Loubna P-492

Indiano, Ilaria P-572 
Inga, Thorsdottir P-440

Ingelsson, Martin P-621

Ingram, Owen P-266

Ingrand, Isabelle P-753

Ingrand, Pierre P-753

Inoue, Kanichi O-37

Inoue, Yasuteru P-366

Inzitari, Marco O-18, P-71, P-311, P-622

Ioan Prada, Gabriel P-397, P-489, P-791

Ionita, Giulia P-283

Iozzo, Patricia O-27

Irazusta, Jon P-237

Irigoyen, RA P-264

Irina, Osipovskaya P-744

Irita, Hirofumi P-377

Isabelle, Baumgaertner P-376

Isabelle, Bereder P-118

Isaia, Giovanni C. O-8

Ishikawa, Shogo O-89

Isik, Ahmet Turan P-588

Isoguchi, Aito P-366

Ispirli, Gul P-773

Itani, Mohamad P-252, P-487

Itikawa, Tetsuo P-662

Ito, Fuhka O-34

Ito, Yoshitaka O-37

Ito, Yuki P-675

Iuliana Pislaru, Anca P-80

Izuka, Yumiko P-12

Izutsu, Miku O-34, P-616

Jaatinen, Roope P-646

Jacobs, Bobbi P-786

Jacobs, Jeremy M. P-890

Jacobshagen, Claudius P-19

Jagadish, Mallya Ullal P-466

Jager-Wittenaar, Harriët P-142

Jagger, C. P-181

Jagger, Carol P-435

Jakavonyte-Akstinienè, Agne P-16

Jakupaj, Adriana P-836

Jalil, H. P-480, P-482

Jamel, Koubaa P-761

James, Kirstyn O-63

Jameson, Karen P-460

Jamet, Amelie P-329

Jan Blauw, Gerard P-837

Jan Peter, Thomas P-617

Janavičiūté, Audronè P-478

Jandova, Tereza P-183

Jansen, C. J. P-530

Jansen, R. W. M. M. P-598

Janssen, D. J. A. P-869

Jaramillo-Hidalgo, Javier P-454

Jarmusch, Stefanie O-23

Jarolimova, Eva P-128

Järvinen, Heli P-611

Jarvis, Aron P-735

Jarzebowski, Witold P-51, P-371

Jason, Guertin P-53

Jaurrieta, Juan Jose Solano P-66, P-281, P-282, P-346

Javier López Rodríguez, Francisco P-832

Javier Martin-Sánchez, F. P-569

Jean Baptiste, Beuscart P-225, P-497, P-593

Jean-Claude, Leners P-880
Jean-Pierre, Baeyens P-523

Jefferson-Loveday, Charles O-31

Jennings, Emma P-56

Jeong, Jinkyu P-415

Jepsen, Ditte P-167

Jeremy, Fallot O-72

Jeuring, Hans W. P-137

Jevons, Gary P-293

Jguirim, Mahbouba P-360, P-760

Jguirim, Mahoua P-761

Jia, Ruiyi P-121

Jimenez Rodriguez, A. P-174

Jiménez, Encarna Rodríguez P-543

Jiménez, Rocío Malfeito P-114

Jing, Remigereau P-439

Jo P-346

Joaquim, Prud'homm P-509

Joël, Ankri O-25

Johanne, Monette P-70

Johansson, Björn P-657

John, Morley P-138

Johnell, Kristina O-120, P-29, P-345, P-385

Johnsen, Marc P-535

Johnson, James P-710

Johnson, Kelly D. P-112

Johnston, James Noble P-545

Joly, L. P-208

Jonkman, Nini H. O-28, P-421

Jonsson, Palmi V. O-5, P-169, P-209, P-406, P-440, P-442, P-462

Jonveaux, Therese P-671

Jordan, Emmet O-93

Jordan, Stephanie Louise P-268

Jordhøy, Marit Slaaen P-82

Jørgensen, LM O-113, P-737, P-738

Jørgensen, Martin Grønbech P-391

Jørgensen, Niklas Rye P-167

José Betancourt, Alvaro P-845

José Cruz Jentoft, Alfonso P-709

Jose, Matthew P-3

Jost, J. P-38

Jouanny, Pierre P-24

Jukema, Wouter O-104

Julian, Adrien P-329

Julien, Dekoninck P-523

Juliette, Podevin P-79

Jurašková, Božena P-600

Jürgen, Bauer O-19

Just, Katja S. P-49

Justine, Trimouillas P-420

Juul-Larsen, H.G. P-737, P-738, O-113

Jyväkorpi, Satu O-30, O-32, P-643

Kaae, S. O-113

Kader, Nayaab Abdul P-405

Kagan, Ella P-483, P-707, P-780, P-781

Kaipainen, Tarja P-438

Kaisu, Pitkälä P-445

Kalbe, Elke P-667

Kalisvaart, K.J. P-838

Kamali, Mohammad P-289

Kamaruzzaman, S.B O-7

Kameyama, Yumi P-412

Kamfar, Daniah M. P-688

Kampe, Karin O-17

Kanenawa, Kyosuke P-366 
Kang, Min-gu P-107

Kanso, Fatme P-787

Kańtoch, Anna P-214

Kapoor, Rachna P-520

Kar, İrem P-607

Kara, Guzin P-774

Kara, Ozgur P-216

Karabıyık, Esra P-265

Karabulut, Erdem P-217, P-390, P-667

Karan, Ayşe P-147

Kåreholt, Ingemar P-486

Karg, Daniela P-798

Karin, Maley P-376

Karine, Moreau O-98

Karnoe Knudsen, Anna P-155

Karolczak, Kamil P-398

Karttunen, Niina P-612, P-642

Kaş, Atiye P-305

Kashtanova, Daria P-120

Kasim, Zaid P-172, P-187

Kasiukiewicz, Agnieszka P-142

Kaspar, Kala O-2

Kasper, J. O-33, P-818

Kässmann, Helmut P-140

Katia, Muller P-499

Kato, Masato P-12

Kato, Nanae P-382

Kaufman-Szymczyk, Agnieszka P-136

Kautiainen, H. P-591

Kautiainen, Hannu P-589

Kavaliauskaite, Indre Marija P-339

Kawashima, Shuji P-458

Kay Falson, Mary P-464

Kazanavičius, Egidijus P-478

Kazanavičius, Vygintas P-478

Keage, Hannah P-660

Kearney, Patricia O-1

Kearney-Schwartz, Anna P-556, P-671

Keeble, E. P-179, P-181

Keelan, R. P-525

Keelan, Ray P-259

Keijser, Bart O-108

Keijsers, C. J. P. W. O-51

Keller, Dagmar I. P-533

Kellert, Benedikt P-633

Kelly, Mary O-1, O-9

Kemal Kilic, Mustafa O-41, P-216, P-619, P-808

Kennelly, S. P-777

Kennelly, Sean O-62, O-93, P-651, P-664, P-897

Kennelly, Siobhan M. P-628

Kenny, Rose Anne O-62, P-897

Kent, Jacqui P-711

Kerminen, H. P-254

Keršienè, Milda P-426

Kessler, Josef P-667

Khamis Al Hamad, Hanadi P-560

Khamis Mubarak Alhamad, Hanadi P-757

Khamis, Hanadi P-275

Khan, A. P-802

Khan, Haroon O-12, P-90, P-96, P-97

Khan, Hassan P-507

Khanfir, Monia P-372

Kharicha, Kalpa P-175, P-449

Khaskia, Mona P-471

Khaw, Rachel P-97

Kheder Messa, Ben P-748
Khera, Akhil P-538

Khiami, Frederic P-84, P-85

Khoo, E. P-586

Kiesswetter, Eva O-1, O-3, O-9, O-108, P-821,

Kilander, Lena O-26, P-621

Kilavuz, Asli P-756

Kilbane, Lorna P-651

Kilıc, Cihan P-147, P-157, P-158, P-468, P-827

Kilic, Dogukan P-232

Kim, Cheol-Ho P-107, P-144

Kim, Jae Hak P-363

Kim, Jongsung P-415

Kim, Joonghyun P-555

Kim, Kwang Min P-200

Kim, Kwang-Il P-107, P-144

Kim, Kyu Nam P-200

Kim, Kyunam P-428

Kim, Sung Kyu P-555

Kim, Sungsoo P-415

Kim, Sunjin P-415

Kim, Sun-Wook P-144

Kimura, Masayuki O-106

Kinoshita, Kaori P-458, P-881

Kinowski, J. M. P-542

Kinugawa, Kiyoka P-857

Kioh, S. H. O-7

Kirdi, Nuray P-638

Kiriyama, Shinya O-89

Kirk, Craig P-553

Kirshner, Dani P-687

Kiss, Rainer O-16, O-56, P-301, P-302, P-310

Kisvetrová, Helena P-600

Kiyoka, Kinugawa P-274

Kızılarslanoğlu, Muhammed Cemal O-41, P-176, P-216, P-219,

P-247, P-619, P-808, P-826

Kizmaz, Erhan P-306

Klaus, Wingenfeld P-297

Klein, Bracha P-430

Klenk, Jochen O-28

Klich-Rączka, Alicja P-815

Klimek, Ewa P-825

Knapskog, Anne-Brita O-85, O-86, P-410

Knašiene, Jurgita P-426, P-427, P-461, P-478, P-689

Knol, Wilma P-57

Knoop, Veerle P-212

Kobayashi, Tomoharu P-12

Kobeh, David P-573

Koçak, Fatma Özge Kayhan P-20

Kocak, Ozge Kayhan P-773

Kocatürk, Celalettin P-505

Kockelkoren Remko, R. P-402

Kocyigit, Suleyman Emre P-436

Kodate, Naonori O-67

Kodavali, Kali P-288

Koek Huiberdina, H. L. P-402

Koek, Dineke P-602

Koek, Huiberdina P-57

Koelé, M. C. P-404, P-422

Koenig, Wolfgang P-448

Kohler, Michaela O-17

Kojima, Taro P-412

Koker, Yusuf Can P-232

Kolarić, Branko P-271, P-272, P-273, P-490

Kollhorst, Bianca O-109, P-14

Komarov, Roman P-483

Komissarov, Elena P-867 
Konan, Ali P-808

Kondo, Izumi P-196

Königkamp, Barbara P-798

Konstantynowicz, Jerzy O-24

Konttila, Tarja P-870

Koo, E. P-586

Koopmans Raymond, T. C. M. P-389

Koponen, Marjaana O-112, P-74, P-611, P-665

Korc-Grodzicki, Beatriz P-258

Korhonen, Maarit P-33

Kostka, Joanna P-136, P-398

Kostka, Tomasz P-136, P-398, P-451, P-796

Kotovskaya, Y.V. P-636

Kotovskaya, Yliya P-26

Kotovskaya, Yuliya P-5, P-47, P-120, P-690

Kotsani, M. O-53, P-208

Kotsani, Marina P-234, P-740, P-758, P-795,

Koubaa, Nadia P-432

Koutsouri, A. P-254

Koutsouri, Natassa P-455

Koyama, Jiro O-37

Kozaki, Koichi P-159

Kozak-Szkopek, Elzbieta P-823, P-824

Kozma, A. P-358, P-362, P-763

Kozma, Andrei P-489

Kozmic, Sarah P-464

Krähenmann, Kathrin P-533

Krammel, Sonja P-778

Krasteva, Elisaveta O-105, P-127, P-407

Krause, Olaf O-35

Kreutzer, Martina P-140

Kristiina, Tuukkanen P-445

Kroc, Lukasz P-796

Królczyk, Jarosław O-97

Kroon, P. P-130

Kropinska, Sylwia P-510

Kruger, Claire P-685

Kruger, K. P-695

Krulová, Petra P-600

Krupp, S. O-33, P-818

Krupp, Sonja P-790

Kucukdagli, Pinar P-835

Kudret, Hüseyin P-607

Kuhn-Thiel, Alexandra P-10

Kuipers, Ernst P-878

Kuitert, Marloes O-61

Kul, Seval P-178, P-180

Kumar Agrawal, Anil P-727

Kumwenda, Mick P-584

Kurowska, Iwona P-705

Kusz, Halina P-119

Kvæl, Linda P-300

Kwon, In Soon P-192

Kyri, Vanhecke P-523

Laakkonen, M.L. P-591

Laakkonen, Marja Liisa, O-118, P-589, P-870

Labat, Carlos O-106, P-125, P-234, P-740, P-795

Labayen, Idoia P-828

Lacour, A. P-38

Lacroix, Andrè O-56, P-18

Lafargue, Aurelie P-812

Laforet, Jeremy P-492

Lafuente, Carmelo P-371, P-51

Laghi, Luca P-579
Lagrange, Jean-Léon O-58

Laing, K. O-44

Lajes, Marlene P-813, P-814

Lakkappa, Bharath P-288

Lakomy, Cécilé P-125

Lambertucci, Lorella P-244

Lamers, Scott O-20

Lamloum, M. P-751

Lamloum, Mounir P-372

Lamoth, Claudine O-61

Lamppu, Pauli O-118

Lampshire, Zoe P-735

Landelle, Caroline O-71

Landi, Francesco P-142, P-156

Landow, Wendy P-464

Lang, T. P-249

Langaas, Anne P-300

Langeveld, A. P-817

Langová, Kateřina P-600

Lapenna, Maria P-446, P-703

Larijani, Bagher P-151, P-606

Larsen, Anders Holsgaard P-167

Larsen, Martin P-129

Lasco, Antonino P-226

Lassen, Annmarie P-571

Lasserson, Daniel S. P-564

Lasseur, Catherine P-812

Latini, Ester P-848

Latronico, Nicola P-854, P-855

Lattuada, Marco P-572

Lau, S. H. G. P-76

Launer, Lenore P-249

Laure, Marie P-871

Laurence, Genton O-55

Laurent, Marie O-107, O-58

Laurentius, Thea O-35, P-803, P-806

Lauretani, Fulvio O-42, P-218, P-805, P-811, P-819

Lauriane, Segaux P-901

Laurila, Jouko O-118

Laušin, Veronika P-272

Lavikainen, Piia O-112, P-74, P-604, P-665

Law, N. C. P-132

Law, Ngie P-131

Léa, Hoisnard P-260

Leal Seabra, Fatima P-236

Leal, Agnieszka Bozanic P-629, P-630

Lecbychova, Karolina P-27

Lech, Sonia P-640

Leclercq, Pierre P-498

Lee, Hyun Suk P-131, P-132

Lee, Ji Eun P-192

Lee, Jungyoon P-415

Lee, Sami P-415

Lee, Seo Kyong P-555

Lee, Yanggyun P-374

Leeming, Michael P-785

Lefeber, Geert P-57

Legout, Laurence P-575

Legué, Catherine P-457

Leguelinel-Blache, G. P-542

Lehours, Philippe P-625

Leithner, Andreas P-83

Lelli, Diana O-18, P-311

Lelubre, C. P-13, P-55

Lemaire, Pierre P-742

Lemke, N. C. P-645 
Lemke, Nele C. P-637

Lemper, Jean-Claude P-21

Lena, Pickert P-472

Lenzen, M. J. P-72

León Ortiz, Matilde P-494

Leon, Lagrange Jean P-829

Leonid, Dvoretsky P-744

Leonidas, Georgalis P-710

Lepetit, Kevin P-492

Lera, Lydia P-239, P-679

Lesauskaite, Vita P-426, P-478, P-479

Lesende, Iñaki Martin P-718, P-730

Leshabari, Kelvin P-755

Leskauskaitè, Daiva P-426

Letenneur, Luc P-625

Levy, Itay P-309

Levy, Pierre P-457

Lewis, Jacintha P-757

Lewis, Mathew P-419

LI, F. Y. P-532

Li, Linxin O-90, P-682

Li, Rebecca P-717

Liljas, Ann P-175, P-449

Lilleheie, Ingvild O-64

Lim, S. P. W. P-532

Lim, Stephen P-511

Lim, W. S. P-134

Lim, Z. P-586

Lin, Shujing P-119

Lin, Y. C. P-409

Lin, Y. F. P-409

Linard, Morgane P-625

Lindgren, Britt-Marie O-84

Lindhardt, Mette P-783

Lindner, Karolina P-727

Line, Guénette P-53

Ling Hong, Ai P-466

Linkohr, Birgit P-154

Linscott, Nicola O-49

Lionel, Collet O-83

Lionel, Couzi O-98

Lips, P. T. A. P-422

Lisa, Götze P-617

Lisa, Kouladjian O'Donnell O-45

Lisk, Clifford P-255, P-64

Lisk, R. P-328

Lisk, Radcliffe O-65, P-73

Liu, Yuansheng P-1

Liu-Ambrose, Teresa P-388

Liuu, Evelyne P-329, P-820

Lo Forte, Aldo O-78, P-559, P-563

Lobo-Marín, Maria P-425

Lochlainn, Mary Ni P-465

Locquet, Médéa P-143

Loetscher, Tobias P-660

Logan, P. A. P-609

Logan, P. P-601

Logan, Pip P-327

Loganathan, SB P-91

Logroscino, Giancarlo P-714

Logue, G. P-480, P-482

Logue, Genna O-12, P-90, P-96, P-97

Loïc, Josseran P-233

Lok, Dirk J. A. P-298

Lökk, Johan O-88

Lola, Corsin P-829
Lona, Murielle P-498

Long, G. P-188

Long, S.L. P-532

Longobucco, Yari P-218, P-805

Looman, Natasja O-52

Lopes, Joana O-115, P-331, P-332

Lopes-dos-Santos, Ana P-331, P-332

Lopez Alvarez, Eva P-346

López Castellanos, Genoveva P-354

López Diez-Picazo, Andrea P-669

López Tatis, Myriel P-680

López, Adriana P-287, P-592

Lopez, Clara O-74

Lopez, Elsa Nelly Baca P-670

López, Jana Albéniz P-709

Lopez, Marta Checa O-101

López-Giménez, Esther P-197, P-198

López-Sendón, José Luis P-577, P-578

López-Utiel, Melisa P-197, P-198, P-494

Lorenzi, C. P-386

Lorenzi, Chiara O-40, P-365, P-784

Loughlin, E. P-215

Loughlin, Elaine P-793

Loureiro, Helena P-330, P-813, P-814

Loureiro, Isabel P-713

Lourenço, Filipa P-557

Lovett, Nicola O-50, O-96, P-647

Lowthian, Judy P-500

Loyens, Silke P-22

Lozada, María Soledad Chiriboga P-670

Lozano-M, Isabel P-454

Lozoya Moreno, Silvia P-197, P-198, P-227, P-494

Lu, Qing P-163

Lu, Xiaoyan P-112

Lubbers, S. P-530

Luc, Mateusz P-727

Lucarelli, Giulia P-559

Lucas, Morin P-883

Lucenteforte, E. P-386

Luciano, Francesco P-722

Lucke, J. A. O-73, P-521, P-522

Lucke, Jacinta A. P-569

Lucke, Jacinta P-585, P-837

Ludwig, C. P-752

Lueje Alonso, Esther O-11, P-102, P-104

Luengo-Fernandez, Ramon P-682

Luigi, Bergamaschini P-315

Luiking, Yvette P-318

Luiting, Stephan P-25

Luk, James Ka Hay P-864

Lukas, Albert P-280

Lukić, Marica P-271, P-273

Lukinac, Petra P-875

Lull Yoon, Jong P-192

Lunardelli, M.L. P-98, P-335

Lunardelli, Maria Lia O-10

Lund, C. M. P-777

Luthy, C. P-752

Luyckx, Michel P-526, P-539

Luzina, Alexandra P-743

Lynch, Marie P-685

Lyons, Imogen P-336

Ly-Yang, Fernando P-230

M P-638 
M. Antunes, A. P-68

Mabuza, Ziyanda P-704

Machado, Inês P-222, P-223

Machelle, Wilchesky P-70

Machin, R. P-503

Machón, Mónica O-100, P-207

Macijauskiene, Jurate P-16, P-479

Madruga Galán, Felipe P-661

Maggi, Stefania P-588, P-799

Maggio, Marcello Giuseppe P-218

Maggio, Marcello P-805, P-811, P-819

Magny, Emmanuelle P-371

Mahmood, R. P-328

Mahmood, Rashid O-65

Maier, Andrea B. O-28, P-122, P-123, P-162, P-421

Mainwaring, Cathryn P-308

Mair, Alpana O-117

Maître, Isabelle O-1

Majid, Zeinab P-78

Malafarina, Vincenzo P-250, P-341, P-467

Malara, Alba O-94, P-722

Mallya, J. U. P-76, P-91

Maloberti, Daniela P-572

Maltarić, Manuela P-271, P-272, P-273

Mamarelis, A. P-659

Mamun, Kaysar P-587

Mañas, Leocadio Rodriguez O-101

Manckoundia, Patrick P-24

Mandell, Mark P-836

Manevich, T. M. P-636

Manjavong, Manchumad P-886

Manna, Ruth P-258

Mannion, E. P-215

Mannion, Edel P-793

Mansour, N. Ben P-751

Manthorpe, Jill P-175

Mantovani, G. P-335, P-98

Manzanares, Sonia Castaño P-718

Manzoor, Amir O-65

Maracchini, Gianfranco O-80

Maray Gondra, Batirtze P-730

Marc, Bonnefoy O-83

Marcel, Arcand P-70

Marcel, Émond O-45

Marcel, Goldberg O-25

Marcel, Rarek P-472

Marcello, Maggio P-183

March, A. P-335

March, Albert O-10

Marchion, Niccolò P-559

Marchionni, Niccolò O-78, P-568

Marco, Ester P-142, P-156

Maréchal, N. P-347

Marengoni, A. P-177

Marengoni, Alessandra O-43, P-854, P-855

María Barral Varela, Ana P-832

Maria Canelas, Ana P-242

Maria de Andrés, Ana P-71

María López Álvarez, Eva P-66, P-281, P-282

Maria Mannarino, Giulio P-784

Maria Mello, Anna P-502, P-570, P-798, P-799

Maria Rempe, Hanna O-3

Maria Trovão, Milena P-475

Maria, Zdoca Rucsandra P-749

Marianne, Giroux O-45

Mariano Juárez, Lorenzo P-437, P-456
Marie Herghelegiu, Anna P-397, P-791

Marie Odile, Hager P-439

Marie, Herr O-25, O-102

Marie, Toussaint-Hacquard P-65

Marie, Zins O-25

Marie-France, Demers P-53

Marie-France, Nguyen O-83

Marie-Josée, Sirois O-45

Marien, S. P-254

Marie-Nelly, Nathalie P-67

Marilyn, Simard O-45

Marin Epelde, Itxaso P-199

Marin, Frédéric P-492

Marina, Orloff P-499

Marine, Gueugnon O-83

Marine, Sanchez P-764

Marín-Epelde, Itxaso P-15

Mark Doherty, T. P-106

Markert, Bernd P-803

Marks, Morgan A. P-112

Markus, Langhans P-693

Maronnat, Florian P-371

Marozzi, Irene P-696

Marques Veríssimo, Manuel Teixeira P-794

Marques, Bárbara P-692

Marques, Consuelo Penha Castro P-475

Marques, P. P-519

Marquez, Carlos P-239, P-679

Marseglia, Anna P-615

Martella, L. P-386

Martella, Letizia O-40

Martello, Valentina P-283

Martín- Sánchez, Francisco Javier P-548, P-549

Martín, María Redondo P-548

Martín, Mercedes P-779

Martin, Saul P-204

Martin, Schimmel P-648

Martina, Rimmele P-297

Martínez Álvarez, Mariana P-456

Martinez Ve, Nicolas P-199

Martinez, Juan García P-115

Martinez, Santos P-94

Martinez-Alvarez, Mariana P-437

Martínez-Fernández, María Isabel P-343, P-350, P-359

Martínez-García, Beatriz P-494

Martinez-Tapia, Claudia O-58, P-871

Martínez-Velilla, Nicolás P-15

Martín-Maestre, Isabel P-227, P-577, P-801, P-822

Martins, Raul P-813, P-814

Martins, Rita P-759, P-858

Martins, Sónia P-210, P-235

Martos, Lola O-50

Marum, R. J. v. P-31

Mary, Diya P-651

Masanes, Ferran P-467

Mascaró Lamarca, J. P-754

Massaia, Massimiliano O-8

Massucci, Maurizio P-185, P-716

Mastaviciute, Asta P-156, P-224, P-337

Masuch, Johanna P-778

Masud, T. P-601, P-802

Masud, Tahir P-167, P-391, P-644

Masuda, Teruaki P-366

Masuyama, Shigeru O-67

Matala, Rina P-873

Matejka, Jan P-375 
Mateo-Abad, Maider O-100, P-207

Mateus, J. E. P-108, P-518, P-519

Mateus, José E. P-534, P-692

Mathilde, Gisselbrecht P-79, P-439, P-444, P-901

Mathoulin-Pélissier, Simone O-107

Matovelle, Priscila P-801, P-822

Matsui, Yasumoto P-196, P-881

Mattace Raso, Francesco P-878

Mattace-Raso, F. U. S. P-72

Mattace-Raso, Francesco O-91

Matteo, Cesari O-102

Matthias, Ried P-693

Matthieu, Durand O-72, P-909

Mattiazzo, G. F. P-317

Maufort, L. P-13

Mauger, Dominique O-28

May, Polly P-547

Mayr-Pirker, Brigitte P-140

Mazur, Grzegorz P-373

Mazzarolo, Giulia P-747

Mazzone, Andrea P-855

Mazzone, Antonino P-251

Mbululo, Luqmaan P-755

McCann, Adrian P-632

McCartan, Deirdre P-408, P-664

McCarthy, Christine P-793

McCluskey, Lauren O-48, P-276

McColl, Aubretia O-90, P-682

McCullagh, Ruth P-597

McHale, Cathy P-664

McHugh, Sheena P-417

McKee, Kevin P-621

McKercher, Charlotte P-3

Meaume, Sylvie P-457

Mecocci, Patrizia P-185, P-393, P-446, P-703, P-716

Medioli, A. P-631

Meendering, A. L. O-82, P-639

Mégraud, Francis P-625

Mehmet P-505

Meinke, Peter O-23

Mele, Francesca P-885, P-888

Mella, Barbara Peña P-346

Mellingsæter, M. R. P-254

Mellingsæter, Marte P-844

Mello, Anna Maria P-512

Mellone, Sabato P-421, P-816

Melone, Sabato O-56, P-310

Melzer, David O-92

Memelink, Robert G. P-164

Memon, Anjum P-48

Mendes, Francisca P-431

Mendez, T. P-580

Mendonça, Nuno P-435

Mendoza, Soledad Dominguez P-115

Mendoza-De la Garza, Maria P-113

Menenakos, Evangelos P-125

Menendez, Sara Artola O-101

Menéndez-Colino, Rocío P-227, P-230, P-577, P-578, P-800, P-801, P-822

Menezes, Rikitha P-595

Mercalli, Franco O-69

Merchant, R. A. P-586

Merello de Miguel, Ana P-709

Merello, Ana P-142

Merello-De Miguel, A. P-243, Merello-De Miguel, A. P-95

Mergy, Jean P-820
Merja, Suominen P-445

Merlin, Larson P-883

Merve Oren, Meryem P-157, P-158, P-827

Mesa-Lampré, María Pilar P-86

Mesas, Mónica P-311, P-71

Meschi, Tiziana O-42

Meskers, Carel P-162

Messa, P. P-804

Mestre, Thibault P-498

Methven, Lisa P-433

Meuwissen, A. P-347

Meyer, Anna Maria P-797

Meyer, Claudia P-500

Meyer, Klaus P-632

Meyrignac, Lucille O-74

Mezera, Vojtech P-375

Meziere, Anthony P-84, P-85, P-857

Michael, Atef P-75, P-368, P-704, P-899

Michael, Denkinger O-19

Michael, Drey O-23

Michel, Harel P-420

Michel, Mazerolles P-883

Michèle, Aubin P-70

Michèle, Morin P-70

Mickley, Frank P-515, P-516

Middleton, L. P-540

Midlöv, P. P-30

Midttun, Oivind P-632

Miggiani, Timothy P-902

Migliorini, Marta P-696

Miguéis-Ferreira, Inês P-331, P-332

Miguel-Alhambra, Luciana O-12, P-90, P-96, P-97

Mikolaizak, Stefanie P-421

Milan, Chang P-440

Millán-Calenti, José C. P-287

Millenaar Joany, K. P-389

Miller, Alan B. P-694

Miller, Fiona P-529

Minder, Christoph E. O-29

Minetti, Loredana P-278

Minetti, Loredana P-807

Miptah, H.N. P-594

Miralles, Oriol P-546

Miralles, Ramon P-296, P-348, P-610

Miranda, Óscar P-94

Miret-Corchado, C. P-243

Mirghani Sayed Abbas, Asma P-757

Mirzadeh, Fatemeh P-370

Misumi, Yohei P-23, P-366

Mitchell, Emma P-77

Mitchell, Sophie P-849, P-850

Mitha, Nathalie O-74

Mitsui, Yurika P-377

Mitter, Pamina O-50

Miura, Hisayuki P-196, P-868, P-881

Mizokami, Fumihiro P-12

Mizukoshi, Manami P-382

Mkhitaryan, E. A. P-634, P-635, P-636

Moatari, Mohammad O-12, P-96

Mobaraki, Hossein P-289

Mohamadi, Masoumeh P-369, P-370

Mohamed Habib, Houman P-46, P-731, P-748

Mohamed, Hammami P-741

Mohamed, Mohamed P-381, P-383

Moisi, Laura O-79

Mola, Sonia Jiménez P-105, P-99 
Molin, Marianne P-484

Molina-Recio, G. P-424, P-425

Mollinedo-Cardalda, Irimia P-287, P-592

Molnár, Christian O-36

Monacelli, Fiammetta P-100, P-101, P-856

Mongars, L. P-208

Monia, Khanfir P-46

Monllau, Juan Carlos P-94

Monnier, Anna P-906

Montalbán, Paula Fernández P-709

Monteiro, Mário P-813, P-814

Monteiro, Sally Cristina Moutinho P-351

Montenegro, Mercedes P-220, P-669

Montero Errasquín, Beatriz P-709

Monti, Elena P-183

Montiel-Luque, Alonso O-100

Mooijaart, S. P. O-73, P-521, P-522, P-817

Mooijaart, Simon P. P-569, P-585, P-837

Morabito, Nunziata P-226

Moral-Cuesta, Debora P-800, P-801, P-822

Moralejo, Miriam P-354

Morandi, Alessandro O-18, P-854, P-855

Moraros, John P-396

Moreira, Emília P-210, P-235

Moreira, V. G. P-865

Morelli, Ilaria O-42

Moreno, Pedro A. P-669

Moreno, Pedro Antonio P-333, P-334

Moreno, Pedro P-220

Moreno, Silvia Lozoya O-22

Moreno-Gonzalez, Rafael O-91

Moreno-Iturriaga, Marta P-87

Moride, Yola P-112

Moriguchi, Emilio H. P-762

Morin, Lucas O-120, O-121, P-29, P-345, P-385

Morishita, Ryuichi P-675

Moser, André P-831

Mossakowska, Małgorzata O-97, P-814, P-815, P-903

Mossello, Enrico O-95, P-244, P-784, P-848, P-854, P-855

Motamed, Gita P-67

Mounir, Lamloum P-46, P-731, P-748

Moussallem, Toufic P-125

Mponzi, Shadrack P-755

Mravak, Stela P-271, P-273

Mrčela, Nada Tomasović P-490

Mubarak, Essa P-275

Mueller, Christoph P-588

Mugelli, A. P-386

Mühlbauer, Viktoria P-241, P-349

Mulkerrin, E. P-215

Mullarkey, Daniel P-266

Müller, M. J. P-135

Müller, Michael P-798

Mundt, Marion P-803

Muñoz, Javier Montero P-66, P-281, P-282

Muñoz-Díaz, Belén P-424, P-425

Muñoz-García, M. P-95

Muñoz-Gost, Neus P-87

Münzer, Thomas P-455

Murali, Srinivasan P-648

Murphy, Kevin P-56

Murphy, R. P-215

Murphy, Robert P-793

Murteira, Fábio P-236

Musacchio, C. P-833, P-853

Mussi, C. P-335, P-98
Mussi, Chiara O-10

Musta P-619

Myint, Phyo Kyaw O-126

Mylène, Allain P-829

Myrstad, Christian P-844

Myrstad, Marius O-81, P-844

Nabil, Zerhouni P-60, P-61, P-62, P-63, P-491

Nabipour, Iraj P-151, P-606

Nacer, Bergaoui P-360, P-361, P-761

Nacu, R. M. O-124, P-357, P-358, P-362, P-763

Nacu, Raluca P-791

Naddukkandiyil, Navas P-364

Nadia, Antoniotti P-315

Nadukkandiyil, Navas P-117

Næss, Halvor O-123, P-410

Nagaratnam, K. P-54, P-463

Nagase, Daisuke P-377

Nagel, Gabriele O-1

Nagyova, Beatrix P-485, P-538, P-711, P-786

Naik, Mala P-410

Naja, Nabil P-252, P-487

Najeh, Boussetta P-342, P-379

Nakajima, Tsuneo P-206, P-675

Nakbi, Amel P-384, P-432

Nakling, Arne O-123

Namazi Shabestari, Alireza P-151, P-369, P-370

Nanayakkara, Parabath W. B. P-569

Nani, Carolina O-38

Nano, Dennis P-798

Naourez, Kolsi P-761

Naqvi, Huma P-78

Narančić, Nina Smolej P-272

Narciso, Marco Ribeiro P-245

Narici, Marco P-183

Nassima, Toumi P-420

Natalya, Balyasnikova P-744

Nathalie, Champoux P-70

Nathalie, Denewet P-683

Nathalie, Mitha P-60, P-61, P-62, P-63, P-491

Nathalie, Tesquet P-40

Nathan, Theill P-627

Natori, Kazuhiko P-377

Natsch, Stephanie P-904

Nau, Roland P-633

Naughton, Corina O-87

Naumann, Christina P-394

Nava-del Val, Antonia O-100

Navarro Ceballos, Carmen P-115

Ndonde, Wazir P-755

Nebe, Thomas P-691

Nebo, Vivien P-308

Neerland, Bjørn Erik O-81, P-844

Neilson, Victoria P-269

Neri, Maria Cristina P-315

Nesbakken, Arild P-82

Nesrine, Zaghbi P-46

Nesti, N. P-386

Nestor, G. O-44

Neuburger, J. P-179, P-181

Neumann, Lilli O-29, P-304

Neumann-Podczaska, Agnieszka O-122, P-815

Newton, Julia L. P-312

Newton, Paul P-470

Ng, C. P-188 
Ng, H. M P-76

Ng, P. P-91

$\mathrm{Ng}$, Priscilla P-466

Nguyen, Kim-Huong O-111

Nguyen-Thi, Phi Linh P-493

Nickel, Christian H. P-569

Nicolaou, Mary O-3

Nicolas, Hoertel P-901

Nicolas, Noetzel P-472

Nicolas, Saffon P-883

Nicoleta, Reinald P-901

Nielsen, Dorthe P-170

Nigro, Francesca Maria P-696

Nishihara, Keiji P-458

Nisoli, Enzo O-8

Nitschke, Ina O-54

Nlooto, Manimbulu P-4

Noale, Marianna P-747

Nodimar, Celine P-812

Noémie, Champigny P-764

Nogueres-Rimblas, Carme P-87

Noguerón-García, Alicia P-198

Nomoto, Keiji P-12

Nomura, Toshiya P-23, P-366

Noonan, Claire P-651

Nordheim, Johanna P-640

Nordrehaug, Jan Erik P-632

Nori, Nicole O-116

Norman, Doron P-309

Norman, K. P-135

North, Matthew P-64

Notarelli, Antonella P-502, P-512

Nötzel, Nicolas P-798

Nouhaud, Charlotte P-666

Nour, Guedich P-342

Nour, Guediche P-379

Nourhashemi, Fati O-121

Nouvenne, Antonio O-42

Nowak, Tomasz O-122

Noy, Dani P-471

Nucci, Veronica P-848

Nugteren, Ineke C. P-876

Nuotio, Maria P-646

Nuria Cristina, Herrera-Fernandez P-171

Nuta, C. O-124

Nuta, C. P-357, P-358, P-362, P-763

Nygård, Ottar P-632

Nykänen, Irma P-438

O’Brien, B. P-608

O'Brien, Helen P-651

O’Caoimh, Rónán O-87, P-594, P-619

O'Callaghan, M. P-860

O'Connor, Kieran O-87

O'Connell, Eimear P-597

O'Connor, Eibhlís O-9

O'Connor, Eibhlis M. O-1

O'Donnell, Desmond O-63

O’Donnell, Lisa Kouladjian P-34

O'Herlihy, Eileen O-1

O'Keeffe, Mary O-1, O-9

O'Mahony, B. P-860

O'Mahony, D. P-860

O'Mahony, Denis O-63, P-56

O'Meara, Sarah P-597
O’Neill, Des P-651

O'Neill, Desmond O-93, P-408

O'Philbin, Laura O-87

O'Regan, Niamh O-87

O'Reilly, Susan P-628

O'Shea, Emma O-1

O'Shea, G. P-328, P-215

O'Sullivan, Hugh P-651

O'Toole, Paul W. O-1

Obayashi, Kazuko O-67

Obi, I. T. P-809, P-852

Obi, Ijeoma Tonia P-529

Obukofe, Ross P-405

Ocké, Marga C. P-435

Ogawa, Sumito P-412

Ogita, Mihoko P-190, P-194

Ogrin, Rajna P-500

Öhman, H. P-591

Öhman, Hanna P-589

Oigawa, Hitomi P-416

Oikonomidis, Dimitrios P-740

Okayama, Naoki P-382

Okoli, Unoma P-485, P-786

Okura, Mika P-190, P-194

Olde Rikkert, M.G.M. P-88, P-89

Olfa, Saidi P-751

Olga, Vakulenko P-744

Oliveira Brito, Haissa P-116, P-351, P-444

Oliveira, Agripino P-236, P-242

Oliveira, Diana P-431

Oliveira, J. P-108

Oliveira, Pedro P-236

Oliveira, Renato P-596

Oliveira, Valdiane Dutra P-32

Olivia, Dalleur P-225

Olivier, Dubreuil P-376

Olivier, Guerin O-72, P-118, P-356, P-499, P-590, P-764, P-909

Olof G., Geirsdottir P-440

Ommundsen, Nina P-82

On behalf of Frailtools Team, P-214

Oncul, Ali P-176, P-219, P-247, P-826

Onder, G. P-789

Onder, Graziano O-18, O-59, P-11, P-29, P-139, P-349

Onuchina, Yliya P-26, P-213

Onwuteaka-Philipsen, B.D. P-884

Ooi, Choon Ean O-111

Oosthuizen, Frasia P-4

Opris, S. P-776

Ordoñez Estebañez, Gloria P-346

Ordóñez, Miguel Araújo P-543

Oren, Merve P-775

Orlien Lindskov, Filippa P-155

Orozco, A. P-191

Orso, Francesco O-78, P-559, P-563, P-568, P-697

Ortega Morente, J. M. P-174

Ortega, Raúl Ruiz P-354

Ortolani, Elena O-18

Ortonobes-Roig, Sara P-87

Ostapenko, V.S. P-634, P-635

Ostapenko, Valentina P-26

Östgren, C. J. P-30

Ostovar, Afshin P-151, P-606

Oubaya, Nadia O-107

Oueslati, Hakim P-372

Overall, Andy O-105, P-127

Oviedo Briones, Myriam P-220, P-333, P-334 
Oviedo, Myriam P-204

Oviedo-Briones, Myriam P-669

Owen, Lucy P-872

Owusu-Agyei, Peter P-529

Oyama, Akane P-206, P-675

Ozcan, Münevver P-390

Ozcelik, Duygu P-232

Ozkan, Ozlem Persil P-147

Ozkan, Serkan P-232

Ozkok, Serdar P-447, P-719

Ozsurekci, Cemile P-217, P-390, P-638, P-667, P-808

Özsürekci, Cemile P-663

Oztop, Mucahit P-771, P-792

Ozturk, E. O-51

Öztürk, Yasemin Kılıç P-20

Öztürk, Zeynel Abidin P-178, P-180, P-265, P-452, P-725, P-728,

P-729, P-734, P-750

Ozturk, Zeynel Abidin P-505

Paccalin, Marc P-329, P-575, P-753, P-820

Pagacz, Pierre P-498

Page, Amy P-33

Page, Nigel P-78

Paillaud, Elena O-58, O-107

Pakpahan, Eduwin P-238

Palese, Francesca P-714

Palicio Martínez, C. P-174

Palin, A. P-540

Palomeras Fanegas, Elisabet P-895

Palumbo, E. P-631

Pamoukdjian, Frederic P-871

Panarra, A. P-68

Panarra, António P-557, P-765

Pang, Kuan-Yu O-43

Paoli, Martina P-622

Paolillo, Ciro P-579, P-854

Paracha, Areej O-12, P-90, P-96, P-97

Pardo, Armando P-204

Parekh, Nikesh O-103, O-105, P-48, P-127, P-407

Parigger, Laura P-184

Park, Kyoung Un P-107

Park, Sae Mi P-200

Park, Semi P-428

Parker, Patricia P-257

Parker, S. G. O-44, P-179, P-181

Parlapiano, Silvia O-78, P-559, P-563, P-568

Parnicka, Agnieszka P-204, P-214

Parretti, Luca P-185, P-716

Parro-Martín, M. A. P-95

Partridge, Jamie P-464

Pascal, Jarno P-509

Pascaline, Boudou-Rouquette P-901

Passeri, Giovanni P-811

Patching, Allan O-111

Patel, Ashish P-279, P-898

Patel, Monique P-676

Patricia, Pérez-Rodríguez P-230

Patrick, Cherin P-182

Patrizio, E. O-77

Pattinson, J. P-503

Pattison, Tim P-336

Paul-Emile, Haÿ P-233

Pauline, Anrys P-225, P-504

Pauline, Occeli P-79

Pavić, Tajana P-272
Pawelec, Pawelec P-122

Pawlaczyk, Mariola P-153, P-788

Pazan, Farhad P-10, P-42

Pearce, Ruth M. P-312

Ped P-197

Pedersen, A. P-480, P-482

Pedersen, Amanda O-12, P-90, P-96, P-97

Pedersen, Jette Lindegaard P-453

Pedersen, Preben Ulrich P-453

Pedone, Claudio O-18

Pedrazzoni, Mario P-811, P-819

Pedro Monfort, Clara P-841

Pedroso, Ana O-115, P-331, P-332

Peeterbroeck, J. P-13, P-55

Peinado, Concha P-745

Pelà, Giovanna P-819

Pelà, Maria Giovanna P-805

Peleg, Roni P-780

Pelgröm, Anouk D.A. P-434

Pelligra, Victoria Landaluce P-841

Peltier, Alex P-498

Pendlebury, Sarah T. O-90, O-96, P-564, P-647, P-656, P-682

Pendleton, N. P-620

Penzo, Jacopo P-185, P-716

Perälä, Mia-Maria O-27

Pereira, H. P-721

Peres, Karine P-625

Pereverzev, Anton P-47

Pereverzeva, Anastasia P-47

Pérez Rodríguez, Rodrigo P-333, P-334

Pérez Fernández-Rius, Ana P-197, P-494

Pérez, Encarna Martín P-105

Pérez, Ion Negrete P-718

Perez, Laura Mónica O-18, P-71, P-311, P-622

Pérez-Rodríguez, Rodrígo P-220, P-669

Perez-Zepeda, Mario Ulises O-39, P-145, P-528

Perichon, Renaud P-526, P-539

Perkisas, Stany O-20, P-187

Perret-Guillaume, Christine P-493, P-626, P-671

Perrino, Felice P-819

Persson, Karin O-85, O-86

Perttila, Niko P-589

Pervaiz, Annie P-64

Pessina, Michele P-218

Pessoa, Ana Sofia O-96

Pestana, E. A. P-152

Petermans, Jean P-143, P-160, P-496, P-498

Petermeise, Sophie P-154, P-195, P-448

Petersen, J. O-113, P-737, P-738

Peterson, Gregory P-3

Petrovic, Mirko P-349

Pfaffinger, Ingrid P-140

Pfeiffer, Klaus O-17, P-295, P-299

Pfister, Roman P-799

Philippe, Caillet P-79, P-829

Philippe, Cestac P-40

Philippe, Robert P-590

Philippe, Voyer P-53, P-70

Piasek, Joanna P-510

Piau, Antoine P-496

Picavet, H. S. J. O-57

Piccard, Sebastien P-496, P-498

Pickert, Lena P-570, P-797, P-798

Pieczyńska, Anna P-788

Pienovi, Roberta P-572

Pierre, Cattan P-79 
Pierre, Merville O-98

Pierre-Hugues, Carmichael O-45, P-53, P-70

Pierrick, Bedouch P-491, P-60, P-61, P-62, P-63

Piers, Ruth O-119, P-866

Pietko da Cunha, Daniel P-401

Pigłowska, Małgorzata P-136, P-451

Pijnappels, Mirjam O-28, P-421

Pijnenburg Yolande, A. L. P-389

Pili, Fausto Giordano O-8

Pilnick, A. P-608

Pilot, Alberto P-798

Pilotto, A. P-72, P-833, P-853

Pilotto, Alberto O-70, P-141, P-570, P-572, P-766, P-797, P-799

Pinard, Pauline P-812

Pincher, Bethan P-544

Pinho, Diana Lucia Moura P-32

Piotrowicz, Karolina O-97, P-142, P-205

Pirich, Chrisitan P-140

Pisa, Federica Edith P-14

Pisa, Federica P-714

Pislaru, A. I. O-124, P-357, P-358, P-362, P-763

Pislaru, Anca Iuliana P-397

Pitiyarn, Sutisa P-887

Pitkälä, K. H. P-591

Pitkälä, Kaisu O-6, O-32, O-118, P-589, P-643

Pizzonia, M. P-98, P-335

Pizzonia, Monica O-10, P-100

Plaza Carmona, Laura P-198, P-197, P-494

Plontke, Stefan P-394

Pluderi, Mauro O-38

Pocinho, Margarida P-814

Pohjolainen, Pertti O-27

Poincen P-79

Polidori, Maria Cristina P-280, P-799

PolSenior Study Group P-825

Pomiersky, Rebekka P-295, P-299

Poolman, Jan P-710

Poon, M. H. P-802

Popescu, M. E. P-598

Popović-Grle, Sanja P-875

Poręba, Małgorzata P-373

Poręba, Rafał P-373

Porras-Guerra, Maria Isabel P-605

Porrino, Paola O-8

Porta Gonzalez, Leonel A. P-841

Porter, Kerry P-561

Porto Hormaza, Beatriz P-730

Porto, J. P-518, P-519

Portugal-Nunes, Carlos P-641

Pottegård, A. O-113

Pourhassan, M. P-135

Pouw, Maaike A. P-481

Poydenot, P. P-152

Poyraz, Burc Cagr1 P-623

Prachi, Prachi P-524

Prada, A. G. O-124, P-357, P-358, P-362, P-763

Prada, David P-220, P-669

Prada, G. I. O-124, P-357, P-358, P-362, P-763

Praino, Francesco O-94

Prashchayeu, Kiryl P-736

Pratesi, Alessandra O-78, O-95, P-559, P-563, P-568, P-697

Prati, Beatrice O-42

Predavec, Sanja P-271, P-272, P-273

Prenni, Valentina P-185, P-446, P-703, P-716

Press, Yan P-430, P-483, P-707, P-780, P-781, P-867

Presse, Nancy P-435
Pressel, Eckart P-155

Price, Angeline O-12, P-90, P-96

Prudence, Gibert P-491, P-60, P-61, P-62, P-63

Prud'homm, Joaquim O-47

Pruszczyk, Piotr P-823, P-824

Puente, Pablo Herrero P-548, P-549

Puerta, Letizia Suescun P-250

Puha, Bogdan P-81

PUISIEUX, François P-593

Puisieux, Francois P-202, P-497, P-526, P-539

Pulford, E. C. O-14

Pumares, Maria P-354

Pun, Gajendra P-524

Punchik, Boris P-483, P-707, P-780, P-781, P-867

Puntoni, M. P-833, P-853

Pupo, Simone P-696

Putrino, Luana P-722

Puzianowska-Kuźnicka, Monika O-97, P-815

Quaderi, André P-590

Quaresma, Filipa O-96, P-647

Quigg, Stephanie P-113

Quilliot, Didier P-125

Quintin, Cécile P-674

Quirke, Lyntara O-111

Qureshi Abdulwaris, Mahvesh P-757

Rabes, Laura P-230

Rabia, Boulahssass P-444

Rachel, Rouleau P-53

Rafanelli, Martina O-40, O-96, P-365, P-712

Rahamimov, Irina P-687

Rahiman Kammadath, Abdul P-364

Rainbow, James P-77

Rainey, Conor P-544

Rainey, Katie P-77

Rainfray, Muriel O-107, P-812

Raiteri, R. P-833, P-853

Raivio, M. P-591

Raivio, Minna P-589

Rajcoomar, Nisha P-562

Rajkumar, Chakravarthi O-103, O-105, P-48, P-127, P-407

Rakugi, Hiromi P-206, P-675

Ralf, C. O-33, P-818

Ralhan, S. O-14

Ramadan, Marwan P-117

Ramel, Alfons O-5, P-169, P-209, P-406, P-442, P-462, P-486

Ramon Espinoza, Maria Fernanda P-15, P-199

Ramos, I. P-108

Randles, M. P-860

Ranhoff, Anette Hylen P-395

Ranieri, Piera O-10

Rantanen, Taina O-27

Rapazzini, P. P-98, P-335

Rapazzini, Piero O-10

Rapp, Kilian O-28

Rarek, Marcel P-570, P-797

Rashedi, Vahid P-892

Rastegar, Neguine P-418

Ratnayake, Madhavi P-524

Ratsimbazafy, V. P-38

Ratte-Henn, Susanne P-798

Rauff, Karine Vigestad P-41

Raul, M. P-152

Raux, Mathieu P-84, P-85 
Ravensbergen, W.M. P-317

Ravetta, Claudia O-8

Razzano, M. P-833, P-853

Rea, Jennifer P-449

Redling, Theresa P-595

Redondo-Sánchez, Juana P-424, P-425

Refaee, Mahmoud P-560

Refsum, Helga P-632

Reginster, Jean-Yves O-24, P-143, P-160, P-161

Regnersgaard, Signe P-155

Regula, Gmünder P-627

Rehem, Tania Cristina S. B. P-32

Rehman, Joe O-76

Reichardt, L. A. P-340

Reijnierse, Esmee P-162

Reilev, M. O-113

Reinald, Nicoleta O-107

Reinold, Jonas O-109, P-14, P-28

Reiss, Jens P-140

Religa, Dorota P-657

Remy, Collomp P-499

Renom, Anna P-296, P-610

Revnic, Cristian Romeo P-326, P-558, P-653

Revnic, Floarea P-326, P-558, P-653

Rexach, José Antonio Serra P-467

Rezgui, Lamia P-757

Riad, Nermine P-381, P-383

Riadh, Batikh P-378

Ribera, Ivon P-779

Riboux, Sophie-Charlotte P-573

Ricevuti, Giovanni O-69, P-283, P-579

Richard, M. P-542

Rico, Leonor P-207

Rico-Martin, Sergio P-437, P-456

Riedel, Oliver P-28

Riedl, Regina P-83

Riganti, Chiara O-8

Riley, Katie P-464

Rim, Abid P-378

Rinonapoli, Giuseppe P-185, P-716

Riou, Bruno P-84, P-85

Riso, P. P-130

Rispoli, Vincenzo O-94

Rivadeneira, Fernando P-25

Rivasi, G. P-386

Rivasi, Giulia O-40, P-244, P-712, P-365

Rivas-Ruiz, Francisco O-100

Rivera, Carlos Amorós P-832

Rizzoli, René P-160

Rizzuto, D. P-177

Robert Johnson on behalf of the ZOE-50/70 study group, P-110

Robert, Laurine P-526, P-539

Roberts, H. C. P-179

Roberts, Helen C. P-149, P-433

Roberts, Helen P-511

Robertson, K. P-609

Robinson, A. L. P-181

Robinson, David O-66

Robinson, Lisa J. P-312

Robinson, S. P-215

Robinson, Sian P-433, P-460

Robinson, Stephanie P-793

Robles, María José P-94

Robles, María Verónica P-622

Robles, Rocío Molás P-680

Robson, Leonie P-33
Rocco Villani, Emanuele O-59

Rodenas, Isabel Huedo O-22

Rodrigo, Ivan Anton P-199

Rodrigues, Belina P-641

Rodríguez Mañas, Leocadio P-333, P-334

Rodríguez Rosa, Antonio P-99, P-105

Rodriguez, Ana P-237

Rodríguez, Jakobe Pisón P-718

Rodríguez, Javier Jacob P-548, P-549

Rodriguez, MLuz P-296, P-610

Rodríguez-García, WD P-187

Rodríguez-Mañas, Leocadio O-22, P-139, P-214, P-220, P-669

Rodríguez-Sánchez, Isabel P-227

Rogers, Anne P-460, P-511

Roitto, H. M. P-254, P-591

Rolland, Yves O-121, P-160

Roller-Wirnsberger, Regina P-139

Rollot-Trad, Florence P-871

Rollwagen, Lena P-633

Rolph, Giles P-186

Romain, Bosc P-829

Romain, Coriat P-376

Roman-Sicilia, Heriberto P-145

Romero Carrete, C. J. P-754

Romero, Luis O-22

Romero-Saldaña, M. P-424, P-425

Ronderos, Rocio Garcia P-346

Roqué, Marta P-622

Roqueta, Cristina P-343, P-350, P-359

Rosada, Adrian P-280

Rosenborg, Annica P-834

Rosendahl, Erik P-621

Rosholm, Jens-Ulrik P-17, P-571

Rosner, R. P-645

Rossi, Andrea P. O-116

Rossi, Emanuela P-854

Rossi, Paolo Dionigi O-38, P-393

Rossi, Pascal M. P-125

Rossignol, Patrick O-106

Rostagno, C. P-98

Rostagno, Carlo P-244

Rostoft, Siri P-82, P-355

Roszak, Magdalena P-153

Rotariu, Cristian P-489

Roth, Anja P-18

Rothenbacher, Dietrich O-28, P-49

Rothwell, Peter M. O-90, P-682

Rotman, Johanna P-430

Rottenkolber, Marietta P-154, P-195

Rotteveel, Ilse P-164

Roubaud Baudron, Claire P-625

Roubaud, Claire P-575, P-753

Roubaud-Baudron, Claire P-513, P-573

Rousselot, Hubert O-107, P-493

Rowley, Mollie O-12, P-90, P-96, P-97

Royle, Kimberley P-810

Royles, C. P-188

Rozeta, Draghici P-380

Rozing, Maarten O-104

Ruengsinpinya, Ploy P-228

Ruth, Paz-Maya P-171

Rubele, Sofia O-116

Rudaityte, Gertruda P-461

Rudolf, Schelling Hans P-627

Rüffert, Danny P-387

Ruggiero, Carmelinda P-185, P-446, P-703, P-716 
Ruiz Hidalgo, D. P-754

Runikhina, N. K. P-634, P-635

Runikhina, Nadezda P-26, P-47, P-743

Rutten, Jeanine P-904

Ryan, Dan P-651

Ryan, E. P-525

Ryg, Jesper J. P-41, P-167, P-170, P-391, P-571

Sa P-563

Sá, R. P-108

Saad, Robert P-441, P-787

Saber, Sadia P-545, P-849, P-850

Sabin, Irina P-471

Sabine, Drevet P-109

Sabrina, Maniewicz P-648

Sacco, Flavia Carla P-712

Sá-Couto, Pedro P-222, P-223

Sáez López, Pilar P-105

Sáez, Enrique Pérez P-680

Sáez-Nieto, Carmen P-230

Saffon, Nicolas O-121

Saglia, Jody O-70

Sagnier, Marie-Christine P-67

Saguez, Rodrigo P-239

Sahin, Kezban Ulku P-638

Şahin, Sevnaz P-20

Sahin, Sevnaz P-506, P-773

Sahutoglu, sercan P-773

Saïd, Fatma P-372

Saiz, Neus P-221

Sajko, Dalma P-271, P-273

Saka, B. P-254

Saka, Bülent P-455

Saka, Sule Ajibola P-4

Saks, K. P-777

Sala, Jacopo P-283

Salazar, Jaime Rodríguez P-115

Saleh, Haroon P-560

Salem, Thouraya Ben P-731, P-748

Salgueiro, F. P-721

Salisbury, Helen O-50

Salmenius-Suominen, Heli P-643

Salomon, L. P-542

Salonen, Minna K. O-27

Saltvedt, Ingvild O-85, O-86, P-844

Salvador, Pedro P-236, P-242

Sameh, Sayhi P-342, P-378, P-379

Sammon, Tonya P-582

Sammut, Christopher P-417

Sampietro, Lorenzo P-807

Samson, Tali P-867

Samuelsson, O. P-777

Samuelsson, Olafur P-501

Samul, Judyta P-823, P-824

Sanche, Elisabeth P-142

Sanchez, Hugo P-679

Sanchéz, Juan Luis Sanchéz P-199

Sánchez-Carpintero, Andrea P-343, P-350

Sánchez-Castellano, C. P-243

Sanchez-Castellano, C. P-95

Sánchez-González, Benedicta P-494

Sánchez-Jurado, Pedro O-22

Sanchez-Rodriguez, Dolores P-156

Sandra, Higuet P-683

Sandra, Lind P-65
Sandra, Mavoungou P-274

Sandrine, Roussel P-504

Sandu, I. A. O-124, P-357, P-358, P-362, P-763

Sandu, Ioana Alexandra P-81, P-93, P-450

Sanfilippo, Carlo O-70

Sanna-Maria, Hongisto P-445

Santano Mogena, Esperanza P-456, P-437

Santolaria, Javier P-845, P-846

Santos Gutiérrez, A. P-754

Santos, Ana Lopes O-115

Santos, Lèlita P-534

Santos, Luis María García P-661, P-841, P-847

Santos, Nadine Correia P-641

Santos, Rui M. P-692

Santos-Eggimmann, Brigitte P-260

Saoussen, Zrour P-360, P-361, P-761

Sappa, Matteo P-58

Sapru, Sunil P-520

Sarac, Fulden P-231, P-443, P-756

Sarac, Z. Fulden P-773

Saraceni, Vincenzo Maria P-248

Sarah, Hilmer O-45

Saraiva da Cunha, J. P-108

Sardella, Alberto P-226

Sarduy Azcoaga, Iraide P-718

Sargent, Samantha P-561

Sarıpınarlı, Begüm P-285

Sartori, Arianna P-714

Sarycheva, Tatyana P-604

Sasaki, Yuki O-89

Saskia, Kruiskamp P-165, P-182

Sassi, Francesca O-8

Satake, Shosuke O-60, P-12, P-196, P-458, P-881

Sathanandan, Krishanthi P-545, P-849, P-850

Sato, Misaki P-382

Satu, Jyväkorpi P-445

Sauce, Delphine P-129

Saucedo-Figueredo, Carmen O-100

Savas, Sumru P-231, P-232, P-443, P-505, P-756

Savci, Sercan P-447

Savikko, N. P-591

Savikko, Nina P-589

Savva, George P-613

Sawadogo, Lynda P-371

Saxton, N. P-256

Sayer, A. O-44

Sayer, Avan A. P-149

Sayer, Avan Aihie P-511

Sayer, Avan P-238, P-240

Sayiner, Zeynel Abidin P-178

Scaglia, L. P-631

Scamosci, Michela P-393

Schaap, L. A. O-57

Schadegg-Peña, Daniel P-86

Schäufele, Martina P-295, P-299

Schellevis, Francois O-114, P-43

Schena, Chiara P-686

Scherr, Franziska P-723

Schiff, Rebekah O-103, P-48, P-407

Schimberg, A.S. P-817

Schink, Tania O-109, P-14

Schmidmaier, Ralf P-195

Schmidt, Laura P-803, P-806

Schmidt, Pia P-469

Schmidt, T. O-33

Schneider, Andrea P-448 
Schober, Daniela P-413

Schoenenberger, Andreas P-831

Schofield, Patricia P-588

Schönfeldt-Lecuona, Carlos P-349

Schoon, Yvonne P-569

Schoser, Benedikt O-23

Schuit, Stephanie P-585, P-837

Schülein, Samuel P-325

Schulz, Ralf P-797

Schumacher, Axel P-556

Schütze, Sandra P-633

Schuurmans, E. P. P-88, P-89

Schvartzman, Paulo R. P-762

Schwertner, Emilia P-657

Scutt, Greg O-105, P-127, P-407

Sebastian, Voigt-Radloff O-19

Sébastien, Czernichow O-25

Sebastien, Gonfrier O-72, P-909

Secnik, Juraj P-657

Seco, Maria Alonso P-841

Seibert, Moritz P-349

Seiger, A. O-21

Seiger, Åke P-201

Seißler, Jochen P-154

Sekeroz, Serbay P-792

Selbæk, Geir O-85, O-86

Selem, Thouraya Ben P-46

Selte, Canan P-154

Selvarajah, Sujitha P-268

Selvi Öztorun, Hande P-168, P-782

Selvi Oztorun, Hande P-352, P-353, P-367

Semenov, Anna P-483

Senda, Kazuyoshi O-60, P-196, P-868

Senesi, Barbara O-70

Sengul Aycicek, Gozde P-390, P-619, P-638

Şengül Ayçiçek, Gözde P-663, P-720, P-894

Sengul Aycicek, Gözde P-808

Senneville, Eric P-513

Sepyani, Alireza P-151

Seppälä, Lotta P-25

Sequeira, Mafalda O-115, P-331, P-332

Serap İnal, H. P-285

Sérézal, Irène Gallais P-345

Servais, Alexandre P-539

Sette, Stefania P-435

Settembrini, Vincenzo O-94

Seven, Umran P-667

Severine, Henrard P-225

Sevilla, Eulalia P-296, P-610

Sevillano, Arturo P-307

Sezgin, Gulbuz P-505

Sfikakis, P. P. O-53

Sgrilli, Federica P-502, P-512

Shabanzadeh, Alireza P-369

Shaffeiee, Gita P-151, P-606

Shafiq, Shahriar P-849, P-850

Shah, Devesh P-545

Shah, K. O-14

Shakersain, Behnaz O-2

Shanagher, Deirdre P-685

Sharashkina, N. V. P-634, P-635

Sharashkina, Natalia P-26, P-743

Sharifi, Farshd P-151, P-606

Sharma, Jagdish P-676

Shchedrina, Anna P-743

Sheriff, Shahul P-767
Shevchenko, Daniella P-47

Shibuya, Akiko P-377

Shimizu, Atsuya P-12

Shimokata, Hiroshi O-60

Shioiri, Hiroki O-37

Shipway, David P-77

Sialino, Lena D. O-57

Sidhu, G. K. P-532

Sieber, Cornel C. P-821

Sieber, Cornel Christian O-3

Siegfried, Negatsch P-627

Siggeirsdottir, Kristin P-249

Sigurdardottir, Sigurveig H. P-486

Sigurdsson, Gunnar P-249

Silva, C. P-518, P-519

Silva, Carlos D. P-692

Silva, Isabel P-765

Silva, Marco P-813, P-814

Silva-Cardoso, José P-235

Simão, Adélia P-431

Simon, Pernot P-376

Simona, Opris P-380

Simone, Brefka O-19

Simonen, Mika O-27

Simsek, S. P-598

Singh, Raunak P-279, P-898

Singler, K. P-254

Singler, Katrin P-778

Siri, G. P-833

Siri, Giacomo P-797

Skalska, Anna O-97, P-205, P-825

Škarić-Jurić, Tatjana P-272

Skelton, Dawn A. P-388

Školoudík, David P-600

Skovlund, Eva O-85, P-82

Slankamenac, Ksenija P-533

Slema, Fathi Ben P-751

Slingerland, M. P-817

Sloane, Philip O-46, P-904

Sluggett, Janet O-111, P-29, P-33

Slusarczyk, Przemyslaw P-903

Smalbrugge, Martin P-904

Smidt, Nynke P-52, P-481

Smith, Karen P-336

Smith, Lee P-166

Smith, Michael O-49

Smith, Sarah O-96, P-647

Smithard, David G. P-882

Smyth, Hannah P-651

Snijder, Marieke B. O-3

Sobieszczańska, Małgorzata P-373, P-727

Socha, Krzysztof P-796

Socorro, Alberto P-779

Söderström, Hugo P-657

Sofia Pessoa, Ana P-647

Solano, Antonia P-745

Soledad Álamo, S. P-204

Soliman, Kamal O-65

Solmi, Marco P-588

Solórzano-Santos, F. P-583

Sołtysik, Bartłomiej P-136, P-398, P-796

Somme, Dominique O-47

Song, Kyoung-Ho P-107

Sønnesyn, Hogne O-123

Sophie Denise Otter, Anne P-718

Sophie, Boyer P-229 
Soraghan, Christopher O-66

Soraya, Qassemi P-40

Sørensen, Gustav Valentin Blichfeldt P-391

Soriano, Pere LLorens P-548, P-549

Sorina Maria, Aurelian P-749

Sosa, Raquel P-307

Šostar, Zvonimir P-271, P-272, P-273

Soto, Luis P-622

Soubeyran, Pierre O-107

Souissi, Marwa P-698

Soulis, G. O-53

Soulis, George P-740, P-758

Sourdet, Sandrine O-121

Sousa, Débora P-116

Sousa, Nuno P-641

Soylu, Gülçimen P-189, P-728, P-729, P-734, P-750

Soysal, Pinar P-133, P-436, P-588, P-830, P-891

Soytas, Rabia Bag P-447, P-719

Spadea, Fausto O-94, P-722

Spallarossa, Franca P-807

Spanjers, Kiki P-624

Spinewine, Anne P-746

Spitz, Elisabeth P-626

Spriet, Isabel P-21, P-22

Springall, Nicholas O-12, P-90, P-96, P-97

Sproesser, Gudrun O-3

Squeri, Valentina O-70

Srinonprasert, Varalak P-886, P-887

Sriram, Krishnan P-464

Stachnik, Katarzyna P-510

Staley, Jessica P-257

Stam, F. P-598

Stannard, G. P-344

Starmans, Tom, P-715

Stefan, Dazert P-617

Stefan, Mehaffey O-23

Stefani, Ilario P-251

Stefaniu, R. O-124, P-357, P-358, P-763

Stefaniu, Ramona P-80, P-93, P-450

Stefanoiu, R. P-362

Steffens, Michael P-49

Steffl, Michal P-681

Steingrimsdottir, Laufey P-249

Steinmetz, Jean-Paul P-418

Stella, M. P-833, P-853

Stenveld, Fiona P-52

Stephens, Christopher P-145

Šter, Marija Petek P-726

Stevenson, Jennifer O-103, P-48, P-407

Steves, Claire J. P-465

Steyer, Elisabeth P-556

Stingl, Julia C. P-49

Stival, Marina Morato P-32

Stokes, C. L. P-59

Stokes, Maria P-150

Stompór, Małgorzata P-772

Stompór, Tomasz P-705, P-772

Stortecky, Stefan P-831

Strandberg, T.E P-591

Strandberg, Timo O-6, O-30, O-32, P-589

Strauß, Thomas P-821

Strauven, Goedele P-746

Street, Joe P-419

Streicher, Melanie O-1, O-9

Streppel, Martinet T. P-164

Stricker, Bruno P-25
Strike, Gayle P-511

Strotzka, Stefan P-790

Stubbs, Brendon P-141, P-166, P-588

Stuck, Andreas P-831

Stuffken, R. P-838

Stukas, Rimantas P-337, P-339

Stuurman, Anke P-710

Styszyński, Arkadiusz O-122, P-815

Su, S. P-76, P-91, P-466, P-616

Suarez, Eva Martinez P-895

Suárez, Pablo Enrique Solla P-66, P-281, P-282

Subba, Karuna P-529

Subramonian, Santosh P-529

Suchankova, Hana P-27

Sugimoto, Ken P-206, P-675

Sullivan-Solte, Julia O. P-640

Sulmont-Rossé, Claire O-1

Sulo, Suela P-464

Sumaiyah, M. O-7

Sumaway, Jonathan P-560

Sumer, Fatih P-216, P-217, P-390, P-638, P-808

Sümer, Fatih P-663

Sunwar, Nisha P-676

Suominen, M. P-643

Suppiah, Malathi P-288

Suraarunsumrit, Patumporn P-887

Surkiene, Gene P-339

Sürmeli, Deniz Mut P-168, P-367, P-782

Surmeli, Deniz Mut P-352, P-353

Surmeli, Zeki Gokhan P-352

Surquin, Murielle P-156

Svensson, Olle O-84

Svetlana, Topolyanskaya P-744

Swagerty, D. L. P-862

Swart, K. M. A. P-422

Sweed, Hala P-381, P-383

Swingewood, Nathan P-75

Syamala, Shirmila P-560

Syrmos, Nikolaos P-399, P-724

Szybalska, Aleksandra O-97, P-815, P-903

Szymanska, Monika P-824

Tabish Zaidi, Syed P-3

Tadros, George P-899

Tagliaferri, Sara P-183, P-218, P-805, P-811, P-819

Taglini, Alessandra P-885, P-888

Taha, Hind P-381, P-383

Tahmi, Fella P-857

Taipale, Heidi O-112, P-29, P-74, P-604, P-611, P-612, P-642, P-665

Tait, Z. Fulden P-344

Takada, Shuko P-206

Takahashi, Kyo P-159

Takahashi, Paul P-113

Takahashi, Yuhka O-34

Takami, Yoichi P-206

Takami, Youichi P-675

Takanashi, Sanae P-881

Takasou, Marin P-889

Takeda, Shuko P-675

Takeya, Miyuki P-206

Takeya, Yasushi P-206, P-675

Takx Richard, RAP P-402

Talme, Toomas P-345

Tamburini, Paola P-816

Tamulaitiene, Marija P-224, P-337, P-339 
Tamulaitienè, Marija P-423

Tamulaityte-Morozoviené, Inga P-423

Tan, A. W. B. P-532

Tan, Bryan P-685

Tan, Chew Sia P-466

Tan, Edwin P-29, P-33

Tan, G. J. O-7

Tan, M. P. O-7

Tan, Wallace O-76

Tana, Claudio O-42

Tanaka, Tomoki P-159

Tanganelli, Fabiana O-23

Tanskanen, Antti O-112, P-29, P-74, P-604, P-611, P-612, P-642, P-665

Tao, Xuefei P-163

Tapanainen, Heli P-435

Tapianen, Vesa P-665

Tarazona-Santabalbina, F. J. P-254, P-455

Tasci, Ilker P-505

Taşkın, Harun P-306

Tassitro, Elena O-10

Tasso, Emilia P-278, P-807

Tatis, Myriel López P-832

Tatyana, Eliseeva P-744

Tavares, João P-222, P-223, P-330

Taxis, Katja O-61, P-34

Tay, L. P-134

Taylor, C. P-256

Taylor, M. P-338

Tchalla, A. P-38

Ted, Malmstrom P-138

Teh, Ruth O-1

Tell, Grethe S. P-632

Temnenko, Natalia P-47

Teodoro, Tomás P-596

ter Maaten, J. C. P-530

ter Maaten, Jan C. P-481

Teresa Vilarmau-Dolcet, $\mathrm{M}^{\mathrm{a}}$. P-87

Terranova, Claudio P-747

Tesfaye, Wubshet P-3

Tesi, Francesca P-712, P-855

Tessari, Annalisa P-599

Tewari, Reena P-660

Then, Cornelia P-154

Thierry, Dantoine P-229, P-420

Thierry, Fosse P-356

Thietart, Sara P-84, P-85

Thomas, Benzing P-472

Thomas, Cario P-509

Thomas, Caroline O-79

Thomas, Gaertner P-693

Thompson, S. P-344

Thompson, Sanja O-50, P-717, P-785, P-875

Thorand, Barbara P-154, P-448

Thomson, William P-405

Thorsdottir, Inga O-5, P-209, P-406, P-442, P-462

Throm, Christina P-10

Tichelaar, J. O-51

Ticinesi, Andrea O-42, P-183

Tieland, Michael P-164

Tiihonen, Jari O-112, P-74, P-604, P-611, P-612, P-642, P-665

Tiihonen, Miia P-438

Tillemans, M. P. H. P-838

Tilvis, Reijo P-589

Timmons, Suzanne O-1, P-417, P-597, P-685

Tinarelli, Clara P-185
Tingley, Danielle P-735

Tiskute, Vaiva P-461

Tkacheva, O. N. P-634, P-635, P-636

Tkacheva, Olga P-5, P-26, P-47, P-120, P-690, P-743

Toba, Kenji O-60, P-159

Tobis, Slawomir P-510

Todd, Chris O-28

Tokuda, Haruhiko P-196

Tolppanen, Anna-Maija O-112, P-611, P-612, P-642

Tomasović-Mrčela, Nada P-271, P-273

Tomasz Gr, Tomasz P-204

Tomek-Roksandić, Spomenka P-271, P-272, P-490, P-273

Tomeo, Charlotte O-13, P-859

Tomioka, Tomoko O-37

Tomiyama, Naoki P-400

Tonarelli, Francesco O-95

Tonon, Michele P-747

Topinková, Eva O-24

Topinkova, Eva O-59

Torbahn, Gabriel O-1, O-9, P-241, P-349, P-821

Toro, Pablo P-629, P-630

Torregrosa, Roberto Petidier P-199

Torrents, Nuria P-311

Torres Bonafonte, O. H. P-754

Torres, M. T. P-68

Torres, Margarida P-557

Torriglia, D. P-833

Torrini, Monica P-855

Tortù, V. P-386

Tortù, Virginia O-40, P-244

Torvi, Anneli P-643

Toscano-Rico, Miguel P-156

Tosun, Pinar P-505

Toubal, S. P-542

Toupance, Simon O-106, P-125, P-126

Tournigand, Christophe P-871

Tournoy, Jos P-21, P-22, P-294

Touzi, Mongi P-360, P-761

Tozzetti, Camilla O-78

Trajanoska, Kate P-25

Trappenburg, Marijke P-162

Trevas, Sara O-115

Trevas, Sara P-331, P-332

Tricerri, Francesca P-572

Tristan, Cudennec P-79

Trompet, Stella O-104

Trushkova, Ekaterina P-684

Trushkova, Nina P-684

Trzmiel, Tomasz P-153, P-788

Trzmielewska, Ewa P-373

Tsabar, Nir P-430

Tsartsara, Stella I. P-514

Tsekoura, Maria O-24

Tsirulnicov, Tatyana P-284

Tsuda, Yukimoto P-366

Tubach, Florence P-536

Tuck, Julie P-561

Tufan, Asli P-505, P-768

Tuna Dogrul, Rana O-41

Tung, Ericka P-113

Turan Isik, Ahmet P-133, P-436, P-830, P-891

Turgut, Tuğba P-168, P-782

Turgut, Tugba P-352, P-353, P-367

Turin, Jimmy Martin Gonzales O-101

Türkbeyler, İbrahim Halil P-452

Turkbeyler, Ibrahim Halil P-725 
Turkmen, Banu Ozulu P-468, P-827, P-896

Turnbull, Douglass P-240

Turner, Nicola P-711

Turrin, G. P-386

Turrin, Giada O-40

Turrini, Aida P-435

Tuttle, Camilla P-121, P-123

Tuukkanen, Kristiina P-643

Twisk, J. W. R P-340

Tzafestas, C. S. O-68

Tzanetakou, Irini P-125, P-740, P-758

Udina, Cristina P-311, P-622

Ueda, Mitsuharu P-366

Ueland, Per Magne P-632

Uitterlinden, A.G. P-422

Uitterlinden, Andre P-25

Ulbrich, Lisa P-799

Ullrich, Phoebe O-16, O-56, P-302, P-310, P-900

Umeda, Tomohiro P-416

Umeki, Haruhi P-382

Ünalan, Pemra P-768

Ungar, A. P-98, P-335, P-386

Ungar, Andrea O-10, O-40, O-78, O-95, P-244, P-365, P-563, P-579,

P-697, P-712, P-784, P-799, P-848

Unneby, Anna O-84

Unsal, Pelin P-638, P-667

Ünsal, Pelin P-663, P-720

Urbano, Sandra P-350, P-359

Urbiete, Lina P-479

Urien, Maria P-341

Uriz Otano, Francisco P-250

Urquiza, Miriam P-237, P-828

Urtamo, Annele O-6, O-30, O-32

USAREL, Cansu P-891

Ushkalova, Elena P-47

Utiel, Melisa López O-22

Uzun, Özge P-263

Vaartjes, Ilonca P-602

Vahabi, Zahra P-151, P-369

Vaillant Ciszewicz, Anne-Julie P-590

Vainshtein-Tal, Maya P-430

Vaio, Teresa P-692

Valappil, Sameer Acharath P-364

Valdés-Aragonés, Myriam P-220, P-669

Valdivieso, Esteban Toral P-548, P-549

Valdiviesso, Rui P-210, P-235

Valecchi, Rita P-446

Valente, Debora P-765

Valentini, V. P-789

Valeria, Buttò P-183

Vališ, Martin P-600

Valle Calonge, Elena P-281, P-282, P-346

Vallet, Helane P-857

Vallet, Hélène O-79, P-129

Vallone, Francesco O-70

Valoti, Paolo P-365

Valverde, Paula Vicent P-661

van Agtmael, M.A. O-51

van Ancum, Jeanine M. P-421

van Asselt, Dieneke O-52

van Baarle, F.E.H.P. P-522

van Balen, R. P-320, P-317

van Balen, Romke P-291 van Bruchem-Visser, Rozemarijn P-878

van Buul, L. W. P-554

van Buul, Laura P-904

van Campen, J.P. P-404

van Campen, Jos O-61

van Dam van Isselt, Eléonore F. P-286, P-298

van de Pol, Alma P-906

van de Pol, Marjolein O-52

van de Vorst, Irene P-602

van den Berg, A. A. P-530

van den Berg, Claudia P-318

van den Berg, E. P-530

van den Hanenberg, F. O-51

van den Helder, Jantine P-164

van den Ingh, L. M. O-82, P-639

Van den Noortgate, Nele O-119, P-156, P-866

Van der Cammen, Tischa O-103, P-407, P-495

Van der Esch, Martin P-340

Van der Linden, Lorenz P-21, P-22

van der Putten, Gert-Jan P-324

van der Roest, Henriëtte O-59

Van der Schaaf, Joerg P-693

Van der Schaaf, M. P-340

van der Sijp, M. P. L. P-317

van der Steen, Jenny O-87

van der Velde, Marije P-569

van der Velde, N. P-404

van der Velde, Nathalie P-25

van der Velden, L. P-817

Van der Wardt, V. P-601, P-609, P-614

Van der Wardt, Veronika P-414, P-650

van der Wouden, Hans P-904

van der Wouden, JC P-320

van der, T. J. M. P-422

van Deudekom, F. J. P-817

van Dijk, Gert P-878

van Dijk, Liset O-114

van Dijk, Margaretha P-294

van Dijk, S. C. P-422

van Dronkelaar, Carliene P-164

van Eijk, M. P-317

van Eijk, Monica P-286

Van Emden, Jan O-111

van Goor, H. O-51

van Haastrecht, M. O-51

van Haelst, S. A. E. P-404

van Hout, Hein P-11

Van Humbeeck, Liesbeth O-119, P-866

van Iersel, Marianne O-52

Van Lerberge, Eva P-708

van Marum, Rob P-11

Van Mieghem, Eugénie O-20

van Mieghem, N. M. P-72

van Munster, B. C. P-530

van Munster, Barbara C. P-481, P-52

van Oostrom, S. H. O-57

van Oppen, James D. P-569

van Rijn, Marjon P-876, P-877

van Schoor, N. M. P-422

van Schoor, Natasja M. O-28

van Schoor, Natasja P-25

Van Seben, Rosanne P-340

van Tol, Lisa S. P-569

van Tulder, M. W. P-554

van Wijngaarden, J. P. P-422

van Wijngaarden, Jan P-298 
van Wijngaarden, Janneke P-318

Van Wymelbeke, Virginie O-1

van Zwienen-Pot, Judith O-1

Vançon, Guy P-234

Vandael, Eline P-746

VanDerBosch, Gretchen P-464

Vandevijver, Celine P-708

Vandewoude, Maurits O-20, P-187

VanDiepen, Erik P-898

Vanina, Bongard P-883

Vankova, Hana P-128

Vankova, Marketa P-128

Vannacci, A. P-386

Vanni, Valerio P-365

VanSwearingen, Jessie P-622

Vaquerizo, Beatriz P-343, P-350

Vaquero-Pinto, M.N. P-243

Varan, Hacer Doğan O-41

Varan, Hacer Dogan P-216, P-219, P-247, P-619

Varli, Murat P-168, P-352, P-353, P-367, P-505, P-607, P-782

Varman, Surendra Doraiswamy P-532

Varnier, Gwénaëlle P-871

Vasishta, Sanjeev P-767

Vassallo, M. A. P-313, P-777

Vassilev, Ivaylo P-460

Vaubourdolle, Michel P-457

Vazquez, Olga P-296, P-610

Vázquez, Olga P-94, P-343, P-348, P-350

Vedeler, Christian A. P-632

Veenhuizen, R. B. P-554, P-884

Veenhuizen, Ruth P-904

Veerle, Foulon P-225, P-504

Velasco, Rocío P-800

Veliceasa, Bogdan P-81

Vella Azzopardi, Roberta P-37

Vella Seychell, Elaine P-37

Vella, Antoine P-37, P-488

Vella, Antoine P-6

Vella, Robert P-212

Vella-Brincat, Jane P-582

Velniceasa, Bogdan P-80

Velonika, V. S. P-777

Venegas-Sanabria, Luis-Carlos P-197

Veninsek, G. P-777

Ventura, Maria Teresa P-574

Venturini, Letizia O-69

Verdejo, Carlos P-307, P-605

Vereijken, Beatrix O-28

Vergara, Itziar O-100, P-207

Verhaak, Peter O-114, P-43

Verheij, T. J. M. P-554

Verheij, Theo P-906

Verhey Frans, R. J. P-389

Verheyden, Geert P-294

Veríssimo, M. T. P-108, P-518, P-519

Veríssimo, Manuel P-330, P-431, P-534, P-813, P-814

Veríssimo, Manuel T. P-692

Veríssimo, Rafaela P-236

Verissimo, Rafaela P-242

Verlohr, Dagmar P-790

Vermeiren, Sofie P-212

Vernocchi, Simonetta P-686

Verny, Marc O-13, P-84, P-85, P-859

Veronese, Nicola P-141, P-166, P-588, P-599, P-766

Veronique, Bigorie P-829

Véronique, Mailland-Putegnat P-764
Veronique, Mondain P-118, P-356, P-499

Verreijen, Amely M. P-164

Verschuren, W. M. M. O-57

Verschuur, E. M. L. P-884

Verstraeten, Thomas P-710

Verté, Dominique P-212

Vertrano, Davide P-29

Vetrano, D. L. P-177

Vetrano, Davide O-43

Vetrano, Davide Liborio O-59

Vettasseri, M. Z. P-503, P-659

Vettoretti, S. P-804

Vezza, C. P-804

Vicari, Stefano P-747

Vidal, Flávia Castello Branco P-116

Vidal, MAngels P-296, P-610

Vidal, Vanessa Álvarez P-114

Viitanen, Matti P-646

Vijayakumar, B. P-851

Vikertaite, Egle P-337

Vikström, S. O-21

Vikström, Sofia P-201

Viktoria, Mühlbauer O-19

Vilches-Moraga, A. P-480, P-482

Vilches-Moraga, Arturo O-12, P-90, P-96, P-97, P-336, P-507

Villain, Cedric O-13, P-859

Villain, Cédric P-84, P-85

Villani, E. R. P-789

Villareal, Juan Jose P-237

Villemonais, Denis P-126

Viñas, María Piar López P-99, P-105

Virtanen, Suvi M. P-435

Visser, M. O-57

Visser, Marjolein O-1, O-3, O-9, O-108, P-164, P-434

Vistnes, Maria O-86

Visvanathan, Renuka P-33

Vitali, FRANCESCA P-278

VITALI, Francesca P-807

Vitkus, Dovydas P-423

Vivian, Lilian P-762

Voigt-Antons, Jan P-640

Voigt-Radloff, Sebastian P-241, P-349

Voinea, Silviu P-326, P-558, P-653

Volkert, Dorothee O-1, O-108, O-3, P-821

Volpato, S. P-335, P-98

Volpe, Cris Renata Grou P-32

von Arnim, Christine A. F. P-349

von Bonsdorff, Mikaela O-30

von Bonsdorff, Mikaela B. O-27

von Campe, Hugo P-625

von Renteln-Kruse, Wolfgang O-29

Vos, Marianne P-715

Voshaar, Richard Oude O-114, P-43

Vrancken, Jerome P-498

Vreeswijk, R. P-838

Vrotsou, Kalliopi O-100, P-207

Vyse, Adriana P-836

Waaijer, Mariette P-122, P-123

Wada, Tadashi P-881

Walgers, J. J. P-598

Walgraeve, Karolien P-21, P-22

Walter, Grit P-394

Walter, Stefan P-204, P-220, P-333, P-334, P-669

Walters, Chasity P-257 
Walters, Kate P-175, P-449

Wan, Bettina P-541

Wan, G. P-134

Wang, Dan P-163

Wang, Hui Xin O-43, P-615

Wang-Hansen, Marte Sofie P-844

Ward, Kimberly O-46

Warren, Charlotte P-240

Wastesson, Jonas O-120, P-29, P-385

Watała, Cezary P-398

Watanabe, Hidehiro P-382

Watanabe, Masanori O-50, P-717

Watne, Leiv Otto O-81

Wattel, L. M. P-320

Watters, Hazel P-73

Wautrecht, J. C. P-55

Wearing, Julia P-150

Wegener, Martina P-403

Wehbe, Amine P-261, P-441, P-787

Wehling, Martin P-10, P-42

Wei, Peter J.M. P-164

Weiss, Christel P-10, P-42

Weiß, Stefanie P-195

Welch, Lindsay P-460

Wells, Adrian P-717

Wells, P. P-614

Welsh, Tomas P-268, P-269, P-654, P-735

Wenaweser, Peter P-831

Wentworth, L. P-188, P-540

Wentworth, Lauren P-293, P-419

Werle, Berenice M. P-762

Werner, C. O-68, P-645

Werner, Christian O-16, P-301, P-310, P-319, P-637

Wernham, Cathy P-735

Westendorp, Rudi O-104

Westendorp, Rudi P-122, P-123

Wester, W. P-31

Westerlind, B. P-30

Westphal, M. P-152

Wetzels, Roland P-715

White, Katherine O-49

Whitmore, Lara P-541

Wieczorowska-Tobis, Katarzyna O-122, P-510, P-815

Wienke, Andreas P-394

Wifek, Bani P-46

Wijnhoven, H. A. H. O-57

Wijnhoven, Hanneke A. H. O-3, P-434

Wilkinson, Chris P-246

Wilkinson, Emma O-65

Willems, Dick L. P-876, P-877

Willems, H. C. P-404

William Molloy, David P-619

Williams, Edward P-565

Williams, J. P-540

Williams, Joanna P-293

Willkomm, M. O-33, P-818

Wiloth, S. P-645

Wiloth, Stefanie P-637

Wilson, Tom P-266

Wimmer, Barbara P-3

Wimo, A. O-21

Wimo, Anders O-88, P-201

Winblad, Bengt P-657

Windecker, Stephan P-831

Winkowski, Filip P-727

Winterborn, Rebecca P-77
Wirth, R. P-135

Wizner, Barbara P-204, P-214

Wizner, Barbara P-825

Wojkowska-Mach, Jadwiga P-655

Wojzischke, Julia P-318, P-321, P-322, P-323

Wolf, Ursula P-392, P-394, P-403

Won, Chang Won P-193

Woo, Seon Hee P-893

Wordliczek, Jerzy P-705, P-772

Wouters, Hans O-61, O-114, P-34, P-43

Woźniak, Marta P-727

Wretman, Christopher O-46

Wright, David P-613, P-873

Wyller, Torgeir Bruun P-82

Xavier, Perrot O-83

$\mathrm{Xu}$, Weili P-615

$\mathrm{Xu}$, Xue P-1

Ya, Su O-34

Yağc1, Nesrin P-305

Yagci, Nesrin P-771, P-774, P-792

Yakaryılmaz, Funda Datli P-750

Yakhno, N. N. P-634, P-635

Yalçin, Ahmet P-168

Yalman, Ali P-306

Yalvaç, Yusuf P-728, P-729, P-734

Yamada, Yoko P-412

Yamamoto, Akiko P-12

Yamamoto, Koichi P-206

Yamamoto, Kouichi P-675

Yamashita, Taro P-366

Yang, Lin P-166

Yared, Pierre P-125

Yaşa, Cenk P-147

Yasmin Latief, YL P-69

Yavuz, Bunyamin O-41

Yavuz, Bünyamin P-663

Yavuz, Burcu Balam O-41, P-216, P-217, P-390, P-663, P-720, P-894

Yavuzer, Hakan P-447, P-505, P-623, P-719

Yazid, Belkacemi P-829

Yeo, A. P-134

Yeong, K. P-328

Yeong, Keefai O-65, P-73

Yeung, Suey P-162

Yew, S. P-134

Yi, J. M. P-76

Yılmaz, Ayşenur P-306

Y1lmaz, Merve P-231, P-443

Yilmaz, Ozlem P-157, P-158, P-468, P-581, P-827

Yllmaz, Sevda P-305

Yilmaz, Tamer P-473

Yin Tan, Rui P-466

Yiu, B. O-14

Yong, R. P-91

Yoon, Seokjun P-415

Yoshida, Akihiro P-382

Youn, Kanwoo P-374

Youness, Mohamed P-360

Yuki, Michiko O-34, P-616

Yuruyen, Mehmet P-623

Yves, Passadori P-509

Yves, Rolland P-36, P-39, P-883

Zafra, Gabriel Ariza P-494

Zaki, Peter P-477 
Zalar, Barbara Jemec P-726

Zambom Ferraresi, Fabricio P-199

Zambon, Antonella P-855

Zamboni, Mauro O-116

Zanclar, Hazal P-232

Zárate-Sáez, C.M. P-95

Zarrouk, Amira P-146

Zasadzka, Ewa P-153, P-788

Zebachi, Sonia O-107

Zekry, D. P-752

Zelano, Giovanni P-684

Zeldez, Vladimir P-867

Zerah, Lorène P-85, P-857

Zerbib, Lucas P-536

Zerhouni, Nabil O-74

Zethelius, Björn O-26

Zhang, Jiali P-656

Zheludkov, Svetlana P-687
Ziere, Gijsbertus O-91

Zigoura, Ekaterini O-70

Zijl, W. H. P-817

Zimmerman, Sheryl O-46

Zirabe, Valentine P-671

Zoghlami, N P-751

Zoukar, Olfa P-698, P-700

Zrour, Saoussen P-211, P-741, P-760

Zubačiūtè, Indrè P-427

Zubavičiūtè, Indrè P-689

Zucchelli, A. P-177

Zuidema, Sytse P-34

Zupiria, Xabier P-207

Zurlo, A. P-98, P-335

Zurlo, Amedeo O-10

Zwahlen, Marcel P-831

Zyryanov, Sergey P-47 